\title{
Selectivity Control in 3d Transition Metal-Catalyzed C-H Activation
}

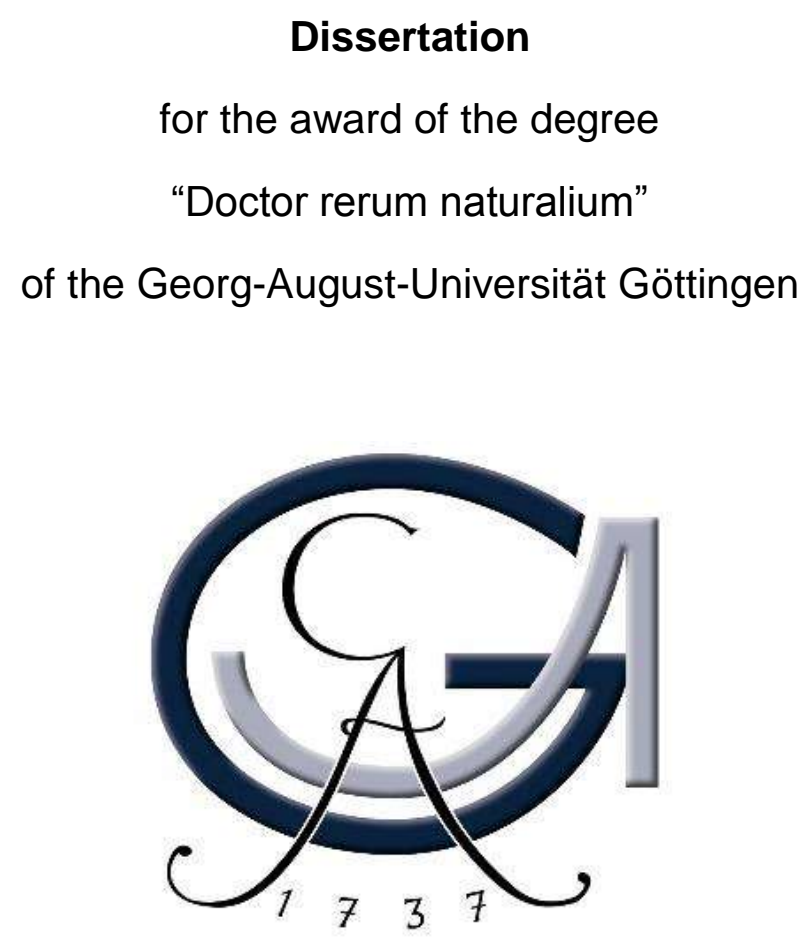

within the doctoral program of chemistry of the Georg-August-University School of Science (GAUSS)

submitted by

Joachim Loup

from Vully-les-Lacs (Montmagny) VD, Switzerland 



\section{Thesis Committee}

Prof. Dr. Lutz Ackermann, Institute of Organic and Biomolecular Chemistry, Göttingen

Prof. Dr. Alexander Breder, Institut für Organische Chemie, Regensburg/Institute of Organic and Biomolecular Chemistry, Göttingen

\section{Members of the Examination Board}

Reviewer: Prof. Dr. Lutz Ackermann, Institute of Organic and Biomolecular Chemistry, Göttingen

Second Reviewer: Prof. Dr. Alexander Breder, Institut für Organische Chemie, Regensburg/Institute of Organic and Biomolecular Chemistry, Göttingen

\section{Further Members of the Examination Board}

Prof. Dr. Manuel Alcarazo, Institute of Organic and Biomolecular Chemistry, Göttingen

Dr. Shoubhik Das, Institute of Organic and Biomolecular Chemistry, Göttingen

Prof. Dr. Dietmar Stalke, Institute of Inorganic Chemistry, Göttingen

Prof. Dr. Dr. h.c.mult. Lutz F. Tietze, Institute of Organic and Biomolecular Chemistry, Göttingen

Date of the Oral Examination: 16.08.2019 



\section{Table of Contents}

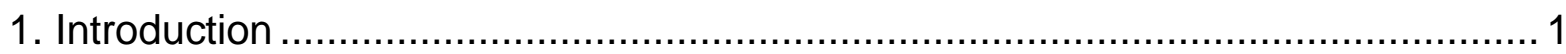

1.1. Transition Metal-Catalyzed C-H Activation ............................................. 1

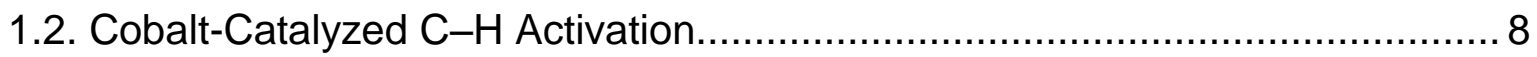

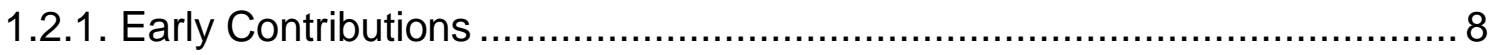

1.2.2. Cobalt(III)-Catalyzed C-H Activation ...................................................... 10

1.2.3. Enantioselective Cobalt-Catalyzed C-H Functionalizations under

Reductive Conditions .......................................................................... 19

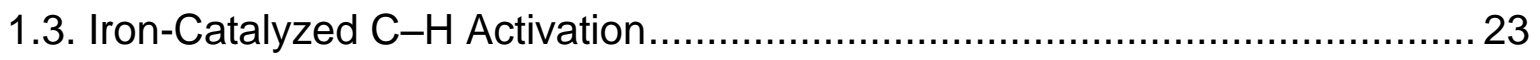

1.4. Nickel-Catalyzed C-H Activation ......................................................... 32

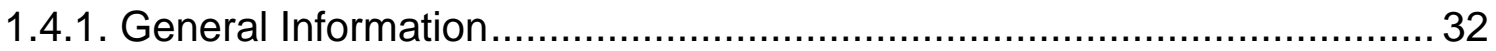

1.4.2. Nickel-Catalyzed C-H Activation by Alkene Hydroarylation .................... 35

1.4.3. Enantioselective Nickel-Catalyzed C-H Activation..................................39

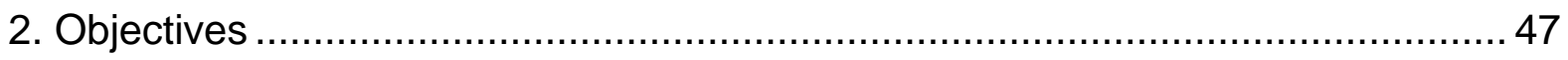

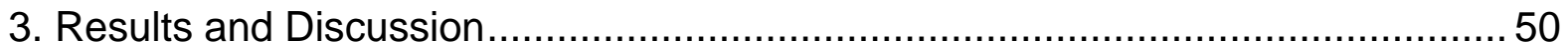

3.1. Cobalt(III)-Catalyzed C-H Amidation by Oxazoline Assistance .....................5 50

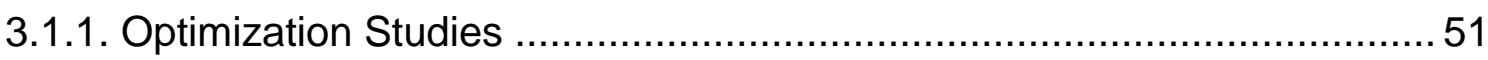

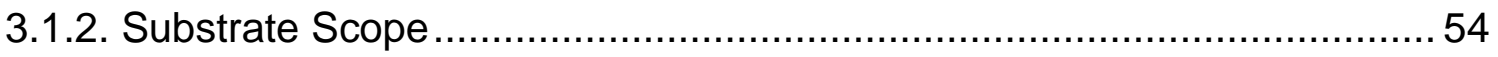

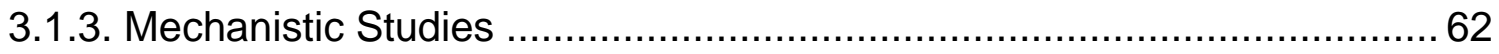

3.2. Asymmetric Iron-Catalyzed Hydroarylations by $\mathrm{C}-\mathrm{H}$ Activation .................... 66

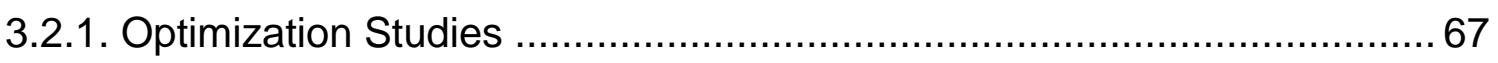

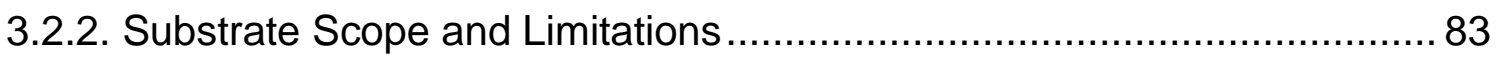

3.2.3. Determination of the Absolute Configuration ......................................... 94

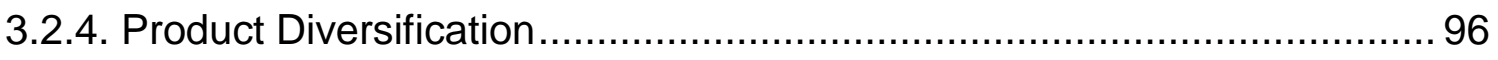




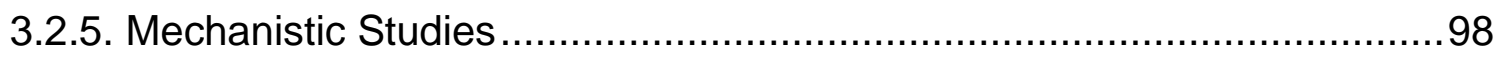

3.2.6. Proposed Mechanism ............................................................... 117

3.2.7. Iron-Catalyzed Alkyne Hydroarylations ….........................................119

3.3. Asymmetric Nickel-Catalyzed Hydroarylations by C-H Activation ...............122

3.3.1. Preliminary Studies towards Asymmetric Intermolecular

Hydroarylations by C-H Activation ..........................................................123

3.3.2. Optimization Studies of Enantioselective Intramolecular Nickel-

Catalyzed Hydroarylations via C-H Activation............................................127

3.3.3. Substrate Scope and Limitations ...................................................134

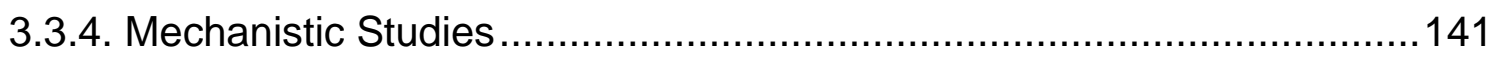

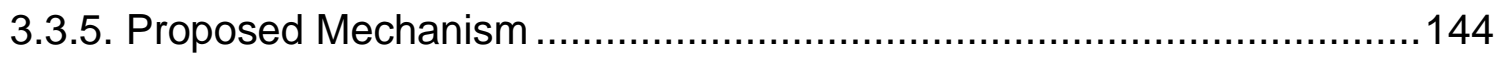

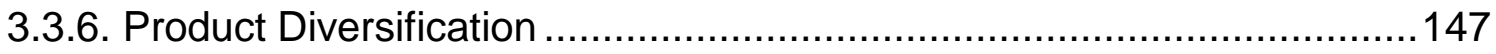

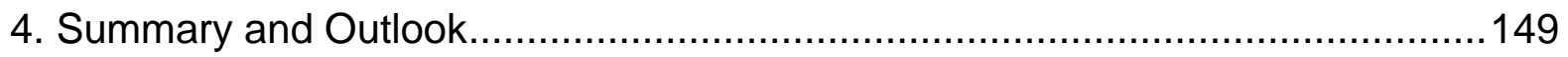

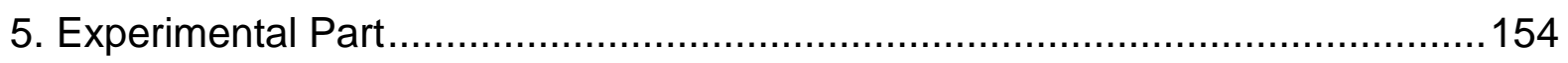

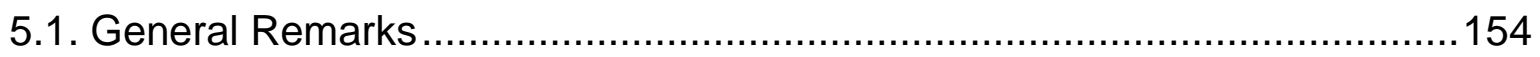

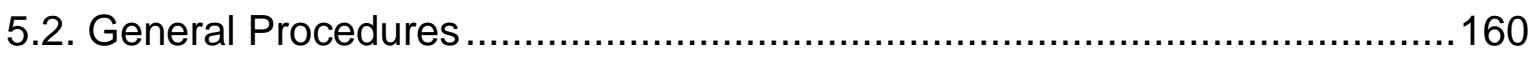

5.3. Cobalt(III)-Catalyzed Directed C-H Amidation...........................................167

5.3.1. Experimental Procedures and Analytical Data.....................................167

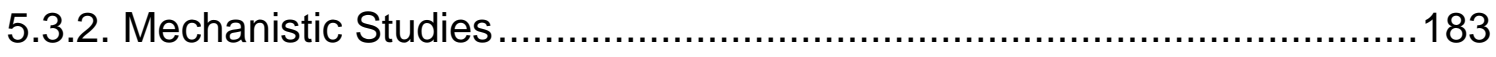

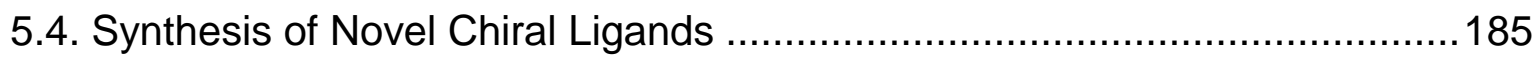

5.4.1. Experimental Procedures and Analytical Data of Novel Chiral NHC

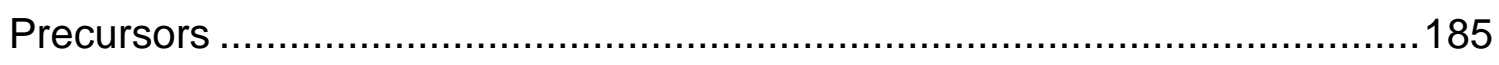

5.4.2. Experimental Procedures and Analytical Data of Novel Chiral

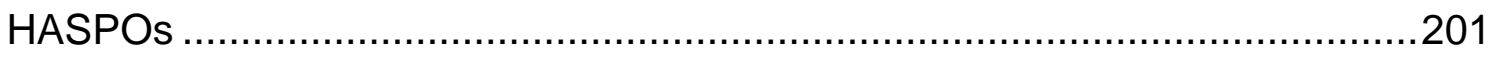

5.5. Iron-Catalyzed Enantioselective C-H Alkylation with Alkenes ....................204 
5.5.1. Experimental Procedures and Analytical Data .................................... 204

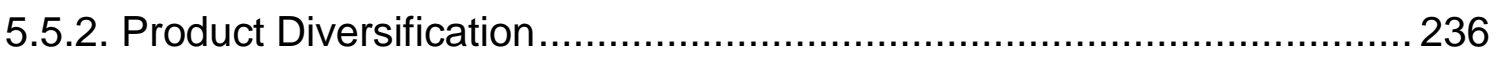

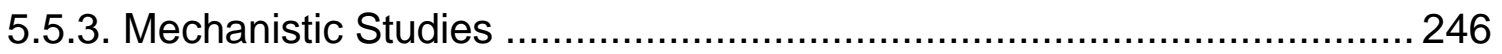

5.6. Asymmetric Nickel-Catalyzed Hydroarylations by C-H Activation ............... 262

5.6.1. Experimental Procedures and Analytical Data for the Intermolecular Nickel-Catalyzed Hydroarylation of Alkenes................................................. 262

5.6.2. Mechanistic Studies for the Intermolecular Nickel-Catalyzed

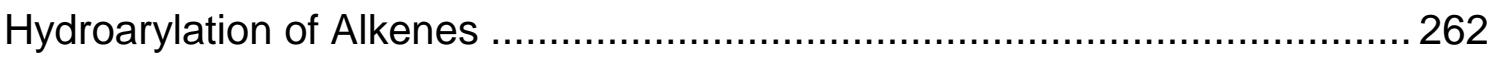

5.6.3 Experimental Procedures and Analytical Data for the Asymmetric Intramolecular Nickel-Catalyzed Hydroarylation of Alkenes ........................... 264

5.6.4. Mechanistic Studies for the Asymmetric Intramolecular Nickel-

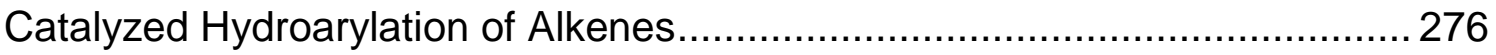

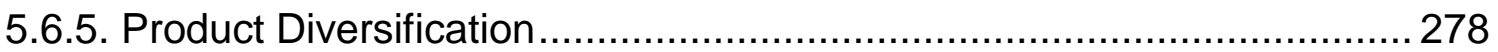

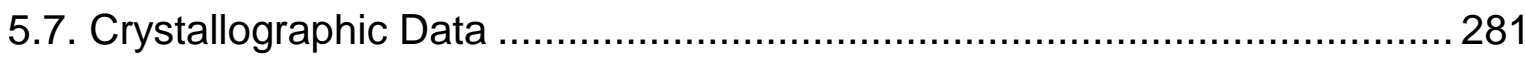

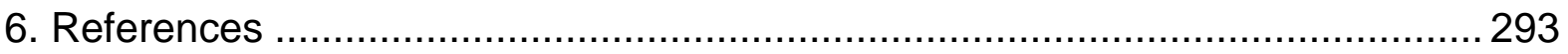

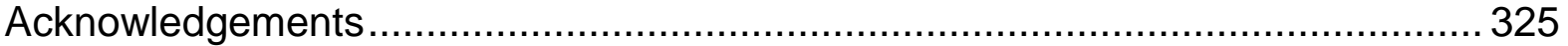

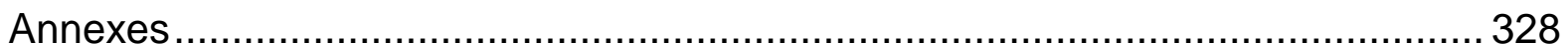




\section{List of Abbreviations}

$\AA$

Ac

acac

$[\alpha]_{\mathrm{D}}$

Ad

Alk

Am

AMLA

app

aq.

$\mathrm{Ar}$

atm

ATR

$b$

BDE

BDMAEE

BDPP

$\mathrm{BHT}$

BIES

BINAP

BINOL

$\mathrm{Bn}$

Boc

BOX ångström

acetyl

acetyl acetonate

specific rotation at $589 \mathrm{~nm}$

adamantyl

alkyl

amyl

ambiphilic metal ligand activation

apparent

aqueous

aryl

atmospheric pressure

attenuated total reflection

branched

bond dissociation energy (at $298 \mathrm{~K}$ )

bis-(2-dimethylaminoethyl)ether

bis-(diphenylphosphino)pentane

2,6-di-tert-butyl-4-methylphenol

base-assisted internal electrophilic substitution

2,2'-bis(diphenylphosphino)-1,1'-binaphthyl

[1,1'-binaphthalene]-2,2'-diol

benzyl

tert-butyloxycarbonyl

bis(oxazoline) 


\begin{tabular}{|c|c|}
\hline bpy & 2,2'-bipyridine \\
\hline $\mathrm{Bu}$ & butyl \\
\hline br & broad \\
\hline $\mathrm{Bz}$ & benzoyl \\
\hline $\mathrm{C}$ & Celsius \\
\hline c & concentration (in g / $100 \mathrm{~mL}$ ) \\
\hline CAAC & cyclic alkyl amino carbene \\
\hline calc. & calculated \\
\hline cat. & catalytic \\
\hline CMD & concerted metalation deprotonation \\
\hline $\operatorname{cod}$ & 1,5-cyclooctadiene \\
\hline $\mathrm{Cp}$ & cyclopentadienyl \\
\hline $\mathrm{Cp}^{*}$ & pentamethylcyclopentadienyl \\
\hline CPME & cyclopentyl methyl ether \\
\hline $\mathrm{Cp}^{t}$ & 1,3-di-tert-butylcyclopentadienyl \\
\hline $\mathrm{C}_{\mathrm{q}}$ & quaternary carbon \\
\hline Cy & cyclohexyl \\
\hline Сур & cyclopentyl \\
\hline$\delta$ & $\begin{array}{l}\text { chemical shift (NMR) or isomer shift (Mössbauer } \\
\text { spectroscopy) }\end{array}$ \\
\hline$d$ & doublet \\
\hline $\mathrm{DACH}$ & 1,2-diaminocyclohexane \\
\hline $\mathrm{dba}$ & dibenzylideneacetone \\
\hline 2,3-DCB & 2,3-dichlorobutane \\
\hline DCE & 1,2-dichloroethane \\
\hline $\mathrm{DCIB}$ & 1,2-dichloroisobutane \\
\hline
\end{tabular}


$\Delta E_{\mathrm{Q}}$

DFT

DG

Dipp

DMF

DMPU

DMSO

DoM

DPEN

dppb

dppbz

dppe

dppf

dppm

dppp

d.r.

ee

EI

equiv

e.r.

ESI

Et

EWG

Fc

FGI quadrupole splitting

density functional theory

directing group

2,6-diisopropylphenyl

$\mathrm{N}, \mathrm{N}$-dimethylformamide

1,3-dimethyl-1,3-diazinan-2-one

dimethyl sulfoxide

directed ortho-metalation

1,2-diphenylethane-1,2-diamine

1,3-bis(diphenylphosphino)butane

1,2-bis(diphenylphosphino)benzene

1,3-bis(diphenylphosphino)ethane

1,1'-bis(diphenylphosphino)ferrocene

1,3-bis(diphenylphosphino)methane

1,3-bis(diphenylphosphino)propane

diastereomeric ratio

enantiomeric excess

electron ionization

equivalents

enantiomeric ratio

electrospray ionization

ethyl

electron-withdrawing group

ferrocenyl

functional group interconversion 


\begin{tabular}{|c|c|}
\hline g & gram \\
\hline GC & gas chromatography \\
\hline gem & geminal \\
\hline $\mathrm{h}$ & hour or hexet \\
\hline HASPO & heteroatom-substituted secondary phosphine oxide \\
\hline Hept & heptyl \\
\hline hept & heptet \\
\hline Het & heteroaryl or heteroatom \\
\hline HFIP & 1,1,1,3,3,3-hexafluoro-2-propanol \\
\hline HPLC & high-performance liquid chromatography \\
\hline HR-MS & high resolution mass spectrometry \\
\hline $\mathrm{Hz}$ & hertz \\
\hline$i$ & iso \\
\hline $\mathrm{ICy} \cdot \mathrm{HCl}$ & 1,3-dicyclohexyl-1H-imidazol-3-ium chloride \\
\hline Ile & isoleucine \\
\hline IMes. $\mathrm{HCl}$ & 1,3-dimesityl-1H-imidazol-3-ium chloride \\
\hline $\mathrm{IPr} \cdot \mathrm{HCl}$ & $\begin{array}{l}\text { 1,3-bis(2,6-diisopropylphenyl)-1H-imidazol-3-ium } \\
\text { chloride }\end{array}$ \\
\hline$|\mathrm{Xy}| \cdot \mathrm{HCl}$ & $\begin{array}{l}\text { 1,3-bis(2,6-dimethylphenyl)-1H-imidazol-3-ium } \\
\text { chloride }\end{array}$ \\
\hline IR & infrared \\
\hline$J$ & coupling constant \\
\hline K & Kelvin \\
\hline k & reaction rate constant \\
\hline kcal & kilocalorie \\
\hline $\mathrm{KIE}$ & kinetic isotope effect \\
\hline
\end{tabular}




\begin{tabular}{|c|c|}
\hline $\mathrm{L}$ & liter or (pre-)ligand \\
\hline I & linear \\
\hline LED & light-emitting diode \\
\hline LiHMDS & lithium bis(trimethylsilyl)amide \\
\hline LLHT & ligand-to-ligand hydrogen transfer \\
\hline M & metal or molar \\
\hline$m$ & meta \\
\hline $\mathrm{m}$ & multiplet \\
\hline M. p. & melting point \\
\hline$m / z$ & mass-to-charge ratio \\
\hline MAD & $\begin{array}{l}\text { methylaluminium bis(2,6-di-tert-butyl-4- } \\
\text { methylphenoxide) }\end{array}$ \\
\hline Mc & metallocenyl \\
\hline $\mathrm{Me}$ & methyl \\
\hline Mes & mesityl \\
\hline $\mathrm{mg}$ & milligram \\
\hline $\mathrm{MHz}$ & megahertz \\
\hline $\min$ & minutes \\
\hline $\mathrm{mm}$ & millimeter \\
\hline $\mathrm{mmol}$ & millimole \\
\hline MOM & methoxymethyl \\
\hline MPAA & mono- $N$-protected amino acid \\
\hline MS & mass spectrometry or molecular sieves \\
\hline Ms & methanesulfonyl (mesyl) \\
\hline $\mathrm{Np}$ & naphthyl \\
\hline NBS & $N$-bromosuccinimide \\
\hline
\end{tabular}




\begin{tabular}{|c|c|}
\hline $\mathrm{NHC}$ & N-heterocyclic carbene \\
\hline $\mathrm{NHO}$ & N-heterocyclic olefin \\
\hline NLE & non-linear effect \\
\hline NMP & $N$-methylpyrrolidinone \\
\hline NMR & nuclear magnetic resonance \\
\hline n.r. & no reaction \\
\hline 0 & ortho \\
\hline$\varnothing$ & diameter \\
\hline ODCB & 1,2-dichlorobenzene \\
\hline $\mathrm{p}$ & pentet \\
\hline$p$ & para \\
\hline Pent & pentyl \\
\hline $\mathrm{Ph}$ & phenyl \\
\hline Ph-BPE & 1,2-bis-(2,5-diphenylphospholano)ethane \\
\hline phen & 1,10-phenanthroline \\
\hline Piv & pivaloyl (trimethylacetyl) \\
\hline PMB & para-methoxybenzyl \\
\hline PMDETA & $N, N, N^{\prime}, N^{\prime \prime}, N "-$ pentamethyldiethylenetriamine \\
\hline PMP & para-methoxyphenyl \\
\hline ppm & parts-per-million \\
\hline $\operatorname{Pr}$ & propyl \\
\hline py & pyridyl \\
\hline РувOX & 2,6-bis(oxazolinyl)pyridine \\
\hline pym & pyrimidyl \\
\hline Q & 8-quinolyl \\
\hline
\end{tabular}




\begin{tabular}{|c|c|}
\hline$q$ & quartet \\
\hline $\mathrm{R}$ & (organic) rest \\
\hline $\mathrm{R}_{\mathrm{L}}$ & large substituent \\
\hline rac & racemic \\
\hline $\mathrm{Rc}$ & ruthenocenyl \\
\hline$r D G$ & removable directing group \\
\hline rel. int. & relative intensity \\
\hline rt & room temperature \\
\hline s & singlet or second \\
\hline sat. & saturated \\
\hline SET & single electron transfer \\
\hline Si & $\mathrm{R}_{3} \mathrm{Si}$ \\
\hline slMes. $\mathrm{HCl}$ & 1,3-dimesitylimidazolidinium chloride \\
\hline $\mathrm{slXyl} \cdot \mathrm{HCl}$ & 1,3-bis(2,6-dimethylphenyl)-imidazolinium chloride \\
\hline SN & nucleophilic substitution \\
\hline SPO & secondary phosphine oxide \\
\hline SPS & solvent purification system \\
\hline$T$ & temperature \\
\hline $\mathrm{t}$ & triplet or time \\
\hline$t$ & tert \\
\hline TADDOL & $\begin{array}{l}\alpha, \alpha, \alpha^{\prime}, \alpha^{\prime} \text {-tetraaryl-2,2-disubstituted 1,3-dioxolane- } \\
\text { 4,5-dimethanol }\end{array}$ \\
\hline TAM & triazolyldimethylmethyl \\
\hline TBS & tert-butyldimethylsilyl \\
\hline TEMPO & 2,2,6,6-tetramethylpiperidine- $N$-oxide \\
\hline Tf & trifluoromethanesulfonyl (triflyl) \\
\hline
\end{tabular}




$\begin{array}{ll}\text { TFA } & \text { trifluoroacetic acid } \\ \text { TFE } & \text { 2,2,2-trifluoroethanol } \\ \text { THF } & \text { tetrahydrofuran } \\ \text { TLC } & \text { thin layer chromatography } \\ \text { TM } & \text { transition metal } \\ \text { TMEDA } & N, N, N^{\prime}, N^{\prime} \text {-tetramethylethane-1,2-diamine } \\ \text { TMS } & \text { trimethylsilyl } \\ \text { Ts } & \text { para-toluenesulfonyl (tosyl) } \\ \text { TPP } & \text { tetraphenylporphyrin dianion } \\ t_{r} & \text { retention time } \\ \text { UV } & \text { ultraviolet } \\ X & \text { (pseudo)halide } \\ \text { Xantphos } & 4,5 \text {-bis(diphenylphosphino)-9,9-dimethylxanthene }\end{array}$





\section{Introduction}

The tremendous development of organic synthesis within the last century has allowed for the preparation of life-saving pharmaceuticals, crop-protection agents, functional materials such as polymers, and dyes, among others, affecting the life of billions of people. Despite its transformative nature, which has resulted in diverse applications with countless benefits for the society, chemistry continues to be perceived as a polluting science due to resource and energy consumption, waste generation, and the use of toxic chemicals.

In order to obviate or at least reduce these drawbacks, the development of more environment-friendly, resource- ${ }^{[1]}$ step- and atom-economical ${ }^{[2]}$ synthetic methodologies is highly desirable. In this perspective, Anastas and Warner proposed the "12 Principles of Green Chemistry", ${ }^{[3]}$ which are meant to guide the synthetic chemist towards environmentally-benign chemical processes. Among those, catalysis, that is the use of catalytic rather than stoichiometric amounts of reagents, and the direct use of readily available chemicals without the need of pre-functionalization are particularly attractive approaches to reduce the formation of byproducts and thereby chemical waste. Furthermore, the use of less-toxic compounds and mild reaction conditions is also expected to enable safer chemical processes.

\subsection{Transition Metal-Catalyzed C-H Activation}

"A dream of organic chemists has been the discovery of coupling reactions with no prefunctionalization of the coupling partners." (V. Snieckus) ${ }^{[4]}$

Organic synthesis, including catalytic reactions, has long been dominated by the transformation of functional groups, hence requiring pre-functionalized starting materials. In this context, a major achievement of catalysis in the past five decades 
has been the development of transition metal-catalyzed cross-couplings, forming carbon-carbon (C-C) and carbon-heteroatom (C-Het) bonds. ${ }^{[4]}$ Interestingly, pioneering results were obtained as early as in the late $19^{\text {th }}$ century by, inter alia, Glaser $^{[5]}$ and Ullmann ${ }^{[6]}$ using stoichiometric or catalytic amounts of copper. Nevertheless, transition metal-mediated coupling reactions have only found broad applications since the development of palladium-catalyzed cross-couplings between organometallic reagents with organic electrophiles. ${ }^{[4]}$

Major successes in this field have been realized for the formation of $\mathrm{C}-\mathrm{C}$ bonds using diverse coupling partners, resulting in the development of numerous name reactions, such as the Suzuki-Miyaura, ${ }^{[7]}$ Negishi ${ }^{\left[{ }^{[8]}\right.}$ Mizoroki-Heck, ${ }^{\left[{ }^{[0]}\right.}$ KumadaCorriu, ${ }^{[10]} H_{i y a m a}{ }^{[11]}$ Stille ${ }^{[12]}$ and Sonogashira-Hagihara ${ }^{[13]}$ cross-coupling reactions. Additionally, while not always $\mathrm{C}-\mathrm{C}$ bond forming processes, the TsujiTrost reaction ${ }^{[14]}$ as well as the Buchwald-Hartwig amination ${ }^{[15]}$ should be mentioned as other significant milestones in palladium coupling catalysis. Palladium-catalyzed cross-couplings are nowadays a routine tool in organic synthesis, with applications ranging from material sciences to the late-stage diversification of biologically active compounds, ${ }^{[16]}$ and their importance was recognized by the Nobel Prize in Chemistry awarded collectively to Heck, Negishi and Suzuki in 2010..$^{[4,17]}$

However, those processes still suffer from various drawbacks which significantly affect their ecological footprint. Indeed, the need for rare noble transition metal catalysts, pre-functionalized substrates and sensitive organometallic reagents, as well as the generation of stoichiometric amounts of harmful waste, render those processes hazardous and harmful to the environment.

Significant achievements have been made to address those limitations, which include the use of sustainable non-noble metal catalysts such as nicke ${ }^{[18]}$ and iron, ${ }^{[19]}$ the use of biomass-derived solvents, ${ }^{[20]}$ and the development of reusable ${ }^{[21]}$ or highly active catalysts operating at low loadings. ${ }^{[22]}$ However, those approaches do not tackle the main issues of cross-coupling chemistry, namely the need for pre-functionalized starting materials and the generation of stoichiometric waste byproducts. 
Therefore, the direct functionalization of omnipresent $\mathrm{C}-\mathrm{H}$ bonds would appear as a highly desirable alternative to conventional cross-couplings due to the improved step- and atom-economy (Scheme 1.1). In this context, catalytic $\mathrm{C}-\mathrm{H}$ activation has experienced a tremendous development in recent years, ${ }^{[23]}$ and has now surfaced as a transformative tool for molecular syntheses, with notable applications in pharmaceutical industries, ${ }^{[24]}$ as well as the synthesis of complex bioactive natural products $^{[25]}$ and material sciences, ${ }^{[26]}$ among others. Nevertheless, the direct functionalization of $\mathrm{C}-\mathrm{H}$ bonds with organic electrophiles still requires the prefunctionalization of one of the coupling partners, generating a stoichiometric amount of (pseudo)halogenated byproducts (Scheme 1.1b). In contrast, hydroarylations ${ }^{[27]}$ would be perfectly atom-economical, redox-neutral, and more step-economical as well since no pre-functionalization is required. Crossdehydrogenative $\mathrm{C}-\mathrm{H}$ activation would also, in theory, be a fully atom-economical approach, as only molecular hydrogen is formally generated as a byproduct (Scheme 1.1c). However, those reactions usually require a stoichiometric oxidant, which results in stoichiometric waste generation, and typically suffer from a rather narrow substrate scope.

a) 'traditional' cross-coupling
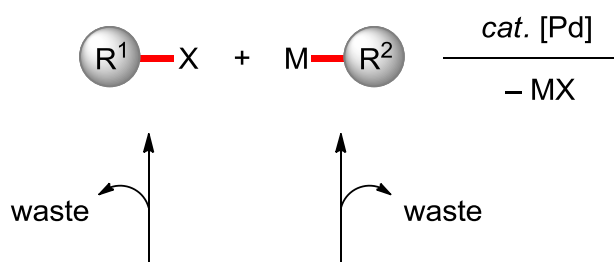

b) $\mathrm{C}-\mathrm{H}$ bond activation

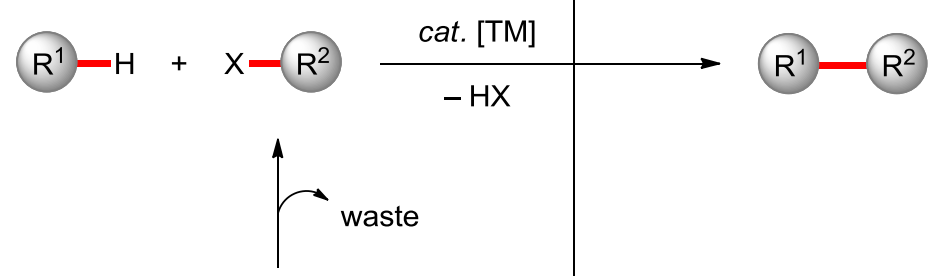

c) cross-dehydrogenative C-H functionalization

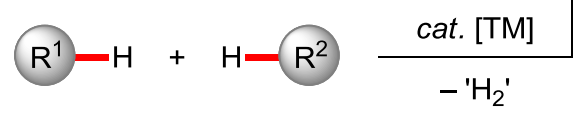

Scheme 1.1. Comparison of traditional cross-coupling vs. C-H activation. 
Nevertheless, several challenges which need to be overcome are associated with synthetically useful $\mathrm{C}-\mathrm{H}$ activation. First, the $\mathrm{C}-\mathrm{H}$ bond is typically significantly more stable than the $\mathrm{C}-\mathrm{X}$ bond of common cross-coupling partners (e.g. $\quad \mathrm{BDE}(\mathrm{Ph}-\mathrm{H}) \approx 113 \mathrm{kcal} \mathrm{mol}^{-1}$ vs. $\quad \mathrm{BDE}(\mathrm{Ph}-\mathrm{Cl}) \approx 97 \mathrm{kcal} \mathrm{mol}^{-1}$, $\left.\mathrm{BDE}(\mathrm{Ph}-\mathrm{Br}) \approx 84 \mathrm{kcal} \mathrm{mol}^{-1}, \mathrm{BDE}(\mathrm{Ph}-\mathrm{l}) \approx 67 \mathrm{kcal} \mathrm{mol}^{-1}\right) \cdot{ }^{[28]}$ While early examples of $\mathrm{C}-\mathrm{H}$ activations required harsh reaction conditions which strongly limited their applications to the synthesis of complex and sensitive molecules, recent progress has focused on the development of milder ${ }^{[29]}$ and more selective processes. The mechanism of the key $\mathrm{C}-\mathrm{H}$ cleavage step has been studied extensively as its understanding is particularly important for the design of efficient catalytic processes. Excluding outer-sphere mechanisms (e.g. carbene/nitrene insertions ${ }^{[30]}$ or radical reactions $\left.{ }^{[31]}\right)$, five general modes of action have been proposed for the $\mathrm{C}-\mathrm{H}$ metalation step depending on the nature of the substrate, the metal catalyst, its ligands and oxidation state (Scheme 1.2). ${ }^{[32]}$ These pathways consist of oxidative addition, electrophilic substitution, $\sigma$-bond metathesis, 1,2-addition and baseassisted metalation. The oxidative addition pathway is typical for electron-rich, lowvalent complexes of late transition metals, such as rhenium, ruthenium, osmium, iridium, platinum and even iron, ${ }^{[32 b]}$ from which higher oxidation states are readily accessible (Scheme 1.2a). While this mechanism has also been proposed for early transition metals, later findings provided support for $\sigma$-bond metathesis, typically involving an alkyl- or hydride-metal complex (vide infra). Late transition metals in high oxidation states, such as $\mathrm{Pd}(\mathrm{II}), \mathrm{Pt}(\mathrm{II}), \mathrm{Pt}(\mathrm{IV})$, or $\mathrm{Hg}(\mathrm{II})$, tend to undergo $\mathrm{C}-\mathrm{H}$ activation by an electrophilic substitution in which the metal acts as a Lewis acid. In those processes, the putative intermediate is formed by electrophilic attack of the metal, usually in a strongly polar medium (Scheme 1.2b). For early transition metals, as well as lanthanides and actinides, $\sigma$-bond metathesis tend to be the preferred pathway. A key feature this mechanism is the concerted formation and breaking of $\mathrm{C}-\mathrm{H}$ and $\mathrm{C}-\mathrm{M}$ bonds in the transition state (Scheme $1.2 \mathrm{C}$ ). ${ }^{[32 \mathrm{~b}]}$ The 1,2-addition route is observed for metals with an unsaturated $M=Y$ bond, typically imido, oxo and alkylidene complexes. Those transformations occur via a $\left[2_{\sigma}+2_{\pi}\right]$-type reaction where the $\mathrm{Y}$ group serves as the formal hydrogen acceptor (Scheme 1.2d). Finally, 
another category of $\mathrm{C}-\mathrm{H}$ cleavage processes is the base-assisted $\mathrm{C}-\mathrm{H}$ activation. Here, the base, most commonly a carboxylate, ${ }^{[32 a]}$ facilitates the proton abstraction during the $\mathrm{C}-\mathrm{H}$ scission step.

a) oxidative addition

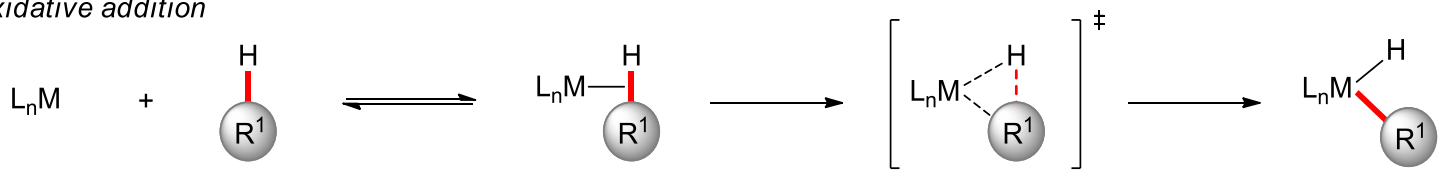

b) electrophilic substitution

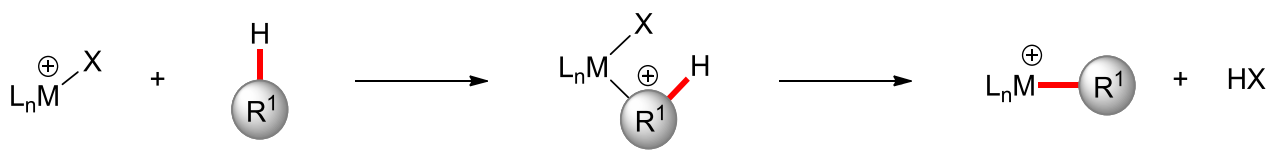

c) $\sigma$-bond metathesis

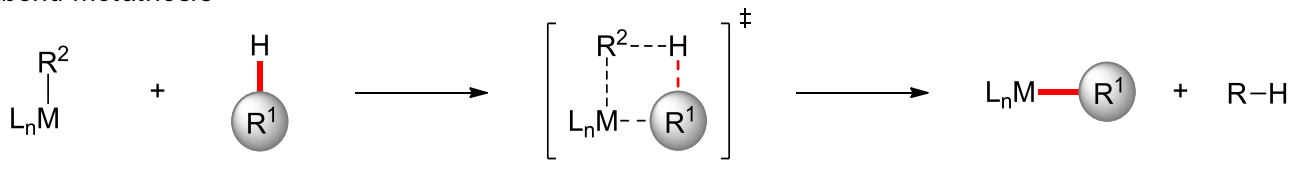

d) 1,2-addition

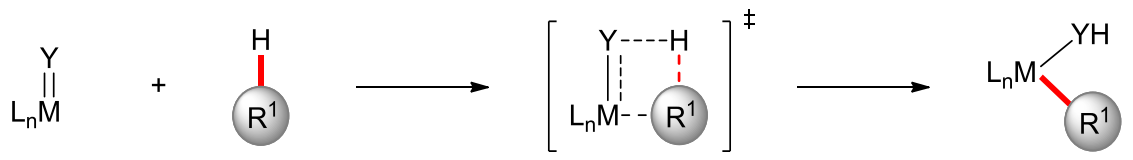

e) base-assisted metalation

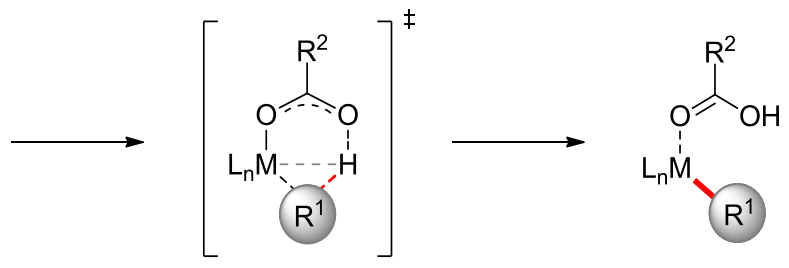

Scheme 1.2. Different pathways for organometallic $\mathrm{C}-\mathrm{H}$ activation.

Further investigations on base-assisted $\mathrm{C}-\mathrm{H}$ activations unravelled several different possible pathways (Scheme 1.3). Following the pioneering theoretical studies of Sakaki, ${ }^{[33]}$ the synergistic interaction between the metal center, carboxylate-ligand and $\mathrm{C}-\mathrm{H}$ bond was rationalized by Gorelsky and the late Fagnou, leading to the 
concept of concerted metalation-deprotonation (CMD) occurring via a six-membered transition state. ${ }^{[34]}$ Subsequent computational studies by Macgregor suggested the relevance of an agostic metal-hydrogen interaction in a mechanism named ambiphilic metal-ligand activation (AMLA). ${ }^{[32 c, 35]}$ Those processes are typically characterized by a considerable kinetic isotope effect (KIE) and a preference for electron-deficient substrates. In contrast, the term internal electrophilic substitution $(\mathrm{IES})^{[36]}$ describes a mechanism occurring through a highly strained four-membered ring transition state. This process has been proposed for reactions involving alkoxide bases. Recently, the concept of base-assisted internal electrophilic substitution $(B I E S)^{[37]}$ has emerged in order to explain the preference for electron-rich substrates in several catalytic transformations.

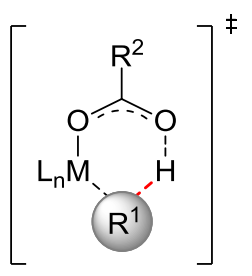

CMD

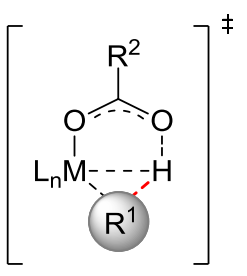

AMLA

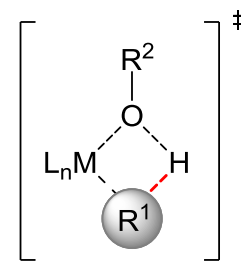

IES

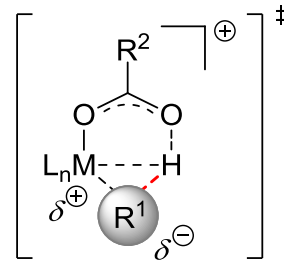

BIES

Scheme 1.3. Proposed transition states for base-assisted $\mathrm{C}-\mathrm{H}$ metalations.

Another challenge of $\mathrm{C}-\mathrm{H}$ activation chemistry is the fact that $\mathrm{C}-\mathrm{H}$ bonds are omnipresent in organic compounds and have almost identical bond dissociation energies. The control of selectivity in those transformations is therefore a task of key importance. Various approaches have been developed to tackle this issue, namely the use of substrate's electronic bias, steric control, or a Lewis-basic group that coordinates to the transition metal catalyst and directs the $\mathrm{C}-\mathrm{H}$ activation at the desired position (Scheme 1.4). Since electronic and steric biases depend on the substrate itself, those approaches usually result in a rather narrow substrate scope. In contrast, the introduction of a directing group ${ }^{[38]}$ (DG) allows for a broad variety of substrates to be selectively functionalized. Nevertheless, a major limitation of this approach is the need to incorporate the directing group in the substrate. However, 
the use of weakly coordinating, ${ }^{[39]}$ removable ${ }^{[40]}$ or transient ${ }^{[41]}$ directing groups has considerably expanded the possibilities of this approach.
a) electronic bias
b) steric bias
c) directing group

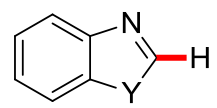

$Y=N R, O, S$
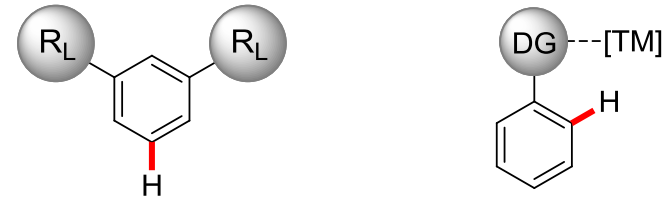

Scheme 1.4. Methods to achieve positional selectivity in $\mathrm{C}-\mathrm{H}$ activation.

Major progress in the field of $\mathrm{C}-\mathrm{H}$ activation has been achieved with late transition metal catalysts. However, due to their high cost, ${ }^{[42]}$ low abundance ${ }^{[43]}$ and high toxicity, ${ }^{[44]}$ this approach is rather not sustainable. Therefore, the development of catalytic methods for the functionalization of otherwise inert $\mathrm{C}-\mathrm{H}$ bonds employing non-noble $3 \mathrm{~d}$ metals has attracted considerable interest in the last decade. ${ }^{[45]}$ Inter alia, the development of cobalt-,${ }^{[46]}$ iron- ${ }^{[47]}$ nickel- ${ }^{[48]}$ manganese $-{ }^{[49]}$ and coppercatalyzed ${ }^{[50]} \mathrm{C}-\mathrm{H}$ activations has been particularly successful.

Despite those major advances, full selectivity control in enantioselective $\mathrm{C}-\mathrm{H}$ functionalizations continues to heavily rely on precious $4 d$ and $5 d$ transition metals, prominently featuring toxic and expensive palladium, rhodium, and iridium complexes. ${ }^{[51]}$ Indeed, only a few extremely rare examples of enantioselective $\mathrm{C}-\mathrm{H}$ functionalizations utilizing first-row transition metal catalysts had been published at the outset of this thesis. However, several additional contributions to this burgeoning field of research would later be disclosed in the course of the present work, by

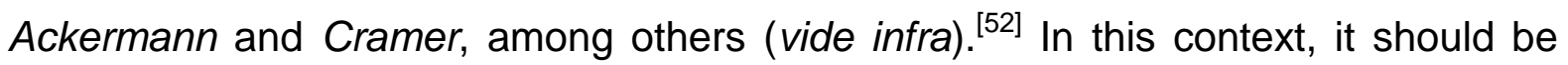
noted that the development of catalytic enantioselective methodologies in organic synthesis is a topic of extremely high interest, as best exemplified by the Nobel Prize in Chemistry awarded in 2001 to Noyori, Knowles and Sharpless for their seminal contributions to asymmetric catalysis. ${ }^{[53]}$ Therefore, given the sustainable nature and transformative power of $3 \mathrm{~d}$ metal-catalyzed $\mathrm{C}-\mathrm{H}$ activations, further exciting 
developments are expected in the near future in this rapidly-evolving research area. $^{[52]}$

\subsection{Cobalt-Catalyzed C-H Activation}

The potential of cobalt complexes in catalysis has long been recognized. Indeed, during the course of World War II, Roelen discovered, while studying the FischerTropsch process, that cobalt was able to promote the hydrocarbonylation of ethylene. ${ }^{[54]}$ The reaction was found to occur in solution and, consequently, is sometimes considered as the birth of homogeneous catalysis. ${ }^{[54]}$

Thereafter, Kharasch reported on the cobalt-catalyzed homo-coupling of aryl Grignard reagents. ${ }^{[55]}$ Later notable developments of cobalt chemistry in organic synthesis include the well-known Pauson-Khand ${ }^{[56]}$ and Nicholas ${ }^{[57]}$ reactions, cross-couplings, ${ }^{[58]}$ hydrogenations, ${ }^{[59]}$ cycloadditions, annulations, and many more. ${ }^{[60]}$

\subsubsection{Early Contributions}

In the 1950s, Murahashi disclosed the synthesis of 2-phenylphthalimidine 2 from imine 1a and carbon monoxide in the presence of catalytic dicobalt octacarbonyl (Scheme 1.5). ${ }^{[61]}$ The scope of the transformation was soon extended to include azobenzenes. ${ }^{[62]}$ This work is absolutely remarkable as it not only represents the first cobalt-catalyzed organometallic $\mathrm{C}-\mathrm{H}$ activation, but also one of the first examples of directing group assisted $\mathrm{C}-\mathrm{H}$ activation, if not the first. 


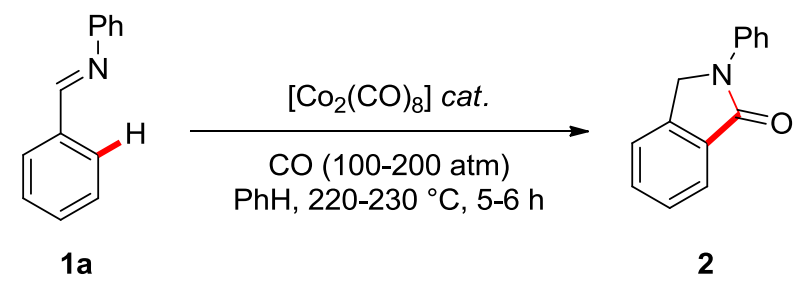

Scheme 1.5. Cobalt-catalyzed carbonylative cyclization of imines. ${ }^{[61]}$

This work remained largely neglected until the early 1990s with Klein's studies on the preparation of cyclometalated cobalt complexes from $\left[\mathrm{Co}\left(\mathrm{CH}_{3}\right)\left(\mathrm{PMe}_{3}\right)_{4}\right](3)$ and various chelating substrates (Scheme 1.6). ${ }^{[63]}$ Experimental evidence suggested the reaction to be initiated by the dissociation of a $\mathrm{PMe}_{3}$ ligand, which is replaced by azobenzene 4 , followed by ortho-metalation with elimination of methane to deliver 5. ${ }^{[63 f]}$ Interestingly, unlike in Murahashis work, no cyclized product was obtained upon exposure to carbon monoxide. Remarkably, due to the high reactivity of complex 3, even 4-membered metallacycles could be obtained. ${ }^{[63 e]}$

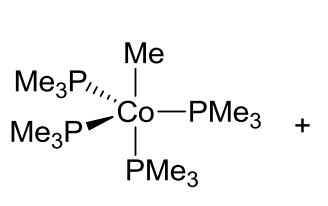

3

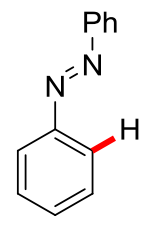

4

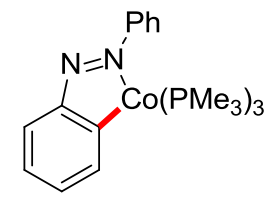

5

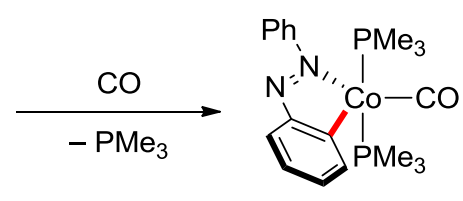

6

Scheme 1.6. Stoichiometric cyclocobaltation of azobenzene $4{ }^{[33]]}$

A major contribution in cobalt catalysis was achieved in 1994 by Kisch, who reported on the hydroarylation of tolane $7 \mathrm{a}$ with azobenzene $\mathbf{4}$ using a catalytic amount of cobalt-hydride complexes 8 or 9 (Scheme 1.7). ${ }^{[64]}$ This work represents the first cobalt-catalyzed $\mathrm{C}-\mathrm{H}$ hydroarylation of alkynes, where the authors proposed the active catalyst to be generated in situ after the loss of $\mathrm{N}_{2}$ or $\mathrm{H}_{2}$, respectively. Following the seminal work of Kisch, Petit used a related well-defined, low-valent phosphine-cobalt complex $\mathrm{Co}\left(\mathrm{PMe}_{3}\right)_{4}$ as catalyst for various hydroarylations of alkynes and alkenes. ${ }^{[65]}$ 


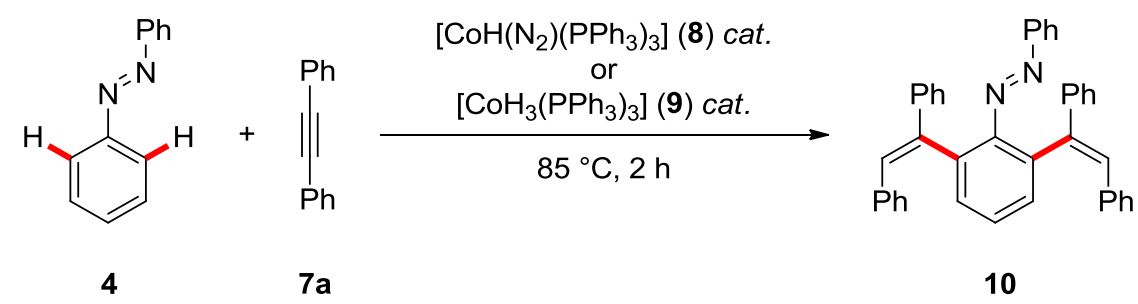

Scheme 1.7. Cobalt-catalyzed hydroarylation of tolane 7a with azobenzene $4 .^{[64]}$

Another key finding which would pave the way to future developments was subsequently disclosed by Brookhart, ${ }^{[6]]}$ who observed $\mathrm{H} / \mathrm{D}$ scrambling in $\mathrm{Cp}{ }^{*} \mathrm{Co}(\mathrm{I})$ complex 11 upon heating in deuterated benzene (Scheme 1.8). Interestingly, the authors proposed the $\mathrm{C}\left(\mathrm{sp}^{2}\right)-\mathrm{H}$ bonds to be activated through oxidative addition of a 16-electron cobalt species.

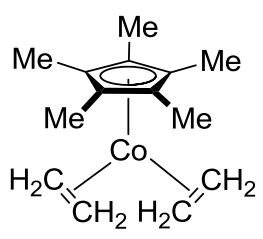

11

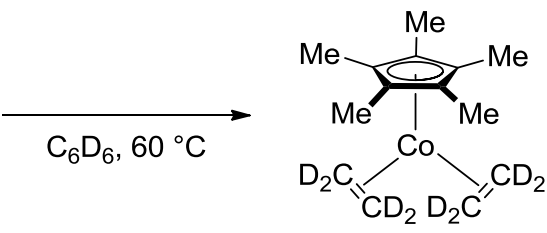

$[\mathrm{D}]_{8}-11$

Scheme 1.8. $\mathrm{H} / \mathrm{D}$ scrambling of complex 11 in $\mathrm{C}_{6} \mathrm{D}_{6}{ }^{[66]}$

Based on the contributions discussed above, cobalt-catalyzed $\mathrm{C}-\mathrm{H}$ activation was for years dominated by the use of "low-valent" cobalt complexes or reductive conditions, with key contributions to the field by Nakamura, ${ }^{[67]}$ Yoshikai $^{[46 a, 46 d, 68]}$ and Ackermann, ${ }^{[69]}$ among others. ${ }^{[45,46 c]}$ It is noteworthy that, in most of these studies, the active catalyst is ill-defined, being generated in situ from a cobalt salt, a (pre-)ligand and an organometallic species.

\subsubsection{Cobalt(III)-Catalyzed C-H Activation}

The use of cyclopentadienyl-rhodium(III) catalysts allowed for remarkable advancements in the field of transition metal-catalyzed $\mathrm{C}-\mathrm{H}$ activation. ${ }^{[70]}$ However, 
due to the low natural abundance and high cost of rhodium, the development of alternatives employing earth-abundant metals is highly desirable. In this context, a major progress was achieved in 2013 by Yoshino, Matsunaga and Kanai, who discovered the previously known cationic $\mathrm{Cp}^{*} \mathrm{Co}(\mathrm{III})$ complex $14^{[71]}$ to be a potent catalyst for $\mathrm{C}-\mathrm{H}$ activation (Scheme 1.9). ${ }^{[72]} \mathrm{It}$ is noteworthy that, while various cyclopentadienyl-cobalt(III) complexes had been described previously, ${ }^{[71,73]}$ their use in $\mathrm{C}-\mathrm{H}$ activation had remained unprecedented until then. Among the various cyclopentadienyl derivatives that were investigated, $\mathrm{Cp}^{*}$ was identified as the best ligand, while other derivatives fell short in the envisioned transformation. ${ }^{\text {[72] }}$ Furthermore, no conversion was obtained with simple cobalt salts.

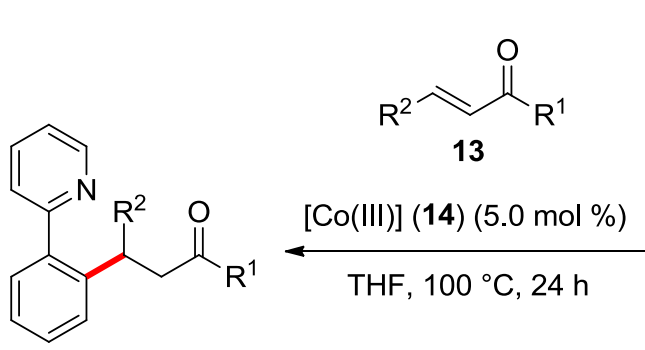

15

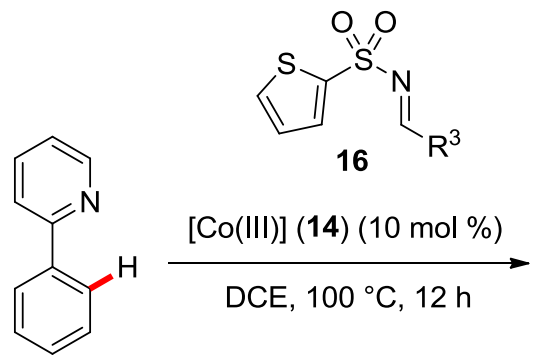

12

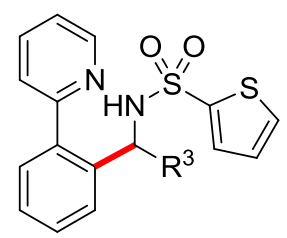

17

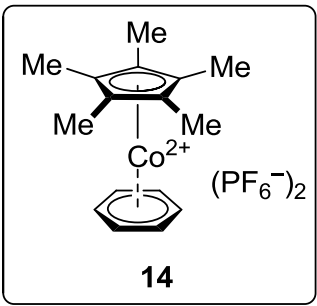

Scheme 1.9. Cobalt(III)-catalyzed hydroarylation of $\alpha, \beta$-unsaturated ketones 13 and $\mathrm{N}$-sulfonyl imines $16{ }^{[72]}$

Based on related rhodium(III)-catalyzed transformations, ${ }^{[74]}$ the mechanism of the cobalt(III)-catalyzed hydroarylation was proposed to be initiated by the displacement of the labile benzene ligand by the phenylpyridine substrate 12 to form 18 (Scheme 1.10). ${ }^{[72]}$ The authors suggested the subsequent $\mathrm{C}-\mathrm{H}$ activation step to occur via an electrophilic aromatic substitution or a concerted metalationdeprotonation mechanism to form the cyclometalated intermediate 19, which is 
assumed to be the active catalyst. After ligand exchange with imine $\mathbf{1 6}$ to give $\mathbf{2 0}$, insertion of the latter to give intermediate $\mathbf{2 1}$, and proto-demetalation, the product $\mathbf{1 7}$ is released while the active catalyst is regenerated. In a subsequent report, this chemistry was extended to indole substrates by Kanai and Matsunaga using carboxylate additives which allowed for high catalytic efficacy. ${ }^{[75]}$

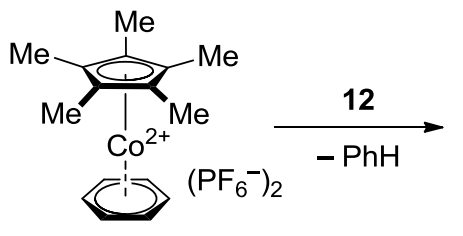

14

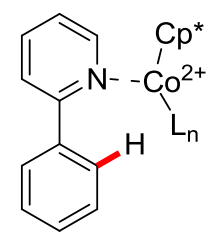

$(L=12)$<smiles>[R]C(NS(=O)(=O)OCc1ccccn1)c1ccccc1-c1ccccc1</smiles>

17

$\mathrm{L}$
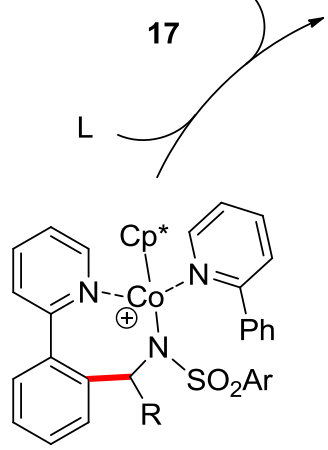

18<smiles>CC(C)CC(C)C(=O)F</smiles><smiles></smiles>

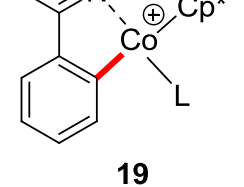

19<smiles>CC(C)C(C)I</smiles>

22
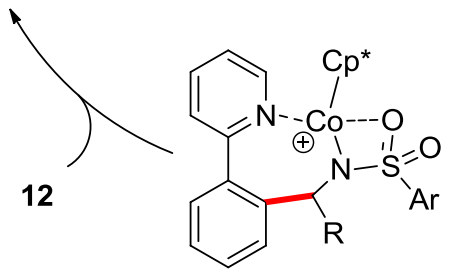

21

Scheme 1.10. Proposed mechanism of the $\mathrm{Cp}^{*} \mathrm{Co}(\mathrm{III})$-catalyzed hydroarylation of imines $16 .^{[72]}$

Taking inspiration from this elegant work, numerous reports on $\mathrm{Cp}^{*} \mathrm{Co}$ (III)-catalyzed $\mathrm{C}-\mathrm{H}$ activation were disclosed in the following years. ${ }^{[45,46,76]}$ Only selected relevant 
examples are discussed in this thesis, with a focus on enantioselective transformations.

The insertion reactions were not limited to activated $\mathrm{C}-\mathrm{C}$ and $\mathrm{C}-\mathrm{N}$ double bonds. Indeed, Matsunaga, Kanai and coworkers developed procedures for the selective cobalt-catalyzed hydroarylation of alkynes 7 with $\mathrm{N}$-carbamoyl indoles and pyrroles 23 (Scheme 1.11). ${ }^{[7]}$ Remarkably, small changes of the directing group or the reaction conditions had a profound impact on the selectivity of the reaction. Indeed, while a judicious choice of the carbamate directing group and reaction temperature allowed for either the formation of the hydroarylated product 24 or the thermodynamically more stable cyclized product 25 (Scheme 1.11a-b), ${ }^{[77 d]}$ the use of the cationic complexes $\left[\mathrm{Cp}{ }^{\star} \mathrm{Co}(\mathrm{MeCN})_{3}\right]^{2+}$ provided access to tetrasubstituted alkenes 27 (Scheme 1.11c). ${ }^{[77 a]}$ DFT calculations suggested the $\mathrm{C}-\mathrm{H}$ cleavage step to occur through an acetate-enabled CMD manifold. Interestingly, $\mathrm{Cp}{ }^{\star} \mathrm{Rh}(\mathrm{III})$ catalysts were found to be inefficient in the envisioned transformations, giving only small amounts of the alkenylated product 24. The findings highlighted the different reactivity and complementarity of cobalt as compared to rhodium. Thus, the high nucleophilicity of the C-Co bond allowed for a unique reactivity. ${ }^{[46 a, 46 b, 77 a, 77 d]}$ This difference may, partly, be explained by the higher electronegativity of cobalt compared to rhodium. ${ }^{[78]}$ The unique reactivity of cobalt(III) catalysts was also exploited by Ackermann in an allylation by $\mathrm{C}-\mathrm{H} / \mathrm{C}-\mathrm{C}$ activation, where the less stable Z-isomer was selectively obtained, using $\mathrm{Cp}^{*} \mathrm{Co}(\mathrm{III})$ catalysts, while the corresponding rhodium(III) complex gave unselective mixtures of diastereomers. ${ }^{[79]}$ 
a) hydroarylation

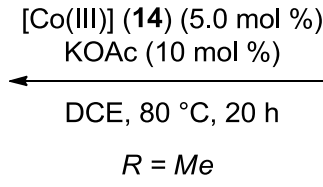

24

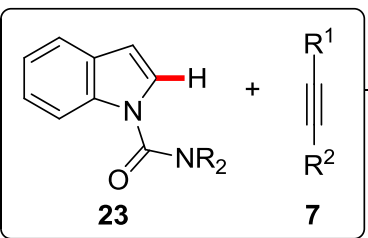

[Co(III)] (14) (5.0 mol \%)

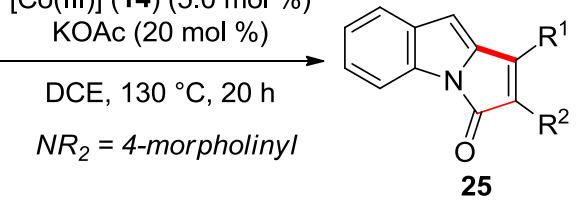

c) DG migration

$\left[\mathrm{Cp}{ }^{*} \mathrm{Co}(\mathrm{MeCN})_{3}\right]\left(\mathrm{SbF}_{6}\right)_{2}$

(26) $(5.0 \mathrm{~mol} \%)$

$\operatorname{KOAC}(5.0 \mathrm{~mol} \%)$

DCE, $90^{\circ} \mathrm{C}, 20 \mathrm{~h}$

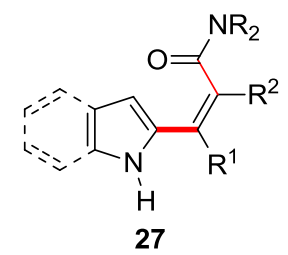

Scheme 1.11. Selectivity control in $\mathrm{Cp}{ }^{*} \mathrm{Co}(\mathrm{III})$-catalyzed $\mathrm{C}-\mathrm{H}$ activation of indoles 23 with alkynes $7 .^{[77 \mathrm{a}, 77 \mathrm{~d}]}$

The scope of $\mathrm{Cp}^{\star} \mathrm{Co}(\mathrm{III})$-catalyzed $\mathrm{C}-\mathrm{H}$ activations by hydroarylation is not limited to alkynes. Indeed, unactivated alkenes, and even allenes, ${ }^{[80]}$ have been employed as coupling partners in $\mathrm{C}-\mathrm{H}$ activations. However, in the case of alkenes 29, the control of linear vs. branched selectivities represents a challenging issue. Ackermann and coworkers disclosed in 2017 a procedure for the selective hydroarylation of unactivated alkenes (Scheme 1.12a). ${ }^{[37 b]}$ Remarkably, while the anti-Markovnikov isomer $\mathbf{3 0}$ was obtained in the absence of additives, the introduction of 1.0 equivalent of $1-\mathrm{AdCO}_{2} \mathrm{H}$ was found to promote the selective formation of the branched product 31. Detailed experimental and computational mechanistic studies revealed the switch of selectivity to be caused by a change of mechanism. Indeed, in the absence of the carboxylic acid additive, the $\mathrm{C}-\mathrm{H}$ cleavage step was found to proceed via a ligand-to-ligand hydrogen transfer (LLHT) ${ }^{[32 a, 37 b, 81]}$ manifold involving two substrates 28 and delivering the linear product 30 . In contrast, $1-\mathrm{AdCO}_{2} \mathrm{H}$ was found to enable a base-assisted internal electrophilic type substitution (BIES) delivering the branched product 31. This work also provided a proof-of-concept for an asymmetric version of this reaction. Thus, various chiral carboxylic acids were 
tested in the transformation, with $N$-phthaloyl protected isoleucine (32) providing product 31aa with 62.5:37.5 e.r. (Scheme 1.12b).

a) selectivity control in cobalt-catalyzed hydroarylations

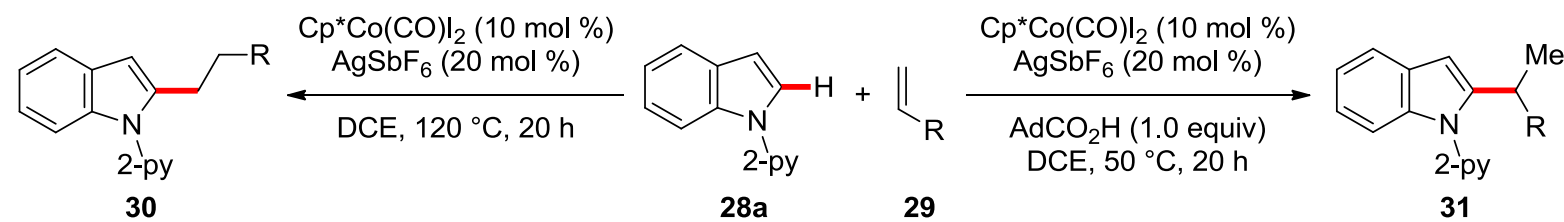

b) preliminary results towards an asymmetric transformation
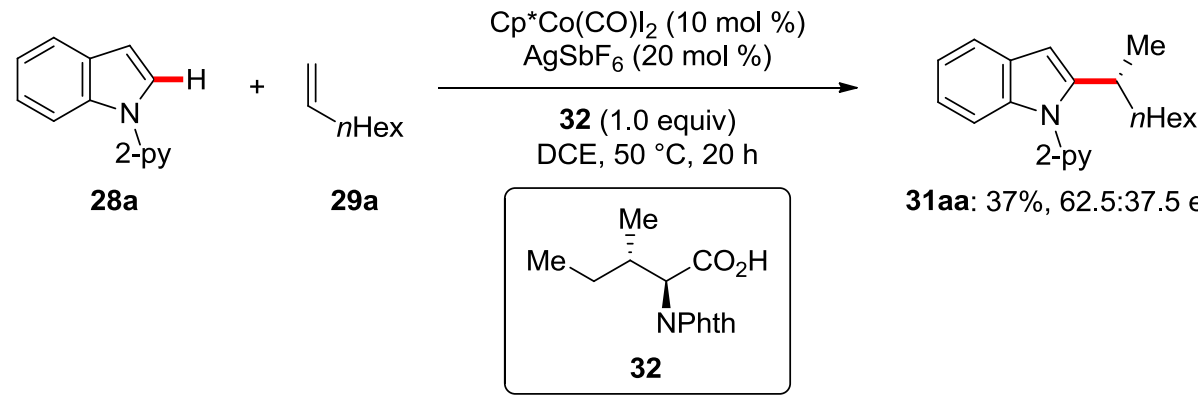

31aa: $37 \%, 62.5: 37.5$ e.r.

Scheme 1.12. Selectivity control in cobalt-catalyzed C-H alkylations. ${ }^{[37 b]}$

Triggered by this seminal report, the Ackermann group disclosed in 2018 the first highly enantioselective cyclopentadienyl-cobalt(III)-catalyzed $\mathrm{C}-\mathrm{H}$ activation (Scheme 1.13). ${ }^{[82]}$ Essential to success was the design of the new chiral carboxylic acid 34. Under the reaction conditions, various indoles 28b and allylbenzene derivatives $\mathbf{3 3}$ were smoothly converted to the alkylated products $\mathbf{3 5}$ with excellent enantioselectivity. Computational studies and H/D-exchange experiments suggested the enantio-determining step to be an irreversible proto-demetalation promoted by the chiral acid 34. 


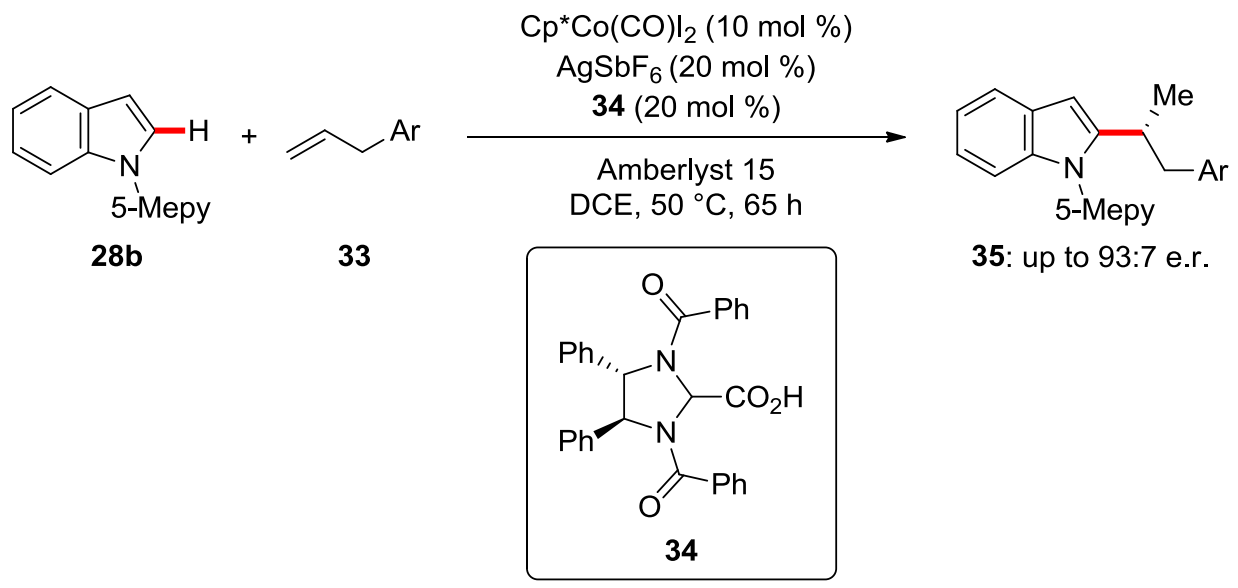

Scheme 1.13. Enantioselective cobalt(III)-catalyzed alkylation of indoles $\mathbf{2 8 b}{ }^{\left[{ }^{82]}\right.}$

Furthermore, $\mathrm{Cp}^{*} \mathrm{Co}(\mathrm{III})$ catalysis is not restricted to hydroarylations. Other $\mathrm{C}-\mathrm{C}$ bond forming reactions include inter alia alkynylations, ${ }^{[83]}$ allylations, ${ }^{[84]}$ aminocarbonylations $^{[85]}$ and various annulations. ${ }^{[45]}$ Besides $\mathrm{C}-\mathrm{C}$ bond forming processes, the formation of $\mathrm{C}-\mathrm{X}$ and $\mathrm{C}-\mathrm{N}$ bonds has proven quite successful as well. In this context, Matsunaga and Kanai established a protocol for the $\mathrm{C}-\mathrm{H}$ amination of indoles 36 with azides 37 using $\mathrm{Cp}^{*} \mathrm{Co}(\mathrm{CO}) \mathrm{I}_{2}$ as an air-stable pre-catalyst (Scheme 1.14). ${ }^{[86]}$ Using $\mathrm{Cp}^{*} \mathrm{Co}(\mathrm{CO}) \mathrm{I}_{2}$ and a silver salt to generate the cationic catalyst in situ, rather than using the highly sensitive sandwich complex $\mathbf{1 4}$, has since been a widely applied approach due to its user-friendly nature. However, due to the inherently unsafe handling of usually explosive and toxic ${ }^{[87]}$ azides, protocols employing safer aminating reagents would be highly desirable. Other $\mathrm{Cp}^{*} \mathrm{Co}$ (III)-catalyzed $\mathrm{C}$-(pseudo)Het bond-forming reactions include halogenations, ${ }^{[84 \mathrm{~d}, 88]}$ cyanations $^{[84 \mathrm{~d}, 89]}$ and thiolations, ${ }^{[00]}$ among others. ${ }^{[45,46,76]}$

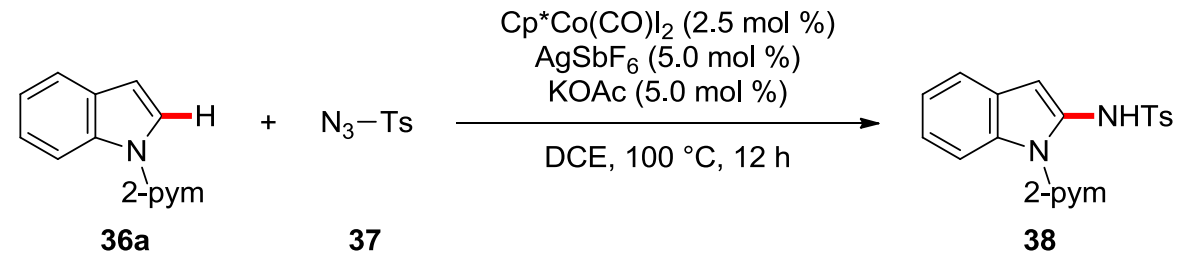

Scheme 1.14. Cobalt-catalyzed $\mathrm{C}-\mathrm{H}$ amination of indoles 36 with azides $37{ }^{[86]}$ 
Inspired by this report and the topical interest for $\mathrm{C}-\mathrm{H}$ aminations, ${ }^{[23 \mathrm{e}]}$ several research groups developed additional cobalt-catalyzed transformations to forge $\mathrm{C}-\mathrm{N}$ bonds. Among other findings, the research groups of Chang, ${ }^{[91]} \mathrm{Jiao}^{[92]}$ and Ackermann ${ }^{[37 e]}$ independently pioneered the use of dioxazolones $\mathbf{4 0}$ as safer but potent amidating reagents in cobalt catalysis (see Chapter 3.1). Two years later, Dixon and Seayad reported a thioamide-assisted amidation of $\mathrm{C}\left(\mathrm{sp}^{3}\right)-\mathrm{H}$ bonds. ${ }^{[93]}$ This work remains one of the rare examples of $\mathrm{C}\left(\mathrm{sp}^{3}\right)-\mathrm{H}$ activation with a cobalt catalyst. DFT calculations provided support for the $\mathrm{C}-\mathrm{H}$ activation step to proceed via an external carboxylate-assisted concerted metalation/deprotonation mechanism. Subsequently, Matsunaga and coworkers developed an asymmetric version of this protocol (Scheme 1.15). ${ }^{\left[{ }^{[4]}\right.}$ Here, the highly enantioselective $\mathrm{C}\left(\mathrm{sp}^{3}\right)-\mathrm{H}$ amidation of thioamides $\mathbf{3 9}$ was achieved by the combination of the chiral carboxylic acid 42 with an achiral cobalt(III) complex. A remarkable feature of this work was the identification of the novel (tert-butyl-tetramethylcyclopentadienyl)Co(III) complex 41, which promoted the reaction with higher enantioselectivity than the standard $\mathrm{Cp}^{*} \mathrm{Co}(\mathrm{III})$ complexes. Interestingly, while the tuning of the cyclopentadienyl ligand has been intensively investigated in rhodium catalysis, ${ }^{[70 a]}$ variations of the $\mathrm{Cp}^{*}$ ligand remain underdeveloped in $\mathrm{Co}(\mathrm{III})$-catalyzed $\mathrm{C}-\mathrm{H}$ activation. It is noteworthy that this approach represents the first example of enantioselective inner-sphere $\mathrm{C}\left(\mathrm{sp}^{3}\right)-\mathrm{H}$ activation with a $3 \mathrm{~d}$ transition metal catalyst.
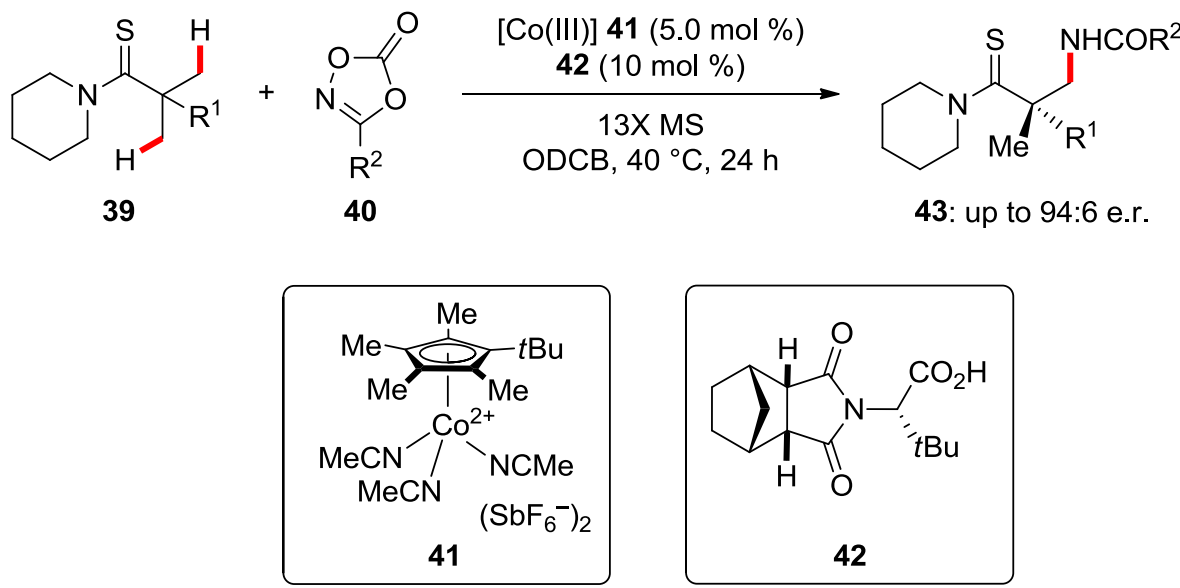

Scheme 1.15. Enantioselective cobalt(III)-catalyzed C $\left(\mathrm{sp}^{3}\right)-\mathrm{H}$ amidation of thioamides $39 .{ }^{[94]}$ 
The concept of cooperation between an achiral cobalt(III) catalyst and a chiral carboxylic acid was further exploited by Shi and coworkers, who, taking inspiration from previous works on the cobalt-catalyzed amidation of ferrocenes by the same research group ${ }^{[95]}$ and Ackermann, ${ }^{[96]}$ achieved the enantioselective amidation of ferrocenes 44 with dioxazolones 40 (Scheme 1.16). ${ }^{[97]}$ Here, the design of the novel chiral monoprotected amino acid (MPAA) ligand 45 allowed for the synthesis of amidated ferrocene thioamides $\mathbf{4 6}$ with high yield but moderate enantioselectivity. Interestingly, the modest optical purity could be improved by a single crystallization to afford the amidated products in $>99 \%$ ee.

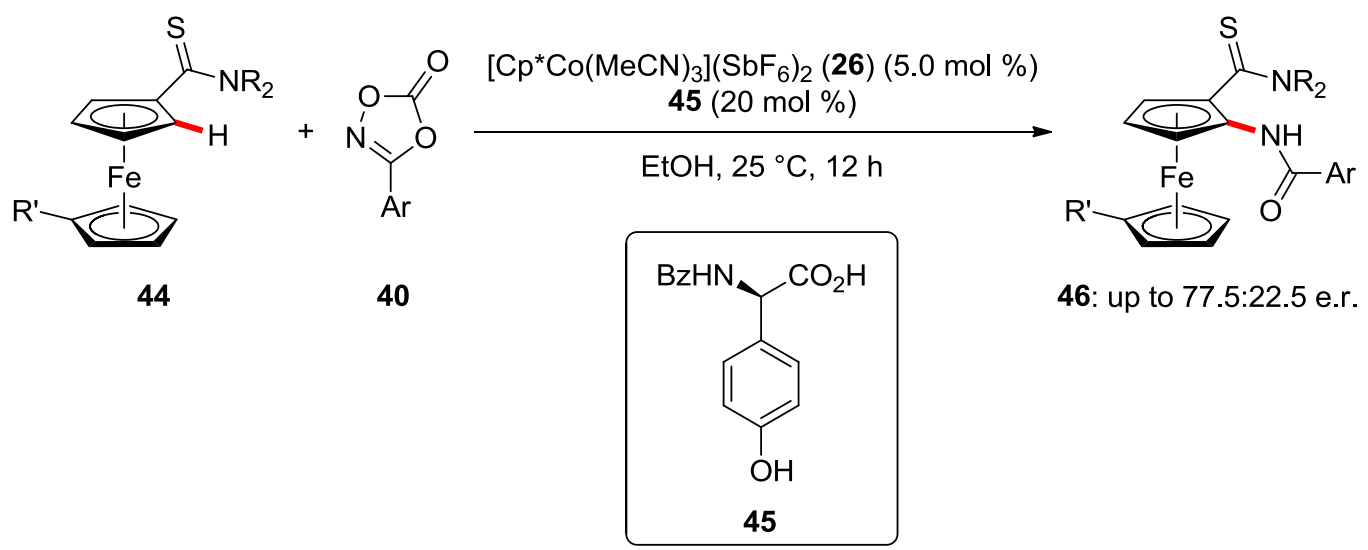

Scheme 1.16. Enantioselective cobalt(III)-catalyzed C-H amidation of ferrocenes $44 .^{[97]}$

As discussed above, the pioneering examples of enantioselective cyclopentadienylcobalt(III)-catalyzed $\mathrm{C}-\mathrm{H}$ transformations relied on the use of a simple achiral $\mathrm{Cp}^{*} \mathrm{Co}$ (III)-catalyst in combination with an external chiral carboxylic acid. Very recently, Cramer reported a complementary approach based on the use of the finely designed trisubstituted ${ }^{[98]}$ chiral cyclopentadienyl-cobalt complex 49. ${ }^{[99]}$ This catalyst proved highly efficient for the asymmetric synthesis of dihydroisoquinolones $\mathbf{5 0}$ from $\mathrm{N}$-chlorobenzamides 47 and a diverse set of alkenes $48^{[100]}$ (Scheme 1.17). ${ }^{[99]}$ The introduction of a bulky tert-butyl group on the chiral $\mathrm{Cp}$ ligand was essential to achieve high enantioselectivities, and was found to affect the dihedral angle of the binaphthyl backbone. A remarkably diverse set of alkenes was fully tolerated in the transformation, including styrenes, unactivated alkenes, acrylates and 
$\mathrm{N}$-vinylphtalimide, providing the cyclized product as a single regioisomer. In contrast, rhodium(III) complexes provided the products $\mathbf{5 0}$ with both lower regio- and enantioselectivities. ${ }^{[101]}$

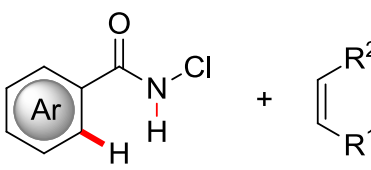

47

48

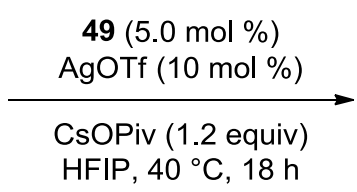

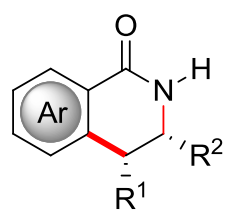

50: up to $99.5: 0.5$ e.r.

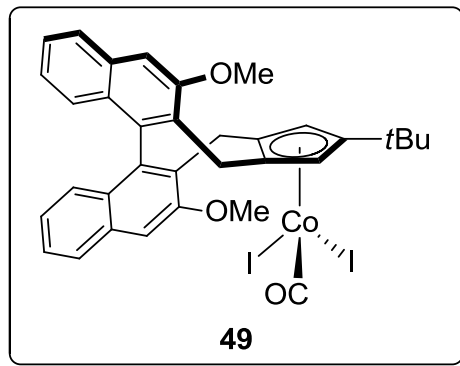

Scheme 1.17. Chiral cyclopentadienyl cobalt(III)-catalyzed $\mathrm{C}-\mathrm{H}$ functionalizations with

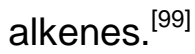

\subsubsection{Enantioselective Cobalt-Catalyzed $\mathrm{C}-\mathrm{H}$ Functionalizations under Reductive Conditions}

In addition to the reports discussed above employing high-valent $\mathrm{Cp}^{*} \mathrm{Co}$ (III) complexes (Schemes 1.12-13, 1.15-17), several protocols employing cobalt catalysts for asymmetric $\mathrm{C}-\mathrm{H}$ functionalizations under reductive conditions have been recently disclosed as well, but remain rare. Those reports highlight the current interest for enantioselective $\mathrm{C}-\mathrm{H}$ activation with earth-abundant $3 \mathrm{~d}$ metals, ${ }^{[52]}$ and the need to conduct further research in this burgeoning field of research.

In 2014, the Yoshikai group disclosed an elegant enantioselective intramolecular hydroacylation of 2-acylbenzaldehydes 51 and 2-alkenylbenzaldehydes 54 for the synthesis of synthetically meaningful chiral phthalide 53 and indanone 56 building blocks (Scheme 1.18a-b). ${ }^{[102]}$ Previously, this type of asymmetric transformations had only been achieved with costly rhodium catalysts. ${ }^{[103]}$ The combination of $\mathrm{CoBr}_{2}$ 
with $(R, R)$-Ph-BPE (52) was found to provide optimal results for the intramolecular hydroacylation of 2-acylbenzaldehydes 51 (Scheme 1.18a). The protocol could be extended to 2-alkenylbenzaldehydes 54 when using $\mathrm{CoCl}_{2}$ and $(R, R)$-BDPP (55) as the optimal catalytic system to deliver indanones 56 (Scheme 1.18b). Mechanistic studies provided evidence for a relatively facile $\mathrm{C}-\mathrm{H}$ activation step and a subsequent rate-limiting reductive elimination step. In a later report, Yoshikai and Gosmini expanded this approach from disubstituted alkenes $\mathbf{5 4}$ to more challenging trisubstituted alkenes 57, allowing for the expedient synthesis of highly functionalized chiral cyclic ketones 58 (Scheme 1.18c). ${ }^{[104]}$ Here, the combination of $\mathrm{CoBr}_{2}$ and $(R, R)$-BDPP (55) provided optimal results. Interestingly, the authors noted that the $E / Z$ ratio of the starting materials 57 only had a minor effect on the enantiomeric excess of the products 58. 
a) hydroacylation of ketones

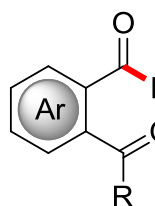

51
$\mathrm{CoBr}_{2}(10 \mathrm{~mol} \%)$ $(R, R)-\mathrm{Ph}-\mathrm{BPE}(\mathbf{5 2})(10 \mathrm{~mol} \%)$

In $(20 \mathrm{~mol} \%)$ $\mathrm{MeCN}, 80^{\circ} \mathrm{C}, 12 \mathrm{~h}$<smiles>[R6]C1CC[C@@H](P)[C@@H]1Pc1ccccc1</smiles>

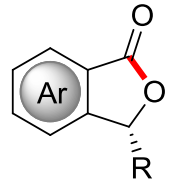

53: up to $98 \%$ ee

b) hydroacylation of disubstituted olefins

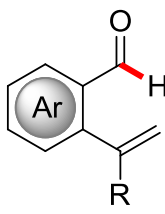

54

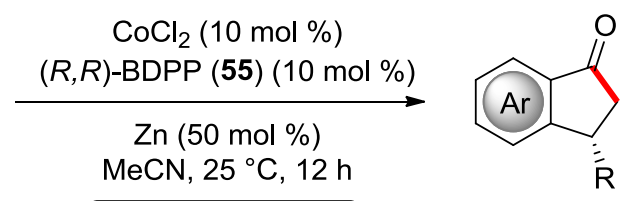

56: up to $97 \%$ ee

c) hydroacylation of trisubstituted olefins<smiles>[R]C=C([R])c1ccccc1C=O</smiles>

57

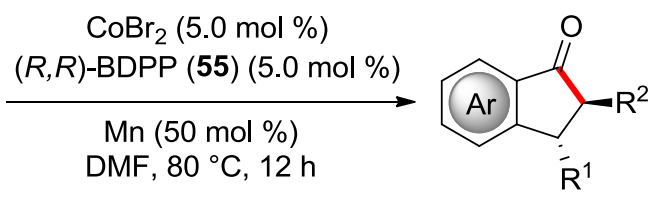

58: up to $>20: 1$ d.r., up to $98 \%$ ee

Scheme 1.18. Enantioselective cobalt-catalyzed intramolecular hydroacylations. ${ }^{[102,104]}$

Yoshikai had previously disclosed a cobalt-catalyzed $\mathrm{C}-\mathrm{H}$ alkylation of indoles with alkenes. ${ }^{[105]}$ In 2015, the same research group developed an enantioselective variation of this reaction (Scheme 1.19). ${ }^{[106]}$ Remarkably, this work represents the first asymmetric intermolecular transformation by cobalt-catalyzed $\mathrm{C}-\mathrm{H}$ activation. While simple BINOL-derived phosphoramidites provided the desired alkylated product 62 in low yield and enantiomeric excess, variations of the chiral diol backbone significantly increased the enantioselectivity of the transformation. Thereby, diversely substituted indoles 59a and styrene derivatives 60 furnished the alkylated products 62 in good yields and high enantioselectivities in the presence of $\mathrm{TMSCH}_{2} \mathrm{MgCl}$. 


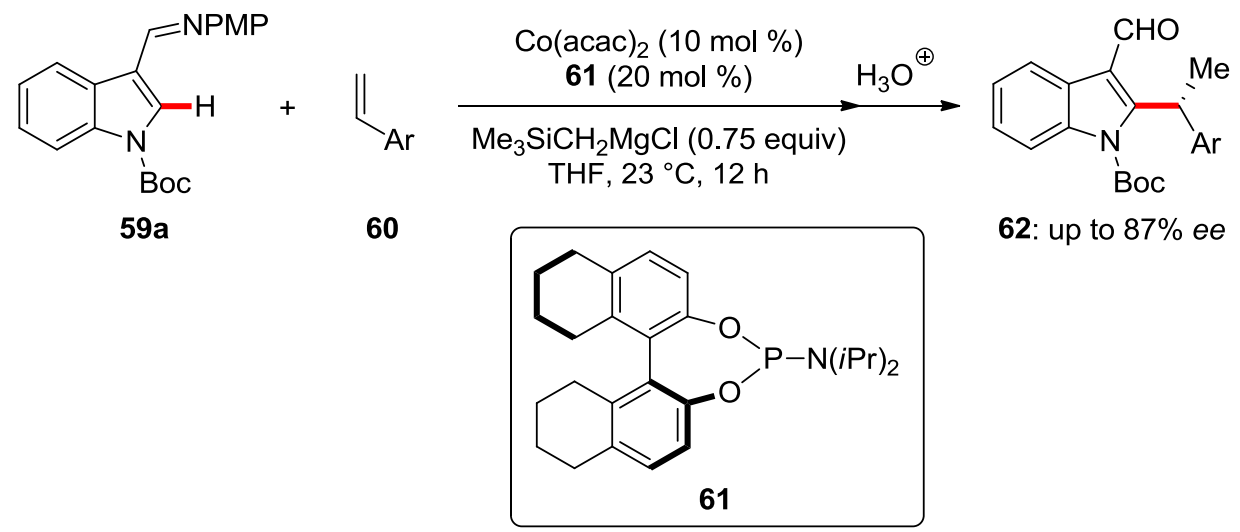

Scheme 1.19. Enantioselective cobalt-catalyzed hydroarylation of styrenes 60 with indoles 59a. ${ }^{[106]}$

In 2017, the Dong group disclosed a unique desymmetrization strategy for the intramolecular enantioselective hydroacylation of alkenes 63 to construct chiral cyclobutanone derivatives 64 (Scheme 1.20). ${ }^{[107]}$ A cobalt catalyst with (S,S)-BDPP (ent-55) as the chiral ligand enabled the highly selective synthesis of unusual strained four-membered rings $\mathbf{6 4}$ rather than their five-membered regioisomers. The authors tested diversely $\alpha$-substituted dienyl aldehydes 63 which underwent the cyclization with high regio- and enantio-selectivities, with sensitive functionalities such as TMS or chloro being fully tolerated in the transformation. Mechanistic studies provided support for a cobalt(0)/cobalt(II) catalytic cycle.

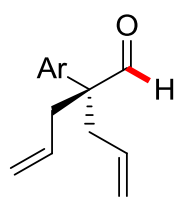

63

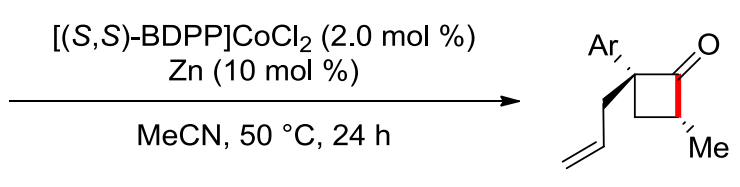

64: up to $96 \%$ ee

Scheme 1.20. Enantioselective hydroacylation for the synthesis of cyclobutanones $64 .^{[107]}$ 


\subsection{Iron-Catalyzed C-H Activation}

Iron is by far the most abundant metal on Earth. ${ }^{[43]}$ Applications of iron catalysts in molecular syntheses benefit from comparatively low costs and toxicities, as well as a broad array of available oxidation states. ${ }^{[108]}$ Furthermore, the considerable increase of prices of many late transition metals in recent years created a demand for less expensive alternatives. ${ }^{[108 b]}$

The independent synthesis of pentacarbonyliron in 1891 by $M o n d^{[109]}$ and Berthelot ${ }^{[110]}$ is usually considered as the birth of organoiron chemistry. ${ }^{[108 c]} \mathrm{A}$ subsequent milestone of iron chemistry was the identification of iron salts as potent catalysts in reactions with Grignard reagents, including homocouplings, by Kharasch in 1941. ${ }^{[55,111]}$ The serendipitous discovery of ferrocene, reported by Pauson and Kealy in $1951^{[112]}$ and whose transformative applications would change the face of chemistry, ${ }^{[113]}$ was another major contribution in organometallic iron chemistry. The 1950s also marked the first use of well-defined organoiron species in organic synthesis with the preparation of hydroquinone by Reppe from acetylene and iron carbonyl complexes. ${ }^{[14]}$

A breakthrough in organoiron catalysis was the identification of iron salts as catalysts in cross-couplings between Grignard reagents and vinyl bromides by Kochi in $1971,{ }^{[115]}$ which, remarkably, predated subsequent works with palladium and nickel catalysts. ${ }^{[4]}$ Interestingly, the authors probed various metal halides and identified iron as "one of the most effective metal catalysts for the promotion of the reactions between Grignard reagents and organic halides. ${ }^{\text {[115a] }}$ Although the use of iron catalysts in cross-couplings was first overlooked due to the development of palladium catalysis, ${ }^{[4]}$ it has since experienced a renaissance. However, despite major progress, ${ }^{[19]}$ good mechanistic understanding is lacking and the design of novel ligands is required for further advancements. ${ }^{[47 b]}$

From a historical point of view, it should be noted that stoichiometric $\mathrm{C}-\mathrm{H}$ activation with iron complexes were reported as early as 1968 by Hata. ${ }^{[16]}$ Irradiation of the 
iron(0) complex 65 resulted in the loss of ethylene and oxidative addition into a $\mathrm{C}\left(\mathrm{sp}^{2}\right)-\mathrm{H}$ bond of the phosphine ligand (Scheme 1.21).

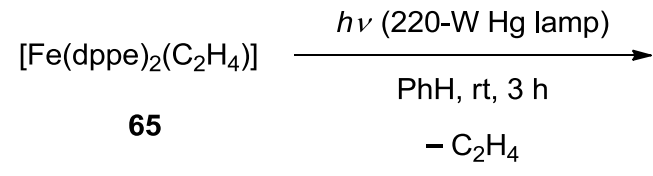

Scheme 1.21. Synthesis of cyclometalated complex activation. ${ }^{[16]}$

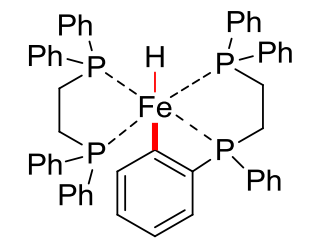

66

66 by photoinduced $\mathrm{C}\left(\mathrm{sp}^{2}\right)-\mathrm{H}$

Later works further demonstrated the feasibility of stoichiometric $\mathrm{C}-\mathrm{H}$ activation with highly reactive low-valent iron complexes. ${ }^{[117]}$ Among others, $\mathrm{Fe}\left(\mathrm{PMe}_{3}\right)_{4}$ (67), first independently prepared by Muetterties ${ }^{[117 b]}$ and Schmidbaur ${ }^{[118]}$ in 1975, has been particularly effective in directing group-assisted cyclometalations (Scheme 1.22). ${ }^{[119]}$ Interestingly, $\mathrm{Fe}\left(\mathrm{PMe}_{3}\right)_{4}$ was found to predominantly exist as an iron(II) species due to $\mathrm{C}-\mathrm{H}$ activation of the phosphine ligand. ${ }^{[117 b, 118]}$

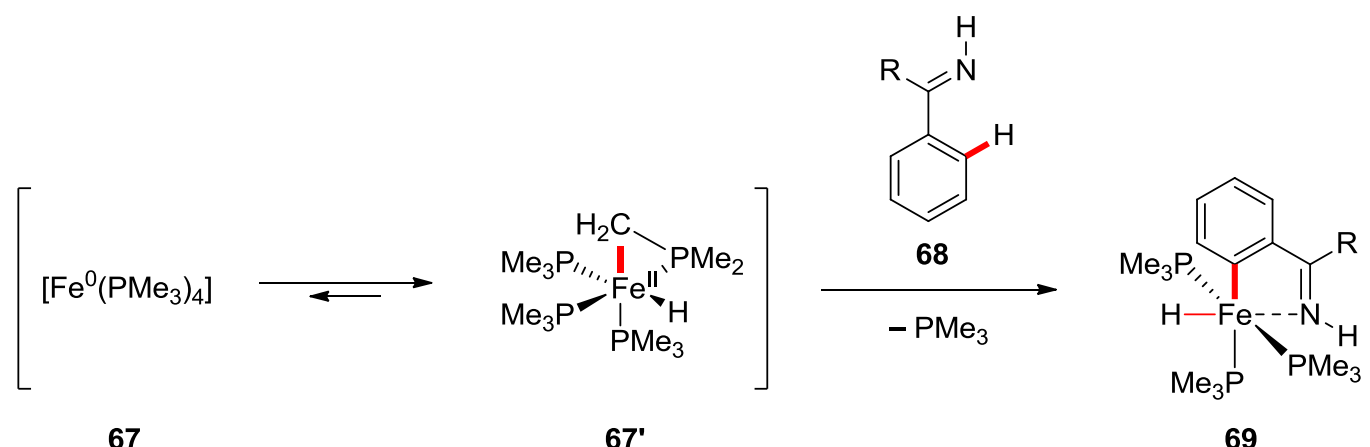

Scheme 1.22. Stoichiometric ortho- $\mathrm{C}-\mathrm{H}$ metalation of ketimines with $\mathrm{Fe}\left(\mathrm{PMe}_{3}\right)_{4} \cdot{ }^{[119]}$

$\mathrm{Fe}\left(\mathrm{PMe}_{3}\right)_{4}$ would later prove instrumental to the development of catalytic $\mathrm{C}-\mathrm{H}$ activations. Indeed, the first efforts towards a catalytic $\mathrm{C}-\mathrm{H}$ activation employing an iron complex were reported by Jones in 1987. ${ }^{[120]}$ Here, the use of a catalyst generated from $\mathrm{Fe}\left(\mathrm{PMe}_{3}\right)_{4}(67)$ and isocyanide ligands (70) allowed for the synthesis of imines 1 from benzene under UV irradiation (Scheme 1.23). A reaction conducted 
in $\mathrm{C}_{6} \mathrm{D}_{6}$ showed the solvent - and not the $\mathrm{PMe}_{3}$ ligand - to be the source of the aldimine's hydrogen. Low concentrations were required due to substrate inhibition, and the authors suggested that light was needed to induce the dissociation of an isocyanide ligand to generate a reactive species able to insert into a $\mathrm{C}-\mathrm{H}$ bond. Furthermore, in a later report over four decades after its original discovery, $\mathrm{Fe}\left(\mathrm{PMe}_{3}\right)_{4}$ was finally found to be effective in iron-catalyzed $\mathrm{C}-\mathrm{H}$ activations without the need of additional ligands, as elegantly demonstrated by Kakiuchi for the carbonyl-assisted hydroarylation of alkenes. ${ }^{[121]}$ On the same line, a carbonyldirected $\mathrm{C}-\mathrm{H}$ methylation had been reported by $E$. Nakamura shortly before. ${ }^{[122]}$

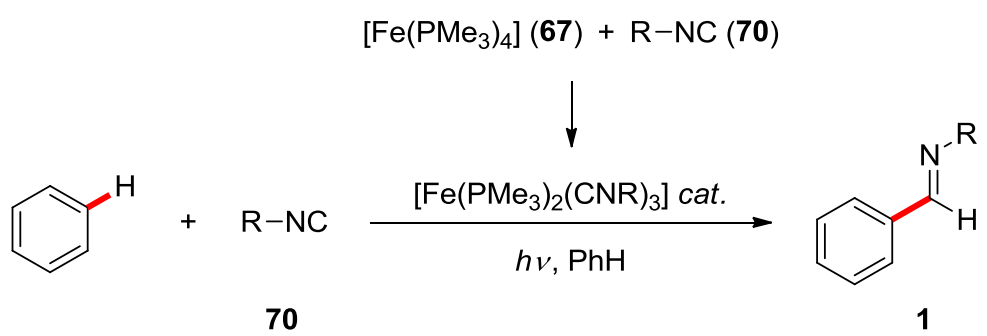

Scheme 1.23. Aldimine synthesis by iron-catalyzed C-H activation. ${ }^{[120]}$

In 2008, a breakthrough in the field of iron-catalyzed inner-sphere $\mathrm{C}-\mathrm{H}$ activation was made by $E$. Nakamura, who disclosed an iron-catalyzed oxidative $\mathrm{C}-\mathrm{H}$ arylation. ${ }^{[123]}$ Interestingly, this discovery was made by serendipity by an undergraduate student working on an iron-catalyzed cross-coupling reaction. ${ }^{[124]}$ In addition to the expected product 12, a small amount of the ortho-arylated phenylpyridine 72 was obtained as well (Scheme 1.24). Oxygen traces, as well as 2,2'-bipyridine (bpy) - another byproduct of the reaction - were later identified as essential to the formation of the $\mathrm{C}-\mathrm{H}$ arylated product. 


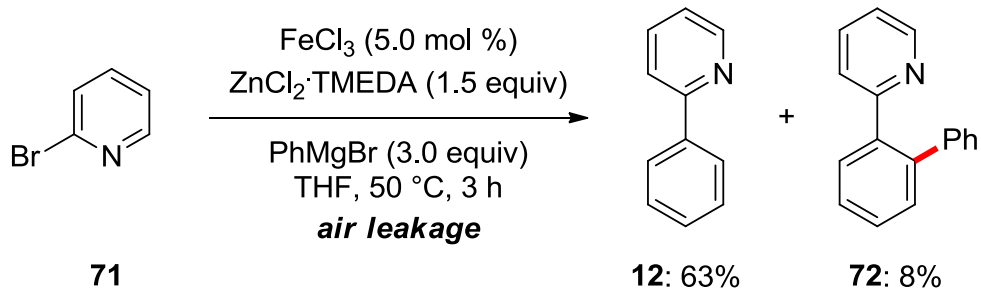

Scheme 1.24. Discovery of iron-catalyzed $\mathrm{C}-\mathrm{H}$ arylation as a byproduct of crosscoupling. ${ }^{[123]}$

After extensive optimization of this iron-catalyzed $\mathrm{C}-\mathrm{H}$ arylation, 1,2-dichloro-2methylpropane (DCIB) was identified as the optimal oxidant and phenanthroline as the best ligand (Scheme 1.25). ${ }^{[123]}$ Interestingly, the zinc additive was essential for the reaction to occur. While its role has been proposed to be the in situ generation of arylzinc species, $\mathrm{Mg}$-free $\mathrm{Ph}_{2} \mathrm{Zn}$ and $\mathrm{PhZnBr}$ fell short in delivering any arylated product $\mathbf{7 2}$, either in the absence or in the presence of TMEDA.

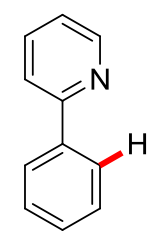

12

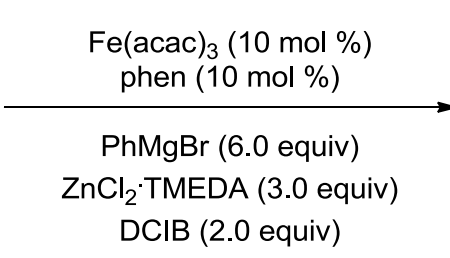

THF, $0{ }^{\circ} \mathrm{C}, 16 \mathrm{~h}$

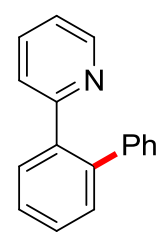

72

Scheme 1.25. First example of iron-catalyzed C-H arylation. ${ }^{[123]}$

Subsequent achievements by Nakamura and others in the research area of ironcatalyzed $\mathrm{C}\left(\mathrm{sp}^{2}\right)-\mathrm{H}$ arylation include, inter alia, the use of more synthetically useful imines $^{[125]}$ and amides ${ }^{[126]}$ as the directing groups, the use of environmentally benign oxygen as oxidant, ${ }^{[127]}$ the arylation of non-aromatic $\mathrm{C}\left(\mathrm{sp}^{2}\right)-\mathrm{H}$ bonds, ${ }^{[128]}$ the direct use of Grignard reagents in the absence of zinc additives, ${ }^{[129]}$ and the use of metallic magnesium to prevent the handling of sensitive and dangerous organometallic reagents. ${ }^{[130]}$

While the authors did not propose a mechanism in their original reports, a plausible catalytic cycle was later suggested by Nakamura and coworkers based on KIE 
studies and stoichiometric reactions (Scheme 1.26). ${ }^{[129]}$ A possible cycle is initiated by the formation of an aryliron species (73) by transmetalation of the aryl Grignard reagent to the iron center. Then, after a reversible coordination of the iron center to the pyridyl group of 12 , an irreversible $\mathrm{C}-\mathrm{H}$ metalation with concomitant elimination of an arene can happen. Subsequently, the cyclometalated intermediate 75 can undergo a $\mathrm{C}-\mathrm{C}$ bond forming reductive elimination upon reaction with $\mathrm{DCIB}$ to generate the desired arylated product $\mathbf{7 2}$, isobutene, and dichloroiron species $\mathbf{7 6}$. Finally, a transmetalation of $\mathbf{7 6}$ with the Grignard reagent regenerates the active species and closes the catalytic cycle.

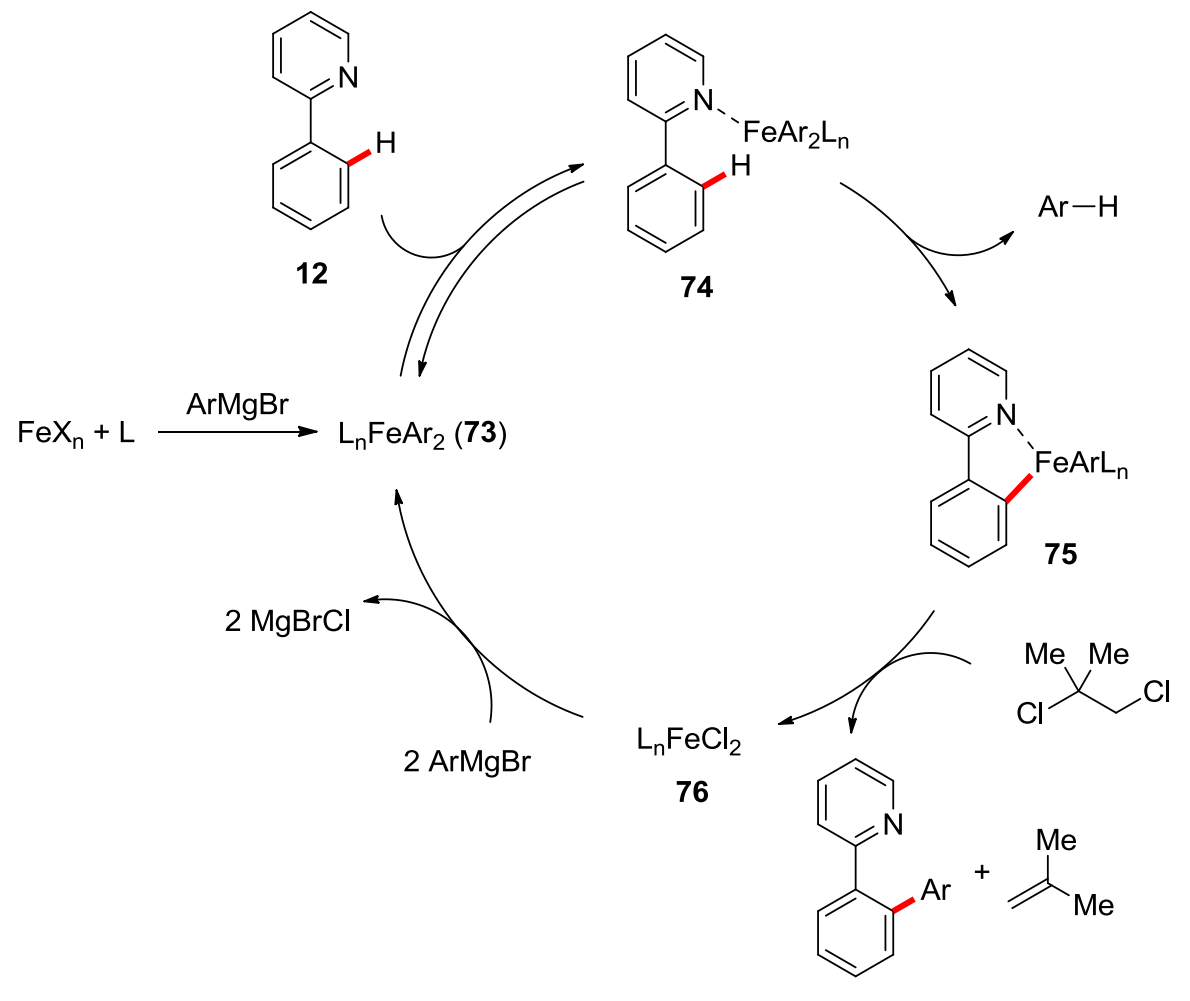

72

Scheme 1.26. Proposed mechanism of the oxidative iron-catalyzed $\mathrm{C}-\mathrm{H}$ arylation. ${ }^{[129]}$

Thereafter, theoretical calculations on the mechanism of this reaction were reported by Shaik and Chen. ${ }^{[131]}$ Their findings suggest that both $\mathrm{Fe}(\mathrm{II})$ and $\mathrm{Fe}(\mathrm{III})$ can promote the $\mathrm{C}-\mathrm{H}$ activation by means of an uncommon two-state reactivity ${ }^{[132]}$ (TSR) scenario. In this way, the initially excited low-spin singlet and doublet states 
crossover through the high-spin ground states to promote the $\mathrm{C}-\mathrm{H}$ scission. Furthermore, the authors suggested the $\mathrm{C}-\mathrm{H}$ cleavage step to occur through a type of $\sigma$-bond metathesis. The key $\mathrm{C}-\mathrm{C}$ bond forming step was proposed to occur via reductive elimination from an iron(III) species, after which the iron catalyst is reoxidized by DCIB via a single electron transfer (SET) mechanism.

A breakthrough in the field was the introduction of bidentate ${ }^{[133]}$ directing groups, which not only allowed for unprecedented iron-catalyzed $\mathrm{C}\left(\mathrm{sp}^{3}\right)-\mathrm{H}$ activations, but also significantly expanded the scope of possible transformations beyond oxidative arylations with organometallic reagents. In this context, E. Nakamura reported on an iron-catalyzed $\mathrm{C}\left(\mathrm{sp}^{3}\right)-\mathrm{H}$ arylation of carboxamides 77 under the assistance of the 8-aminoquinoline directing group initially introduced by Daugulis ${ }^{[134]}$ for palladium catalysis (Scheme 1.27a). ${ }^{[135]}$ The important KIE and preference for terminal methyl groups over internal alkyl substituents provided support for an inner-sphere $\mathrm{C}-\mathrm{H}$ activation process rather than a radical pathway. Shortly afterwards, Ackermann designed a modular triazole directing group which proved effective for the ironcatalyzed arylation of both $\mathrm{C}\left(\mathrm{sp}^{2}\right)-\mathrm{H}$ and $\mathrm{C}\left(\mathrm{sp}^{3}\right)-\mathrm{H}$ positions (Scheme 1.27b). ${ }^{[136]}$ It should be noted that all those transformations require bidentate phosphines as the ligands. 
a) E. Nakamura

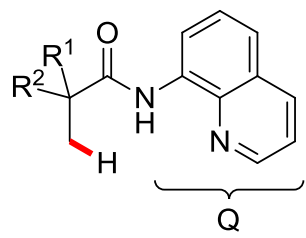

77

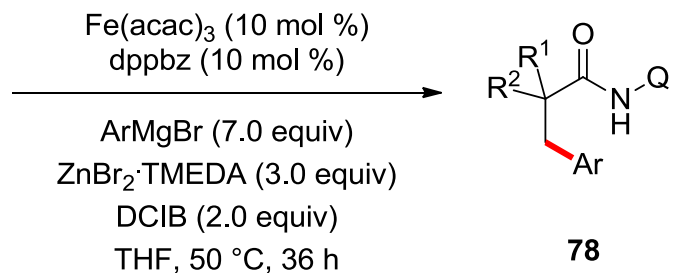

b) Ackermann

i. $C\left(s p^{2}\right)-H$ arylation<smiles>[R]/C=C(/[Y10])C(=O)NC(C)(C)c1cn([R])nn1</smiles>

79

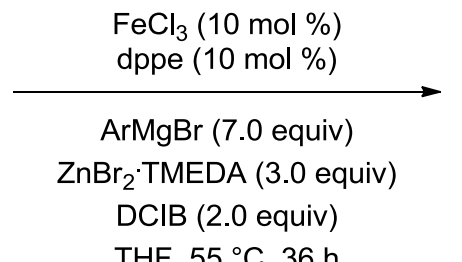

$\mathrm{THF}, 55^{\circ} \mathrm{C}, 36 \mathrm{~h}$<smiles>[Y16]NC(=O)C([R])=C([R])[Al]</smiles>

80

81
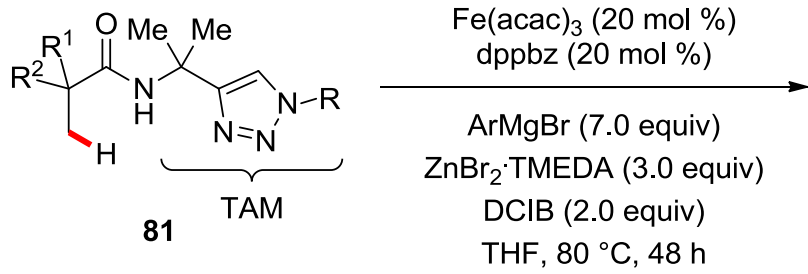

iii. traceless removal of the TAM directing group<smiles>Cc1ccccc1C(=O)N[Te]</smiles>

83

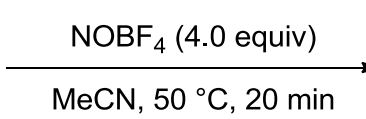

$\mathrm{MeCN}, 50^{\circ} \mathrm{C}, 20 \mathrm{~min}$<smiles>Cc1ccccc1C(=O)O</smiles>

84: $87 \%$

Scheme 1.27. Iron-catalyzed $\mathrm{C}-\mathrm{H}$ arylation under bidentate directing group assistance. ${ }^{[135-137]}$

Other significant advances were subsequently achieved under the assistance of bidentate directing groups by the research groups of E. Nakamura, Ackermann and Cook, among others. Major progresses include, but are not limited to, C-H alkylations with Grignard reagents or alkyl halides, ${ }^{[138]} \mathrm{C}\left(\mathrm{sp}^{2}\right)-\mathrm{H}$ allylations, ${ }^{[137,139]}$ $\mathrm{C}\left(\mathrm{sp}^{2}\right)-\mathrm{H}$ benzylations, ${ }^{[137,138 \mathrm{~d}]} \mathrm{C}\left(\mathrm{sp}^{2}\right)-\mathrm{H}$ alkynylations with alkynyl bromides, ${ }^{[140]}$ 
$\mathrm{C}\left(\mathrm{sp}^{2}\right)-\mathrm{H}$ aminations with $\mathrm{N}$-chloroamines, ${ }^{[141]} \mathrm{C}-\mathrm{H}$ alkenylations and arylations with organoboron reagents, ${ }^{[142]} \mathrm{C}\left(\mathrm{sp}^{2}\right)-\mathrm{H}$ and $\mathrm{C}\left(\mathrm{sp}^{3}\right)-\mathrm{H}$ methylations with $\mathrm{AlMe}_{3},{ }^{[143]}$ various annulation reactions with alkynes ${ }^{[144]}$ and allenes, ${ }^{[145]}$ the $\mathrm{C}\left(\mathrm{sp}^{2}\right)-\mathrm{H}$ alkylation of benzylamine derivatives ${ }^{[146]}$ and the two-fold $\mathrm{C}-\mathrm{H}$ activation/cross-coupling of heteroarenes. ${ }^{[147]}$

A rare iron-catalyzed $\mathrm{C}-\mathrm{H}$ activation by hydroarylation was disclosed by Yoshikai in $2015 .^{[148]}$ Taking inspiration from their previous work on cobalt catalysis ${ }^{[105,106]}$ (Scheme 1.19) and from the similar reactivity of iron and cobalt complexes in stoichiometric $\mathrm{C}-\mathrm{H}$ activations, ${ }^{[63 \mathrm{c}]}$ the authors devised an iron-NHC catalyst for the addition of 3-iminoindoles 59 to styrenes 60 (Scheme 1.28a). ${ }^{[148]}$ Slight modifications of the reaction conditions allowed for the use of alkynes 7 as well (Scheme 1.28b).

a) iron-catalyzed hydroarylation of styrenes

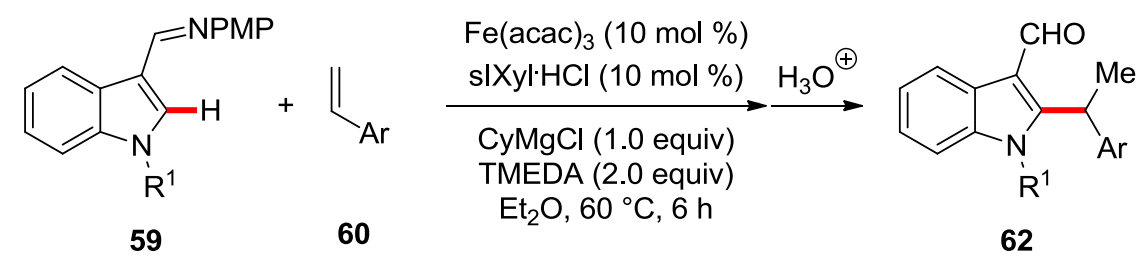

b) iron-catalyzed hydroarylation of internal alkynes

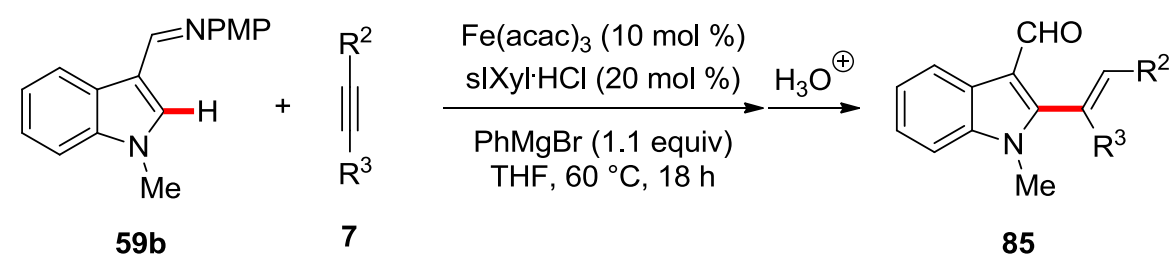

Scheme 1.28. Iron-catalyzed hydroarylations of styrenes and alkynes with indoles 59. ${ }^{[148]}$

Despite major progress, iron-catalyzed $\mathrm{C}-\mathrm{H}$ activation is still a recent field of research. Among other expected advances, the development of enantioselective $\mathrm{C}-\mathrm{H}$ functionalizations is highly desirable. In this context, it should be noted that examples of asymmetric transformations by organometallic iron catalysts remain extremely rare. Indeed, only a single example had been reported at the outset of the 
present work, namely an iron-catalyzed cross-coupling of $\alpha$-chloroesters 86 with aryl Grignard reagents by $M$. Nakamura (Scheme 1.29). ${ }^{[149]}$ This approach would later be extended to arylborates. ${ }^{[150]}$

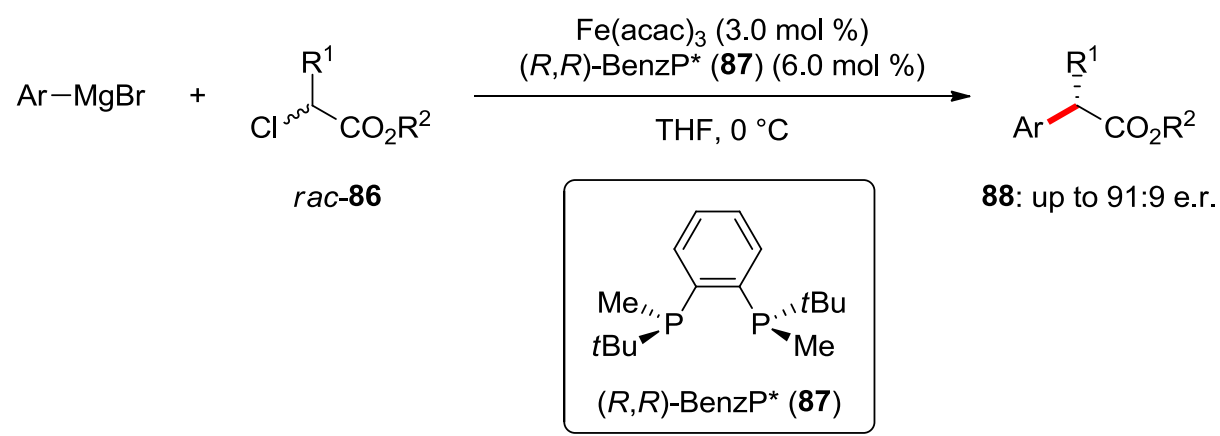

Scheme 1.29. Iron-catalyzed enantioselective cross-coupling of $\alpha$-chloroesters 86 with Grignard reagents. ${ }^{[149]}$

While our work ${ }^{[151]}$ represents the very first enantioselective functionalization by ironcatalyzed inner-sphere C-H activation (see Chapter 3.2), it is noteworthy that Butenschön reported shortly afterwards an asymmetric arylation of ferrocene derivatives 89, providing the planar-chiral product 91 in moderate enantiomeric excess (Scheme 1.30). ${ }^{[152]}$ It should be noted that Ackermann had previously identified ferrocene amides $\mathbf{8 9}$ as viable substrates in iron-catalyzed $\mathrm{C}-\mathrm{H}$

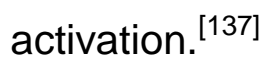

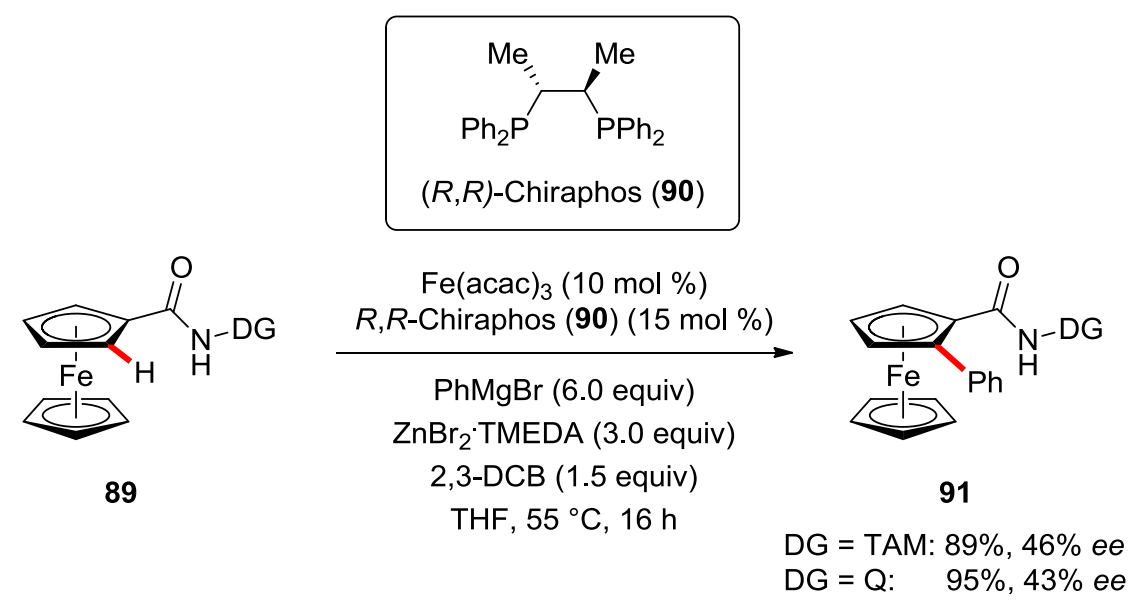

Scheme 1.30. Enantioselective iron-catalyzed $\mathrm{C}-\mathrm{H}$ arylation of ferrocenes $89 .{ }^{[152]}$ 


\subsection{Nickel-Catalyzed C-H Activation}

\subsubsection{General Information}

Nickel catalysts have been recognized as powerful tools in molecular syntheses, with numerous applications in $\mathrm{C}-\mathrm{C}$ bond forming processes. ${ }^{[153]}$ Among others, applications to cross-coupling chemistry, ${ }^{[18 a, 18 b, 18 d, 154]}$ and the functionalization of otherwise inert $\mathrm{C}-\mathrm{O},{ }^{[18 \mathrm{c}, 18 \mathrm{e}, 155]} \mathrm{C}-\mathrm{F},{ }^{[156]}$ and $\mathrm{C}-\mathrm{H}$ bonds ${ }^{[45,48]}$ have gained significant momentum in recent years. While nickel has been considered as the "impoverished younger sibling of palladium", ${ }^{[153 \mathrm{~d}]}$ its high reactivity and unique properties, such as facile oxidative addition and a number of readily available oxidation states, render it particularly attractive in catalysis. ${ }^{[153 \mathrm{c}, 153 \mathrm{~d}]}$ Furthermore, it should be noted that the use of nickel in catalysis, notably in cross-couplings, actually pre-dates many noble

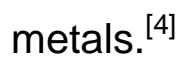

The potential of nickel for $\mathrm{C}-\mathrm{H}$ activation was first demonstrated by Dubeck and Kleiman in $1963,{ }^{[157]}$ who prepared the cyclometalated complex 93 by the reaction of nickelocene 92 with azobenzene 4 (Scheme 1.31a). Thereafter, no other stoichiometric nickelation of non-activated ${ }^{[158]} \mathrm{C}-\mathrm{H}$ bonds was reported for several decades, until Liang discovered in 2006 that the pincer nickel complex 94 could react with benzene to deliver 95 without the need of a directing group (Scheme 1.31b). ${ }^{[159]}$ 
a) cyclometalation of azobenzene $\mathbf{4}$ with nickelocene 92

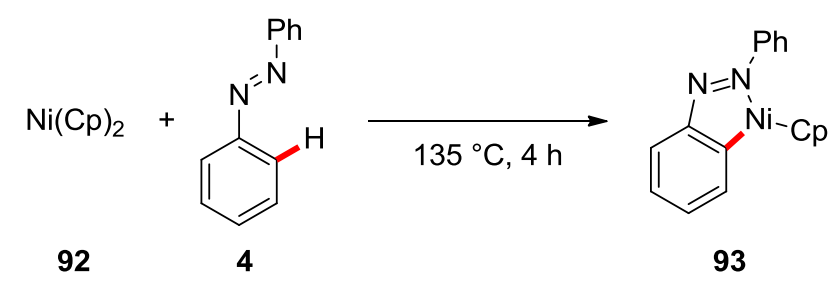

b) undirected $\mathrm{C}-\mathrm{H}$ nickelation of benzene

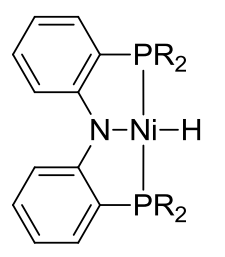

$94(\mathrm{R}=i \mathrm{Pr}, \mathrm{Cy})$

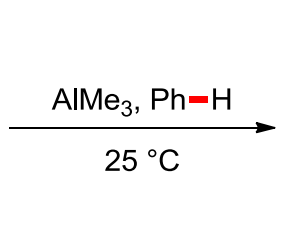

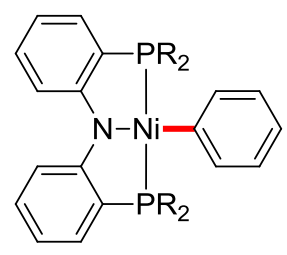

95

Scheme 1.31. Stoichiometric nickel-mediated C-H activations. ${ }^{[157,159]}$

In the same year, Nakao and Hiyama discovered by serendipity that nickel(0) species could catalyze the functionalization of activated, that is somewhat acidic, C-H bond with alkynes 7 (Scheme 1.32). ${ }^{[160]}$ Tsuda and Saegusa had previously demonstrated the potential of nickel catalysts in related hydroacylation reactions. ${ }^{[158 f]}$ The selective activation of a $\mathrm{C}-\mathrm{H}$ bond over a $\mathrm{C}-\mathrm{CN}$ bond of $\mathrm{N}$-protected 3-cyanoindoles 96 could be controlled by the proper choice of the ligand and $N$-protecting group of the indole. ${ }^{[160]}$ Under the optimized reaction conditions, diverse azoles 99, including benzimidazoles, electron-poor indoles, caffeine, benzofuran, benzothiophene, benzoxazole and thiazole, could be alkenylated selectively at the C2 position. Based on preliminary experimental mechanistic studies, the authors proposed the $\mathrm{C}-\mathrm{H}$ cleavage step to occur via oxidative addition. However, a later study by Zimmerman and Montgomery suggested the possibility of a ligand-to-ligand hydrogen transfer (LLHT) manifold. ${ }^{[161]}$ 
a) serendipitous discovery of nickel-catalyzed $\mathrm{C}-\mathrm{H}$ alkenylation

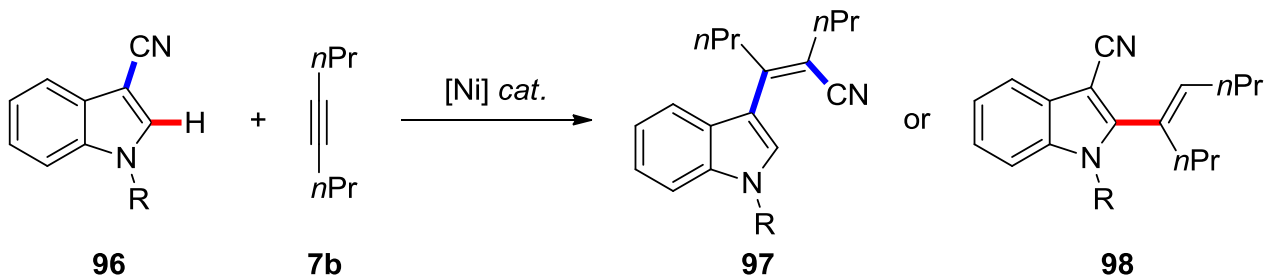

b) nickel-catalyzed $\mathrm{C}-\mathrm{H}$ alkenylation under optimized conditions

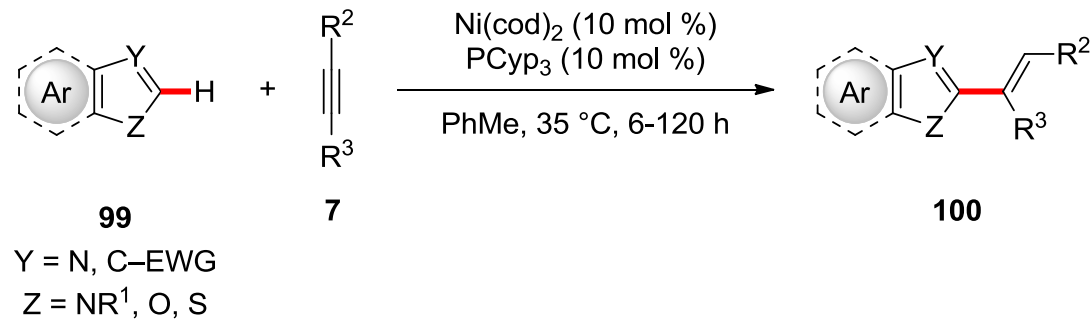

Scheme 1.32. Nickel-catalyzed hydroheteroarylation of alkynes with azoles. ${ }^{[160]}$

Afterwards, the scope of the hydroheteroarylation of alkynes was expanded to other heterocycles, with major contributions of Nakao/Hiyama, Ong/Yap and Miura. Among others, oxadiazoles, ${ }^{[162]}$ pyrazoles, ${ }^{[163]}$ simple imidazoles, ${ }^{[164]}$ pyridine oxides,${ }^{[165]}$ pyridines ${ }^{[166]}$ pyridones $^{[167]}$ and pentafluorobenzene ${ }^{[168]}$ were identified as viable substrates in the nickel-catalyzed hydro(hetero)arylation of alkynes. For substrates bearing less acidic $\mathrm{C}-\mathrm{H}$ bonds, the addition of co-catalytic Lewis acidic organoaluminium additives was needed for the reaction to occur. In addition to azoles 99, formamides proved to be suitable substrates as well. ${ }^{[169]}$ Taking inspiration of the success of nickel catalysts in the hydroarylation of alkynes, this chemistry was later expanded to alkenes (vide infra) and even allenes. ${ }^{[170]}$

While not directly relevant to the topic of this thesis, it should be mentioned that other non-hydroarylation-type nickel-catalyzed $\mathrm{C}-\mathrm{H}$ activations have been reported as well. Among other transformations, the arylation, ${ }^{[171]}$ alkylation with alkyl (pseudo)halides, ${ }^{[172]}$ alkynylation with alkynyl bromides ${ }^{[173]}$ or terminal alkynes ${ }^{[174]}$ and alkenylation ${ }^{[171 a, 171 d, 175]}$ of $\mathrm{C}-\mathrm{H}$ acidic azoles are nowadays well established processes. Furthermore, nickel-catalyzed $\mathrm{C}-\mathrm{H}$ activations are not limited to $\mathrm{C}-\mathrm{H}$ 
acidic heteroarenes. Indeed, truly unactivated $\mathrm{C}-\mathrm{H}$ bonds have been functionalized as well, but this chemistry remains so far mostly restricted to the use of bidentate directing groups. ${ }^{[176]}$ In this context, the $\mathrm{C}\left(\mathrm{sp}^{2}\right)-\mathrm{H}$ arylation, ${ }^{[177]}$ alkylation, ${ }^{[178]}$ alkynylation with alkynyl bromides ${ }^{[179]}$ or terminal alkynes, ${ }^{[180]}$ alkyne annulation, ${ }^{[181]}$ allylation, ${ }^{[178 d, 182]}$ carbonylation $^{[183]}$ and thiolation ${ }^{[184]}$ of benzamide derivatives have inter alia been achieved with nickel catalysts. The use of bidentate directing groups also allowed the functionalization of $\mathrm{C}\left(\mathrm{sp}^{3}\right)-\mathrm{H}$ positions. ${ }^{[45,48 \mathrm{c}, 176]}$ While $\mathrm{C}-\mathrm{H}$ transformations of unactivated arenes were long restricted to the use of bidentate directing groups, major progress in the field was reported by Ackermann who recently introduced the simple and easily removable 2-pyrimidyl directing group for the ortho-functionalization of aniline derivatives. ${ }^{[185]}$ Similarly, Punji disclosed several nickel-catalyzed $\mathrm{C}-\mathrm{H}$ functionalizations of indoles bearing monodentate directing groups. ${ }^{[186]}$

\subsubsection{Nickel-Catalyzed C-H Activation by Alkene Hydroarylation}

The use of alkenes has gained considerable attention in transition metal-catalyzed C-H activation. ${ }^{[187]}$ Indeed, due to their low cost, availability and sustainability (no need for pre-functionalization), alkenes are attractive coupling partners for the formation of $\mathrm{C}-\mathrm{C}$ bonds. In this context, nickel catalysts have proven particularly powerful for the hydrofunctionalization of $\mathrm{C}-\mathrm{C}$ multiple bonds. ${ }^{[48 \mathrm{a}, 188]}$ Furthermore, the hydroarylation of alkenes generates $\mathrm{C}\left(\mathrm{sp}^{3}\right)$ positions, offering opportunities for the development of asymmetric transformations employing chiral nickel catalysts.

Taking inspiration from their pioneering works on the hydroarylation of alkynes, ${ }^{[188 a]}$ Nakao and Hiyama reported in 2008 the unprecedented hydroarylation of conjugated alkenes with pentafluorobenzene (101) using reaction conditions nearly identical to those used for alkynes (Scheme 1.33a). ${ }^{[168]}$ Interestingly, the branched product was selectively obtained. Miura disclosed in 2009 the hydroarylation of styrenes 60 with oxadiazoles 104 using $\mathrm{Ni}(\operatorname{cod})_{2}$ and the bidentate phosphine ligand Xantphos as the catalytic system (Scheme 1.33b). ${ }^{[162]}$ Similar findings were reported 
shortly afterwards by Nakao and Hiyama who identified the NHC ligand IMes as optimal for the envisioned transformation (Scheme 1.33c). ${ }^{[189]}$ Remarkably, the authors were also able to use simple alkyl-substituted alkenes for the first time under more forcing conditions. In sharp contrast to conjugated alkenes, the linear product was obtained in this case. The nickel/NHC manifold would later prove broadly applicable in the hydroarylation of alkenes with (hetero)arenes. ${ }^{[45,48 a]}$

a) Nakao/Hiyama

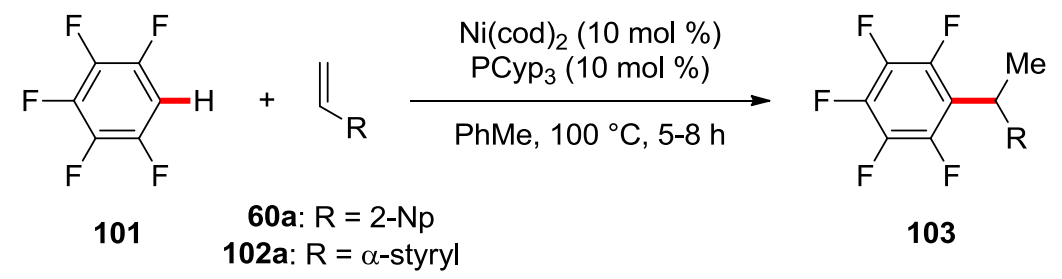

b) Miura

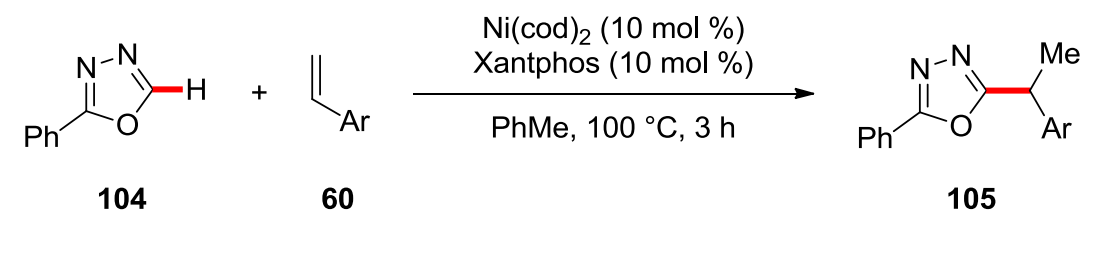

c) Nakao/Hiyama

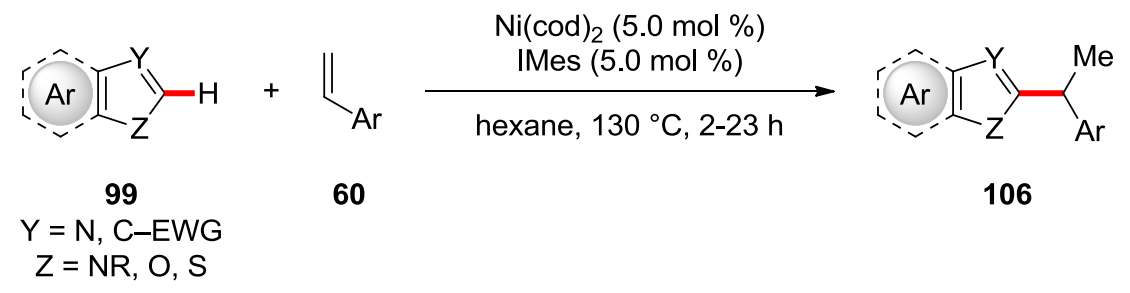

Scheme 1.33. Early examples of nickel-catalyzed hydroarylations of alkenes with (hetero)arenes. ${ }^{[162,168,189]}$

Based on deuterium-labeling experiments, the $\mathrm{C}-\mathrm{H}$ cleavage step was proposed to occur via a reversible oxidative addition of a nickel( 0$)$ complex into the $\mathrm{C}-\mathrm{H}$ bond (Scheme 1.34). ${ }^{[162,168,189]}$ Then, coordination of the alkene followed by hydronickelation of the latter produces intermediate 110. Those steps are proposed to be reversible, thus explaining the observed $H / D$ scrambling. Thereafter, an 
irreversible and rate-determining reductive elimination delivers product 106 and regenerates intermediate 107. Furthermore, Nakao/Hiyama and Miura proposed the formation of the Markovnikov product to be favored because of the formation of $\pi$-benzyl or $\pi$-allyl nickel intermediates, which would also explain the poor performance of alkyl-substituted alkenes and acrylates in these reactions. ${ }^{[162,168,189]}$

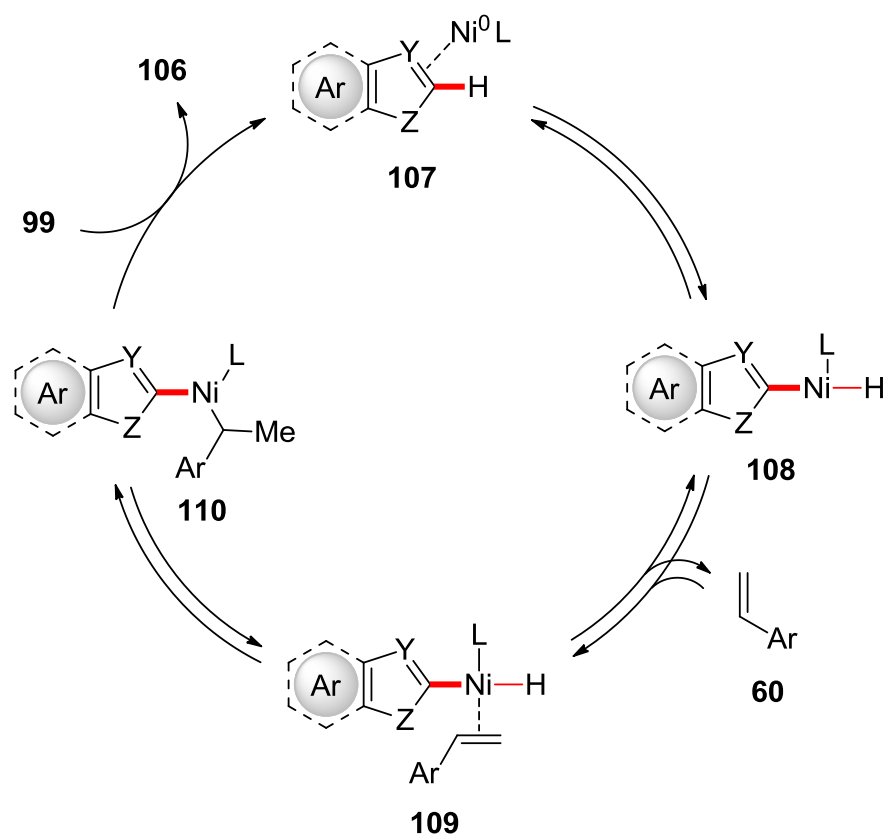

Scheme 1.34. Proposed catalytic cycle of the nickel-catalyzed hydroarylation of vinylarenes 60 with azoles $99 .^{[189]}$

Subsequently, the origin of the regioselectivity of the reaction was investigated in detail by DFT by Shi. ${ }^{[190]}$ This study provided support to the mechanism proposed by Nakao, Hiyama and Miura, and revealed a secondary orbital overlap between the styrene's aryl group and the nickel center. This interaction was found to accelerate the rate-limiting $\mathrm{C}-\mathrm{C}$ bond forming reductive elimination and favor the formation of the Markovnikov product from conjugated alkenes. In case of simple alkylsubstituted olefins, this interaction is absent and the sterically less hindered linear product is thus formed favorably.

A major breakthrough in the nickel-catalyzed hydroarylation of alkenes was the introduction of Lewis acidic organoaluminium additives, which not only allowed 
transformations of unactivated heteroarenes and alkenes, but also proved critical to control the selectivity of the reaction. Among other substrates, pyridones ${ }^{[167,191]}$ and pyridines ${ }^{[192]}$ could be coupled with alkenes in the presence of $\mathrm{AlMe}_{3}$ or MAD as additive, as observed previously for the hydroarylation of alkynes. While nonconjugated alkenes otherwise remain very challenging in nickel-catalyzed hydroarylations, organoaluminium additives were found to facilitate their use in either inter- or intramolecular reactions with various heteroarenes. ${ }^{[167,191-193]}$ The regioselectivity of those transformations is of particular interest. While the branched product $\mathbf{1 0 6}$ is normally obtained from conjugated alkenes, aliphatic olefins usually provide the linear product. ${ }^{[190]}$ In this context, Ong was able to reverse the usual selectivity of the hydroarylation of styrene 60 with benzimidazoles 99 by the addition of co-catalytic $\mathrm{AlMe}_{3}$ (Scheme 1.35). ${ }^{[194]}$ Unselective mixtures were obtained with other Lewis acids.

a) branched-selectivity under Al-free conditions

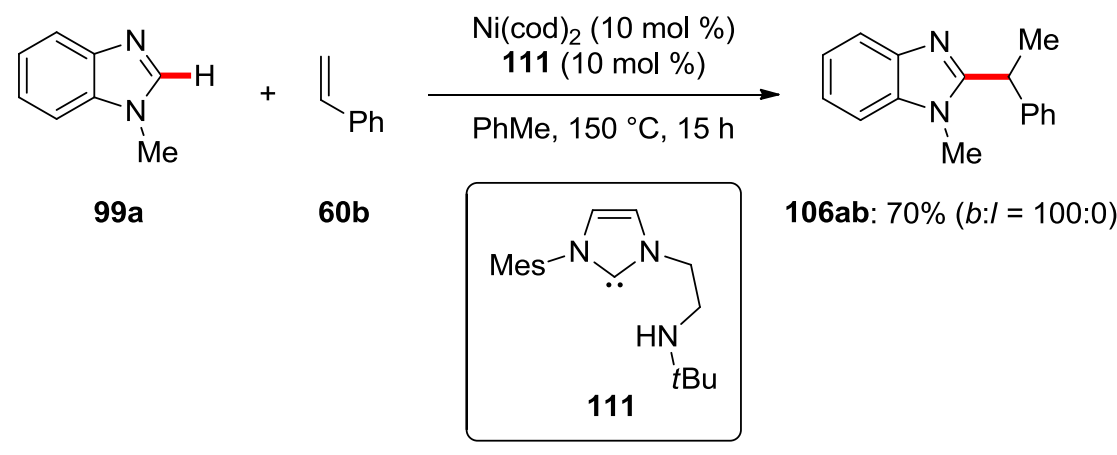

b) linear-selectivity with $\mathrm{AlMe}_{3}$

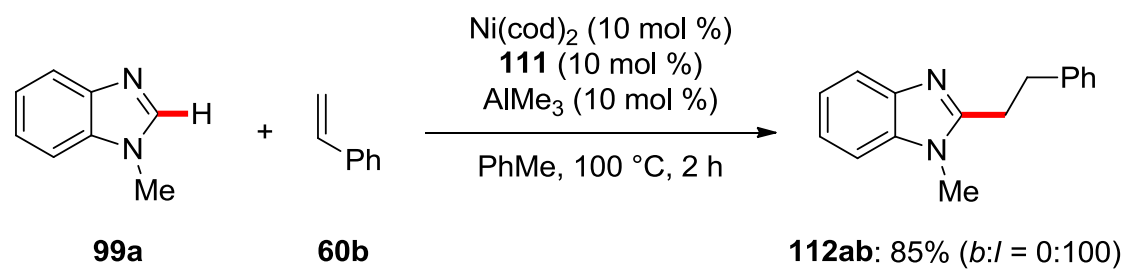

Scheme 1.35. Regioselectivity control in nickel-catalyzed hydroarylations of styrenes 60 with benzimidazoles $99 .{ }^{[194]}$ 
Detailed mechanistic studies were conducted by Ong and coworkers to delineate the mechanism of this process and the role of $\mathrm{AlMe}_{3}{ }^{[194 a]}$ Their findings revealed that $\mathrm{AIMe}_{3}$ not only controlled the regioselectivity of the transformation, but also significantly increased the rate of product formation. In contrast, the aluminium-free reaction was found to be much slower, still being in its induction period after $3 \mathrm{~h}$. The authors were also able to observe a $\mathrm{Ni}-\mathrm{H}$ species, which may indicate an oxidative addition pathway to be involved in the transformation. Furthermore, the adduct of the benzimidazole substrate and $\mathrm{AlMe}_{3}$ was isolated. Based on those findings, the linear selectivity was proposed to result from steric control during the insertion of the styrene into the $\mathrm{Ni}-\mathrm{H}$ bond (Scheme 1.36). In contrast, in the absence of the organoaluminium additive, hydride insertion at the $\beta$-carbon of styrene is electronically favored, giving the branched product.

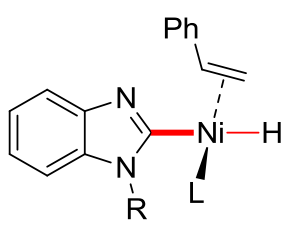

109

electronic control vs.

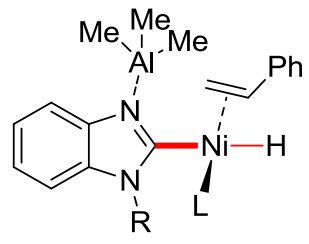

109'

steric control

Scheme 1.36. Model for selectivity switch in the nickel-catalyzed hydroarylation of styrenes. ${ }^{[194]}$

\subsubsection{Enantioselective Nickel-Catalyzed C-H Activation}

While nickel-catalyzed inner-sphere $\mathrm{C}-\mathrm{H}$ activations, especially hydroarylations, are nowadays well established, asymmetric transformations remain scarce. Thus far, all known examples involve the asymmetric functionalization of alkenes. ${ }^{[52]}$

Taking inspiration from previous works by Nakao and Hiyama, ${ }^{[169]}$ Cramer reported an enantioselective nickel-catalyzed intramolecular hydrocarbamoylation of homoallylic formamides 113 (Scheme 1.37). ${ }^{[195]}$ In this context, it should be mentioned that $(\mathrm{C}=\mathrm{O})-\mathrm{H}$ activations have been accomplished with a broad range of 
catalysts, with the hydroacylations of alkenes and alkynes being extensively documented. ${ }^{[196]}$ A significant fraction of formyl C-H activations has been proposed to occur through radical mechanisms, ${ }^{[31 \mathrm{a}]}$ taking advantage of the low bond dissociation energy (BDE) of the formyl $\mathrm{C}-\mathrm{H}$ bond. ${ }^{[28]}$ The chiral heteroatomsubstituted secondary phosphine oxide ${ }^{[197]}$ (HASPO) 114 preligand developed by Cramer enabled the asymmetric synthesis of pyrrolidinones 115 , featuring a Ni/Al ${ }^{[198]}$ heterobimetallic ${ }^{[23 b]}$ activation mode. While a nickel catalyst solely prepared from the chiral SPO performed poorly, the addition of a co-catalytic amount of phosphine led to an increased efficacy, presumably by assisting the displacement of the cod ligand from the precatalyst. This work is truly remarkable as it represents the first enantioselective transformation by inner-sphere $\mathrm{C}-\mathrm{H}$ activation with a $3 \mathrm{~d}$ transition metal catalyst. ${ }^{[52]}$
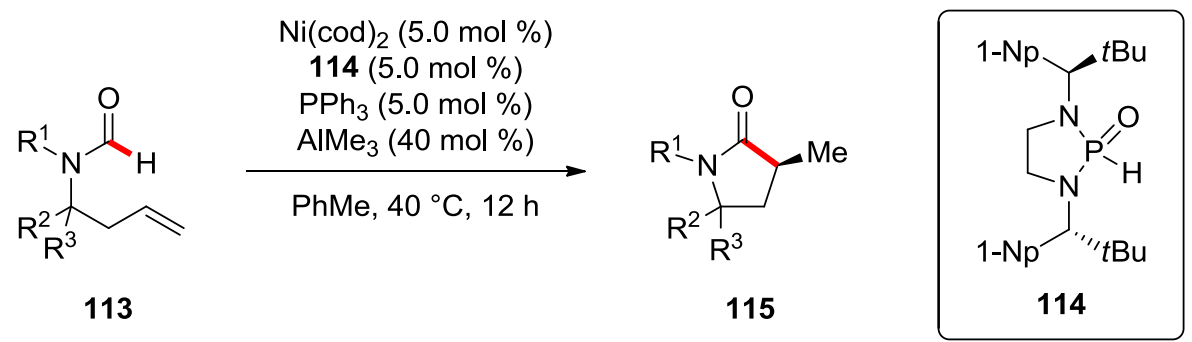

Scheme 1.37. Enantioselective intramolecular nickel-catalyzed hydrocarbamoylations of alkenes. ${ }^{[195]}$

A reasonable catalytic cycle was proposed to begin with the formation of the Al/SPO adduct 116, a bifunctional ligand whose aluminium center retains its Lewis acidity while the Lewis basic phosphorous atom can coordinate to the nickel center (Scheme 1.38). The aluminium center then activates the carbonyl group of 113, providing intermediate 118. Thereafter, oxidative of the $\mathrm{C}-\mathrm{H}$ bond on nickel generates the six-membered hetero-bimetallacycle 119. Migratory insertion then leads to complex 120, and reductive elimination releases lactam 115 and regenerates the heterobimetallic catalyst 117. This mechanism was further supported by the independent synthesis of the Lewis acid/SPO adduct 116, which 
was found to promote the cyclization with excellent yield and enantioselectivity without additional $\mathrm{AlMe}_{3}$ or $\mathrm{PPh}_{3}$.

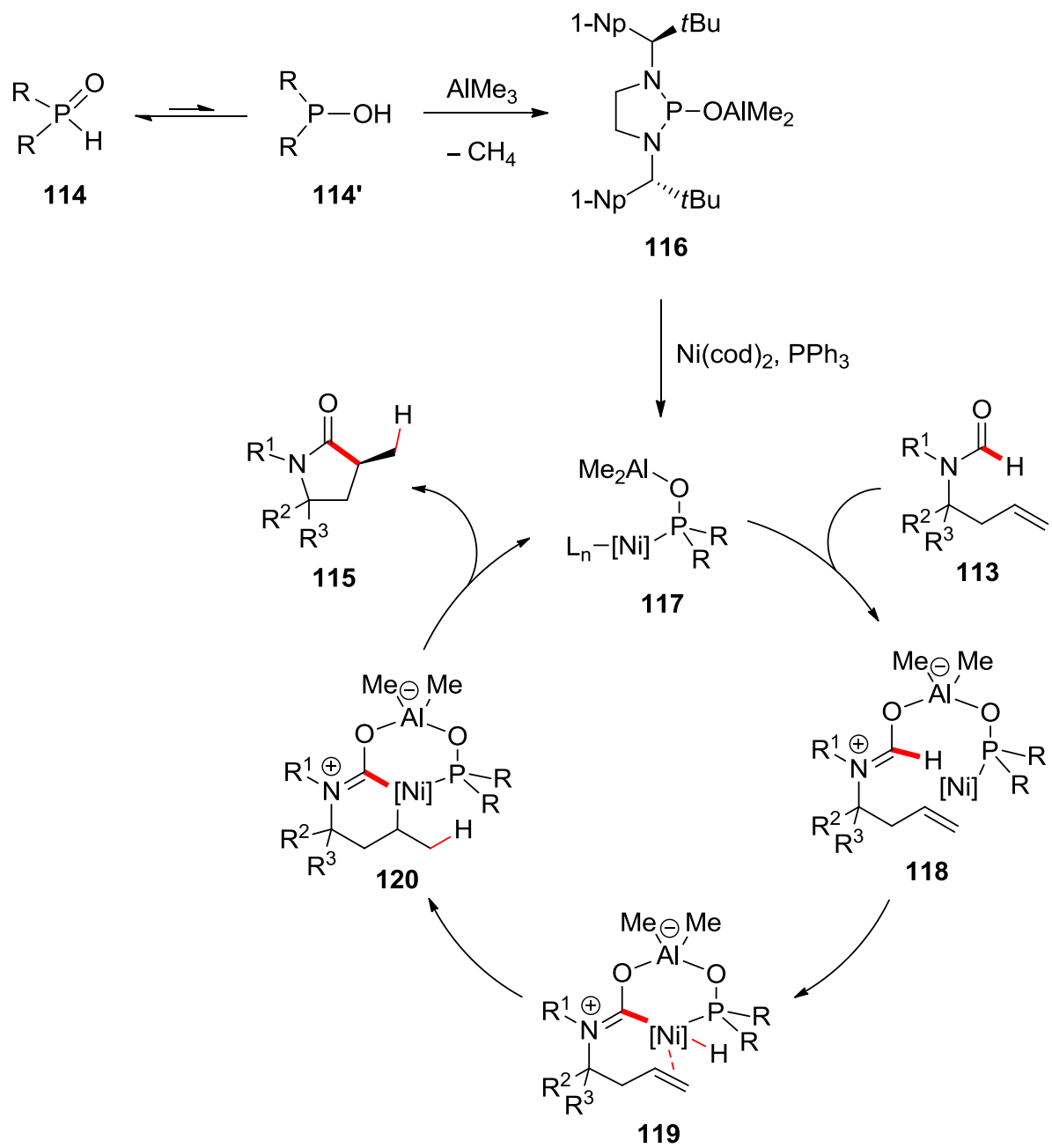

Scheme 1.38. Plausible mechanism of the nickel-catalyzed hydrocarbamoylation. ${ }^{[195]}$

As previously mentioned, Nakao and Hiyama developed an intramolecular $\mathrm{C}-\mathrm{H}$ alkylation of pyridones with unactivated tethered alkenes using a nickel-P(iPr $)_{3}$ catalytic system in the presence of $\mathrm{AlMe}_{3} .^{[167]}$ Inspired by these results, Cramer subsequently developed a ligand-controlled regiodivergent annulation of pyridones 121, with IPr giving selectively the endo-cyclized product, while the exo-product was obtained with cod as the ligand. ${ }^{[193]}$ Preliminary efforts towards an asymmetric version of this reaction were also disclosed (Scheme 1.39a). The chiral NHC ligand 
122, based on the design of Hong and coworkers, ${ }^{[199]}$ provided the endo-cyclized products 123 in 78.5:21.5 e.r.

a) Cramer, 2015

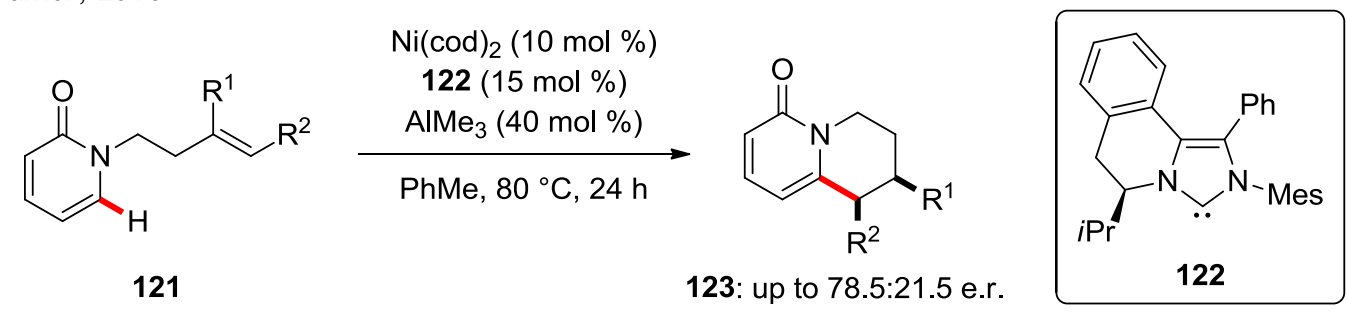

b) Cramer, 2018
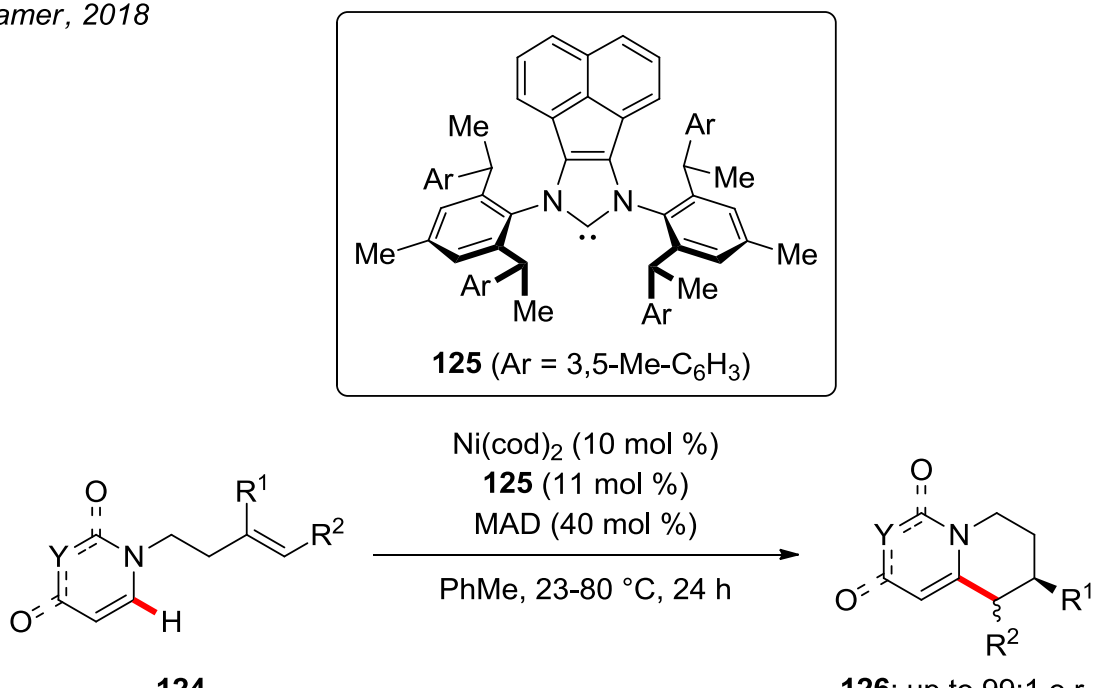

Scheme 1.39. Enantioselective nickel-catalyzed hydroarylation with pyridones. ${ }^{[193,200]}$

Further investigations by Cramer on the asymmetric cyclization of pyridones with tethered olefins led to the discovery of the novel chiral NHC 125, inspired from a previous ligand design by Gawley ${ }^{[201]}$ with a modified acenaphthene backbone (Scheme 1.39b). ${ }^{[200]}$ Under the optimized reaction conditions, the endo-cyclized annulated pyridones and uracils 126 were obtained from diversely decorated alkenes 124 in excellent yields and enantiomeric excesses at mild reaction temperatures in the presence of MAD. Based on literature precedents, ${ }^{[202]}$ the authors proposed the $\mathrm{C}-\mathrm{H}$ cleavage step to occur through a LLHT manifold. This approach was later extended to pyridines 127 by Shi under similar reaction 
conditions (Scheme 1.40). ${ }^{[203]}$ Thus, the corresponding tetrahydro(iso)quinolines 129 were obtained with excellent diastereo- and regio-selectivities.
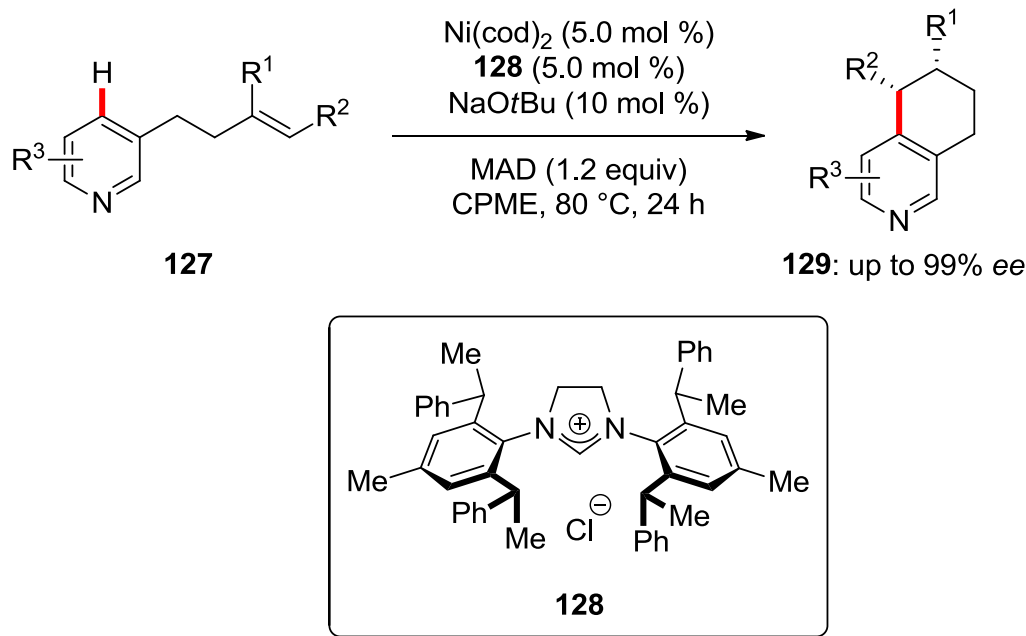

Scheme 1.40. Enantioselective nickel-catalyzed hydroarylation with pyridines $127 .{ }^{[203]}$

Following the elegant studies of Bergman and Ellman, ${ }^{[204]}$ the undirected cyclization of azoles with tethered alkenes has long been dominated by rhodium(I) catalysts, with a notable exception by Cavell for the nickel-catalyzed cyclization of highly activated (benz)imidazolium salts. ${ }^{[205]}$ In this context, $Y e$ reported on the unprecedented nickel-catalyzed enantio- and exo-selective hydroarylation of olefins 130 with tethered imidazoles (Scheme 1.41). ${ }^{[206]}$ The TADDOL-derived HASPO preligand 131 enabled a nickel-aluminum bimetallic catalysis. Such TADDOLHASPOs had previously been exploited in asymmetric organocatalysis, ${ }^{[207]}$ but their use in enantioselective transition-metal catalysis had remained rare. ${ }^{[208]}$ Thus, diverse polycyclic azoles 132 with $\beta$-stereocenters were obtained in outstanding yields and enantioselectivities. Interestingly, sensitive functional groups, including bromo-substituents, as well as diversely substituted alkenes, proved viable in the nickel catalysis. 

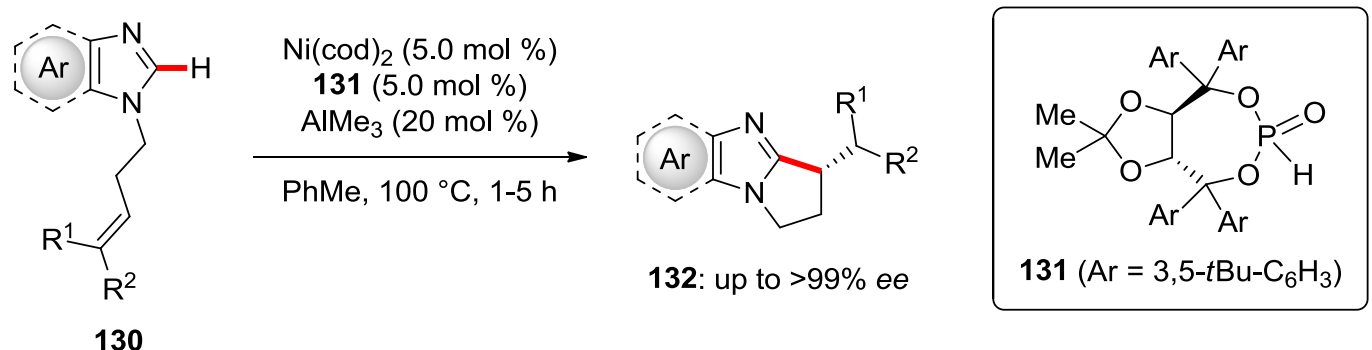

Scheme 1.41. Asymmetric nickel-catalyzed exo-selective hydroarylation of alkenes $130 .{ }^{[206]}$

In analogy to the hydrocarbamoylation presented above (Schemes 1.37-1.38), a plausible catalytic cycle begins with the formation of an Al/SPO adduct, which can coordinate the nickel precursor to deliver complex 133 (Scheme 1.42). Then, through a heterobimetallic mode of activation, the aluminium center can be coordinated by the imidazole's nitrogen, while the nickel center binds the alkene to give intermediate 134. The authors suggested the $\mathrm{C}-\mathrm{H}$ cleavage step to occur through a direct LLHT from the imidazole to the olefin, but an oxidative addition pathway could not be entirely ruled out. 


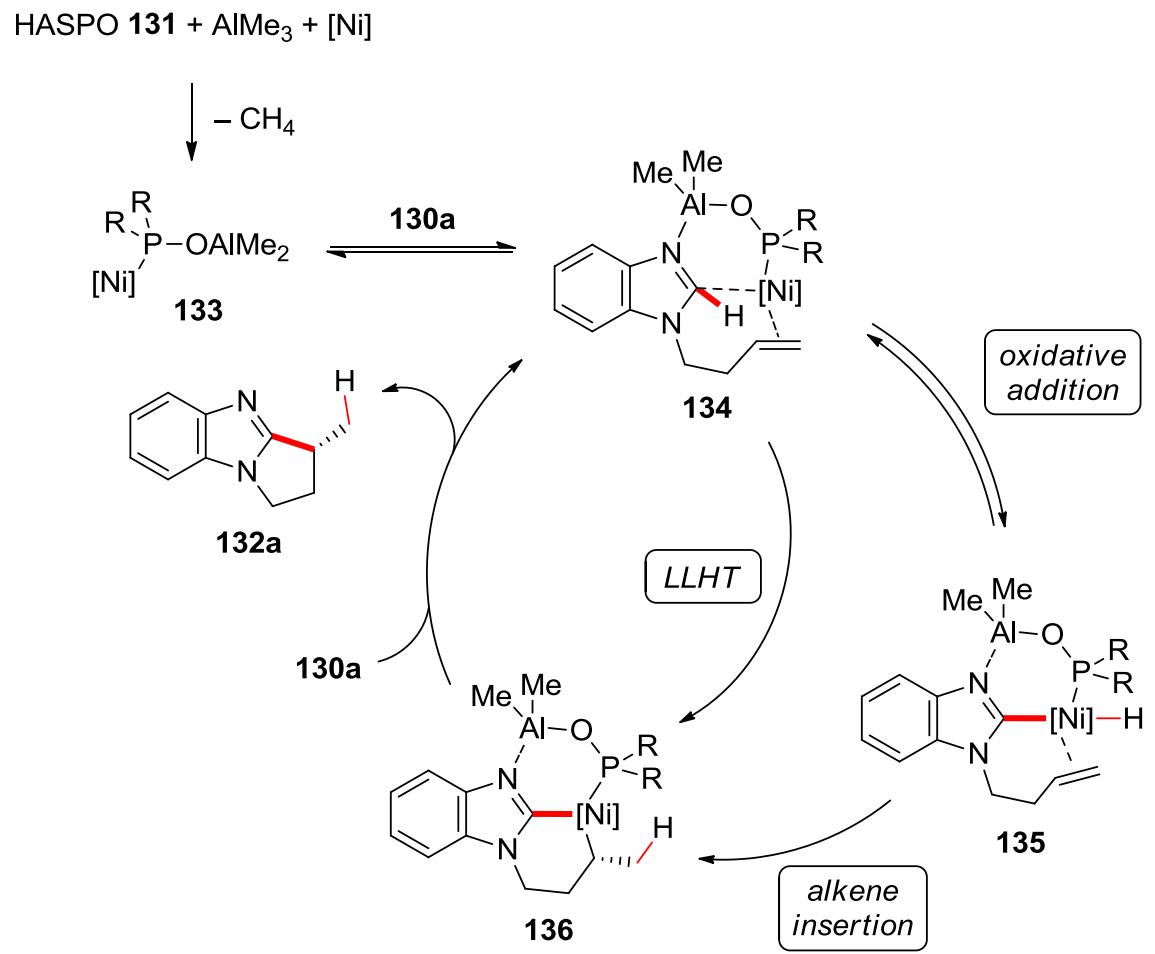

Scheme 1.42. Proposed mechanism of the asymmetric nickel-catalyzed exo-selective hydroarylation. ${ }^{[206]}$

While nickel-catalyzed intramolecular asymmetric hydroarylations have been recognized as a powerful tool for the synthesis of important polycyclic bioactive scaffolds, enantioselective intermolecular versions remain hitherto largely unknown.

In 2011, Fukuzawa disclosed an elegant nickel/NHC-catalyzed three-component reaction between benzaldehydes 138, norbornenes 137, and silanes 139 leading to polycyclic indanols 141. ${ }^{[209]}$ Thereafter, Cramer designed the chiral NHC ligand 140 to achieve this transformation in an enantioselective fashion (Scheme 1.43). ${ }^{[210]}$ Interestingly, while the flanking $\mathrm{N}$-aryl substituents of Grubbs-type chiral $\mathrm{NHCs}^{[211]}$ have been extensively investigated, modifications of the chiral backbone remain underexplored. This transformation allowed for the expedient diastereoselective synthesis of annulated indanols 141 bearing five contiguous stereocenters. 


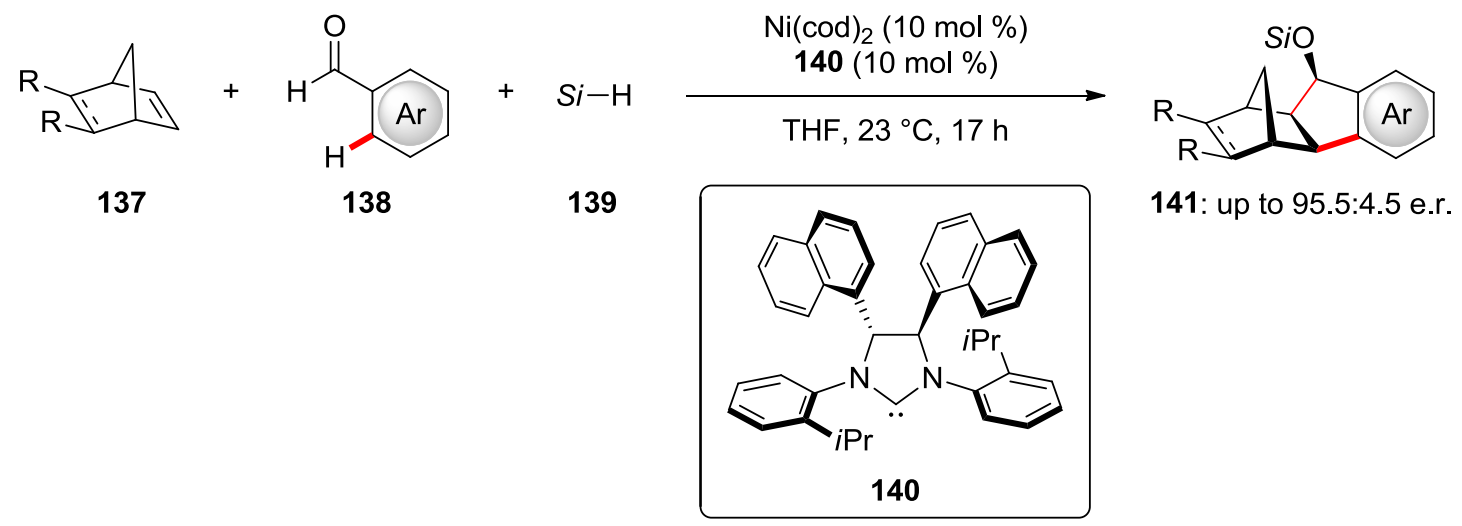

Scheme 1.43. Nickel-catalyzed asymmetric reductive three-component coupling. ${ }^{[210]}$

Despite significant progress in very recent years with non-noble metals, ${ }^{[52]}$ such as nickel $^{[193,200,203,206]}$ and cobalt ${ }^{[106]}$ (Schemes 1.13, 1.19, 1.39-1.41), enantioselective hydroarylation-type $\mathrm{C}-\mathrm{H}$ activations ${ }^{[23 i]}$ remain vastly dominated by costly noble $4 \mathrm{~d}$ and $5 d$ transition metals, such as iridium ${ }^{[212]}$, rhodium, ${ }^{[213]}$ and others, ${ }^{[214]}$ or rareearth complexes. ${ }^{[215]}$ Therefore, the development of new chiral catalysts based on earth-abundant, inexpensive and less-toxic $3 d$ transition metals is highly desirable. 


\section{Objectives}

Methods for the selective functionalization of otherwise inert $\mathrm{C}-\mathrm{H}$ bonds have been recognized as a transformative tool in synthetic organic chemistry, with applications ranging from the synthesis of complex bioactive compounds to material sciences. ${ }^{[23 k, 23 n, 23 p, 40 a, 216]}$ In particular, 3d metal catalysts have emerged in recent years as inexpensive, earth-abundant and less toxic alternatives to their heavier counterparts. ${ }^{[45]}$ However, full selectivity control in base metal-catalyzed $\mathrm{C}-\mathrm{H}$ activation continues to be challenging. ${ }^{[52]}$ In this context, the development of novel $3 d$ transition metal catalysts enabling chemo- and stereo-selective $\mathrm{C}-\mathrm{H}$ functionalizations should be investigated.

As catalytic $\mathrm{C}-\mathrm{H}$ aminations typically rely on noble metal catalysts or require harsh reaction conditions, ${ }^{[23 e, 217]}$ we became interested in the development of a userfriendly and broadly applicable protocol for the cobalt-catalyzed $\mathrm{C}-\mathrm{H}$ amidation of synthetically useful 2-aryloxazolines 142 employing dioxazolones 40 as versatile amidating reagents (Scheme 2.1). ${ }^{[37 \mathrm{e}]}$ Mechanistic studies were performed to delineate the mode of action of the $\mathrm{C}-\mathrm{H}$ activation.

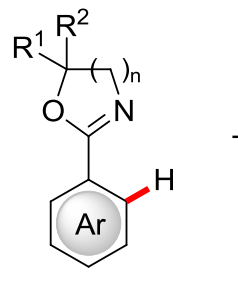

142

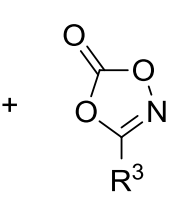

40

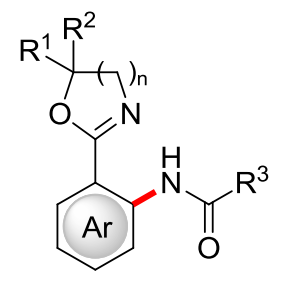

143

Scheme 2.1. Cobalt-catalyzed C-H amidation.

The enantioselective functionalization of $\mathrm{C}-\mathrm{H}$ bonds remains largely dominated by noble transition metal catalysts such as palladium, rhodium and iridium. ${ }^{[51]}$ While significant progress has been very recently achieved by employing earth-abundant non-precious $3 d$ metals, ${ }^{[52]}$ iron-catalyzed asymmetric functionalizations by innersphere $\mathrm{C}-\mathrm{H}$ activation remained completely unprecedented at the outset of this 
work, ${ }^{[47]}$ and represent an exceptional challenge in asymmetric catalysis. In this context, we initiated the development of the first enantioselective iron-catalyzed $\mathrm{C}-\mathrm{H}$ alkylation by alkene hydroarylation (Scheme 2.2). ${ }^{[151]}$ The design of novel chiral NHC ligands proved to be crucial to achieve high enantioselectivities. Furthermore, detailed studies by ${ }^{57} \mathrm{Fe}$ Mössbauer spectroscopy and electrospray-ionization mass spectrometry were conducted to unravel the nature of the in situ generated catalyst. ${ }^{[218]}$

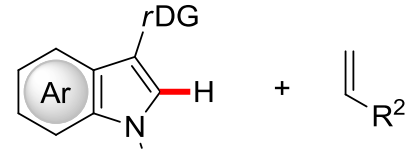

$\mathrm{R}^{1}$

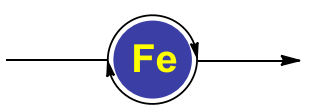

29

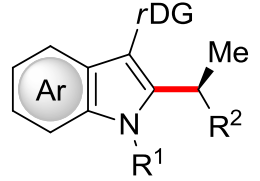

62

Scheme 2.2. Iron-catalyzed enantioselective $\mathrm{C}-\mathrm{H}$ secondary alkylation.

Inspired by the success of nickel/NHC complexes as catalysts for alkene hydroarylations via undirected heteroaromatic $\mathrm{C}-\mathrm{H}$ activation, ${ }^{[27,188 \mathrm{a}]}$ we decided to probe the newly designed chiral NHC ligands in the nickel-catalyzed secondary alkylation of benzimidazoles 99 with styrenes 60 . While nickel-catalyzed intermolecular hydroarylations are well-documented, the development of an asymmetric protocol remains unprecedented. Promising enantioselectivities were observed for the first time in this preliminary work.

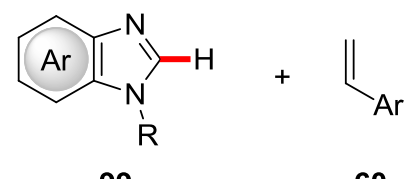

99

60

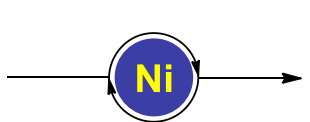

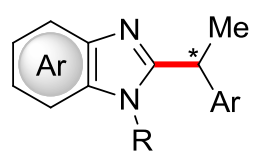

106

Scheme 2.3. Nickel-catalyzed enantioselective intermolecular $\mathrm{C}-\mathrm{H}$ alkylation.

The cyclization of heteroarenes with tethered alkenes has long been dominated by rhodium(I) catalysts, following the elegant pioneering studies by Bergman and Ellman. ${ }^{[204,213 d, 219]}$ Recently, nickel-catalyzed hydroarylation-type C-H activation has 
emerged as a cost-efficient alternative. ${ }^{[27,48 a]}$ However, the intramolecular hydroarylations of unactivated alkenes remain strongly limited by the requirement of pyrophoric organoaluminium additives, significantly compromising their functional group tolerance and synthetic utility. ${ }^{[200,203,206]}$ This observation prompted us to investigate the asymmetric cyclization of $\mathrm{N}$-homoallylimidazoles 144 under aluminium-free conditions (Scheme 2.4). ${ }^{[220]}$ Interestingly, the endo product 145 was selectively obtained, which in sharp contrast to previously reported methods. ${ }^{[206]}$ Mechanistic studies were then conducted in order to delineate the unique reactivity of the developed catalytic system.
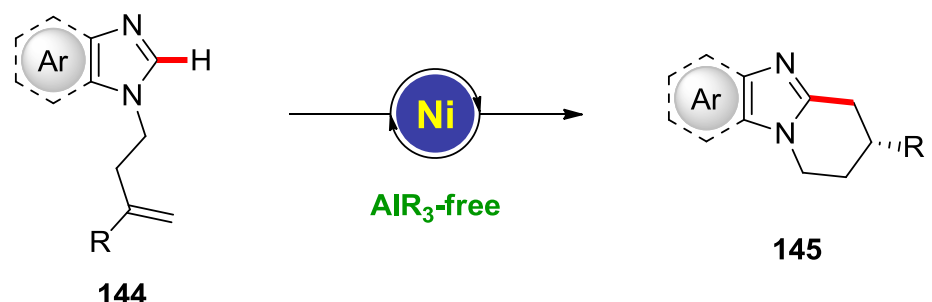

Scheme 2.4. Nickel-catalyzed enantioselective intramolecular C-H alkylation. 


\section{Results and Discussion}

\subsection{Cobalt(III)-Catalyzed C-H Amidation by Oxazoline Assistance}

Diversely decorated 2-aryloxazolines are key structural motifs in bioactive natural products and compounds relevant to the pharmaceutical and agrochemical industries, among others (Scheme 3.1). ${ }^{[221]}$ In the context of this work, it is noteworthy that several ortho-amidated 2-phenyloxazolines have been patented or even commercialized as pesticides or fungicides, with applications to crop protection. ${ }^{[222]}$ Furthermore, oxazolines are versatile and readily accessible synthetic intermediates which can easily be transformed into a wealth of diverse functional groups. ${ }^{[223]}$ Additionally, oxazolines are powerful ligands in transition metal-catalyzed transformations. ${ }^{[224]}$<smiles>CCOc1cc(C(C)(C)C)ccc1C1COC(c2c(F)cccc2F)=N1</smiles>

Etoxazole acaricide/insecticide<smiles>COc1cc([C@H]2CN=C(c3ccc4c(ccn4C)c3)O2)cc(OC)c1OC</smiles>

A-289099 tubulin polymerization inhibitor

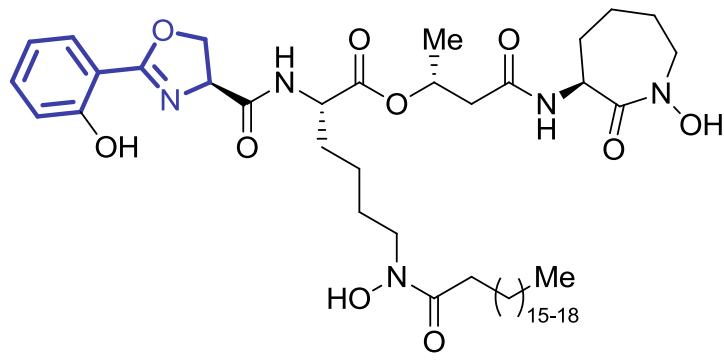

Mycobactin T siderophore/growth factor of Mycobacterium tuberculosis<smiles>Cc1cccc(C2=NCCO2)c1NC(=O)c1cc(C(F)(F)F)nn1-c1ncccc1Cl</smiles>

invertebrate pest control for crop protection<smiles>COc1ccc(C(=O)Nc2ccccc2C2=NCCO2)cc1OC</smiles>

antifungal activity against E. graminis (powdery mildew)<smiles>O=C(Nc1ccccc1C1=NCCO1)c1ccccc1F</smiles>

antifungal activity against L. nodorum (glume blotch)

Scheme 3.1. Selected examples of bioactive 2-aryloxazolines. 
Hence, methods to access highly functionalized oxazoline derivatives are highly sought after by the synthetic community. The ortho-functionalization of 2-aryloxazolines through directed ortho-metalation (DoM) is well documented. ${ }^{[225]}$ However, such methods require relatively harsh reaction conditions, such as an excess of organolithium bases, which strongly limit their functional group tolerance. Therefore, catalytic processes operating under mild conditions and avoiding cryogenic conditions are highly desirable.

Thereby, we became interested in the development of an atom- and stepeconomical method for the diversification of oxazolines. Despite the significant progress of oxidative $\mathrm{C}-\mathrm{H}$ amination reactions in recent years, most pre-existing methodologies either required harsh reaction conditions or are restricted to noble transition metal catalysts, such as iridium, rhodium, palladium and ruthenium. ${ }^{[23 e, 217]}$ Also, many of these processes typically employed azides as aminating reagents, bearing major safety concerns. We hence decided to explore the feasibility of cobalt(III)-catalyzed amidations of 2-aryloxazolines 142 using dioxazolones 40 as user-friendly and safer ${ }^{[226]}$ amination reagents.

\subsubsection{Optimization Studies}

After initial results were obtained by Dr. Ruhuai Mei for the $\mathrm{Cp}^{*} \mathrm{Co}(\mathrm{III})$-catalyzed amidation of various substrates bearing Lewis-basic directing groups, ${ }^{[227]}$ it was decided to focus on 2-aryloxazolines due to their importance as synthetic intermediates and key motifs in bioactive compounds. 2-Tolyloxazoline 142a and dioxazolone 40a were selected as model substrates for optimization studies towards the envisioned $\mathrm{C}-\mathrm{H}$ transformation (Table 3.1). A preliminary solvent optimization performed by Dr. R. Mei identified DCE as the optimal reaction medium (entries 1-2). Increasing the amount of silver salts and carboxylate additives to $20 \mathrm{~mol} \%$ proved beneficial (entry 3). Additional solvents were probed in the transformation, giving the desired amidated arene 143aa in low to moderate yields (entries 4-9). The effect of additives on the $\mathrm{C}-\mathrm{H}$ amidation was then extensively 
studied (entries 10-22). Interestingly, several carboxylic acids, carboxylates, carbonates, phosphates, and mono-protected amino acids were found to promote the desired transformation, albeit with lower efficacy than NaOAc. However, sodium mesitylcarboxylate and trichloroacetate gave remarkably poor results, presumably due to excessive steric hindrance and lower basicity, respectively. Lower reaction temperatures were detrimental to the reaction outcome (entry 23). Other cobalt precursors were tested in the oxazoline-assisted $\mathrm{C}-\mathrm{H}$ amidation (entries 24-27), revealing $\mathrm{Cp}^{*} \mathrm{Co}(\mathrm{CO}) \mathrm{I}_{2}$ to be the most potent catalyst. While cationic $\left[\mathrm{Cp}{ }^{*} \mathrm{Co}(\mathrm{MeCN})_{3}\right]\left(\mathrm{SbF}_{6}\right)_{2}$ or dimeric $\left[\mathrm{Cp}^{*} \mathrm{Col}_{2}\right]_{2}$ were only slightly less efficient than $\mathrm{Cp}^{*} \mathrm{Co}(\mathrm{CO}) \mathrm{I}_{2}$, simple cobalt salts such as $\mathrm{CoCl}_{2}$ or unsubstituted $\mathrm{CpCo}$ (III) complexes in contrast fell short in the desired catalytic transformation. Finally, control experiments confirmed the importance of the $\mathrm{Cp}^{*} \mathrm{Co}(\mathrm{III})$-catalyst and the $\mathrm{NaOAc}$ additive (entries 28-29).

Table 3.1. Optimization of the oxazoline-assisted cobalt(III)-catalyzed amidation. ${ }^{[a]}$
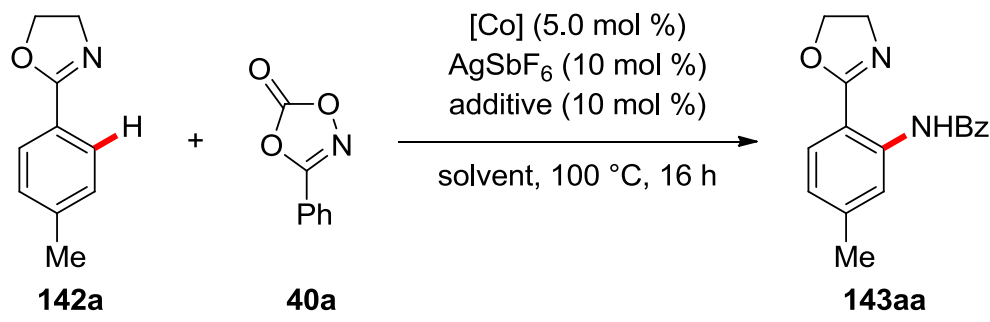

\begin{tabular}{|c|c|c|c|c|}
\hline Entry & [Co] & Solvent & Additive & Yield [\%] ${ }^{[b]}$ \\
\hline $1^{[\mathrm{c}]}$ & $\mathrm{Cp}^{\star} \mathrm{Co}(\mathrm{CO}) \mathrm{I}_{2}$ & TFE & $\mathrm{NaOAc}$ & n.r. \\
\hline $2^{[c]}$ & $\mathrm{Cp}{ }^{\star} \mathrm{Co}(\mathrm{CO}) \mathrm{I}_{2}$ & DCE & $\mathrm{NaOAc}$ & 65 \\
\hline $3^{[c, d]}$ & $\mathrm{Cp}^{*} \mathrm{Co}(\mathrm{CO}) \mathrm{I}_{2}$ & DCE & $\mathrm{NaOAc}$ & 68 \\
\hline $4^{[\mathrm{d}]}$ & $\mathrm{Cp}^{\star} \mathrm{Co}(\mathrm{CO}) \mathrm{I}_{2}$ & $\mathrm{PhCF}_{3}$ & $\mathrm{NaOAc}$ & 53 \\
\hline $5^{[\mathrm{d}]}$ & $\mathrm{Cp}^{\star} \mathrm{Co}(\mathrm{CO}) \mathrm{I}_{2}$ & 1,4-dioxane & $\mathrm{NaOAc}$ & 24 \\
\hline $6^{[\mathrm{d}]}$ & $\mathrm{Cp}{ }^{\star} \mathrm{Co}(\mathrm{CO}) \mathrm{I}_{2}$ & EtOAc & $\mathrm{NaOAc}$ & $<10$ \\
\hline $7^{[\mathrm{d}]}$ & $\mathrm{Cp}^{\star} \mathrm{Co}(\mathrm{CO}) \mathrm{I}_{2}$ & $n \mathrm{Bu}_{2} \mathrm{O}$ & $\mathrm{NaOAc}$ & 21 \\
\hline
\end{tabular}




\begin{tabular}{|c|c|c|c|c|}
\hline $8^{[d]}$ & $\mathrm{Cp}^{*} \mathrm{Co}(\mathrm{CO}) \mathrm{I}_{2}$ & tAmOH & $\mathrm{NaOAc}$ & 6 \\
\hline $9^{[d]}$ & $\mathrm{Cp}^{*} \mathrm{Co}(\mathrm{CO}) \mathrm{I}_{2}$ & $\mathrm{PhMe}$ & $\mathrm{NaOAc}$ & 31 \\
\hline $10^{[c]}$ & $\mathrm{Cp}^{*} \mathrm{Co}(\mathrm{CO}) \mathrm{I}_{2}$ & DCE & KOAC & 47 \\
\hline $11^{[c]}$ & $\mathrm{Cp}^{*} \mathrm{Co}(\mathrm{CO}) \mathrm{I}_{2}$ & DCE & $\mathrm{PivOH}$ & 58 \\
\hline $12^{[c]}$ & $\mathrm{Cp}^{*} \mathrm{Co}(\mathrm{CO}) \mathrm{I}_{2}$ & DCE & NaOPiv & 63 \\
\hline $13^{[d]}$ & $\mathrm{Cp}^{*} \mathrm{Co}(\mathrm{CO}) \mathrm{I}_{2}$ & DCE & $\mathrm{NaO}_{2} \mathrm{CMes}$ & 3 \\
\hline $14^{[\mathrm{d}]}$ & $\mathrm{Cp}^{*} \mathrm{Co}(\mathrm{CO}) \mathrm{I}_{2}$ & DCE & $\mathrm{NaO}_{2} \mathrm{CAd}$ & 44 \\
\hline $15^{[d]}$ & $\mathrm{Cp}^{*} \mathrm{Co}(\mathrm{CO}) \mathrm{I}_{2}$ & DCE & Ac-lle- $\mathrm{CO}_{2} \mathrm{Na}$ & 17 \\
\hline $16^{[d]}$ & $\mathrm{Cp}^{*} \mathrm{Co}(\mathrm{CO}) \mathrm{I}_{2}$ & DCE & $\mathrm{NaO}_{2} \mathrm{CCCl}_{3}$ & 4 \\
\hline $17^{[\mathrm{e]}}$ & $\mathrm{Cp}^{*} \mathrm{Co}(\mathrm{CO}) \mathrm{I}_{2}$ & DCE & $\mathrm{Zn}(\mathrm{OAc})_{2}$ & 29 \\
\hline $18^{[\mathrm{d}]}$ & $\mathrm{Cp}^{*} \mathrm{Co}(\mathrm{CO}) \mathrm{I}_{2}$ & DCE & $\mathrm{NaHCO}_{3}$ & 40 \\
\hline $19^{[d]}$ & $\mathrm{Cp}^{*} \mathrm{Co}(\mathrm{CO}) \mathrm{I}_{2}$ & DCE & $\mathrm{NaOBz}$ & 51 \\
\hline $20^{[d]}$ & $\mathrm{Cp}^{*} \mathrm{Co}(\mathrm{CO}) \mathrm{I}_{2}$ & DCE & $\mathrm{Na}_{2} \mathrm{HPO}_{4}$ & 40 \\
\hline $21^{[d]}$ & $\mathrm{Cp}^{*} \mathrm{Co}(\mathrm{CO}) \mathrm{I}_{2}$ & DCE & LiOAc & 49 \\
\hline $22^{[d]}$ & $\mathrm{Cp}^{*} \mathrm{Co}(\mathrm{CO}) \mathrm{I}_{2}$ & DCE & $\mathrm{HCO}_{2} \mathrm{Na}$ & 47 \\
\hline $23^{[d, f]}$ & $\mathrm{Cp}^{*} \mathrm{Co}(\mathrm{CO}) \mathrm{I}_{2}$ & DCE & $\mathrm{NaOAc}$ & 39 \\
\hline $24^{[\mathrm{c}, \mathrm{d}]}$ & {$\left[\mathrm{Cp}^{*} \mathrm{Col}_{2}\right]_{2}$} & DCE & $\mathrm{NaOAc}$ & 61 \\
\hline $25^{[d]}$ & $\mathrm{CpCo}(\mathrm{CO}) \mathrm{I}_{2}$ & DCE & $\mathrm{NaOAc}$ & traces \\
\hline $26^{[c]}$ & $\mathrm{CoCl}_{2}$ & DCE & $\mathrm{NaOAc}$ & n.r. \\
\hline $27^{[c]}$ & {$\left[\mathrm{Cp}^{*} \mathrm{Co}(\mathrm{MeCN})_{3}\right]\left(\mathrm{SbF}_{6}\right)_{2}$} & DCE & - & 54 \\
\hline $28^{[c]}$ & $\mathrm{Cp}{ }^{*} \mathrm{Co}(\mathrm{CO}) \mathrm{I}_{2}$ & DCE & - & 35 \\
\hline $29^{[c]}$ & - & DCE & $\mathrm{NaOAc}$ & n.r. \\
\hline
\end{tabular}


$30^{[\mathrm{g}]} \quad\left[\mathrm{Cp}{ }^{*} \mathrm{Co}(\mathrm{MeCN})_{3}\right]\left(\mathrm{SbF}_{6}\right)_{2} \quad \mathrm{DCE} \quad \mathrm{NaOAc} \quad 3$

${ }^{[a]}$ Reaction conditions: 142a (0.50 mmol), 40a (1.2 equiv), [Co] $(5.0 \mathrm{~mol} \%), \mathrm{AgSbF}_{6}(10 \mathrm{~mol} \%)$, additive $(10 \mathrm{~mol} \%)$, solvent $(2.0 \mathrm{~mL}), 100{ }^{\circ} \mathrm{C}, 16 \mathrm{~h} .{ }^{[\mathrm{b}]}$ Isolated yield. ${ }^{[\mathrm{c}]}$ Performed by Dr. R. Mei. ${ }^{[d]}$ Additive (20 mol \%) and $\mathrm{AgSbF}_{6}(20 \mathrm{~mol} \%) .{ }^{[\mathrm{e}]}$ Additive (10 mol \%) and $\mathrm{AgSbF}_{6}(20 \mathrm{~mol} \%) .{ }^{[\mathrm{f}]} \mathrm{At}$ $85^{\circ} \mathrm{C}$. ${ }^{[g]}$ Under blue LED irradiation at $23^{\circ} \mathrm{C}$ in the presence of $\operatorname{RuTPP}(\mathrm{CO})(1.0 \mathrm{~mol} \%)$ as co-catalyst. $\mathrm{AgSbF}_{6}$ was omitted.

It should be noted that no 2,6-diamidated product was detected, presumably due to intramolecular hydrogen bonds between the directing group's nitrogen and the amide's $\mathrm{N}-\mathrm{H}$ group, preventing the functionalization of the second ortho-position. Furthermore, it is noteworthy that no aminocarbonylation product was observed in any of those reactions either, despite dioxazolones being reported to decompose to the corresponding isocyanates via heat- or light-induced Lossen rearrangements. ${ }^{[228]}$ These findings are complementary to a previous report by Ackermann on the use of acylazides in cobalt(III)-catalyzed $\mathrm{C}-\mathrm{H}$ aminocarbonylations, where the authors proposed isocyanates to be formed in situ via a Curtius rearrangement, ${ }^{[85]}$ and highlight the unique reactivity of dioxazolones 40 as aminating reagents. Along the same lines, dioxazolones are documented to undergo light-induced $\mathrm{N}-\mathrm{O}$ cleavage with $\mathrm{CO}_{2}$ loss in the presence of ruthenium(II) porphyrin catalysts to form rutheno $\mathrm{N}$-acycl nitrene intermediates. ${ }^{[229]}$ We hence decided to investigate whether the desired $\mathrm{C}-\mathrm{H}$ amidation would be feasible at ambient temperature under light irradiation in the presence of catalytic [Ru(TPP)CO] (Table 3.1, entry 30). Unfortunately, a significant conversion of the starting material was not detected under those conditions.

\subsubsection{Substrate Scope}

With the optimized catalytic system in hand, the versatility and robustness of the $\mathrm{Cp}^{*} \mathrm{Co}$ (III)-catalyzed oxazolinyl-directed $\mathrm{C}-\mathrm{H}$ amination was then investigated. Various 2-phenyloxazolines 142 were efficiently converted to the amide products 143 with high efficacy (Table 3.2). Among others, a wealth of alkyl, aryl, ether and 
halogenated substituents were fully tolerated in the transformation, affording products 143 in moderate to good yield (entries 1-7). Remarkably, even the secondary amide $142 \mathrm{~h}$ bearing free $\mathrm{N}-\mathrm{H}$ groups was successfully transformed into the desired product 143ha with complete site-selectively (entry 8). meta-Substituted 2-phenyloxazolines $\mathbf{1 4 2 i - k}$ were also viable substrates for the cobalt-catalyzed $\mathrm{C}-\mathrm{H}$ nitrogenation, and were functionalized on the less sterically hindered ortho-position with complete positional selectivity (entries 9-11). Moreover, the $\mathrm{C}-\mathrm{H}$ amidation was found to be scalable. Indeed, a gram-scale reaction was carried and provided product 143aa without any loss of efficacy.

Table 3.2. Substrate scope of 2-aryloxazolines 142 in the cobalt-catalyzed C-H amidation. ${ }^{[a]}$

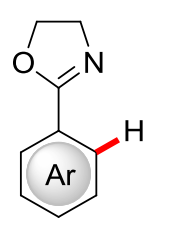

142

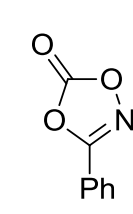

$40 a$

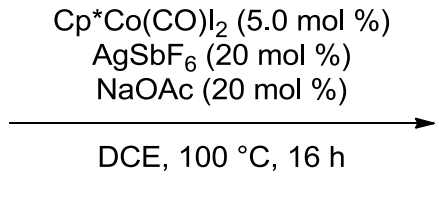

Entry Oxazoline


3<smiles>CCc1ccc(C2=NCCO2)cc1</smiles>

142c

4

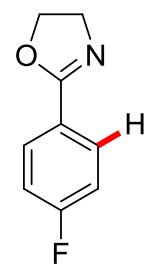

142d

5

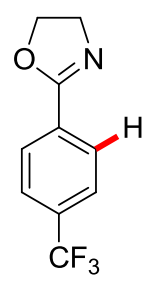

$142 \mathrm{e}$

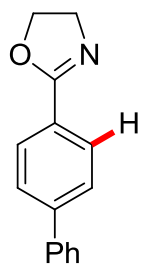

$142 f$

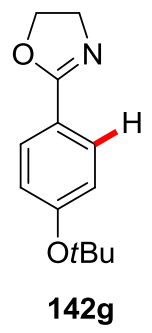

7<smiles>CC(=O)Nc1ccc(C2=NCCO2)cc1</smiles>

$142 \mathrm{~h}$

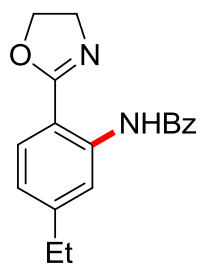

143ca

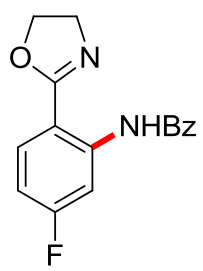

143da

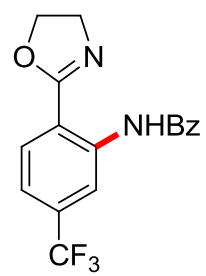

143ea

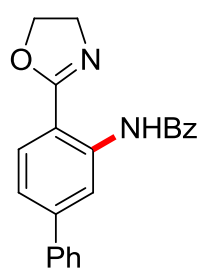

$143 f a$

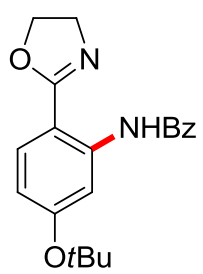

143ga

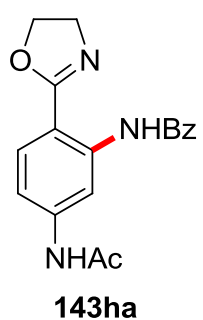

65

71

63

74

50

58 
9<smiles>FC(F)(F)c1cccc(C2=NCCO2)c1</smiles>

10<smiles>Clc1cccc(C2=NCCO2)c1</smiles>

142j

11<smiles>COc1cc(F)cc(C2=NCCO2)c1</smiles>

142k<smiles>O=[N+]([O-])c1ccc(C2=NCCO2)cc1</smiles>

12<smiles>CC(C)(C)Nc1ccc(C(F)(F)F)cc1C1=NCCO1</smiles>

65<smiles>O=C(Nc1ccc(Cl)cc1C1=NCCO1)c1ccccc1</smiles><smiles>COc1cc(C2=NCCO2)c(NC(=O)c2ccccc2)cc1F</smiles>

---
74

n.r.

\footnotetext{
${ }^{[a]}$ Reaction conditions: 142 (0.50 mmol), 40 (1.2 equiv), $\mathrm{Cp}^{*} \mathrm{Co}(\mathrm{CO}) \mathrm{I}_{2}(5.0 \mathrm{~mol} \%), \mathrm{AgSbF}_{6}$ (20 mol \%), NaOAc $(20 \mathrm{~mol} \%)$, DCE $(2.0 \mathrm{~mL}), 100{ }^{\circ} \mathrm{C}, 16 \mathrm{~h} .{ }^{[\mathrm{b}]}$ Isolated yields. ${ }^{[\mathrm{c}]}$ Reaction carried on $5.6 \mathrm{mmol}$ scale. ${ }^{[\mathrm{d}]}$ Reaction carried on $0.25 \mathrm{mmol}$ scale. ${ }^{[\mathrm{e}]}$ Performed by $D r . R$. Mei. ${ }^{[\mathrm{f}]}$ Using $\left[\mathrm{Cp}^{*} \mathrm{RhCl}_{2}\right]_{2}(2.5 \mathrm{~mol} \%)$ instead of $\mathrm{Cp}{ }^{*} \mathrm{Co}(\mathrm{CO}) \mathrm{I}_{2}$.
}

The versatile $\mathrm{Cp}{ }^{*} \mathrm{Co}(\mathrm{III})$-catalyst however encountered also limitations. 2-Phenyloxazolines bearing ortho-substituents could only be converted to the desired amides in low to moderate yield. Also, no conversion of the starting material was detected when using nitro-substituted oxazoline 142I (Table 3.2, entry 12).

Furthermore, $\left[\mathrm{Cp}^{*} \mathrm{RhCl}_{2}\right]_{2}$ was also tested as catalyst in the transformation of 2-(meta-chlorophenyl)-oxazoline 142j. No significant difference of chemo- or site- 
selectivity compared to $\mathrm{Cp}^{\star} \mathrm{Co}(\mathrm{CO}) \mathrm{I}_{2}$ was observed, providing product $143 \mathrm{ja}$ in a similar yield (Table 3.2, entry 10).

Additional substrates 142, including 2-phenyloxazolines bearing sensitive halogen, ester and cyano substituents, as well as differently substituted dioxazolones 40, were also investigated by Dr. R. Mei in the transformation, and thus were found to give the desired amidated products. ${ }^{[37 e, 227 a]}$

Thereafter, the effect of the substitution pattern of the oxazoline moiety was studied (Table 3.3). Diversely functionalized oxazolines as well as six-membered dihydrooxazines were efficiently converted to amides 143 in good to excellent yield (entries 1-5). However, additional substituents adjacent to the nitrogen atom of the oxazolinyl group were not tolerated, with 4-ethyloxazoline 142r remaining untouched when submitted to the reaction conditions (entry 6). This outcome is likely explained by an excessive steric hindrance near the catalyst-coordinating nitrogen atom of the oxazoline directing group. Indeed, the reactivity of cobalt(III) is greatly affected by steric factors due to its small ionic radius. ${ }^{[46 a, 46 b]}$

Table 3.3. Substrate scope of substituted oxazolines and oxazines 142 in the cobaltcatalyzed $\mathrm{C}-\mathrm{H}$ amidation. ${ }^{[\mathrm{a}]}$

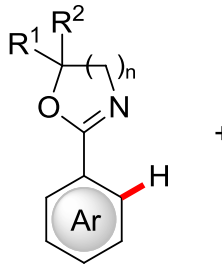

142

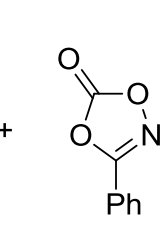

$40 a$

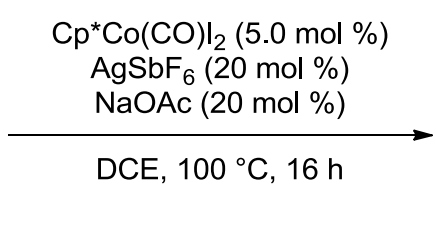

DCE, $100^{\circ} \mathrm{C}, 16 \mathrm{~h}$

Product

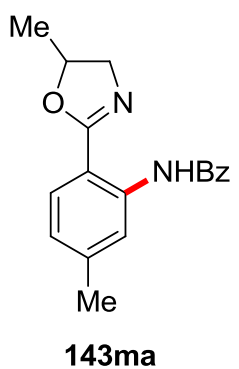


2<smiles>Cc1ccc(C2=NCC(c3ccccc3)O2)cc1</smiles>

$142 n$

3

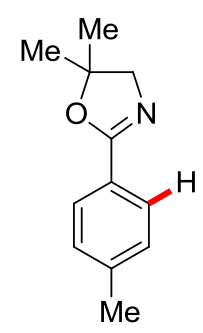

1420

4<smiles>Cc1cccc(C2=NCCCO2)c1</smiles>

5

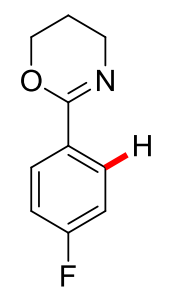

$142 q$

6

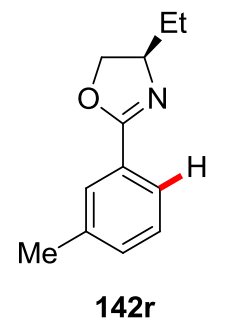

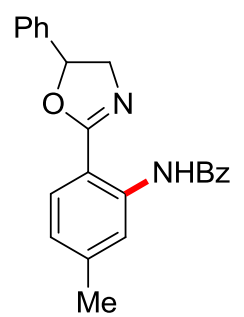

143na

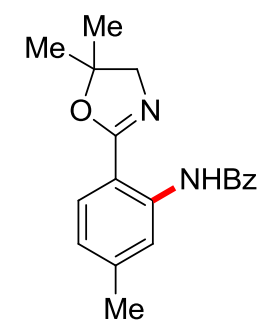

$1430 a$<smiles>Cc1ccc(NC(=O)c2ccccc2)c(C2=NCCCO2)c1</smiles><smiles>O=C(Nc1cc(F)ccc1C1=NCCCO1)c1ccccc1</smiles>

143qa
74

86

66

71

$$
\begin{array}{ll}
--- & \text { traces }
\end{array}
$$

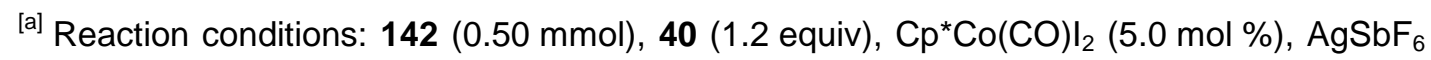
(20 mol \%), NaOAc (20 mol \%), DCE $(2.0 \mathrm{~mL}), 100{ }^{\circ} \mathrm{C}, 16 \mathrm{~h} .{ }^{[\mathrm{b}]}$ Isolated yields. 
Gratifyingly, the scope of the $\mathrm{Cp}^{*} \mathrm{Co}(\mathrm{III})$-catalyzed $\mathrm{C}-\mathrm{H}$ amidation was not limited to 2-phenyloxazolines 142. Indeed, various indoles 28/36 were efficiently and selectively functionalized at the C2-position under the assistance of removable pyridyl (py) and pyrimidyl (pym) directing groups in the presence of diverse alkyl, alkoxy and ester substituents, highlighting the versatility and the robustness of the developed methodology (Table 3.4). Remarkably, due to the higher inherent reactivity of indoles 28/36 compared to 2-aryloxazolines 142, the synthesis of amidated products $146 / 147$ was found to be viable at a low catalyst loading of $2.5 \mathrm{~mol} \%$ and a rather mild reaction temperature of $70{ }^{\circ} \mathrm{C}$, after further optimization by Dr. R. Mei. ${ }^{[227 b]} \mathrm{Cp}^{t} \mathrm{Co}(\mathrm{CO}) \mathrm{I}_{2}\left(\mathrm{Cp}^{t}=1,3\right.$-di-tert-butylcyclopentadienyl) was also tested as the catalyst in the cobalt-catalyzed $\mathrm{C}-\mathrm{H}$ amidation of indoles due to recent reports indicating the superiority of $\mathrm{Cp}^{t}$ over $\mathrm{Cp}^{*}$ in rhodium(III)-catalyzed transformations. ${ }^{[230]}$ However, in the present case, the desired product 147aa was only formed in a moderate yield of $27 \%$ when using the $\mathrm{Cp}^{t}$ catalyst, compared to the $86 \%$ obtained when the $\mathrm{Cp}^{*}$ complex was employed (entry 1 ). For more challenging substrates, such as sterically hindered C3-alkylated indole 36b, increasing the reaction temperature as well as the catalyst loading improved the reaction outcome to afford the desired product $147 \mathrm{ba}$ in synthetically useful yields (entry 2). Furthermore, it is noteworthy that, while 2-aryloxazolines 142 absolutely required carboxylate additives for the transformation to occur with high catalytic efficacy, the more reactive indole substrates $\mathbf{2 8 / 3 6}$ could be amidated in high yield even in the absence of the base additive (entry 2). However, weakly coordinating directing groups such as carbamates and ureas fell short in the envisioned cobaltcatalyzed amidation (entry 10). 
Table 3.4. Substrate scope of substituted indoles $28 / 36$ in the cobalt-catalyzed $\mathrm{C}-\mathrm{H}$ amidation. ${ }^{\text {[a] }}$

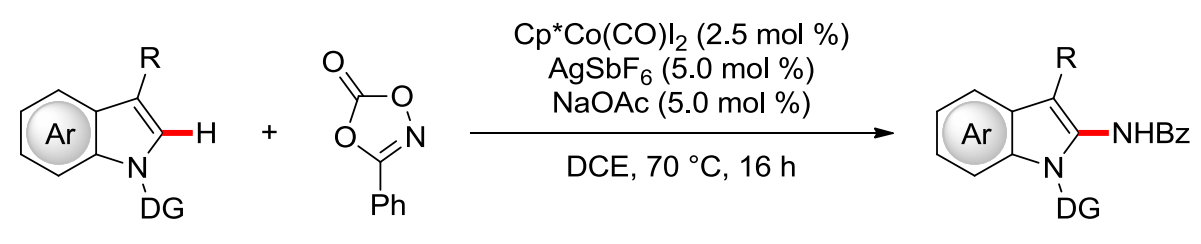

Entry Indole

$36 \mathrm{~b}$<smiles>[R20]n1c(C)cc2ccc(C(=O)OC)cc21</smiles>

$28 \mathrm{c}$<smiles></smiles>

28d

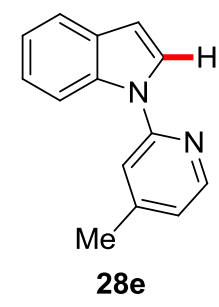<smiles>[R6]c1cc2ccccc2n1[R6]O</smiles>

147 aa

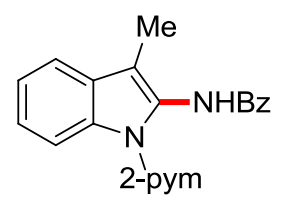

147ba<smiles>[R6]n1c(N)cc2ccc(C(C)=O)cc21</smiles>

$146 \mathrm{ca}$<smiles>COc1cccc2c1cc(NC(=O)OCc1ccccc1)n2[TeH]</smiles>

146da<smiles>CC(C)(C)OC(=O)Nc1cc2ccccc2n1-c1cc(C(C)(C)C)ccn1</smiles>

86

$27^{[c]}$

39

$65^{[d]}$

$61^{[\mathrm{d}, \mathrm{e}]}$

91

96

97 
6<smiles>Cc1ccc(-n2ccc3ccccc32)nc1</smiles>

7

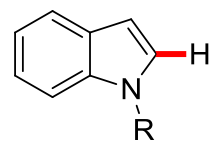

$R=\operatorname{Boc}(\mathbf{1 4 8})$

$\mathrm{R}=\mathrm{C}(\mathrm{O}) \mathrm{NMe}_{2}(\mathbf{2 3 a})$

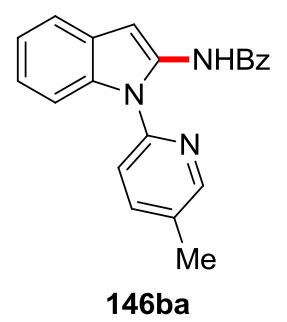

92

n.r.

${ }^{[a]}$ Reaction conditions: 28/36 (0.50 mmol), 40 (1.2 equiv), $\mathrm{Cp}^{*} \mathrm{Co}(\mathrm{CO}) \mathrm{I}_{2}$ (2.5 mol \%), $\mathrm{AgSbF}_{6}$ $(5.0 \mathrm{~mol} \%), \quad \mathrm{NaOAc}(5.0 \mathrm{~mol} \%), \quad \mathrm{DCE}(2.0 \mathrm{~mL}), 70^{\circ} \mathrm{C}, 16 \mathrm{~h} .{ }^{[\mathrm{b}]}$ Isolated yields. ${ }^{[\mathrm{c}]}$ Using $\mathrm{Cp}^{t} \mathrm{Co}(\mathrm{CO}) \mathrm{I}_{2}$ instead of $\mathrm{Cp}^{*} \mathrm{Co}(\mathrm{CO}) \mathrm{I}_{2}$. ${ }^{[\mathrm{d}]}$ Using $\mathrm{Cp}^{*} \mathrm{Co}(\mathrm{CO}) \mathrm{I}_{2}(5.0 \mathrm{~mol} \%), \mathrm{AgSbF}_{6}(10 \mathrm{~mol} \%)$ and $\mathrm{NaOAc}(10 \mathrm{~mol} \%)$ at $100^{\circ} \mathrm{C} .{ }^{[\mathrm{e}]} \mathrm{NaOAc}$ was omitted.

Moreover, the reactions of additional indole substrates 28 bearing sensitive electrophilic functional groups, such as bromo and iodo, as well as differently substituted dioxazolones $\mathbf{4 0}$, including thiophene moieties, were studied in detail by Dr. R. Mei, and showcased the chemo-selectivity and robustness of the developed $\mathrm{C}-\mathrm{H}$ functionalization methodology. ${ }^{[37 \mathrm{e}, 227 \mathrm{a}]}$ In addition to 2-aryloxazonlines 142 and $\mathrm{N}$-pyri(mi)dylindoles $\mathbf{2 8 / 3 6}$, other substrate classes were smoothly amidated employing the developed methodology. Indeed, 2-phenylpyridines, $\mathrm{N}$-phenylpyrazoles, $\mathrm{N}$-phenylindazoles and 2-phenylpyrimidines were among other efficiently converted to the corresponding amides by the $\mathrm{Cp}^{*} \mathrm{Co}(\mathrm{CO}) \mathrm{I}_{2}$ catalyst, as reported by Dr. R. Mei. ${ }^{[227 a]}$

\subsubsection{Mechanistic Studies}

Given the unique features of the versatile cobalt-catalyzed $\mathrm{C}-\mathrm{H}$ amidation, we became interested in delineating its mode of action. Towards this objective, experimental mechanistic studies were conducted. 
First, the effect on the reaction of various radical scavengers, such as TEMPO and $\mathrm{BHT}$, was probed (Scheme 3.2a). Here, only a mild reduction of the yield was observed, supporting the catalytic transformation not to occur via a radical-based mechanism. This observation contrasts with mechanistic studies performed for cobalt-catalyzed $\mathrm{C}-\mathrm{H}$ alkoxylations, where a SET process has been proposed. ${ }^{[231]} \mathrm{A}$ mercury poisoning test was conducted to probe the homogeneity of the reaction (Scheme 3.2b). Here, no significant reduction of the catalytic activity was observed in the presence of a stoichiometric amount of metallic mercury, which supports the homogenous nature of the catalytic process.

a) radical scavengers
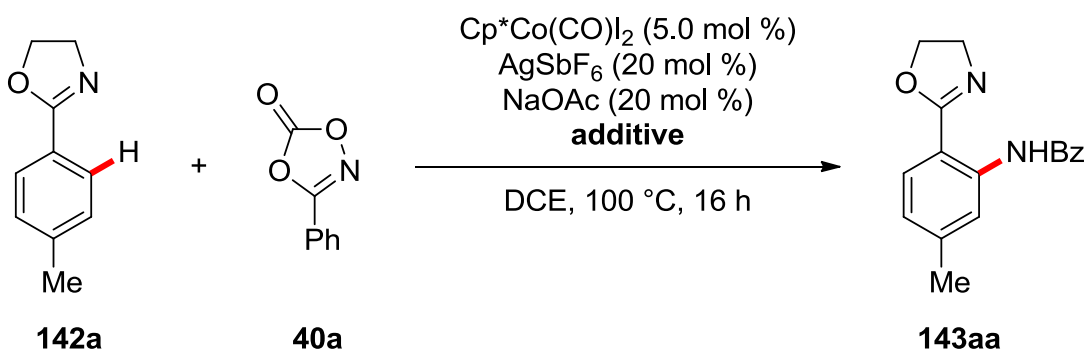

\begin{tabular}{|lr|}
\hline Additive & Yield \\
\hline none: & $68 \%$ \\
TEMPO (1.0 equiv): & $46 \%$ \\
TEMPO (2.0 equiv): & $49 \%$ \\
BHT (1.0 equiv): & $48 \%$ \\
$(E)-$ stilbene (1.0 equiv): & $63 \%$ \\
Ph $_{2} \mathrm{C}=\mathrm{CH}_{2}(1.0$ equiv): & $67 \%$ \\
galvinoxyl (1.0 equiv): & $14 \%$ \\
\hline
\end{tabular}

b) mercury drop test

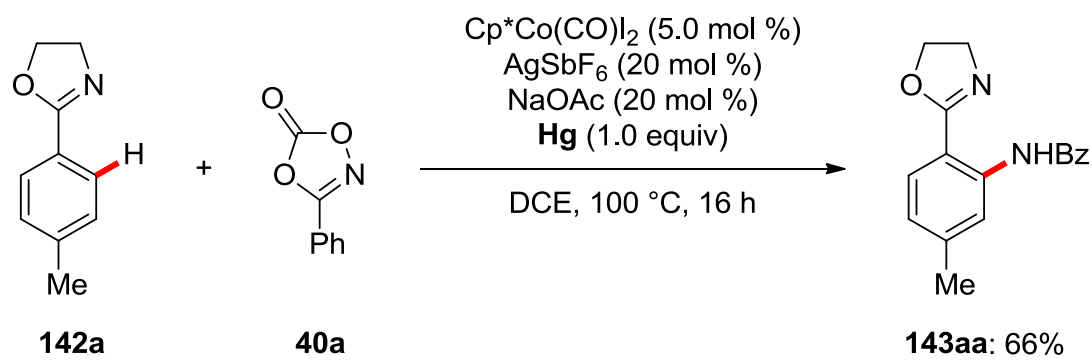

Scheme 3.2. Effect of radical scavengers on the cobalt(III)-catalyzed $\mathrm{C}-\mathrm{H}$ amidation and mercury drop test. 
Reactions with an isotopically labeled protic co-solvent were performed by $\operatorname{Dr}$. $R$. Mei. ${ }^{[227 a]}$ A considerable $H / D$ scrambling was observed in the absence of dioxazolones 40. Yet, no deuterium incorporation was detected in the presence of the amidation reagent 40. Furthermore, kinetic studies were conducted by $D r . R$. Mei. ${ }^{[227 a]}$ A kinetic isotope effect (KIE) of $k_{H} / k_{D} \approx 2.3$ revealed the $\mathrm{C}-\mathrm{H}$ cleavage step to be kinetically relevant. Additionally, competition experiments between differently substituted substrates indicated more electron-rich 2-aryloxazolines 142 to react preferentially, which supports the $\mathrm{C}-\mathrm{H}$ activation event to occur through a baseassisted, intermolecular electrophilic substitution-type (BIES) $\mathrm{C}-\mathrm{H}$ metalation manifold. ${ }^{[37]}$

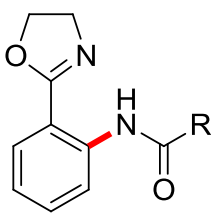

143

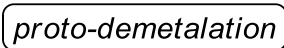

$\mathrm{AcOH}$
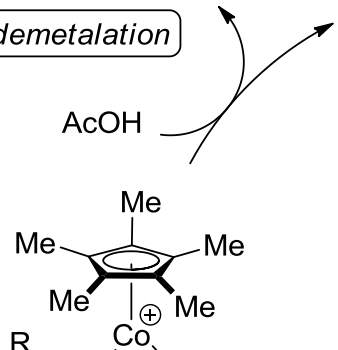

$\mathrm{R}$<smiles>[R]C(=O)N(C)c1ccccc1C1=[N+](C)CCO1</smiles>

151

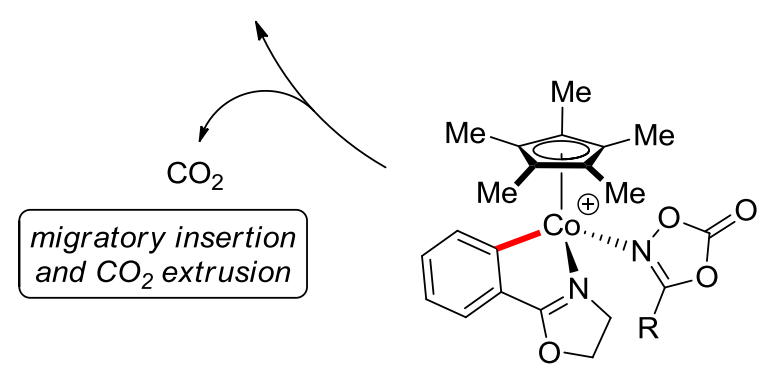

150

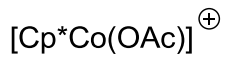

$\mathrm{Cp}^{*} \mathrm{Co}(\mathrm{CO}) \mathrm{I}_{2}$

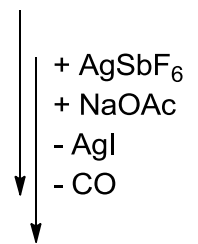<smiles>c1ccc(C2=NCCO2)cc1</smiles>

142
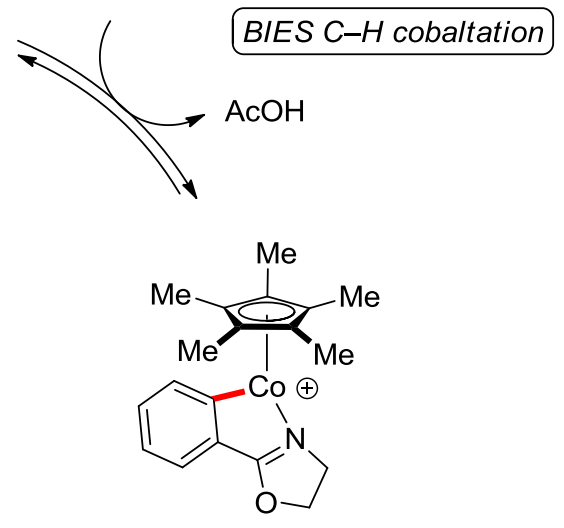

149

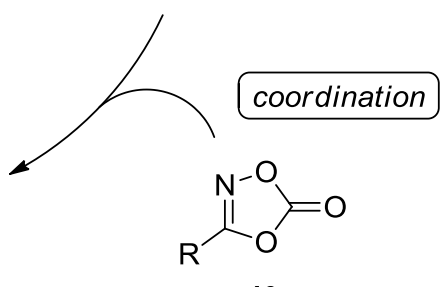

40

Scheme 3.3. Plausible catalytic cycle of the $\mathrm{Cp}{ }^{*} \mathrm{Co}(\mathrm{III})$-catalyzed $\mathrm{C}-\mathrm{H}$ amidation. 
Based on the conducted mechanistic studies and previous reports, the transformation is believed to be initiated by the formation of the catalytically active cationic complex $\mathrm{Cp}^{*} \mathrm{Co}(\mathrm{OAc})^{+}$through halogen abstraction by the silver salt and coordination by the carboxylate additive (Scheme 3.3). Then, a plausible catalytic cycle begins with a kinetically relevant, acetate-assisted $\mathrm{C}-\mathrm{H}$ metalation to generate cobaltacycle 149 , which is believed to occur via a BIES mechanism. ${ }^{[37]}$ Thereafter, coordination of the dioxazolone $\mathbf{4 0}$ provides intermediate 150. Finally, $\mathrm{CO}_{2}$ extrusion and protodemetalation by the formed acetic acid provide the desired amidated product 143 and regenerate the active cobalt(III)-carboxylate catalyst. 


\subsection{Asymmetric Iron-Catalyzed Hydroarylations by $\mathrm{C}-\mathrm{H}$ Activation}

While major achievements in the research area of $\mathrm{C}-\mathrm{H}$ activation have been realized with the aid of precious, rather toxic noble transition metals, recent focus has shifted to earth-abundant and inexpensive $3 d$ metals. ${ }^{[45]}$ However, despite substantial advances, full selectivity control in enantioselective $\mathrm{C}-\mathrm{H}$ transformations continues to heavily rely on noble $4 \mathrm{~d}$ and $5 \mathrm{~d}$ transition metals, mostly palladium, rhodium and iridium complexes. ${ }^{[51]}$ In sharp contrast, enantioselective $\mathrm{C}-\mathrm{H}$ functionalizations with sustainable $3 d$ metals remain underdeveloped, but have gained significant momentum during the course of this doctoral thesis. ${ }^{[52]}$

In this context, enantioselective transformations by inner-sphere iron-catalyzed $\mathrm{C}-\mathrm{H}$ activation remained unprecedented at the outset of this work. Drawing inspiration from the work of Yoshikai on the racemic iron-catalyzed hydroarylation of styrenes 60 and alkynes 7 with indoles 59 (Scheme 1.28), ${ }^{[148]}$ the possibility to develop an asymmetric iron-catalyzed $\mathrm{C}-\mathrm{H}$ alkylation was investigated. The envisioned transformation is highly desirable due to its perfect atom-economy ${ }^{[2]}$ and the use of readily available, non-prefunctionalized alkenes, making the process environmentally benign.

While our work represents the first enantioselective iron-catalyzed transformation via organometallic $\mathrm{C}-\mathrm{H}$ activation, it should be noted that a few examples of ironcatalyzed enantioselective $\mathrm{C}-\mathrm{H}$ functionalizations via outer-sphere mechanisms have been previously reported. ${ }^{[232]}$ Atroposelective oxidative couplings of 2-naphthols ${ }^{[233]}$ and biomimetic $\mathrm{C}\left(\mathrm{sp}^{3}\right)-\mathrm{H}$ hydroxylations ${ }^{[234]}$ have indeed been achieved with chiral iron catalysts in moderate to excellent enantioselectivities. Furthermore, it should be duly noted that, to the best of our knowledge, ${ }^{[232,235]}$ chiral $\mathrm{NHC}$ ligands had never been used in asymmetric iron catalysis prior to this work. 


\subsubsection{Optimization Studies}

The optimization studies began by probing the effect of various (pre-)ligands on the envisioned enantioselective C2-alkylation of indole 59b with styrene 60b (Table 3.5). It should be emphasized that, in addition to chiral $\mathrm{NHC}^{[235 d, 236]}$ precursors, different ligand classes, including achiral ligands, were tested in order to understand the requirements of the transformation. Indeed, only a very limited number of ligands were reportedly tested by Yoshikai in the racemic transformation, ${ }^{[148]}$ which remains the sole example of this kind of reaction under iron catalysis. Yoshikai and coworkers investigated a narrow class of bis-aryl substituted NHC precursors, with the best reaction outcome being obtained with $s|X y| \cdot H C l$ while more sterically hindered NHC ligands gave poor results. A few simple phosphine and bipyridyl ligands were also tested, but fell short in providing the $\mathrm{C}-\mathrm{H}$ alkylated indole in synthetically useful yields. We hence became interested in the performance of different ligands or differently substituted NHC precursors in this transformation.

The desired $\mathrm{C}-\mathrm{H}$ alkylated product $152 \mathrm{bb}$ could be obtained in excellent yield using a slight modification of the reported conditions, ${ }^{[148]}$ and a control experiment demonstrated the importance of the ligand (entries 1-2). No significant enantioinduction was observed when TMEDA was replaced with (-)-sparteine (entry 3). The simple chiral NHC precursor 153, originally introduced by Grubbs as a chiral sIMes analogue for enantioselective alkene metathesis, ${ }^{[211]}$ provided product $\mathbf{1 5 2 \mathbf { b } b}$ in low yield but promising enantioselectivity (entry 4). Various additives were tested in combination with 153, but did not improve the reaction outcome (entries 5-8). Interestingly, the bulkier ligand $\mathbf{1 5 4}$ gave even poorer results, with the opposite enantiomer being obtained as the "major" product (entry 9). Intrigued by whether the poor results of 153 , which closely resembles the capable $|X y| \cdot H C l$ pre-ligand, were due to the chiral diphenyl backbone or to the lacking ortho-methyl substituent, 155 was tested in the transformation (entry 10). While the alkylated product 152bb was formed in good yield, no significant enantioselectivity was observed. Different carbene classes, such as Berkesse/s triazolium-based $\mathrm{NHC}$ pre-ligand $156^{[237]}$ and $\mathrm{CAAC}^{[238]}$ precursor 157 , were also tested in the reaction (entries 11-12). While 
moderate to good conversions were observed, their performance was not superior to the common IXyl. $\mathrm{HCl}$ or IMes. $\mathrm{HCl} \mathrm{NHC}$ precursors. Then, chiral alkyl-substituted NHC precursors 158-160 were investigated in the enantioselective alkylation, but unfortunately gave extremely poor results (entries 13-15).

To further uncover the required ligand properties, different types of (pre-)ligands were tested in the transformation. No significant conversion or enantio-induction was observed with phosphine or phosphoramidite ligands (entries 16-18). A rather promising enantioselectivity was observed with the prototypical chiral secondary phosphine oxide (SPO) 164, despite the poor conversion (entry 19). Other simple, non-chiral SPOs were hence investigated, but only provided the alkylated product 152bb in low yields (entries 20-22). Additional (HA)SPOs were then probed, without major success (entries 23-24). Phosphine chlorides $^{[239]}$ 170-171 were tested as well, but failed to deliver any desired product (entries 25-26). It should be noted that phosphine chloride pre-ligands may react with the Grignard reagent to generate the corresponding phosphine in situ, although findings by Ackermann in the context of palladium-catalyzed Kumada-Corriu couplings provide support for the formation of tertiary phosphines not to occur under similar conditions. ${ }^{[239 a]}$ The performance of nitrogen-based ligands in the envisioned iron-catalyzed asymmetric hydroarylation was then explored. As promising results were obtained with the bidentate ligand 172, its chiral analogue 173, which had been previously successfully employed in iron-catalyzed hydrofunctionalizations of alkenes, ${ }^{[240]}$ was probed in the $\mathrm{C}-\mathrm{H}$ alkylation, but no significant enantio-induction was observed (entries 27-28). Furthermore, PyBOX ${ }^{[2249]} 174$ and diamine 175 were tested, but failed to deliver any promising results (entries 29-30). 
Table 3.5. Preliminary ligand optimization for the iron-catalyzed hydroarylation of styrene $60 \mathrm{~b}$ with indole $59 \mathrm{~b} .^{[\mathrm{a}]}$

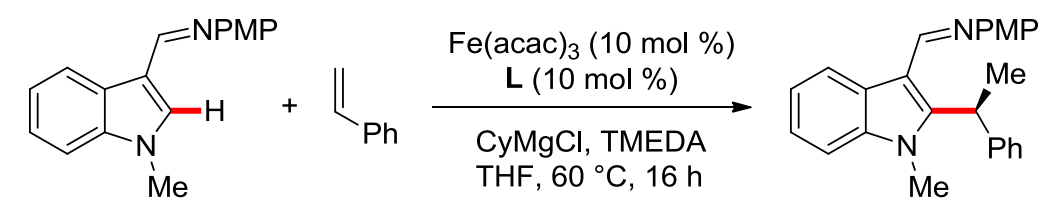

$59 \mathrm{~b}$

$60 \mathrm{~b}$

$152 \mathrm{bb}$

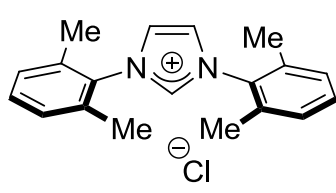

$\mathrm{IXyl} \cdot \mathrm{HCl}$

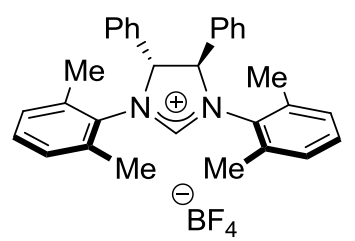

155

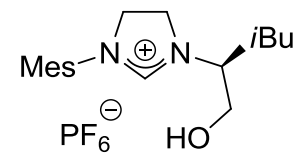

158

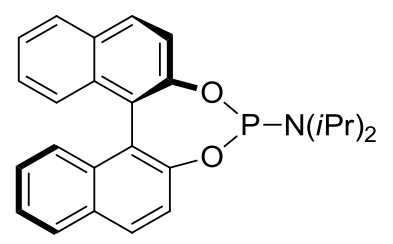

161

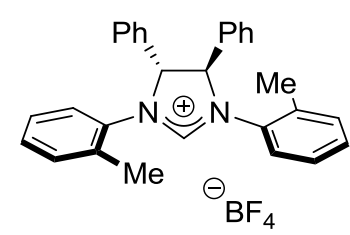

153

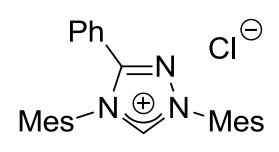

156

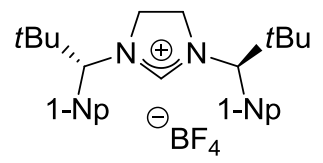

159<smiles>COc1c(Cl)ccc(P)c1-c1c(-c2ccccc2)ccc(Cl)c1OC</smiles>

162
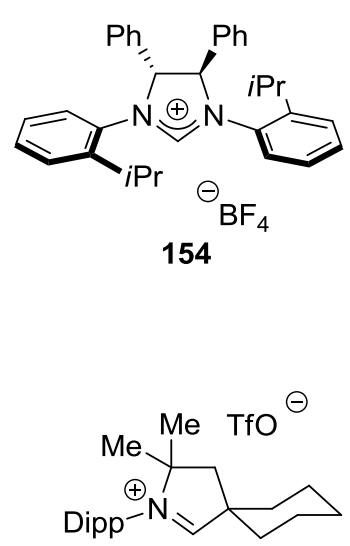

157

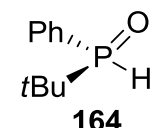

164

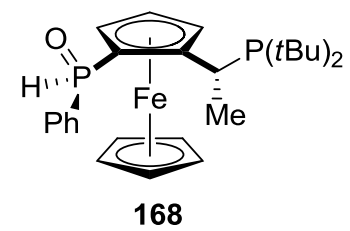

$(1-\mathrm{Ad})_{2} \mathrm{P}(\mathrm{O}) \mathrm{H}$

165

$$
\mathrm{Cy}_{2} \mathrm{P}(\mathrm{O}) \mathrm{H}
$$

166<smiles>CC(C)c1cccc(Pc2ccccc2)c1OP1OC(c2ccccc2)=C(c2ccccc2)C([PH2+](c2ccccc2)c2ccccc2)=C2OC(C)(C)O[C@H]21</smiles>

163

(1)

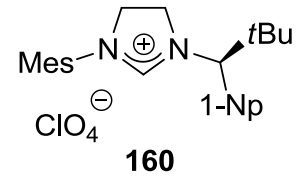


<smiles>Clp1oc2ccc3ccccc3c2c2c(ccc3ccccc32)o1</smiles>

171

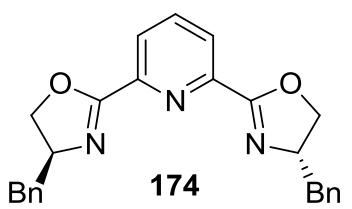

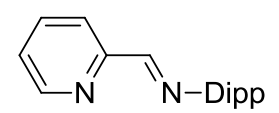

172

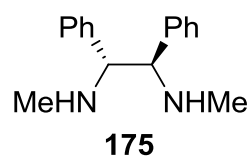

\begin{tabular}{|c|c|c|c|}
\hline Entry & $\mathbf{L}$ & Yield [\%] $]^{[b]}$ & e.r. ${ }^{[c]}$ \\
\hline 1 & $\mid \mathrm{Xy|} \cdot \mathrm{HCl}$ & 92 & $50: 50$ \\
\hline 2 & --- & $<2 \%$ & - \\
\hline $3^{[\mathrm{d}]}$ & $|X y| \cdot H C l$ & 53 & $<5 \%$ ee \\
\hline 4 & 153 & 11 & $60: 40$ \\
\hline $5^{[\mathrm{e}]}$ & 153 & traces & - \\
\hline $6^{[f]}$ & 153 & n.r. & - \\
\hline $7^{[f, g]}$ & 153 & n.r. & - \\
\hline $8^{[\mathrm{h}]}$ & 153 & traces & - \\
\hline 9 & 154 & 6 & $45: 55$ \\
\hline 10 & 155 & 61 & $54: 46$ \\
\hline 11 & 156 & 66 & - \\
\hline 12 & 157 & 23 & - \\
\hline 13 & 158 & 4 & $<5 \%$ ee \\
\hline 14 & 159 & 5 & $<5 \%$ ee \\
\hline 15 & 160 & 8 & $<5 \%$ ee \\
\hline
\end{tabular}




\begin{tabular}{cccc}
\hline 16 & 161 & 12 & $<5 \%$ ee \\
17 & 162 & 15 & $<5 \%$ ee \\
18 & 163 & 2 & $<5 \%$ ee \\
19 & 164 & 8 & $59: 41$ \\
20 & 165 & 20 & - \\
21 & 166 & 18 & - \\
22 & 167 & 20 & $<5 \%$ ee \\
23 & 168 & 22 & $<5 \%$ ee \\
24 & 169 & 5 & - \\
25 & 170 & traces & - \\
26 & 171 & traces & - \\
27 & 172 & 32 & $<5 \%$ ee \\
$28^{[i]}$ & 173 & 17 & $55: 45$ \\
29 & 174 & 12 & $<\%$ \\
30 & 175 & 10 & $(15 \%$ ee \\
\hline
\end{tabular}

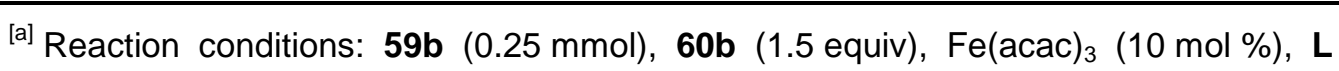
(10 mol \%), CyMgCl (1.0 equiv), TMEDA (2.0 equiv), THF $(0.50 \mathrm{~mL}), 60{ }^{\circ} \mathrm{C}, 16 \mathrm{~h}$. ${ }^{[b]}$ Yield of the isolated product. ${ }^{[c]}$ Determined by chiral HPLC analysis. ${ }^{[d]}$ (-)-Sparteine (2.0 equiv) instead of TMEDA. ${ }^{[e]}$ THF/NMP (5/1) as solvent. ${ }^{[f]}\left[\mathrm{ZnBr}_{2} \cdot \mathrm{TMEDA}\right]$ (2.0 equiv) was added. ${ }^{[\mathrm{g}]} \mathrm{TMEDA}$ was omitted. ${ }^{[\mathrm{h}]} \mathrm{LiCl}$ (1.0 equiv) was added. ${ }^{\left[{ }^{[}\right]} \mathrm{Fe}(\mathrm{acac})_{3}$ was omitted.

Based on the results discussed above (Table 3.5), it became apparent that only bis-aryl NHCs seem to be suitable ligands in this transformation, as no significant enantio-induction and/or conversion was observed with other ligand classes or differently decorated $\mathrm{NHC}$ precursors. Hence, we attempted to rationalize the effect of the substituents of the flanking phenyl groups of the $\mathrm{NHC}$ core in order to design 
more selective and effective ligands for this transformation (Scheme 3.4a). It should be noted that the development of chiral analogues of the common monodentate $\mathrm{NHC}$ ligands IMes and IPr has been described as an extremely challenging task. Indeed, the differences of steric and electronic properties of chiral NHC ligands compared to IMes or IPr often render them incompetent for the envisioned transformations. ${ }^{[241]}$

First, the comparison of xylyl-substituted pre-ligands $(\mathrm{IXy|} \cdot \mathrm{HCl}$ and 155) to $\mathrm{NHC}$ precursor 153 lacking an ortho-methyl group clearly indicated that both substituents in 2-and 6-positions of the $\mathrm{N}$-aryl groups are essential for the reaction to provide the desired alkylated product in good yield (Table 3.5, entries 1, 4, 10). However, no significant enantioselectivity was ever observed with this design. The introduction of bulkier ortho-substituents on the aryl groups of Grubbs-type ${ }^{[211]} \mathrm{NHC}$ ligands would seem highly desirable, as it has been a successful approach in many previous reports. ${ }^{[242]}$ However, additional steric bulk on the ortho-position is not tolerated in the present case, as exemplified by the extremely poor performances of 154 (Table 3.5, entry 9) or IPr in Yoshikais racemic C-H alkylation. ${ }^{[148]}$

Therefore, it appeared clearly that a novel ligand design was required here. Based on the results discussed above, it emerged that two small (i.e. primary) orthosubstituents at the 2- and 6-positions of the flanking aryl groups are necessary in order to achieve high conversions, although no significant enantio-induction was observed with this substitution pattern. Hence, the design of a NHC ligand able to provide the alkylated product with both high yield and enantioselectivity seemed to be an unsolvable problem at first. However, we reasoned that replacing the second methyl substituent at the 6-position of the $\mathrm{N}$-aryl group with a bulky substituent at the adjacent meta 5-position might just do the trick, providing enough steric bulk on both sides of the aryl group, but not too close to the metal center. Interestingly, the envisioned design would create a wide and rather flexible (due to free rotation about the $\mathrm{C}-\mathrm{N}$ bond) $\mathrm{C}_{2}$-symmetric chiral pocket (Fig. 3.4b). Indeed, it is expected, based on the original design by Grubbs, ${ }^{[211]}$ that the chiral DPEN backbone will repel the ortho-methyl substituent of the $\mathrm{N}$-aryl groups. The flanking aryl groups then act as 
chiral relays to position the large meta-substituents on opposite sides of the NHC core, transferring the stereo-information of the chiral backbone to the substrates bound to the metal center during the reaction. This assumption was subsequently supported by a recent publication by Michon and coworkers in which the X-ray crystal structure of a rhodium(I) complex with a NHC ligand following the same design was reported. ${ }^{[243]}$ While the introduction of bulky meta-substituents had been described previously, it should be noted that the reported 2,5-disubstituted $\mathrm{N}$-aryl groups always bore large ortho-substituents. ${ }^{[236,244]}$

a) inspiration for meta-substituted NHC ligands

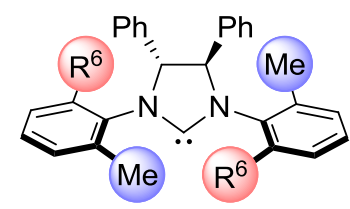

The problem with $\mathrm{R}^{6}$ :

- $\mathrm{R}^{6}=\mathrm{H}$ (153): moderate enantio-induction, poor conversion

- $\mathrm{R}^{6}=\mathrm{Me}(\mathbf{1 5 5})$ : poor enantio-induction, high conversion

- Bulkier (i.e. $2^{\circ}$ alkyl) ortho-substituents: poor enantio-induction and conversion

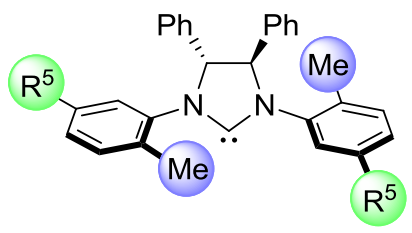

What about bulky meta-substituents?

b) stereochemical model

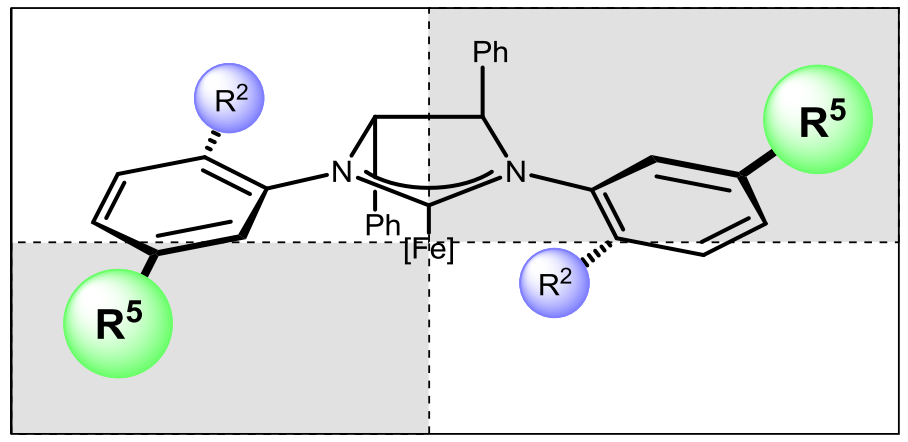

Scheme 3.4. Rationale for remote meta-substituted NHC ligands.

Thereby, various $\mathrm{NHC}$ precursors bearing $\mathrm{N}$-aryl groups with meta-substituents were prepared and probed in the envisioned transformation (Table 3.6). While the prototypical chiral NHC precursor 153 only provided poor results (Table 3.5, entry 1 and Table 3.6, entry 4), the introduction of a simple meta-methyl substituent exhibited a huge beneficial effect on the reaction outcome (Table 3.6, entry 2). 
Extensive variations of the meta-substituent (entries 3-9) revealed an isopropyl group to be optimal (entry 3). Variations of the ortho-substituent were probed as well, but a methoxy substituent provided inferior results compared to a simple methyl group (entry 5). N-9-phenanthryl-NHC precursor 186 was tested as well. Despite the promising conversion, no significant enantio-induction was observed (entry 10). Variations of the chiral DPEN backbone were then investigated. Remarkably, a NHC precursor derived from $\mathrm{DACH}(\mathbf{1 8 7}$, entry 11$)$ promoted the hydroarylation with an isolated yield similar to its DPEN-derived analogue (177, entry 3 ), but no significant enantioselectivity was observed. Changing the ligand-to-metal ratio from 1:1 to $2: 1$ improved the reaction outcome considerably, increasing the enantiomeric ratio to 75:25 (entry 12). Finally, replacing TMEDA with other amine additives had a rather limited effect on the outcome of the transformation (entries 13-14).

Table 3.6. Optimization of chiral NHC pre-ligands for the enantioselective hydroarylation of styrene. $^{[\mathrm{a}]}$
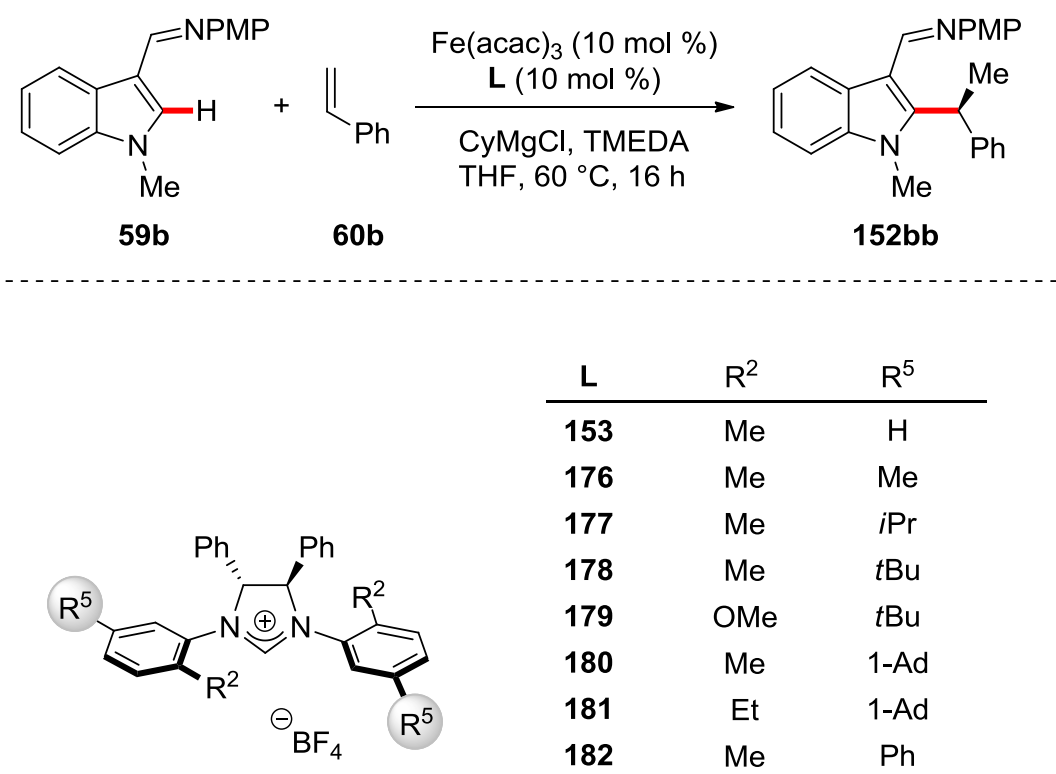

\begin{tabular}{ccc}
$\mathrm{L}$ & $\mathrm{R}^{2}$ & $\mathrm{R}^{5}$ \\
\hline 153 & $\mathrm{Me}$ & $\mathrm{H}$ \\
176 & $\mathrm{Me}$ & $\mathrm{Me}$ \\
177 & $\mathrm{Me}$ & $i \mathrm{Pr}$ \\
178 & $\mathrm{Me}$ & $t \mathrm{Bu}$ \\
179 & $\mathrm{OMe}$ & $t \mathrm{Bu}$ \\
180 & $\mathrm{Me}$ & $1-\mathrm{Ad}$ \\
181 & $\mathrm{Et}$ & $1-\mathrm{Ad}$ \\
182 & $\mathrm{Me}$ & $\mathrm{Ph}$ \\
183 & $\mathrm{Me}$ & $4-t \mathrm{Bu}-\mathrm{C}_{6} \mathrm{H}_{4}$ \\
184 & $\mathrm{Me}$ & $\mathrm{CH}\left(4-\mathrm{F}-\mathrm{C}_{6} \mathrm{H}_{4}\right)_{2}$ \\
185 & $\mathrm{Me}$ & $4-\mathrm{Hept}$
\end{tabular}




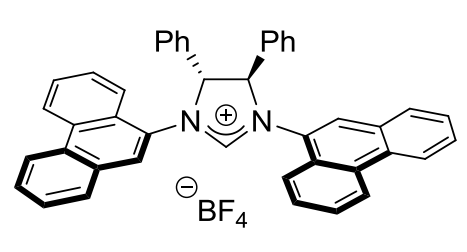

186

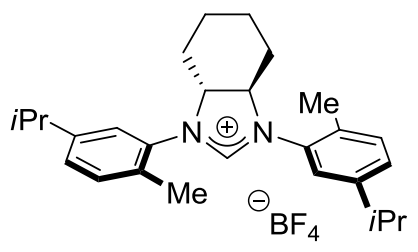

187

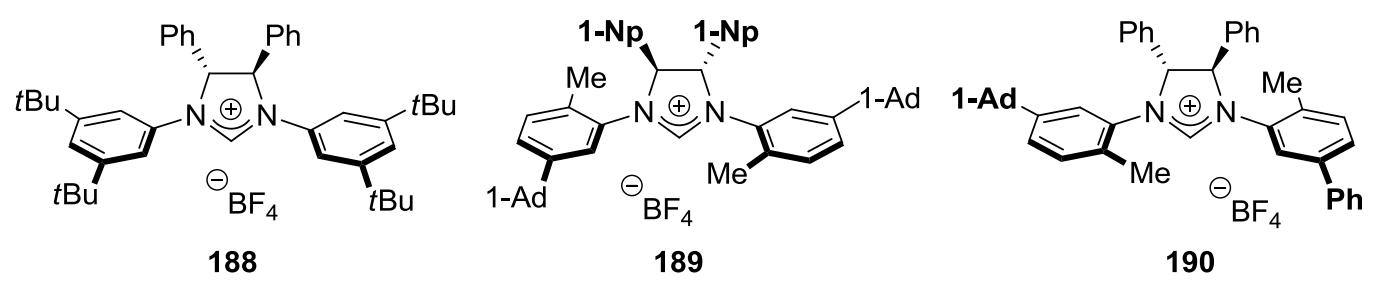

\begin{tabular}{|c|c|c|c|}
\hline Entry & $\mathbf{L}$ & Yield [\%] ${ }^{[b]}$ & e.r. ${ }^{[c]}$ \\
\hline 1 & 153 & 11 & $60: 40$ \\
\hline 2 & 176 & 48 & $67: 33$ \\
\hline 3 & 177 & 67 & $71: 29$ \\
\hline 4 & 178 & 53 & $70: 30$ \\
\hline 5 & 179 & 21 & $58: 42$ \\
\hline 6 & 180 & 58 & 69:31 \\
\hline 7 & 182 & 23 & $64: 36$ \\
\hline 8 & 184 & 33 & $66: 34$ \\
\hline 9 & 185 & 36 & $66: 34$ \\
\hline 10 & 186 & 62 & $55: 45$ \\
\hline 11 & 187 & 57 & $<5 \%$ ee \\
\hline $12^{[\mathrm{d}]}$ & 177 & 65 & $75: 25$ \\
\hline
\end{tabular}




\begin{tabular}{|c|c|c|c|}
\hline $13^{[\mathrm{d}, \mathrm{e}]}$ & 177 & 68 & $72: 28$ \\
\hline $14^{[\mathrm{d}, \mathrm{f}]}$ & 177 & 58 & $68: 32$ \\
\hline \multicolumn{4}{|c|}{$\begin{array}{l}\text { [a] Reaction conditions: } 59 \mathrm{~b}(0.25 \mathrm{mmol}), 60 \mathrm{~b} \text { (1.5 equiv), Fe(acac) } 3(10 \mathrm{~mol} \%), \mathbf{L} \\
(10 \mathrm{~mol} \%), \mathrm{CyMgCl}\left(1.0 \text { equiv), TMEDA (2.0 equiv), THF }(0.50 \mathrm{~mL}), 60{ }^{\circ} \mathrm{C}, 16 \mathrm{~h} \text {. }\right. \\
\text { [b] Yield of the isolated product. }{ }^{[\mathrm{c}]} \text { Determined by chiral HPLC analysis. }{ }^{[\mathrm{d}]} \mathbf{L}(20 \mathrm{~mol} \%) \\
\text { and CyMgCl (1.1 equiv). }{ }^{[\mathrm{e}]} \text { PMDETA instead of TMEDA. }{ }^{[\mathrm{f}]} \text { BDMAEE instead of } \\
\text { TMEDA. }\end{array}$} \\
\hline
\end{tabular}

As asymmetric catalysis can sometimes be very substrate-specific, differently decorated indoles and styrenes were probed in the transformation (Table 3.7). First, the impact of the indole's $N$-protecting group was investigated (entries 1-5). Whereas different $N$-alkyl substituents proved viable in the reaction, Boc and $p$-tosyl protecting groups fell short in delivering any $\mathrm{C}-\mathrm{H}$ alkylated product, in complete agreement with the previous report of Yoshikai. ${ }^{[148]}$ Interestingly, those findings are in stark contrast to the cobalt-catalyzed hydroarylation of styrenes with indoles developed by the same research group, where electron-withdrawing groups such as Boc were not only tolerated, but superior to alkyl or benzyl moieties. ${ }^{[106]}$ MOM-protected indole $\mathbf{5 9 g}$ provided nearly identical results compared to $\mathrm{N}$-methylindole $\mathbf{5 9 b}$, while the introduction of a bulkier benzyl group had a critical effect. Indeed, both the yield and the enantioselectivity were significantly improved (entry 5). Further optimization studies were conducted using this substrate (entries 6-9). Different Grignard reagents or lower catalyst loadings did not afford satisfactory results. Remarkably, in sharp contrast to the work of Yoshikai ${ }^{[148]}$ the transformation was found to occur even in the absence of TMEDA, albeit in lower efficiency and selectivity. With the optimal indole substrate 59c in hand, various styrene derivatives $\mathbf{6 0}$ were tested in the iron-catalyzed asymmetric hydroarylation. 2-Vinylnaphthalene 60a showed poor performance in the transformation, while parafluorostyrene $60 \mathrm{~g}$ did not give better results than simple styrene $60 \mathrm{~b}$ (entries 10-11). Gratifyingly, the use of electron-rich 4-methoxystyrene $60 \mathrm{c}$ provided a far better yield and enantioselectivity compared to other styrene analogues (entry 12). The excellent conversion allowed us to lower the reaction temperature to $45^{\circ} \mathrm{C}$, improving the 
enantioselectivity to $89: 11$ e.r., while maintaining a synthetically useful yield (entry 13). While these conditions were initially used to study the substrate scope and limitations of the transformation (vide infra), a subsequent ligand screening revealed the superiority of meta-adamantyl ligand 180, which provided the hydroarylation product 62cc with an enantiomeric ratio of 92:8 (entries 14-19). Interestingly, the importance of the small ortho-methyl group was confirmed, as poor results were obtained with either a bulkier or no substituent (entries 15-16). Additionally, the introduction of a bulkier substituents in the chiral backbone did not improve the enantioselectivity (entry 17), in contrast to a previous work of Cramer and coworkers (Scheme 1.43). ${ }^{[210]}$ Outstanding conversions were obtained with $C_{2^{-}}$and $C_{1}$-symmetrical ${ }^{[241]}$ precursors bearing meta-aryl substituents, but lower enantiomeric excesses were observed (entries 18-19). Finally, $\mathrm{FeF}_{3}$ fell short in delivering any hydroarylation product (entry 20), despite its documented superiority over other iron precursors in iron/NHC-catalyzed Kumada-Corriu-type crosscouplings. ${ }^{[245]}$

Table 3.7. Substrate engineering and final optimization for the enantioselective hydroarylation of styrenes. ${ }^{[a]}$ (Ligand structures are given in Table 3.6.)

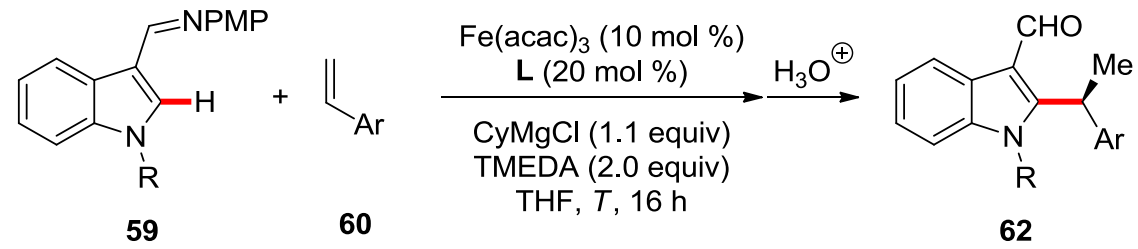

\begin{tabular}{|c|c|c|c|c|c|c|}
\hline Entry & $\mathbf{R}$ & $\mathrm{Ar}$ & $\mathbf{L}$ & $T\left[{ }^{\circ} \mathrm{C}\right]$ & Yield [\%] $^{[\mathrm{b}]}$ & e.r. ${ }^{[c]}$ \\
\hline $1^{[\mathrm{d}]}$ & $\mathrm{Me}$ & $\mathrm{Ph}$ & 177 & 60 & 65 & $75: 25$ \\
\hline $2^{[\mathrm{d}]}$ & Boc & $\mathrm{Ph}$ & 177 & 60 & n.r. & - \\
\hline $3^{[\mathrm{d}]}$ & Ts & $\mathrm{Ph}$ & 177 & 60 & n.r. & - \\
\hline 4 & MOM & $\mathrm{Ph}$ & 177 & 60 & 64 & $75: 25$ \\
\hline $5^{[\mathrm{d}]}$ & $\mathrm{Bn}$ & $\mathrm{Ph}$ & 177 & 60 & 73 & $80: 20$ \\
\hline
\end{tabular}




\begin{tabular}{|c|c|c|c|c|c|c|}
\hline $6^{[\mathrm{d}, \mathrm{e}]}$ & $\mathrm{Bn}$ & $\mathrm{Ph}$ & 177 & 60 & 43 & $78: 22$ \\
\hline $7^{[\mathrm{d}, \mathrm{f}]}$ & $\mathrm{Bn}$ & $\mathrm{Ph}$ & 177 & 60 & n.r. & - \\
\hline $8^{[d, g]}$ & $\mathrm{Bn}$ & $\mathrm{Ph}$ & 177 & 60 & 42 & $68: 32$ \\
\hline $9^{[\mathrm{d}, \mathrm{h}]}$ & $\mathrm{Bn}$ & $\mathrm{Ph}$ & 177 & 60 & 52 & $76: 24$ \\
\hline 10 & $\mathrm{Bn}$ & $2-N p$ & 177 & 60 & 46 & $56: 44$ \\
\hline 11 & $\mathrm{Bn}$ & $4-\mathrm{F}-\mathrm{C}_{6} \mathrm{H}_{4}$ & 177 & 60 & 72 & $81: 19$ \\
\hline 12 & $\mathrm{Bn}$ & PMP & 177 & 60 & 93 & $85: 15$ \\
\hline 13 & $\mathrm{Bn}$ & PMP & 177 & 45 & 76 & $89: 11$ \\
\hline 14 & $\mathrm{Bn}$ & PMP & 180 & 45 & 95 & $92: 8$ \\
\hline 15 & $\mathrm{Bn}$ & PMP & 181 & 45 & 78 & $90: 10$ \\
\hline 16 & $\mathrm{Bn}$ & PMP & 188 & 45 & 10 & $58: 42$ \\
\hline 17 & $\mathrm{Bn}$ & PMP & 189 & 45 & 87 & $11: 89$ \\
\hline 18 & $\mathrm{Bn}$ & PMP & 183 & 45 & 98 & $75: 25$ \\
\hline 19 & $\mathrm{Bn}$ & PMP & 190 & 45 & 98 & $87: 13$ \\
\hline $20^{[i]}$ & $\mathrm{Bn}$ & PMP & 180 & 45 & traces & - \\
\hline
\end{tabular}

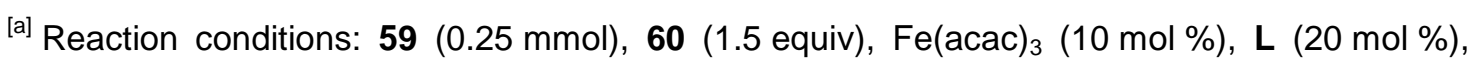
CyMgCl (1.1 equiv), TMEDA (2.0 equiv), THF $(0.50 \mathrm{~mL}), 60^{\circ} \mathrm{C}, 16 \mathrm{~h} .{ }^{[\mathrm{b}]}$ Yield of the isolated product. ${ }^{[c]}$ Determined by chiral HPLC analysis. ${ }^{[d]}$ No hydrolysis, the product was isolated as the imine. ${ }^{[e]} \mathrm{PhMgCl}$ instead of $\mathrm{CyMgCl}$. ${ }^{[]]} \mathrm{TMSCH}_{2} \mathrm{MgCl}$ instead of $\mathrm{CyMgCl}$. ${ }^{[g]} \mathrm{Fe}(\mathrm{acac})_{3}$ (3.0 mol \%) and 177 (6.0 mol \%). ${ }^{[\mathrm{h}]}$ TMEDA was omitted. ${ }^{[\mathrm{i}]} \mathrm{FeF}_{3}$ instead of $\mathrm{Fe}(\mathrm{acac})_{3}$.

While studying the substrate scope of the reaction (vide infra), vinylferrocene 191a was identified as a suitable coupling partner in this transformation, providing the hydroarylation product with unprecedented enantioselectivities. Those findings prompted us to re-optimize the reaction for this unique class of substrates. Ferrocene derivatives are particularly important in asymmetric catalysis, ${ }^{[246]}$ material 
sciences, ${ }^{[247]}$ and bioinorganic chemistry, ${ }^{[248]}$ among others. ${ }^{[113]}$ First, a representative set of chiral NHC precursors was probed in the asymmetric alkylation of $N$-benzylindole 59c with vinylferrocene 191a (Table 3.8). The simple Grubbs' carbenes 153 and 154 performed poorly in the envisioned reaction, but confirmed the importance of the ortho-methyl groups (entries 1-2). An excellent conversion but only a moderate enantio-induction were observed with xylyl-substituted $\mathrm{NHC}$ precursor 155 (entry 3). Once more, the introduction of meta-substituents on the flanking aryl group of the NHC had a dramatic effect and allowed for high enantioselectivities (entries 4-8). Remarkably, a clear trend was observed depending on the size of the meta-alkyl substituent, with 1-adamantyl groups giving optimal results (entry 7). Interestingly, meta-phenyl substituents gave the highest conversion despite the moderate enantio-induction (entry 8). Kündig's $N, N$-alkylsubstituted $\mathrm{NHCs}^{[249]}$ were probed as well, but performed extremely poorly (entries 9-10), in agreement with previous work for the hydroarylation of styrenes (Table 3.5).

Table 3.8. Ligand optimization of the enantioselective hydroarylation of vinylferrocene $191 a^{[a]}$

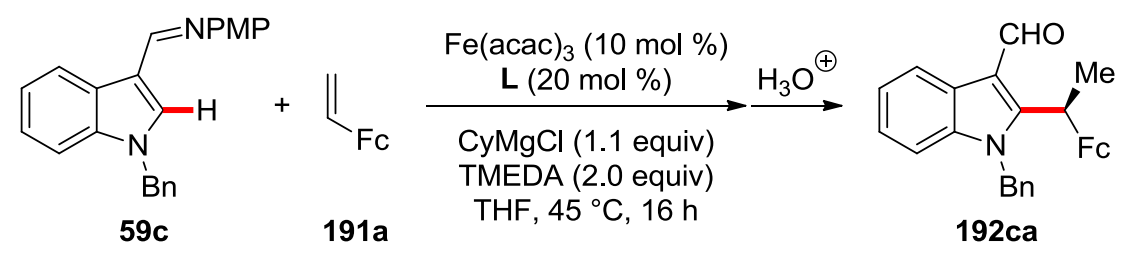

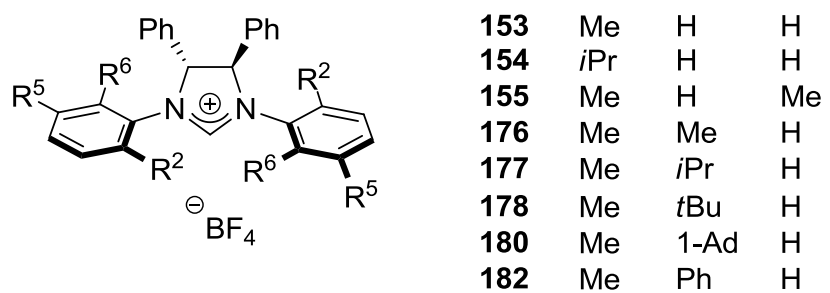
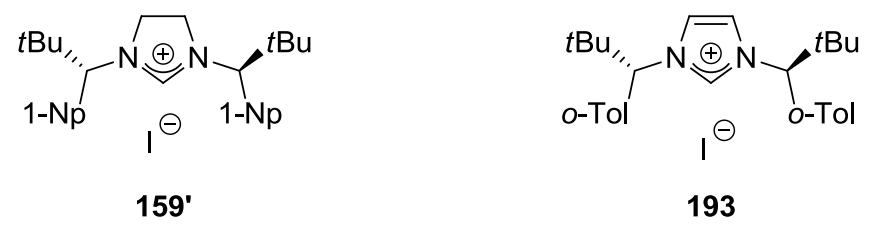


\begin{tabular}{cccc}
\hline Entry & $\mathbf{L}$ & Yield [\%] $^{[\mathrm{b}]}$ & e.r. $^{[\mathrm{c}]}$ \\
\hline $1^{[\mathrm{d}]}$ & $\mathbf{1 5 3}$ & 5.5 & $25: 75$ \\
$2^{[\mathrm{d}]}$ & $\mathbf{1 5 4}$ & 3.3 & $54: 46$ \\
3 & $\mathbf{1 5 5}$ & 81 & $41: 59$ \\
4 & $\mathbf{1 7 6}$ & 69 & $11: 89$ \\
5 & $\mathbf{1 7 7}$ & 69 & $7: 93$ \\
6 & $\mathbf{1 7 8}$ & 53 & $5: 95$ \\
7 & $\mathbf{1 8 0}$ & 69 & $4: 96$ \\
8 & $\mathbf{1 8 2}$ & 76 & $12: 88$ \\
9 & $\mathbf{1 5 9}$ & n.r. & - \\
10 & $\mathbf{1 9 3}$ & n.r. & - \\
\hline
\end{tabular}

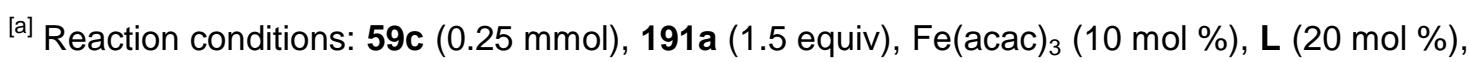
CyMgCl (1.1 equiv), TMEDA (2.0 equiv), THF $(0.50 \mathrm{~mL}), 45^{\circ} \mathrm{C}, 16 \mathrm{~h} .{ }^{[b]}$ Yield of the isolated product. ${ }^{[\mathrm{c}]}$ Determined by HPLC analysis. ${ }^{[\mathrm{d}]}$ At $60^{\circ} \mathrm{C}$.

Additional optimization studies and control experiments were then conducted (Table 3.9). A Job plot ${ }^{[250]}$ of the ligand-to-metal ratio confirmed a $2: 1$ ratio to be optimal (entries 1-4). However, the only slightly lower conversion obtained using a 1:1 ligand-to-metal ratio, along with the previous work of Yoshikai, ${ }^{[148]}$ would rather indicate a mono-ligated iron/NHC catalyst to be operative in the transformation. Moreover, detailed mechanistic studies provided further support for a mono-NHC ligated iron species to be catalytically active (vide infra). Sub-stoichiometric amounts of $\mathrm{CyMgCl}$ only provided the desired alkylated product 192ca in low yields, supporting the Grignard reagent to play a bigger role than just serving as a base to generate the free NHC in situ (entries 5-7). Other organomagnesium reagents were tested in the transformation, but proved to be inferior to $\mathrm{CyMgCl}$ (entries 8-11). Interestingly, while the highest conversions were obtained with organometallic 
species prone to $\beta$-hydride elimination after transmetalation to iron ${ }^{[240 b, 251]}$ (entries 1 and 9), significant conversion was observed with $\mathrm{PhMgCl}$ as well (entry 8). In contrast, no conversion was observed using $\mathrm{NaOtBu}$ as the base (entry 12), which supports an in situ generated organoiron species to be involved in the catalysis. Despite the different oxidation states and counter-ions, $\mathrm{FeCl}_{2}$ provided results comparable to $\mathrm{Fe}(\mathrm{acac})_{3}$ (entry 13). A reaction conducted at ambient temperature furnished the alkylated product 192ca with a slightly higher enantiomeric ratio of $97: 3$, but significantly lower yield (entry 14). In contrast, increasing the temperature to $60^{\circ} \mathrm{C}$ improved the yield but lowered the enantioselectivity (entry 15). Once again, in stark contrast to the work of Yoshikai, ${ }^{[148]}$ the transformation was found to occur even in the absence of TMEDA, albeit in lower efficiency (entry 16). Finally, control experiments confirmed the key role of the iron catalyst, as no product formation was detected in the absence of $\mathrm{Fe}(\mathrm{acac})_{3}$ or using other first-row transition metal salts (entries 17-20).

Table 3.9. Further optimization studies and control experiments for the enantioselective hydroarylation of vinylferrocene $191 a^{[a]}$
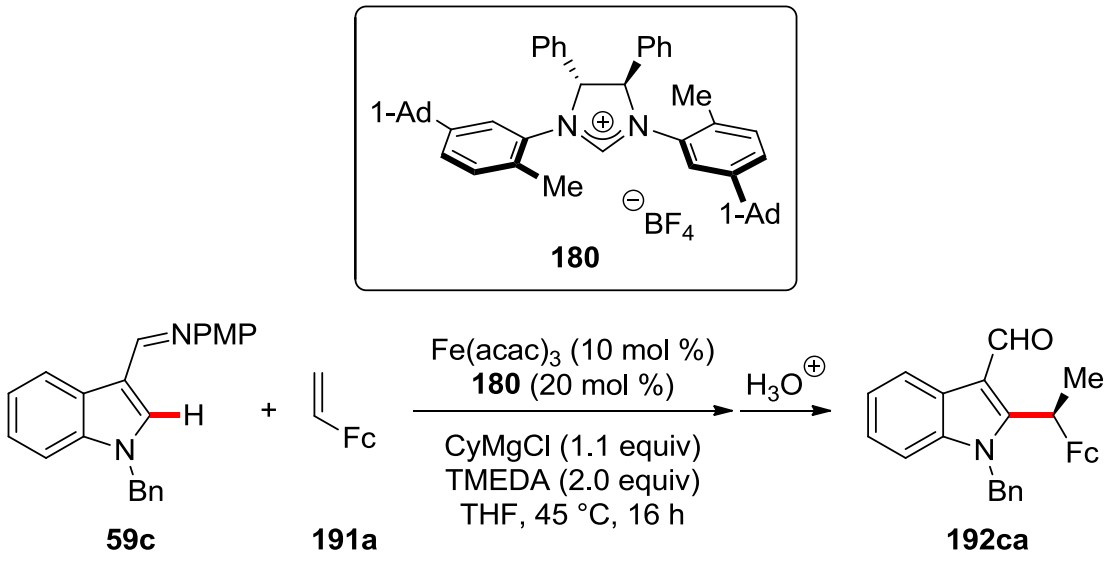

\begin{tabular}{cccc}
\hline Entry & Deviation from the standard conditions & Yield [\%] $^{[\mathrm{b}]}$ & e.r. $^{[\mathrm{c}]}$ \\
\hline 1 & none & 69 & $4: 96$ \\
$2^{[\mathrm{d}]}$ & $\mathbf{1 8 0}(5.0 \mathrm{~mol} \%)$ & 36 & $8: 92$ \\
$3^{[\mathrm{d}]}$ & $\mathbf{1 8 0}(10 \mathrm{~mol} \%)$ & 53 & $5: 95$ \\
\hline
\end{tabular}




\begin{tabular}{|c|c|c|c|}
\hline $4^{[\mathrm{e}]}$ & $180(30 \mathrm{~mol} \%)$ & 65 & $4: 96$ \\
\hline 5 & CyMgCl was omitted & n.r. & - \\
\hline 6 & CyMgCl ( 0.50 equiv) & 18 & $4: 96$ \\
\hline 7 & CyMgCl (0.60 equiv) & 26 & $4: 96$ \\
\hline 8 & $\mathrm{PhMgCl}$ instead of $\mathrm{CyMgCl}$ & 33 & $4: 96$ \\
\hline 9 & iPrMgCl instead of CyMgCl & 55 & $5: 95$ \\
\hline 11 & $\mathrm{TMSCH}_{2} \mathrm{MgCl}$ instead of $\mathrm{CyMgCl}$ & n.r. & - \\
\hline 12 & $\mathrm{NaOtBu}$ instead of $\mathrm{CyMgCl}$ & n.r. & - \\
\hline 13 & $\mathrm{FeCl}_{2}$ instead of $\mathrm{Fe}(\mathrm{acac})_{3}$ & 50 & $5: 95$ \\
\hline 14 & at $23^{\circ} \mathrm{C}$ & 40 & $3: 97$ \\
\hline 15 & at $60^{\circ} \mathrm{C}$ & 72 & $6: 94$ \\
\hline 16 & TMEDA was omitted & 39 & $5: 95$ \\
\hline 17 & $\mathrm{Fe}(\mathrm{acac})_{3}$ was omitted & - & - \\
\hline 18 & $\mathrm{Co}(\mathrm{acac})_{2}$ instead of $\mathrm{Fe}(\mathrm{acac})_{3}$ & - & - \\
\hline 19 & $\mathrm{Mn}(\mathrm{acac})_{2}$ instead of $\mathrm{Fe}(\mathrm{acac})_{3}$ & - & - \\
\hline 20 & $\mathrm{Ni}(\text { acac })_{2}$ instead of $\mathrm{Fe}(\text { acac })_{3}$ & - & - \\
\hline
\end{tabular}

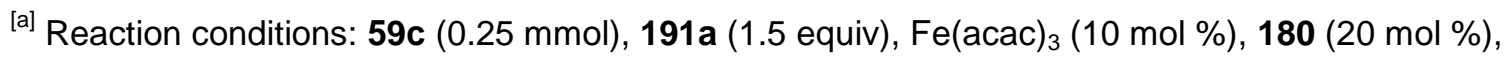
CyMgCl (1.1 equiv), TMEDA (2.0 equiv), THF $(0.50 \mathrm{~mL}), 45^{\circ} \mathrm{C}, 16 \mathrm{~h} .{ }^{[b]}$ Yield of the isolated product. ${ }^{[c]}$ Determined by chiral HPLC analysis. ${ }^{[d]} \mathrm{CyMgCl}\left(1.0\right.$ equiv). ${ }^{[\mathrm{e}]} \mathrm{CyMgCl}$ (1.2 equiv).

It should be highlighted that such high enantioselectivities are remarkable in organometallic iron catalysis and remain unmatched as of today. Indeed, at the outset of this work, the only previous example of such an asymmetric transformation was an iron-catalyzed Kumada-Corriu cross-coupling employing a complex chiral bidentate phosphine, which provided the coupled products in up to 91:9 e.r. 
(Scheme 1.29). ${ }^{[149]}$ Later works on cross-couplings ${ }^{[150]}$ and $\mathrm{C}-\mathrm{H}$ activation ${ }^{[152]}$ provided the corresponding products with even lower enantioselectivities. Thus, enantiomeric excesses over $90 \%$, furthermore obtained with a monodentate chiral ligand, are absolutely outstanding in this burgeoning field of catalysis. Indeed, the present work not only represents the first enantioselective transformation by innersphere iron-catalyzed $\mathrm{C}-\mathrm{H}$ activation, but also the first use of chiral $\mathrm{NHC}$ ligands in asymmetric iron catalysis.

\subsubsection{Substrate Scope and Limitations}

With the optimized iron catalyst in hands, its performance in the intermolecular asymmetric $\mathrm{C}-\mathrm{H}$ alkylation of indole derivatives 59 with the electron-rich 4-methoxystyrene (60c) was explored (Table 3.10). The desired carbaldehydes 62 were obtained with excellent yields and high levels of enantiocontrol from diversely substituted indoles 59 (entries 1-12). Indeed, electron-rich as well as electron-poor indoles 59 were amenable to the present reaction. Furthermore, the transformation was fully compatible with numerous functionalities on the indole nitrogen, including alkyl and benzyl substituents bearing synthetically useful functional groups. It is noteworthy that NHC precursor 177 , bearing meta-IPr substituents, was found to furnish products $62 \mathrm{fc}, 62 \mathrm{gc}$ and $\mathbf{6 2 \mathrm { bc }}$ with a slightly improved enantioselectivity compared to pre-ligand 180 (entries $4,5,8$ ). Remarkably, the pharmacologically relevant 7-azaindole 59n was also identified as a viable substrate for the first time in iron-catalyzed $\mathrm{C}-\mathrm{H}$ activations, although the alkylated product $62 \mathrm{nc}$ was only obtained with a moderate enantiomeric ratio of 76:24 (entry 13). A single recrystallization however improved the enantiomeric ratio to 90:10. Yet, other azaindoles gave unsatisfactory results so far (entries 14-15). Furthermore, no significant conversion was observed using pyrrole 59q or benzothiophene 194 (entries 16-17). 
Table 3.10. Substrate scope of substituted (aza)indoles 59 in the iron-catalyzed enantioselective $\mathrm{C}-\mathrm{H}$ alkylation with styrene $60 \mathrm{c}^{\text {[a] }}$

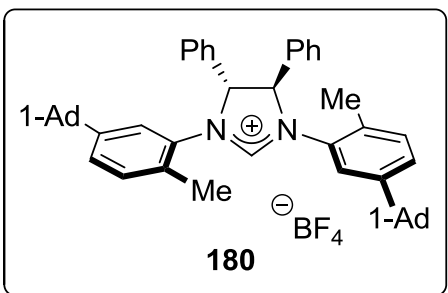

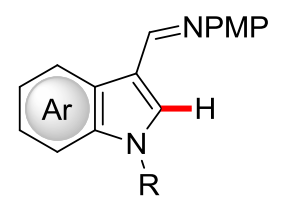

59

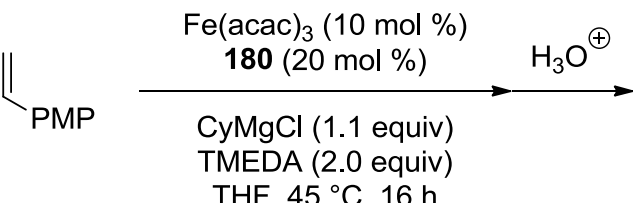

60c

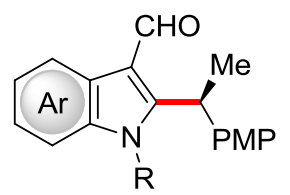

62

Entry


5<smiles>COn1c(C=N)c(C=[N+]=[N-])c2ccccc21</smiles>

$59 \mathrm{~g}$

6<smiles>[Y17]N=Cc1cn(CC(F)F)c2ccccc12</smiles>

$59 h$

7<smiles>N=Cc1c(C=[N+]=P)c2ccccc2n1CC1OCCC1=O</smiles>

8<smiles>Cn1cc(C=NN=[W])c2ccccc21</smiles>

$59 b$<smiles>COc1ccc2c(c1)c(/C=N/N=[W])c(Cc1ccccc1)n2Cc1ccccc1</smiles>

59j<smiles>[Y7]N=Cc1cn(Cc2ccccc2)c2ccc(F)cc12</smiles>

59k<smiles>C[C@H](P)c1c(C=O)c2ccccc2n1[O+]</smiles>

80 $86: 14$

$84^{[\mathrm{d}]}$ $87: 13^{[d]}$

62gc<smiles>C[C@@H](P)c1c(C=O)c2ccccc2n1CC(F)F</smiles>

96 94:6 $81^{[\mathrm{d}]}$ $91: 9^{[d]}$<smiles>[Y6]C(P)c1c(C=O)c2ccccc2n1CC1OCCC1OC(C)=O</smiles><smiles>C[C@H](P)c1c(C=O)c2ccccc2n1C</smiles>

$62 b c$<smiles>COc1ccc2c(c1)c(C=O)c(C(C)CNP)n2Cc1ccccc1</smiles>

62jc<smiles>CC(P)c1c(C=O)c2cc(F)ccc2n1Cc1ccccc1</smiles>

62kc
93 $76^{[\mathrm{d}]}$ $84: 16$

82

$76^{[\mathrm{d}]}$

94:6

$91: 9^{[d]}$

98

$85^{[\mathrm{d}]}$ $90: 10^{[\mathrm{dd}]}$

95

$76^{[\mathrm{d}]}$ 93:7

$91: 9^{[\mathrm{d}]}$ 
11<smiles>Cc1cc(C)cc(Cn2c(/C=N/N)c(/C=N/P)c3ccccc32)c1</smiles><smiles></smiles>

90

$91: 9$

59I

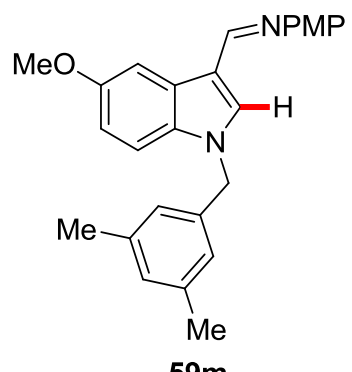<smiles>COc1cc(C)cc(Cn2c(C(C)[Pb])c(C=O)c3cc(OC)ccc32)c1</smiles>

96

$91: 9$

12

$59 m$

$13^{[\mathrm{e}]}$<smiles>[N+]=NC=Cc1c(Cc2ccccc2)n(Cc2ccccc2)c2ncccc12</smiles>

$59 n$

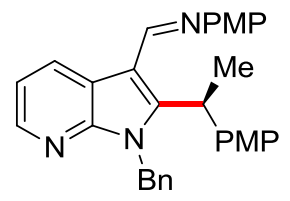

84

$76: 24$

$51^{[\mathrm{f}]}$

$90: 10^{[\mathrm{f}]}$

$14^{[\mathrm{e}]}$<smiles>N=Cc1c(N=[W+]=N)c2cnccc2n1Cc1ccccc1</smiles>

$--$

traces

590

$15^{[\mathrm{e}]}$<smiles></smiles>

$<10 \%{ }^{[\mathrm{g}]}$

$59 p$

16<smiles></smiles>
$--$
$<5 \%$ [g]

$59 q$ 
17

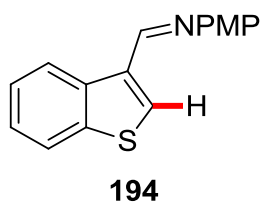

n.r.

194

${ }^{[a]}$ Reaction conditions: 59 (0.25 mmol), 60c (1.5 equiv), Fe(acac) $3(10 \mathrm{~mol} \%), 180$ (20 mol \%), CyMgCl (1.1 equiv), TMEDA (2.0 equiv), THF $(0.50 \mathrm{~mL}), 45^{\circ} \mathrm{C}, 16 \mathrm{~h}$. ${ }^{[\mathrm{b}]}$ Yield of the isolated product. ${ }^{[c]}$ Determined by chiral HPLC analysis. ${ }^{[\mathrm{d}]}$ Using 177 instead of $180 .{ }^{[\mathrm{e}]}$ No hydrolysis, product isolated as the imine. ${ }^{[\mathrm{fl}]}$ After recrystallization from $\mathrm{PrOH} .{ }^{[9]}$ Determined by ${ }^{1} \mathrm{H}$ NMR.

Thereafter, a variety of styrenes 60 were tested in the $\mathrm{C}-\mathrm{H}$ transformation (Table 3.11). Diverse vinylarenes 60 with electron-rich and electron-poor substituents smoothly underwent the iron-catalyzed hydroarylation with good to excellent levels of enantioselectivity, providing exclusively the branched products 62 (entries 1-8). The high chemoselectivity and broad substrate scope of the transformation were however met with some limitations. No significant conversion was observed with styrenes bearing perfluorinated groups or additional substituents on the alkene (entries 9-11). Additionally, several vinylheteroarenes 60 were probed in the intermolecular hydroarylation, but no desired alkylated product could be obtained (entries 12-13). Unactivated alkenes such as 1-octene 29a also failed to provide any $\mathrm{C}-\mathrm{H}$ alkylated indole (entry 14). Vinyl ethers, vinyl silanes and dienes 102 also fell short in the reaction (entries 15-17).

Table 3.11. Substrate scope of vinylarenes 60 in the iron-catalyzed enantioselective $\mathrm{C}-\mathrm{H}$ alkylation. ${ }^{[a]}$

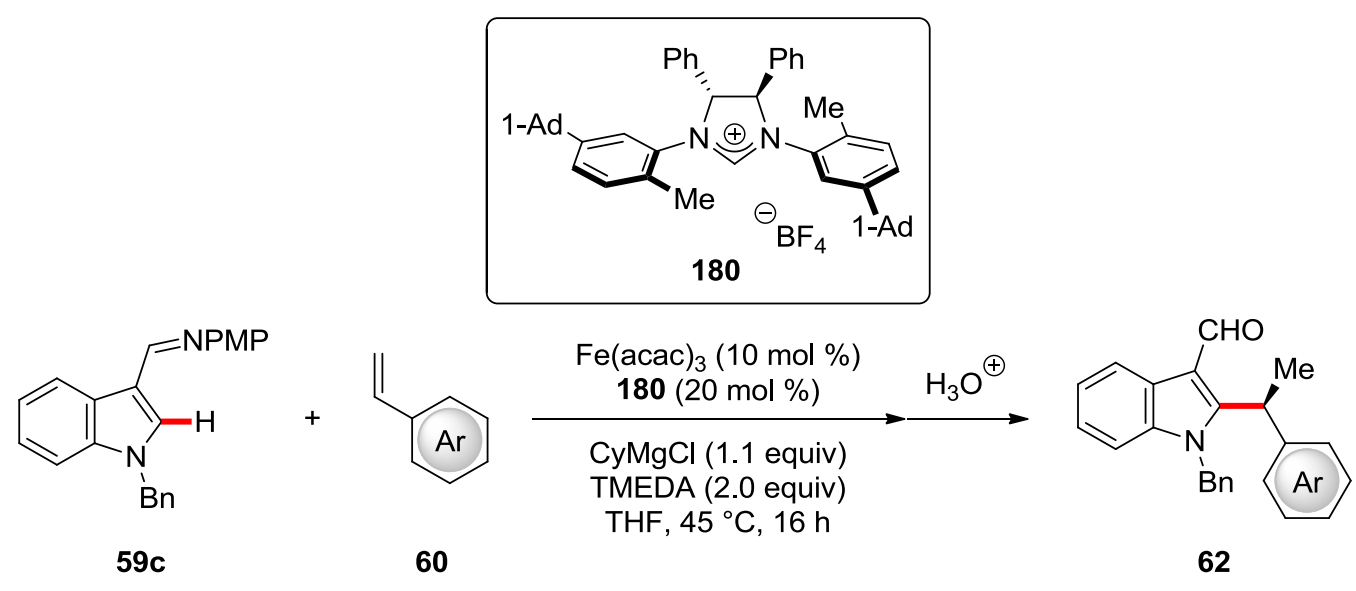




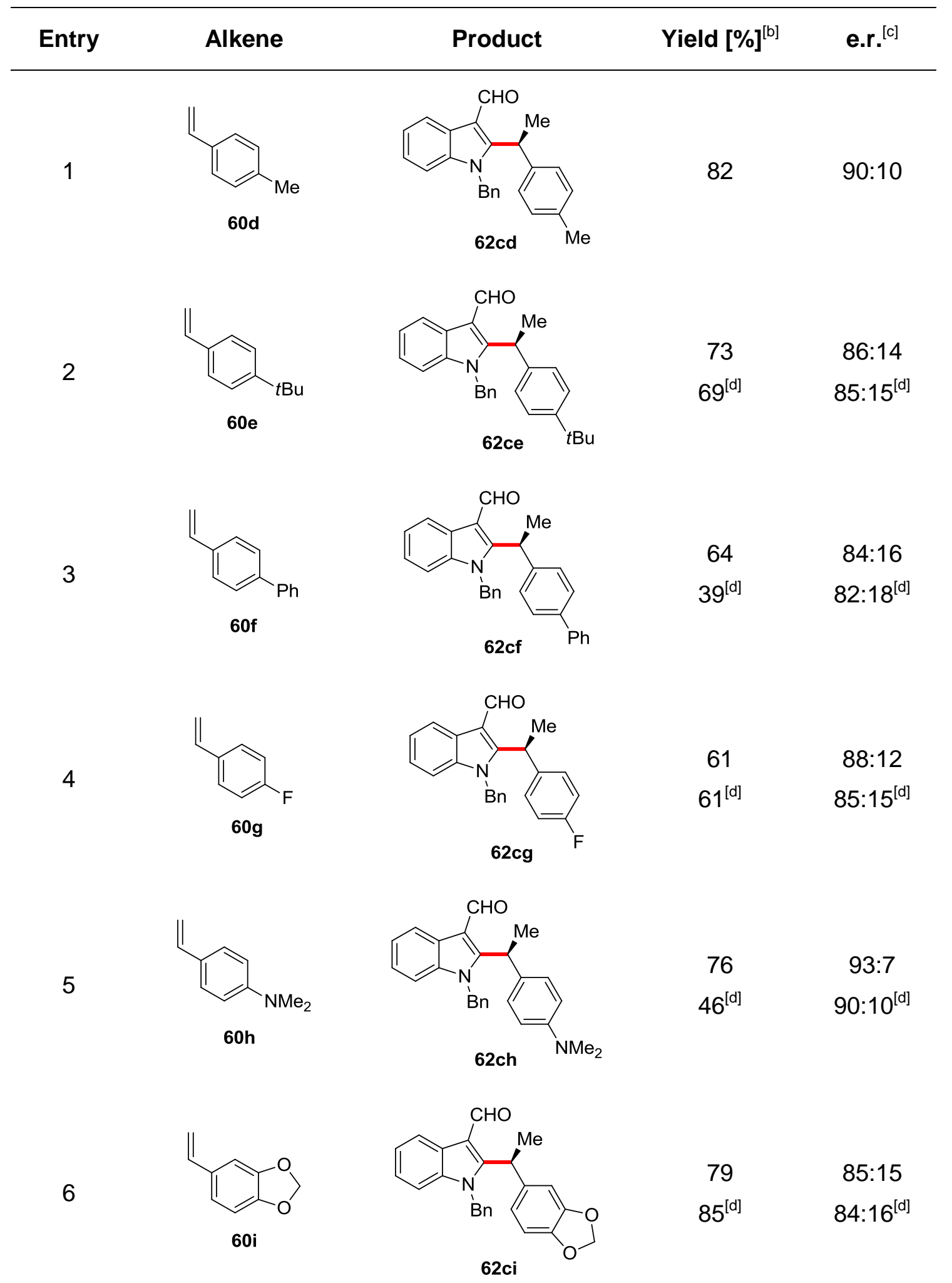


7<smiles>C=Cc1ccc(OC)c(OC)c1</smiles>

$60 \mathrm{j}$

8<smiles>C=Cc1ccc(C)cc1C</smiles>

9<smiles>C=Cc1ccc(C(F)(F)F)cc1</smiles>

601

10<smiles>C=Cc1c(F)c(F)c(F)c(F)c1F</smiles>

$60 \mathrm{~m}$

11<smiles></smiles>

$60 n$

12

$$
\mathrm{N}^{N}
$$

600

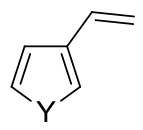

13

$$
Y=S(60 p)
$$$$
Y=\operatorname{NBn}(60 q)
$$

14

15<smiles>COc1ccc(C(C)c2c(C=O)c3ccccc3n2Cc2ccccc2)cc1OC</smiles>

56

$90: 10$

$81^{[\mathrm{d}]}$

$87: 13^{[d]}$
41

$62^{[d]}$

90:10

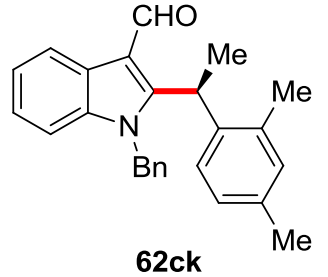

$--<10 \%{ }^{[\mathrm{d}]}$

$--$

n.r. ${ }^{[\mathrm{d}]}$

$---<10 \%$ [d]

---

n.r. ${ }^{[\mathrm{d}]}$

$---$

$<10 \%$ [d]

$--$

$---$

n.r. ${ }^{[\mathrm{d}]}$ 
16

17

$$
\begin{gathered}
\mathrm{Si}(\mathrm{OEt})_{3} \\
196
\end{gathered}
$$

$$
\begin{aligned}
& R=H(102 a) \\
& R=P h(102 b)
\end{aligned}
$$

n.r. ${ }^{[\mathrm{d}]}$

n.r. ${ }^{[\mathrm{d}]}$

${ }^{[a]}$ Reaction conditions: $59 \mathrm{c}$ (0.25 mmol), 60 (1.5 equiv), Fe(acac) $)_{3}(10 \mathrm{~mol} \%), 180$ (20 mol \%), CyMgCl (1.1 equiv), TMEDA (2.0 equiv), THF $(0.50 \mathrm{~mL}), 45^{\circ} \mathrm{C}, 16 \mathrm{~h} .{ }^{[b]}$ Yield of the isolated product. ${ }^{[c]}$ Determined by chiral HPLC analysis. ${ }^{[d]}$ Using 177 instead of 180.

While various vinylheteroarenes failed to deliver any $\mathrm{C}-\mathrm{H}$ alkylated product in the envisioned iron-catalyzed enantioselective hydroarylation (vide supra), vinylferrocene 191a was in contrast identified as a viable substrate in this transformation. To our delight, differently substituted indoles 59 bearing electronrich, electron-poor and various $\mathrm{N}$-substituents were functionalized in moderate to good yields and outstanding levels of enantioselectivity, usually over $90 \%$ ee (Table 3.12, entries 1-12). Pharmacologically meaningful azaindole 59n participated in the reaction as well, providing the alkylated product 192 in moderate yield, but excellent positional selectivity (entry 13).

Table 3.12. Substrate scope of substituted (aza)indoles 59 in the iron-catalyzed enantioselective $\mathrm{C}-\mathrm{H}$ alkylation with vinylferrocene $191 \mathrm{a} .{ }^{[a]}$

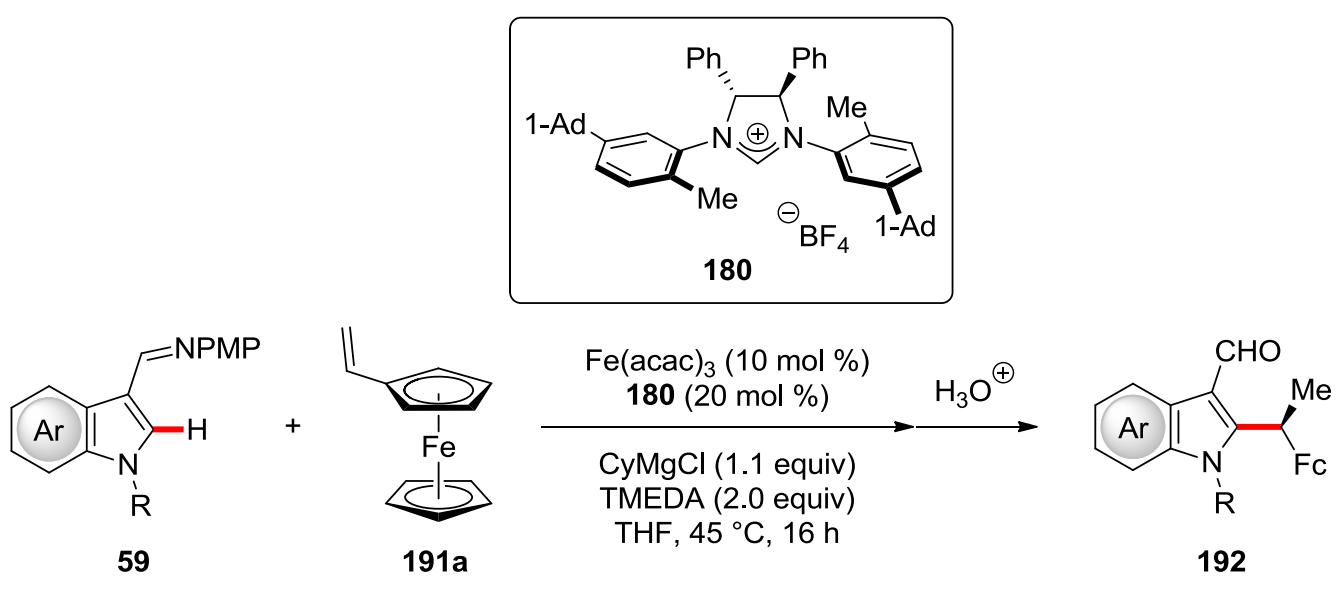




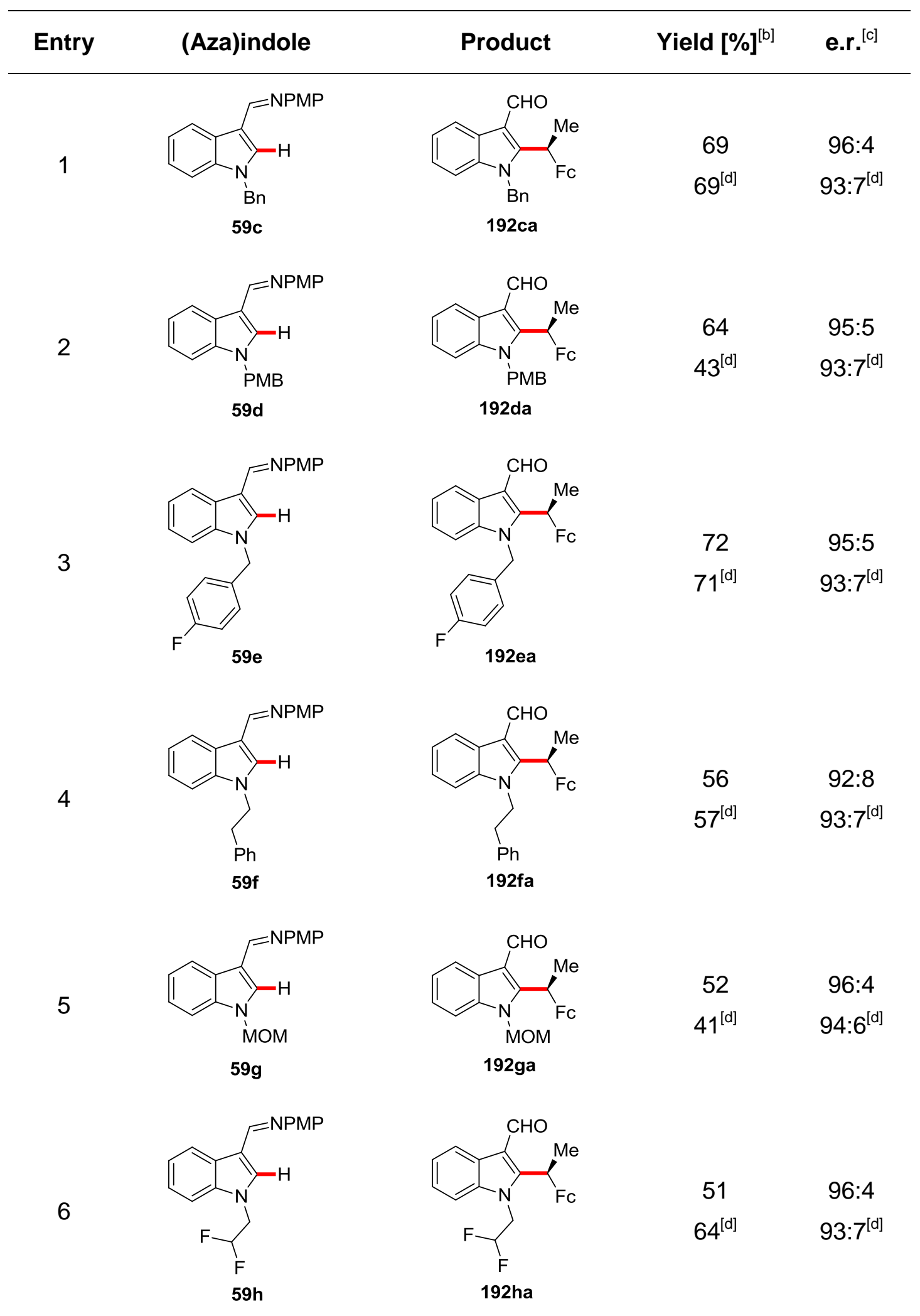


7

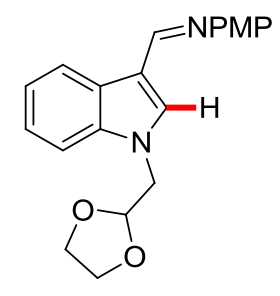

$59 i$

8<smiles></smiles>

$59 b$

9<smiles>COc1ccc2c(c1)c(C=N[P+]#N)c(Cc1ccccc1)n2Cc1ccccc1</smiles>

59j

10<smiles>[Y16]N=Cc1c(Cc2ccccc2)n(Cc2ccccc2)c2ccc(F)cc12</smiles>

$59 k$

11<smiles>Cc1cc(C)cc(Cn2c(C=N)c(C=N)c3ccccc32)c1</smiles>

591<smiles>COc1cc(C)cc(Cn2c(C=N)c(C=[N+]=[Nb])c3cc(OC)ccc32)c1</smiles>

12
$59 \mathrm{~m}$

$$
\text { 每 }
$$<smiles>CC(C)(F)c1c(C=O)c2ccccc2n1CC1OCCO1</smiles><smiles>CC(F)c1c(C=O)c2ccccc2n1C</smiles>

192ba<smiles>COc1ccc2c(c1)c(C=O)c(C(C)(F)F)n2Cc1ccccc1</smiles>

192ja<smiles>CC(F)c1c(C=O)c2cc(F)ccc2n1Cc1ccccc1</smiles>

192ka<smiles>Cc1cc(C)cc(Cn2c(C(C)F)c(C=O)c3ccccc32)c1</smiles>

49

$43^{[\mathrm{d}]}$ 95:5

192la<smiles>COc1ccc(Cn2c(C(C)F)c(C=O)c3cc(C)cc(C)c32)c(C)c1</smiles>

192ma
77

$96: 4$

$79^{[\mathrm{d}]}$ $94: 6^{[d]}$

42 95:5

$62^{[\mathrm{d}]}$ $92: 8^{[d]}$ $93: 7^{[d]}$ 
$13^{[\mathrm{e}]}$

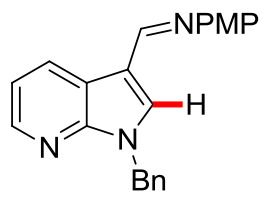

$59 n$<smiles>CC(F)c1c(/C=N\N)c2cccnc2n1Cc1ccccc1</smiles>

53

$90: 10$

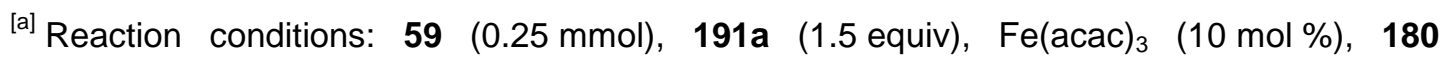
(20 mol \%), CyMgCl (1.1 equiv), TMEDA (2.0 equiv), THF $(0.50 \mathrm{~mL}), 45^{\circ} \mathrm{C}, 16 \mathrm{~h} .{ }^{[\mathrm{b}]}$ Yield of the isolated product. ${ }^{[c]}$ Determined by chiral HPLC analysis. ${ }^{[d]}$ Using 177 instead of 180. ${ }^{[\mathrm{e}]}$ Product isolated as the imine.

Differently substituted vinylmetallocenes 191 were then tested in the iron-catalyzed C2-alkylation of indoles (Table 3.13). In addition to ferrocenylalkenes, the isoelectronic but larger ruthenocenyl-substituted olefin 191b smoothly underwent the asymmetric hydroarylation (entry 1 ). Furthermore, the sterically hindered pentamethylferrocene-derived olefin 191c proved to be a viable substrate in the transformation as well, providing the highly congested Markovnikov product 192 in good yield and excellent enantioselectivity (entry 2). The preparation of highly enantiomerically-enriched ferrocenyl- and ruthenocenyl-indoles once more showcased the remarkable selectivity and versatility and of the developed catalytic system.

Table 3.13. Substrate scope of vinylmetallocenes 191 in the iron-catalyzed enantioselective C-H alkylation. ${ }^{[a]}$
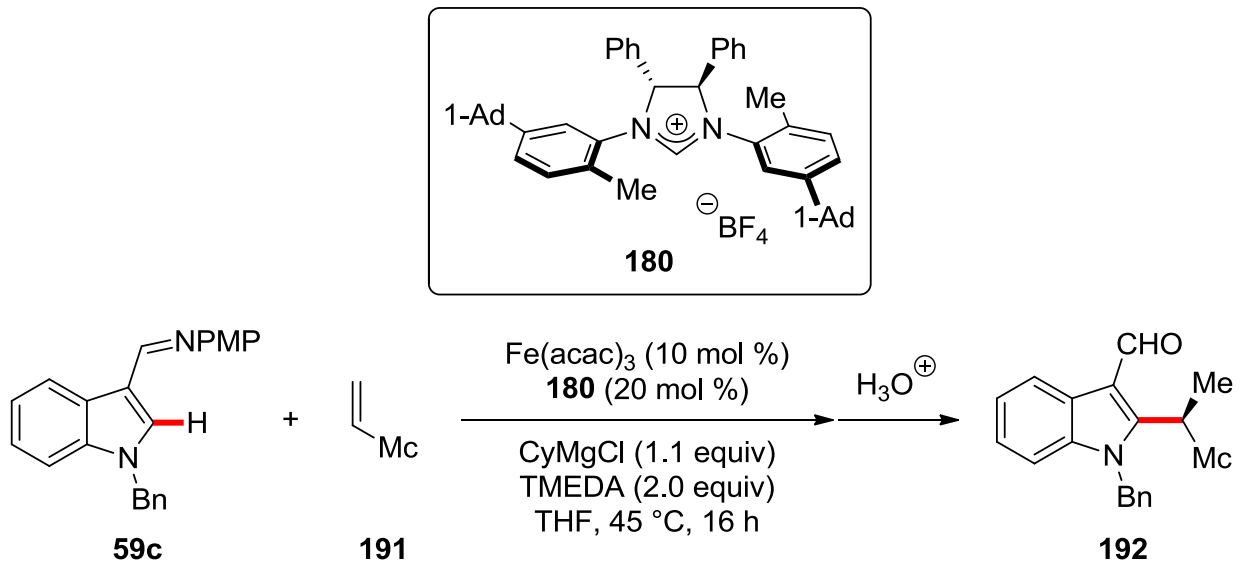


Entry Vinylmetallocene

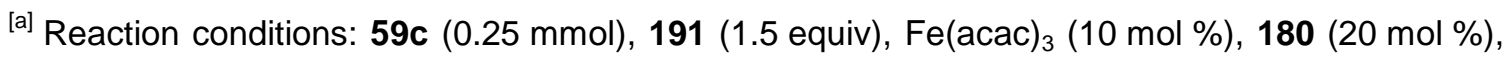
CyMgCl (1.1 equiv), TMEDA (2.0 equiv), THF $(0.50 \mathrm{~mL}), 45{ }^{\circ} \mathrm{C}, 16 \mathrm{~h}$. ${ }^{[\mathrm{b}]}$ Yield of the isolated product. ${ }^{[c]}$ Determined by chiral HPLC analysis. ${ }^{[d]}$ Using 177 instead of 180.

\subsubsection{Determination of the Absolute Configuration}

The determination of the absolute configuration is of primordial importance in asymmetric catalysis. Since styrene derivatives 60 are amorphous solids and lack heavy atoms, the highly crystalline bromo-substituted imine 197 was prepared in one step and obtained in $99 \%$ ee after a single crystallization (Scheme 3.5). Crystals suitable for X-ray diffraction were then grown by slow evaporation, allowing for the assignment of the absolute configuration. The product was found to be the S-enantiomer. 
a) synthesis of imine 197<smiles>CC(NP)c1c(C=O)c2ccccc2n1Cc1ccccc1</smiles>

62cc (92:8 e.r.)

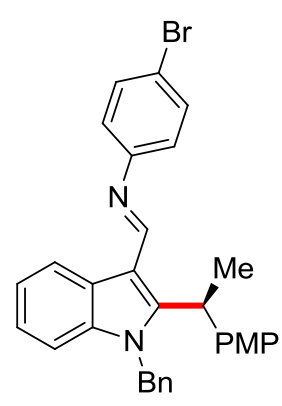

197: $53 \%, 99.5: 0.5$ e.r.

b) molecular structure of imine 197

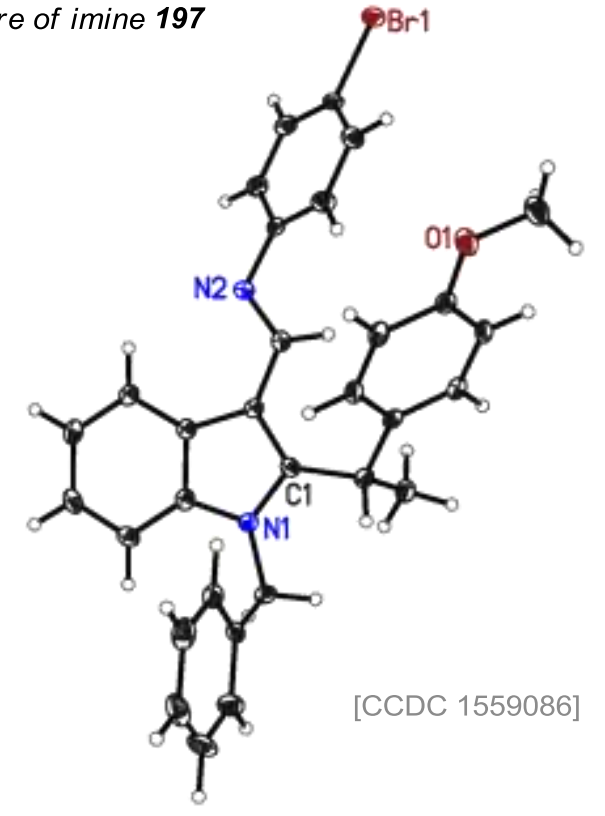

Scheme 3.5. a) Synthesis and b) ORTEP plot of imine 197. Anisotropic displacement parameters are depicted at the $50 \%$ probability level. The crystal structure was measured and solved by $H$. Keil and approved by Prof. D. Stalke.

Likewise, ferrocene derivative 192ca could be crystallized directly, providing the product in $>99 \%$ ee (Scheme 3.6). Due to the iron atom of the ferrocene moiety, no additional heavy atom had to be installed in the compound, and crystals suitable for $X$-ray diffraction analysis could be grown directly by slow evaporation from benzene. The $R$-enantiomer was found to be selectively formed in the transformation. The different $R / S$ configuration observed with vinylmetallocenes compared to styrenes is only due to different relative priorities of aryl and metallocenyl groups compared to the indole core according to the Cahn-Ingold-Prelog sequence rules. ${ }^{[252]}$ Interestingly, $\mathrm{C}-\mathrm{H} \cdots \pi$ interactions can be observed between the $\mathrm{N}$-benzyl 
substituent of the indole and the ferrocenyl group, which might explain the importance of the benzyl group in order to obtain high enantioselectivities.

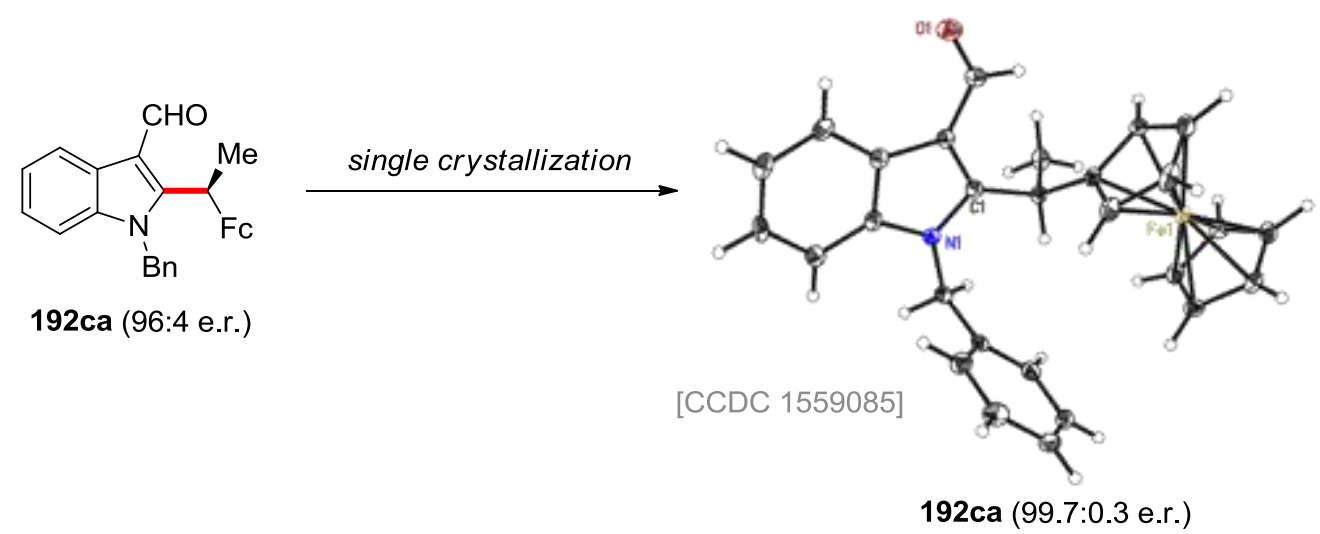

Scheme 3.6. Preparation and ORTEP plot of 192ca. Anisotropic displacement parameters are depicted at the $50 \%$ probability level. The crystal structure was measured and solved by $H$. Keil and approved by Prof. D. Stalke.

\subsubsection{Product Diversification}

The synthetic utility of the iron-catalyzed $\mathrm{C}-\mathrm{H}$ alkylation was further illustrated by late-stage diversification of the thus-obtained products (Scheme 3.7). The formyl group could be removed in a traceless fashion under palladium catalysis without significant loss of the enantiomeric excess (Scheme 3.7a). It is noteworthy that higher catalyst loadings or reaction temperatures provided the deformylated product in higher yields, but substantial racemization was then observed. The weakly coordinating $^{[39,253]}$ formyl motif could also be used as directing group to promote $\mathrm{C}-\mathrm{H}$ functionalization at the indole's C4-position. Using the methodology reported by Ramaiah Prabhu, ${ }^{[254]}$ the benzene core could be alkenylated with methyl acrylate using a ruthenium catalyst,${ }^{[255]}$ giving access to highly functionalized indoles through position-selective twofold $\mathrm{C}-\mathrm{H}$ activation (Scheme 3.7b). 


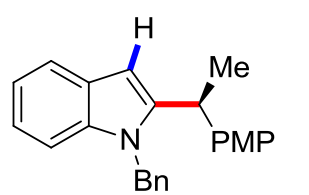

198: $66 \%, 88: 12$ e.r.

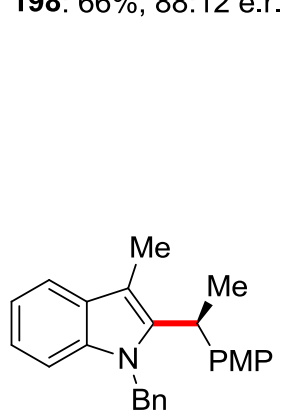

200: $61 \%, 89: 11$ e.r.

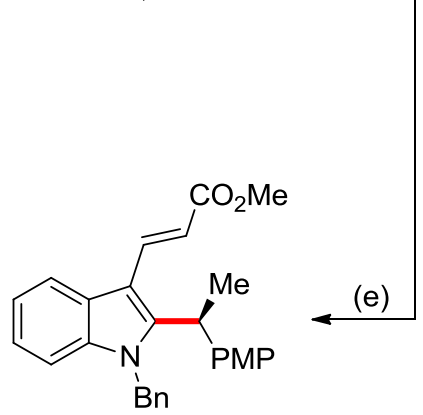

202: $81 \%, 88: 12$ e.r.

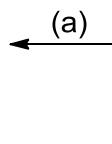

(c)
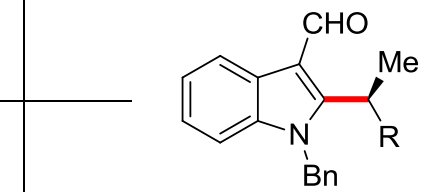

62cc: $R=P M P, 90: 10$ e.r. 192ca: $R=F c,>99: 1$ e.r. (b)<smiles>COC(C)(C)C</smiles><smiles></smiles>

$R=P M P$ (199a): 74\%, 89:11 e.r.

$R=F c(199 b): \quad 64 \%, 98: 2$ e.r.

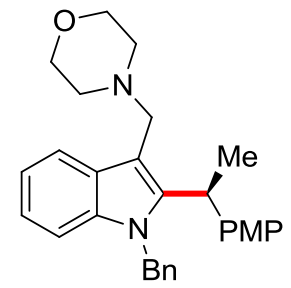

201: $82 \%, 89: 11$ e.r.

$\stackrel{(f)}{\longrightarrow}$

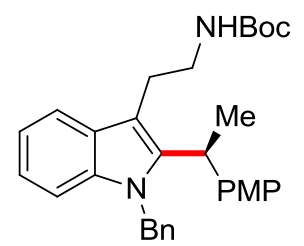

203: $60 \%, 88: 12$ e.r.

Scheme 3.7. Product diversification. a) $\mathrm{Pd}(\mathrm{OAc})_{2}(8.0 \mathrm{~mol} \%)$, cyclohexane, $4 \AA \mathrm{AS}, 140{ }^{\circ} \mathrm{C}$. b) $\left[\mathrm{RuCl}_{2}(p \text {-cymene })\right]_{2}(5.0 \mathrm{~mol} \%), \mathrm{AgSbF}_{6}(20 \mathrm{~mol} \%), \mathrm{Cu}(\mathrm{OAc})_{2} \cdot \mathrm{H}_{2} \mathrm{O}$, methyl acrylate, DCE, $120^{\circ} \mathrm{C}$. c) $\mathrm{H}_{2}, \mathrm{Pd} / \mathrm{C}$, EtOH, $23^{\circ} \mathrm{C}$. d) Morpholine, $\mathrm{NaBH}(\mathrm{OAc})_{3}, \mathrm{AcOH}, \mathrm{DCE}, 23^{\circ} \mathrm{C}$. e) $\mathrm{Ph}_{3} \mathrm{PCHCO}_{2} \mathrm{Me}, \mathrm{PhMe}, 130{ }^{\circ} \mathrm{C}$. f) i. $\mathrm{NH}_{4} \mathrm{OAc}, \mathrm{MeNO}_{2}, 90^{\circ} \mathrm{C}$; ii. $\mathrm{LiAlH}_{4}, \mathrm{THF}, 85^{\circ} \mathrm{C}$; iii. $\mathrm{Boc}_{2} \mathrm{O}, \mathrm{Et}_{3} \mathrm{~N}, 1,4$-dioxane, $23^{\circ} \mathrm{C}$.

Various transformations of the synthetically useful formyl group were also conducted (Scheme 3.7c-f). The formyl group could be reduced to a methyl group, or converted to other functional groups via reductive amination or Wittig reaction. Additionally, the pharmacologically relevant protected tryptamine $\mathbf{2 0 3}$ could also be obtained in 3 steps without significant racemization. 


\subsubsection{Mechanistic Studies}

Given the unique features of the developed asymmetric iron-catalyzed $\mathrm{C}-\mathrm{H}$ alkylation, we became interested in delineating its mode of action. Hence, experimental and computational ${ }^{[256]}$ mechanistic studies were performed in order to gain insights into the reaction's mechanism.

\subsubsection{Deuterium Labeling Experiments}

First, experiments with isotopically labelled indole substrate $[D]_{1}-59 b$ were conducted (Scheme 3.8). The deuterium atom was found to be selectively transferred to the terminal position of the alkene, which provides support for an inner-sphere $\mathrm{C}-\mathrm{H}$ activation. This observation can be rationalized with the $\mathrm{C}-\mathrm{H}$ scission occurring by ligand-to-ligand hydrogen-transfer (LLHT) or $\mathrm{C}-\mathrm{H}$ oxidative addition to a low-valent iron species. Interestingly, no deuterium incorporation was observed at the methine position as it was the case in the related work of Yoshikai. ${ }^{[148]}$ 


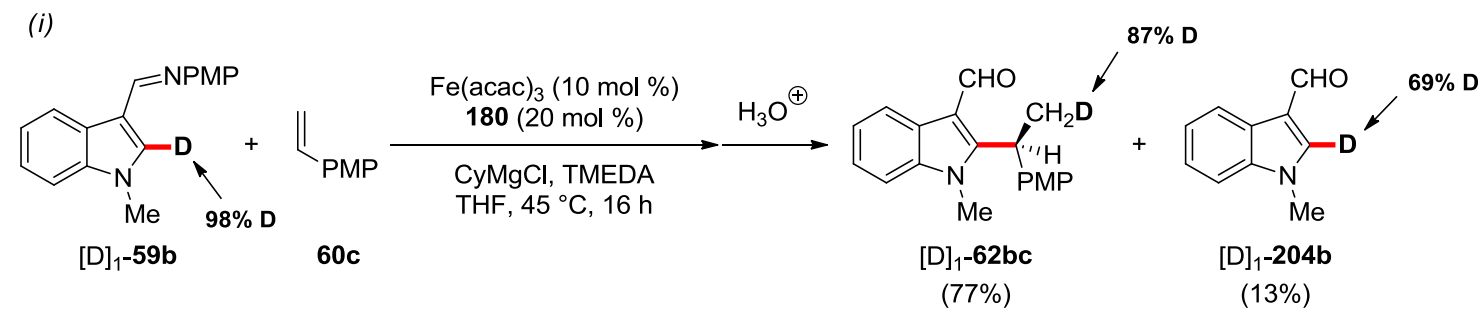

(ii)
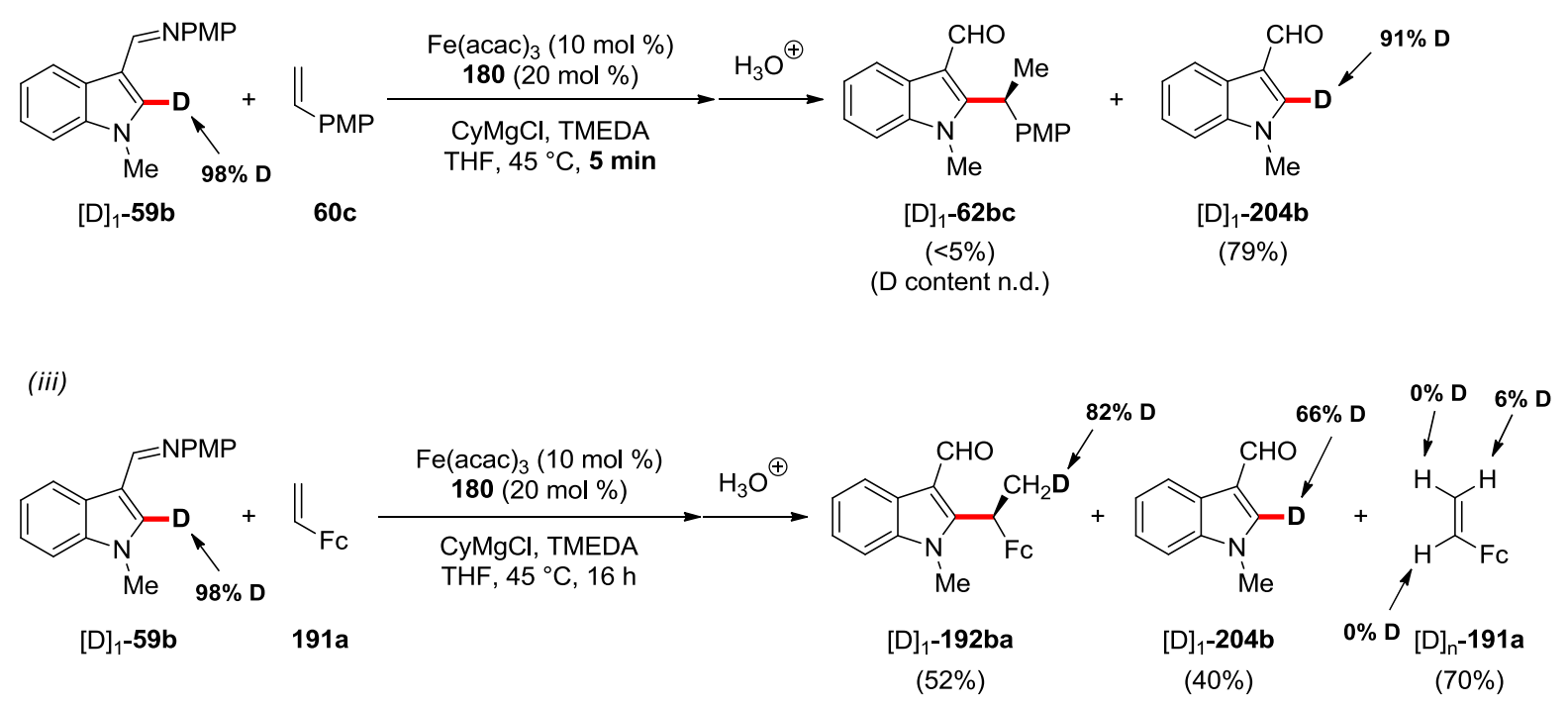

Scheme 3.8. Reactions with isotopically labeled substrate $[D]_{1}-59 b$.

Thereafter, a crossover experiment was conducted between deuterated and nondeuterated substrates (Scheme 3.9). [D $]_{1}-59 \mathrm{~b}$ and $59 \mathrm{f}$ were selected due to their similar efficacy in the transformation and the easy separation of their corresponding products by silica gel chromatography. Remarkably, a nearly identical deuterium incorporation was observed in both products $[D]_{n}-62 b c$ and $[D]_{n}-62 f c$. This finding seemingly rules out the oxidative addition/reductive elimination pathway initially proposed by Yoshikai for the racemic reaction ${ }^{[148]}$ and provides support for a LLHTmanifold. ${ }^{[32 a, 37 b, 81]}$ 
<smiles>Cn1c(OCC(=O)O)c(C=N)c2ccccc21</smiles>

$[D]_{1}-59 b$ (0.50 equiv)<smiles>N=Cc1cn(CCc2ccccc2)c2ccccc12</smiles>

$59 f$ ( 0.50 equiv)<smiles>C[C@@H](P)c1c(C=O)c2ccccc2n1C</smiles>

$[D]_{n}-62 b c: 92 \%$

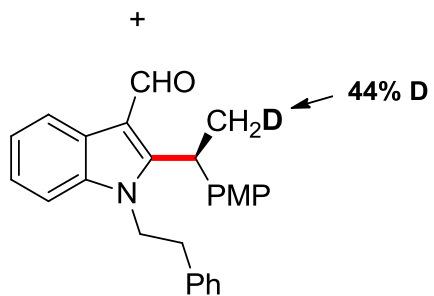

$[D]_{n}-62 f c: 78 \%$

Scheme 3.9. Crossover experiment between deuterated and non-deuterated substrates 59.

Furthermore, additional experiments with deuterated substrate $[D]_{1}-59 b$ revealed a kinetic isotope effect $(\mathrm{KIE})$ of $k_{\mathrm{H}} / k_{\mathrm{D}} \approx 1.0$ (Scheme 3.10). A KIE of this magnitude suggests that the $\mathrm{C}-\mathrm{H}$ cleavage step is not turnover-limiting, ${ }^{[257]}$ and provides evidence for a facile and reversible $\mathrm{C}-\mathrm{H}$ activation event.

a) with 4-methoxystyrene 60c

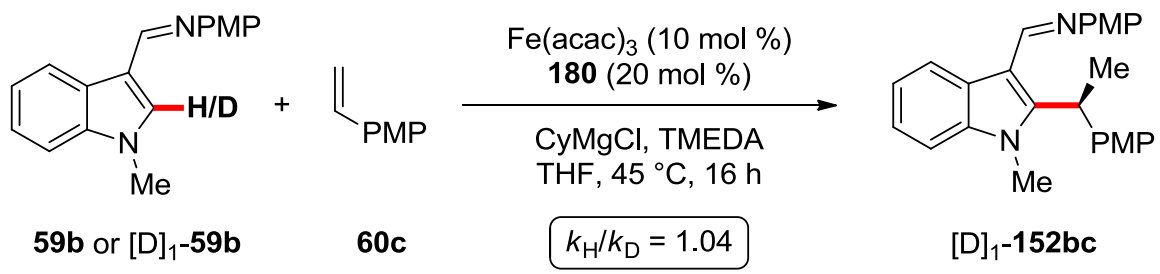

b) with 4-fluorostyrene $60 \mathrm{~g}$

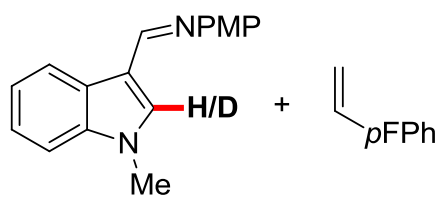

$59 b$ or $[D]_{1}-59 b$

$60 \mathrm{~g}$

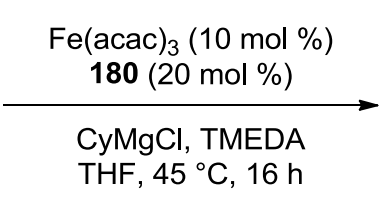

$k_{\mathrm{H}} / k_{\mathrm{D}}=1.02$

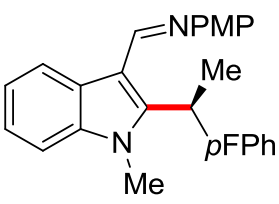

$[D]_{1}-152 b g$

Scheme 3.10. KIE studies. Experiment $b$ was conducted by Dr. D. Zell. 


\subsubsection{Kinetics}

Subsequently, detailed kinetic studies of the enantioselective $\mathrm{C}-\mathrm{H}$ alkylation were conducted by Dr. D. Zell. ${ }^{[151]}$ A first-order dependence on the concentration of the indole substrate 59b was observed, along with a saturation kinetics behavior for the styrene $60 \mathrm{~g}$ (Scheme 3.11).

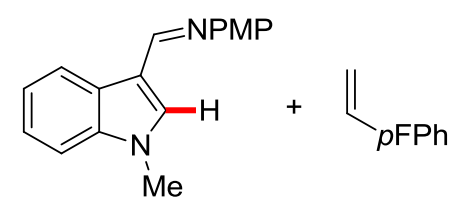

$59 b$

$60 \mathrm{~g}$

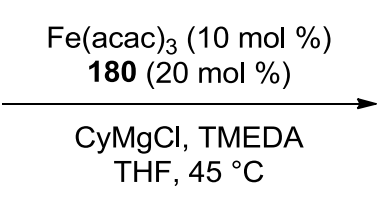

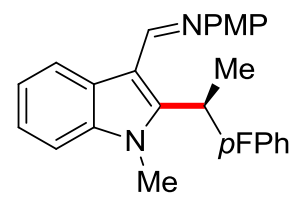

$152 \mathrm{bg}$ a) $1^{\text {st }}$ order in indole $\mathbf{5 9 b}$

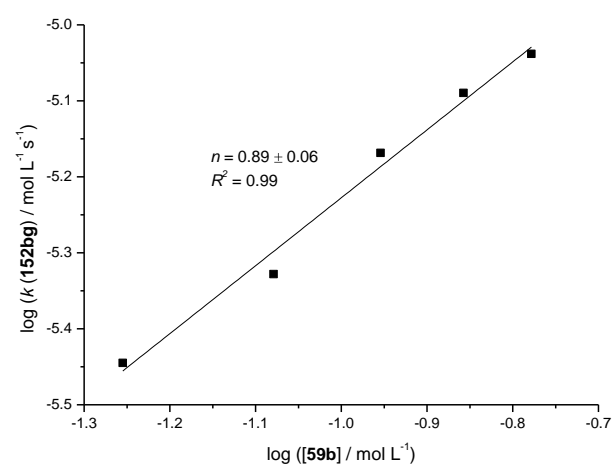

b) $0^{\text {th }}$ order in styrene $60 \mathrm{~g}$

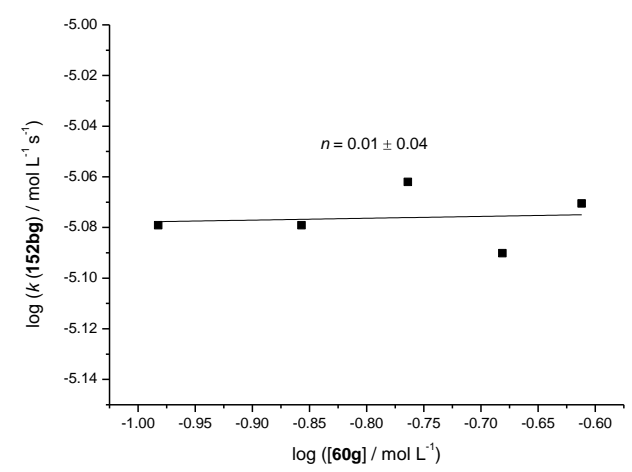

Scheme 3.11. Kinetic analysis. The experiments were conducted by Dr. D. Zell.

\subsubsection{Mercury Drop Test}

A mercury poisoning test was conducted to probe the homogeneity of the catalytic process (Scheme 3.12). No significant reduction of the catalytic activity was observed in the presence of an excess of metallic mercury, which confirms the homogenous nature of the transformation. 


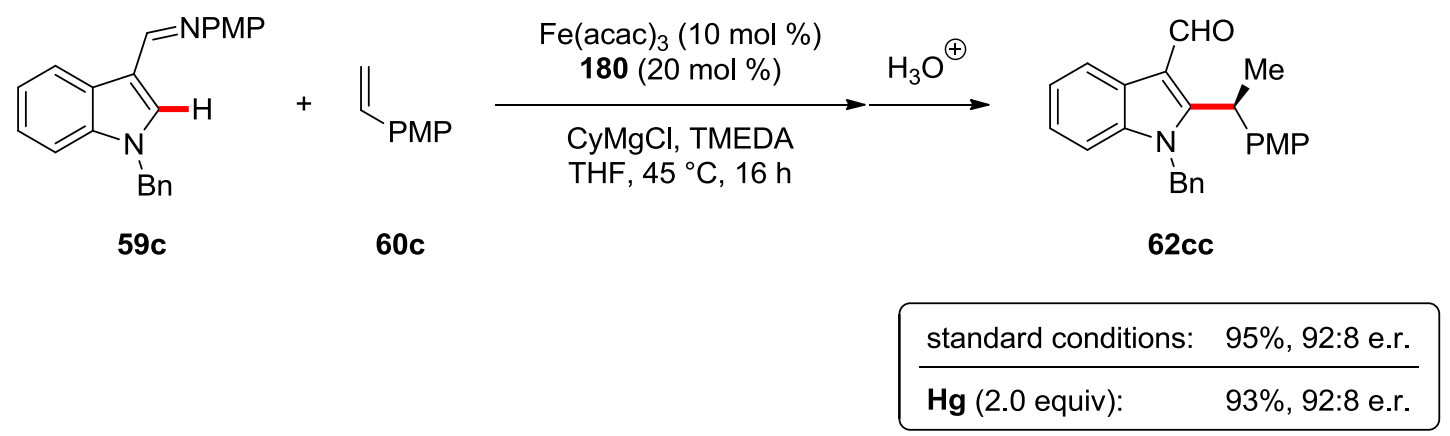

Scheme 3.12. Mercury drop test for the iron-catalyzed $\mathrm{C}-\mathrm{H}$ alkylation.

This finding is especially important since iron(0) nanoparticles are known to form upon exposure of iron salts to Grignard reagents, ${ }^{[258]}$ and their presence in the reaction mixture has been indirectly supported by ${ }^{57} \mathrm{Fe}$ Mössbauer spectroscopic studies (vide infra). Nevertheless, iron(0) nanoparticles do not appear to play a role in the catalyzed transformation.

\subsubsection{Non-Linear Effect Studies}

The effect of the enantiomeric excess of ligand $\mathbf{1 8 0}$ over the enantiomeric induction of the transformation was investigated (Scheme 3.13). The absence of a non-linear effect (NLE) renders a multi-ligand containing catalyst or catalytically competent oligomers unlikely to be operative in the asymmetric $\mathrm{C}-\mathrm{H}$ secondary alkylation. ${ }^{[259]}$ Hence, the beneficial effect of a ligand-to-metal ratio of 2:1 is apparently not due to the formation of bis-NHC-ligated iron species. 

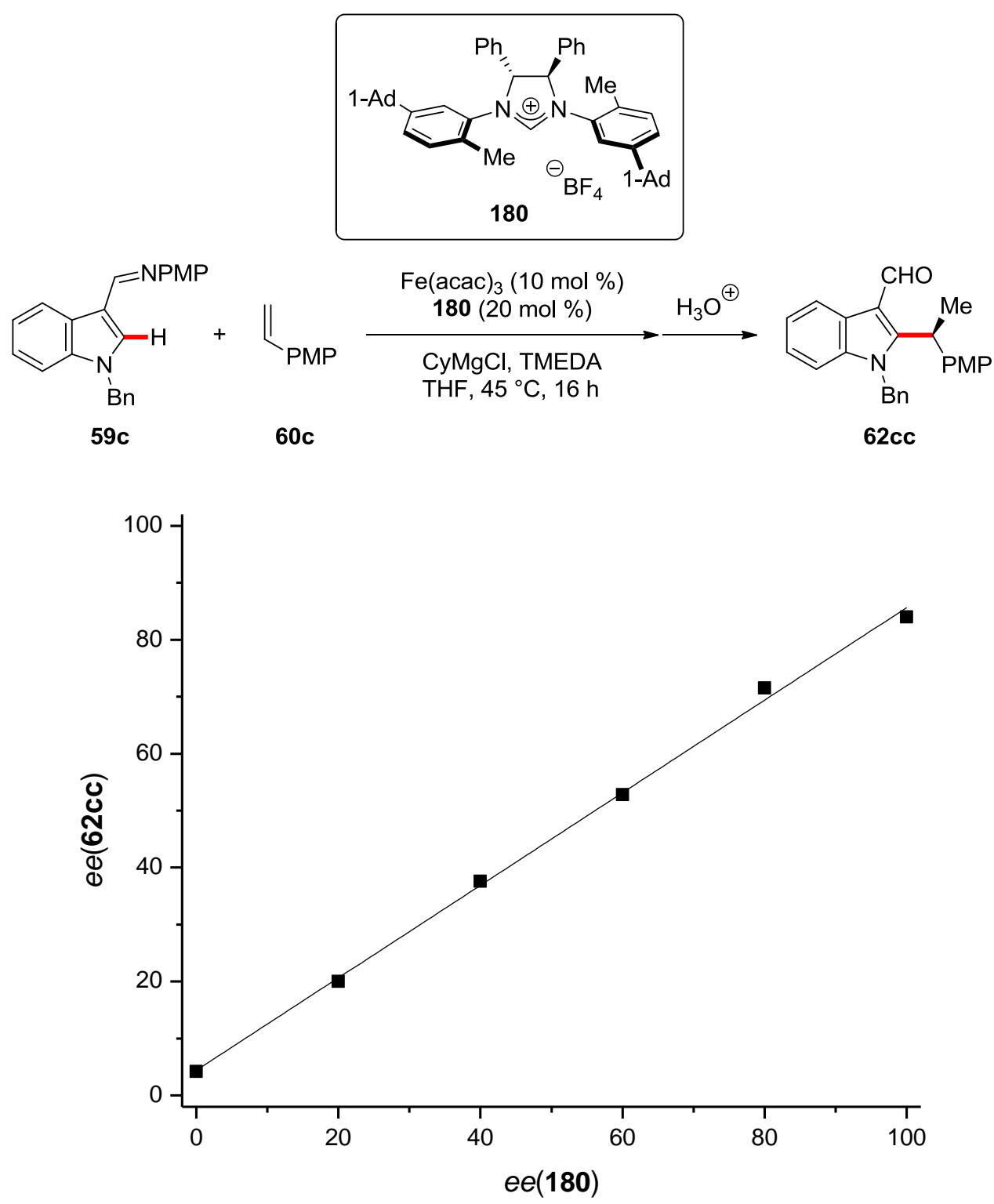

Scheme 3.13. Non-linear effect studies.

\subsubsection{In situ Analysis by Mass Spectrometry and Mössbauer Spectroscopy}

While detailed kinetic studies, deuterium labelling experiments and non-linear effect studies were performed to delineate the modus operandi of the unique ironcatalyzed enantioselective $\mathrm{C}-\mathrm{H}$ alkylation (vide supra), the oxidation state and coordination sphere of the active catalyst have remained thus far speculative. Indeed, in iron/ $\mathrm{NHC}$-catalyzed $\mathrm{C}-\mathrm{H}$ activations, ${ }^{[148,151,260]}$ as well as in related 
"low-valent" cobalt/NHC-catalyzed C-H activations, ${ }^{[46 c, 46 d, 69 a, 69 c]}$ the active catalyst is usually generated in situ from a metal salt and an imidazol(in)ium NHC precursor in the presence of a Grignard reagent. The use of well-defined iron/ $\mathrm{NHC}$ complexes as catalysts is well documented in molecular syntheses in different contexts. ${ }^{[235 a-c]}$ In contrast, no well-defined iron catalysts or intermediates have so far been isolated or characterized in the context of $\mathrm{C}-\mathrm{H}$ activation, with the notable exception of Tatsumi and Ohki employing a half sandwich iron(II)/NHC complex for undirected $\mathrm{C}-\mathrm{H}$ borylations of heteroarenes. ${ }^{[261]}$ In the reactions employing in situ generated iron catalysts, the organometallic reagent has been proposed to play a dual role, serving both as the base and a potential reductant. The mechanism of the generation of the catalytically active species, as well as possible side-reactions occurring during this process, have thus far been overlooked. ${ }^{[262]}$ Furthermore, iron species with formal oxidation states ranging from $-2^{[263]}$ to $+4^{[264]}$ have been observed in reactions of iron precursors with Grignard reagents. ${ }^{[108 a, 265]}$ It is noteworthy that some of these species have been postulated as intermediates in iron-catalyzed Kumada-Corriutype cross-couplings ${ }^{[19]}$ operating under reaction conditions similar to iron-catalyzed $\mathrm{C}-\mathrm{H}$ activations. So far, all information on the oxidation state of the in situ generated iron catalysts enabling the $\mathrm{C}-\mathrm{H}$ activation has been gained through DFT calculations regarding iron-catalyzed oxidative $\mathrm{C}-\mathrm{H}$ functionalizations with alkyl halides. ${ }^{[131]}$ Hence, the nature of the catalytically active species in the asymmetric iron-catalyzed hydroarylation has remained unknown until now.

Interestingly, Yoshikai originally proposed a "low-valent"[266] Fe/NHC complex generated in situ through the reduction of the iron(III) pre-catalyst by the Grignard reagent to be operative in the racemic hydroarylation of vinylarenes and alkynes with indoles, and the $\mathrm{C}-\mathrm{H}$ activation step to occur via oxidative addition into the $\mathrm{C}-\mathrm{H}$ bond. ${ }^{[148]}$ Yoshikai attributed the requirement of an excess of the Grignard reagent to the possible formation of ferrate species. In the course of the optimization studies for the enantioselective secondary alkylation of indoles 59, it was observed that $\mathrm{Fe}(\mathrm{acac})_{3}$ and $\mathrm{FeCl}_{2}$ pre-catalysts, despite their different oxidation states and counterions, gave comparable conversions and enantioselectivities (Table 3.9). This observation suggested that the iron precursors were transformed by the Grignard 
reagent to the same catalytically competent iron species. Another possibility is the in situ formation of organoferrates. Indeed, Koszinowski recently reported that the nature of the iron precursor has very little effect on the transmetalation reactions with organometallic species to form such complexes. ${ }^{[267]}$ These observations raised the question as to the nature of the active iron catalyst and its mode of action, and highlight the need for detailed, comprehensive mechanistic studies to unravel fundamental aspects of iron-catalyzed $\mathrm{C}-\mathrm{H}$ activations. Such mechanistic insights have recently been gained for iron-catalyzed Kumada-Corriu-type cross-coupling reactions via Mössbauer spectroscopy and mass spectrometry, among other analytical methods. ${ }^{[265,267,268]}$ These reports highlighted the dynamic nature and remarkable complexity of organometallic iron chemistry.

Therefore, we became interested in the application of electrospray-ionization (ESI) mass spectrometry and ${ }^{57} \mathrm{Fe}$ Mössbauer spectroscopy to unravel the key intermediates formed in situ in the enantioselective iron-catalyzed $\mathrm{C}-\mathrm{H}$ alkylation. ${ }^{[218]}$ In contrast to ESI-MS, ${ }^{57} \mathrm{Fe}$ Mössbauer spectroscopy has the advantage of probing the entire population of iron species, regardless of their individual charge. This research work was conducted in collaboration with the research groups of Prof. Dr. K. Koszinowski (ESI-MS) and Prof. Dr. F. Meyer (Mössbauer spectroscopy).

We decided to follow a step-by-step approach and therefore initiated our investigations by probing the species formed in a stoichiometric reaction between the iron precatalyst, the Grignard reagent and TMEDA in THF, without the NHC precursor 180 or the indole substrate 59. Negative-ion mode ESI-MS of a solution of $\mathrm{Fe}(\mathrm{acac})_{3}$ treated with 8.0 equiv of $\mathrm{CyMgCl}$ in the presence of TMEDA (4.0 equiv) showed a mixture of various organoferrate species, among which $\mathrm{Cy}_{3} \mathrm{Fe}(\mathrm{II})^{-}$and $\mathrm{Cy}_{4} \mathrm{Fe}(\mathrm{III})^{-}$were dominant (Fig. 3.1a). Previous reports had already demonstrated the formation of abundant organoferrates upon transmetalation of iron precursors with Grignard reagents under similar reaction conditions. ${ }^{[267]}$ Although ESI-MS cannot directly detect any neutral species, the observation of small amounts of $\mathrm{Cy}_{5} \mathrm{Fe}_{2}{ }^{-}$and $\mathrm{Cy}_{4} \mathrm{Fe}_{2} \mathrm{Cl}^{-}$, both with iron in an average oxidation state of +2 , may indicate the presence of neutral organoiron complexes, such as $\mathrm{Cy}_{2} \mathrm{Fe}$ or $\mathrm{CyFeCl}$, 
which supposedly reacted with $\mathrm{Cy}_{3} \mathrm{Fe}(\mathrm{II})^{-}$to afford the dinuclear aggregates. The low abundance of the dinuclear anions can be attributed to TMEDA, which has previously been shown to prevent the formation of polynuclear organoferrates. ${ }^{[267,269]}$ All the observed organoferrates were found to be highly unstable, presumably due to $\beta$-hydride elimination, and completely vanished within 2 minutes. Experiments conducted with the better-behaved $\mathrm{PhMgCl}$ were therefore performed as well. $\mathrm{PhMgCl}$ has previously been shown to promote the desired $\mathrm{C}-\mathrm{H}$ alkylation as well, albeit with a slightly diminished performance (vide supra, Tables 3.7, 3.9). It should also be noted that phenyl Grignard reagents have proven instrumental in other iron/NHC-catalyzed C-H activations. ${ }^{[148,260]}$ Likewise, iron(II) and iron(III) phenylferrates were detected by ESI-MS in the reaction of $\mathrm{Fe}(\mathrm{acac})_{3}$ with $\mathrm{PhMgCl}$ in the presence of TMEDA (Fig. 3.1b), being in full agreement with previous findings. ${ }^{[267,269 b, 270]}$
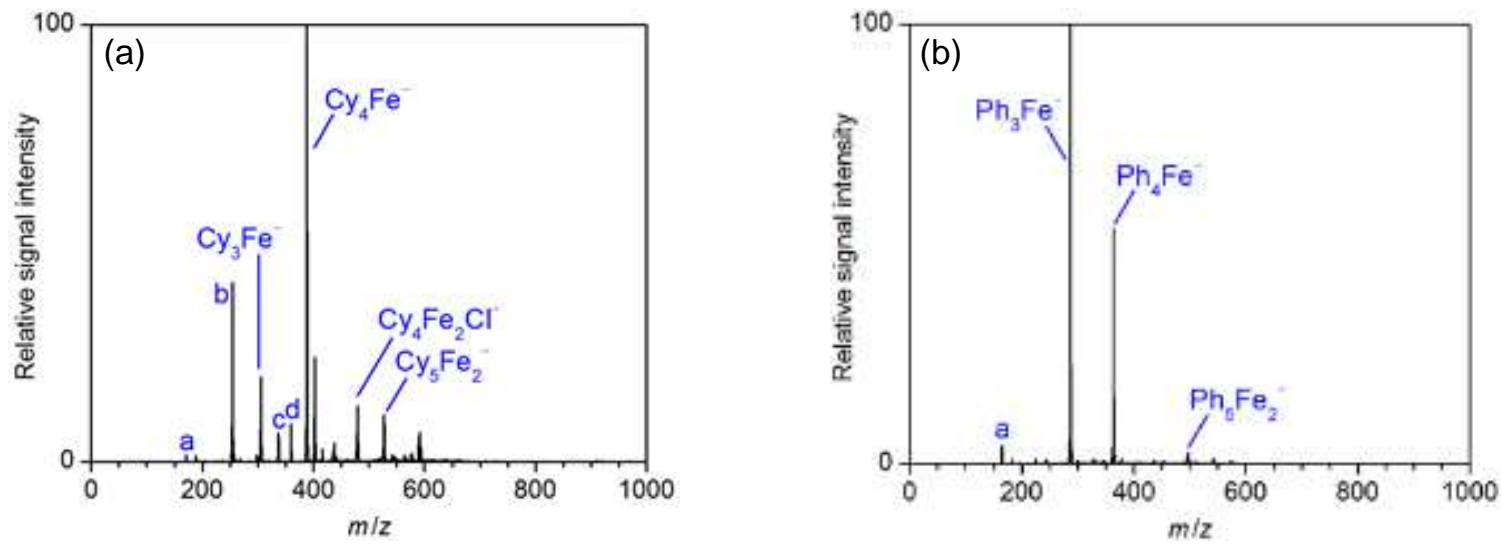

Figure 3.1. (a) Negative-ion mode ESI mass spectrum of a solution of the products formed in the reaction of $\mathrm{Fe}(\mathrm{acac})_{3}(10 \mathrm{mM})$ with TMEDA (4.0 equiv) and $\mathrm{CyMgCl}$ (8.0 equiv) in THF; $a=\left[\mathrm{Cy}, \mathrm{Fe}, \mathrm{O}_{2}\right]^{-}, b=\left[\mathrm{Cy}_{2}, \mathrm{Fe}, \mathrm{O}_{2}\right]^{-}, c=\left[\mathrm{Cy}_{3}, \mathrm{Fe}, \mathrm{O}_{2}\right]^{-}, d=\mathrm{Cy}_{4} \mathrm{Al}^{-}$. Ions $a-c$ resulted from reactions with residual traces of oxygen, $d$ from an aluminum contamination. (b) Negativeion mode ESI mass spectrum of a solution of the products formed in the reaction of $\mathrm{Fe}(\mathrm{acac})_{3}(10 \mathrm{mM})$ with TMEDA (4.0 equiv) and $\mathrm{PhMgCl}$ (8.0 equiv) in THF; $a=\left[\mathrm{Ph}, \mathrm{Fe}, \mathrm{O}_{2}\right]^{-}$. lons a resulted from reactions with residual traces of oxygen. The experiments were conducted by Dr. T. Parchomyk.

A frozen solution of ${ }^{57} \mathrm{FeCl}_{2} / \mathrm{CyMgCl} / \mathrm{TMEDA}$ in THF was next analyzed by ${ }^{57} \mathrm{Fe}$ Mössbauer spectroscopy at $80 \mathrm{~K}$ (Fig. 3.2a). The obtained spectrum featured the 
signatures of two iron species, which were assigned to a major high-spin iron(III) species and a minor low-coordinate iron(II) species, in line with the formation of $\mathrm{Cy}_{4} \mathrm{Fe}(\mathrm{III})^{-}$and $\mathrm{Cy}_{3} \mathrm{Fe}(\mathrm{II})^{-}$observed by ESI-MS. Interestingly, we did not detect any $\mathrm{Cy}_{4} \mathrm{Fe}(\mathrm{IV})$, which has been observed by Fürstner in a related setting. ${ }^{[264]}$ The remarkable formation of a dominating iron(III) species from the iron(II) precursor in the presence of Grignard reagents and the absence of any external oxidant can be attributed to disproportionation with concomitant formation of low-valent iron species, as previously reported. ${ }^{[267]}$ Yet, no low-valent iron species could be detected in our experiments. Therefore, a Mössbauer spectrum was recorded at $7 \mathrm{~K}$ (Fig. 3.2b), but was essentially identical to the spectrum recorded at $80 \mathrm{~K}$. No signal for iron(0) nanoparticles ${ }^{[258]}$ or other low-valent iron species could be observed by ${ }^{57} \mathrm{Fe}$ Mössbauer spectroscopy. However, unfavorable relaxation dynamics ${ }^{[271]}$ may lead to pronounced line broadening, which prevents detection of iron nanoparticles.
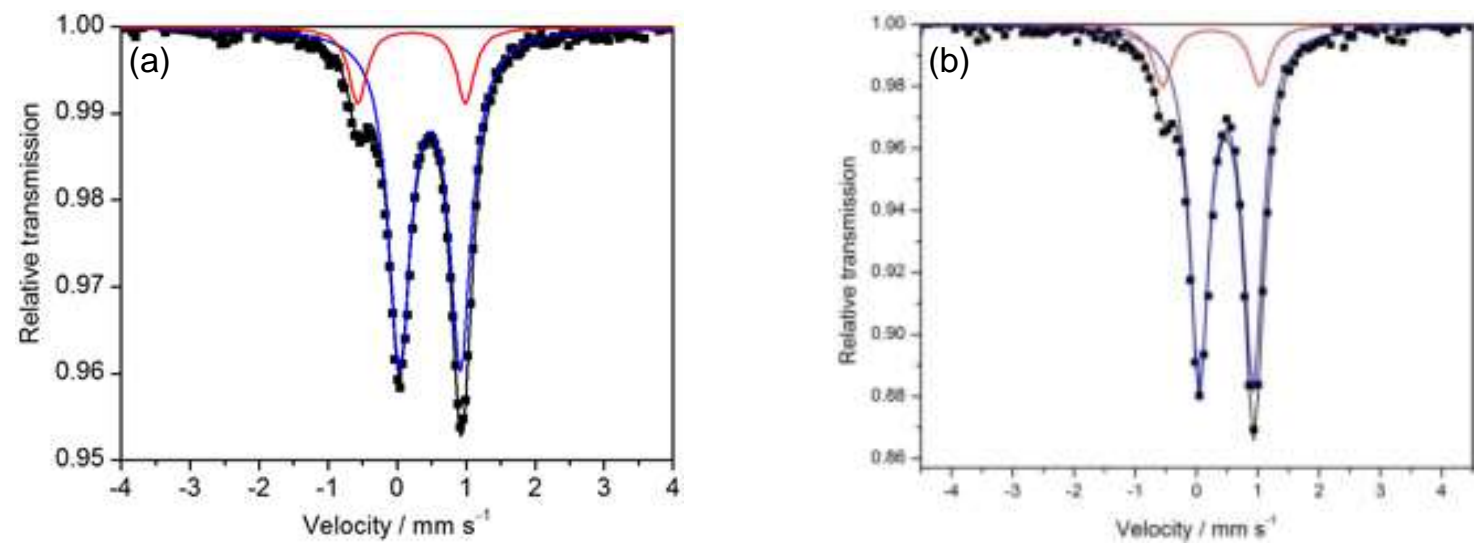

Figure 3.2. (a) Mössbauer spectrum of a frozen solution ( $T=80 \mathrm{~K}$ ) of the products formed in the reaction of ${ }^{57} \mathrm{FeCl}_{2}(5.0 \mathrm{mM})$, TMEDA (4.0 equiv) and $\mathrm{CyMgCl}$ (8.0 equiv) in THF; components of the fit: $\delta$ (blue $)=0.48 \mathrm{~mm} \mathrm{~s}^{-1}, \Delta E_{Q}$ (blue $)=0.89 \mathrm{~mm} \mathrm{~s}^{-1}$, rel. int. $=84 \%$; $\delta($ red $)=0.21 \mathrm{~mm} \mathrm{~s}^{-1}, \Delta E_{Q}($ red $)=1.56 \mathrm{~mm} \mathrm{~s}^{-1}$, rel. int. $=16 \%$. (b) Mössbauer spectrum and components of the fit of a frozen solution $(T=7 \mathrm{~K})$ of the products formed in the reaction of ${ }^{57} \mathrm{FeCl}_{2}(5.0 \mathrm{mM}$ ), TMEDA (4.0 equiv) and $\mathrm{CyMgCl}$ (8.0 equiv) in THF; components of the fit: $\delta$ (blue $)=0.48 \mathrm{~mm} \mathrm{~s}^{-1}, \quad \Delta E_{Q}$ (blue $)=0.88 \mathrm{~mm} \mathrm{~s}^{-1}, \quad$ rel. int. $=85 \% ; \quad \delta($ red $)=0.24 \mathrm{~mm} \mathrm{~s}^{-1}$, $\Delta E_{\mathrm{Q}}($ red $)=1.59 \mathrm{~mm} \mathrm{~s}^{-1}$, rel. int. $=15 \%$. The spectra were recorded and interpreted by $\operatorname{Dr}$. $S$. Demeshko. 
The instability of the cyclohexylferrates was further highlighted by ${ }^{57} \mathrm{Fe}$ Mössbauer spectroscopic analysis of the same reaction after it was allowed to warm to $23^{\circ} \mathrm{C}$ (Fig. 3.3). The spectrum showed the complete disappearance of the iron(II) ate complex, a reduced amount of $\mathrm{Cy}_{4} \mathrm{Fe}(\mathrm{III})^{-}$and the emergence of a new dominant species, whose unspecific doublet unfortunately does not allow for assignment.

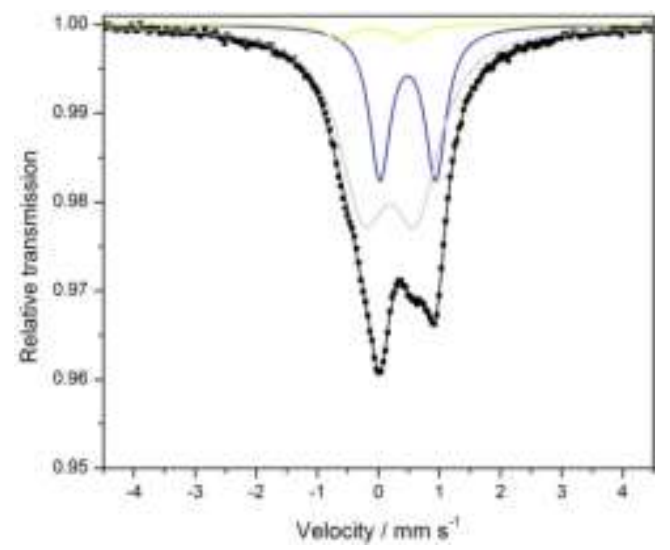

Figure 3.3. Mössbauer spectrum and components of the fit of a frozen solution ( $T=80 \mathrm{~K}$ ) of the products formed in the reaction of ${ }^{57} \mathrm{FeCl}_{2}(5.0 \mathrm{mM})$, TMEDA (4.0 equiv) and $\mathrm{CyMgCl}$ (8.0 equiv) in THF at $23^{\circ} \mathrm{C}$; components of the fit: $\delta$ (gray) $=0.19 \mathrm{~mm} \mathrm{~s}^{-1}$, $\Delta E_{\mathrm{Q}}$ (gray) $=0.86 \mathrm{~mm} \mathrm{~s}^{-1}$, rel. int. $=68 \% ; \delta$ (blue $)=0.48 \mathrm{~mm} \mathrm{~s}^{-1}, \Delta E_{Q}$ (blue $)=0.91 \mathrm{~mm} \mathrm{~s}^{-1}$, rel. int. $=28 \% ; \quad \delta$ (yellow) $=-0.10 \mathrm{~mm} \mathrm{~s}^{-1}, \Delta E_{Q}$ (yellow) $=1.10 \mathrm{~mm} \mathrm{~s}^{-1}$, rel. int. $=3 \%$. The spectrum was recorded and interpreted by Dr. S. Demeshko.

A similar spectrum was obtained from the reaction of ${ }^{57} \mathrm{FeCl}_{2}$ with $\mathrm{PhMgCl}$ in the presence of TMEDA (Fig. 3.4), indicating the formation of the phenylferrates $\mathrm{Ph}_{3} \mathrm{Fe}(\mathrm{II})^{-}$and $\mathrm{Ph}_{4} \mathrm{Fe}(\mathrm{III})^{-}$, being in line with the ESI-MS results (Fig. 3.1b) and previous reports. ${ }^{[267,269 b]}$ As the catalyzed $\mathrm{C}-\mathrm{H}$ activation was found to completely shut down in the absence of the NHC ligand (vide supra, Table 3.5), the observed $\mathrm{NHC}$ ligand-free organoferrates are assumed to be catalytically inactive, but are plausible intermediates of the generation of the catalytically competent species. 


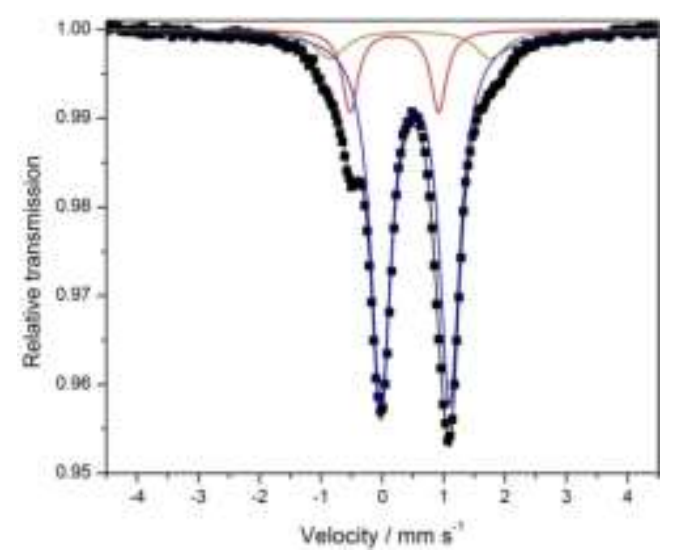

Figure 3.4. Mössbauer spectrum of a frozen solution $(T=80 \mathrm{~K}$ ) of the products formed in the reaction of ${ }^{57} \mathrm{FeCl}_{2}(5.0 \mathrm{mM})$, TMEDA (4.0 equiv) and $\mathrm{PhMgCl}$ (8.0 equiv) in THF; components of the fit: $\delta$ (blue $)=0.54 \mathrm{~mm} \mathrm{~s}^{-1}, \Delta E_{\mathrm{Q}}$ (blue) $=1.12 \mathrm{~mm} \mathrm{~s}^{-1}$, rel. int. $=78 \%$; $\delta($ red $)=0.20 \mathrm{~mm} \mathrm{~s}^{-1}, \Delta E_{\mathrm{Q}}($ red $)=1.44 \mathrm{~mm} \mathrm{~s}^{-1}$, rel. int. $=13 \% ; \delta$ (dark yellow) $=0.46 \mathrm{~mm} \mathrm{~s}^{-1}$, $\Delta E_{\mathrm{Q}}($ dark yellow $)=2.61 \mathrm{~mm} \mathrm{~s}^{-1}$, rel. int. $=9 \%$. The spectrum was recorded and interpreted by Dr. S. Demeshko.

Subsequently, similar experiments were performed in the presence of the chiral $\mathrm{NHC}$ precursor 180. While the homoleptic ferrates remained present in the solution, two newly formed iron(II) species could also be detected by ESI-MS, namely $\mathrm{Cy}_{3} \mathrm{Fe}(\mathrm{NHC})^{-}$and $\mathrm{Cy}_{2} \mathrm{FeH}(\mathrm{NHC})^{-}$(Fig. 3.5). The latter, with a significantly higher intensity, is believed to result from $\beta$-hydride elimination of the former. Interestingly, no NHC complexes of iron(III) or low-valent iron were detected, suggesting the selective formation of $\mathrm{Fe}(\mathrm{II}) / \mathrm{NHC}$ species in the reaction. It should be noted that the peak corresponding to the $\mathrm{Cy}_{4} \mathrm{Al}^{-}$contamination only appears so intense because of the relative low intensity of the anionic iron species. 


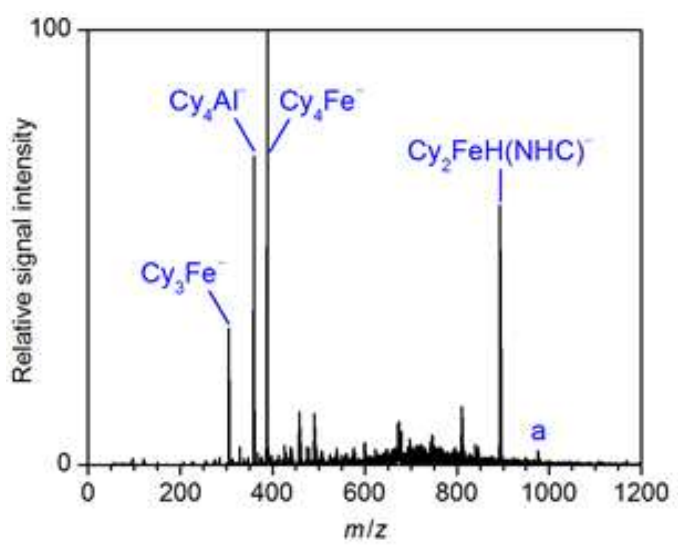

Figure 3.5. Negative-ion mode ESI mass spectrum of a solution of the products formed in the reaction of $\mathrm{Fe}(\mathrm{acac})_{3}(10 \mathrm{mM})$ with TMEDA (4.0 equiv), $\mathrm{CyMgCl}$ (8.0 equiv) and 180 (1.0 equiv) in THF; $a=\left[\mathrm{Fe}(\mathrm{NHC}) \mathrm{Cy}_{3}\right]^{-}$. $\mathrm{NHC}=\mathrm{C}_{49} \mathrm{H}_{54} \mathrm{~N}_{2}$. The experiment was conducted by S. Lülf.

When a similar experiment was performed using $\mathrm{PhMgCl}$, $\mathrm{Fe} / \mathrm{NHC}$ species could not be observed (Fig. 3.6a). Yet, the relative intensity of the iron(II) ate complex was noticeably reduced, which indicates its consumption for the formation of neutral species not detectable by ESI mass spectrometry. Besides, no magnesium-NHC complexes or residual imidazolinium salt could be observed by positive-mode ESI-MS in any of the experiments (Fig. 3.6b). In this context, it should be mentioned that organomagnesium(II)/NHC complexes are known ${ }^{[272]}$ and relevant in asymmetric catalysis. ${ }^{[273]}$ Also, a recent study by Bedford on iron-catalyzed Negishitype reactions suggests that the phosphine ligand binds to the zinc rather than the iron atom. ${ }^{[274]}$ In contrast, the present findings provide evidence for the NHC to coordinate to the iron catalyst, and not to magnesium(II). 

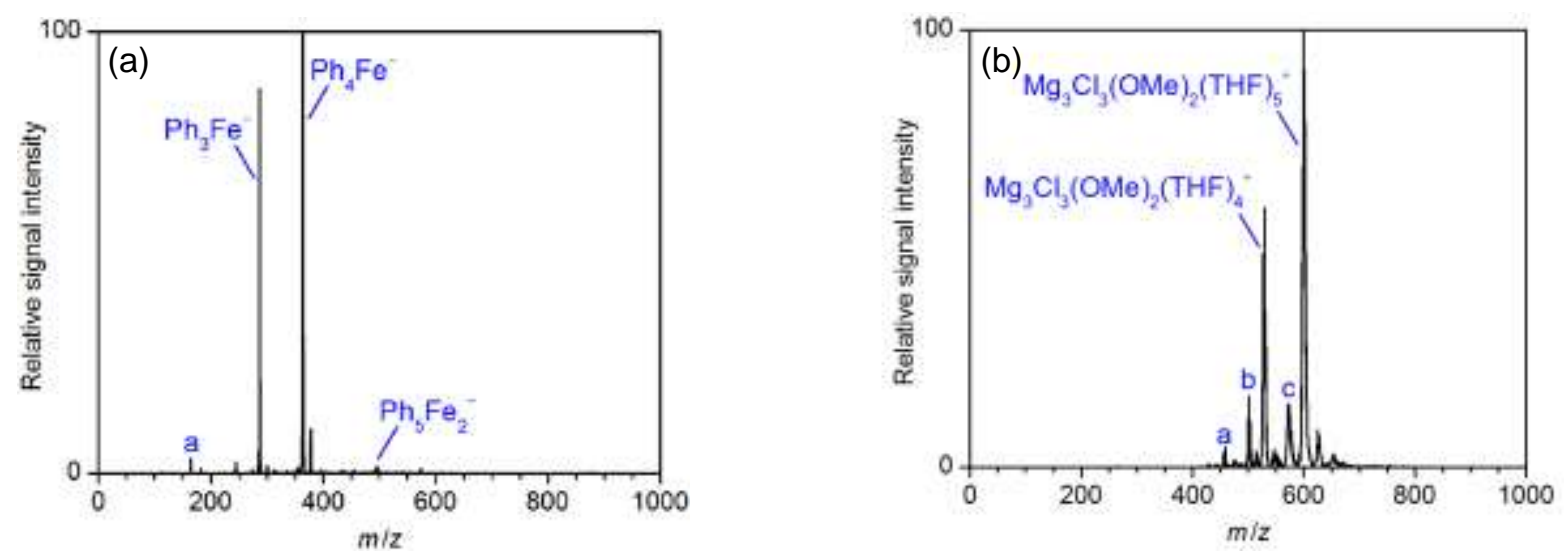

Figure 3.6. (a) Negative-ion mode ESI spectrum of a solution of the products formed in the reaction of $\mathrm{Fe}(\mathrm{acac})_{3}(10 \mathrm{mM})$ with TMEDA (4.0 equiv), $\mathrm{PhMgCl}$ (8.0 equiv) and 180 (1.0 equiv) in THF; $a=\left[\mathrm{Ph}, \mathrm{Fe}, \mathrm{O}_{2}\right]^{-}$. (b) Positive-ion mode ESI spectrum representative of all experiments; $\quad a=\mathrm{Mg}_{3} \mathrm{Cl}_{3}(\mathrm{OMe})(\mathrm{OH})(\mathrm{TMEDA})^{2+}, \quad b=\mathrm{Mg}_{3} \mathrm{Cl}_{3}(\mathrm{OMe})_{2}(\mathrm{THF})_{2}(\mathrm{TMEDA})^{+}$, $c=\mathrm{Mg}_{3} \mathrm{Cl}_{3}(\mathrm{OMe})_{2}(\mathrm{THF})_{3}(\mathrm{TMEDA})^{+}$. The incorporated methoxides originate from traces of methanol as reported previously. ${ }^{[267,268 c, 275]}$ The experiments were conducted by $\operatorname{Dr}$. T. Parchomyk.

When a frozen solution of ${ }^{57} \mathrm{FeCl}_{2} / \mathbf{1 8 0} / \mathrm{CyMgCl} / \mathrm{TMEDA}$ was analyzed by ${ }^{57} \mathrm{Fe}$ Mössbauer spectroscopy, a rather intricate spectrum could be observed, which has been simulated well assuming five subspectra (Fig. 3.7a). ${ }^{[276]}$ Two subspectra (Fig. 3.7a, blue and red) are almost identical to the previously detected ferrates (Fig. 3.2a). The most pronounced new signal (Fig. 3.7a, green) can be attributed to a low-coordinate iron(II) high-spin complex, most likely trigonal-planar $\mathrm{Cy}_{2} \mathrm{Fe}(\mathrm{NHC}),{ }^{[277]}$ in good agreement with ESI-MS (Fig. 3.5). Another newly formed species (Fig. 3.7a, magenta), with a higher isomer shift of $0.54 \mathrm{~mm} \mathrm{~s}^{-1}$ together with a lower quadrupole splitting of $2.04 \mathrm{~mm} \mathrm{~s}^{-1}$, may indicate a more symmetric iron(II) high-spin species with a higher coordination number such as $\mathrm{Cy}_{3} \mathrm{Fe}(\mathrm{NHC})^{-}$, as detected by ESI-MS (Fig. 3.5). An additional minor species (Fig. 3.7a, cyan) was also observed in the ${ }^{57} \mathrm{Fe}$ Mössbauer spectrum of the reaction with $\mathrm{CyMgCl}$, but its non-characteristic doublet does not allow for further assignment. 

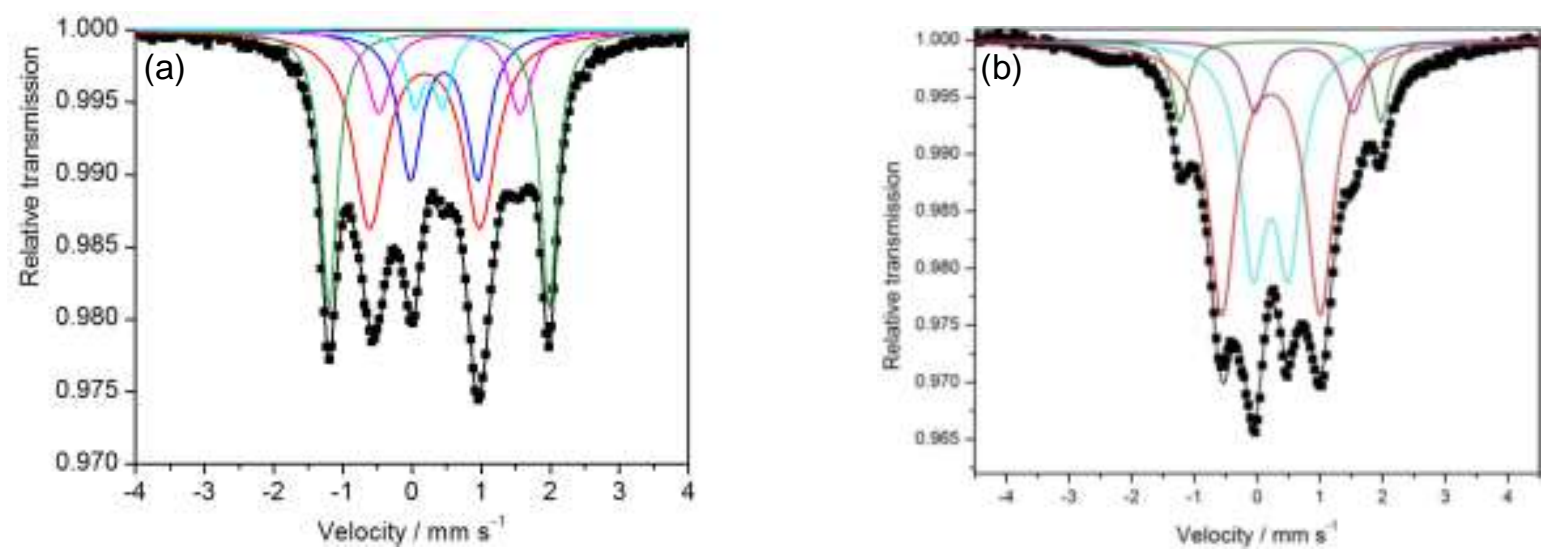

Figure 3.7. (a) Mössbauer spectrum of a frozen solution ( $T=80 \mathrm{~K})$ of the products formed in the reaction of ${ }^{57} \mathrm{FeCl}_{2}(5.0 \mathrm{mM})$, TMEDA (4.0 equiv), 180 (1.0 equiv) and $\mathrm{CyMgCl}$ (8.0 equiv) in THF; components of the fit: $\delta($ red $)=0.18 \mathrm{~mm} \mathrm{~s}^{-1}, \Delta E_{\mathrm{Q}}(\mathrm{red})=1.59 \mathrm{~mm} \mathrm{~s}^{-1}$, rel. int. $=36 \% ; \quad \delta$ (green) $=0.39 \mathrm{~mm} \mathrm{~s}^{-1}, \quad \Delta E_{\mathrm{Q}}$ (green) $=3.19 \mathrm{~mm} \mathrm{~s}^{-1}, \quad$ rel. int. $=27 \%$; $\delta$ (blue $)=0.46 \mathrm{~mm} \mathrm{~s}^{-1}, \Delta E_{Q}$ (blue $)=0.98 \mathrm{~mm} \mathrm{~s}^{-1}$, rel. int. $=19 \% ; \delta$ (magenta) $=0.54 \mathrm{~mm} \mathrm{~s}^{-1}$, $\Delta E_{\mathrm{Q}}$ (magenta) $=2.04 \mathrm{~mm} \mathrm{~s}^{-1}, \quad \quad$ rel. int. $=11 \% ; \quad \delta($ cyan $)=0.24 \mathrm{~mm} \mathrm{~s}^{-1}$, $\Delta E_{\mathrm{Q}}$ (cyan) $=0.40 \mathrm{~mm} \mathrm{~s}^{-1}$, rel. int. $=7 \%{ }^{[276]}$ (b) Mössbauer spectrum and components of the fit of a frozen solution $(T=80 \mathrm{~K})$ of the products formed in the reaction of ${ }^{57} \mathrm{FeCl}_{2}(5.0 \mathrm{mM})$, TMEDA (4.0 equiv), CyMgCl (8.0 equiv) and 180 (1.0 equiv) in THF at $23^{\circ} \mathrm{C}$; components of the fit: $\delta($ red $)=0.22 \mathrm{~mm} \mathrm{~s}^{-1}, \Delta E_{\mathrm{Q}}($ red $)=1.57 \mathrm{~mm} \mathrm{~s}^{-1}$, rel. int. $=47 \% ; \delta$ (cyan $)=0.22 \mathrm{~mm} \mathrm{~s}^{-1}$, $\Delta E_{\mathrm{Q}}($ cyan $)=0.57 \mathrm{~mm} \mathrm{~s}^{-1}, \quad$ rel. int. $=34 \% ; \quad \delta$ (purple $)=0.75 \mathrm{~mm} \mathrm{~s}^{-1}, \quad \Delta E_{Q}($ purple $)=$ $1.57 \mathrm{~mm} \mathrm{~s}^{-1}, \quad$ rel. int. $=10 \% ; \quad \delta$ (green) $=0.37 \mathrm{~mm} \mathrm{~s}^{-1}, \quad \Delta E_{\mathrm{Q}}$ (green) $=3.20 \mathrm{~mm} \mathrm{~s}^{-1}$, rel. int. $=9 \%$. The spectra were recorded and interpreted by Dr. S. Demeshko.

Two related iron(II) species were also observed in the corresponding reaction with $\mathrm{PhMgCl}$ (Fig. 3.8). However, no species related to the minor uncharacteristic signal observed before (Fig. 3.7a, cyan) was detected in this experiment. It is hence believed that this species was formed via $\beta$-hydride elimination from the $\mathrm{Cy}_{2} \mathrm{Fe}(\mathrm{NHC})$ complex. This hypothesis is further corroborated by the observation that, when the sample was prepared at higher temperatures (Fig. 3.7b), this species (Fig. 3.7a, cyan) became more pronounced, while the intensity of the $\mathrm{Cy}_{2} \mathrm{Fe}(\mathrm{NHC})$ signal was reduced (Fig. 3.7b, green). 


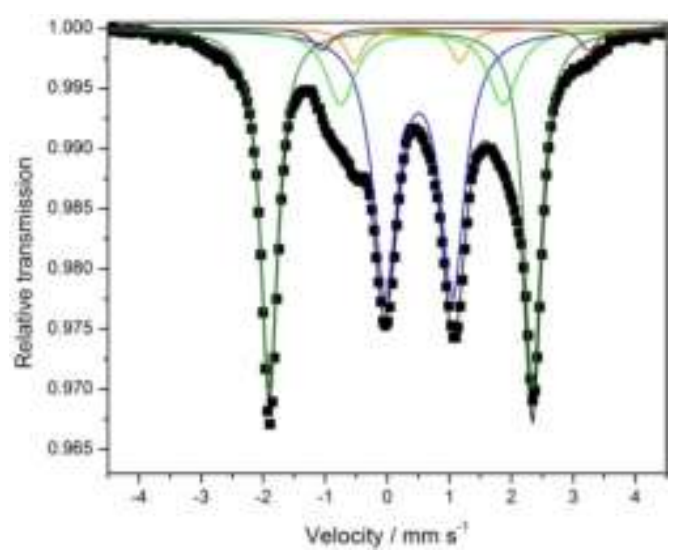

Figure 3.8. Mössbauer spectrum and components of the fit of a frozen solution $(T=80 \mathrm{~K})$ of the products formed in the reaction of ${ }^{57} \mathrm{FeCl}_{2}(5.0 \mathrm{mM})$, TMEDA (4.0 equiv), $\mathrm{PhMgCl}$ (8.0 equiv) and 180 (1.0 equiv) in THF; components of the fit: $\delta$ (blue) $=0.51 \mathrm{~mm} \mathrm{~s}^{-1}$, $\Delta E_{\mathrm{Q}}$ (blue) $=1.09 \mathrm{~mm} \mathrm{~s}^{-1}$, rel. int. $=40 \% ; \delta$ (green) $=0.22 \mathrm{~mm} \mathrm{~s}^{-1}, \Delta E_{\mathrm{Q}}$ (green) $=4.25 \mathrm{~mm} \mathrm{~s}^{-1}$, rel. int. $=39 \% ; \delta($ light green $)=0.56 \mathrm{~mm} \mathrm{~s}^{-1}, \Delta E_{Q}$ (light green $)=2.62 \mathrm{~mm} \mathrm{~s}^{-1}$, rel. int. $=14 \%$; $\delta$ (orange $)=0.32 \mathrm{~mm} \mathrm{~s}^{-1}, \Delta E_{\mathrm{Q}}$ (orange) $=1.70 \mathrm{~mm} \mathrm{~s}^{-1}$, rel. int. $=4 \% ; \delta($ wine $)=1.10 \mathrm{~mm} \mathrm{~s}^{-1}$, $\Delta E_{Q}($ wine $)=4.30 \mathrm{~mm} \mathrm{~s}^{-1}$, rel. int. $=3 \%$. The spectrum was recorded and interpreted by Dr. S. Demeshko.

Thereafter, additional experiments in the presence of the indole substrate $59 \mathrm{~b}$ were performed. In addition to the previously observed species, $\left[\mathrm{Cy}_{4} \mathrm{Fe} \text { (indole)] }\right]^{-}$was observed by ESI-MS analysis of the reaction of ${ }^{57} \mathrm{FeCl}_{2} / \mathrm{CyMgCl} / \mathrm{TMEDA} / \mathbf{1 8 0} / \mathbf{5 9} \mathbf{b}$ (Fig. 3.9a). Yet, this species is believed to be catalytically irrelevant in the $\mathrm{C}-\mathrm{H}$ activation due to the absence of the NHC ligand. Again, the apparent high intensity of the $\mathrm{Cy}_{4} \mathrm{Al}^{-}$peak is due to the relative low intensity of the anionic iron species. ESI-MS analysis of the reaction of ${ }^{57} \mathrm{FeCl}_{2} / \mathrm{PhMgCl} / \mathrm{TMEDA} / \mathbf{1 8 0} / 59 \mathrm{~b}$ did not reveal any new species, but showed the almost complete disappearance of the $\mathrm{Ph}_{3} \mathrm{Fe}(\mathrm{II})^{-}$ ferrate (Fig. 3.9b). Its consumption may suggest a reaction between the iron(II) ate complex, or a species in equilibrium with it, and the substrate 59 to form a neutral species. Therefore, this observation is suggestive of an organometallic iron(II) species to be involved in the catalysis as an intermediate or in the generation of the active catalyst. 

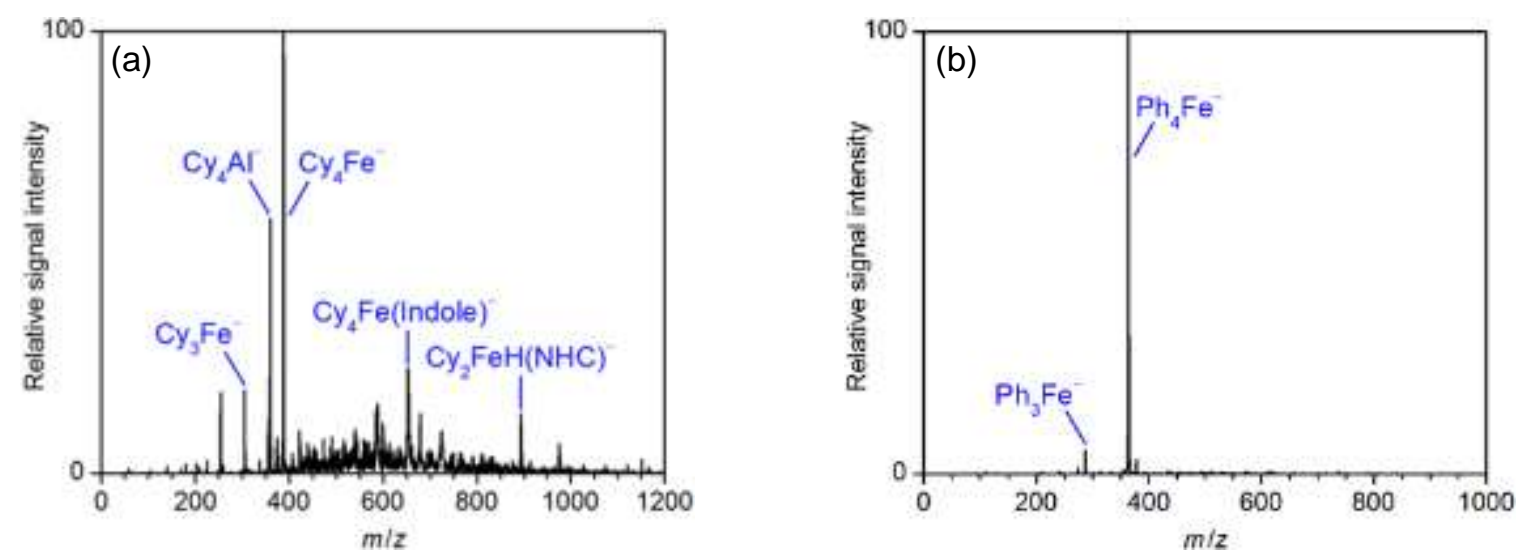

Figure 3.9. (a) Negative-ion mode ESI spectrum of a solution of the products formed in the reaction of $\mathrm{Fe}(\mathrm{acac})_{3}(10 \mathrm{mM}$ ) with TMEDA (4.0 equiv), CyMgCl (8.0 equiv), 180 (1.0 equiv) and 59b (1.0 equiv) in THF. (b) Negative-ion mode ESI spectrum of a solution of the products formed in the reaction of $\mathrm{Fe}(\mathrm{acac})_{3}(10 \mathrm{mM})$ with TMEDA (4.0 equiv), $\mathrm{PhMgCl}$ (8.0 equiv), 180 (1.0 equiv) and $59 \mathrm{~b}$ (1.0 equiv) in THF. The experiments were conducted by S. Lülf and Dr. T. Parchomyk, respectively.

Further, no new species or significant changes upon the addition of substrate $59 \mathrm{~b}$ were observed by ${ }^{57} \mathrm{Fe}$ Mössbauer spectroscopy analysis of the analogous reactions with either $\mathrm{CyMgCl}$ or $\mathrm{PhMgCl}$ (Fig. 3.10). Yet, a slight reduction of the intensity of the species believed to be $\mathrm{R}_{2} \mathrm{Fe}(\mathrm{NHC})$ was observed (Fig. 3.10a-b, green), possibly indicating its consumption in a reaction with $\mathbf{5 9 b}$.
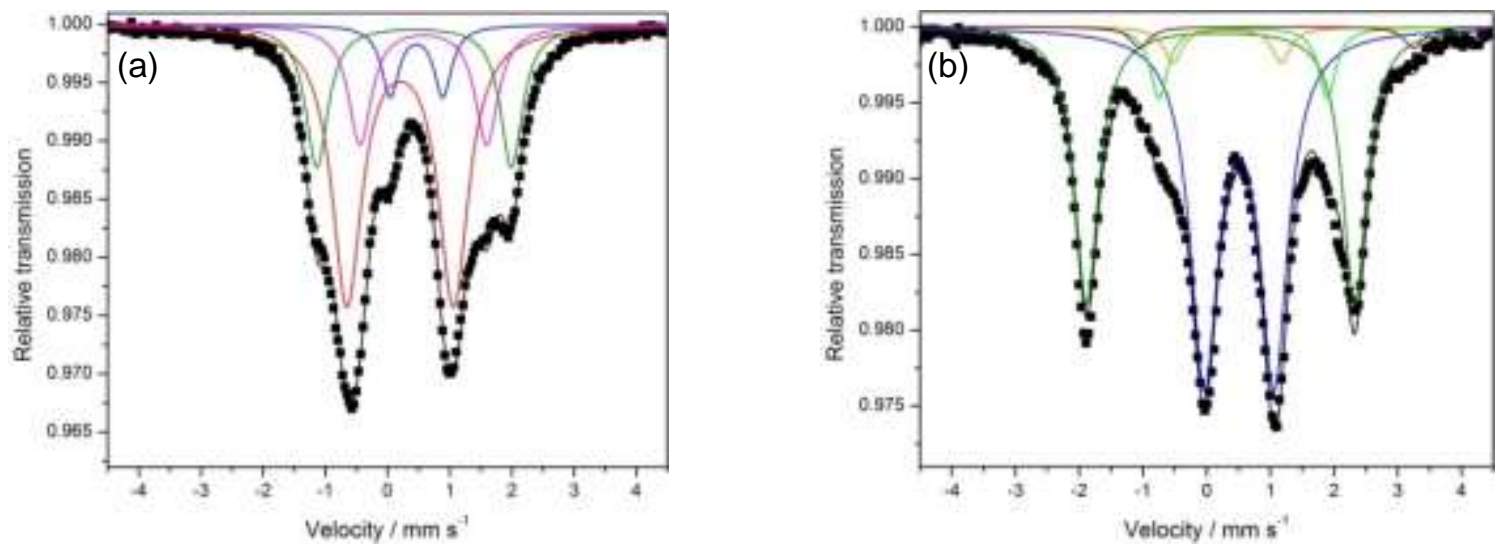

Figure 3.10. (a) Mössbauer spectrum and components of the fit of a frozen solution ( $T=80 \mathrm{~K}$ ) of the products formed in the reaction of ${ }^{57} \mathrm{FeCl}_{2}(5.0 \mathrm{mM})$, TMEDA (4.0 equiv), $\mathrm{CyMgCl}$ (8.0 equiv), 180 (1.0 equiv) and 59b (1.0 equiv) in THF; components of the fit: $\delta($ red $)=0.20 \mathrm{~mm} \mathrm{~s}^{-1}, \quad \Delta E_{Q}($ red $)=1.71 \mathrm{~mm} \mathrm{~s}^{-1}, \quad$ rel. int. $=53 \% ; \quad \delta$ (green $)=0.43 \mathrm{~mm} \mathrm{~s}^{-1}$, 
$\Delta E_{\mathrm{Q}}($ green $)=3.13 \mathrm{~mm} \mathrm{~s}^{-1}$

rel. int. $=21 \%$;

$\delta$ (magenta $)=0.58 \mathrm{~mm} \mathrm{~s}^{-1}$,

$\Delta E_{\mathrm{Q}}$ (magenta) $=2.04 \mathrm{~mm} \mathrm{~s}^{-1}$,

rel. int. $=18 \% ; \quad \delta$ (blue $)=0.47 \mathrm{~mm} \mathrm{~s}^{-1}$,

$\Delta E_{\mathrm{Q}}($ blue $)=$

$0.84 \mathrm{~mm} \mathrm{~s}^{-1}$, rel. int. $=8 \%$. (b) Mössbauer spectrum and components of the fit of a frozen solution $(T=80 \mathrm{~K})$ of the products formed in the reaction of ${ }^{57} \mathrm{FeCl}_{2}(5.0 \mathrm{mM})$, TMEDA (4.0 equiv), $\mathrm{PhMgCl}$ (8.0 equiv), $\mathbf{1 8 0}$ (1.0 equiv) and $\mathbf{5 9 b}$ (1.0 equiv) in THF; components of the fit: $\quad \delta$ (blue $)=0.51 \mathrm{~mm} \mathrm{~s}^{-1}, \quad \Delta E_{Q}($ blue $)=1.09 \mathrm{~mm} \mathrm{~s}^{-1}, \quad$ rel. int. $=52 \%$; $\delta($ green $)=0.22 \mathrm{~mm} \mathrm{~s}^{-1}, \quad \Delta E_{\mathrm{Q}}$ (green $)=4.21 \mathrm{~mm} \mathrm{~s}^{-1}, \quad$ rel. int. $=36 \%$; $\delta$ (light green $)=0.57 \mathrm{~mm} \mathrm{~s}^{-1}, \quad \Delta E_{\mathrm{Q}}$ (light green $)=2.64 \mathrm{~mm} \mathrm{~s}^{-1}, \quad$ rel. int. $=7 \%$; $\delta$ (orange $)=0.32 \mathrm{~mm} \mathrm{~s}^{-1}, \Delta E_{\mathrm{Q}}$ (orange) $=1.70 \mathrm{~mm} \mathrm{~s}^{-1}$, rel. int. $=4 \% ; \delta($ wine $)=1.10 \mathrm{~mm} \mathrm{~s}^{-1}$, $\Delta E_{Q}$ (wine $)=4.30 \mathrm{~mm} \mathrm{~s}^{-1}$, rel. int. $=2 \%$. The spectra were recorded and interpreted by $\operatorname{Dr}$. $S$. Demeshko.

In summary, our experimental findings suggest the formation of organometallic iron(II)/NHC complexes as intermediates in the iron-catalyzed enantioselective $\mathrm{C}-\mathrm{H}$ alkylation of indoles 59 with vinylarenes 60/191. No interaction between iron and TMEDA was observed in any of the experiments, which suggests that TMEDA coordinates to the magnesium(II) ions and does not interact with the iron catalyst. Additionally, our observations provide support to the long-proposed role of Grignard reagents to serve as both reductant and base in iron-catalyzed $\mathrm{C}-\mathrm{H}$ activations.

The major limitation of mass spectrometry is, obviously, the difficulty to observe neutral species. As a consequence, we reasoned that the use of a charge-tagged $\mathrm{NHC}$ ligand would allow for the detection of otherwise neutral $\mathrm{R}_{2} \mathrm{Fe}(\mathrm{II}) \mathrm{NHC}$ species. For example, phosphonium-tags have previously been employed by Koszinowski to detect species formed in iron-catalyzed cross-couplings. ${ }^{[269 a]}$ We hence became interested in the synthesis of a positively charged NHC precursor resembling pre-ligand 180. Starting from the amino-tagged NHC precursor 206 reported by Grela and coworkers, ${ }^{[278]}$ the ammonium-tagged imidazolinium 207 was prepared readily in two steps via salt metathesis and a Menshutkin reaction with methyl iodide (Scheme 3.14). A test reaction revealed 207 to be a potent pre-ligand for the ironcatalyzed hydroarylation, giving the alkylated product with an efficacy comparable to IMes $\cdot \mathrm{HCl}$ (Scheme 3.15). ${ }^{[148]}$ However, ESI-MS measurements performed by Dr. T. Parchomyk and $F$. Kraft of the Koszinowski research group have so far remained unsuccessful. Indeed, problems of very poor solubility and loss of Mel in solution 
have so far prevented us from observing the expected $\mathrm{NHC}$-ligated iron species by ESI-MS. ${ }^{[279]}$ Interestingly, Grela and coworkers prepared the ammonium-tagged $\mathrm{NHC} /$ ruthenium complex by a late-stage methylation of the corresponding neutral amino-NHC/ruthenium complex. ${ }^{[278]}$ In a later work, it was revealed that the direct complexation of the ammonium-tagged NHC precursor with ruthenium had also been unsuccessful. ${ }^{[280]}$

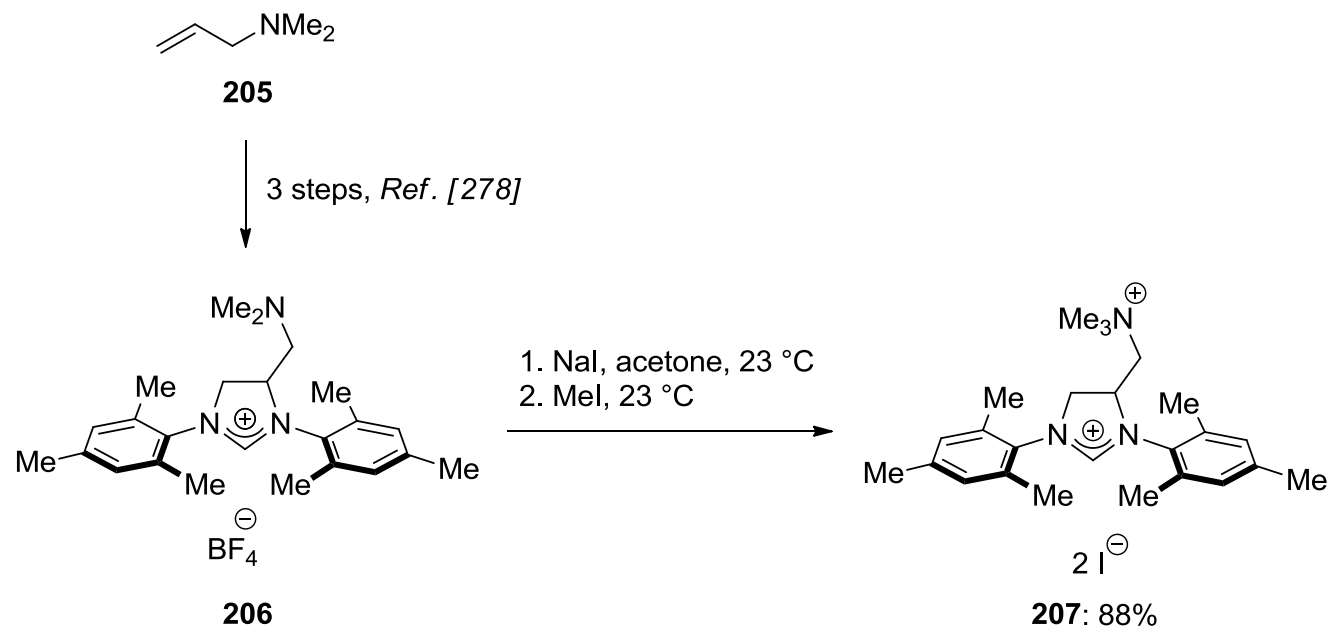

Scheme 3.14. Synthesis of charge-tagged NHC precursor 207.
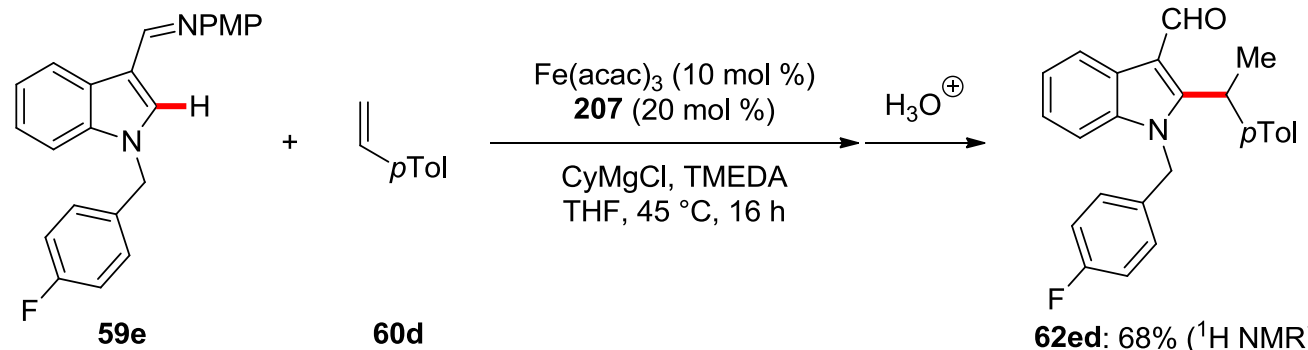

62ed: $68 \%\left({ }^{1} \mathrm{H}\right.$ NMR)

Scheme 3.15. Reaction with charge-tagged NHC precursor 207 as the pre-ligand. 


\subsubsection{Proposed Mechanism}

Based on the conducted mechanistic studies and literature precedents, the catalytic cycle is proposed to begin with the formation of an active organometallic iron catalyst through the reduction of the iron(III) precursor by the action of the Grignard reagent (Scheme 3.16). While $\beta$-hydride elimination certainly plays a role in the reduction of the iron precursor when $\mathrm{CyMgCl}$ is employed, this process does not seem to be essential since significant conversions were observed when using $\mathrm{PhMgCl}$ (Tables 3.7, 3.9). In the latter case, the reduction process likely occurs via reductive elimination from a $\mathrm{Ph}_{2}[\mathrm{Fe}]$ species, as alternative processes through radical expulsion are disfavored. ${ }^{[267]}$ Nevertheless, ${ }^{57} \mathrm{Fe}$ Mössbauer spectroscopy strongly supports a facile $\beta$-hydride elimination to occur even at ambient temperature (Fig. 3.8b). Additionally, iron(II)-hydride species were detected by ESI-MS as well (Fig. 3.5, 3.9a). Hence, the $\mathrm{Cy}_{2} \mathrm{Fe}(\mathrm{NHC})$ species observed by ${ }^{57} \mathrm{Fe}$ Mössbauer spectroscopy (Fig. 3.7) is most likely only an intermediate of the catalyst generation process. Furthermore, in consideration of our mechanistic studies and previous reports, ${ }^{[267,269 b, 281]}$ the TMEDA additive is believed to induce deaggregation and coordinate to the organomagnesium reagent rather than the active $\mathrm{NHC}$-ligated iron catalyst. 


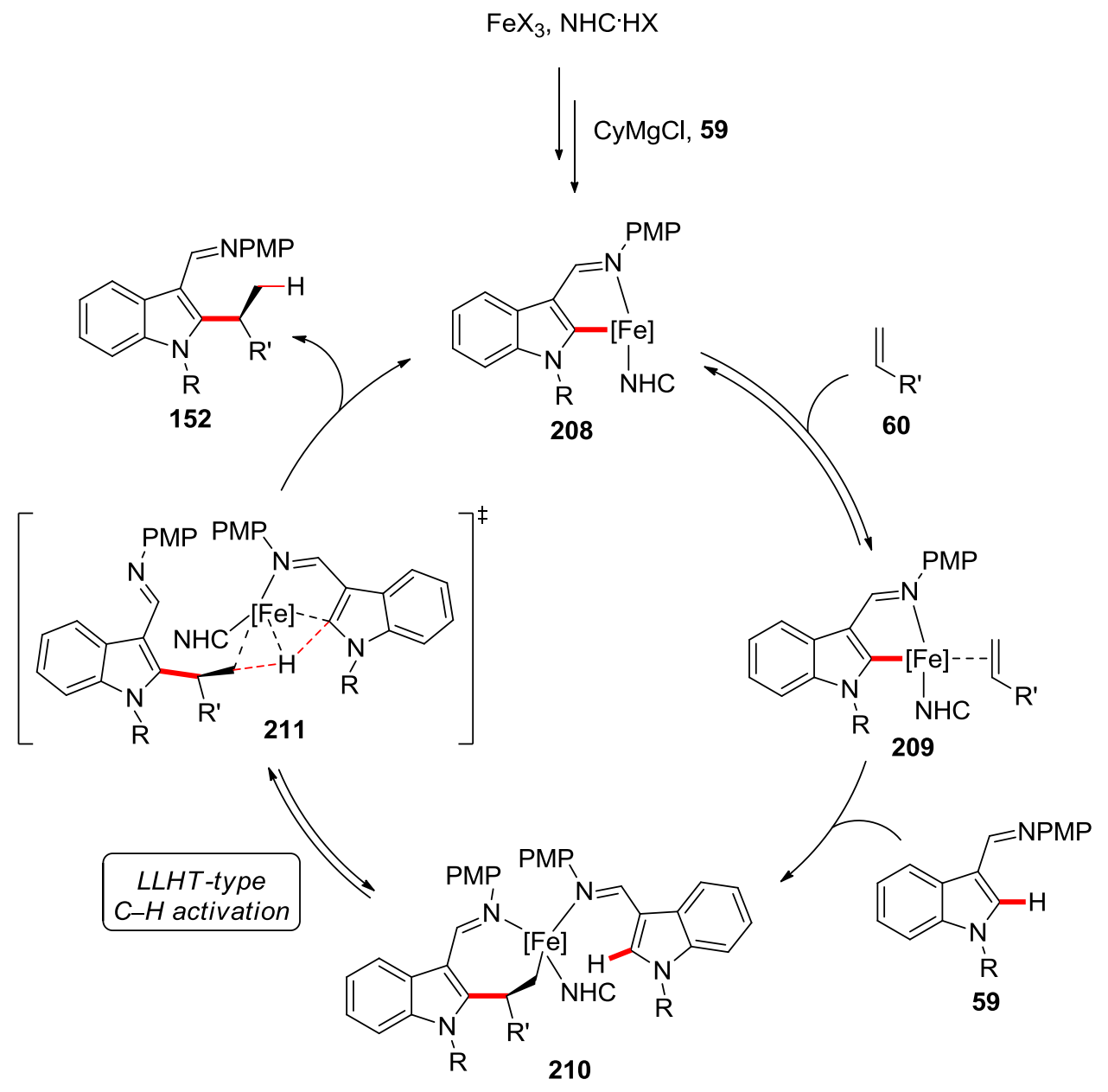

Scheme 3.16. Proposed mechanism of the asymmetric iron-catalyzed alkylation.

After the in situ formation of an organometallic mono-NHC-iron catalyst, the latter can be coordinated by the alkene $\mathbf{6 0}$ in a reversible fashion leading to its zerothorder dependence (Scheme 3.11). The subsequent kinetically relevant migratory insertion is assisted by coordination of the substrate $59,{ }^{[282]}$ explaining its first-order rate law. Given the facile inner-sphere $\mathrm{C}-\mathrm{H}$ cleavage, the selective deuterium transfer to the methyl group in product $[D]_{1}-62$ (Schemes 3.8 ) and the crossover experiment (Scheme 3.9), the $\mathrm{C}-\mathrm{H}$ metalation step is proposed to occur via a ligandto-ligand hydrogen transfer (LLHT) manifold, by the action of the coordinated substrate 59. It should be noted that a turnover-limiting initial coordination of substrate 59 , followed by $\mathrm{C}-\mathrm{H}$ activation by oxidative addition, would also be in 
agreement with our kinetic findings. However, the crossover experiment, along with preliminary computational studies by DFT, ${ }^{[256]}$ seem to favor a LLHT regime.

\subsubsection{Iron-Catalyzed Alkyne Hydroarylations}

In the original report by Yoshikai and coworkers, the substrate scope of the racemic iron-catalyzed hydroarylations was not limited to vinylarenes. Indeed, internal alkynes proved viable as well. ${ }^{[148]}$ While the alkenylated products do not possess any chiral center, we speculated that axial chirality might exist across the $\mathrm{C}-\mathrm{C}$ bond generated in the transformation. This hypothesis was confirmed by the synthesis of compound 85ca and the separation of its atropisomers by chiral HPLC (Figure 3.11). Methods for the synthesis of axially chiral compounds are of high interest for synthetic chemists due to the importance of axially chiral natural products ${ }^{[283]}$ and chiral ligands, ${ }^{[70 c, 284]}$ among others. Interestingly, while several examples of atroposelective $\mathrm{C}-\mathrm{H}$ activations employing noble transition metal catalysts are reported in the literature, ${ }^{[213 \mathrm{~h}, 285]}$ the selective synthesis of axially chiral compounds via $3 \mathrm{~d}$ transition metal-catalyzed $\mathrm{C}-\mathrm{H}$ activation remains thus far unprecedented. ${ }^{[52]}$

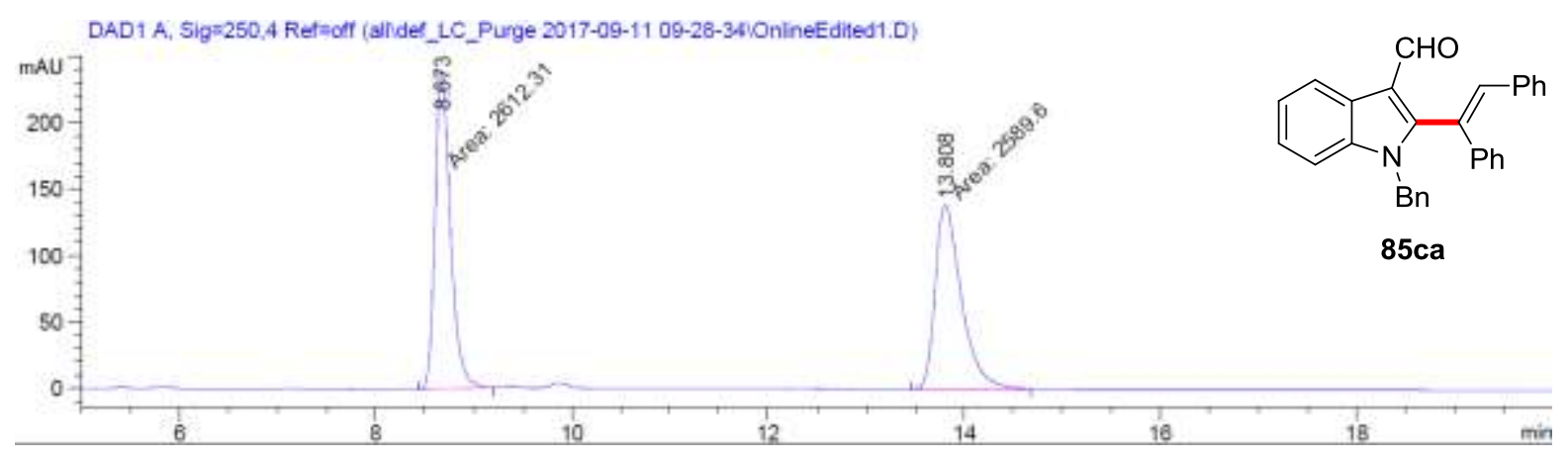

Figure 3.11. Chiral HPLC chromatogram of 85ca (Daicel CHIRALPAK ${ }^{\circledR}$ IB-3, $n$-hexane/ $\mathrm{PrOH}=80 / 20$, flow rate $1.0 \mathrm{~mL} / \mathrm{min}$ ).

Hence, intrigued by the possibility to develop an enantioselective version, a representative set of chiral $\mathrm{NHC}$ ligands were tested in the envisioned asymmetric transformation employing $\mathrm{N}$-benzylindole $59 \mathrm{c}$ and tolane $7 \mathrm{a}$ (Table 3.14). Sadly, 
while low conversion was obtained under a slight modification of Yoshikais conditions (entry 1), no product was detected with any of the tested chiral pre-ligands (entries 2-4). Since the racemic reaction is only reported on $\mathrm{N}$-methylindole $59 \mathrm{~b}$, we reasoned that the extra steric bulk of the benzyl substituent might hinder the catalysis. Therefore, various NHC precursors were tested for the alkenylation of $N$-methylindole $59 \mathrm{~b}$. While the expected product $85 \mathrm{ba}$ was obtained in moderate yield using IXyl (entry 5), no conversion was obtained when employing chiral ligands (entries 6-8). Furthermore, the analysis by chiral HPLC of product 85ba revealed the existence of a plateau between the peaks of the two atropisomers, which clearly indicates their interconversion at ambient temperature (Figure 3.12$){ }^{[286]}$ The observed lack of reactivity, as well as the conformational instability of the formed atropisomers, prompted us to discontinue this project.

Table 3.14. Iron-catalyzed hydroarylation of alkyne $7 a$ with indoles 59 . $^{[a]}$

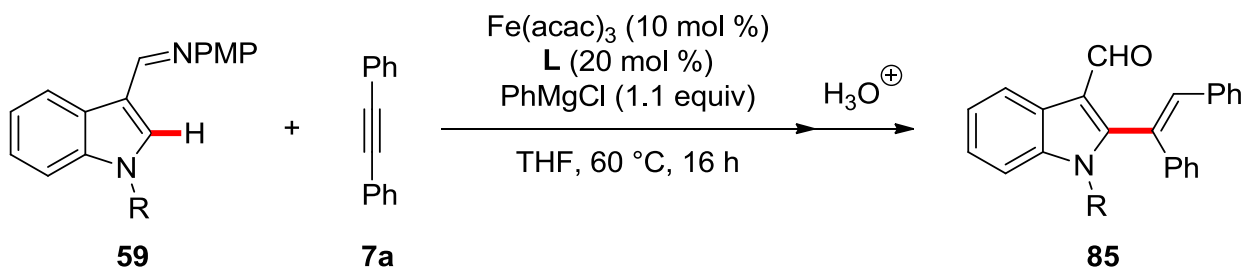

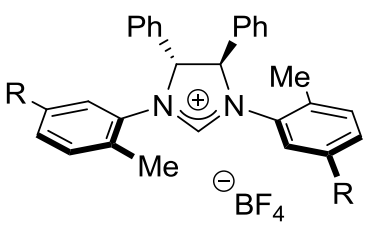

180: $R=1-A d$

176: $R=M e$

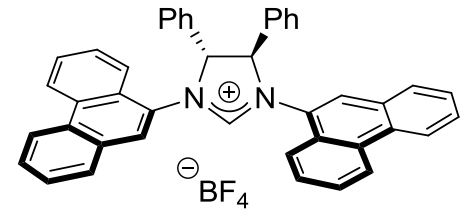

188

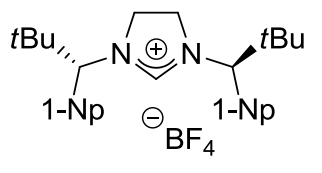

159

\begin{tabular}{ccccc}
\hline Entry & $\mathbf{R}$ & $\mathbf{L}$ & Yield [\%] $^{[\mathrm{b}]}$ & e.r. \\
\hline 1 & Bn & $\mid \mathrm{IXy| \cdot HCl}$ & 13 & - \\
2 & $\mathrm{Bn}$ & $\mathbf{1 8 0}$ & n.r. & - \\
3 & $\mathrm{Bn}$ & $\mathbf{1 7 6}$ & n.r. & - \\
4 & $\mathrm{Bn}$ & $\mathbf{1 8 6}$ & n.r. & -
\end{tabular}




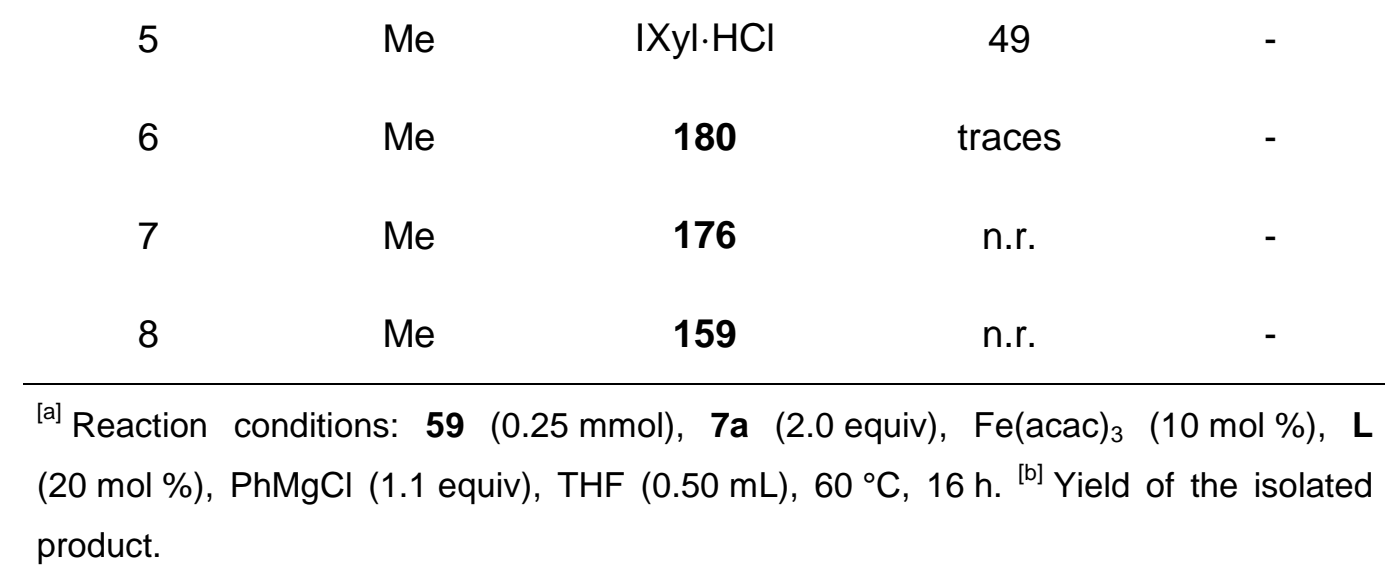

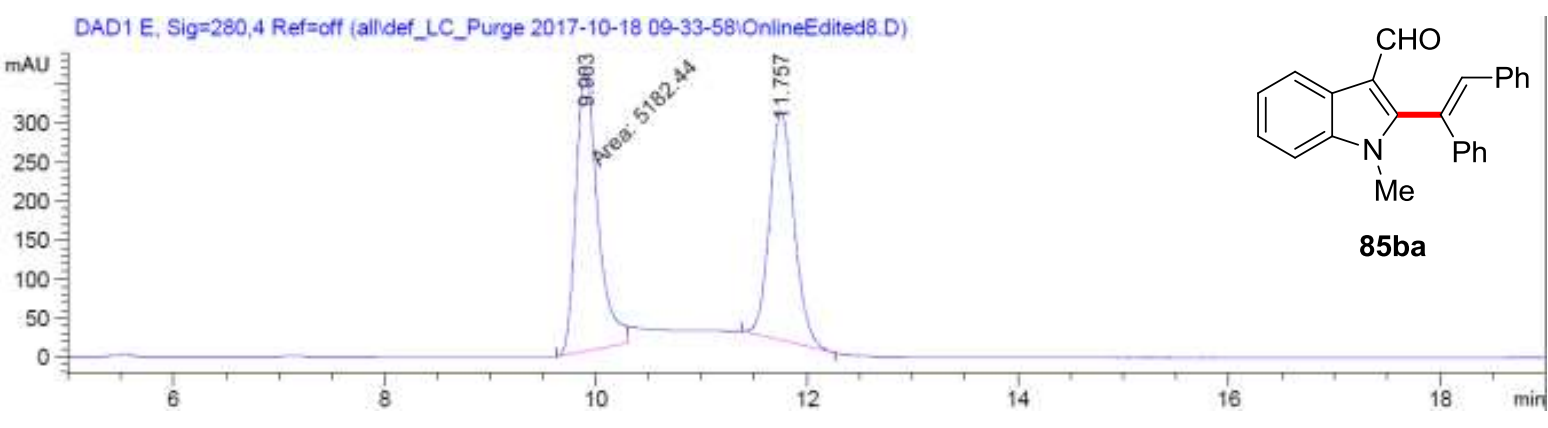

Figure 3.12. Chiral HPLC chromatogram of $85 \mathrm{ba}$. The plateau between the two peaks indicates on-column interconversion of the two atropisomers ${ }^{[286]}$ (Daicel CHIRALPAK ${ }^{\circledR}$ IC-3, $n$-hexane/ $\mathrm{PrOH}=70 / 30$, flow rate $1.0 \mathrm{~mL} / \mathrm{min}$ ). 


\subsection{Asymmetric Nickel-Catalyzed Hydroarylations by C-H Activation}

Nickel complexes have been recognized as powerful tools in molecular syntheses, with numerous applications to $C-C$ bond forming processes. ${ }^{[153 d, 153 e]}$ Due to its high abundance in the Earth's crust, broad range of accessible oxidation states, and comparatively low cost, ${ }^{[287]}$ nickel catalysis has experienced a considerable development in recent years. Among others, applications to cross-coupling chemistry $^{[18]}$ and the functionalization of otherwise inert $\mathrm{C}-\mathrm{H}$ bonds ${ }^{[45,48 b, 48 c]}$ have attracted significant interest. In particular, nickel catalysts have emerged as an especially powerful tool for hydroarylation-type $\mathrm{C}-\mathrm{H}$ activations. ${ }^{[27,188 a]}$

However, asymmetric nickel-catalyzed hydroarylations remain rare. While major progress has been recently achieved for intramolecular hydroarylations employing nickel/aluminium ${ }^{[288]}$ heterobimetallic ${ }^{[23 b]}$ catalysis, $^{[193,200,203,206]}$ enantioselective intermolecular processes are unprecedented. In a broader context, asymmetric intermolecular nickel-catalyzed $\mathrm{C}-\mathrm{H}$ functionalizations remain unknown, with only one single exception by Cramer for the reductive three-component coupling of benzaldehydes, norbornenes and silanes (Scheme 1.43). ${ }^{[210]}$

In this context, the association of $\mathrm{N}$-heterocyclic carbene (NHC) ligands with nickel is remarkably versatile and has been intensively exploited for, inter alia, $\mathrm{C}-\mathrm{C}$ forming processes via hydroarylation reactions or cross-couplings. ${ }^{[289]}$ These literature precedents prompted us to investigate the performance of the novel chiral $\mathrm{NHC}$ precursors that were developed for asymmetric iron-catalyzed hydroarylations (Chapter 3.2) in the unprecedented nickel-catalyzed asymmetric secondary alkylation of benzimidazoles 99 with styrenes 60 . 


\subsubsection{Preliminary Studies towards Asymmetric Intermolecular Hydroarylations by $\mathrm{C}-\mathrm{H}$ Activation}

Initially, we selected the well-documented branched-selective hydroarylation of

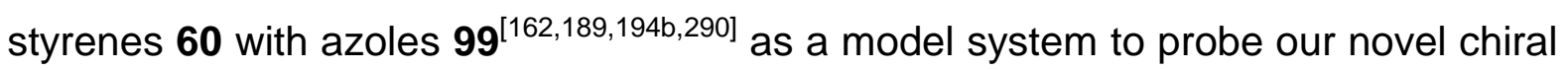
$\mathrm{NHC}$ precursors. $\mathrm{NaOtBu}$ was used as a base to generate the free $\mathrm{NHC}$ in situ, as was previously reported. ${ }^{[170,203]}$ A preliminary ligand optimization ${ }^{[291]}$ identified $\mathrm{N}, \mathrm{N}$-diaryl NHCs, similar to those used in the enantioselective iron-catalyzed hydroarylation (Chapter 3.2), as optimal ligands for the transformation in terms of conversion. Other ligand classes, including $N, N$-dialkyl NHCs, phosphines, phosphine oxides, diamines, BOX ligands, dienes and phosphoramidites, gave all conversions lower than $25 \%$. However, enantiomeric excesses were found to be highly irreproducible. Intrigued by those issues, we decided to investigate their cause. A base-induced racemization was immediately suspected to occur since the chiral center, a bis-benzylic position substituted with an electron-withdrawing benzimidazole moiety, is rather activated. Furthermore, 2-allylbenzimidazoles are reported to isomerize in a similar setting, even at a lower temperature of $100{ }^{\circ} \mathrm{C} .{ }^{[170]}$ Therefore, the effect of the amount of base and various additives on the transformation was studied in order to prevent the suspected racemization (Table 3.15). First, an increase of the amount of base led to a decreased enantiomeric ratio (entries 1-2). Lowering the amount of base was found to improve the enantioselectivity of the transformation, but resulted in low conversions (entries 3-4). These findings provide strong support for the suspected base-induced racemization. Thereafter, the effect of various additives was probed in the asymmetric hydroarylation. As inconsistent enantioselectivities were obtained when using toluene from different sources (SPS, distilled over $\mathrm{Na}$, etc.), the trace water content was suspected to play a key role in the irreproducibility of the reaction. Hence, co-catalytic amounts of water were added into the reaction. Remarkably, traces of water were found to strongly improve the enantiomeric excess of product 106bc (entries 5-8). Other protic or Lewis acidic additives were then tested in the reaction (entries 9-12), the best results being obtained in the presence of a 
co-catalytic amount of BHT (entry 12). While the beneficial effect of protic additives is not fully understood yet, it is suspected that they might "neutralize" the excess of $\mathrm{NaOtBu}$, thus preventing the racemization of product $106 \mathrm{bc}$. Meanwhile, the linear product was obtained selectively in the presence of a co-catalytic amount of $\mathrm{AlMe}_{3}$, as expected from literature precedents (entry 13). ${ }^{[194]}$

Table 3.15. Effect of the base and other additives of the enantioselective nickel-catalyzed intermolecular hydroarylation of styrene $60 \mathrm{c}$ with benzimidazole $99 \mathrm{~b}$. $^{[a]}$

${ }_{2^{[\mathrm{d}]}}^{[\mathrm{ej}}$


10

$t \mathrm{AmOH}^{[\mathrm{h}]} \quad 60$

$61: 39$

11

$\mathrm{BHT}(50 \mathrm{~mol} \%)$

53

$80: 20$

12

$\mathrm{BHT}(20 \mathrm{~mol} \%)$

57

80:20

13

$\mathrm{AlMe}_{3}(40 \mathrm{~mol} \%)$

$0^{[i]}$

${ }^{[a]}$ Reaction conditions: 99b (0.50 mmol), 60c (2.0 equiv), Ni(cod $)_{2} \quad(10 \mathrm{~mol} \%)$, L (10 mol \%), NaOtBu (20 mol \%), additive, PhMe $(2.0 \mathrm{~mL}), 130{ }^{\circ} \mathrm{C}, 16 \mathrm{~h} .{ }^{[\mathrm{b}]}$ Isolated yields. ${ }^{[c]}$ Determined by chiral HPLC. ${ }^{[\mathrm{d}]}$ Using NaOtBu (40 mol \%). ${ }^{[\mathrm{e}]}$ Using $\mathrm{NaOtBu}$ (15 mol \%). ${ }^{[\mathrm{f}]}$ Using NaOtBu (10 mol \%). ${ }^{[\mathrm{g}]}$ Degassed $\mathrm{H}_{2} \mathrm{O} .{ }^{[\mathrm{h}]} \mathrm{PhMe} / \mathrm{AmOH}=15 / 1$ was used as the reaction medium. ${ }^{[i]}$ The linear product 112 was obtained in $91 \%$ yield.

With the issues of racemization and irreproducibility being solved, a representative set of chiral NHC precursors was then tested in the envisioned asymmetric hydroarylation (Table 3.16). Somewhat surprisingly, all the pre-ligands probed in the reaction provided the alkylated product $106 \mathrm{bc}$ with almost identical enantioselectivities and similar yields.

Table 3.16. Further ligand optimization in the presence of BHT as additive. ${ }^{[\mathrm{a}]}$

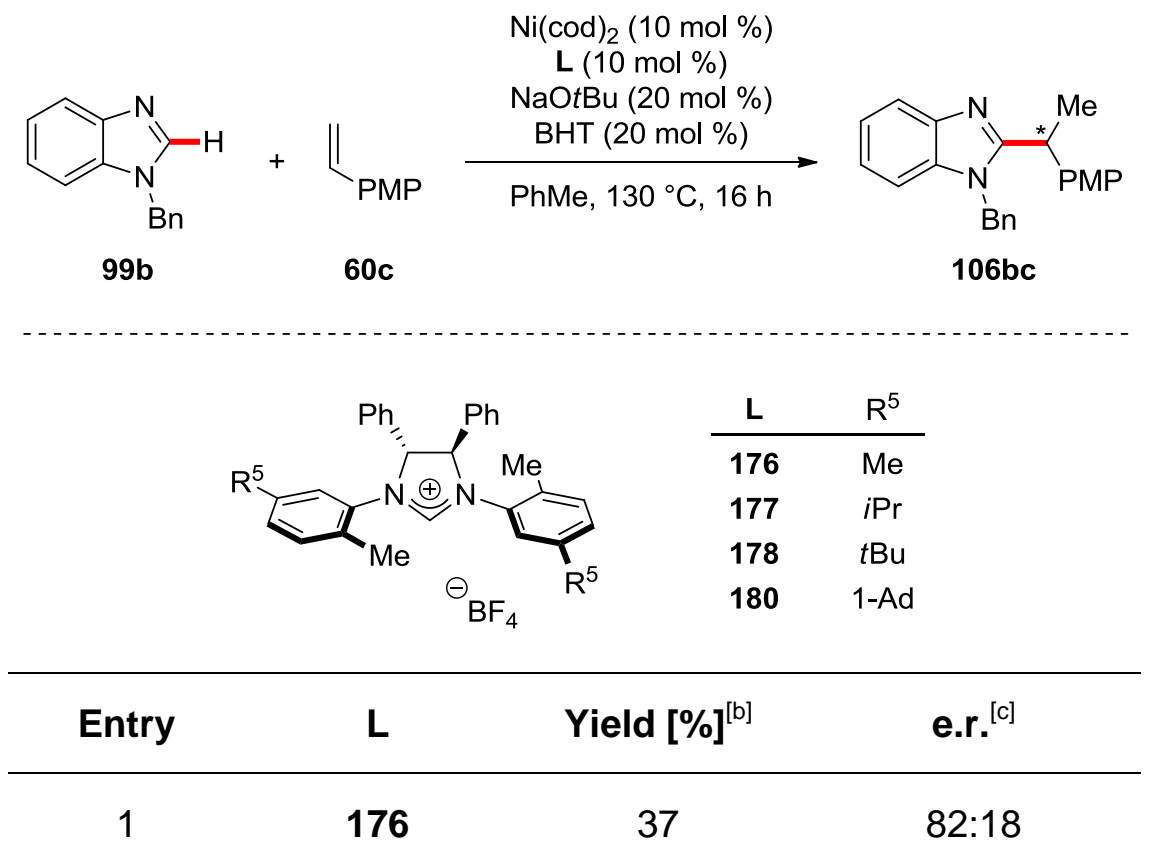


2

3

4
177

178

180
52

56

57
79.5:20.5

80.5:19.5

80:20

${ }^{[a]}$ Reaction conditions: 99b (0.50 mmol), 60c (2.0 equiv), $\mathrm{Ni}(\mathrm{cod})_{2}$ (10 mol \%), L (10 mol \%), NaOtBu (20 mol \%), BHT (20 mol \%), PhMe $(2.0 \mathrm{~mL}), 130{ }^{\circ} \mathrm{C}, 16 \mathrm{~h} .{ }^{[\mathrm{b}]}$ Isolated yields. ${ }^{[\mathrm{c}]}$ Determined by chiral HPLC.

Preliminary mechanistic studies were conducted to delineate the mode of action of the $\mathrm{C}-\mathrm{H}$ activation. A reaction with the deuterated substrate $[D]_{1}-99 \mathrm{~b}$ was performed. Interestingly, while most of the deuterium was selectively transferred to the methyl group, indicating an organometallic $\mathrm{C}-\mathrm{H}$ bond cleavage, significant scrambling was observed. This observation can be rationalized with a facile and reversible $\mathrm{C}-\mathrm{H}$ scission step.

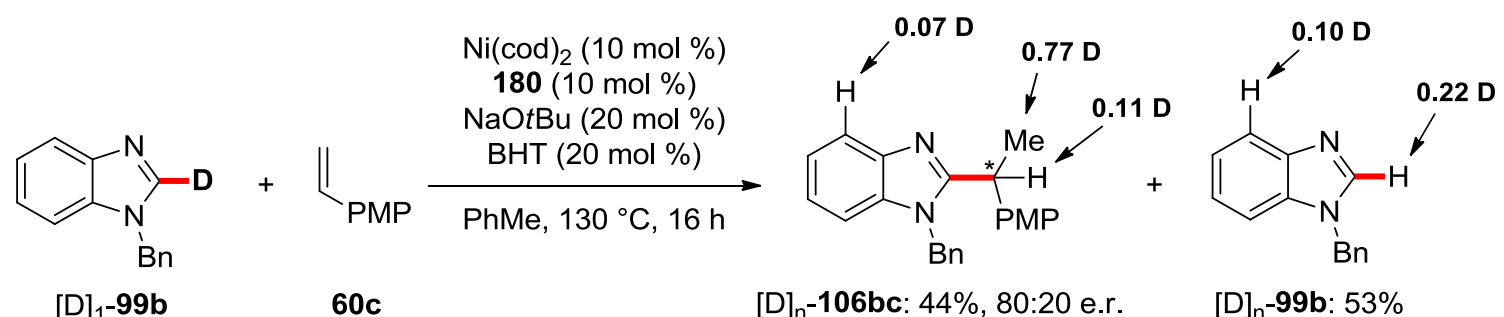

Scheme 3.17. Reaction with deuterium-labeled substrate $[D]_{1}-99 b$.

Meanwhile, as asymmetric catalysis is known to be substrate-specific, different types of heteroarenes and alkenes were probed in the envisioned asymmetric hydroarylation. Notably, promising results were obtained for intramolecular transformations. Those findings motivated us to investigate enantioselective nickelcatalyzed cyclizations by $\mathrm{C}-\mathrm{H}$ activation (vide infra). 


\subsubsection{Optimization Studies of Enantioselective Intramolecular Nickel- Catalyzed Hydroarylations via C-H Activation}

Chemo-, regio- and enantio-selective cyclizations are of key importance in synthetic chemistry, with applications to, inter alia, the synthesis of pharmaceuticals and bioactive natural products. ${ }^{[56 a, 193,292]}$

In this context, intramolecular hydroarylations ${ }^{[27]}$ represent a particularly attractive cyclization strategy due to its perfect atom economy. ${ }^{[2]}$ While nickel catalysts have emerged as powerful tools for the hydroarylations of C-C multiple bonds, ${ }^{[48 a, 188 a]}$ intramolecular processes have long been restricted to the functionalization of highly activated azolium salts. ${ }^{[205]}$ In recent years, the development of heterobimetallic nickel/aluminium catalysis ${ }^{[198]}$ has allowed for the use of unactivated alkenes and substrates bearing less acidic $\mathrm{C}-\mathrm{H}$ bonds. Despite being highly desirable, enantioselective methodologies remained extremely scarce at the outset of this work. ${ }^{[193,195]}$ However, this research area has attracted significant attention during the course of this thesis, as several examples were independently reported by other research groups. ${ }^{[200,203,206]}$ It is noteworthy that all those works absolutely require pyrophoric Lewis acidic organoaluminium additives, such as $\mathrm{AIMe}_{3}$ or $\mathrm{MAD},{ }^{[293]}$ for the reaction to occur.

Therefore, we decided to investigate the asymmetric cyclization of $\mathrm{N}$-homoallylimidazoles $\mathbf{1 4 4}$ by nickel-catalyzed hydroarylation (Table 3.17). This model substrate was selected due to literature precedents with rhodium(I) catalysts $^{[219 b]}$ and the importance of the benzimidazole scaffold in bioactive compounds. ${ }^{[294]}$ Our optimization studies began by testing a wealth of chiral ligands for the envisioned asymmetric alkene hydroarylation. First, various representative NHC precursors and phosphoramidites were tested in the transformation, but fell short in delivering any product (entries 1-5). In agreement with literature precedents (vide supra), the addition of $\mathrm{AlMe}_{3}$ was found to enable the desired cyclization (entry 6). Interestingly, the endo product was selectively obtained, in sharp contrast to another methodology independently reported by $Y e$ around the same time. ${ }^{[206]}$ 
Table 3.17. Optimization of the ligand for the enantioselective Ni-catalyzed cyclization. ${ }^{[a]}$
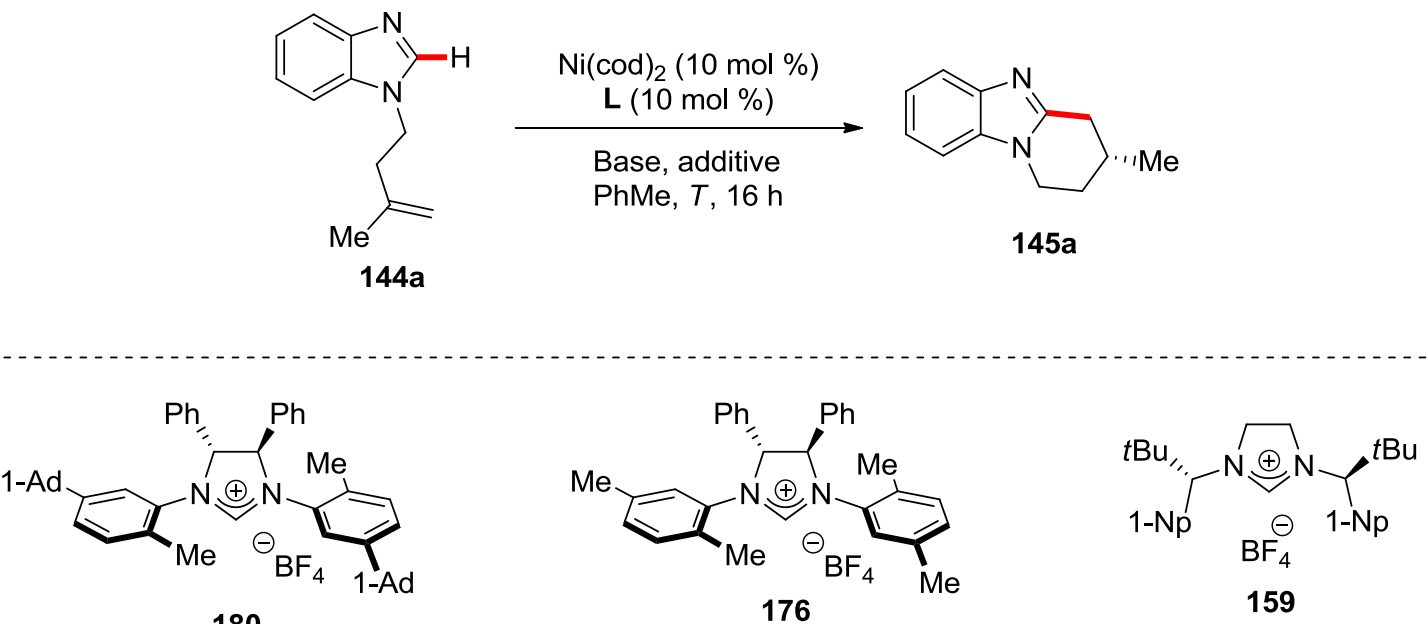<smiles>CC1(C)OC2C(c3ccccc3)C(c3ccccc3)(c3ccccc3)OP(N(c3ccccc3)c3ccccc3)OC2(c2ccccc2)O1</smiles>

212<smiles>[14CH3]P(=O)(c1ccccc1)c1ccccc1</smiles><smiles></smiles>

219

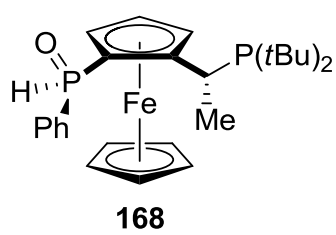<smiles>CCCCCCCN(CCCC)p1oc2ccc3ccccc3c2c2c(ccc3ccccc32)o1</smiles>

161<smiles>CC(c1ccccc1)N1CCN(C(C)c2ccccc2)P1Cl</smiles>

170

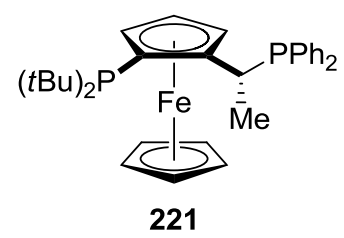

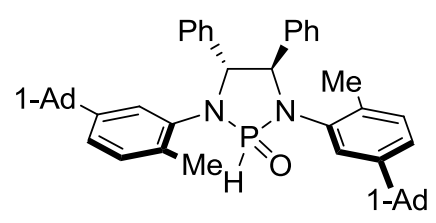

213

214: $\mathrm{Ar}=2-\mathrm{Me}-\mathrm{C}_{6} \mathrm{H}_{4}$

215: $\mathrm{Ar}=4-\mathrm{F}-\mathrm{C}_{6} \mathrm{H}_{4}$

131: $\mathrm{Ar}=3,5-\mathrm{Bu}-\mathrm{C}_{6} \mathrm{H}_{3}$

216: $\mathrm{Ar}=3,5-\mathrm{Bu}-4-\mathrm{OMe}-\mathrm{C}_{6} \mathrm{H}_{2}$

217: $\mathrm{Ar}=2-\mathrm{Np}$

218: $\mathrm{Ar}=2-\mathrm{Me}-5-(1-\mathrm{Ad})-\mathrm{C}_{6} \mathrm{H}_{3}$

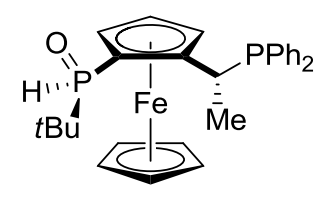

220

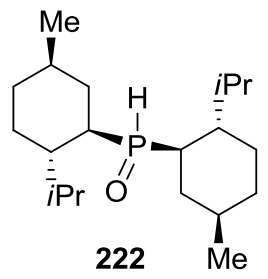




\begin{tabular}{|c|c|c|c|c|c|c|}
\hline Entry & Ligand & Base & Additive & $T\left[{ }^{\circ} \mathrm{C}\right]$ & Yield [\%] ${ }^{[\mathrm{b}]}$ & e.r..$^{[c]}$ \\
\hline 1 & 180 & $\mathrm{NaOtBu}(20 \mathrm{~mol} \%)$ & - & 130 & n.r. & - \\
\hline 2 & 176 & $\mathrm{NaOtBu}(20 \mathrm{~mol} \%)$ & - & 130 & n.r. & - \\
\hline 3 & 159 & $\mathrm{NaOtBu}(20 \mathrm{~mol} \%)$ & - & 130 & n.r. & - \\
\hline 4 & 212 & - & - & 130 & n.r. & - \\
\hline 5 & 161 & - & - & 130 & n.r. & - \\
\hline 6 & 180 & $\mathrm{NaOtBu}(20 \mathrm{~mol} \%)$ & $\mathrm{AlMe}_{3}(40 \mathrm{~mol} \%)$ & 130 & 53 & $67: 33$ \\
\hline 7 & 180 & $\mathrm{NaOtBu}(20 \mathrm{~mol} \%)$ & $\mathrm{AlMe}_{3}(40 \mathrm{~mol} \%)$ & 100 & n.r. & - \\
\hline 8 & 180 & $\mathrm{NaOtBu}(20 \mathrm{~mol} \%)$ & $\mathrm{AIMe}_{3}$ (1.0 equiv) & 130 & 43 & $67: 33$ \\
\hline $9^{[\mathrm{d}]}$ & 180 & $\mathrm{NaOtBu}(20 \mathrm{~mol} \%)$ & $\mathrm{AlMe}_{3}(40 \mathrm{~mol} \%)$ & 130 & n.r. & - \\
\hline 10 & 213 & - & $\mathrm{AlMe}_{3}(40 \mathrm{~mol} \%)$ & 130 & 88 & $32: 68$ \\
\hline 11 & 213 & - & $\begin{array}{c}\mathrm{AlMe}_{3}(40 \mathrm{~mol} \%)+ \\
\mathrm{PPh}_{3}(10 \mathrm{~mol} \%)\end{array}$ & 130 & n.r. & - \\
\hline 12 & 213 & - & MAD (40 mol \%) & 130 & n.r. & - \\
\hline 13 & 213 & - & $\mathrm{BPh}_{3}(40 \mathrm{~mol} \%)$ & 130 & n.r. & - \\
\hline 14 & 213 & - & $\mathrm{AIMe}_{3}(40 \mathrm{~mol} \%)$ & 110 & n.r. & - \\
\hline 15 & 213 & - & $\mathrm{AlMe}_{3}(40 \mathrm{~mol} \%)$ & 95 & n.r. & - \\
\hline 16 & 161 & - & $\mathrm{AlMe}_{3}(40 \mathrm{~mol} \%)$ & 130 & 22 & $50: 50$ \\
\hline 17 & 164 & - & $\mathrm{AlMe}_{3}(40 \mathrm{~mol} \%)$ & 130 & 67 & $53: 47$ \\
\hline 18 & - & - & $\mathrm{AlMe}_{3}(40 \mathrm{~mol} \%)$ & 130 & traces & - \\
\hline 19 & 214 & - & $\mathrm{AlMe}_{3}(40 \mathrm{~mol} \%)$ & 130 & 70 & $32: 68$ \\
\hline 20 & 214 & - & $\mathrm{AlMe}_{3}(40 \mathrm{~mol} \%)$ & 110 & 65 & $33: 67$ \\
\hline 21 & 214 & - & $\mathrm{AlMe}_{3}(40 \mathrm{~mol} \%)$ & 95 & 66 & $32: 68$ \\
\hline 22 & 214 & - & $\mathrm{AlMe}_{3}(40 \mathrm{~mol} \%)$ & 80 & traces & - \\
\hline 23 & 214 & - & $\mathrm{AlMe}_{3}(20 \mathrm{~mol} \%)$ & 95 & 50 & $33: 67$ \\
\hline 24 & 214 & - & $\mathrm{AlMe}_{3}(60 \mathrm{~mol} \%)$ & 95 & 47 & $33: 67$ \\
\hline
\end{tabular}




\begin{tabular}{|c|c|c|c|c|c|c|}
\hline 25 & 214 & - & - & 95 & n.r. & - \\
\hline $26^{[\mathrm{d}]}$ & 214 & - & $\mathrm{AlMe}_{3}(40 \mathrm{~mol} \%)$ & 95 & n.r. & - \\
\hline $27^{[\mathrm{e}]}$ & 214 & - & $\mathrm{AlMe}_{3}(40 \mathrm{~mol} \%)$ & 95 & 60 & $39: 61$ \\
\hline $28^{[f]}$ & 214 & - & $\mathrm{AlMe}_{3}(40 \mathrm{~mol} \%)$ & 95 & traces & - \\
\hline 29 & 215 & - & $\mathrm{AlMe}_{3}(40 \mathrm{~mol} \%)$ & 130 & 12 & $50: 50$ \\
\hline 30 & 131 & - & $\mathrm{AlMe}_{3}(40 \mathrm{~mol} \%)$ & 95 & 89 & $48: 52$ \\
\hline 31 & 216 & - & $\mathrm{AlMe}_{3}(40 \mathrm{~mol} \%)$ & 95 & 56 & $53: 47$ \\
\hline 32 & 217 & - & $\mathrm{AlMe}_{3}(40 \mathrm{~mol} \%)$ & 95 & n.r. & - \\
\hline 33 & 218 & - & $\mathrm{AlMe}_{3}(40 \mathrm{~mol} \%)$ & 95 & traces & - \\
\hline 34 & 218 & - & $\mathrm{AlMe}_{3}(40 \mathrm{~mol} \%)$ & 110 & 55 & $54: 46$ \\
\hline 35 & 219 & - & $\mathrm{AlMe}_{3}(40 \mathrm{~mol} \%)$ & 130 & n.r. & - \\
\hline 36 & 170 & - & $\mathrm{AlMe}_{3}(40 \mathrm{~mol} \%)$ & 130 & n.r. & - \\
\hline 37 & 170 & $\mathrm{NaOtBu}(10 \mathrm{~mol} \%)$ & $\mathrm{AlMe}_{3}(40 \mathrm{~mol} \%)$ & 130 & 16 & $56: 44$ \\
\hline 38 & 220 & - & $\mathrm{AlMe}_{3}(40 \mathrm{~mol} \%)$ & 95 & 88 & $>99: 1$ \\
\hline $39^{[d]}$ & 220 & - & $\mathrm{AlMe}_{3}(40 \mathrm{~mol} \%)$ & 95 & n.r. & - \\
\hline 40 & 168 & - & $\mathrm{AlMe}_{3}(40 \mathrm{~mol} \%)$ & 95 & 86 & $23: 77$ \\
\hline $41^{[\mathrm{g}]}$ & 220 & - & $\mathrm{AlMe}_{3}(10 \mathrm{~mol} \%)$ & 95 & 95 & $>99: 1$ \\
\hline $42^{[\mathrm{g}]}$ & 221 & - & $\mathrm{AlMe}_{3}(10 \mathrm{~mol} \%)$ & 95 & 13 & $55: 45$ \\
\hline 43 & 221 & - & - & 95 & n.r. & - \\
\hline 44 & 222 & - & $\mathrm{AlMe}_{3}(40 \mathrm{~mol} \%)$ & 95 & 85 & $80: 20$ \\
\hline $45^{[\mathrm{h}]}$ & 220 & - & $\mathrm{AlMe}_{3}(4.0 \mathrm{~mol} \%)$ & 95 & 97 & $>99: 1$ \\
\hline 46 & 220 & - & $\mathrm{AlMe}_{3}(10 \mathrm{~mol} \%)$ & 85 & 95 & $>99: 1$ \\
\hline 47 & 220 & - & $\mathrm{AlMe}_{3}(10 \mathrm{~mol} \%)$ & 75 & 94 & $>99: 1$ \\
\hline 48 & 220 & - & - & 95 & 91 & $96: 4$ \\
\hline
\end{tabular}




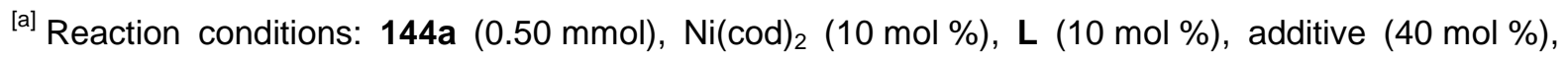
base $(20 \mathrm{~mol} \%)$, PhMe $(2.0 \mathrm{~mL}), 16 \mathrm{~h} .{ }^{[\mathrm{b}]}$ Yield of the isolated product. ${ }^{[\mathrm{c}]}$ Determined by chiral HPLC analysis. ${ }^{[\mathrm{d}]} \mathrm{Ni}(\mathrm{cod})_{2}$ was omitted. ${ }^{[\mathrm{e}]} 214(15 \mathrm{~mol} \%) .{ }^{[\mathrm{fl}} 214(5.0 \mathrm{~mol} \%) .{ }^{[\mathrm{g}]}$ Using Ni(cod $)_{2}(2.5 \mathrm{~mol} \%)$ and $\mathbf{L}(2.5 \mathrm{~mol} \%)$ in PhMe $(1.0 \mathrm{~mL}) .{ }^{[\mathrm{h}]}$ Using Ni(cod) ${ }_{2}(1.0 \mathrm{~mol} \%)$ and $220(1.0 \mathrm{~mol} \%)$ in $\mathrm{PhMe}$ (1.0 mL).

The desired product was obtained in moderate yield and enantiomeric excess when using the previously developed NHC precursor 180 (entry 6). Lowering the temperature or increasing the amount of $\mathrm{AlMe}_{3}$ did not improve the outcome of the reaction (entries 7-8). A control experiment proved the importance of the nickel catalyst (entry 9).

Inspired by the success of (HA)SPO pre-ligands in nickel catalysis, ${ }^{[155 a, 195,206,208,239 b]}$ this ligand class was investigated in the envisioned transformation. The novel chiral HASPO 213, derived from the same diamine intermediate as $\mathbf{1 8 0}$, gave promising results (entry 10). The introduction of additional co-catalytic $\mathrm{PPh}_{3}$ completely suppressed the reactivity (entry 11), despite being reported as beneficial in other transformations occurring under a Ni/Al/SPO regime (see Scheme 1.37). ${ }^{[195,206,208]}$ Interestingly, $\mathrm{MAD}$ or $\mathrm{BPh}_{3}$ fell short in delivering any cyclized product (entries 12-13). Again, lower reaction temperatures proved detrimental to the transformation (entries 14-15). Other ligand types were tested as well. Monodentate phosphoramidites, such as ligands 161 , provided product 145 a in poor yield without significant enantio-induction (entry 16). Then, the prototypical P-chiral secondary phosphine oxide (SPO) 164 provided the product in good yield, but only poor enantioselectivity (entry 17). Furthermore, a control experiment confirmed the importance of the ligand (entry 18). As (HA)SPOs seemed to be the most promising ligand class at this point, we probed next other HASPO pre-ligands, including TADDOL-derived 214 (entry 19). Interestingly, while TADDOL-based phosphine oxides had previously been employed in asymmetric organocatalysis, ${ }^{[207]}$ their use in transition metal catalysis remains rare. ${ }^{[206,208]}$ Remarkably, $\mathbf{2 1 4}$ proved to be superior to previously investigated ligands, as the reaction temperature could be lowered to $95^{\circ} \mathrm{C}$ (entries 20-22). A Job plot of the amount of $\mathrm{AlMe}_{3}$ confirmed $40 \mathrm{~mol} \%$ to be optimal (entries 23-25), and a control experiment confirmed the key role of the 
nickel catalyst (entry 26). Variations of the ligand-to-metal ratio suggested a 1:1 ratio to be optimal under those conditions (entries 27-28). While the ortho-tolyl derivative 214 gave promising results even at lower temperatures, HASPO derived from differently substituted TADDOLs performed poorly in the desired transformation (entries 29-34). As phosphine chlorides are reported to exhibit a reactivity similar to (HA)SPOs, ${ }^{[239 a]} 219$ and 170 were probed in the desired asymmetric cyclization, but no conversion of the alkene 144a was observed (entries 35-36). However, some product formation could be observed after the addition of co-catalytic $\mathrm{NaO}$ tBu (entry 37), presumably due to the in situ formation of the corresponding diaminooxophosphine. ${ }^{[239 b]}$

Thereafter, JoSPOphos-type ligands were tested due to their well-documented efficacy in rhodium(I)-catalyzed enantioselective hydrofunctionalization reactions. ${ }^{[295]}$ Applications of the JoSPOphos ligand family besides rhodium(I) catalysis remain scarce, with notable exceptions in ruthenium- ${ }^{[296]}$ and cobalt-catalyzed ${ }^{[297]}$ asymmetric hydrogenations. To our delight, JoSPOphos 220 afforded the desired product 145a in excellent yield and perfect enantioselectivity (entry 38). In this context, it is noteworthy that this transformation represents the first use of JoSPOphos pre-ligand $\mathbf{2 2 0}$ with a transition metal other than rhodium(I). Once again, a control experiment confirmed the importance of the nickel catalyst (entry 39). The related ligand $\mathbf{1 6 8}$ provided the cyclized product in similar yield but only moderate enantioselectivity (entry 40 ). The catalyst loading could be lowered to $2.5 \mathrm{~mol} \%$ without losing any activity (entry 41). Meanwhile, poor results were obtained with the corresponding JosiPhos ligand 221, highlighting the superiority of the secondary phosphine oxide moiety (entries 42-43). The dimenthyl-substituted SPO 222 recently disclosed by Hintermann ${ }^{[298]}$ was probed as well in the asymmetric $\mathrm{C}-\mathrm{H}$ alkylation (entry 44). Remarkably, the obtained enantiomeric excess of $60 \%$ is the highest observed with a monodentate chiral ligand. Thereafter, 220 was found to promote the reaction with catalyst loadings as low as $1.0 \mathrm{~mol} \%$ (entry 45). Moreover, the excellent performance of the JoSPOphos-derived catalyst at lower temperature once again showcased its remarkable activity (entries 46-47). Extraordinarily, control experiments revealed the JoSPOphos-enabled alkene 
hydroarylation to occur smoothly even in the absence of pyrophoric $\mathrm{AlMe}_{3}$ as additive (entry 48). It should be noted that nickel-catalyzed hydroarylations of nonactivated alkenes in the absence of organoaluminium reagents are extremely rare ${ }^{[189,299]}$ and had never been achieved in an enantioselective fashion previously.

Given the unique opportunity for improved functional group tolerance, we decided to further optimize the $\mathrm{AlMe}_{3}$-free conditions (Table 3.18). Control experiments confirmed the importance of the nickel catalyst and of pre-ligand 220 (entry 1-3). Lower catalyst loadings did not significantly affect the conversion, but lower enantioselectivities were observed here (entries 4-7). Lower temperatures did not prove beneficial to the reaction outcome (entries 8-9). Thereafter, variations of the ligand-to-metal ratio provided informative results (entries 10-15). A ligand-to-nickel ratio of 2:1 was found to significantly reduce the enantioselectivity of the reaction, as well as the yield at lower catalyst loadings. Somewhat surprisingly, the use of an excess of nickel provided the highest enantioselectivities, the best results being obtained with a $220 / \mathrm{Ni}$ ratio of $1: 2$ (entry 13). A larger excess of nickel did not considerably alter the enantioselectivity of the reaction, but lower conversions were observed. Possibly, an excess of nickel may be required in order to prevent the formation of less selective bis-ligated nickel species. ${ }^{[300]}$

Table 3.18. Optimization of the additive-free hydroarylation. ${ }^{[a]}$

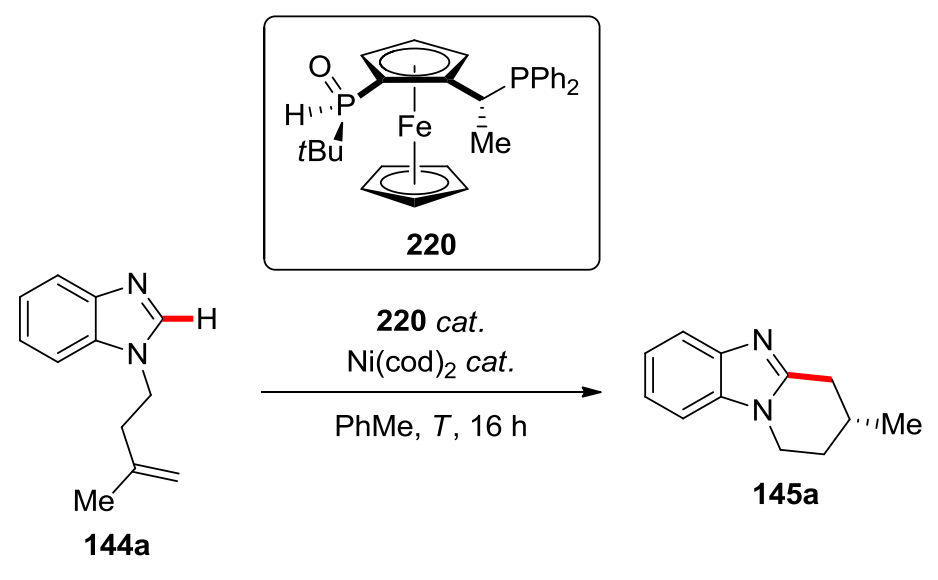




\begin{tabular}{|c|c|c|c|c|c|}
\hline Entry & $\mathrm{Ni}(\mathrm{cod})_{2}[\mathrm{~mol} \%]$ & 220 [mol \%] & $T\left[{ }^{\circ} \mathrm{C}\right]$ & Yield [\%] ${ }^{[b]}$ & e.r. ${ }^{[c]}$ \\
\hline 1 & 10 & 10 & 95 & 91 & $97: 3$ \\
\hline 2 & 0 & 10 & 95 & n.r. & - \\
\hline 3 & 10 & 0 & 95 & traces & - \\
\hline 4 & 5.0 & 5.0 & 95 & 98 & $89: 11$ \\
\hline 5 & 2.5 & 2.5 & 95 & 88 & $90: 10$ \\
\hline $6^{[\mathrm{d}]}$ & 2.5 & 2.5 & 95 & n.r. & - \\
\hline 7 & 1.0 & 1.0 & 95 & 82 & $91: 9$ \\
\hline 8 & 2.5 & 2.5 & 85 & 73 & $87: 13$ \\
\hline 9 & 2.5 & 2.5 & 75 & 16 & $80: 20$ \\
\hline 10 & 10 & 20 & 95 & 59 & $76: 24$ \\
\hline 11 & 10 & 5.0 & 95 & 89 & $97: 3$ \\
\hline 12 & 5.0 & 10 & 95 & 38 & $77: 23$ \\
\hline 13 & 5.0 & 2.5 & 95 & 96 & $96: 4$ \\
\hline 14 & 5.0 & 1.25 & 95 & 31 & $94: 6$ \\
\hline 15 & 2.5 & 1.0 & 95 & 78 & $96: 4$ \\
\hline
\end{tabular}

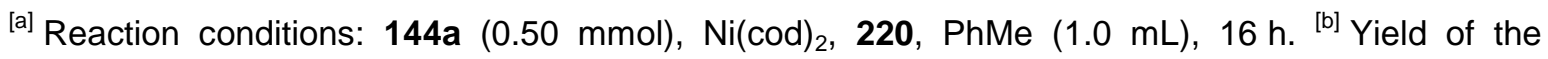
isolated product. ${ }^{[\mathrm{c}]}$ Determined by chiral HPLC analysis of the isolated product. ${ }^{[\mathrm{d}]}$ Using $\mathrm{Ni}(\mathrm{acac})_{2}$ instead of $\mathrm{Ni}(\operatorname{cod})_{2}$.

\subsubsection{Substrate Scope and Limitations}

With the optimized conditions in hand, we next decided to explore the versatility and robustness of the nickel-catalyzed asymmetric hydroarylation. The remarkably simple catalytic system proved able to cyclize various alkene-tethered heteroarenes 
144 in outstanding yields and levels of enantioselectivity (Table 3.19). The substrate scope of the transformation was not limited to benzannulated azoles. Indeed, even the simple ester-substituted imidazole $\mathbf{1 4 4 b}$ was efficiently converted in a highly enantioselective fashion (entry 2). Moreover, a variety of highly functionalized tethered prochiral alkenes $\mathbf{1 4 4}$ were fully tolerated in the transformation, providing the desired cyclized products $\mathbf{1 4 5}$ in high yields and excellent levels of enantiocontrol (entries 3-10). Extended alkyl chains, bulky cycloalkyl and benzyl groups, cyclic acetals and silyl ethers were smoothly converted, proving the $\mathrm{C}-\mathrm{H}$ alkylated products 145 with high selectivity control. Remarkably, additional double bonds in the tethered alkene were compatible with the transformation, with the distal olefins left completely untouched. Indeed, $\mathrm{N}-\gamma$-geranyl- $144 \mathrm{~h}$ and $\mathrm{N}-\gamma$-farnesylbenzimidazole 144i were selectively cyclized to the desired products 145 , with only the proximal exo-double bond reacting. Additionally, even unhindered terminal alkenes, such as $\mathbf{1 4 4} \mathbf{j}$, proved to be viable substrates for the $\mathrm{C}-\mathrm{H}$ alkylation, without significant formation of polycyclic byproducts.

Table 3.19. Substrate scope of gem-substituted olefins 144 and heterocycles in the nickelcatalyzed intramolecular hydroarylation. ${ }^{[a]}$
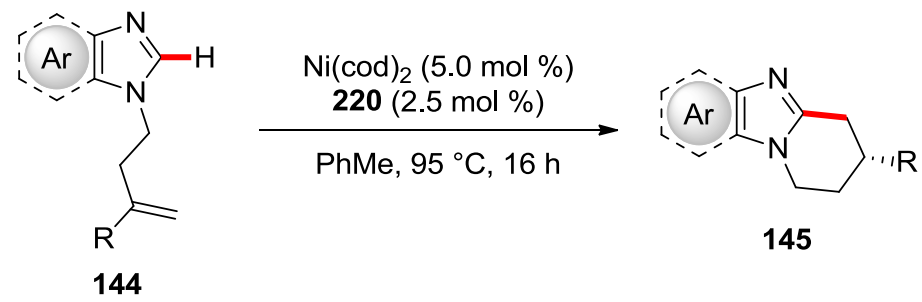

145

Entry Substrate


2<smiles>C=C(C)CCn1c(C(C)=O)cnc1-c1ccccc1</smiles>

3

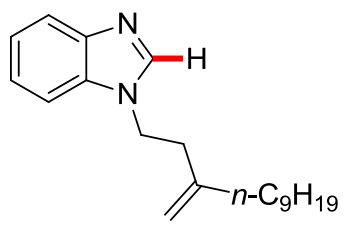

144c

4

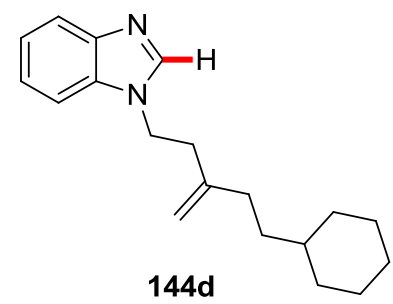

5

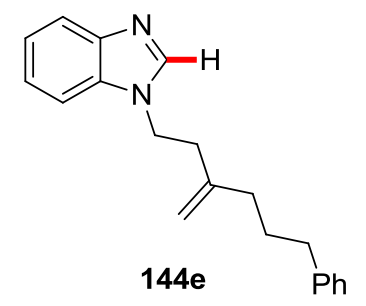

6

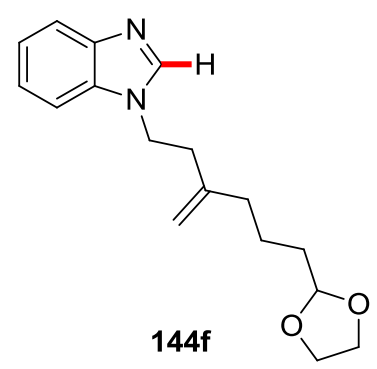

7

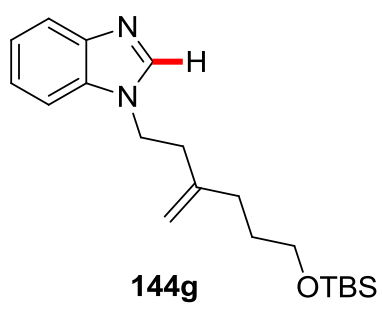

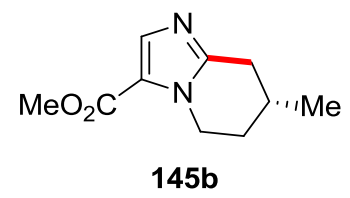

39

$66^{[d]} \quad 99: 1^{[d]}$
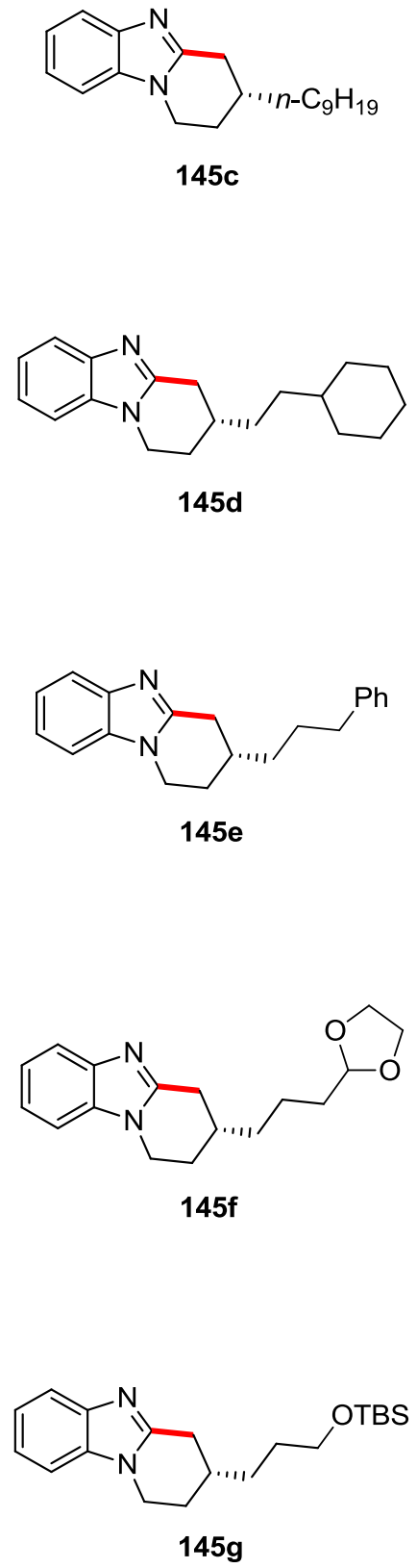

$87^{[\mathrm{d}]} \quad 98: 2^{[\mathrm{d}]}$
$88 \quad 96: 4$

85

98:2

81

$97: 3$

83

$95: 5$

$145 \mathrm{~g}$ 
8

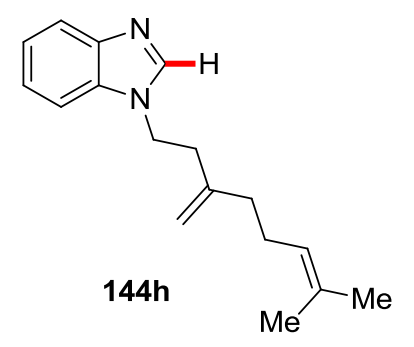

9

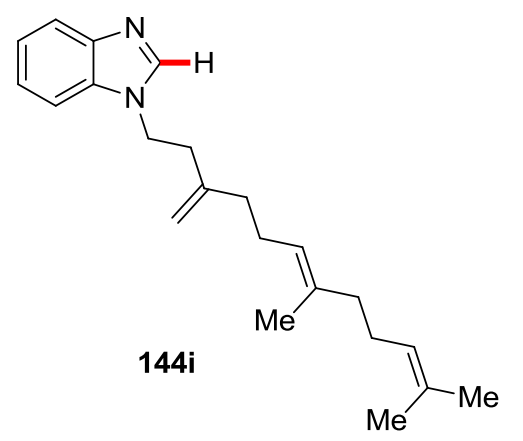

10

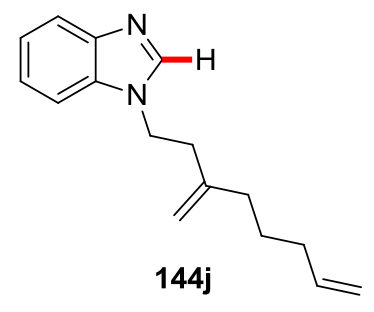

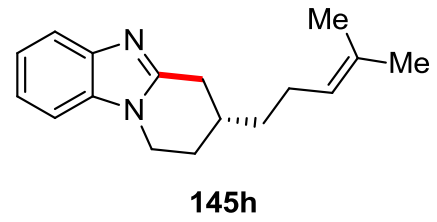

84

$97: 3$

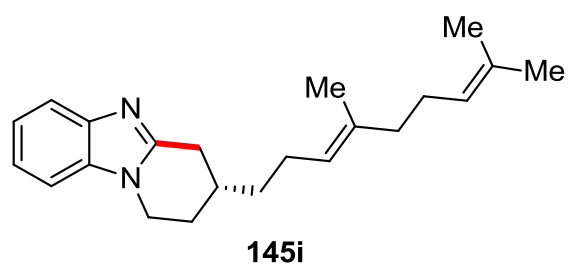

84

99:1

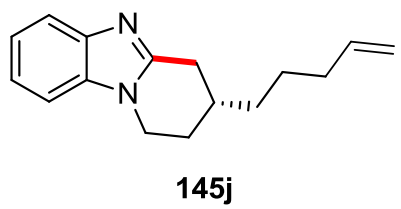

29

$54^{[\mathrm{d}]}$
$97: 3$

$97: 3^{[d]}$

${ }^{[a]}$ Reaction conditions: $144(0.50 \mathrm{mmol}), \mathrm{Ni}(\mathrm{cod})_{2}(5.0 \mathrm{~mol} \%), 220$ (2.5 mol \%), PhMe $(1.0 \mathrm{~mL})$, $95{ }^{\circ} \mathrm{C}, 16 \mathrm{~h} .{ }^{[b]}$ Isolated yields. ${ }^{[\mathrm{c}]}$ Determined by chiral HPLC analysis. ${ }^{[\mathrm{d}]}$ With $\mathrm{Ni}(\operatorname{cod})_{2}(10 \mathrm{~mol} \%)$ and $220(5.0 \mathrm{~mol} \%)$.

Moreover, numerous additional substrates 144 were tested by $V$. Müller and $D r$. $D$. Ghorai in the nickel-catalyzed intramolecular hydroarylation, with a focus on the heteroarene moiety. Various substituted benzimidazoles, including electron-rich, electron-poor and polychlorinated derivatives performed well in the reaction, once again showcasing the chemo-selectivity and versatility of the developed organoaluminium-free $\mathrm{C}-\mathrm{H}$ functionalization methodology (Scheme 3.18). Beside diversely decorated benzimidazoles, a variety of pharmaceutically relevant motifs, including aza-benzimidazoles, highly functionalized purines and theophylline derivatives, were efficiently converted to the cyclized products 145 with high selectivity control. 


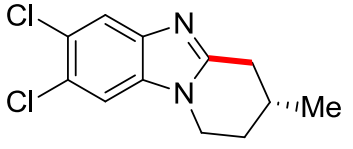

145k: $84 \%, 98: 2$ e.r. ${ }^{[a, c]}$

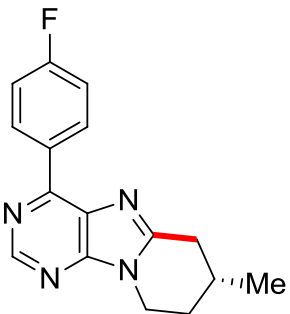

145n: $81 \%, 95: 5$ e.r. ${ }^{[b]}$

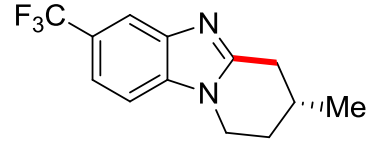

145I: $91 \%, 99: 1$ e.r. ${ }^{[a]}$

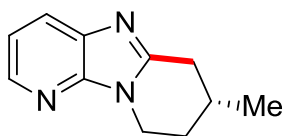

145m: $89 \%, 97: 3$ e.r. ${ }^{[b]}$

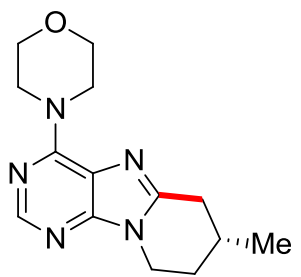

145o: $75 \%, 95: 5$ e.r. ${ }^{[b, c]}$

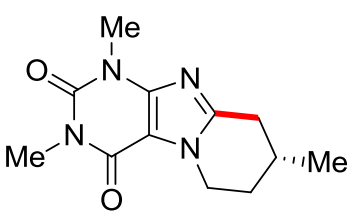

145p: $81 \%, 95: 5$ e.r. ${ }^{[b, c]}$

Scheme 3.18. Selected additional examples of the substrate scope. ${ }^{[a]}$ Performed by Dr. D. Ghorai. ${ }^{[\mathrm{b}]}$ Performed by V. Müller. ${ }^{[\mathrm{c}]}$ With Ni(cod $)_{2}(10 \mathrm{~mol} \%)$ and $220(5.0 \mathrm{~mol} \%)$.

Further studies were conducted to understand the effect of the substitution pattern of the tethered alkene 144 (Table 3.20). The findings revealed that 1,1-disubstituted alkenes were essential to achieve high conversion and stereo-selectivity, as substrates lacking the gem-disubstitution pattern, despite high enantioselectivities, were found to give the 5-membered exo-cyclized product 145 in low to moderate yields (entries 1-2). More sterically encumbered substrates, such as 144s and 144t, fell short in delivering any cyclized product (entries 3-4). Aryl-substituted alkenes, or alkenes with shorter or longer tethers, gave unsatisfactory results too (entries 5-7). Interestingly, in the case of the unsubstituted substrate $144 x$, the isomerized alkene 223 was obtained as the major product of the transformation, which could be supportive of the formation of a nickel-hydride and/or a $\pi$-allyl-nickel intermediate (entry 8). ${ }^{[299 b, 301]}$ This hypothesis was further substantiated by performing the same reaction with the deuterated analog $[D]_{1}-144 x$, as $H / D$ scrambling was observed along the double bond and the allylic methyl group (entry 9 ). 
Table 3.10. Effect of the substitution pattern of the olefin. ${ }^{\text {[a] }}$

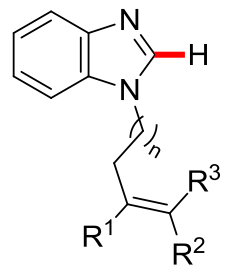

144

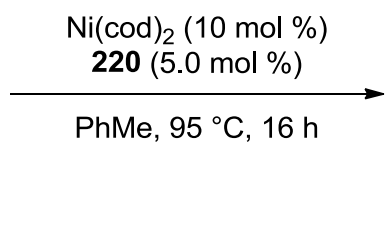

$\mathrm{Ni}(\operatorname{cod})_{2}(10 \mathrm{~mol} \%)$

PhMe, $95^{\circ} \mathrm{C}, 16 \mathrm{~h}$<smiles>[Y6]C[C@]([R])(c1nc2ccccc2n1[Y4])C([R])[R]</smiles>

145

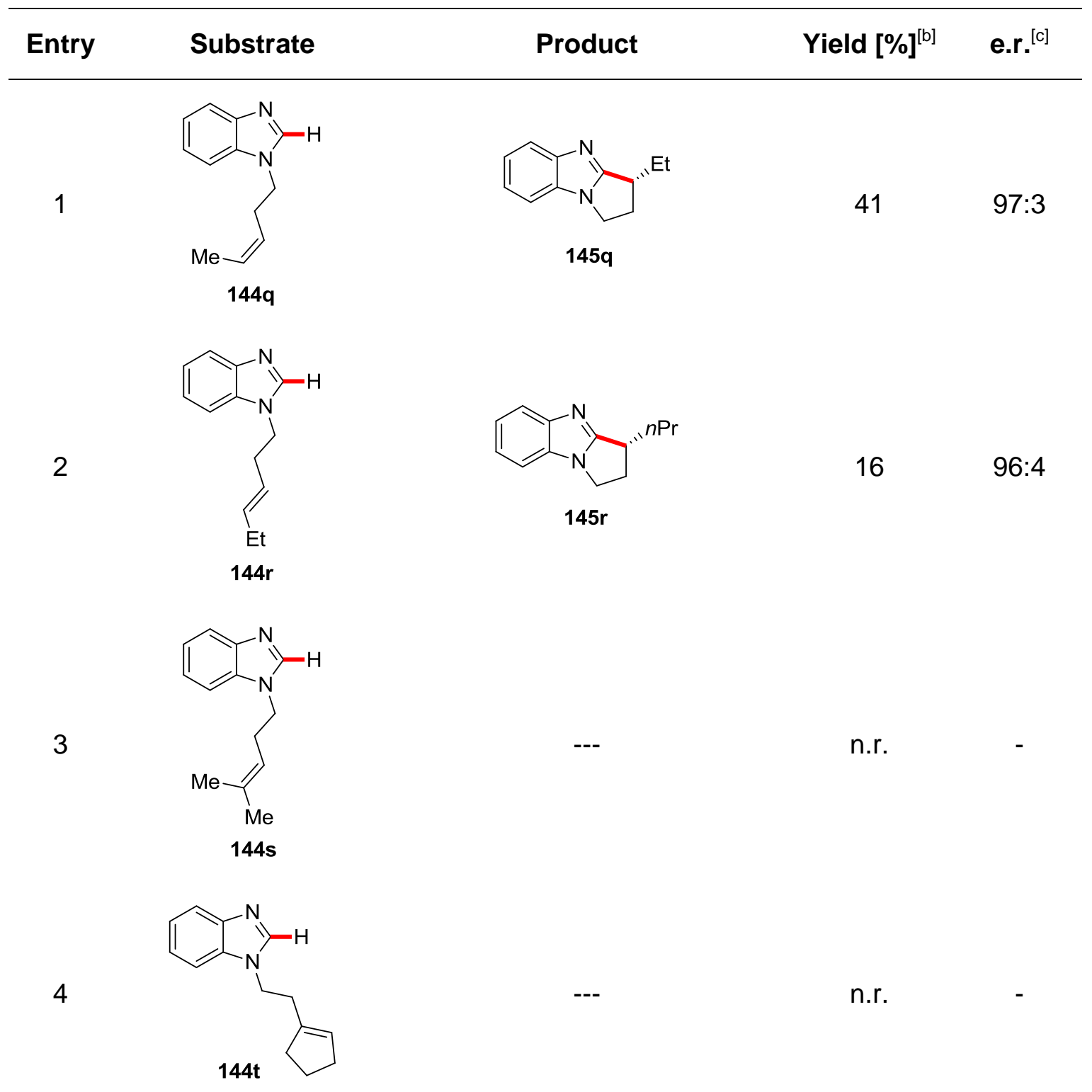


5<smiles>C=C(CCn1cnc2ccccc21)C(C)(C)c1ccccc1</smiles>

6<smiles>C=C(C)Cn1c(C)nc2ccccc21</smiles>

$7^{[\mathrm{d}]}$<smiles>C=C(C)CCCn1c(C)nc2ccccc21</smiles>

8<smiles>C=CCCn1cc2ccccc2n1</smiles>

9<smiles>C=CCCn1c(O[13CH3])nc2ccccc21</smiles>

$[D]_{1}-144 x$

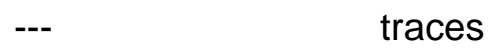

n.r.

n.r.

74

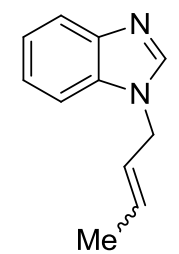

$223(E / Z=4: 1)$

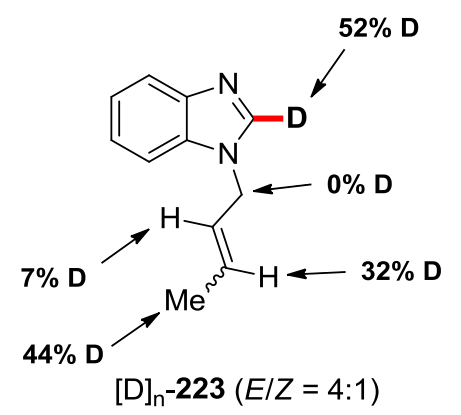

76

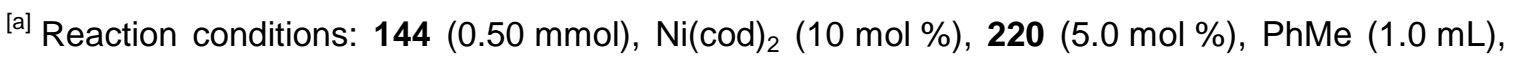
$95^{\circ} \mathrm{C}, 16$ h. ${ }^{[b]}$ Yield of the isolated product. ${ }^{[c]}$ Determined by chiral HPLC analysis. ${ }^{[\mathrm{d}]}$ Experiment conducted by V. Müller. 


\subsubsection{Mechanistic Studies}

\subsubsection{Deuterium Labeling Experiments and Kinetic Studies}

Given the unique features of the asymmetric aluminium-free nickel-catalyzed $\mathrm{C}-\mathrm{H}$ alkylation, we became attracted to unravel its mode of action. To this end, experiments with deuterated compounds and detailed kinetic studies were conducted by $V$. Müller. ${ }^{[220]}$ First, a reaction performed with deuterated substrate $[D]_{1}-144 a$ revealed $H / D$ scrambling at the methyl group and positions of the former olefin (Scheme 3.20a). This observation can be rationalized with a facile and reversible $\mathrm{C}-\mathrm{H}$ cleavage step, and is strikingly different from Ye's nickel-catalyzed exo-selective cyclization in which no scrambling was observed. ${ }^{[206]}$ Then, $\mathrm{C}-\mathrm{H}$ activation performed with isotopically labeled compound $[D]_{1}-144 a$ showed a kinetic isotope effect (KIE) of $k_{H} / k_{\mathrm{D}} \approx 1.1$ (Scheme $3.20 \mathrm{~b}$ ), suggesting the $\mathrm{C}-\mathrm{H}$ scission step not to be turnover limiting. ${ }^{[257]}$

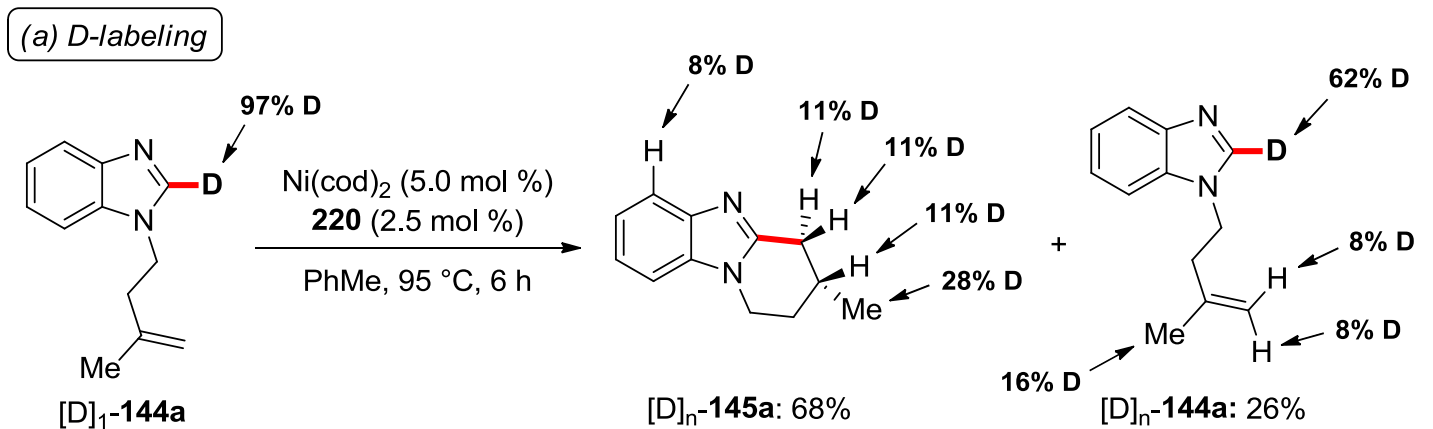

(b) Kinetic isotope effect

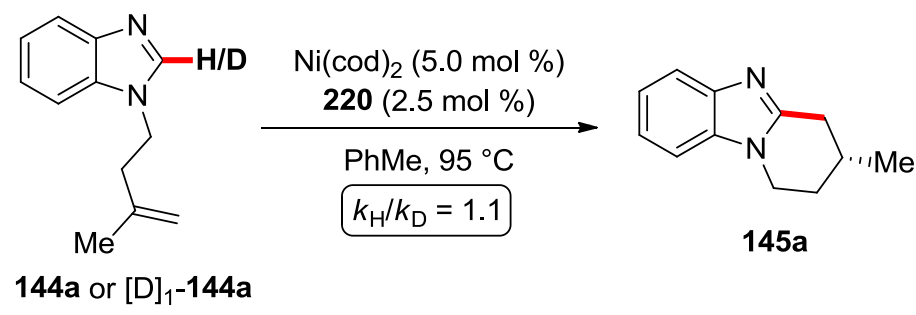

Scheme 3.20. Deuterium labeling experiments. Experiments conducted by V. Müller. 
Furthermore, detailed kinetic studies conducted by $V$. Müller revealed a first-order rate dependence in the substrate 144, the pre-ligand 220 and the nickel precursor, in the latter case with an inhibition at higher nickel concentrations (Scheme 3.21a-c). A possible interpretation to this rather unusual finding could be the existence of a critical nickel concentration beyond which an autocatalytic deactivation of the catalyst occurs, possibly via aggregation of nickel, as was previously proposed for palladium catalysis. ${ }^{[302]}$ Another explanation to the detrimental effect of higher concentrations of $\mathrm{Ni}(\mathrm{cod})_{2}$ could be the competitive coordination of free cod to the nickel center, resulting in off-cycle intermediates decelerating the catalysis, as previously reported by Zimmerman/Montgomery. ${ }^{[161,202 b]}$ This hypothesis is further substantiated by the observation that, in the presence of additional free cod, the transformation was found to indeed proceed at a lower rate. This finding provides additional support to the hypothesis that inhibition of the active nickel catalyst is caused by free cod originating from the consumption/degradation of the added $\mathrm{Ni}(\mathrm{cod})_{2}\left(\right.$ Scheme 3.21d). ${ }^{[220]}$ 

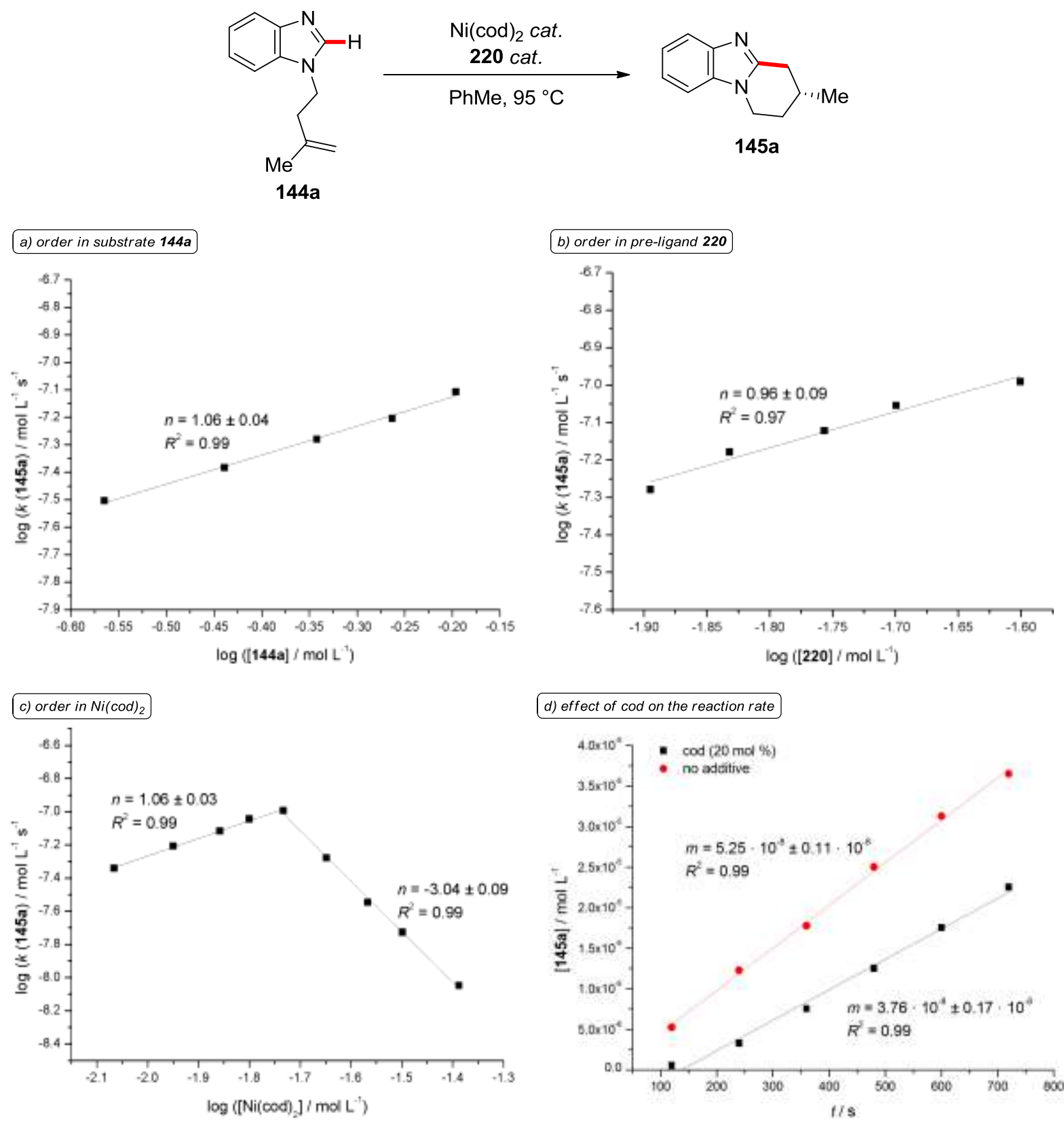

Scheme 3.21. Kinetic analysis nickel-catalyzed hydroarylation. Experiments conducted by V. Müller.

\subsubsection{Non-Linear Effect Studies}

In order to get some insights into the unusual ligand-to-metal ratio of 1:2, the effect of the enantiomeric excess of JoSPOphos pre-ligand 220 over the enantiomeric induction of the transformation was investigated (Scheme 3.22). Here, the absence 
of a non-linear effect (NLE) apparently excludes a multi-ligand containing catalyst or catalytically competent oligomers to be operative in the asymmetric nickel-catalyzed intramolecular hydroarylation. ${ }^{\text {[259] }}$
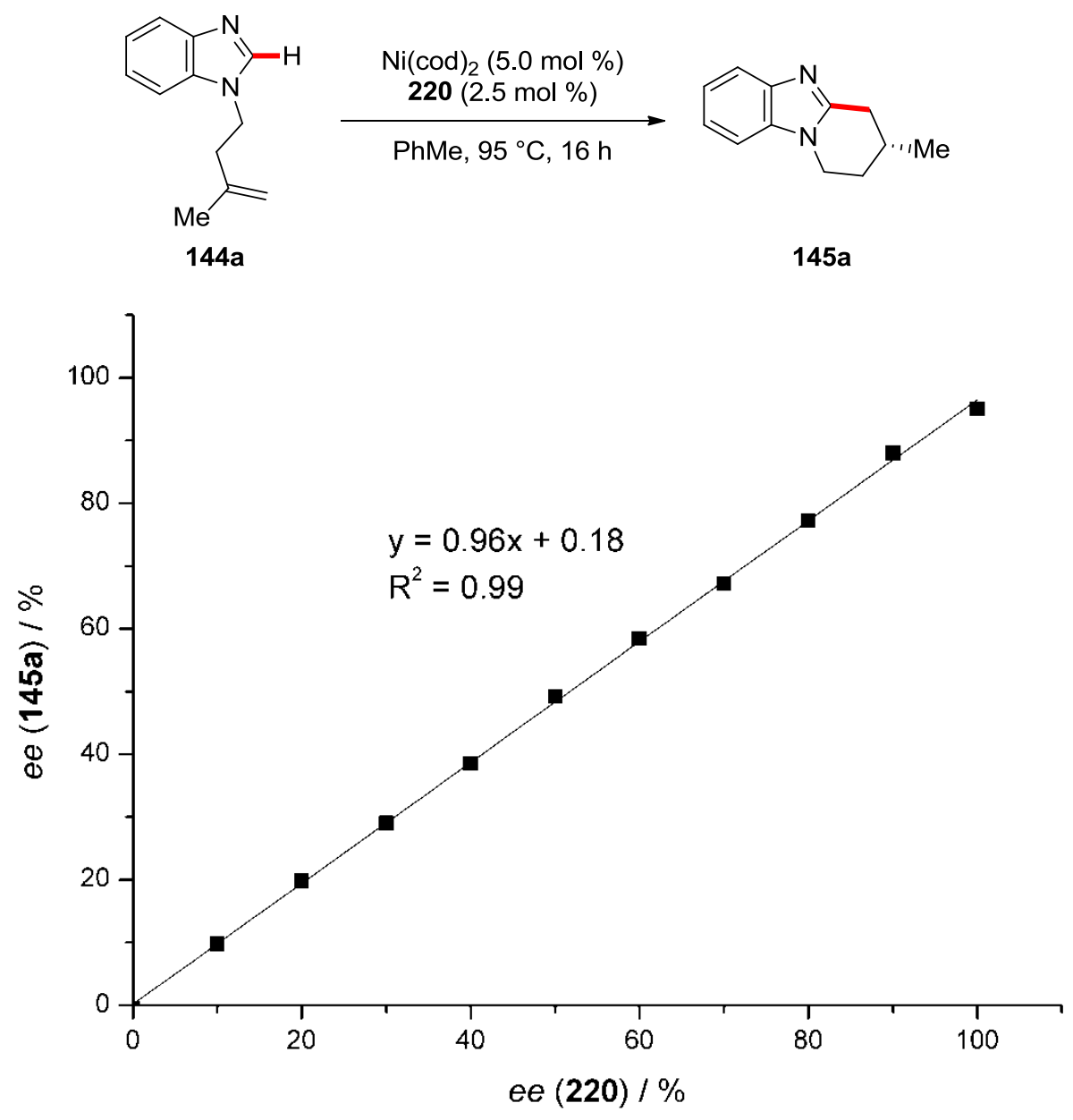

Scheme 3.22. Non-linear effect studies.

\subsubsection{Proposed Mechanism}

Based on our detailed mechanistic studies and previous literature reports, ${ }^{[81,190,202 a, 303]}$ the catalytic reaction is proposed to be initiated by the formation of the organometallic nickel(II) complex 224 (Scheme 3.23). Complex 224 was prepared by $\operatorname{Dr}$. $D$. Ghorai from a stoichiometric reaction of $\mathrm{Ni}(\mathrm{cod})_{2}$ with pre-ligand 
220, and found to be active in both stoichiometric and catalytic reactions with 144a. ${ }^{[220]}$ A plausible pathway for the generation of complex 224 could be the oxidative addition of nickel $(0)$ into the $\mathrm{P}(\mathrm{O})-\mathrm{H}$ bond, as it has been previously reported in the literature, ${ }^{[304]}$ followed by hydride migration to the bound cod and chain walking. ${ }^{[161]}$ It should also be noted that this coordination mode of bidentate ligands containing a phosphine oxide moiety has previously been reported for iridium(I) complexes. ${ }^{[305]}$ Interestingly, Pfaltz and coworkers described the ligand as a $P$-coordinating, $O$-anionic phosphinite rather than a $P$-anionic deprotonated phosphine oxide. ${ }^{[305]}$

Complex 224 is then coordinated by substrate 144 to form intermediate 225, which after loss of a cyclooctene molecule yields intermediate 226, the proposed active catalyst. Intermediate 226 then undergoes the stereo-determining and $\mathrm{C}-\mathrm{C}$ bond forming migratory insertion to deliver the cyclized intermediate 227. After a kinetically relevant coordination of a second substrate 144, explaining its first-order kinetic dependence, the key $\mathrm{C}-\mathrm{H}$ activation event occurs. In view of our mechanistic studies, we propose the facile $\mathrm{C}-\mathrm{H}$ cleavage to occur via a ligand-to-ligand hydrogen transfer (LLHT) manifold. ${ }^{[81,156 a, 202 c, 299 b, 306]}$ The observed H/D scrambling and olefin isomerization are believed to be caused by side-reactions of the catalyst, presumably involving a nickel- $\pi$-allyl or a nickel-hydride species which may possibly result from oxidative addition into the $\mathrm{P}(\mathrm{O})-\mathrm{H}$ bond during the catalyst generation process.

According to the proposed catalytic cycle, complex 224 is a plausible off-cycle intermediate, or a resting state, whose reversible formation is favored by higher concentrations of cod, explaining the negative order in $\mathrm{Ni}(\operatorname{cod})_{2}$ above a certain concentration (Scheme 3.21c) and rationalizing the detrimental effect of adding an excess of free cod to the catalytic reaction (Schemes 3.21d). Indeed, such codincorporating $\pi$-allyl complexes are documented to be stable off-cycle intermediates whose formation diminishes the catalytic efficiency. ${ }^{[161,202 b]}$ 


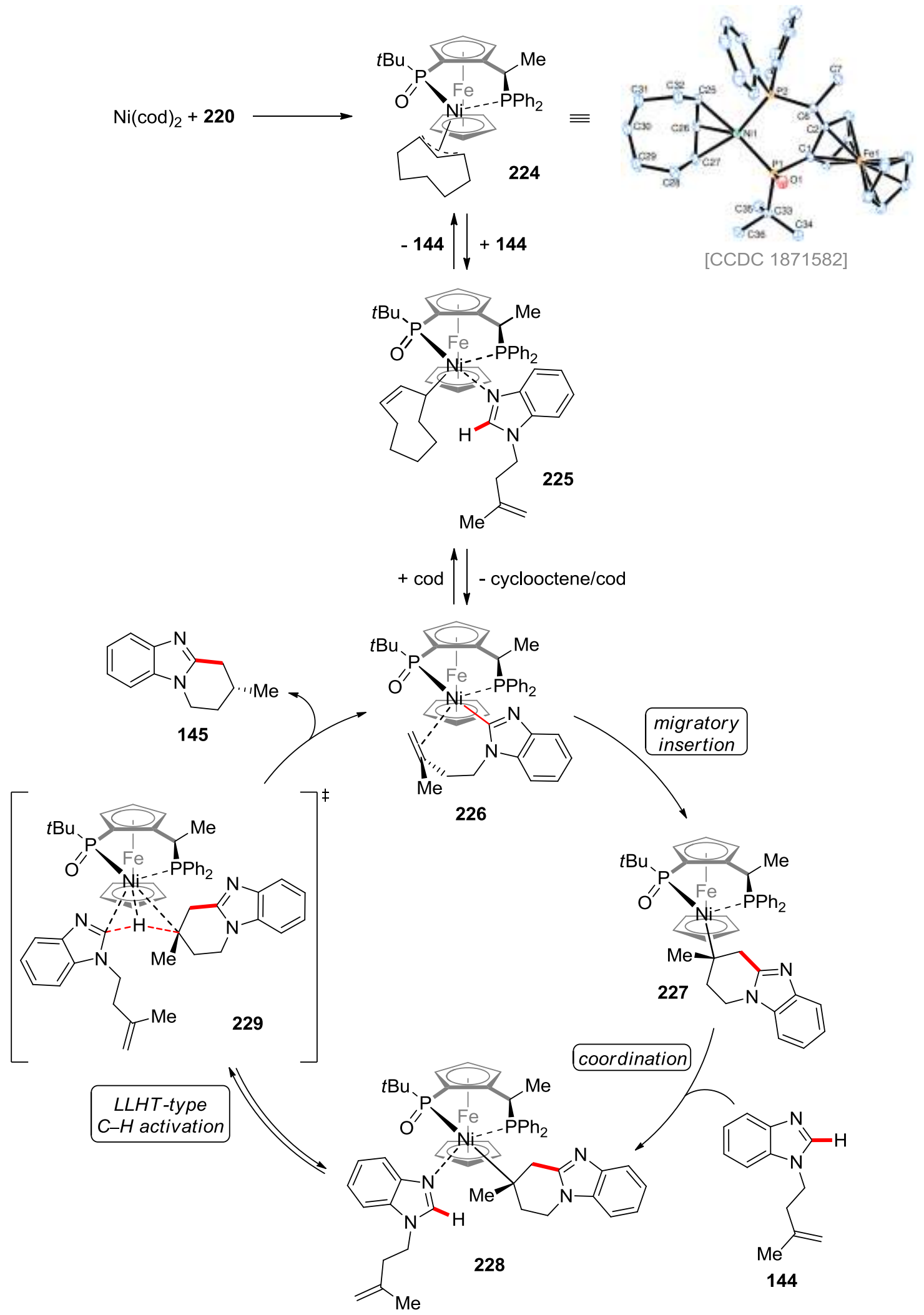

Scheme 3.23. Proposed catalytic cycle. Complex 224 was prepared and crystallized by $D r$. D. Ghorai. The crystal structure was measured and solved by Dr. C. Golz. 


\subsubsection{Product Diversification}

The synthetic value of the nickel-catalyzed $\mathrm{C}-\mathrm{H}$ alkylation was further demonstrated by late-stage diversification of the thus-obtained products (Scheme 3.24). In an attempt to introduce a heavy atom in order to determine the absolute configuration via X-ray diffraction crystallography, benzimidazolium iodide $\mathbf{2 3 0}$ was prepared by treating the standard product 145 a with methyl iodide. The $R$-configuration of the cyclized product was confirmed by X-ray diffraction analysis.
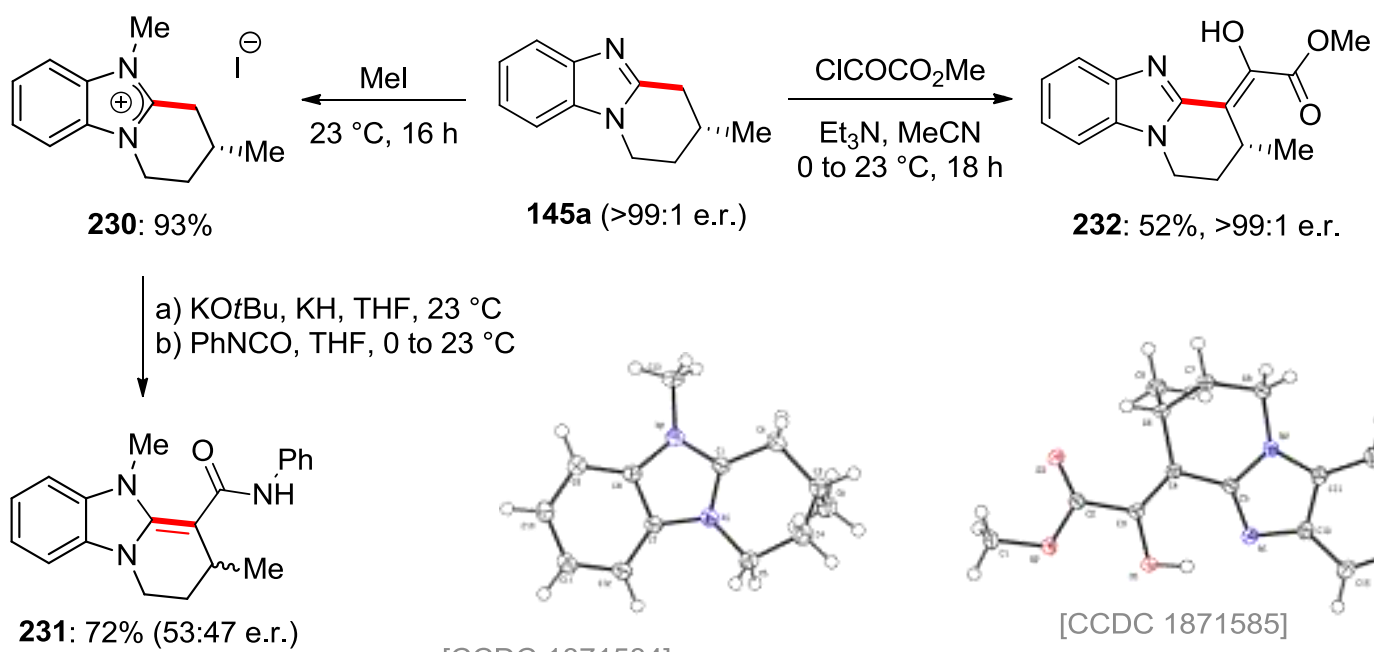

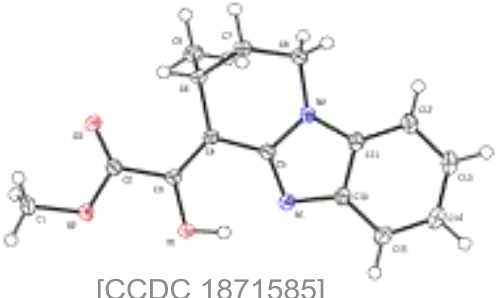

[CCDC 1871585]

rac-232

230

Scheme 3.24. Diversification of $145 a$ and molecular structure of products 230 and 232 with thermal ellipsoids at $50 \%$ probability level. The crystal structures were measured and solved by Dr. C. Golz.

Thereafter, further transformations of the activated 2-substituent of the benzimidazolium moiety were attempted. The formation of reactive $\mathrm{N}$-heterocyclic olefins $(\mathrm{NHO})$ upon treatment of 2-alkylimidazolium with a base is indeed well documented. ${ }^{[307]}$ The resulting electron-rich $\mathrm{NHO}$ intermediate was successfully trapped using phenyl isocyanate as the electrophile to provide amide $\mathbf{2 3 1}$ in good yield. Unfortunately, the product was obtained as a racemic mixture, presumably due to base-induced racemization of the activated allylic position during the process. 
Therefore, we aimed to functionalize the same position under milder conditions. To our delight, the cyclized product $145 \mathrm{a}$ could be directly functionalized under racemization-free conditions using a procedure reported by $R o u s h^{[308]}$ to provide $\alpha$-keto-ester 232, whose structure was unambiguously confirmed by X-ray diffraction analysis. Interestingly, such polycyclic $\alpha$-keto esters are regarded as valuable synthetic intermediates for the synthesis of antiviral agents. ${ }^{[308]}$

Overall, the synthetic utility of the developed enantioselective nickel-catalyzed hydroarylation was further showcased by late-stage diversification of the obtained products, as pharmaceutically relevant motifs could be obtained under racemizationfree conditions. 


\section{Summary and Outlook}

Organic synthesis has long relied on time- and energy-consuming prefunctionalization strategies which generate stoichiometric amounts of waste. Recently, the direct functionalization of $\mathrm{C}-\mathrm{H}$ bonds has emerged as an environmentally benign alternative that avoids lengthy syntheses, and has attracted substantial interest from both academia and the chemical industries. However, the selective functionalization of omnipresent $\mathrm{C}-\mathrm{H}$ bonds remains challenging and hence a research topic of high interest. While major progress was initially achieved with noble transition metal catalysts, the use of inexpensive and earth-abundant $3 d$ metals has gained significant momentum within the last decade.

In the first project, the first cobalt-catalyzed $\mathrm{C}-\mathrm{H}$ functionalization by the assistance of synthetically useful oxazolines was developed (Scheme 4.1). ${ }^{[3 \mathrm{e}, 227 \mathrm{a}]}$ Previous work on catalytic $\mathrm{C}-\mathrm{H}$ aminations had relied on noble transition metal catalysts or harsh reaction conditions. ${ }^{[23 e, 217]}$ The versatile, robust and user-friendly $\mathrm{Cp}^{*} \mathrm{Co}$ (III) catalyst allowed for the direct $\mathrm{C}-\mathrm{H}$ amidation of arenes and indoles using dioxazolones $\mathbf{4 0}$ as amidating reagents under the assistance of, inter alia, oxazolinyl, pyridyl and pyrimidyl directing groups. Mechanistic studies provided strong support for a kinetically relevant $\mathrm{C}-\mathrm{H}$ cobaltation via an acetate-enabled $\mathrm{BIES}$ manifold, and revealed the transformation to be homogenous in nature. Radical intermediates were excluded by reactions conducted in the presence of radical scavengers. 


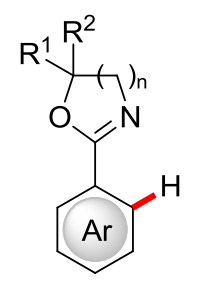

142

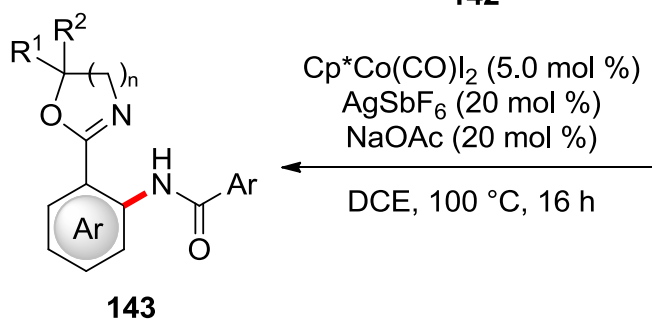

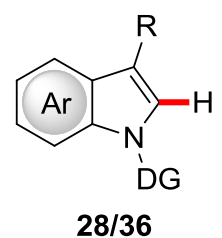
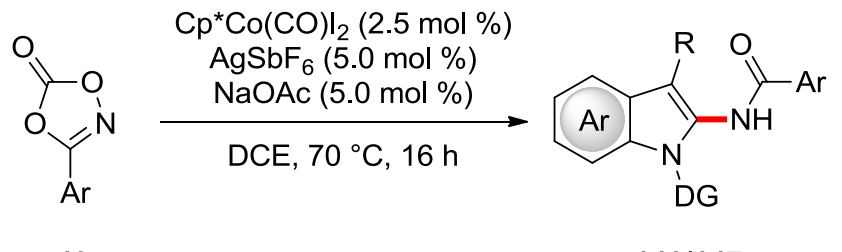

40

$146 / 147$

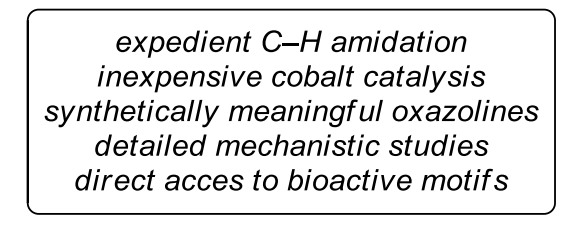

Scheme 4.1. Cobalt(III)-catalyzed C-H amidation.

While significant progress in the field of first-row transition metal-catalyzed $\mathrm{C}-\mathrm{H}$ activation was achieved within the last decade, ${ }^{[45]}$ enantioselective transformations continue to heavily rely on noble $4 d$ and $5 d$ metals. ${ }^{[51]}$ However, the last few years have witnessed the emergence of $3 \mathrm{~d}$-metal catalyzed asymmetric $\mathrm{C}-\mathrm{H}$ activations, but examples remain scarce as this research area is still in its infancy. ${ }^{[52]}$ In this context, the first enantioselective iron-catalyzed $\mathrm{C}-\mathrm{H}$ functionalization by innersphere $\mathrm{C}-\mathrm{H}$ activation was developed (Scheme 4.2).$^{[151]}$ In the course of extensive optimization studies, the design of the novel meta-substituted NHC precursor 180 was found to be critical to success. A broad range of indoles and azaindoles 59 proved viable substrates in the hydroarylation of diversely substituted styrenes 60 and vinylmetallocenes 191, providing the products 62/192 in up to $96: 4$ e.r. and complete branched-selectivity. Remarkably, this work constitutes the first and, so far, only highly enantioselective transformation via inner-sphere iron-catalyzed $\mathrm{C}-\mathrm{H}$ activation. Moreover, this work also represents the unprecedented use of a chiral $\mathrm{NHC}$ ligand in enantioselective iron catalysis. 


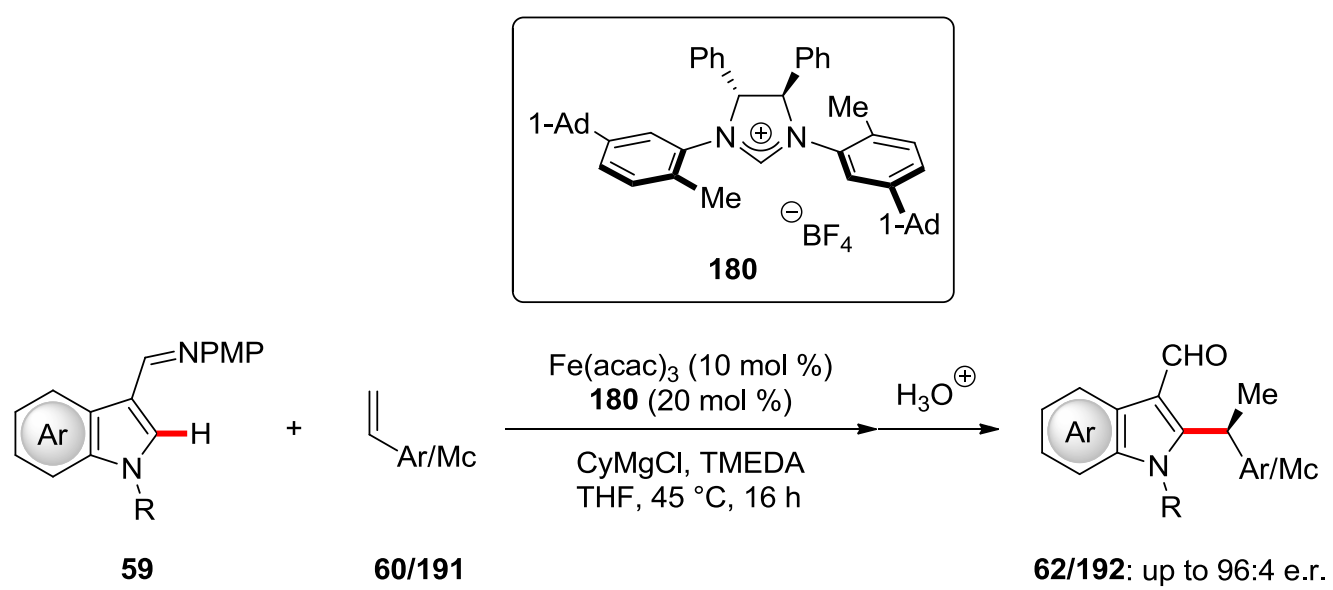

Scheme 4.2. Enantioselective iron-catalyzed C-H alkylation.

Detailed mechanistic studies were conducted to delineate the mechanism of the transformation. Our findings support a facile $\mathrm{C}-\mathrm{H}$ cleavage occurring through a ligand-to-ligand hydrogen transfer manifold, and revealed the reaction to be homogenous in nature. Furthermore, kinetic studies unravelled a first-order dependence in the indole substrate 59 and a rather uncommon zeroth order in the alkene 60 . Additionally, an in situ analysis of the transformation by electrosprayionization mass spectrometry and ${ }^{57} \mathrm{Fe}$ Mössbauer spectroscopy was performed and supports an organometallic mono-NHC-ligated iron(II) species to be involved in the catalysis. ${ }^{[218]}$ Remarkably, while "low-valent" iron species have initially been proposed to be active in iron-catalyzed $\mathrm{C}-\mathrm{H}$ activations in the presence of Grignard reagents, this work provides the first direct evidence of an iron(II) species in such processes.

Thereafter, we then became interested in the development of new enantioselective transformations employing our novel chiral NHC pre-ligands. Among the attempted reactions, asymmetric nickel-catalyzed hydroarylations appeared most promising. Significant enantioselectivities were observed in preliminary studies on intermolecular undirected $\mathrm{C}-\mathrm{H}$ alkylations of benzimidazoles 99 with styrenes 60 (Scheme 4.3). While the observed enantioselectivities are only moderate, this transformation represents the first example of enantioselective undirected intermolecular nickel-catalyzed $\mathrm{C}-\mathrm{H}$ activations. 


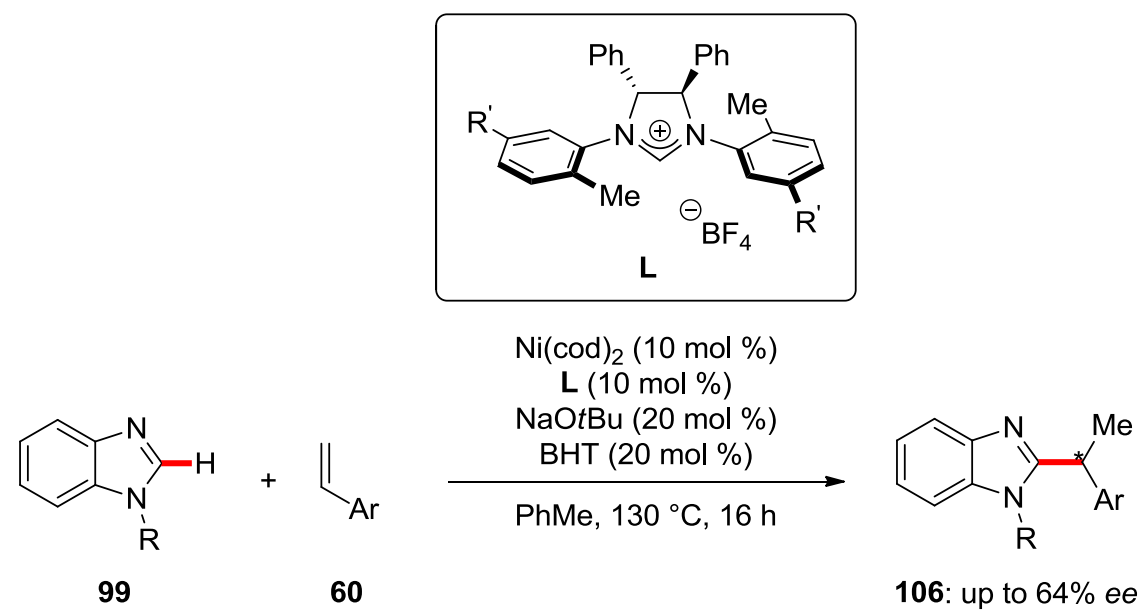

Scheme 4.3. Enantioselective nickel-catalyzed intermolecular hydroarylations.

Finally, drawing inspiration from those preliminary results, we devised the first asymmetric endo-selective cyclization of azoles with alkenes (Scheme 4.4). ${ }^{[220]}$ Remarkably, in sharp contrast to other nickel-catalyzed intramolecular hydroarylations with unactivated alkenes, ${ }^{[193,195,200,203,206]}$ the developed methodology obviates the need for pyrophoric organoaluminium reagents. ${ }^{[309]}$ Various substituted benzimidazoles 144, including electron-rich, electron-poor and polychlorinated derivatives performed well in the transformation. Moreover, the reaction was not limited to benzimidazoles since a broad variety of bioactive heterocyclic motifs, including highly functionalized purines and theophylline derivatives, were smoothly converted to the cyclized products $\mathbf{1 4 5}$ under the optimized reaction conditions. Furthermore, the transformation was found to be highly chemoselective, as for substrates bearing several olefinic motifs solely reacted at the proximal alkene, with the distal olefins remaining untouched. The detailed mechanistic studies provide support to the formation of an organometallic nickel(II) species as the active catalyst and a facile $\mathrm{C}-\mathrm{H}$ cleavage step occurring through a LLHT manifold. Finally, the absence of a non-linear effect is indicative of a mono-ligated nickel catalyst to be operative in the asymmetric hydroarylation. 


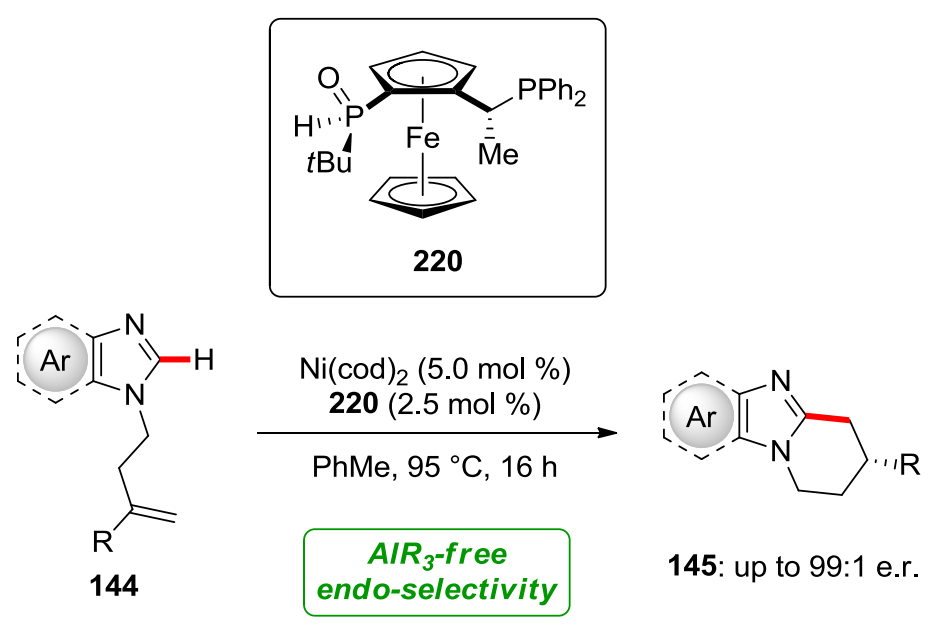

Scheme 4.4. Enantioselective nickel-catalyzed intramolecular hydroarylations.

Given the topical interest for $3 \mathrm{~d}$ metal-catalyzed $\mathrm{C}-\mathrm{H}$ activation, further exciting developments in this rapidly-evolving research area are anticipated in the near future. 


\section{Experimental Part}

\subsection{General Remarks}

All reactions involving air- and/or moisture-sensitive compounds were conducted under a dry nitrogen atmosphere using pre-dried glassware and standard Schlenk techniques. If not otherwise noted, yields refer to isolated compounds which were estimated to be $>95 \%$ pure based on ${ }^{1} \mathrm{H}$ NMR and/or GC analysis.

\section{Vacuum}

The following average pressure was measured on the used rotary vane pump RD4 from Vacuubrand ${ }^{\circledR}$ : 0.8·10 ${ }^{-1}$ mbar (uncorrected value).

\section{Melting Points}

Melting points were measured on a Stuart ${ }^{\circledR}$ Melting Point Apparatus SMP3 from Barloworld Scientific. Values are uncorrected.

\section{Chromatography}

Analytical thin layer chromatography (TLC) was performed on silica gel 60 F254 aluminium sheets from Merck. Plates were either visualized under irradiation at $254 \mathrm{~nm}$ or $365 \mathrm{~nm}$ or developed by treatment with a potassium permanganate solution followed by careful warming. Chromatographic purifications were accomplished by column chromatography on Merck Geduran ${ }^{\circledR}$ silica gel, grade 60 (40-63 $\mu \mathrm{m}, 70-230$ mesh ASTM). 


\section{Infrared Spectroscopy}

IR spectra were recorded using a Bruker ${ }^{\circledR}$ Alpha-P ATR spectrometer. Liquid samples were measured as film and solid samples neat. Spectra were recorded in the range from 4000 to $400 \mathrm{~cm}^{-1}$. Analysis of the spectral data was carried out using Opus 6. Absorption is given in wave numbers $\left(\mathrm{cm}^{-1}\right)$.

\section{Nuclear Magnetic Resonance Spectroscopy}

NMR spectra were recorded on Mercury Plus 300, VNMRS 300, Inova 500 and 600 from Varian ${ }^{\circledR}$, or Avance 300, Avance III 300 and 400, Avance III HD 400 and 500 from Bruker $^{\circledR}$. Chemical shifts are reported in $\delta$-values in ppm relative to the residual proton peak or carbon peak of the deuterated solvent.

\begin{tabular}{l|cc} 
& ${ }^{1} \mathbf{H}$ NMR & ${ }^{13} \mathbf{C}$ NMR \\
\hline $\mathrm{CDCl}_{3}$ & 7.26 & 77.16 \\
DMSO- $_{6}$ & 2.50 & 39.52 \\
Benzene- $_{6}$ & 7.16 & 128.06 \\
MeOH-d $_{4}$ & 3.31 & 49.00
\end{tabular}

The coupling constants $J$ are reported in hertz $(\mathrm{Hz})$. Analysis of the recorded spectra was carried out using MestReNova 10.0 software.

\section{Gas Chromatography}

Monitoring of reaction process via gas chromatography or coupled gas chromatography-mass spectrometry was performed using a 7890 GC-system with/without mass detector 5975C (Triple-Axis-Detector) or a 7890B GC-system coupled with a 5977A mass detector, both from Agilent Technologies ${ }^{\circledR}$. 


\section{Mass Spectrometry}

Electron ionization (EI) and El high resolution mass spectra (HR-MS) were measured on a time-of-flight mass spectrometer AccuTOF from JEOL. Electrospray ionization (ESI) mass spectra were recorded on an lo-Trap mass spectrometer LCQ from Finnigan, a quadrupole time-of-flight maXis from Bruker Daltonic or on a timeof-flight mass spectrometer microTOF from Bruker Daltonic. ESI-HR-MS spectra were recorded on a Bruker Apex IV or Bruker Daltonic 7T, Fourier transform ion cyclotron resonance (FTICR) mass spectrometer. The ratios of mass to charge $(\mathrm{m} / \mathrm{z})$ are indicated, intensities relative to the base peak $(I=100)$ are written in parentheses.

\section{Chiral HPLC}

Chiral HPLC chromatograms were recorded on an Agilent 1290 Infinity using CHIRALPAK $^{\circledR}$ IA-3, IB-3, IC-3, ID-3, IE-3 and IF-3 columns (3.0 $\mu$ m particle size; ø: $4.6 \mathrm{~mm}$ and $250 \mathrm{~mm}$ length) at ambient temperature.

\section{Specific Rotations}

Optical rotations were measured on a Jasco P-2000 or an Anton Paar MCP 150 polarimeter using a $10 \mathrm{~cm}$ cell with a $\mathrm{Na} 589 \mathrm{~nm}$ filter. Concentrations are indicated in $\mathrm{g} / 100 \mathrm{~mL}$.

\section{Mössbauer Spectroscopy}

Mössbauer spectra were recorded with a ${ }^{57}$ Co source in a Rh matrix using an alternating constant acceleration Wissel Mössbauer spectrometer operated in the transmission mode and equipped with a Janis closed-cycle helium cryostat. Isomer shifts are given relative to iron metal at ambient temperature. Simulation of the 
experimental data was performed with the Mfit program using Lorentzian line doublets. ${ }^{[310]}$

\section{Solvents}

All solvents for reactions involving air- and/or moisture-sensitive reagents were dried, distilled and stored under an inert atmosphere (dry nitrogen) according to the following standard procedures.

1,2-Dichloroethane (DCE), N-methyl-2-pyrrolidone (NMP) and 1,3-dimethyl-3,4,5,6tetrahydro-2 $\left(1 \mathrm{H}\right.$ )-pyrimidinone (DMPU) were dried over $\mathrm{CaH}_{2}$ for $8 \mathrm{~h}$, degassed and distilled under reduced pressure. 1,4-Dioxane, di-n-butylether $\left(n \mathrm{Bu}_{2} \mathrm{O}\right)$ and tert-amyl alcohol $(\mathrm{AmOH})$ were dried over $\mathrm{Na}$ for $8 \mathrm{~h}$, degassed and distilled under reduced pressure.

$\mathrm{CH}_{2} \mathrm{Cl}_{2}$, DMF, THF, $\mathrm{Et}_{2} \mathrm{O}$ and PhMe were obtained from a MBRAUN MB SPS-800 solvent purification system.

\section{Chemicals}

Chemicals obtained from commercial sources with a purity $>95 \%$ were used as received without further purification. $\mathrm{CyMgCl}$ was prepared from chlorocyclohexane and magnesium turnings in anhydrous THF under nitrogen atmosphere, and titrated before use with $\mathrm{I}_{2} / \mathrm{LiCl}^{\left[{ }^{[111]}\right.}$ Pre-ligands 220 (commercial name: SL-J681-1), ent-220 (SL-J681-2), and 168 (SL-J688-1) were obtained from Solvias AG.

The following compounds were known from the literature and synthesized according to previously known methods:

Dioxazolone 40a, ${ }^{[229 b]} 2$-aryl oxazines 142, ${ }^{[312]}$ (pyridin-2-yl)-1H-indoles 28 and (pyrimidin-2-yl)-1 $H$-indoles 36, ${ }^{[313]} \mathrm{Cp}^{*} \mathrm{Co}(\mathrm{CO}) \mathrm{I}_{2},{ }^{[86]} \mathrm{RuTPP}(\mathrm{CO}),{ }^{\left[{ }^{[14]}\right]}$ indoles 59c, 59b and $[D]_{1}-59 b,{ }^{[105]}$ indole $59 a,{ }^{[106]}$ chiral NHC precursors 153 and $154,{ }^{[211]} 158,{ }^{[315]}$ 
$159,{ }^{[316]} 160^{[241]}$ and $179,{ }^{[243]}$ phosphoramidites $161^{[106]}$ and $212,{ }^{[317]}$ SchmalzPhos $163,{ }^{[318]}$ HASPO 169 and phosphine chloride $170,{ }^{[195]}$ iron complex $173,{ }^{[319]}$ diamine $175,{ }^{[320]}$ styrenes $60 \mathrm{f}, 60 \mathrm{~h}, 60 \mathrm{i}$ and $60 \mathrm{j},{ }^{[321]}(Z)$-trimethyl(styryl)silane $60 \mathrm{n},{ }^{[322]}$ vinylthiophene $60 \mathrm{p},{ }^{[323]}$ vinylferrocene $191 \mathrm{a},{ }^{[324]}$ vinylruthenocene $191 \mathrm{~b},{ }^{[325]} 1$-vinyl$1^{\prime}, 2^{\prime}, 3^{\prime}, 4^{\prime}, 5^{\prime}$-pentamethylferrocene $191 c^{[326]}$ benzimidazole $144 a^{[219 b]}$ benzimidazoles 144r, 144s, 144x, and $[D]_{1}-144 x,{ }^{[206]}$ and TADDOL-SPOs 131, 216 and 217. ${ }^{[206,207]}$

The following compounds were kindly synthesized and provided by the persons listed below:

Karsten Rauch: IMes. HCl, IPr.HCl, (HA)SPOs 164-167, 214 and 215, $\left[\mathrm{RuCl}_{2}(p \text {-cymene) }]_{2},\left[\mathrm{Cp}^{*} \mathrm{RhCl}_{2}\right]_{2}\right.$, dry and/or degassed solvents (DCE, tAmOH, 1,4-dioxane, NMP, $\left.\mathrm{H}_{2} \mathrm{O}\right)$.

Dr. Ruhuai Mei: oxazolines 142j, 142p and 142r, indole 36a, [Cp $\left.{ }^{\star} \mathrm{Co}(\mathrm{CO}) \mathrm{I}_{2}\right]$.

Sven C. Richter: IXy|· HCl.

Dr. Sebastian Lackner: PyBOX ligand 174.

Dr. Marc Moselage: CAAC precursor 157, dry DMPU.

Dr. Gianpiero Cera: ligand 172.

Dr. Tobias Parchomyk (Koszinowski research group): ${ }^{57} \mathrm{FeCl}_{2}$.

Dr. Nicolas Sauermann: $\left[\mathrm{Cp}^{*} \mathrm{Col}_{2}\right]_{2}$, dry DMPU.

Dr. Thomas Müller: N-Bn-benzimidazole 99b.

Dr. Hui Wang: $\left[\mathrm{Cp}^{*} \mathrm{Co}(\mathrm{MeCN})_{3}\right]\left(\mathrm{SbF}_{6}\right)_{2}(26)$ and $\mathrm{NaO}_{2} \mathrm{C}$-lle-Ac.

Dr. Alexander Bechtoldt: dienes 112a and 112b.

Prof. Dr. E. Peter Kündig and coworkers (Université de Genève): chiral NHC precursors 159' and 193. 
Prof. Dr. Hintermann and coworkers (Technische Universität München): SPO 222.

Prof. Dr. Albrecht Berkessel and coworkers (Universität zu Köln): NHC precursor 156. 


\subsection{General Procedures}

General Procedure for the Cobalt-Catalyzed $\mathrm{C}-\mathrm{H}$ Amidation of 2-Aryloxazolines 142 (GP1)

2-Aryloxazoline 142 ( $0.50 \mathrm{mmol}, 1.0$ equiv), dioxazolone 40 (0.60 mmol, 1.2 equiv), $\mathrm{Cp}^{*} \mathrm{Co}(\mathrm{CO}) \mathrm{I}_{2} \quad(11.9 \mathrm{mg}, \quad 0.025 \mathrm{mmol}, 5.0 \mathrm{~mol} \%), \mathrm{AgSbF}_{6} \quad(34 \mathrm{mg}, 0.10 \mathrm{mmol}$, $20 \mathrm{~mol} \%)$ and $\mathrm{NaOAc}(8.2 \mathrm{mg}, 0.10 \mathrm{mmol}, 20 \mathrm{~mol} \%)$ were placed into an ovendried $25 \mathrm{~mL}$ Schlenk tube equipped with a septum under $\mathrm{N}_{2}$ atmosphere. DCE $(2.0 \mathrm{~mL})$ was introduced via cannula. The reaction mixture was stirred at $100{ }^{\circ} \mathrm{C}$ for $16 \mathrm{~h}$. After cooling to ambient temperature, the reaction mixture was dry loaded onto silica gel and purified by flash column chromatography ( $n$-hexane/EtOAc) to afford the desired product 143.

General Procedure for the Cobalt-Catalyzed C-H Amidation of Indoles 28/36 (GP2)

Indole $28 / 36$ ( $0.50 \mathrm{mmol}, 1.0$ equiv), dioxazolone 40 (0.60 mmol, 1.2 equiv), $\mathrm{Cp}^{*} \mathrm{Co}(\mathrm{CO}) \mathrm{I}_{2}(5.9 \mathrm{mg}, 0.0125 \mathrm{mmol}, 2.5 \mathrm{~mol} \%), \mathrm{AgSbF}_{6}(8.6 \mathrm{mg}, 0.025 \mathrm{mmol}$, $5.0 \mathrm{~mol} \%)$ and $\mathrm{NaOAc}(2.1 \mathrm{mg}, 0.025 \mathrm{mmol}, 5.0 \mathrm{~mol} \%)$ were placed into an ovendried $25 \mathrm{~mL}$ Schlenk tube equipped with a septum under $\mathrm{N}_{2}$ atmosphere. DCE $(2.0 \mathrm{~mL})$ was introduced via cannula. The reaction mixture was stirred at $70{ }^{\circ} \mathrm{C}$ for $16 \mathrm{~h}$. After cooling to ambient temperature, the reaction mixture was dry loaded onto silica gel and purified by flash column chromatography ( $n$-hexane/EtOAc) to afford the desired product 146/147.

\section{General Procedure for the Synthesis of Indoles 59 (GP3)}

A solution of 3 -formylindole ( $3.44 \mathrm{mmol}, 1.0$ equiv) in anhydrous DMF ( $35 \mathrm{~mL}$ ) was cooled to $0{ }^{\circ} \mathrm{C}$. $\mathrm{NaH}$ (165 mg, $4.13 \mathrm{mmol}, 1.2$ equiv, $60 \%$ in mineral oil) was then added portionwise under ice-cooling. The resulting suspension was stirred at $0{ }^{\circ} \mathrm{C}$ 
for $15 \mathrm{~min}$, then allowed to warm up to ambient temperature and stirred for $1 \mathrm{~h}$. The reaction mixture was then cooled to $0^{\circ} \mathrm{C}$, and the corresponding alkyl halide (4.13 mmol, 1.2 equiv) was added slowly under ice cooling. The resulting suspension was stirred at $0^{\circ} \mathrm{C}$ for $15 \mathrm{~min}$, then stirred overnight at the indicated temperature $\left(23^{\circ} \mathrm{C}\right.$ for benzyl bromides, methoxymethyl chloride and 2-bromo-1,1difluoroethane, $60^{\circ} \mathrm{C}$ for other alkyl halides). The reaction mixture was cooled to $0{ }^{\circ} \mathrm{C}$, poured into sat. aqueous $\mathrm{NH}_{4} \mathrm{Cl}(100 \mathrm{~mL})$, and extracted with EtOAC $(3 \times 100 \mathrm{~mL})$. The combined organic phases were washed with $\mathrm{H}_{2} \mathrm{O}(3 \times 100 \mathrm{~mL})$ and brine $(50 \mathrm{~mL})$, dried over $\mathrm{Na}_{2} \mathrm{SO}_{4}$, and concentrated under reduced pressure. The residue was triturated with $n$-hexane to provide the product 204 , which was used in the next step without further purification.

The crude product 204 was suspended in PhMe $(15 \mathrm{~mL})$ at ambient temperature. p-Anisidine (467 mg, $3.79 \mathrm{mmol}, 1.1$ equiv) and activated $4 \AA \mathrm{MS}(5.0 \mathrm{~g})$ were then added in one portion. The resulting suspension was stirred at $60^{\circ} \mathrm{C}$ for $16 \mathrm{~h}$. The suspension was allowed to cool to ambient temperature and filtered. The filtrate was concentrated under reduced pressure. The residue was recrystallized from $n$-hexane $/ \mathrm{CH}_{2} \mathrm{Cl}_{2}$ /isopropanol. The crystals were collected by filtration, washed with cold pentane and dried under vacuum to provide the pure imine 59.

The analytical data of (aza)indoles 59 have been reported elsewhere ${ }^{[151]}$ and are not included in this thesis.

\section{General Procedure for the Synthesis of Chiral Imidazolinium Salts (GP4)}

Following a modified procedure, ${ }^{[211]} \mathrm{Pd}(\mathrm{OAc})_{2}$ (16.0 mg, $71 \mu \mathrm{mol}, 5.0 \mathrm{~mol} \%$ ), (+/-)-BINAP (88.0 mg, $0.14 \mathrm{mmol}, 10 \mathrm{~mol} \%$ ), and $\mathrm{NaOtBu}(410 \mathrm{mg}, 4.26 \mathrm{mmol}$, 3.0 equiv) were added under nitrogen atmosphere to anhydrous $\mathrm{PhMe}(25 \mathrm{~mL})$ and stirred for $30 \mathrm{~min}$ at ambient temperature. $(R, R)$-Diphenylethylenediamine (300 $\mathrm{mg}$, $1.42 \mathrm{mmol}, 1.0$ equiv) and the corresponding aryl bromide ( $2.98 \mathrm{mmol}, 2.1$ equiv) were then added and the solution was stirred at $100^{\circ} \mathrm{C}$ for $16 \mathrm{~h}$. The solution was cooled to ambient temperature, diluted with $n$-hexane $(75 \mathrm{~mL})$, and filtered through a 
plug of silica. The silica was washed with $n$-hexane $/ \mathrm{CH}_{2} \mathrm{Cl}_{2}=1 / 1$ to elute the product. The volatiles were removed in vacuo. The residue was purified by rapid flash chromatography ( $n$-hexane/ $\mathrm{CH}_{2} \mathrm{Cl}_{2}$ ) to provide the crude $N, N$ '-diarylated diamine. The crude $N, N^{\prime}$-diarylated diamine (1.0 equiv), ammonium tetrafluoroborate (1.2 equiv) were stirred in triethyl orthoformate at $120^{\circ} \mathrm{C}$ for $5 \mathrm{~h}$. The solution was then allowed to cool to ambient temperature. The reaction mixture was dry loaded onto silica gel and purified by column chromatography $\left(\mathrm{CH}_{2} \mathrm{Cl}_{2} /\right.$ acetone or $n$-hexane/acetone).

General Procedures for the Iron-catalyzed Enantioselective C-H Alkylation with Solid Alkenes (GP5)

In an oven-dried $25 \mathrm{~mL}$ Schlenk tube were placed the indole substrate 59 (0.25 mmol, 1.0 equiv), Fe(acac) $)_{3}(8.8 \mathrm{mg}, 0.025 \mathrm{mmol}, 10 \mathrm{~mol} \%), 180$ (38 mg, $0.050 \mathrm{mmol}, 20 \mathrm{~mol} \%)$ and vinylarene 60 or 191 (0.38 mmol, 1.5 equiv). The Schlenk tube was closed with a rubber septum, then evacuated and backfilled with nitrogen 3 times. THF $(0.50 \mathrm{~mL}$ ) and TMEDA (58 mg, $0.50 \mathrm{mmol}, 2.0$ equiv) were added via syringe. CyMgCl $(0.28 \mathrm{mmol}, 1.1$ equiv, typically $1 \mathrm{M})$ was then added dropwise at ambient temperature. The resulting mixture was stirred at $45^{\circ} \mathrm{C}$ for $16 \mathrm{~h}$. The reaction mixture was allowed to cool to ambient temperature and diluted with THF $(1.5 \mathrm{~mL}) . \mathrm{HCl}(3.0 \mathrm{M}, 2.0 \mathrm{~mL})$ was added in a single portion, and the resulting mixture was stirred at ambient temperature for $2 \mathrm{~h}$. The mixture was poured into sat. aqueous $\mathrm{NH}_{4} \mathrm{Cl}(10 \mathrm{~mL})$ and extracted with EtOAc $(3 \times 10 \mathrm{~mL})$. The combined organic layers were washed with brine $(10 \mathrm{~mL})$, dried over $\mathrm{Na}_{2} \mathrm{SO}_{4}$, and concentrated under reduced pressure. The residue was purified by silica gel chromatography ( $n$-hexane/EtOAc) to afford the desired product 62/192. 


\section{General Procedures for the Iron-catalyzed Enantioselective C-H Alkylation with Liquid Alkenes (GP6)}

In an oven-dried $25 \mathrm{~mL}$ Schlenk tube were placed the indole substrate 59 (0.25 mmol, 1.0 equiv), Fe(acac) $)_{3}(8.8 \mathrm{mg}, 0.025 \mathrm{mmol}, 10 \mathrm{~mol} \%$ ) and 180 (38 mg, $0.050 \mathrm{mmol}, 20 \mathrm{~mol} \%)$. The Schlenk tube was closed with a rubber septum, then evacuated and backfilled with nitrogen 3 times. THF $(0.50 \mathrm{~mL}$ ), TMEDA (58 mg, $0.50 \mathrm{mmol}, 2.0$ equiv) and vinylarene 60 (0.38 mmol, 1.5 equiv) were added via syringe. $\mathrm{CyMgCl}(0.28 \mathrm{mmol}, 1.1$ equiv, typically $1 \mathrm{M})$ was then added dropwise at ambient temperature. The resulting mixture was stirred at $45^{\circ} \mathrm{C}$ for $16 \mathrm{~h}$. The reaction mixture was allowed to cool to ambient temperature and diluted with THF $(1.5 \mathrm{~mL}) . \mathrm{HCl}(3.0 \mathrm{M}, 2.0 \mathrm{~mL})$ was added in a single portion, and the resulting mixture was stirred at ambient temperature for $2 \mathrm{~h}$. The mixture was poured into sat. aqueous $\mathrm{NH}_{4} \mathrm{Cl}(10 \mathrm{~mL})$ and extracted with EtOAc $(3 \times 10 \mathrm{~mL})$. The combined organic layers were washed with brine $(10 \mathrm{~mL})$, dried over $\mathrm{Na}_{2} \mathrm{SO}_{4}$, and concentrated under reduced pressure. The residue was purified by silica gel chromatography ( $n$-hexane/EtOAc) to afford the desired product $\mathbf{6 2}$.

\section{General Procedure for the Synthesis of Racemic Alkylated Indoles 62/192 (GP7)}

General procedures GP5 or GP6 were followed using 1,3-bis(2,6-dimethylphenyl)$1 \mathrm{H}$-imidazol-3-ium chloride (IXyl. $\mathrm{HCl})(7.8 \mathrm{mg}, 25 \mu \mathrm{mol}, 10 \mathrm{~mol} \%)$ instead of 180.

\section{General Procedure for the Iron-Catalyzed Hydroarylation of Alkynes 7 (GP8)}

In an oven-dried $25 \mathrm{~mL}$ Schlenk tube were placed the indole substrate 59 (0.25 mmol, 1.0 equiv), $\mathrm{Fe}(\mathrm{acac})_{3}(8.8 \mathrm{mg}, 0.025 \mathrm{mmol}, 10 \mathrm{~mol} \%), \mathbf{L}(0.050 \mathrm{mmol}$, $20 \mathrm{~mol} \%)$ and tolane 7 (0.50 mmol, 2.0 equiv). The Schlenk tube was closed with a rubber septum, then evacuated and backfilled with nitrogen 3 times. THF $(0.50 \mathrm{~mL})$ was added via syringe. $\mathrm{PhMgCl}(0.14 \mathrm{~mL}, 0.28 \mathrm{mmol}, 1.1$ equiv, $1.9 \mathrm{M})$ was then 
added dropwise at ambient temperature. The resulting mixture was stirred at $60{ }^{\circ} \mathrm{C}$ for $16 \mathrm{~h}$. The reaction mixture was allowed to cool to ambient temperature and diluted with THF $(1.5 \mathrm{~mL})$. $\mathrm{HCl}(3.0 \mathrm{M}, 2.0 \mathrm{~mL})$ was added in a single portion, and the resulting mixture was stirred at ambient temperature for $2 \mathrm{~h}$. The mixture was poured into sat. aqueous $\mathrm{NH}_{4} \mathrm{Cl}(10 \mathrm{~mL})$ and extracted with EtOAc $(3 \times 10 \mathrm{~mL})$. The combined organic layers were washed with brine $(10 \mathrm{~mL})$, dried over $\mathrm{Na}_{2} \mathrm{SO}_{4}$, and concentrated under reduced pressure. The residue was purified by silica gel chromatography ( $n$-hexane/EtOAc) to afford the desired product.

The analytical data are in accordance with those previously reported in the literature. ${ }^{[148]}$

\section{General Procedure for the Enantioselective Nickel-Catalyzed Intermolecular Hydroarylation (GP9)}

An oven-dried $25 \mathrm{~mL}$ Schlenk tube was charged with $\mathrm{N}$-benzylbenzimidazole $99 \mathrm{~b}$ (104 mg, $0.50 \mathrm{mmol}, 1.0$ equiv) and $\mathbf{L}(10 \mathrm{~mol} \%, 50 \mu \mathrm{mol}$ ), and introduced into a nitrogen-filled glovebox. $\mathrm{Ni}(\mathrm{cod})_{2}(13.8 \mathrm{mg}, 50 \mu \mathrm{mol}, 10 \mathrm{~mol} \%), \mathrm{NaOtBu}(9.6 \mathrm{mg}$, $0.10 \mathrm{mmol}, 20 \mathrm{~mol} \%)$ and PhMe $(2.0 \mathrm{~mL})$ were then added. The resulting mixture was stirred at ambient temperature for $3 \mathrm{~min}$. BHT (22.0 $\mathrm{mg}, 0.10 \mathrm{mmol}, 20 \mathrm{~mol} \%)$ was then added, followed by 4-methoxystyrene 60c (134 mg, $1.0 \mathrm{mmol}, 2.0$ equiv). The Schlenk tube was closed, taken out of the glovebox, placed in a pre-heated oil bath at $130{ }^{\circ} \mathrm{C}$ and stirred for $16 \mathrm{~h}$. The reaction mixture was allowed to cool to ambient temperature, diluted with EtOAc $(5.0 \mathrm{~mL})$, filtered through a short plug of $\mathrm{SiO}_{2}$, rinsed with $\mathrm{EtOAc}(3 \times 10 \mathrm{~mL})$ and concentrated under reduced pressure. The residue was purified by column chromatography on silica gel $(n$-hexane/EtOAc $=$ $4.5 / 1 \rightarrow 3 / 1$ ) to yield $106 \mathrm{bc}$ as a colorless oil. 


\section{General Procedure for the Synthesis of Alkene-Tethered Azoles 144 (GP10)}

Following a modified procedure, ${ }^{[193]} \mathrm{MsCl}$ (1.2 equiv) was added dropwise to a solution of the unsaturated alcohol (1.0 equiv), $\mathrm{Et}_{3} \mathrm{~N}$ (1.5 equiv) and DMAP $(5.0 \mathrm{~mol} \%)$ in dry $\mathrm{CH}_{2} \mathrm{Cl}_{2}(0.10 \mathrm{M})$ at $0{ }^{\circ} \mathrm{C}$. The reaction mixture was allowed to warm to $23^{\circ} \mathrm{C}$, stirred for $16 \mathrm{~h}$, and diluted with $\mathrm{CH}_{2} \mathrm{Cl}_{2}$ to $4 \times$ the initial volume. The obtained solution was washed successively with $2 \mathrm{M} \mathrm{HCl}$ (twice), sat. aqueous $\mathrm{NaHCO}_{3}$, brine and dried $\left(\mathrm{Na}_{2} \mathrm{SO}_{4}\right)$. Concentration under reduced pressure afforded the crude mesylate which was used in the next step without further purification.

A solution of benzimidazole (500 mg, $4.23 \mathrm{mmol}, 1.00$ equiv) in anhydrous DMF $\left(40 \mathrm{~mL}\right.$ ) was cooled to $0^{\circ} \mathrm{C}$. $\mathrm{NaH}(203 \mathrm{mg}, 5.08 \mathrm{mmol}, 1.20$ equiv, $60 \%$ in mineral oil) was then added portionwise under ice-cooling. The resulting suspension was stirred at $0^{\circ} \mathrm{C}$ for $15 \mathrm{~min}$, then allowed to warm up to ambient temperature and stirred for $1 \mathrm{~h}$. The reaction mixture was then cooled to $0^{\circ} \mathrm{C}$, and the corresponding mesylate (5.08 mmol, 1.2 equiv) was added slowly under ice cooling. The resulting suspension was stirred at $0{ }^{\circ} \mathrm{C}$ for $15 \mathrm{~min}$, then stirred at $23^{\circ} \mathrm{C}$ for $16 \mathrm{~h}$. The reaction mixture was cooled to $0{ }^{\circ} \mathrm{C}$, poured into sat. aqueous $\mathrm{NH}_{4} \mathrm{Cl}(100 \mathrm{~mL})$, and extracted with EtOAc $(3 \times 100 \mathrm{~mL})$. The combined organic phases were washed with $\mathrm{H}_{2} \mathrm{O}$ $(4 \times 100 \mathrm{~mL})$ and brine $(50 \mathrm{~mL})$, dried over $\mathrm{Na}_{2} \mathrm{SO}_{4}$, and concentrated under reduced pressure. The residue was purified by column chromatography on silica gel (n-hexane/EtOAc).

The analytical data of alkene-tethered azoles 144 have been reported elsewhere ${ }^{[220]}$ and are not included in this thesis.

\section{General Procedure for the Nickel-Catalyzed Enantioselective Intramolecular Hydroarylation (GP11)}

An oven-dried $25 \mathrm{~mL}$ Schlenk tube was charged with substrate $144(0.50 \mathrm{mmol}$, 1.0 equiv), $\mathrm{Ni}(\mathrm{cod})_{2}(6.9 \mathrm{mg}, 25 \mu \mathrm{mol}, 5.0 \mathrm{~mol} \%), 220 \quad(6.3 \mathrm{mg}, \quad 12.5 \mu \mathrm{mol}$, $2.5 \mathrm{~mol} \%)$ and $\mathrm{PhMe}(1.0 \mathrm{~mL})$ under $\mathrm{N}_{2}$. The Schlenk tube was closed with a rubber 
septum and placed in a pre-heated oil bath at $95^{\circ} \mathrm{C}$. The reaction mixture was stirred at $95{ }^{\circ} \mathrm{C}$ for $16 \mathrm{~h}$, then cooled to ambient temperature and diluted with EtOAc $(5 \mathrm{~mL})$. The mixture was filtered through a short plug of silica gel, rinsed with EtOAc $(4 \times 10 \mathrm{~mL})$ and concentrated under reduced pressure. The residue was purified by column chromatography on silica gel ( $n$-hexane/EtOAc) to provide the product 145.

\section{General Procedure for the Nickel-Catalyzed Racemic Intramolecular Hydroarylation (GP12)}

The general procedure GP11 was followed using Ni(cod) $)_{2}(13.8 \mathrm{mg}, 50 \mu \mathrm{mol}$, $10 \mathrm{~mol} \%), \mathrm{AlMe}_{3}(0.10 \mathrm{~mL}, \quad 0.20 \mathrm{mmol}, 0.40$ equiv, $2 \mathrm{M}$ in $\mathrm{PhMe})$ and rac- $\mathrm{Ph}(\mathrm{tBu}) \mathrm{P}(\mathrm{O}) \mathrm{H}(9.1 \mathrm{mg}, 50 \mu \mathrm{mol}, 10 \mathrm{~mol} \%)$ instead of 220. 


\subsection{Cobalt(III)-Catalyzed Directed C-H Amidation}

\subsubsection{Experimental Procedures and Analytical Data}

\section{$N$-[2-(4,5-Dihydrooxazol-2-yl)-5-methylphenyl]benzamide (143aa)}

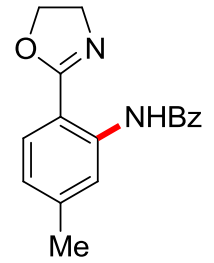

The general procedure GP1 was followed using oxazoline 142a $(81 \mathrm{mg}, 0.25 \mathrm{mmol}$, 1.0 equiv), dioxazolone $40 \mathrm{a}$ ( $49 \mathrm{mg}, 0.30 \mathrm{mmol}, 1.2$ equiv), $\mathrm{Cp}^{*} \mathrm{Co}(\mathrm{CO}) \mathrm{I}_{2}(6.0 \mathrm{mg}$, $0.013 \mathrm{mmol}, 5.0 \mathrm{~mol} \%), \mathrm{AgSbF}_{6}(17 \mathrm{mg}, 0.050 \mathrm{mmol}, 20 \mathrm{~mol} \%)$ and $\mathrm{NaOAc}$ $(4.1 \mathrm{mg}, \quad 0.050 \mathrm{mmol}, 20 \mathrm{~mol} \%)$ in DCE $(1.0 \mathrm{~mL})$. Purification by column chromatography on silica gel ( $n$-hexane/EtOAc $=5 / 1)$ yielded 143aa $(39 \mathrm{mg}, 56 \%)$ as a white solid. Reaction carried on $5.6 \mathrm{mmol}: 905 \mathrm{mg}, 58 \%$. Reaction performed by Dr. R. Mei on $0.50 \mathrm{mmol}: 95 \mathrm{mg}, 68 \%$. M. p. $=149-150{ }^{\circ} \mathrm{C}$. ${ }^{1} \mathrm{H}$ NMR $(400 \mathrm{MHz}$, $\left.\mathrm{CDCl}_{3}\right): \delta=13.00(\mathrm{~s}, 1 \mathrm{H}), 8.82(\mathrm{~s}, 1 \mathrm{H}), 8.10(\mathrm{~d}, J=8.1 \mathrm{~Hz}, 2 \mathrm{H}), 7.77(\mathrm{~d}, J=8.0 \mathrm{~Hz}$, $1 \mathrm{H}), 7.58-7.45(\mathrm{~m}, 3 \mathrm{H}), 6.92(\mathrm{dd}, J=8.0,1.4 \mathrm{~Hz}, 1 \mathrm{H}), 4.41-4.33(\mathrm{~m}, 2 \mathrm{H}), 4.19-4.14$ $(\mathrm{m}, 2 \mathrm{H}), 2.43(\mathrm{~s}, 3 \mathrm{H}) .{ }^{13} \mathrm{C}$ NMR $\left(100 \mathrm{MHz}, \mathrm{CDCl}_{3}\right): \delta=166.0\left(\mathrm{C}_{\mathrm{q}}\right), 164.9\left(\mathrm{C}_{\mathrm{q}}\right), 143.4$ $\left(\mathrm{C}_{\mathrm{q}}\right), 140.0\left(\mathrm{C}_{\mathrm{q}}\right), 135.3\left(\mathrm{C}_{\mathrm{q}}\right), 131.5(\mathrm{CH}), 129.1(\mathrm{CH}), 128.5(\mathrm{CH}), 127.7(\mathrm{CH}), 123.3$ $(\mathrm{CH}), 120.3(\mathrm{CH}), 111.0\left(\mathrm{C}_{\mathrm{q}}\right), 66.1\left(\mathrm{CH}_{2}\right), 54.6\left(\mathrm{CH}_{2}\right), 22.1\left(\mathrm{CH}_{3}\right)$. IR (ATR): 3055, 2982, 2915, 1677, 1363, 1155, 1103, 1055, 817, 754, $\mathrm{cm}^{-1}$. MS (EI) $\mathrm{m} / \mathrm{z}$ (relative intensity) 281 (20) [M+H $\mathrm{H}^{+}$, 280 (55), 263 (25), 203 (100), 160 (50). HR-MS (EI) m/z calcd for $\mathrm{C}_{17} \mathrm{H}_{16} \mathrm{~N}_{2} \mathrm{O}_{2}\left[\mathrm{M}^{+}\right]$280.1212, found 280.1212.

\section{$N$-[2-(4,5-Dihydrooxazol-2-yl)phenyl]benzamide (143ba)}

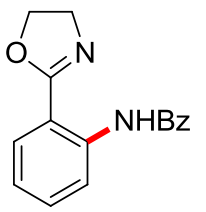


The general procedure GP1 was followed using oxazoline 142b $(74 \mathrm{mg}, 0.50 \mathrm{mmol}$, 1.0 equiv) and dioxazolone $40 \mathrm{a}$ (98 $\mathrm{mg}, 0.60 \mathrm{mmol}, 1.2$ equiv). Purification by column chromatography on silica gel $(n$-hexane/EtOAc $=11 / 2)$ yielded 143ba (95 mg, 71\%) as a white solid. M. p. $=143-145{ }^{\circ} \mathrm{C} .{ }^{1} \mathbf{H}$ NMR $\left(400 \mathrm{MHz}, \mathrm{CDCl}_{3}\right)$ : $\delta=13.03(\mathrm{~s}, 1 \mathrm{H}), 8.97(\mathrm{dd}, J=8.5,1.2 \mathrm{~Hz}, 1 \mathrm{H}), 8.10(\mathrm{~d}, J=8.1 \mathrm{~Hz}, 2 \mathrm{H}), 7.91$ (dd, $J=7.9,1.7 \mathrm{~Hz}, 1 \mathrm{H}$ ), 7.59-7.45 (m, 4H), 7.12 (ddd, $J=7.9,7.3,1.2 \mathrm{~Hz}, 1 \mathrm{H}$ ), 4.42 (td, $J=9.4,1.1 \mathrm{~Hz}, 2 \mathrm{H}), 4.21(\mathrm{td}, J=9.4,1.1 \mathrm{~Hz}, 2 \mathrm{H}) .{ }^{13} \mathrm{C}$ NMR $\left(100 \mathrm{MHz}, \mathrm{CDCl}_{3}\right)$ : $\delta=166.2\left(\mathrm{C}_{\mathrm{q}}\right), 165.1\left(\mathrm{C}_{\mathrm{q}}\right), 140.4\left(\mathrm{C}_{\mathrm{q}}\right), 135.5\left(\mathrm{C}_{\mathrm{q}}\right), 132.8(\mathrm{CH}), 131.8(\mathrm{CH}), 129.4$ $(\mathrm{CH}), 128.7(\mathrm{CH}), 127.9(\mathrm{CH}), 122.5(\mathrm{CH}), 120.1(\mathrm{CH}), 113.7\left(\mathrm{C}_{\mathrm{q}}\right), 66.4\left(\mathrm{CH}_{2}\right), 54.9$ $\left(\mathrm{CH}_{2}\right)$. IR (ATR): 3026, 1614, 1446, 1303, 1058, 943, 748, 703, $674 \mathrm{~cm}^{-1}$. MS (EI) m/z (relative intensity) 266 (56) [M+'], 189 (96), 146 (42), 105 (100), 77 (75), 51 (12). HR-MS (ESI) $\mathrm{m} / z$ calcd for $\mathrm{C}_{16} \mathrm{H}_{13} \mathrm{~N}_{2} \mathrm{O}_{2}[\mathrm{M}-\mathrm{H}]^{-}: 265.0983$, found: 265.0991.

The analytical data are in accordance with those previously reported in the literature. ${ }^{[327]}$

\section{N-[2-(4,5-Dihydrooxazol-2-yl)-5-ethylphenyl]benzamide (143ca)}<smiles>CCc1ccc(C2=NCCO2)c(NC(=O)c2ccccc2)c1</smiles>

The general procedure GP1 was followed using oxazoline 142c $(88 \mathrm{mg}, 0.50 \mathrm{mmol}$, 1.0 equiv) and dioxazolone 40a (98 $\mathrm{mg}, 0.60 \mathrm{mmol}, 1.2$ equiv). Purification by column chromatography on silica gel $(n$-hexane/EtOAc $=7 / 1)$ yielded $143 \mathrm{ca}(95 \mathrm{mg}$, $65 \%)$ as a white solid. M. p. $=122-124{ }^{\circ} \mathrm{C} .{ }^{1} \mathbf{H}$ NMR $\left(300 \mathrm{MHz}, \mathrm{CDCl}_{3}\right): \delta=13.02$ (s, $1 \mathrm{H}), 8.87(\mathrm{dq}, J=1.8,0.6 \mathrm{~Hz}, 1 \mathrm{H}), 8.17-8.05(\mathrm{~m}, 2 \mathrm{H}), 7.81(\mathrm{~d}, J=8.1 \mathrm{~Hz}, 1 \mathrm{H})$, 7.61-7.43 (m, 3H), 6.96 (ddt, $J=8.1,1.7,0.6 \mathrm{~Hz}, 1 \mathrm{H}), 4.40$ (td, $J=9.1,1.0 \mathrm{~Hz}, 2 \mathrm{H}$ ), 4.19 (td, $J=9.1,1.0 \mathrm{~Hz}, 2 \mathrm{H}$ ), $2.74(\mathrm{q}, J=7.6 \mathrm{~Hz}, 2 \mathrm{H}$ ), 1.30 (t, $J=7.6 \mathrm{~Hz}, 3 \mathrm{H}$ ). ${ }^{13} \mathrm{C}$ NMR $\left(125 \mathrm{MHz}, \mathrm{CDCl}_{3}\right): \delta=166.1\left(\mathrm{C}_{\mathrm{q}}\right), 165.0\left(\mathrm{C}_{\mathrm{q}}\right), 149.8\left(\mathrm{C}_{\mathrm{q}}\right), 140.3\left(\mathrm{C}_{\mathrm{q}}\right), 135.5$ $\left(\mathrm{C}_{\mathrm{q}}\right), 131.6(\mathrm{CH}), 129.3(\mathrm{CH}), 128.6(\mathrm{CH}), 127.8(\mathrm{CH}), 122.2(\mathrm{CH}), 119.4(\mathrm{CH}), 111.3$ 
$\left(\mathrm{C}_{\mathrm{q}}\right)$, $66.3\left(\mathrm{CH}_{2}\right), 54.8\left(\mathrm{CH}_{2}\right), 29.6\left(\mathrm{CH}_{2}\right), 15.5\left(\mathrm{CH}_{3}\right)$. IR (ATR): 3065, 2962, 1620, 1580, 1296, 1242, 1052, 698, $678 \mathrm{~cm}^{-1}$. MS (EI) $\mathrm{m} / z$ (relative intensity) 294 (49) $\left[\mathrm{M}^{+}\right], 277$ (14), 217 (100), 174 (36), 105 (47), 77 (49). HR-MS (EI) m/z calcd for $\mathrm{C}_{18} \mathrm{H}_{18} \mathrm{~N}_{2} \mathrm{O}_{2}\left[\mathrm{M}^{+}\right]$: 294.1368, found: 294.1376.

\section{$N$-[2-(4,5-Dihydrooxazol-2-yl)-5-fluorophenyl]benzamide (143da)}

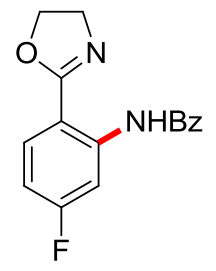

The general procedure GP1 was followed using oxazoline 142d $(83 \mathrm{mg}, 0.50 \mathrm{mmol}$, 1.0 equiv) and dioxazolone 40a (98 $\mathrm{mg}, 0.60 \mathrm{mmol}, 1.2$ equiv). Purification by column chromatography on silica gel ( $n$-hexane/EtOAc $=85 / 15$ ) yielded 143da (101 mg, 71\%) as a white solid. M. p. $=163-165^{\circ} \mathrm{C} .{ }^{1} \mathbf{H}$ NMR $\left(300 \mathrm{MHz}, \mathrm{CDCl}_{3}\right)$ : $\delta=13.16(\mathrm{~s}, 1 \mathrm{H}), 8.79$ (dd, $J=12.2,2.6 \mathrm{~Hz}, 1 \mathrm{H}$ ), 8.08 (d, $J=8.0 \mathrm{~Hz}, 2 \mathrm{H}$ ), 7.88 (dd, $J=8.9,6.5 \mathrm{~Hz}, 1 \mathrm{H}$ ), 7.62-7.43 (m, 3H), 6.80 (ddd, $J=8.9,7.6,2.6 \mathrm{~Hz}, 1 \mathrm{H}$ ), 4.41 (td, $J=9.2,1.3 \mathrm{~Hz}, 2 \mathrm{H}), 4.19(\mathrm{td}, J=9.2,1.3 \mathrm{~Hz}, 2 \mathrm{H}) .{ }^{13} \mathrm{C}$ NMR $\left(100 \mathrm{MHz}, \mathrm{CDCl}_{3}\right)$ : $\delta=166.2\left(\mathrm{C}_{\mathrm{q}}\right), 165.0\left(\mathrm{~d},{ }^{1} \mathrm{~J}_{\mathrm{C}-\mathrm{F}}=249.0 \mathrm{~Hz}, \mathrm{C}_{\mathrm{q}}\right), 164.4\left(\mathrm{C}_{\mathrm{q}}\right), 142.1\left(\mathrm{~d},{ }^{3} \mathrm{~J}_{\mathrm{C}-\mathrm{F}}=12.8 \mathrm{~Hz}\right.$, $\left.\mathrm{C}_{\mathrm{q}}\right), 134.9\left(\mathrm{C}_{\mathrm{q}}\right), 131.9(\mathrm{CH}), 131.1\left(\mathrm{~d},{ }^{3} \mathrm{~J}_{\mathrm{C}-\mathrm{F}}=10.4 \mathrm{~Hz}, \mathrm{CH}\right), 128.6(\mathrm{CH}), 127.8(\mathrm{CH})$, $109.8\left(\mathrm{~d},{ }^{4} \mathrm{~J}_{\mathrm{C}-\mathrm{F}}=2.9 \mathrm{~Hz}, \mathrm{C}_{\mathrm{q}}\right), 109.5\left(\mathrm{~d},{ }^{2} \mathrm{~J}_{\mathrm{C}-\mathrm{F}}=22.5 \mathrm{~Hz}, \mathrm{CH}\right), 107.3(\mathrm{~d}$, $\left.{ }^{2} J_{\mathrm{C}-\mathrm{F}}=28.8 \mathrm{~Hz}, \quad \mathrm{CH}\right), \quad 66.3 \quad\left(\mathrm{CH}_{2}\right), \quad 54.6 \quad\left(\mathrm{CH}_{2}\right) .{ }^{19} \mathrm{~F} \mathrm{NMR} \quad\left(282 \mathrm{MHz}, \quad \mathrm{CDCl}_{3}\right)$ : $\delta=-104.2$ (m). IR (ATR): 3111, 2975, 1614, 1599, 1544, 1430, 1255, $705 \mathrm{~cm}^{-1}$. MS (EI) $\mathrm{m} / z$ (relative intensity) 284 (33) [M+], 207 (84), 164 (30), 105 (100), 77 (45), 44 (10). HR-MS (ESI) $\mathrm{m} / z$ calcd for $\mathrm{C}_{16} \mathrm{H}_{14} \mathrm{~N}_{2} \mathrm{O}_{2} \mathrm{~F}\left[\mathrm{M}+\mathrm{H}^{+}\right]$: 285.1034, found: 285.1038 . 
$N$-[2-(4,5-Dihydrooxazol-2-yl)-5-(trifluoromethyl)phenyl]benzamide (143ea)<smiles>O=C(Nc1cc(C(F)(F)F)ccc1C1=NCCO1)c1ccccc1</smiles>

The general procedure GP1 was followed using oxazoline 142e (108 mg, 0.50 mmol, 1.0 equiv) and dioxazolone 40a (98 mg, $0.60 \mathrm{mmol}, 1.2$ equiv). Purification by column chromatography on silica gel ( $n$-hexane/EtOAc $=85 / 15)$ yielded 143ea (106 mg, 63\%) as a white solid. M. p. $=191-193^{\circ} \mathrm{C} .{ }^{1} \mathbf{H}$ NMR $\left(300 \mathrm{MHz}, \mathrm{CDCl}_{3}\right)$ : $\delta=13.10(\mathrm{~s}, 1 \mathrm{H}), 9.34(\mathrm{~d}, J=1.8 \mathrm{~Hz}, 1 \mathrm{H}), 8.08(\mathrm{~d}, J=8.0 \mathrm{~Hz}, 2 \mathrm{H}), 8.02-7.93(\mathrm{~m}$, $1 \mathrm{H}$ ), 7.63-7.44 (m, 3H), 7.33 (ddd, $J=8.3,1.8,0.7 \mathrm{~Hz}, 1 \mathrm{H}$ ), $4.46(\mathrm{td}, J=9.3,1.3 \mathrm{~Hz}$, 2H), 4.24 (td, $J=9.3,1.3 \mathrm{~Hz}, 2 \mathrm{H}) .{ }^{13} \mathrm{C}$ NMR $\left(100 \mathrm{MHz}, \mathrm{CDCl}_{3}\right): \delta=166.2\left(\mathrm{C}_{\mathrm{q}}\right), 164.2$ $\left(\mathrm{C}_{\mathrm{q}}\right), 140.6\left(\mathrm{C}_{\mathrm{q}}\right), 134.7\left(\mathrm{C}_{\mathrm{q}}\right), 134.1\left(\mathrm{q},{ }^{2} \mathrm{~J}_{\mathrm{C}-\mathrm{F}}=32.7 \mathrm{~Hz}, \mathrm{C}_{\mathrm{q}}\right), 132.0(\mathrm{CH}), 129.8(\mathrm{CH})$, $128.7(\mathrm{CH}), 127.7(\mathrm{CH}), 123.6\left(\mathrm{q},{ }^{1} J_{\mathrm{C}-\mathrm{F}}=273.1 \mathrm{~Hz}, \mathrm{C}_{\mathrm{q}}\right), 118.7\left(\mathrm{q},{ }^{3} J_{\mathrm{C}-\mathrm{F}}=3.8 \mathrm{~Hz}\right.$, $\mathrm{CH}), 116.9\left(\mathrm{q},{ }^{3} \mathrm{~J}_{\mathrm{C}-\mathrm{F}}=4.2 \mathrm{~Hz}, \mathrm{CH}\right), 116.0\left(\mathrm{C}_{\mathrm{q}}\right), 66.5\left(\mathrm{CH}_{2}\right), 54.8\left(\mathrm{CH}_{2}\right) \cdot{ }^{19} \mathrm{~F}$ NMR $\left(282 \mathrm{MHz}, \mathrm{CDCl}_{3}\right): \delta=-63.2$ (s). IR (ATR): 3016, 1622, 1588, 1426, 1333, 1118, 1081, 1055, 922, 899, $701 \mathrm{~cm}^{-1}$. MS (EI) $\mathrm{m} / z$ (relative intensity) 334 (28) [M+], 257 (36), 214 (14), 158 (7), 105 (100), 77 (44), 51 (6). HR-MS (ESI) m/z calcd for $\mathrm{C}_{17} \mathrm{H}_{14} \mathrm{~N}_{2} \mathrm{O}_{2} \mathrm{~F}_{3}\left[\mathrm{M}+\mathrm{H}^{+}\right]$: 335.1002, found: 335.1005.

\section{N-[4-(4,5-Dihydrooxazol-2-yl)-[1,1'-biphenyl]-3-yl]benzamide (143fa)}

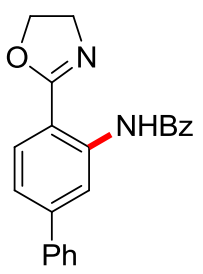

The general procedure GP1 was followed using oxazoline $142 \mathrm{f}(112 \mathrm{mg}, 0.50 \mathrm{mmol}$, 1.0 equiv) and dioxazolone $40 \mathrm{a}$ (98 $\mathrm{mg}, 0.60 \mathrm{mmol}, 1.2$ equiv). Purification by column chromatography on silica gel $(n$-hexane/EtOAc $=6 / 1$ ) yielded 143fa (127 mg, 74\%) as a white solid. M. p. $=170-171^{\circ} \mathrm{C} .{ }^{1} \mathbf{H}$ NMR $\left(300 \mathrm{MHz}, \mathrm{CDCl}_{3}\right)$ : 
$\delta=13.08(\mathrm{~s}, 1 \mathrm{H}), 9.34(\mathrm{~d}, J=1.8 \mathrm{~Hz}, 1 \mathrm{H}), 8.12(\mathrm{~d}, J=8.1 \mathrm{~Hz}, 2 \mathrm{H}), 7.95(\mathrm{~d}$, $J=8.2 \mathrm{~Hz}, 1 \mathrm{H}), 7.80-7.69(\mathrm{~m}, 2 \mathrm{H}), 7.62-7.32(\mathrm{~m}, 7 \mathrm{H}), 4.41(\mathrm{td}, J=9.2,0.9 \mathrm{~Hz}, 2 \mathrm{H})$, 4.21 (td, $J=9.2,0.9 \mathrm{~Hz}, 2 \mathrm{H}) .{ }^{13} \mathrm{C}$ NMR $\left(100 \mathrm{MHz}, \mathrm{CDCl}_{3}\right): \delta=166.3\left(\mathrm{C}_{\mathrm{q}}\right), 165.0$ $\left(\mathrm{C}_{\mathrm{q}}\right), 145.3\left(\mathrm{C}_{\mathrm{q}}\right), 140.7\left(\mathrm{C}_{\mathrm{q}}\right), 140.2\left(\mathrm{C}_{\mathrm{q}}\right), 135.4\left(\mathrm{C}_{\mathrm{q}}\right), 131.8(\mathrm{CH}), 129.8(\mathrm{CH}), 128.9$ $(\mathrm{CH}), 128.7(\mathrm{CH}), 128.2(\mathrm{CH}), 127.9(\mathrm{CH}), 127.5(\mathrm{CH}), 121.1(\mathrm{CH}), 118.5(\mathrm{CH})$, $112.5\left(\mathrm{C}_{\mathrm{q}}\right), 66.4\left(\mathrm{CH}_{2}\right), 54.9\left(\mathrm{CH}_{2}\right)$. IR (ATR): 3056, 1618, 1569, 1409, 1249, 1064, $697,678 \mathrm{~cm}^{-1}$. MS (EI) $\mathrm{m} / z$ (relative intensity) $342(64)\left[\mathrm{M}^{+}\right], 265$ (100), 222 (35), 166 (15), 105 (55), 77 (57). HR-MS (EI) $m / z$ calcd for $\mathrm{C}_{22} \mathrm{H}_{18} \mathrm{~N}_{2} \mathrm{O}_{2}\left[\mathrm{M}^{+}\right]$: 342.1368, found: 342.1364 .

\section{$N$-[5-(tert-Butoxy)-2-(4,5-dihydrooxazol-2-yl)phenyl]benzamide (143ga)}

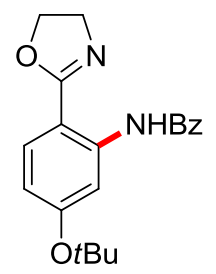

The general procedure GP1 was followed using oxazoline 142g (110 mg, $0.50 \mathrm{mmol}, 1.0$ equiv) and dioxazolone $40 \mathrm{a}$ (98 $\mathrm{mg}, 0.60 \mathrm{mmol}, 1.2$ equiv). Purification by column chromatography on silica gel $(n$-hexane/EtOAc $=7.5 / 1$ ) yielded 143ga (84 mg, 50\%) as a white solid. M. p. = 120-122 ${ }^{\circ} \mathrm{C}$. ${ }^{1} \mathbf{H}$ NMR $\left(500 \mathrm{MHz}, \mathrm{CDCl}_{3}\right): \delta=13.05(\mathrm{~s}, 1 \mathrm{H}), 8.76(\mathrm{~d}, J=2.4 \mathrm{~Hz}, 1 \mathrm{H}), 8.13-8.07(\mathrm{~d}$, $J=8.1 \mathrm{~Hz}, 2 \mathrm{H}), 7.77(\mathrm{~d}, J=8.7 \mathrm{~Hz}, 1 \mathrm{H}), 7.57-7.45(\mathrm{~m}, 3 \mathrm{H}), 6.70(\mathrm{dd}, J=8.7$, $2.4 \mathrm{~Hz}, 1 \mathrm{H}$ ), 4.36 (td, $J=9.3,0.9 \mathrm{~Hz}, 2 \mathrm{H}), 4.15(\mathrm{td}, J=9.3,0.9 \mathrm{~Hz}, 2 \mathrm{H}), 1.49(\mathrm{~s}, 9 \mathrm{H})$. ${ }^{13} \mathrm{C}$ NMR $\left(125 \mathrm{MHz}, \mathrm{CDCl}_{3}\right): \delta=166.3\left(\mathrm{C}_{\mathrm{q}}\right), 164.9\left(\mathrm{C}_{\mathrm{q}}\right), 159.8\left(\mathrm{C}_{\mathrm{q}}\right), 141.2\left(\mathrm{C}_{\mathrm{q}}\right), 135.4$ $\left(\mathrm{C}_{\mathrm{q}}\right), 131.7(\mathrm{CH}), 130.0(\mathrm{CH}), 127.8(\mathrm{CH}), 127.8(\mathrm{CH}), 116.8(\mathrm{CH}), 113.1(\mathrm{CH}), 108.0$ $\left(\mathrm{C}_{\mathrm{q}}\right), 79.6\left(\mathrm{C}_{\mathrm{q}}\right), 66.2\left(\mathrm{CH}_{2}\right), 54.6\left(\mathrm{CH}_{2}\right), 29.1\left(\mathrm{CH}_{3}\right)$. IR (ATR): 2978, 2935, 2877, 1630, 1578, 1362, 1257, 1239, $707 \mathrm{~cm}^{-1}$. MS (EI) $\mathrm{m} / z$ (relative intensity) 338 (7) [M+'], 282 (67), 205 (100), 162 (19), 105 (83), 77 (53), 57 (24). HR-MS (EI) m/z calcd for $\mathrm{C}_{20} \mathrm{H}_{22} \mathrm{~N}_{2} \mathrm{O}_{3}\left[\mathrm{M}^{+}\right]$: 338.1630, found: 338.1627. 
N-[5-Acetamido-2-(4,5-dihydrooxazol-2-yl)phenyl]benzamide (143ha)

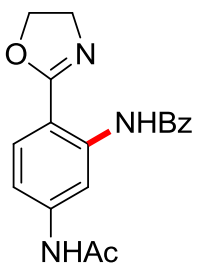

The general procedure GP1 was followed using oxazoline 142h (102 mg, $0.50 \mathrm{mmol}, 1.0$ equiv) and dioxazolone $40 \mathrm{a}$ (98 $\mathrm{mg}, 0.60 \mathrm{mmol}, 1.2$ equiv). Purification by column chromatography on silica gel $(n$-hexane/EtOAc $=2 / 1)$ yielded 143ha (94 mg, 58\%) as a white solid. M. p. $=189-191{ }^{\circ} \mathrm{C} .{ }^{1} \mathbf{H} \mathbf{~ N M R}(300 \mathrm{MHz}$, DMSO- $\left.\mathrm{d}_{6}\right): \delta=13.07(\mathrm{~s}, 1 \mathrm{H}), 10.28(\mathrm{~s}, 1 \mathrm{H}), 8.96(\mathrm{~d}, J=2.1 \mathrm{~Hz}, 1 \mathrm{H}), 8.02(\mathrm{~d}$, $J=7.8 \mathrm{~Hz}, 2 \mathrm{H}), 7.80(\mathrm{~d}, J=8.7 \mathrm{~Hz}, 1 \mathrm{H}), 7.72-7.53(\mathrm{~m}, 4 \mathrm{H}), 4.42(\mathrm{td}, J=9.1,1.1 \mathrm{~Hz}$, $2 \mathrm{H}), 4.16(\mathrm{td}, J=9.1,1.1 \mathrm{~Hz}, 2 \mathrm{H}), 2.09(\mathrm{~s}, 3 \mathrm{H}) .{ }^{13} \mathrm{C} \mathrm{NMR}\left(100 \mathrm{MHz}, \mathrm{CDCl}_{3}\right)$ : $\delta=168.8\left(\mathrm{C}_{\mathrm{q}}\right), 166.6\left(\mathrm{C}_{\mathrm{q}}\right), 164.9\left(\mathrm{C}_{\mathrm{q}}\right), 142.0(\mathrm{CH}), 140.7\left(\mathrm{C}_{\mathrm{q}}\right), 135.2(\mathrm{CH}), 132.0$ $(\mathrm{CH}), 130.8(\mathrm{CH}), 128.8(\mathrm{CH}), 127.9(\mathrm{CH}), 113.8\left(\mathrm{C}_{\mathrm{q}}\right), 109.8\left(\mathrm{C}_{\mathrm{q}}\right), 109.6\left(\mathrm{C}_{\mathrm{q}}\right), 66.4$ $\left(\mathrm{CH}_{2}\right), 54.7\left(\mathrm{CH}_{2}\right), 24.9\left(\mathrm{CH}_{3}\right)$. IR (ATR): 3312, 3278, 1621, 1518, 1402, 1360, 1286, 1240, 1061, $704 \mathrm{~cm}^{-1}$. MS (EI) $\mathrm{m} / z$ (relative intensity) 323 (23) [M+], 246 (38), 204 (11), 161 (10), 105 (44), 77 (39), 58 (15), 43 (100). HR-MS (EI) m/z calcd for $\mathrm{C}_{18} \mathrm{H}_{17} \mathrm{~N}_{3} \mathrm{O}_{3}\left[\mathrm{M}^{+}\right]:$323.1270, found: 323.1266.

\section{N-[2-(4,5-Dihydrooxazol-2-yl)-4-(trifluoromethyl)phenyl]benzamide (143ia)}<smiles>O=C(Nc1ccc(C(F)(F)F)cc1C1=NCCO1)c1ccccc1</smiles>

The general procedure GP1 was followed using oxazoline $142 \mathbf{i}(108 \mathrm{mg}, 0.50 \mathrm{mmol}$, 1.0 equiv) and dioxazolone $40 \mathrm{a}$ (98 $\mathrm{mg}, 0.60 \mathrm{mmol}, 1.2$ equiv). Purification by column chromatography on silica gel $(n$-hexane/EtOAc $=7 / 1)$ yielded 143ia $(108 \mathrm{mg}$, $65 \%)$ as a white solid. M. p. $=184-187^{\circ} \mathrm{C} .{ }^{1} \mathbf{H}$ NMR $\left(300 \mathrm{MHz}, \mathrm{CDCl}_{3}\right): \delta=13.17(\mathrm{~s}$, $1 \mathrm{H}), 9.11(\mathrm{dt}, J=8.9,0.7 \mathrm{~Hz}, 1 \mathrm{H}), 8.19(\mathrm{dt}, J=2.3,0.6 \mathrm{~Hz}, 1 \mathrm{H}), 8.09(\mathrm{~d}, J=8.1 \mathrm{~Hz}$, 
2H), 7.75 (ddt, $J=8.9,2.3,0.61 \mathrm{H}), 7.64-7.45(\mathrm{~m}, 3 \mathrm{H}), 4.48$ (td, $J=9.1,1.6 \mathrm{~Hz}$, $2 \mathrm{H}), 4.26(\mathrm{td}, J=9.1,1.6 \mathrm{~Hz}, 2 \mathrm{H}) .{ }^{13} \mathrm{C}$ NMR $\left(125 \mathrm{MHz}, \mathrm{CDCl}_{3}\right): \delta=166.2\left(\mathrm{C}_{\mathrm{q}}\right), 164.0$ $\left(\mathrm{C}_{\mathrm{q}}\right), 142.8\left(\mathrm{C}_{\mathrm{q}}\right), 134.7\left(\mathrm{C}_{\mathrm{q}}\right), 132.0(\mathrm{CH}), 129.2\left(\mathrm{q},{ }^{3} \mathrm{~J}_{\mathrm{C}-\mathrm{F}}=3.4 \mathrm{~Hz}, \mathrm{CH}\right), 128.6(\mathrm{CH})$, $127.7(\mathrm{CH}), 126.5\left(\mathrm{q},{ }^{3} \mathrm{~J}_{\mathrm{C}-\mathrm{F}}=4.0 \mathrm{~Hz}, \mathrm{CH}\right), 124.9$ (q, $\left.{ }^{1} \mathrm{~J}_{\mathrm{C}-\mathrm{F}}=271.3 \mathrm{~Hz}, \mathrm{C}_{\mathrm{q}}\right), 124.2$ (q, $\left.{ }^{2} J_{\mathrm{C}-\mathrm{F}}=33.4 \mathrm{~Hz}, \mathrm{C}_{\mathrm{q}}\right), 119.9(\mathrm{CH}), 113.4\left(\mathrm{C}_{\mathrm{q}}\right), 66.6\left(\mathrm{CH}_{2}\right), 54.8\left(\mathrm{CH}_{2}\right) .{ }^{19} \mathrm{~F}$ NMR $\left(282 \mathrm{MHz}, \mathrm{CDCl}_{3}\right): \delta=-62.2$ (s). IR (ATR): 3013, 1627, 1308, 1237, 1107, 1082, 1058, 952, $695 \mathrm{~cm}^{-1}$. MS (EI) $\mathrm{m} / z$ (relative intensity) 334 (38) $\left[\mathrm{M}^{+}\right], 257$ (57), 214 (21), 158 (10), 105 (100), 77 (64). HR-MS (EI) $m / z$ calcd for $\mathrm{C}_{17} \mathrm{H}_{13} \mathrm{~N}_{2} \mathrm{O}_{2} \mathrm{~F}_{3}\left[\mathrm{M}^{+}\right]$: 334.0929, found: 334.0923 .

\section{N-[4-Chloro-2-(4,5-dihydrooxazol-2-yl)phenyl]benzamide (143ja)}<smiles>O=C(Nc1ccccc1)c1cccc(Cl)c1</smiles>

The general procedure GP1 was followed using oxazoline 142j $(91 \mathrm{mg}, 0.50 \mathrm{mmol}$, 1.0 equiv), dioxazolone $40 \mathrm{a}$ (98 mg, $0.60 \mathrm{mmol}, 1.2$ equiv), [ $\left.\mathrm{Cp}^{*} \mathrm{RhCl}_{2}\right]_{2}(7.7 \mathrm{mg}$, $0.013 \mathrm{mmol}, 2.5 \mathrm{~mol} \%), \mathrm{AgSbF}_{6}$ (34 mg, $\left.0.10 \mathrm{mmol}, 20 \mathrm{~mol} \%\right)$ and $\mathrm{NaOAc}$ ( $8.2 \mathrm{mg}, 0.10 \mathrm{mmol}, 20 \mathrm{~mol} \%$ ). Purification by column chromatography on silica gel $(n$-hexane/EtOAc $=5 / 1)$ yielded $\mathbf{1 4 3 j a}(111 \mathrm{mg}, 74 \%)$ as a white solid. (Reaction performed by Dr. R. Mei using $\mathrm{Cp}^{*} \mathrm{Co}(\mathrm{CO}) \mathrm{I}_{2}: 101 \mathrm{mg}, 67 \%$.) M. p. $=140-141^{\circ} \mathrm{C}$. ${ }^{1} \mathrm{H}$ NMR $\left(400 \mathrm{MHz}, \mathrm{CDCl}_{3}\right): \delta=12.88(\mathrm{~s}, 1 \mathrm{H}), 8.90(\mathrm{~d}, J=9.0 \mathrm{~Hz}, 1 \mathrm{H}), 8.01(\mathrm{~d}$, $J=8.2 \mathrm{~Hz}, 2 \mathrm{H}), 7.77(\mathrm{~d}, J=2.6 \mathrm{~Hz}, 1 \mathrm{H}), 7.54-7.45(\mathrm{~m}, 1 \mathrm{H}), 7.49-7.40(\mathrm{~m}, 2 \mathrm{H}), 7.39$ (dd, $J=9.0,2.6 \mathrm{~Hz}, 1 \mathrm{H}$ ), 4.34 (td, $J=9.4,1.0 \mathrm{~Hz}, 2 \mathrm{H}$ ), 4.12 (td, $J=9.4,1.0 \mathrm{~Hz}, 2 \mathrm{H}$ ). ${ }^{13} \mathrm{C}$ NMR $\left(100 \mathrm{MHz}, \mathrm{CDCl}_{3}\right): \delta=165.8\left(\mathrm{C}_{\mathrm{q}}\right), 163.8\left(\mathrm{C}_{\mathrm{q}}\right), 138.6\left(\mathrm{C}_{\mathrm{q}}\right), 134.8\left(\mathrm{C}_{\mathrm{q}}\right), 132.2$ $(\mathrm{CH}), 131.7(\mathrm{CH}), 128.8(\mathrm{CH}), 128.5(\mathrm{CH}), 127.6(\mathrm{CH}), 127.2\left(\mathrm{C}_{\mathrm{q}}\right), 121.0(\mathrm{CH}), 114.7$ $\left(\mathrm{C}_{\mathrm{q}}\right), 66.3\left(\mathrm{CH}_{2}\right), 54.6\left(\mathrm{CH}_{2}\right)$. IR (ATR): 3048, 2969, 1666, 1615, 1579, 1474, 1230, 1057, 950, $693 \mathrm{~cm}^{-1}$. MS (EI) $\mathrm{m} / z$ (relative intensity) $300(30)\left[\mathrm{M}^{+}\right]\left({ }^{35} \mathrm{Cl}\right), 307(5)$, 223 (30), 180 (15), 124 (15), 105 (100). HR-MS (EI) $m / z$ calcd for $\mathrm{C}_{16} \mathrm{H}_{13}{ }^{35} \mathrm{CIN}_{2} \mathrm{O}_{2}$ $\left[\mathrm{M}^{+}\right]$300.0666, found 300.0655 . 
N-[2-(4,5-Dihydrooxazol-2-yl)-5-fluoro-4-methoxyphenyl]benzamide (143ka)<smiles>COc1cc(C2=NCCO2)c(NC(=O)c2ccccc2)cc1F</smiles>

The general procedure GP1 was followed using oxazoline 142k (98 mg, $0.50 \mathrm{mmol}$, 1.0 equiv) and dioxazolone $40 \mathrm{a}$ (98 $\mathrm{mg}, 0.60 \mathrm{mmol}, 1.2$ equiv). Purification by column chromatography on silica gel $(n$-hexane/EtOAc $=4.5 / 1)$ yielded 143ka (116 mg, 74\%) as a white solid. M. p. $=161-163{ }^{\circ} \mathrm{C} .{ }^{1} \mathbf{H}$ NMR $\left(300 \mathrm{MHz}, \mathrm{CDCl}_{3}\right)$ : $\delta=12.93(\mathrm{~s}, 1 \mathrm{H}), 8.86(\mathrm{~d}, J=14.3 \mathrm{~Hz}, 1 \mathrm{H}), 8.06(\mathrm{~d}, J=8.0 \mathrm{~Hz}, 2 \mathrm{H}), 7.61-7.43(\mathrm{~m}$, $4 \mathrm{H}$ ), 4.43 (td, $J=9.3,1.4 \mathrm{~Hz}, 2 \mathrm{H}$ ), 4.21 (td, $J=9.3,1.4 \mathrm{~Hz}, 2 \mathrm{H}), 3.91$ (s, 3H). ${ }^{13} \mathrm{C}$ NMR $\left(125 \mathrm{MHz}, \mathrm{CDCl}_{3}\right): \delta=165.6\left(\mathrm{C}_{\mathrm{q}}\right), 164.1\left(\mathrm{C}_{\mathrm{q}}\right), 154.3\left(\mathrm{~d},{ }^{1} \mathrm{~J}_{\mathrm{C}-\mathrm{F}}=250.9 \mathrm{~Hz}\right.$, $\left.\mathrm{C}_{\mathrm{q}}\right), 142.5\left(\mathrm{~d},{ }^{2} \mathrm{~J}_{\mathrm{C}-\mathrm{F}}=11.6 \mathrm{~Hz}, \mathrm{C}_{\mathrm{q}}\right), 135.0\left(\mathrm{~d},{ }^{4} \mathrm{~J}_{\mathrm{C}-\mathrm{F}}=10.9 \mathrm{~Hz}, \mathrm{C}_{\mathrm{q}}\right), 135.0\left(\mathrm{C}_{\mathrm{q}}\right), 131.6$ $(\mathrm{CH}), 128.5(\mathrm{CH}), 127.6(\mathrm{CH}), 113.9\left(\mathrm{~d},{ }^{3} \mathrm{~J}_{\mathrm{C}-\mathrm{F}}=3.8 \mathrm{~Hz}, \mathrm{CH}\right), 109.4\left(\mathrm{~d},{ }^{3} J_{\mathrm{C}-\mathrm{F}}=3.4 \mathrm{~Hz}\right.$, $\left.\mathrm{C}_{\mathrm{q}}\right), 108.8\left(\mathrm{~d},{ }^{2} \mathrm{~J}_{\mathrm{C}-\mathrm{F}}=25.5 \mathrm{~Hz}, \mathrm{CH}\right), 66.3\left(\mathrm{CH}_{2}\right), 56.6\left(\mathrm{CH}_{3}\right), 54.7\left(\mathrm{CH}_{2}\right) \cdot{ }^{19} \mathrm{~F}$ NMR (282 MHz, $\mathrm{CDCl}_{3}$ ): $\delta=-125.8$ (ddd, $J=14.3,9.2,1.5 \mathrm{~Hz}$ ). IR (ATR): 2968, 1614, 1544, 1271, 1206, 1020, 944, 877, $702 \mathrm{~cm}^{-1}$. MS (EI) $\mathrm{m} / \mathrm{z}$ (relative intensity) 314 (26) [M+], 297 (5), 237 (18), 194 (6), 105 (100), 77 (21). HR-MS (El) m/z calcd for $\mathrm{C}_{17} \mathrm{H}_{15} \mathrm{~N}_{2} \mathrm{O}_{3} \mathrm{~F}\left[\mathrm{M}^{+}\right]: 314.1067$, found: 314.1071 .

\section{N-[5-Methyl-2-(5-methyl-4,5-dihydrooxazol-2-yl)phenyl]benzamide (143ma)}

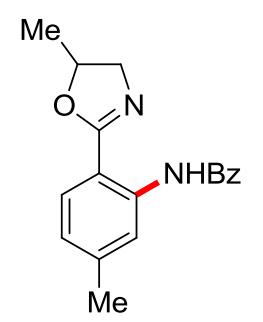

The general procedure GP1 was followed using oxazoline $142 \mathrm{~m}$ (88 mg, $0.50 \mathrm{mmol}$, 1.0 equiv) and dioxazolone $40 \mathrm{a}$ (98 $\mathrm{mg}, 0.60 \mathrm{mmol}, 1.2$ equiv). Purification by column chromatography on silica gel ( $n$-hexane/EtOAc $=8 / 1$ ) yielded 143ma 
$(125 \mathrm{mg}, 85 \%)$ as a white solid. M. p. $=126-128{ }^{\circ} \mathrm{C} .{ }^{1} \mathbf{H}$ NMR $\left(400 \mathrm{MHz}, \mathrm{CDCl}_{3}\right)$ : $\delta=13.06(\mathrm{~s}, 1 \mathrm{H}), 8.83(\mathrm{dq}, J=1.1,0.6 \mathrm{~Hz}, 1 \mathrm{H}), 8.16-8.06(\mathrm{~m}, 2 \mathrm{H}), 7.78(\mathrm{~d}$, $J=8.0 \mathrm{~Hz}, 1 \mathrm{H}), 7.59-7.45(\mathrm{~m}, 3 \mathrm{H}), 6.97-6.88(\mathrm{~m}, 1 \mathrm{H}), 4.81$ (ddq, $J=9.4,7.4$, $6.2 \mathrm{~Hz}, 1 \mathrm{H}$ ), 4.26 (dd, $J=14.2,9.4 \mathrm{~Hz}, 1 \mathrm{H}$ ), 3.73 (dd, $J=14.2,7.4 \mathrm{~Hz}, 1 \mathrm{H}$ ), 2.44 (s, $3 \mathrm{H}), 1.44(\mathrm{~d}, J=6.2 \mathrm{~Hz}, 3 \mathrm{H}) .{ }^{13} \mathrm{C}$ NMR $\left(100 \mathrm{MHz}, \mathrm{CDCl}_{3}\right): \delta=166.2\left(\mathrm{C}_{\mathrm{q}}\right), 164.5$ $\left(\mathrm{C}_{\mathrm{q}}\right), 143.5\left(\mathrm{C}_{\mathrm{q}}\right), 140.2\left(\mathrm{C}_{\mathrm{q}}\right), 135.6\left(\mathrm{C}_{\mathrm{q}}\right), 131.7(\mathrm{CH}), 129.3(\mathrm{CH}), 128.7(\mathrm{CH}), 127.9$ $(\mathrm{CH}), 123.4(\mathrm{CH}), 120.5(\mathrm{CH}), 111.4\left(\mathrm{C}_{\mathrm{q}}\right), 75.0(\mathrm{CH}), 61.3\left(\mathrm{CH}_{2}\right), 22.2\left(\mathrm{CH}_{3}\right), 21.1$ $\left(\mathrm{CH}_{3}\right)$. IR (ATR): 3063, 2974, 1622, 1585, 1295, 1243, 1062, 1049, 696, $677 \mathrm{~cm}^{-1}$. MS (EI) $\mathrm{m} / z$ (relative intensity) 294 (22) [M+], 217 (100), 160 (29), 105 (34), 77 (44). HR-MS (El) $\mathrm{m} / z$ calcd for $\mathrm{C}_{18} \mathrm{H}_{18} \mathrm{~N}_{2} \mathrm{O}_{2}\left[\mathrm{M}^{+}\right]$: 294.1368, found: 294.1370 .

\section{N-[5-Methyl-2-(5-phenyl-4,5-dihydrooxazol-2-yl)phenyl]benzamide (143na)}

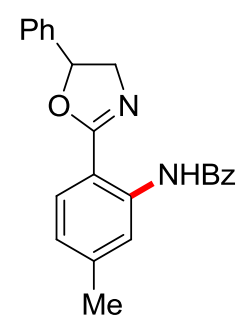

The general procedure GP1 was followed using oxazoline 142n (119 mg, $0.50 \mathrm{mmol}$, 1.0 equiv) and dioxazolone $40 \mathrm{a}$ (98 $\mathrm{mg}, 0.60 \mathrm{mmol}, 1.2$ equiv). Purification by column chromatography on silica gel ( $n$-hexane/EtOAc $=8 / 1$ ) yielded 143na (131 mg, 74\%) as a white solid. M. p. = 131-133 ${ }^{\circ} \mathrm{C} .{ }^{1} \mathbf{H} \mathbf{~ N M R}(400 \mathrm{MHz}$, $\left.\mathrm{CDCl}_{3}\right): \delta=12.99(\mathrm{~s}, 1 \mathrm{H}), 8.88(\mathrm{t}, J=1.1 \mathrm{~Hz}, 1 \mathrm{H}), 8.16-8.06(\mathrm{~m}, 2 \mathrm{H}), 7.90(\mathrm{~d}$, $J=8.0 \mathrm{~Hz}, 1 \mathrm{H}$ ), 7.61-7.45 (m, 3H), 7.45-7.30 (m, 5H), 6.96 (ddd, $J=8.0,1.7$, $0.8 \mathrm{~Hz}, 1 \mathrm{H}), 5.64$ (dd, $J=10.1,7.7 \mathrm{~Hz}, 1 \mathrm{H}), 4.60$ (dd, $J=14.5,10.1 \mathrm{~Hz}, 1 \mathrm{H}), 4.13$ $(\mathrm{dd}, J=14.5,7.7 \mathrm{~Hz}, 1 \mathrm{H}), 2.46(\mathrm{~s}, 3 \mathrm{H}) .{ }^{13} \mathrm{C}$ NMR $\left(100 \mathrm{MHz}, \mathrm{CDCl}_{3}\right): \delta=166.2\left(\mathrm{C}_{\mathrm{q}}\right)$, $164.6\left(\mathrm{C}_{\mathrm{q}}\right), 143.8\left(\mathrm{C}_{\mathrm{q}}\right), 140.7\left(\mathrm{C}_{\mathrm{q}}\right), 140.4\left(\mathrm{C}_{\mathrm{q}}\right), 135.5\left(\mathrm{C}_{\mathrm{q}}\right), 131.7(\mathrm{CH}), 129.5(\mathrm{CH})$, $129.0(\mathrm{CH}), 128.7(\mathrm{CH}), 128.6(\mathrm{CH}), 127.9(\mathrm{CH}), 125.8(\mathrm{CH}), 123.6(\mathrm{CH}), 120.6$ $(\mathrm{CH}), 111.0\left(\mathrm{C}_{\mathrm{q}}\right), 79.6(\mathrm{CH}), 62.9\left(\mathrm{CH}_{2}\right), 22.3\left(\mathrm{CH}_{3}\right)$. IR $(\mathrm{ATR}):$ 3066, 2965, 1618, 1294, 1055, 759, 707, 694, $677 \mathrm{~cm}^{-1}$. MS (EI) $\mathrm{m} / z$ (relative intensity) $356(40)\left[\mathrm{M}^{+}\right]$, 
279 (45), 238 (25), 160 (61), 119 (27), 105 (99), 77 (100). HR-MS (EI) m/z calcd for $\mathrm{C}_{23} \mathrm{H}_{20} \mathrm{~N}_{2} \mathrm{O}_{2}\left[\mathrm{M}^{+}\right]: 356.1525$, found: 356.1533 .

\section{N-[2-(5,5-Dimethyl-4,5-dihydrooxazol-2-yl)-5-methylphenyl]benzamide (1430a)}

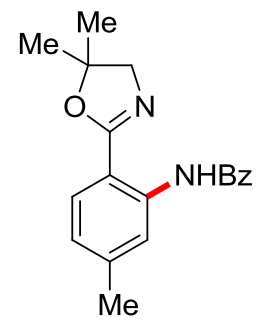

The general procedure GP1 was followed using oxazoline 1420 (95 mg, $0.50 \mathrm{mmol}$, 1.0 equiv) and dioxazolone $40 \mathrm{a}$ (98 $\mathrm{mg}, 0.60 \mathrm{mmol}, 1.2$ equiv). Purification by column chromatography on silica gel $(n$-hexane/EtOAc $=8 / 1)$ yielded 1430a (132 mg, 86\%) as a white solid. M. p. $=103-105{ }^{\circ} \mathrm{C} .{ }^{1} \mathbf{H}$ NMR $\left(400 \mathrm{MHz}, \mathrm{CDCl}_{3}\right)$ : $\delta=13.13(\mathrm{~s}, 1 \mathrm{H}), 8.87-8.81(\mathrm{~m}, 1 \mathrm{H}), 8.16-8.07(\mathrm{~m}, 2 \mathrm{H}), 7.78(\mathrm{~d}, J=8.0 \mathrm{~Hz}, 1 \mathrm{H})$, 7.58-7.45 (m, 3H), 6.93 (ddd, $J=8.0,1.7,0.8 \mathrm{~Hz}, 1 \mathrm{H}), 3.89(\mathrm{~s}, 2 \mathrm{H}), 2.44(\mathrm{~s}, 3 \mathrm{H})$, $1.50(\mathrm{~s}, 6 \mathrm{H}) .{ }^{13} \mathrm{C}$ NMR $\left(100 \mathrm{MHz}, \mathrm{CDCl}_{3}\right): \delta=166.1\left(\mathrm{C}_{\mathrm{q}}\right), 163.9\left(\mathrm{C}_{\mathrm{q}}\right), 143.3\left(\mathrm{C}_{\mathrm{q}}\right)$, $140.2\left(\mathrm{C}_{\mathrm{q}}\right), 135.6\left(\mathrm{C}_{\mathrm{q}}\right), 131.6(\mathrm{CH}), 129.2(\mathrm{CH}), 128.6(\mathrm{CH}), 127.9(\mathrm{CH}), 123.3(\mathrm{CH})$, $120.4(\mathrm{CH}), 111.7\left(\mathrm{C}_{\mathrm{q}}\right), 82.9\left(\mathrm{C}_{\mathrm{q}}\right), 66.6\left(\mathrm{CH}_{2}\right), 27.4\left(\mathrm{CH}_{3}\right), 22.2\left(\mathrm{CH}_{3}\right)$. IR $(\mathrm{ATR})$ : 3065, 2974, 2873, 1620, 1583, 1298, 1059, 695, $677 \mathrm{~cm}^{-1}$. MS (EI) $\mathrm{m} / \mathrm{z}$ (relative intensity) 308 (53) [ $\left.\mathrm{M}^{+}\right], 275$ (21), 231 (100), 160 (93), 134 (14), 105 (72), 77 (69), 51 (9). HR-MS (ESI) $m / z$ calcd for $\mathrm{C}_{19} \mathrm{H}_{21} \mathrm{~N}_{2} \mathrm{O}_{2}\left[\mathrm{M}+\mathrm{H}^{+}\right]$: 309.1598, found: 309.1598.

\section{$N$-[2-(5,6-Dihydro-4H-1,3-oxazin-2-yl)-5-fluorophenyl]benzamide (143pa)}<smiles>Cc1ccc(NC(=O)c2ccccc2)c(C2=NCCCO2)c1</smiles> 
The general procedure GP1 was followed using oxazine 142p (88 mg, $0.50 \mathrm{mmol}$, 1.0 equiv) and dioxazolone $40 \mathrm{a}$ (98 $\mathrm{mg}, 0.60 \mathrm{mmol}, 1.2$ equiv). Purification by column chromatography on silica gel ( $n$-hexane/EtOAc $=8 / 1)$ yielded 143pa $(97 \mathrm{mg}$, $66 \%)$ as a white solid. M. p. $=127-129^{\circ} \mathrm{C} .{ }^{1} \mathrm{H}$ NMR $\left(300 \mathrm{MHz}, \mathrm{CDCl}_{3}\right): \delta=13.87(\mathrm{~s}$, $1 \mathrm{H}), 8.81(\mathrm{~d}, J=8.5 \mathrm{~Hz}, 1 \mathrm{H}), 8.07-7.97(\mathrm{~m}, 2 \mathrm{H}), 7.72(\mathrm{~d}, J=2.1 \mathrm{~Hz}, 1 \mathrm{H}), 7.58-7.41$ (m, 3H), 7.27 (dd, $J=8.5,2.1 \mathrm{~Hz}, 1 \mathrm{H}$ ), 4.42 (t, $J=5.4 \mathrm{~Hz}, 2 \mathrm{H}$ ), 3.73 (t, $J=5.9 \mathrm{~Hz}$, 2H), $2.34(\mathrm{~s}, 3 \mathrm{H}), 2.04(\mathrm{tt}, J=5.9,5.4 \mathrm{~Hz}, 2 \mathrm{H}) .{ }^{13} \mathrm{C}$ NMR $\left(125 \mathrm{MHz}, \mathrm{CDCl}_{3}\right)$ : $\delta=165.7\left(\mathrm{C}_{\mathrm{q}}\right), 157.1\left(\mathrm{C}_{\mathrm{q}}\right), 137.9\left(\mathrm{C}_{\mathrm{q}}\right), 136.1\left(\mathrm{C}_{\mathrm{q}}\right), 132.3(\mathrm{CH}), 131.5\left(\mathrm{C}_{\mathrm{q}}\right), 131.4$ $(\mathrm{CH}), 128.6(\mathrm{CH}), 128.4(\mathrm{CH}), 127.6(\mathrm{CH}), 120.1(\mathrm{CH}), 118.3\left(\mathrm{C}_{\mathrm{q}}\right), 65.5\left(\mathrm{CH}_{2}\right), 42.1$ $\left(\mathrm{CH}_{2}\right), 21.7\left(\mathrm{CH}_{2}\right), 21.0\left(\mathrm{CH}_{3}\right)$. IR (ATR): 2853, 1641, 1595, 1525, 1349, 1237, 822, $701,543 \mathrm{~cm}^{-1}$. MS (EI) $\mathrm{m} / z$ (relative intensity) $294(54)\left[\mathrm{M}^{+}\right], 217$ (100), 189 (11), 160 (61), 105 (52), 77 (61). HR-MS (ESI) $m / z$ calcd for $\mathrm{C}_{18} \mathrm{H}_{19} \mathrm{~N}_{2} \mathrm{O}_{2}\left[\mathrm{M}+\mathrm{H}^{+}\right]$: 295.1441, found: 295.1444 .

\section{$N$-[2-(5,6-Dihydro-4H-1,3-oxazin-2-yl)-4-methylphenyl]benzamide (143qa)}

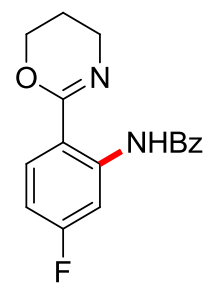

The general procedure GP1 was followed using oxazine $142 \mathrm{q}(90 \mathrm{mg}, 0.50 \mathrm{mmol}$, 1.0 equiv) and dioxazolone $40 \mathrm{a}$ (98 $\mathrm{mg}, 0.60 \mathrm{mmol}, 1.2$ equiv). Purification by column chromatography on silica gel ( $n$-hexane/EtOAc $=85 / 15$ ) yielded 143qa (106 mg, 71\%) as a white solid. M. p. $=144-147^{\circ} \mathrm{C} .{ }^{1} \mathbf{H}$ NMR $\left(400 \mathrm{MHz}, \mathrm{CDCl}_{3}\right)$ : $\delta=14.19(\mathrm{~s}, 1 \mathrm{H}), 8.75(\mathrm{dd}, J=12.1,2.7 \mathrm{~Hz}, 1 \mathrm{H}), 8.06-7.96(\mathrm{~m}, 2 \mathrm{H}), 7.89$ (dd, $J=8.9,6.6 \mathrm{~Hz}, 1 \mathrm{H}), 7.58-7.43(\mathrm{~m}, 3 \mathrm{H}), 6.74(\mathrm{ddd}, J=8.9,7.5,2.7 \mathrm{~Hz}, 1 \mathrm{H}), 4.41$ (tt, $J=5.2,0.8 \mathrm{~Hz}, 2 \mathrm{H}$ ), $3.72(\mathrm{t}, J=5.9 \mathrm{~Hz}, 2 \mathrm{H}), 2.04(\mathrm{tt}, J=5.9,5.2 \mathrm{~Hz}, 2 \mathrm{H}) .{ }^{13} \mathrm{C}$ NMR $\left(100 \mathrm{MHz}, \mathrm{CDCl}_{3}\right): \delta=166.0\left(\mathrm{C}_{\mathrm{q}}\right), 164.3\left(\mathrm{~d},{ }^{1} \mathrm{~J}_{\mathrm{C}-\mathrm{F}}=249.0 \mathrm{~Hz}, \mathrm{C}_{\mathrm{q}}\right), 156.6\left(\mathrm{C}_{\mathrm{q}}\right), 142.2$ $\left(\mathrm{d},{ }^{3} \mathrm{~J}_{\mathrm{C}-\mathrm{F}}=12.5 \mathrm{~Hz}, \mathrm{C}_{\mathrm{q}}\right), 135.5\left(\mathrm{C}_{\mathrm{q}}\right), 131.6(\mathrm{CH}), 129.8\left(\mathrm{~d},{ }^{3} \mathrm{~J}_{\mathrm{C}-\mathrm{F}}=10.2 \mathrm{~Hz}, \mathrm{CH}\right)$, $128.5(\mathrm{CH}), 127.6(\mathrm{CH}), 114.4\left(\mathrm{~d},{ }^{4} J_{\mathrm{C}-\mathrm{F}}=2.9 \mathrm{~Hz}, \mathrm{C}_{\mathrm{q}}\right), 108.9\left(\mathrm{~d},{ }^{2} J_{\mathrm{C}-\mathrm{F}}=22.1 \mathrm{~Hz}, \mathrm{CH}\right)$, 
$107.2\left(\mathrm{~d},{ }^{2} \mathrm{~J}_{\mathrm{C}-\mathrm{F}}=28.2 \mathrm{~Hz}, \mathrm{CH}\right), \quad 65.5\left(\mathrm{CH}_{2}\right), 41.9\left(\mathrm{CH}_{2}\right), 21.6\left(\mathrm{CH}_{2}\right) .{ }^{19} \mathbf{F} \mathbf{~ N M R}$ $\left(282 \mathrm{MHz}, \mathrm{CDCl}_{3}\right): \delta=-(106.36-106.53)(\mathrm{m})$. IR (ATR): 2894, 2859, 1642, 1533, 1261, 1134, 703, $676 \mathrm{~cm}^{-1}$. MS (EI) $\mathrm{m} / \mathrm{z}$ (relative intensity) 298 (34) [M+1, 221 (100), 193 (9), 164 (62), 105 (55), 77 (49), 51 (7). HR-MS (ESI) $m / z$ calcd for $\mathrm{C}_{17} \mathrm{H}_{16} \mathrm{~N}_{2} \mathrm{O}_{2} \mathrm{~F}$ $\left[\mathrm{M}+\mathrm{H}^{+}\right]: 299.1190$, found: 299.1192 .

\section{N-[1-(Pyrimidin-2-yl)-1H-indol-2-yl]benzamide (147aa)}

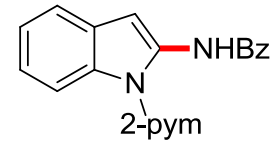

The general procedure GP2 was followed using indole substrate $36 \mathbf{a}$ (98 $\mathrm{mg}$, $0.50 \mathrm{mmol}, 1.0$ equiv) and dioxazolone $40 \mathrm{a}$ (98 $\mathrm{mg}, 0.60 \mathrm{mmol}, 1.2$ equiv). Purification by column chromatography on silica gel ( $n$-hexane/EtOAc $=2 / 1$ ) yielded 147aa (148 mg, 94\%) as a pale yellow solid. M. p. $=137-138{ }^{\circ} \mathrm{C} .{ }^{1} \mathbf{H}$ NMR $\left(300 \mathrm{MHz}, \mathrm{CDCl}_{3}\right): \delta=12.99(\mathrm{~s}, 1 \mathrm{H}), 8.63-8.59(\mathrm{~m}, 1 \mathrm{H}), 8.57-8.55(\mathrm{~m}, 2 \mathrm{H}), 7.94-$ $7.87(\mathrm{~m}, 2 \mathrm{H}), 7.55-7.41(\mathrm{~m}, 4 \mathrm{H}), 7.37(\mathrm{~s}, 1 \mathrm{H}), 7.17(\mathrm{pd}, J=7.2,1.5 \mathrm{~Hz}, 2 \mathrm{H}), 6.95(\mathrm{t}$, $J=4.8 \mathrm{~Hz}, 1 \mathrm{H}) \cdot{ }^{13} \mathrm{C}$ NMR $\left(75 \mathrm{MHz}, \mathrm{CDCl}_{3}\right): \delta=163.5\left(\mathrm{C}_{\mathrm{q}}\right), 158.6\left(\mathrm{C}_{\mathrm{q}}\right), 157.3(\mathrm{CH})$, $135.5\left(\mathrm{C}_{\mathrm{q}}\right), 134.4\left(\mathrm{C}_{\mathrm{q}}\right), 132.5\left(\mathrm{C}_{\mathrm{q}}\right), 131.7(\mathrm{CH}), 129.9\left(\mathrm{C}_{\mathrm{q}}\right), 128.7(\mathrm{CH}), 126.9(\mathrm{CH})$, $123.0(\mathrm{CH}), 122.4(\mathrm{CH}), 119.7(\mathrm{CH}), 116.5(\mathrm{CH}), 115.9(\mathrm{CH}), 95.6(\mathrm{CH})$. IR (ATR): $3015,1667,1587,1492,1348,1253,791,703,588,444 \mathrm{~cm}^{-1}$. MS (EI) $\mathrm{m} / \mathrm{z}$ (relative

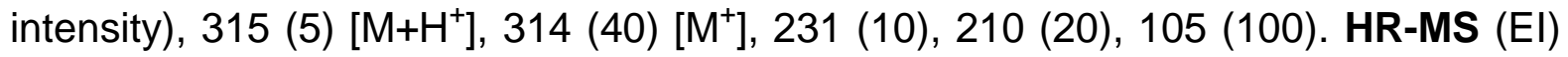
$\mathrm{m} / z$ calcd for $\mathrm{C}_{19} \mathrm{H}_{14} \mathrm{~N}_{4} \mathrm{O}\left[\mathrm{M}^{+}\right]$314.1168, found 314.1165.

\section{$N$-[3-Methyl-1-(pyrimidin-2-yl)-1H-indol-2-yl]benzamide (147ba)}

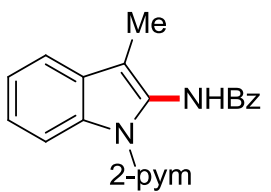


The general procedure GP2 was followed using indole $36 \mathrm{~b}(105 \mathrm{mg}, 0.50 \mathrm{mmol}$, 1.0 equiv), dioxazolone $40 \mathrm{a}$ (98 mg, $0.60 \mathrm{mmol}, 1.2$ equiv), [ $\mathrm{Cp}{ }^{*} \mathrm{Co}(\mathrm{CO}) \mathrm{I}_{2}$ ] $(11.9 \mathrm{mg}$, $0.025 \mathrm{mmol}, 5.0 \mathrm{~mol} \%), \mathrm{AgSbF}_{6}(17 \mathrm{mg}, 0.050 \mathrm{mmol}, 10 \mathrm{~mol} \%)$ and $\mathrm{NaOAc}$ $\left(4.1 \mathrm{mg}, 0.050 \mathrm{mmol}, 10 \mathrm{~mol} \%\right.$ ) at $100^{\circ} \mathrm{C}$. Purification by column chromatography on silica gel ( $n$-hexane/EtOAc $=8: 2 \rightarrow 6: 4)$ yielded $147 \mathrm{ba}(106 \mathrm{mg}, 65 \%)$ as a white solid. (Without NaOAc: $101 \mathrm{mg}, 61 \%$.) M. p. $=159-160{ }^{\circ} \mathrm{C} .{ }^{1} \mathrm{H} \mathrm{NMR}(300 \mathrm{MHz}$, $\left.\mathrm{CDCl}_{3}\right): \delta=10.88(\mathrm{~s}, 1 \mathrm{H}), 8.70(\mathrm{~d}, J=4.8 \mathrm{~Hz}, 2 \mathrm{H}), 8.56-8.46(\mathrm{~m}, 1 \mathrm{H}), 7.99(\mathrm{~d}$, $J=6.9 \mathrm{~Hz}, 2 \mathrm{H}), 7.61-7.43(\mathrm{~m}, 4 \mathrm{H}), 7.34-7.20(\mathrm{~m}, 2 \mathrm{H}), 7.04(\mathrm{t}, J=4.8 \mathrm{~Hz}, 1 \mathrm{H}), 2.31$ (s, 3H). ${ }^{13} \mathrm{C}$ NMR $\left(125 \mathrm{MHz}, \mathrm{CDCl}_{3}\right): \delta=158.1\left(\mathrm{C}_{\mathrm{q}}\right), 157.9(\mathrm{CH}), 157.9\left(\mathrm{C}_{\mathrm{q}}\right), 134.5$ $\left(\mathrm{C}_{\mathrm{q}}\right), 134.2\left(\mathrm{C}_{\mathrm{q}}\right), 132.0(\mathrm{CH}), 130.0\left(\mathrm{C}_{\mathrm{q}}\right), 129.1\left(\mathrm{C}_{\mathrm{q}}\right), 128.8(\mathrm{CH}), 127.5(\mathrm{CH}), 123.6$ $(\mathrm{CH}), 122.5(\mathrm{CH}), 118.5(\mathrm{CH}), 116.1(\mathrm{CH}), 114.9(\mathrm{CH}), 110.3\left(\mathrm{C}_{\mathrm{q}}\right), 10.2\left(\mathrm{CH}_{3}\right)$. IR (ATR): 1673, 1562, 1503, 1429, 1272, 740, 710, $624 \mathrm{~cm}^{-1}$. MS (EI) $\mathrm{m} / \mathrm{z}$ (relative intensity) 328 (58) [M+], 223 (95), 207 (26), 153 (12) 105 (100), 77 (51), 44 (55). HR-MS (ESI) $m / z$ calcd for $\mathrm{C}_{20} \mathrm{H}_{17} \mathrm{~N}_{4} \mathrm{O}\left[\mathrm{M}+\mathrm{H}^{+}\right]$: 329.1397, found: 329.1396.

\section{Methyl 2-Benzamido-1-(pyridin-2-yl)-1H-indole-6-carboxylate (146ca)}

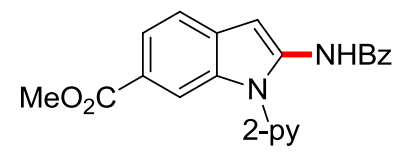

The general procedure GP2 was followed using indole 28c $(126 \mathrm{mg}, 0.50 \mathrm{mmol}$, 1.0 equiv) and dioxazolone $2 \mathrm{a}$ ( $98 \mathrm{mg}, 0.60 \mathrm{mmol}, 1.2$ equiv). Purification by column chromatography on silica gel ( $n$-hexane/EtOAc $=3: 1 \rightarrow 3: 2)$ yielded 146ca $(169 \mathrm{mg}$, $91 \%)$ as an off-white solid. M. p. $=180-183{ }^{\circ} \mathrm{C} .{ }^{1} \mathbf{H}$ NMR $\left(500 \mathrm{MHz}, \mathrm{CDCl}_{3}\right)$ : $\delta=11.95(\mathrm{~s}, 1 \mathrm{H}), 8.67(\mathrm{ddd}, J=5.0,2.0,0.9 \mathrm{~Hz}, 1 \mathrm{H}), 8.32(\mathrm{dt}, J=1.5,0.8 \mathrm{~Hz}, 1 \mathrm{H})$, 8.02 (ddd, $J=8.3,7.5,1.9 \mathrm{~Hz}, 1 \mathrm{H}), 7.97-7.87(\mathrm{~m}, 3 \mathrm{H}), 7.82(\mathrm{dt}, J=8.2,0.9 \mathrm{~Hz}, 1 \mathrm{H})$, 7.63 (dd, $J=8.2,0.6 \mathrm{~Hz}, 1 \mathrm{H}$ ), 7.57 (ddt, $J=8.2,6.4,1.3 \mathrm{~Hz}, 1 \mathrm{H}$ ), 7.52 (ddt, $J=8.2$, 6.6, $1.4 \mathrm{~Hz}, 2 \mathrm{H}), 7.39-7.32(\mathrm{~m}, 2 \mathrm{H}), 3.93(\mathrm{~s}, 3 \mathrm{H}) .{ }^{13} \mathrm{C} \mathrm{NMR}\left(125 \mathrm{MHz}, \mathrm{CDCl}_{3}\right)$ : $\delta=168.0\left(\mathrm{C}_{\mathrm{q}}\right), 163.9\left(\mathrm{C}_{\mathrm{q}}\right), 151.7\left(\mathrm{C}_{\mathrm{q}}\right), 148.4(\mathrm{CH}), 140.1(\mathrm{CH}), 137.9\left(\mathrm{C}_{\mathrm{q}}\right), 134.1$ $\left(\mathrm{C}_{\mathrm{q}}\right), 133.9\left(\mathrm{C}_{\mathrm{q}}\right), 132.3(\mathrm{CH}), 131.5\left(\mathrm{C}_{\mathrm{q}}\right), 129.0(\mathrm{CH}), 127.3(\mathrm{CH}), 123.6(\mathrm{CH}), 123.4$ $\left(\mathrm{C}_{\mathrm{q}}\right), 121.5(\mathrm{CH}), 120.1(\mathrm{CH}), 118.5(\mathrm{CH}), 112.6(\mathrm{CH}), 93.9(\mathrm{CH}), 52.1\left(\mathrm{CH}_{3}\right)$. 
IR (ATR): 3059, 2952, 1703, 1673, 1530, 1436, 1262, 1219, 998, $786 \mathrm{~cm}^{-1}$. MS (EI) m/z (relative intensity) 371 (34) [M+], 281 (17), 253 (8), 207 (54), 105 (100), 77 (30), 44 (18). HR-MS (ESI) $\mathrm{m} / z$ calcd for $\mathrm{C}_{22} \mathrm{H}_{18} \mathrm{~N}_{3} \mathrm{O}_{3}\left[\mathrm{M}+\mathrm{H}^{+}\right]$: 372.1343 , found: 372.1332 .

\section{N-[4-Methoxy-1-(pyridin-2-yl)-1 H-indol-2-yl]benzamide (146da)}

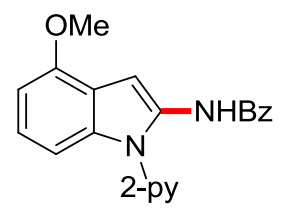

The general procedure GP2 was followed using indole $28 \mathrm{~d}(112 \mathrm{mg}, 0.50 \mathrm{mmol}$, 1.0 equiv) and dioxazolone $40 \mathrm{a}$ (98 $\mathrm{mg}, 0.60 \mathrm{mmol}, 1.2$ equiv). Purification by column chromatography on silica gel $(n$-hexane/EtOAc $=3 / 1)$ yielded 146da $(165 \mathrm{mg}, 96 \%)$ as a pale yellow solid. M. p. $=152-154{ }^{\circ} \mathrm{C} .{ }^{1} \mathbf{H} \mathbf{N M R}(500 \mathrm{MHz}$, $\left.\mathrm{CDCl}_{3}\right): \delta=11.75(\mathrm{~s}, 1 \mathrm{H}), 8.61$ (ddd, $\left.J=4.9,2.0,0.9 \mathrm{~Hz}, 1 \mathrm{H}\right), 7.97-7.86(\mathrm{~m}, 3 \mathrm{H})$, $7.75(\mathrm{dt}, J=8.3,1.0 \mathrm{~Hz}, 1 \mathrm{H}), 7.58-7.45(\mathrm{~m}, 3 \mathrm{H}), 7.41(\mathrm{~s}, 1 \mathrm{H}), 7.30-7.18(\mathrm{~m}, 2 \mathrm{H})$, $7.11(\mathrm{t}, J=8.1 \mathrm{~Hz}, 1 \mathrm{H}), 6.66(\mathrm{dd}, J=7.9,0.6 \mathrm{~Hz}, 1 \mathrm{H}), 3.98(\mathrm{~s}, 3 \mathrm{H}) .{ }^{13} \mathrm{C}$ NMR $\left(125 \mathrm{MHz}, \mathrm{CDCl}_{3}\right): \delta=163.5\left(\mathrm{C}_{\mathrm{q}}\right), 153.4\left(\mathrm{C}_{\mathrm{q}}\right), 152.2\left(\mathrm{C}_{\mathrm{q}}\right), 148.2(\mathrm{CH}), 139.5(\mathrm{CH})$, $134.5\left(\mathrm{C}_{\mathrm{q}}\right), 133.5\left(\mathrm{C}_{\mathrm{q}}\right), 133.2\left(\mathrm{C}_{\mathrm{q}}\right), 131.9(\mathrm{CH}), 128.8(\mathrm{CH}), 127.1(\mathrm{CH}), 122.7(\mathrm{CH})$, $120.9(\mathrm{CH}), 119.9\left(\mathrm{C}_{\mathrm{q}}\right), 118.2(\mathrm{CH}), 104.0(\mathrm{CH}), 102.7(\mathrm{CH}), 91.2(\mathrm{CH}), 55.7\left(\mathrm{CH}_{3}\right)$. IR (ATR): 3057, 2954, 1666, 1538, 1470, 1437, 1250, 1090, 764, $686 \mathrm{~cm}^{-1}$. MS (EI) m/z (relative intensity) 343 (69) [M+], 281 (6), 238 (27), 207 (20), 169 (7), 105 (100), 77 (40), 44 (11). HR-MS (ESI) $\mathrm{m} / z$ calcd for $\mathrm{C}_{21} \mathrm{H}_{18} \mathrm{~N}_{3} \mathrm{O}_{2}\left[\mathrm{M}+\mathrm{H}^{+}\right]$: 344.1394, found: 344.1394 . 


\section{N-[1-(4-Methylpyridin-2-yl)-1 H-indol-2-yl]benzamide (146ea)}

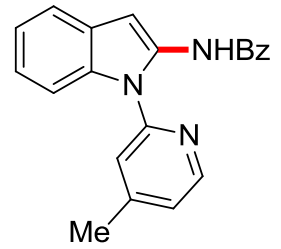

The general procedure GP2 was followed using indole $28 \mathrm{e}(104 \mathrm{mg}, 0.50 \mathrm{mmol}$, 1.0 equiv) and dioxazolone $40 \mathrm{a}$ (98 $\mathrm{mg}, 0.60 \mathrm{mmol}, 1.2$ equiv). Purification by column chromatography on silica gel ( $n$-hexane/EtOAc $=5 / 1$ ) yielded 146ea $(159 \mathrm{mg}, 97 \%)$ as an off-white solid. M. p. $=166-169{ }^{\circ} \mathrm{C} .{ }^{1} \mathbf{H} \mathrm{NMR}(500 \mathrm{MHz}$, $\left.\mathrm{CDCl}_{3}\right): \delta=11.92(\mathrm{~s}, 1 \mathrm{H}), 8.52-8.46(\mathrm{~m}, 1 \mathrm{H}), 7.97-7.91(\mathrm{~m}, 2 \mathrm{H}), 7.66-7.58(\mathrm{~m}, 3 \mathrm{H})$, 7.58-7.50 (m, 2H), 7.50-7.46 (m, 1H), $7.29(\mathrm{~s}, 1 \mathrm{H}), 7.28-7.16(\mathrm{~m}, 2 \mathrm{H}), 7.11$ (ddd, $J=5.2,1.5,0.8 \mathrm{~Hz}, 1 \mathrm{H}), 2.49(\mathrm{~s}, 3 \mathrm{H}) .{ }^{13} \mathrm{C}$ NMR $\left(125 \mathrm{MHz}, \mathrm{CDCl}_{3}\right): \delta=163.8\left(\mathrm{C}_{\mathrm{q}}\right)$, $152.2\left(\mathrm{C}_{\mathrm{q}}\right), 151.4\left(\mathrm{C}_{\mathrm{q}}\right), 147.9(\mathrm{CH}), 135.1\left(\mathrm{C}_{\mathrm{q}}\right), 134.4\left(\mathrm{C}_{\mathrm{q}}\right), 132.0(\mathrm{CH}), 132.0\left(\mathrm{C}_{\mathrm{q}}\right)$, $129.8\left(\mathrm{C}_{\mathrm{q}}\right), 128.9(\mathrm{CH}), 127.2(\mathrm{CH}), 122.2(\mathrm{CH}), 122.1(\mathrm{CH}), 121.7(\mathrm{CH}), 120.8(\mathrm{CH})$, $118.6(\mathrm{CH}), 110.7(\mathrm{CH}), 93.6(\mathrm{CH}), 21.6\left(\mathrm{CH}_{3}\right)$. IR $(\mathrm{ATR}):$ 3228, 3044, 1672, 1523, 1459, 1259, 805, 685, 636, $447 \mathrm{~cm}^{-1}$. MS (EI) $\mathrm{m} / z$ (relative intensity) $327(60)\left[\mathrm{M}^{+}\right]$, 222 (26), 195 (26), 105 (100), 77 (41), 44 (33). HR-MS (ESI) m/z calcd for $\mathrm{C}_{21} \mathrm{H}_{18} \mathrm{~N}_{3} \mathrm{O}\left[\mathrm{M}+\mathrm{H}^{+}\right]: 328.1444$, found: 328.1447.

\section{$N$-[1-(5-Methylpyridin-2-yl)-1H-indol-2-yl]benzamide (146ba)}

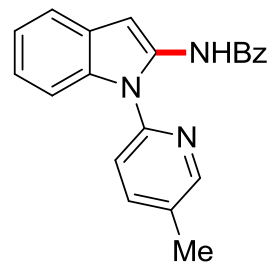

The general procedure GP2 was followed using indole 28b $(104 \mathrm{mg}, 0.50 \mathrm{mmol}$, 1.0 equiv) and dioxazolone 40a (98 $\mathrm{mg}, 0.60 \mathrm{mmol}, 1.2$ equiv). Purification by column chromatography on silica gel ( $n$-hexane/EtOAc $=5 / 1$ ) yielded $146 \mathrm{ba}$ $(151 \mathrm{mg}, 92 \%)$ as a pale yellow solid. M. p. $=171-173{ }^{\circ} \mathrm{C} .{ }^{1} \mathbf{H} \mathbf{N M R}(300 \mathrm{MHz}$, $\left.\mathrm{CDCl}_{3}\right): \delta=11.80(\mathrm{~s}, 1 \mathrm{H}), 8.45(\mathrm{dp}, J=2.3,0.8 \mathrm{~Hz}, 1 \mathrm{H}), 8.00-7.86(\mathrm{~m}, 2 \mathrm{H}), 7.75$ 
(ddd, $J=8.4,2.4,0.7 \mathrm{~Hz}, 1 \mathrm{H}), 7.67(\mathrm{dd}, J=8.4,0.8 \mathrm{~Hz}, 1 \mathrm{H}), 7.65-7.61(\mathrm{~m}, 1 \mathrm{H})$, 7.60-7.47 (m, 4H), $7.28(\mathrm{t}, J=0.5 \mathrm{~Hz}, 1 \mathrm{H}), 7.24-7.13(\mathrm{~m}, 2 \mathrm{H}), 2.44(\mathrm{~s}, 3 \mathrm{H})$. ${ }^{13} \mathrm{C}$ NMR $\left(125 \mathrm{MHz}, \mathrm{CDCl}_{3}\right): \delta=163.6\left(\mathrm{C}_{\mathrm{q}}\right), 149.9\left(\mathrm{C}_{\mathrm{q}}\right), 148.2(\mathrm{CH}), 140.1(\mathrm{CH})$, $134.9\left(\mathrm{C}_{\mathrm{q}}\right), 134.4\left(\mathrm{C}_{\mathrm{q}}\right), 132.0\left(\mathrm{C}_{\mathrm{q}}\right), 131.9(\mathrm{CH}), 130.7\left(\mathrm{C}_{\mathrm{q}}\right), 129.6\left(\mathrm{C}_{\mathrm{q}}\right), 128.8(\mathrm{CH})$, $127.2(\mathrm{CH}), 121.9(\mathrm{CH}), 121.7(\mathrm{CH}), 120.7(\mathrm{CH}), 117.6(\mathrm{CH}), 110.5(\mathrm{CH}), 93.5(\mathrm{CH})$, $18.2\left(\mathrm{CH}_{3}\right)$. IR (ATR): 3183, 3049, 1683, 1539, 1477, 1455, 783, 690, 650, $637 \mathrm{~cm}^{-1}$. MS (EI) $m / z$ (relative intensity) $327(60)\left[\mathrm{M}^{+}\right], 281$ (24), 253 (13), 207 (76), 105 (100), 77 (35), 44 (22). HR-MS (ESI) $\mathrm{m} / z$ calcd for $\mathrm{C}_{21} \mathrm{H}_{18} \mathrm{~N}_{3} \mathrm{O}\left[\mathrm{M}+\mathrm{H}^{+}\right]$: 328.1444 , found: 328.1444 . 


\subsubsection{Mechanistic Studies}

\section{Reactions with Radical Scavengers}

142a (81 mg, $0.50 \mathrm{mmol}, \quad 1.0$ equiv), $\quad 40 \mathrm{a} \quad(98 \mathrm{mg}, \quad 0.60 \mathrm{mmol}, \quad 1.2$ equiv), $\mathrm{Cp}^{*} \mathrm{Co}(\mathrm{CO}) \mathrm{I}_{2} \quad(11.9 \mathrm{mg}, \quad 0.025 \mathrm{mmol}, 5.0 \mathrm{~mol} \%), \mathrm{AgSbF}_{6} \quad(34 \mathrm{mg}, 0.10 \mathrm{mmol}$, $20 \mathrm{~mol} \%), \mathrm{NaOAc}(8.2 \mathrm{mg}, 0.10 \mathrm{mmol}, 20 \mathrm{~mol} \%)$ and the radical scavenger were placed into an oven-dried $25 \mathrm{~mL}$ Schlenk tube equipped with a septum under $\mathrm{N}_{2}$ atmosphere. DCE $(2.0 \mathrm{~mL})$ was introduced via cannula. The reaction mixture was stirred at $100{ }^{\circ} \mathrm{C}$ for $16 \mathrm{~h}$. After cooling to ambient temperature, the reaction mixture was dry loaded onto silica gel and purified by flash column chromatography ( $n$-hexane/EtOAc $=7 / 1)$ to yield the product 143aa as an off-white solid.

Table 5.1. Effect of radical scavengers on the cobalt-catalyzed $\mathrm{C}-\mathrm{H}$ amidation.

\begin{tabular}{ccc}
\hline Entry & Radical scavenger & Yield [\%] $^{[\mathrm{a}]}$ \\
\hline 1 & none & 68 \\
3 & TEMPO (1.0 equiv) & 46 \\
4 & TEMPO (2.0 equiv) & 49 \\
5 & BHT (1.0 equiv) & 48 \\
6 & $(E)$-stilbene (1.0 equiv) & 63 \\
7 & $\mathrm{Ph}_{2} \mathrm{C}=\mathrm{CH}_{2}(1.0$ equiv) & 67 \\
\hline
\end{tabular}

${ }^{[a]}$ Isolated yield.

\section{Mercury Poisoning Test}

142a (81 mg, $0.50 \mathrm{mmol}, \quad 1.0$ equiv), $\quad 40 \mathrm{a} \quad(98 \mathrm{mg}, \quad 0.60 \mathrm{mmol}, \quad 1.2$ equiv), $\mathrm{Cp}^{*} \mathrm{Co}(\mathrm{CO}) \mathrm{I}_{2} \quad(12 \mathrm{mg}, \quad 0.025 \mathrm{mmol}, \quad 5.0 \mathrm{~mol} \%), \mathrm{AgSbF}_{6} \quad(34 \mathrm{mg}, \quad 0.10 \mathrm{mmol}$, 
$20 \mathrm{~mol} \%$ ), and $\mathrm{NaOAc}(8.2 \mathrm{mg}, 0.10 \mathrm{mmol}, 20 \mathrm{~mol} \%$ ) were placed into a $25 \mathrm{~mL}$ Schlenk tube equipped with a septum under $\mathrm{N}_{2}$ atmosphere. DCE $(2.0 \mathrm{~mL})$ was introduced via cannula. The reaction mixture was stirred at $100{ }^{\circ} \mathrm{C}$ for $2.5 \mathrm{~min}$ and mercury (100 mg, $0.50 \mathrm{mmol}, 1.0$ equiv) was added via syringe. At this point, no detectable formation of 143aa was observed by GC-MS analysis of an aliquot of the reaction mixture. The reaction mixture was stirred at $100^{\circ} \mathrm{C}$ for $16 \mathrm{~h}$. After cooling to ambient temperature, the reaction mixture was dry loaded onto silica gel and purified by flash column chromatography $(n$-hexane/EtOAc $=7 / 1$ ) to yield the product 143aa (92 $\mathrm{mg}, 66 \%$ ) as a white solid. 


\subsection{Synthesis of Novel Chiral Ligands}

\subsubsection{Experimental Procedures and Analytical Data of Novel Chiral NHC Precursors}

(4R,5R)-1,3-Bis(2,6-dimethylphenyl)-4,5-diphenyl-4,5-dihydro-1 H-imidazol-3ium Tetrafluoroborate (155)

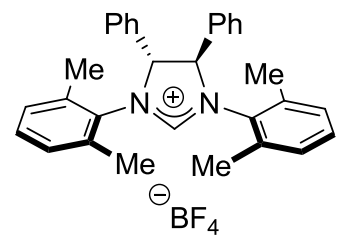

The general procedure GP4 was followed using $(R, R)$-diphenylethylenediamine (200 mg, $0.94 \mathrm{mmol}, \quad 1.0$ equiv), $\mathrm{Pd}(\mathrm{OAc})_{2} \quad(21.1 \mathrm{mg}, \quad 94 \mu \mathrm{mol}, \quad 10 \mathrm{~mol} \%)$, (+/-)-BINAP (117 mg, 188 umol, 20 mol \%), NaOtBu (271 mg, 2.82 mmol, 3.0 equiv) and 2-bromo-1,3-dimethylbenzene (366 mg, $1.98 \mathrm{mmol}, 2.1$ equiv) in PhMe (20 mL). Short column chromatography ( $n$-hexane/ $\mathrm{CH}_{2} \mathrm{Cl}_{2}=3 / 2 \rightarrow 1 / 1$ ) yielded the crude diarylated diamine (285 mg, 72\%). The crude $N, N^{\prime}$-diarylated diamine (280 mg, $0.67 \mathrm{mmol}, 1.0$ equiv) was treated with ammonium tetrafluoroborate (84 mg, $0.80 \mathrm{mmol}, 1.2$ equiv) and triethyl orthoformate $(1.0 \mathrm{~mL})$. Purification by column chromatography $\left(\mathrm{CH}_{2} \mathrm{Cl}_{2} /\right.$ acetone $\left.=9 / 1 \rightarrow 4 / 1\right)$ yielded $155(277 \mathrm{mg}, 80 \%)$ as a white solid. M. p. $=149-151^{\circ} \mathrm{C} .{ }^{1} \mathbf{H}$ NMR $\left(300 \mathrm{MHz}, \mathrm{CDCl}_{3}\right): \delta=8.78(\mathrm{~s}, 1 \mathrm{H}), 7.44-7.32$ (m, 10H), $7.18(\mathrm{~d}, J=3.9 \mathrm{~Hz}, 4 \mathrm{H}), 6.94(\mathrm{dd}, J=6.7,2.7 \mathrm{~Hz}, 2 \mathrm{H}), 6.04(\mathrm{~d}, J=0.7 \mathrm{~Hz}$, 2H), $2.72(\mathrm{~s}, 6 \mathrm{H}), 2.00(\mathrm{~s}, 6 \mathrm{H}) .{ }^{13} \mathrm{C}$ NMR $\left(125 \mathrm{MHz}, \mathrm{CDCl}_{3}\right): \delta=158.2(\mathrm{CH}), 136.6$ $\left(\mathrm{C}_{\mathrm{q}}\right), 134.5\left(\mathrm{C}_{\mathrm{q}}\right), 131.2\left(\mathrm{C}_{\mathrm{q}}\right), 131.1(\mathrm{CH}), 130.8(\mathrm{CH}), 130.3(\mathrm{CH}), 129.5(\mathrm{CH}), 129.5$ $(\mathrm{CH}), 129.4(\mathrm{CH}), 128.8(\mathrm{CH}), 72.9(\mathrm{CH}), 19.1\left(\mathrm{CH}_{3}\right), 18.4\left(\mathrm{CH}_{3}\right),{ }^{19} \mathrm{~F}$ NMR $\left(282 \mathrm{MHz}, \mathrm{CDCl}_{3}\right): \delta=-152.84(\mathrm{~s}),-152.90(\mathrm{dd}, J=2.5,0.8 \mathrm{~Hz})$. IR (ATR): 3059, 1613, 1222, 1050, 1031, $699 \mathrm{~cm}^{-1}$. MS (ESI) $\mathrm{m} / z$ (relative intensity): 431 (100) $\left[\mathrm{M}-\mathrm{BF}_{4}\right]^{+}$. HR-MS (ESI) $\mathrm{m} / z$ calcd for $\mathrm{C}_{31} \mathrm{H}_{31} \mathrm{~N}_{2}\left[\mathrm{M}-\mathrm{BF}_{4}\right]^{+}$431.2482, found 431.2479. $[\alpha]_{\mathrm{D}}^{20}:+303.1\left(\mathrm{c}=1.05, \mathrm{CHCl}_{3}\right)$. 
The analytical data are in accordance with those previously reported in the literature. ${ }^{[328]}$

(4R,5R)-1,3-Bis(2,5-dimethylphenyl)-4,5-diphenyl-4,5-dihydro-1 H-imidazol-3ium Tetrafluoroborate (176)

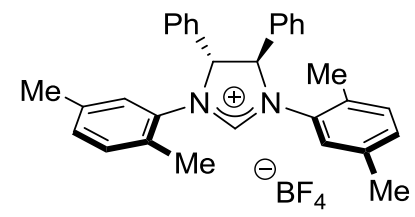

The general procedure GP4 was followed using $(R, R)$-diphenylethylenediamine (150 mg, $\quad 0.71 \mathrm{mmol}, \quad 1.0$ equiv), $\mathrm{Pd}(\mathrm{OAc})_{2} \quad(7.9 \mathrm{mg}, \quad 35 \mu \mathrm{mol}, \quad 5.0 \mathrm{~mol} \%)$, (+/-)-BINAP (44 mg, $71 \mu \mathrm{mol}, 10 \mathrm{~mol} \%$ ), NaOtBu (204 mg, $2.12 \mathrm{mmol}, 3.0$ equiv) and 2-bromo-1,4-dimethylbenzene ( $275 \mathrm{mg}, 1.48 \mathrm{mmol}, 2.1$ equiv) in PhMe (15 mL). Short column chromatography ( $n$-hexane/ $\mathrm{CH}_{2} \mathrm{Cl}_{2}=2 / 1$ ) yielded the crude diarylated diamine (300 mg, quantitative). The crude $N, N^{\prime}$-diarylated diamine (300 mg, $0.71 \mathrm{mmol}, 1.0$ equiv) was treated with ammonium tetrafluoroborate (90 mg, $0.86 \mathrm{mmol}, 1.2$ equiv) and triethyl orthoformate $(1.5 \mathrm{~mL})$. Purification by column chromatography $\left(\mathrm{CH}_{2} \mathrm{Cl}_{2} /\right.$ acetone $\left.=7 / 1\right)$ yielded $176(313 \mathrm{mg}, 85 \%)$ as a white solid. M. p. $=125-127{ }^{\circ} \mathrm{C} .{ }^{1} \mathrm{H}$ NMR $\left(400 \mathrm{MHz}, \mathrm{CDCl}_{3}\right): \delta=8.45(d, J=0.7 \mathrm{~Hz}, 1 \mathrm{H}), 7.44-$ $7.32(\mathrm{~m}, 10 \mathrm{H}), 7.28(\mathrm{t}, J=1.1 \mathrm{~Hz}, 2 \mathrm{H}), 7.09-6.98(\mathrm{~m}, 4 \mathrm{H}), 5.78(\mathrm{~d}, J=0.7 \mathrm{~Hz}, 2 \mathrm{H})$, $2.39(\mathrm{~s}, 6 \mathrm{H}), 2.24(\mathrm{~s}, 6 \mathrm{H}) .{ }^{13} \mathrm{C}$ NMR $\left(100 \mathrm{MHz}, \mathrm{CDCl}_{3}\right): \delta=157.4(\mathrm{CH}), 138.0\left(\mathrm{C}_{\mathrm{q}}\right)$, $133.7\left(\mathrm{C}_{\mathrm{q}}\right), 132.4\left(\mathrm{C}_{\mathrm{q}}\right), 131.4(\mathrm{CH}), 130.9(\mathrm{CH}), 130.3(\mathrm{CH}), 129.8\left(\mathrm{C}_{\mathrm{q}}\right), 129.7(\mathrm{CH})$, $128.1(\mathrm{CH}), 127.9(\mathrm{CH}), 76.1(\mathrm{CH}), 20.6\left(\mathrm{CH}_{3}\right), 17.9\left(\mathrm{CH}_{3}\right) .{ }^{19} \mathrm{~F}$ NMR $(282 \mathrm{MHz}$, $\left.\mathrm{CDCl}_{3}\right): \delta=-151.31(\mathrm{~s}),-151.36$ (d, $J=2.5 \mathrm{~Hz}$ ). IR (ATR): 3066, 1613, 1049, 1035, 756, $700 \mathrm{~cm}^{-1}$. MS (ESI) $\mathrm{m} / \mathrm{z}$ (relative intensity): 431 (100) [M-BF $]^{+}$. HR-MS (ESI)

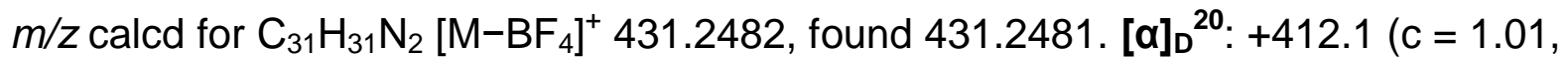
$\left.\mathrm{CHCl}_{3}\right)$. 


\section{(4R,5R)-1,3-Bis(5-isopropyl-2-methylphenyl)-4,5-diphenyl-4,5-dihydro-1 H-}

\section{imidazol-3-ium Tetrafluoroborate (177)}

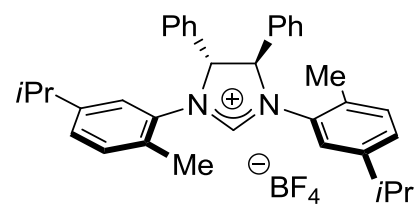

Under inert atmosphere, an oven-dried $100 \mathrm{~mL}$ Schlenk flask was charged with $(R, R)$-diphenylethylenediamine (350 mg, $1.65 \mathrm{mmol}, 1.0$ equiv), $\mathrm{Pd}(\mathrm{dba})_{2}$ (95 mg, $0.16 \mathrm{mmol}, 10 \mathrm{~mol} \%$ ), dppf (183 mg, $0.33 \mathrm{mmol}, 20 \mathrm{~mol} \%$ ) and $\mathrm{NaOtBu}(475 \mathrm{mg}$, $4.94 \mathrm{mmol}, 3.0$ equiv). The flask was evacuated and backfilled with nitrogen 3 times. PhMe $(20 \mathrm{~mL})$ and freshly prepared 5-isopropyl-2-methylphenyl trifluoromethanesulfonate ${ }^{[329]}$ (980 mg, $3.46 \mathrm{mmol}, 2.1$ equiv) were then added via syringe. The flask was then placed in a pre-heated oil bath at $100^{\circ} \mathrm{C}$, and stirred for $16 \mathrm{~h}$. The solution was then cooled to ambient temperature, diluted with $n$-hexane $(60 \mathrm{~mL})$, filtered through a plug of silica and washed with $n$-hexane $/ \mathrm{CH}_{2} \mathrm{Cl}_{2}=1 / 1$. The filtrate was concentrated under reduced pressure. Short column chromatography ( $n$-hexane/ $\mathrm{CH}_{2} \mathrm{Cl}_{2}=4 / 1$ ) yielded the crude $N, N^{\prime}$-diarylated diamine as a pale yellow foam (605 mg, $78 \%)$. The crude $N, N^{\prime}$-diarylated diamine $(600 \mathrm{mg}$, $1.28 \mathrm{mmol}, 1.0$ equiv), ammonium tetrafluoroborate (161 mg, $1.54 \mathrm{mmol}, 1.2$ equiv), and triethyl orthoformate $(5.0 \mathrm{~mL})$ were stirred at $120^{\circ} \mathrm{C}$ for $5 \mathrm{~h}$. The solution was then allowed to cool to ambient temperature. The reaction mixture was dry loaded onto silica gel and purified by column chromatography $\left(\mathrm{CH}_{2} \mathrm{Cl}_{2} /\right.$ acetone = $15 / 1 \rightarrow 10 / 1)$. The obtained product was washed with $\mathrm{Et}_{2} \mathrm{O}$ until colorless to yield 177 $(529 \mathrm{mg}, 72 \%)$ as a colorless powder. M. p. $=133-135{ }^{\circ} \mathrm{C} .{ }^{1} \mathbf{H} \mathbf{~ N M R}(500 \mathrm{MHz}$, $\left.\mathrm{CDCl}_{3}\right): \delta=8.52(\mathrm{~s}, 1 \mathrm{H}), 7.45-7.35(\mathrm{~m}, 10 \mathrm{H}), 7.25(\mathrm{~d}, J=1.7 \mathrm{~Hz}, 2 \mathrm{H}), 7.10$ (qd, $J=8.0,1.4 \mathrm{~Hz}, 4 \mathrm{H}$ ), 5.81 (s, 2H), 2.82 (hept, $J=6.9 \mathrm{~Hz}, 2 \mathrm{H}$ ), 2.44 (s, 6H), 1.11 (dd, $J=6.9,6.2 \mathrm{~Hz}, 12 \mathrm{H}) .{ }^{13} \mathrm{C}$ NMR $\left(125 \mathrm{MHz}, \mathrm{CDCl}_{3}\right): \delta=157.1(\mathrm{CH}), 149.0\left(\mathrm{C}_{\mathrm{q}}\right), 133.6$ $\left(\mathrm{C}_{\mathrm{q}}\right), 132.4\left(\mathrm{C}_{\mathrm{q}}\right), 131.4(\mathrm{CH}), 130.3(\mathrm{CH}), 130.0\left(\mathrm{C}_{\mathrm{q}}\right), 129.6(\mathrm{CH}), 128.3(\mathrm{CH}), 128.2$ $(\mathrm{CH}), 125.9(\mathrm{CH}), 76.0(\mathrm{CH}), 33.3(\mathrm{CH}), 23.6\left(\mathrm{CH}_{3}\right), 23.4\left(\mathrm{CH}_{3}\right), 17.9\left(\mathrm{CH}_{3}\right)$. ${ }^{19} \mathrm{~F}$ NMR $\left(282 \mathrm{MHz}, \mathrm{CDCl}_{3}\right): \delta=-151.20(\mathrm{~s}),-151.25$ (dd, $\left.J=2.1,0.9 \mathrm{~Hz}\right)$. IR (ATR): 2962, 1624, 1613, 1212, 1049, $701 \mathrm{~cm}^{-1}$. MS (ESI) $\mathrm{m} / \mathrm{z}$ (relative intensity): 
487 (100) $\left[\mathrm{M}-\mathrm{BF}_{4}\right]^{+}$. HR-MS (ESI) $\mathrm{m} / z$ calcd for $\mathrm{C}_{35} \mathrm{H}_{39} \mathrm{~N}_{2}\left[\mathrm{M}-\mathrm{BF}_{4}\right]^{+} 487.3108$, found 487.3106. [a] $]_{\mathrm{D}}^{20}:+379.9\left(\mathrm{c}=1.03, \mathrm{CHCl}_{3}\right)$.

(4R,5R)-1,3-Bis[5-(tert-butyl)-2-methylphenyl]-4,5-diphenyl-4,5-dihydro-1 Himidazol-3-ium Tetrafluoroborate (178)

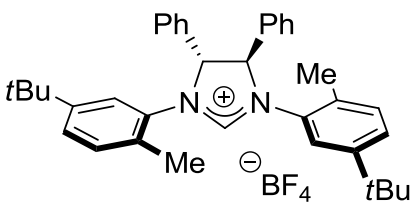

The general procedure GP4 was followed using $(R, R)$-diphenylethylenediamine (100 mg, $\quad 0.47 \mathrm{mmol}, \quad 1.0$ equiv), $\mathrm{Pd}(\mathrm{OAc})_{2} \quad$ (5.3 mg, $24 \mu \mathrm{mol}, \quad 5.0 \mathrm{~mol} \%$ ), (+/-)-BINAP (29 mg, $47 \mu \mathrm{mol}, 10 \mathrm{~mol} \%$ ), NaOtBu (136 mg, $1.42 \mathrm{mmol}, 3.0$ equiv) and 2-bromo-4-(tert-butyl)-1-methylbenzene ${ }^{[330]}$ (225 mg, $0.99 \mathrm{mmol}, 2.1$ equiv) in PhMe (10 mL). Short column chromatography ( $n$-hexane $/ \mathrm{CH}_{2} \mathrm{Cl}_{2}=4 / 1$ ) yielded the crude diarylated diamine ( $240 \mathrm{mg}$, quantitative). The crude $N, N^{\prime}$-diarylated diamine (240 mg, $0.47 \mathrm{mmol}, 1.0$ equiv) was treated with ammonium tetrafluoroborate (60 mg, $0.56 \mathrm{mmol}, 1.2$ equiv) and triethyl orthoformate $(4.0 \mathrm{~mL})$. Purification by column chromatography $\left(\mathrm{CH}_{2} \mathrm{Cl}_{2} /\right.$ acetone $\left.=15 / 1 \rightarrow 5 / 1\right)$ yielded $178(210 \mathrm{mg}, 74 \%)$ as a white solid. M. p. $=125-128^{\circ} \mathrm{C} .{ }^{1} \mathbf{H}$ NMR $\left(300 \mathrm{MHz}, \mathrm{CDCl}_{3}\right): \delta=8.52(\mathrm{~s}, 1 \mathrm{H})$, 7.47-7.32 (m, 12H), 7.24 (dd, $J=8.0,2.0 \mathrm{~Hz}, 2 \mathrm{H}), 7.13(\mathrm{dd}, J=8.0,0.7 \mathrm{~Hz}, 2 \mathrm{H}$ ), $5.81(\mathrm{~s}, 2 \mathrm{H}), 2.46(\mathrm{~s}, 6 \mathrm{H}), 1.18(\mathrm{~s}, 18 \mathrm{H}) \cdot{ }^{13} \mathrm{C}$ NMR $\left(125 \mathrm{MHz}, \mathrm{CDCl}_{3}\right): \delta=157.0$ $(\mathrm{CH}), 151.2\left(\mathrm{C}_{\mathrm{q}}\right), 133.5\left(\mathrm{C}_{\mathrm{q}}\right), 132.2\left(\mathrm{C}_{\mathrm{q}}\right), 131.1(\mathrm{CH}), 130.2(\mathrm{CH}), 129.7\left(\mathrm{C}_{\mathrm{q}}\right), 129.5$ $(\mathrm{CH}), 128.3(\mathrm{CH}), 126.8(\mathrm{CH}), 125.1(\mathrm{CH}), 76.1(\mathrm{CH}), 34.6\left(\mathrm{C}_{\mathrm{q}}\right), 31.0\left(\mathrm{CH}_{3}\right), 17.8$ $\left(\mathrm{CH}_{3}\right) .{ }^{19} \mathrm{~F} \mathrm{NMR}\left(282 \mathrm{MHz}, \mathrm{CDCl}_{3}\right): \delta=-151.14(\mathrm{~s}),-151.19(\mathrm{~d}, J=2.2 \mathrm{~Hz})$. IR (ATR): 2962, 1604, 1214, 1050, 1031, $700 \mathrm{~cm}^{-1}$. MS (ESI) $\mathrm{m} / \mathrm{z}$ (relative intensity): 515 (100) $\left[\mathrm{M}-\mathrm{BF}_{4}\right]^{+}$. HR-MS (ESI) $\mathrm{m} / z$ calcd for $\mathrm{C}_{37} \mathrm{H}_{43} \mathrm{~N}_{2}$ [M-BF $]^{+} 515.3421$, found 515.3420. [a $]_{\mathrm{D}}^{20}:+326.0\left(\mathrm{c}=1.00, \mathrm{CHCl}_{3}\right)$. 


\section{Synthesis of 180}

\section{1-(p-Tolyl)adamantane (233)}<smiles>Cc1ccc([Al])cc1</smiles>

Following a modified procedure,${ }^{[331]}$ a suspension of 1-bromoadamantane $(15.0 \mathrm{~g}$, $69.7 \mathrm{mmol}, 1.00$ equiv), $\mathrm{Pd} / \mathrm{C} 10 \mathrm{wt} \%$ ( $3.70 \mathrm{~g}, 3.50 \mathrm{mmol}, 5.00 \mathrm{~mol} \%$ ), $\mathrm{K}_{2} \mathrm{CO}_{3}$ (11.6 g, $83.6 \mathrm{mmol}, 1.20$ equiv) and PhMe $(200 \mathrm{~mL})$ was refluxed for $16 \mathrm{~h}$ under a slow stream of $\mathrm{N}_{2}$. The mixture was allowed to cool to ambient temperature, diluted with $n$-hexane $(200 \mathrm{~mL})$, filtered through a plug of Celite ${ }^{\circledR}$ and concentrated under reduced pressure. The residue was recrystallized from $n$-hexane at $-30^{\circ} \mathrm{C}$, collected by filtration, washed with cold $n$-pentane and dried under high vacuum to provide 1-(p-tolyl)adamantane $233(13.8 \mathrm{~g}, \mathbf{8 7 \%})$ as a colorless crystalline solid.

The analytical data are in agreement with those previously reported in the literature. ${ }^{[331]}$

\section{1-(3-Bromo-4-methylphenyl)adamantane (234)}<smiles>[Mg]c1ccc(Br)c(Br)c1</smiles>

Following a modified procedure, ${ }^{[330]} 1$-(p-tolyl)adamantane $233(5.57 \mathrm{~g}, 24.6 \mathrm{mmol}$, 1.0 equiv) was dissolved in chloroform $(12.2 \mathrm{~mL})$. The flask was wrapped with aluminum foil and placed in an ice bath. Under stirring, bromine $(1.27 \mathrm{~mL}$, $24.6 \mathrm{mmol}, 1.0$ equiv) was added dropwise via syringe. The mixture was stirred at ambient temperature for $16 \mathrm{~h}$. Then, the solution was poured into $\mathrm{NaHSO}_{3}(0.1 \mathrm{M}$, $100 \mathrm{~mL})$ and extracted with $n$-hexane $(3 \times 100 \mathrm{~mL})$. The combined organic layer was washed with brine $(50 \mathrm{~mL})$, dried over $\mathrm{Na}_{2} \mathrm{SO}_{4}$, and concentrated under reduced 
pressure. The residue was taken into $n$-hexane and filtered through a short plug of silica gel. The silica was washed with $n$-hexane. The combined filtrate was concentrated under reduced pressure and the residue recrystallized from $n$-hexane at $-30{ }^{\circ} \mathrm{C}$ to yield the product 234 as a white crystalline solid $(5.82 \mathrm{~g}, 77 \%$ ). M. p. $=115-117{ }^{\circ} \mathrm{C} .{ }^{1} \mathrm{H}$ NMR $\left(400 \mathrm{MHz}, \mathrm{CDCl}_{3}\right): \delta=7.50(\mathrm{~d}, J=1.8 \mathrm{~Hz}, 1 \mathrm{H}), 7.20$ (dd, $J=8.0,1.8 \mathrm{~Hz}, 1 \mathrm{H}$ ), 7.17 (dt, $J=8.0,0.6 \mathrm{~Hz}, 1 \mathrm{H}), 2.36(\mathrm{~s}, 3 \mathrm{H}), 2.09$ (h, $J=2.7 \mathrm{~Hz}, 3 \mathrm{H}), 1.94-1.85(\mathrm{~m}, 6 \mathrm{H}), 1.84-1.68(\mathrm{~m}, 6 \mathrm{H}) .{ }^{13} \mathrm{C}$ NMR $\left(100 \mathrm{MHz}, \mathrm{CDCl}_{3}\right)$ : $\delta=151.1\left(\mathrm{C}_{\mathrm{q}}\right), 134.7\left(\mathrm{C}_{\mathrm{q}}\right), 130.4(\mathrm{CH}), 129.0(\mathrm{CH}), 124.9\left(\mathrm{C}_{\mathrm{q}}\right), 123.8(\mathrm{CH}), 43.1$ $\left(\mathrm{CH}_{2}\right)$, $36.7\left(\mathrm{CH}_{2}\right), 36.0\left(\mathrm{C}_{\mathrm{q}}\right), 28.9(\mathrm{CH}), 22.3\left(\mathrm{CH}_{3}\right)$. IR (ATR): 2902, 2845, 1493, 1447, 1031, 801, $675 \mathrm{~cm}^{-1}$. MS (EI) $\mathrm{m} / \mathrm{z}$ (relative intensity): $306(65)[\mathrm{M}]^{+}\left({ }^{81} \mathrm{Br}\right.$ ), 304 (65) $[\mathrm{M}]{ }^{+}\left({ }^{79} \mathrm{Br}\right), 249(34)\left({ }^{81} \mathrm{Br}\right), 247$ (33) $\left({ }^{79} \mathrm{Br}\right), 168$ (100), 94 (24). HR-MS (El) m/z calcd for $\mathrm{C}_{17} \mathrm{H}_{21}{ }^{79} \mathrm{Br}[\mathrm{M}]^{+} 304.0827$, found 304.0834 .

(4R,5R)-1,3-Bis[5-(adamantan-1-yl)-2-methylphenyl]-4,5-diphenyl-4,5-dihydro$1 \mathrm{H}$-imidazol-3-ium Tetrafluoroborate (180)

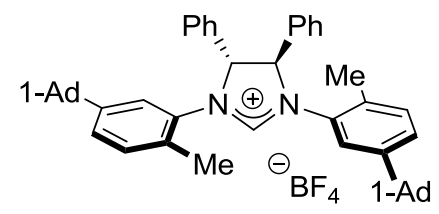

The general procedure GP4 was followed using $(R, R)$-diphenylethylenediamine (200 mg, $0.94 \mathrm{mmol}, \quad 1.0$ equiv), $\quad \mathrm{Pd}(\mathrm{OAc})_{2} \quad(10.6 \mathrm{mg}, \quad 47 \mu \mathrm{mol}, \quad 5.0 \mathrm{~mol} \%$ ), (+/-)-BINAP (59 mg, $94 \mu \mathrm{mol}, 10 \mathrm{~mol} \%$ ), NaOtBu (272 mg, $2.83 \mathrm{mmol}, 3.0$ equiv) and 1-(3-bromo-4-methylphenyl)adamantane 234 (604 mg, $1.98 \mathrm{mmol}, 2.1$ equiv) in PhMe $(15 \mathrm{~mL})$. Short column chromatography ( $n$-hexane $/ \mathrm{CH}_{2} \mathrm{Cl}_{2}=6 / 1$ ) yielded the crude diarylated diamine (602 mg, 91\%). The crude $N, N^{\prime}$-diarylated diamine (565 mg, $0.85 \mathrm{mmol}, 1.0$ equiv) was treated with ammonium tetrafluoroborate (108 mg, $1.03 \mathrm{mmol}, 1.2$ equiv) and triethyl orthoformate $(4.0 \mathrm{~mL})$. Purification by column chromatography ( $n$-hexane/acetone $=3 / 1 \rightarrow 1 / 1)$ yielded $180(522 \mathrm{mg}, 81 \%)$ as an off-white solid. M. p. $=196-198{ }^{\circ} \mathrm{C} .{ }^{1} \mathbf{H}$ NMR $\left(300 \mathrm{MHz}, \mathrm{CDCl}_{3}\right): \delta=8.59$ (s, $1 \mathrm{H}), 7.49-7.34(\mathrm{~m}, 10 \mathrm{H}), 7.28(\mathrm{~d}, J=1.9 \mathrm{~Hz}, 2 \mathrm{H}), 7.22(\mathrm{dd}, J=8.0,2.0 \mathrm{~Hz}, 2 \mathrm{H})$, 
$7.15(\mathrm{~d}, J=8.1 \mathrm{~Hz}, 2 \mathrm{H}), 5.81(\mathrm{~d}, J=0.6 \mathrm{~Hz}, 2 \mathrm{H}), 2.47(\mathrm{~s}, 6 \mathrm{H}), 2.10-2.00(\mathrm{~m}, 6 \mathrm{H})$, $1.80-1.68(\mathrm{~m}, 24 \mathrm{H}) .{ }^{13} \mathrm{C}$ NMR $\left(125 \mathrm{MHz}, \mathrm{CDCl}_{3}\right): \delta=157.0(\mathrm{CH}), 151.3\left(\mathrm{C}_{\mathrm{q}}\right), 133.7$ $\left(\mathrm{C}_{\mathrm{q}}\right), 132.3\left(\mathrm{C}_{\mathrm{q}}\right), 131.2(\mathrm{CH}), 130.1(\mathrm{CH}), 129.8\left(\mathrm{C}_{\mathrm{q}}\right), 129.5(\mathrm{CH}), 128.3(\mathrm{CH}), 126.5$ $(\mathrm{CH}), 124.5(\mathrm{CH}), 76.1(\mathrm{CH}), 42.7\left(\mathrm{CH}_{2}\right), 36.6\left(\mathrm{CH}_{2}\right), 36.1\left(\mathrm{C}_{\mathrm{q}}\right), 28.9(\mathrm{CH}), 17.9$ $\left(\mathrm{CH}_{3}\right) .{ }^{19} \mathrm{~F} \mathrm{NMR}\left(282 \mathrm{MHz}, \mathrm{CDCl}_{3}\right): \delta=-151.11$ (s), -151.17 (d, J=2.2 Hz). IR (ATR): 2899, 2846, 1604, 1213, 1052, 755, $699 \mathrm{~cm}^{-1}$. MS (ESI) $\mathrm{m} / \mathrm{z}$ (relative intensity): 671 (100) $\left[\mathrm{M}-\mathrm{BF}_{4}\right]^{+}$. HR-MS (ESI) $\mathrm{m} / z$ calcd for $\mathrm{C}_{49} \mathrm{H}_{55} \mathrm{~N}_{2}\left[\mathrm{M}-\mathrm{BF}_{4}\right]^{+}$

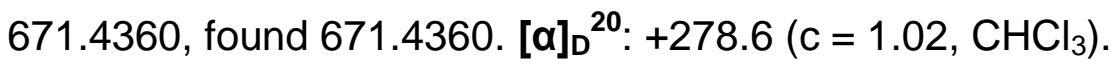

(4R,5R)-1,3-Bis[5-(adamantan-1-yl)-2-ethylphenyl]-4,5-diphenyl-4,5-dihydro-1 $H$ imidazol-3-ium Tetrafluoroborate (181)

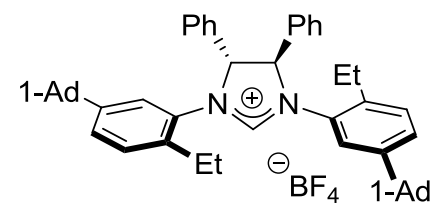

1-(3-Bromo-4-ethylphenyl)adamantane was prepared in 2 steps from ethylbenzene following the same procedure as for the synthesis of $234 .^{[330,331]}$ The crude product was used without purification.

The general procedure GP4 was followed using $(R, R)$-diphenylethylenediamine (202 mg, $0.95 \mathrm{mmol}, \quad 1.0$ equiv), $\mathrm{Pd}(\mathrm{OAc})_{2} \quad$ (21.4 mg, $\left.95 \mu \mathrm{mol}, \quad 10 \mathrm{~mol} \%\right)$, (+/-)-BINAP (119 mg, $191 \mu \mathrm{mol}, 20 \mathrm{~mol} \%$ ), NaOtBu (275 mg, $2.86 \mathrm{mmol}, 3.0$ equiv) and crude 1-(3-bromo-4-ethylphenyl)adamantane (638 mg, $2.0 \mathrm{mmol}, 2.1$ equiv) in PhMe $(20 \mathrm{~mL})$. Short column chromatography ( $n$-hexane $\left./ \mathrm{CH}_{2} \mathrm{Cl}_{2}=7 / 1 \rightarrow 3 / 1\right)$ yielded the crude diarylated diamine $(470 \mathrm{mg}, 72 \%$ ) as a yellow foam. The crude $N, N^{\prime}$-diarylated diamine (470 mg, $0.68 \mathrm{mmol}, 1.0$ equiv) was treated with ammonium tetrafluoroborate ( $86 \mathrm{mg}, 0.82 \mathrm{mmol}, 1.2$ equiv) and triethyl orthoformate $(3.0 \mathrm{~mL})$. Purification by column chromatography $\left(\mathrm{CH}_{2} \mathrm{Cl}_{2} /\right.$ acetone $\left.=35 / 1 \rightarrow 20 / 1\right)$ yielded 181 $(364 \mathrm{mg}, 68 \%)$ as a pale yellow solid. M. p. $=167-169{ }^{\circ} \mathrm{C} .{ }^{1} \mathbf{H} \mathbf{~ N M R}(500 \mathrm{MHz}$, $\left.\mathrm{CDCl}_{3}\right): \delta=8.41(\mathrm{~s}, 1 \mathrm{H}), 7.46-7.37(\mathrm{~m}, 10 \mathrm{H}), 7.36(\mathrm{~d}, J=2.0 \mathrm{~Hz}, 2 \mathrm{H}), 7.27$ (dd, $J=8.1,1.8 \mathrm{~Hz}, 2 \mathrm{H}), 7.19(\mathrm{~d}, J=8.2 \mathrm{~Hz}, 2 \mathrm{H}), 5.81(\mathrm{~s}, 2 \mathrm{H}), 2.81-2.71(\mathrm{~m}, 4 \mathrm{H}), 2.09$ 
$2.00(\mathrm{~m}, 6 \mathrm{H}), 1.76-1.71(\mathrm{~m}, 24 \mathrm{H}), 1.32(\mathrm{t}, J=7.4 \mathrm{~Hz}, 6 \mathrm{H}) .{ }^{13} \mathrm{C}$ NMR $(125 \mathrm{MHz}$, $\left.\mathrm{CDCl}_{3}\right): \delta=157.3(\mathrm{CH}), 151.4\left(\mathrm{C}_{\mathrm{q}}\right), 136.0\left(\mathrm{C}_{\mathrm{q}}\right), 133.6\left(\mathrm{C}_{\mathrm{q}}\right), 131.8\left(\mathrm{C}_{\mathrm{q}}\right), 130.4(\mathrm{CH})$, $129.7(\mathrm{CH}), 129.1(\mathrm{CH}), 128.7(\mathrm{CH}), 126.9(\mathrm{CH}), 125.3(\mathrm{CH}), 76.6(\mathrm{CH}), 42.7\left(\mathrm{CH}_{2}\right)$, $36.7\left(\mathrm{CH}_{2}\right), 36.2\left(\mathrm{C}_{\mathrm{q}}\right), 28.9(\mathrm{CH}), 23.7\left(\mathrm{CH}_{2}\right), 15.2\left(\mathrm{CH}_{3}\right) .{ }^{19} \mathrm{~F} \mathrm{NMR}(471 \mathrm{MHz}$, $\left.\mathrm{CDCl}_{3}\right): \delta=-150.93,-150.99$ (d, $J=2.3 \mathrm{~Hz}$ ). IR (ATR): 2900, 2847, 1603, 1454, 1268, 1211, 1052, $699 \mathrm{~cm}^{-1}$. MS (ESI) $\mathrm{m} / \mathrm{z}$ (relative intensity): 699 (100) [M-BF $]^{+}$. HR-MS (ESI) $\mathrm{m} / \mathrm{z}$ calcd for $\mathrm{C}_{51} \mathrm{H}_{59} \mathrm{~N}_{2}\left[\mathrm{M}-\mathrm{BF}_{4}\right]^{+}$699.4673, found 699.4674. [a] ${ }_{\mathrm{D}}{ }^{20}$ : $-237.7\left(\mathrm{c}=0.97, \mathrm{CHCl}_{3}\right)$.

(4R,5R)-1,3-Bis(4-methyl-[1,1'-biphenyl]-3-yl)-4,5-diphenyl-4,5-dihydro-1 Himidazol-3-ium Tetrafluoroborate (182)

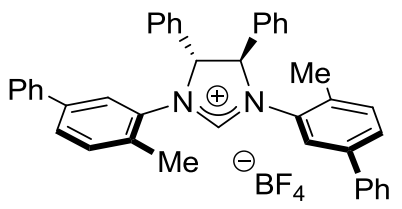

The general procedure GP4 was followed using $(R, R)$-diphenylethylenediamine (180 mg, $\quad 0.85 \mathrm{mmol}, \quad 1.0$ equiv), $\mathrm{Pd}(\mathrm{OAc})_{2} \quad(9.5 \mathrm{mg}, \quad 42 \mu \mathrm{mol}, \quad 5.0 \mathrm{~mol} \%)$, (+/-)-BINAP (53 mg, $85 \mu \mathrm{mol}, 10 \mathrm{~mol} \%$ ), NaOtBu (244 mg, $2.54 \mathrm{mmol}, 3.0$ equiv) and 3-bromo-4-methyl-1,1'-biphenyl ${ }^{[332]}$ (440 mg, $1.78 \mathrm{mmol}, 2.1$ equiv) in $\mathrm{PhMe}$ $\left(15 \mathrm{~mL}\right.$ ). Short column chromatography ( $n$-hexane $/ \mathrm{CH}_{2} \mathrm{Cl}_{2}=4 / 1 \rightarrow 2.5 / 1$ ) yielded the crude diarylated diamine (395 mg, 85\%). The crude $N, N^{\prime}$-diarylated diamine (375 mg, $0.69 \mathrm{mmol}, 1.0$ equiv) was treated with ammonium tetrafluoroborate (87 mg, $0.83 \mathrm{mmol}, 1.2$ equiv) and triethyl orthoformate $(2.5 \mathrm{~mL})$. Purification by column chromatography $\left(\mathrm{CH}_{2} \mathrm{Cl}_{2} /\right.$ acetone $\left.=15 / 1 \rightarrow 5 / 1\right)$ yielded $182(379 \mathrm{mg}, 85 \%)$ as an off-white solid. M. p. $=162-164{ }^{\circ} \mathrm{C} .{ }^{1} \mathbf{H}$ NMR $\left(300 \mathrm{MHz}, \mathrm{CDCl}_{3}\right): \delta=8.58$ (s, $1 \mathrm{H}), 7.64(\mathrm{~d}, J=1.8 \mathrm{~Hz}, 2 \mathrm{H}), 7.55-7.48(\mathrm{~m}, 4 \mathrm{H}), 7.47-7.27(\mathrm{~m}, 18 \mathrm{H}), 7.23(\mathrm{~d}$, $J=8.0 \mathrm{~Hz}, 2 \mathrm{H}), 5.85(\mathrm{~s}, 2 \mathrm{H}), 2.50(\mathrm{~s}, 6 \mathrm{H}) \cdot{ }^{13} \mathrm{C} \mathrm{NMR}\left(125 \mathrm{MHz}, \mathrm{CDCl}_{3}\right): \delta=157.7$ $(\mathrm{CH}), 140.8\left(\mathrm{C}_{\mathrm{q}}\right), 138.7\left(\mathrm{C}_{\mathrm{q}}\right), 133.3\left(\mathrm{C}_{\mathrm{q}}\right), 133.0\left(\mathrm{C}_{\mathrm{q}}\right), 132.0(\mathrm{CH}), 131.9\left(\mathrm{C}_{\mathrm{q}}\right), 130.3$ $(\mathrm{CH}), 129.7(\mathrm{CH}), 128.8(\mathrm{CH}), 128.5(\mathrm{CH}), 128.2(\mathrm{CH}), 127.7(\mathrm{CH}), 127.0(\mathrm{CH})$, $125.8(\mathrm{CH}), 76.3(\mathrm{CH}), 18.2\left(\mathrm{CH}_{3}\right) .{ }^{19} \mathrm{~F}$ NMR (282 MHz, $\left.\mathrm{CDCl}_{3}\right): \delta=-150.45(\mathrm{~s})$, 
-150.50 (d, $J=2.2 \mathrm{~Hz}$ ). IR (ATR): 3060, 1600, 1212, 1052, 759, $697 \mathrm{~cm}^{-1}$. MS (ESI) $\mathrm{m} / \mathrm{z}$ (relative intensity): 555 (100) $\left[\mathrm{M}-\mathrm{BF}_{4}\right]^{+}$. HR-MS (ESI) $\mathrm{m} / z$ calcd for $\mathrm{C}_{41} \mathrm{H}_{35} \mathrm{~N}_{2}$ $\left[\mathrm{M}-\mathrm{BF}_{4}\right]^{+}$555.2795, found 555.2796. $[\alpha]_{\mathrm{D}}^{20}:+383.6\left(\mathrm{c}=1.10, \mathrm{CHCl}_{3}\right)$.

(4R,5R)-1,3-Bis(4'-(tert-butyl)-4-methyl-[1,1'-biphenyl]-3-yl)-4,5-diphenyl-4,5dihydro-1 H-imidazol-3-ium Tetrafluoroborate (183)

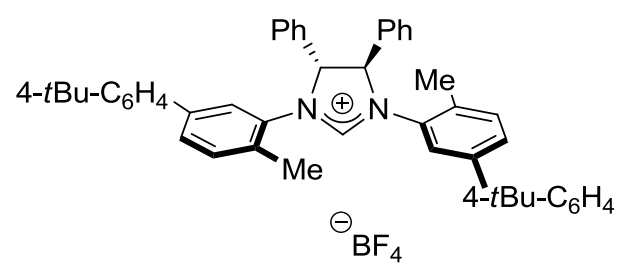

3-Bromo-4'-(tert-butyl)-4-methyl-1,1'-biphenyl was prepared following reported procedures. $^{[332]}$

The general procedure GP4 was followed using $(R, R)$-diphenylethylenediamine (300 mg, $1.41 \mathrm{mmol}, \quad 1.0$ equiv), $\mathrm{Pd}(\mathrm{OAc})_{2} \quad(15.9 \mathrm{mg}, \quad 71 \mu \mathrm{mol}, \quad 5.0 \mathrm{~mol} \%)$, (+/-)-BINAP (88 mg, $14.1 \mu \mathrm{mol}, 10 \mathrm{~mol} \%$ ), NaOtBu (407 mg, $4.24 \mathrm{mmol}, 3.0$ equiv) and 3-bromo-4'-(tert-butyl)-4-methyl-1,1'-biphenyl (900 mg, $2.97 \mathrm{mmol}, 2.1$ equiv, $70 \%$ purity) in PhMe (12.0 mL). Short column chromatography ( $n$-hexane $/ \mathrm{CH}_{2} \mathrm{Cl}_{2}=$ $10 / 1 \rightarrow 2.5 / 1)$ yielded the crude diarylated diamine $(853 \mathrm{mg}, 92 \%)$ as an off-white foam. The crude $N, N^{\prime}$-diarylated diamine ( $853 \mathrm{mg}, 1.30 \mathrm{mmol}, 1.0$ equiv) was treated with ammonium tetrafluoroborate $(170 \mathrm{mg}, 1.62 \mathrm{mmol}, 1.25$ equiv) and triethyl orthoformate $(5.0 \mathrm{~mL})$. Purification by column chromatography $\left(\mathrm{CH}_{2} \mathrm{Cl}_{2} /\right.$ acetone $=$ $30 / 1 \rightarrow 7 / 1)$ followed by recrystallization from $i \mathrm{PrOH}(8.0 \mathrm{~mL})$ yielded $183(911 \mathrm{mg}$, $93 \%)$ as an off-white solid. M. p. $=325-326{ }^{\circ} \mathrm{C} .{ }^{1} \mathbf{H}$ NMR $\left(400 \mathrm{MHz}, \mathrm{CDCl}_{3}\right): \delta=8.60$ (s, $1 \mathrm{H}), 7.67(\mathrm{~d}, J=1.9 \mathrm{~Hz}, 2 \mathrm{H}), 7.51-7.46(\mathrm{~m}, 4 \mathrm{H}), 7.46-7.42(\mathrm{~m}, 10 \mathrm{H}), 7.42-7.34$ $(\mathrm{m}, 6 \mathrm{H}), 7.22(\mathrm{~d}, J=8.0 \mathrm{~Hz}, 2 \mathrm{H}), 5.86(\mathrm{~s}, 2 \mathrm{H}), 2.49(\mathrm{~s}, 6 \mathrm{H}), 1.34(\mathrm{~s}, 18 \mathrm{H}) .{ }^{13} \mathrm{C}$ NMR $\left(100 \mathrm{MHz}, \mathrm{CDCl}_{3}\right): \delta=157.9(\mathrm{CH}), 151.0\left(\mathrm{C}_{\mathrm{q}}\right), 140.9\left(\mathrm{C}_{\mathrm{q}}\right), 136.0\left(\mathrm{C}_{\mathrm{q}}\right), 133.6\left(\mathrm{C}_{\mathrm{q}}\right)$, $133.2\left(\mathrm{C}_{\mathrm{q}}\right), 132.2(\mathrm{CH}), 131.7\left(\mathrm{C}_{\mathrm{q}}\right), 130.5(\mathrm{CH}), 129.9(\mathrm{CH}), 128.5(\mathrm{CH}), 128.4(\mathrm{CH})$, $126.8(\mathrm{CH}), 126.0(\mathrm{CH}), 125.8(\mathrm{CH}), 76.5(\mathrm{CH}), 34.7\left(\mathrm{C}_{\mathrm{q}}\right), 31.5\left(\mathrm{CH}_{3}\right), 18.2\left(\mathrm{CH}_{3}\right)$. ${ }^{19}$ F NMR $\left(376 \mathrm{MHz}, \mathrm{CDCl}_{3}\right): \delta=-150.61,-150.66(\mathrm{~d}, J=2.2 \mathrm{~Hz})$. IR (ATR): 2962, 
1607, 1495, 1213, 1053, 818, 699, $567 \mathrm{~cm}^{-1}$. MS (ESI) $\mathrm{m} / \mathrm{z}$ (relative intensity): 667 (100) $\left[\mathrm{M}-\mathrm{BF}_{4}\right]^{+}$. HR-MS (ESI) $\mathrm{m} / z$ calcd for $\mathrm{C}_{49} \mathrm{H}_{51} \mathrm{~N}_{2}\left[\mathrm{M}-\mathrm{BF}_{4}\right]^{+}$667.4047, found 667.4047. $[\alpha]_{\mathrm{D}}^{20}:+333.5(\mathrm{c}=1.0$, acetone).

\section{Synthesis of 184}

\section{4,4'-[(3-Bromo-4-methylphenyl)methylene]bis(fluorobenzene) (235)}

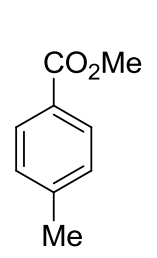

1. ArMgBr, THF, 0 to $23^{\circ} \mathrm{C}$

2. $\mathrm{Et}_{3} \mathrm{SiH}$ (3.0 equiv), TFA (5.0 equiv), $\mathrm{CH}_{2} \mathrm{Cl}_{2}, 23^{\circ} \mathrm{C}, 30 \mathrm{~min}$

3. $\mathrm{Br}_{2}$ (1.0 equiv), $\mathrm{CHCl}_{3}, 0$ to $23^{\circ} \mathrm{C}$

$\left(\mathrm{Ar}=4-\mathrm{F}-\mathrm{C}_{6} \mathrm{H}_{4}\right)$<smiles>Cc1ccc(C([Al])(Cl)Cl)cc1Br</smiles>

235

235 was prepared from methyl $p$-toluate following reported procedures. ${ }^{[330,333]}$ The crude product 235 was used in the next step without further purification.

(4R,5R)-1,3-Bis\{5-[bis(4-fluorophenyl)methyl]-2-methylphenyl\}-4,5-diphenyl4,5-dihydro-1 $\mathrm{H}$-imidazol-3-ium Tetrafluoroborate (184)

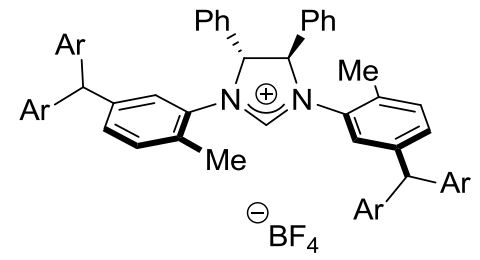

$$
\mathrm{Ar}=4-\mathrm{F}-\mathrm{C}_{6} \mathrm{H}_{4}
$$

The general procedure GP4 was followed using $(R, R)$-diphenylethylenediamine (120 mg, $\quad 0.57 \mathrm{mmol}, \quad 1.0$ equiv), $\mathrm{Pd}(\mathrm{dba})_{2} \quad$ (36 mg, $\left.63 \mu \mathrm{mol}, \quad 11 \mathrm{~mol} \%\right)$, dppf (63 mg, $114 \mu \mathrm{mol}, 20 \mathrm{~mol} \%$ ), NaOtBu (163 mg, $1.70 \mathrm{mmol}, 3.0$ equiv) and crude 4,4'-[(3-bromo-4-methylphenyl)methylene]bis(fluorobenzene) (235) (591 mg, $1.20 \mathrm{mmol}, 2.1$ equiv, 70\% purity) in PhMe (8.0 mL). Short column chromatography ( $n$-hexane/ $\mathrm{CH}_{2} \mathrm{Cl}_{2}=9 / 1 \rightarrow 4 / 1$ ) yielded the crude diarylated diamine (402 $\mathrm{mg}, 90 \%$ ) as an off-white foam. The crude $N, N^{\prime}$-diarylated diamine $(380 \mathrm{mg}, 0.48 \mathrm{mmol}$, 
1.0 equiv) was treated with ammonium tetrafluoroborate $(60 \mathrm{mg}, 0.57 \mathrm{mmol}$, 1.2 equiv) and triethyl orthoformate $(4.0 \mathrm{~mL})$. Purification by column chromatography $\left(\mathrm{CH}_{2} \mathrm{Cl}_{2} /\right.$ acetone $\left.=13 / 1\right)$ yielded $184(394 \mathrm{mg}, 92 \%)$ as an off-white solid. M. p. $=132-133^{\circ} \mathrm{C} .{ }^{1} \mathrm{H}$ NMR $\left(500 \mathrm{MHz}, \mathrm{CDCl}_{3}\right): \delta=8.78(\mathrm{~s}, 1 \mathrm{H}), 7.38-7.31(\mathrm{~m}$, 2H), 7.30-7.25 (m, 4H), 7.20-7.15 (m, 4H), $7.14(\mathrm{~d}, J=7.9 \mathrm{~Hz}, 2 \mathrm{H}), 6.91(\mathrm{~m}, 20 \mathrm{H})$, $5.56(\mathrm{~s}, 2 \mathrm{H}), 5.44(\mathrm{~s}, 2 \mathrm{H}), 2.43(\mathrm{~s}, 6 \mathrm{H}) .{ }^{13} \mathrm{C}$ NMR $\left(125 \mathrm{MHz}, \mathrm{CDCl}_{3}\right): \delta=161.6(\mathrm{~d}$, $\left.{ }^{1} J_{\mathrm{C}-\mathrm{F}}=245.2 \mathrm{~Hz}, \mathrm{C}_{\mathrm{q}}\right), 161.6\left(\mathrm{~d},{ }^{1} \mathrm{~J}_{\mathrm{C}-\mathrm{F}}=245.2 \mathrm{~Hz}, \mathrm{C}_{\mathrm{q}}\right), 157.8(\mathrm{CH}), 143.9\left(\mathrm{C}_{\mathrm{q}}\right), 138.6$ $\left(\mathrm{d},{ }^{4} \mathrm{~J}_{\mathrm{C}-\mathrm{F}}=3.2 \mathrm{~Hz}, \mathrm{C}_{\mathrm{q}}\right), 138.5\left(\mathrm{~d},{ }^{4} \mathrm{~J}_{\mathrm{C}-\mathrm{F}}=3.2 \mathrm{~Hz}, \mathrm{C}_{\mathrm{q}}\right), 133.6\left(\mathrm{C}_{\mathrm{q}}\right), 132.6\left(\mathrm{C}_{\mathrm{q}}\right), 132.0$ $(\mathrm{CH}), 131.4\left(\mathrm{C}_{\mathrm{q}}\right), 130.9(\mathrm{CH}), 130.9\left(\mathrm{~d},{ }^{3} \mathrm{~J}_{\mathrm{C}-\mathrm{F}}=8.1 \mathrm{~Hz}, \mathrm{CH}\right), 130.9\left(\mathrm{~d},{ }^{3} \mathrm{~J}_{\mathrm{C}-\mathrm{F}}=8.1 \mathrm{~Hz}\right.$, $\mathrm{CH}), 130.5(\mathrm{CH}), 129.8(\mathrm{CH}), 128.6(\mathrm{CH}), 128.0(\mathrm{CH}), 115.4\left(\mathrm{~d},{ }^{2} \mathrm{~J}_{\mathrm{C}-\mathrm{F}}=21.3 \mathrm{~Hz}\right.$, $\mathrm{CH}), 115.4\left(\mathrm{~d},{ }^{2} \mathrm{~J}_{\mathrm{C}-\mathrm{F}}=21.3 \mathrm{~Hz}, \mathrm{CH}\right), 76.1(\mathrm{CH}), 54.2(\mathrm{CH}), 18.1\left(\mathrm{CH}_{3}\right) .{ }^{19} \mathrm{~F}$ NMR $\left(282 \mathrm{MHz}, \mathrm{CDCl}_{3}\right): \delta=-116.48$ (tdd, $\left.J=13.8,8.2,5.6 \mathrm{~Hz}\right),-151.03(\mathrm{~d}, J=1.5 \mathrm{~Hz}$ ), -151.08 (d, $J=2.7 \mathrm{~Hz}$ ). IR (ATR): 1602, 1504, 1219, 1158, 1050, 822, 755, 699, $565 \mathrm{~cm}^{-1}$. MS (ESI) $\mathrm{m} / \mathrm{z}$ (relative intensity): 807 (100) $\left[\mathrm{M}-\mathrm{BF}_{4}\right]^{+}$. HR-MS (ESI) $\mathrm{m} / \mathrm{z}$

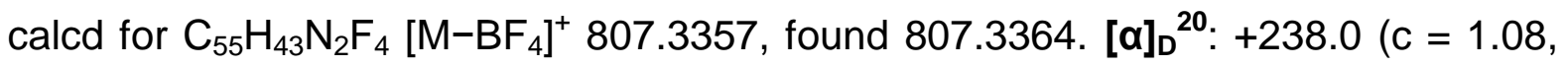
$\left.\mathrm{CHCl}_{3}\right)$.

\section{Synthesis of 185}

\section{2-Bromo-4-(heptan-4-yl)-1-methylbenzene (236)}

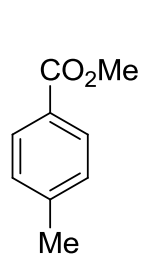

1. $n \mathrm{PrMgBr}, \mathrm{THF}, 0$ to $23^{\circ} \mathrm{C}$
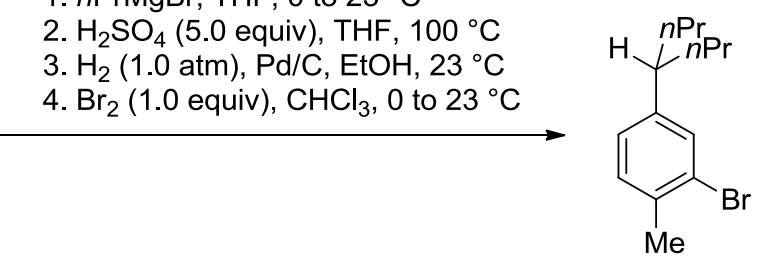

236

236 was prepared from methyl $p$-toluate following reported procedures. ${ }^{[330,334]}$ The crude product 236 was used in the next step without further purification. 
(4R,5R)-1,3-Bis[5-(heptan-4-yl)-2-methylphenyl]-4,5-diphenyl-4,5-dihydro-1Himidazol-3-ium Tetrafluoroborate (185)

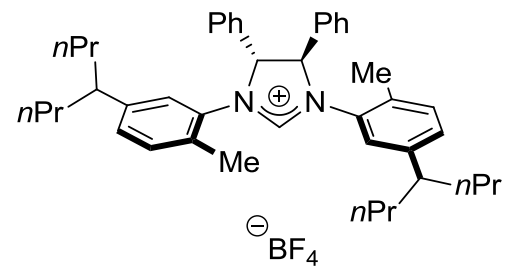

The general procedure GP4 was followed using $(R, R)$-diphenylethylenediamine (150 mg, $\quad 0.71 \mathrm{mmol}, \quad 1.0$ equiv), $\mathrm{Pd}(\mathrm{OAc})_{2} \quad(7.9 \mathrm{mg}, \quad 35 \mu \mathrm{mol}, \quad 5.0 \mathrm{~mol} \%)$, (+/-)-BINAP (44 mg, $71 \mu \mathrm{mol}, 10 \mathrm{~mol} \%$ ), NaOtBu (204 mg, $2.12 \mathrm{mmol}, 3.0$ equiv) and crude 2-bromo-4-(heptan-4-yl)-1-methylbenzene (236) $(615 \mathrm{mg}, 1.49 \mathrm{mmol}$, 2.1 equiv, $65 \%$ purity) in $\mathrm{PhMe}(12 \mathrm{~mL})$. Short column chromatography ( $n$-hexane $\left./ \mathrm{CH}_{2} \mathrm{Cl}_{2}=12 / 1\right)$ yielded the crude diarylated diamine (379 $\mathrm{mg}, 90 \%$ ). The crude $N, N^{\prime}$-diarylated diamine $(378 \mathrm{mg}, 0.64 \mathrm{mmol}, 1.0$ equiv) was treated with ammonium tetrafluoroborate (81 $\mathrm{mg}, 0.77 \mathrm{mmol}, 1.2$ equiv) and triethyl orthoformate (4.0 mL). Purification by column chromatography $\left(\mathrm{CH}_{2} \mathrm{Cl}_{2} /\right.$ acetone $\left.=19 / 1 \rightarrow 10 / 1\right)$ yielded 185 (190 mg, 43\%) as an off-white foam. M. p. $=88-90{ }^{\circ} \mathrm{C} .{ }^{1} \mathbf{H}$ NMR (500 $\left.\mathrm{MHz}, \mathrm{CDCl}_{3}\right): \delta=8.51(\mathrm{~s}, 1 \mathrm{H}), 7.44-7.29(\mathrm{~m}, 10 \mathrm{H}), 7.13(\mathrm{~d}, J=1.7 \mathrm{~Hz}, 2 \mathrm{H}), 7.09(\mathrm{~d}$, $J=7.8 \mathrm{~Hz}, 2 \mathrm{H}$ ), 6.97 (dd, $J=7.8,1.8 \mathrm{~Hz}, 2 \mathrm{H}), 5.82(\mathrm{~s}, 2 \mathrm{H}), 2.46(\mathrm{~s}, 6 \mathrm{H}), 2.45-2.36$ $(\mathrm{m}, 2 \mathrm{H}), 1.50-1.39(\mathrm{~m}, 4 \mathrm{H}), 1.39-1.29(\mathrm{~m}, 4 \mathrm{H}), 1.00-0.87(\mathrm{~m}, 4 \mathrm{H}), 0.87-0.75(\mathrm{~m}$, 4H), 0.73 (t, $J=7.2 \mathrm{~Hz}, 6 \mathrm{H}), 0.72(\mathrm{t}, J=7.2 \mathrm{~Hz}, 6 \mathrm{H}) .{ }^{13} \mathrm{C}$ NMR $\left(100 \mathrm{MHz}, \mathrm{CDCl}_{3}\right)$ : $\delta=156.9(\mathrm{CH}), 146.6\left(\mathrm{C}_{\mathrm{q}}\right), 133.6\left(\mathrm{C}_{\mathrm{q}}\right), 132.5\left(\mathrm{C}_{\mathrm{q}}\right), 131.5(\mathrm{CH}), 130.3(\mathrm{CH}), 130.2$ $\left(\mathrm{C}_{\mathrm{q}}\right), 129.7(\mathrm{CH}), 129.5(\mathrm{CH}), 128.5(\mathrm{CH}), 127.2(\mathrm{CH}), 76.1(\mathrm{CH}), 45.0(\mathrm{CH}), 39.1$ $\left(\mathrm{CH}_{2}\right), 38.8\left(\mathrm{CH}_{2}\right), 20.6\left(\mathrm{CH}_{2}\right), 20.6\left(\mathrm{CH}_{2}\right), 18.1\left(\mathrm{CH}_{3}\right), 14.2\left(\mathrm{CH}_{3}\right), 14.2\left(\mathrm{CH}_{3}\right)$. ${ }^{19} \mathrm{~F}$ NMR $\left(377 \mathrm{MHz}, \mathrm{CDCl}_{3}\right): \delta=-151.15,-151.20$ (d, $\left.J=2.1 \mathrm{~Hz}\right)$. IR (ATR): 2955, 2928, 1606, 1456, 1214, 1051, $700 \mathrm{~cm}^{-1}$. MS (ESI) $\mathrm{m} / z$ (relative intensity): 599 (100) [M-BF $\left.]_{4}\right]^{+}$. HR-MS (ESI) $\mathrm{m} / z$ calcd for $\mathrm{C}_{43} \mathrm{H}_{55} \mathrm{~N}_{2}\left[\mathrm{M}-\mathrm{BF}_{4}\right]^{+} 599.4360$, found 599.4359 . $[\alpha]_{\mathrm{D}}^{20}:+331.2\left(\mathrm{C}=0.51, \mathrm{CHCl}_{3}\right)$. 


\section{(4R,5R)-1,3-Di(phenanthren-9-yl)-4,5-diphenyl-4,5-dihydro-1 $H$-imidazol-3-ium}

\section{Tetrafluoroborate (186)}

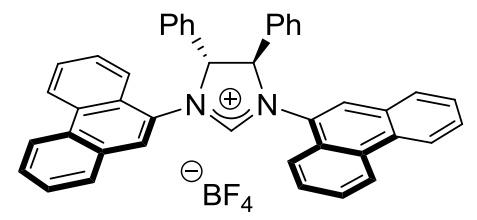

The general procedure GP4 was followed using $(R, R)$-diphenylethylenediamine (100 mg, $\quad 0.47 \mathrm{mmol}, \quad 1.0$ equiv), $\quad \mathrm{Pd}(\mathrm{OAc})_{2} \quad(5.3 \mathrm{mg}, \quad 24 \mu \mathrm{mol}, \quad 5.0 \mathrm{~mol} \%)$, (+/-)-BINAP (29 mg, $47 \mu \mathrm{mol}, 10 \mathrm{~mol} \%$ ), NaOtBu (136 mg, $1.42 \mathrm{mmol}, 3.0$ equiv) and 9-bromophenanthrene (254 mg, $0.99 \mathrm{mmol}, 2.1$ equiv) in PhMe (8.0 mL). Short column chromatography ( $n$-hexane $/ \mathrm{CH}_{2} \mathrm{Cl}_{2}=16 / 1 \rightarrow 3 / 1$ ) yielded the crude diarylated diamine (284 mg, quantitative) as a yellow foam. The crude $N, N^{\prime}$-diarylated diamine (280 mg, $0.50 \mathrm{mmol}, 1.0$ equiv) was treated with ammonium tetrafluoroborate (62 mg, $0.59 \mathrm{mmol}, 1.2$ equiv) and triethyl orthoformate $(8.0 \mathrm{~mL})$. Purification by column chromatography $\left(\mathrm{CH}_{2} \mathrm{Cl}_{2} /\right.$ acetone $\left.=15 / 1 \rightarrow 3 / 1\right)$ yielded $186(275 \mathrm{mg}, 83 \%)$ as a yellow solid. M. p. $=221-223^{\circ} \mathrm{C} .{ }^{1} \mathbf{H}$ NMR $\left(300 \mathrm{MHz}, \mathrm{CDCl}_{3}\right): \delta=8.59$ (dd, $J=8.5,1.1 \mathrm{~Hz}, 2 \mathrm{H}), 8.50(\mathrm{~d}, J=8.3 \mathrm{~Hz}, 2 \mathrm{H}), 8.31(\mathrm{~s}, 2 \mathrm{H}), 8.13-8.01(\mathrm{~m}, 3 \mathrm{H}), 7.97$ (dd, $J=8.1,1.3 \mathrm{~Hz}, 2 \mathrm{H}$ ), 7.76 (ddd, $J=8.1,7.0,1.1 \mathrm{~Hz}, 2 \mathrm{H}$ ), 7.61 (ddd, $J=8.4,7.0$, $1.3 \mathrm{~Hz}, 4 \mathrm{H}), 7.57-7.50(\mathrm{~m}, 4 \mathrm{H}), 7.48-7.38(\mathrm{~m}, 2 \mathrm{H}), 7.32-7.18(\mathrm{~m}, 6 \mathrm{H}), 6.26(\mathrm{~s}, 2 \mathrm{H})$. ${ }^{13} \mathrm{C}$ NMR $\left(125 \mathrm{MHz}, \mathrm{CDCl}_{3}\right): \delta=158.8(\mathrm{CH}), 132.5\left(\mathrm{C}_{\mathrm{q}}\right), 131.3\left(\mathrm{C}_{\mathrm{q}}\right), 130.5\left(\mathrm{C}_{\mathrm{q}}\right)$, $130.4(\mathrm{CH}), 130.3\left(\mathrm{C}_{\mathrm{q}}\right), 130.2(\mathrm{CH}), 129.6(\mathrm{CH}), 129.6(\mathrm{CH}), 128.7(\mathrm{CH}), 128.7(\mathrm{CH})$, $128.1\left(\mathrm{C}_{\mathrm{q}}\right) 128.0(\mathrm{CH}), 127.8(\mathrm{CH}), 127.7(\mathrm{CH}), 127.1\left(\mathrm{C}_{\mathrm{q}}\right), 123.8(\mathrm{CH}), 122.4(\mathrm{CH})$, $121.6(\mathrm{CH}), 77.3(\mathrm{CH}) .{ }^{19} \mathrm{~F} \mathrm{NMR}\left(282 \mathrm{MHz}, \mathrm{CDCl}_{3}\right): \delta=-149.68(\mathrm{~m})$. IR (ATR): 3065, 1605, 1028, 749, 726, $698 \mathrm{~cm}^{-1}$. MS (ESI) $\mathrm{m} / \mathrm{z}$ (relative intensity): 575 (100) $\left[\mathrm{M}-\mathrm{BF}_{4}\right]^{+}$. HR-MS (ESI) $\mathrm{m} / z$ calcd for $\mathrm{C}_{43} \mathrm{H}_{31} \mathrm{~N}_{2}\left[\mathrm{M}-\mathrm{BF}_{4}\right]^{+}$575.2482, found 575.2475. $[\alpha]_{\mathrm{D}}^{20}:+427.1\left(\mathrm{c}=0.99, \mathrm{CHCl}_{3}\right)$. 
(3a $R, 7 \mathrm{a} R$ )-1,3-Bis(5-isopropyl-2-methylphenyl)-3a,4,5,6,7,7a-hexahydro-1 Hbenzo[d]imidazol-3-ium Tetrafluoroborate (187)

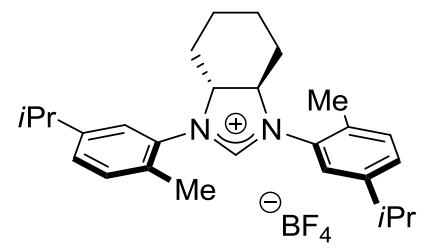

The general procedure GP4 was followed using (1R,2R)-cyclohexane-1,2-diamine (116 mg, $1.02 \mathrm{mmol}, 1.0$ equiv), $\mathrm{Pd}(\mathrm{dba})_{2}$ (64 mg, $0.11 \mathrm{mmol}, 11 \mathrm{~mol} \%$ ), dppf (112 mg, $0.20 \mathrm{mmol}, 20 \mathrm{~mol} \%$ ), NaOtBu (292 mg, $3.04 \mathrm{mmol}, 3.0$ equiv) and 5-isopropyl-2-methylphenyl trifluoromethanesulfonate ${ }^{[329]} \quad(600 \mathrm{mg}, \quad 2.13 \mathrm{mmol}$, 2.1 equiv) in PhMe (10.0 mL). Short column chromatography ( $n$-hexane/ $\mathrm{CH}_{2} \mathrm{Cl}_{2}=$ $8 / 1 \rightarrow 4 / 1)$ yielded the crude diarylated diamine $(90 \mathrm{mg}, 23 \%)$ as a yellow foam. The crude $N, N^{\prime}$-diarylated diamine $(90 \mathrm{mg}, 0.24 \mathrm{mmol}, 1.0$ equiv) was treated with ammonium tetrafluoroborate (30 mg, $0.29 \mathrm{mmol}, 1.2$ equiv) and triethyl orthoformate $(1.5 \mathrm{~mL})$. Purification by column chromatography $\left(\mathrm{CH}_{2} \mathrm{Cl}_{2} /\right.$ acetone $\left.=9 / 1 \rightarrow 5 / 1\right)$ yielded $187(65.8 \mathrm{mg}, 73 \%)$ as a pale yellow foam. M. p. $=90-92{ }^{\circ} \mathrm{C} .{ }^{1} \mathbf{H}$ NMR (500 MHz, $\left.\mathrm{CDCl}_{3}\right): \delta=7.97(\mathrm{~s}, 1 \mathrm{H}), 7.49(\mathrm{~s}, 2 \mathrm{H}), 7.24$ (app s, 4H), $4.31(\mathrm{~s}, 2 \mathrm{H}), 2.96$ (p, $J=6.8 \mathrm{~Hz}, 2 \mathrm{H}), 2.35$ (s, 6H), 2.09 (d, $J=10.6 \mathrm{~Hz}, 2 \mathrm{H}), 1.96(\mathrm{~d}, J=8.5 \mathrm{~Hz}, 2 \mathrm{H}$ ), 1.86 (app s, 2H), 1.51-1.39 (m, 2H), $1.26(\mathrm{~d}, J=6.7 \mathrm{~Hz}, 12 \mathrm{H}) .{ }^{13} \mathrm{C}$ NMR $(125 \mathrm{MHz}$, $\left.\mathrm{CDCl}_{3}\right): \delta=159.0(\mathrm{CH}), 149.7\left(\mathrm{C}_{\mathrm{q}}\right), 132.9\left(\mathrm{C}_{\mathrm{q}}\right), 131.6(\mathrm{CH}), 131.0\left(\mathrm{C}_{\mathrm{q}}\right), 128.5(\mathrm{CH})$, $125.9(\mathrm{CH}), 71.4(\mathrm{CH}), 33.7(\mathrm{CH}), 27.6\left(\mathrm{CH}_{2}\right), 24.0\left(\mathrm{CH}_{3}\right), 23.9\left(\mathrm{CH}_{3}\right), 23.9\left(\mathrm{CH}_{2}\right)$, $17.6\left(\mathrm{CH}_{3}\right) .{ }^{19} \mathrm{~F}$ NMR $\left(471 \mathrm{MHz}, \mathrm{CDCl}_{3}\right): \delta=-151.33,-151.39(\mathrm{~d}, J=2.2 \mathrm{~Hz})$. IR (ATR): 2900, 2847, 1591, 1449, 1256, 1038, 942, $755 \mathrm{~cm}^{-1}$. MS (ESI) $\mathrm{m} / \mathrm{z}$ (relative intensity): 389 (100) $\left[\mathrm{M}-\mathrm{BF}_{4}\right]^{+}$. HR-MS (ESI) $\mathrm{m} / z$ calcd for $\mathrm{C}_{27} \mathrm{H}_{37} \mathrm{~N}_{2}$ $\left[\mathrm{M}-\mathrm{BF}_{4}\right]^{+}$389.2951, found 389.2952. [a $]_{\mathrm{D}}^{20}:+33.9\left(\mathrm{c}=0.81, \mathrm{CHCl}_{3}\right)$. 
(4R,5R)-1,3-Bis(3,5-di-tert-butylphenyl)-4,5-diphenyl-4,5-dihydro-1 H-imidazol-3ium Tetrafluoroborate (188)

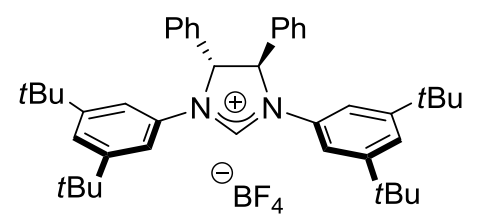

The general procedure GP4 was followed using $(R, R)$-diphenylethylenediamine (200 mg, $0.94 \mathrm{mmol}, \quad 1.0$ equiv), $\quad \mathrm{Pd}(\mathrm{OAc})_{2} \quad(11.0 \mathrm{mg}, \quad 49 \mu \mathrm{mol}, \quad 5.0 \mathrm{~mol} \%)$, (+/-)-BINAP (59 mg, $95 \mu \mathrm{mol}, 10 \mathrm{~mol} \%$ ), NaOtBu (272 mg, $2.83 \mathrm{mmol}, 3.0$ equiv) and 1-bromo-3,5-di-tert-butylbenzene (533 mg, $1.98 \mathrm{mmol}, 2.1$ equiv) in $\mathrm{PhMe}$ $\left(15 \mathrm{~mL}\right.$ ). Short column chromatography ( $n$-hexane $/ \mathrm{CH}_{2} \mathrm{Cl}_{2}=8 / 1 \rightarrow 4 / 1$ ) yielded the crude diarylated diamine (557 mg, quantitative). The crude $N, N^{\prime}$-diarylated diamine (557 mg, $0.95 \mathrm{mmol}, 1.0$ equiv) was treated with ammonium tetrafluoroborate (119 mg, $1.14 \mathrm{mmol}, 1.2$ equiv) and triethyl orthoformate $(3.0 \mathrm{~mL})$. Purification by column chromatography $\left(\mathrm{CH}_{2} \mathrm{Cl}_{2} /\right.$ acetone $\left.=20 / 1 \rightarrow 15 / 1\right)$ yielded $188(545 \mathrm{mg}, 87 \%)$ as a white solid. M. p. $=275-277^{\circ} \mathrm{C} .{ }^{1} \mathbf{H}$ NMR $\left(500 \mathrm{MHz}, \mathrm{CDCl}_{3}\right): \delta=9.37(\mathrm{~s}, 1 \mathrm{H})$, 7.46-7.37 (m, 10H), 7.31 (t, $J=1.6 \mathrm{~Hz}, 2 \mathrm{H}), 7.17(\mathrm{~s}, 2 \mathrm{H}), 7.16(\mathrm{~s}, 2 \mathrm{H}), 5.71(\mathrm{~d}$, $J=0.5 \mathrm{~Hz}, 2 \mathrm{H}), 1.22(\mathrm{~s}, 36 \mathrm{H}) .{ }^{13} \mathrm{C}$ NMR $\left(125 \mathrm{MHz}, \mathrm{CDCl}_{3}\right): \delta=153.4(\mathrm{CH}), 153.2$ $\left(\mathrm{C}_{\mathrm{q}}\right), 135.0\left(\mathrm{C}_{\mathrm{q}}\right), 134.1\left(\mathrm{C}_{\mathrm{q}}\right), 130.2(\mathrm{CH}), 130.1(\mathrm{CH}), 127.5(\mathrm{CH}), 122.8(\mathrm{CH}), 116.1$ $(\mathrm{CH}), 75.4(\mathrm{CH}), 35.3\left(\mathrm{C}_{\mathrm{q}}\right), 31.2\left(\mathrm{CH}_{3}\right) .{ }^{19} \mathrm{~F}$ NMR $\left(471 \mathrm{MHz}, \mathrm{CDCl}_{3}\right): \delta=-150.31$, -150.37 (d, $J=2.2 \mathrm{~Hz}$ ). IR (ATR): 2902, 1619, 1583, 1054, 756, $700 \mathrm{~cm}^{-1}$. MS (ESI) $\mathrm{m} / z$ (relative intensity): 599 (100) $\left[\mathrm{M}-\mathrm{BF}_{4}\right]^{+}$. HR-MS (ESI) $\mathrm{m} / z$ calcd for $\mathrm{C}_{43} \mathrm{H}_{55} \mathrm{~N}_{2}$

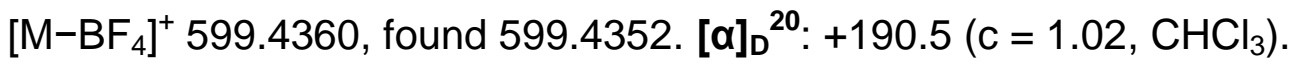

(4S,5S)-1,3-Bis[5-(adamantan-1-yl)-2-methylphenyl]-4,5-di(naphthalen-1-yl)-4,5dihydro-1 $H$-imidazol-3-ium Tetrafluoroborate (189)

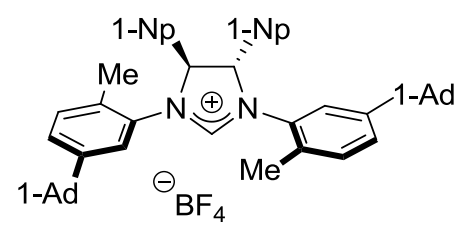


The general procedure GP4 was followed using (1S,2S)-1,2-di(naphthalen1-yl)ethane-1,2-diamine ${ }^{[335]}$ (480 mg, $1.54 \mathrm{mmol}, 1.0$ equiv), $\mathrm{Pd}(\mathrm{OAc})_{2} \quad(34.5 \mathrm{mg}$, $0.15 \mathrm{mmol}, 10 \mathrm{~mol} \%), \quad(+/-)$-BINAP $\quad(191 \mathrm{mg}, 0.31 \mathrm{mmol}, 20 \mathrm{~mol} \%), \quad \mathrm{NaOtBu}$ (443 mg, $4.61 \mathrm{mmol}, 3.0$ equiv) and 1-(3-bromo-4-methylphenyl)adamantane 234 (985 mg, $3.23 \mathrm{mmol}, 2.1$ equiv) in PhMe $(20 \mathrm{~mL})$. Short column chromatography ( $n$-hexane/ $\mathrm{CH}_{2} \mathrm{Cl}_{2}=7 / 1 \rightarrow 1 / 1$ ) yielded the crude diarylated diamine $(737 \mathrm{mg}, 63 \%$ ) as an off-white foam. The crude $N, N^{\prime}$-diarylated diamine $(737 \mathrm{mg}, 0.97 \mathrm{mmol}$, 1.0 equiv) was treated with ammonium tetrafluoroborate $(122 \mathrm{mg}, 1.16 \mathrm{mmol}$, 1.2 equiv) and triethyl orthoformate $(3.0 \mathrm{~mL})$. Purification by column chromatography $\left(\mathrm{CH}_{2} \mathrm{Cl}_{2} /\right.$ acetone $\left.=25 / 1 \rightarrow 10 / 1\right)$, followed by recrystallization from $\mathrm{PrOH}(5.0 \mathrm{~mL})$, yielded 189 (656 mg, 79\%) as a white solid. M. p. $=292-293{ }^{\circ} \mathrm{C} .{ }^{1} \mathbf{H}$ NMR $(600 \mathrm{MHz}$, $\left.\mathrm{CDCl}_{3}\right): \delta=9.59-8.25$ (brm, 2H), 8.24-7.27 (brm, 11H), 7.23-7.03 (brm, 8H), 7.046.08 (brm, 2H), 2.69-2.41 (m, 6H), 2.14-1.84 (m, 6H), 1.83-1.34 (m, 24H). As previously reported in a similar situation, ${ }^{[210]}$ peaks were noticeably broadened and difficult to integrate, likely due to slow rotation. ${ }^{13} \mathrm{C} \mathrm{NMR}\left(125 \mathrm{MHz}, \mathrm{CDCl}_{3}\right)$ : $\delta=157.7(\mathrm{CH}), 151.4\left(\mathrm{C}_{\mathrm{q}}\right), 133.7\left(\mathrm{C}_{\mathrm{q}}\right), 132.8\left(\mathrm{C}_{\mathrm{q}}\right), 132.0(\mathrm{CH}), 131.6(\mathrm{CH}), 131.3$ $\left(\mathrm{C}_{\mathrm{q}}\right), 130.6(\mathrm{CH}), 130.1\left(\mathrm{C}_{\mathrm{q}}\right), 129.5\left(\mathrm{C}_{\mathrm{q}}\right), 129.1(\mathrm{CH}), 128.2(\mathrm{CH}), 127.3(\mathrm{CH}), 126.3$ $(\mathrm{CH}), 126.0(\mathrm{CH}), 123.9(\mathrm{CH}), 121.1(\mathrm{CH}), 71.0(\mathrm{CH}), 42.6\left(\mathrm{CH}_{2}\right), 36.7\left(\mathrm{CH}_{2}\right), 36.1$ $\left(\mathrm{C}_{\mathrm{q}}\right), 28.9(\mathrm{CH}), 18.4\left(\mathrm{CH}_{3}\right) .{ }^{19} \mathrm{~F}$ NMR $\left(376 \mathrm{MHz}, \mathrm{CDCl}_{3}\right): \delta=-151.19,-151.24(\mathrm{~d}$, $J=2.5 \mathrm{~Hz}$ ). IR (ATR): 2900, 1592, 1260, 1052, 800, 773, $477 \mathrm{~cm}^{-1}$. MS (ESI) m/z (relative intensity): 771 (100) $\left[\mathrm{M}-\mathrm{BF}_{4}\right]^{+}$. HR-MS (ESI) $\mathrm{m} / \mathrm{z}$ calcd for $\mathrm{C}_{57} \mathrm{H}_{59} \mathrm{~N}_{2}$

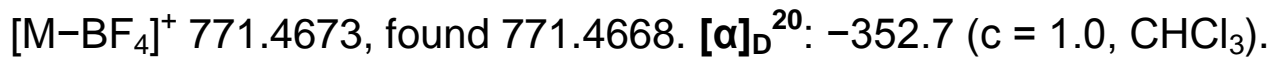

(4R,5R)-1-[5-(Adamantan-1-yl)-2-methylphenyl]-3-(4-methyl-[1,1'-biphenyl]-3yl)-4,5-diphenyl-4,5-dihydro-1 $\mathrm{H}$-imidazol-3-ium Tetrafluoroborate (190)

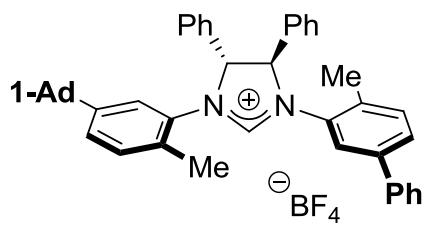


190 was prepared following a procedure reported in the literature ${ }^{[242 c]}$ from $(R, R)$-diphenylethylenediamine (368 mg, $1.73 \mathrm{mmol}, 1.0$ equiv), 3-bromo-4-methyl1,1'-biphenyl ${ }^{[330]} \quad(450 \mathrm{mg}, \quad 1.82 \mathrm{mmol}, \quad 1.05$ equiv) and 1-(3-bromo-4methylphenyl)adamantane 234 (1.2 equiv in the $2^{\text {nd }}$ step). After purification by column chromatography $\left(\mathrm{CH}_{2} \mathrm{Cl}_{2} /\right.$ acetone $\left.=16 / 1 \rightarrow 6 / 1\right)$ and recrystallization from iPrOH (3.0 mL) at $-30{ }^{\circ} \mathrm{C}, 190$ (556 mg, 46\% over 3 steps) was obtained as a white solid. M. p. $=154-156{ }^{\circ} \mathrm{C} .{ }^{1} \mathrm{H}$ NMR $\left(400 \mathrm{MHz}, \mathrm{CDCl}_{3}\right): \delta=8.54(\mathrm{~s}, 1 \mathrm{H}), 7.62(\mathrm{~d}$, $J=1.8 \mathrm{~Hz}, 1 \mathrm{H}), 7.54-7.45(\mathrm{~m}, 4 \mathrm{H}), 7.45-7.34(\mathrm{~m}, 11 \mathrm{H}), 7.33-7.27(\mathrm{~m}, 2 \mathrm{H}), 7.24(\mathrm{~d}$, $J=4.0 \mathrm{~Hz}, 1 \mathrm{H}), 7.22(\mathrm{dd}, J=8.1,2.0 \mathrm{~Hz}, 1 \mathrm{H}), 7.14(\mathrm{~d}, J=8.1 \mathrm{~Hz}, 1 \mathrm{H}), 5.93(\mathrm{~d}$, $J=10.4 \mathrm{~Hz}, 1 \mathrm{H}), 5.74(\mathrm{~d}, J=10.4 \mathrm{~Hz}, 1 \mathrm{H}), 2.49(\mathrm{~s}, 3 \mathrm{H}), 2.46(\mathrm{~s}, 3 \mathrm{H}), 2.09-2.00(\mathrm{~m}$, $3 \mathrm{H}), 1.78-1.66(\mathrm{~m}, 12 \mathrm{H}) .{ }^{13} \mathrm{C}$ NMR $\left(100 \mathrm{MHz}, \mathrm{CDCl}_{3}\right): \delta=157.5(\mathrm{CH}), 151.6\left(\mathrm{C}_{q}\right)$, $141.0\left(\mathrm{C}_{\mathrm{q}}\right), 139.0\left(\mathrm{C}_{\mathrm{q}}\right), 133.7\left(\mathrm{C}_{\mathrm{q}}\right), 133.6\left(\mathrm{C}_{\mathrm{q}}\right), 133.3\left(\mathrm{C}_{\mathrm{q}}\right), 132.4\left(\mathrm{C}_{\mathrm{q}}\right), 132.3(\mathrm{CH})$, $132.1\left(\mathrm{C}_{\mathrm{q}}\right), 131.4(\mathrm{CH}), 130.5(\mathrm{CH}), 130.4(\mathrm{CH}), 130.0\left(\mathrm{C}_{\mathrm{q}}\right), 129.9(\mathrm{CH}), 129.8(\mathrm{CH})$, $129.0(\mathrm{CH}), 128.7(\mathrm{CH}), 128.6(\mathrm{CH}), 128.3(\mathrm{CH}), 127.9(\mathrm{CH}), 127.2(\mathrm{CH}), 126.8$ $(\mathrm{CH}), 125.6(\mathrm{CH}), 125.2(\mathrm{CH}), 76.4(\mathrm{CH}), 76.1(\mathrm{CH}), 42.7\left(\mathrm{CH}_{2}\right), 36.7\left(\mathrm{CH}_{2}\right), 36.2$ $\left(\mathrm{C}_{\mathrm{q}}\right), 28.9(\mathrm{CH}), 18.2\left(\mathrm{CH}_{3}\right), 18.0\left(\mathrm{CH}_{3}\right) .{ }^{19} \mathrm{~F} \mathrm{NMR}\left(376 \mathrm{MHz}, \mathrm{CDCl}_{3}\right): \delta=-150.81$, -150.86 (d, $J=2.4 \mathrm{~Hz}$ ). IR (ATR): 2901, 2847, 1604, 1213, 1052, 760, $698 \mathrm{~cm}^{-1}$.

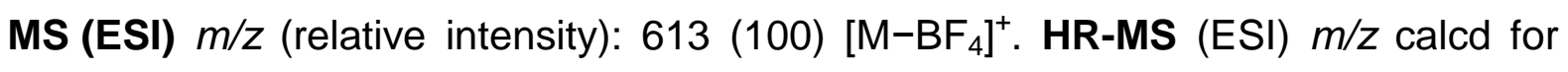

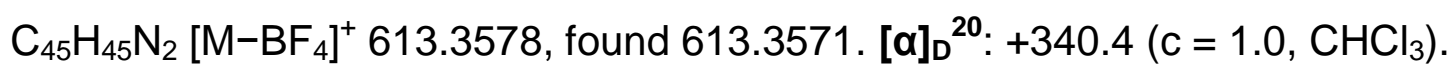

\subsubsection{Experimental Procedures and Analytical Data of Novel Chiral HASPOs}

(4R,5R)-1,3-Bis[5-(adamantan-1-yl)-2-methylphenyl]-4,5-diphenyl-1,3,2diazaphospholidine 2-Oxide (213)

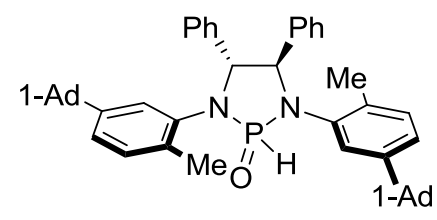

Following a modified procedure, ${ }^{[239 \mathrm{~b}]} \mathrm{PCl}_{3}(45 \mu \mathrm{L}, 0.51 \mathrm{mmol}, 1.0$ equiv) was added dropwise at $0^{\circ} \mathrm{C}$ to a solution of the $N, N^{\prime}$-diarylated diamine (see the synthesis of 
pre-ligand 180) (340 mg, $0.51 \mathrm{mmol}, 1.0$ equiv) and $\mathrm{Et}_{3} \mathrm{~N}(0.54 \mathrm{~mL}, 3.85 \mathrm{mmol}$, 7.5 equiv) in $\mathrm{CH}_{2} \mathrm{Cl}_{2}(2.1 \mathrm{~mL})$. After stirring at ambient temperature for $1 \mathrm{~h}, \mathrm{H}_{2} \mathrm{O}$ (9.3 $\mu \mathrm{L}, 0.51 \mathrm{mmol}, 1.0$ equiv) was added at $0{ }^{\circ} \mathrm{C}$. The resulting mixture was stirred for $20 \mathrm{~h}$ at $23^{\circ} \mathrm{C}$, filtered through Celite ${ }^{\circledR}$ and concentrated under reduced pressure. The residue was purified by column chromatography on silica gel ( $n$-hexane/EtOAc $=19 / 1 \rightarrow 9 / 1)$ to yield $213(211 \mathrm{mg}, 58 \%)$ as a yellow foam. M. p. $=147-149^{\circ} \mathrm{C} .{ }^{1} \mathrm{H}$ NMR $\left(300 \mathrm{MHz}, \mathrm{CDCl}_{3}\right): \delta=8.07\left(\mathrm{~d}, J_{\mathrm{H}-\mathrm{P}}=603.3 \mathrm{~Hz}, 1 \mathrm{H}\right)$, $7.44(\mathrm{dd}, J=7.9,1.7 \mathrm{~Hz}, 2 \mathrm{H}), 7.34-7.17(\mathrm{~m}, 9 \mathrm{H}), 7.05-6.94(\mathrm{~m}, 5 \mathrm{H}), 5.12$ (ddd, $J=6.1,3.1,1.0 \mathrm{~Hz}, 1 \mathrm{H}), 4.90(\mathrm{t}, J=6.0 \mathrm{~Hz}, 1 \mathrm{H}), 2.46(\mathrm{~s}, 3 \mathrm{H}), 2.41(\mathrm{~s}, 3 \mathrm{H}), 2.12-$ $1.93(\mathrm{~m}, 6 \mathrm{H}), 1.84-1.62(\mathrm{~m}, 24 \mathrm{H}) .{ }^{13} \mathrm{C}$ NMR $\left(125 \mathrm{MHz}, \mathrm{CDCl}_{3}\right): \delta=150.1\left(\mathrm{C}_{\mathrm{q}}\right), 149.8$ $\left(\mathrm{C}_{\mathrm{q}}\right), 139.4\left(\mathrm{C}_{\mathrm{q}}\right), 139.4\left(\mathrm{C}_{\mathrm{q}}\right), 138.6\left(\mathrm{C}_{\mathrm{q}}\right), 138.5\left(\mathrm{C}_{\mathrm{q}}\right), 136.7\left(\mathrm{C}_{\mathrm{q}}\right), 136.6\left(\mathrm{C}_{\mathrm{q}}\right), 136.0$ $\left(\mathrm{C}_{\mathrm{q}}\right), 135.9\left(\mathrm{C}_{\mathrm{q}}\right), 134.3\left(\mathrm{C}_{\mathrm{q}}\right), 134.2\left(\mathrm{C}_{\mathrm{q}}\right), 132.4\left(\mathrm{C}_{\mathrm{q}}\right), 132.4\left(\mathrm{C}_{\mathrm{q}}\right), 130.9(\mathrm{CH}), 128.6$ $(\mathrm{CH}), 128.5(\mathrm{CH}), 128.3(\mathrm{CH}), 128.2(\mathrm{CH}), 128.1(\mathrm{CH}), 127.7(\mathrm{CH}), 126.2(\mathrm{CH})$, $124.5(\mathrm{CH}), 123.7(\mathrm{CH}), 123.0(\mathrm{CH}), 72.6(\mathrm{~d}, J=9.0 \mathrm{~Hz}, \mathrm{CH}), 72.5$ (d, J = $10.2 \mathrm{~Hz}$, $\mathrm{CH}), 43.2\left(\mathrm{CH}_{2}\right), 43.2\left(\mathrm{CH}_{2}\right), 37.0\left(\mathrm{CH}_{2}\right), 37.0\left(\mathrm{CH}_{2}\right), 36.0\left(\mathrm{C}_{\mathrm{q}}\right), 36.0\left(\mathrm{C}_{\mathrm{q}}\right), 29.1(\mathrm{CH})$, $19.1\left(\mathrm{CH}_{3}\right), 18.7\left(\mathrm{CH}_{3}\right) \cdot{ }^{31} \mathrm{P}$ NMR (162 MHz, $\left.\mathrm{CDCl}_{3}\right): \delta=9.94$. IR (ATR): 2899, 2846, 1450, 1240, 1137, 1005, 751, $698 \mathrm{~cm}^{-1}$. MS (ESI) $\mathrm{m} / z$ (relative intensity): 2143 (100) $[3 \mathrm{M}+\mathrm{Na}]^{+}, 1437(37)[2 \mathrm{M}+\mathrm{Na}]^{+}, 729(35)[\mathrm{M}+\mathrm{Na}]^{+}$. HR-MS (ESI) $\mathrm{m} / z$ calcd for

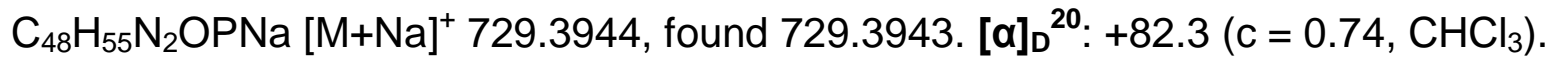

(3a $R, 8 \mathrm{a} R$ )-2,2-Dimethyl-4,4,8,8-tetra(2-methyl-5-adamant-1-ylphenyl)tetrahydro$[1,3]$ dioxolo[4,5-e][1,3,2]dioxaphosphepine 6-Oxide (218)
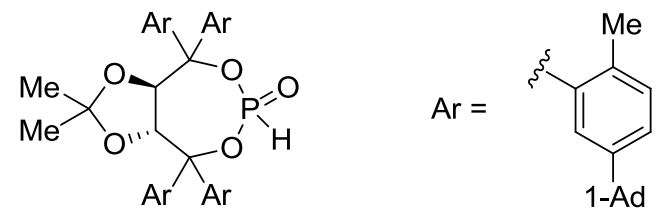

The corresponding $(R, R)$-TADDOL was prepared in 2 steps from $\mathrm{L}-(+)$-diethyl tartrate and $\mathbf{2 3 4}$ following reported procedures. ${ }^{[336]}$

Following a modified procedure, ${ }^{[207]} \mathrm{PCl}_{3}(158 \mu \mathrm{L}, 1.81 \mathrm{mmol}, 1.2$ equiv) was added dropwise at $0{ }^{\circ} \mathrm{C}$ to a solution of the diol $\left(1.60 \mathrm{~g}, 1.51 \mathrm{mmol}, 1.0\right.$ equiv) and $\mathrm{Et}_{3} \mathrm{~N}$ 
(0.63 mL, $4.53 \mathrm{mmol}, 3.0$ equiv) in THF $(9.0 \mathrm{~mL})$. After stirring at ambient temperature for $16 \mathrm{~h}, \mathrm{Et}_{3} \mathrm{~N}\left(0.21 \mathrm{~mL}, 1.51 \mathrm{mmol}, 1.0\right.$ equiv) and $\mathrm{H}_{2} \mathrm{O}(27 \mu \mathrm{L}$, $1.51 \mathrm{mmol}, 1.0$ equiv) were added at $0^{\circ} \mathrm{C}$. The resulting mixture was stirred for $8 \mathrm{~h}$ at $23^{\circ} \mathrm{C}$, filtered through Celite ${ }^{\circledR}$ and concentrated under reduced pressure. The residue was purified by column chromatography on silica gel $(n$-hexane/EtOAc $=$ $25 / 1 \rightarrow 6 / 1)$ to yield $218\left(835 \mathrm{mg}, 50 \%\right.$ ) as a colorless solid. M. p. $=229-231^{\circ} \mathrm{C}$. ${ }^{1} \mathrm{H}$ NMR $\left(400 \mathrm{MHz}, \mathrm{CDCl}_{3}\right): \delta=8.54-7.35$ (brm, 4H), 7.26-6.78 (brm, 9H), 6.395.48 (brm, $2 \mathrm{H}), 2.31-1.32(\mathrm{~m}, 72 \mathrm{H}), 1.18-0.17(\mathrm{~m}, 6 \mathrm{H})$. As previously reported in a similar situation, ${ }^{[207]}$ peaks were noticeably broadened and difficult to integrate, likely due to slow rotation. ${ }^{13} \mathrm{C}$ NMR $\left(100 \mathrm{MHz}, \mathrm{CDCl}_{3}\right): \delta=148.9\left(\mathrm{C}_{\mathrm{q}}\right), 147.9\left(\mathrm{C}_{\mathrm{q}}\right), 147.5$ $\left(\mathrm{C}_{\mathrm{q}}\right), 140.1\left(\mathrm{C}_{\mathrm{q}}\right), 136.4\left(\mathrm{C}_{\mathrm{q}}\right), 136.1\left(\mathrm{C}_{\mathrm{q}}\right), 135.8\left(\mathrm{C}_{\mathrm{q}}\right), 134.8\left(\mathrm{C}_{\mathrm{q}}\right), 132.9(\mathrm{CH}), 132.5$ $(\mathrm{CH}), 132.5(\mathrm{CH}), 132.3(\mathrm{CH}), 126.1(\mathrm{CH}), 125.1(\mathrm{CH}), 124.6(\mathrm{CH}), 124.4(\mathrm{CH})$, $113.4\left(\mathrm{C}_{\mathrm{q}}\right), 91.3\left(\mathrm{C}_{\mathrm{q}}\right), 90.5\left(\mathrm{C}_{\mathrm{q}}\right), 81.3(\mathrm{CH}), 80.7(\mathrm{CH}), 77.4(\mathrm{CH}), 43.3\left(\mathrm{CH}_{2}\right), 43.3$ $\left(\mathrm{CH}_{2}\right), 37.0\left(\mathrm{CH}_{2}\right), 36.9\left(\mathrm{CH}_{2}\right), 36.8\left(\mathrm{CH}_{2}\right), 36.8\left(\mathrm{CH}_{2}\right), 36.3(\mathrm{CH}), 36.1\left(\mathrm{C}_{\mathrm{q}}\right), 36.0$ $\left(\mathrm{C}_{q}\right), 29.1(\mathrm{CH}), 29.1(\mathrm{CH}), 29.0(\mathrm{CH}), 29.0(\mathrm{CH}), 27.1\left(\mathrm{CH}_{3}\right), 25.9\left(\mathrm{CH}_{3}\right), 23.0\left(\mathrm{CH}_{3}\right)$, $22.5\left(\mathrm{CH}_{3}\right), 21.2\left(\mathrm{CH}_{3}\right), 21.0\left(\mathrm{CH}_{3}\right) .{ }^{31} \mathrm{P}$ NMR (162 MHz, $\left.\mathrm{CDCl}_{3}\right): \delta=-7.14$. IR (ATR): 2900, 2847, 1449, 1084, 975, 941, 806, $755 \mathrm{~cm}^{-1}$. MS (ESI) $\mathrm{m} / \mathrm{z}$ (relative intensity): $1128(100)[\mathrm{M}+\mathrm{Na}]^{+}$. HR-MS (ESI) $\mathrm{m} / z$ calcd for $\mathrm{C}_{75} \mathrm{H}_{93} \mathrm{O}_{5} \mathrm{PNa}[\mathrm{M}+\mathrm{Na}]^{+}$

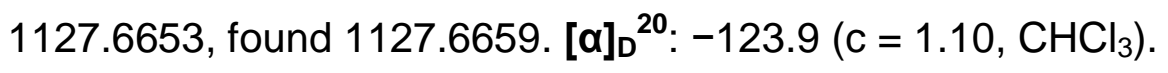




\subsection{Iron-Catalyzed Enantioselective C-H Alkylation with Alkenes}

\subsubsection{Experimental Procedures and Analytical Data}

(S)-1-Benzyl-2-[1-(4-methoxyphenyl)ethyl]-1 H-indole-3-carbaldehyde (62cc)

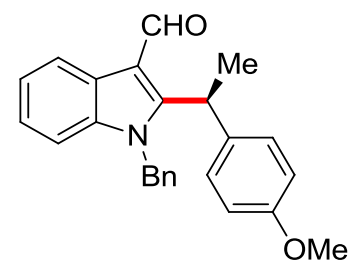

The general procedure GP6 was followed using indole $59 \mathrm{c}(85 \mathrm{mg}, 0.25 \mathrm{mmol}$, 1.0 equiv) and styrene $60 \mathrm{c}$ ( $50 \mathrm{mg}, 0.38 \mathrm{mmol}, 1.5$ equiv). Purification by column chromatography on silica gel $(n$-hexane/EtOAc $=8 / 1 \rightarrow 6 / 1)$ yielded 62cc $(88.0 \mathrm{mg}$, 95\%) as a yellow solid. M. p. $=58-59{ }^{\circ} \mathrm{C} .{ }^{1} \mathbf{H}$ NMR $\left(400 \mathrm{MHz}, \mathrm{CDCl}_{3}\right): \delta=10.22(\mathrm{~s}$, $1 \mathrm{H}$ ), 8.43 (ddd, $J=7.9,1.3,0.7 \mathrm{~Hz}, 1 \mathrm{H}$ ), 7.31 (ddd, $J=8.0,7.1,1.1 \mathrm{~Hz}, 1 \mathrm{H}$ ), 7.29 $7.20(\mathrm{~m}, 4 \mathrm{H}), 7.14(\mathrm{dt}, J=8.1,0.9 \mathrm{~Hz}, 1 \mathrm{H}), 7.11(\mathrm{dd}, J=8.9,0.9 \mathrm{~Hz}, 2 \mathrm{H}), 6.90$ (dddd, $J=6.7,2.7,2.0,1.1 \mathrm{~Hz}, 2 \mathrm{H}$ ), $6.80(\mathrm{~d}, J=8.8 \mathrm{~Hz}, 2 \mathrm{H}$ ), $5.25(\mathrm{~d}, J=17.1 \mathrm{~Hz}$, 1H), $5.21(\mathrm{~d}, J=17.1 \mathrm{~Hz}, 1 \mathrm{H}), 4.99(\mathrm{q}, J=7.4 \mathrm{~Hz}, 1 \mathrm{H}), 3.77(\mathrm{~s}, 3 \mathrm{H}), 1.72(\mathrm{~d}$, $J=7.4 \mathrm{~Hz}, 3 \mathrm{H}) .{ }^{13} \mathrm{C}$ NMR $\left(100 \mathrm{MHz}, \mathrm{CDCl}_{3}\right): \delta=185.4(\mathrm{CH}), 158.5\left(\mathrm{C}_{\mathrm{q}}\right), 154.2\left(\mathrm{C}_{\mathrm{q}}\right)$, $137.0\left(\mathrm{C}_{\mathrm{q}}\right), 136.0\left(\mathrm{C}_{\mathrm{q}}\right), 133.2\left(\mathrm{C}_{\mathrm{q}}\right), 128.9(\mathrm{CH}), 128.0(\mathrm{CH}), 127.6(\mathrm{CH}), 126.0\left(\mathrm{C}_{\mathrm{q}}\right)$, $125.8(\mathrm{CH}), 123.7(\mathrm{CH}), 123.1(\mathrm{CH}), 121.7(\mathrm{CH}), 114.8\left(\mathrm{C}_{\mathrm{q}}\right), 114.2(\mathrm{CH}), 110.1(\mathrm{CH})$, $55.3\left(\mathrm{CH}_{3}\right), 47.5\left(\mathrm{CH}_{2}\right), 34.3(\mathrm{CH}), 20.0\left(\mathrm{CH}_{3}\right)$. IR (ATR): 2929, 1643, 1510, 1246, 1179, 1029, $744 \mathrm{~cm}^{-1}$. MS (EI) $\mathrm{m} / z$ (relative intensity): 369 (79) [M] $]^{+}, 261$ (30), 197 (84), 91 (100). HR-MS (EI) $\mathrm{m} / z$ calcd for $\mathrm{C}_{25} \mathrm{H}_{23} \mathrm{NO}_{2}[\mathrm{M}]^{+} 369.1729$, found 369.1735. $[\alpha]_{D}^{23}$ : $-54.9\left(\mathrm{c}=1.74, \mathrm{CHCl}_{3}\right)$. HPLC separation (Chiralpak ${ }^{\circledR} \mathrm{IA}-3, n$-hexane/iPrOH $80: 20,1.0 \mathrm{~mL} / \mathrm{min}$, detection at $273 \mathrm{~nm}): t_{r}$ (major) $=8.3 \mathrm{~min}, t_{r}($ minor $)=9.5 \mathrm{~min}$, 92:8 e.r. 


\section{(S)-1-(4-Methoxybenzyl)-2-[1-(4-methoxyphenyl)ethyl]-1H-indole-3-}

\section{carbaldehyde (62dc)}

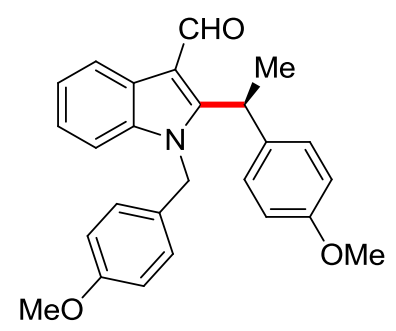

The general procedure GP6 was followed using indole $59 \mathrm{~d}(93 \mathrm{mg}, 0.25 \mathrm{mmol}$, 1.0 equiv) and styrene $60 \mathrm{c}$ (50 mg, $0.38 \mathrm{mmol}, 1.5$ equiv). Purification by column chromatography on silica gel $(n$-hexane/EtOAc $=8 / 1 \rightarrow 5.5 / 1)$ yielded 62dc $(79.4 \mathrm{mg}$, $80 \%$ ) as a yellow solid. M. p. $=56-57{ }^{\circ} \mathrm{C} .{ }^{1} \mathbf{H}$ NMR $\left(500 \mathrm{MHz}, \mathrm{CDCl}_{3}\right): \delta=10.20(\mathrm{~s}$, $1 \mathrm{H}$ ), 8.42 (dt, $J=7.9,1.0 \mathrm{~Hz}, 1 \mathrm{H}$ ), 7.30 (ddd, $J=8.1,7.1,1.1 \mathrm{~Hz}, 1 \mathrm{H}$ ), 7.23 (ddd, $J=8.3,7.1,1.3 \mathrm{~Hz}, 1 \mathrm{H}), 7.16(\mathrm{dt}, J=8.2,1.0 \mathrm{~Hz}, 1 \mathrm{H}), 7.11(\mathrm{~d}, J=8.4 \mathrm{~Hz}, 2 \mathrm{H})$, 6.86-6.74 (m, 6H), $5.19(\mathrm{~d}, J=17.1 \mathrm{~Hz}, 1 \mathrm{H}), 5.15(\mathrm{~d}, J=17.1 \mathrm{~Hz}, 1 \mathrm{H}), 4.99(\mathrm{q}$, $J=7.4 \mathrm{~Hz}, 1 \mathrm{H}), 3.77(\mathrm{~s}, 3 \mathrm{H}), 3.76(\mathrm{~s}, 3 \mathrm{H}), 1.72(\mathrm{~d}, J=7.4 \mathrm{~Hz}, 3 \mathrm{H}) .{ }^{13} \mathrm{C}$ NMR $\left(125 \mathrm{MHz}, \mathrm{CDCl}_{3}\right): \delta=185.2(\mathrm{CH}), 158.9\left(\mathrm{C}_{\mathrm{q}}\right), 158.3\left(\mathrm{C}_{\mathrm{q}}\right), 154.1\left(\mathrm{C}_{\mathrm{q}}\right), 137.0\left(\mathrm{C}_{\mathrm{q}}\right)$, $133.3\left(\mathrm{C}_{\mathrm{q}}\right), 128.0(\mathrm{CH}), 127.9\left(\mathrm{C}_{\mathrm{q}}\right), 127.0(\mathrm{CH}), 126.0\left(\mathrm{C}_{\mathrm{q}}\right), 123.5(\mathrm{CH}), 123.0(\mathrm{CH})$, $121.7(\mathrm{CH}), 114.7\left(\mathrm{C}_{\mathrm{q}}\right), 114.2(\mathrm{CH}), 114.1(\mathrm{CH}), 110.2(\mathrm{CH}), 55.3\left(\mathrm{CH}_{3}\right), 55.3\left(\mathrm{CH}_{3}\right)$, $47.1\left(\mathrm{CH}_{2}\right), 34.5(\mathrm{CH}), 20.2\left(\mathrm{CH}_{3}\right)$. IR (ATR): 2929, 1643, 1509, 1244, 1175, 1030, $739 \mathrm{~cm}^{-1}$. MS (EI) m/z (relative intensity): 399 (31) [M] ${ }^{+}, 278$ (40), 263 (10), 250 (13), 227 (24), 121 (100). HR-MS (EI) $\mathrm{m} / z$ calcd for $\mathrm{C}_{26} \mathrm{H}_{25} \mathrm{NO}_{3}[\mathrm{M}]^{+} 399.1834$, found 399.1826. $[\alpha]_{D}{ }^{23}:-52.4\left(\mathrm{C}=1.17, \mathrm{CHCl}_{3}\right)$. HPLC separation (Chiralpak ${ }^{\circledR} \mathrm{IA}-3$, $n$-hexane/PrOH 80:20, $1.0 \mathrm{~mL} / \mathrm{min}$, detection at $250 \mathrm{~nm}$ ): $t_{r}$ (major) $=10.4 \mathrm{~min}$, $t_{r}(\operatorname{minor})=11.7 \mathrm{~min}, 92: 8$ e.r. 
(S)-1-(4-Fluorobenzyl)-2-[1-(4-methoxyphenyl)ethyl]-1 $H$-indole-3-carbaldehyde $(62 \mathrm{ec})$

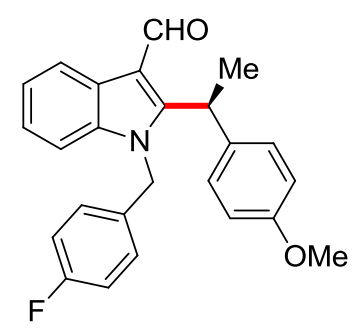

The general procedure GP6 was followed using indole $59 \mathrm{e}(90 \mathrm{mg}, 0.25 \mathrm{mmol}$, 1.0 equiv) and styrene $60 \mathrm{c}$ (50 mg, $0.38 \mathrm{mmol}, 1.5$ equiv). Purification by column chromatography on silica gel $(n$-hexane/EtOAc $=8 / 1 \rightarrow 5.5 / 1)$ yielded 62ec $(90.9 \mathrm{mg}$, 94\%) as a yellow solid. M. p. $=60-61{ }^{\circ} \mathrm{C} .{ }^{1} \mathrm{H}$ NMR $\left(500 \mathrm{MHz}, \mathrm{CDCl}_{3}\right): \delta=10.24(\mathrm{~s}$, $1 \mathrm{H}$ ), 8.43 (ddd, $J=7.8,1.2,0.7 \mathrm{~Hz}, 1 \mathrm{H}$ ), 7.32 (ddd, $J=8.0,7.1,1.0 \mathrm{~Hz}, 1 \mathrm{H}$ ), 7.23 (ddd, $J=8.3,7.2,1.2 \mathrm{~Hz}, 1 \mathrm{H}$ ), 7.14-7.08 (m, 3H), $6.94(\mathrm{t}, J=8.6 \mathrm{~Hz}, 2 \mathrm{H}), 6.84$ (dd, $J=8.9,5.1 \mathrm{~Hz}, 2 \mathrm{H}), 6.80(\mathrm{~d}, J=8.8 \mathrm{~Hz}, 2 \mathrm{H}), 5.19(\mathrm{~s}, 2 \mathrm{H}), 5.02(\mathrm{q}, J=7.4 \mathrm{~Hz}, 1 \mathrm{H})$, $3.77(\mathrm{~s}, 3 \mathrm{H}), 1.72(\mathrm{~d}, J=7.4 \mathrm{~Hz}, 3 \mathrm{H}) .{ }^{13} \mathrm{C}$ NMR $\left(125 \mathrm{MHz}, \mathrm{CDCl}_{3}\right): \delta=185.3(\mathrm{CH})$, $162.0\left(\mathrm{~d},{ }^{1} \mathrm{~J}_{\mathrm{C}-\mathrm{F}}=246.5 \mathrm{~Hz}, \mathrm{C}_{\mathrm{q}}\right), 158.4\left(\mathrm{C}_{\mathrm{q}}\right), 154.0\left(\mathrm{C}_{\mathrm{q}}\right), 136.9\left(\mathrm{C}_{\mathrm{q}}\right), 132.9\left(\mathrm{C}_{\mathrm{q}}\right), 131.6$ $\left(\mathrm{d},{ }^{4} \mathrm{~J}_{\mathrm{C}-\mathrm{F}}=3.1 \mathrm{~Hz}, \mathrm{C}_{\mathrm{q}}\right), 128.0(\mathrm{CH}), 127.4\left(\mathrm{~d},{ }^{3} \mathrm{~J}_{\mathrm{C}-\mathrm{F}}=8.2 \mathrm{~Hz}, \mathrm{CH}\right), 125.9\left(\mathrm{C}_{\mathrm{q}}\right), 123.7$ $(\mathrm{CH}), 123.2(\mathrm{CH}), 121.7(\mathrm{CH}), 115.7\left(\mathrm{~d},{ }^{2} \mathrm{~J}_{\mathrm{C}-\mathrm{F}}=21.7 \mathrm{~Hz}, \mathrm{CH}\right), 114.8\left(\mathrm{C}_{\mathrm{q}}\right), 114.1$ $(\mathrm{CH}), 110.0(\mathrm{CH}), 55.2\left(\mathrm{CH}_{3}\right), 46.9\left(\mathrm{CH}_{2}\right), 34.2(\mathrm{CH}), 19.9\left(\mathrm{CH}_{3}\right) \cdot{ }^{19} \mathbf{F}$ NMR $\left(471 \mathrm{MHz}, \mathrm{CDCl}_{3}\right): \delta=-114.54$ (tt, $J=8.5,5.2 \mathrm{~Hz}$ ). IR (ATR): 2932, 1644, 1508, 1247, 823, $743 \mathrm{~cm}^{-1}$. MS (ESI) $\mathrm{m} / z$ (relative intensity): 797 (28) [2M+Na] $]^{+}, 410$ (42) $[\mathrm{M}+\mathrm{Na}]^{+}, 388(100)[\mathrm{M}+\mathrm{H}]^{+}, 280$ (9). HR-MS (ESI) $\mathrm{m} / z$ calcd for $\mathrm{C}_{25} \mathrm{H}_{23} \mathrm{NO}_{2} \mathrm{~F}[\mathrm{M}+\mathrm{H}]^{+}$ 388.1707, found 388.1696. [ []$_{D}{ }^{23}$ : $-58.1\left(\mathrm{c}=1.47, \mathrm{CHCl}_{3}\right)$. HPLC separation (Chiralpak ${ }^{\circledR}$ IA-3, $n$-hexane/PrOH $80: 20,1.0 \mathrm{~mL} / \mathrm{min}$, detection at $250 \mathrm{~nm}$ ): $t_{r}($ major $)=8.6 \mathrm{~min}, t_{r}($ minor $)=10.0 \mathrm{~min}, 92: 8$ e.r. 
(S)-2-[1-(4-Methoxyphenyl)ethyl]-1-phenethyl-1H-indole-3-carbaldehyde (62fc)

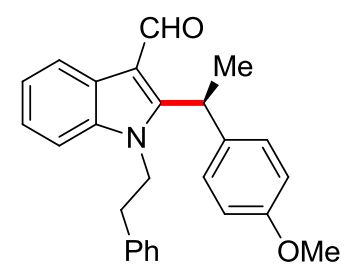

The general procedure GP6 was followed using indole $59 f(89 \mathrm{mg}, 0.25 \mathrm{mmol}$, 1.0 equiv) and styrene $60 \mathrm{c}$ (50 mg, $0.38 \mathrm{mmol}, 1.5$ equiv). Purification by column chromatography on silica gel $(n$-hexane/EtOAc $=9 / 1 \rightarrow 7 / 1)$ yielded 62fc $(83.4 \mathrm{mg}$, $87 \%)$ as a yellow solid. Using 177: $67.5 \mathrm{mg}(70 \%), 89: 11$ e.r. M. p. $=48-49{ }^{\circ} \mathrm{C}$. ${ }^{1} \mathrm{H}$ NMR $\left(300 \mathrm{MHz}, \mathrm{CDCl}_{3}\right): \delta=10.22(\mathrm{~s}, 1 \mathrm{H}), 8.49-8.37(\mathrm{~m}, 1 \mathrm{H}), 7.44-7.22(\mathrm{~m}, 6 \mathrm{H})$, 7.18 (dd, $J=8.9,0.9 \mathrm{~Hz}, 2 \mathrm{H}$ ), 7.02 (dd, $J=7.9,1.6 \mathrm{~Hz}, 2 \mathrm{H}$ ), 6.89 (d, $J=8.8 \mathrm{~Hz}, 2 \mathrm{H}$ ), 4.95 (q, $J=7.4 \mathrm{~Hz}, 1 \mathrm{H}$ ), 4.22 (ddd, $J=8.9,6.9,1.4 \mathrm{~Hz}, 2 \mathrm{H}$ ), 3.80 (s, 3H), 2.94 (ddd, $J=13.3,8.8,6.7 \mathrm{~Hz}, 1 \mathrm{H}), 2.64-2.51(\mathrm{~m}, 1 \mathrm{H}), 1.79(\mathrm{~d}, J=7.4 \mathrm{~Hz}, 3 \mathrm{H}) .{ }^{13} \mathrm{C}$ NMR $\left(125 \mathrm{MHz}, \mathrm{CDCl}_{3}\right): \delta=185.0(\mathrm{CH}), 158.4\left(\mathrm{C}_{\mathrm{q}}\right), 153.4\left(\mathrm{C}_{\mathrm{q}}\right), 137.4\left(\mathrm{C}_{\mathrm{q}}\right), 136.1\left(\mathrm{C}_{\mathrm{q}}\right)$, $133.4\left(\mathrm{C}_{\mathrm{q}}\right), 128.7(\mathrm{CH}), 128.5(\mathrm{CH}), 128.1(\mathrm{CH}), 126.9(\mathrm{CH}), 126.2\left(\mathrm{C}_{\mathrm{q}}\right), 123.4(\mathrm{CH})$, $122.9(\mathrm{CH}), 121.8(\mathrm{CH}), 114.4\left(\mathrm{C}_{\mathrm{q}}\right), 114.2(\mathrm{CH}), 109.7(\mathrm{CH}), 55.3\left(\mathrm{CH}_{3}\right), 46.1\left(\mathrm{CH}_{2}\right)$, $35.3\left(\mathrm{CH}_{2}\right), 34.4(\mathrm{CH}), 20.0\left(\mathrm{CH}_{3}\right)$. IR (ATR): 2931, 1643, 1510, 1246, 745, $699 \mathrm{~cm}^{-1}$. MS (ESI) $\mathrm{m} / \mathrm{z}$ (relative intensity): $789(16)[2 \mathrm{M}+\mathrm{Na}]^{+}, 767(5)[2 \mathrm{M}+\mathrm{H}]^{+}, 398$ (22), $384(100)[\mathrm{M}+\mathrm{H}]^{+}, 117$ (12). HR-MS (ESI) $\mathrm{m} / z$ calcd for $\mathrm{C}_{26} \mathrm{H}_{26} \mathrm{NO}_{2}[\mathrm{M}+\mathrm{H}]^{+}$ 384.1958, found 384.1969. $[\alpha]_{D}{ }^{20}:-19.6\left(\mathrm{c}=1.20, \mathrm{CHCl}_{3}\right)$. HPLC separation (Chiralpak ${ }^{\circledR}$ IA-3, $n$-hexane/PrOH $80: 20,1.0 \mathrm{~mL} / \mathrm{min}$, detection at $250 \mathrm{~nm}$ ): $t_{r}($ major $)=7.6 \mathrm{~min}, t_{r}($ minor $)=8.6 \mathrm{~min}, 86: 14$ e.r.

(S)-1-(Methoxymethyl)-2-[1-(4-methoxyphenyl)ethyl]-1H-indole-3-carbaldehyde (62gc)

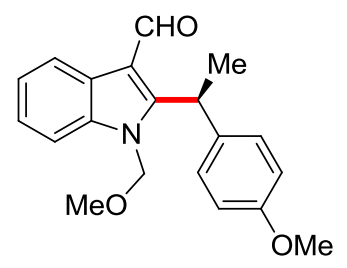


The general procedure GP6 was followed using indole $59 \mathrm{~g}(74 \mathrm{mg}, 0.25 \mathrm{mmol}$, 1.0 equiv) and styrene $60 \mathrm{c}$ (50 mg, $0.38 \mathrm{mmol}, 1.5$ equiv). Purification by column chromatography on silica gel ( $n$-hexane/EtOAc $=8 / 1 \rightarrow 6 / 1)$ yielded 62gc $(64.9 \mathrm{mg}$, $80 \%)$ as a yellow oil. Using 177: $68.0 \mathrm{mg}(84 \%), 87: 13$ e.r. ${ }^{1} \mathbf{H} \mathrm{NMR}(500 \mathrm{MHz}$, $\left.\mathrm{CDCl}_{3}\right): \delta=10.20(\mathrm{~s}, 1 \mathrm{H}), 8.41-8.34(\mathrm{~m}, 1 \mathrm{H}), 7.48-7.42(\mathrm{~m}, 1 \mathrm{H}), 7.31$ (ddd, $J=8.0$, 7.2, $0.9 \mathrm{~Hz}, 2 \mathrm{H}), 7.21(\mathrm{~d}, J=8.0 \mathrm{~Hz}, 2 \mathrm{H}), 6.86(\mathrm{~d}, J=8.8 \mathrm{~Hz}, 2 \mathrm{H}), 5.35(\mathrm{~d}$, $J=11.0 \mathrm{~Hz}, 1 \mathrm{H}), 5.31(\mathrm{~d}, J=11.0 \mathrm{~Hz}, 1 \mathrm{H}), 5.07(\mathrm{q}, J=7.3 \mathrm{~Hz}, 1 \mathrm{H}), 3.79(\mathrm{~s}, 3 \mathrm{H})$, $3.21(\mathrm{~s}, 3 \mathrm{H}), 1.89(\mathrm{~d}, J=7.4 \mathrm{~Hz}, 3 \mathrm{H}) .{ }^{13} \mathrm{C}$ NMR $\left(125 \mathrm{MHz}, \mathrm{CDCl}_{3}\right): \delta=185.8(\mathrm{CH})$, $158.5\left(\mathrm{C}_{\mathrm{q}}\right), 154.6\left(\mathrm{C}_{\mathrm{q}}\right), 137.1\left(\mathrm{C}_{\mathrm{q}}\right), 133.5\left(\mathrm{C}_{\mathrm{q}}\right), 128.1(\mathrm{CH}), 125.8\left(\mathrm{C}_{\mathrm{q}}\right), 123.8(\mathrm{CH})$, $123.3(\mathrm{CH}), 121.8(\mathrm{CH}), 115.3\left(\mathrm{C}_{\mathrm{q}}\right), 114.1(\mathrm{CH}), 109.9(\mathrm{CH}), 74.4\left(\mathrm{CH}_{2}\right), 56.1\left(\mathrm{CH}_{3}\right)$, $55.3\left(\mathrm{CH}_{3}\right), 34.2(\mathrm{CH}), 20.5\left(\mathrm{CH}_{3}\right)$. IR (ATR): 2932, 2836, 1647, 1510, 1247, 1029, $748 \mathrm{~cm}^{-1}$. MS (EI) $\mathrm{m} / z$ (relative intensity): 323 (100) [M], 276 (40), 263 (27), 215 (29), 151 (44), 121 (50). HR-MS (EI) $\mathrm{m} / z$ calcd for $\mathrm{C}_{20} \mathrm{H}_{21} \mathrm{NO}_{3}[\mathrm{M}]^{+} 323.1521$, found 323.1530. $[\alpha]_{\mathrm{D}}{ }^{20}:-51.0\left(\mathrm{C}=1.01, \mathrm{CHCl}_{3}\right)$. HPLC separation (Chiralpak ${ }^{\circledR}$ IB-3, $n$-hexane/iPrOH 80:20, $1.0 \mathrm{~mL} / \mathrm{min}$, detection at $273 \mathrm{~nm}): t_{r}$ (major) $=9.6 \mathrm{~min}$, $t_{r}($ minor $)=10.4 \min , 86: 14$ e.r.

(S)-1-(2,2-Difluoroethyl)-2-[1-(4-methoxyphenyl)ethyl]-1 H-indole-3carbaldehyde (62hc)

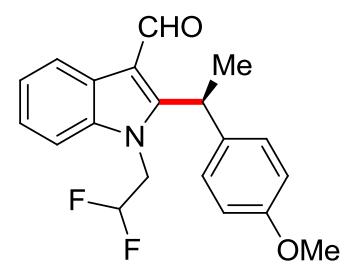

The general procedure GP6 was followed using indole $59 \mathrm{~h}(79 \mathrm{mg}, 0.25 \mathrm{mmol}$, 1.0 equiv) and styrene 60c (50 mg, $0.38 \mathrm{mmol}, 1.5$ equiv). Purification by column chromatography on silica gel $(n$-hexane/EtOAc $=8 / 1 \rightarrow 6 / 1)$ yielded 62hc $(82.4 \mathrm{mg}$, 96\%) as a yellow solid. M. p. $=49-50{ }^{\circ} \mathrm{C} .{ }^{1} \mathrm{H}$ NMR $\left(500 \mathrm{MHz}, \mathrm{CDCl}_{3}\right): \delta=10.22$ (s, $1 \mathrm{H}$ ), 8.39 (dt, $J=5.4,2.1 \mathrm{~Hz}, 1 \mathrm{H}), 7.38-7.29(\mathrm{~m}, 3 \mathrm{H}), 7.20(\mathrm{dd}, J=8.9,0.9 \mathrm{~Hz}, 2 \mathrm{H})$, $6.88(\mathrm{~d}, J=8.8 \mathrm{~Hz}, 2 \mathrm{H}), 5.44(\mathrm{tt}, J=55.2,4.2 \mathrm{~Hz}, 1 \mathrm{H}), 5.05(\mathrm{q}, J=7.4 \mathrm{~Hz}, 1 \mathrm{H})$, 4.47-4.33 (m, 2H), $3.80(\mathrm{~s}, 3 \mathrm{H}), 1.88(\mathrm{~d}, J=7.5 \mathrm{~Hz}, 3 \mathrm{H}) .{ }^{13} \mathrm{C} \mathrm{NMR}(125 \mathrm{MHz}$, 
$\left.\mathrm{CDCl}_{3}\right): \delta=185.5(\mathrm{CH}), 158.7\left(\mathrm{C}_{\mathrm{q}}\right), 153.9\left(\mathrm{C}_{\mathrm{q}}\right), 136.9\left(\mathrm{C}_{\mathrm{q}}\right), 133.0\left(\mathrm{C}_{\mathrm{q}}\right), 128.1(\mathrm{CH})$, $125.9\left(\mathrm{C}_{\mathrm{q}}\right), 124.0(\mathrm{CH}), 123.5(\mathrm{CH}), 121.8(\mathrm{CH}), 115.3\left(\mathrm{C}_{\mathrm{q}}\right), 114.4(\mathrm{CH}), 113.2(\mathrm{t}$, $\left.{ }^{1} J_{\mathrm{C}-\mathrm{F}}=245.3 \mathrm{~Hz}, \mathrm{CH}\right), 109.7(\mathrm{CH}), 55.3\left(\mathrm{CH}_{3}\right), 46.4\left(\mathrm{t},{ }^{2} \mathrm{~J}_{\mathrm{C}-\mathrm{F}}=28.9 \mathrm{~Hz}, \mathrm{CH}_{2}\right), 34.2$ $(\mathrm{CH}), 20.2\left(\mathrm{CH}_{3}\right) .{ }^{19} \mathrm{~F}$ NMR $\left(471 \mathrm{MHz}, \mathrm{CDCl}_{3}\right): \delta=-119.04(\mathrm{dt}, J=55.3,13.2 \mathrm{~Hz})$. IR (ATR): 2960, 2838, 1644, 1511, 1248, 1057, $747 \mathrm{~cm}^{-1}$. MS (EI) $\mathrm{m} / \mathrm{z}$ (relative intensity): 343 (100) [M] $]^{+}, 328$ (44), 326 (33), 311 (25), 235 (56), 121 (30). HR-MS (El) $\mathrm{m} / \mathrm{z}$ calcd for $\mathrm{C}_{20} \mathrm{H}_{19} \mathrm{~F}_{2} \mathrm{NO}_{2}[\mathrm{M}]^{+} 343.1384$, found 343.1386. [a] ${ }_{\mathrm{D}}{ }^{23}:-77.5$ ( $\mathrm{C}=1.50, \mathrm{CHCl}_{3}$ ). HPLC separation (Chiralpak ${ }^{\circledR} \mathrm{IA}-3$, $n$-hexane/PrOH 80:20, $1.0 \mathrm{~mL} / \mathrm{min}$, detection at $250 \mathrm{~nm}$ ): $t_{r}$ (major) $=8.4 \mathrm{~min}, t_{r}($ minor $)=9.1 \mathrm{~min}, 94: 6$ e.r.

\section{(S)-1-[(1,3-Dioxolan-2-yl)methyl]-2-[1-(4-methoxyphenyl)ethyl]-1 H-indole-3-}

\section{carbaldehyde (62ic)}

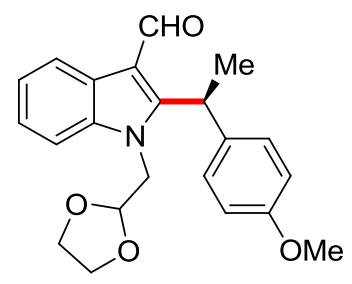

The general procedure GP6 was followed using indole $59 \mathrm{i}(84 \mathrm{mg}, 0.25 \mathrm{mmol}$, 1.0 equiv) and styrene $60 \mathrm{c}$ ( $50 \mathrm{mg}, 0.38 \mathrm{mmol}, 1.5$ equiv). Purification by column chromatography on silica gel $(n$-hexane/EtOAc $=6 / 1 \rightarrow 3 / 1)$ yielded 62ic $(75.2 \mathrm{mg}$, $82 \%)$ as a yellow solid. $\mathbf{M} . \mathbf{p} .=129-132{ }^{\circ} \mathrm{C} .{ }^{1} \mathbf{H}$ NMR $\left(300 \mathrm{MHz}, \mathrm{CDCl}_{3}\right): \delta=10.01$ (s, 1H), 8.40-8.33 (m, 1H), 7.50-7.43 (m, 1H), 7.33-7.26 (m, 2H), 7.20 (dd, $J=8.9$, $0.9 \mathrm{~Hz}, 2 \mathrm{H}), 6.84(\mathrm{~d}, J=8.8 \mathrm{~Hz}, 2 \mathrm{H}), 5.13(\mathrm{t}, J=3.4 \mathrm{~Hz}, 1 \mathrm{H}), 4.93(\mathrm{q}, J=7.3 \mathrm{~Hz}$, $1 \mathrm{H}), 4.34(\mathrm{dd}, J=3.4,1.0 \mathrm{~Hz}, 2 \mathrm{H}), 3.86-3.81(\mathrm{~m}, 4 \mathrm{H}), 3.79(\mathrm{~s}, 3 \mathrm{H}), 1.88(\mathrm{~d}$, $J=7.3 \mathrm{~Hz}, 3 \mathrm{H}) .{ }^{13} \mathrm{C}$ NMR $\left(125 \mathrm{MHz}, \mathrm{CDCl}_{3}\right): \delta=185.9(\mathrm{CH}), 158.3\left(\mathrm{C}_{\mathrm{q}}\right), 155.2\left(\mathrm{C}_{\mathrm{q}}\right)$, $137.0\left(\mathrm{C}_{\mathrm{q}}\right), 134.2\left(\mathrm{C}_{\mathrm{q}}\right), 128.1(\mathrm{CH}), 125.9\left(\mathrm{C}_{\mathrm{q}}\right), 123.3(\mathrm{CH}), 123.0(\mathrm{CH}), 121.8(\mathrm{CH})$, $114.6\left(\mathrm{C}_{\mathrm{q}}\right), 114.1(\mathrm{CH}), 110.5(\mathrm{CH}), 101.9(\mathrm{CH}), 65.3\left(\mathrm{CH}_{2}\right), 65.3\left(\mathrm{CH}_{2}\right), 55.3\left(\mathrm{CH}_{3}\right)$, $46.7\left(\mathrm{CH}_{2}\right), 34.9(\mathrm{CH}), 21.3\left(\mathrm{CH}_{3}\right)$. IR (ATR): 2925, 1633, 1511, 1247, 1030, $745 \mathrm{~cm}^{-1}$. MS (ESI) $\mathrm{m} / z$ (relative intensity): 753 (16) [2M+Na] $]^{+}, 471$ (36), 388 (16) $[\mathrm{M}+\mathrm{Na}]^{+}, 366(100)[\mathrm{M}+\mathrm{H}]^{+}, 216$ (22), 124 (16). HR-MS (ESI) $\mathrm{m} / \mathrm{z}$ calcd for 


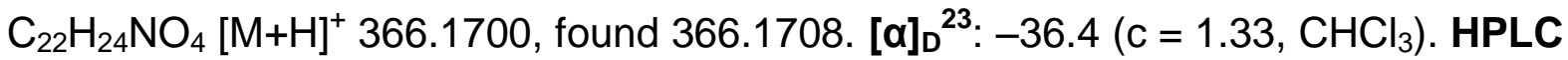
separation (Chiralpak ${ }^{\circledR}$ IF-3, $n$-hexane/iPrOH $80: 20,0.75 \mathrm{~mL} / \mathrm{min}$, detection at $250 \mathrm{~nm}): t_{r}($ minor $)=18.3 \mathrm{~min}, t_{r}($ major $)=21.1 \mathrm{~min}, 6: 94$ e.r.

(S)-2-[1-(4-Methoxyphenyl)ethyl]-1-methyl-1H-indole-3-carbaldehyde (62bc)

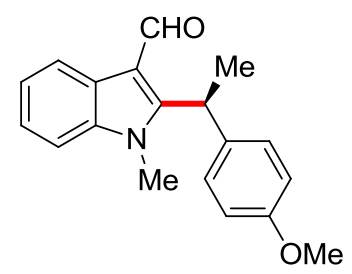

The general procedure GP6 was followed using indole 59b $(66 \mathrm{mg}, 0.25 \mathrm{mmol}$, 1.0 equiv) and styrene $60 \mathrm{c}$ ( $50 \mathrm{mg}, 0.38 \mathrm{mmol}, 1.5$ equiv). Purification by column chromatography on silica gel $(n$-hexane/EtOAc $=8 / 1 \rightarrow 5 / 1)$ yielded 62 bc $(68.2 \mathrm{mg}$, 93\%) as a yellow oil. Using 177: $55.7 \mathrm{mg}$ (76\%), 85:15 e.r. ${ }^{1} \mathbf{H} \mathrm{NMR}(500 \mathrm{MHz}$, $\left.\mathrm{CDCl}_{3}\right): \delta=10.26(\mathrm{~s}, 1 \mathrm{H}), 8.38(\mathrm{ddd}, J=7.6,3.1,1.5 \mathrm{~Hz}, 1 \mathrm{H}), 7.34-7.26(\mathrm{~m}, 3 \mathrm{H})$, 7.17 (dd, $J=8.9,1.0 \mathrm{~Hz}, 2 \mathrm{H}$ ), $6.87(\mathrm{~d}, J=8.8 \mathrm{~Hz}, 2 \mathrm{H}$ ), 5.16 (q, $J=7.4 \mathrm{~Hz}, 1 \mathrm{H}$ ), 3.80 (s, 3H), $3.47(\mathrm{~s}, 3 \mathrm{H}), 1.86(\mathrm{~d}, J=7.4 \mathrm{~Hz}, 3 \mathrm{H}) .{ }^{13} \mathrm{C} \mathrm{NMR}\left(125 \mathrm{MHz}, \mathrm{CDCl}_{3}\right): \delta=184.8$ $(\mathrm{CH}), 158.4\left(\mathrm{C}_{\mathrm{q}}\right), 153.8\left(\mathrm{C}_{\mathrm{q}}\right), 137.3\left(\mathrm{C}_{\mathrm{q}}\right), 132.9\left(\mathrm{C}_{\mathrm{q}}\right), 128.0(\mathrm{CH}), 125.7\left(\mathrm{C}_{\mathrm{q}}\right), 123.4$ $(\mathrm{CH}), 123.0(\mathrm{CH}), 121.4(\mathrm{CH}), 114.4\left(\mathrm{C}_{\mathrm{q}}\right), 114.2(\mathrm{CH}), 109.2(\mathrm{CH}), 55.3\left(\mathrm{CH}_{3}\right), 33.7$ (CH), $31.1\left(\mathrm{CH}_{3}\right), 18.8\left(\mathrm{CH}_{3}\right)$. IR (ATR): 2933, 1643, 1510, 1468, 1246, $750 \mathrm{~cm}^{-1}$. MS (ESI) $m / z$ (relative intensity): 609 (20) $[2 \mathrm{M}+\mathrm{Na}]^{+}, 316(26)[\mathrm{M}+\mathrm{Na}]^{+}, 294(100)$ $[\mathrm{M}+\mathrm{H}]^{+}, 186$ (22). HR-MS (ESI) $\mathrm{m} / z$ calcd for $\mathrm{C}_{19} \mathrm{H}_{20} \mathrm{NO}_{2}[\mathrm{M}+\mathrm{H}]^{+} 294.1489$, found 294.1489. $[\alpha]_{D}^{20}:-70.6\left(\mathrm{c}=0.94, \mathrm{CHCl}_{3}\right)$. HPLC separation (Chiralpak ${ }^{\circledR}$ IA-3, $n$-hexane/iPrOH 80:20, $1.0 \mathrm{~mL} / \mathrm{min}$, detection at $250 \mathrm{~nm}$ ): $t_{r}$ (major) $=10.6 \mathrm{~min}$, $t_{r}(\operatorname{minor})=11.5 \mathrm{~min}, 84: 16$ e.r.

The analytical data are in accordance with those previously reported in the literature for the racemic compound. ${ }^{[148]}$ 
(S)-1-Benzyl-5-methoxy-2-[1-(4-methoxyphenyl)ethyl]-1H-indole-3-

\section{carbaldehyde (62jc)}

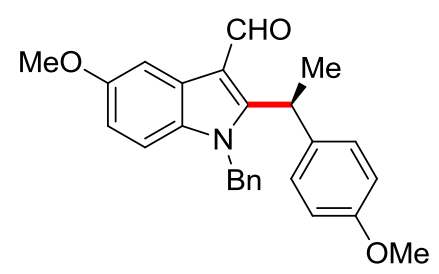

The general procedure GP6 was followed using indole 59j $(93 \mathrm{mg}, 0.25 \mathrm{mmol}$, 1.0 equiv) and styrene $60 \mathrm{c}$ ( $50 \mathrm{mg}, 0.38 \mathrm{mmol}, 1.5$ equiv). Purification by column chromatography on silica gel $(n$-hexane/EtOAc $=7 / 1 \rightarrow 5 / 1)$ yielded 62jc $(97.9 \mathrm{mg}$, $98 \%)$ as a yellow solid. M. p. $=59-60{ }^{\circ} \mathrm{C} .{ }^{1} \mathrm{H}$ NMR $\left(300 \mathrm{MHz}, \mathrm{CDCl}_{3}\right): \delta=10.16(\mathrm{~s}$, $1 \mathrm{H}), 7.94(\mathrm{~d}, J=2.5 \mathrm{~Hz}, 1 \mathrm{H}), 7.33-7.17(\mathrm{~m}, 3 \mathrm{H}), 7.11(\mathrm{dd}, J=9.0,0.9 \mathrm{~Hz}, 2 \mathrm{H}), 7.02$ (dd, $J=8.9,0.5 \mathrm{~Hz}, 1 \mathrm{H}), 6.94-6.87(\mathrm{~m}, 2 \mathrm{H}), 6.85(\mathrm{dd}, J=8.9,2.6 \mathrm{~Hz}, 1 \mathrm{H}), 6.80(\mathrm{~d}$, $J=8.8 \mathrm{~Hz}, 2 \mathrm{H}), 5.26-5.13(\mathrm{~m}, 2 \mathrm{H}), 4.91(\mathrm{q}, J=7.3 \mathrm{~Hz}, 1 \mathrm{H}), 3.90(\mathrm{~s}, 3 \mathrm{H}), 3.77(\mathrm{~s}$, $3 \mathrm{H}), 1.71$ (d, $J=7.4 \mathrm{~Hz}, 3 \mathrm{H}) .{ }^{13} \mathrm{C}$ NMR $\left(125 \mathrm{MHz}, \mathrm{CDCl}_{3}\right): \delta=185.2(\mathrm{CH}), 158.3$ $\left(\mathrm{C}_{\mathrm{q}}\right), 156.8\left(\mathrm{C}_{\mathrm{q}}\right), 154.2\left(\mathrm{C}_{\mathrm{q}}\right), 135.9\left(\mathrm{C}_{\mathrm{q}}\right), 133.2\left(\mathrm{C}_{\mathrm{q}}\right), 131.7\left(\mathrm{C}_{\mathrm{q}}\right), 128.8(\mathrm{CH}), 127.9$ $(\mathrm{CH}), 127.6(\mathrm{CH}), 126.6\left(\mathrm{C}_{\mathrm{q}}\right), 125.7(\mathrm{CH}), 114.7\left(\mathrm{C}_{\mathrm{q}}\right), 114.1(\mathrm{CH}), 113.8(\mathrm{CH}), 110.9$ $(\mathrm{CH}), 103.4(\mathrm{CH}), 55.9\left(\mathrm{CH}_{3}\right), 55.3\left(\mathrm{CH}_{3}\right), 47.7\left(\mathrm{CH}_{2}\right), 34.5(\mathrm{CH}), 20.2\left(\mathrm{CH}_{3}\right)$. IR (ATR): 2933, 1642, 1510, 1453, 1246, 1031, $696 \mathrm{~cm}^{-1}$. MS (EI) $\mathrm{m} / \mathrm{z}$ (relative intensity): 399 (76) [M] , 308 (42), 291 (26), 278 (23), 200 (22), 197 (74), 91 (100).

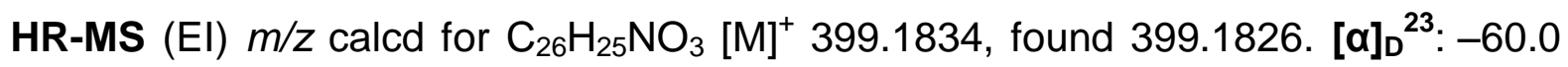
(c $=1.68, \mathrm{CHCl}_{3}$ ). HPLC separation (Chiralpak ${ }^{\circledR} \mathrm{IA}-3$, $n$-hexane/PrOH 80:20, $1.0 \mathrm{~mL} / \mathrm{min}$, detection at $273 \mathrm{~nm}): t_{r}($ major $)=9.4 \mathrm{~min}, t_{r}($ minor $)=11.1 \mathrm{~min}, 93: 7$ e.r.

(S)-1-Benzyl-5-fluoro-2-[1-(4-methoxyphenyl)ethyl]-1 H-indole-3-carbaldehyde (62kc)

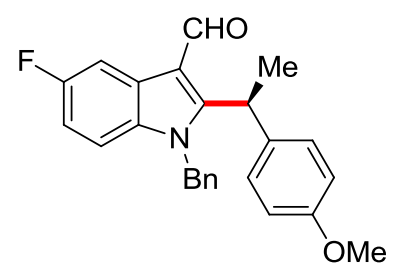


The general procedure GP6 was followed using indole 59k $(90 \mathrm{mg}, 0.25 \mathrm{mmol}$, 1.0 equiv) and styrene $60 \mathrm{c}$ (50 mg, $0.38 \mathrm{mmol}, 1.5$ equiv). Purification by column chromatography on silica gel $(n$-hexane/EtOAc $=8 / 1 \rightarrow 6 / 1)$ yielded 62kc $(92.0 \mathrm{mg}$, 95\%) as a yellow solid. M. p. $=54-56{ }^{\circ} \mathrm{C} .{ }^{1} \mathrm{H}$ NMR $\left(400 \mathrm{MHz}, \mathrm{CDCl}_{3}\right): \delta=10.16(\mathrm{~s}$, $1 \mathrm{H}), 8.11$ (dd, $J=9.4,2.6 \mathrm{~Hz}, 1 \mathrm{H}), 7.33-7.21(\mathrm{~m}, 3 \mathrm{H}), 7.11$ (d, $J=8.1 \mathrm{~Hz}, 2 \mathrm{H}), 7.05$ (dd, $J=8.9,4.2 \mathrm{~Hz}, 1 \mathrm{H}$ ), 6.94 (ddd, $J=8.9,2.6,2.6 \mathrm{~Hz}, 1 \mathrm{H}), 6.92-6.85(\mathrm{~m}, 2 \mathrm{H})$, $6.81(\mathrm{~d}, J=8.8 \mathrm{~Hz}, 2 \mathrm{H}), 5.25(\mathrm{~d}, J=17.3 \mathrm{~Hz}, 1 \mathrm{H}), 5.19$ (d, $J=17.2 \mathrm{~Hz}, 1 \mathrm{H}), 4.94$ (q, $J=7.3 \mathrm{~Hz}, 1 \mathrm{H}), 3.77(\mathrm{~s}, 3 \mathrm{H}), 1.72(\mathrm{~d}, J=7.4 \mathrm{~Hz}, 3 \mathrm{H}) .{ }^{13} \mathrm{C} \mathrm{NMR}\left(100 \mathrm{MHz}, \mathrm{CDCl}_{3}\right)$ : $\delta=185.1(\mathrm{CH}), 160.0\left(\mathrm{~d},{ }^{1} \mathrm{~J}_{\mathrm{C}-\mathrm{F}}=238.8 \mathrm{~Hz}, \mathrm{C}_{\mathrm{q}}\right), 158.5\left(\mathrm{C}_{\mathrm{q}}\right), 155.3\left(\mathrm{C}_{\mathrm{q}}\right), 135.7\left(\mathrm{C}_{\mathrm{q}}\right)$, $133.4\left(\mathrm{C}_{\mathrm{q}}\right), 132.9\left(\mathrm{C}_{\mathrm{q}}\right), 128.9(\mathrm{CH}), 128.0(\mathrm{CH}), 127.8(\mathrm{CH}), 126.6(\mathrm{~d}$, $\left.{ }^{3} J_{\mathrm{C}-\mathrm{F}}=11.0 \mathrm{~Hz}, \mathrm{C}_{\mathrm{q}}\right), 125.7(\mathrm{CH}), 114.8\left(\mathrm{~d},{ }^{4} \mathrm{~J}_{\mathrm{C}-\mathrm{F}}=4.3 \mathrm{~Hz}, \mathrm{C}_{\mathrm{q}}\right), 114.2(\mathrm{CH}), 111.8(\mathrm{~d}$, $\left.{ }^{2} J_{\mathrm{C}-\mathrm{F}}=26.2 \mathrm{~Hz}, \mathrm{CH}\right), 111.0\left(\mathrm{~d},{ }^{3} J_{\mathrm{C}-\mathrm{F}}=9.6 \mathrm{~Hz}, \mathrm{CH}\right), 107.4\left(\mathrm{~d},{ }^{2} J_{\mathrm{C}-\mathrm{F}}=24.9 \mathrm{~Hz}, \mathrm{CH}\right)$, $55.3\left(\mathrm{CH}_{3}\right), 47.7\left(\mathrm{CH}_{2}\right), \quad 34.5(\mathrm{CH}), 20.0\left(\mathrm{CH}_{3}\right) .{ }^{19} \mathrm{~F} \mathrm{NMR}\left(376 \mathrm{MHz}, \mathrm{CDCl}_{3}\right)$ : $\delta=-120.24$ (ddd, $J=9.3,4.3,4.3 \mathrm{~Hz}$ ). IR (ATR): 2928, 1646, 1510, 1454, 1247, $1026 \mathrm{~cm}^{-1}$. MS (ESI) $\mathrm{m} / z$ (relative intensity): $410(24)[\mathrm{M}+\mathrm{Na}]^{+}, 388(100)[\mathrm{M}+\mathrm{H}]^{+}$, 372 (30), 289 (27), 280 (9). HR-MS (ESI) $\mathrm{m} / \mathrm{z}$ calcd for $\mathrm{C}_{25} \mathrm{H}_{23} \mathrm{NO}_{2} \mathrm{~F}[\mathrm{M}+\mathrm{H}]^{+}$ 388.1707, found 388.1701. [a $]_{\mathrm{D}}^{23}$ : $-54.9\left(\mathrm{c}=1.53, \mathrm{CHCl}_{3}\right)$. HPLC separation (Chiralpak ${ }^{\circledR}$ IA-3, $n$-hexane/PrOH $80: 20,1.0 \mathrm{~mL} / \mathrm{min}$, detection at $250 \mathrm{~nm}$ ): $t_{r}($ major $)=8.1 \mathrm{~min}, t_{r}($ minor $)=9.4 \mathrm{~min}, 93: 7$ e.r.

(S)-1-(3,5-Dimethylbenzyl)-2-[1-(4-methoxyphenyl)ethyl]-1 H-indole-3carbaldehyde (62Ic)

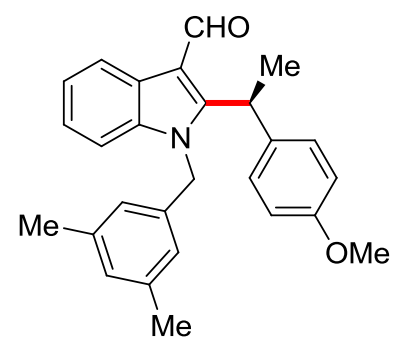

The general procedure GP6 was followed using indole 591 (92 $\mathrm{mg}, 0.25 \mathrm{mmol}$, 1.0 equiv) and styrene $60 \mathrm{c}$ (50 mg, $0.38 \mathrm{mmol}, 1.5$ equiv). Purification by column chromatography on silica gel $(n$-hexane/EtOAc $=9 / 1 \rightarrow 7 / 1)$ yielded 62lc $(89.5 \mathrm{mg}$, 
90\%) as a yellow solid. M. p. $=56-58^{\circ} \mathrm{C} .{ }^{1} \mathbf{H}$ NMR $\left(300 \mathrm{MHz}, \mathrm{CDCl}_{3}\right): \delta=10.22(\mathrm{~s}$, $1 \mathrm{H}$ ), 8.43 (ddd, $J=7.8,1.3,0.8 \mathrm{~Hz}, 1 \mathrm{H}$ ), 7.31 (ddd, $J=7.9,6.9,1.3 \mathrm{~Hz}, 1 \mathrm{H}$ ), 7.23 (ddd, $J=8.2,6.9,1.4 \mathrm{~Hz}, 1 \mathrm{H}$ ), 7.17 (ddd, $J=8.1,1.4,0.8 \mathrm{~Hz}, 1 \mathrm{H}$ ), 7.13 (dd, $J=9.0$, $0.9 \mathrm{~Hz}, 2 \mathrm{H}$ ), 6.87 (ddd, $J=1.6,0.9,0.9 \mathrm{~Hz}, 1 \mathrm{H}$ ), 6.81 (d, $J=8.8 \mathrm{~Hz}, 2 \mathrm{H}$ ), 6.55-6.47 (m, 2H), $5.18(\mathrm{~s}, 2 \mathrm{H}), 4.97$ (q, J = $7.4 \mathrm{~Hz}, 1 \mathrm{H}$ ), $3.78(\mathrm{~s}, 3 \mathrm{H}), 2.21(\mathrm{~s}, 3 \mathrm{H}), 2.21$ (s, $3 \mathrm{H}), 1.75(\mathrm{~d}, J=7.4 \mathrm{~Hz}, 3 \mathrm{H}) .{ }^{13} \mathrm{C}$ NMR $\left(125 \mathrm{MHz}, \mathrm{CDCl}_{3}\right): \delta=185.3(\mathrm{CH}), 158.3$ $\left(\mathrm{C}_{\mathrm{q}}\right), 154.2\left(\mathrm{C}_{\mathrm{q}}\right), 138.4\left(\mathrm{C}_{\mathrm{q}}\right), 137.0\left(\mathrm{C}_{\mathrm{q}}\right), 135.8\left(\mathrm{C}_{\mathrm{q}}\right), 133.3\left(\mathrm{C}_{\mathrm{q}}\right), 129.2(\mathrm{CH}), 128.0$ $(\mathrm{CH}), 125.9\left(\mathrm{C}_{\mathrm{q}}\right), 123.5(\mathrm{CH}), 123.5(\mathrm{CH}), 123.0(\mathrm{CH}), 121.6(\mathrm{CH}), 114.7\left(\mathrm{C}_{\mathrm{q}}\right), 114.0$ $(\mathrm{CH}), 110.2(\mathrm{CH}), 55.3\left(\mathrm{CH}_{3}\right), 47.6\left(\mathrm{CH}_{2}\right), 34.5(\mathrm{CH}), 21.3\left(\mathrm{CH}_{3}\right), 20.3\left(\mathrm{CH}_{3}\right)$. IR (ATR): 2922, 1644, 1510, 1458, 1247, 1179, $745 \mathrm{~cm}^{-1}$. MS (EI) $\mathrm{m} / \mathrm{z}$ (relative intensity): 397 (77) [M] ${ }^{+}, 289$ (26), 278 (30), 263 (22), 225 (100), 119 (77), 91 (24).

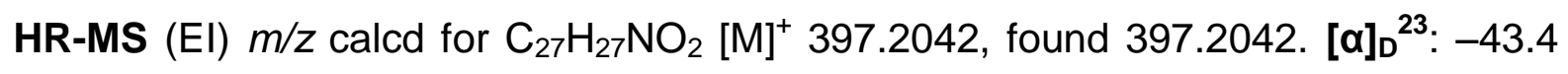
(c $=1.62, \mathrm{CHCl}_{3}$ ). HPLC separation (Chiralpak ${ }^{\circledR} \mathrm{IA}-3, n$-hexane/PrOH 80:20, $1.0 \mathrm{~mL} / \mathrm{min}$, detection at $273 \mathrm{~nm}): t_{r}($ major $)=6.6 \mathrm{~min}, t_{r}($ minor $)=7.6 \mathrm{~min}, 91: 9$ e.r.

\section{(S)-1-(3,5-Dimethylbenzyl)-5-methoxy-2-[1-(4-methoxyphenyl)ethyl]-1 H-indole-}

\section{3-carbaldehyde (62mc)}

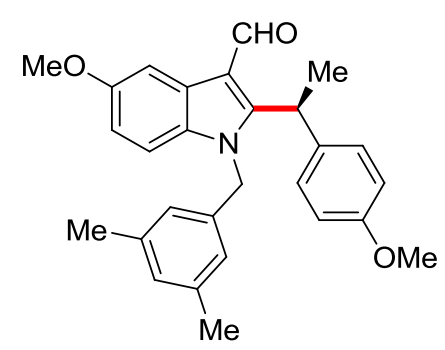

The general procedure GP6 was followed using indole $59 \mathrm{~m}(100 \mathrm{mg}, 0.25 \mathrm{mmol}$, 1.0 equiv) and styrene $60 \mathrm{c}$ ( $50 \mathrm{mg}, 0.38 \mathrm{mmol}, 1.5$ equiv). Purification by column chromatography on silica gel $(n$-hexane/EtOAc $=10 / 1 \rightarrow 6 / 1)$ yielded $62 \mathrm{mc}$ (102.6 mg, 96\%) as a yellow solid. M. p. $=57-58{ }^{\circ} \mathrm{C} .{ }^{1} \mathbf{H}$ NMR $\left(300 \mathrm{MHz}, \mathrm{CDCl}_{3}\right)$ : $\delta=10.16(\mathrm{~s}, 1 \mathrm{H}), 7.95(\mathrm{~d}, J=2.5 \mathrm{~Hz}, 1 \mathrm{H}), 7.11(\mathrm{~d}, J=8.8 \mathrm{~Hz}, 2 \mathrm{H}), 7.04(\mathrm{~d}$, $J=8.9 \mathrm{~Hz}, 1 \mathrm{H}), 6.87(\mathrm{~s}, 1 \mathrm{H}), 6.85(\mathrm{dd}, J=8.9,2.5 \mathrm{~Hz}, 1 \mathrm{H}), 6.80(\mathrm{~d}, J=8.8 \mathrm{~Hz}, 2 \mathrm{H})$, 6.54-6.46 (m, 2H), $5.14(\mathrm{~s}, 2 \mathrm{H}), 4.88(\mathrm{q}, J=7.3 \mathrm{~Hz}, 1 \mathrm{H}), 3.91(\mathrm{~s}, 3 \mathrm{H}), 3.77(\mathrm{~s}, 3 \mathrm{H})$, $2.21(\mathrm{~s}, 6 \mathrm{H}), 1.74(\mathrm{~d}, J=7.4 \mathrm{~Hz}, 3 \mathrm{H}) .{ }^{13} \mathrm{C}$ NMR $\left(125 \mathrm{MHz}, \mathrm{CDCl}_{3}\right): \delta=185.2(\mathrm{CH})$, 
$158.3\left(\mathrm{C}_{\mathrm{q}}\right), 156.7\left(\mathrm{C}_{\mathrm{q}}\right), 154.2\left(\mathrm{C}_{\mathrm{q}}\right), 138.4\left(\mathrm{C}_{\mathrm{q}}\right), 135.8\left(\mathrm{C}_{\mathrm{q}}\right), 133.4\left(\mathrm{C}_{\mathrm{q}}\right), 131.8\left(\mathrm{C}_{\mathrm{q}}\right)$, $129.2(\mathrm{CH}), 128.0(\mathrm{CH}), 126.6\left(\mathrm{C}_{\mathrm{q}}\right), 123.5(\mathrm{CH}), 114.5\left(\mathrm{C}_{\mathrm{q}}\right), 114.0(\mathrm{CH}), 113.7(\mathrm{CH})$, $111.0(\mathrm{CH}), 103.3(\mathrm{CH}), 55.9\left(\mathrm{CH}_{3}\right), 55.3\left(\mathrm{CH}_{3}\right), 47.7\left(\mathrm{CH}_{2}\right), 34.6(\mathrm{CH}), 21.3\left(\mathrm{CH}_{3}\right)$, $20.4\left(\mathrm{CH}_{3}\right)$. IR (ATR): 2932, 1643, 1510, 1457, 1246, 1034, $831 \mathrm{~cm}^{-1}$. MS (ESI) $\mathrm{m} / \mathrm{z}$ (relative intensity): $855(39)[2 \mathrm{M}+\mathrm{H}]^{+}, 428(100)[\mathrm{M}+\mathrm{H}]^{+}$. HR-MS (ESI) $\mathrm{m} / z$ calcd for

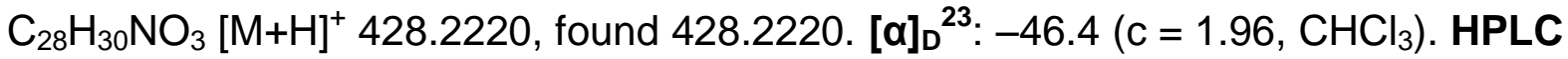
separation (Chiralpak ${ }^{\circledR}$ IA-3, $n$-hexane/PrOH $80: 20,1.0 \mathrm{~mL} / \mathrm{min}$, detection at $250 \mathrm{~nm}): t_{r}($ major $)=7.5 \mathrm{~min}, t_{r}($ minor $)=8.9 \mathrm{~min}, 91: 9$ e.r.

(S)-N-\{[1-Benzyl-2-(1-(4-methoxyphenyl)ethyl)-1 H-pyrrolo[2,3-b]pyridin-3yl]methylene\}-4-methoxyaniline (152nc)

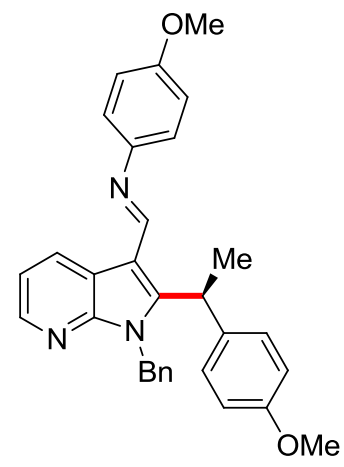

The general procedure GP6 was followed using azaindole $59 \mathrm{n}(85 \mathrm{mg}, 0.25 \mathrm{mmol}$, 1.0 equiv) and styrene $60 \mathrm{c}$ (50 $\mathrm{mg}, 0.38 \mathrm{mmol}, 1.5$ equiv). The reaction mixture was diluted with sat. aqueous $\mathrm{NaHCO}_{3}(10 \mathrm{~mL})$ and extracted with EtOAc $(3 \times 10 \mathrm{~mL})$. The combined organic layers were washed with brine $(10 \mathrm{~mL})$, dried over $\mathrm{Na}_{2} \mathrm{SO}_{4}$, and concentrated under reduced pressure. The residue was purified by silica gel chromatography ( $n$-hexane/EtOAc $=8 / 1 \rightarrow 6 / 1)$ to yield 152nc $(100.2 \mathrm{mg}, 84 \%$, 24:76 e.r.) as an off-white solid. The product was recrystallized from $\mathrm{PrOH}(5 \mathrm{~mL})$ to provide further enantioenriched $152 \mathrm{nc}(60.6 \mathrm{mg}, 51 \%, 10: 90$ e.r.) as a colorless crystalline solid. M. p. $=170-171^{\circ} \mathrm{C} .{ }^{1} \mathbf{H}$ NMR $\left(600 \mathrm{MHz}, \mathrm{CDCl}_{3}\right): \delta=8.91$ (dd, $J=7.8,1.7 \mathrm{~Hz}, 1 \mathrm{H}$ ), 8.50 (s, 1H), 8.37 (dd, $J=4.7,1.6 \mathrm{~Hz}, 1 \mathrm{H}), 7.28-7.19(\mathrm{~m}, 4 \mathrm{H})$, $7.07(\mathrm{dd}, J=8.9,0.8 \mathrm{~Hz}, 2 \mathrm{H}), 7.02-6.98(\mathrm{~m}, 4 \mathrm{H}), 6.86(\mathrm{~d}, J=8.9 \mathrm{~Hz}, 2 \mathrm{H}), 6.81(\mathrm{~d}$, $J=8.8 \mathrm{~Hz}, 2 \mathrm{H}), 5.62(\mathrm{~d}, J=16.5 \mathrm{~Hz}, 1 \mathrm{H}), 5.50(\mathrm{~d}, J=16.5 \mathrm{~Hz}, 1 \mathrm{H}), 4.70(\mathrm{q}$, 
$J=7.3 \mathrm{~Hz}, 1 \mathrm{H}), 3.80(\mathrm{~s}, 3 \mathrm{H}), 3.77(\mathrm{~s}, 3 \mathrm{H}), 1.60(\mathrm{~d}, J=7.4 \mathrm{~Hz}, 3 \mathrm{H}) .{ }^{13} \mathrm{C}$ NMR $\left(125 \mathrm{MHz}, \mathrm{CDCl}_{3}\right): \delta=158.3\left(\mathrm{C}_{\mathrm{q}}\right), 157.4\left(\mathrm{C}_{\mathrm{q}}\right), 153.0(\mathrm{CH}), 149.5\left(\mathrm{C}_{\mathrm{q}}\right), 148.5\left(\mathrm{C}_{\mathrm{q}}\right)$, $146.4\left(\mathrm{C}_{\mathrm{q}}\right), 143.9(\mathrm{CH}), 137.5\left(\mathrm{C}_{\mathrm{q}}\right), 134.0\left(\mathrm{C}_{\mathrm{q}}\right), 131.1(\mathrm{CH}), 128.7(\mathrm{CH}), 128.2(\mathrm{CH})$, $127.4(\mathrm{CH}), 126.3(\mathrm{CH}), 121.8(\mathrm{CH}), 118.8\left(\mathrm{C}_{\mathrm{q}}\right), 118.1(\mathrm{CH}), 114.2(\mathrm{CH}), 114.0(\mathrm{CH})$, $110.4\left(\mathrm{C}_{\mathrm{q}}\right), 55.5\left(\mathrm{CH}_{3}\right), 55.3\left(\mathrm{CH}_{3}\right), 45.3\left(\mathrm{CH}_{2}\right), 35.0(\mathrm{CH}), 20.4\left(\mathrm{CH}_{3}\right)$. IR (ATR): 2934, 2834, 1497, 1427, 1242, 1031, 826, $728 \mathrm{~cm}^{-1}$. MS (ESI) $\mathrm{m} / \mathrm{z}$ (relative intensity): $476(100)[\mathrm{M}+\mathrm{H}]^{+}$. HR-MS (ESI) $\mathrm{m} / z$ calcd for $\mathrm{C}_{31} \mathrm{H}_{30} \mathrm{~N}_{3} \mathrm{O}_{2}[\mathrm{M}+\mathrm{H}]^{+}$ 476.2333, found 476.2332. [ $\alpha]_{\mathrm{D}}^{23}:+192.7\left(\mathrm{c}=1.11, \mathrm{CHCl}_{3}\right)$. HPLC separation (Chiralpak ${ }^{\circledR}$ IF-3, $n$-hexane/PrOH 90:10, $1.0 \mathrm{~mL} / \mathrm{min}$, detection at $273 \mathrm{~nm}$ ): $t_{r}($ minor $)=9.4 \mathrm{~min}, t_{r}$ (major) $=10.8 \mathrm{~min}, 24: 76$ e.r., 10:90 e.r. after recrystallization .

\section{(S)-1-Benzyl-2-[1-(p-tolyl)ethyl]-1 H-indole-3-carbaldehyde (62cd)}

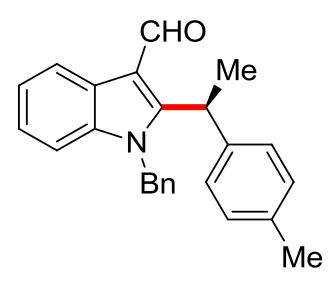

The general procedure GP6 was followed using indole $59 \mathrm{c}(85 \mathrm{mg}, 0.25 \mathrm{mmol}$, 1.0 equiv) and styrene $60 \mathrm{~d}$ ( $44 \mathrm{mg}, 0.38 \mathrm{mmol}, 1.5$ equiv). Purification by column chromatography on silica gel $(n$-hexane/EtOAc $=11 / 1)$ yielded $62 \mathrm{~cd}(72.7 \mathrm{mg}, 82 \%)$ as a yellow solid. M. p. $=69-71{ }^{\circ} \mathrm{C} .{ }^{1} \mathrm{H}$ NMR $\left(400 \mathrm{MHz}, \mathrm{CDCl}_{3}\right): \delta=10.23(\mathrm{~s}, 1 \mathrm{H})$, 8.44 (ddd, $J=7.9,1.3,0.8 \mathrm{~Hz}, 1 \mathrm{H}$ ), 7.31 (ddd, $J=8.0,7.1,1.0 \mathrm{~Hz}, 1 \mathrm{H}$ ), 7.29-7.23 (m, 3H), 7.22 (dd, $J=7.1,1.2 \mathrm{~Hz}, 1 \mathrm{H}$ ), $7.15(\mathrm{dt}, J=8.2,1.0 \mathrm{~Hz}, 1 \mathrm{H}$ ), 7.09 (app s, 4H), 6.91 (dd, $J=7.1,2.6 \mathrm{~Hz}, 2 \mathrm{H}$ ), $5.26(\mathrm{~d}, J=17.2 \mathrm{~Hz}, 1 \mathrm{H}), 5.21(\mathrm{~d}, J=17.2 \mathrm{~Hz}$, $1 \mathrm{H}), 5.00(\mathrm{q}, J=7.4 \mathrm{~Hz}, 1 \mathrm{H}), 2.31(\mathrm{~s}, 3 \mathrm{H}), 1.73(\mathrm{~d}, J=7.4 \mathrm{~Hz}, 3 \mathrm{H}) .{ }^{13} \mathrm{C}$ NMR $\left(100 \mathrm{MHz}, \mathrm{CDCl}_{3}\right): \delta=185.4(\mathrm{CH}), 154.2\left(\mathrm{C}_{\mathrm{q}}\right), 138.2\left(\mathrm{C}_{\mathrm{q}}\right), 137.0\left(\mathrm{C}_{\mathrm{q}}\right), 136.6\left(\mathrm{C}_{\mathrm{q}}\right)$, $136.0\left(\mathrm{C}_{\mathrm{q}}\right), 129.4(\mathrm{CH}), 128.8(\mathrm{CH}), 127.6(\mathrm{CH}), 126.9(\mathrm{CH}), 126.0\left(\mathrm{C}_{\mathrm{q}}\right), 125.8(\mathrm{CH})$, $123.6(\mathrm{CH}), 123.1(\mathrm{CH}), 121.7(\mathrm{CH}), 114.8\left(\mathrm{C}_{\mathrm{q}}\right), 110.1(\mathrm{CH}), 47.5\left(\mathrm{CH}_{2}\right), 34.7(\mathrm{CH})$, $20.9\left(\mathrm{CH}_{3}\right), 19.9\left(\mathrm{CH}_{3}\right)$. IR (ATR): 2912, 1644, 1421, 1395, 1039, 806, $729 \mathrm{~cm}^{-1}$. MS (EI) $\mathrm{m} / z$ (relative intensity): 353 (75) [M] ${ }^{+}, 261$ (22), 218 (35), 181 (75), 91 (100).

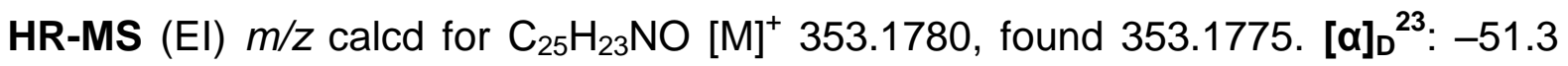


(c $=1.33, \mathrm{CHCl}_{3}$ ). HPLC separation $\left(\right.$ Chiralpak $^{\circledR} \mathrm{IA}-3$, $n$-hexane/IPrOH 80:20, $1.0 \mathrm{~mL} / \mathrm{min}$, detection at $250 \mathrm{~nm}$ ): $t_{r}$ (major) $=6.5 \mathrm{~min}, t_{r}($ minor $)=7.2 \mathrm{~min}, 90: 10$ e.r.

(S)-1-Benzyl-2-\{1-[4-(tert-butyl)phenyl]ethyl\}-1 H-indole-3-carbaldehyde (62ce)

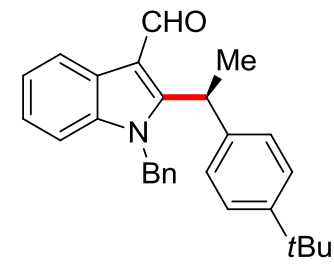

The general procedure GP6 was followed using indole $59 \mathrm{c}(85 \mathrm{mg}, 0.25 \mathrm{mmol}$, 1.0 equiv) and styrene $60 \mathrm{e}$ ( $60 \mathrm{mg}, 0.38 \mathrm{mmol}, 1.5$ equiv). Purification by column chromatography on silica gel $(n$-hexane/EtOAc $=15 / 1 \rightarrow 11 / 1)$ yielded 62ce $(72.2 \mathrm{mg}$, $73 \%)$ as a yellow oil. ${ }^{1} \mathrm{H}$ NMR $\left(300 \mathrm{MHz}, \mathrm{CDCl}_{3}\right): \delta=10.24$ (s, $\left.1 \mathrm{H}\right), 8.44$ (ddd, $J=7.9,1.4,0.8 \mathrm{~Hz}, 1 \mathrm{H}), 7.35-7.19(\mathrm{~m}, 7 \mathrm{H}), 7.18-7.09(\mathrm{~m}, 3 \mathrm{H}), 6.96-6.83(\mathrm{~m}, 2 \mathrm{H})$, $5.27(\mathrm{~s}, 2 \mathrm{H}), 5.00(\mathrm{q}, J=7.4 \mathrm{~Hz}, 1 \mathrm{H}), 1.75(\mathrm{~d}, J=7.4 \mathrm{~Hz}, 3 \mathrm{H}), 1.28(\mathrm{~s}, 9 \mathrm{H}) .{ }^{13} \mathrm{C}$ NMR $\left(125 \mathrm{MHz}, \mathrm{CDCl}_{3}\right): \delta=185.3(\mathrm{CH}), 154.1\left(\mathrm{C}_{\mathrm{q}}\right), 149.8\left(\mathrm{C}_{\mathrm{q}}\right), 138.0\left(\mathrm{C}_{\mathrm{q}}\right), 136.9\left(\mathrm{C}_{\mathrm{q}}\right)$, $135.8\left(\mathrm{C}_{\mathrm{q}}\right), 128.7(\mathrm{CH}), 127.5(\mathrm{CH}), 126.6(\mathrm{CH}), 125.9\left(\mathrm{C}_{\mathrm{q}}\right), 125.7(\mathrm{CH}), 125.6(\mathrm{CH})$, $123.6(\mathrm{CH}), 123.1(\mathrm{CH}), 121.7(\mathrm{CH}), 114.8\left(\mathrm{C}_{\mathrm{q}}\right), 110.1(\mathrm{CH}), 47.6\left(\mathrm{CH}_{2}\right), 34.8(\mathrm{CH})$, $34.4\left(\mathrm{C}_{\mathrm{q}}\right), 31.3\left(\mathrm{CH}_{3}\right), 20.0\left(\mathrm{CH}_{3}\right)$. IR (ATR): 2961, 1645, 1454, 1421, 1395, $745 \mathrm{~cm}^{-1}$. MS (EI) $\mathrm{m} / \mathrm{z}$ (relative intensity): 395 (57) [M] ${ }^{+}, 338$ (25), 261 (19), 248 (23), 223 (67), 91 (100). HR-MS (EI) $\mathrm{m} / z$ calcd for $\mathrm{C}_{28} \mathrm{H}_{29} \mathrm{NO}[\mathrm{M}]^{+} 395.2249$, found 395.2239. $[\alpha]_{D}{ }^{23}:-34.8\left(\mathrm{C}=1.34, \mathrm{CHCl}_{3}\right)$. HPLC separation (Chiralpak ${ }^{\circledR} \mathrm{IA}-3$, $n$-hexane/iPrOH 80:20, $1.0 \mathrm{~mL} / \mathrm{min}$, detection at $250 \mathrm{~nm}$ ): $t_{r}$ (minor) $=5.6 \mathrm{~min}$, $t_{r}($ major $)=6.1 \mathrm{~min}, 14: 86$ e.r. 
(S)-2-\{1-([1,1'-Biphenyl]-4-yl)ethyl\}-1-benzyl-1 H-indole-3-carbaldehyde (62cf)

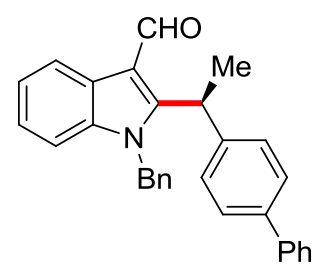

The general procedure GP5 was followed using indole $59 \mathrm{c}(85 \mathrm{mg}, 0.25 \mathrm{mmol}$, 1.0 equiv) and styrene $60 \mathrm{f}$ (68 $\mathrm{mg}, 0.38 \mathrm{mmol}, 1.5$ equiv). Purification by column chromatography on silica gel $(n$-hexane/EtOAc $=15 / 1 \rightarrow 10 / 1)$ yielded 62cf $(66.9 \mathrm{mg}$, $64 \%)$ as a yellow solid. M. p. $=84-85^{\circ} \mathrm{C}$. ${ }^{1} \mathrm{H}$ NMR $\left(300 \mathrm{MHz}, \mathrm{CDCl}_{3}\right): \delta=10.29(\mathrm{~s}$, $1 \mathrm{H}$ ), 8.44 (ddd, $J=7.8,1.3,0.7 \mathrm{~Hz}, 1 \mathrm{H}), 7.58-7.39(\mathrm{~m}, 6 \mathrm{H}), 7.38-7.20(\mathrm{~m}, 8 \mathrm{H}), 7.17$ (dt, $J=8.1,1.1 \mathrm{~Hz}, 1 \mathrm{H}), 6.97-6.86(\mathrm{~m}, 2 \mathrm{H}), 5.27(\mathrm{~s}, 2 \mathrm{H}), 5.10(\mathrm{q}, J=7.4 \mathrm{~Hz}, 1 \mathrm{H})$, $1.79(\mathrm{~d}, J=7.4 \mathrm{~Hz}, 3 \mathrm{H}) .{ }^{13} \mathrm{C}$ NMR $\left(125 \mathrm{MHz}, \mathrm{CDCl}_{3}\right): \delta=185.1(\mathrm{CH}), 153.6\left(\mathrm{C}_{\mathrm{q}}\right)$, $140.3\left(\mathrm{C}_{\mathrm{q}}\right), 140.2\left(\mathrm{C}_{\mathrm{q}}\right), 139.8\left(\mathrm{C}_{\mathrm{q}}\right), 137.0\left(\mathrm{C}_{\mathrm{q}}\right), 135.8\left(\mathrm{C}_{\mathrm{q}}\right), 128.8(\mathrm{CH}), 128.7(\mathrm{CH})$, $127.6(\mathrm{CH}), 127.4(\mathrm{CH}), 127.4(\mathrm{CH}), 127.3(\mathrm{CH}), 126.9(\mathrm{CH}), 126.0\left(\mathrm{C}_{\mathrm{q}}\right), 125.7(\mathrm{CH})$, $123.7(\mathrm{CH}), 123.2(\mathrm{CH}), 121.6(\mathrm{CH}), 114.8\left(\mathrm{C}_{\mathrm{q}}\right), 110.2(\mathrm{CH}), 47.7\left(\mathrm{CH}_{2}\right), 34.9(\mathrm{CH})$, $20.0\left(\mathrm{CH}_{3}\right)$. IR (ATR): 2927, 1644, 1453, 1395, 1039, 746, $694 \mathrm{~cm}^{-1}$. MS (EI) $\mathrm{m} / \mathrm{z}$ (relative intensity): 415 (92) [M] ${ }^{+}, 280$ (42), 261 (39), 243 (96), 91 (100). HR-MS (EI) $\mathrm{m} / \mathrm{z}$ calcd for $\mathrm{C}_{30} \mathrm{H}_{25} \mathrm{NO}[\mathrm{M}]^{+}$415.1936, found 415.1934. [ $\left.\alpha\right]_{D}{ }^{23}$ : -49.0 (c=1.18, $\mathrm{CHCl}_{3}$ ). HPLC separation (Chiralpak ${ }^{\circledR} \mathrm{IB}-3, n$-hexane/PrOH 70:30, $1.0 \mathrm{~mL} / \mathrm{min}$, detection at $250 \mathrm{~nm}): t_{r}$ (major) $=10.1 \mathrm{~min}, t_{r}($ minor $)=14.3 \mathrm{~min}, 84: 16$ e.r.

\section{(S)-1-Benzyl-2-[1-(4-fluorophenyl)ethyl]-1H-indole-3-carbaldehyde (62cg)}

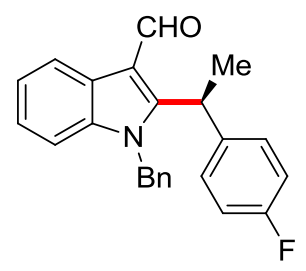

The general procedure GP6 was followed using indole $59 \mathrm{c}(85 \mathrm{mg}, 0.25 \mathrm{mmol}$, 1.0 equiv) and styrene $60 \mathrm{~g}$ ( $46 \mathrm{mg}, 0.38 \mathrm{mmol}, 1.5$ equiv). Purification by column chromatography on silica gel $(n$-hexane/EtOAc $=12 / 1 \rightarrow 9 / 1)$ yielded $62 \mathrm{cg}(54.2 \mathrm{mg}$, 
$61 \%)$ as a yellow solid. M. p. $=56-57^{\circ} \mathrm{C} .{ }^{1} \mathrm{H}$ NMR $\left(500 \mathrm{MHz}, \mathrm{CDCl}_{3}\right): \delta=10.22$ (s, $1 \mathrm{H}$ ), 8.41 (dt, $J=7.9,1.0 \mathrm{~Hz}, 1 \mathrm{H}$ ), 7.32 (ddd, $J=8.0,7.1,1.1 \mathrm{~Hz}, 1 \mathrm{H}), 7.30-7.21$ (m, $4 \mathrm{H}$ ), 7.17 (ddd, $J=6.6,1.6,0.9 \mathrm{~Hz}, 2 \mathrm{H}), 7.15(\mathrm{dd}, J=5.3,1.0 \mathrm{~Hz}, 1 \mathrm{H}), 6.95$ (t, $J=8.6 \mathrm{~Hz}, 2 \mathrm{H}$ ), 6.89 (ddt, $J=6.2,1.8,0.8 \mathrm{~Hz}, 2 \mathrm{H}$ ), $5.23(\mathrm{~s}, 2 \mathrm{H}$ ), 5.03 (q, $J=7.4 \mathrm{~Hz}$, $1 \mathrm{H}), 1.74(\mathrm{~d}, J=7.4 \mathrm{~Hz}, 3 \mathrm{H}) .{ }^{13} \mathrm{C}$ NMR $\left(125 \mathrm{MHz}, \mathrm{CDCl}_{3}\right): \delta=185.2(\mathrm{CH}), 161.6(\mathrm{~d}$, $\left.{ }^{1} J_{\mathrm{C}-\mathrm{F}}=246.4 \mathrm{~Hz}, \mathrm{C}_{\mathrm{q}}\right), 153.3\left(\mathrm{C}_{\mathrm{q}}\right), 137.0\left(\mathrm{C}_{\mathrm{q}}\right), 137.0\left(\mathrm{~d},{ }^{4} \mathrm{~J}_{\mathrm{C}-\mathrm{F}}=3.2 \mathrm{~Hz}, \mathrm{C}_{\mathrm{q}}\right), 135.7$ $\left(\mathrm{C}_{\mathrm{q}}\right), 128.9(\mathrm{CH}), 128.5\left(\mathrm{~d},{ }^{3} \mathrm{~J}_{\mathrm{C}-\mathrm{F}}=7.8 \mathrm{~Hz}, \mathrm{CH}\right), 127.7(\mathrm{CH}), 126.0\left(\mathrm{C}_{\mathrm{q}}\right), 125.7(\mathrm{CH})$, $123.8(\mathrm{CH}), 123.2(\mathrm{CH}), 121.6(\mathrm{CH}), 115.6\left(\mathrm{~d},{ }^{2} \mathrm{~J}_{\mathrm{C}-\mathrm{F}}=21.3 \mathrm{~Hz}, \mathrm{CH}\right), 114.7\left(\mathrm{C}_{\mathrm{q}}\right)$, $110.2(\mathrm{CH}), 47.5\left(\mathrm{CH}_{2}\right), 34.4(\mathrm{CH}), 20.0\left(\mathrm{CH}_{3}\right) .{ }^{19} \mathrm{~F} \mathrm{NMR}\left(471 \mathrm{MHz}, \mathrm{CDCl}_{3}\right)$ : $\delta=-115.64$ (ttd, $J=8.5,5.3,1.5 \mathrm{~Hz}$ ). IR (ATR): 2926, 1644, 1507, 1396, 743, $695 \mathrm{~cm}^{-1}$. MS (ESI) $\mathrm{m} / \mathrm{z}$ (relative intensity): 1092 (26), 913 (16), 737 (67) [2M+Na] 380 (38) $[\mathrm{M}+\mathrm{Na}]^{+}, 358(100)[\mathrm{M}+\mathrm{H}]^{+}, 214$ (9). HR-MS (ESI) $\mathrm{m} / z$ calcd for $\mathrm{C}_{24} \mathrm{H}_{21} \mathrm{NOF}$ $[\mathrm{M}+\mathrm{H}]^{+}$358.1602, found 358.1608. $[\alpha]_{\mathrm{D}}{ }^{23}:-61.9$ (c $\left.=0.99, \mathrm{CHCl}_{3}\right)$. HPLC separation (Chiralpak ${ }^{\circledR} \mid \mathrm{A}-3, n$-hexane/ $\mathrm{PrOH} 80: 20,1.0 \mathrm{~mL} / \mathrm{min}$, detection at $250 \mathrm{~nm}): t_{r}($ major $)=7.0 \mathrm{~min}, t_{r}($ minor $)=7.6 \mathrm{~min}, 88: 12$ e.r.

(S)-1-Benzyl-2-\{1-[4-(dimethylamino]phenyl)ethyl\}-1 $H$-indole-3-carbaldehyde (62ch)

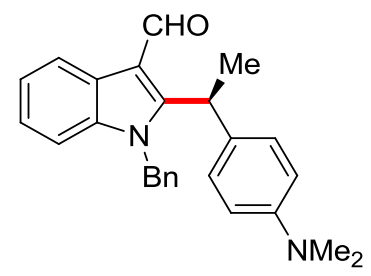

The general procedure GP6 was followed using indole $59 \mathrm{c}(85 \mathrm{mg}, 0.25 \mathrm{mmol}$,

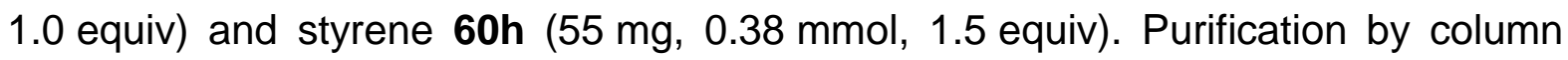
chromatography on silica gel $(n$-hexane/EtOAc $=9 / 1 \rightarrow 6 / 1)$ yielded 62ch $(72.4 \mathrm{mg}$, $76 \%)$ as a yellow solid. M. p. $=55-57{ }^{\circ} \mathrm{C} .{ }^{1} \mathbf{H}$ NMR $\left(300 \mathrm{MHz}, \mathrm{CDCl}_{3}\right): \delta=10.23(\mathrm{~s}$, $1 \mathrm{H}$ ), 8.44 (ddd, $J=7.8,1.3,0.7 \mathrm{~Hz}, 1 \mathrm{H}$ ), $7.35-7.17$ (m, $5 \mathrm{H}$ ), 7.13 (dt, $J=8.1,1.0 \mathrm{~Hz}$, $1 \mathrm{H}), 7.06(\mathrm{dd}, J=9.0,0.9 \mathrm{~Hz}, 2 \mathrm{H}), 6.98-6.85(\mathrm{~m}, 2 \mathrm{H}), 6.64(\mathrm{~d}, J=8.8 \mathrm{~Hz}, 2 \mathrm{H}), 5.27$ (d, $J=17.3 \mathrm{~Hz}, 1 \mathrm{H}$ ), $5.21(\mathrm{~d}, J=17.3 \mathrm{~Hz}, 1 \mathrm{H}), 4.95(\mathrm{q}, J=7.4 \mathrm{~Hz}, 1 \mathrm{H}), 2.92(\mathrm{~s}, 6 \mathrm{H})$, $1.70(\mathrm{~d}, J=7.4 \mathrm{~Hz}, 3 \mathrm{H}) .{ }^{13} \mathrm{C}$ NMR $\left(100 \mathrm{MHz}, \mathrm{CDCl}_{3}\right): \delta=185.6(\mathrm{CH}), 155.0\left(\mathrm{C}_{\mathrm{q}}\right)$, 
$149.4\left(\mathrm{C}_{\mathrm{q}}\right), 137.1\left(\mathrm{C}_{\mathrm{q}}\right), 136.2\left(\mathrm{C}_{\mathrm{q}}\right), 128.8(\mathrm{CH}), 128.7\left(\mathrm{C}_{\mathrm{q}}\right), 127.6(\mathrm{CH}), 127.5(\mathrm{CH})$, $126.0\left(\mathrm{C}_{\mathrm{q}}\right), 125.8(\mathrm{CH}), 123.5(\mathrm{CH}), 123.0(\mathrm{CH}), 121.8(\mathrm{CH}), 114.8\left(\mathrm{C}_{\mathrm{q}}\right), 112.8(\mathrm{CH})$, $110.1(\mathrm{CH}), 47.5\left(\mathrm{CH}_{2}\right), 40.5\left(\mathrm{CH}_{3}\right), 34.2(\mathrm{CH}), 20.0\left(\mathrm{CH}_{3}\right)$. IR (ATR): 2924, 1643, 1519, 1453, 1395, $746 \mathrm{~cm}^{-1}$. MS (EI) $\mathrm{m} / \mathrm{z}$ (relative intensity): 382 (100) [M] ${ }^{+}, 365$ (48), 291 (34), 261 (73), 247 (36), 210 (48), 91 (69). HR-MS (EI) m/z calcd for $\mathrm{C}_{26} \mathrm{H}_{26} \mathrm{~N}_{2} \mathrm{O}[\mathrm{M}]^{+}$382.2045, found 382.2041. [a] ${ }_{\mathrm{D}}^{23}$ : -55.2 (c=1.31, $\mathrm{CHCl}_{3}$ ). HPLC separation (Chiralpak ${ }^{\circledR} \mathrm{IA}-3, \quad n$-hexane/ $\mathrm{PrOH} 80: 20,1.0 \mathrm{~mL} / \mathrm{min}$, detection at $273 \mathrm{~nm}): t_{r}($ major $)=7.9 \mathrm{~min}, t_{r}(\operatorname{minor})=9.1 \mathrm{~min}, 93: 7$ e.r.

\section{(S)-2-\{1-(Benzo[d][1,3]dioxol-5-yl)ethyl\}-1-benzyl-1 H-indole-3-carbaldehyde} (62ci)

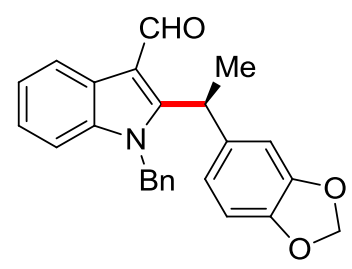

The general procedure GP6 was followed using indole $59 \mathrm{c}(85 \mathrm{mg}, 0.25 \mathrm{mmol}$, 1.0 equiv) and styrene $60 \mathrm{i}$ (56 mg, $0.38 \mathrm{mmol}, 1.5$ equiv). Purification by column chromatography on silica gel $(n$-hexane/EtOAc $=8 / 1 \rightarrow 5 / 1)$ yielded 62ci $(75.4 \mathrm{mg}$, $79 \%)$ as a yellow solid. M. p. $=62-63{ }^{\circ} \mathrm{C} .{ }^{1} \mathbf{H}$ NMR $\left(300 \mathrm{MHz}, \mathrm{CDCl}_{3}\right): \delta=10.24(\mathrm{~s}$, $1 \mathrm{H}$ ), 8.42 (ddd, $J=7.8,1.4,0.7 \mathrm{~Hz}, 1 \mathrm{H}), 7.35-7.29(\mathrm{~m}, 1 \mathrm{H}), 7.29-7.19(\mathrm{~m}, 4 \mathrm{H}), 7.15$ (dt, $J=7.6,0.7 \mathrm{~Hz}, 1 \mathrm{H}), 6.94-6.85(\mathrm{~m}, 2 \mathrm{H}), 6.74-6.60(\mathrm{~m}, 3 \mathrm{H}), 5.90$ (ddd, $J=7.2$, $1.4,0.5 \mathrm{~Hz}, 2 \mathrm{H}), 5.26$ (s, 2H), 4.97 (q, $J=7.4 \mathrm{~Hz}, 1 \mathrm{H}), 1.70(\mathrm{~d}, J=7.4 \mathrm{~Hz}, 3 \mathrm{H})$. ${ }^{13} \mathrm{C}$ NMR $\left(125 \mathrm{MHz}, \mathrm{CDCl}_{3}\right): \delta=185.1(\mathrm{CH}), 153.6\left(\mathrm{C}_{\mathrm{q}}\right), 148.0\left(\mathrm{C}_{\mathrm{q}}\right), 146.4\left(\mathrm{C}_{\mathrm{q}}\right)$, $137.0\left(\mathrm{C}_{\mathrm{q}}\right), 135.8\left(\mathrm{C}_{\mathrm{q}}\right), 135.0\left(\mathrm{C}_{\mathrm{q}}\right), 128.8(\mathrm{CH}), 127.5(\mathrm{CH}), 125.9\left(\mathrm{C}_{\mathrm{q}}\right), 125.7(\mathrm{CH})$, $123.7(\mathrm{CH}), 123.1(\mathrm{CH}), 121.6(\mathrm{CH}), 119.8(\mathrm{CH}), 114.7\left(\mathrm{C}_{\mathrm{q}}\right), 110.1(\mathrm{CH}), 108.2(\mathrm{CH})$, $107.9(\mathrm{CH}), 101.1\left(\mathrm{CH}_{2}\right), 47.6\left(\mathrm{CH}_{2}\right), 34.8(\mathrm{CH}), 20.1\left(\mathrm{CH}_{3}\right)$. IR (ATR): 2926, 1644, 1486, 1233, 1035, $745 \mathrm{~cm}^{-1}$. MS (ESI) $\mathrm{m} / \mathrm{z}$ (relative intensity): $767(11)[2 \mathrm{M}+\mathrm{H}]^{+}, 489$ (9), 384 (100) $[\mathrm{M}+\mathrm{H}]^{+}, 269$ (6). HR-MS (ESI) $\mathrm{m} / z$ calcd for $\mathrm{C}_{25} \mathrm{H}_{22} \mathrm{NO}_{3}[\mathrm{M}+\mathrm{H}]^{+}$

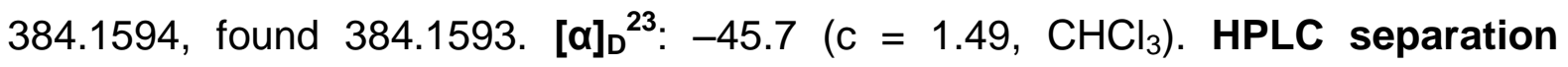


(Chiralpak ${ }^{\circledR}$ IA-3, $n$-hexane/PrOH $80: 20,1.0 \mathrm{~mL} / \mathrm{min}$, detection at $250 \mathrm{~nm}$ ): $t_{r}($ major $)=9.7 \min , t_{r}($ minor $)=10.5 \min , 85: 15$ e.r.

(S)-1-Benzyl-2-[1-(3,4-dimethoxyphenyl)ethyl]-1 H-indole-3-carbaldehyde (62cj)

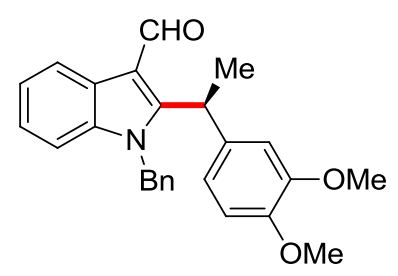

The general procedure GP6 was followed using indole $59 \mathrm{c}(85 \mathrm{mg}, 0.25 \mathrm{mmol}$, 1.0 equiv) and styrene $60 \mathrm{j}$ (62 $\mathrm{mg}, 0.38 \mathrm{mmol}, 1.5$ equiv). Purification by column chromatography on silica gel $(n$-hexane/EtOAc $=8 / 1 \rightarrow 5 / 1)$ yielded 62cj $(55.9 \mathrm{mg}$, $56 \%)$ as a yellow solid. M. p. $=57-58{ }^{\circ} \mathrm{C} .{ }^{1} \mathrm{H}$ NMR $\left(300 \mathrm{MHz}, \mathrm{CDCl}_{3}\right): \delta=10.23(\mathrm{~s}$, $1 \mathrm{H}$ ), 8.43 (ddd, $J=7.8,1.4,0.8 \mathrm{~Hz}, 1 \mathrm{H}$ ), 7.32 (ddd, $J=7.8,7.0,1.3 \mathrm{~Hz}, 1 \mathrm{H}$ ), 7.28$7.20(\mathrm{~m}, 4 \mathrm{H}$ ), 7.16 (d, J = 7.9 Hz, 1H), 6.90 (ddt, J=5.9, 2.5, $0.8 \mathrm{~Hz}, 2 \mathrm{H}$ ), 6.80 (ddd, $J=8.3,1.9,0.8 \mathrm{~Hz}, 1 \mathrm{H}), 6.76(\mathrm{~d}, J=8.3 \mathrm{~Hz}, 1 \mathrm{H}), 6.60(\mathrm{~d}, J=1.8 \mathrm{~Hz}, 1 \mathrm{H}), 5.26(\mathrm{~s}$, 2H), 5.00 (q, $J=7.3 \mathrm{~Hz}, 1 \mathrm{H}$ ), $3.84(\mathrm{~s}, 3 \mathrm{H}), 3.70(\mathrm{~s}, 3 \mathrm{H}), 1.73(\mathrm{~d}, J=7.4 \mathrm{~Hz}, 3 \mathrm{H}$ ). ${ }^{13} \mathrm{C}$ NMR $\left(125 \mathrm{MHz}, \mathrm{CDCl}_{3}\right): \delta=185.2(\mathrm{CH}), 153.9\left(\mathrm{C}_{\mathrm{q}}\right), 149.1\left(\mathrm{C}_{\mathrm{q}}\right), 148.0\left(\mathrm{C}_{\mathrm{q}}\right)$, $137.0\left(\mathrm{C}_{\mathrm{q}}\right), 135.9\left(\mathrm{C}_{\mathrm{q}}\right), 133.6\left(\mathrm{C}_{\mathrm{q}}\right), 128.7(\mathrm{CH}), 127.6(\mathrm{CH}), 125.9\left(\mathrm{C}_{\mathrm{q}}\right), 125.7(\mathrm{CH})$, $123.6(\mathrm{CH}), 123.1(\mathrm{CH}), 121.7(\mathrm{CH}), 118.8(\mathrm{CH}), 114.7\left(\mathrm{C}_{\mathrm{q}}\right), 111.2(\mathrm{CH}), 110.8(\mathrm{CH})$, $110.1(\mathrm{CH}), 55.9\left(\mathrm{CH}_{3}\right), 55.9\left(\mathrm{CH}_{3}\right), 47.5\left(\mathrm{CH}_{2}\right), 34.8(\mathrm{CH}), 20.2\left(\mathrm{CH}_{3}\right)$. IR (ATR): 2930, 1644, 1513, 1453, 1237, 1144, 1024, $745 \mathrm{~cm}^{-1}$. MS (ESI) $\mathrm{m} / z$ (relative intensity): 1220 (8) $[3 \mathrm{M}+\mathrm{Na}]^{+}, 821$ (20) $[2 \mathrm{M}+\mathrm{Na}]^{+}, 799$ (16) $[2 \mathrm{M}+\mathrm{H}]^{+}, 422(20)$ $[\mathrm{M}+\mathrm{Na}]^{+}, 400(100)[\mathrm{M}+\mathrm{H}]^{+}, 293$ (10). HR-MS (ESI) $\mathrm{m} / z$ calcd for $\mathrm{C}_{26} \mathrm{H}_{26} \mathrm{NO}_{3}[\mathrm{M}+\mathrm{H}]^{+}$ 400.1907, found 400.1903. [ $[\alpha]_{D}^{23}:-63.5\left(\mathrm{c}=0.94, \mathrm{CHCl}_{3}\right)$. HPLC separation (Chiralpak ${ }^{\circledR}$ IA-3, $n$-hexane/PrOH $80: 20,1.0 \mathrm{~mL} / \mathrm{min}$, detection at $250 \mathrm{~nm}$ ): $t_{r}($ major $)=10.4 \mathrm{~min}, t_{r}(\operatorname{minor})=12.3 \mathrm{~min}, 90: 10$ e.r. 
(S)-1-Benzyl-2-[1-(2,4-dimethylphenyl)ethyl]-1H-indole-3-carbaldehyde (62ck)

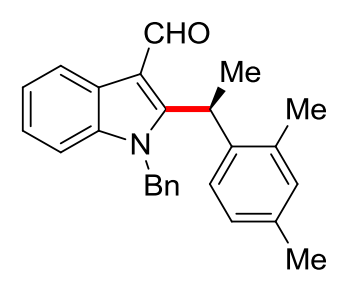

The general procedure GP6 was followed using indole $59 \mathrm{c}(85 \mathrm{mg}, 0.25 \mathrm{mmol}$, 1.0 equiv) and styrene $60 \mathrm{k}$ ( $50 \mathrm{mg}, 0.38 \mathrm{mmol}, 1.5$ equiv). Purification by column chromatography on silica gel ( $n$-hexane/EtOAc $=17 / 1 \rightarrow 12 / 1)$ yielded 62ck $(37.7 \mathrm{mg}$, $41 \%)$ as a light yellow oil. ${ }^{1} \mathrm{H}$ NMR $\left(500 \mathrm{MHz}, \mathrm{CDCl}_{3}\right): \delta=10.20(\mathrm{~s}, 1 \mathrm{H}), 8.41$ (dt, $J=7.9,1.0 \mathrm{~Hz}, 1 \mathrm{H}$ ), 7.31 (ddd, $J=8.0,7.0,1.1 \mathrm{~Hz}, 1 \mathrm{H}), 7.25(\mathrm{~d}, J=7.9 \mathrm{~Hz}, 1 \mathrm{H}$ ), 7.24-7.20 (m, 4H), $7.18(\mathrm{dt}, J=8.2,1.0 \mathrm{~Hz}, 1 \mathrm{H}), 6.99$ (dd, $J=7.9,1.9 \mathrm{~Hz}, 1 \mathrm{H})$, 6.88-6.76 (m, 3H), 5.25 (s, 2H), 4.94 (q, J = 7.3 Hz, 1H), 2.25 (s, 3H), 1.95 (s, 3H), $1.70(\mathrm{~d}, J=7.3 \mathrm{~Hz}, 3 \mathrm{H}) .{ }^{13} \mathrm{C}$ NMR $\left(125 \mathrm{MHz}, \mathrm{CDCl}_{3}\right): \delta=185.7(\mathrm{CH}), 153.3\left(\mathrm{C}_{\mathrm{q}}\right)$, $137.0\left(\mathrm{C}_{\mathrm{q}}\right), 136.9\left(\mathrm{C}_{\mathrm{q}}\right), 136.4\left(\mathrm{C}_{\mathrm{q}}\right), 136.2\left(\mathrm{C}_{\mathrm{q}}\right), 135.7\left(\mathrm{C}_{\mathrm{q}}\right), 131.9(\mathrm{CH}), 128.7(\mathrm{CH})$, $127.5(\mathrm{CH}), 127.0(\mathrm{CH}), 126.3(\mathrm{CH}), 126.1\left(\mathrm{C}_{q}\right), 125.8(\mathrm{CH}), 123.6(\mathrm{CH}), 123.1(\mathrm{CH})$, $121.6(\mathrm{CH}), 114.8\left(\mathrm{C}_{\mathrm{q}}\right), 110.0(\mathrm{CH}), 47.2\left(\mathrm{CH}_{2}\right), 34.2(\mathrm{CH}), 20.9\left(\mathrm{CH}_{3}\right), 20.8\left(\mathrm{CH}_{3}\right)$, $19.7\left(\mathrm{CH}_{3}\right)$. IR (ATR): 2921, 1643, 1453, 1395, 1039, $743 \mathrm{~cm}^{-1}$. MS (ESI) m/z (relative intensity): $757(31)\left[2 \mathrm{M}+\mathrm{Na}^{+}, 473\right.$ (19), $390(24)[\mathrm{M}+\mathrm{Na}]^{+}, 368$ (100) $[\mathrm{M}+\mathrm{H}]^{+}$. HR-MS (ESI) $\mathrm{m} / z$ calcd for $\mathrm{C}_{26} \mathrm{H}_{26} \mathrm{NO}[\mathrm{M}+\mathrm{H}]^{+}$368.2009, found 368.2006.

$[\alpha]_{\mathrm{D}}{ }^{23}$ : $-66.8\left(\mathrm{C}=0.52, \mathrm{CHCl}_{3}\right)$. HPLC separation (Chiralpak ${ }^{\circledR} \mathrm{IA}-3, n$-hexane/PrOH $80: 20,1.0 \mathrm{~mL} / \mathrm{min}$, detection at $250 \mathrm{~nm}): t_{r}($ major $)=5.9 \mathrm{~min}, t_{r}($ minor $)=6.5 \mathrm{~min}$, $91: 9$ e.r.

(R)-1-Benzyl-2-(1-ferrocenylethyl)-1 H-indole-3-carbaldehyde (192ca)

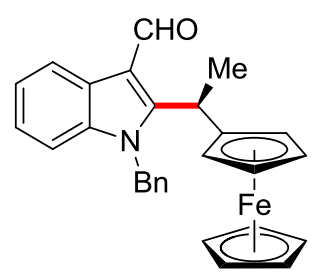


The general procedure GP5 was followed using indole $59 \mathrm{c}(85 \mathrm{mg}, 0.25 \mathrm{mmol}$, 1.0 equiv) and vinylferrocene (191a) $(80 \mathrm{mg}, 0.38 \mathrm{mmol}, 1.5$ equiv). Purification by column chromatography on silica gel $(n$-hexane/EtOAc $=12 / 1 \rightarrow 9 / 1)$ yielded 192ca (76.9 mg, 69\%) as an orange solid. M. p. $=61-63^{\circ} \mathrm{C} .{ }^{1} \mathbf{H}$ NMR $\left(300 \mathrm{MHz}, \mathrm{CDCl}_{3}\right)$ : $\delta=10.17(\mathrm{~s}, 1 \mathrm{H}), 8.37(\mathrm{~d}, J=7.6 \mathrm{~Hz}, 1 \mathrm{H}), 7.38-7.27(\mathrm{~m}, 4 \mathrm{H}), 7.26-7.18(\mathrm{~m}, 1 \mathrm{H})$, $7.16(\mathrm{~d}, J=8.0 \mathrm{~Hz}, 1 \mathrm{H}), 7.02-6.92(\mathrm{~m}, 2 \mathrm{H}), 5.39(\mathrm{~d}, J=17.3 \mathrm{~Hz}, 1 \mathrm{H}), 5.29(\mathrm{~d}$, $J=17.1 \mathrm{~Hz}, 1 \mathrm{H}), 4.93(\mathrm{brs}, 1 \mathrm{H}), 4.36(\mathrm{dt}, J=2.7,1.4, \mathrm{~Hz}, 1 \mathrm{H}), 4.27-4.10(\mathrm{~m}, 1 \mathrm{H})$, 4.09-4.04 (m, 6H), $3.84(\mathrm{~s}, 1 \mathrm{H}), 1.68(\mathrm{~d}, J=7.4 \mathrm{~Hz}, 3 \mathrm{H}) .{ }^{13} \mathrm{C}$ NMR $(125 \mathrm{MHz}$, $\left.\mathrm{CDCl}_{3}\right): \delta=185.3(\mathrm{CH}), 154.8\left(\mathrm{C}_{\mathrm{q}}\right), 136.8\left(\mathrm{C}_{\mathrm{q}}\right), 136.3\left(\mathrm{C}_{\mathrm{q}}\right), 128.9(\mathrm{CH}), 127.8(\mathrm{CH})$, $125.9(\mathrm{CH}), 125.9\left(\mathrm{C}_{\mathrm{q}}\right), 123.5(\mathrm{CH}), 123.1(\mathrm{CH}), 121.7(\mathrm{CH}), 113.7\left(\mathrm{C}_{\mathrm{q}}\right), 109.9(\mathrm{CH})$, $90.0\left(\mathrm{C}_{\mathrm{q}}\right), 69.0(\mathrm{CH}), 68.7(\mathrm{CH}), 68.7(\mathrm{CH}), 67.0(\mathrm{CH}), 66.6(\mathrm{CH}), 47.4\left(\mathrm{CH}_{2}\right), 31.5$ (CH), $20.1\left(\mathrm{CH}_{3}\right)$. IR (ATR): 2926, 1643, 1422, 1398, 813, 744, $480 \mathrm{~cm}^{-1}$. MS (EI) $\mathrm{m} / z$ (relative intensity): 447 (100) $[\mathrm{M}]^{+}, 382$ (64), 381 (75), 290 (50), 262 (45). HR-MS (El) $\mathrm{m} / \mathrm{z}$ calcd for $\mathrm{C}_{28} \mathrm{H}_{25} \mathrm{FeNO}[\mathrm{M}]^{+}$447.1286, found 447.1263. [a] ${ }_{\mathrm{D}}^{23}$ : $+220.0\left(\mathrm{c}=1.19, \mathrm{CHCl}_{3}\right)$. HPLC separation $\left(\right.$ Chiralpak $^{\circledR} \mathrm{IF}-3, n$-hexane/iPrOH $80: 20,0.75 \mathrm{~mL} / \mathrm{min}$, detection at $250 \mathrm{~nm}): t_{r}($ minor $)=18.3 \mathrm{~min}, t_{r}$ (major) $=21.6 \mathrm{~min}$, 4:96 e.r.

192ca (190 mg, $0.42 \mathrm{mmol}, 96: 4$ e.r.) was recrystallized from $n$-hexane $/ \mathrm{CH}_{2} \mathrm{Cl}_{2}$ to furnish the optically pure product (116 $\mathrm{mg}, 0.26 \mathrm{mmol}, 61 \%$ ) as a dark yellow solid. $[\alpha]_{D}^{23}:+347.8 \quad\left(\mathrm{c}=1.17, \quad \mathrm{CHCl}_{3}\right) . \quad$ HPLC separation $\left(\right.$ Chiralpak $^{\circledR} \quad$ IF-3, $n$-hexane/iPrOH 80:20, $0.75 \mathrm{~mL} / \mathrm{min}$, detection at $273 \mathrm{~nm}): t_{r}$ (minor) $=18.7 \mathrm{~min}$, $t_{r}$ (major) $=22.1 \mathrm{~min}, 0.3: 99.7$ e.r. Crystals suitable for X-ray crystallography were grown by slow evaporation from a solution of 192ca in benzene. 
(R)-2-(1-Ferrocenylethyl)-1-(4-methoxybenzyl)-1 H-indole-3-carbaldehyde (192da)

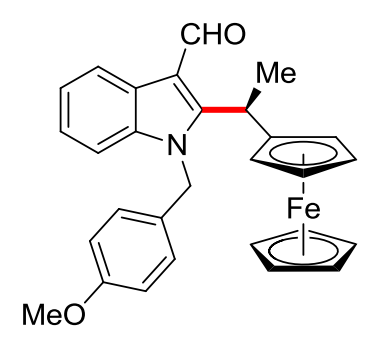

The general procedure GP5 was followed using indole $59 \mathrm{~d}(93 \mathrm{mg}, 0.25 \mathrm{mmol}$, 1.0 equiv) and vinylferrocene (191a) $(80 \mathrm{mg}, 0.38 \mathrm{mmol}, 1.5$ equiv). Purification by column chromatography on silica gel $(n$-hexane/EtOAc $=10 / 1 \rightarrow 7.5 / 1)$ yielded 192da (75.8 mg, 64\%) as an orange solid. M. p. $=67-69{ }^{\circ} \mathrm{C} .{ }^{1} \mathbf{H}$ NMR $\left(400 \mathrm{MHz}, \mathrm{CDCl}_{3}\right)$ : $\delta=10.17(\mathrm{~s}, 1 \mathrm{H}), 8.36(\mathrm{dt}, J=7.9,1.1 \mathrm{~Hz}, 1 \mathrm{H}), 7.32-7.21(\mathrm{~m}, 1 \mathrm{H}), 7.24-7.18(\mathrm{~m}$, $1 \mathrm{H}), 7.16(\mathrm{~d}, J=8.2 \mathrm{~Hz}, 1 \mathrm{H}), 6.89(\mathrm{~d}, J=8.4 \mathrm{~Hz}, 2 \mathrm{H}), 6.83(\mathrm{~d}, J=8.8 \mathrm{~Hz}, 2 \mathrm{H}), 5.32$ (d, $J=16.8 \mathrm{~Hz}, 1 \mathrm{H}$ ), 5.24 (d, $J=16.8 \mathrm{~Hz}, 1 \mathrm{H}$ ), 4.94 (brs, $1 \mathrm{H}$ ), 4.36 (dt, $J=2.6$, $1.3 \mathrm{~Hz}, 1 \mathrm{H}), 4.11-4.04(\mathrm{~m}, 7 \mathrm{H}), 3.86(\mathrm{~s}, 1 \mathrm{H}), 3.79(\mathrm{~s}, 3 \mathrm{H}), 1.67(\mathrm{~d}, J=7.4 \mathrm{~Hz}, 3 \mathrm{H})$. ${ }^{13} \mathrm{C}$ NMR $\left(100 \mathrm{MHz}, \mathrm{CDCl}_{3}\right): \delta=185.2(\mathrm{CH}), 159.1\left(\mathrm{C}_{\mathrm{q}}\right), 154.8\left(\mathrm{C}_{\mathrm{q}}\right), 136.8\left(\mathrm{C}_{\mathrm{q}}\right)$, $128.2\left(\mathrm{C}_{\mathrm{q}}\right), 127.1(\mathrm{CH}), 125.9\left(\mathrm{C}_{\mathrm{q}}\right), 123.4(\mathrm{CH}), 123.0(\mathrm{CH}), 121.7(\mathrm{CH}), 114.3(\mathrm{CH})$, $113.6\left(\mathrm{C}_{\mathrm{q}}\right), 110.0(\mathrm{CH}), 90.2\left(\mathrm{C}_{\mathrm{q}}\right), 69.0(\mathrm{CH}), 68.7(\mathrm{CH}), 68.7(\mathrm{CH}), 67.1(\mathrm{CH}), 66.7$ $(\mathrm{CH}), 55.3\left(\mathrm{CH}_{3}\right), 47.0\left(\mathrm{CH}_{2}\right), 31.5(\mathrm{CH}), 20.1\left(\mathrm{CH}_{3}\right)$. IR (ATR): 2961, 2929, 1642, 1510, 1245, 1026, 814, $742 \mathrm{~cm}^{-1}$. MS (ESI) $\mathrm{m} / z$ (relative intensity): 577 (60), 572 (62), 535 (45), 500 (94) [M+Na] $]^{+}, 478$ (100) $[\mathrm{M}+\mathrm{H}]^{+}, 460$ (32). HR-MS (ESI) m/z calcd for $\mathrm{C}_{29} \mathrm{H}_{28} \mathrm{FeNO}_{2}[\mathrm{M}+\mathrm{H}]^{+}$478.1464, found 478.1444. [ $\left.\alpha\right]_{D}{ }^{23}$ : +152.1 (c = 1.33, $\mathrm{CHCl}_{3}$ ). HPLC separation (Chiralpak ${ }^{\circledR} \mathrm{IF}-3, n$-hexane/PrOH 80:20, $0.75 \mathrm{~mL} / \mathrm{min}$, detection at $250 \mathrm{~nm}): t_{r}($ minor $)=23.3 \mathrm{~min}, t_{r}($ major $)=28.9 \mathrm{~min}, 5: 95$ e.r. 
(R)-2-(1-Ferrocenylethyl)-1-(4-fluorobenzyl)-1 H-indole-3-carbaldehyde (192ea)

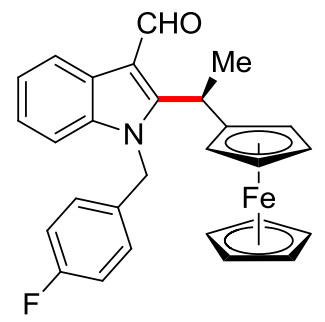

The general procedure GP5 was followed using indole $59 \mathrm{e}(90 \mathrm{mg}, 0.25 \mathrm{mmol}$, 1.0 equiv) and vinylferrocene (191a) $(80 \mathrm{mg}, 0.38 \mathrm{mmol}, 1.5$ equiv). Purification by column chromatography on silica gel $(n$-hexane/EtOAc $=13 / 1 \rightarrow 7.5 / 1)$ yielded 192ea (83.2 $\mathrm{mg}, 72 \%)$ as an orange solid. M. p. $=64-66{ }^{\circ} \mathrm{C} .{ }^{1} \mathbf{H}$ NMR $\left(500 \mathrm{MHz}, \mathrm{CDCl}_{3}\right)$ : $\delta=10.22(\mathrm{~s}, 1 \mathrm{H}), 8.36(\mathrm{dt}, J=7.9,1.0 \mathrm{~Hz}, 1 \mathrm{H}), 7.28$ (ddd, $J=8.0,7.1,1.0 \mathrm{~Hz}, 1 \mathrm{H}$ ), 7.21 (ddd, $J=8.3,7.2,1.3 \mathrm{~Hz}, 1 \mathrm{H}$ ), $7.10(\mathrm{~d}, J=8.3 \mathrm{~Hz}, 1 \mathrm{H}), 6.98(\mathrm{t}, J=8.6 \mathrm{~Hz}, 2 \mathrm{H}$ ), 6.91-6.86 (m, 2H), $5.33(\mathrm{~d}, J=17.2 \mathrm{~Hz}, 1 \mathrm{H}), 5.25$ (d, $J=16.9 \mathrm{~Hz}, 1 \mathrm{H}), 4.99$ (brs, $1 \mathrm{H}$ ), 4.35 (dt, $J=2.6,1.4 \mathrm{~Hz}, 1 \mathrm{H}$ ), 4.09 (s, $5 \mathrm{H}$ ), 4.08 (ddd, $J=2.5,1.3,1.3 \mathrm{~Hz}, 1 \mathrm{H}$ ), 4.06 (ddd, $J=2.5,1.3,1.3 \mathrm{~Hz}, 1 \mathrm{H}$ ), $3.87(\mathrm{~s}, 1 \mathrm{H}), 1.66(\mathrm{~d}, J=7.4 \mathrm{~Hz}, 3 \mathrm{H}) .{ }^{13} \mathrm{C}$ NMR $\left(125 \mathrm{MHz}, \mathrm{CDCl}_{3}\right): \delta=185.1(\mathrm{CH}), 162.1\left(\mathrm{~d},{ }^{1} \mathrm{~J}_{\mathrm{C}-\mathrm{F}}=246.8 \mathrm{~Hz}, \mathrm{C}_{\mathrm{q}}\right), 154.4\left(\mathrm{C}_{\mathrm{q}}\right), 136.7$ $\left(\mathrm{C}_{\mathrm{q}}\right), 131.9\left(\mathrm{~d},{ }^{4} \mathrm{~J}_{\mathrm{C}-\mathrm{F}}=3.1 \mathrm{~Hz}, \mathrm{C}_{\mathrm{q}}\right), 127.5\left(\mathrm{~d},{ }^{3} \mathrm{~J}_{\mathrm{C}-\mathrm{F}}=8.2 \mathrm{~Hz}, \mathrm{CH}\right), 125.9\left(\mathrm{C}_{\mathrm{q}}\right), 123.6$ $(\mathrm{CH}), 123.1(\mathrm{CH}), 121.7(\mathrm{CH}), 115.8\left(\mathrm{~d},{ }^{2} \mathrm{~J}_{\mathrm{C}-\mathrm{F}}=21.6 \mathrm{~Hz}, \mathrm{CH}\right), 113.7\left(\mathrm{C}_{\mathrm{q}}\right), 109.9$ $(\mathrm{CH}), 89.8\left(\mathrm{C}_{\mathrm{q}}\right), 69.0(\mathrm{CH}), 68.8(\mathrm{CH}), 68.7(\mathrm{CH}), 67.1(\mathrm{CH}), 66.6(\mathrm{CH}), 46.9\left(\mathrm{CH}_{2}\right)$, $31.4(\mathrm{CH}), 19.9\left(\mathrm{CH}_{3}\right) .{ }^{19} \mathrm{~F} \mathrm{NMR}\left(471 \mathrm{MHz}, \mathrm{CDCl}_{3}\right): \delta=-114.36$ (s). IR (ATR): 2904, 1643, 1509, 1222, 1039, 819, $743 \mathrm{~cm}^{-1}$. MS (EI) $\mathrm{m} / \mathrm{z}$ (relative intensity): 465 (100) $\left[\mathrm{M}^{+}, 400\right.$ (61), 399 (86), 290 (38), 109 (50). HR-MS (ESI) m/z calcd for

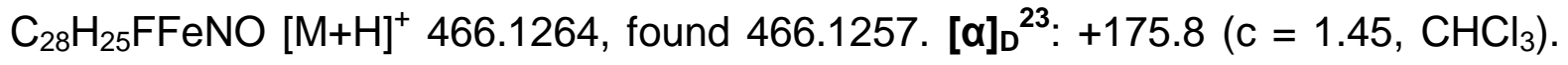
HPLC separation (Chiralpak ${ }^{\circledR}$ IF-3, $n$-hexane/ $/ \mathrm{PrOH} 80: 20,0.75 \mathrm{~mL} / \mathrm{min}$, detection at $250 \mathrm{~nm}): t_{r}($ minor $)=19.2 \mathrm{~min}, t_{r}($ major $)=22.4 \mathrm{~min}, 5: 95$ e.r. 


\section{(R)-2-(1-Ferrocenylethyl)-1-phenethyl-1 $H$-indole-3-carbaldehyde (192fa)}

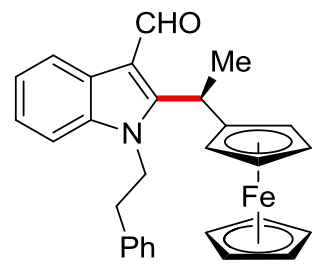

The general procedure GP5 was followed using indole $59 \mathrm{f}(89 \mathrm{mg}, 0.25 \mathrm{mmol}$, 1.0 equiv) and vinylferrocene (191a) $(80 \mathrm{mg}, 0.38 \mathrm{mmol}, 1.5$ equiv). Purification by column chromatography on silica gel $(n$-hexane/EtOAc $=15 / 1 \rightarrow 10 / 1)$ yielded 192fa (64.6 mg, 56\%) as an orange solid. Using 177: $65.4 \mathrm{mg}$ (57\%), 7:93 e.r. M. p. $=62-$ $63^{\circ} \mathrm{C} .{ }^{1} \mathrm{H}$ NMR $\left(300 \mathrm{MHz}, \mathrm{CDCl}_{3}\right): \delta=10.20(\mathrm{~s}, 1 \mathrm{H}), 8.40-8.32(\mathrm{~m}, 1 \mathrm{H}), 7.38-7.27$ (m, 6H), 7.13 (d, $J=6.3 \mathrm{~Hz}, 2 \mathrm{H}$ ), 5.03 (brs, $1 \mathrm{H}$ ), 4.46 (dt, $J=2.6,1.3 \mathrm{~Hz}, 1 \mathrm{H}$ ), $4.41-$ $4.18(\mathrm{~m}, 8 \mathrm{H}), 4.17$ (ddd, $J=2.4,1.2,1.2 \mathrm{~Hz}, 1 \mathrm{H}), 4.10-4.05(\mathrm{~m}, 1 \mathrm{H}), 2.95$ (td, $J=12.0,10.3,5.3 \mathrm{~Hz}, 1 \mathrm{H}), 2.58$ (brs, $1 \mathrm{H}), 1.73$ (d, $J=7.4 \mathrm{~Hz}, 3 \mathrm{H}) .{ }^{13} \mathbf{C}$ NMR $\left(125 \mathrm{MHz}, \mathrm{CDCl}_{3}\right): \delta=184.6(\mathrm{CH}), 153.6\left(\mathrm{C}_{\mathrm{q}}\right), 137.6\left(\mathrm{C}_{\mathrm{q}}\right), 136.0\left(\mathrm{C}_{\mathrm{q}}\right), 128.7(\mathrm{CH})$, $128.6(\mathrm{CH}), 126.9(\mathrm{CH}), 126.1\left(\mathrm{C}_{\mathrm{q}}\right), 123.3(\mathrm{CH}), 122.9(\mathrm{CH}), 121.6(\mathrm{CH}), 113.2\left(\mathrm{C}_{\mathrm{q}}\right)$, $109.7(\mathrm{CH}), 90.0\left(\mathrm{C}_{\mathrm{q}}\right), 69.1(\mathrm{CH}), 69.1(\mathrm{CH}), 68.9(\mathrm{CH}), 67.3(\mathrm{CH}), 66.9(\mathrm{CH}), 46.1$ $\left(\mathrm{CH}_{2}\right), 35.3\left(\mathrm{CH}_{2}\right), 31.2(\mathrm{CH}), 19.5\left(\mathrm{CH}_{3}\right)$. IR (ATR): 2932, 1636, 1423, 1038, 809, 740, $695 \mathrm{~cm}^{-1}$. MS (ESI) $\mathrm{m} / z$ (relative intensity): 484 (94) $[\mathrm{M}+\mathrm{Na}]^{+}, 462$ (100) $[\mathrm{M}+\mathrm{H}]^{+}, 444$ (72). HR-MS (ESI) $\mathrm{m} / z$ calcd for $\mathrm{C}_{29} \mathrm{H}_{28} \mathrm{FeNO}[\mathrm{M}+\mathrm{H}]^{+} 462.1515$, found 462.1507. $[\alpha]_{D}{ }^{23}:+185.3\left(\mathrm{c}=1.16, \mathrm{CHCl}_{3}\right.$ ). HPLC separation (Chiralpak ${ }^{\circledR}$ IA-3, $n$-hexane/PrOH 80:20, $1.0 \mathrm{~mL} / \mathrm{min}$, detection at $250 \mathrm{~nm}): t_{r}($ minor $)=8.0 \mathrm{~min}$, $t_{r}($ major $)=10.6 \mathrm{~min}, 8: 92$ e.r.

(R)-2-(1-Ferrocenylethyl)-1-(methoxymethyl)-1 $H$-indole-3-carbaldehyde (192ga)

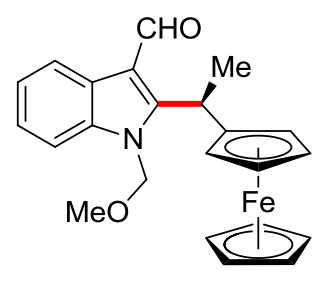


The general procedure GP5 was followed using indole $59 \mathrm{~g}(74 \mathrm{mg}, 0.25 \mathrm{mmol}$, 1.0 equiv) and vinylferrocene (191a) $(80 \mathrm{mg}, 0.38 \mathrm{mmol}, 1.5$ equiv). Purification by column chromatography on silica gel $(n$-hexane/EtOAc $=9 / 1 \rightarrow 7 / 1)$ yielded 192ga (52.2 $\mathrm{mg}, 52 \%)$ as an orange oil. ${ }^{1} \mathrm{H}$ NMR $\left(300 \mathrm{MHz}, \mathrm{CDCl}_{3}\right): \delta=10.25(\mathrm{~s}, 1 \mathrm{H})$, 8.37-8.27 (m, 1H), 7.48-7.36 (m, 1H), 7.34-7.23 (m, 2H), $5.40(\mathrm{~s}, 2 \mathrm{H}), 5.06(\mathrm{q}$, $J=7.3 \mathrm{~Hz}, 1 \mathrm{H}$ ), 4.47 (dt, $J=2.7,1.3 \mathrm{~Hz}, 1 \mathrm{H}), 4.25-4.17(\mathrm{~m}, 5 \mathrm{H}), 4.16$ (td, $J=2.4$, $1.2 \mathrm{~Hz}, 1 \mathrm{H}), 4.13(\mathrm{td}, J=2.4,1.3 \mathrm{~Hz}, 1 \mathrm{H}), 4.07(\mathrm{dt}, J=2.6,1.3 \mathrm{~Hz}, 1 \mathrm{H}), 3.23(\mathrm{~s}, 3 \mathrm{H})$, $1.82(\mathrm{~d}, J=7.4 \mathrm{~Hz}, 3 \mathrm{H}) .{ }^{13} \mathrm{C}$ NMR $\left(125 \mathrm{MHz}, \mathrm{CDCl}_{3}\right): \delta=185.3(\mathrm{CH}), 154.7\left(\mathrm{C}_{\mathrm{q}}\right)$, $136.8\left(\mathrm{C}_{\mathrm{q}}\right), 125.7\left(\mathrm{C}_{\mathrm{q}}\right), 123.6(\mathrm{CH}), 123.1(\mathrm{CH}), 121.6(\mathrm{CH}), 114.3\left(\mathrm{C}_{\mathrm{q}}\right), 109.8(\mathrm{CH})$, $90.2\left(\mathrm{C}_{\mathrm{q}}\right), 74.3\left(\mathrm{CH}_{2}\right), 69.1(\mathrm{CH}), 68.8(\mathrm{CH}), 68.8(\mathrm{CH}), 67.1(\mathrm{CH}), 66.8(\mathrm{CH}), 56.1$ $\left(\mathrm{CH}_{3}\right), 31.4(\mathrm{CH}), 20.5\left(\mathrm{CH}_{3}\right)$. IR (ATR): 2902, 1647, 1386, 1038, 817, $749 \mathrm{~cm}^{-1}$. MS (ESI) $m / z$ (relative intensity): $825(44)[2 \mathrm{M}+\mathrm{Na}]^{+}, 424(75)[\mathrm{M}+\mathrm{Na}]^{+}, 402(24)$ $[\mathrm{M}+\mathrm{H}]^{+}, 384$ (14), 213 (100). HR-MS (ESI) $\mathrm{m} / z$ calcd for $\mathrm{C}_{23} \mathrm{H}_{24} \mathrm{FeNO}_{2}[\mathrm{M}+\mathrm{H}]^{+}$ 402.1151, found 402.1162. [ $\alpha]_{\mathrm{D}}{ }^{23}:+206.3\left(\mathrm{c}=0.79, \mathrm{CHCl}_{3}\right)$. HPLC separation (Chiralpak ${ }^{\circledR}$ ID-3, $n$-hexane/ $\mathrm{PrOH} 70: 30,1.0 \mathrm{~mL} / \mathrm{min}$, detection at $273 \mathrm{~nm}$ ): $t_{r}($ minor $)=12.2 \mathrm{~min}, t_{r}($ major $)=12.8 \mathrm{~min}, 4: 96$ e.r.

(R)-1-(2,2-Difluoroethyl)-2-(1-ferrocenylethyl)-1H-indole-3-carbaldehyde (192ha)

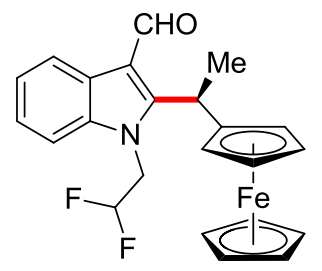

The general procedure GP5 was followed using indole $59 \mathrm{~h}(79 \mathrm{mg}, 0.25 \mathrm{mmol}$, 1.0 equiv) and vinylferrocene (191a) $(80 \mathrm{mg}, 0.38 \mathrm{mmol}, 1.5$ equiv). Purification by column chromatography on silica gel ( $n$-hexane/EtOAc $=10 / 1)$ yielded 192ha (53.7 mg, 51\%) as an orange solid. M. p. $=62-64{ }^{\circ} \mathrm{C} .{ }^{1} \mathbf{H}$ NMR $\left(500 \mathrm{MHz}, \mathrm{CDCl}_{3}\right)$ : $\delta=10.26(\mathrm{~s}, 1 \mathrm{H}), 8.34-8.29(\mathrm{~m}, 1 \mathrm{H}), 7.35-7.27(\mathrm{~m}, 3 \mathrm{H}), 5.40(\mathrm{t}, J=53.2 \mathrm{~Hz}, 1 \mathrm{H})$, $5.13(\mathrm{~s}, 1 \mathrm{H}), 4.53-4.35(\mathrm{~m}, 3 \mathrm{H}), 4.25-4.19(\mathrm{~m}, 6 \mathrm{H}), 4.18(\mathrm{td}, J=2.5,1.3 \mathrm{~Hz}, 1 \mathrm{H})$, $4.11(\mathrm{dt}, J=2.6,1.4 \mathrm{~Hz}, 1 \mathrm{H}), 1.80(\mathrm{~d}, J=7.5 \mathrm{~Hz}, 3 \mathrm{H}) .{ }^{13} \mathrm{C} \mathbf{N M R}\left(125 \mathrm{MHz}, \mathrm{CDCl}_{3}\right)$ : 
$\delta=185.1(\mathrm{CH}), 154.0\left(\mathrm{C}_{\mathrm{q}}\right), 136.8\left(\mathrm{C}_{\mathrm{q}}\right), 125.8\left(\mathrm{C}_{\mathrm{q}}\right), 123.8(\mathrm{CH}), 123.4(\mathrm{CH}), 121.4$ $(\mathrm{CH}), 114.1\left(\mathrm{C}_{\mathrm{q}}\right), 113.5\left(\mathrm{t},{ }^{1} \mathrm{~J}_{\mathrm{C}-\mathrm{F}}=245.2 \mathrm{~Hz}, \mathrm{CH}\right), 109.8(\mathrm{CH}), 89.7\left(\mathrm{C}_{\mathrm{q}}\right), 69.2(\mathrm{CH})$, $69.2(\mathrm{CH}), 68.9(\mathrm{CH}), 67.5(\mathrm{CH}), 66.8(\mathrm{CH}), 46.4\left(\mathrm{t},{ }^{2} \mathrm{~J}_{\mathrm{C}-\mathrm{F}}=29.0 \mathrm{~Hz}, \mathrm{CH}_{2}\right), 31.0$ $(\mathrm{CH}), 19.7\left(\mathrm{CH}_{3}\right) .{ }^{19} \mathrm{~F} \mathrm{NMR}\left(471 \mathrm{MHz}, \mathrm{CDCl}_{3}\right): \delta=-119.18(\mathrm{~d}, J=55.3 \mathrm{~Hz})$. IR (ATR): 2903, 1653, 1396, 1042, 1019, 812, $747 \mathrm{~cm}^{-1}$. MS (EI) $\mathrm{m} / \mathrm{z}$ (relative intensity): 421 (100) [M] ${ }^{+}, 356$ (42), 355 (93), 230 (31), 138 (30), 121 (45). HR-MS (ESI) $\mathrm{m} / \mathrm{z}$ calcd for $\mathrm{C}_{23} \mathrm{H}_{22} \mathrm{~F}_{2} \mathrm{FeNO}[\mathrm{M}+\mathrm{H}]^{+} 422.1014$, found 422.1011. [a $]_{\mathrm{D}}{ }^{23}:+230.1$ (c $=0.63, \mathrm{CHCl}_{3}$ ). HPLC separation (Chiralpak ${ }^{\circledR} \mathrm{IA}-3, n$-hexane/PrOH 80:20, $1.0 \mathrm{~mL} / \mathrm{min}$, detection at $273 \mathrm{~nm}$ ): $t_{r}($ minor $)=8.3 \mathrm{~min}, t_{r}$ (major) $=10.0 \mathrm{~min}, 4: 96$ e.r.

\section{(R)-1-[(1,3-Dioxolan-2-yl)methyl]-2-(1-ferrocenylethyl)-1 H-indole-3- carbaldehyde (192ia)}

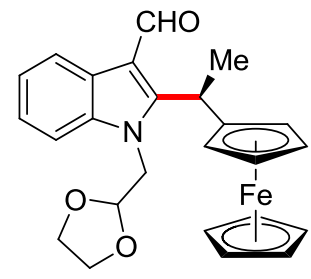

The general procedure GP5 was followed using indole 59i $(84 \mathrm{mg}, 0.25 \mathrm{mmol}$, 1.0 equiv) and vinylferrocene (191a) $(80 \mathrm{mg}, 0.38 \mathrm{mmol}, 1.5$ equiv). Purification by column chromatography on silica gel $(n$-hexane/EtOAc $=6 / 1 \rightarrow 4 / 1$ ) yielded 192ia (43.2 mg, 39\%) as an orange solid. M. p. $=75-77^{\circ} \mathrm{C} .{ }^{1} \mathbf{H}$ NMR $\left(500 \mathrm{MHz}, \mathrm{CDCl}_{3}\right)$ : $\delta=10.08(\mathrm{~s}, 1 \mathrm{H}), 8.33-8.28(\mathrm{~m}, 1 \mathrm{H}), 7.47-7.39(\mathrm{~m}, 1 \mathrm{H}), 7.29-7.23(\mathrm{~m}, 2 \mathrm{H}), 5.13(\mathrm{~s}$, 1H), 4.92 (brs, 1H), 4.44 (dt, $J=2.5,1.4 \mathrm{~Hz}, 1 \mathrm{H}$ ), 4.40 (dd, $J=15.3,2.9 \mathrm{~Hz}, 1 \mathrm{H}$ ), 4.35 (dd, $J=15.3,3.9 \mathrm{~Hz}, 1 \mathrm{H}$ ), 4.17 (s, $5 \mathrm{H}$ ), 4.15 (td, $J=2.5,1.3 \mathrm{~Hz}, 1 \mathrm{H}$ ), 4.12 (td, $J=2.5,1.2 \mathrm{~Hz}, 1 \mathrm{H}), 4.09(\mathrm{dt}, J=2.5,1.3 \mathrm{~Hz}, 1 \mathrm{H}), 3.93-3.84(\mathrm{~m}, 4 \mathrm{H}), 1.80(\mathrm{~d}$, $J=7.3 \mathrm{~Hz}, 3 \mathrm{H}) .{ }^{13} \mathrm{C}$ NMR $\left(125 \mathrm{MHz}, \mathrm{CDCl}_{3}\right): \delta=185.9(\mathrm{CH}), 155.8\left(\mathrm{C}_{\mathrm{q}}\right), 136.8\left(\mathrm{C}_{\mathrm{q}}\right)$, $125.8\left(\mathrm{C}_{\mathrm{q}}\right), 123.2(\mathrm{CH}), 123.0(\mathrm{CH}), 121.7(\mathrm{CH}), 113.7\left(\mathrm{C}_{\mathrm{q}}\right), 110.5(\mathrm{CH}), 102.1(\mathrm{CH})$, $91.0\left(\mathrm{C}_{\mathrm{q}}\right), 68.9(\mathrm{CH}), 68.8(\mathrm{CH}), 68.8(\mathrm{CH}), 67.1(\mathrm{CH}), 66.9(\mathrm{CH}), 65.3\left(\mathrm{CH}_{2}\right), 65.2$ $\left(\mathrm{CH}_{2}\right), 46.6\left(\mathrm{CH}_{2}\right), 31.6(\mathrm{CH}), 20.8\left(\mathrm{CH}_{3}\right)$. IR (ATR): 2917, 1642, 1460, 1041, 817, $745 \mathrm{~cm}^{-1}$. MS (ESI) $\mathrm{m} / \mathrm{z}$ (relative intensity): $909(47)[2 \mathrm{M}+\mathrm{Na}]^{+}, 466(100)[\mathrm{M}+\mathrm{Na}]^{+}$, 
444 (39) $[\mathrm{M}+\mathrm{H}]^{+}$. HR-MS (ESI) $\mathrm{m} / z$ calcd for $\mathrm{C}_{25} \mathrm{H}_{26} \mathrm{FeNO}_{3}[\mathrm{M}+\mathrm{H}]^{+} 444.1257$, found 444.1255. $[\alpha]_{\mathrm{D}}^{23}$ : +176.1 ( $\mathrm{C}=0.67, \mathrm{CHCl}_{3}$ ). HPLC separation (Chiralpak ${ }^{\circledR}$ IF-3, $n$-hexane/iPrOH 70:30, $1.0 \mathrm{~mL} / \mathrm{min}$, detection at $250 \mathrm{~nm}$ ): $t_{r}($ minor $)=10.5 \mathrm{~min}$, $t_{r}($ major $)=13.1 \mathrm{~min}, 5: 95$ e.r.

(R)-2-(1-Ferrocenylethyl)-1-methyl-1H-indole-3-carbaldehyde (192ba)

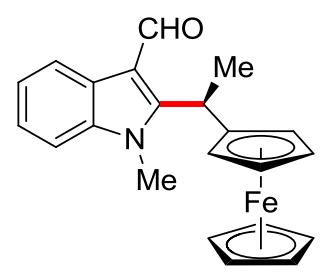

The general procedure GP5 was followed using indole 59b $(66 \mathrm{mg}, 0.25 \mathrm{mmol}$, 1.0 equiv) and vinylferrocene (191a) $(80 \mathrm{mg}, 0.38 \mathrm{mmol}, 1.5$ equiv). Purification by column chromatography on silica gel $(n$-hexane/EtOAc $=11 / 1 \rightarrow 8.5 / 1)$ yielded 192ba $(49.3 \mathrm{mg}, 53 \%)$ as an orange oil. ${ }^{1} \mathrm{H}$ NMR $\left(300 \mathrm{MHz}, \mathrm{CDCl}_{3}\right): \delta=10.25(\mathrm{~s}, 1 \mathrm{H})$, 8.39-8.26 (m, 1H), 7.36-7.18 (m, 3H), 5.17 (q, $J=7.3 \mathrm{~Hz}, 1 \mathrm{H}$ ), 4.44 (dtd, $J=2.7$, 1.3, $0.7 \mathrm{~Hz}, 1 \mathrm{H}), 4.25-4.17(\mathrm{~m}, 5 \mathrm{H}), 4.18-4.14(\mathrm{~m}, 1 \mathrm{H}), 4.13$ (ddd, $J=2.5,1.3$, $1.3 \mathrm{~Hz}, 1 \mathrm{H}$ ), 4.03 (dtd, $J=2.6,1.4,0.3 \mathrm{~Hz}, 1 \mathrm{H}$ ), 3.56 (s, 3H), 1.77 (d, $J=7.4 \mathrm{~Hz}$, $3 \mathrm{H}) .{ }^{13} \mathrm{C}$ NMR $\left(125 \mathrm{MHz}, \mathrm{CDCl}_{3}\right): \delta=184.3(\mathrm{CH}), 153.8\left(\mathrm{C}_{\mathrm{q}}\right), 137.1\left(\mathrm{C}_{\mathrm{q}}\right), 125.7\left(\mathrm{C}_{\mathrm{q}}\right)$, $123.2(\mathrm{CH}), 122.8(\mathrm{CH}), 121.1(\mathrm{CH}), 113.2\left(\mathrm{C}_{\mathrm{q}}\right), 109.1(\mathrm{CH}), 89.6\left(\mathrm{C}_{\mathrm{q}}\right), 69.1(\mathrm{CH})$, $68.7(\mathrm{CH}), 68.6(\mathrm{CH}), 67.1(\mathrm{CH}), 66.8(\mathrm{CH}), 31.2(\mathrm{CH}), 31.0\left(\mathrm{CH}_{3}\right), 19.0\left(\mathrm{CH}_{3}\right)$. IR (ATR): 2903, 1642, 1391, 1039, 815, 746, $479 \mathrm{~cm}^{-1}$. MS (EI) $\mathrm{m} / \mathrm{z}$ (relative intensity): 371 (100) [M] ${ }^{+}, 306$ (53), 305 (94), 278 (73). HR-MS (EI) m/z calcd for $\mathrm{C}_{22} \mathrm{H}_{21} \mathrm{FeNO}[\mathrm{M}]^{+}$371.0973, found 371.0962. [ $\left.\alpha\right]_{\mathrm{D}}{ }^{23}:+217.3\left(\mathrm{c}=0.85, \mathrm{CHCl}_{3}\right)$. HPLC separation (Chiralpak ${ }^{\circledR}$ IF-3, $n$-hexane/iPrOH $80: 20,0.75 \mathrm{~mL} / \mathrm{min}$, detection at $250 \mathrm{~nm}): t_{r}($ minor $)=19.0 \mathrm{~min}, t_{r}($ major $)=21.7 \mathrm{~min}, 6: 94$ e.r. 


\section{(R)-1-Benzyl-2-(1-ferrocenylethyl)-5-methoxy-1H-indole-3-carbaldehyde (192ja)}

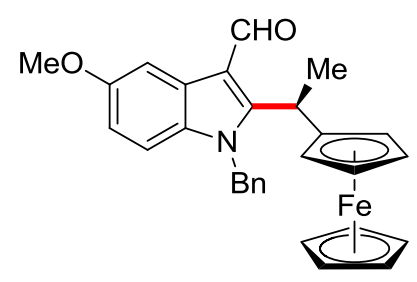

The general procedure GP5 was followed using indole 59j $(93 \mathrm{mg}, 0.25 \mathrm{mmol}$, 1.0 equiv) and vinylferrocene (191a) $(80 \mathrm{mg}, 0.38 \mathrm{mmol}, 1.5$ equiv). Purification by column chromatography on silica gel $(n$-hexane/EtOAc $=10 / 1 \rightarrow 7 / 1)$ yielded 192ja (91.9 mg, 77\%) as an orange solid. M. p. $=77-79{ }^{\circ} \mathrm{C} .{ }^{1} \mathbf{H}$ NMR $\left(400 \mathrm{MHz}, \mathrm{CDCl}_{3}\right)$ : $\delta=10.13(\mathrm{~s}, 1 \mathrm{H}), 7.89(\mathrm{~d}, J=2.5 \mathrm{~Hz}, 1 \mathrm{H}), 7.37-7.26(\mathrm{~m}, 3 \mathrm{H}), 7.04(\mathrm{~d}, J=8.8 \mathrm{~Hz}$, $1 \mathrm{H}), 6.97(\mathrm{~d}, J=6.5 \mathrm{~Hz}, 2 \mathrm{H}), 6.84(\mathrm{dd}, J=8.9,2.5 \mathrm{~Hz}, 1 \mathrm{H}), 5.35(\mathrm{~d}, J=17.2 \mathrm{~Hz}$, $1 \mathrm{H}), 5.26(\mathrm{~d}, J=17.1 \mathrm{~Hz}, 1 \mathrm{H}), 4.85(\mathrm{brs}, 1 \mathrm{H}), 4.35$ (dt, $J=2.6,1.3 \mathrm{~Hz}, 1 \mathrm{H}), 4.08$ (td, $J=2.5,1.3 \mathrm{~Hz}, 1 \mathrm{H}), 4.07-4.02(\mathrm{~m}, 6 \mathrm{H}), 3.88(\mathrm{~s}, 3 \mathrm{H}), 3.83(\mathrm{~s}, 1 \mathrm{H}), 1.66(\mathrm{~d}$, $J=7.4 \mathrm{~Hz}, 3 \mathrm{H}) .{ }^{13} \mathrm{C}$ NMR $\left(100 \mathrm{MHz}, \mathrm{CDCl}_{3}\right): \delta=185.2(\mathrm{CH}), 156.8\left(\mathrm{C}_{\mathrm{q}}\right), 154.8\left(\mathrm{C}_{\mathrm{q}}\right)$, $136.3\left(\mathrm{C}_{\mathrm{q}}\right), 131.6\left(\mathrm{C}_{\mathrm{q}}\right), 128.9(\mathrm{CH}), 127.8(\mathrm{CH}), 126.5\left(\mathrm{C}_{\mathrm{q}}\right), 125.9(\mathrm{CH}), 113.7(\mathrm{CH})$, $113.6\left(\mathrm{C}_{\mathrm{q}}\right), 110.8(\mathrm{CH}), 103.3(\mathrm{CH}), 90.1\left(\mathrm{C}_{\mathrm{q}}\right), 68.9(\mathrm{CH}), 68.7(\mathrm{CH}), 68.7(\mathrm{CH}), 67.0$ $(\mathrm{CH}), 66.6(\mathrm{CH}), 55.8\left(\mathrm{CH}_{3}\right), 47.6\left(\mathrm{CH}_{2}\right), 31.6(\mathrm{CH}), 20.2\left(\mathrm{CH}_{3}\right)$. IR (ATR): 2930, 1640, 1453, 1041, 799, 750, $697 \mathrm{~cm}^{-1}$. MS (ESI) $\mathrm{m} / \mathrm{z}$ (relative intensity): 1932 (17) $[4 \mathrm{M}+\mathrm{Na}]^{+}, 1454(51)[3 \mathrm{M}+\mathrm{Na}]^{+}, 977(100)[2 \mathrm{M}+\mathrm{Na}]^{+}, 500(97)[\mathrm{M}+\mathrm{Na}]^{+}, 478(57)$ $[\mathrm{M}+\mathrm{H}]^{+}$. HR-MS (ESI) $\mathrm{m} / z$ calcd for $\mathrm{C}_{29} \mathrm{H}_{28} \mathrm{FeNO}_{2}[\mathrm{M}+\mathrm{H}]^{+}$478.1464, found 478.1452. $[\alpha]_{D}^{23}:+259.9\left(\mathrm{c}=1.75, \mathrm{CHCl}_{3}\right) . \quad$ HPLC separation $\left(\right.$ Chiralpak $^{\circledR}$ ID-3, $n$-hexane $/ \mathrm{PrOH} 70: 30,1.0 \mathrm{~mL} / \mathrm{min}$, detection at $273 \mathrm{~nm}): t_{r}$ (major) $=19.1 \mathrm{~min}$, $t_{r}(\operatorname{minor})=22.9 \min , 96: 4$ e.r. 
(R)-1-Benzyl-2-(1-ferrocenylethyl)-5-fluoro-1 $H$-indole-3-carbaldehyde (192ka)

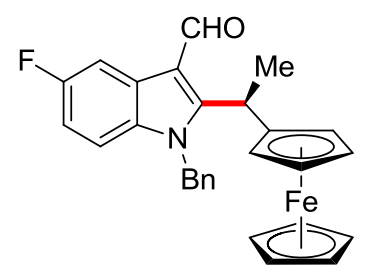

The general procedure GP5 was followed using indole $59 \mathrm{k}(90 \mathrm{mg}, 0.25 \mathrm{mmol}$, 1.0 equiv) and vinylferrocene (191a) (80 mg, $0.38 \mathrm{mmol}, 1.5$ equiv). Purification by column chromatography on silica gel $(n$-hexane/EtOAc $=12 / 1 \rightarrow 9.5 / 1)$ yielded 192ka $(48.9 \mathrm{mg}, 42 \%)$ as an orange solid. $\mathbf{M} . \mathbf{p} .=62-64{ }^{\circ} \mathrm{C} .{ }^{1} \mathrm{H}$ NMR $\left(500 \mathrm{MHz}, \mathrm{CDCl}_{3}\right)$ : $\delta=10.11(\mathrm{~s}, 1 \mathrm{H}), 8.04(\mathrm{dd}, J=9.4,2.6 \mathrm{~Hz}, 1 \mathrm{H}), 7.38-7.27(\mathrm{~m}, 3 \mathrm{H}), 7.09-7.03(\mathrm{~m}$, 1H), 6.98-6.89 (m, 3H), $5.36(\mathrm{~d}, J=17.3 \mathrm{~Hz}, 1 \mathrm{H}), 5.29(\mathrm{~d}, J=17.0 \mathrm{~Hz}, 1 \mathrm{H}), 4.87$ (brs, $1 \mathrm{H}), 4.35(\mathrm{dt}, J=2.7,1.3 \mathrm{~Hz}, 1 \mathrm{H}), 4.16-4.02(\mathrm{~m}, 7 \mathrm{H}), 3.85-3.81(\mathrm{~m}, 1 \mathrm{H}), 1.67$ $(\mathrm{d}, J=7.4 \mathrm{~Hz}, 3 \mathrm{H}) .{ }^{13} \mathrm{C}$ NMR $\left(125 \mathrm{MHz}, \mathrm{CDCl}_{3}\right): \delta=185.0(\mathrm{CH}), 160.0(\mathrm{~d}$, $\left.{ }^{1} J_{\mathrm{C}-\mathrm{F}}=238.6 \mathrm{~Hz}, \mathrm{C}_{\mathrm{q}}\right), 155.8\left(\mathrm{C}_{\mathrm{q}}\right), 136.0\left(\mathrm{C}_{\mathrm{q}}\right), 133.2\left(\mathrm{C}_{\mathrm{q}}\right), 129.0(\mathrm{CH}), 127.9(\mathrm{CH})$, $126.5\left(\mathrm{~d},{ }^{3} \mathrm{~J}_{\mathrm{C}-\mathrm{F}}=11.0 \mathrm{~Hz}, \mathrm{C}_{\mathrm{q}}\right), 125.8(\mathrm{CH}), 113.7\left(\mathrm{~d},{ }^{4} \mathrm{~J}_{\mathrm{C}-\mathrm{F}}=4.4 \mathrm{~Hz}, \mathrm{C}_{\mathrm{q}}\right), 111.7(\mathrm{~d}$, $\left.{ }^{2} J_{\mathrm{C}-\mathrm{F}}=26.2 \mathrm{~Hz}, \mathrm{CH}\right), 110.8\left(\mathrm{~d},{ }^{3} J_{\mathrm{C}-\mathrm{F}}=9.5 \mathrm{~Hz}, \mathrm{CH}\right), 107.4\left(\mathrm{~d},{ }^{2} J_{\mathrm{C}-\mathrm{F}}=25.2 \mathrm{~Hz}, \mathrm{CH}\right)$, $89.8\left(\mathrm{C}_{\mathrm{q}}\right), 69.0(\mathrm{CH}), 68.8(\mathrm{CH}), 68.7(\mathrm{CH}), 67.1(\mathrm{CH}), 66.6(\mathrm{CH}), 47.7\left(\mathrm{CH}_{2}\right), 31.7$ $(\mathrm{CH}), 20.1\left(\mathrm{CH}_{3}\right) .{ }^{19} \mathrm{~F}$ NMR $\left(471 \mathrm{MHz}, \mathrm{CDCl}_{3}\right): \delta=-120.36$ (td, $\left.J=9.2,4.2 \mathrm{~Hz}\right)$. IR (ATR): 2903, 1645, 1104, 1024, 1001, $797 \mathrm{~cm}^{-1}$. MS (ESI) $\mathrm{m} / \mathrm{z}$ (relative intensity): $953(10)[2 \mathrm{M}+\mathrm{Na}]^{+}, 488(47)[\mathrm{M}+\mathrm{Na}]^{+}, 466(8)[\mathrm{M}+\mathrm{H}]^{+}, 253$ (18), 213 (100). HR-MS

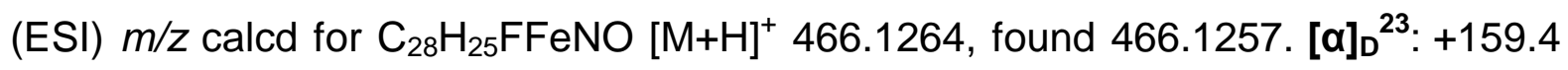
(c $=0.45, \mathrm{CHCl}_{3}$ ). HPLC separation (Chiralpak ${ }^{\circledR}$ ID-3, $n$-hexane/IPrOH 70:30, $1.0 \mathrm{~mL} / \mathrm{min}$, detection at $250 \mathrm{~nm}): t_{r}($ major $)=15.7 \mathrm{~min}, t_{r}($ minor $)=17.2 \mathrm{~min}$, 95:5 e.r. 


\section{(R)-1-(3,5-Dimethylbenzyl)-2-(1-ferrocenylethyl)-1 $H$-indole-3-carbaldehyde}

\section{(192la)}

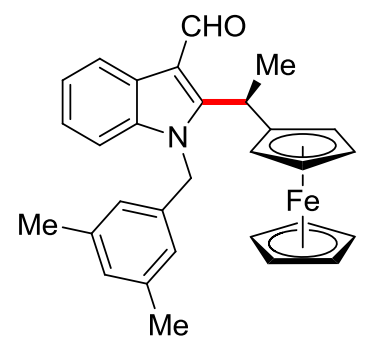

The general procedure GP5 was followed using indole 591 (92 mg, $0.25 \mathrm{mmol}$, 1.0 equiv) and vinylferrocene (191a) ( $80 \mathrm{mg}, 0.38 \mathrm{mmol}, 1.5$ equiv). Purification by column chromatography on silica gel $(n$-hexane/EtOAc $=12.5 / 1)$ yielded 192la $(57.9 \mathrm{mg}, 49 \%)$ as an orange solid. M. p. $=54-56{ }^{\circ} \mathrm{C} .{ }^{1} \mathbf{H}$ NMR $\left(500 \mathrm{MHz}, \mathrm{CDCl}_{3}\right)$ : $\delta=10.14(\mathrm{~s}, 1 \mathrm{H}), 8.36(\mathrm{dt}, J=7.9,1.0 \mathrm{~Hz}, 1 \mathrm{H}), 7.28(\mathrm{ddd}, J=7.9,6.6,1.5 \mathrm{~Hz}, 1 \mathrm{H}$ ), 7.22 (td, $J=7.4,6.6,1.3 \mathrm{~Hz}, 1 \mathrm{H}), 7.19(\mathrm{~d}, J=8.5 \mathrm{~Hz}, 1 \mathrm{H}), 6.93(\mathrm{~s}, 1 \mathrm{H}), 6.60(\mathrm{~s}, 2 \mathrm{H})$, $5.34(\mathrm{~d}, J=17.2 \mathrm{~Hz}, 1 \mathrm{H}), 5.21(\mathrm{~d}, J=16.9 \mathrm{~Hz}, 1 \mathrm{H}$ ), 4.88 (brs, $1 \mathrm{H}), 4.36$ (dt, $J=2.6$, $1.4 \mathrm{~Hz}, 1 \mathrm{H}$ ), $4.08(\mathrm{td}, J=2.5,1.3 \mathrm{~Hz}, 1 \mathrm{H}), 4.07-4.01(\mathrm{~m}, 6 \mathrm{H}), 3.84(\mathrm{~s}, 1 \mathrm{H}), 2.26(\mathrm{~s}$, $6 \mathrm{H}), 1.69(\mathrm{~d}, J=7.4 \mathrm{~Hz}, 3 \mathrm{H}) .{ }^{13} \mathrm{C}$ NMR $\left(125 \mathrm{MHz}, \mathrm{CDCl}_{3}\right): \delta=185.4(\mathrm{CH}), 155.0$ $\left(\mathrm{C}_{\mathrm{q}}\right), 138.6\left(\mathrm{C}_{\mathrm{q}}\right), 136.9\left(\mathrm{C}_{\mathrm{q}}\right), 136.3\left(\mathrm{C}_{\mathrm{q}}\right), 129.4(\mathrm{CH}), 125.9\left(\mathrm{C}_{\mathrm{q}}\right), 123.7(\mathrm{CH}), 123.5$ $(\mathrm{CH}), 123.0(\mathrm{CH}), 121.7(\mathrm{CH}), 113.6\left(\mathrm{C}_{\mathrm{q}}\right), 110.0(\mathrm{CH}), 90.3\left(\mathrm{C}_{\mathrm{q}}\right), 68.9(\mathrm{CH}), 68.7$ $(\mathrm{CH}), 68.7(\mathrm{CH}), 67.0(\mathrm{CH}), 66.7(\mathrm{CH}), 47.4\left(\mathrm{CH}_{2}\right), 31.5(\mathrm{CH}), 21.3\left(\mathrm{CH}_{3}\right), 20.3$ $\left(\mathrm{CH}_{3}\right)$. IR (ATR): 2920, 1643, 1459, 1040, 815, 746, $479 \mathrm{~cm}^{-1}$. MS (ESI) $\mathrm{m} / \mathrm{z}$ (relative intensity): $973(51)[2 \mathrm{M}+\mathrm{Na}]^{+}, 498(100)[\mathrm{M}+\mathrm{Na}]^{+}, 476(67)[\mathrm{M}+\mathrm{H}]^{+}, 335(25), 213$ (80). HR-MS (ESI) $\mathrm{m} / \mathrm{z}$ calcd for $\mathrm{C}_{30} \mathrm{H}_{30} \mathrm{FeNO}[\mathrm{M}+\mathrm{H}]^{+}$476.1672, found 476.1666. $[\alpha]_{D}{ }^{23}:+180.7\left(\mathrm{c}=1.06, \mathrm{CHCl}_{3}\right)$. HPLC separation (Chiralpak ${ }^{\circledR}$ IF-3, $n$-hexane/ $\mathrm{PrOH} 80: 20,0.75 \mathrm{~mL} / \mathrm{min}$, detection at $250 \mathrm{~nm}): t_{r}($ minor $)=17.5 \mathrm{~min}$, $t_{r}$ (major) $=22.2 \mathrm{~min}, 5: 95$ e.r. 
(R)-1-(3,5-Dimethylbenzyl)-2-(1-ferrocenylethyl)-5-methoxy-1 H-indole-3carbaldehyde (192ma)

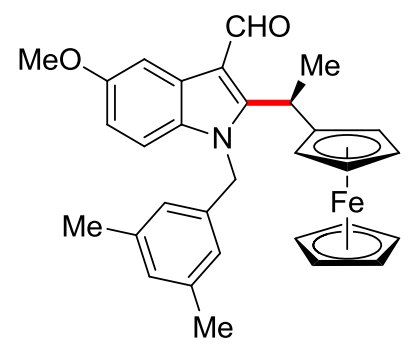

The general procedure GP5 was followed using indole $59 \mathrm{~m}(100 \mathrm{mg}, 0.25 \mathrm{mmol}$, 1.0 equiv) and vinylferrocene (191a) $(80 \mathrm{mg}, 0.38 \mathrm{mmol}, 1.5$ equiv). Purification by column chromatography on silica gel $(n$-hexane/EtOAc $=12 / 1 \rightarrow 7 / 1)$ yielded $192 \mathrm{ma}$ $(87.2 \mathrm{mg}, 69 \%)$ as an orange solid. $\mathbf{M} . \mathbf{p} .=79-81^{\circ} \mathrm{C} .{ }^{1} \mathbf{H} \mathbf{~ N M R}\left(300 \mathrm{MHz}, \mathrm{CDCl}_{3}\right)$ : $\delta=10.10(\mathrm{~s}, 1 \mathrm{H}), 7.89(\mathrm{~d}, J=2.5 \mathrm{~Hz}, 1 \mathrm{H}), 7.07(\mathrm{~d}, J=8.9 \mathrm{~Hz}, 1 \mathrm{H}), 6.93(\mathrm{~s}, 1 \mathrm{H})$, $6.84(\mathrm{dd}, J=8.9,2.6 \mathrm{~Hz}, 1 \mathrm{H}), 6.59(\mathrm{~s}, 2 \mathrm{H}), 5.30(\mathrm{~d}, J=17.0 \mathrm{~Hz}, 1 \mathrm{H}), 5.18(\mathrm{~d}$, $J=17.0 \mathrm{~Hz}, 1 \mathrm{H}$ ), 4.81 (brs, $1 \mathrm{H}), 4.37-4.34(\mathrm{~m}, 1 \mathrm{H}), 4.08$ (td, $J=2.5,1.3 \mathrm{~Hz}, 1 \mathrm{H}$ ), 4.24-3.91 (m, 6H), 3.89 (s, 3H), 3.86-3.83 (m, 1H), 2.26 (s, 6H), 1.68 (d, J = 7.4 Hz, 3H). ${ }^{13} \mathrm{C}$ NMR $\left(125 \mathrm{MHz}, \mathrm{CDCl}_{3}\right): \delta=185.1(\mathrm{CH}), 156.7\left(\mathrm{C}_{\mathrm{q}}\right), 154.9\left(\mathrm{C}_{\mathrm{q}}\right), 138.5\left(\mathrm{C}_{\mathrm{q}}\right)$, $136.2\left(\mathrm{C}_{\mathrm{q}}\right), 131.6\left(\mathrm{C}_{\mathrm{q}}\right), 129.3(\mathrm{CH}), 126.5\left(\mathrm{C}_{\mathrm{q}}\right), 123.6(\mathrm{CH}), 113.6(\mathrm{CH}), 113.5\left(\mathrm{C}_{\mathrm{q}}\right)$, $110.8(\mathrm{CH}), 103.3(\mathrm{CH}), 90.3\left(\mathrm{C}_{\mathrm{q}}\right), 68.9(\mathrm{CH}), 68.7(\mathrm{CH}), 68.7(\mathrm{CH}), 67.0(\mathrm{CH}), 66.7$ $(\mathrm{CH}), 55.9\left(\mathrm{CH}_{3}\right), 47.6\left(\mathrm{CH}_{2}\right), 31.7(\mathrm{CH}), 21.4\left(\mathrm{CH}_{3}\right), 20.3\left(\mathrm{CH}_{3}\right)$. IR (ATR): 2914, 1641, 1457, 1424, 1042, 798, $751 \mathrm{~cm}^{-1}$. MS (ESI) $\mathrm{m} / \mathrm{z}$ (relative intensity): 661 (13), $528(100)[\mathrm{M}+\mathrm{Na}]^{+}, 506(58)[\mathrm{M}+\mathrm{H}]^{+}, 289$ (15), 213 (59). HR-MS (ESI) $\mathrm{m} / z$ calcd for

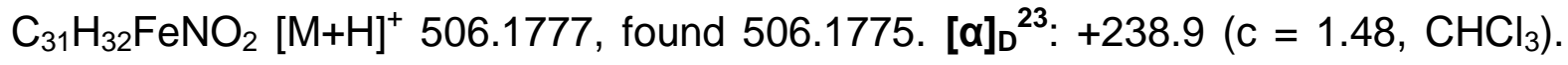
HPLC separation (Chiralpak ${ }^{\circledR}$ ID-3, $n$-hexane/PrOH 70:30, $1.0 \mathrm{~mL} / \mathrm{min}$, detection at $273 \mathrm{~nm}): t_{r}($ major $)=18.6 \mathrm{~min}, t_{r}($ minor $)=22.6 \mathrm{~min}, 96: 4$ e.r. 
(R)-N-\{[1-Benzyl-2-(1-ferrocenylethyl)-1 H-pyrrolo[2,3-b]pyridin-3-yl]methylene\}-

\section{4-methoxyaniline (192na)}

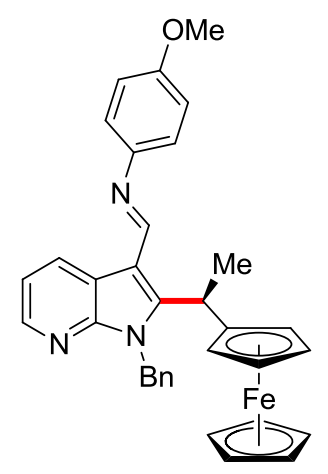

The general procedure GP5 was followed using azaindole $59 \mathrm{n}(85 \mathrm{mg}, 0.25 \mathrm{mmol}$, 1.0 equiv) and vinylferrocene (191a) $(50 \mathrm{mg}, 0.38 \mathrm{mmol}, 1.5$ equiv). The reaction mixture was diluted with sat. aqueous $\mathrm{NaHCO}_{3}(10 \mathrm{~mL})$ and extracted with EtOAc $(3 \times 10 \mathrm{~mL})$. The combined organic layers were washed with brine $(10 \mathrm{~mL})$, dried over $\mathrm{Na}_{2} \mathrm{SO}_{4}$, and concentrated under reduced pressure. The residue was purified by silica gel chromatography ( $n$-hexane/EtOAc $=10 / 1+0.5 \% \mathrm{Et}_{3} \mathrm{~N}$ ) to yield 192na (72.7 mg, 53\%) as an orange solid. M. p. $=63-64{ }^{\circ} \mathrm{C} .{ }^{1} \mathbf{H} \mathbf{~ N M R}\left(600 \mathrm{MHz}, \mathrm{CDCl}_{3}\right)$ : $\delta=8.85(\mathrm{dd}, J=7.8,1.6 \mathrm{~Hz}, 1 \mathrm{H}), 8.48(\mathrm{~s}, 1 \mathrm{H}), 8.35(\mathrm{dd}, J=4.7,1.7 \mathrm{~Hz}, 1 \mathrm{H}), 7.40-$ $7.30(\mathrm{~m}, 3 \mathrm{H}), 7.21(\mathrm{dd}, J=7.8,4.7 \mathrm{~Hz}, 1 \mathrm{H}), 7.11(\mathrm{~d}, J=7.2 \mathrm{~Hz}, 2 \mathrm{H}), 6.98(\mathrm{~d}$, $J=8.8 \mathrm{~Hz}, 2 \mathrm{H}), 6.85(\mathrm{~d}, J=8.9 \mathrm{~Hz}, 2 \mathrm{H}), 6.07(\mathrm{~d}, J=16.3 \mathrm{~Hz}, 1 \mathrm{H}), 5.28(\mathrm{~d}$, $J=16.3 \mathrm{~Hz}, 1 \mathrm{H}$ ), $4.58(\mathrm{q}, J=7.3 \mathrm{~Hz}, 1 \mathrm{H}), 4.38$ (dt, $J=2.6,1.3 \mathrm{~Hz}, 1 \mathrm{H}$ ), 4.12 (td, $J=2.5,1.3 \mathrm{~Hz}, 1 \mathrm{H}$ ), 4.06 (td, $J=2.5,1.3 \mathrm{~Hz}, 1 \mathrm{H}$ ), 3.98 (s, 5H), $3.81(\mathrm{~s}, 3 \mathrm{H}), 3.64$ (dd, $J=2.5,1.3 \mathrm{~Hz}, 1 \mathrm{H}), 1.60(\mathrm{~d}, J=7.3 \mathrm{~Hz}, 3 \mathrm{H}) .{ }^{13} \mathrm{C}$ NMR $\left(125 \mathrm{MHz}, \mathrm{CDCl}_{3}\right)$ : $\delta=157.2\left(\mathrm{C}_{\mathrm{q}}\right), 153.0(\mathrm{CH}), 150.1\left(\mathrm{C}_{\mathrm{q}}\right), 148.2\left(\mathrm{C}_{\mathrm{q}}\right), 146.4\left(\mathrm{C}_{\mathrm{q}}\right), 143.6(\mathrm{CH}), 137.9$ $\left(\mathrm{C}_{\mathrm{q}}\right), 131.1(\mathrm{CH}), 128.8(\mathrm{CH}), 127.6(\mathrm{CH}), 126.4(\mathrm{CH}), 121.9(\mathrm{CH}), 118.8\left(\mathrm{C}_{\mathrm{q}}\right), 118.0$ $(\mathrm{CH}), 114.1(\mathrm{CH}), 109.5\left(\mathrm{C}_{\mathrm{q}}\right), 90.8\left(\mathrm{C}_{\mathrm{q}}\right), 68.9(\mathrm{CH}), 68.9(\mathrm{CH}), 68.9(\mathrm{CH}), 66.8(\mathrm{CH})$, $66.7(\mathrm{CH}), 55.5\left(\mathrm{CH}_{3}\right), 45.2\left(\mathrm{CH}_{2}\right), 32.2(\mathrm{CH}), 20.3\left(\mathrm{CH}_{3}\right)$. IR (ATR): 2934, 1613, 1498, 1428, 1242, $728 \mathrm{~cm}^{-1}$. MS (ESI) $\mathrm{m} / \mathrm{z}$ (relative intensity): $554(100)[\mathrm{M}+\mathrm{H}]^{+}$. HR-MS (ESI) $\mathrm{m} / z$ calcd for $\mathrm{C}_{34} \mathrm{H}_{32} \mathrm{~N}_{3} \mathrm{OFe}[\mathrm{M}+\mathrm{H}]^{+}$554.1890, found 554.1884. $[\alpha]_{D}^{23}:+309.1 \quad\left(c=1.16, \quad \mathrm{CHCl}_{3}\right) . \quad$ HPLC separation (Chiralpak ${ }^{\circledR} \quad$ IF-3, $n$-hexane/PrOH 90:10, $1.0 \mathrm{~mL} / \mathrm{min}$, detection at $273 \mathrm{~nm}): t_{r}($ minor $)=11.3 \mathrm{~min}$, $t_{r}($ major $)=11.8 \mathrm{~min}, 10: 90$ e.r. 
(R)-1-Benzyl-2-(1-ruthenocenylethyl)-1 H-indole-3-carbaldehyde (192cb)

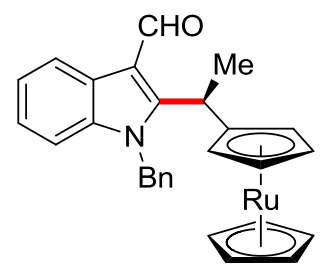

The general procedure GP5 was followed using indole $59 \mathrm{c}(93 \mathrm{mg}, 0.25 \mathrm{mmol}$, 1.0 equiv) and vinylruthenocene (191b) (96 mg, $0.38 \mathrm{mmol}, 1.5$ equiv). Purification by column chromatography on silica gel ( $n$-hexane/EtOAc $=13 / 1 \rightarrow 7.5 / 1)$ yielded 192cb (59.1 mg, 48\%) as a dark yellow solid. M. p. = 52-54 ${ }^{\circ} \mathrm{C}$. ${ }^{1} \mathrm{H}$ NMR $(500 \mathrm{MHz}$, $\left.\mathrm{CDCl}_{3}\right): \delta=10.28(\mathrm{~s}, 1 \mathrm{H}), 8.39(\mathrm{dt}, J=7.9,1.0 \mathrm{~Hz}, 1 \mathrm{H}), 7.34-7.25(\mathrm{~m}, 4 \mathrm{H}), 7.22(\mathrm{td}$, $J=7.6,1.3 \mathrm{~Hz}, 1 \mathrm{H}), 7.18(\mathrm{~d}, J=8.1 \mathrm{~Hz}, 1 \mathrm{H}), 6.97(\mathrm{dd}, J=7.9,1.7 \mathrm{~Hz}, 2 \mathrm{H}), 5.43(\mathrm{~d}$, $J=17.2 \mathrm{~Hz}, 1 \mathrm{H}), 5.39(\mathrm{~d}, J=17.1 \mathrm{~Hz}, 1 \mathrm{H}), 4.74(\mathrm{dt}, J=2.4,1.1 \mathrm{~Hz}, 1 \mathrm{H}), 4.61$ (brs, $1 \mathrm{H}), 4.48(\mathrm{~s}, 5 \mathrm{H}), 4.44(\mathrm{td}, J=2.3,1.1 \mathrm{~Hz}, 1 \mathrm{H}), 4.42(\mathrm{td}, J=2.3,1.1 \mathrm{~Hz}, 1 \mathrm{H}), 4.33$ (brs, $1 \mathrm{H}$ ), 1.56 (d, $J=7.5 \mathrm{~Hz}, 3 \mathrm{H}) .{ }^{13} \mathrm{C}$ NMR $\left(125 \mathrm{MHz}, \mathrm{CDCl}_{3}\right): \delta=185.3(\mathrm{CH})$, $154.6\left(\mathrm{C}_{\mathrm{q}}\right), 136.8\left(\mathrm{C}_{\mathrm{q}}\right), 136.2\left(\mathrm{C}_{\mathrm{q}}\right), 128.9(\mathrm{CH}), 127.8(\mathrm{CH}), 125.9\left(\mathrm{C}_{\mathrm{q}}\right), 125.8(\mathrm{CH})$, $123.6(\mathrm{CH}), 123.1(\mathrm{CH}), 121.9(\mathrm{CH}), 113.9\left(\mathrm{C}_{\mathrm{q}}\right), 110.0(\mathrm{CH}), 94.0\left(\mathrm{C}_{\mathrm{q}}\right), 71.2(\mathrm{CH})$, $71.0(\mathrm{CH}), 70.7(\mathrm{CH}), 69.6(\mathrm{CH}), 69.5(\mathrm{CH}), 47.5\left(\mathrm{CH}_{2}\right), 31.5(\mathrm{CH}), 20.9\left(\mathrm{CH}_{3}\right)$. IR (ATR): 2904, 1643, 1453, 1022, 805, $744 \mathrm{~cm}^{-1}$. MS (EI) $\mathrm{m} / \mathrm{z}$ (relative intensity): 493 (39) [M] ${ }^{+}\left({ }^{102} \mathrm{Ru}\right), 384$ (28), 317 (40), 300 (28), 259 (24), 91 (100). HR-MS (ESI)

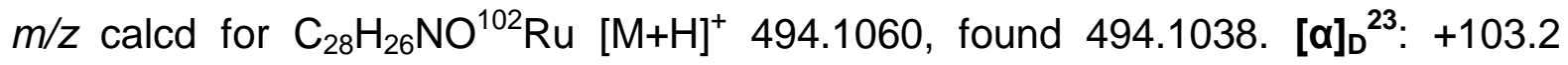
(c $=0.95, \mathrm{CHCl}_{3}$ ). HPLC separation (Chiralpak ${ }^{\circledR} \mathrm{IF}-3, n$-hexane/PrOH 80:20, $0.75 \mathrm{~mL} / \mathrm{min}$, detection at $273 \mathrm{~nm}): t_{r}($ minor $)=20.8 \mathrm{~min}, t_{r}$ (major) $=21.9 \mathrm{~min}$, 4:96 e.r. 
(R)-1-Benzyl-2-[1-(1',2', 3',4',5'-pentamethylferrocenyl)ethyl]-1H-indole-3-

\section{carbaldehyde (192cc)}

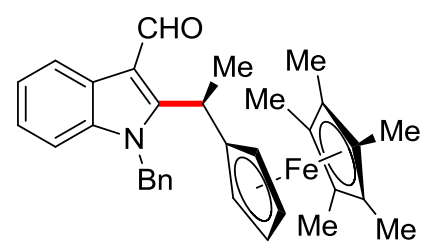

The general procedure GP6 was followed using indole 59c $(93 \mathrm{mg}, 0.25 \mathrm{mmol}$, 1.0 equiv) and vinylferrocene $191 \mathrm{c}$ (106 mg, $0.38 \mathrm{mmol}, 1.5$ equiv). Purification by column chromatography on silica gel $(n$-hexane/EtOAc $=20 / 1)$ yielded 192cc (75.0 mg, 58\%) as an orange solid. M. p. $=91-94{ }^{\circ} \mathrm{C} .{ }^{1} \mathbf{H}$ NMR $\left(500 \mathrm{MHz}, \mathrm{CDCl}_{3}\right)$ : $\delta=10.25$ (brs, $1 \mathrm{H}), 8.37(\mathrm{~d}, J=7.9 \mathrm{~Hz}, 1 \mathrm{H}), 7.34-7.21(\mathrm{~m}, 4 \mathrm{H}), 7.18(\mathrm{t}, J=7.6 \mathrm{~Hz}$, 1H), 7.14-6.99 (m, 1H), 6.99-6.70 (m, 2H), 5.25 (brs, 3H), $3.81(\mathrm{~s}, 1 \mathrm{H}), 3.63$ (td, $J=2.4,1.1 \mathrm{~Hz}, 1 \mathrm{H}$ ), 3.59 (td, $J=2.4,1.1 \mathrm{~Hz}, 1 \mathrm{H}), 3.36(\mathrm{~s}, 1 \mathrm{H}), 1.83(\mathrm{~s}, 15 \mathrm{H}), 1.55$ $(\mathrm{d}, J=7.4 \mathrm{~Hz}, 3 \mathrm{H}) .{ }^{13} \mathrm{C}$ NMR $\left(125 \mathrm{MHz}, \mathrm{CDCl}_{3}\right): \delta=185.1(\mathrm{CH}), 155.5\left(\mathrm{C}_{\mathrm{q}}\right), 136.9$ $\left(\mathrm{C}_{\mathrm{q}}\right), 136.2\left(\mathrm{C}_{\mathrm{q}}\right), 128.8(\mathrm{CH}), 127.5(\mathrm{CH}), 125.8\left(\mathrm{C}_{\mathrm{q}}\right), 125.6(\mathrm{CH}), 123.4(\mathrm{CH}), 123.0$ $(\mathrm{CH}), 121.6(\mathrm{CH}), 113.7\left(\mathrm{C}_{\mathrm{q}}\right), 110.0(\mathrm{CH}), 88.9\left(\mathrm{C}_{\mathrm{q}}\right), 80.0\left(\mathrm{C}_{\mathrm{q}}\right), 73.4(\mathrm{CH}), 71.4(\mathrm{CH})$, $71.3(\mathrm{CH}), 68.6(\mathrm{CH}), 47.2\left(\mathrm{CH}_{2}\right), 28.8(\mathrm{CH}), 19.4\left(\mathrm{CH}_{3}\right), 11.0\left(\mathrm{CH}_{3}\right)$. IR (ATR): 2898, 1638, 1423, 1028, 819, $729 \mathrm{~cm}^{-1}$. MS (EI) $\mathrm{m} / z$ (relative intensity): 517 (100) [M] ${ }^{+}$, 382 (32), 381 (39), 290 (23), 262 (19). HR-MS (EI) m/z calcd for $\mathrm{C}_{33} \mathrm{H}_{35} \mathrm{FeNO}[\mathrm{M}]^{+}$

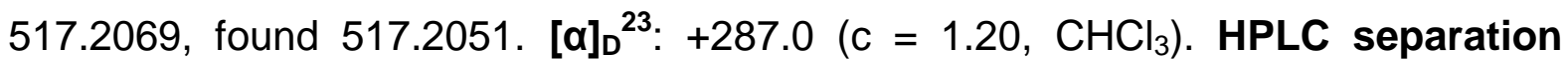
(Chiralpak ${ }^{\circledR}$ IF-3, $n$-hexane/PrOH 90:10, $1.0 \mathrm{~mL} / \mathrm{min}$, detection at $250 \mathrm{~nm}$ ): $t_{r}($ major $)=12.7 \mathrm{~min}, t_{r}($ minor $)=13.5 \mathrm{~min}, 95: 5$ e.r. 


\subsubsection{Product Diversification}

(S)-N-\{[1-Benzyl-2-(1-(4-methoxyphenyl)ethyl)-1 H-indol-3-yl]methylene\}-4bromoaniline (197)

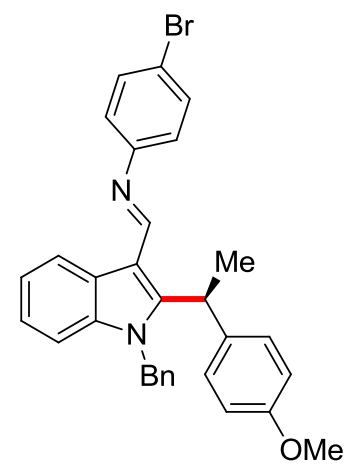

62cc (90 mg, $0.24 \mathrm{mmol}, 1.0$ equiv, 92:8 e.r.), 4-bromoaniline (46 mg, $0.27 \mathrm{mmol}$, 1.1 equiv) and activated $4 \AA$ molecular sieves $(1.0 \mathrm{~g})$ were suspended in $\mathrm{PhMe}$ $(5.0 \mathrm{~mL})$ and stirred at $50^{\circ} \mathrm{C}$ for $16 \mathrm{~h}$. The suspension was allowed to cool to ambient temperature, filtered and concentrated under reduced pressure. The residue was recrystallized from $n$-hexane/isopropanol to provide the optically pure imine 197 as a white crystalline solid $(67 \mathrm{mg}, 53 \%)$. M. p. $=179-181{ }^{\circ} \mathrm{C} .{ }^{1} \mathbf{H}$ NMR $\left(300 \mathrm{MHz}, \mathrm{CDCl}_{3}\right): \delta=8.66$ (ddd, $\left.J=7.8,1.4,0.7 \mathrm{~Hz}, 1 \mathrm{H}\right), 8.61(\mathrm{~s}, 1 \mathrm{H}), 7.43$ (d, $J=8.7 \mathrm{~Hz}, 2 \mathrm{H}$ ), 7.29 (ddd, $J=7.8,6.8,1.5 \mathrm{~Hz}, 1 \mathrm{H}$ ), 7.26-7.19 (m, 4H), 7.17 (ddd, $J=8.0,1.5,0.8 \mathrm{~Hz}, 1 \mathrm{H}), 7.12(\mathrm{~d}, J=8.2 \mathrm{~Hz}, 2 \mathrm{H}), 6.96(\mathrm{~d}, J=8.7 \mathrm{~Hz}, 2 \mathrm{H}), 6.94-6.88$ $(\mathrm{m}, 2 \mathrm{H}), 6.81(\mathrm{~d}, J=8.8 \mathrm{~Hz}, 2 \mathrm{H}), 5.27(\mathrm{~s}, 2 \mathrm{H}), 4.85(\mathrm{q}, J=7.3 \mathrm{~Hz}, 1 \mathrm{H}), 3.78(\mathrm{~s}, 3 \mathrm{H})$, $1.67(\mathrm{~d}, J=7.4 \mathrm{~Hz}, 3 \mathrm{H}) \cdot{ }^{13} \mathrm{C}$ NMR $\left(125 \mathrm{MHz}, \mathrm{CDCl}_{3}\right): \delta=158.3\left(\mathrm{C}_{\mathrm{q}}\right), 155.0(\mathrm{CH})$, $152.8\left(\mathrm{C}_{\mathrm{q}}\right), 149.8\left(\mathrm{C}_{\mathrm{q}}\right), 137.3\left(\mathrm{C}_{\mathrm{q}}\right), 136.6\left(\mathrm{C}_{\mathrm{q}}\right), 133.9\left(\mathrm{C}_{\mathrm{q}}\right), 131.8(\mathrm{CH}), 128.7(\mathrm{CH})$, $128.1(\mathrm{CH}), 127.4(\mathrm{CH}), 126.0\left(\mathrm{C}_{\mathrm{q}}\right), 125.8(\mathrm{CH}), 123.2(\mathrm{CH}), 122.6(\mathrm{CH}), 122.5(\mathrm{CH})$, $122.1(\mathrm{CH}), 117.7\left(\mathrm{C}_{\mathrm{q}}\right), 114.0(\mathrm{CH}), 111.9\left(\mathrm{C}_{\mathrm{q}}\right), 109.8(\mathrm{CH}), 55.4\left(\mathrm{CH}_{3}\right), 47.4\left(\mathrm{CH}_{2}\right)$, $34.5(\mathrm{CH}), 20.0\left(\mathrm{CH}_{3}\right)$. IR (ATR): 2923, 2827, 1604, 1570, 1421, 752, $725 \mathrm{~cm}^{-1}$. MS (ESI) $m / z$ (relative intensity): $525(100)[\mathrm{M}+\mathrm{H}]]^{+}\left({ }^{81} \mathrm{Br}\right), 523(100)[\mathrm{M}+\mathrm{H}]^{+}\left({ }^{79} \mathrm{Br}\right)$, 475 (8). HR-MS (ESI) $\mathrm{m} / z$ calcd for $\mathrm{C}_{31} \mathrm{H}_{28} \mathrm{~N}_{2} \mathrm{O}^{81} \mathrm{Br}[\mathrm{M}+\mathrm{H}]^{+}$525.1361, found 525.1360. $[\alpha]_{D}{ }^{23}:+60.1\left(\mathrm{c}=0.84, \mathrm{CHCl}_{3}\right)$. HPLC separation (Chiralpak ${ }^{\circledR} \mathrm{IA}-3$, $n$-hexane/iPrOH 80:20, $1.0 \mathrm{~mL} / \mathrm{min}$, detection at $273 \mathrm{~nm}): t_{r}($ major $)=5.9 \mathrm{~min}$, $t_{r}($ minor $)=6.7 \min , 99.5: 0.5$ e.r. 
Crystals suitable for X-ray crystallography were grown by slow evaporation of a solution of 197 in a mixture of $n$-hexane/EtOAc/ $\mathrm{CH}_{2} \mathrm{Cl}_{2}$.

\section{(S)-1-Benzyl-2-[1-(4-methoxyphenyl)ethyl]-1H-indole (198)}

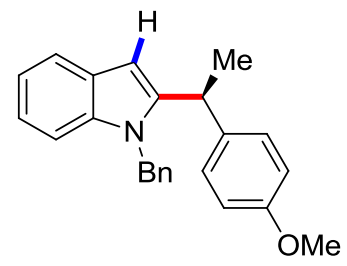

Following a modified procedure, ${ }^{[337]}$ a $20 \mathrm{~mL}$ oven-dried screw cap reaction tube was charged with $4 \AA$ molecular sieves $(60 \mathrm{mg}), 62 \mathrm{cc}(50 \mathrm{mg}, 0.135 \mathrm{mmol}, 1.0$ equiv, 90:10 e.r.), $\mathrm{Pd}(\mathrm{OAc})_{2}(2.5 \mathrm{mg}, 11 \mu \mathrm{mol}, 8.0 \mathrm{~mol} \%)$ and cyclohexane $(0.75 \mathrm{~mL})$. The tube was tightly closed by screw cap and placed in a pre-heated oil bath at $140{ }^{\circ} \mathrm{C}$. The reaction mixture was vigorously stirred for $24 \mathrm{~h}$. The reaction mixture was allowed to cool to ambient temperature and filtered through a short plug of Celite ${ }^{\circledR}$. The Celite ${ }^{\circledR}$ was washed with EtOAc. The filtrate was concentrated under reduced pressure. Purification of the residue by column chromatography on silica gel ( $n$-hexane/EtOAc $=25 / 1)$ yielded deformylated product $198(30.5 \mathrm{mg}, 66 \%)$ as a colorless oil. ${ }^{1} \mathrm{H}$ NMR $\left(500 \mathrm{MHz}, \mathrm{CDCl}_{3}\right): \delta=7.68$ (ddd, $J=5.9,3.1,0.8 \mathrm{~Hz}, 1 \mathrm{H}$ ), 7.26-7.20 (m, 3H), 7.20-7.15 (m, 1H), $7.13(\mathrm{dd}, J=6.1,3.1 \mathrm{~Hz}, 2 \mathrm{H}), 7.04(\mathrm{~d}$, $J=8.6 \mathrm{~Hz}, 2 \mathrm{H}$ ), 6.85 (ddd, $J=6.9,1.8,0.8 \mathrm{~Hz}, 2 \mathrm{H}), 6.79(\mathrm{~d}, J=8.7 \mathrm{~Hz}, 2 \mathrm{H}), 6.62$ (t, $J=0.9 \mathrm{~Hz}, 1 \mathrm{H}), 5.20(\mathrm{~d}, J=17.2 \mathrm{~Hz}, 1 \mathrm{H}), 4.96(\mathrm{~d}, J=17.2 \mathrm{~Hz}, 1 \mathrm{H}), 4.04(\mathrm{q}$, $J=7.3 \mathrm{~Hz}, 1 \mathrm{H}), 3.78(\mathrm{~s}, 3 \mathrm{H}), 1.64(\mathrm{~d}, J=7.1 \mathrm{~Hz}, 3 \mathrm{H}) .{ }^{13} \mathrm{C} \mathrm{NMR}\left(125 \mathrm{MHz}, \mathrm{CDCl}_{3}\right)$ : $\delta=158.1\left(\mathrm{C}_{\mathrm{q}}\right), 144.1\left(\mathrm{C}_{\mathrm{q}}\right), 138.0\left(\mathrm{C}_{\mathrm{q}}\right), 137.6\left(\mathrm{C}_{\mathrm{q}}\right), 136.9\left(\mathrm{C}_{\mathrm{q}}\right), 128.6(\mathrm{CH}), 128.2$ $(\mathrm{CH}), 127.8\left(\mathrm{C}_{\mathrm{q}}\right), 127.0(\mathrm{CH}), 125.8(\mathrm{CH}), 121.2(\mathrm{CH}), 120.1(\mathrm{CH}), 119.5(\mathrm{CH}), 114.0$ $(\mathrm{CH}), 109.3(\mathrm{CH}), 99.8(\mathrm{CH}), 55.2\left(\mathrm{CH}_{3}\right), 46.3\left(\mathrm{CH}_{2}\right), 37.1(\mathrm{CH}), 22.9\left(\mathrm{CH}_{3}\right)$. IR (ATR): 2928, 1509, 1452, 1241, 1031, 830, $727 \mathrm{~cm}^{-1}$. MS (EI) $\mathrm{m} / z$ (relative intensity): 341 (94) [M] ${ }^{+}, 326$ (100), 91 (70). HR-MS (ESI) $\mathrm{m} / z$ calcd for $\mathrm{C}_{24} \mathrm{H}_{24} \mathrm{NO}$ $[\mathrm{M}+\mathrm{H}]^{+}$342.1852, found 342.1851. $[\alpha]_{\mathrm{D}}{ }^{23}:+58.3\left(\mathrm{c}=0.62, \mathrm{CHCl}_{3}\right)$. HPLC separation (Chiralpak ${ }^{\circledR} \mid \mathrm{A}-3, n$-hexane/ $\mathrm{PrOH} 80: 20,1.0 \mathrm{~mL} / \mathrm{min}$, detection at $273 \mathrm{~nm}): t_{r}($ major $)=4.2 \mathrm{~min}, t_{r}(\operatorname{minor})=4.8 \mathrm{~min}, 88: 12$ e.r. 
(S,E)-Methyl 3-\{1-Benzyl-3-formyl-2-[1-(4-methoxyphenyl)ethyl]-1 $H$-indol-4yl\}acrylate (199a)

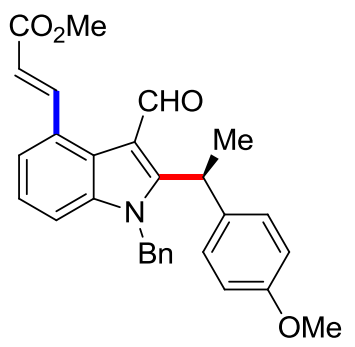

Following a modified procedure, ${ }^{[254]}$ a $5 \mathrm{~mL}$ round-bottom flask was charged under air with $62 \mathrm{cc}$ (74 mg, $0.20 \mathrm{mmol}, 1.0$ equiv, 90:10 e.r.), $\left[\mathrm{RuCl}_{2}(p \text {-cymene) }]_{2}(6.1 \mathrm{mg}\right.$, $10 \mu \mathrm{mol}, \quad 5.0 \mathrm{~mol} \%), \mathrm{AgSbF}_{6} \quad(13.7 \mathrm{mg}, \quad 40 \mu \mathrm{mol}, 20 \mathrm{~mol} \%), \quad \mathrm{Cu}(\mathrm{OAc})_{2} \cdot \mathrm{H}_{2} \mathrm{O}$ (20.0 mg, $0.10 \mathrm{mmol}, 0.50$ equiv) and 1,2-dichloroethane $(2.0 \mathrm{~mL})$. Methyl acrylate $(0.15 \mathrm{~mL}, 1.60 \mathrm{mmol}, 8.0$ equiv) was then added in a single portion. The flask was equipped with a condenser and placed in a pre-heated oil bath at $120^{\circ} \mathrm{C}$. The reaction mixture was stirred at $120^{\circ} \mathrm{C}$ for $16 \mathrm{~h}$. The suspension was allowed to cool down to ambient temperature, diluted with $n$-hexane/EtOAc 1/1 (5 mL), filtered through a short plug of silica gel, and eluted with $n$-hexane/EtOAc 1/1 $(3 \times 20 \mathrm{~mL})$. The filtrate was concentrated under reduced pressure and purified by column chromatography on silica gel ( $n$-hexane/EtOAc $=7 / 1 \rightarrow 3 / 1)$ to yield $199 \mathrm{a}(67.3 \mathrm{mg}$, $74 \%)$ as a yellow foam. M. p. $=65-67^{\circ} \mathrm{C} .{ }^{1} \mathrm{H}$ NMR $\left(400 \mathrm{MHz}, \mathrm{CDCl}_{3}\right): \delta=10.37(\mathrm{~s}$, $1 \mathrm{H}), 8.99(\mathrm{~d}, J=15.8 \mathrm{~Hz}, 1 \mathrm{H}), 7.49(\mathrm{dt}, J=7.5,0.9 \mathrm{~Hz}, 1 \mathrm{H}), 7.28-7.20(\mathrm{~m}, 3 \mathrm{H}), 7.20$ (ddd, $J=8.1,7.4,0.6 \mathrm{~Hz}, 1 \mathrm{H}$ ), $7.12(\mathrm{dd}, J=8.2,1.0 \mathrm{~Hz}, 1 \mathrm{H}$ ), 7.09 (dd, $J=9.0$, $1.0 \mathrm{~Hz}, 2 \mathrm{H}), 6.86-6.81(\mathrm{~m}, 2 \mathrm{H}), 6.79(\mathrm{~d}, J=8.8 \mathrm{~Hz}, 2 \mathrm{H}), 6.40(\mathrm{~d}, J=15.8 \mathrm{~Hz}, 1 \mathrm{H})$, $5.45(\mathrm{q}, J=7.4 \mathrm{~Hz}, 1 \mathrm{H}), 5.24(\mathrm{~d}, J=17.3 \mathrm{~Hz}, 1 \mathrm{H}), 5.17(\mathrm{~d}, J=17.3 \mathrm{~Hz}, 1 \mathrm{H}), 3.86(\mathrm{~s}$, $3 \mathrm{H}), 3.76(\mathrm{~s}, 3 \mathrm{H}), 1.67(\mathrm{~d}, J=7.4 \mathrm{~Hz}, 3 \mathrm{H}) .{ }^{13} \mathrm{C} \mathrm{NMR}\left(100 \mathrm{MHz}, \mathrm{CDCl}_{3}\right): \delta=185.3$ $(\mathrm{CH}), 167.4\left(\mathrm{C}_{\mathrm{q}}\right), 158.4\left(\mathrm{C}_{\mathrm{q}}\right), 155.5\left(\mathrm{C}_{\mathrm{q}}\right), 145.6(\mathrm{CH}), 137.9\left(\mathrm{C}_{\mathrm{q}}\right), 135.6\left(\mathrm{C}_{\mathrm{q}}\right), 132.8$ $\left(\mathrm{C}_{\mathrm{q}}\right), 129.3\left(\mathrm{C}_{\mathrm{q}}\right), 128.8(\mathrm{CH}), 127.9(\mathrm{CH}), 127.6(\mathrm{CH}), 125.6(\mathrm{CH}), 124.7\left(\mathrm{C}_{\mathrm{q}}\right), 123.5$ $(\mathrm{CH}), 122.0(\mathrm{CH}), 119.3(\mathrm{CH}), 115.5\left(\mathrm{C}_{\mathrm{q}}\right), 114.1(\mathrm{CH}), 112.0(\mathrm{CH}), 55.3\left(\mathrm{CH}_{3}\right), 51.7$ $\left(\mathrm{CH}_{3}\right), 47.7\left(\mathrm{CH}_{2}\right), 33.7(\mathrm{CH}), 18.7\left(\mathrm{CH}_{3}\right)$. IR (ATR): 2948, 1712, 1654, 1510, 1247, 1163, 1031, $725 \mathrm{~cm}^{-1}$. MS (EI) $\mathrm{m} / z$ (relative intensity): 453 (44) [M] ${ }^{+}, 394$ (35), 393 (57), 392 (44), 302 (42), 197 (88), 91 (100). HR-MS (EI) m/z calcd for $\mathrm{C}_{29} \mathrm{H}_{27} \mathrm{NO}_{4}$ $[\mathrm{M}]^{+}$453.1940, found 453.1931. [ $\left.\alpha\right]_{D}^{23}:-93.8\left(\mathrm{c}=0.53, \mathrm{CHCl}_{3}\right)$. HPLC separation 
(Chiralpak ${ }^{\circledR}$ IA-3, $n$-hexane/PrOH $80: 20,1.0 \mathrm{~mL} / \mathrm{min}$, detection at $273 \mathrm{~nm}$ ): $t_{r}($ major $)=11.1 \mathrm{~min}, t_{r}($ minor $)=15.4 \min , 89: 11$ e.r.

(R,E)-Methyl 3-[1-Benzyl-3-formyl-2-(1-ferrocenylethyl)-1H-indol-4-yl]acrylate (199b)

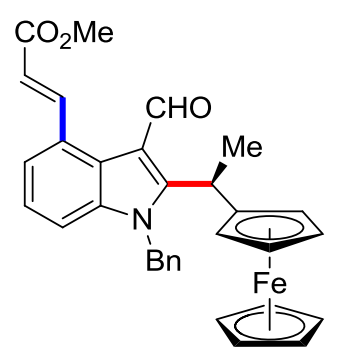

Following a modified procedure, ${ }^{[254]}$ a $5 \mathrm{~mL}$ round-bottom flask was charged under air with recrystallized 192ca (89 $\mathrm{mg}, 0.20 \mathrm{mmol}, 1.0$ equiv, 99.7:0.3 e.r.), $\left[\mathrm{RuCl}_{2}(p \text {-cymene })\right]_{2}(6.1 \mathrm{mg}, 10 \mu \mathrm{mol}, 5.0 \mathrm{~mol} \%), \mathrm{AgSbF}_{6}(13.7 \mathrm{mg}, 40 \mu \mathrm{mol}$, $20 \mathrm{~mol} \%), \mathrm{Cu}(\mathrm{OAc})_{2} . \mathrm{H}_{2} \mathrm{O}$ (20 mg, $0.10 \mathrm{mmol}, 0.50$ equiv) and 1,2-dichloroethane $(2.0 \mathrm{~mL})$. Methyl acrylate $(0.15 \mathrm{~mL}, 1.60 \mathrm{mmol}, 8.0$ equiv) was then added in a single portion. The flask was equipped with a condenser and placed in a pre-heated oil bath at $120{ }^{\circ} \mathrm{C}$. The reaction mixture was stirred at $120{ }^{\circ} \mathrm{C}$ for $16 \mathrm{~h}$. The suspension was allowed to cool down to ambient temperature, diluted with $n$-hexane/EtOAc 1/1 (5 mL), filtered through a short plug of silica gel, and eluted with $n$-hexane/EtOAc 1/1 $(3 \times 20 \mathrm{~mL})$. The filtrate was concentrated under reduced pressure and purified by column chromatography on silica gel ( $n$-hexane/EtOAc $=$ $7 / 1 \rightarrow 4.5 / 1)$ to yield $199 \mathrm{~b}(67.9 \mathrm{mg}, 64 \%)$ as an orange solid. M. p. $=79-80^{\circ} \mathrm{C}$. ${ }^{1} \mathrm{H}$ NMR $\left(300 \mathrm{MHz}, \mathrm{CDCl}_{3}\right): \delta=10.36(\mathrm{~s}, 1 \mathrm{H}), 8.98(\mathrm{~d}, J=15.8 \mathrm{~Hz}, 1 \mathrm{H}), 7.47(\mathrm{~d}$, $J=7.1 \mathrm{~Hz}, 1 \mathrm{H}), 7.36-7.03(\mathrm{~m}, 5 \mathrm{H}), 6.95-6.79(\mathrm{~m}, 2 \mathrm{H}), 6.38(\mathrm{~d}, J=15.8 \mathrm{~Hz}, 1 \mathrm{H})$, 5.52-5.19 (m, 3H), 4.33 (dt, $J=2.6,1.3 \mathrm{~Hz}, 1 \mathrm{H}), 4.10$ (brs, 5H), 4.06 (td, $J=2.4$, $1.3 \mathrm{~Hz}, 1 \mathrm{H}), 4.04(\mathrm{td}, J=2.4,1.2 \mathrm{~Hz}, 1 \mathrm{H}), 3.93-3.86(\mathrm{~m}, 1 \mathrm{H}), 3.86(\mathrm{~s}, 3 \mathrm{H}), 1.63(\mathrm{~d}$, $J=7.4 \mathrm{~Hz}, 3 \mathrm{H}) .{ }^{13} \mathrm{C}$ NMR $\left(125 \mathrm{MHz}, \mathrm{CDCl}_{3}\right): \delta=184.9(\mathrm{CH}), 167.3\left(\mathrm{C}_{\mathrm{q}}\right), 145.6(\mathrm{CH})$, $137.7\left(\mathrm{C}_{\mathrm{q}}\right), 135.8\left(\mathrm{C}_{\mathrm{q}}\right), 129.2\left(\mathrm{C}_{\mathrm{q}}\right), 128.8(\mathrm{CH}), 127.6(\mathrm{CH}), 125.7(\mathrm{CH}), 124.6\left(\mathrm{C}_{\mathrm{q}}\right)$, $123.4(\mathrm{CH}), 121.8(\mathrm{CH}), 119.1(\mathrm{CH}), 114.6\left(\mathrm{C}_{\mathrm{q}}\right), 111.8(\mathrm{CH}), 89.8\left(\mathrm{C}_{\mathrm{q}}\right), 69.1(\mathrm{CH})$, $68.8(\mathrm{CH}), 68.7(\mathrm{CH}), 67.0(\mathrm{CH}), 66.6(\mathrm{CH}), 51.7\left(\mathrm{CH}_{3}\right), 47.7\left(\mathrm{CH}_{2}\right), 31.3(\mathrm{CH}), 19.1$ 
$\left(\mathrm{CH}_{3}\right)$. One $\mathrm{C}_{\mathrm{q}}$ could not be observed. IR (ATR): 2925, 1712, 1655, 1259, 1164, 1018, 794, $727 \mathrm{~cm}^{-1}$. MS (ESI) $\mathrm{m} / z$ (relative intensity): 1085 (33) $[2 \mathrm{M}+\mathrm{Na}]^{+}, 554$ (100) $[\mathrm{M}+\mathrm{Na}]^{+}, 532(20)[\mathrm{M}+\mathrm{H}]^{+}, 281$ (23), 213 (22). HR-MS (ESI) $\mathrm{m} / \mathrm{z}$ calcd for

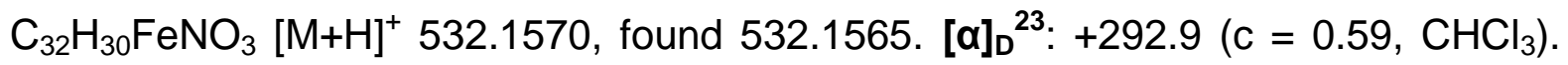
HPLC separation (Chiralpak ${ }^{\circledR} \mathrm{IF}-3, n$-hexane/ $\mathrm{PrOH} 70: 30,1.0 \mathrm{~mL} / \mathrm{min}$, detection at $273 \mathrm{~nm}): t_{r}($ major $)=12.8 \mathrm{~min}, t_{r}($ minor $)=14.1 \mathrm{~min}, 98: 2$ e.r.

(S)-1-Benzyl-2-[1-(4-methoxyphenyl)ethyl]-3-methyl-1H-indole (200)

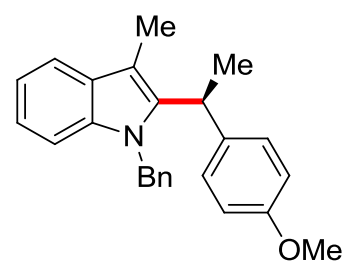

A $100 \mathrm{~mL}$ two-necked flask was charged with $62 \mathrm{cc}(50 \mathrm{mg}, 0.135 \mathrm{mmol}, 1.0$ equiv, 90:10 e.r.), Pd/C 10 wt. \% (15 mg, $0.014 \mathrm{mmol}, 10 \mathrm{~mol} \%$ ) and EtOH (15 mL). The flask was rapidly evacuated and backfilled with $\mathrm{H}_{2} 3$ times. The suspension was then vigorously stirred under $\mathrm{H}_{2}$ atmosphere for $16 \mathrm{~h}$ at ambient temperature. The suspension was diluted with $n$-hexane $(50 \mathrm{~mL})$ and filtered through a short plug of Celite $^{\circledR}$. The Celite ${ }^{\circledR}$ was washed with $n$-hexane/EtOAc (1/1). The filtrate was concentrated under reduced pressure, and the residue purified by column chromatography on silica gel ( $n$-hexane/EtOAc $=15 / 1)$ to yield $200(29.3 \mathrm{mg}, 61 \%)$ as a colorless solid. M. p. $=91-93^{\circ} \mathrm{C} .{ }^{1} \mathbf{H}$ NMR $\left(300 \mathrm{MHz}, \mathrm{CDCl}_{3}\right): \delta=7.66-7.53(\mathrm{~m}$, $1 \mathrm{H}$ ), 7.27-7.16 (m, 3H), 7.15-7.10 (m, 3H), 7.07 (dd, $J=8.9,0.8 \mathrm{~Hz}, 2 \mathrm{H}), 6.90$ (ddd, $J=6.8,1.9,0.9 \mathrm{~Hz}, 2 \mathrm{H}), 6.78(\mathrm{~d}, J=8.8 \mathrm{~Hz}, 2 \mathrm{H}), 5.20(\mathrm{~d}, J=17.5 \mathrm{~Hz}, 1 \mathrm{H}), 5.12(\mathrm{~d}$, $J=17.5 \mathrm{~Hz}, 1 \mathrm{H}), 4.44(\mathrm{q}, J=7.3 \mathrm{~Hz}, 1 \mathrm{H}), 3.78(\mathrm{~s}, 3 \mathrm{H}), 2.30(\mathrm{~s}, 3 \mathrm{H}), 1.62(\mathrm{~d}$, $J=7.4 \mathrm{~Hz}, 3 \mathrm{H}) .{ }^{13} \mathrm{C}$ NMR $\left(125 \mathrm{MHz}, \mathrm{CDCl}_{3}\right): \delta=157.8\left(\mathrm{C}_{\mathrm{q}}\right), 139.2\left(\mathrm{C}_{\mathrm{q}}\right), 138.3\left(\mathrm{C}_{\mathrm{q}}\right)$, $136.5\left(\mathrm{C}_{\mathrm{q}}\right), 135.5\left(\mathrm{C}_{\mathrm{q}}\right), 128.9\left(\mathrm{C}_{\mathrm{q}}\right), 128.5(\mathrm{CH}), 128.0(\mathrm{CH}), 126.9(\mathrm{CH}), 125.8(\mathrm{CH})$, $121.2(\mathrm{CH}), 118.9(\mathrm{CH}), 118.1(\mathrm{CH}), 113.7(\mathrm{CH}), 109.2(\mathrm{CH}), 107.6\left(\mathrm{C}_{\mathrm{q}}\right), 55.3\left(\mathrm{CH}_{3}\right)$, $46.9\left(\mathrm{CH}_{2}\right), 34.9(\mathrm{CH}), 19.9\left(\mathrm{CH}_{3}\right), 9.5\left(\mathrm{CH}_{3}\right)$. IR (ATR): 2924, 1508, 1466, 1241, 1173, 1034, $736 \mathrm{~cm}^{-1}$. MS (EI) $\mathrm{m} / z$ (relative intensity): 355 (100) [M] $]^{+}, 340$ (75), 234 (22), 91 (55). HR-MS (EI) $\mathrm{m} / z$ calcd for $\mathrm{C}_{25} \mathrm{H}_{25} \mathrm{NO}[\mathrm{M}]^{+} 355.1936$, found 355.1940. 
$[\alpha]_{D}^{23}:-22.8\left(\mathrm{c}=0.532, \mathrm{CHCl}_{3}\right)$. HPLC separation $\left(\right.$ Chiralpak $^{\circledR} \quad \mathrm{A}-3$, $n$-hexane $/ \mathrm{PrOH} 99: 1,1.0 \mathrm{~mL} / \mathrm{min}$, detection at $273 \mathrm{~nm}): t_{r}($ major $)=6.3 \mathrm{~min}$, $t_{r}(\operatorname{minor})=7.0 \mathrm{~min}, 89: 11$ e.r.

(S)-4-\{[1-Benzyl-2-(1-(4-methoxyphenyl)ethyl)-1 H-indol-3-yl]methyl\}morpholine (201)

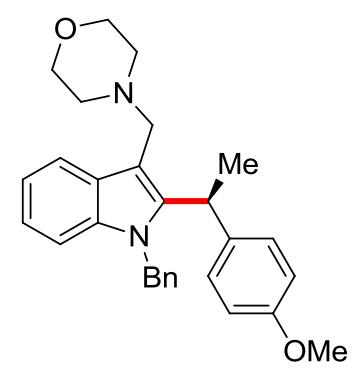

A solution of $62 \mathrm{cc}(75 \mathrm{mg}, 0.20 \mathrm{mmol}, 1.0$ equiv, $90: 10$ e.r.), morpholine (35 mg, $0.41 \mathrm{mmol}, 2.0$ equiv), $\mathrm{NaBH}(\mathrm{OAc})_{3}(172 \mathrm{mg}, 0.81 \mathrm{mmol}, 4.0$ equiv) and $\mathrm{AcOH}$ (1 drop) in DCE $(2.0 \mathrm{~mL})$ was stirred at ambient temperature for $18 \mathrm{~h}$. The reaction mixture was diluted with $\mathrm{CH}_{2} \mathrm{Cl}_{2}(10 \mathrm{~mL})$, washed with sat. aqueous $\mathrm{NaHCO}_{3}$ $(10 \mathrm{~mL})$, and dried over $\mathrm{Na}_{2} \mathrm{SO}_{4}$. After removal of volatiles under reduced pressure, the residue was purified by column chromatography on silica gel ( $n$-hexane/EtOAc $=$ $7.5 / 1 \rightarrow 5.5 / 1)$ to yield $201(73.3 \mathrm{mg}, 82 \%)$ as a colorless oil. ${ }^{1} \mathrm{H} \mathbf{N M R}(300 \mathrm{MHz}$, $\left.\mathrm{CDCl}_{3}\right): \delta=7.79-7.72(\mathrm{~m}, 1 \mathrm{H}), 7.25-7.17(\mathrm{~m}, 3 \mathrm{H}), 7.17-7.02(\mathrm{~m}, 5 \mathrm{H}), 6.84(\mathrm{ddt}$, $J=6.2,2.5,0.9 \mathrm{~Hz}, 2 \mathrm{H}), 6.76(\mathrm{~d}, J=8.8 \mathrm{~Hz}, 2 \mathrm{H}), 5.13(\mathrm{~s}, 2 \mathrm{H}), 4.63(\mathrm{q}, J=7.3 \mathrm{~Hz}$, 1H), $3.77(\mathrm{~s}, 3 \mathrm{H}), 3.72-3.50(\mathrm{~m}, 6 \mathrm{H}), 2.52-2.40(\mathrm{~m}, 4 \mathrm{H}), 1.63(\mathrm{~d}, J=7.4 \mathrm{~Hz}, 3 \mathrm{H})$. ${ }^{13} \mathrm{C}$ NMR $\left(125 \mathrm{MHz}, \mathrm{CDCl}_{3}\right): \delta=157.9\left(\mathrm{C}_{\mathrm{q}}\right), 142.1\left(\mathrm{C}_{\mathrm{q}}\right), 137.9\left(\mathrm{C}_{\mathrm{q}}\right), 136.4\left(\mathrm{C}_{\mathrm{q}}\right), 134.9$ $\left(\mathrm{C}_{\mathrm{q}}\right), 128.9\left(\mathrm{C}_{\mathrm{q}}\right), 128.5(\mathrm{CH}), 128.2(\mathrm{CH}), 126.9(\mathrm{CH}), 125.7(\mathrm{CH}), 121.2(\mathrm{CH}), 119.3$ $(\mathrm{CH}), 118.7(\mathrm{CH}), 113.6(\mathrm{CH}), 109.4(\mathrm{CH}), 108.5\left(\mathrm{C}_{\mathrm{q}}\right), 67.3\left(\mathrm{CH}_{2}\right), 55.3\left(\mathrm{CH}_{3}\right), 53.6$ $\left(\mathrm{CH}_{2}\right), 52.8\left(\mathrm{CH}_{2}\right), 47.1\left(\mathrm{CH}_{2}\right), 34.6(\mathrm{CH}), 19.1\left(\mathrm{CH}_{3}\right)$. IR (ATR): 2928, 1510, 1453, 1246, 1114, 1031, $736 \mathrm{~cm}^{-1}$. MS (ESI) $\mathrm{m} / \mathrm{z}$ (relative intensity): $441(6)[\mathrm{M}+\mathrm{H}]^{+}, 392$ (6), 354 (100). HR-MS (ESI) $\mathrm{m} / z$ calcd for $\mathrm{C}_{29} \mathrm{H}_{33} \mathrm{~N}_{2} \mathrm{O}_{2}[\mathrm{M}+\mathrm{H}]^{+} 441.2537$, found 441.2538. $[\alpha]_{D}^{23}$ : $-41.1\left(\mathrm{c}=0.50, \mathrm{CHCl}_{3}\right.$ ). HPLC separation (Chiralpak ${ }^{\circledR}$ IF-3, 
$n$-hexane/iPrOH 90:10, $1.0 \mathrm{~mL} / \mathrm{min}$, detection at $273 \mathrm{~nm}): t_{r}$ (minor) $=6.8 \mathrm{~min}$, $t_{r}($ major $)=7.2 \min , 11: 89$ e.r.

(S,E)-Methyl 3-\{1-Benzyl-2-[1-(4-methoxyphenyl)ethyl]-1H-indol-3-yl\}acrylate (202)

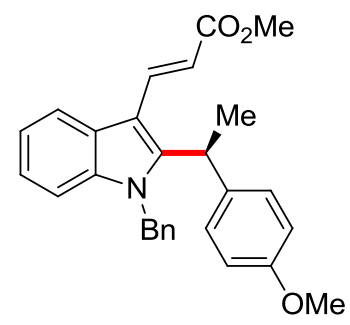

Following a modified procedure ${ }^{[338]} \mathbf{6 2 c c}(75 \mathrm{mg}, 0.20 \mathrm{mmol}, 1.0$ equiv, 90:10 e.r.) and methyl (triphenylphosphoranylidene)acetate (204 mg, $0.61 \mathrm{mmol}, 3.0$ equiv) were suspended in PhMe $(5.0 \mathrm{~mL})$. The resulting mixture was stirred under reflux $\left(130^{\circ} \mathrm{C}\right)$ for $16 \mathrm{~h}$. The reaction mixture was allowed to cool down to ambient temperature, and diluted with EtOAc $(15 \mathrm{~mL})$. The solution was washed with $\mathrm{H}_{2} \mathrm{O}$ $(15 \mathrm{~mL})$ and brine $(15 \mathrm{~mL})$, dried over $\mathrm{Na}_{2} \mathrm{SO}_{4}$, and concentrated under reduced pressure. The residue was purified by column chromatography on silica gel ( $n$-hexane/EtOAc $=12 / 1)$ to provide $202(70.0 \mathrm{mg}, 81 \%)$ as an off-white solid. M. p. $=63-64{ }^{\circ} \mathrm{C} .{ }^{1} \mathbf{H}$ NMR $\left(400 \mathrm{MHz}, \mathrm{CDCl}_{3}\right): \delta=8.09(\mathrm{~d}, J=15.8 \mathrm{~Hz}, 1 \mathrm{H}), 7.99$ (ddd, $J=7.7,1.3,0.7 \mathrm{~Hz}, 1 \mathrm{H}$ ), 7.30-7.17 (m, 5H), 7.17 (ddd, $J=8.2,7.1,1.2 \mathrm{~Hz}$, $1 \mathrm{H}), 7.13-7.04(\mathrm{~m}, 3 \mathrm{H}), 6.88-6.81(\mathrm{~m}, 2 \mathrm{H}), 6.79(\mathrm{~d}, J=8.8 \mathrm{~Hz}, 2 \mathrm{H}), 6.54(\mathrm{~d}$, $J=15.8 \mathrm{~Hz}, 1 \mathrm{H}), 5.16(\mathrm{~d}, J=17.4 \mathrm{~Hz}, 1 \mathrm{H}), 5.10(\mathrm{~d}, J=17.4 \mathrm{~Hz}, 1 \mathrm{H}), 4.82(\mathrm{q}$, $J=7.4 \mathrm{~Hz}, 1 \mathrm{H}), 3.80(\mathrm{~s}, 3 \mathrm{H}), 3.77(\mathrm{~s}, 3 \mathrm{H}), 1.64(\mathrm{~d}, J=7.4 \mathrm{~Hz}, 3 \mathrm{H}) \cdot{ }^{13} \mathrm{C}$ NMR $\left(100 \mathrm{MHz}, \mathrm{CDCl}_{3}\right): \delta=168.8\left(\mathrm{C}_{\mathrm{q}}\right), 158.2\left(\mathrm{C}_{\mathrm{q}}\right), 147.9\left(\mathrm{C}_{\mathrm{q}}\right), 137.9(\mathrm{CH}), 137.8\left(\mathrm{C}_{\mathrm{q}}\right)$, $136.7\left(\mathrm{C}_{\mathrm{q}}\right), 133.5\left(\mathrm{C}_{\mathrm{q}}\right), 128.7(\mathrm{CH}), 128.0(\mathrm{CH}), 127.3(\mathrm{CH}), 125.9\left(\mathrm{C}_{\mathrm{q}}\right), 125.7(\mathrm{CH})$, $122.8(\mathrm{CH}), 121.6(\mathrm{CH}), 120.5(\mathrm{CH}), 114.0(\mathrm{CH}), 113.0(\mathrm{CH}), 110.4(\mathrm{CH}), 109.8\left(\mathrm{C}_{q}\right)$, $55.3\left(\mathrm{CH}_{3}\right), 51.3\left(\mathrm{CH}_{3}\right), 47.5\left(\mathrm{CH}_{2}\right), 34.0(\mathrm{CH}), 19.2\left(\mathrm{CH}_{3}\right)$. IR (ATR): 2963, 1705, 1611, 1245, 1163, 1028, $726 \mathrm{~cm}^{-1}$. MS (ESI) $\mathrm{m} / z$ (relative intensity): 873 (60) $[2 \mathrm{M}+\mathrm{Na}]^{+}, 448(100)[\mathrm{M}+\mathrm{Na}]^{+}, 426(74)[\mathrm{M}+\mathrm{H}]^{+}, 117$ (91). HR-MS (ESI) $\mathrm{m} / \mathrm{z}$ calcd for

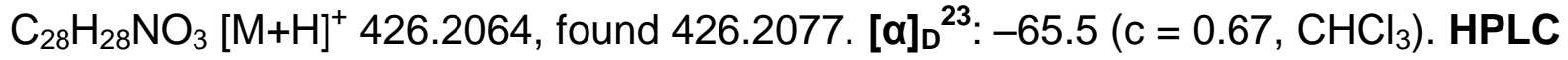


separation (Chiralpak ${ }^{\circledR} \mathrm{IA}-3, \quad n$-hexane/ $\mathrm{PrOH} 80: 20,1.0 \mathrm{~mL} / \mathrm{min}$, detection at $273 \mathrm{~nm}): t_{r}($ major $)=5.9 \mathrm{~min}, t_{r}($ minor $)=6.6 \mathrm{~min}, 88: 12$ e.r.

\section{(S,E)-1-Benzyl-2-[1-(4-methoxyphenyl)ethyl]-3-(2-nitrovinyl)-1H-indole (237)}

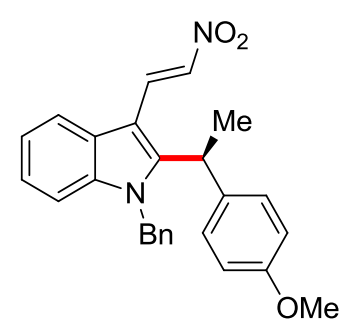

A $5 \mathrm{~mL}$ round-bottom flask was loaded with $62 \mathrm{cc}(80 \mathrm{mg}, 0.22 \mathrm{mmol}, 1.0$ equiv, $90: 10$ e.r.), ammonium acetate ( $33 \mathrm{mg}, 0.43 \mathrm{mmol}, 2.0$ equiv) and nitromethane $(1.5 \mathrm{~mL})$. The resulting solution was stirred at $90^{\circ} \mathrm{C}$ for $16 \mathrm{~h}$. The reaction mixture was concentrated under reduced pressure, and the residue was purified by column chromatography on silica gel ( $n$-hexane/EtOAc $=12 / 1)$ to provide $237(74.8 \mathrm{mg}$, $84 \%)$ as a bright yellow solid. M. p. $=45-47^{\circ} \mathrm{C} .{ }^{1} \mathbf{H}$ NMR $\left(400 \mathrm{MHz}, \mathrm{CDCl}_{3}\right): \delta=8.43$ (d, $J=13.3 \mathrm{~Hz}, 1 \mathrm{H}$ ), 7.85 (d, $J=13.2 \mathrm{~Hz}, 1 \mathrm{H}$ ), 7.81 (dt, $J=8.0,1.1 \mathrm{~Hz}, 1 \mathrm{H}$ ), 7.33 (ddd, $J=8.1,7.1,1.2 \mathrm{~Hz}, 1 \mathrm{H}), 7.29-7.22(\mathrm{~m}, 4 \mathrm{H}), 7.18(\mathrm{dt}, J=8.0,1.0 \mathrm{~Hz}, 1 \mathrm{H}), 7.07$ (dd, $J=9.0,0.9 \mathrm{~Hz}, 2 \mathrm{H}), 6.91-6.86(\mathrm{~m}, 2 \mathrm{H}), 6.82(\mathrm{~d}, J=8.8 \mathrm{~Hz}, 2 \mathrm{H}), 5.25(\mathrm{~d}$, $J=17.4 \mathrm{~Hz}, 1 \mathrm{H}), 5.20(\mathrm{~d}, J=17.4 \mathrm{~Hz}, 1 \mathrm{H}), 4.80(\mathrm{q}, J=7.4 \mathrm{~Hz}, 1 \mathrm{H}), 3.78(\mathrm{~s}, 3 \mathrm{H})$, $1.68(\mathrm{~d}, J=7.4 \mathrm{~Hz}, 3 \mathrm{H}) \cdot{ }^{13} \mathrm{C}$ NMR $\left(100 \mathrm{MHz}, \mathrm{CDCl}_{3}\right): \delta=158.5\left(\mathrm{C}_{\mathrm{q}}\right), 152.2\left(\mathrm{C}_{\mathrm{q}}\right)$, $138.0\left(\mathrm{C}_{\mathrm{q}}\right), 135.9\left(\mathrm{C}_{\mathrm{q}}\right), 132.8(\mathrm{CH}), 132.6\left(\mathrm{C}_{\mathrm{q}}\right), 132.2(\mathrm{CH}), 128.9(\mathrm{CH}), 128.0(\mathrm{CH})$, $127.7(\mathrm{CH}), 125.7(\mathrm{CH}), 125.3\left(\mathrm{C}_{\mathrm{q}}\right), 123.7(\mathrm{CH}), 122.8(\mathrm{CH}), 120.6(\mathrm{CH}), 114.3(\mathrm{CH})$, $111.0(\mathrm{CH}), 106.2\left(\mathrm{C}_{\mathrm{q}}\right), 55.3\left(\mathrm{CH}_{3}\right), 47.8\left(\mathrm{CH}_{2}\right), 34.3(\mathrm{CH}), 19.4\left(\mathrm{CH}_{3}\right)$. IR (ATR): 2965, 1606, 1510, 1245, 1176, 961, $733 \mathrm{~cm}^{-1}$. MS (ESI) $\mathrm{m} / \mathrm{z}$ (relative intensity): 847 (31) $[2 \mathrm{M}+\mathrm{Na}]^{+}, 435(88)[\mathrm{M}+\mathrm{Na}]^{+}, 413(53)[\mathrm{M}+\mathrm{H}]^{+}, 177$ (32), 117 (100). HR-MS

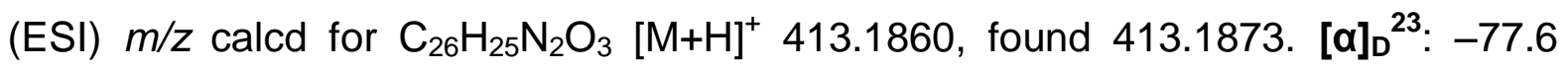
( $\mathrm{C}=0.63, \mathrm{CHCl}_{3}$ ). HPLC separation (Chiralpak ${ }^{\circledR} \mathrm{IF}-3, n$-hexane/PrOH 90:10, $1.0 \mathrm{~mL} / \mathrm{min}$, detection at $273 \mathrm{~nm}): t_{r}($ minor $)=12.4 \mathrm{~min}, t_{r}($ major $)=13.2 \mathrm{~min}$, $11: 89$ e.r. 
(S)-2-\{1-Benzyl-2-[1-(4-methoxyphenyl)ethyl]-1 H-indol-3-yl\}ethanamine (238)

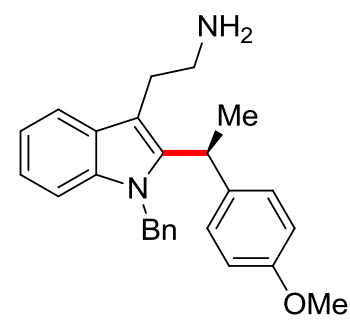

A $5 \mathrm{~mL}$ round-bottom flask was charged with 237 (40 mg, $0.097 \mathrm{mmol}, 1.0$ equiv, 89:11 e.r.). THF (3.0 mL) was then added, followed by $\mathrm{LiAlH}_{4}(11 \mathrm{mg}, 0.29 \mathrm{mmol}$, 3.0 equiv). The resulting suspension was refluxed at $85^{\circ} \mathrm{C}$ for $2.5 \mathrm{~h}$. The reaction mixture was cooled to $0{ }^{\circ} \mathrm{C}$ with an ice bath, and methanol was carefully added dropwise. The mixture was then poured into sat. aqueous $\mathrm{NaHCO}_{3}(10 \mathrm{~mL})$ and extracted with EtOAc $(3 \times 10 \mathrm{~mL})$. The combined organic layer was washed with brine $\left(15 \mathrm{~mL}\right.$ ), dried over $\mathrm{Na}_{2} \mathrm{SO}_{4}$, and concentrated under reduced pressure. The residue was purified by column chromatography on silica gel $\left(\mathrm{CH}_{2} \mathrm{Cl}_{2} / \mathrm{MeOH}=10 / 1+0.5 \% \mathrm{Et}_{3} \mathrm{~N}\right)$ to yield tryptamine $238(28.3 \mathrm{mg}, 76 \%)$ as a colorless solid. M. p. $=71-72{ }^{\circ} \mathrm{C} .{ }^{1} \mathrm{H}$ NMR $\left(400 \mathrm{MHz}, \mathrm{CDCl}_{3}\right): \delta=7.72-7.65(\mathrm{~m}, 1 \mathrm{H})$, 7.25-7.15 (m, 3H), 7.11-7.06 (m, 3H), $7.03(\mathrm{~d}, J=8.6 \mathrm{~Hz}, 2 \mathrm{H}), 6.87$ (dd, $J=7.8$, $1.5 \mathrm{~Hz}, 2 \mathrm{H}$ ), 6.76 (d, $J=8.7 \mathrm{~Hz}, 2 \mathrm{H}$ ), 6.02 (brs, 2H), 5.13 (s, 2H), 4.51 (q, $J=7.3 \mathrm{~Hz}$, $1 \mathrm{H}), 3.71(\mathrm{~s}, 3 \mathrm{H}), 3.18-3.09(\mathrm{~m}, 2 \mathrm{H}), 3.08-3.00(\mathrm{~m}, 1 \mathrm{H}), 2.90(\mathrm{dt}, J=12.2,7.7 \mathrm{~Hz}$, $1 \mathrm{H}), 1.59(\mathrm{~d}, J=7.4 \mathrm{~Hz}, 3 \mathrm{H}) \cdot{ }^{13} \mathrm{C}$ NMR $\left(100 \mathrm{MHz}, \mathrm{CDCl}_{3}\right): \delta=158.0\left(\mathrm{C}_{\mathrm{q}}\right), 140.9$ $\left(\mathrm{C}_{\mathrm{q}}\right), 137.9\left(\mathrm{C}_{\mathrm{q}}\right), 136.8\left(\mathrm{C}_{\mathrm{q}}\right), 134.7\left(\mathrm{C}_{\mathrm{q}}\right), 128.6(\mathrm{CH}), 128.1(\mathrm{CH}), 128.0\left(\mathrm{C}_{\mathrm{q}}\right), 127.0$ $(\mathrm{CH}), 125.8(\mathrm{CH}), 121.6(\mathrm{CH}), 119.6(\mathrm{CH}), 118.3(\mathrm{CH}), 113.9(\mathrm{CH}), 109.6(\mathrm{CH})$, $107.7\left(\mathrm{C}_{\mathrm{q}}\right), 55.2\left(\mathrm{CH}_{3}\right), 47.2\left(\mathrm{CH}_{2}\right), 41.4\left(\mathrm{CH}_{2}\right), 34.2(\mathrm{CH}), 25.3\left(\mathrm{CH}_{2}\right), 19.6\left(\mathrm{CH}_{3}\right)$. IR (ATR): 2927, 1510, 1466, 1244, 1177, 1030, $736 \mathrm{~cm}^{-1}$. MS (ESI) $\mathrm{m} / z$ (relative intensity): 769 (6) [2M+H] $]^{+}, 385(100)[\mathrm{M}+\mathrm{H}]^{+}, 368$ (20), 251 (16), 135 (37). HR-MS (ESI) $\mathrm{m} / \mathrm{z}$ calcd for $\mathrm{C}_{26} \mathrm{H}_{29} \mathrm{~N}_{2} \mathrm{O}[\mathrm{M}+\mathrm{H}]^{+} 385.2274$, found 385.2288.

The two enantiomers could at this stage not be separated by chiral HPLC (vide infra). 
(S)-tert-Butyl \{2-[1-Benzyl-2-(1-(4-methoxyphenyl)ethyl)-1 H-indol-3-

\section{yl]ethyl\}carbamate (203)}

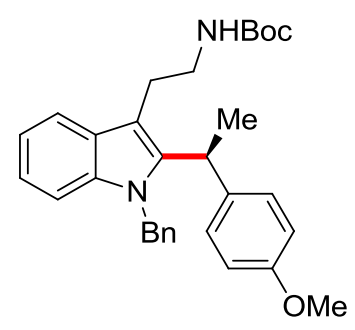

To a solution of tryptamine $238(21.0 \mathrm{mg}, 0.055 \mathrm{mmol}, 1.0$ equiv) in 1,4-dioxane $(1.0 \mathrm{~mL})$ were added $\mathrm{Et}_{3} \mathrm{~N}\left(17 \mathrm{mg}, 0.16 \mathrm{mmol}, 3.0\right.$ equiv) and $\mathrm{Boc}_{2} \mathrm{O}$ (18 mg, $0.080 \mathrm{mmol}, 1.5$ equiv). The resulting solution was stirred at ambient temperature for $16 \mathrm{~h}$. The reaction mixture was then concentrated under reduced pressure and the residue purified by column chromatography on silica gel $(n$-hexane/EtOAc $=8 / 1$ ) to provide 203 (24.9 $\mathrm{mg}, 94 \%)$ as a colorless solid. M. p. $=57-59{ }^{\circ} \mathrm{C} .{ }^{1} \mathbf{H}$ NMR $\left(500 \mathrm{MHz}, \mathrm{CDCl}_{3}\right): \delta=7.63(\mathrm{dd}, J=6.2,2.8 \mathrm{~Hz}, 1 \mathrm{H}), 7.25-7.15(\mathrm{~m}, 3 \mathrm{H}), 7.15-7.05$ (m, 3H), 7.05 (dd, $J=8.9,0.8 \mathrm{~Hz}, 2 \mathrm{H}), 6.85$ (dd, $J=7.7,1.5 \mathrm{~Hz}, 2 \mathrm{H}), 6.77$ (d, $J=8.7 \mathrm{~Hz}, 2 \mathrm{H}), 5.11(\mathrm{~s}, 2 \mathrm{H}), 4.61-4.54(\mathrm{~m}, 1 \mathrm{H}), 4.51(\mathrm{q}, J=7.3 \mathrm{~Hz}, 1 \mathrm{H}), 3.77(\mathrm{~s}$, 3H), 3.41-3.23 (m, 2H), 2.95 (t, J=7.0 Hz, 2H), $1.59(\mathrm{~d}, J=7.4 \mathrm{~Hz}, 3 \mathrm{H}), 1.41$ (s, 9H). ${ }^{13} \mathrm{C}$ NMR $\left(125 \mathrm{MHz}, \mathrm{CDCl}_{3}\right): \delta=158.0\left(\mathrm{C}_{\mathrm{q}}\right), 155.8\left(\mathrm{C}_{\mathrm{q}}\right), 140.6\left(\mathrm{C}_{\mathrm{q}}\right), 138.0\left(\mathrm{C}_{\mathrm{q}}\right)$, $136.8\left(\mathrm{C}_{\mathrm{q}}\right), 134.9\left(\mathrm{C}_{\mathrm{q}}\right), 128.5(\mathrm{CH}), 128.2\left(\mathrm{C}_{\mathrm{q}}\right), 128.0(\mathrm{CH}), 127.0(\mathrm{CH}), 125.8(\mathrm{CH})$, $121.4(\mathrm{CH}), 119.3(\mathrm{CH}), 118.5(\mathrm{CH}), 113.8(\mathrm{CH}), 109.6(\mathrm{CH}), 109.3\left(\mathrm{C}_{\mathrm{q}}\right), 78.9\left(\mathrm{C}_{\mathrm{q}}\right)$, $55.2\left(\mathrm{CH}_{3}\right), 47.2\left(\mathrm{CH}_{2}\right), 41.3\left(\mathrm{CH}_{2}\right), 34.1(\mathrm{CH}), 28.4\left(\mathrm{CH}_{3}\right), 25.3\left(\mathrm{CH}_{2}\right), 19.6\left(\mathrm{CH}_{3}\right)$. IR (ATR): 2927, 1698, 1509, 1466, 1245, 1172, $734 \mathrm{~cm}^{-1}$. MS (EI) $\mathrm{m} / \mathrm{z}$ (relative intensity): 354 (100) [M] ${ }^{+}, 248$ (19), 91 (89). HR-MS (El) $m / z$ calcd for $\mathrm{C}_{31} \mathrm{H}_{36} \mathrm{~N}_{2} \mathrm{O}_{3}$ $[\mathrm{M}]^{+}$484.2726, found 484.2730. [ $\left.\alpha\right]_{\mathrm{D}}{ }^{23}:-53.5\left(\mathrm{c}=0.23, \mathrm{CHCl}_{3}\right)$. HPLC separation (Chiralpak ${ }^{\circledR}$ IB-3, $n$-hexane/PrOH $80: 20,1.0 \mathrm{~mL} / \mathrm{min}$, detection at $273 \mathrm{~nm}$ ): $t_{r}($ minor $)=6.2 \mathrm{~min}, t_{r}($ major $)=6.7 \mathrm{~min}, 12: 88$ e.r. 


\subsubsection{Mechanistic Studies}

\section{Deuterium-Labeling Experiments}

\section{With 4-methoxystyrene (60c):}

In an oven-dried $25 \mathrm{~mL}$ Schlenk tube were placed the indole substrate $[D]_{1}-59 \mathrm{~b}$ (66 mg, $0.25 \mathrm{mmol}, 1.0$ equiv), [Fe(acac) $)_{3}$ ( $\left.8.8 \mathrm{mg}, 0.025 \mathrm{mmol}, 10 \mathrm{~mol} \%\right)$ and 180 (38 $\mathrm{mg}, 0.050 \mathrm{mmol}, 20 \mathrm{~mol} \%$ ). The Schlenk tube was closed with a rubber septum, then evacuated and backfilled with $\mathrm{N}_{2} 3$ times. THF $(0.50 \mathrm{~mL}$ ), TMEDA (58 mg, $0.50 \mathrm{mmol}, 2.0$ equiv) and 4-methoxystyrene (60c) $(50 \mathrm{mg}, 0.38 \mathrm{mmol}, 1.5$ equiv) were added via syringe. CyMgCl $(0.22 \mathrm{~mL}, 0.275 \mathrm{mmol}, 1.1$ equiv, $1.23 \mathrm{M}$ in THF) was then added dropwise at ambient temperature. The resulting mixture was stirred at $45{ }^{\circ} \mathrm{C}$ for $16 \mathrm{~h}$. The reaction mixture was allowed to cool to ambient temperature and diluted with THF $(1.5 \mathrm{~mL}) . \mathrm{HCl}(3.0 \mathrm{M}, 2.0 \mathrm{~mL})$ was added in a single portion, and the resulting mixture was stirred at ambient temperature for $2 \mathrm{~h}$. The mixture was poured into sat. aqueous $\mathrm{NH}_{4} \mathrm{Cl}(10 \mathrm{~mL})$ and extracted with EtOAc $(3 \times 10 \mathrm{~mL})$. The combined organic layers were washed with brine $(10 \mathrm{~mL})$, dried over $\mathrm{Na}_{2} \mathrm{SO}_{4}$, and concentrated under reduced pressure. The yields of recovered starting materials and hydroarylation product were determined by ${ }^{1} \mathrm{H} N \mathrm{NMR}$ analysis of the crude mixture using 1,1,2,2-tetrachloroethane as an internal standard. The crude mixture was purified by silica gel chromatography ( $n$-hexane/EtOAc $10 / 1 \rightarrow 2 / 1$ ) to afford the product and hydrolyzed starting material. Deuterium contents were determined by ${ }^{1} \mathrm{H}$ NMR spectroscopic analysis.

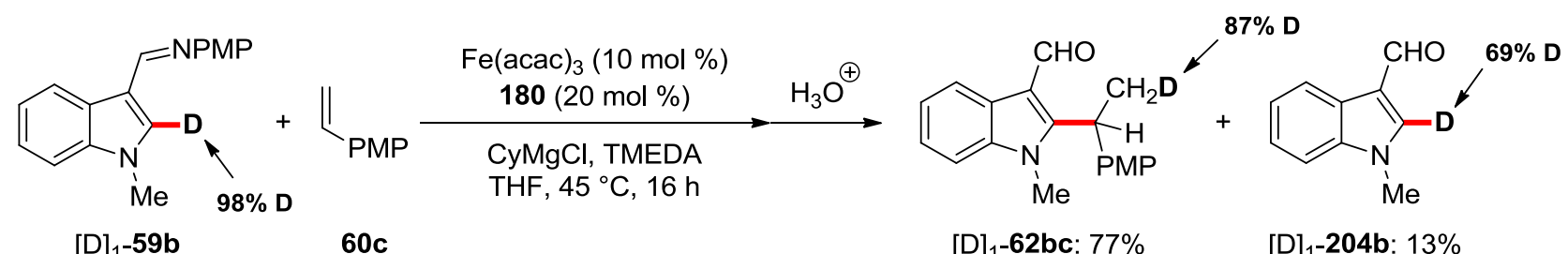




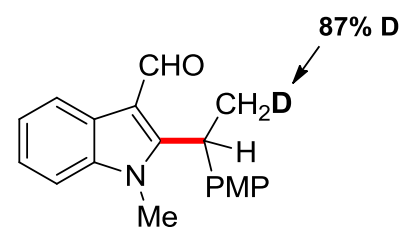

$[\mathrm{D}]_{1}-62 \mathrm{bc}$

$\left(\mathrm{CDCl}_{3}, 300 \mathrm{MHz}\right.$ )

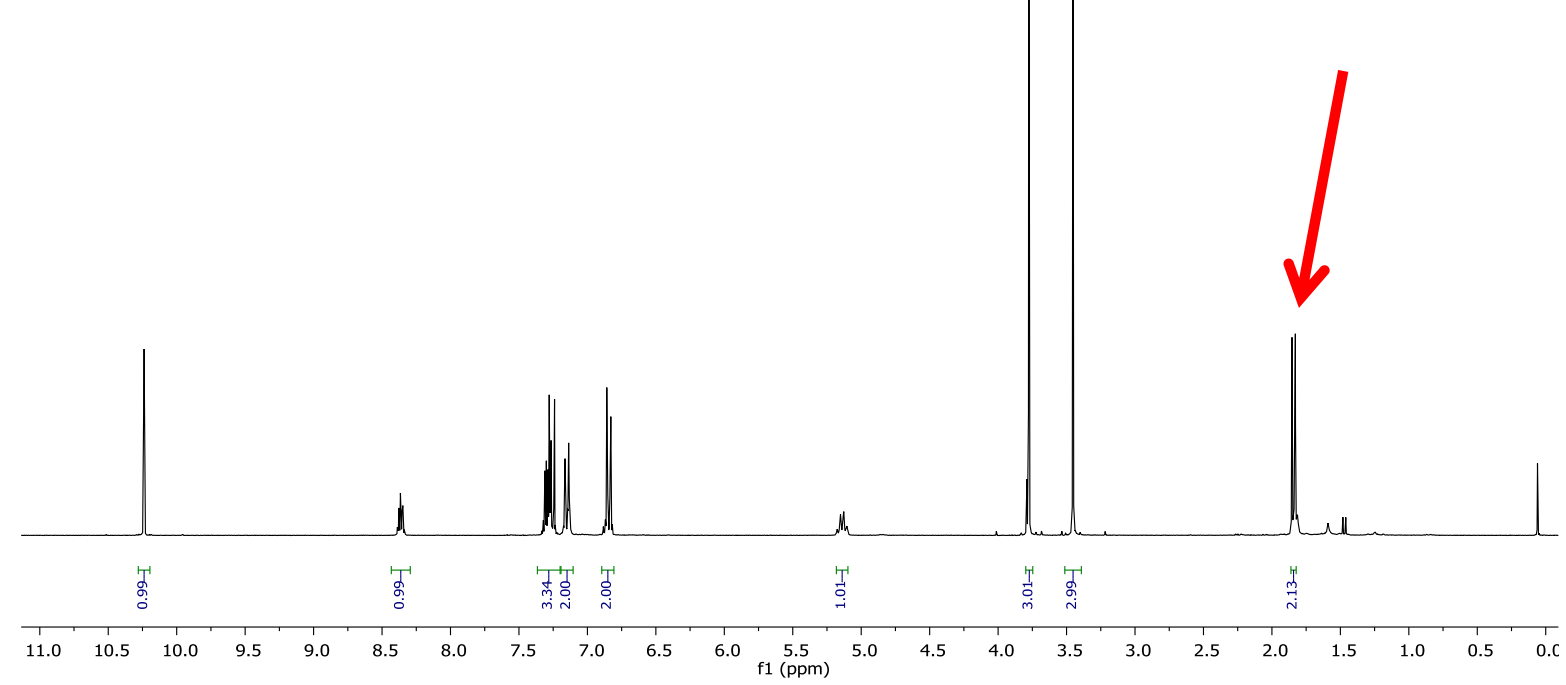

In contrast to the work of Yoshikai, ${ }^{[148]}$ no deuterium incorporation was observed at the methine position. 


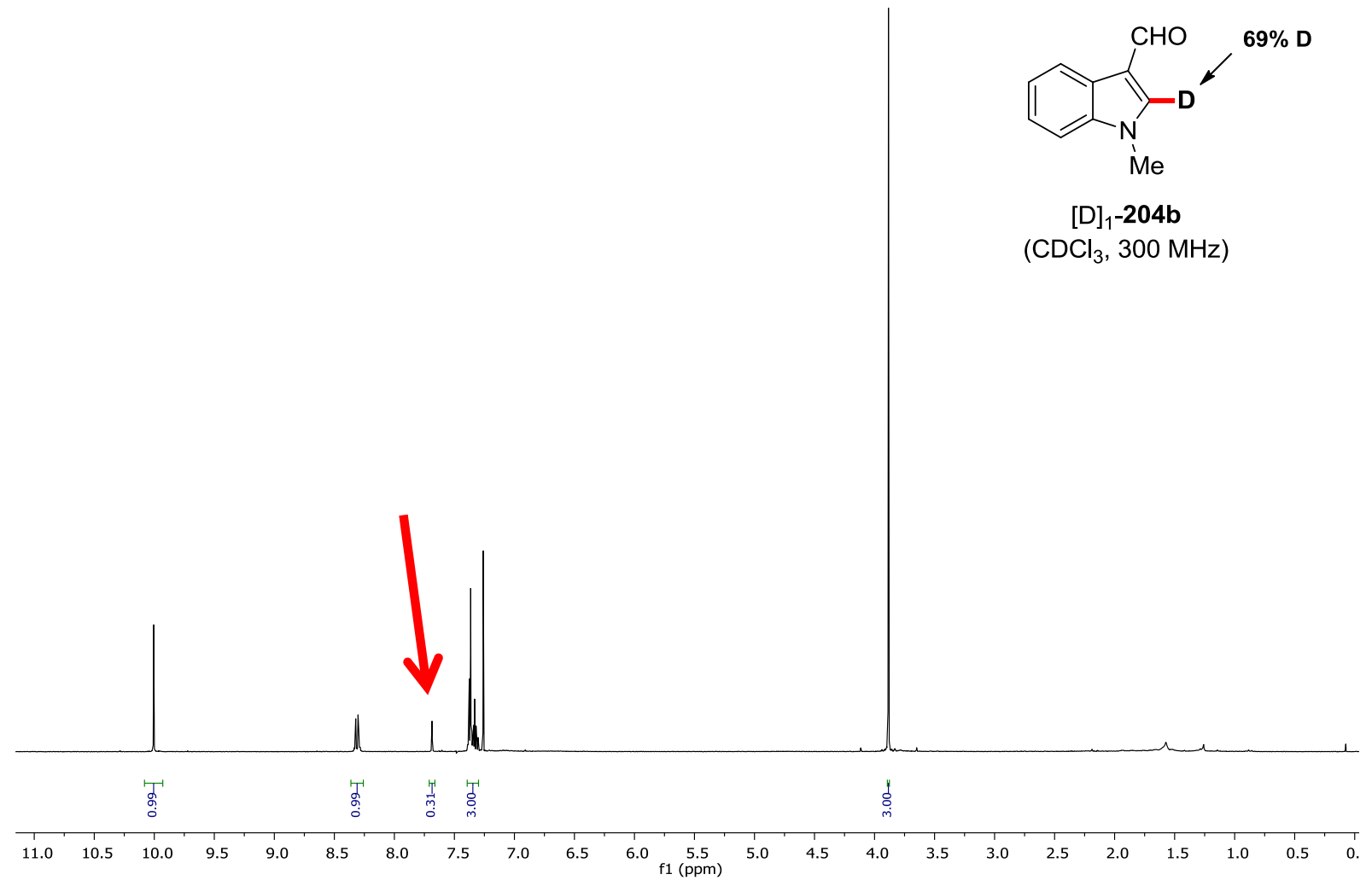

The same procedure as described above was followed, conducting the reaction for $5.0 \mathrm{~min}$.

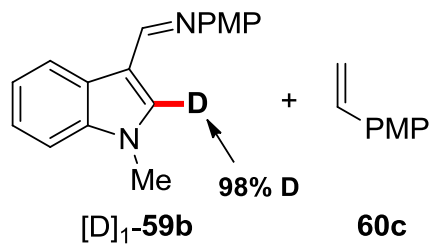

$[D]_{1}-59 b$

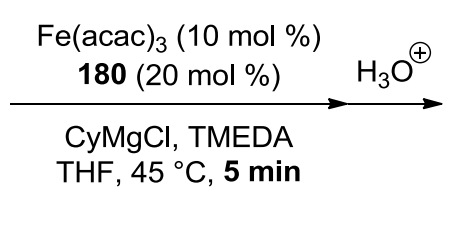

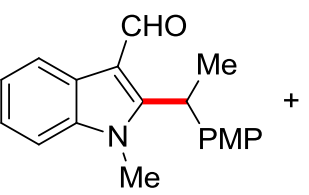

$[D]_{1}-62 b c:<5 \%$ (D content n.d.)<smiles>Cn1c(O[13CH3])c(C=O)c2ccccc21</smiles>

$[D]_{1}-204 b: 79 \%$ 


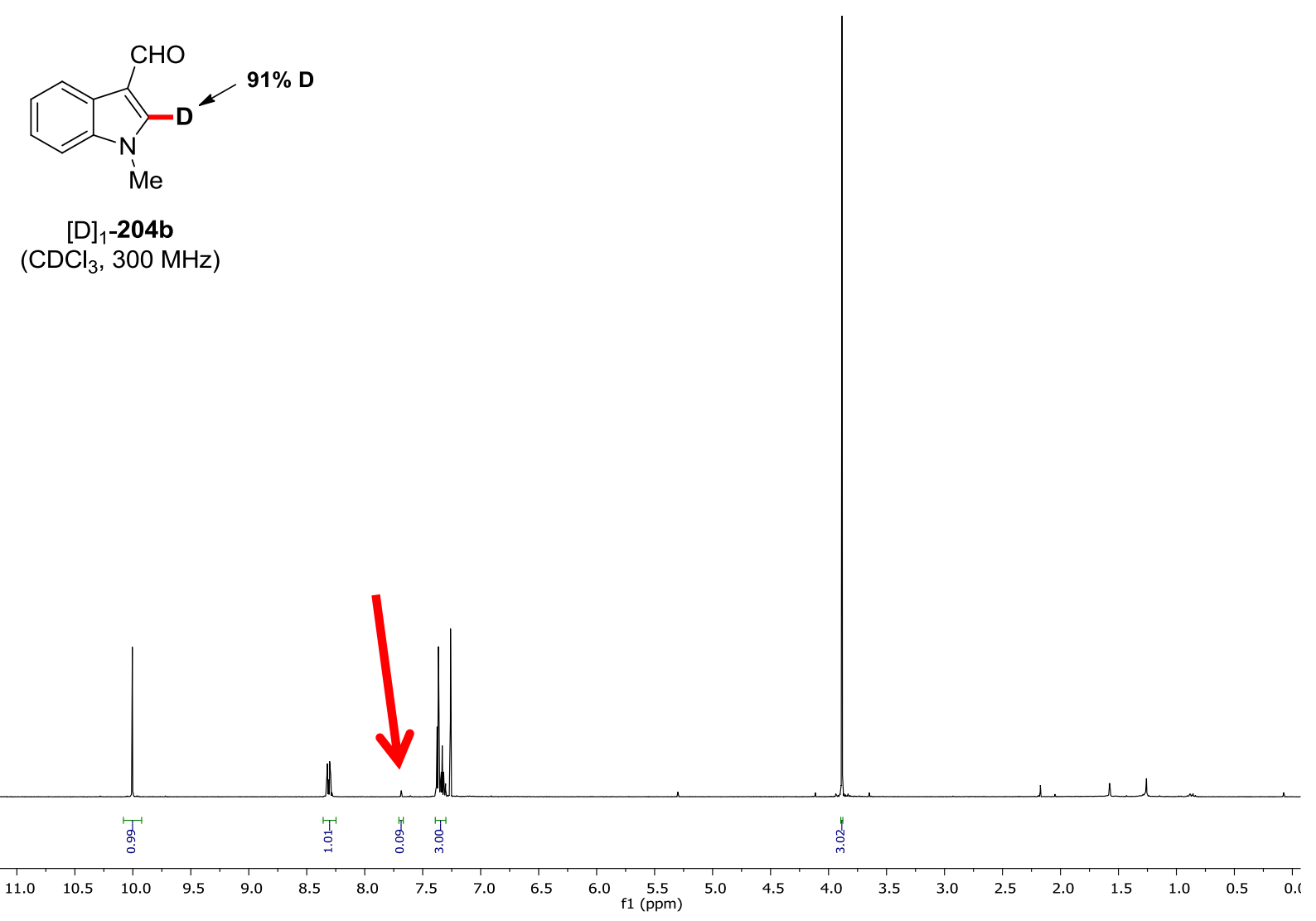

\section{With Vinylferrocene (191a):}

In an oven-dried $25 \mathrm{~mL}$ Schlenk tube were placed the indole substrate $[D]_{1}-59 b$ (66 mg, $0.25 \mathrm{mmol}, 1.0$ equiv), [Fe(acac) $)_{3}$ ( $\left.8.8 \mathrm{mg}, 0.025 \mathrm{mmol}, 10 \mathrm{~mol} \%\right), 180$ (38 mg, $0.050 \mathrm{mmol}, 20 \mathrm{~mol} \%$ ) and vinylferrocene (191a) $(80 \mathrm{mg}, 0.38 \mathrm{mmol}$, 1.5 equiv). The Schlenk tube was closed with a rubber septum, then evacuated and backfilled with $\mathrm{N}_{2} 3$ times. THF (0.50 mL) and TMEDA (58 mg, $0.50 \mathrm{mmol}, 2.0$ equiv) were added via syringe. CyMgCl $(0.28 \mathrm{~mL}, 0.275 \mathrm{mmol}, 1.1$ equiv, $1.0 \mathrm{M}$ in THF) was then added dropwise at ambient temperature. The resulting mixture was stirred at $45^{\circ} \mathrm{C}$ for $16 \mathrm{~h}$. The reaction mixture was allowed to cool to ambient temperature and diluted with $\mathrm{THF}(1.5 \mathrm{~mL}) . \mathrm{HCl}(3.0 \mathrm{M}, 2.0 \mathrm{~mL})$ was added in a single portion, and the resulting mixture was stirred at ambient temperature for $2 \mathrm{~h}$. The mixture was poured into sat. aqueous $\mathrm{NH}_{4} \mathrm{Cl}(10 \mathrm{~mL})$ and extracted with EtOAc $(3 \times 10 \mathrm{~mL})$. The combined organic layers were washed with brine $(10 \mathrm{~mL})$, dried over $\mathrm{Na}_{2} \mathrm{SO}_{4}$, and concentrated under reduced pressure. The yields of recovered starting materials 
and hydroarylation product were determined by ${ }^{1} \mathrm{H}$ NMR analysis of the crude mixture using 1,1,2,2-tetrachloroethane as an internal standard. The crude mixture was purified by silica gel chromatography ( $n$-hexane/EtOAc $25 / 1 \rightarrow 2 / 1$ ) to afford the product and hydrolyzed starting material. Deuterium contents were determined by ${ }^{1} \mathrm{H}$ NMR spectroscopic analysis.

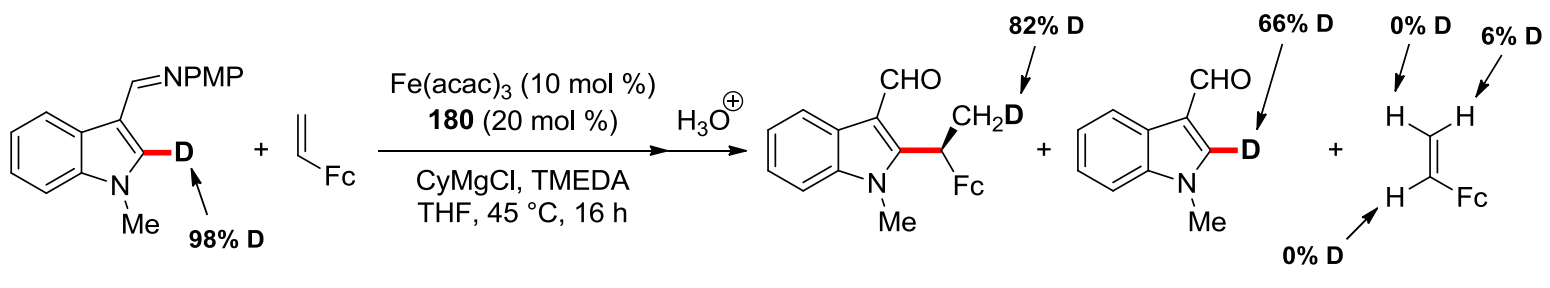

$[D]_{1}-59 b \quad 191 a$

$[D]_{1}-192 b a: 52 \%$

$[D]_{1}-204 b: 40 \%$

$[D]_{n}-191 a: 70 \%$

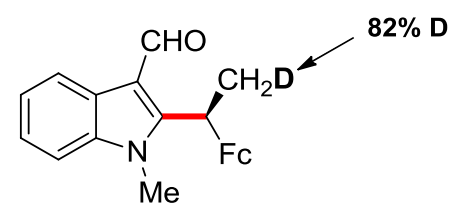

$[\mathrm{D}]_{1}-192 \mathrm{ba}$

$\left(\mathrm{CDCl}_{3}, 600 \mathrm{MHz}\right)$

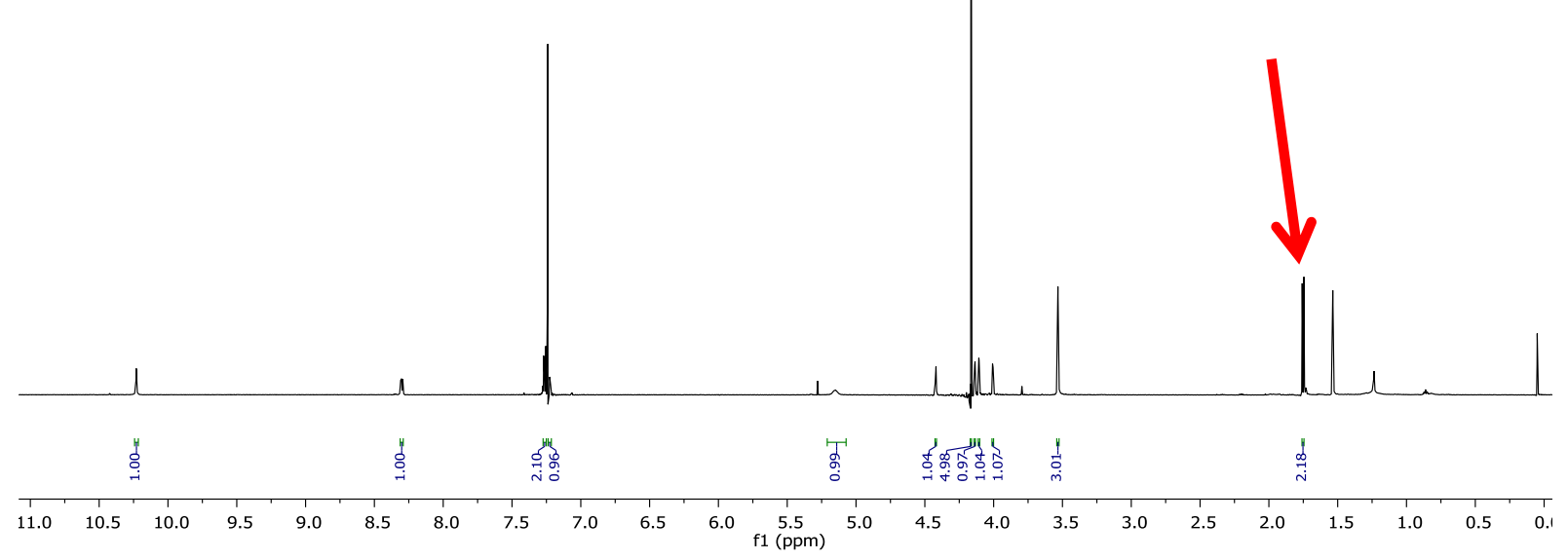



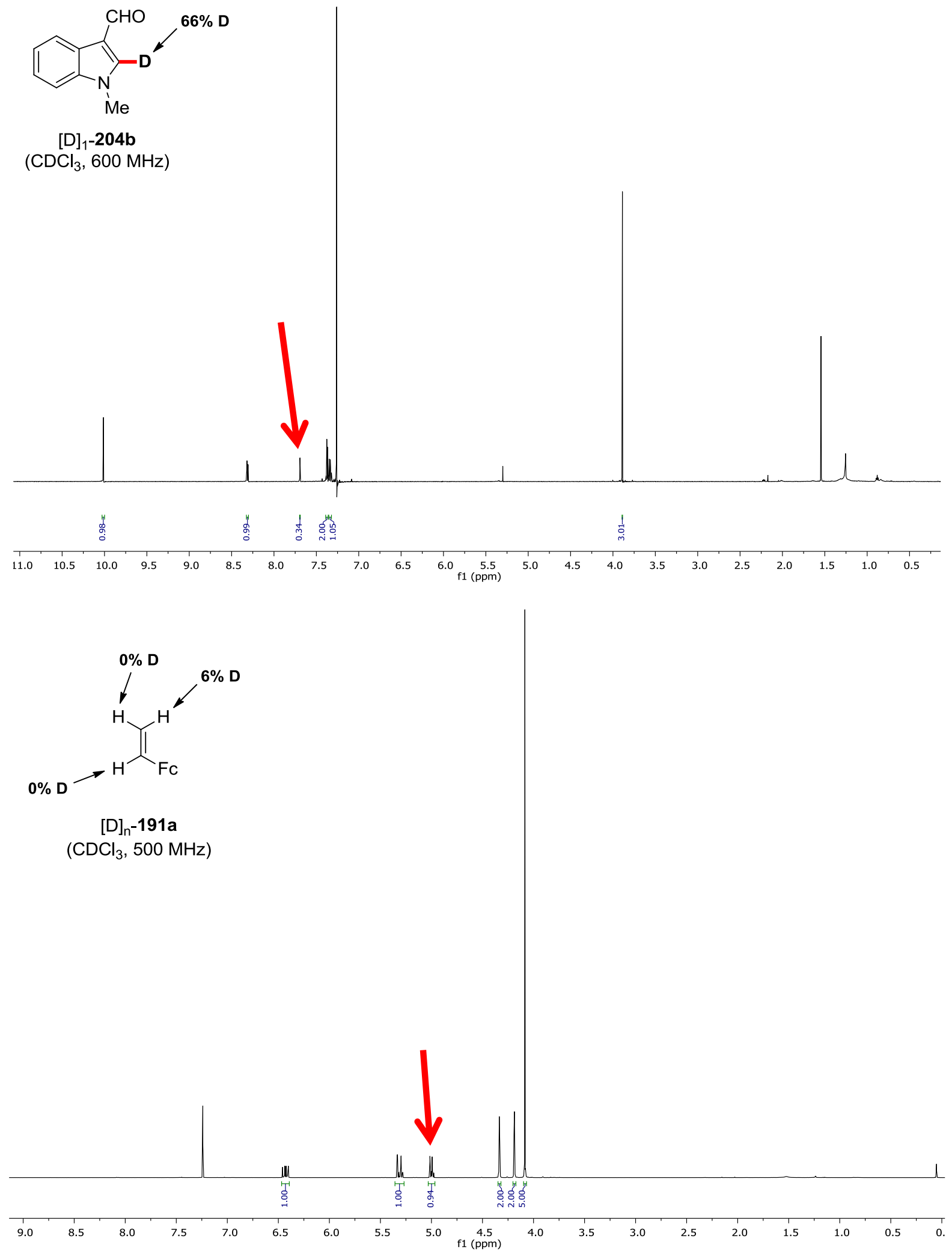


\section{Crossover Experiment}
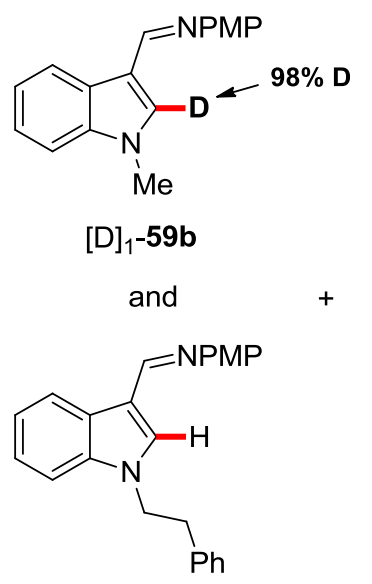

$59 f$

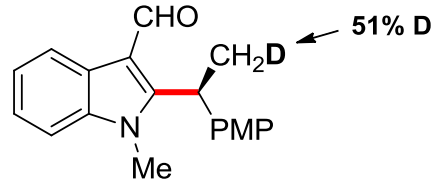

$[D]_{n}-62 b c: ~ 92 \%$

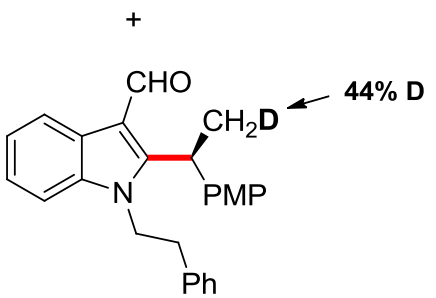

$[D]_{n}-62 f c: ~ 78 \%$

In an oven-dried $25 \mathrm{~mL}$ Schlenk tube were placed indole substrates [D] ${ }_{1}-59 \mathrm{~b}$ (66 mg, $0.25 \mathrm{mmol}, 0.50$ equiv) and $59 f\left(89 \mathrm{mg}, 0.25 \mathrm{mmol}, 0.50\right.$ equiv), $\mathrm{Fe}(\mathrm{acac})_{3}$ (17.6 mg, $0.050 \mathrm{mmol}, 10 \mathrm{~mol} \%)$ and $180(76 \mathrm{mg}, 0.10 \mathrm{mmol}, 20 \mathrm{~mol} \%)$. The Schlenk tube was closed with a rubber septum, then evacuated and backfilled with nitrogen 3 times. THF (1.0 mL), TMEDA (116 mg, $1.0 \mathrm{mmol}, 2.0$ equiv) and styrene 60c (100 mg, $0.75 \mathrm{mmol}, 1.5$ equiv) were added via syringe. CyMgCl $(0.59 \mathrm{~mL}$, $0.55 \mathrm{mmol}$, 1.1 equiv, $0.93 \mathrm{M}$ in THF) was then added dropwise at ambient temperature. The resulting mixture was stirred at $45^{\circ} \mathrm{C}$ for $16 \mathrm{~h}$. The reaction mixture was allowed to cool to ambient temperature and diluted with THF (3.0 mL). $\mathrm{HCl}(3.0 \mathrm{M}, 4.0 \mathrm{~mL})$ was added in a single portion, and the resulting mixture was stirred at ambient temperature for $2 \mathrm{~h}$. The mixture was poured into sat. aqueous $\mathrm{NH}_{4} \mathrm{Cl}(20 \mathrm{~mL})$ and extracted with EtOAc $(3 \times 20 \mathrm{~mL})$. The combined organic layers were washed with brine $(20 \mathrm{~mL})$, dried over $\mathrm{Na}_{2} \mathrm{SO}_{4}$, and concentrated under reduced pressure. The residue was purified by silica gel chromatography ( $n$-hexane/EtOAc $=9 / 15 / 1)$ to afford $[D]_{n}-62 b c(67.5 \mathrm{mg}, 92 \%)$ and $[\mathrm{D}]_{n}-62 \mathrm{fc}$ (75.2 mg, 78\%). Deuterium contents were determined by ${ }^{1} \mathrm{H}$ NMR spectroscopic analysis. 


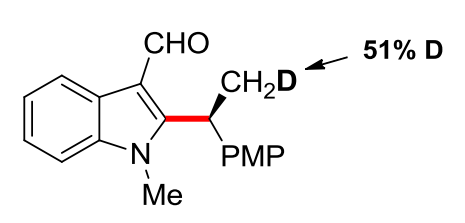

$[D]_{n}-62 b c$

$\left(\mathrm{CDCl}_{3}, 600 \mathrm{MHz}\right)$

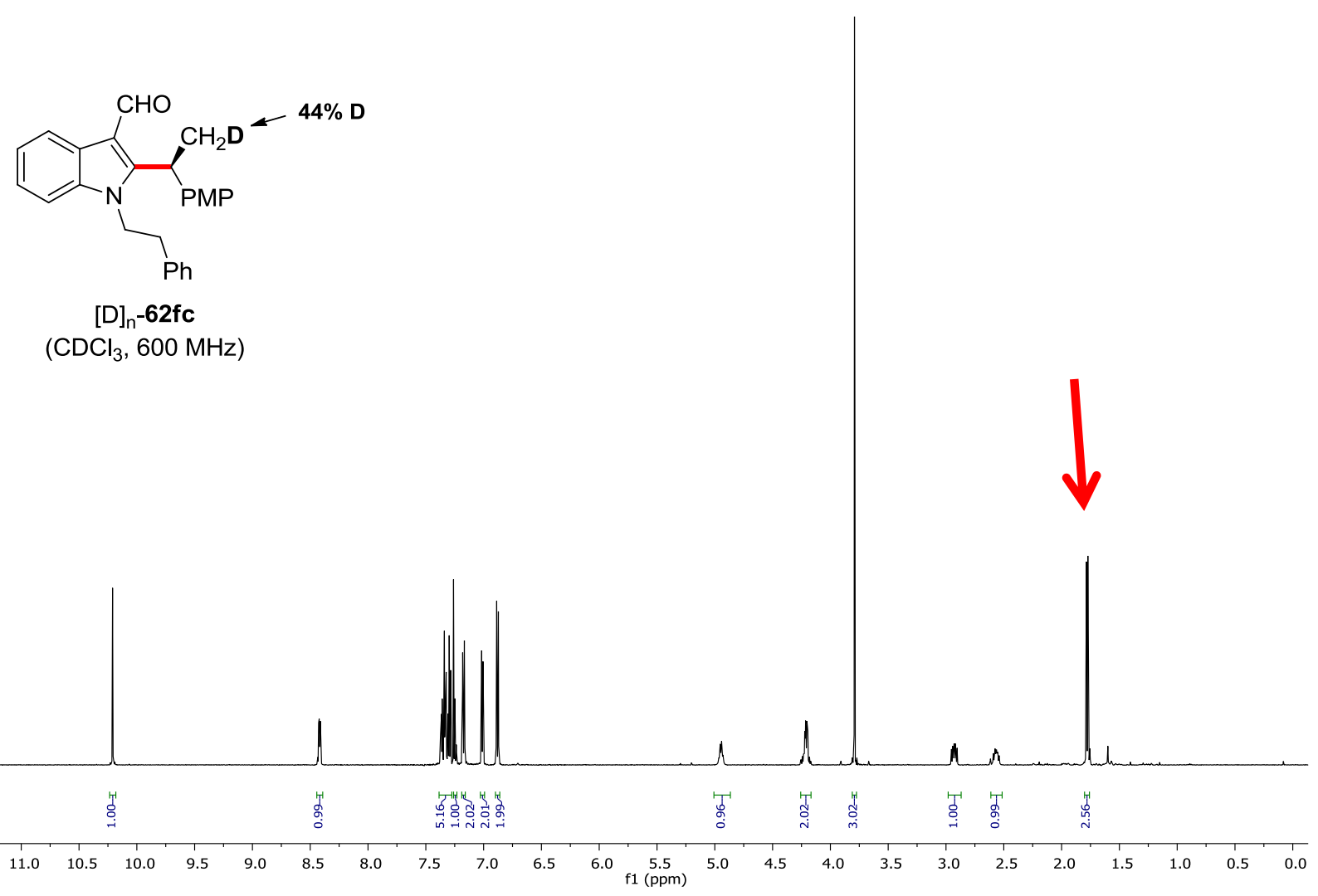

$[\mathrm{D}]_{\mathrm{n}}-62 \mathrm{fc}$

$\left(\mathrm{CDCl}_{3}, 600 \mathrm{MHz}\right)$

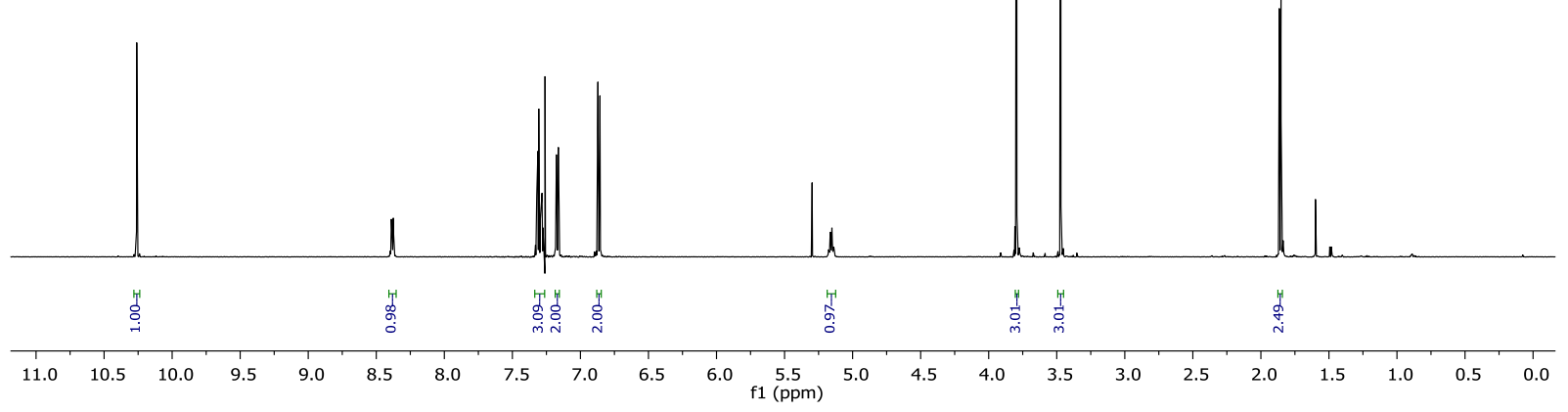




\section{KIE Studies}

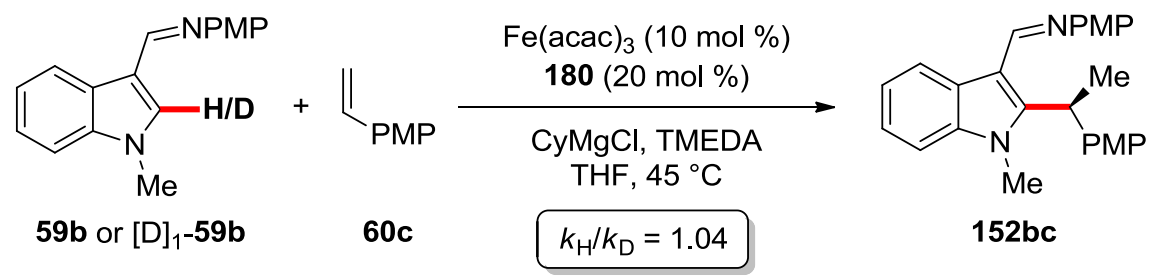

The kinetic isotope effect (KIE) was examined by applying the initial rate method. In an oven-dried $25 \mathrm{~mL}$ Schlenk tube were placed the indole substrates $59 \mathrm{~b}$ or [D] $]_{1}-59 \mathrm{~b}$ (198 or $199 \mathrm{mg}, 0.75 \mathrm{mmol}, 1.0$ equiv), $\mathrm{Fe}(\mathrm{acac})_{3}(26.4 \mathrm{mg}, 0.075 \mathrm{mmol}$, $10 \mathrm{~mol} \%$ ) and 180 (114 mg, $0.15 \mathrm{mmol}, 20 \mathrm{~mol} \%)$. The Schlenk tubes were closed with a rubber septum, then evacuated and backfilled with nitrogen 3 times. THF (1.50 mL), TMEDA (174 mg, $1.50 \mathrm{mmol}, 2.0$ equiv) and 4-methoxystyrene 60c (150 mg, $1.125 \mathrm{mmol}, 1.5$ equiv) were added via syringe. CyMgCl $(0.67 \mathrm{~mL}$, $0.825 \mathrm{mmol}, 1.1$ equiv, $1.23 \mathrm{M}$ in THF) was then added dropwise at ambient temperature. The Schlenk tubes were placed in a pre-heated oil bath at $45^{\circ} \mathrm{C}$ $(t=0 \mathrm{~min})$. Aliquots $(100 \mu \mathrm{L})$ were removed periodically every $5 \mathrm{~min}$ via syringe, diluted with $\mathrm{EtOH}(0.50 \mathrm{~mL})$, then with $\mathrm{CH}_{2} \mathrm{Cl}_{2}(2.5 \mathrm{~mL})$ and filtered through a short plug of silica gel. The plug was washed with EtOAc. The combined filtrates were concentrated in vacuo. Conversion was determined by ${ }^{1} \mathrm{H}$ NMR using $1,1,2,2-$ tetrachloroethane as an internal standard.

Table 5.2. Kinetic Isotope Effect.

\begin{tabular}{c|cc}
$t /$ min & $\mathbf{1 5 2 b c} / \%$ & {$[D]_{1}-\mathbf{1 5 2 b c} / \%$} \\
\hline 5 & 4 & 6 \\
10 & 7 & 8 \\
15 & 9 & 8 \\
20 & 12 & 10 \\
25 & 15 & 15 \\
30 & 17 & 19
\end{tabular}




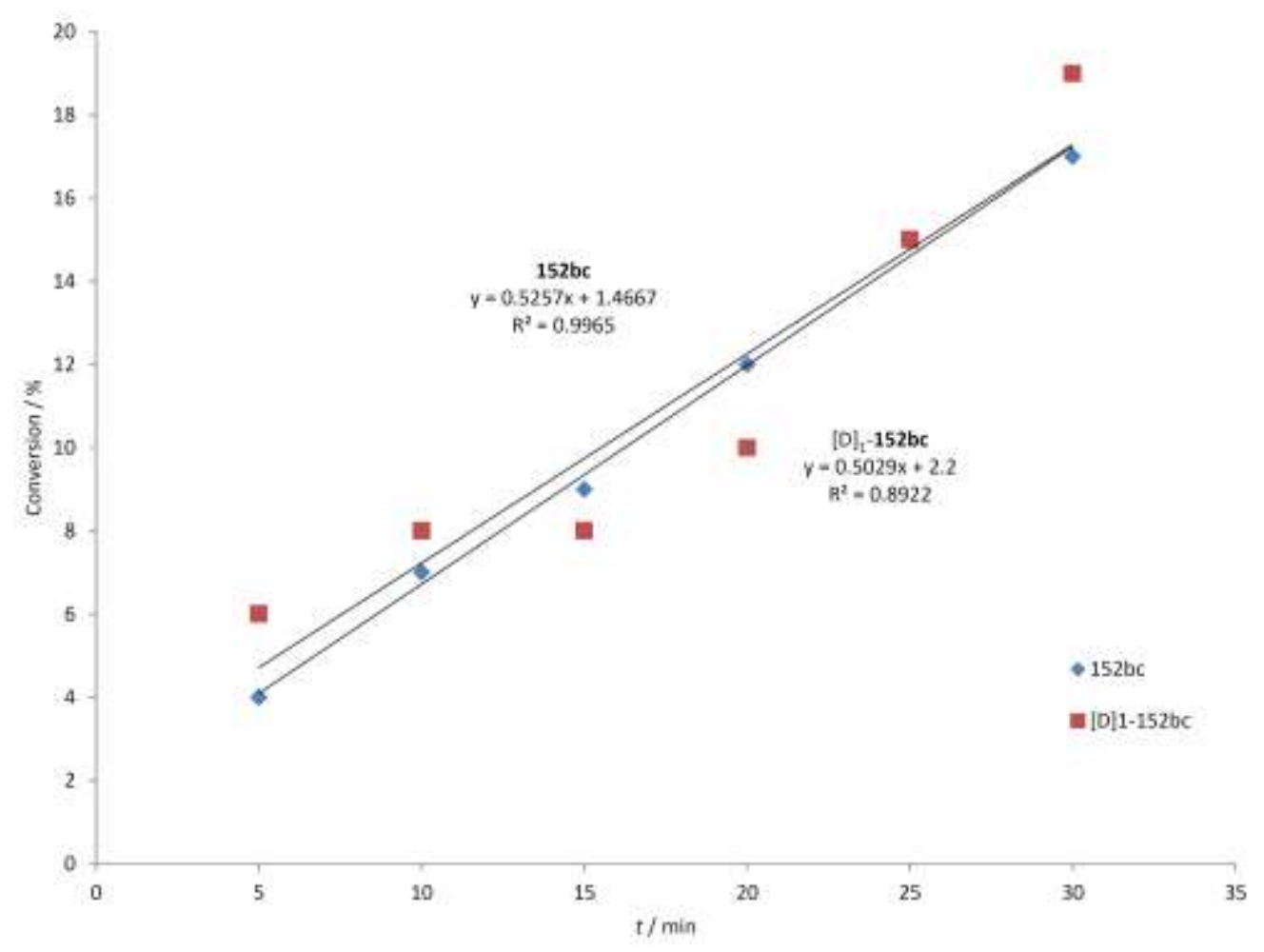

Figure 5.1. Kinetic Isotope Effect.

Additionally, KIE studies of the reaction with 4-fluorostyrene $(\mathbf{6 0 g})$ were conducted by Dr. D. Zell. ${ }^{[151]}$

\section{Mercury Drop Test}

In an oven-dried $25 \mathrm{~mL}$ Schlenk tube were placed indole $59 \mathrm{c}(85 \mathrm{mg}, 0.25 \mathrm{mmol}$, 1.0 equiv), $\mathrm{Fe}(\mathrm{acac})_{3}(8.8 \mathrm{mg}, 0.025 \mathrm{mmol}, 10 \mathrm{~mol} \%$ ) and 180 (38 mg, $0.050 \mathrm{mmol}$, $20 \mathrm{~mol} \%)$. The Schlenk tube was closed with a rubber septum, then evacuated and backfilled with nitrogen 3 times. THF $(0.50 \mathrm{~mL})$, TMEDA $(58 \mathrm{mg}, 0.50 \mathrm{mmol}$, 2.0 equiv) and vinylarene $60 \mathrm{c}(0.38 \mathrm{mmol}, 1.5$ equiv) were added via syringe. CyMgCl $(0.27 \mathrm{~mL}, 0.28 \mathrm{mmol}, 1.1$ equiv, $1.03 \mathrm{M})$ was then added dropwise at ambient temperature. The resulting mixture was stirred at ambient temperature for $1.0 \mathrm{~min} . \mathrm{Hg}$ (100 mg, $0.50 \mathrm{mmol}, 2.0$ equiv) was added via syringe. The reaction mixture was then stirred at $45^{\circ} \mathrm{C}$ for $16 \mathrm{~h}$. The reaction mixture was allowed to cool to ambient temperature and diluted with THF $(1.5 \mathrm{~mL})$. $\mathrm{HCl}(3.0 \mathrm{M}, 2.0 \mathrm{~mL})$ was 
added in a single portion, and the resulting mixture was stirred at ambient temperature for $2 \mathrm{~h}$. The mixture was poured into sat. aqueous $\mathrm{NH}_{4} \mathrm{Cl}(10 \mathrm{~mL})$ and extracted with EtOAc $(3 \times 10 \mathrm{~mL})$. The combined organic layers were washed with brine $(10 \mathrm{~mL})$, dried over $\mathrm{Na}_{2} \mathrm{SO}_{4}$, and concentrated under reduced pressure. The residue was purified by silica gel chromatography ( $n$-hexane/EtOAc $=8 / 1 \rightarrow 6 / 1$ ) to afford $62 \mathrm{cc}(85.7 \mathrm{mg}, 93 \%$, $93: 7$ e.r.).

\section{Non-Linear Effect Studies}

In an oven-dried $25 \mathrm{~mL}$ Schlenk tube were placed the indole substrate 59c (0.25 mmol, 1.0 equiv), $\mathrm{Fe}(\mathrm{acac})_{3}(8.8 \mathrm{mg}, 0.025 \mathrm{mmol}, 10 \mathrm{~mol} \%)$ and a mixture of 180 and ent-180 (total amount: $38 \mathrm{mg}, 0.050 \mathrm{mmol}, 20 \mathrm{~mol} \%$ ). The Schlenk tube was closed with a rubber septum, then evacuated and backfilled with nitrogen 3 times. THF ( $0.50 \mathrm{~mL})$, TMEDA (58 $\mathrm{mg}, 0.50 \mathrm{mmol}, 2.0$ equiv) and vinylarene 60c ( $0.38 \mathrm{mmol}, 1.5$ equiv) were added via syringe. CyMgCl $(0.28 \mathrm{mmol}, 1.1$ equiv, typically $1.0 \mathrm{M}$ ) was then added dropwise at ambient temperature. The resulting mixture was stirred at $45{ }^{\circ} \mathrm{C}$ for $16 \mathrm{~h}$. The reaction mixture was allowed to cool to ambient temperature and diluted with THF $(1.5 \mathrm{~mL}) . \mathrm{HCl}(3.0 \mathrm{M}, 2.0 \mathrm{~mL})$ was added in a single portion, and the resulting mixture was stirred at ambient temperature for $2 \mathrm{~h}$. The mixture was poured into sat. aqueous $\mathrm{NH}_{4} \mathrm{Cl}(10 \mathrm{~mL})$ and extracted with EtOAc $(3 \times 10 \mathrm{~mL})$. The combined organic layers were washed with brine $(10 \mathrm{~mL})$, dried over $\mathrm{Na}_{2} \mathrm{SO}_{4}$, and concentrated under reduced pressure. The conversion was determined by ${ }^{1} \mathrm{H}$ NMR using 1,1,2,2-tetrachloroethane as an internal standard. Enantiomeric excesses were measured by chiral HPLC analysis of the crude product (Chiralpak ${ }^{\circledR}$ IA-3, $n$-hexane//PrOH 80:20, $1.0 \mathrm{~mL} / \mathrm{min}$ ). 
Table 5.3. NLE studies.

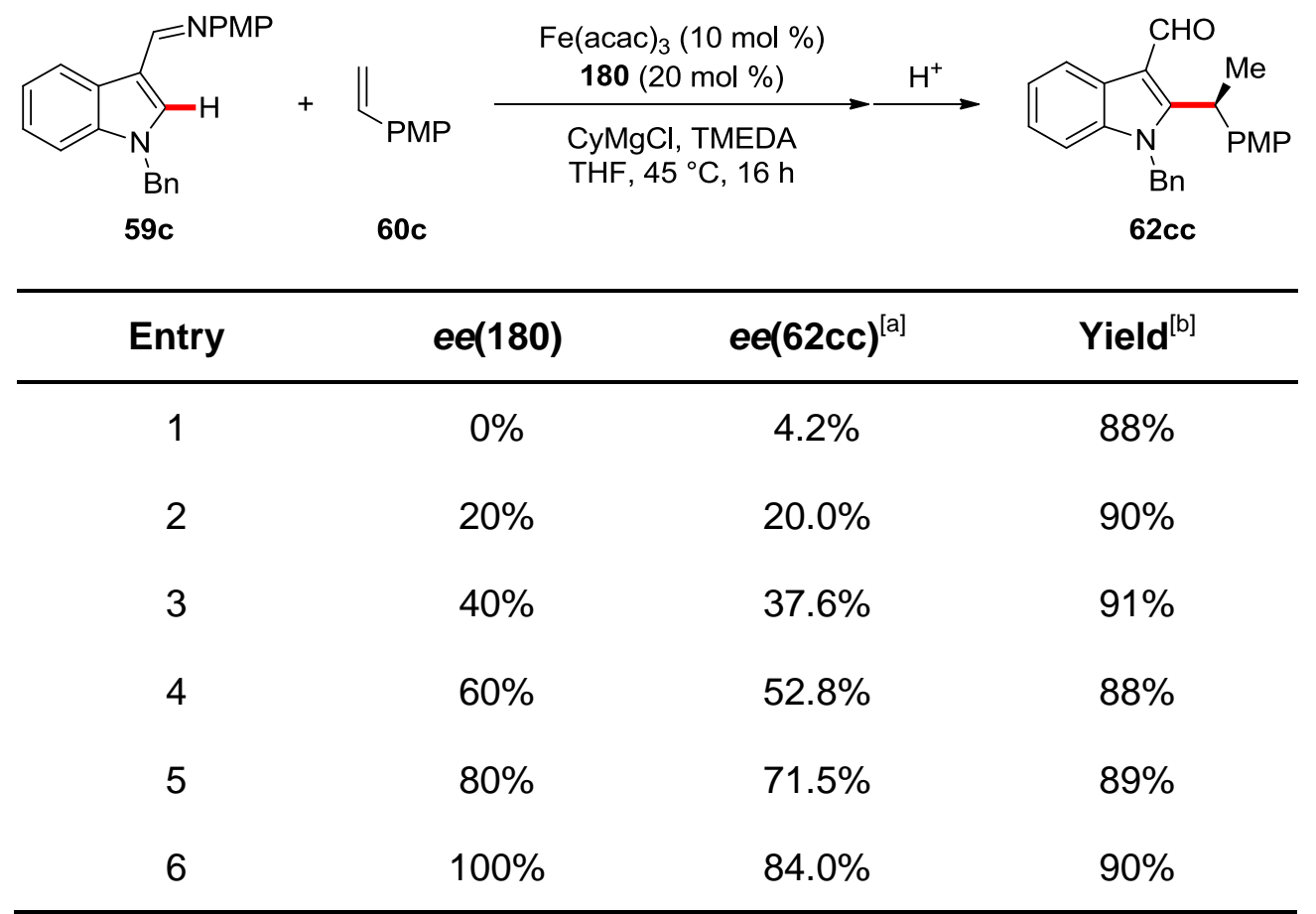

[a] Determined by chiral HPLC analysis of the crude reaction mixture.

${ }^{[b]}$ Determined by crude ${ }^{1} \mathrm{H}$ NMR using 1,1,2,2-tetrachloroethane as internal standard.

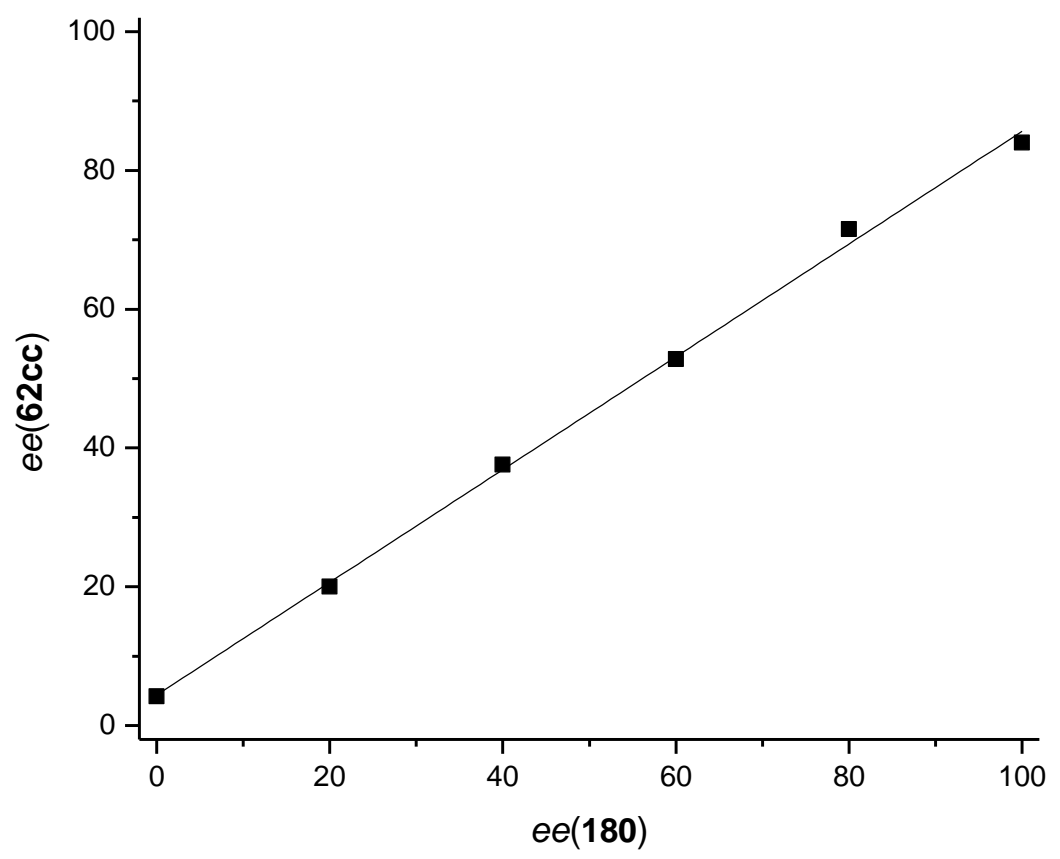

Figure 5.2. Absence of a non-linear effect. 


\section{Mössbauer Spectroscopy}

Mössbauer sample solutions were prepared by the addition of the Grignard reagent (8.0 equiv) to solutions of ${ }^{57} \mathrm{FeCl}_{2}(5.0 \mathrm{mM}, 1.0$ equiv) and TMEDA (4.0 equiv) in $\mathrm{THF}$ in a $\mathrm{N}_{2}$-filled glovebox (unless specified otherwise: at $-20^{\circ} \mathrm{C}$ for $\mathrm{CyMgCl}$, and at $23^{\circ} \mathrm{C}$ for $\mathrm{PhMgCl}$ ) and directly transferred into the Mössbauer sample cell before immediately freezing in liquid nitrogen (outside of the glovebox). 180 and 59b were added before the Grignard reagent.

After preparation, the spectra were recorded and interpreted by Dr. S. Demeshko (Meyer research group). All Mössbauer parameters are summarized in Tables 5.4-5.5. 
Table 5.4. Mössbauer parameters of reactions with $\mathrm{CyMgCl}$.

\begin{tabular}{|c|c|c|c|c|c|c|}
\hline Reaction & Figure & $\delta\left[\mathrm{mm} \mathrm{s}^{-1}\right]$ & $\Delta E_{\mathrm{Q}}\left[\mathrm{mm} \mathrm{s}^{-1}\right]$ & Rel. int. [\%] & Color & Assignment \\
\hline $\begin{array}{l}{ }^{57} \mathrm{FeCl}_{2}(5.0 \mathrm{mM})+ \\
\text { TMEDA (4.0 equiv) + } \\
\text { CyMgCl ( } 8.0 \text { equiv })\end{array}$ & Fig. 3.2a & $\begin{array}{l}0.48 \\
0.21\end{array}$ & $\begin{array}{l}0.89 \\
1.56\end{array}$ & $\begin{array}{l}84 \\
16\end{array}$ & $\begin{array}{l}\text { blue } \\
\text { red }\end{array}$ & $\begin{array}{l}\mathrm{Cy}_{4} \mathrm{Fe}(\mathrm{III})^{-} \\
\mathrm{Cy}_{3} \mathrm{Fe}(\mathrm{II})^{-}\end{array}$ \\
\hline $\begin{array}{l}{ }^{57} \mathrm{FeCl}_{2}(5.0 \mathrm{mM})+ \\
\text { TMEDA (4.0 equiv) }+ \\
\text { CyMgCl (8.0 equiv) }{ }^{[\mathrm{a}]}\end{array}$ & Fig. 3.2b & $\begin{array}{l}0.48 \\
0.24\end{array}$ & $\begin{array}{l}0.88 \\
1.59\end{array}$ & $\begin{array}{l}85 \\
15\end{array}$ & $\begin{array}{l}\text { blue } \\
\text { red }\end{array}$ & $\begin{array}{l}\mathrm{Cy}_{4} \mathrm{Fe}(\mathrm{III})^{-} \\
\mathrm{Cy}_{3} \mathrm{Fe}(\mathrm{II})^{-}\end{array}$ \\
\hline $\begin{array}{l}{ }^{57} \mathrm{FeCl}_{2}(5.0 \mathrm{mM})+ \\
\text { TMEDA }(4.0 \text { equiv })+ \\
\text { CyMgCl }(8.0 \text { equiv })^{[b]}\end{array}$ & Fig. 3.3 & $\begin{array}{r}0.19 \\
0.48 \\
-0.10\end{array}$ & $\begin{array}{l}0.86 \\
0.91 \\
1.10\end{array}$ & $\begin{array}{l}68 \\
28 \\
3\end{array}$ & $\begin{array}{l}\text { gray } \\
\text { blue } \\
\text { yellow }\end{array}$ & $\mathrm{Cy}_{4} \mathrm{Fe}(\mathrm{III})^{-}$ \\
\hline $\begin{array}{c}{ }^{57} \mathrm{FeCl}_{2}(5.0 \mathrm{mM})+ \\
\text { TMEDA (4.0 equiv) + } \\
180 \text { (1.0 equiv })+ \\
\text { CyMgCl (8.0 equiv) }\end{array}$ & Fig. 3.7a & $\begin{array}{l}0.18 \\
0.39 \\
0.46 \\
0.54 \\
0.24\end{array}$ & $\begin{array}{l}1.59 \\
3.19 \\
0.98 \\
2.04 \\
0.40\end{array}$ & $\begin{array}{l}36 \\
27 \\
19 \\
11 \\
7\end{array}$ & $\begin{array}{c}\text { red } \\
\text { green } \\
\text { blue } \\
\text { magenta } \\
\text { cyan }\end{array}$ & $\begin{array}{c}\mathrm{Cy}_{3} \mathrm{Fe}(\mathrm{II})^{-} \\
\mathrm{Cy}_{2} \mathrm{Fe}(\mathrm{II})(\mathrm{NHC}) \\
\mathrm{Cy}_{4} \mathrm{Fe}(\mathrm{III})^{-} \\
\mathrm{Cy}_{3} \mathrm{Fe}(\mathrm{II})(\mathrm{NHC})^{-} \\
--\end{array}$ \\
\hline $\begin{array}{c}{ }^{57} \mathrm{FeCl}_{2}(5.0 \mathrm{mM})+ \\
\mathrm{TMEDA}(4.0 \text { equiv })+ \\
\mathbf{1 8 0}(1.0 \text { equiv })+ \\
\mathrm{CyMgCl}(8.0 \text { equiv })^{[\mathrm{b}]}\end{array}$ & Fig. 3.7b & $\begin{array}{l}0.22 \\
0.22 \\
0.75 \\
0.37\end{array}$ & $\begin{array}{l}1.57 \\
0.57 \\
1.57 \\
3.20\end{array}$ & $\begin{array}{l}47 \\
34 \\
10 \\
9\end{array}$ & $\begin{array}{l}\text { red } \\
\text { cyan } \\
\text { purple } \\
\text { green }\end{array}$ & $\begin{array}{c}\mathrm{Cy}_{3} \mathrm{Fe}(\mathrm{II})^{-} \\
--- \\
--- \\
\mathrm{Cy}_{2} \mathrm{Fe}(\mathrm{II})(\mathrm{NHC})\end{array}$ \\
\hline $\begin{array}{c}{ }^{57} \mathrm{FeCl}_{2}(5.0 \mathrm{mM})+ \\
\mathrm{TMEDA}(4.0 \text { equiv })+ \\
180 \text { ( } 1.0 \text { equiv })+ \\
59 b \text { ( } 1.0 \text { equiv })+ \\
\text { CyMgCl (8.0 equiv) }\end{array}$ & Fig. 3.10a & $\begin{array}{l}0.20 \\
0.43 \\
0.58 \\
0.47\end{array}$ & $\begin{array}{l}1.71 \\
3.13 \\
2.04 \\
0.84\end{array}$ & $\begin{array}{l}53 \\
21 \\
18 \\
8\end{array}$ & $\begin{array}{c}\text { red } \\
\text { green } \\
\text { magenta } \\
\text { blue }\end{array}$ & $\begin{array}{c}\mathrm{Cy}_{3} \mathrm{Fe}(\mathrm{II})^{-} \\
\mathrm{Cy}_{2} \mathrm{Fe}(\mathrm{II})(\mathrm{NHC}) \\
\mathrm{Cy}_{3} \mathrm{Fe}(\mathrm{II})(\mathrm{NHC})^{-} \\
\mathrm{Cy}_{4} \mathrm{Fe}(\mathrm{III})^{-}\end{array}$ \\
\hline
\end{tabular}

${ }^{[a]}$ Recorded at $7 \mathrm{~K} .{ }^{[b]}$ Prepared at $23^{\circ} \mathrm{C} . \mathrm{NHC}=\mathrm{C}_{49} \mathrm{H}_{54} \mathrm{~N}_{2}$. 
Table 5.5. Mössbauer parameters of reactions with $\mathrm{PhMgCl}$.

\begin{tabular}{|c|c|c|c|c|c|c|}
\hline Reaction & Figure & $\delta\left[\mathrm{mm} \mathrm{s}^{-1}\right]$ & $\Delta E_{Q}\left[\mathrm{~mm} \mathrm{~s}^{-1}\right]$ & Rel. int. [\%] & Color & Assignment \\
\hline \multirow{3}{*}{$\begin{array}{c}{ }^{57} \mathrm{FeCl}_{2}(5.0 \mathrm{mM})+ \\
\text { TMEDA (4.0 equiv) } \\
+\mathrm{PhMgCl} \\
\text { (8.0 equiv) }\end{array}$} & \multirow{3}{*}{ Fig. 3.4} & 0.54 & 1.12 & 78 & blue & $\mathrm{Ph}_{4} \mathrm{Fe}(\mathrm{III})^{-}$ \\
\hline & & 0.20 & 1.44 & 13 & red & $\mathrm{Ph}_{3} \mathrm{Fe}(\mathrm{II})^{-}$ \\
\hline & & 0.46 & 2.61 & 9 & dark yellow & --- \\
\hline \multirow{5}{*}{$\begin{array}{l}{ }^{57} \mathrm{FeCl}_{2}(5.0 \mathrm{mM})+ \\
\mathrm{TMEDA}(4.0 \text { equiv }) \\
+\mathbf{1 8 0}(1.0 \text { equiv })+ \\
\mathrm{PhMgCl} \text { (8.0 equiv) }\end{array}$} & \multirow{5}{*}{ Fig. 3.8} & 0.51 & 1.09 & 40 & blue & $\mathrm{Ph}_{4} \mathrm{Fe}(\mathrm{III})^{-}$ \\
\hline & & 0.22 & 4.25 & 39 & green & $\mathrm{Ph}_{2} \mathrm{Fe}(\mathrm{II})(\mathrm{NHC})$ \\
\hline & & 0.56 & 2.62 & 14 & light green & --- \\
\hline & & 0.32 & 1.70 & 4 & orange & --- \\
\hline & & 1.10 & 4.30 & 3 & wine & --- \\
\hline${ }^{57} \mathrm{FeCl}_{2}(5.0 \mathrm{mM})+$ & \multirow{5}{*}{ Fig. 3.10b } & 0.51 & 1.09 & 52 & blue & $\mathrm{Ph}_{4} \mathrm{Fe}(\mathrm{III})^{-}$ \\
\hline TMEDA (4.0 equiv) & & 0.22 & 4.21 & 36 & green & $\mathrm{Ph}_{2} \mathrm{Fe}(\mathrm{II})(\mathrm{NHC})$ \\
\hline$+\mathbf{1 8 0}(1.0$ equiv $)+$ & & 0.57 & 2.64 & 7 & light green & --- \\
\hline $59 b(1.0$ equiv $)+$ & & 0.32 & 1.70 & 4 & orange & --- \\
\hline PhMgCl (8.0 equiv) & & 1.10 & 4.30 & 2 & wine & --- \\
\hline
\end{tabular}

\section{ESI-MS}

Standard sample solutions were prepared by the addition of the Grignard reagent (8.0 equiv) to a solution of $\mathrm{Fe}(\mathrm{acac})_{3}$ (1.0 equiv), TMEDA (4.0 equiv) in THF at $-78{ }^{\circ} \mathrm{C}$, and dilution to $10 \mathrm{mM}$. 180 and $59 \mathrm{~b}$ were added before the Grignard reagent.

Samples were prepared and analyzed by Dr. T. Parchomyk and S. Lülf (Koszinowski research group). 


\section{1,3-Dimesityl-4-[(trimethylammonio)methyl]-4,5-dihydro-1 H-imidazol-3-ium}

\section{lodide (207)}

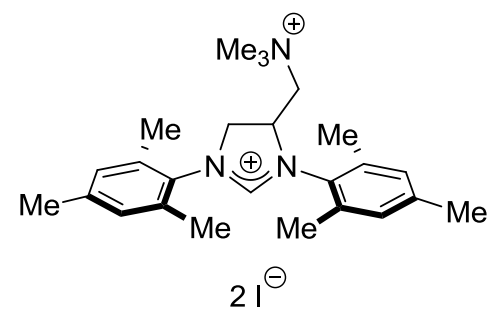

The amino-tagged NHC precursor $206^{[278]}(3.0 \mathrm{~g}, 6.65 \mathrm{mmol}, 1.0$ equiv) was dissolved in acetone $(25 \mathrm{~mL})$ and $\mathrm{Nal}(6.0 \mathrm{~g}, 40 \mathrm{mmol}, 6.0$ equiv) was added. The reaction mixture was stirred at $23^{\circ} \mathrm{C}$ for $14 \mathrm{~h}$ and then evaporated under reduced pressure. The residue was suspended in $\mathrm{CH}_{2} \mathrm{Cl}_{2}$, filtered through a short plug of Celite ${ }^{\circledR}$ and concentrated to yield the crude imidazolinium iodide quantitatively.

The imidazolinium iodide ( $400 \mathrm{mg}, 0.81 \mathrm{mmol}, 1.0$ equiv) was suspended in $\mathrm{Mel}$ (5.1 mL, $81 \mathrm{mmol}, 100$ equiv) and stirred at $23^{\circ} \mathrm{C}$ for $14 \mathrm{~h}$. $\mathrm{Et}_{2} \mathrm{O}(5.0 \mathrm{~mL})$ was added to the reaction mixture. The precipitate was collected by filtration, washed with $\mathrm{Et}_{2} \mathrm{O}$ $(10 \mathrm{~mL})$ and dried in vacuo to provide $207(452 \mathrm{mg}, 88 \%)$ as a colorless solid. M. p. $=308^{\circ} \mathrm{C}$ (decomposition). ${ }^{1} \mathbf{H}$ NMR $\left(300 \mathrm{MHz}, \mathrm{DMSO}-\mathrm{d}_{6}\right): \delta=9.13(\mathrm{~s}, 1 \mathrm{H})$, 7.20-7.13 (m, 2H), 7.12 (app s, 2H), 5.60 (q, J = 10.5 Hz, 1H), $4.92(\mathrm{t}, J=11.7 \mathrm{~Hz}$, $1 \mathrm{H}), 4.56(\mathrm{t}, J=11.1 \mathrm{~Hz}, 1 \mathrm{H}), 4.41(\mathrm{dd}, J=13.1,10.1 \mathrm{~Hz}, 1 \mathrm{H}), 3.39(\mathrm{~d}, J=13.1 \mathrm{~Hz}$, 1H), 3.11 (s, 9H), 2.40 (app s, 9H), 2.35-2.24 (m, 9H). ${ }^{13} \mathrm{C} \mathrm{NMR}(125 \mathrm{MHz}$, DMSO-d $\left.\mathrm{d}_{6}\right): \delta=161.0(\mathrm{CH}), 140.2\left(\mathrm{C}_{\mathrm{q}}\right), 140.2\left(\mathrm{C}_{\mathrm{q}}\right), 136.6\left(\mathrm{C}_{\mathrm{q}}\right), 135.8\left(\mathrm{C}_{\mathrm{q}}\right), 135.7\left(\mathrm{C}_{\mathrm{q}}\right)$, $135.0\left(\mathrm{C}_{\mathrm{q}}\right), 130.3(\mathrm{CH}), 130.2\left(\mathrm{C}_{\mathrm{q}}\right), 130.1(\mathrm{CH}), 129.6(\mathrm{CH}), 129.5(\mathrm{CH}), 127.9\left(\mathrm{C}_{\mathrm{q}}\right)$, $63.7\left(\mathrm{CH}_{2}\right), 57.8(\mathrm{CH}), 56.7\left(\mathrm{CH}_{2}\right), 52.8\left(\mathrm{CH}_{3}\right), 20.7\left(\mathrm{CH}_{3}\right), 20.6\left(\mathrm{CH}_{3}\right), 18.3\left(\mathrm{CH}_{3}\right)$, $18.0\left(\mathrm{CH}_{3}\right), 17.5\left(\mathrm{CH}_{3}\right), 17.4\left(\mathrm{CH}_{3}\right)$. IR (ATR): 2995, 1628, 1482, 1461, 1262, 1021, $871,815 \mathrm{~cm}^{-1}$. MS (ESI) $\mathrm{m} / z$ (relative intensity): 190 (100) [2M-2I] $]^{2+}$. HR-MS (ESI) $\mathrm{m} / z$ calcd for $\mathrm{C}_{25} \mathrm{H}_{37} \mathrm{~N}_{3}[2 \mathrm{M}-2 \mathrm{l}]^{2+} 189.6488$, found 189.6491 . 


\subsection{Asymmetric Nickel-Catalyzed Hydroarylations by C-H Activation}

5.6.1. Experimental Procedures and Analytical Data for the Intermolecular Nickel-Catalyzed Hydroarylation of Alkenes

\section{1-Benzyl-2-[1-(4-methoxyphenyl)ethyl]-1H-benzo[d]imidazole (106bc)}<smiles>CC(NP)c1nc2ccccc2n1Cc1ccccc1</smiles>

The general procedure GP9 was followed using 180 (38 mg, $50 \mu \mathrm{mol}, 10 \mathrm{~mol} \%$ ). Purification by column chromatography on silica gel $(n$-hexane/EtOAc $=4.5 / 1 \rightarrow 3 / 1)$ yielded $106 \mathrm{bc}(91.5 \mathrm{mg}, 53 \%)$ as a thick colorless oil. ${ }^{1} \mathrm{H} \mathbf{~ N M R}\left(400 \mathrm{MHz}, \mathrm{CDCl}_{3}\right)$ : $\delta=7.89(\mathrm{dt}, J=8.1,0.9 \mathrm{~Hz}, 1 \mathrm{H}), 7.29-7.22(\mathrm{~m}, 4 \mathrm{H}), 7.20(\mathrm{td}, J=8.0,1.2 \mathrm{~Hz}, 1 \mathrm{H})$, 7.17-7.14 (m, 1H), $7.10(\mathrm{~d}, J=8.7 \mathrm{~Hz}, 2 \mathrm{H}), 6.93-6.85(\mathrm{~m}, 2 \mathrm{H}), 6.78(\mathrm{~d}, J=8.7 \mathrm{~Hz}$, 2H), $5.19(\mathrm{~d}, J=16.8 \mathrm{~Hz}, 1 \mathrm{H}), 5.03(\mathrm{~d}, J=16.9 \mathrm{~Hz}, 1 \mathrm{H}), 4.16(\mathrm{q}, J=7.0 \mathrm{~Hz}, 1 \mathrm{H})$, $3.76(\mathrm{~s}, 3 \mathrm{H}), 1.79(\mathrm{~d}, J=7.1 \mathrm{~Hz}, 3 \mathrm{H}) .{ }^{13} \mathrm{C}$ NMR $\left(125 \mathrm{MHz}, \mathrm{CDCl}_{3}\right): \delta=158.5\left(\mathrm{C}_{\mathrm{q}}\right)$, $157.2\left(\mathrm{C}_{\mathrm{q}}\right), 142.6\left(\mathrm{C}_{\mathrm{q}}\right), 136.1\left(\mathrm{C}_{\mathrm{q}}\right), 135.9\left(\mathrm{C}_{\mathrm{q}}\right), 135.1\left(\mathrm{C}_{\mathrm{q}}\right), 128.9(\mathrm{CH}), 128.4(\mathrm{CH})$, $127.7(\mathrm{CH}), 126.2(\mathrm{CH}), 122.6(\mathrm{CH}), 122.0(\mathrm{CH}), 119.8(\mathrm{CH}), 114.4(\mathrm{CH}), 109.6$ $(\mathrm{CH}), 55.4\left(\mathrm{CH}_{3}\right), 46.9\left(\mathrm{CH}_{2}\right), 38.3(\mathrm{CH}), 22.3\left(\mathrm{CH}_{3}\right)$. IR (ATR): 2929, 1509, 1453, 1241, 1176, 1030, 831, $727 \mathrm{~cm}^{-1}$. MS (ESI) $\mathrm{m} / \mathrm{z}$ (relative intensity): 365 (33) $[\mathrm{M}+\mathrm{Na}]^{+}, 343(100)[\mathrm{M}+\mathrm{H}]^{+}$. HR-MS (ESI) $\mathrm{m} / z$ calcd for $\mathrm{C}_{23} \mathrm{H}_{23} \mathrm{~N}_{2} \mathrm{O}[\mathrm{M}+\mathrm{H}]^{+} 343.1805$, found 343.1798. [ []$_{D}{ }^{20}$ : $-60.9\left(\mathrm{C}=1.0, \mathrm{CHCl}_{3}\right)$. HPLC separation (Chiralpak ${ }^{\circledR}$ IB-3, $n$-hexane/iPrOH 80:20, $1.0 \mathrm{~mL} / \mathrm{min}$, detection at $250 \mathrm{~nm}): t_{r}($ major $)=6.6 \mathrm{~min}$, $t_{r}(\operatorname{minor})=9.2 \mathrm{~min}, 20: 80$ e.r.

5.6.2. Mechanistic Studies for the Intermolecular Nickel-Catalyzed Hydroarylation of Alkenes

$[D]_{1}-99 b$ was prepared by treating $99 b$ with oxalyl chloride $/ \mathrm{D}_{2} \mathrm{O}$, following $\mathrm{a}$ procedure previously reported in the literature. ${ }^{[339]}$ 
The general procedure GP9 was followed using $[D]_{1}-99 b(105 \mathrm{mg}, 0.50 \mathrm{mmol}$, 1.0 equiv) and 180 (38 mg, $50 \mu \mathrm{mol}, \quad 10 \mathrm{~mol} \%$ ). Purification by column chromatography on silica gel $(n$-hexane/EtOAc $=4.5 / 1 \rightarrow 0 / 1)$ yielded $[D]_{n}-106 b c$ (74.8 $\mathrm{mg}, 44 \%)$ as a thick colorless oil and re-isolated $[D]_{\mathrm{n}}-99 \mathrm{~b}(55.7 \mathrm{mg}, 53 \%)$ as a colorless solid. Deuterium contents were determined by ${ }^{1} \mathrm{H}$ NMR spectroscopic analysis.

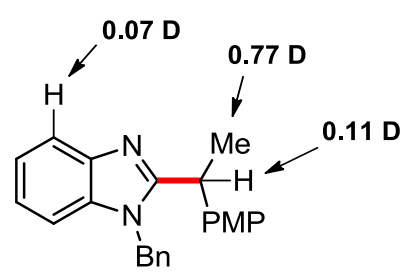

$[\mathrm{D}]_{\mathrm{n}}-106 \mathrm{bc}$

$\left(\mathrm{CDCl}_{3}, 600 \mathrm{MHz}\right)$

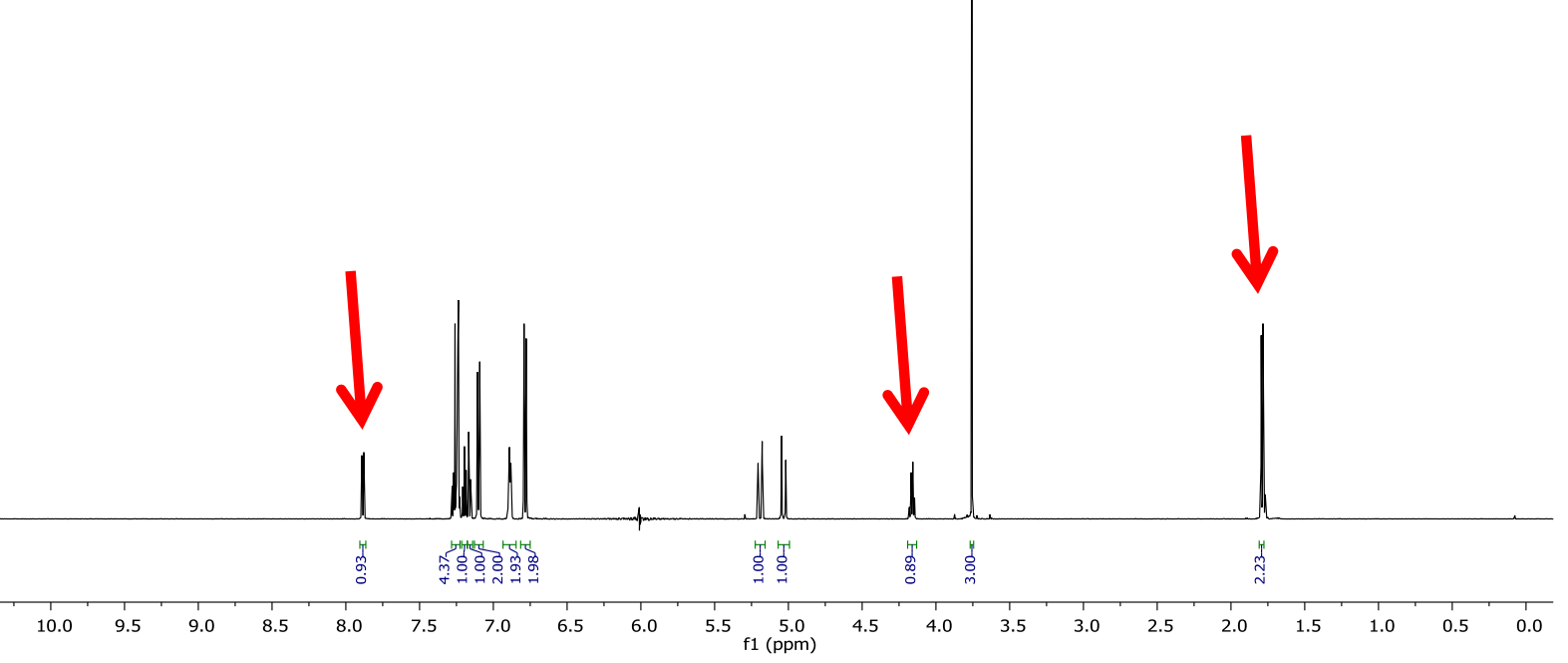




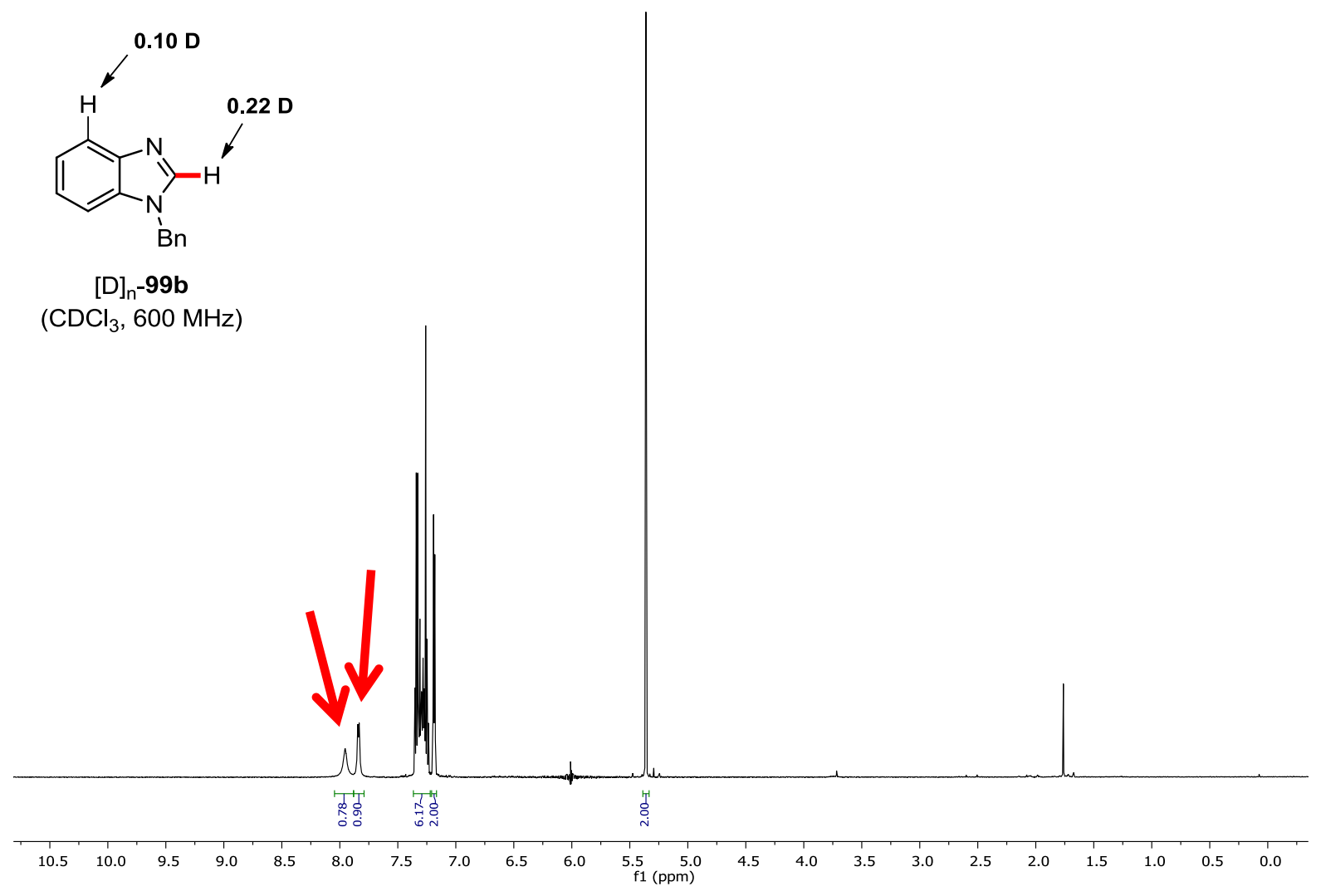

5.6.3 Experimental Procedures and Analytical Data for the Asymmetric Intramolecular Nickel-Catalyzed Hydroarylation of Alkenes

(R)-3-Methyl-1,2,3,4-tetrahydrobenzo[4,5]imidazo[1,2-a]pyridine (145a)

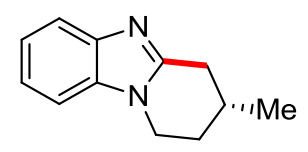

The general procedure GP11 was followed using 144a $(93 \mathrm{mg}, 0.50 \mathrm{mmol}$, 1.0 equiv). Purification by column chromatography on silica gel ( $n$-hexane/EtOAc $=$ $1 / 2 \rightarrow 0 / 1)$ yielded $145 \mathrm{a}(89.5 \mathrm{mg}, 96 \%)$ as a white solid. M. p. $=135-136{ }^{\circ} \mathrm{C}$. ${ }^{1}$ H NMR $\left(300 \mathrm{MHz}, \mathrm{CDCl}_{3}\right): \delta=7.80-7.70(\mathrm{~m}, 1 \mathrm{H}), 7.38-7.21(\mathrm{~m}, 3 \mathrm{H}), 4.24$ (ddd, $J=12.1,5.9,3.0 \mathrm{~Hz}, 1 \mathrm{H}$ ), $3.98(\mathrm{td}, J=11.7,11.3,4.9 \mathrm{~Hz}, 1 \mathrm{H}$ ), 3.25 (ddd, $J=17.1$, 4.8, $1.9 \mathrm{~Hz}, 1 \mathrm{H}$ ), 2.67 (dd, $J=17.1,10.7 \mathrm{~Hz}, 1 \mathrm{H}$ ), 2.29-2.07 (m, 2H), 1.82 (dtd, $J=14.0,10.7,5.7 \mathrm{~Hz}, 1 \mathrm{H}), 1.24(\mathrm{~d}, J=6.5 \mathrm{~Hz}, 3 \mathrm{H}) .{ }^{13} \mathrm{C} \mathrm{NMR}\left(75 \mathrm{MHz}, \mathrm{CDCl}_{3}\right)$ : 
$\delta=151.8\left(\mathrm{C}_{\mathrm{q}}\right), 143.1\left(\mathrm{C}_{\mathrm{q}}\right), 134.6\left(\mathrm{C}_{\mathrm{q}}\right), 122.0(\mathrm{CH}), 121.7(\mathrm{CH}), 118.9(\mathrm{CH}), 108.8$ $(\mathrm{CH}), 41.6\left(\mathrm{CH}_{2}\right), 33.5\left(\mathrm{CH}_{2}\right), 30.5\left(\mathrm{CH}_{2}\right), 27.7(\mathrm{CH}), 21.1\left(\mathrm{CH}_{3}\right)$. IR (ATR): 3050, 2948, 2921, 2864, 1458, 1417, 1285, 739, $437 \mathrm{~cm}^{-1}$. MS (ESI) $\mathrm{m} / \mathrm{z}$ (relative intensity): $187(100)[\mathrm{M}+\mathrm{H}]^{+}$. HR-MS (ESI) $\mathrm{m} / z$ calcd for $\mathrm{C}_{12} \mathrm{H}_{15} \mathrm{~N}_{2}[\mathrm{M}+\mathrm{H}]^{+}$187.1230,

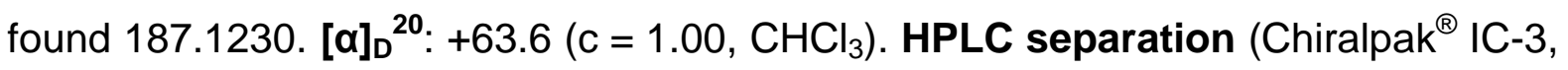
$n$-hexane/PrOH 80:20, $1.0 \mathrm{~mL} / \mathrm{min}$, detection at $273 \mathrm{~nm}): t_{r}($ major $)=16.8 \mathrm{~min}$, $t_{r}(\operatorname{minor})=18.6 \mathrm{~min}, 96: 4$ e.r.

(R)-Methyl 7-Methyl-5,6,7,8-tetrahydroimidazo[1,2-a]pyridine-2-carboxylate (145b)

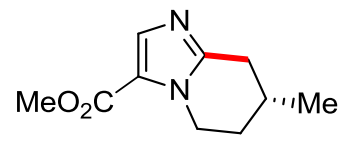

The general procedure GP11 was followed using 144b $(97 \mathrm{mg}, 0.50 \mathrm{mmol}$, 1.0 equiv), $\mathrm{Ni}(\mathrm{cod})_{2}(13.8 \mathrm{mg}, 50.0 \mu \mathrm{mol}, 10.0 \mathrm{~mol} \%$ ) and 220 (12.6 mg, $25.0 \mu \mathrm{mol}$, $5.00 \mathrm{~mol} \%)$ in PhMe (1.0 mL). Purification by column chromatography on silica gel $(n$-hexane/EtOAc $=1.5 / 1 \rightarrow 1 / 2)$ yielded $145 \mathrm{~b}(64.1 \mathrm{mg}, 66 \%)$ as a white solid. M. p. $=74-76{ }^{\circ} \mathrm{C} .{ }^{1} \mathrm{H}$ NMR $\left(300 \mathrm{MHz}, \mathrm{CDCl}_{3}\right): \delta=7.66(\mathrm{~s}, 1 \mathrm{H}), 4.54$ (ddd, $J=13.9$, 5.8, $3.0 \mathrm{~Hz}, 1 \mathrm{H}$ ), 4.01 (ddd, $J=14.4,11.0,4.9 \mathrm{~Hz}, 1 \mathrm{H}$ ), 3.81 (s, 3H), 3.04 (ddd, $J=17.2,4.9,1.8 \mathrm{~Hz}, 1 \mathrm{H}), 2.44(\mathrm{dd}, J=17.2,10.3 \mathrm{~Hz}, 1 \mathrm{H}), 2.13-1.91(\mathrm{~m}, 2 \mathrm{H}), 1.63$ (dtd, $J=14.1,11.2,5.6 \mathrm{~Hz}, 1 \mathrm{H}), 1.12(\mathrm{~d}, J=6.5 \mathrm{~Hz}, 3 \mathrm{H}) .{ }^{13} \mathrm{C} \mathrm{NMR}\left(75 \mathrm{MHz}, \mathrm{CDCl}_{3}\right)$ : $\delta=161.1\left(\mathrm{C}_{\mathrm{q}}\right), 150.3\left(\mathrm{C}_{\mathrm{q}}\right), 137.0(\mathrm{CH}), 121.9\left(\mathrm{C}_{\mathrm{q}}\right), 51.3\left(\mathrm{CH}_{3}\right), 44.6\left(\mathrm{CH}_{2}\right), 33.3$ $\left(\mathrm{CH}_{2}\right), 30.7\left(\mathrm{CH}_{2}\right), 26.8(\mathrm{CH}), 21.1\left(\mathrm{CH}_{3}\right)$. IR (ATR): 2957, 1704, 1443, 1224, 1176, $1141,1072,766 \mathrm{~cm}^{-1}$. MS (ESI) $\mathrm{m} / \mathrm{z}$ (relative intensity): 209 (21), $195(100)[\mathrm{M}+\mathrm{H}]^{+}$, 127 (25). HR-MS (ESI) $\mathrm{m} / z$ calcd for $\mathrm{C}_{10} \mathrm{H}_{15} \mathrm{~N}_{2} \mathrm{O}_{2}[\mathrm{M}+\mathrm{H}]^{+}$195.1128, found 195.1123. $[\alpha]_{\mathrm{D}}{ }^{20}:+72.8\left(\mathrm{c}=0.29, \mathrm{CHCl}_{3}\right)$. HPLC separation (Chiralpak ${ }^{\circledR}$ ID-3, $n$-hexane/IPrOH $60: 40,1.0 \mathrm{~mL} / \mathrm{min}$, detection at $250 \mathrm{~nm}): t_{r}($ major $)=7.6 \mathrm{~min}, t_{r}($ minor $)=8.2 \mathrm{~min}$, 99:1 e.r. 
(R)-3-Nonyl-1,2,3,4-tetrahydrobenzo[4,5]imidazo[1,2-a]pyridine (145c)

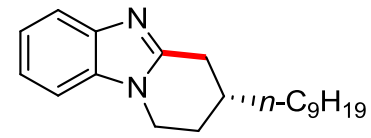

The general procedure GP11 was followed using $144 \mathrm{c}$ (149 mg, $0.50 \mathrm{mmol}$, 1.0 equiv). Purification by column chromatography on silica gel ( $n$-hexane/EtOAc $=$ $3 / 1 \rightarrow 1.5 / 1)$ yielded $145 \mathrm{C}(131.0 \mathrm{mg}, 88 \%)$ as a white solid. M. p. $=85-86^{\circ} \mathrm{C}$. ${ }^{1} \mathrm{H}$ NMR $\left(300 \mathrm{MHz}, \mathrm{CDCl}_{3}\right): \delta=7.72-7.64(\mathrm{~m}, 1 \mathrm{H}), 7.33-7.16(\mathrm{~m}, 3 \mathrm{H}), 4.22$ (ddd, $J=12.0,5.7,3.2 \mathrm{~Hz}, 1 \mathrm{H}$ ), 3.95 (ddd, $J=12.0,10.7,5.0 \mathrm{~Hz}, 1 \mathrm{H}$ ), 3.24 (ddd, $J=17.1$, 4.8, 1.7 Hz, 1H), 2.64 (dd, $J=17.1,10.4 \mathrm{~Hz}, 1 \mathrm{H}), 2.27-2.17(\mathrm{~m}, 1 \mathrm{H}), 2.07-1.93(\mathrm{~m}$, $1 \mathrm{H}), 1.78(\mathrm{dtd}, J=13.4,10.8,5.7 \mathrm{~Hz}, 1 \mathrm{H}), 1.57-1.15(\mathrm{~m}, 16 \mathrm{H}), 0.89(\mathrm{t}, J=6.8 \mathrm{~Hz}$, 3H). ${ }^{13} \mathrm{C}$ NMR $\left(125 \mathrm{MHz}, \mathrm{CDCl}_{3}\right): \delta=152.0\left(\mathrm{C}_{\mathrm{q}}\right), 143.2\left(\mathrm{C}_{\mathrm{q}}\right), 134.6\left(\mathrm{C}_{\mathrm{q}}\right), 122.1(\mathrm{CH})$, $121.7(\mathrm{CH}), 119.0(\mathrm{CH}), 108.8(\mathrm{CH}), 41.8\left(\mathrm{CH}_{2}\right), 35.7\left(\mathrm{CH}_{2}\right), 32.8(\mathrm{CH}), 32.1\left(\mathrm{CH}_{2}\right)$, $32.0\left(\mathrm{CH}_{2}\right), 29.9\left(\mathrm{CH}_{2}\right), 29.8\left(\mathrm{CH}_{2}\right), 29.8\left(\mathrm{CH}_{2}\right), 29.5\left(\mathrm{CH}_{2}\right), 29.0\left(\mathrm{CH}_{2}\right), 27.0\left(\mathrm{CH}_{2}\right)$, $22.9\left(\mathrm{CH}_{2}\right), 14.3\left(\mathrm{CH}_{3}\right)$. IR (ATR): 2920, 2851, 1513, 1459, 1421, 1287, $740 \mathrm{~cm}^{-1}$. MS (ESI) $m / z$ (relative intensity): $299(100)[M+H]^{+}$. HR-MS (ESI) $m / z$ calcd for $\mathrm{C}_{20} \mathrm{H}_{31} \mathrm{~N}_{2}[\mathrm{M}+\mathrm{H}]^{+}$299.2482, found 299.2480. [ $\left.\alpha\right]_{\mathrm{D}}{ }^{20}:+37.2$ (c = 1.01, $\left.\mathrm{CHCl}_{3}\right)$. HPLC separation (Chiralpak ${ }^{\circledR} \mathrm{IC}-3, n$-hexane/ $/ \mathrm{PrOH} 80: 20,1.0 \mathrm{~mL} / \mathrm{min}$, detection at $273 \mathrm{~nm}): t_{r}($ major $)=11.3 \mathrm{~min}, t_{r}($ minor $)=12.4 \mathrm{~min}, 96: 4$ e.r.

(R)-3-(2-Cyclohexylethyl)-1,2,3,4-tetrahydrobenzo[4,5]imidazo[1,2-a]pyridine $(145 d)$

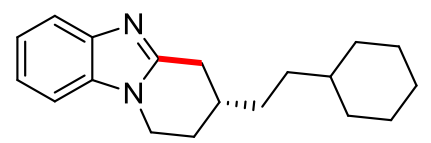

The general procedure GP11 was followed using 144d $(141 \mathrm{mg}, 0.50 \mathrm{mmol}$, 1.0 equiv). Purification by column chromatography on silica gel ( $n$-hexane/EtOAc $=$ $4 / 1 \rightarrow 2 / 1)$ yielded $145 \mathrm{~d}(119.3 \mathrm{mg}, 85 \%)$ as a white solid. M. p. $=134-135{ }^{\circ} \mathrm{C}$. ${ }^{1} \mathrm{H}$ NMR $\left(300 \mathrm{MHz}, \mathrm{CDCl}_{3}\right): \delta=7.72-7.64(\mathrm{~m}, 1 \mathrm{H}), 7.34-7.15(\mathrm{~m}, 3 \mathrm{H}), 4.22$ (ddd, $J=11.9,5.7,3.2 \mathrm{~Hz}, 1 \mathrm{H}$ ), 3.94 (ddd, $J=11.9,10.6,5.0 \mathrm{~Hz}, 1 \mathrm{H}$ ), 3.24 (ddd, $J=17.1$, 
4.8, 1.7 Hz, $1 \mathrm{H}), 2.64(\mathrm{dd}, J=17.1,10.3 \mathrm{~Hz}, 1 \mathrm{H}), 2.28-2.15(\mathrm{~m}, 1 \mathrm{H}), 2.04-1.86(\mathrm{~m}$, $1 \mathrm{H}), 1.86-1.58(\mathrm{~m}, 6 \mathrm{H}), 1.54-1.42(\mathrm{~m}, 2 \mathrm{H}), 1.37-1.07(\mathrm{~m}, 6 \mathrm{H}), 1.01-0.79(\mathrm{~m}, 2 \mathrm{H})$. ${ }^{13} \mathrm{C}$ NMR $\left(125 \mathrm{MHz}, \mathrm{CDCl}_{3}\right): \delta=152.0\left(\mathrm{C}_{\mathrm{q}}\right), 143.2\left(\mathrm{C}_{\mathrm{q}}\right), 134.6\left(\mathrm{C}_{\mathrm{q}}\right), 122.1(\mathrm{CH})$, $121.7(\mathrm{CH}), 119.0(\mathrm{CH}), 108.8(\mathrm{CH}), 41.8\left(\mathrm{CH}_{2}\right), 37.9(\mathrm{CH}), 34.7\left(\mathrm{CH}_{2}\right), 33.6\left(\mathrm{CH}_{2}\right)$, $33.6\left(\mathrm{CH}_{2}\right)$, $33.1(\mathrm{CH}), 32.9\left(\mathrm{CH}_{2}\right), 32.0\left(\mathrm{CH}_{2}\right), 28.9\left(\mathrm{CH}_{2}\right), 26.6\left(\mathrm{CH}_{2}\right)$. IR (ATR): 2916, 2847, 1514, 1455, 1417, 1284, $737 \mathrm{~cm}^{-1}$. MS (ESI) $\mathrm{m} / z$ (relative intensity): $283(100)[\mathrm{M}+\mathrm{H}]^{+}$. HR-MS (ESI) $\mathrm{m} / z$ calcd for $\mathrm{C}_{19} \mathrm{H}_{27} \mathrm{~N}_{2}[\mathrm{M}+\mathrm{H}]^{+}$283.2169, found 283.2161. $[\alpha]_{\mathrm{D}}{ }^{20}:+45.0\left(\mathrm{C}=1.03, \mathrm{CHCl}_{3}\right)$. HPLC separation (Chiralpak ${ }^{\circledR} \mathrm{IC}-3$, $n$-hexane/ $\mathrm{PrOH} 80: 20,1.0 \mathrm{~mL} / \mathrm{min}$, detection at $250 \mathrm{~nm}): t_{r}$ (major) $=14.5 \mathrm{~min}$, $t_{r}(\operatorname{minor})=16.3 \mathrm{~min}, 98: 2$ e.r.

\section{(R)-3-(3-Phenylpropyl)-1,2,3,4-tetrahydrobenzo[4,5]imidazo[1,2-a]pyridine} (145e)

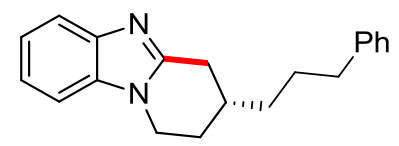

The general procedure GP11 was followed using 144e $(145 \mathrm{mg}, 0.50 \mathrm{mmol}$, 1.0 equiv). Purification by column chromatography on silica gel ( $n$-hexane/EtOAc $=$ $3 / 1 \rightarrow 1 / 1)$ yielded $145 \mathrm{e}(116.9 \mathrm{mg}, 81 \%)$ as a white solid. $\mathbf{M} . \mathbf{p .}=80-83^{\circ} \mathrm{C} .{ }^{1} \mathbf{H}$ NMR $\left(300 \mathrm{MHz}, \mathrm{CDCl}_{3}\right): \delta=7.77-7.66(\mathrm{~m}, 1 \mathrm{H}), 7.40-7.13(\mathrm{~m}, 8 \mathrm{H}), 4.22$ (ddd, $J=12.1$, 5.7, 3.2 Hz, $1 \mathrm{H}$ ), 3.93 (td, $J=11.4,5.0 \mathrm{~Hz}, 1 \mathrm{H}$ ), 3.27 (ddd, $J=17.0,4.8,1.7 \mathrm{~Hz}, 1 \mathrm{H}$ ), 2.76-2.57 (m, 3H), 2.29-2.15 (m, 1H), 2.11-1.94 (m, 1H), 1.88-1.68 (m, 3H), 1.61$1.47(\mathrm{~m}, 2 \mathrm{H}) .{ }^{13} \mathrm{C}$ NMR $\left(125 \mathrm{MHz}, \mathrm{CDCl}_{3}\right): \delta=151.7\left(\mathrm{C}_{\mathrm{q}}\right), 143.1\left(\mathrm{C}_{\mathrm{q}}\right), 142.1\left(\mathrm{C}_{\mathrm{q}}\right)$, $134.5\left(\mathrm{C}_{\mathrm{q}}\right), 128.4(\mathrm{CH}), 128.4(\mathrm{CH}), 125.9(\mathrm{CH}), 122.1(\mathrm{CH}), 121.7(\mathrm{CH}), 119.0(\mathrm{CH})$, $108.8(\mathrm{CH}), 41.7\left(\mathrm{CH}_{2}\right), 36.0\left(\mathrm{CH}_{2}\right), 35.1\left(\mathrm{CH}_{2}\right), 32.7(\mathrm{CH}), 31.9\left(\mathrm{CH}_{2}\right), 28.8\left(\mathrm{CH}_{2}\right)$, $28.8\left(\mathrm{CH}_{2}\right)$. IR (ATR): 3026, 2919, 2856, 1510, 1483, 1455, 1284, 742, $691 \mathrm{~cm}^{-1}$. MS (ESI) $m / z$ (relative intensity): 291 (100) $[\mathrm{M}+\mathrm{H}]^{+}$. HR-MS (ESI) $\mathrm{m} / \mathrm{z}$ calcd for

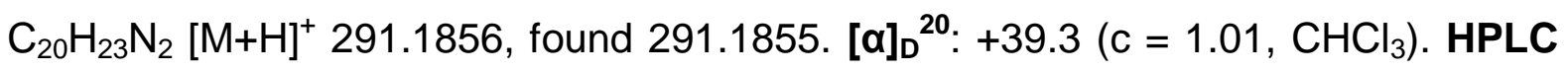
separation (Chiralpak ${ }^{\circledR} \mathrm{IC}-3, n$-hexane/ $\mathrm{PrOH} 80: 20,1.0 \mathrm{~mL} / \mathrm{min}$, detection at $273 \mathrm{~nm}): t_{r}($ major $)=20.6 \mathrm{~min}, t_{r}($ minor $)=25.5 \mathrm{~min}, 97: 3$ e.r. 
(R)-3-[3-(1,3-Dioxolan-2-yl)propyl]-1,2,3,4-tetrahydrobenzo[4,5]imidazo[1,2a]pyridine (145f)

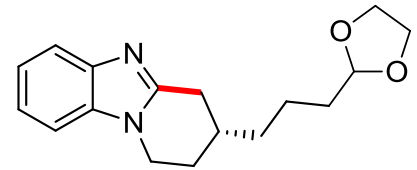

The general procedure GP11 was followed using $144 \mathrm{f}$ (143 $\mathrm{mg}, 0.50 \mathrm{mmol}$, 1.0 equiv). Purification by column chromatography on silica gel ( $n$-hexane/EtOAc $=$ $1 / 1 \rightarrow 0 / 1)$ yielded $145 f(118.5 \mathrm{mg}, 83 \%)$ as a white solid. M. p. $=99-100^{\circ} \mathrm{C}$. ${ }^{1} \mathbf{H}$ NMR $\left(300 \mathrm{MHz}, \mathrm{CDCl}_{3}\right): \delta=7.75-7.61(\mathrm{~m}, 1 \mathrm{H}), 7.35-7.15(\mathrm{~m}, 3 \mathrm{H}), 4.89$ (t, $J=4.6 \mathrm{~Hz}, 1 \mathrm{H}$ ), 4.23 (ddd, $J=12.0,5.7,3.2 \mathrm{~Hz}, 1 \mathrm{H}$ ), 4.04-3.81 (m, 5H), 3.25 (ddd, $J=17.1,4.8,1.7 \mathrm{~Hz}, 1 \mathrm{H}), 2.66(\mathrm{dd}, J=17.1,10.4 \mathrm{~Hz}, 1 \mathrm{H}), 2.32-2.17(\mathrm{~m}, 1 \mathrm{H}), 2.12-$ $1.91(\mathrm{~m}, 1 \mathrm{H}), 1.87-1.65(\mathrm{~m}, 3 \mathrm{H}), 1.65-1.47(\mathrm{~m}, 4 \mathrm{H}) .{ }^{13} \mathrm{C} \mathrm{NMR}\left(125 \mathrm{MHz}, \mathrm{CDCl}_{3}\right)$ : $\delta=151.8\left(\mathrm{C}_{\mathrm{q}}\right), 143.2\left(\mathrm{C}_{\mathrm{q}}\right), 134.6\left(\mathrm{C}_{\mathrm{q}}\right), 122.1(\mathrm{CH}), 121.7(\mathrm{CH}), 119.0(\mathrm{CH}), 108.8$ $(\mathrm{CH}), 104.4(\mathrm{CH}), 65.1\left(\mathrm{CH}_{2}\right), 41.8\left(\mathrm{CH}_{2}\right), 35.5\left(\mathrm{CH}_{2}\right), 34.0\left(\mathrm{CH}_{2}\right), 32.9(\mathrm{CH}), 32.0$ $\left(\mathrm{CH}_{2}\right), 28.8\left(\mathrm{CH}_{2}\right), 21.5\left(\mathrm{CH}_{2}\right)$. IR (ATR): 2922, 2893, 1457, 1415, 1106, 1053, 1021, $741 \mathrm{~cm}^{-1}$. MS (ESI) $\mathrm{m} / \mathrm{z}$ (relative intensity): $287(100)[\mathrm{M}+\mathrm{H}]^{+}$. HR-MS (ESI) $\mathrm{m} / \mathrm{z}$ calcd for $\mathrm{C}_{17} \mathrm{H}_{23} \mathrm{~N}_{2} \mathrm{O}_{2}[\mathrm{M}+\mathrm{H}]^{+}$287.1754, found 287.1753. [ $\left.\alpha\right]_{D}{ }^{20}:+39.1$ (c $=0.42$, $\mathrm{CHCl}_{3}$ ). HPLC separation (Chiralpak ${ }^{\circledR} \mathrm{IC}-3, n$-hexane/PrOH 30:70, $1.0 \mathrm{~mL} / \mathrm{min}$, detection at $273 \mathrm{~nm}): t_{r}($ major $)=12.8 \mathrm{~min}, t_{r}($ minor $)=15.6 \mathrm{~min}, 95: 5$ e.r.

(R)-3-\{3-[(tert-Butyldimethylsilyl)oxy]propyl\}-1,2,3,4-tetrahydrobenzo $[4,5]$ imidazo[1,2-a]pyridine $(145 \mathrm{~g})$

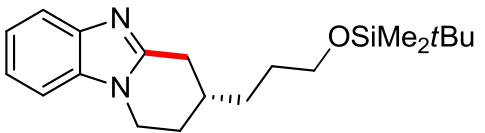

The general procedure GP11 was followed using $\mathbf{1 4 4 \mathrm { g }}(172 \mathrm{mg}, 0.50 \mathrm{mmol}$, 1.0 equiv), Ni(cod) 2 (13.8 mg, $50.0 \mu \mathrm{mol}, 10.0 \mathrm{~mol} \%$ ) and 220 (12.6 mg, $25.0 \mu \mathrm{mol}$, $5.00 \mathrm{~mol} \%)$ in $\mathrm{PhMe}(1.0 \mathrm{~mL})$. Purification by column chromatography on silica gel $(n$-hexane/EtOAc $=3 / 1 \rightarrow 1 / 1.5)$ yielded $145 \mathrm{~g}(149.3 \mathrm{mg}, 87 \%)$ as a white solid. M. p. $=134-135^{\circ} \mathrm{C} .{ }^{1} \mathbf{H}$ NMR $\left(500 \mathrm{MHz}, \mathrm{CDCl}_{3}\right): \delta=7.73-7.64(\mathrm{~m}, 1 \mathrm{H}), 7.33-7.26$ 
(m, 1H), 7.26-7.18 (m, 2H), 4.24 (ddd, $J=12.0,5.7,3.1 \mathrm{~Hz}, 1 \mathrm{H}), 3.95(\mathrm{td}, J=11.4$, $5.0 \mathrm{~Hz}, 1 \mathrm{H}$ ), 3.66 (td, $J=6.3,2.3 \mathrm{~Hz}, 2 \mathrm{H}$ ), 3.25 (ddd, $J=17.1,4.8,1.7 \mathrm{~Hz}, 1 \mathrm{H}$ ), 2.65 (dd, $J=17.1,10.6 \mathrm{~Hz}, 1 \mathrm{H}), 2.23(\mathrm{dtd}, J=12.9,4.9,2.6 \mathrm{~Hz}, 1 \mathrm{H}), 2.08-1.95(\mathrm{~m}, 1 \mathrm{H})$, $1.84-1.75(\mathrm{~m}, 1 \mathrm{H}), 1.72-1.61(\mathrm{~m}, 2 \mathrm{H}), 1.61-1.46(\mathrm{~m}, 2 \mathrm{H}), 0.90(\mathrm{~s}, 9 \mathrm{H}), 0.06(\mathrm{~s}, 6 \mathrm{H})$. ${ }^{13} \mathrm{C}$ NMR $\left(125 \mathrm{MHz}, \mathrm{CDCl}_{3}\right): \delta=151.9\left(\mathrm{C}_{\mathrm{q}}\right), 143.2\left(\mathrm{C}_{\mathrm{q}}\right), 134.6\left(\mathrm{C}_{\mathrm{q}}\right), 122.2(\mathrm{CH})$, $121.8(\mathrm{CH}), 119.1(\mathrm{CH}), 108.9(\mathrm{CH}), 63.1\left(\mathrm{CH}_{2}\right), 41.8\left(\mathrm{CH}_{2}\right), 32.6(\mathrm{CH}), 31.9\left(\mathrm{CH}_{2}\right)$, $31.9\left(\mathrm{CH}_{2}\right), 30.1\left(\mathrm{CH}_{2}\right), 28.9\left(\mathrm{CH}_{2}\right), 26.1\left(\mathrm{CH}_{3}\right), 18.5\left(\mathrm{C}_{\mathrm{q}}\right),-5.1\left(\mathrm{CH}_{3}\right)$. IR (ATR): 2933, 2854, 1457, 1251, 1094, 832, 771, $739 \mathrm{~cm}^{-1}$. MS (ESI) $\mathrm{m} / z$ (relative intensity): $345(100)[\mathrm{M}+\mathrm{H}]^{+}$. HR-MS (ESI) $\mathrm{m} / z$ calcd for $\mathrm{C}_{20} \mathrm{H}_{33} \mathrm{~N}_{2} \mathrm{OSi}[\mathrm{M}+\mathrm{H}]^{+} 345.2357$, found 345.2358. $[\alpha]_{D}{ }^{20}:+37.6\left(\mathrm{c}=0.59, \mathrm{CHCl}_{3}\right)$. HPLC separation (Chiralpak ${ }^{\circledR} \mathrm{IB}-3$, $n$-hexane/THF $80: 20,1.5 \mathrm{~mL} / \mathrm{min}$, detection at $280 \mathrm{~nm}): t_{r}($ major $)=8.4 \mathrm{~min}$, $t_{r}(\operatorname{minor})=10.6 \min , 98: 2$ e.r.

\section{(R)-3-(4-Methylpent-3-en-1-yl)-1,2,3,4-tetrahydrobenzo[4,5]imidazo[1,2-}

\section{a]pyridine (145h)}

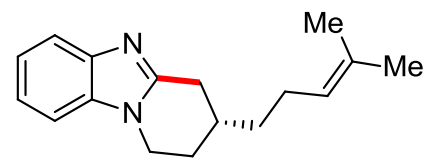

The general procedure GP11 was followed using $144 \mathrm{~h}(127 \mathrm{mg}, 0.50 \mathrm{mmol}$, 1.0 equiv). Purification by column chromatography on silica gel ( $n$-hexane/EtOAc $=$ $3 / 1 \rightarrow 1.5 / 1)$ yielded $145 \mathrm{~h}(106.4 \mathrm{mg}, 84 \%)$ as a white solid. M. p. $=98-99{ }^{\circ} \mathrm{C}$. ${ }^{1} \mathrm{H}$ NMR $\left(300 \mathrm{MHz}, \mathrm{CDCl}_{3}\right): \delta=7.73-7.64(\mathrm{~m}, 1 \mathrm{H}), 7.33-7.18(\mathrm{~m}, 3 \mathrm{H}), 5.17-5.08$ (m, 1H), 4.24 (ddd, $J=12.1,5.7,3.2 \mathrm{~Hz}, 1 \mathrm{H}$ ), 3.96 (td, $J=11.4,5.0 \mathrm{~Hz}, 1 \mathrm{H}$ ), 3.26 (ddd, $J=17.1,4.8,1.7 \mathrm{~Hz}, 1 \mathrm{H}$ ), $2.66(\mathrm{dd}, J=17.1,10.4 \mathrm{~Hz}, 1 \mathrm{H}), 2.28-2.18(\mathrm{~m}, 1 \mathrm{H})$, 2.20-2.07 (m, 2H), 2.08-1.93 (m, 1H), $1.80(\mathrm{dtd}, J=13.4,10.9,5.8 \mathrm{~Hz}, 1 \mathrm{H}), 1.70$ (s, $3 \mathrm{H}), 1.64(\mathrm{~s}, 3 \mathrm{H}), 1.57-1.48(\mathrm{~m}, 2 \mathrm{H}) .{ }^{13} \mathrm{C}$ NMR $\left(100 \mathrm{MHz}, \mathrm{CDCl}_{3}\right): \delta=151.9\left(\mathrm{C}_{\mathrm{q}}\right)$, $143.2\left(\mathrm{C}_{\mathrm{q}}\right), 134.6\left(\mathrm{C}_{\mathrm{q}}\right), 132.3\left(\mathrm{C}_{\mathrm{q}}\right), 123.8(\mathrm{CH}), 122.1(\mathrm{CH}), 121.7(\mathrm{CH}), 119.0(\mathrm{CH})$, $108.8(\mathrm{CH}), 41.7\left(\mathrm{CH}_{2}\right), 35.6\left(\mathrm{CH}_{2}\right), 32.1(\mathrm{CH}), 31.7\left(\mathrm{CH}_{2}\right), 28.8\left(\mathrm{CH}_{2}\right), 25.8\left(\mathrm{CH}_{3}\right)$, $25.2\left(\mathrm{CH}_{2}\right), 17.9\left(\mathrm{CH}_{3}\right)$. IR (ATR): 2957, 2924, 2852, 1486, 1450, 1286, 1228, $742 \mathrm{~cm}^{-1}$. MS (ESI) $\mathrm{m} / \mathrm{z}$ (relative intensity): $255(100)[\mathrm{M}+\mathrm{H}]^{+}$. HR-MS (ESI) $\mathrm{m} / \mathrm{z}$ 
calcd for $\mathrm{C}_{17} \mathrm{H}_{23} \mathrm{~N}_{2}[\mathrm{M}+\mathrm{H}]^{+}$255.1856, found 255.1853. [ $\left.\alpha\right]_{D}^{20}:+45.4$ (c = 1.01, $\mathrm{CHCl}_{3}$ ). HPLC separation (Chiralpak ${ }^{\circledR} \mathrm{IC}-3, n$-hexane/ $/ \mathrm{PrOH} 80: 20,1.0 \mathrm{~mL} / \mathrm{min}$, detection at $273 \mathrm{~nm}): t_{r}($ major $)=13.6 \mathrm{~min}, t_{r}($ minor $)=15.3 \mathrm{~min}, 97: 3$ e.r.

( $R, E)-3-(4,8-D i m e t h y l n o n a-3,7-d i e n-1-y l)-1,2,3,4-t e t r a h y d r o b e n z o$ $[4,5]$ imidazo[1,2-a]pyridine (145i)

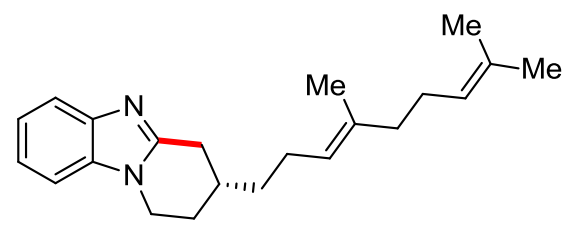

The general procedure GP11 was followed using 144i $(161 \mathrm{mg}, 0.50 \mathrm{mmol}$, 1.0 equiv). Purification by column chromatography on silica gel ( $n$-hexane/EtOAc $=$ $3.5 / 1 \rightarrow 2 / 1$ ) yielded $145 \mathrm{i}(134.6 \mathrm{mg}, 84 \%)$ as a white solid. M. p. $=79-80{ }^{\circ} \mathrm{C}$. ${ }^{1} \mathbf{H}$ NMR $\left(300 \mathrm{MHz}, \mathrm{CDCl}_{3}\right): \delta=7.73-7.64(\mathrm{~m}, 1 \mathrm{H}), 7.33-7.17(\mathrm{~m}, 3 \mathrm{H}), 5.18-5.04$ (m, 2H), 4.23 (ddd, $J=11.9,5.7,3.2 \mathrm{~Hz}, 1 \mathrm{H}$ ), 3.95 (ddd, $J=11.9,10.7,5.0 \mathrm{~Hz}, 1 \mathrm{H}$ ), 3.25 (ddd, $J=17.1,4.8,1.7 \mathrm{~Hz}, 1 \mathrm{H}$ ), 2.66 (dd, $J=17.1,10.4 \mathrm{~Hz}, 1 \mathrm{H}$ ), 2.28-2.19 (m, $1 \mathrm{H}), 2.21-2.09(\mathrm{~m}, 2 \mathrm{H}), 2.10-1.94(\mathrm{~m}, 5 \mathrm{H}), 1.87-1.67(\mathrm{~m}, 1 \mathrm{H}), 1.65(\mathrm{~s}, 3 \mathrm{H}), 1.63(\mathrm{~s}$, $3 \mathrm{H}), 1.59(\mathrm{~s}, 3 \mathrm{H}), 1.58-1.49(\mathrm{~m}, 2 \mathrm{H}) .{ }^{13} \mathrm{C}$ NMR $\left(125 \mathrm{MHz}, \mathrm{CDCl}_{3}\right): \delta=151.9\left(\mathrm{C}_{\mathrm{q}}\right)$, $143.2\left(\mathrm{C}_{\mathrm{q}}\right), 135.9\left(\mathrm{C}_{\mathrm{q}}\right), 134.6\left(\mathrm{C}_{\mathrm{q}}\right), 131.5\left(\mathrm{C}_{\mathrm{q}}\right), 124.3(\mathrm{CH}), 123.6(\mathrm{CH}), 122.1(\mathrm{CH})$, $121.7(\mathrm{CH}), 119.0(\mathrm{CH}), 108.8(\mathrm{CH}), 41.8\left(\mathrm{CH}_{2}\right), 39.9\left(\mathrm{CH}_{2}\right), 35.7\left(\mathrm{CH}_{2}\right), 32.3(\mathrm{CH})$, $31.9\left(\mathrm{CH}_{2}\right), 29.0\left(\mathrm{CH}_{2}\right), 26.9\left(\mathrm{CH}_{2}\right), 25.9\left(\mathrm{CH}_{3}\right), 25.3\left(\mathrm{CH}_{2}\right), 17.9\left(\mathrm{CH}_{3}\right), 16.3\left(\mathrm{CH}_{3}\right)$. IR (ATR): 2962, 2911, 2851, 1510, 1451, 1322, 1107, $742 \mathrm{~cm}^{-1}$. MS (ESI) $\mathrm{m} / \mathrm{z}$ (relative intensity): $323(100)[\mathrm{M}+\mathrm{H}]^{+}$. HR-MS (ESI) $\mathrm{m} / z$ calcd for $\mathrm{C}_{22} \mathrm{H}_{31} \mathrm{~N}_{2}[\mathrm{M}+\mathrm{H}]^{+}$ 323.2482, found 323.2478. [ $\alpha]_{\mathrm{D}}{ }^{20}:+34.2\left(\mathrm{c}=1.22, \mathrm{CHCl}_{3}\right)$. HPLC separation (Chiralpak ${ }^{\circledR}$ IC-3, $n$-hexane/ $\mathrm{PrOH} 80: 20,1.0 \mathrm{~mL} / \mathrm{min}$, detection at $273 \mathrm{~nm}$ ): $t_{r}($ major $)=10.8 \mathrm{~min}, t_{r}($ minor $)=11.9 \min , 99: 1$ e.r. 


\section{(R)-3-(Pent-4-en-1-yl)-1,2,3,4-tetrahydrobenzo[4,5]imidazo[1,2-a]pyridine (145j)}

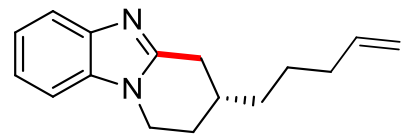

The general procedure GP11 was followed using 144j $(120 \mathrm{mg}, 0.50 \mathrm{mmol}$, 1.0 equiv), Ni(cod) $)_{2}(13.8 \mathrm{mg}, 50.0 \mu \mathrm{mol}, 10.0 \mathrm{~mol} \%$ ) and 220 (12.6 mg, $25.0 \mu \mathrm{mol}$, $5.00 \mathrm{~mol} \%)$ in PhMe (1.0 mL). Purification by column chromatography on silica gel $(n$-hexane/EtOAc $=3 / 1 \rightarrow 1.5 / 1)$ yielded $145 \mathbf{j}(64.3 \mathrm{mg}, 54 \%)$ as a white solid. M. p. $=110-111^{\circ} \mathrm{C} .{ }^{1} \mathrm{H}$ NMR $\left(600 \mathrm{MHz}, \mathrm{CDCl}_{3}\right): \delta=7.71-7.65(\mathrm{~m}, 1 \mathrm{H}), 7.31-7.27$ $(\mathrm{m}, 1 \mathrm{H}), 7.26-7.20(\mathrm{~m}, 2 \mathrm{H}), 5.82$ (ddt, $J=17.1,10.2,6.7 \mathrm{~Hz}, 1 \mathrm{H}$ ), 5.03 (ddt, $J=17.1,1.8,1.6 \mathrm{~Hz}, 1 \mathrm{H}$ ), 4.98 (ddt, $J=10.2,2.3,1.3 \mathrm{~Hz}, 1 \mathrm{H}$ ), 4.23 (ddd, $J=11.9$, 5.7, $3.2 \mathrm{~Hz}, 1 \mathrm{H}$ ), 3.95 (ddd, $J=11.7,10.9,5.0 \mathrm{~Hz}, 1 \mathrm{H}$ ), 3.25 (ddd, $J=17.0,4.8$, $1.7 \mathrm{~Hz}, 1 \mathrm{H}$ ), 2.65 (dd, $J=17.0,10.5 \mathrm{~Hz}, 1 \mathrm{H}), 2.26-2.19(\mathrm{~m}, 1 \mathrm{H}), 2.11$ (q, $J=6.9 \mathrm{~Hz}$, 2H), 2.05-1.96 (m, 1H), 1.79 (dtd, $J=13.5,10.9,5.8 \mathrm{~Hz}, 1 \mathrm{H}), 1.59-1.45(\mathrm{~m}, 4 \mathrm{H})$. ${ }^{13} \mathrm{C}$ NMR $\left(125 \mathrm{MHz}, \mathrm{CDCl}_{3}\right): \delta=151.8\left(\mathrm{C}_{\mathrm{q}}\right), 143.2\left(\mathrm{C}_{\mathrm{q}}\right), 138.5(\mathrm{CH}), 134.6\left(\mathrm{C}_{\mathrm{q}}\right)$, $122.1(\mathrm{CH}), 121.7(\mathrm{CH}), 119.0(\mathrm{CH}), 114.9\left(\mathrm{CH}_{2}\right), 108.8(\mathrm{CH}), 41.8\left(\mathrm{CH}_{2}\right), 35.1$ $\left(\mathrm{CH}_{2}\right), 33.9\left(\mathrm{CH}_{2}\right), 32.8(\mathrm{CH}), 32.0\left(\mathrm{CH}_{2}\right), 28.9\left(\mathrm{CH}_{2}\right), 26.3\left(\mathrm{CH}_{2}\right)$. IR (ATR): 2927, 2856, 1511, 1456, 1414, 1284, 907, $742 \mathrm{~cm}^{-1}$. MS (ESI) $\mathrm{m} / \mathrm{z}$ (relative intensity): 259 (35), 241 (100) $[\mathrm{M}+\mathrm{H}]^{+}$. HR-MS (ESI) $\mathrm{m} / z$ calcd for $\mathrm{C}_{16} \mathrm{H}_{21} \mathrm{~N}_{2}[\mathrm{M}+\mathrm{H}]^{+} 241.1699$, found 241.1697. $[\alpha]_{\mathrm{D}}{ }^{20}:+54.9\left(\mathrm{C}=0.42, \mathrm{CHCl}_{3}\right)$. HPLC separation (Chiralpak ${ }^{\circledR} \mathrm{IC}-3$, $n$-hexane/PrOH 80:20, $1.0 \mathrm{~mL} / \mathrm{min}$, detection at $250 \mathrm{~nm}$ ): $t_{r}$ (major) $=15.4 \mathrm{~min}$, $t_{r}(\operatorname{minor})=18.1 \mathrm{~min}, 97: 3$ e.r.

\section{(R)-3-Ethyl-2,3-dihydro-1 $H$-benzo[d]pyrrolo[1,2-a]imidazole (145q)}

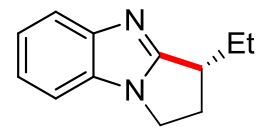

The general procedure GP11 was followed using $144 q$ (93 $\mathrm{mg}, 0.50 \mathrm{mmol}$, 1.0 equiv), Ni(cod) ${ }_{2}(13.8 \mathrm{mg}, 50.0 \mu \mathrm{mol}, 10.0 \mathrm{~mol} \%$ ) and 220 (12.6 mg, $25.0 \mu \mathrm{mol}$, $5.00 \mathrm{~mol} \%)$ in PhMe (1.0 mL). Purification by column chromatography on silica gel 
$(n$-hexane/EtOAc $=1 / 1 \rightarrow 1 / 3)$ yielded $145 q(37.9 \mathrm{mg}, 41 \%)$ as a pale yellow oil. ${ }^{1} \mathrm{H}$ NMR $\left(300 \mathrm{MHz}_{\mathrm{CDCl}}\right): \delta=7.78-7.69(\mathrm{~m}, 1 \mathrm{H}), 7.33-7.17(\mathrm{~m}, 3 \mathrm{H}), 4.12$ (ddd, $J=10.2,8.7,4.2 \mathrm{~Hz}, 1 \mathrm{H}$ ), 4.00 (ddd, $J=10.2,8.1,7.0 \mathrm{~Hz}, 1 \mathrm{H}$ ), 3.28-3.16 (m, 1H), 2.86 (dtd, $J=12.6,8.3,4.2$ Hz, $1 \mathrm{H}$ ), 2.35 (ddt, $J=13.0,8.8,7.0 \mathrm{~Hz}, 1 \mathrm{H}$ ), 2.16-1.92 $(\mathrm{m}, 1 \mathrm{H}), 1.72(\mathrm{dp}, J=13.6,7.5 \mathrm{~Hz}, 1 \mathrm{H}), 1.12(\mathrm{t}, J=7.4 \mathrm{~Hz}, 3 \mathrm{H}) .{ }^{13} \mathrm{C}$ NMR $(125 \mathrm{MHz}$, $\left.\mathrm{CDCl}_{3}\right): \delta=163.6\left(\mathrm{C}_{\mathrm{q}}\right), 148.6\left(\mathrm{C}_{\mathrm{q}}\right), 132.2\left(\mathrm{C}_{\mathrm{q}}\right), 121.8(\mathrm{CH}), 121.6(\mathrm{CH}), 119.7(\mathrm{CH})$, $109.5(\mathrm{CH}), 42.1\left(\mathrm{CH}_{2}\right), 37.7(\mathrm{CH}), 32.9\left(\mathrm{CH}_{2}\right), 26.3\left(\mathrm{CH}_{2}\right), 11.9\left(\mathrm{CH}_{3}\right)$. IR (ATR): 2962, 2931, 1524, 1451, 1415, 1277, 908, $730 \mathrm{~cm}^{-1}$. MS (ESI) $\mathrm{m} / z$ (relative intensity): 187 (100) $[\mathrm{M}+\mathrm{H}]^{+}$. HR-MS (ESI) $\mathrm{m} / z$ calcd for $\mathrm{C}_{12} \mathrm{H}_{15} \mathrm{~N}_{2}[\mathrm{M}+\mathrm{H}]^{+}$187.1230, found 187.1231. $[\alpha]_{D}{ }^{20}:-1.1\left(c=0.60, \mathrm{CHCl}_{3}\right)$. HPLC separation (Chiralpak ${ }^{\circledR}$ IC-3, n-hexane/iPrOH 80:20, $1.0 \mathrm{~mL} / \mathrm{min}$, detection at $250 \mathrm{~nm}$ ): $t_{r}$ (major) $=10.7 \mathrm{~min}$, $t_{r}($ minor $)=15.0 \mathrm{~min}, 97: 3$ e.r.

(R)-3-Propyl-2,3-dihydro-1H-benzo[d]pyrrolo[1,2-a]imidazole (145r)

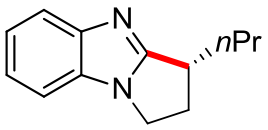

The general procedure GP11 was followed using 144r (100 mg, $0.50 \mathrm{mmol}$, 1.0 equiv), Ni(cod) $)_{2}(13.8 \mathrm{mg}, 50.0 \mu \mathrm{mol}, 10.0 \mathrm{~mol} \%$ ) and 220 (12.6 mg, $25.0 \mu \mathrm{mol}$, $5.00 \mathrm{~mol} \%)$ in PhMe (1.0 mL). Purification by column chromatography on silica gel $(n$-hexane/EtOAc $=2 / 1 \rightarrow 1 / 1.5)$ yielded $145 \mathrm{r}(16.4 \mathrm{mg}, 16 \%)$ as a white solid. M. p. $=63-65^{\circ} \mathrm{C} .{ }^{1} \mathbf{H}$ NMR $\left(300 \mathrm{MHz} \mathrm{CDCl}_{3}\right): \delta=7.77-7.67(\mathrm{~m}, 1 \mathrm{H}), 7.34-7.24(\mathrm{~m}$, $1 \mathrm{H}), 7.24-7.16(\mathrm{~m}, 2 \mathrm{H}), 4.13$ (ddd, $J=10.2,8.7,4.2 \mathrm{~Hz}, 1 \mathrm{H}), 4.00$ (ddd, $J=10.1$, 8.0, 7.0 Hz, 1H), 3.27 (tdd, $J=8.1,7.1,5.8 \mathrm{~Hz}, 1 \mathrm{H}$ ), 2.86 (dtd, $J=12.6,8.2,4.2 \mathrm{~Hz}$, $1 \mathrm{H}), 2.34$ (ddt, $J=12.9,8.7,7.0 \mathrm{~Hz}, 1 \mathrm{H}), 2.10-1.91(\mathrm{~m}, 1 \mathrm{H}), 1.71-1.42(\mathrm{~m}, 3 \mathrm{H})$, $0.99(\mathrm{t}, J=7.2 \mathrm{~Hz}, 3 \mathrm{H}) .{ }^{13} \mathrm{C}$ NMR $\left(100 \mathrm{MHz}, \mathrm{CDCl}_{3}\right): \delta=164.0\left(\mathrm{C}_{\mathrm{q}}\right), 148.8\left(\mathrm{C}_{\mathrm{q}}\right)$, $132.3\left(\mathrm{C}_{\mathrm{q}}\right), 121.8(\mathrm{CH}), 121.7(\mathrm{CH}), 119.8(\mathrm{CH}), 109.5(\mathrm{CH}), 42.1\left(\mathrm{CH}_{2}\right), 36.1(\mathrm{CH})$, $35.5\left(\mathrm{CH}_{2}\right), 33.3\left(\mathrm{CH}_{2}\right), 20.7\left(\mathrm{CH}_{2}\right), 14.1\left(\mathrm{CH}_{3}\right)$. IR (ATR): 2955, 2929, 1522, 1451, 1411, 1275, 1217, $740 \mathrm{~cm}^{-1}$. MS (ESI) $\mathrm{m} / \mathrm{z}$ (relative intensity): 201 (100) $[\mathrm{M}+\mathrm{H}]^{+}$. HR-MS (ESI) $\mathrm{m} / z$ calcd for $\mathrm{C}_{13} \mathrm{H}_{17} \mathrm{~N}_{2}[\mathrm{M}+\mathrm{H}]^{+}$201.1386, found 201.1386. [ $\left.\alpha\right]_{\mathrm{D}}{ }^{20}:-0.4$ 
(c $=0.38, \mathrm{CHCl}_{3}$ ). HPLC separation (Chiralpak ${ }^{\circledR} \mathrm{IC}-3, n$-hexane/PrOH 80:20, $1.0 \mathrm{~mL} / \mathrm{min}$, detection at $250 \mathrm{~nm}$ ): $t_{r}($ major $)=9.7 \mathrm{~min}, t_{r}($ minor $)=13.1 \mathrm{~min}, 96: 4$ e.r.

The analytical data are in accordance with those previously reported in the literature. ${ }^{[206]}$

\section{Alkene Isomerization}
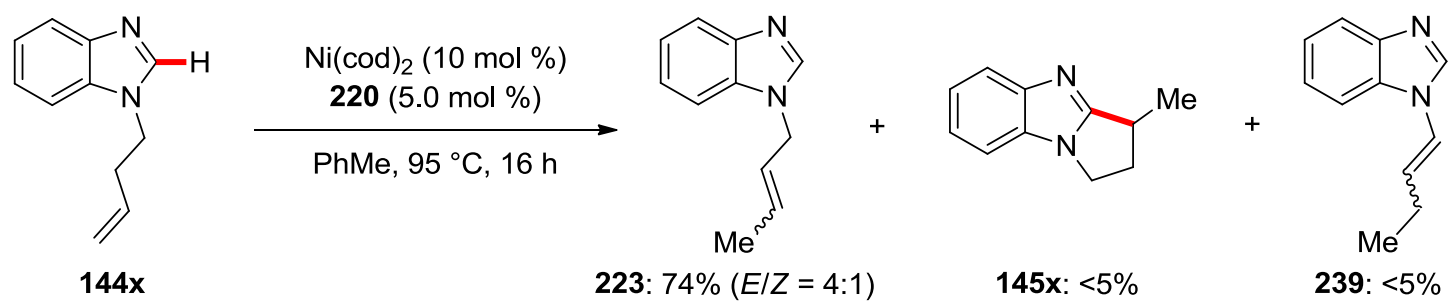

The general procedure GP11 was followed using 144x $(86 \mathrm{mg}, 0.50 \mathrm{mmol}$, 1.0 equiv), Ni(cod) $)_{2}(13.8 \mathrm{mg}, 50.0 \mu \mathrm{mol}, 10.0 \mathrm{~mol} \%$ ) and 220 (12.6 mg, $25.0 \mu \mathrm{mol}$, $5.00 \mathrm{~mol} \%)$ in PhMe $(1.0 \mathrm{~mL})$. Purification by column chromatography on silica gel $(n$-hexane/EtOAc $=1.5 / 1 \rightarrow 1 / 3)$ yielded $223(63.8 \mathrm{mg}, 74 \%)$ as a pale yellow oil. The $\mathrm{E} / \mathrm{Z}$ ratio was determined by ${ }^{1} \mathrm{H}$ NMR analysis of the crude product.

\section{1-(But-2-en-1-yl)-1H-benzo[d]imidazole (223)}

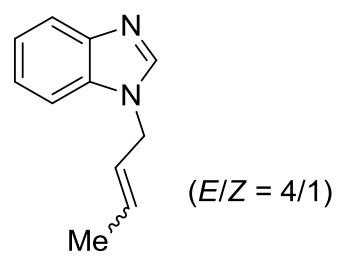

${ }^{1} \mathrm{H}$ NMR $\left(600 \mathrm{MHz}, \mathrm{CDCl}_{3}\right): \delta=7.88(\mathrm{~s}, 1 \mathrm{H}), 7.82-7.78(\mathrm{~m}, 1 \mathrm{H}), 7.39-7.35(\mathrm{~m}, 1 \mathrm{H})$, 7.31-7.24 (m, 2H), 5.86-5.79 (m, 0.16H, Z-isomer), 5.77-5.69 (m, 0.84H, E-isomer), 5.67-5.57 (m, 1H), 4.78 (ddd, $J=6.8,1.7,0.9 \mathrm{~Hz}, 0.34 \mathrm{H}, Z$-isomer), 4.70-4.66 (m, $1.70 \mathrm{H}, E$-isomer), 1.84 (ddt, $J=7.0,1.9,0.9 \mathrm{~Hz}, 0.50 \mathrm{H}, Z$-isomer), 1.72 (dq, $J=6.2$, $1.3 \mathrm{~Hz}, 2.51 \mathrm{H}, E$-isomer). ${ }^{13} \mathrm{C} \mathrm{NMR}\left(75 \mathrm{MHz}, \mathrm{CDCl}_{3}\right): \delta=144.1\left(\mathrm{C}_{\mathrm{q}}\right.$, both isomers), $142.9\left(\mathrm{CH}\right.$, both isomers), $142.7\left(\mathrm{C}_{\mathrm{q}}\right.$, both isomers $), 134.0(\mathrm{CH}, \mathrm{Z}$-isomer $), 130.7$ 
(CH, E-isomer), 129.7 ( $\mathrm{CH}, Z$-isomer), $124.9(\mathrm{CH}, E$-isomer), $124.0(\mathrm{CH}, Z$-isomer $)$, 122.9 ( $\mathrm{CH}, E$-isomer), $122.2(\mathrm{CH}, Z$-isomer $), 122.1(\mathrm{CH}, E$-isomer $), 120.5(\mathrm{CH}$, $Z$-isomer), 120.5 ( $\mathrm{CH}, E$-isomer), 110.0 ( $\mathrm{CH}, E$-isomer), 109.8 ( $\mathrm{CH}, Z$-isomer), 47.0 ( $\mathrm{CH}_{2}, E$-isomer), 41.8 ( $\mathrm{CH}_{2}, Z$-isomer $), 17.7\left(\mathrm{CH}_{3}, E\right.$-isomer $), 13.3\left(\mathrm{CH}_{3}\right.$, Z-isomer $)$. IR (ATR): 2917, 1493, 1458, 1285, 1260, 1198, 963, $743 \mathrm{~cm}^{-1}$. MS (ESI) $\mathrm{m} / \mathrm{z}$ (relative intensity): 195 (3) $[\mathrm{M}+\mathrm{Na}]^{+}, 173(100)[\mathrm{M}+\mathrm{H}]^{+}, 119$ (18). HR-MS (ESI) m/z calcd for $\mathrm{C}_{11} \mathrm{H}_{13} \mathrm{~N}_{2}[\mathrm{M}+\mathrm{H}]^{+}$173.1073, found 173.1072.

The analytical data for the major $E$-isomer are in accordance with those previously reported in the literature. ${ }^{[340]}$

\section{Alkene Isomerization with D-Labeling}

The general procedure GP11 was followed using $\left[\mathrm{D}_{1}-1\right.$ 144x $(86 \mathrm{mg}, 0.50 \mathrm{mmol}$, 1.0 equiv), Ni(cod) $)_{2}(13.8 \mathrm{mg}, 50.0 \mu \mathrm{mol}, 10.0 \mathrm{~mol} \%$ ) and 220 (12.6 mg, $25.0 \mu \mathrm{mol}$, $5.00 \mathrm{~mol} \%)$ in PhMe $(1.0 \mathrm{~mL})$. Purification by column chromatography on silica gel $(n$-hexane/EtOAc $=1.5 / 1 \rightarrow 1 / 3)$ yielded $[\mathrm{D}]_{\mathrm{n}}-223(66.1 \mathrm{mg}, 76 \%)$ as a pale yellow oil. The $E / Z$ ratio was determined by ${ }^{1} \mathrm{H} N \mathrm{NM}$ analysis of the crude product. Deuterium incorporation was determined by ${ }^{1} \mathrm{H}$ NMR spectroscopic analysis of the isolated product.

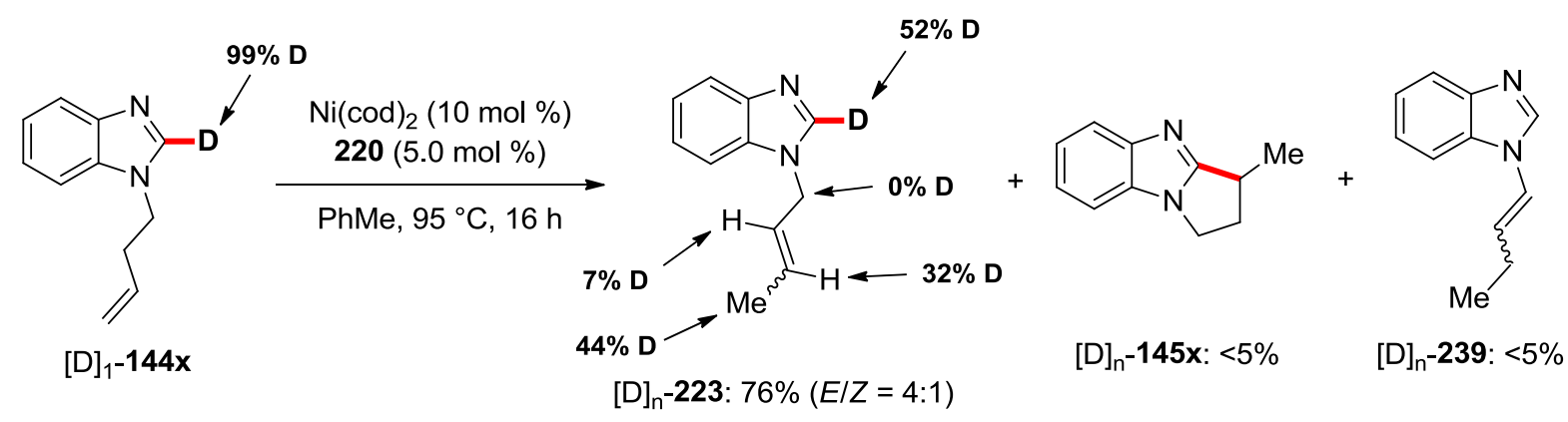



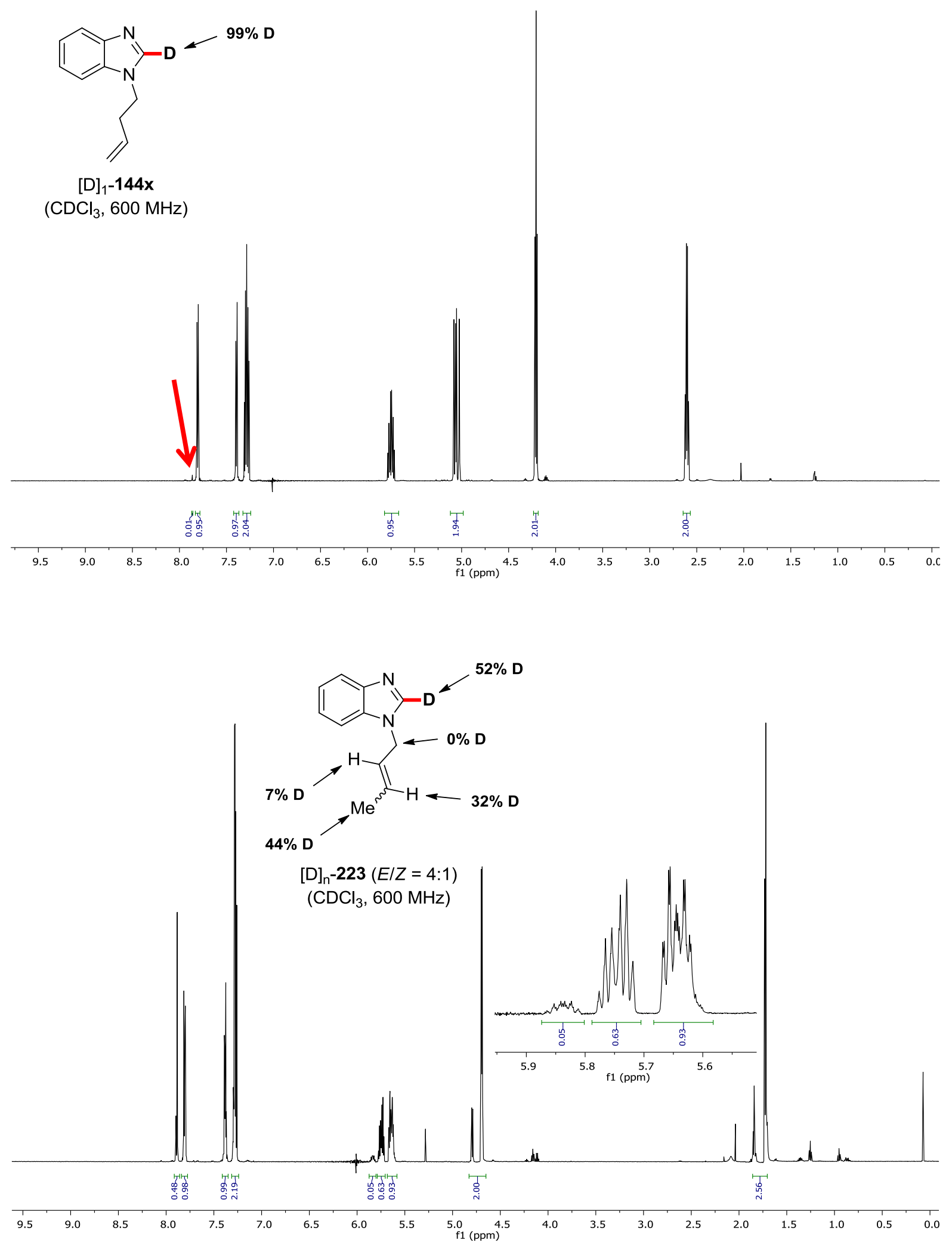
5.6.4. Mechanistic Studies for the Asymmetric Intramolecular Nickel-Catalyzed Hydroarylation of Alkenes

\section{Non-Linear Effect Studies}

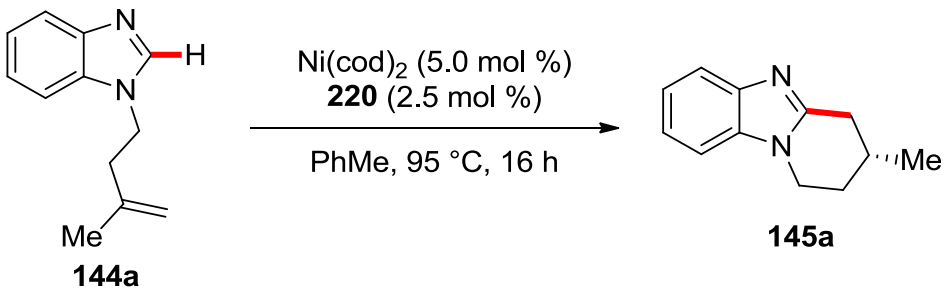

Inside a nitrogen-filled glovebox, an oven-dried $25 \mathrm{~mL}$ Schlenk tube was charged with a mixture of stock solutions of 220 and ent-220 in PhMe (both $\mathrm{c}=6.90 \mathrm{mg} / \mathrm{mL}$ ) for a total volume of $1.0 \mathrm{~mL}$. Ni(cod) $)_{2}(6.9 \mathrm{mg}, 25 \mu \mathrm{mol}, 5.0 \mathrm{~mol} \%)$, and substrate 144a (93 mg, $0.50 \mathrm{mmol}, 1.0$ equiv) were then added. The Schlenk tube was closed with a rubber septum, taken out of the glovebox, and placed in a pre-heated oil bath at $95{ }^{\circ} \mathrm{C}$. The reaction mixture was stirred at $95^{\circ} \mathrm{C}$ for $16 \mathrm{~h}$, then cooled to $23^{\circ} \mathrm{C}$ and diluted with EtOAc $(5.0 \mathrm{~mL})$. The mixture was filtered through a short plug of silica gel, rinsed with EtOAc $(4 \times 10 \mathrm{~mL})$ and concentrated in vacuo. The conversion was determined by ${ }^{1} \mathrm{H}$ NMR using 1,1,2,2-tetrachloroethane as an internal standard. Enantiomeric excesses were measured by chiral HPLC analysis of the crude product (Chiralpak ${ }^{\circledR}$ IC-3, $n$-hexane/PrOH 80:20, $1.0 \mathrm{~mL} / \mathrm{min}$ ).

Table 5.6. Non-Linear Effect Studies.

\begin{tabular}{cccc}
\hline Entry & $\boldsymbol{e e ( 2 2 0 ) ^ { [ \mathrm { a } ] }}$ & $\boldsymbol{e e}(\mathbf{1 4 4 a})^{[\mathrm{b}]}$ & Yield $^{[\mathrm{c}]}$ \\
\hline 1 & $0 \%$ & -0.6 & $>98 \%$ \\
2 & $10 \%$ & 9.8 & $>98 \%$ \\
3 & $20 \%$ & 19.8 & $>98 \%$ \\
4 & $30 \%$ & 29.0 & $>98 \%$ \\
5 & $40 \%$ & 38.6 & $>98 \%$ \\
\hline
\end{tabular}




\begin{tabular}{cccc}
\hline 6 & $50 \%$ & 49.2 & $>98 \%$ \\
7 & $60 \%$ & 58.4 & $>98 \%$ \\
8 & $70 \%$ & 67.2 & $>98 \%$ \\
9 & $80 \%$ & 77.2 & $>98 \%$ \\
10 & $90 \%$ & 88.0 & $>98 \%$ \\
11 & $100 \%$ & 95.0 & $>98 \%$ \\
\hline
\end{tabular}

${ }^{[a]}$ Prepared by mixing two stock solutions of each enantiomer of pre-ligand $\mathbf{2 2 0}$.

${ }^{[b]}$ Determined by chiral HPLC analysis of the crude reaction mixture.

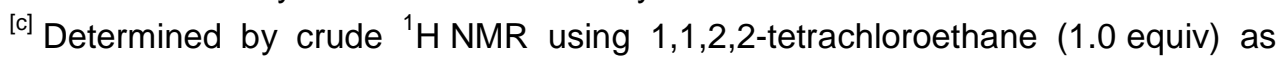
internal standard.

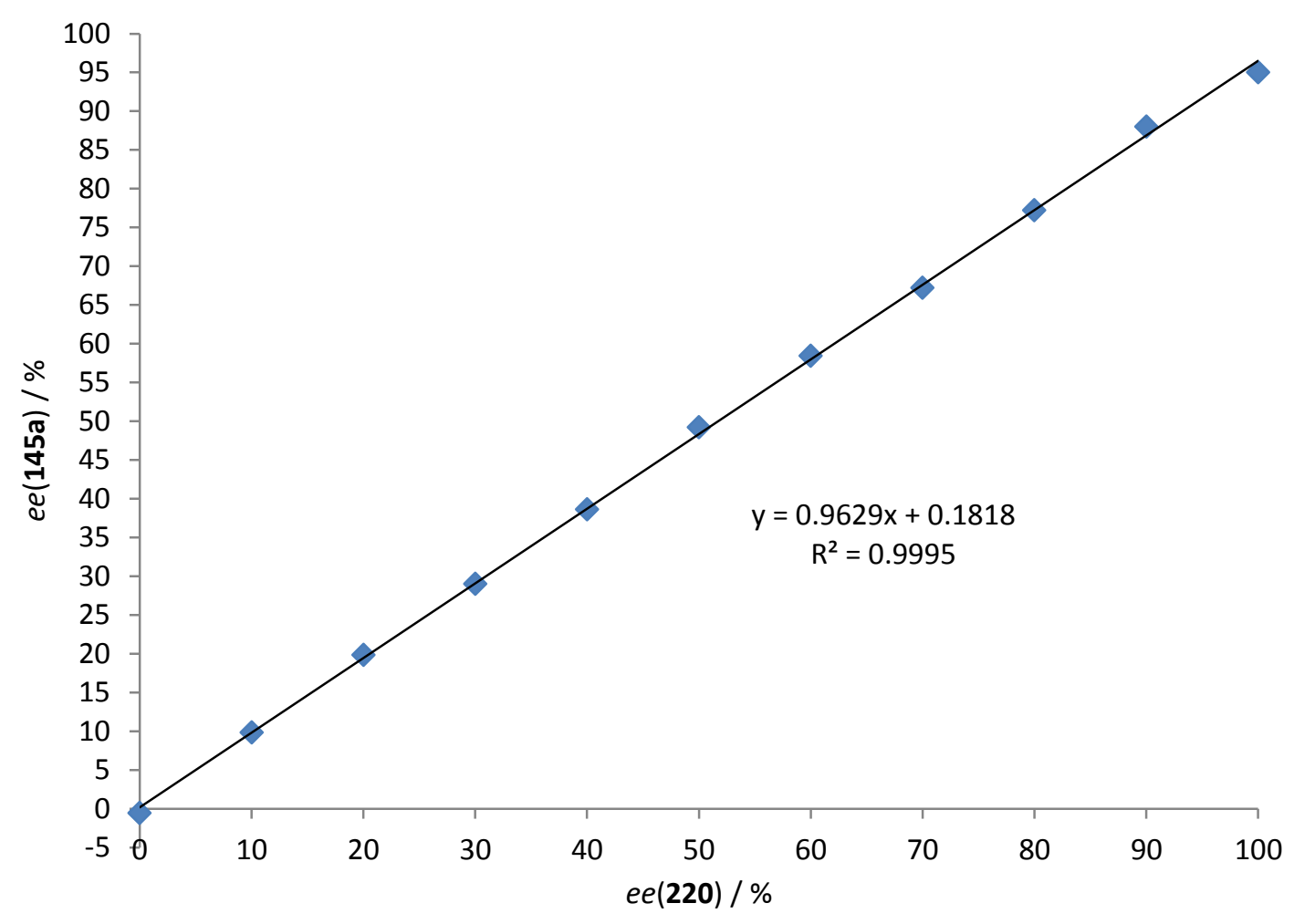

Figure 5.3. Absence of a non-linear effect. 


\subsubsection{Product Diversification}

(R)-3,5-Dimethyl-1,2,3,4-tetrahydrobenzo[4,5]imidazo[1,2-a]pyridin-5-ium lodide (230)

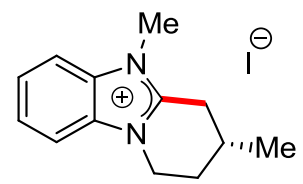

Following a modified procedure ${ }^{[341]}$ 145a $(75 \mathrm{mg}, 0.40 \mathrm{mmol}, 1.0$ equiv, $>99: 1$ e.r. $)$ was dissolved in methyl iodide $(1.5 \mathrm{~mL})$ and the resulting mixture was stirred for $16 \mathrm{~h}$. The resulting suspension was diluted with $\mathrm{Et}_{2} \mathrm{O}(15 \mathrm{~mL})$. The precipitate was collected by filtration, washed with $\mathrm{Et}_{2} \mathrm{O}(3 \times 5 \mathrm{~mL})$ and dried in vacuo to provide benzimidazolium iodide $230(123.5 \mathrm{mg}, 93 \%)$ as a white powder. M. p. $=151^{\circ} \mathrm{C}$ (decomposition). ${ }^{1} \mathbf{H}$ NMR $\left(300 \mathrm{MHz}, \mathrm{CDCl}_{3}\right): \delta=7.72-7.61(\mathrm{~m}, 2 \mathrm{H}), 7.62-7.48(\mathrm{~m}$, 2H), 4.47 (ddd, $J=12.7,5.7,2.5 \mathrm{~Hz}, 1 \mathrm{H}$ ), 4.28 (td, $J=11.9,4.8 \mathrm{~Hz}, 1 \mathrm{H}$ ), 4.02 (s, $3 \mathrm{H}$ ), 3.73 (ddd, $J=18.1,5.4,1.0 \mathrm{~Hz}, 1 \mathrm{H}$ ), 3.04 (dd, $J=18.2,10.3 \mathrm{~Hz}, 1 \mathrm{H}$ ), 2.67$2.49(\mathrm{~m}, 1 \mathrm{H}), 2.36-2.25(\mathrm{~m}, 1 \mathrm{H}), 2.02(\mathrm{dtd}, J=14.0,11.4,5.6 \mathrm{~Hz}, 1 \mathrm{H}), 1.27(\mathrm{~d}$, $J=6.7 \mathrm{~Hz}, 3 \mathrm{H}) .{ }^{13} \mathrm{C}$ NMR $\left(125 \mathrm{MHz}, \mathrm{CDCl}_{3}\right): \delta=151.0\left(\mathrm{C}_{\mathrm{q}}\right), 131.9\left(\mathrm{C}_{\mathrm{q}}\right), 131.0\left(\mathrm{C}_{\mathrm{q}}\right)$, $126.8(\mathrm{CH}), 126.4(\mathrm{CH}), 112.5(\mathrm{CH}), 112.3(\mathrm{CH}), 43.8\left(\mathrm{CH}_{2}\right), 32.7(\mathrm{CH}), 30.8\left(\mathrm{CH}_{2}\right)$, $28.9\left(\mathrm{CH}_{2}\right), 25.7\left(\mathrm{CH}_{3}\right), 21.0\left(\mathrm{CH}_{3}\right)$. IR (ATR): 3017, 2955, 2909, 1537, 1470, 760, $427 \mathrm{~cm}^{-1}$. MS (ESI) $\mathrm{m} / \mathrm{z}$ (relative intensity): 201 (100) [M-I] . HR-MS (ESI) $\mathrm{m} / z$ calcd

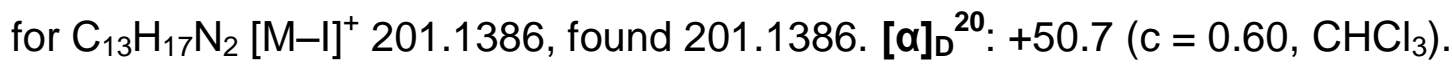

Crystals suitable for X-ray crystallography were grown by vapor diffusion from $\mathrm{Et}_{2} \mathrm{O}$ into a saturated solution of 230 in $\mathrm{EtOH}$.

\section{3,5-Dimethyl-N-phenyl-1,2,3,5-tetrahydrobenzo[4,5]imidazo[1,2-a]pyridine-4- carboxamide (231)}

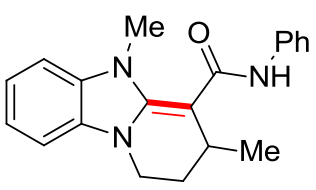


Following a modified procedure, ${ }^{[307 a]}$ benzimidazolium iodide 230 (200 mg, $0.61 \mathrm{mmol}$, 1.0 equiv, >99:1 e.r.), potassium hydride $(29.3 \mathrm{mg}, \quad 0.73 \mathrm{mmol}$, 1.2 equiv), and potassium tert-butoxide ( $1.4 \mathrm{mg}, 12 \mu \mathrm{mol}, 2.0 \mathrm{~mol} \%$ ) were combined with THF $(3.5 \mathrm{~mL})$ inside a nitrogen-filled glovebox. The suspension was stirred at $23^{\circ} \mathrm{C}$ for $20 \mathrm{~h}$, and then filtered through a short plug of Celite ${ }^{\circledR}$. The filter cake was washed with THF $(3 \times 5.0 \mathrm{~mL})$. The filtrate was cooled to $0^{\circ} \mathrm{C}$, and phenyl isocyanate (109 mg, $0.91 \mathrm{mmol}, 1.5$ equiv) was added dropwise under inert atmosphere. The resulting solution was stirred for $1 \mathrm{~h}$ at $0^{\circ} \mathrm{C}$; then at $23^{\circ} \mathrm{C}$ for $16 \mathrm{~h}$. The reaction mixture was concentrated under reduced pressure, and the residue was recrystallized from $\mathrm{Et}_{2} \mathrm{O} / \mathrm{MeOH}=25 / 1$ at $-30^{\circ} \mathrm{C}$ to provide $231(140 \mathrm{mg}, 72 \%$ ) as a pale yellow solid. M. p. $=168{ }^{\circ} \mathrm{C}$ (decomposition). ${ }^{1} \mathbf{H}$ NMR $\left(300 \mathrm{MHz}, \mathrm{C}_{6} \mathrm{D}_{6}\right)$ : $\delta=7.81(\mathrm{~d}, J=7.9 \mathrm{~Hz}, 2 \mathrm{H}), 7.24(\mathrm{dd}, J=8.5,7.3 \mathrm{~Hz}, 2 \mathrm{H}), 6.97-6.81(\mathrm{~m}, 3 \mathrm{H}), 6.74$ (brs, 1H), 6.52-6.40 (m, 2H), $3.18(\mathrm{~s}, 3 \mathrm{H}), 3.15-3.05(\mathrm{~m}, 1 \mathrm{H}), 2.95-2.78(\mathrm{~m}, 2 \mathrm{H})$, 1.43 (tt, $J=12.7,5.1 \mathrm{~Hz}, 1 \mathrm{H}), 1.30-1.18(\mathrm{~m}, 1 \mathrm{H}), 1.01(\mathrm{~d}, J=6.8 \mathrm{~Hz}, 3 \mathrm{H}) .{ }^{13} \mathbf{C}$ NMR $\left(75 \mathrm{MHz}, \mathrm{CDCl}_{3}\right): \delta=165.7\left(\mathrm{C}_{\mathrm{q}}\right), 150.7\left(\mathrm{C}_{\mathrm{q}}\right), 140.2\left(\mathrm{C}_{\mathrm{q}}\right), 135.9\left(\mathrm{C}_{\mathrm{q}}\right), 133.0\left(\mathrm{C}_{\mathrm{q}}\right)$, $129.1(\mathrm{CH}), 122.2(\mathrm{CH}), 121.5(\mathrm{CH}), 121.1(\mathrm{CH}), 119.1(\mathrm{CH}), 108.0(\mathrm{CH}), 105.9$ $(\mathrm{CH}), 79.2\left(\mathrm{C}_{\mathrm{q}}\right)$, $37.6\left(\mathrm{CH}_{2}\right), 35.9\left(\mathrm{CH}_{3}\right), 28.7(\mathrm{CH}), 28.5\left(\mathrm{CH}_{2}\right), 21.8\left(\mathrm{CH}_{3}\right)$. IR (ATR): 3341, 2953, 1539, 1495, 1428, 1307, 1188, 1147, $731 \mathrm{~cm}^{-1}$. MS (ESI) $\mathrm{m} / z$ (relative intensity): 320 (100) $[\mathrm{M}+\mathrm{H}]^{+}$. HR-MS (ESI) $\mathrm{m} / z$ calcd for $\mathrm{C}_{20} \mathrm{H}_{22} \mathrm{~N}_{3} \mathrm{O}[\mathrm{M}+\mathrm{H}]^{+}$ 320.1757, found 320.1758. HPLC separation (Chiralpak ${ }^{\circledR}$ IA-3, $n$-hexane/PrOH $70: 30,1.0 \mathrm{~mL} / \mathrm{min}$, detection at $273 \mathrm{~nm}): t_{r}($ major $)=9.4 \mathrm{~min}, t_{r}($ minor $)=11.2 \mathrm{~min}$, $53: 47$ e.r.

(R)-Methyl 2-(3-Methyl-1,2,3,5-tetrahydrobenzo[4,5]imidazo[1,2-a]pyridin-4-yl)2-oxoacetate (232)

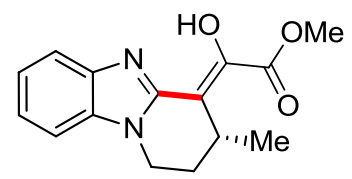

Following a modified procedure, ${ }^{[308]}$ methyl 2-chloro-2-oxoacetate $(122 \mu \mathrm{L}$, $1.34 \mathrm{mmol}, 1.50$ equiv) was added dropwise to an ice-cold solution of $145 \mathrm{a}$ (166 mg, 
$0.89 \mathrm{mmol}, 1.0$ equiv, $>99: 1$ e.r. $)$ and $\mathrm{Et}_{3} \mathrm{~N}(621 \mu \mathrm{L}, 4.46 \mathrm{mmol}, 5.00$ equiv) in acetonitrile $(9.0 \mathrm{~mL})$. The resulting suspension was stirred at $0{ }^{\circ} \mathrm{C}$ for $2 \mathrm{~h}$, then at $23^{\circ} \mathrm{C}$ for $18 \mathrm{~h}$. The mixture was diluted with EtOAc $(20 \mathrm{~mL})$ and filtered through a short plug of Celite ${ }^{\circledR}$, which was rinsed with EtOAc $(3 \times 20 \mathrm{~mL})$. The volatiles were removed under reduced pressure, and the residue was purified by column chromatography ( $n$-hexane/EtOAc $=5 / 1 \rightarrow 2 / 1)$ to provide $232(126 \mathrm{mg}, 52 \%)$ as a yellow solid. M. p. $=137-139{ }^{\circ} \mathrm{C} .{ }^{1} \mathbf{H}$ NMR $\left(500 \mathrm{MHz}, \mathrm{CDCl}_{3}\right): \delta=13.93$ (brs, $\left.1 \mathrm{H}\right)$, 7.66-7.60 (m, 1H), 7.36-7.32 (m, 1H), 7.32-7.28 (m, 2H), 4.21 (ddd, $J=12.3,5.6$, $1.7 \mathrm{~Hz}, 1 \mathrm{H}$ ), 4.09 (td, $J=12.6,4.2 \mathrm{~Hz}, 1 \mathrm{H}$ ), 3.90 (s, 3H), 3.86 (tdd, $J=7.1,5.2$, $3.3 \mathrm{~Hz}, 1 \mathrm{H}$ ), 2.14 (tdd, $J=13.0,5.6,4.1 \mathrm{~Hz}, 1 \mathrm{H}$ ), 2.05 (dddd, $J=13.7,4.5,2.9$, $1.7 \mathrm{~Hz}, 1 \mathrm{H}), 1.27(\mathrm{~d}, J=7.0 \mathrm{~Hz}, 3 \mathrm{H}) .{ }^{13} \mathrm{C}$ NMR $\left(125 \mathrm{MHz}, \mathrm{CDCl}_{3}\right): \delta=164.7\left(\mathrm{C}_{\mathrm{q}}\right)$, $152.6\left(\mathrm{C}_{\mathrm{q}}\right), 150.3\left(\mathrm{C}_{\mathrm{q}}\right), 137.9\left(\mathrm{C}_{\mathrm{q}}\right), 132.9\left(\mathrm{C}_{\mathrm{q}}\right), 123.4(\mathrm{CH}), 123.3(\mathrm{CH}), 117.0(\mathrm{CH})$, $109.1(\mathrm{CH}), 106.4\left(\mathrm{C}_{\mathrm{q}}\right), 52.3\left(\mathrm{CH}_{3}\right), 37.9\left(\mathrm{CH}_{2}\right), 28.5\left(\mathrm{CH}_{2}\right), 26.0(\mathrm{CH}), 19.8\left(\mathrm{CH}_{3}\right)$. IR (ATR): 2951, 1720, 1536, 1292, 1253, 1211, 1153, $748 \mathrm{~cm}^{-1}$. MS (ESI) $\mathrm{m} / \mathrm{z}$ (relative intensity): $567(17)[2 \mathrm{M}+\mathrm{Na}]^{+}, 295(48)[\mathrm{M}+\mathrm{Na}]^{+}, 273(100)[\mathrm{M}+\mathrm{H}]^{+}, 213(7)$ $\left[\mathrm{M}-\mathrm{CO}_{2} \mathrm{Me}\right]^{+}$. HR-MS (ESI) $\mathrm{m} / \mathrm{z}$ calcd for $\mathrm{C}_{15} \mathrm{H}_{17} \mathrm{~N}_{2} \mathrm{O}_{3}[\mathrm{M}+\mathrm{H}]^{+}$273.1234, found 273.1236. $[\alpha]_{D}{ }^{20}:-0.76\left(\mathrm{c}=1.00, \mathrm{CHCl}_{3}\right)$. HPLC separation (Chiralpak ${ }^{\circledR} \mathrm{IC}-3$, $n$-hexane/iPrOH 25:75, $0.50 \mathrm{~mL} / \mathrm{min}$, detection at $290 \mathrm{~nm}$ ): $t_{r}$ (major) $=31.2 \mathrm{~min}$, $t_{r}(\operatorname{minor})=41.5 \mathrm{~min},>99: 1$ e.r.

Crystals suitable for $\mathrm{X}$-ray crystallography were grown by slow evaporation from a solution of rac-232 in $\mathrm{PrOH} / \mathrm{CH}_{2} \mathrm{Cl}_{2}=25 / 1$. 


\subsection{Crystallographic Data}

The crystal structures of 192ca and 197 were measured and solved by $\mathrm{H}$. Keil (Stalke research group).

The data were collected from a shock-cooled crystal at 100(2) K on a 'BRUKER D8' three circle diffractometer equipped with an INCOATEC Mo Microsource with mirror optics (MoK $\mathrm{K}_{\alpha}$ radiation, $\lambda=0.71073 \AA$ ). The data were integrated with SAINT. ${ }^{\left[{ }^{342]} \mathrm{A}\right.}$ multi-scan absorption correction and a $3 \lambda$ correction $^{[343]}$ was applied using SADABS. ${ }^{[344]}$ The structures were solved by SHELXT ${ }^{[345]}$ and refined on $F^{2}$ using SHELXL ${ }^{[346]}$ in the graphical user interface SHELXLE. ${ }^{[347]}$

Table 5.7. Crystal data and structure refinement of $192 \mathrm{ca}$ and 197.

\begin{tabular}{|c|c|c|}
\hline Compound & $192 \mathrm{ca}$ & 197 \\
\hline CCDC number & 1559085 & 1559086 \\
\hline Empirical formula & $\mathrm{C}_{28} \mathrm{H}_{25} \mathrm{FeNO}$ & $\mathrm{C}_{31} \mathrm{H}_{27} \mathrm{BrN}_{2} \mathrm{O}$ \\
\hline Formula weight & 447.34 & 523.45 \\
\hline Temperature $[\mathrm{K}]$ & $100(2)$ & $100(2)$ \\
\hline Wavelength $[\AA ̊]$ & 0.71073 & 0.71073 \\
\hline Crystal system & Orthorhombic & Orthorhombic \\
\hline Space group & $\mathrm{P} 2{ }_{1} 2_{1} 2_{1}$ & $\mathrm{P} 22_{1} 2_{1} 2_{1}$ \\
\hline$a[\AA ̊]$ & $11.061(2)$ & $9.643(2)$ \\
\hline $\mathrm{b}[\AA \AA]$ & $11.652(2)$ & $10.147(2)$ \\
\hline$c[\AA]$ & $16.748(3)$ & $25.036(3)$ \\
\hline$a\left[^{\circ}\right]$ & 90 & 90 \\
\hline $\mathrm{b}\left[^{\circ}\right]$ & 90 & 90 \\
\hline$g\left[^{\circ}\right]$ & 90 & 90 \\
\hline Volume $\left[\AA^{3}\right]$ & $2158.5(7)$ & $2449.7(8)$ \\
\hline Z & 4 & 4 \\
\hline Absorption coefficient $\left[\mathrm{mm}^{-1}\right]$ & 0.719 & 1.706 \\
\hline
\end{tabular}




\begin{tabular}{|c|c|c|}
\hline$F(000)$ & 936 & 1080 \\
\hline Crystal size $\left[\mathrm{mm}^{3}\right]$ & $0.3 \times 0.2 \times 0.2$ & $0.2 \times 0.2 \times 0.1$ \\
\hline Theta range for data collection & 2.129 to $26.382^{\circ}$ & 1.627 to $26.376^{\circ}$ \\
\hline Reflections collected & 25569 & 54351 \\
\hline Independent reflections & 4407 & 5006 \\
\hline $\mathrm{R}_{\text {int }}$ & 0.0366 & 0.0419 \\
\hline Max. and min. transmission & 0.7454 and 0.6670 & 0.7454 and 0.6485 \\
\hline Data / restraints / parameters & $4407 / 0$ / 281 & $5006 / 0 / 318$ \\
\hline Goodness-of-fit on $F^{2}$ & 1.032 & 1.051 \\
\hline Final $R$ indices [l>2sigma(I)] & $\begin{array}{c}R 1=0.0237 \\
w R 2=0.0552\end{array}$ & $\begin{array}{c}R 1=0.0195 \\
w R 2=0.0452\end{array}$ \\
\hline $\mathrm{R}$ indices (all data) & $\begin{array}{c}R 1=0.0272 \\
w R 2=0.0567\end{array}$ & $\begin{array}{c}\mathrm{R} 1=0.0213 \\
\mathrm{wR} 2=0.0457\end{array}$ \\
\hline Absolute structure parameter & $0.013(6)$ & $0.010(3)$ \\
\hline $\begin{array}{l}\text { Largest diff. peak and hole } \\
{\left[\mathrm{e}^{-3}\right]}\end{array}$ & 0.242 and -0.212 & 0.222 and -0.152 \\
\hline
\end{tabular}

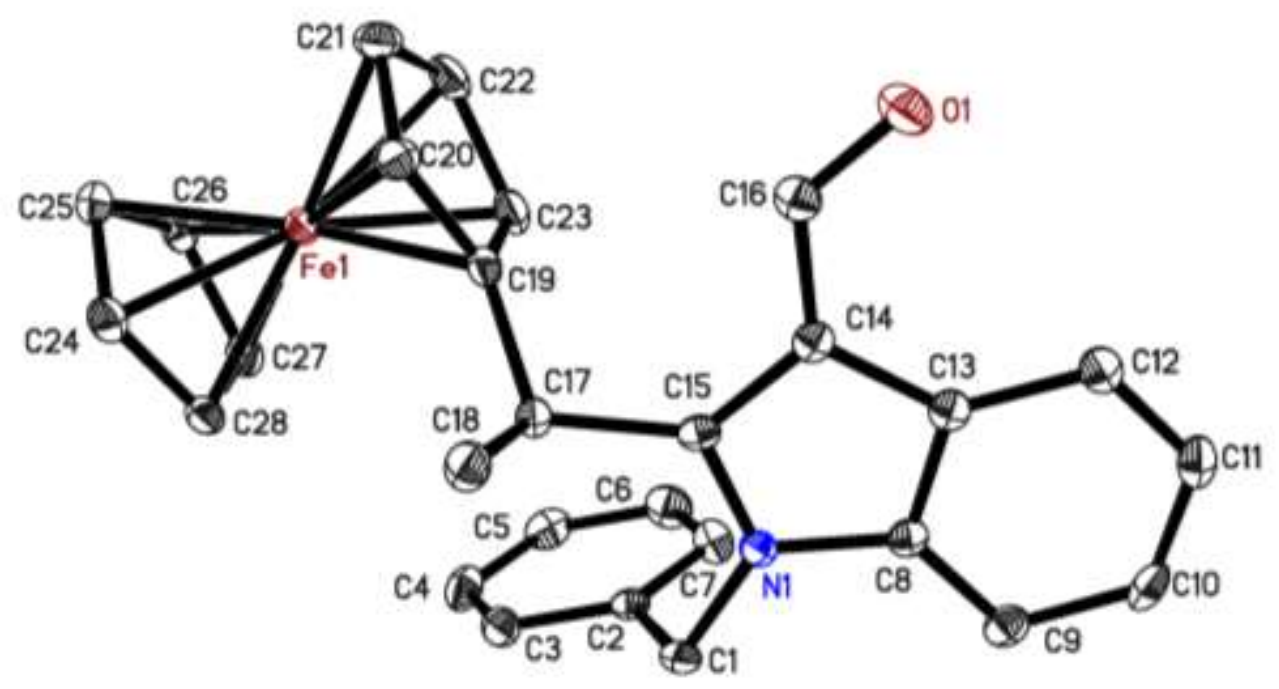

Figure 5.4. Molecular structure of 192ca with thermal ellipsoids at 50\% probability level. The hydrogen atoms are omitted for clarity. 
Table 5.8. Bond lengths $[\AA]$ and angles $\left[^{\circ}\right]$ for $192 \mathrm{ca}$.

\begin{tabular}{|c|c|c|c|}
\hline $\mathrm{Fe}(1)-\mathrm{C}(23)$ & $2.032(2)$ & $C(24)-F e(1)-C(25)$ & $40.67(9)$ \\
\hline $\mathrm{Fe}(1)-\mathrm{C}(27)$ & $2.038(2)$ & $\mathrm{C}(26)-\mathrm{Fe}(1)-\mathrm{C}(25)$ & $40.44(9)$ \\
\hline $\mathrm{Fe}(1)-\mathrm{C}(19)$ & $2.042(2)$ & $C(20)-F e(1)-C(25)$ & $124.86(9)$ \\
\hline $\mathrm{Fe}(1)-\mathrm{C}(28)$ & $2.043(2)$ & $\mathrm{C}(23)-\mathrm{Fe}(1)-\mathrm{C}(21)$ & $68.57(11)$ \\
\hline $\mathrm{Fe}(1)-\mathrm{C}(22)$ & $2.047(3)$ & $\mathrm{C}(27)-\mathrm{Fe}(1)-\mathrm{C}(21)$ & $159.15(10)$ \\
\hline $\mathrm{Fe}(1)-\mathrm{C}(24)$ & $2.047(2)$ & $\mathrm{C}(19)-\mathrm{Fe}(1)-\mathrm{C}(21)$ & $68.67(10)$ \\
\hline $\mathrm{Fe}(1)-\mathrm{C}(26)$ & $2.048(2)$ & $\mathrm{C}(28)-\mathrm{Fe}(1)-\mathrm{C}(21)$ & $159.60(10)$ \\
\hline $\mathrm{Fe}(1)-\mathrm{C}(20)$ & $2.048(2)$ & $C(22)-F e(1)-C(21)$ & $40.39(11)$ \\
\hline $\mathrm{Fe}(1)-\mathrm{C}(25)$ & $2.051(2)$ & $\mathrm{C}(24)-\mathrm{Fe}(1)-\mathrm{C}(21)$ & $124.72(11)$ \\
\hline $\mathrm{Fe}(1)-\mathrm{C}(21)$ & $2.052(2)$ & $\mathrm{C}(26)-\mathrm{Fe}(1)-\mathrm{C}(21)$ & $124.34(10)$ \\
\hline $\mathrm{C}(1)-\mathrm{N}(1)$ & $1.365(3)$ & $C(20)-F e(1)-C(21)$ & $40.71(10)$ \\
\hline$C(1)-C(2)$ & $1.392(3)$ & $C(25)-F e(1)-C(21)$ & $110.06(10)$ \\
\hline$C(1)-C(17)$ & $1.513(3)$ & $N(1)-C(1)-C(2)$ & $108.9(2)$ \\
\hline $\mathrm{O}(1)-\mathrm{C}(9)$ & $1.227(3)$ & $N(1)-C(1)-C(17)$ & $120.4(2)$ \\
\hline$N(1)-C(8)$ & $1.396(3)$ & $C(2)-C(1)-C(17)$ & $130.7(2)$ \\
\hline$N(1)-C(10)$ & $1.464(3)$ & $C(1)-N(1)-C(8)$ & $109.54(19)$ \\
\hline$C(10)-C(11)$ & $1.514(3)$ & $C(1)-N(1)-C(10)$ & $126.35(19)$ \\
\hline$C(11)-C(16)$ & $1.389(3)$ & $C(8)-N(1)-C(10)$ & $123.99(19)$ \\
\hline$C(11)-C(12)$ & $1.395(3)$ & $N(1)-C(10)-C(11)$ & $113.09(19)$ \\
\hline$C(2)-C(9)$ & $1.431(3)$ & $C(16)-C(11)-C(12)$ & $118.9(2)$ \\
\hline$C(2)-C(3)$ & $1.444(3)$ & $C(16)-C(11)-C(10)$ & $119.5(2)$ \\
\hline$C(16)-C(15)$ & $1.388(3)$ & $C(12)-C(11)-C(10)$ & $121.5(2)$ \\
\hline$C(3)-C(8)$ & $1.404(3)$ & $C(1)-C(2)-C(9)$ & $126.2(2)$ \\
\hline$C(3)-C(4)$ & $1.407(3)$ & $C(1)-C(2)-C(3)$ & 107.2(2) \\
\hline$C(15)-C(14)$ & $1.387(4)$ & $C(9)-C(2)-C(3)$ & $126.7(2)$ \\
\hline$C(4)-C(5)$ & $1.383(3)$ & $C(15)-C(16)-C(11)$ & $120.6(2)$ \\
\hline$C(14)-C(13)$ & $1.384(4)$ & $C(8)-C(3)-C(4)$ & 119.2(2) \\
\hline$C(5)-C(6)$ & $1.391(4)$ & $C(8)-C(3)-C(2)$ & $106.6(2)$ \\
\hline$C(13)-C(12)$ & $1.383(3)$ & $C(4)-C(3)-C(2)$ & $134.2(2)$ \\
\hline$C(6)-C(7)$ & $1.384(3)$ & $C(14)-C(15)-C(16)$ & $120.1(2)$ \\
\hline$C(7)-C(8)$ & $1.397(3)$ & $C(5)-C(4)-C(3)$ & $118.0(2)$ \\
\hline$C(17)-C(19)$ & $1.520(3)$ & $C(13)-C(14)-C(15)$ & $119.5(2)$ \\
\hline$C(17)-C(18)$ & $1.533(3)$ & $C(4)-C(5)-C(6)$ & $121.9(2)$ \\
\hline$C(19)-C(23)$ & $1.426(3)$ & $C(12)-C(13)-C(14)$ & $120.5(2)$ \\
\hline$C(19)-C(20)$ & $1.429(3)$ & $C(7)-C(6)-C(5)$ & $121.5(2)$ \\
\hline
\end{tabular}




$\begin{array}{ll}C(20)-C(21) & 1.426(4) \\ C(21)-C(22) & 1.415(4) \\ C(22)-C(23) & 1.424(3) \\ C(24)-C(28) & 1.423(3) \\ C(24)-C(25) & 1.424(3) \\ C(25)-C(26) & 1.416(3) \\ C(26)-C(27) & 1.420(4) \\ C(27)-C(28) & 1.422(3)\end{array}$

$\mathrm{C}(23)-\mathrm{Fe}(1)-\mathrm{C}(27)$

$\mathrm{C}(23)-\mathrm{Fe}(1)-\mathrm{C}(19)$

$\mathrm{C}(27)-\mathrm{Fe}(1)-\mathrm{C}(19)$

$\mathrm{C}(23)-\mathrm{Fe}(1)-\mathrm{C}(28)$

$\mathrm{C}(27)-\mathrm{Fe}(1)-\mathrm{C}(28)$

$\mathrm{C}(19)-\mathrm{Fe}(1)-\mathrm{C}(28)$

$\mathrm{C}(23)-\mathrm{Fe}(1)-\mathrm{C}(22)$

$\mathrm{C}(27)-\mathrm{Fe}(1)-\mathrm{C}(22)$

$\mathrm{C}(19)-\mathrm{Fe}(1)-\mathrm{C}(22)$

$\mathrm{C}(28)-\mathrm{Fe}(1)-\mathrm{C}(22)$

$\mathrm{C}(23)-\mathrm{Fe}(1)-\mathrm{C}(24)$

$\mathrm{C}(27)-\mathrm{Fe}(1)-\mathrm{C}(24)$

$\mathrm{C}(19)-\mathrm{Fe}(1)-\mathrm{C}(24)$

$\mathrm{C}(28)-\mathrm{Fe}(1)-\mathrm{C}(24)$

$\mathrm{C}(22)-\mathrm{Fe}(1)-\mathrm{C}(24)$

$\mathrm{C}(23)-\mathrm{Fe}(1)-\mathrm{C}(26)$

$\mathrm{C}(27)-\mathrm{Fe}(1)-\mathrm{C}(26)$

$\mathrm{C}(19)-\mathrm{Fe}(1)-\mathrm{C}(26)$

$\mathrm{C}(28)-\mathrm{Fe}(1)-\mathrm{C}(26)$

$\mathrm{C}(22)-\mathrm{Fe}(1)-\mathrm{C}(26)$

$\mathrm{C}(24)-\mathrm{Fe}(1)-\mathrm{C}(26)$

$\mathrm{C}(23)-\mathrm{Fe}(1)-\mathrm{C}(20)$

$\mathrm{C}(27)-\mathrm{Fe}(1)-\mathrm{C}(20)$

$\mathrm{C}(19)-\mathrm{Fe}(1)-\mathrm{C}(20)$

$\mathrm{C}(28)-\mathrm{Fe}(1)-\mathrm{C}(20)$

$\mathrm{C}(22)-\mathrm{Fe}(1)-\mathrm{C}(20)$

$\mathrm{C}(24)-\mathrm{Fe}(1)-\mathrm{C}(20)$

$\mathrm{C}(26)-\mathrm{Fe}(1)-\mathrm{C}(20)$
105.29(10)

40.98(9)

120.57(10)

$120.57(10)$

40.76(10)

105.42(10)

40.87(10)

122.15(11)

68.68(10)

$157.51(10)$

157.52(10)

68.42(10)

$122.21(10)$

40.71(10)

160.61(11)

121.69(10)

40.68(10)

157.19(9)

68.59(10)

107.98(10)

68.45(10)

68.77(10)

157.54(10)

40.89(9)

$122.24(10)$

68.35(11)

108.35(10)

160.74(10)
$\begin{array}{ll}C(13)-C(12)-C(11) & 120.4(2) \\ C(6)-C(7)-C(8) & 116.8(2)\end{array}$

$\mathrm{N}(1)-\mathrm{C}(8)-\mathrm{C}(7) 129.6(2)$

$\mathrm{N}(1)-\mathrm{C}(8)-\mathrm{C}(3)$ 107.76(19)

$\mathrm{C}(7)-\mathrm{C}(8)-\mathrm{C}(3) 122.6(2)$

$\mathrm{O}(1)-\mathrm{C}(9)-\mathrm{C}(2)$

125.3(2)

$\mathrm{C}(1)-\mathrm{C}(17)-\mathrm{C}(19) \quad 112.25(19)$

$C(1)-C(17)-C(18) \quad 110.90(19)$

$\mathrm{C}(19)-\mathrm{C}(17)-\mathrm{C}(18) \quad 112.49(19)$

$\mathrm{C}(23)-\mathrm{C}(19)-\mathrm{C}(20) \quad$ 107.6(2)

$\mathrm{C}(23)-\mathrm{C}(19)-\mathrm{C}(17) \quad 125.3(2)$

$\mathrm{C}(20)-\mathrm{C}(19)-\mathrm{C}(17) \quad 127.1(2)$

$\mathrm{C}(23)-\mathrm{C}(19)-\mathrm{Fe}(1) \quad 69.15(12)$

$\mathrm{C}(20)-\mathrm{C}(19)-\mathrm{Fe}(1) \quad 69.79(12)$

$\mathrm{C}(17)-\mathrm{C}(19)-\mathrm{Fe}(1) \quad$ 125.29(16)

$\mathrm{C}(21)-\mathrm{C}(20)-\mathrm{C}(19) \quad$ 108.0(2)

$\mathrm{C}(21)-\mathrm{C}(20)-\mathrm{Fe}(1) \quad 69.79(13)$

$\mathrm{C}(19)-\mathrm{C}(20)-\mathrm{Fe}(1) \quad 69.32(13)$

$\mathrm{C}(22)-\mathrm{C}(21)-\mathrm{C}(20) \quad 108.1(2)$

$\mathrm{C}(22)-\mathrm{C}(21)-\mathrm{Fe}(1) \quad 69.60(15)$

$\mathrm{C}(20)-\mathrm{C}(21)-\mathrm{Fe}(1) \quad 69.50(14)$

$\mathrm{C}(21)-\mathrm{C}(22)-\mathrm{C}(23) \quad$ 108.3(2)

$\mathrm{C}(21)-\mathrm{C}(22)-\mathrm{Fe}(1) \quad 70.02(15)$

$\mathrm{C}(23)-\mathrm{C}(22)-\mathrm{Fe}(1) \quad 69.03(14)$

$C(22)-C(23)-C(19) \quad 108.0(2)$

$\mathrm{C}(22)-\mathrm{C}(23)-\mathrm{Fe}(1) \quad 70.10(14)$

$\mathrm{C}(19)-\mathrm{C}(23)-\mathrm{Fe}(1) \quad 69.87(13)$

$\mathrm{C}(28)-\mathrm{C}(24)-\mathrm{C}(25) \quad$ 107.8(2)

$\mathrm{C}(28)-\mathrm{C}(24)-\mathrm{Fe}(1) \quad 69.51(14)$

$\mathrm{C}(25)-\mathrm{C}(24)-\mathrm{Fe}(1) \quad 69.80(14)$

$\mathrm{C}(26)-\mathrm{C}(25)-\mathrm{C}(24) \quad$ 108.4(2)

$\mathrm{C}(26)-\mathrm{C}(25)-\mathrm{Fe}(1) \quad 69.69(14)$

$\mathrm{C}(24)-\mathrm{C}(25)-\mathrm{Fe}(1) \quad 69.53(13)$

$\mathrm{C}(25)-\mathrm{C}(26)-\mathrm{C}(27) \quad$ 107.7(2)

$\mathrm{C}(25)-\mathrm{C}(26)-\mathrm{Fe}(1) \quad 69.87(14)$

$\mathrm{C}(27)-\mathrm{C}(26)-\mathrm{Fe}(1) \quad 69.29(13)$

$\mathrm{C}(26)-\mathrm{C}(27)-\mathrm{C}(28) \quad$ 108.4(2) 


$\begin{array}{llll}\mathrm{C}(23)-\mathrm{Fe}(1)-\mathrm{C}(25) & 158.92(9) & \mathrm{C}(26)-\mathrm{C}(27)-\mathrm{Fe}(1) & 70.04(14) \\ \mathrm{C}(27)-\mathrm{Fe}(1)-\mathrm{C}(25) & 68.14(10) & \mathrm{C}(28)-\mathrm{C}(27)-\mathrm{Fe}(1) & 69.81(13) \\ \mathrm{C}(19)-\mathrm{Fe}(1)-\mathrm{C}(25) & 159.72(9) & \mathrm{C}(27)-\mathrm{C}(28)-\mathrm{C}(24) & 107.7(2) \\ \mathrm{C}(28)-\mathrm{Fe}(1)-\mathrm{C}(25) & 68.37(9) & \mathrm{C}(27)-\mathrm{C}(28)-\mathrm{Fe}(1) & 69.42(14) \\ \mathrm{C}(22)-\mathrm{Fe}(1)-\mathrm{C}(25) & 124.36(10) & \mathrm{C}(24)-\mathrm{C}(28)-\mathrm{Fe}(1) & 69.78(14)\end{array}$

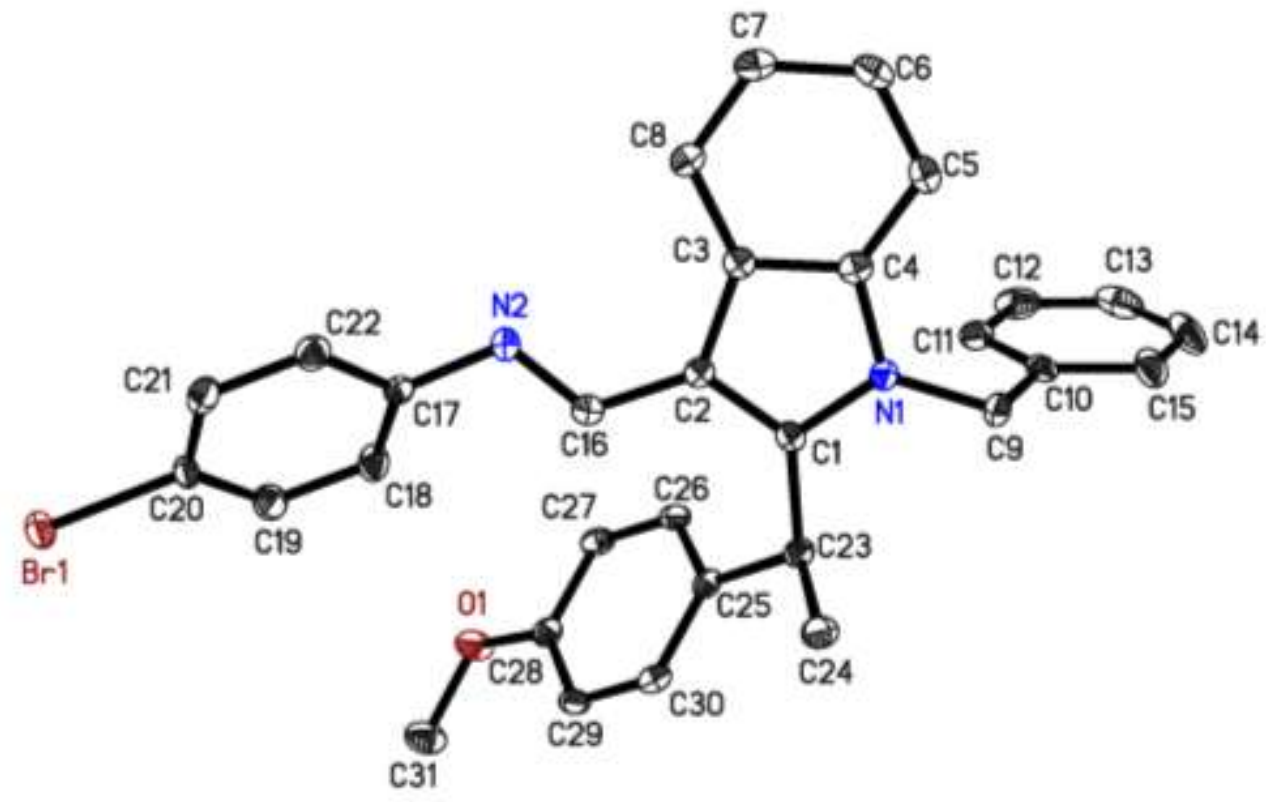

Figure 5.5. Molecular structure of $\mathbf{1 9 7}$ with thermal ellipsoids at $\mathbf{5 0 \%}$ probability level. The hydrogen atoms are omitted for clarity.

Table 5.9. Bond lengths $[\AA ̊]$ and angles $\left[{ }^{\circ}\right]$ for 197.

$\begin{array}{llll}\mathrm{Br}(1)-\mathrm{C}(20) & 1.906(2) & \mathrm{C}(16)-\mathrm{N}(2)-\mathrm{C}(17) & 117.49(19) \\ \mathrm{O}(1)-\mathrm{C}(28) & 1.368(3) & \mathrm{C}(1)-\mathrm{C}(2)-\mathrm{C}(16) & 126.0(2) \\ \mathrm{O}(1)-\mathrm{C}(31) & 1.428(3) & \mathrm{C}(1)-\mathrm{C}(2)-\mathrm{C}(3) & 106.85(18) \\ \mathrm{N}(1)-\mathrm{C}(1) & 1.375(3) & \mathrm{C}(16)-\mathrm{C}(2)-\mathrm{C}(3) & 127.10(19) \\ \mathrm{N}(1)-\mathrm{C}(4) & 1.388(3) & \mathrm{C}(1)-\mathrm{C}(23)-\mathrm{C}(25) & 112.90(17) \\ \mathrm{N}(1)-\mathrm{C}(9) & 1.456(3) & \mathrm{C}(1)-\mathrm{C}(23)-\mathrm{C}(24) & 109.38(18) \\ \mathrm{C}(1)-\mathrm{C}(2) & 1.391(3) & \mathrm{C}(25)-\mathrm{C}(23)-\mathrm{C}(24) & 114.02(19) \\ \mathrm{C}(1)-\mathrm{C}(23) & 1.517(3) & \mathrm{C}(30)-\mathrm{C}(25)-\mathrm{C}(26) & 117.4(2) \\ \mathrm{N}(2)-\mathrm{C}(16) & 1.287(3) & \mathrm{C}(30)-\mathrm{C}(25)-\mathrm{C}(23) & 122.16(18) \\ \mathrm{N}(2)-\mathrm{C}(17) & 1.417(3) & \mathrm{C}(26)-\mathrm{C}(25)-\mathrm{C}(23) & 120.44(19)\end{array}$




$\begin{array}{ll}\mathrm{C}(2)-\mathrm{C}(16) & 1.439(3) \\ \mathrm{C}(2)-\mathrm{C}(3) & 1.447(3) \\ \mathrm{C}(23)-\mathrm{C}(25) & 1.528(3) \\ \mathrm{C}(23)-\mathrm{C}(24) & 1.534(3) \\ \mathrm{C}(25)-\mathrm{C}(30) & 1.386(3) \\ \mathrm{C}(25)-\mathrm{C}(26) & 1.403(3) \\ \mathrm{C}(26)-\mathrm{C}(27) & 1.378(3) \\ \mathrm{C}(27)-\mathrm{C}(28) & 1.401(3) \\ \mathrm{C}(28)-\mathrm{C}(29) & 1.381(3) \\ \mathrm{C}(29)-\mathrm{C}(30) & 1.391(3) \\ \mathrm{C}(3)-\mathrm{C}(8) & 1.403(3) \\ \mathrm{C}(3)-\mathrm{C}(4) & 1.406(3) \\ \mathrm{C}(4)-\mathrm{C}(5) & 1.393(3) \\ \mathrm{C}(5)-\mathrm{C}(6) & 1.384(3) \\ \mathrm{C}(7)-\mathrm{C}(8) & 1.382(3) \\ \mathrm{C}(7)-\mathrm{C}(6) & 1.398(3) \\ \mathrm{C}(17)-\mathrm{C}(22) & 1.396(3) \\ \mathrm{C}(17)-\mathrm{C}(18) & 1.398(3) \\ \mathrm{C}(18)-\mathrm{C}(19) & 1.391(3) \\ \mathrm{C}(9)-\mathrm{C}(10) & 1.520(3) \\ \mathrm{C}(22)-\mathrm{C}(21) & 1.386(3) \\ \mathrm{C}(10)-\mathrm{C}(11) & 1.389(3) \\ \mathrm{C}(10)-\mathrm{C}(15) & 1.390(3) \\ \mathrm{C}(19)-\mathrm{C}(20) & 1.381(3) \\ \mathrm{C}(11)-\mathrm{C}(12) & 1.392(3) \\ \mathrm{C}(20)-\mathrm{C}(21) & 1.381(3) \\ \mathrm{C}(12)-\mathrm{C}(13) & 1.379(4) \\ \mathrm{C}(13)-\mathrm{C}(14) & 1.377(4) \\ \mathrm{C}(15)-\mathrm{C}(14) & 1.401(4)\end{array}$

$\begin{array}{ll}\mathrm{C}(28)-\mathrm{O}(1)-\mathrm{C}(31) & 117.47(17) \\ \mathrm{C}(1)-\mathrm{N}(1)-\mathrm{C}(4) & 109.28(18) \\ \mathrm{C}(1)-\mathrm{N}(1)-\mathrm{C}(9) & 128.11(18) \\ \mathrm{C}(4)-\mathrm{N}(1)-\mathrm{C}(9) & 122.61(18) \\ \mathrm{N}(1)-\mathrm{C}(1)-\mathrm{C}(2) & 109.07(18) \\ \mathrm{N}(1)-\mathrm{C}(1)-\mathrm{C}(23) & 120.08(18) \\ \mathrm{C}(2)-\mathrm{C}(1)-\mathrm{C}(23) & 130.85(19)\end{array}$

$\begin{array}{ll}\mathrm{C}(27)-\mathrm{C}(26)-\mathrm{C}(25) & 121.6(2) \\ \mathrm{C}(26)-\mathrm{C}(27)-\mathrm{C}(28) & 119.81(19) \\ \mathrm{O}(1)-\mathrm{C}(28)-\mathrm{C}(29) & 125.1(2) \\ \mathrm{O}(1)-\mathrm{C}(28)-\mathrm{C}(27) & 115.38(18) \\ \mathrm{C}(29)-\mathrm{C}(28)-\mathrm{C}(27) & 119.5(2) \\ \mathrm{C}(28)-\mathrm{C}(29)-\mathrm{C}(30) & 119.9(2) \\ \mathrm{C}(25)-\mathrm{C}(30)-\mathrm{C}(29) & 121.8(2) \\ \mathrm{C}(8)-\mathrm{C}(3)-\mathrm{C}(4) & 119.0(2) \\ \mathrm{C}(8)-\mathrm{C}(3)-\mathrm{C}(2) & 134.4(2) \\ \mathrm{C}(4)-\mathrm{C}(3)-\mathrm{C}(2) & 106.66(18) \\ \mathrm{N}(1)-\mathrm{C}(4)-\mathrm{C}(5) & 129.2(2) \\ \mathrm{N}(1)-\mathrm{C}(4)-\mathrm{C}(3) & 108.12(19) \\ \mathrm{C}(5)-\mathrm{C}(4)-\mathrm{C}(3) & 122.7(2) \\ \mathrm{C}(6)-\mathrm{C}(5)-\mathrm{C}(4) & 117.1(2) \\ \mathrm{N}(2)-\mathrm{C}(16)-\mathrm{C}(2) & 122.3(2) \\ \mathrm{C}(8)-\mathrm{C}(7)-\mathrm{C}(6) & 121.6(2) \\ \mathrm{C}(5)-\mathrm{C}(6)-\mathrm{C}(7) & 121.2(2) \\ \mathrm{C}(22)-\mathrm{C}(17)-\mathrm{C}(18) & 119.1(2) \\ \mathrm{C}(22)-\mathrm{C}(17)-\mathrm{N}(2) & 117.7(2) \\ \mathrm{C}(18)-\mathrm{C}(17)-\mathrm{N}(2) & 123.2(2) \\ \mathrm{C}(7)-\mathrm{C}(8)-\mathrm{C}(3) & 118.5(2) \\ \mathrm{C}(19)-\mathrm{C}(18)-\mathrm{C}(17) & 119.9(2) \\ \mathrm{N}(1)-\mathrm{C}(9)-\mathrm{C}(10) & 113.54(18) \\ \mathrm{C}(21)-\mathrm{C}(22)-\mathrm{C}(17) & 121.0(2) \\ \mathrm{C}(11)-\mathrm{C}(10)-\mathrm{C}(15) & 119.3(2) \\ \mathrm{C}(11)-\mathrm{C}(10)-\mathrm{C}(9) & 121.7(2) \\ \mathrm{C}(15)-\mathrm{C}(10)-\mathrm{C}(9) & 119.1(2) \\ \mathrm{C}(20)-\mathrm{C}(19)-\mathrm{C}(18) & 119.6(2) \\ \mathrm{C}(10)-\mathrm{C}(11)-\mathrm{C}(12) & 120.4(2) \\ \mathrm{C}(19)-\mathrm{C}(20)-\mathrm{C}(21) & 121.6(2) \\ \mathrm{C}(19)-\mathrm{C}(20)-\mathrm{B}(1) & 119.98(18) \\ \mathrm{C}(21)-\mathrm{C}(20)-\mathrm{B}(1) & 118.46(17) \\ \mathrm{C}(13)-\mathrm{C}(12)-\mathrm{C}(11) & 120.1(2) \\ \mathrm{C}(20)-\mathrm{C}(21)-\mathrm{C}(22) & 118.8(2) \\ \mathrm{C}(14)-\mathrm{C}(13)-\mathrm{C}(12) & 120.0(2) \\ \mathrm{C}(10)-\mathrm{C}(15)-\mathrm{C}(14) & 119.9(2) \\ \mathrm{C}(13)-\mathrm{C}(14)-\mathrm{C}(15) & 120.3(2) \\ & \end{array}$


The crystal structures of $\mathbf{2 3 0}$ and rac-232 were measured and solved by Dr. C. Golz.

A suitable crystal was selected and mounted on a 'Bruker APEX-II CCD' diffractometer. The crystal was kept at $100 \mathrm{~K}$ during data collection. Using Olex2, ${ }^{[348]}$ the structure was solved with the $\mathrm{XT}^{[345]}$ structure solution program using intrinsic phasing and refined with the $\mathrm{XL}^{[349]}$ refinement package using least squares minimization.

\section{X-Ray Crystallographic Data of 230}

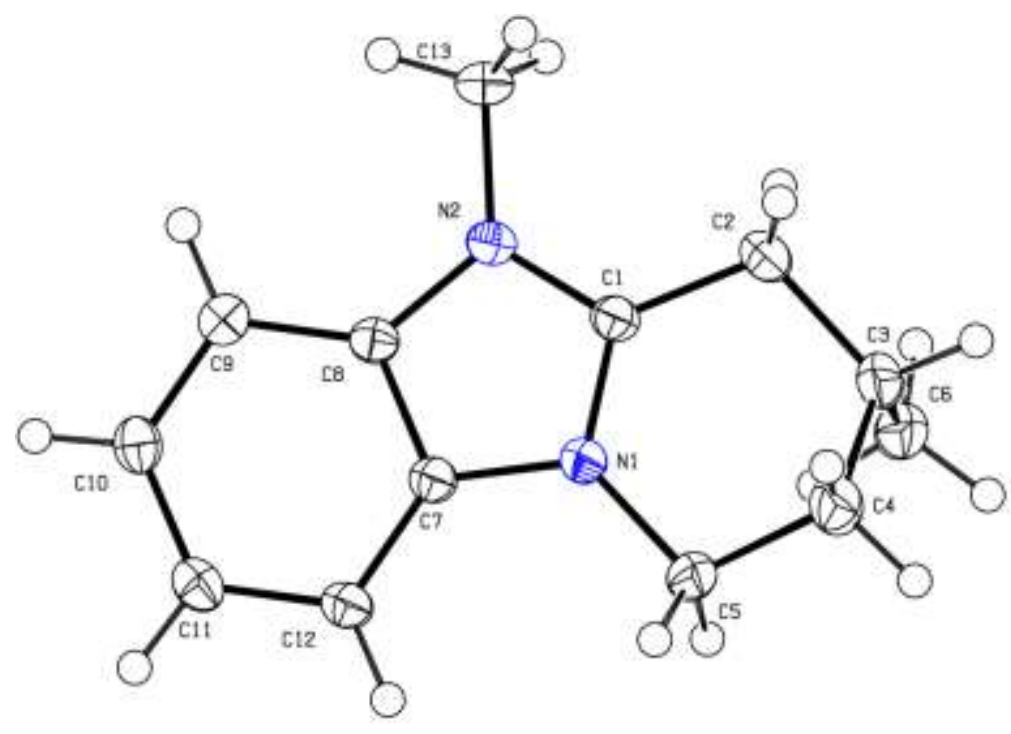

Figure 5.6. Molecular structure of $\mathbf{2 3 0}$ with thermal ellipsoids at $\mathbf{5 0 \%}$ probability level.

Table 5.10. Crystal data and structure refinement for 230 .

Compound

CCDC number

Empirical formula
230

1871584

$\mathrm{C}_{13} \mathrm{H}_{17} / \mathrm{N}_{2}$ 
Formula weight

Temperature/K

Crystal system

Space group

a/Å

$\mathrm{b} / \AA ̊$

$c / A ̊$

$\alpha /^{\circ}$

$\beta /{ }^{\circ}$

$\mathrm{Y} /{ }^{\circ}$

Volume $/ \AA^{3}$

Z

$\rho_{\text {cald }} \mathrm{g} \cdot \mathrm{cm}^{-3}$

$\mu / \mathrm{mm}^{-1}$

$\mathrm{F}(000)$

Crystal size $/ \mathrm{mm}^{3}$

Radiation

$2 \Theta$ range for data collection $/^{\circ}$

Index ranges

Reflections collected

Independent reflections

Data/restraints/parameters

Goodness-of-fit on $\mathrm{F}^{2}$

Final $R$ indexes $[\mid>=2 \sigma(I)]$

Final $R$ indexes [all data]

Largest diff. peak/hole / e $\AA^{-3}$

Flack parameter
328.18

100.0

orthorhombic

$\mathrm{P} 2{ }_{1} 2_{1} 2_{1}$

6.4848(3)

12.6132(6)

15.9161(8)

90

90

90

1301.84(11)

4

1.674

2.436

648.0

$0.237 \times 0.166 \times 0.138$

$\operatorname{MoK} \alpha(\lambda=0.71073)$

5.118 to 65.194

$-9 \leq \mathrm{h} \leq 9,-19 \leq \mathrm{k} \leq 15,-24 \leq \mathrm{I} \leq 23$

19301

$4738\left[R_{\text {int }}=0.0180, R_{\text {sigma }}=0.0153\right]$

$4738 / 0 / 147$

1.104

$\mathrm{R}_{1}=0.0144, \mathrm{wR}_{2}=0.0389$

$R_{1}=0.0146, \mathrm{wR}_{2}=0.0391$

$0.46 /-0.46$

$-0.009(6)$ 
Table 5.11. Bond lengths for 230 .

\begin{tabular}{|c|c|c|c|c|c|}
\hline Atom & Atom & Length/A & Atom & Atom & Length/̊ \\
\hline N1 & $\mathrm{C} 1$ & $1.3398(17)$ & C3 & C6 & $1.525(3)$ \\
\hline $\mathrm{N} 1$ & C5 & $1.4742(19)$ & $\mathrm{C} 4$ & C5 & $1.526(3)$ \\
\hline $\mathrm{N} 1$ & $\mathrm{C7}$ & $1.3886(18)$ & $\mathrm{C7}$ & $\mathrm{C} 8$ & $1.3973(19)$ \\
\hline $\mathrm{N} 2$ & $\mathrm{C} 1$ & $1.3389(18)$ & $\mathrm{C7}$ & C12 & $1.3887(19)$ \\
\hline N2 & $\mathrm{C} 8$ & $1.3897(17)$ & $\mathrm{C} 8$ & $\mathrm{C9}$ & $1.394(2)$ \\
\hline $\mathrm{N} 2$ & C13 & $1.4643(18)$ & C9 & C10 & $1.386(2)$ \\
\hline C1 & $\mathrm{C} 2$ & $1.484(2)$ & C10 & C11 & $1.404(2)$ \\
\hline $\mathrm{C} 2$ & $\mathrm{C} 3$ & $1.541(3)$ & C11 & C12 & $1.385(2)$ \\
\hline C3 & $\mathrm{C} 4$ & $1.523(3)$ & & & \\
\hline
\end{tabular}

Table 5.12. Bond angles for 230 .

$\begin{array}{llllllll}\text { Atom } & \text { Atom } & \text { Atom } & \text { Angle }^{\circ} & & \text { Atom } & \text { Atom } & \text { Atom } \\ \text { C1 } & \text { N1 } & \text { C5 } & 125.02(12) & \text { C3 } & \text { C4 } & \text { C5 } & 112.16(16) \\ \text { C1 } & \text { N1 } & \text { C7 } & 108.58(12) & \text { N1 } & \text { C5 } & \text { C4 } & 109.25(13) \\ \text { C7 } & \text { N1 } & \text { C5 } & 126.39(12) & \text { N1 } & \text { C7 } & \text { C8 } & 106.56(11) \\ \text { C1 } & \text { N2 } & \text { C8 } & 108.52(11) & \text { N1 } & \text { C7 } & \text { C12 } & 131.02(13) \\ \text { C1 } & \text { N2 } & \text { C13 } & 124.57(12) & \text { C12 } & \text { C7 } & \text { C8 } & 122.41(13) \\ \text { C8 } & \text { N2 } & \text { C13 } & 126.92(12) & \text { N2 } & \text { C8 } & \text { C7 } & 106.61(12) \\ \text { N1 } & \text { C1 } & \text { C2 } & 124.23(13) & \text { N2 } & \text { C8 } & \text { C9 } & 131.80(13) \\ \text { N2 } & \text { C1 } & \text { N1 } & 109.72(12) & \text { C9 } & \text { C8 } & \text { C7 } & 121.58(12) \\ \text { N2 } & \text { C1 } & \text { C2 } & 126.06(13) & \text { C10 } & \text { C9 } & \text { C8 } & 116.04(13) \\ \text { C1 } & \text { C2 } & \text { C3 } & 110.14(13) & \text { C9 } & \text { C10 } & \text { C11 } & 122.10(13) \\ \text { C4 } & \text { C3 } & \text { C2 } & 109.30(16) & \text { C12 } & \text { C11 } & \text { C10 } & 121.86(13) \\ \text { C4 } & \text { C3 } & \text { C6 } & 112.71(17) & \text { C11 } & \text { C12 } & \text { C7 } & 116.00(13) \\ \text { C6 } & \text { C3 } & \text { C2 } & 111.66(16) & & & & \end{array}$




\section{X-Ray Crystallographic Data of rac-232}

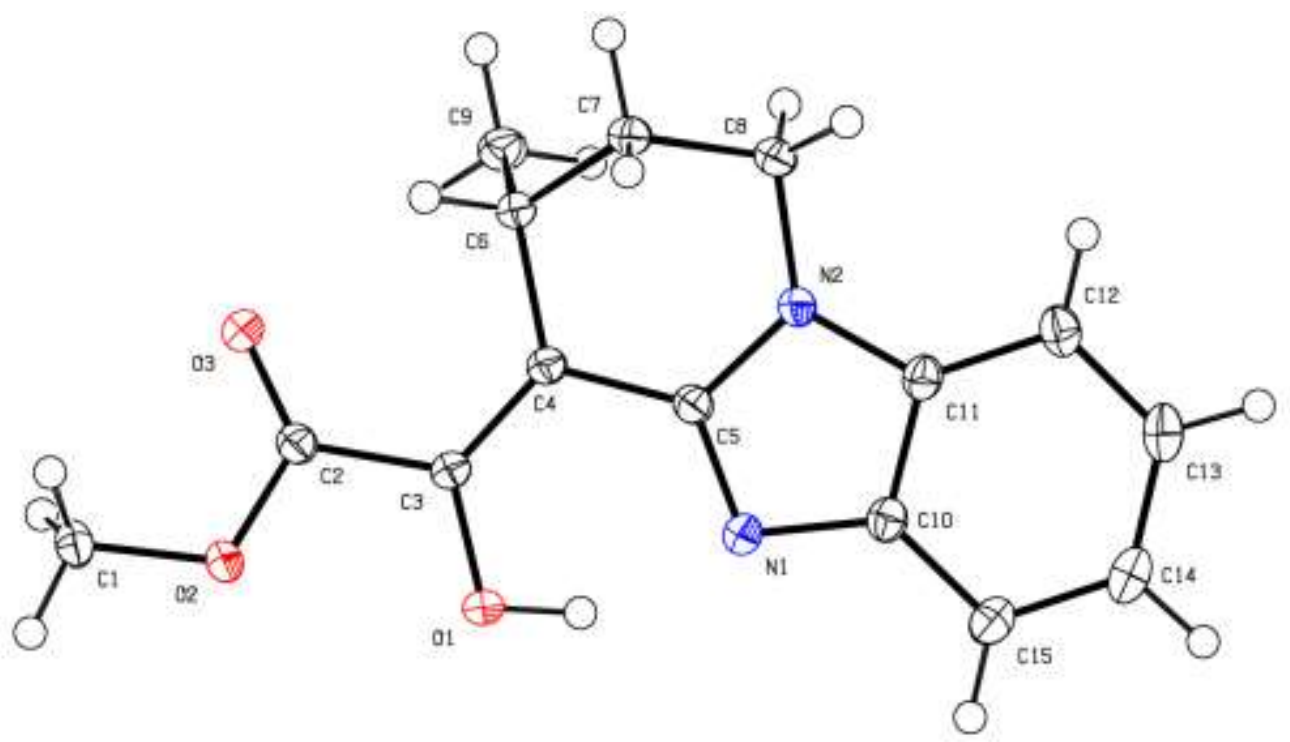

Figure 5.7. Molecular structure of rac-232 with thermal ellipsoids at 50\% probability level.

Table 5.13. Crystal data and structure refinement for rac-232.

Compound

CCDC number

Empirical formula

Formula weight

Temperature/K

Crystal system

Space group

$\mathrm{a} / \mathrm{A}$

b/Å

$c / \AA ̊$

$\alpha /{ }^{\circ}$

$\beta /{ }^{\circ}$

$\mathrm{Y}^{\circ}$

Volume/ $\AA^{3}$ rac-232

1871585

$\mathrm{C}_{15} \mathrm{H}_{16} \mathrm{~N}_{2} \mathrm{O}_{3}$

272.30

99.98

monoclinic

$\mathrm{P} 2$ / $\mathrm{c}$

7.3560(4)

15.9260(7)

11.3561(5)

90

107.168(2)

90

1271.11(11) 


\begin{tabular}{|c|c|}
\hline Z & 4 \\
\hline$\rho_{\text {cald }} / \mathrm{g} \cdot \mathrm{cm}^{-3}$ & 1.423 \\
\hline$\mu / \mathrm{mm}^{-1}$ & 0.100 \\
\hline$F(000)$ & 576.0 \\
\hline Crystal size $/ \mathrm{mm}^{3}$ & $0.273 \times 0.2 \times 0.11$ \\
\hline Radiation & $\operatorname{MoKa}(\lambda=0.71073)$ \\
\hline $2 \Theta$ range for data collection $/^{\circ}$ & 5.116 to 59.474 \\
\hline Index ranges & $-10 \leq h \leq 10,-22 \leq k \leq 22,-15 \leq l \leq 15$ \\
\hline Reflections collected & 20336 \\
\hline Independent reflections & $3597\left[R_{\text {int }}=0.0287, R_{\text {sigma }}=0.0209\right]$ \\
\hline Data/restraints/parameters & $3597 / 0 / 187$ \\
\hline Goodness-of-fit on $\mathrm{F}^{2}$ & 1.072 \\
\hline Final $R$ indexes $[l>=2 \sigma(I)]$ & $R_{1}=0.0418, w R_{2}=0.1038$ \\
\hline Final $R$ indexes [all data] & $R_{1}=0.0484, w R_{2}=0.1084$ \\
\hline Largest diff. peak/hole / e $\AA^{-3}$ & $0.38 /-0.25$ \\
\hline
\end{tabular}

Table 5.14. Bond Lengths for rac-232.

\begin{tabular}{|c|c|c|c|c|c|}
\hline Atom & Atom & Length/Å & Atom & Atom & Length/Å \\
\hline 01 & C3 & $1.3502(13)$ & $\mathrm{C} 4$ & C5 & $1.4492(16)$ \\
\hline $\mathrm{O} 2$ & $\mathrm{C} 1$ & $1.4460(14)$ & $\mathrm{C} 4$ & C6 & $1.5200(15)$ \\
\hline $\mathrm{O} 2$ & $\mathrm{C} 2$ & $1.3393(13)$ & C6 & $\mathrm{C7}$ & $1.5367(16)$ \\
\hline $\mathrm{O} 3$ & $\mathrm{C} 2$ & $1.2086(14)$ & C6 & C9 & $1.5277(17)$ \\
\hline $\mathrm{N} 1$ & C5 & $1.3367(14)$ & $\mathrm{C} 7$ & C8 & $1.5259(17)$ \\
\hline $\mathrm{N} 1$ & C10 & $1.3886(15)$ & C10 & C11 & $1.4081(16)$ \\
\hline $\mathrm{N} 2$ & C5 & $1.3676(14)$ & C10 & C15 & $1.3967(16)$ \\
\hline N2 & $\mathrm{C} 8$ & $1.4674(14)$ & C11 & C12 & $1.3938(16)$ \\
\hline N2 & C11 & $1.3806(14)$ & C12 & C13 & $1.3857(18)$ \\
\hline $\mathrm{C} 2$ & C3 & $1.4936(16)$ & C13 & C14 & $1.4055(19)$ \\
\hline C3 & $\mathrm{C} 4$ & $1.3608(15)$ & C14 & C15 & $1.3896(18)$ \\
\hline
\end{tabular}


Table 5.15. Bond Angles for rac-232.

\begin{tabular}{llllllll} 
Atom & Atom & Atom & Angle $^{\circ}$ & Atom & Atom & Atom & \multicolumn{2}{l}{ Angle ${ }^{\circ}$} \\
C2 & O2 & C1 & $114.79(9)$ & N2 & C5 & C4 & $121.96(10)$ \\
C5 & N1 & C10 & $105.11(9)$ & C4 & C6 & C7 & $109.72(9)$ \\
C5 & N2 & C8 & $124.93(10)$ & C4 & C6 & C9 & $110.71(10)$ \\
C5 & N2 & C11 & $107.07(9)$ & C9 & C6 & C7 & $112.00(10)$ \\
C11 & N2 & C8 & $127.88(10)$ & C8 & C7 & C6 & $112.45(10)$ \\
O2 & C2 & C3 & $111.44(9)$ & N2 & C8 & C7 & $108.96(9)$ \\
O3 & C2 & O2 & $123.31(11)$ & N1 & C10 & C11 & $109.62(10)$ \\
O3 & C2 & C3 & $125.24(10)$ & N1 & C10 & C15 & $130.27(11)$ \\
O1 & C3 & C2 & $113.33(9)$ & C15 & C10 & C11 & $120.10(11)$ \\
O1 & C3 & C4 & $123.98(10)$ & N2 & C11 & C10 & $105.71(10)$ \\
C4 & C3 & C2 & $122.69(10)$ & N2 & C11 & C12 & $131.55(11)$ \\
C3 & C4 & C5 & $117.81(10)$ & C12 & C11 & C10 & $122.72(11)$ \\
C3 & C4 & C6 & $126.20(10)$ & C13 & C12 & C11 & $116.38(11)$ \\
C5 & C4 & C6 & $115.97(9)$ & C12 & C13 & C14 & $121.70(12)$ \\
N1 & C5 & N2 & $112.48(10)$ & C15 & C14 & C13 & $121.61(11)$ \\
N1 & C5 & C4 & $125.56(10)$ & C14 & C15 & C10 & $117.48(11)$
\end{tabular}




\section{References}

[1] T. H. Meyer, L. H. Finger, P. Gandeepan, L. Ackermann, Trends Chem. 2019, 1, 63-76.

[2] a) B. M. Trost, Angew. Chem. Int. Ed. 1995, 34, 259-281; b) B. M. Trost, Science 1991, 254, 1471-1477.

[3] a) P. T. Anastas, M. M. Kirchhoff, Acc. Chem. Res. 2002, 35, 686-694; b) P. T. Anastas, J. C. Warner, Green chemistry: theory and practice, Oxford University Press, Oxford, 1998.

[4] C. C. C. Johansson Seechurn, M. O. Kitching, T. J. Colacot, V. Snieckus, Angew. Chem. Int. Ed. 2012, 51, 5062-5085.

[5] C. Glaser, Ber. Dtsch. Chem. Ges. 1869, 2, 422-424.

[6] a) P. E. Fanta, Synthesis 1974, 9-21; b) F. Ullmann, J. Bielecki, Ber. Dtsch. Chem. Ges. 1901, 34, 2174-2185.

[7] a) N. Miyaura, A. Suzuki, Chem. Rev. 1995, 95, 2457-2483; b) N. Miyaura, A. Suzuki, J. Chem. Soc., Chem. Commun. 1979, 866-867; c) N. Miyaura, K. Yamada, A. Suzuki, Tetrahedron Lett. 1979, 20, 3437-3440.

[8] a) A. O. King, N. Okukado, E.-i. Negishi, J. Chem. Soc., Chem. Commun. 1977, 683-684; b) E. Negishi, A. O. King, N. Okukado, J. Org. Chem. 1977, $42,1821-1823$.

[9] a) R. F. Heck, J. P. Nolley, J. Org. Chem. 1972, 37, 2320-2322; b) T. Mizoroki, K. Mori, A. Ozaki, Bull. Chem. Soc. Jpn. 1971, 44, 581.

[10] a) K. Tamao, K. Sumitani, M. Kumada, J. Am. Chem. Soc. 1972, 94, 43744376; b) R. J. P. Corriu, J. P. Masse, J. Chem. Soc., Chem. Commun. 1972, 144.

[11] a) T. Hiyama, J. Organomet. Chem. 2002, 653, 58-61; b) Y. Hatanaka, T. Hiyama, J. Org. Chem. 1988, 53, 918-920.

[12] a) J. K. Stille, Angew. Chem. Int. Ed. 1986, 25, 508-524; b) D. Milstein, J. K. Stille, J. Am. Chem. Soc. 1979, 101, 4992-4998; c) D. Milstein, J. K. Stille, J. Am. Chem. Soc. 1978, 100, 3636-3638. 
[13] a) R. Chinchilla, C. Nájera, Chem. Soc. Rev. 2011, 40, 5084-5121; b) R. Chinchilla, C. Nájera, Chem. Rev. 2007, 107, 874-922; c) K. Sonogashira, J. Organomet. Chem. 2002, 653, 46-49; d) K. Sonogashira, Y. Tohda, N. Hagihara, Tetrahedron Lett. 1975, 16, 4467-4470.

[14] a) B. M. Trost, D. L. Van Vranken, Chem. Rev. 1996, 96, 395-422; b) B. M. Trost, P. E. Strege, J. Am. Chem. Soc. 1977, 99, 1649-1651; c) B. M. Trost, T. J. Fullerton, J. Am. Chem. Soc. 1973, 95, 292-294; d) J. Tsuji, H. Takahashi, M. Morikawa, Tetrahedron Lett. 1965, 6, 4387-4388.

[15] a) F. Paul, J. Patt, J. F. Hartwig, J. Am. Chem. Soc. 1994, 116, 5969-5970; b) A. S. Guram, S. L. Buchwald, J. Am. Chem. Soc. 1994, 116, 7901-7902.

[16] a) A. de Meijere, S. Bräse, M. Oestreich, Metal-Catalyzed Cross-Coupling Reactions and More, Wiley-VCH, Weinheim, 2014; b) G. A. Molander, J. P. Wolfe, M. Larhed, Cross Coupling and Heck-Type Reactions, Georg Thieme, Stuttgart, 2013.

[17] The Nobel Prize in Chemistry 2010 - Press Release: https://www.nobelprize.org/nobel prizes/chemistry/laureates/2010/press.html (accessed on 10.04.2019).

[18] a) L. N. Cavalcanti, G. A. Molander, Top. Curr. Chem. 2016, 374, 39; b) T. Iwasaki, N. Kambe, Top. Curr. Chem. 2016, 374, 66; c) M. Tobisu, N. Chatani, Top. Curr. Chem. 2016, 374, 41; d) X. Hu, Chem. Sci. 2011, 2 , 1867-1886; e) B. M. Rosen, K. W. Quasdorf, D. A. Wilson, N. Zhang, A.-M. Resmerita, N. K. Garg, V. Percec, Chem. Rev. 2011, 111, 1346-1416.

[19] a) A. Piontek, E. Bisz, M. Szostak, Angew. Chem. Int. Ed. 2018, 57, 1111611128; b) T. L. Mako, J. A. Byers, Inorg. Chem. Front. 2016, 3, 766-790; c) C. Cassani, G. Bergonzini, C.-J. Wallentin, ACS Catal. 2016, 6, 1640-1648; d) E. Nakamura, T. Hatakeyama, S. Ito, K. Ishizuka, L. Ilies, M. Nakamura, Org. React. 2014, 83, 1-210; e) R. Jana, T. P. Pathak, M. S. Sigman, Chem. Rev. 2011, 111, 1417-1492; f) E. Nakamura, N. Yoshikai, J. Org. Chem. 2010, 75, 6061-6067; g) W. M. Czaplik, M. Mayer, J. Cvengroš, A. J. von Wangelin, ChemSusChem 2009, 2, 396-417; h) B. D. Sherry, A. Fürstner, Acc. Chem. Res. 2008, 41, 1500-1511. 
[20] a) P. Gandeepan, N. Kaplaneris, S. Santoro, L. Vaccaro, L. Ackermann, ACS Sustain. Chem. Eng. 2019, 7, 8023-8040; b) E. Bisz, M. Szostak, ChemSusChem 2018, 11, 1290-1294; c) S. Santoro, F. Ferlin, L. Luciani, L. Ackermann, L. Vaccaro, Green Chem. 2017, 19, 1601-1612; d) V. Pace, P. Hoyos, L. Castoldi, P. Domínguez de María, A. R. Alcántara, ChemSusChem 2012, 5, 1369-1379.

[21] a) S. Santoro, S. I. Kozhushkov, L. Ackermann, L. Vaccaro, Green Chem. 2016, 18, 3471-3493; b) Á. Molnár, Chem. Rev. 2011, 111, 2251-2320; c) M. Benaglia, Recoverable and Recyclable Catalysts, John Wiley \& Sons, Chichester, 2009.

[22] a) T. Gensch, M. J. James, T. Dalton, F. Glorius, Angew. Chem. Int. Ed. 2018, 57, 2296-2306; b) J.-C. Hierso, M. Beaupérin, P. Meunier, Eur. J. Inorg. Chem. 2007, 3767-3780.

[23] Selected reviews: a) J. C. K. Chu, T. Rovis, Angew. Chem. Int. Ed. 2018, 57, 62-101; b) M. M. Lorion, K. Maindan, A. R. Kapdi, L. Ackermann, Chem. Soc. Rev. 2017, 46, 7399-7420; c) Y. Wei, P. Hu, M. Zhang, W. Su, Chem. Rev. 2017, 117, 8864-8907; d) J. A. Leitch, C. G. Frost, Chem. Soc. Rev. 2017, 46, 7145-7153; e) Y. Park, Y. Kim, S. Chang, Chem. Rev. 2017, 117, 92479301; f) J. He, M. Wasa, K. S. L. Chan, Q. Shao, J.-Q. Yu, Chem. Rev. 2017, 117, 8754-8786; g) Q.-Z. Zheng, N. Jiao, Chem. Soc. Rev. 2016, 45, 45904627; h) A. Dey, S. Maity, D. Maiti, Chem. Commun. 2016, 52, 12398-12414; i) G. E. M. Crisenza, J. F. Bower, Chem. Lett. 2016, 45, 2-9; j) O. Daugulis, J. Roane, L. D. Tran, Acc. Chem. Res. 2015, 48, 1053-1064; k) J. WencelDelord, F. Glorius, Nat. Chem. 2013, 5, 369-375; I) G. Rouquet, N. Chatani, Angew. Chem. Int. Ed. 2013, 52, 11726-11743; m) L. Ackermann, R. Vicente, A. R. Kapdi, Angew. Chem. Int. Ed. 2009, 48, 9792-9826; n) L. Ackermann, Modern Arylation Methods, Wiley-VCH, Weinheim, 2009; o) X. Chen, K. M. Engle, D.-H. Wang, J.-Q. Yu, Angew. Chem. Int. Ed. 2009, 48, 5094-5115; p) R. G. Bergman, Nature 2007, 446, 391-393.

[24] a) M. Seki, Org. Process Res. Dev. 2016, 20, 867-877; b) L. Ackermann, Org. Process Res. Dev. 2015, 19, 260-269. 
[25] a) S. K. Sinha, G. Zanoni, D. Maiti, Asian J. Org. Chem. 2018, 7, 1178-1192; b) J. Yamaguchi, A. D. Yamaguchi, K. Itami, Angew. Chem. Int. Ed. 2012, 51, 8960-9009.

[26] a) J. Zhang, L. J. Kang, T. C. Parker, S. B. Blakey, C. K. Luscombe, S. R. Marder, Molecules 2018, 23, 922; b) J.-R. Pouliot, F. Grenier, J. T. Blaskovits, S. Beaupré, M. Leclerc, Chem. Rev. 2016, 116, 14225-14274.

[27] L. Ackermann, L. G. Habgood, T. B. Gunnoe, Catalytic Hydroarylation of Carbon-Carbon Multiple Bonds, Wiley-VCH, Weinheim, 2018.

[28] S. J. Blanksby, G. B. Ellison, Acc. Chem. Res. 2003, 36, 255-263.

[29] T. Gensch, M. N. Hopkinson, F. Glorius, J. Wencel-Delord, Chem. Soc. Rev. 2016, 45, 2900-2936.

[30] a) H. M. L. Davies, K. Liao, Nat. Rev. Chem. 2019, 3, 347-360; b) M. P. Doyle, R. Duffy, M. Ratnikov, L. Zhou, Chem. Rev. 2010, 110, 704-724; c) H. M. L. Davies, J. R. Manning, Nature 2008, 451, 417-424; d) H. M. L. Davies, X. Dai, in Comprehensive Organometallic Chemistry III (Eds.: D. M. P. Mingos, R. H. Crabtree), Elsevier, Oxford, 2007, pp. 167-212; e) H. M. L. Davies, R. E. J. Beckwith, Chem. Rev. 2003, 103, 2861-2904.

[31] a) H. Yi, G. Zhang, H. Wang, Z. Huang, J. Wang, A. K. Singh, A. Lei, Chem. Rev. 2017, 117, 9016-9085; b) J.-T. Yu, C. Pan, Chem. Commun. 2016, 52, 2220-2236.

[32] a) L. Ackermann, Chem. Rev. 2011, 111, 1315-1345; b) D. Balcells, E. Clot, O. Eisenstein, Chem. Rev. 2010, 110, 749-823; c) Y. Boutadla, D. L. Davies, S. A. Macgregor, A. I. Poblador-Bahamonde, Dalton Trans. 2009, 5820-5831.

[33] B. Biswas, M. Sugimoto, S. Sakaki, Organometallics 2000, 19, 3895-3908.

[34] a) D. Lapointe, K. Fagnou, Chem. Lett. 2010, 39, 1118-1126; b) S. I. Gorelsky, D. Lapointe, K. Fagnou, J. Am. Chem. Soc. 2008, 130, 1084810849; c) L.-C. Campeau, M. Parisien, A. Jean, K. Fagnou, J. Am. Chem. Soc. 2006, 128, 581-590; d) D. García-Cuadrado, A. A. C. Braga, F. Maseras, A. M. Echavarren, J. Am. Chem. Soc. 2006, 128, 1066-1067. 
[35] a) R. A. Alharis, C. L. McMullin, D. L. Davies, K. Singh, S. A. Macgregor, J. Am. Chem. Soc. 2019, 141, 8896-8906; b) Y. Boutadla, D. L. Davies, S. A. Macgregor, A. I. Poblador-Bahamonde, Dalton Trans. 2009, 5887-5893.

[36] J. Oxgaard, W. J. Tenn, R. J. Nielsen, R. A. Periana, W. A. Goddard, Organometallics 2007, 26, 1565-1567.

[37] a) E. Tan, O. Quinonero, M. Elena de Orbe, A. M. Echavarren, ACS Catal. 2018, 8, 2166-2172; b) D. Zell, M. Bursch, V. Müller, S. Grimme, L. Ackermann, Angew. Chem. Int. Ed. 2017, 56, 10378-10382; c) D. Santrač, S. Cella, W. Wang, L. Ackermann, Eur. J. Org. Chem. 2016, 5429-5436; d) H. Wang, M. Moselage, M. J. González, L. Ackermann, ACS Catal. 2016, 6, 2705-2709; e) R. Mei, J. Loup, L. Ackermann, ACS Catal. 2016, 6, 793-797; f) C. Tirler, L. Ackermann, Tetrahedron 2015, 71, 4543-4551; g) W. Ma, R. Mei, G. Tenti, L. Ackermann, Chem. Eur. J. 2014, 20, 15248-15251.

[38] a) C. Sambiagio, D. Schönbauer, R. Blieck, T. Dao-Huy, G. Pototschnig, P. Schaaf, T. Wiesinger, M. F. Zia, J. Wencel-Delord, T. Besset, B. U. W. Maes, M. Schnürch, Chem. Soc. Rev. 2018, 47, 6603-6743; b) Z. Chen, B. Wang, J. Zhang, W. Yu, Z. Liu, Y. Zhang, Org. Chem. Front. 2015, 2, 1107-1295.

[39] S. De Sarkar, W. Liu, S. I. Kozhushkov, L. Ackermann, Adv. Synth. Catal. 2014, 356, 1461-1479.

[40] a) W. Ma, P. Gandeepan, J. Li, L. Ackermann, Org. Chem. Front. 2017, 4, 1435-1467; b) F. Zhang, D. R. Spring, Chem. Soc. Rev. 2014, 43, 69066919.

[41] P. Gandeepan, L. Ackermann, Chem 2018, 4, 199-222.

[42] a) http://www.infomine.com/investment/metal-prices/ (consulted on 11.04.2019); b) https://mineralprices.com/ (consulted on 11.04.2019).

[43] J. W. Morgan, E. Anders, Proc. Natl. Acad. Sci. USA 1980, 77, 6973-6977.

[44] a) P. B. Tchounwou, C. G. Yedjou, A. K. Patlolla, D. J. Sutton, in Molecular, clinical, and environmental toxicology (Ed.: A. Luch), Springer, Basel, 2009, pp. 133-164; b) S. H. Gilani, Y. Alibhai, J. Toxicol. Environ. Health 1990, 30 , 23-31. 
[45] P. Gandeepan, T. Müller, D. Zell, G. Cera, S. Warratz, L. Ackermann, Chem. Rev. 2019, 119, 2192-2452.

[46] a) T. Yoshino, S. Matsunaga, Synlett 2019, DOI:10.1055/s-0037-1611814; b) T. Yoshino, S. Matsunaga, Adv. Synth. Catal. 2017, 359, 1245-1262; c) M. Moselage, J. Li, L. Ackermann, ACS Catal. 2016, 6, 498-525; d) K. Gao, N. Yoshikai, Acc. Chem. Res. 2014, 47, 1208-1219.

[47] a) N. Yoshikai, Isr. J. Chem. 2017, 57, 1117-1130; b) R. Shang, L. Ilies, E. Nakamura, Chem. Rev. 2017, 117, 9086-9139; c) G. Cera, L. Ackermann, Top. Curr. Chem. 2016, 374, 57.

[48] a) Y. Nakao, in Catalytic Hydroarylation of Carbon-Carbon Multiple Bonds (Eds.: L. Ackermann, T. B. Gunnoe, L. G. Habgood), Wiley-VCH, Weinheim, 2017, pp. 175-192; b) J. Yamaguchi, K. Muto, K. Itami, Top. Curr. Chem. 2016, 374, 55; c) L. C. M. Castro, N. Chatani, Chem. Lett. 2015, 44, 410-421.

[49] a) Y. Hu, B. Zhou, C. Wang, Acc. Chem. Res. 2018, 51, 816-827; b) W. Liu, L. Ackermann, ACS Catal. 2016, 6, 3743-3752.

[50] a) X. Zhu, S. Chiba, Chem. Soc. Rev. 2016, 45, 4504-4523; b) S. D. McCann, S. S. Stahl, Acc. Chem. Res. 2015, 48, 1756-1766; c) S. E. Allen, R. R. Walvoord, R. Padilla-Salinas, M. C. Kozlowski, Chem. Rev. 2013, 113, 6234-6458.

[51] a) T. G. Saint-Denis, R.-Y. Zhu, G. Chen, Q.-F. Wu, J.-Q. Yu, Science 2018, 359, eaao4798; b) C. G. Newton, S.-G. Wang, C. C. Oliveira, N. Cramer, Chem. Rev. 2017, 117, 8908-8976.

[52] a) J. Loup, U. Dhawa, F. Pesciaioli, J. Wencel-Delord, L. Ackermann, Angew. Chem. Int. Ed. 2019, DOI:10.1002/anie.201904214; b) Ł. Woźniak, N. Cramer, Trends Chem. 2019, DOI:10.1016/j.trechm.2019.03.013.

[53] The Nobel Prize in Chemistry 2001 - Press Release: https://www.nobelprize.org/prizes/chemistry/2001/press-release/ (accessed on 11.04.2019).

[54] F. Hebrard, P. Kalck, Chem. Rev. 2009, 109, 4272-4282.

[55] M. S. Kharasch, E. K. Fields, J. Am. Chem. Soc. 1941, 63, 2316-2320. 
[56] a) H.-W. Lee, F.-Y. Kwong, Eur. J. Org. Chem. 2010, 789-811; b) I. U. Khand, G. R. Knox, P. L. Pauson, W. E. Watts, J. Chem. Soc., Perkin Trans. 1 1973, 975-977; c) I. U. Khand, G. R. Knox, P. L. Pauson, W. E. Watts, J. Chem. Soc. D 1971, 36a.

[57] a) R. F. Lockwood, K. M. Nicholas, Tetrahedron Lett. 1977, 18, 4163-4165;

b) K. M. Nicholas, R. Pettit, J. Organomet. Chem. 1972, 44, C21-C24.

[58] G. Cahiez, A. Moyeux, Chem. Rev. 2010, 110, 1435-1462.

[59] a) W. Liu, B. Sahoo, K. Junge, M. Beller, Acc. Chem. Res. 2018, 51, 18581869; b) P. J. Chirik, Acc. Chem. Res. 2015, 48, 1687-1695.

[60] P. Gandeepan, C.-H. Cheng, Acc. Chem. Res. 2015, 48, 1194-1206.

[61] S. Murahashi, J. Am. Chem. Soc. 1955, 77, 6403-6404.

[62] S. Murahashi, S. Horiie, J. Am. Chem. Soc. 1956, 78, 4816-4817.

[63] a) S. Camadanli, R. Beck, U. Flörke, H.-F. Klein, Dalton Trans. 2008, 57015704; b) R. Beck, H. Sun, X. Li, S. Camadanli, H.-F. Klein, Eur. J. Inorg. Chem. 2008, 3253-3257; c) H.-F. Klein, S. Camadanli, R. Beck, D. Leukel, U. Flörke, Angew. Chem. Int. Ed. 2005, 44, 975-977; d) H.-F. Klein, R. Beck, U. Flörke, H.-J. Haupt, Eur. J. Inorg. Chem. 2003, 1380-1387; e) H.-F. Klein, S. Schneider, M. He, U. Floerke, H.-J. Haupt, Eur. J. Inorg. Chem. 2000, 22952301; f) H.-F. Klein, M. Helwig, U. Koch, U. Flörke, H.-J. Haupt, Z. Naturforsch. B. 1993, 48, 778-784.

[64] G. Halbritter, F. Knoch, A. Wolski, H. Kisch, Angew. Chem. Int. Ed. 1994, 33, 1603-1605.

[65] a) B. J. Fallon, E. Derat, M. Amatore, C. Aubert, F. Chemla, F. Ferreira, A. Perez-Luna, M. Petit, Org. Lett. 2016, 18, 2292-2295; b) B. J. Fallon, J.-B. Garsi, E. Derat, M. Amatore, C. Aubert, M. Petit, ACS Catal. 2015, 5, 74937497; c) B. J. Fallon, E. Derat, M. Amatore, C. Aubert, F. Chemla, F. Ferreira, A. Perez-Luna, M. Petit, J. Am. Chem. Soc. 2015, 137, 2448-2451.

[66] C. P. Lenges, M. Brookhart, B. E. Grant, J. Organomet. Chem. 1997, 528, 199-203. 
[67] a) Q. Chen, L. Ilies, N. Yoshikai, E. Nakamura, Org. Lett. 2011, 13, 32323234; b) L. Ilies, Q. Chen, X. Zeng, E. Nakamura, J. Am. Chem. Soc. 2011, 133, 5221-5223.

[68] N. Yoshikai, Bull. Chem. Soc. Jpn. 2014, 87, 843-857.

[69] a) N. Sauermann, J. Loup, D. Kootz, V. R. Yatham, A. Berkessel, L. Ackermann, Synthesis 2017, 49, 3476-3484; b) J. Li, L. Ackermann, Chem. Eur. J. 2015, 21, 5718-5722; c) L. Ackermann, J. Org. Chem. 2014, 79, 8948-8954; d) B. Punji, W. Song, G. A. Shevchenko, L. Ackermann, Chem. Eur. J. 2013, 19, 10605-10610; e) W. Song, L. Ackermann, Angew. Chem. Int. Ed. 2012, 51, 8251-8254.

[70] Selected reviews: a) T. Piou, T. Rovis, Acc. Chem. Res. 2018, 51, 170-180; b) X. Qi, Y. Li, R. Bai, Y. Lan, Acc. Chem. Res. 2017, 50, 2799-2808; c) B. Ye, N. Cramer, Acc. Chem. Res. 2015, 48, 1308-1318; d) G. Song, X. Li, Acc. Chem. Res. 2015, 48, 1007-1020.

[71] U. Koelle, B. Fuss, M. V. Rajasekharan, B. L. Ramakrishna, J. H. Ammeter, M. C. Boehm, J. Am. Chem. Soc. 1984, 106, 4152-4160.

[72] T. Yoshino, H. Ikemoto, S. Matsunaga, M. Kanai, Angew. Chem. Int. Ed. 2013, 52, 2207-2211.

[73] Selected examples: a) W. Li, L.-H. Weng, G.-X. Jin, Inorg. Chem. Commun. 2004, 7, 1174-1177; b) C. D. Ontiveros, J. A. Morrison, Organometallics 1986, 5, 1446-1448; c) R. F. Heck, Inorg. Chem. 1965, 4, 855-857.

[74] a) M. E. Tauchert, C. D. Incarvito, A. L. Rheingold, R. G. Bergman, J. A. Ellman, J. Am. Chem. Soc. 2012, 134, 1482-1485; b) Y. Li, X.-S. Zhang, H. Li, W.-H. Wang, K. Chen, B.-J. Li, Z.-J. Shi, Chem. Sci. 2012, 3, 1634-1639.

[75] T. Yoshino, H. Ikemoto, S. Matsunaga, M. Kanai, Chem. Eur. J. 2013, 19, 9142-9146.

[76] a) P. G. Chirila, C. J. Whiteoak, Dalton Trans. 2017, 46, 9721-9739; b) S. Wang, S.-Y. Chen, X.-Q. Yu, Chem. Comm. 2017, 53, 3165-3180.

[77] a) H. Ikemoto, R. Tanaka, K. Sakata, M. Kanai, T. Yoshino, S. Matsunaga, Angew. Chem. Int. Ed. 2017, 56, 7156-7160; b) K. Sakata, M. Eda, Y. 
Kitaoka, T. Yoshino, S. Matsunaga, J. Org. Chem. 2017, 82, 7379-7387; c) R. Tanaka, H. Ikemoto, M. Kanai, T. Yoshino, S. Matsunaga, Org. Lett. 2016, 18, 5732-5735; d) H. Ikemoto, T. Yoshino, K. Sakata, S. Matsunaga, M. Kanai, J. Am. Chem. Soc. 2014, 136, 5424-5431.

[78] A. L. Allred, J. Inorg. Nucl. Chem. 1961, 17, 215-221.

[79] D. Zell, Q. Bu, M. Feldt, L. Ackermann, Angew. Chem. Int. Ed. 2016, 55, 7408-7412.

[80] S. Nakanowatari, R. Mei, M. Feldt, L. Ackermann, ACS Catal. 2017, 7, 25112515.

[81] S. Tang, O. Eisenstein, Y. Nakao, S. Sakaki, Organometallics 2017, 36, 2761-2771.

[82] F. Pesciaioli, U. Dhawa, J. C. A. Oliveira, R. Yin, M. John, L. Ackermann, Angew. Chem. Int. Ed. 2018, 57, 15425-15429.

[83] a) N. Sauermann, M. J. González, L. Ackermann, Org. Lett. 2015, 17, 53165319; b) Z.-Z. Zhang, B. Liu, C.-Y. Wang, B.-F. Shi, Org. Lett. 2015, 17, 4094-4097.

[84] a) M. Moselage, N. Sauermann, J. Koeller, W. Liu, D. Gelman, L. Ackermann, Synlett 2015, 26, 1596-1600; b) T. Gensch, S. Vásquez-Céspedes, D.-G. Yu, F. Glorius, Org. Lett. 2015, 17, 3714-3717; c) Y. Suzuki, B. Sun, K. Sakata, T. Yoshino, S. Matsunaga, M. Kanai, Angew. Chem. Int. Ed. 2015, 54, 99449947; d) D.-G. Yu, T. Gensch, F. de Azambuja, S. Vásquez-Céspedes, F. Glorius, J. Am. Chem. Soc. 2014, 136, 17722-17725.

[85] J. Li, L. Ackermann, Angew. Chem. Int. Ed. 2015, 54, 8551-8554.

[86] B. Sun, T. Yoshino, S. Matsunaga, M. Kanai, Adv. Synth. Catal. 2014, 356, 1491-1495.

[87] https://pubchem.ncbi.nlm.nih.gov/compound/Tosyl azide (consulted on 15.04.2019).

[88] A. B. Pawar, D. M. Lade, Org. Biomol. Chem. 2016, 14, 3275-3283.

[89] a) J. Li, L. Ackermann, Angew. Chem. Int. Ed. 2015, 54, 3635-3638; b) A. B. Pawar, S. Chang, Org. Lett. 2015, 17, 660-663. 
[90] T. Gensch, F. J. R. Klauck, F. Glorius, Angew. Chem. Int. Ed. 2016, 55, 11287-11291.

[91] J. Park, S. Chang, Angew. Chem. Int. Ed. 2015, 54, 14103-14107.

[92] Y. Liang, Y.-F. Liang, C. Tang, Y. Yuan, N. Jiao, Chem. Eur. J. 2015, 21, 16395-16399.

[93] P. W. Tan, A. M. Mak, M. B. Sullivan, D. J. Dixon, J. Seayad, Angew. Chem. Int. Ed. 2017, 56, 16550-16554.

[94] S. Fukagawa, Y. Kato, R. Tanaka, M. Kojima, T. Yoshino, S. Matsunaga, Angew. Chem. Int. Ed. 2019, 58, 1153-1157.

[95] D.-Y. Huang, Q.-J. Yao, S. Zhang, X.-T. Xu, K. Zhang, B.-F. Shi, Org. Lett. 2019, 21, 951-954.

[96] S. R. Yetra, Z. Shen, H. Wang, L. Ackermann, Beilstein J. Org. Chem. 2018, 14, 1546-1553.

[97] Y.-H. Liu, P.-X. Li, Q.-J. Yao, Z.-Z. Zhang, D.-Y. Huang, M. D. Le, H. Song, L. Liu, B.-F. Shi, Org. Lett. 2019, 21, 1895-1899.

[98] Y. Sun, N. Cramer, Chem. Sci. 2018, 9, 2981-2985.

[99] K. Ozols, Y.-S. Jang, N. Cramer, J. Am. Chem. Soc. 2019, 141, 5675-5680.

[100] X. Yu, K. Chen, Q. Wang, W. Zhang, J. Zhu, Org. Chem. Front. 2018, 5, 994997.

[101] B. Audic, M. D. Wodrich, N. Cramer, Chem. Sci. 2019, 10, 781-787.

[102] J. Yang, N. Yoshikai, J. Am. Chem. Soc. 2014, 136, 16748-16751.

[103] a) D. H. T. Phan, B. Kim, V. M. Dong, J. Am. Chem. Soc. 2009, 131, 1560815609; b) K. Kundu, J. V. McCullagh, A. T. Morehead, J. Am. Chem. Soc. 2005, 127, 16042-16043.

[104] J. Yang, A. Rérat, Y. J. Lim, C. Gosmini, N. Yoshikai, Angew. Chem. Int. Ed. 2017, 56, 2449-2453.

[105] T. Yamakawa, N. Yoshikai, Chem. Asian J. 2014, 9, 1242-1246.

[106] P.-S. Lee, N. Yoshikai, Org. Lett. 2015, 17, 22-25. 
[107] D. K. Kim, J. Riedel, R. S. Kim, V. M. Dong, J. Am. Chem. Soc. 2017, 139, 10208-10211.

[108] a) A. Fürstner, ACS Cent. Sci. 2016, 2, 778-789; b) I. Bauer, H.-J. Knölker, Chem. Rev. 2015, 115, 3170-3387; c) B. Plietker, Iron Catalysis in Organic Chemistry, Wiley-VCH, Weinheim, 2008; d) S. Enthaler, K. Junge, M. Beller, Angew. Chem. Int. Ed. 2008, 47, 3317-3321; e) C. Bolm, J. Legros, J. Le Paih, L. Zani, Chem. Rev. 2004, 104, 6217-6254.

[109] L. Mond, F. Quincke, J. Chem. Soc., Trans. 1891, 59, 604-607.

[110] M. Berthelot, Hebd. Séances Acad. Sci. 1891, 112, 1343-1349.

[111] M. S. Kharasch, P. O. Tawney, J. Am. Chem. Soc. 1941, 63, 2308-2316.

[112] T. J. Kealy, P. L. Pauson, Nature 1951, 168, 1039-1040.

[113] A. Togni, T. Hayashi, Ferrocenes: Homogeneous Catalysis, Organic Synthesis, Materials Science, Wiley-VCH, Weinheim, 1995.

[114] W. Reppe, H. Vetter, Liebigs Ann. Chem. 1953, 582, 133-161.

[115] a) M. Tamura, J. K. Kochi, J. Am. Chem. Soc. 1971, 93, 1487-1489; b) M. Tamura, J. Kochi, Synthesis 1971, 303-305.

[116] G. Hata, H. Kondo, A. Miyake, J. Am. Chem. Soc. 1968, 90, 2278-2281.

[117] a) M. V. Baker, L. D. Field, J. Am. Chem. Soc. 1987, 109, 2825-2826; b) J. W. Rathke, E. L. Muetterties, J. Am. Chem. Soc. 1975, 97, 3272-3273.

[118] H. H. Karsch, H.-F. Klein, H. Schmidbaur, Angew. Chem. Int. Ed. 1975, 14, 637-638.

[119] S. Camadanli, R. Beck, U. Flörke, H.-F. Klein, Organometallics 2009, 28, 2300-2310.

[120] W. D. Jones, G. P. Foster, J. M. Putinas, J. Am. Chem. Soc. 1987, 109, 5047-5048.

[121] N. Kimura, T. Kochi, F. Kakiuchi, J. Am. Chem. Soc. 2017, 139, 1484914852.

[122] R. Shang, L. llies, E. Nakamura, J. Am. Chem. Soc. 2016, 138, 1013210135. 
[123] J. Norinder, A. Matsumoto, N. Yoshikai, E. Nakamura, J. Am. Chem. Soc. 2008, 130, 5858-5859.

[124] E. Nakamura, Two stories of iron https://chemistrycommunity.nature.com/users/209773-eiichinakamura/posts/246018-two-stories-of-iron (consulted on 17.04.2019).

[125] a) J. J. Sirois, R. Davis, B. DeBoef, Org. Lett. 2014, 16, 868-871; b) N. Yoshikai, A. Matsumoto, J. Norinder, E. Nakamura, Angew. Chem. Int. Ed. 2009, 48, 2925-2928.

[126] L. Ilies, E. Konno, Q. Chen, E. Nakamura, Asian J. Org. Chem. 2012, 1, $142-$ 145.

[127] N. Yoshikai, A. Matsumoto, J. Norinder, E. Nakamura, Synlett 2010, 313-316.

[128] L. llies, S. Asako, E. Nakamura, J. Am. Chem. Soc. 2011, 133, 7672-7675.

[129] N. Yoshikai, S. Asako, T. Yamakawa, L. Ilies, E. Nakamura, Chem. Asian J. 2011, 6, 3059-3065.

[130] L. Ilies, M. Kobayashi, A. Matsumoto, N. Yoshikai, E. Nakamura, Adv. Synth. Catal. 2012, 354, 593-596.

[131] Y. Sun, H. Tang, K. Chen, L. Hu, J. Yao, S. Shaik, H. Chen, J. Am. Chem. Soc. 2016, 138, 3715-3730.

[132] a) P. Ma, H. Chen, ACS Catal. 2019, 9, 1962-1972; b) J. N. Harvey, R. Poli, K. M. Smith, Coord. Chem. Rev. 2003, 238-239, 347-361; c) D. Schröder, S. Shaik, H. Schwarz, Acc. Chem. Res. 2000, 33, 139-145.

[133] H. Tang, X.-R. Huang, J. Yao, H. Chen, J. Org. Chem. 2015, 80, 4672-4682.

[134] V. G. Zaitsev, D. Shabashov, O. Daugulis, J. Am. Chem. Soc. 2005, 127, 13154-13155.

[135] R. Shang, L. Ilies, A. Matsumoto, E. Nakamura, J. Am. Chem. Soc. 2013, $135,6030-6032$.

[136] Q. Gu, H. H. Al Mamari, K. Graczyk, E. Diers, L. Ackermann, Angew. Chem. Int. Ed. 2014, 53, 3868-3871. 
[137] G. Cera, T. Haven, L. Ackermann, Angew. Chem. Int. Ed. 2016, 55, 14841488.

[138] a) L. Ilies, S. Ichikawa, S. Asako, T. Matsubara, E. Nakamura, Adv. Synth. Catal. 2015, 357, 2175-2179; b) K. Graczyk, T. Haven, L. Ackermann, Chem. Eur. J. 2015, 21, 8812-8815; c) L. Ilies, T. Matsubara, S. Ichikawa, S. Asako, E. Nakamura, J. Am. Chem. Soc. 2014, 136, 13126-13129; d) E. R. Fruchey, B. M. Monks, S. P. Cook, J. Am. Chem. Soc. 2014, 136, 13130-13133; e) B. M. Monks, E. R. Fruchey, S. P. Cook, Angew. Chem. Int. Ed. 2014, 53, 11065-11069.

[139] S. Asako, L. llies, E. Nakamura, J. Am. Chem. Soc. 2013, 135, 17755-17757.

[140] G. Cera, T. Haven, L. Ackermann, Chem. Eur. J. 2017, 23, 3577-3582.

[141] T. Matsubara, S. Asako, L. Ilies, E. Nakamura, J. Am. Chem. Soc. 2014, 136, 646-649.

[142] a) L. Ilies, Y. Itabashi, R. Shang, E. Nakamura, ACS Catal. 2017, 7, 89-92; b) R. Shang, L. llies, S. Asako, E. Nakamura, J. Am. Chem. Soc. 2014, 136, 14349-14352.

[143] R. Shang, L. llies, E. Nakamura, J. Am. Chem. Soc. 2015, 137, 7660-7663.

[144] a) L. Ilies, Y. Arslanoglu, T. Matsubara, E. Nakamura, Asian J. Org. Chem. 2018, 7, 1327-1329; b) G. Cera, T. Haven, L. Ackermann, Chem. Commun. 2017, 53, 6460-6463; c) T. Matsubara, L. Ilies, E. Nakamura, Chem. Asian J. 2016, 11, 380-384.

[145] J. Mo, T. Müller, J. C. A. Oliveira, L. Ackermann, Angew. Chem. Int. Ed. 2018, $57,7719-7723$.

[146] Z. Shen, G. Cera, T. Haven, L. Ackermann, Org. Lett. 2017, 19, 3795-3798.

[147] T. Doba, T. Matsubara, L. llies, R. Shang, E. Nakamura, Nat. Catal. 2019, 2, 400-406.

[148] M. Y. Wong, T. Yamakawa, N. Yoshikai, Org. Lett. 2015, 17, 442-445.

[149] M. Jin, L. Adak, M. Nakamura, J. Am. Chem. Soc. 2015, 137, 7128-7134. 
[150] T. Iwamoto, C. Okuzono, L. Adak, M. Jin, M. Nakamura, Chem. Commun. 2019, 55, 1128-1131.

[151] J. Loup, D. Zell, J. C. A. Oliveira, H. Keil, D. Stalke, L. Ackermann, Angew. Chem. Int. Ed. 2017, 56, 14197-14201.

[152] D. Schmiel, H. Butenschön, Organometallics 2017, 36, 4979-4989.

[153] a) R. Shi, Z. Zhang, X. Hu, Acc. Chem. Res. 2019, 52, 1471-1483; b) E. A. Standley, S. Z. Tasker, K. L. Jensen, T. F. Jamison, Acc. Chem. Res. 2015, 48, 1503-1514; c) V. P. Ananikov, ACS Catal. 2015, 5, 1964-1971; d) S. Z. Tasker, E. A. Standley, T. F. Jamison, Nature 2014, 509, 299-309; e) Y. Tamaru, Modern organonickel chemistry, Wiley-VCH, Weinheim, 2005.

[154] E. Richmond, J. Moran, Synthesis 2018, 50, 499-513.

[155] a) D. Ghorai, J. Loup, G. Zanoni, L. Ackermann, Synlett 2019, 30, 429-432;

b) C. Zarate, M. van Gemmeren, R. J. Somerville, R. Martin, Adv. Organomet. Chem. 2016, 66, 143-222; c) M. Tobisu, N. Chatani, Acc. Chem. Res. 2015, 48, 1717-1726; d) E. J. Tollefson, L. E. Hanna, E. R. Jarvo, Acc. Chem. Res. 2015, 48, 2344-2353; e) J. Cornella, C. Zarate, R. Martin, Chem. Soc. Rev. 2014, 43, 8081-8097.

[156] a) O. Eisenstein, J. Milani, R. N. Perutz, Chem. Rev. 2017, 117, 8710-8753;

b) T. Ahrens, J. Kohlmann, M. Ahrens, T. Braun, Chem. Rev. 2015, 115, 931972; c) L. Ackermann, R. Born, J. H. Spatz, D. Meyer, Angew. Chem. Int. Ed. 2005, 44, 7216-7219.

[157] J. P. Kleiman, M. Dubeck, J. Am. Chem. Soc. 1963, 85, 1544-1545.

[158] For examples of $\mathrm{C}-\mathrm{H}$ nickelation of activated positions, see: a) A. L. Keen, S. A. Johnson, J. Am. Chem. Soc. 2006, 128, 1806-1807; b) N. M. Brunkan, D. M. Brestensky, W. D. Jones, J. Am. Chem. Soc. 2004, 126, 3627-3641; c) N. D. Clement, K. J. Cavell, C. Jones, C. J. Elsevier, Angew. Chem. Int. Ed. 2004, 43, 1277-1279; d) N. D. Clement, K. J. Cavell, Angew. Chem. Int. Ed. 2004, 43, 3845-3847; e) S. Ogoshi, M. Ueta, M.-a. Oka, H. Kurosawa, Chem. Commun. 2004, 2732-2733; f) T. Tsuda, T. Kiyoi, T. Saegusa, J. Org. Chem. 1990, 55, 2554-2558. 
[159] L.-C. Liang, P.-S. Chien, Y.-L. Huang, J. Am. Chem. Soc. 2006, 128, 1556215563.

[160] Y. Nakao, K. S. Kanyiva, S. Oda, T. Hiyama, J. Am. Chem. Soc. 2006, 128, 8146-8147.

[161] A. J. Nett, W. Zhao, P. M. Zimmerman, J. Montgomery, J. Am. Chem. Soc. 2015, 137, 7636-7639.

[162] T. Mukai, K. Hirano, T. Satoh, M. Miura, J. Org. Chem. 2009, 74, 6410-6413.

[163] K. S. Kanyiva, Y. Nakao, T. Hiyama, Heterocycles 2007, 72, 677-680.

[164] K. S. Kanyiva, F. Löbermann, Y. Nakao, T. Hiyama, Tetrahedron Lett. 2009, 50, 3463-3466.

[165] K. S. Kanyiva, Y. Nakao, T. Hiyama, Angew. Chem. Int. Ed. 2007, 46, 88728874.

[166] a) C.-C. Tsai, W.-C. Shih, C.-H. Fang, C.-Y. Li, T.-G. Ong, G. P. A. Yap, J. Am. Chem. Soc. 2010, 132, 11887-11889; b) Y. Nakao, K. S. Kanyiva, T. Hiyama, J. Am. Chem. Soc. 2008, 130, 2448-2449.

[167] Y. Nakao, H. Idei, K. S. Kanyiva, T. Hiyama, J. Am. Chem. Soc. 2009, 131, 15996-15997.

[168] Y. Nakao, N. Kashihara, K. S. Kanyiva, T. Hiyama, J. Am. Chem. Soc. 2008, 130, 16170-16171.

[169] Y. Nakao, H. Idei, K. S. Kanyiva, T. Hiyama, J. Am. Chem. Soc. 2009, 131, 5070-5071.

[170] S. Nakanowatari, T. Müller, J. C. A. Oliveira, L. Ackermann, Angew. Chem. Int. Ed. 2017, 56, 15891-15895.

[171] a) K. Muto, T. Hatakeyama, J. Yamaguchi, K. Itami, Chem. Sci. 2015, 6, 6792-6798; b) K. Muto, J. Yamaguchi, K. Itami, J. Am. Chem. Soc. 2012, 134, 169-172; c) K. Amaike, K. Muto, J. Yamaguchi, K. Itami, J. Am. Chem. Soc. 2012, 134, 13573-13576; d) H. Hitoshi, H. Koji, S. Tetsuya, M. Masahiro, ChemCatChem 2010, 2, 1403-1406; e) J. Canivet, J. Yamaguchi, I. Ban, K. Itami, Org. Lett. 2009, 11, 1733-1736; f) H. Hachiya, K. Hirano, T. Satoh, M. Miura, Org. Lett. 2009, 11, 1737-1740. 
[172] a) T. Yao, K. Hirano, T. Satoh, M. Miura, Angew. Chem. Int. Ed. 2012, 51, 775-779; b) L. Ackermann, B. Punji, W. Song, Adv. Synth. Catal. 2011, 353, 3325-3329; c) O. Vechorkin, V. Proust, X. Hu, Angew. Chem. Int. Ed. 2010, 49, 3061-3064; d) T. Yao, K. Hirano, T. Satoh, M. Miura, Chem. Eur. J. 2010, $16,12307-12311$.

[173] N. Matsuyama, K. Hirano, T. Satoh, M. Miura, Org. Lett. 2009, 11, 41564159.

[174] N. Matsuyama, M. Kitahara, K. Hirano, T. Satoh, M. Miura, Org. Lett. 2010, 12, 2358-2361.

[175] H. Hachiya, K. Hirano, T. Satoh, M. Miura, Angew. Chem. Int. Ed. 2010, 49, 2202-2205.

[176] N. Chatani, Top. Organomet. Chem. 2016, 56, 19-46.

[177] A. Yokota, Y. Aihara, N. Chatani, J. Org. Chem. 2014, 79, 11922-11932.

[178] a) T. Uemura, M. Yamaguchi, N. Chatani, Angew. Chem. Int. Ed. 2016, 55, 3162-3165; b) Y. Aihara, J. Wuelbern, N. Chatani, Bull. Chem. Soc. Jpn. 2015, 88, 438-446; c) W. Song, S. Lackner, L. Ackermann, Angew. Chem. Int. Ed. 2014, 53, 2477-2480; d) Y. Aihara, N. Chatani, J. Am. Chem. Soc. 2013, 135, 5308-5311.

[179] a) V. G. Landge, C. H. Shewale, G. Jaiswal, M. K. Sahoo, S. P. Midya, E. Balaraman, Catal. Sci. Technol. 2016, 6, 1946-1951; b) Y.-J. Liu, Y.-H. Liu, S.-Y. Yan, B.-F. Shi, Chem. Commun. 2015, 51, 6388-6391; c) J. Yi, L. Yang, C. Xia, F. Li, J. Org. Chem. 2015, 80, 6213-6221.

[180] Y.-H. Liu, Y.-J. Liu, S.-Y. Yan, B.-F. Shi, Chem. Commun. 2015, 51, 1165011653.

[181] H. Shiota, Y. Ano, Y. Aihara, Y. Fukumoto, N. Chatani, J. Am. Chem. Soc. 2011, 133, 14952-14955.

[182] a) N. Barsu, D. Kalsi, B. Sundararaju, Chem. Eur. J. 2015, 21, 9364-9368; b) X. Cong, Y. Li, Y. Wei, X. Zeng, Org. Lett. 2014, 16, 3926-3929.

[183] X. Wu, Y. Zhao, H. Ge, J. Am. Chem. Soc. 2015, 137, 4924-4927. 
[184] a) V. P. Reddy, R. Qiu, T. Iwasaki, N. Kambe, Org. Biomol. Chem. 2015, 13, 6803-6813; b) S.-Y. Yan, Y.-J. Liu, B. Liu, Y.-H. Liu, B.-F. Shi, Chem. Commun. 2015, 51, 4069-4072; c) K. Yang, Y. Wang, X. Chen, A. A. Kadi, H.-K. Fun, H. Sun, Y. Zhang, H. Lu, Chem. Commun. 2015, 51, 3582-3585.

[185] a) D. Ghorai, L. H. Finger, G. Zanoni, L. Ackermann, ACS Catal. 2018, 8, 11657-11662; b) Z. Ruan, D. Ghorai, G. Zanoni, L. Ackermann, Chem. Commun. 2017, 53, 9113-9116; c) Z. Ruan, S. Lackner, L. Ackermann, Angew. Chem. Int. Ed. 2016, 55, 3153-3157; d) Z. Ruan, S. Lackner, L. Ackermann, ACS Catal. 2016, 6, 4690-4693; e) T. Müller, L. Ackermann, Chem. Eur. J. 2016, 22, 14151-14154.

[186] a) R. A. Jagtap, C. P. Vinod, B. Punji, ACS Catal. 2019, 9, 431-441; b) S. M. Khake, S. Jain, U. N. Patel, R. G. Gonnade, K. Vanka, B. Punji, Organometallics 2018, 37, 2037-2045; c) S. M. Khake, V. Soni, R. G. Gonnade, B. Punji, Chem. Eur. J. 2017, 23, 2907-2914; d) V. Soni, R. A. Jagtap, R. G. Gonnade, B. Punji, ACS Catal. 2016, 6, 5666-5672.

[187] Z. Dong, Z. Ren, S. J. Thompson, Y. Xu, G. Dong, Chem. Rev. 2017, 117, 9333-9403.

[188] a) Y. Nakao, Chem. Rec. 2011, 11, 242-251; b) P. W. N. M. van Leeuwen, in Stereoselective Synthesis 1, Vol. 1 (Ed.: J. G. de Vries), Georg Thieme Verlag, Stuttgart, 2011, pp. 409-475.

[189] Y. Nakao, N. Kashihara, K. S. Kanyiva, T. Hiyama, Angew. Chem. Int. Ed. 2010, 49, 4451-4454.

[190] Y.-Y. Jiang, Z. Li, J. Shi, Organometallics 2012, 31, 4356-4366.

[191] R. Tamura, Y. Yamada, Y. Nakao, T. Hiyama, Angew. Chem. Int. Ed. 2012, $51,5679-5682$.

[192] Y. Nakao, Y. Yamada, N. Kashihara, T. Hiyama, J. Am. Chem. Soc. 2010, 132, 13666-13668.

[193] P. A. Donets, N. Cramer, Angew. Chem. Int. Ed. 2015, 54, 633-637. 
[194] a) W.-C. Chen, Y.-C. Lai, W.-C. Shih, M.-S. Yu, G. P. A. Yap, T.-G. Ong, Chem. Eur. J. 2014, 20, 8099-8105; b) W.-C. Shih, W.-C. Chen, Y.-C. Lai, M.-S. Yu, J.-J. Ho, G. P. A. Yap, T.-G. Ong, Org. Lett. 2012, 14, 2046-2049.

[195] P. A. Donets, N. Cramer, J. Am. Chem. Soc. 2013, 135, 11772-11775.

[196] a) S. K. Murphy, V. M. Dong, Chem. Comm. 2014, 50, 13645-13649; b) J. C. Leung, M. J. Krische, Chem. Sci. 2012, 3, 2202-2209; c) M. C. Willis, Chem. Rev. 2010, 110, 725-748.

[197] a) T. M. Shaikh, C.-M. Weng, F.-E. Hong, Coord. Chem. Rev. 2012, 256, 771-803; b) L. Ackermann, Isr. J. Chem. 2010, 50, 652-663; c) L. Ackermann, in Trivalent Phosphorus Compounds in Asymmetric Catalysis, Sythesis and Applications (Ed.: A. Börner), Wiley-VCH, Weinheim, 2008, pp. 831-847; d) L. Ackermann, Synthesis 2006, 1557-1571.

[198] Y.-X. Wang, M. Ye, Sci. China Chem. 2018, 61, 1004-1013.

[199] D. Hirsch-Weil, K. A. Abboud, S. Hong, Chem. Commun. 2010, 46, 75257527.

[200] J. Diesel, A. M. Finogenova, N. Cramer, J. Am. Chem. Soc. 2018, 140, 44894493.

[201] a) A. Albright, D. Eddings, R. Black, C. J. Welch, N. N. Gerasimchuk, R. E. Gawley, J. Org. Chem. 2011, 76, 7341-7351; b) A. Albright, R. E. Gawley, J. Am. Chem. Soc. 2011, 133, 19680-19683.

[202] a) V. Singh, Y. Nakao, S. Sakaki, M. M. Deshmukh, J. Org. Chem. 2017, 82, 289-301; b) A. J. Nett, J. Montgomery, P. M. Zimmerman, ACS Catal. 2017, 7, 7352-7362; c) J. Guihaumé, S. Halbert, O. Eisenstein, R. N. Perutz, Organometallics 2012, 31, 1300-1314.

[203] W.-B. Zhang, X.-T. Yang, J.-B. Ma, Z.-M. Su, S.-L. Shi, J. Am. Chem. Soc. 2019, 141, 5628-5634.

[204] a) D. A. Colby, R. G. Bergman, J. A. Ellman, Chem. Rev. 2010, 110, 624655; b) J. C. Lewis, R. G. Bergman, J. A. Ellman, Acc. Chem. Res. 2008, 41, 1013-1025. 
[205] A. T. Normand, S. K. Yen, H. V. Huynh, T. S. A. Hor, K. J. Cavell, Organometallics 2008, 27, 3153-3160.

[206] Y.-X. Wang, S.-L. Qi, Y.-X. Luan, X.-W. Han, S. Wang, H. Chen, M. Ye, J. Am. Chem. Soc. 2018, 140, 5360-5364.

[207] X. Linghu, J. R. Potnick, J. S. Johnson, J. Am. Chem. Soc. 2004, 126, 30703071.

[208] Q.-S. Liu, D.-Y. Wang, Z.-J. Yang, Y.-X. Luan, J.-F. Yang, J.-F. Li, Y.-G. Pu, M. Ye, J. Am. Chem. Soc. 2017, 139, 18150-18153.

[209] K. Ogata, Y. Atsuumi, D. Shimada, S.-i. Fukuzawa, Angew. Chem. Int. Ed. 2011, 50, 5896-5899.

[210] J. S. E. Ahlin, N. Cramer, Org. Lett. 2016, 18, 3242-3245.

[211] T. J. Seiders, D. W. Ward, R. H. Grubbs, Org. Lett. 2001, 3, 3225-3228.

[212] a) S. Grélaud, P. Cooper, L. J. Feron, J. F. Bower, J. Am. Chem. Soc. 2018, 140, 9351-9356; b) T. Shibata, M. Michino, H. Kurita, Y.-k. Tahara, K. S. Kanyiva, Chem. Eur. J. 2017, 23, 88-91; c) M. Hatano, Y. Ebe, T. Nishimura, H. Yorimitsu, J. Am. Chem. Soc. 2016, 138, 4010-4013; d) T. Shibata, N. Ryu, H. Takano, Adv. Synth. Catal. 2015, 357, 1131-1135; e) Y. Ebe, T. Nishimura, J. Am. Chem. Soc. 2015, 137, 5899-5902; f) C. S. Sevov, J. F. Hartwig, J. Am. Chem. Soc. 2013, 135, 2116-2119; g) S. Pan, N. Ryu, T. Shibata, J. Am. Chem. Soc. 2012, 134, 17474-17477; h) R. Dorta, A. Togni, Chem. Commun. 2003, 760-761.

[213] a) C. M. Filloux, T. Rovis, J. Am. Chem. Soc. 2015, 137, 508-517; b) B. Ye, P. A. Donets, N. Cramer, Angew. Chem. Int. Ed. 2014, 53, 507-511; c) B. Ye, N. Cramer, J. Am. Chem. Soc. 2013, 135, 636-639; d) A. S. Tsai, R. M. Wilson, H. Harada, R. G. Bergman, J. A. Ellman, Chem. Commun. 2009, 3910-3912; e) H. Harada, R. K. Thalji, R. G. Bergman, J. A. Ellman, J. Org. Chem. 2008, 73, 6772-6779; f) R. M. Wilson, R. K. Thalji, R. G. Bergman, J. A. Ellman, Org. Lett. 2006, 8, 1745-1747; g) R. K. Thalji, J. A. Ellman, R. G. Bergman, J. Am. Chem. Soc. 2004, 126, 7192-7193; h) F. Kakiuchi, P. Le Gendre, A. Yamada, H. Ohtaki, S. Murai, Tetrahedron: Asymmetry 2000, 11, 
2647-2651; i) N. Fujii, F. Kakiuchi, A. Yamada, N. Chatani, S. Murai, Chem. Lett. 1997, 26, 425-426.

[214] S. Rodewald, R. F. Jordan, J. Am. Chem. Soc. 1994, 116, 4491-4492.

[215] G. Song, W. W. N. O, Z. Hou, J. Am. Chem. Soc. 2014, 136, 12209-12212.

[216] a) Y. Segawa, T. Maekawa, K. Itami, Angew. Chem. Int. Ed. 2015, 54, 66-81;

b) L. McMurray, F. O'Hara, M. J. Gaunt, Chem. Soc. Rev. 2011, 40, 18851898; c) O. Baudoin, Chem. Soc. Rev. 2011, 40, 4902-4911.

[217] a) D. Hazelard, P.-A. Nocquet, P. Compain, Org. Chem. Front. 2017, 4, 2500-2521; b) P. Subramanian, G. C. Rudolf, K. P. Kaliappan, Chem. Asian J. 2016, 11, 168-192; c) M.-L. Louillat, F. W. Patureau, Chem. Soc. Rev. 2014, 43, 901-910; d) F. Collet, C. Lescot, P. Dauban, Chem. Soc. Rev. 2011, 40, 1926-1936.

[218] J. Loup, T. Parchomyk, S. Lülf, S. Demeshko, F. Meyer, K. Koszinowski, L. Ackermann, Dalton Trans. 2019, 48, 5135-5139.

[219] a) J. C. Rech, M. Yato, D. Duckett, B. Ember, P. V. LoGrasso, R. G. Bergman, J. A. Ellman, J. Am. Chem. Soc. 2007, 129, 490-491; b) K. L. Tan, A. Vasudevan, R. G. Bergman, J. A. Ellman, A. J. Souers, Org. Lett. 2003, 5, 2131-2134; c) K. L. Tan, R. G. Bergman, J. A. Ellman, J. Am. Chem. Soc. 2002, 124, 3202-3203; d) K. L. Tan, R. G. Bergman, J. A. Ellman, J. Am. Chem. Soc. 2001, 123, 2685-2686.

[220] J. Loup, V. Müller, D. Ghorai, L. Ackermann, Angew. Chem. Int. Ed. 2019, 58, 1749-1753.

[221] a) K. M. Nelson, C. E. Salomon, C. C. Aldrich, J. Nat. Prod. 2012, 75, 10371043; b) Q. Li, K. W. Woods, A. Claiborne, I. I. S. L. Gwaltney, K. J. Barr, G. Liu, L. Gehrke, R. B. Credo, Y. H. Hui, J. Lee, R. B. Warner, P. Kovar, M. A. Nukkala, N. A. Zielinski, S. K. Tahir, M. Fitzgerald, K. H. Kim, K. Marsh, D. Frost, S.-C. Ng, S. Rosenberg, H. L. Sham, Bioorg. Med. Chem. Lett. 2002, 12, 465-469; c) G. A. Snow, Biochem J. 1965, 97, 166-175.

[222] a) D. Clark, B. Finkelstein, G. Lahm, T. Selby, T. Stevenson, WO 2003016304 A1, 2003; b) J. Suzuki, T. Ishida, I. Shibuya, K. Toda, J. Pestic. Sci. 2001, 26, 215-223. 
[223] a) T. G. Gant, A. I. Meyers, Tetrahedron 1994, 50, 2297-2360; b) A. I. Meyers, E. D. Mihelich, Angew. Chem. Int. Ed. 1976, 15, 270-281; c) J. A. Frump, Chem. Rev. 1971, 71, 483-505.

[224] a) G. Yang, W. Zhang, Chem. Soc. Rev. 2018, 47, 1783-1810; b) G. Desimoni, G. Faita, K. A. Jørgensen, Chem. Rev. 2011, 111, PR284-PR437; c) C. C. Bausch, A. Pfaltz, in Privileged Chiral Ligands and Catalysts (Ed.: Q. L. Zhou), Wiley-VCH, Weinheim, 2011, pp. 221-256; d) G. C. Hargaden, P. J. Guiry, Chem. Rev. 2009, 109, 2505-2550; e) G. Desimoni, G. Faita, K. A. Jørgensen, Chem. Rev. 2006, 106, 3561-3651; f) H. A. McManus, P. J. Guiry, Chem. Rev. 2004, 104, 4151-4202; g) G. Desimoni, G. Faita, P. Quadrelli, Chem. Rev. 2003, 103, 3119-3154.

[225] a) L. Gupta, A. C. Hoepker, K. J. Singh, D. B. Collum, J. Org. Chem. 2009, 74, 2231-2233; b) C. G. Hartung, V. Snieckus, in Modern Arene Chemistry (Ed.: D. Astruc), Wiley-VCH, Weinheim, 2002, pp. 330-367; c) E. Wehman, G. Van Koten, J. T. B. H. Jastrzebski, M. A. Rotteveel, C. H. Stam, Organometallics 1988, 7, 1477-1485.

[226] Y. Park, S. Jee, J. G. Kim, S. Chang, Org. Process Res. Dev. 2015, 19, 1024-1029.

[227] a) R. Mei, Doctoral thesis, Georg-August-Universität Göttingen, 2017; b) R. Mei, L. Ackermann, unpublished results.

[228] a) P. Dubé, N. F. F. Nathel, M. Vetelino, M. Couturier, C. L. Aboussafy, S. Pichette, M. L. Jorgensen, M. Hardink, Org. Lett. 2009, 11, 5622-5625; b) E. Eibler, J. Käsbauer, H. Pohl, J. Sauer, Tetrahedron Lett. 1987, 28, 1097$1100 ;$ c) J. Sauer, K. K. Mayer, Tetrahedron Lett. 1968, 9, 319-324.

[229] a) V. Bizet, C. Bolm, Eur. J. Org. Chem. 2015, 2854-2860; b) V. Bizet, L. Buglioni, C. Bolm, Angew. Chem. Int. Ed. 2014, 53, 5639-5642.

[230] T. K. Hyster, D. M. Dalton, T. Rovis, Chem. Sci. 2015, 6, 254-258.

[231] X.-K. Guo, L.-B. Zhang, D. Wei, J.-L. Niu, Chem. Sci. 2015, 6, 7059-7071.

[232] a) H. Pellissier, Coord. Chem. Rev. 2019, 386, 1-31; b) K. Gopalaiah, Chem. Rev. 2013, 113, 3248-3296. 
[233] a) N. V. Tkachenko, O. Y. Lyakin, D. G. Samsonenko, E. P. Talsi, K. P. Bryliakov, Catal. Commun. 2018, 104, 112-117; b) S. Narute, D. Pappo, Org. Lett. 2017, 19, 2917-2920; c) S. Narute, R. Parnes, F. D. Toste, D. Pappo, J. Am. Chem. Soc. 2016, 138, 16553-16560; d) H. Egami, K. Matsumoto, T. Oguma, T. Kunisu, T. Katsuki, J. Am. Chem. Soc. 2010, 132, 13633-13635.

[234] J. T. Groves, P. Viski, J. Am. Chem. Soc. 1989, 111, 8537-8538.

[235] a) C. Johnson, M. Albrecht, Coord. Chem. Rev. 2017, 352, 1-14; b) K. Riener, S. Haslinger, A. Raba, M. P. Högerl, M. Cokoja, W. A. Herrmann, F. E. Kühn, Chem. Rev. 2014, 114, 5215-5272; c) D. Bézier, J.-B. Sortais, C. Darcel, Adv. Synth. Catal. 2013, 355, 19-33; d) F. Wang, L.-j. Liu, W. Wang, S. Li, M. Shi, Coord. Chem. Rev. 2012, 256, 804-853.

[236] D. Janssen-Müller, C. Schlepphorst, F. Glorius, Chem. Soc. Rev. 2017, 46, 4845-4854.

[237] V. R. Yatham, W. Harnying, D. Kootz, J.-M. Neudörfl, N. E. Schlörer, A. Berkessel, J. Am. Chem. Soc. 2016, 138, 2670-2677.

[238] V. Lavallo, Y. Canac, C. Präsang, B. Donnadieu, G. Bertrand, Angew. Chem. Int. Ed. 2005, 44, 5705-5709.

[239] a) L. Ackermann, A. R. Kapdi, C. Schulzke, Org. Lett. 2010, 12, 2298-2301; b) L. Ackermann, R. Born, Angew. Chem. Int. Ed. 2005, 44, 2444-2447; c) L. Ackermann, Org. Lett. 2005, 7, 3123-3125.

[240] a) E. McNeill, T. Ritter, Acc. Chem. Res. 2015, 48, 2330-2343; b) M. D. Greenhalgh, A. S. Jones, S. P. Thomas, ChemCatChem 2015, 7, 190-222.

[241] J. S. E. Ahlin, P. A. Donets, N. Cramer, Angew. Chem. Int. Ed. 2014, 53, 13229-13233.

[242] Selected examples: a) H. Wang, G. Lu, G. J. Sormunen, H. A. Malik, P. Liu, J. Montgomery, J. Am. Chem. Soc. 2017, 139, 9317-9324; b) R. Savka, M. Bergmann, Y. Kanai, S. Foro, H. Plenio, Chem. Eur. J. 2016, 22, 9667-9675; c) C.-Y. Ho, C.-W. Chan, L. He, Angew. Chem. Int. Ed. 2015, 54, 4512-4516; d) L. Wu, L. Falivene, E. Drinkel, S. Grant, A. Linden, L. Cavallo, R. Dorta, Angew. Chem. Int. Ed. 2012, 51, 2870-2873; e) T. W. Funk, J. M. Berlin, R. 
H. Grubbs, J. Am. Chem. Soc. 2006, 128, 1840-1846; f) J. M. Berlin, S. D. Goldberg, R. H. Grubbs, Angew. Chem. Int. Ed. 2006, 45, 7591-7595.

[243] M.-A. Abadie, K. MacIntyre, C. Boulho, P. Hoggan, F. Capet, F. AgbossouNiedercorn, C. Michon, Organometallics 2019, 38, 536-543.

[244] Selected references: a) Y. Zhou, Y. Shi, S. Torker, A. H. Hoveyda, J. Am. Chem. Soc. 2018, 140, 16842-16854; b) V. Pace, J. P. Rae, H. Y. Harb, D. J. Procter, Chem. Commun. 2013, 49, 5150-5152; c) K.-s. Lee, A. H. Hoveyda, J. Org. Chem. 2009, 74, 4455-4462.

[245] a) R. Agata, T. Iwamoto, N. Nakagawa, K. Isozaki, T. Hatakeyama, H. Takaya, M. Nakamura, Synthesis 2015, 47, 1733-1740; b) T. Hatakeyama, S. Hashimoto, K. Ishizuka, M. Nakamura, J. Am. Chem. Soc. 2009, 131, 1194911963; c) T. Hatakeyama, M. Nakamura, J. Am. Chem. Soc. 2007, 129, 9844-9845.

[246] a) H. Blaser, B. Pugin, F. Spindler, E. Mejía, A. Togni, in Privileged Chiral Ligands and Catalysts (Ed.: Q. L. Zhou), Wiley-VCH, Weinheim, 2011, pp. 93-136; b) L.-X. Dai, X.-L. Hou, Chiral Ferrocenes in Asymmetric Catalysis: Synthesis and Applications, Wiley-VCH, Weinheim, 2010.

[247] a) R. Pietschnig, Chem. Soc. Rev. 2016, 45, 5216-5231; b) S. Barlow, S. R. Marder, in Functional Organic Materials (Eds.: T. J. J. Müller, U. H. F. Bunz), Wiley-VCH, Weinheim, 2007, pp. 393-437.

[248] a) M. Patra, G. Gasser, Nat. Rev. Chem. 2017, 1, 0066; b) K. Kowalski, Coord. Chem. Rev. 2016, 317, 132-156; c) G. Jaouen, A. Vessières, S. Top, Chem. Soc. Rev. 2015, 44, 8802-8817; d) D. R. van Staveren, N. MetzlerNolte, Chem. Rev. 2004, 104, 5931-5986.

[249] a) D. Katayev, E. Larionov, M. Nakanishi, C. Besnard, E. P. Kündig, Chem. Eur. J. 2014, 20, 15021-15030; b) E. P. Kündig, T. M. Seidel, Y.-x. Jia, G. Bernardinelli, Angew. Chem. Int. Ed. 2007, 46, 8484-8487.

[250] J. S. Renny, L. L. Tomasevich, E. H. Tallmadge, D. B. Collum, Angew. Chem. Int. Ed. 2013, 52, 11998-12013.

[251] a) M. D. Greenhalgh, S. P. Thomas, J. Am. Chem. Soc. 2012, 134, 11900 11903; b) E. Shirakawa, D. Ikeda, S. Masui, M. Yoshida, T. Hayashi, J. Am. 
Chem. Soc. 2012, 134, 272-279; c) E. Shirakawa, D. Ikeda, S. Yamaguchi, T. Hayashi, Chem. Commun. 2008, 1214-1216; d) J. Vela, S. Vaddadi, T. R. Cundari, J. M. Smith, E. A. Gregory, R. J. Lachicotte, C. J. Flaschenriem, P. L. Holland, Organometallics 2004, 23, 5226-5239; e) J. Vela, J. M. Smith, R. J. Lachicotte, P. L. Holland, Chem. Commun. 2002, 2886-2887; f) D. L. Reger, E. C. Culbertson, Inorg. Chem. 1977, 16, 3104-3107.

[252] V. Prelog, G. Helmchen, Angew. Chem. Int. Ed. 1982, 21, 567-583.

[253] K. M. Engle, T.-S. Mei, M. Wasa, J.-Q. Yu, Acc. Chem. Res. 2012, 45, 788802.

[254] V. Lanke, K. Ramaiah Prabhu, Org. Lett. 2013, 15, 6262-6265.

[255] R. Manikandan, M. Jeganmohan, Chem. Commun. 2017, 53, 8931-8947.

[256] J. C. A. Oliveira, L. Ackermann, unpublished results.

[257] E. M. Simmons, J. F. Hartwig, Angew. Chem. Int. Ed. 2012, 51, 3066-3072.

[258] R. B. Bedford, M. Betham, D. W. Bruce, S. A. Davis, R. M. Frost, M. Hird, Chem. Commun. 2006, 1398-1400.

[259] T. Satyanarayana, S. Abraham, H. B. Kagan, Angew. Chem. Int. Ed. 2009, 48, 456-494.

[260] B. Zhou, H. Sato, L. Ilies, E. Nakamura, ACS Catal. 2018, 8, 8-11.

[261] T. Hatanaka, Y. Ohki, K. Tatsumi, Chem. Asian J. 2010, 5, 1657-1666.

[262] A. A. Danopoulos, A. Massard, G. Frison, P. Braunstein, Angew. Chem. Int. Ed. 2018, 57, 14550-14554.

[263] a) R. Martin, A. Fürstner, Angew. Chem. Int. Ed. 2004, 43, 3955-3957; b) A. Fürstner, A. Leitner, Angew. Chem. Int. Ed. 2002, 41, 609-612; c) A. Fürstner, A. Leitner, M. Méndez, H. Krause, J. Am. Chem. Soc. 2002, 124, 13856-13863.

[264] A. Casitas, J. A. Rees, R. Goddard, E. Bill, S. DeBeer, A. Fürstner, Angew. Chem. Int. Ed. 2017, 56, 10108-10113.

[265] R. B. Bedford, Acc. Chem. Res. 2015, 48, 1485-1493. 
[266] In this context, low-valent iron species have been defined as having an oxidation state below 2+ (See Ref. [268b]).

[267] T. Parchomyk, S. Demeshko, F. Meyer, K. Koszinowski, J. Am. Chem. Soc. 2018, 140, 9709-9720.

[268] a) M. L. Neidig, S. H. Carpenter, D. J. Curran, J. C. DeMuth, V. E. Fleischauer, T. E. Iannuzzi, P. G. N. Neate, J. D. Sears, N. J. Wolford, Acc. Chem. Res. 2019, 52, 140-150; b) J. D. Sears, P. G. N. Neate, M. L. Neidig, J. Am. Chem. Soc. 2018, 140, 11872-11883; c) S. B. Muñoz III, S. L. Daifuku, J. D. Sears, T. M. Baker, S. H. Carpenter, W. W. Brennessel, M. L. Neidig, Angew. Chem. Int. Ed. 2018, 57, 6496-6500; d) S. H. Carpenter, T. M. Baker, S. B. Muñoz III, W. W. Brennessel, M. L. Neidig, Chem. Sci. 2018, 9, 7931-7939; e) T. Parchomyk, K. Koszinowski, Synthesis 2017, 49, 32693280; f) S. H. Carpenter, M. L. Neidig, Isr. J. Chem. 2017, 57, 1106-1116; g) J. L. Kneebone, W. W. Brennessel, M. L. Neidig, J. Am. Chem. Soc. 2017, 139, 6988-7003; h) S. B. Muñoz III, S. L. Daifuku, W. W. Brennessel, M. L. Neidig, J. Am. Chem. Soc. 2016, 138, 7492-7495; i) S. L. Daifuku, M. H. AlAfyouni, B. E. R. Snyder, J. L. Kneebone, M. L. Neidig, J. Am. Chem. Soc. 2014, 136, 9132-9143; j) M. H. Al-Afyouni, K. L. Fillman, W. W. Brennessel, M. L. Neidig, J. Am. Chem. Soc. 2014, 136, 15457-15460.

[269] a) T. Parchomyk, K. Koszinowski, Chem. Eur. J. 2017, 23, 3213-3219; b) T. Parchomyk, K. Koszinowski, Chem. Eur. J. 2016, 22, 15609-15613.

[270] T. Parchomyk, K. Koszinowski, Chem. Eur. J. 2018, 24, 16342-16347.

[271] P. Gütlich, E. Bill, A. X. Trautwein, Mössbauer Spectroscopy and Transition Metal Chemistry, Springer, Heidelberg, 2011.

[272] a) Y. O. Wong, L. A. Freeman, A. D. Agakidou, D. A. Dickie, C. E. Webster, R. J. Gilliard, Organometallics 2019, 38, 688-696; b) A. R. Kennedy, R. E. Mulvey, S. D. Robertson, Dalton Trans. 2010, 39, 9091-9099; and references therein.

[273] a) D. Grassi, A. Alexakis, Adv. Synth. Catal. 2015, 357, 3171-3186; b) O. Jackowski, A. Alexakis, Angew. Chem. Int. Ed. 2010, 49, 3346-3350; and references therein. 
[274] A. M. Messinis, S. L. J. Luckham, P. P. Wells, D. Gianolio, E. K. Gibson, H. M. O'Brien, H. A. Sparkes, S. A. Davis, J. Callison, D. Elorriaga, O. Hernandez-Fajardo, R. B. Bedford, Nat. Catal. 2019, 2, 123-133.

[275] C. Schnegelsberg, T. D. Blümke, K. Koszinowski, J. Mass Spectrom. 2015, 50, 1393-1395.

[276] Different fits with five subspectra are possible.

[277] a) S. B. Muñoz III, V. E. Fleischauer, W. W. Brennessel, M. L. Neidig, Organometallics 2018, 37, 3093-3101; b) V. E. Fleischauer, S. B. Muñoz III, P. G. N. Neate, W. W. Brennessel, M. L. Neidig, Chem. Sci. 2018, 9, 18781891; c) K. L. Fillman, J. A. Przyojski, M. H. Al-Afyouni, Z. J. Tonzetich, M. L. Neidig, Chem. Sci. 2015, 6, 1178-1188; d) Y. Liu, L. Wang, L. Deng, Organometallics 2015, 34, 4401-4407.

[278] K. Skowerski, C. Wierzbicka, G. Szczepaniak, Ł. Gułajski, M. Bieniek, K. Grela, Green Chem. 2012, 14, 3264-3268.

[279] F. Kraft, T. Parchomyk, K. Koszinowski, unpublished results.

[280] A. Jana, K. Grela, Chem. Commun. 2018, 54, 122-139.

[281] a) R. B. Bedford, P. B. Brenner, D. Elorriaga, J. N. Harvey, J. Nunn, Dalton Trans. 2016, 45, 15811-15817; b) R. B. Bedford, P. B. Brenner, E. Carter, P. M. Cogswell, M. F. Haddow, J. N. Harvey, D. M. Murphy, J. Nunn, C. H. Woodall, Angew. Chem. Int. Ed. 2014, 53, 1804-1808.

[282] a) J. Zhao, S. Zhang, W.-X. Zhang, Z. Xi, Coord. Chem. Rev. 2014, 270-271, 2-13; b) M. J. Wax, R. G. Bergman, J. Am. Chem. Soc. 1981, 103, 70287030 .

[283] a) G. Bringmann, T. Gulder, T. A. M. Gulder, M. Breuning, Chem. Rev. 2011, 111, 563-639; b) M. C. Kozlowski, B. J. Morgan, E. C. Linton, Chem. Soc. Rev. 2009, 38, 3193-3207.

[284] M. Berthod, G. Mignani, G. Woodward, M. Lemaire, Chem. Rev. 2005, 105, 1801-1836.

[285] Selected examples: a) H. Li, X. Yan, J. Zhang, W. Guo, J. Jiang, J. Wang, Angew. Chem. Int. Ed. 2019, 58, 6732-6736; b) J. Luo, T. Zhang, L. Wang, 
G. Liao, Q.-J. Yao, Y.-J. Wu, B.-B. Zhan, Y. Lan, X.-F. Lin, B.-F. Shi, Angew. Chem. Int. Ed. 2019, 58, 6708-6712; c) C. G. Newton, E. Braconi, J. Kuziola, M. D. Wodrich, N. Cramer, Angew. Chem. Int. Ed. 2018, 57, 11040-11044; d) Y.-S. Jang, Ł. Woźniak, J. Pedroni, N. Cramer, Angew. Chem. Int. Ed. 2018, 57, 12901-12905; e) Z.-J. Jia, C. Merten, R. Gontla, C. G. Daniliuc, A. P. Antonchick, H. Waldmann, Angew. Chem. Int. Ed. 2017, 56, 2429-2434; f) Q.-J. Yao, S. Zhang, B.-B. Zhan, B.-F. Shi, Angew. Chem. Int. Ed. 2017, 56, 6617-6621; g) J. Zheng, W.-J. Cui, C. Zheng, S.-L. You, J. Am. Chem. Soc. 2016, 138, 5242-5245; h) Y. Kuninobu, K. Yamauchi, N. Tamura, T. Seiki, K. Takai, Angew. Chem. Int. Ed. 2013, 52, 1520-1522; i) K. Yamaguchi, J. Yamaguchi, A. Studer, K. Itami, Chem. Sci. 2012, 3, 2165-2169; for a recent review, see: j) G. Liao, T. Zhou, Q.-J. Yao, B.-F. Shi, Chem. Commun. 2019, DOI:10.1039/C9CC03967H.

[286] X. Bu, P. J. Skrdla, P. G. Dormer, Y. Bereznitski, J. Chromatogr. A 2010, 1217, 7255-7264.

[287] K. S. Egorova, V. P. Ananikov, Angew. Chem. Int. Ed. 2016, 55, 1215012162.

[288] Y.-X. Wang, M. Ye, Sci. China Chem. 2018, 61, 1004-1013.

[289] a) V. Ritleng, M. Henrion, M. J. Chetcuti, ACS Catal. 2016, 6, 890-906; b) A. P. Prakasham, P. Ghosh, Inorg. Chim. Acta 2015, 431, 61-100.

[290] G. Vijaykumar, A. Jose, P. K. Vardhanapu, S. P, S. K. Mandal, Organometallics 2017, 36, 4753-4758.

[291] Results not discussed in this thesis due to issues of reproducibility.

[292] a) F. De Simone, J. Waser, Chimia 2012, 66, 233-236; b) A. Whiting, in Advanced asymmetric synthesis (Ed.: G. R. Stephenson), Chapman \& Hall, London, 1996, pp. 126-145.

[293] K. Maruoka, T. Itoh, H. Yamamoto, J. Am. Chem. Soc. 1985, 107, 45734576.

[294] a) D. Song, S. Ma, ChemMedChem 2016, 11, 646-659; b) P. Singla, V. Luxami, K. Paul, RSC Adv. 2014, 4, 12422-12440; c) M. Gaba, S. Singh, C. 
Mohan, Eur. J. Med. Chem. 2014, 76, 494-505; d) Y. Bansal, O. Silakari, Biorg. Med. Chem. 2012, 20, 6208-6236.

[295] a) T.-T. Gao, W.-W. Zhang, X. Sun, H.-X. Lu, B.-J. Li, J. Am. Chem. Soc. 2019, 141, 4670-4677; b) D. Berthold, B. Breit, Org. Lett. 2018, 20, 598-601; c) X.-H. Yang, V. M. Dong, J. Am. Chem. Soc. 2017, 139, 1774-1777; d) A. M. Haydl, L. J. Hilpert, B. Breit, Chem. Eur. J. 2016, 22, 6547-6551; e) X. Wu, Z. Chen, Y.-B. Bai, V. M. Dong, J. Am. Chem. Soc. 2016, 138, 1201312016; f) A. M. Haydl, K. Xu, B. Breit, Angew. Chem. Int. Ed. 2015, 54, 71497153; g) K. Xu, W. Raimondi, T. Bury, B. Breit, Chem. Commun. 2015, 51, 10861-10863; h) K. Xu, N. Thieme, B. Breit, Angew. Chem. Int. Ed. 2014, 53, 7268-7271; i) H. Landert, F. Spindler, A. Wyss, H.-U. Blaser, B. Pugin, Y. Ribourduoille, B. Gschwend, B. Ramalingam, A. Pfaltz, Angew. Chem. Int. Ed. 2010, 49, 6873-6876.

[296] M. Christensen, A. Nolting, M. Shevlin, M. Weisel, P. E. Maligres, J. Lee, R. K. Orr, C. W. Plummer, M. T. Tudge, L.-C. Campeau, R. T. Ruck, J. Org. Chem. 2016, 81, 824-830.

[297] M. R. Friedfeld, H. Zhong, R. T. Ruck, M. Shevlin, P. J. Chirik, Science 2018, 360, 888-893.

[298] S. Koller, J. Gatzka, K. M. Wong, P. J. Altmann, A. Pöthig, L. Hintermann, J. Org. Chem. 2018, 83, 15009-15028.

[299] a) Y. Schramm, M. Takeuchi, K. Semba, Y. Nakao, J. F. Hartwig, J. Am. Chem. Soc. 2015, 137, 12215-12218; b) J. S. Bair, Y. Schramm, A. G. Sergeev, E. Clot, O. Eisenstein, J. F. Hartwig, J. Am. Chem. Soc. 2014, 136, 13098-13101.

[300] For examples of detrimental effects or changes of enantioselectivity with higher ligand-to-metal ratios, see: a) A. Schätz, R. Rasappan, M. Hager, A. Gissibl, O. Reiser, Chem. Eur. J. 2008, 14, 7259-7265; b) Z. Shao, J. Wang, K. Ding, A. S. C. Chan, Adv. Synth. Catal. 2007, 349, 2375-2379; c) M. I. Burguete, M. Collado, J. Escorihuela, S. V. Luis, Angew. Chem. Int. Ed. 2007, 46, 9002-9005; d) H. Danjo, M. Higuchi, M. Yada, T. Imamoto, Tetrahedron Lett. 2004, 45, 603-606. 
[301] For examples of nickel-catalyzed alkene isomerization, see: a) Y. He, Y. Cai, S. Zhu, J. Am. Chem. Soc. 2017, 139, 1061-1064; b) M. Gaydou, T. Moragas, F. Juliá-Hernández, R. Martin, J. Am. Chem. Soc. 2017, 139, 12161-12164; c) I. Buslov, J. Becouse, S. Mazza, M. Montandon-Clerc, X. Hu, Angew. Chem. Int. Ed. 2015, 54, 14523-14526; d) W.-C. Lee, C.-H. Wang, Y.-H. Lin, W.-C. Shih, T.-G. Ong, Org. Lett. 2013, 15, 5358-5361.

[302] A. F. Schmidt, V. V. Smirnov, A. Al-Halaiga, Kinet. Catal. 2007, 48, 390-397.

[303] a) Y. Gao, K. N. Houk, C.-Y. Ho, X. Hong, Org. Biomol. Chem. 2017, 15, 7131-7139; b) X. Hong, J. Wang, Y.-F. Yang, L. He, C.-Y. Ho, K. N. Houk, ACS Catal. 2015, 5, 5545-5555.

[304] a) L.-B. Han, Y. Ono, H. Yazawa, Org. Lett. 2005, 7, 2909-2911; b) L.-B. Han, C. Zhang, H. Yazawa, S. Shimada, J. Am. Chem. Soc. 2004, 126, 5080-5081.

[305] M. Liniger, B. Gschwend, M. Neuburger, S. Schaffner, A. Pfaltz, Organometallics 2010, 29, 5953-5958.

[306] L.-J. Xiao, X.-N. Fu, M.-J. Zhou, J.-H. Xie, L.-X. Wang, X.-F. Xu, Q.-L. Zhou, J. Am. Chem. Soc. 2016, 138, 2957-2960.

[307] a) L. H. Finger, J. Guschlbauer, K. Harms, J. Sundermeyer, Chem. Eur. J. 2016, 22, 16292-16303; b) R. D. Crocker, T. V. Nguyen, Chem. Eur. J. 2016, 22, 2208-2213.

[308] W. Roush, N. A. N. Zheng, V. St Georgiev, US 87833904 A1, 2005.

[309] For another example of aluminium-free nickel-catalyzed $\mathrm{C}-\mathrm{H}$ alkylation by hydroarylation published after our work, see: J. Diesel, D. Grosheva, S. Kodama, N. Cramer, Angew. Chem. Int. Ed. 2019, DOI:10.1002/anie.201904774.

[310] E. Bill, Mfit Program; Max-Planck Institute for Chemical Energy Conversion: Mülheim/Ruhr, Germany, 2008.

[311] A. Krasovskiy, P. Knochel, Synthesis 2006, 890-891.

[312] M. Ishihara, H. Togo, Tetrahedron 2007, 63, 1474-1480.

[313] L. Ackermann, A. V. Lygin, Org. Lett. 2011, 13, 3332-3335. 
[314] D. P. Rillema, J. K. Nagle, L. F. Barringer, T. J. Meyer, J. Am. Chem. Soc. 1981, 103, 56-62.

[315] a) C. Jahier-Diallo, M. S. T. Morin, P. Queval, M. Rouen, I. Artur, P. Querard, L. Toupet, C. Crévisy, O. Baslé, M. Mauduit, Chem. Eur. J. 2015, 21, $993-$ 997; b) H. Clavier, L. Coutable, L. Toupet, J.-C. Guillemin, M. Mauduit, J. Organomet. Chem. 2005, 690, 5237-5254.

[316] D. Katayev, Y.-X. Jia, A. K. Sharma, D. Banerjee, C. Besnard, R. B. Sunoj, E. P. Kündig, Chem. Eur. J. 2013, 19, 11916-11927.

[317] A. Alexakis, J. Burton, J. Vastra, C. Benhaim, X. Fournioux, A. van den Heuvel, J.-M. Levêque, F. Mazé, S. Rosset, Eur. J. Org. Chem. 2000, 40114027.

[318] M. Dindaroğlu, A. Falk, H.-G. Schmalz, Synthesis 2013, 45, 527-535.

[319] B. Moreau, J. Y. Wu, T. Ritter, Org. Lett. 2009, 11, 337-339.

[320] V. F. Kuznetsov, G. R. Jefferson, G. P. A. Yap, H. Alper, Organometallics 2002, 21, 4241-4248.

[321] a) I. Colomer, R. Coura Barcelos, T. J. Donohoe, Angew. Chem. Int. Ed. 2016, 55, 4748-4752; b) Z. Segaoula, J. Leclercq, V. Verones, N. Flouquet, M. Lecoeur, L. Ach, N. Renault, A. Barczyk, P. Melnyk, P. Berthelot, X. Thuru, N. Lebegue, J. Med. Chem. 2016, 59, 8422-8440; c) W. Liu, L. Li, Z. Chen, C.-J. Li, Org. Biomol. Chem. 2015, 13, 6170-6174; d) C. Bao, G. Fan, Q. Lin, B. Li, S. Cheng, Q. Huang, L. Zhu, Org. Lett. 2012, 14, 572-575.

[322] A. E. Sheshenev, M. S. Baird, I. G. Bolesov, A. S. Shashkov, Tetrahedron 2009, 65, 10552-10564.

[323] S. Nakamura, D. Hayama, Angew. Chem. Int. Ed. 2017, 56, 8785-8789.

[324] a) S. Movahhed, J. Westphal, M. Dindaroğlu, A. Falk, H.-G. Schmalz, Chem. Eur. J. 2016, 22, 7381-7384; b) R. Sanders, U. T. Mueller-Westerhoff, J. Organomet. Chem. 1996, 512, 219-224.

[325] a) A. R. Kenaree, T. J. Cuthbert, S. M. Barbon, P. D. Boyle, E. R. Gillies, P. J. Ragogna, J. B. Gilroy, Organometallics 2015, 34, 4272-4280; b) M. R. 
Malachowski, M. F. Grau, J. M. Thomas, A. L. Rheingold, C. E. Moore, Inorg. Chim. Acta 2010, 364, 132-137.

[326] a) F. M. Geisler, G. Helmchen, Synthesis 2006, 2201-2205; b) B. Bildstein, A. Hradsky, H. Kopacka, R. Malleier, K.-H. Ongania, J. Organomet. Chem. 1997, 540, 127-145.

[327] M. Shang, S.-Z. Sun, H.-X. Dai, J.-Q. Yu, J. Am. Chem. Soc. 2014, 136, 3354-3357.

[328] M. R. Chaulagain, G. J. Sormunen, J. Montgomery, J. Am. Chem. Soc. 2007, 129, 9568-9569.

[329] R. S. Shaikh, S. J. S. Düsel, B. König, ACS Catal. 2016, 6, 8410-8414.

[330] A. Podgoršek, S. Stavber, M. Zupan, J. Iskra, Eur. J. Org. Chem. 2006, 483488.

[331] S. Bräse, B. Waegell, A. de Meijere, Synthesis 1998, 148-152.

[332] R. D. Grigg, R. Van Hoveln, J. M. Schomaker, J. Am. Chem. Soc. 2012, 134, 16131-16134.

[333] a) H. Yang, X. Zhang, L. Zhou, P. Wang, J. Org. Chem. 2011, 76, $2040-$ 2048; b) E. Alvarez-Manzaneda, R. Chahboun, E. Cabrera, E. Alvarez, R. Alvarez-Manzaneda, M. Hmamouchi, H. Es-Samti, Tetrahedron Lett. 2007, 48, 8930-8934.

[334] S. Meiries, G. Le Duc, A. Chartoire, A. Collado, K. Speck, K. S. A. Arachchige, A. M. Z. Slawin, S. P. Nolan, Chem. Eur. J. 2013, 19, 1735817368.

[335] H. Kim, Y. Nguyen, C. P.-H. Yen, L. Chagal, A. J. Lough, B. M. Kim, J. Chin, J. Am. Chem. Soc. 2008, 130, 12184-12191.

[336] a) D. Seebach, A. K. Beck, A. Heckel, Angew. Chem. Int. Ed. 2001, 40, $92-$ 138; b) A. Hafner, R. O. Duthaler, R. Marti, G. Rihs, P. Rothe-Streit, F. Schwarzenbach, J. Am. Chem. Soc. 1992, 114, 2321-2336.

[337] A. Modak, A. Deb, T. Patra, S. Rana, S. Maity, D. Maiti, Chem. Commun. 2012, 48, 4253-4255. 
[338] K. D. Schleicher, Y. Sasaki, A. Tam, D. Kato, K. K. Duncan, D. L. Boger, J. Med. Chem. 2013, 56, 483-495.

[339] J. Moran, P. Dornan, A. M. Beauchemin, Org. Lett. 2007, 9, 3893-3896.

[340] C. Seipelt, P. López, T. Kirschgen, A. Dölle, M. D. Zeidler, I. Fonseca, F. H. Cano, B. Paloma, Tetrahedron Lett. 1999, 40, 1739-1742.

[341] A. Purkait, S. K. Roy, H. K. Srivastava, C. K. Jana, Org. Lett. 2017, 19, 2540 2543.

[342] Bruker AXS Inc., in Bruker Apex CCD, SAINT v8.30C (Ed.: Bruker AXS Inst. Inc.), WI, USA, Madison, 2013.

[343] L. Krause, R. Herbst-Irmer, D. Stalke, J. Appl. Crystallogr. 2015, 48, 19071913.

[344] L. Krause, R. Herbst-Irmer, G. M. Sheldrick, D. Stalke, J. Appl. Crystallogr. 2015, 48, 3-10.

[345] G. M. Sheldrick, Acta Crystallogr. 2015, A71, 3-8.

[346] G. M. Sheldrick, Acta Crystallogr. 2015, C71, 3-8.

[347] C. B. Hübschle, G. M. Sheldrick, B. Dittrich, J. Appl. Crystallogr. 2011, 44, $1281-1284$.

[348] O. V. Dolomanov, L. J. Bourhis, R. J. Gildea, J. A. K. Howard, H. Puschmann, J. Appl. Crystallogr. 2009, 42, 339-341.

[349] G. M. Sheldrick, Acta Crystallogr. 2008, A64, 112-122. 


\section{Acknowledgements}

First, I would like to express my deepest gratitude to Prof. Dr. Lutz Ackermann for giving me the opportunity to conduct my $\mathrm{PhD}$ in his research group. Thank you for your support, your guidance and your interest in my projects.

I am grateful to Prof. Dr. Alexander Breder for accepting to be my second supervisor. I also would like to thank Prof. Dr. Manuel Alcarazo, Dr. Shoubhik Das, Prof. Dr. Dietmar Stalke and Prof. Dr. Dr. h.c.mult. Lutz F. Tietze for agreeing to take part in my defense.

I would like to thank the people from our research group with whom I had the opportunity to collaborate: Dr. Ruhuai Mei, Valentin Müller, Dr. João C. A. de Oliveira, Dr. Daniel Zell, Dr. Debasish Ghorai, Dr. Nicolas Sauermann, Uttam Dhawa and Dr. Fabio Pescaioli. My deepest thanks also go to Dr. Tobias Parchomyk, Stefan Lülf, Prof. Dr. Konrad Koszinowski, Dr. Serhiy Demeshko and Prof. Dr. Franc Meyer for their invaluable contributions in the context of our collaboration on iron chemistry. Thank you all!

I would also like to extend my gratitude to Dr. Christopher Golz, Helena Keil and Prof. Dr. Dietmar Stalke for their assistance with X-ray diffraction analysis, as well as to all the members of the analytical departments (NMR and mass spectrometry) at the IOBC for their continuous support to our research work. I would also like to thank Felix Krätzschmar and Prof. Dr. Alexander Breder for their assistance and advice on the measurement of specific rotations.

I would like to thank Stefan Beußhausen for taking care of the instruments of our research group, especially the chiral HPLC, the glovebox and the SPS, which I used almost on a daily basis in the last four years. Thank you for your invaluable help!

My gratitude also goes to Karsten Rauch for his continuous support to our lab work, and especially for the preparation of various (HA)SPOs and carbene precursors.

I would like to thank Mrs. Gabriele Keil-Knepel for her continuous assistance with administrative tasks. 
I also would like to express my gratitude to all past and present members of the Ackermann research group, especially to members of Lab 308 (a.k.a. "the Best Lab"): Dr. Gianpiero Cera, Dr. Lars Finger, Dr. Thomas Müller, Torben Rogge, Sachiyo Nakanowatari, Zhigao Shen, Dr. Wei-Jun Kong, Dr. Zhixiong “Jenson” Ruan, Dr. Nicolas Sauermann, Dr. Ramesh Chandra Samanta, Jiayu Mo, Dr. Huawen Huang, Renato Lucio De Carvalho, Talita Barbara Gontijo, Dr. Antonis Messinis, Antoine Bigot and Karsten Rauch. It has been an honor working with you guys! In this context, I would like to particularly thank Dr. Gianpiero Cera for his precious advices and suggestions on iron catalysis, Dr. Thomas Müller and Sachiyo Nakanowatari for their advices on nickel catalysis, and Torben Rogge for his help with various instruments.

I also would like to thank Prof. Dr. Albrecht Berkessel, Prof. Dr. Lukas Hintermann, and Prof. Dr. E. Peter Kündig for the generous donation of various NHC precursors and SPOs. I would also like to express my gratitude to my coworkers and students from whom I obtained chemicals.

I would like to sincerely thank all the people who proofread this thesis: Dr. Thomas Müller, Nikolaos Kaplaneris, Torben Rogge, Valentin Müller, Dr. João C. A. de Oliveira, Dr. Elżbieta Gońka, Isaac Choi, Maximilian Stangier, Dr. Lars Finger, Dr. Jongwoo Son, Julia Struwe, Wei Wang, Cuiju Zhu, Tjark Meyer and Leonardo Massignan. I also would like to thank all the people who previously corrected manuscripts, supporting information, posters, abstracts and proposals for me: Dr. Thomas Müller, Torben Rogge, Dr. Fabio Pescaioli, Dr. Lars Finger, Dr. Yu-Feng Liang, Valentin Müller, Dr. Hui Wang, Dr. Weiping Liu, Ralf Steinbock, Dr. João C. A. de Oliveira, Dr. Parthasarathi Subramanian, Dr. Nicolas Sauermann, Dr. Marc Moselage, Dr. Vladislav Kotek, and Cuiju Zhu. Thank you all for your time and your patience!

I also would like to express my gratitude to my former supervisors for teaching me so much about chemistry and giving me the opportunity to conduct research within their laboratories, particularly Prof. Dr. Jérôme Waser, Dr. Florian de Nanteuil, Prof. Dr. Sandrine Gerber, Dr. Holger Sellner and Dr. Kenji Namoto. 
Last but not least, I would like to thank my family for their continuous support and their love throughout my life. 


\section{Curriculum Vitae}

\section{Personal Information}

$\begin{array}{ll}\text { Name: } & \text { Joachim Loup } \\ \text { Date of Birth: } & 11.04 .1990 \\ \text { Place of Birth: } & \text { Lausanne VD, Switzerland } \\ \text { Place of Origin: } & \text { Vully-les-Lacs (Montmagny) VD, Switzerland } \\ \text { Nationality: } & \text { Switzerland }\end{array}$

ORCID: 0000-0002-0033-5033

\section{Academic Education}

05.2015-08.2019

PhD Thesis (Supervisor: Prof. Dr. Lutz Ackermann) at the Georg-August-University Göttingen, Germany.

Title: "Selectivity Control in 3d Transition Metal-Catalyzed C-H Activation"

$09.2011-04.2013$

Master of Science MSc in Molecular and Biological Chemistry at the École Polytechnique Fédérale de Lausanne (EPFL), Switzerland. Final grade: 5.51/6.

Master Thesis: "Catalytic Friedel-Crafts Reaction of Aminocyclopropanes" (Supervisor: Prof. Dr. Jérôme Waser).

$09.2008-07.2011$ Bachelor of Science BSc in Chemistry and Chemical Engineering at the École Polytechnique Fédérale de Lausanne (EPFL), Switzerland. Final grade: 5.78/6; final ranking: $1 / 50$.

08.2010-05.2011

Academic exchange (2 semesters) at the National University of Singapore (NUS), Singapore. 
$08.2005-07.2008$

Maturity certificate at the Gymnase intercantonal de la Broye in Payerne VD, Switzerland.

08.1995-07.2005

Kindergarten, primary and secondary schools in Payerne VD, Switzerland.

\section{Industrial Experience}

$02.2014-11.2014$

Internship at Novartis Institutes for BioMedical Research (NIBR), Novartis AG, Basel, Switzerland.

Topic: Synthesis of protease inhibitors.

$04.2013-09.2013$

Internship at Pharma Research and Early Development (pRED), F. Hoffmann-La Roche Ltd, Basel, Switzerland.

Topic: Synthesis of transcription factor enhancers and serine protease inhibitors.

\section{Teaching Experience}

2017-2018

Assistant of the course "Methoden der Modernen Organischen und Biomolekularen Chemie" (2 semesters).

2016-2018

Assistant of the practical course "Chemisches Praktikum für Studierende der Human- und Zahnmedizin" (3 semesters).

2017-2018

Assistant of the practical course "Organisch-ChemischesFortgeschrittenenpraktikum" (1 semester). 


\section{Publications}

9) J. Loup, ${ }^{\ddagger}$ U. Dhawa, ${ }^{\ddagger}$ F. Pesciaioli, J. Wencel-Delord, L. Ackermann, "Enantioselective C-H Activation with Earth-Abundant 3d Transition Metals", Angew. Chem. Int. Ed. 2019, DOI:10.1002/anie.201904214. ( Both authors contributed equally.)

8) J. Loup, T. Parchomyk, S. Lülf, S. Demeshko, F. Meyer, K. Koszinowski, L. Ackermann, "Mössbauer and mass spectrometry support for iron(II) catalysts in enantioselective C-H activation", Dalton Trans. 2019, 48, 5135-5139.

7) D. Ghorai, J. Loup, G. Zanoni, L. Ackermann, "Air-Stable Secondary Phosphine Oxides (SPOs) for Nickel-Catalyzed Cross-Couplings of Aryl Ethers by C-O Activation" Synlett 2019, 30, 429-432.

6) J. Loup,${ }^{\ddagger}$ V. Müller, D. Ghorai, L. Ackermann, "Enantioselective Aluminum-Free Alkene Hydroarylations through C-H Activation by a Chiral Nickel/JoSPOphos Manifold" Angew. Chem. Int. Ed. 2019, 58, 1749-1753. ( ${ }^{\ddagger}$ Both authors contributed equally.)

5) K. Namoto, F. Sirockin, H. Sellner, C. Wiesmann, F. Villard, R. J. Moreau, E. Valeur, Stephanie C. Paulding, S. Schleeger, K. Schipp, J. Loup, L. Andrews, R. Swale, M. Robinson, C. J. Farady, "Structure-based design and synthesis of macrocyclic human rhinovirus 3C protease inhibitors" Bioorg. Med. Chem. Lett. 2018, 28, 906-909.

4) J. Loup, D. Zell, J. C. A. Oliveira, H. Keil, D. Stalke, L. Ackermann, "Asymmetric IronCatalyzed C-H Alkylation Enabled by Remote Ligand meta-Substitution" Angew. Chem. Int. Ed. 2017, 56, 14197-14201. (Highlighted in Synfacts, ChemistryViews, SigmaAldrich and Nachrichten aus der Chemie, Trendbericht Organische Chemie)

3) N. Sauermann, J. Loup, D. Kootz, A. Berkessel, L. Ackermann, "Triazolylidene Ligands Allow Cobalt-Catalyzed C-H/C-O Alkenylations at Ambient Temperature" Synthesis 2017, 49, 3476-3484.

2) R. Mei, ${ }^{*}$ J. Loup,${ }^{\ddagger}$ L. Ackermann, "Oxazolinyl-Assisted C-H Amidation by Cobalt(III) Catalysis" ACS Catal. 2016, 6, 793-797. ("Both authors contributed equally.) (Cited $>130$ times as of 07.2019) 
1) F. de Nanteuil, J. Loup, J. Waser, "Catalytic Friedel-Crafts Reaction of Aminocyclopropanes" Org. Lett. 2013, 15, 3738-3741.

\section{Conferences}

02.2019

02.2019

09.2018

07.2018

11.2017

07.2017

10.2016

05.2016

10.2015 $3^{\text {rd }}$ Workshop of the SPP 1807, Erlangen, Germany (Poster presentation)

$12^{\text {th }}$ CaRLa Winter School, Heidelberg, Germany (Poster presentation and flash talk)

ORCHEM 2018, Berlin, Germany (Poster presentation)

$3^{\text {rd }}$ Summer School of the SPP 1807, DESY Hamburg, Germany (Oral presentation)

Evaluation of the SPP 1807, Gießen, Germany (Poster presentation)

$2^{\text {nd }}$ Summer School of the SPP 1807, Rostock, Germany (Oral presentation)

$2^{\text {nd }}$ Workshop of the SPP 1807, Cologne, Germany (Poster presentation)

$1^{\text {st }}$ Summer School of the SPP 1807, Bremen, Germany (Oral presentation)

$1^{\text {st }}$ Workshop of the SPP 1807, Göttingen, Germany (Poster presentation) 


\section{Erklärung}

Ich versichere, dass ich die vorliegende Dissertation in dem Zeitraum von Mai 2015 bis Juli 2019 am Institut für Organische und Biomolekulare Chemie der Georg-August-

Universität Göttingen

auf Anregung und unter Anleitung von

\section{Herrn Prof. Dr. Lutz Ackermann}

selbstständig durchgeführt und keine anderen als die angegebenen Hilfsmittel und Quellen verwendet habe.

Göttingen, den 08.07.2019

\section{Disclaimer}

Elements of the following publications are reproduced for non-commercial purposes with permission of the editors:

- Ref. [37e]: Reprinted with permission from R. Mei, J. Loup, L. Ackermann, ACS Catal. 2016, 6, 793-797. Copyright 2015 American Chemical Society.

- Ref. [151]: J. Loup, D. Zell, J. C. A. Oliveira, H. Keil, D. Stalke, L. Ackermann, Angew. Chem. Int. Ed. 2017, 56, 14197-14201. Copyright Clearance Center's RightsLink ${ }^{\circledR}$ order: 4560300036116.

- Ref. [218]: J. Loup, T. Parchomyk, S. Lülf, S. Demeshko, F. Meyer, K. Koszinowski, L. Ackermann, Dalton Trans. 2019, 48, 5135-5139 - Published by The Royal Society of Chemistry.

- Ref. [220]: J. Loup, V. Müller, D. Ghorai, L. Ackermann, Angew. Chem. Int. Ed. 2019, 58, 1749-1753. Copyright Clearance Center's RightsLink ${ }^{\circledR}$ order: 4560300144210 . 


\section{NMR Spectra and HPLC Chromatograms}
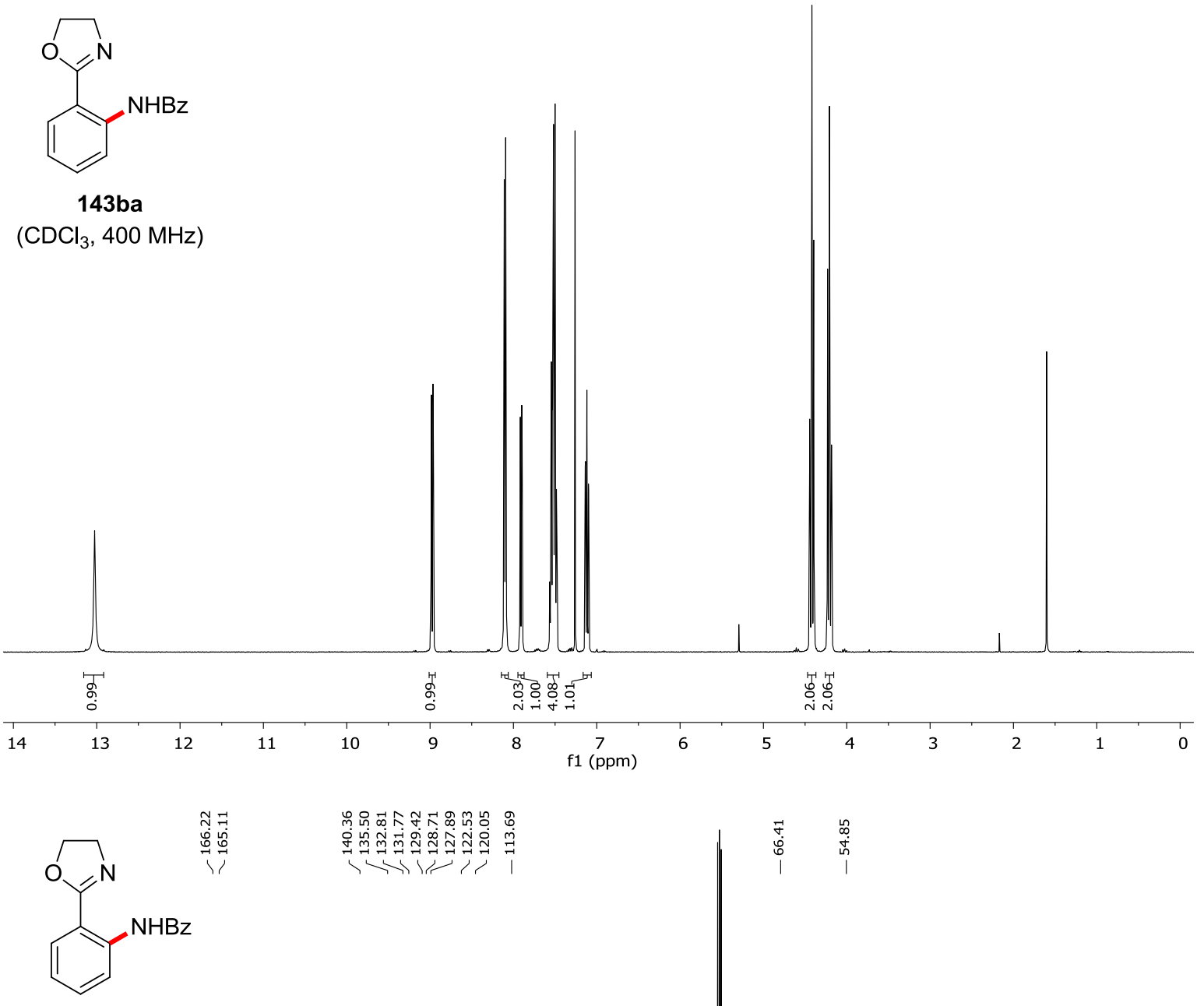

等

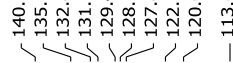

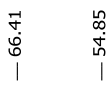

$\left(\mathrm{CDCl}_{3}, 100 \mathrm{MHz}\right)$

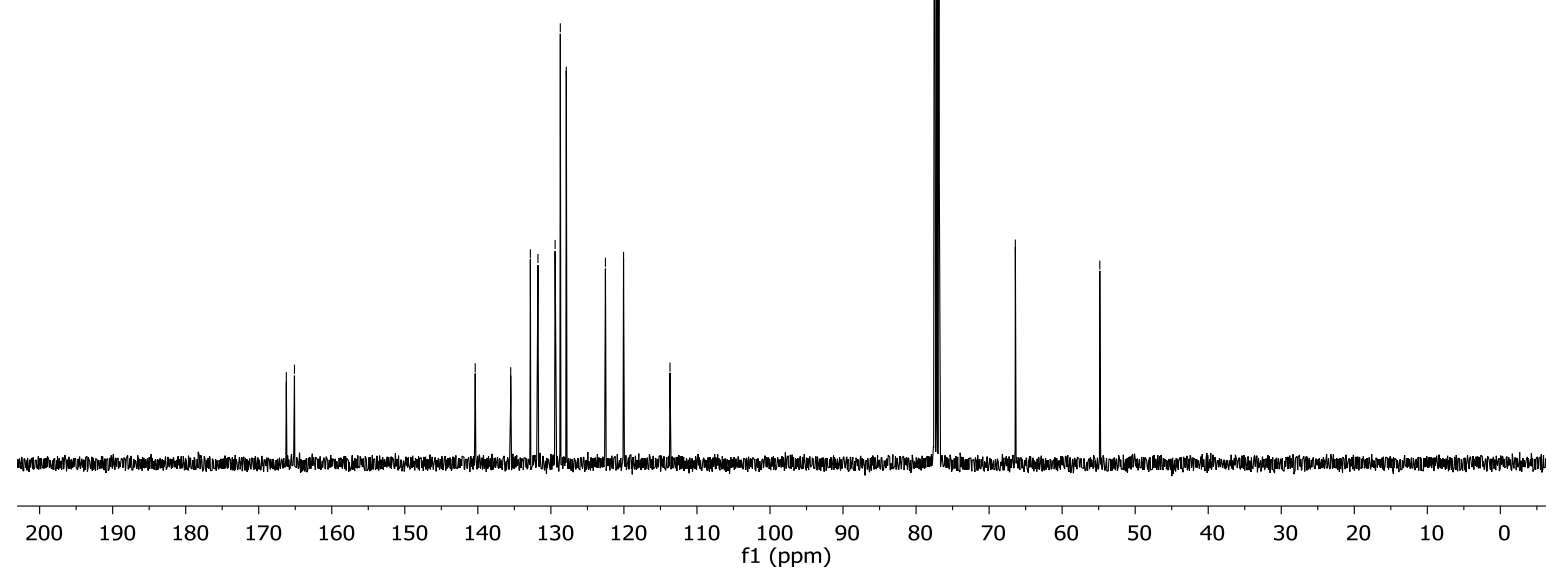




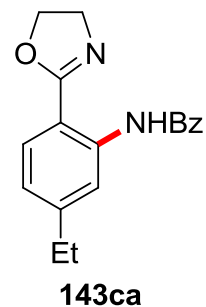

$\left(\mathrm{CDCl}_{3}, 300 \mathrm{MHz}\right)$
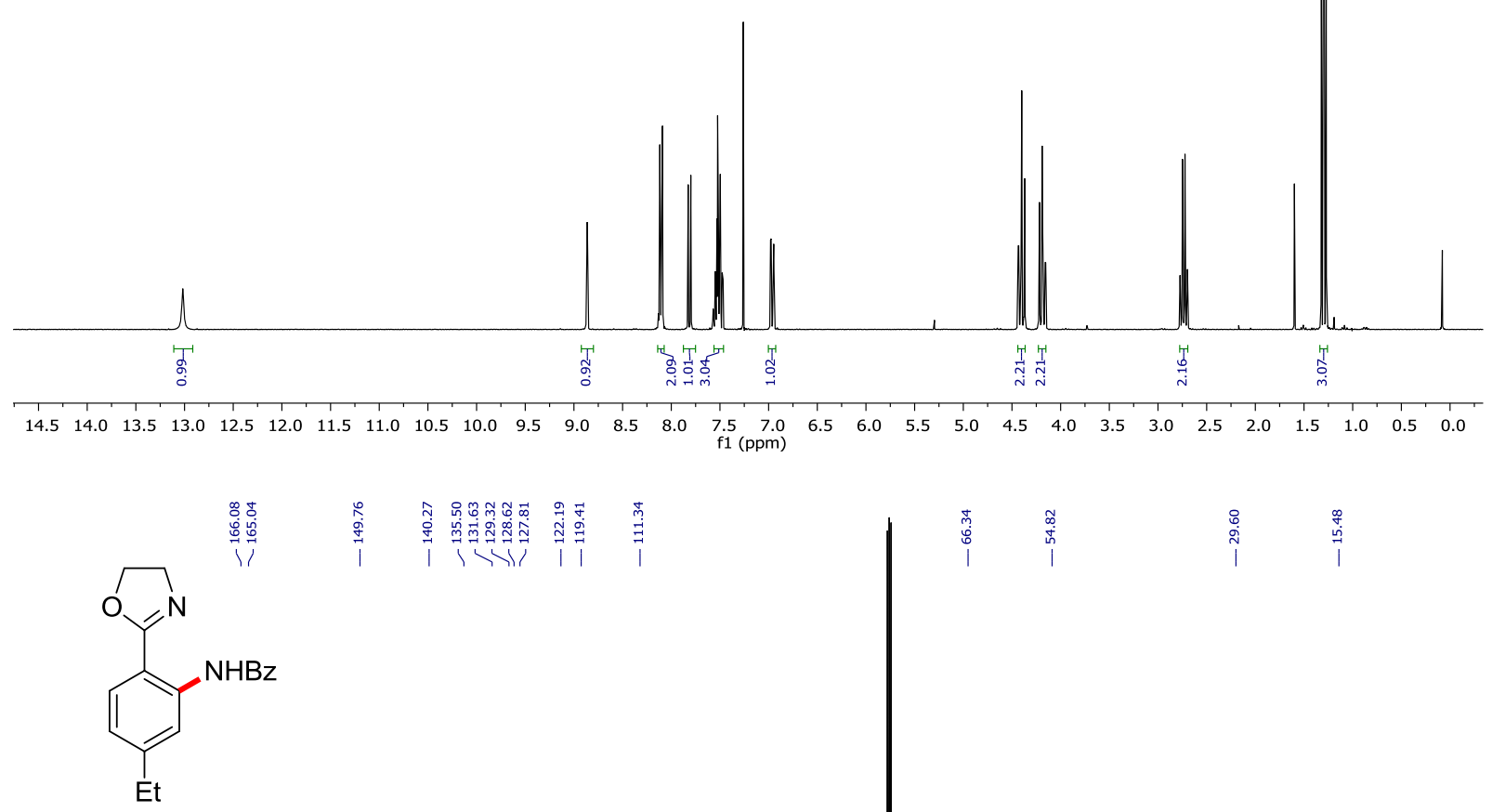

143ca

$\left(\mathrm{CDCl}_{3}, 125 \mathrm{MHz}\right)$

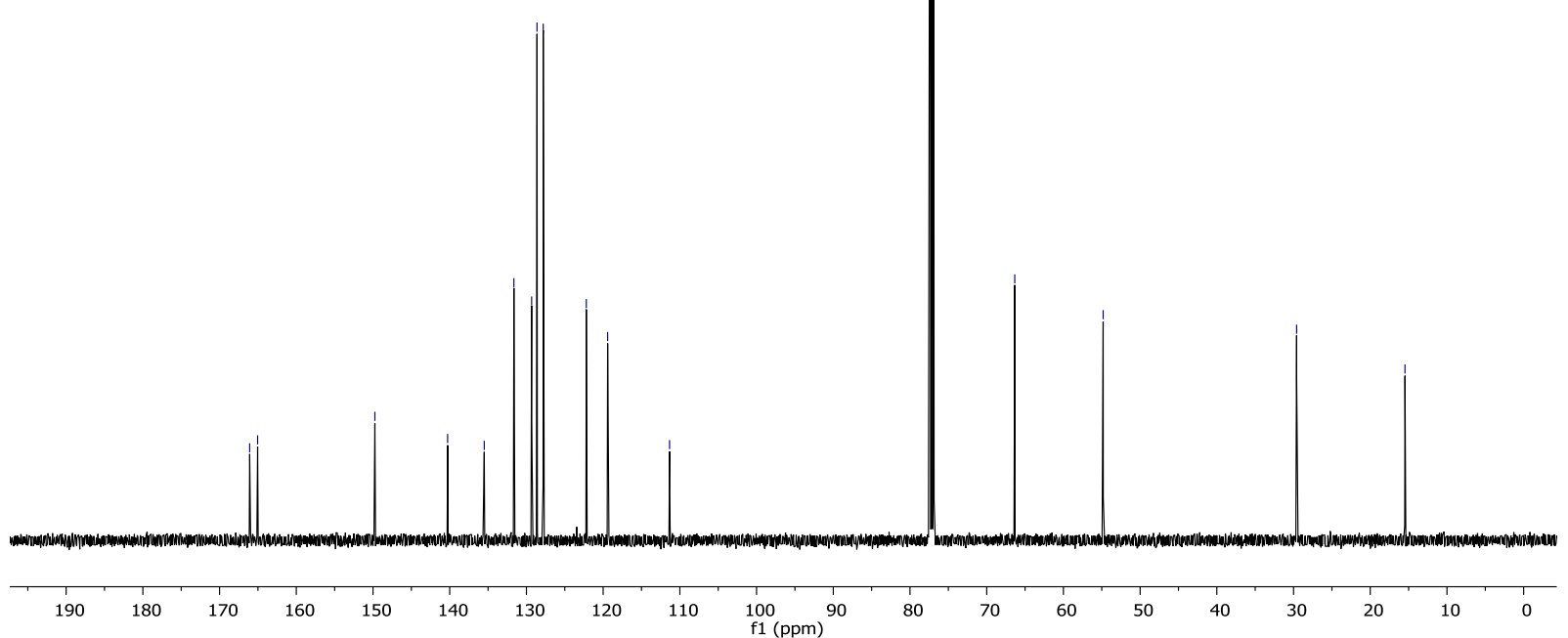




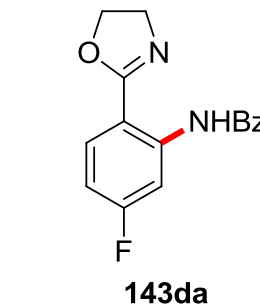

$\left(\mathrm{CDCl}_{3}, 300 \mathrm{MHz}\right)$
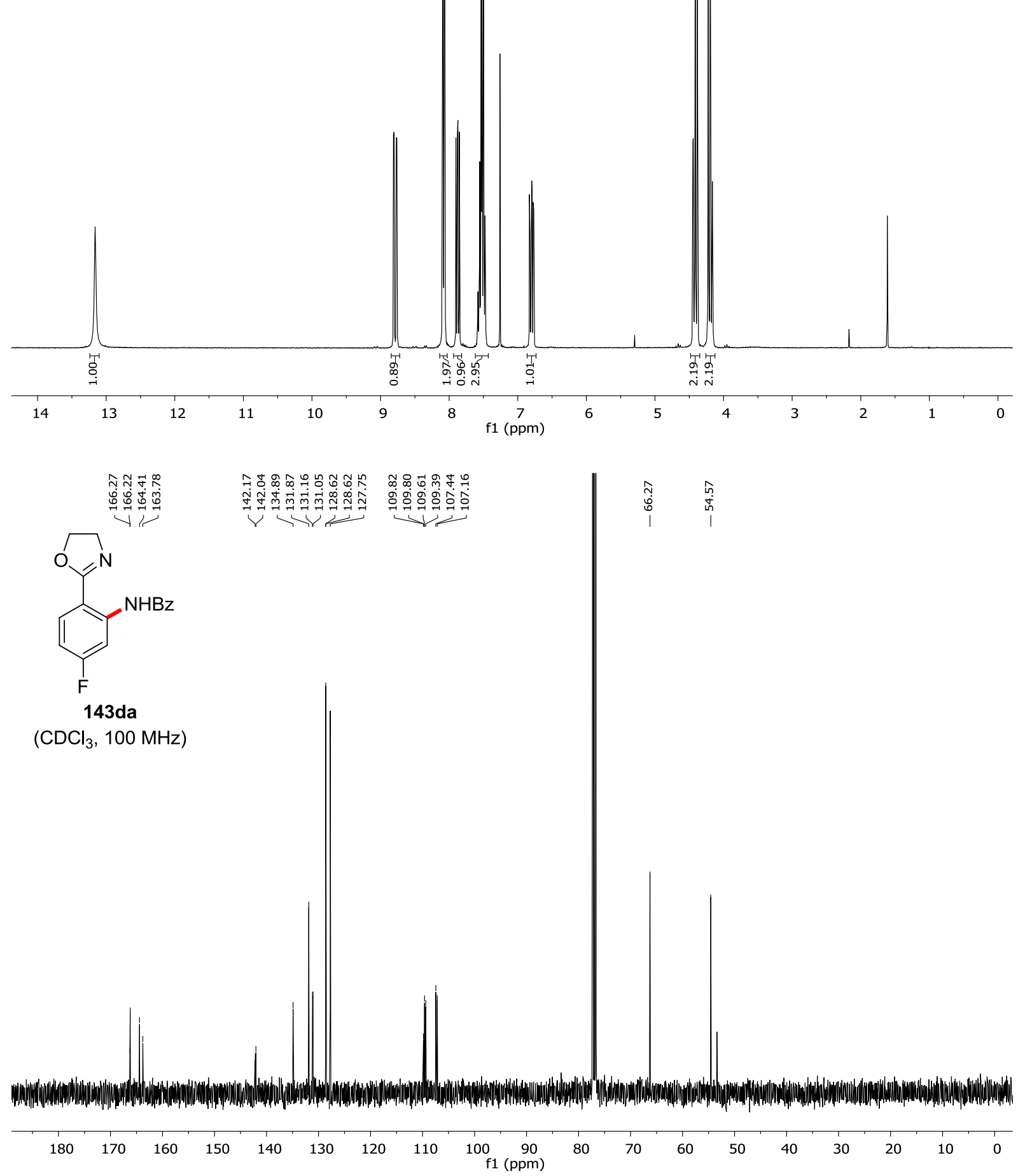


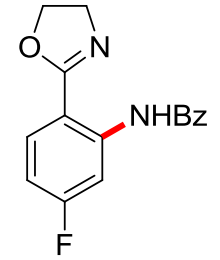

143da

$\left(\mathrm{CDCl}_{3}, 282 \mathrm{MHz}\right)$

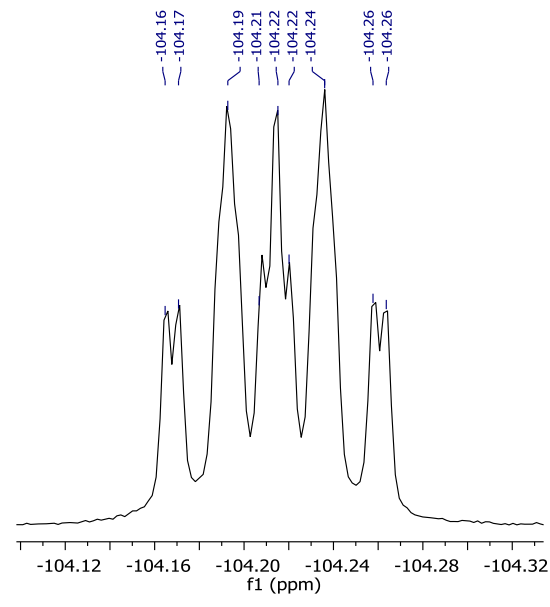

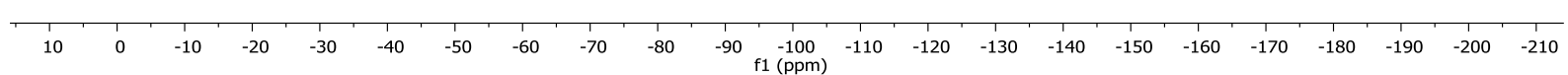




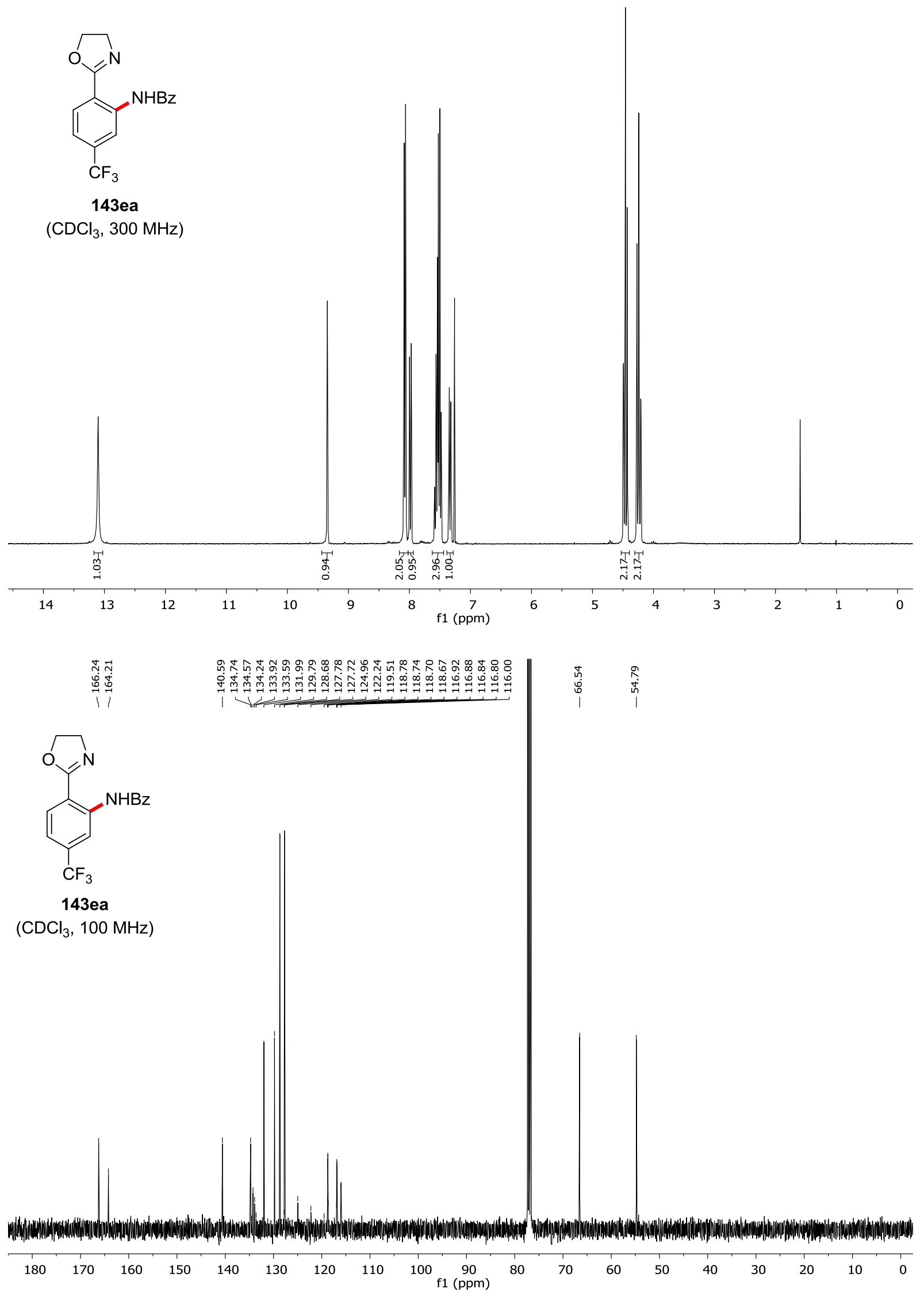




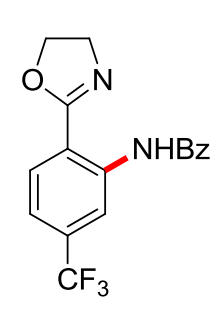

143ea

$\left(\mathrm{CDCl}_{3}, 282 \mathrm{MHz}\right)$

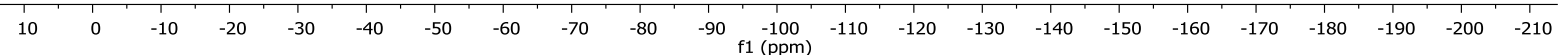



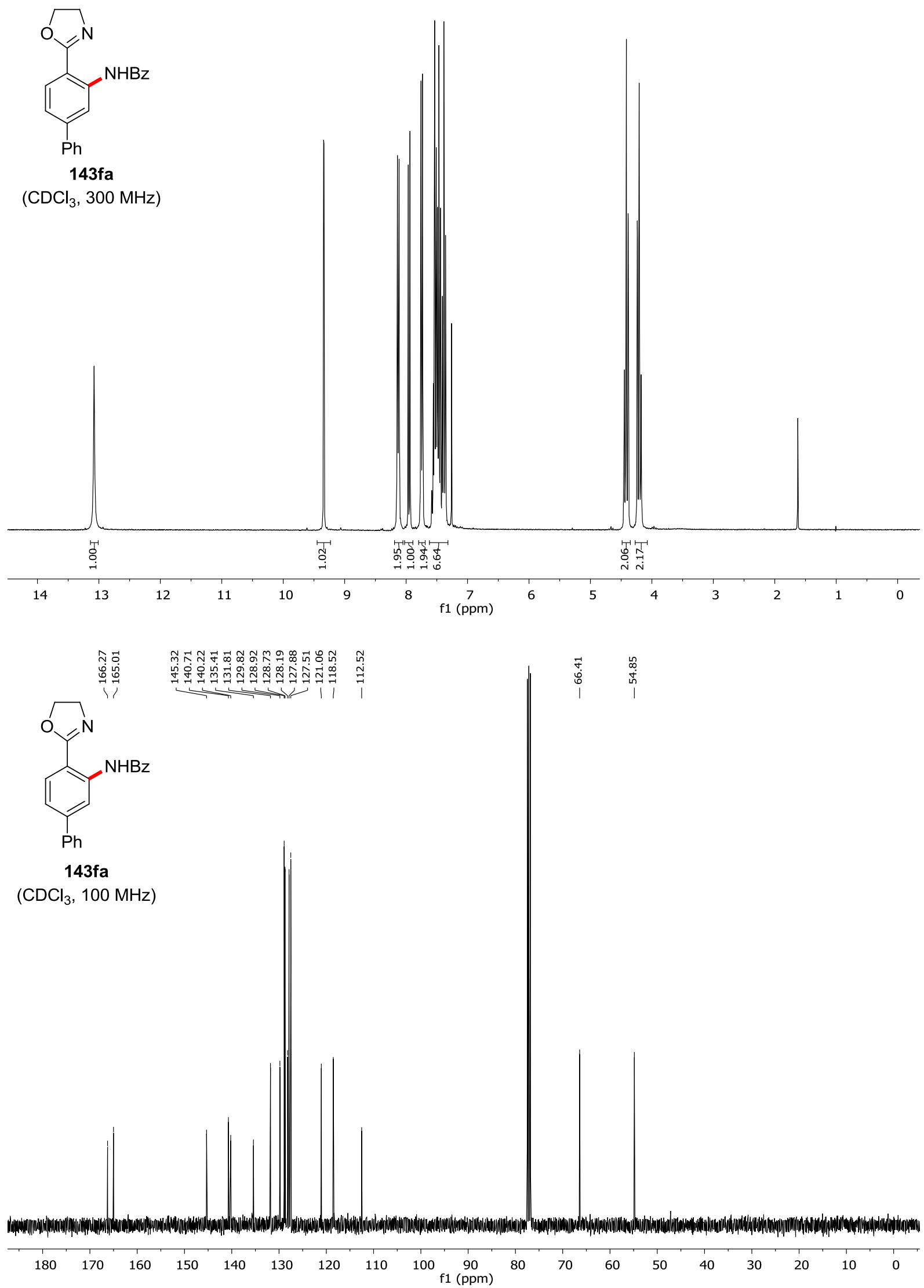


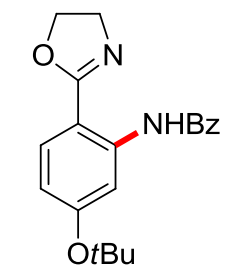

143ga
$\left(\mathrm{CDCl}_{3}, 500 \mathrm{MHz}\right)$

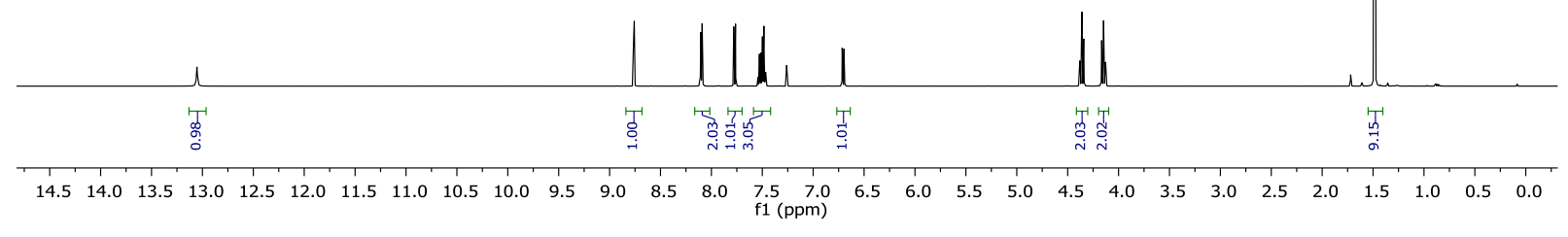

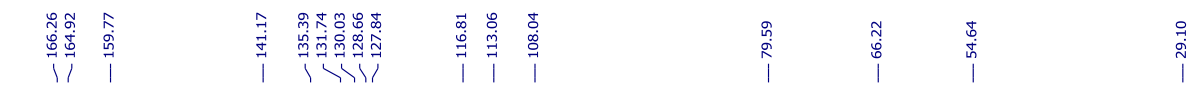

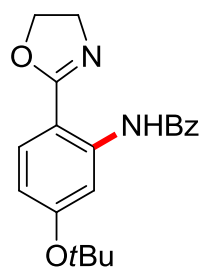

143ga

$\left(\mathrm{CDCl}_{3}, 125 \mathrm{MHz}\right)$

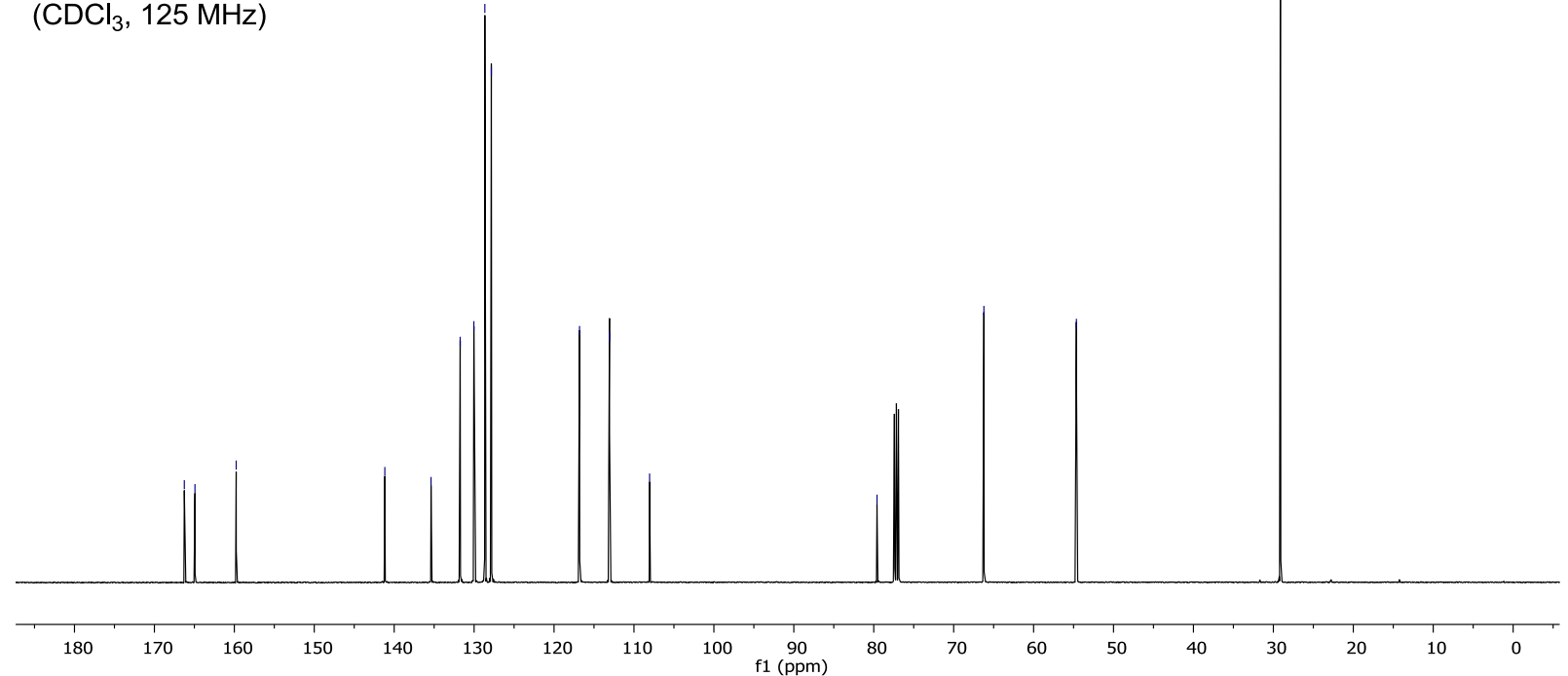




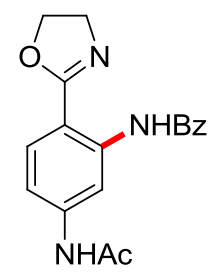

143ha

(DMSO-d, $300 \mathrm{MHz}$ )
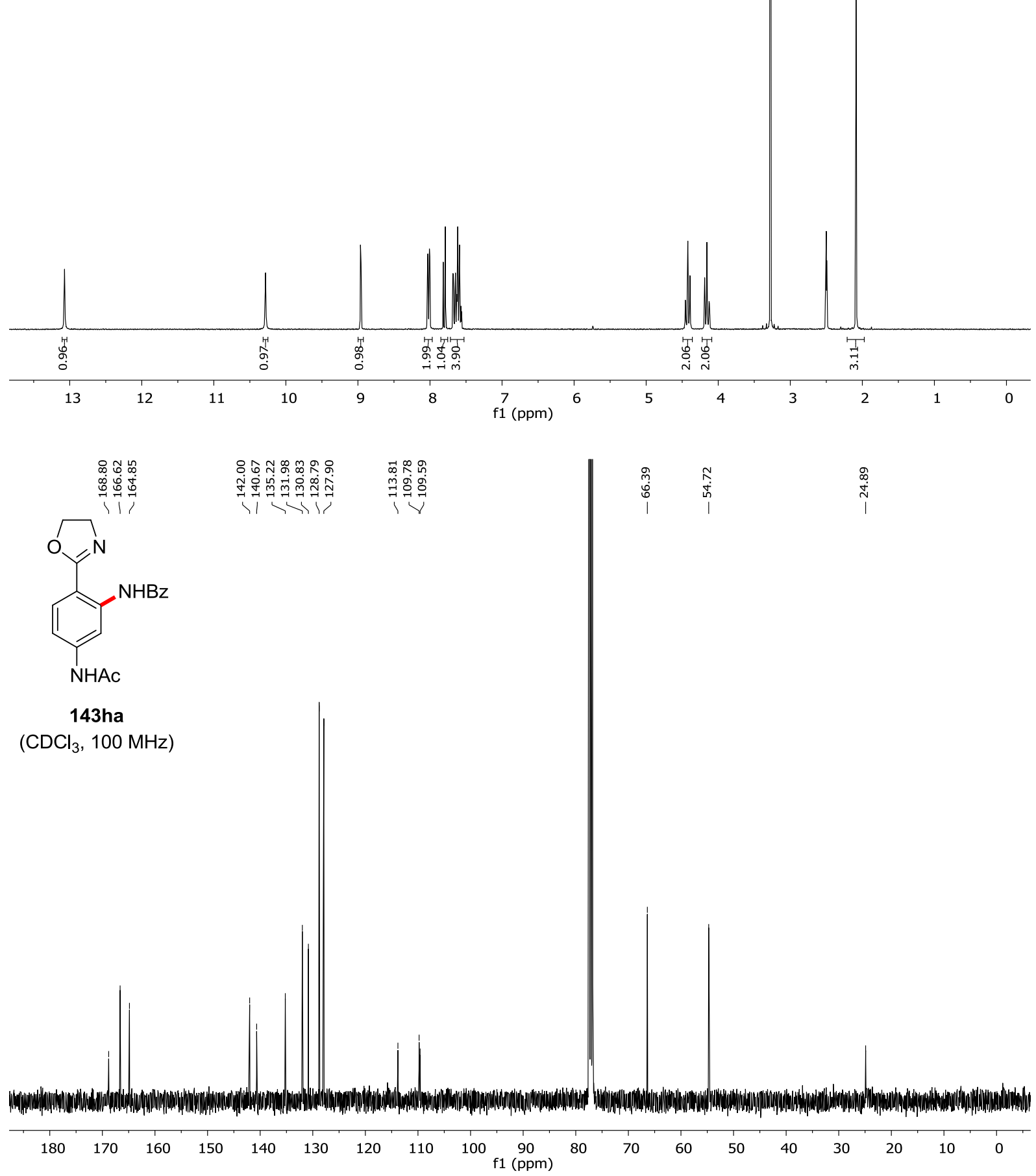

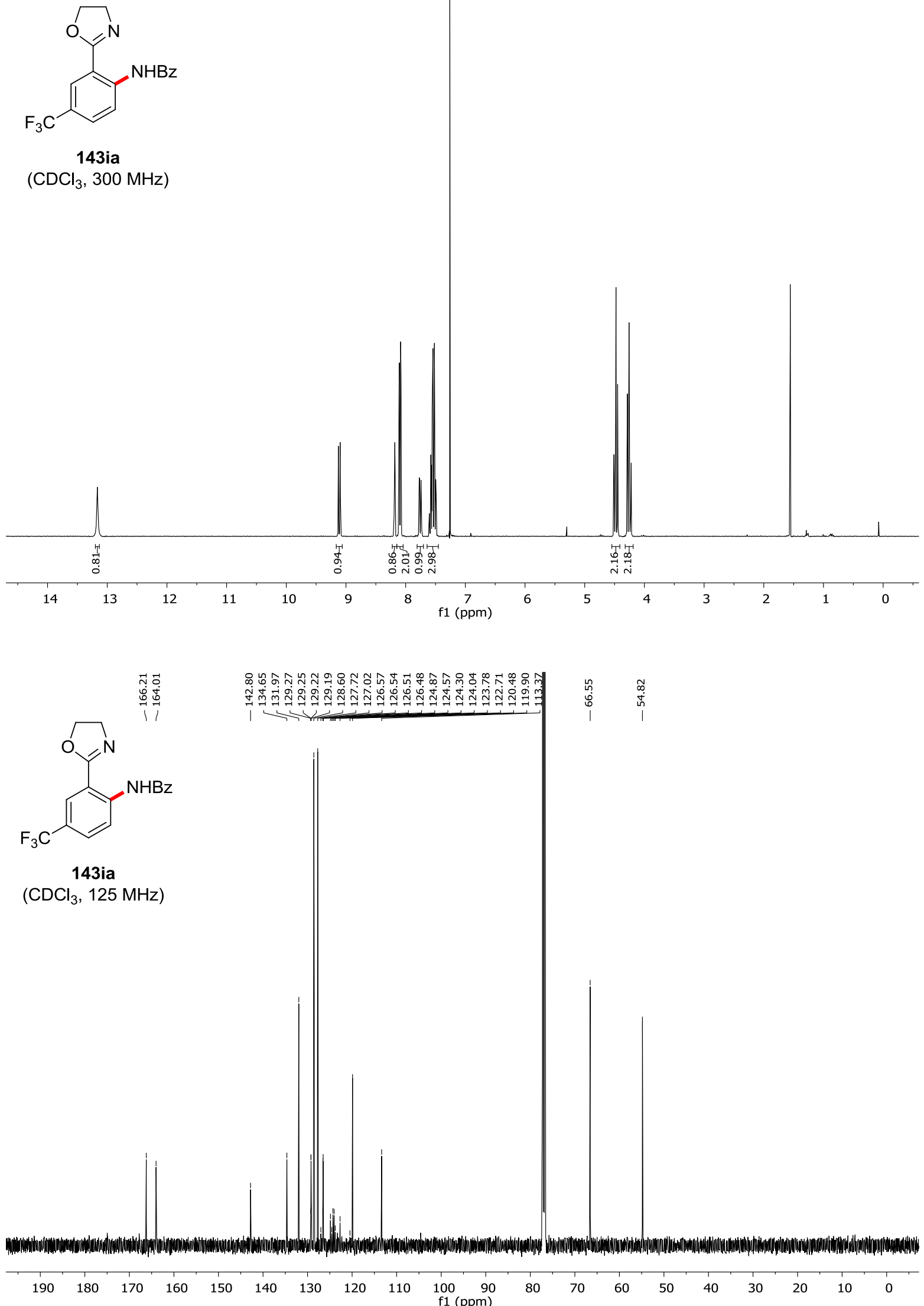


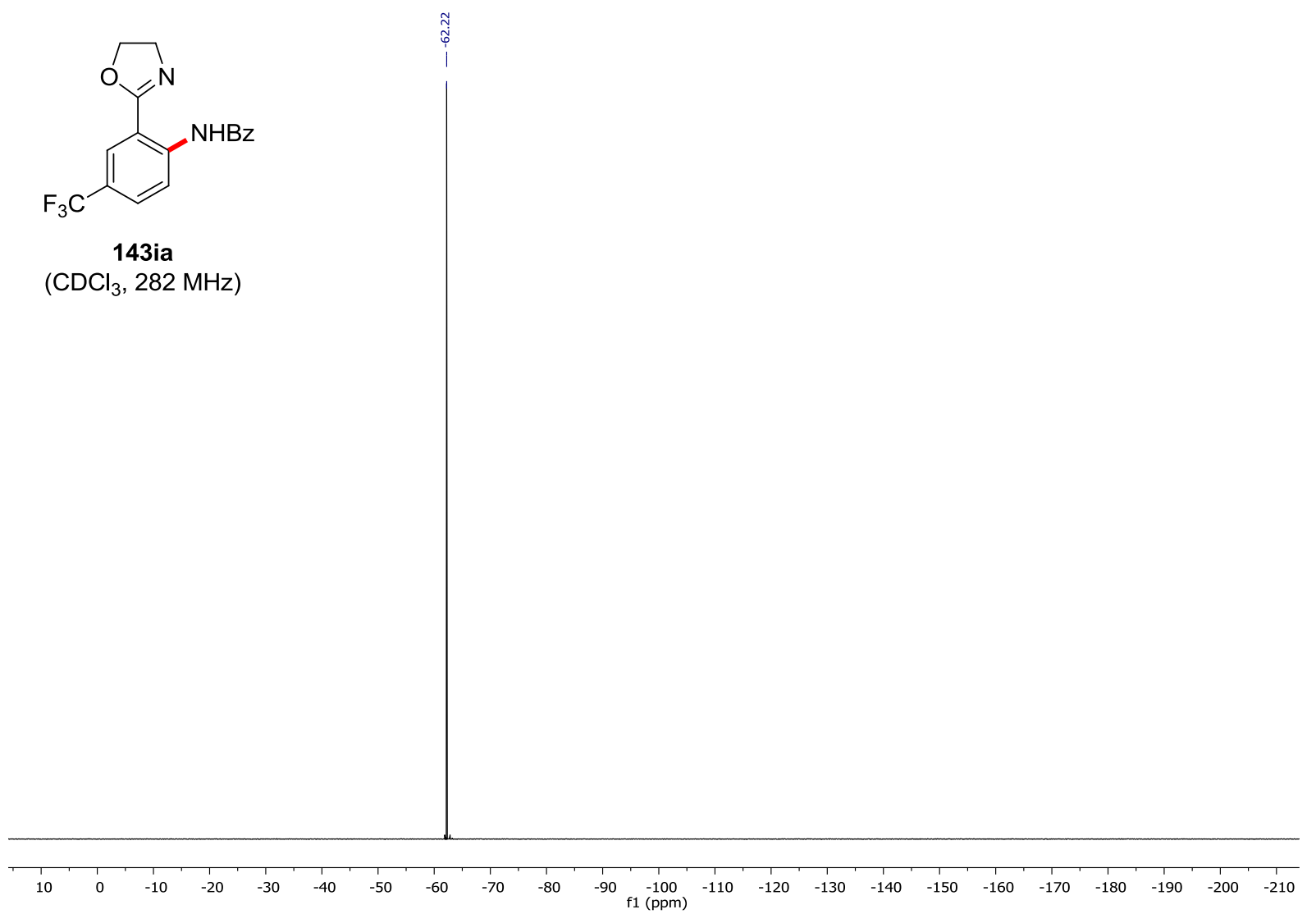



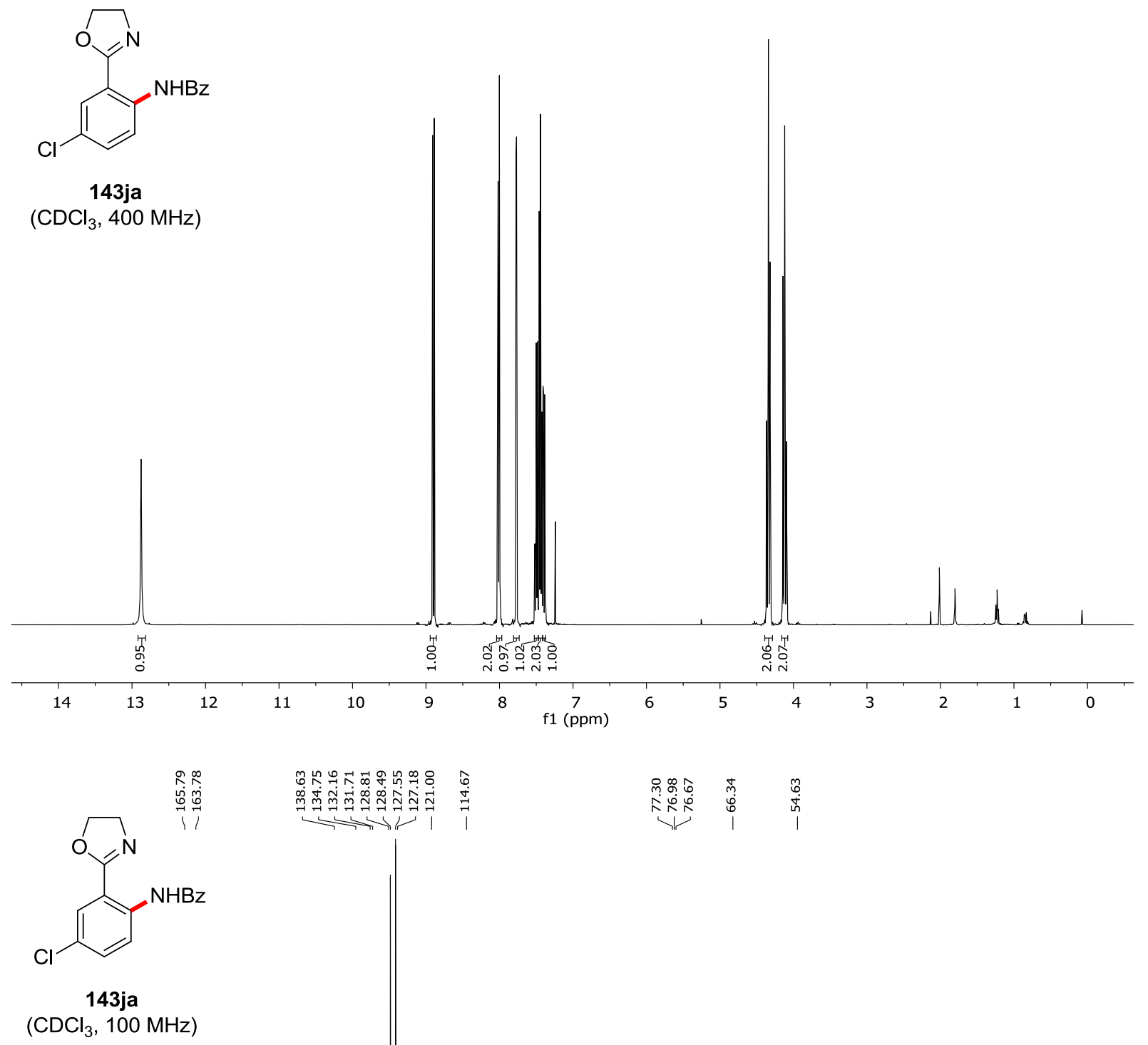

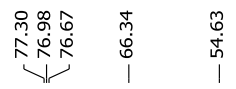

$\left(\mathrm{CDCl}_{3}, 100 \mathrm{MHz}\right)$

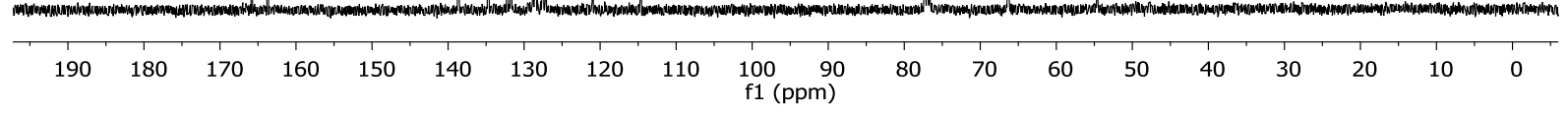


<smiles>COc1cc(C2=NCCO2)c(NC(=O)c2ccccc2)cc1F</smiles>

143ka

$\left(\mathrm{CDCl}_{3}, 300 \mathrm{MHz}\right)$

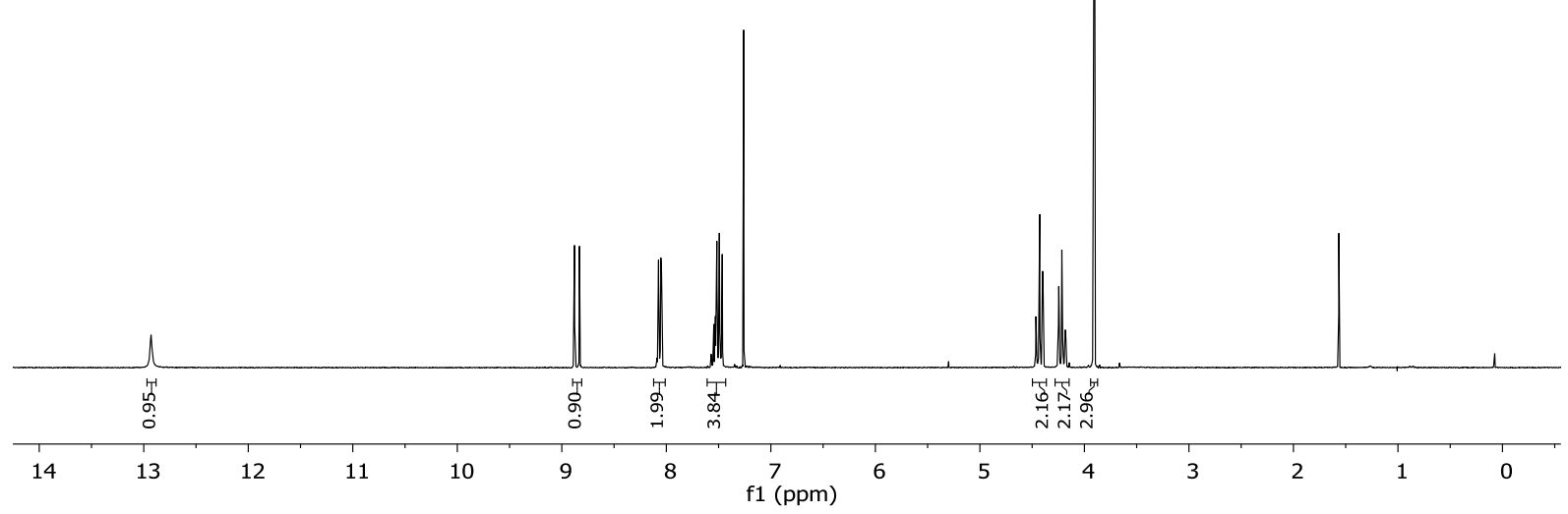<smiles>COc1cc(C2=NCCO2)c(NC(=O)c2ccccc2)cc1F</smiles>

143ka

$\left(\mathrm{CDCl}_{3}, 125 \mathrm{MHz}\right)$

\section{(19)}
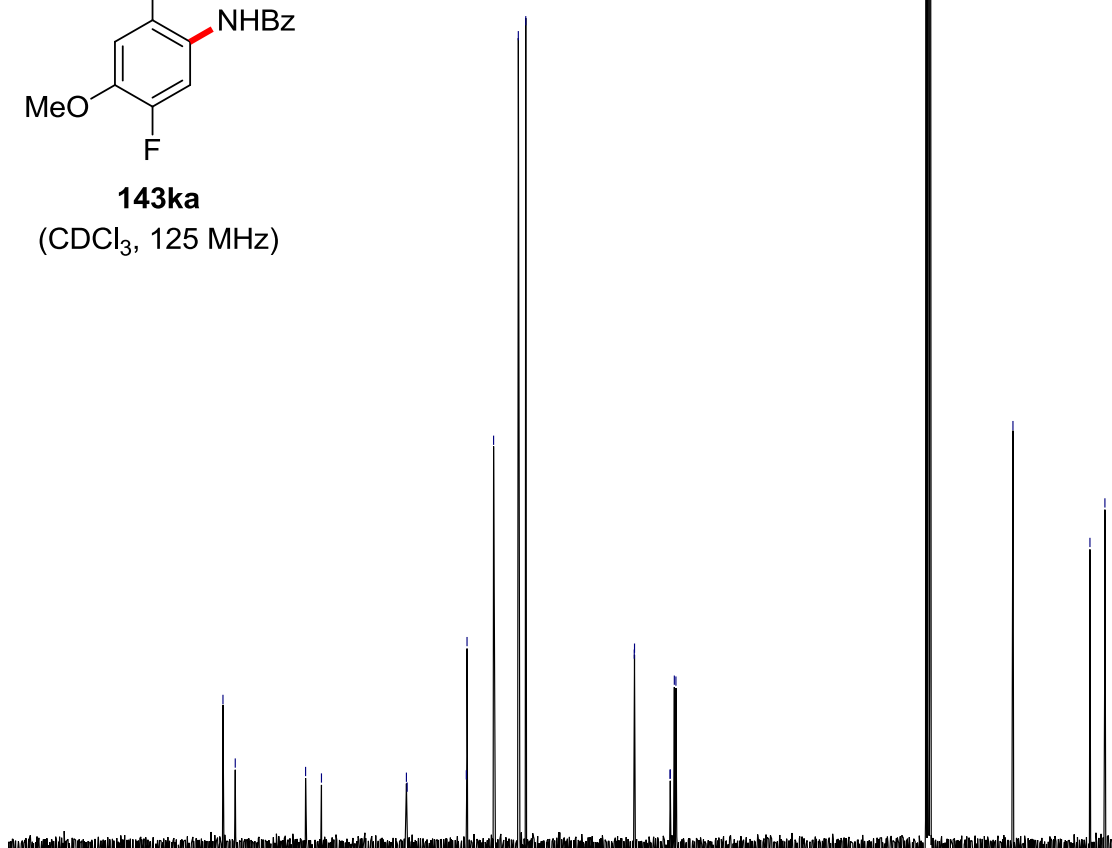


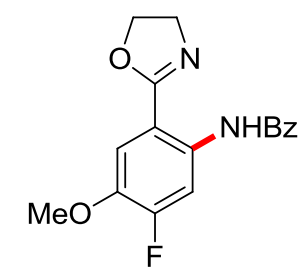

요요

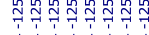

143ka

$\left(\mathrm{CDCl}_{3}, 282 \mathrm{MHz}\right)$
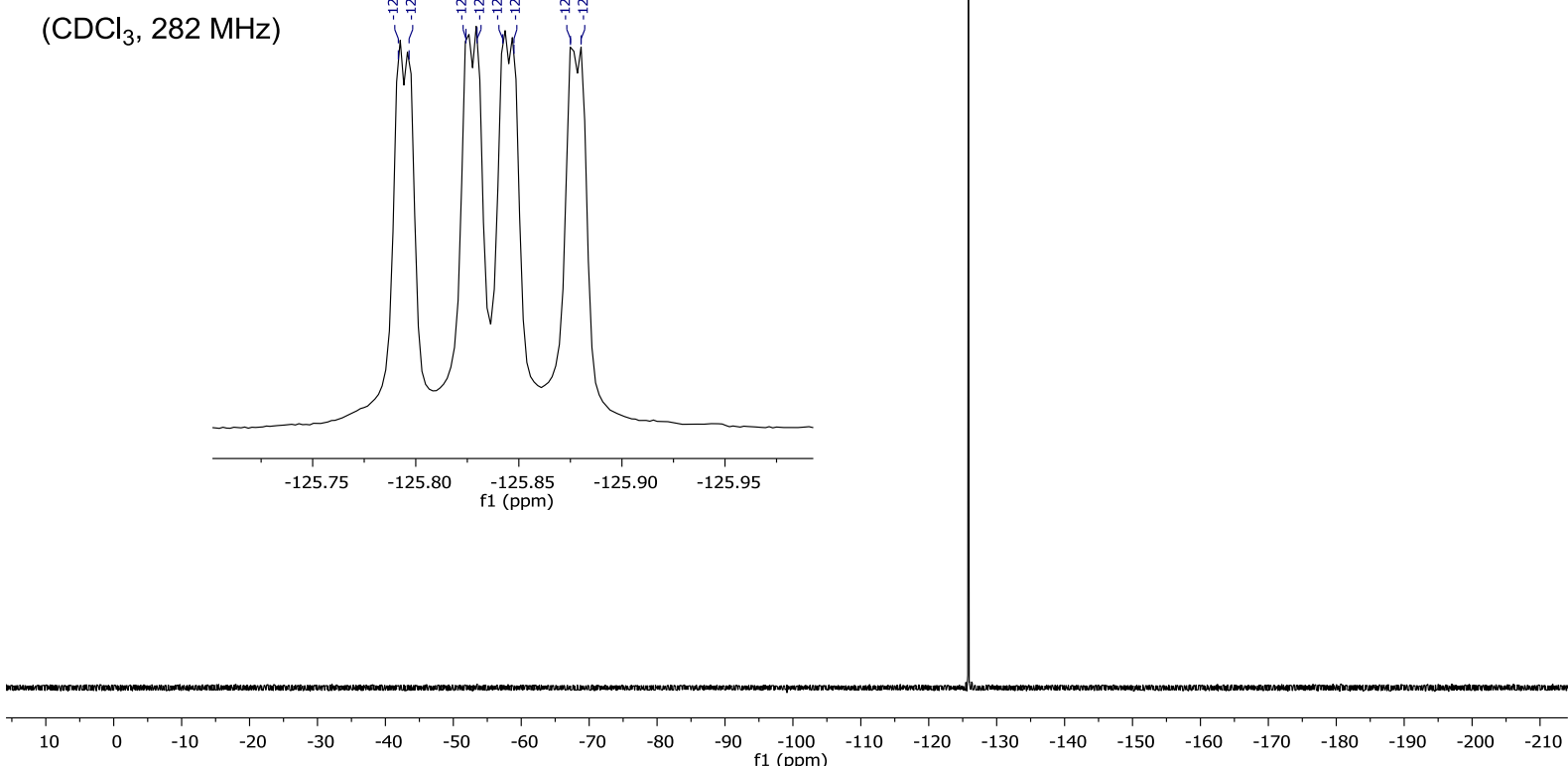

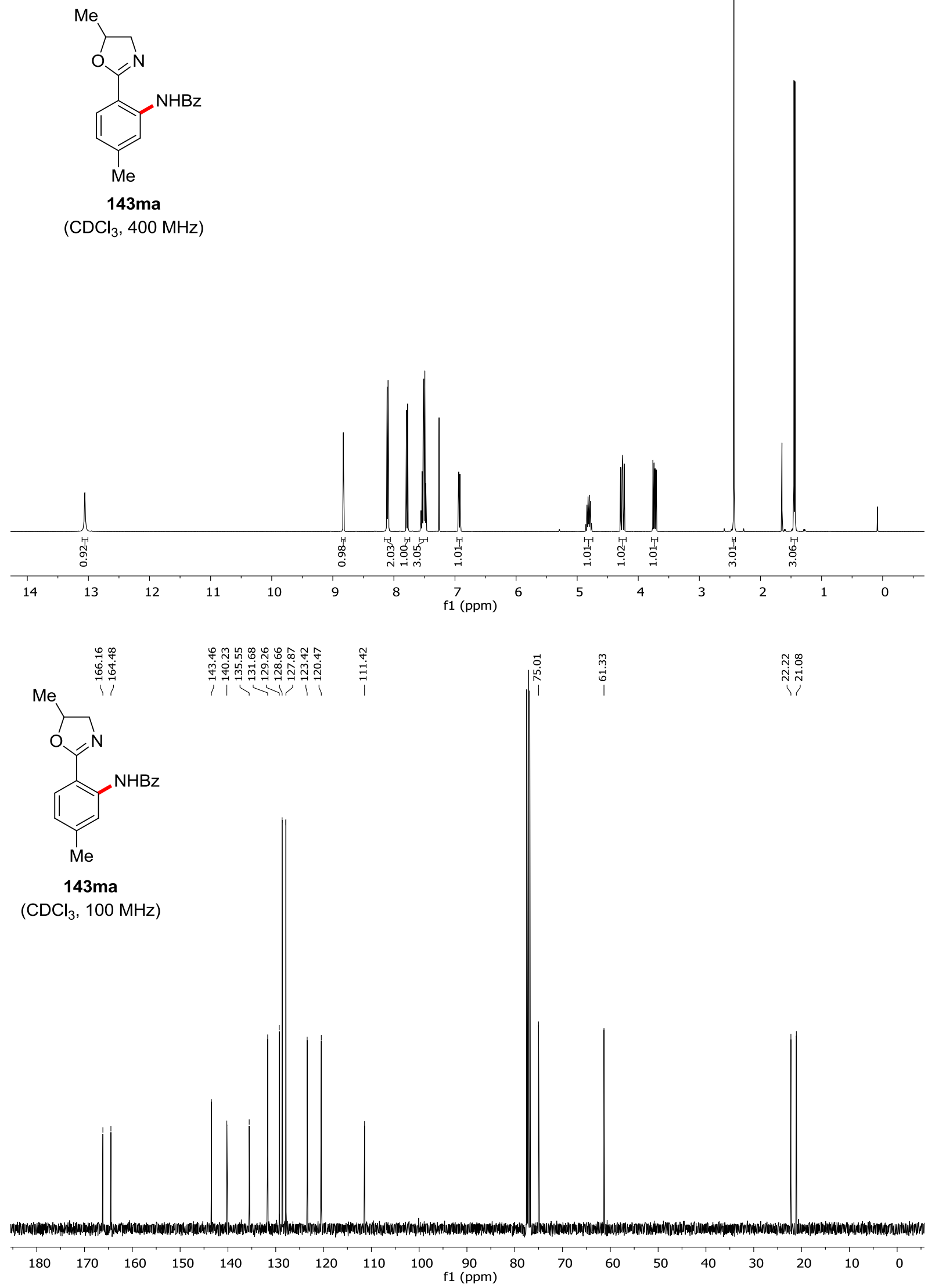


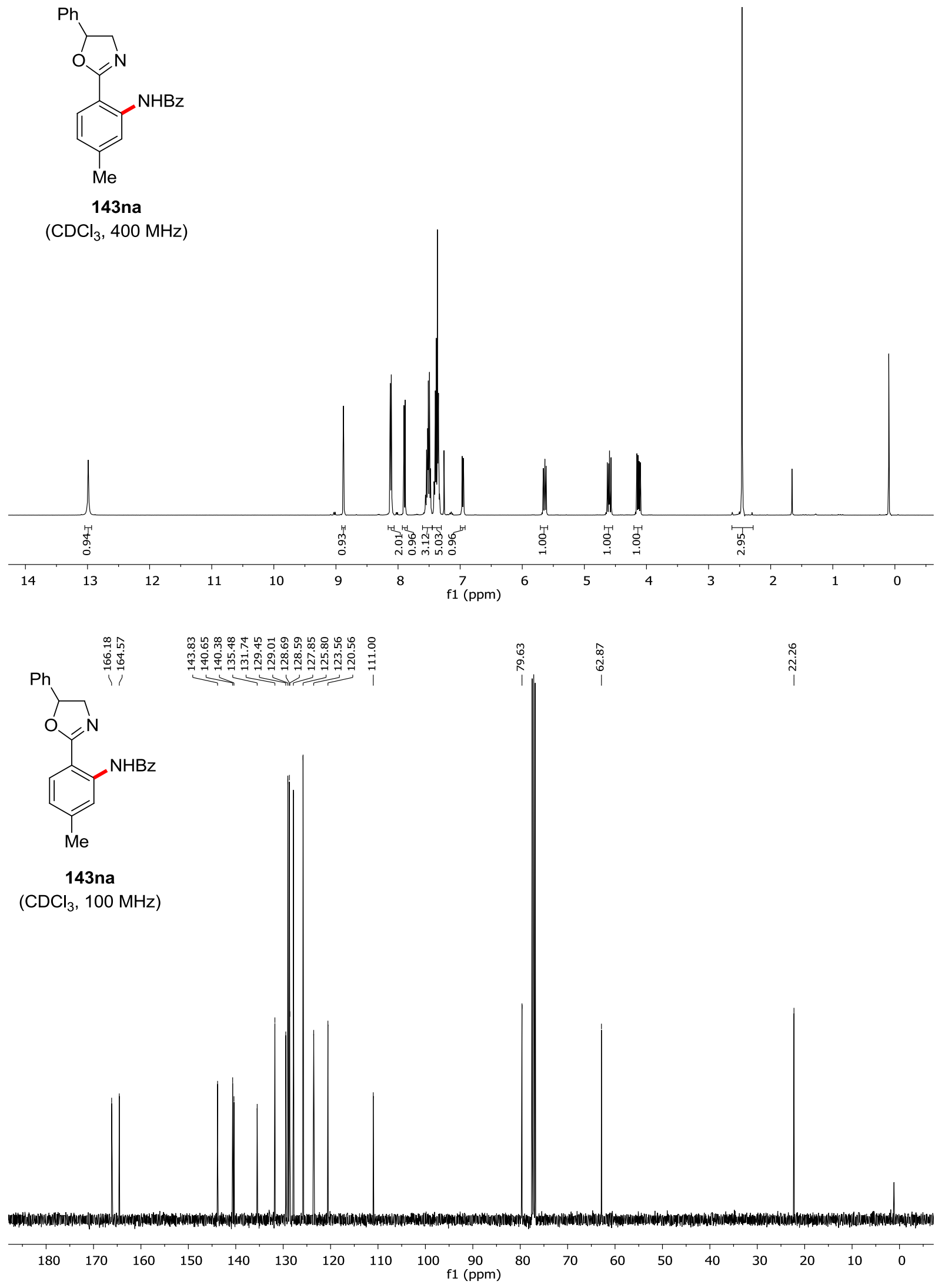




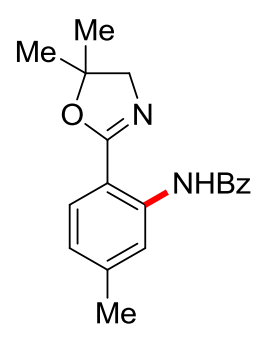

143oa

$\left(\mathrm{CDCl}_{3}, 400 \mathrm{MHz}\right)$
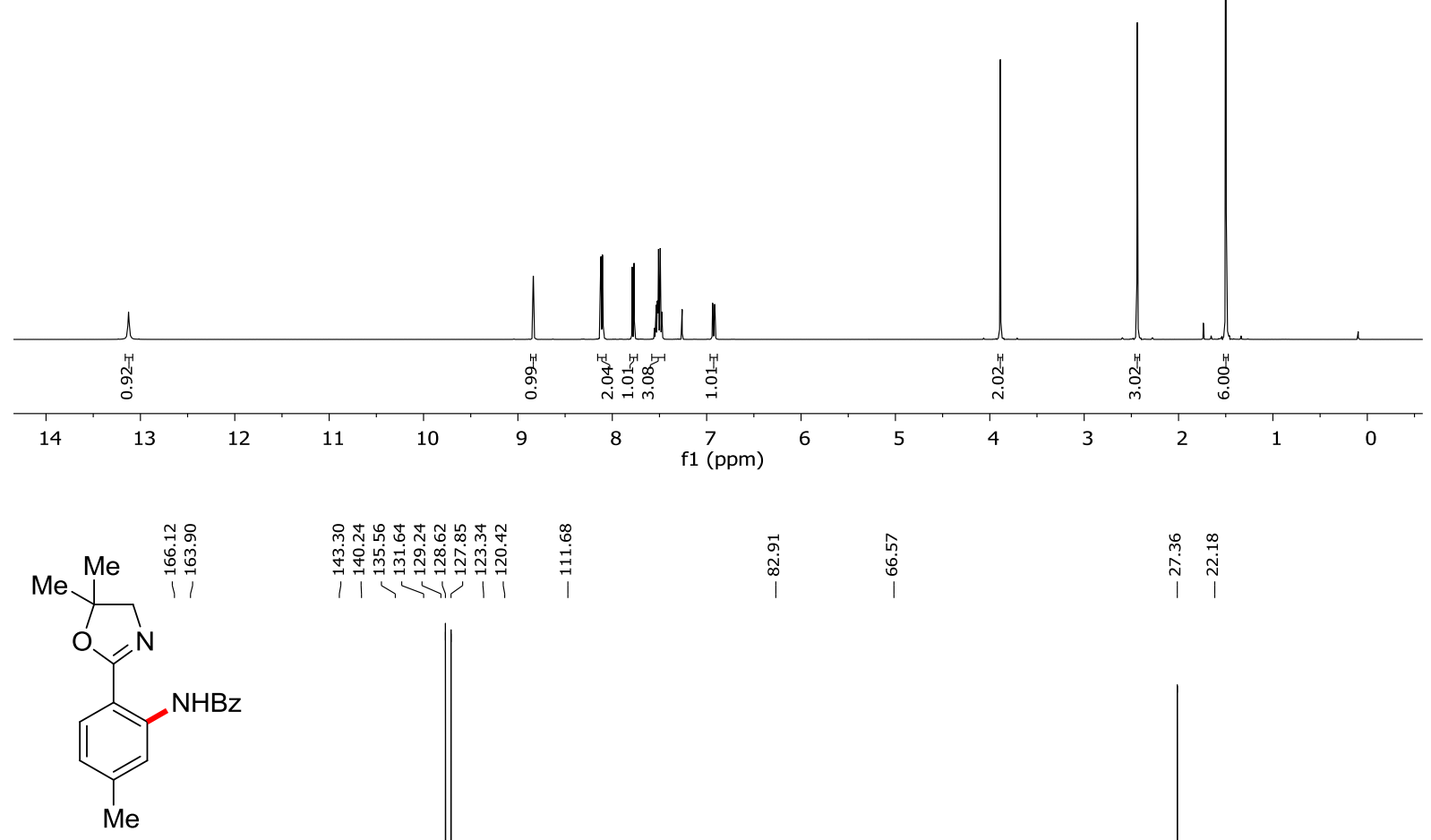

$1430 a$

$\left(\mathrm{CDCl}_{3}, 100 \mathrm{MHz}\right)$

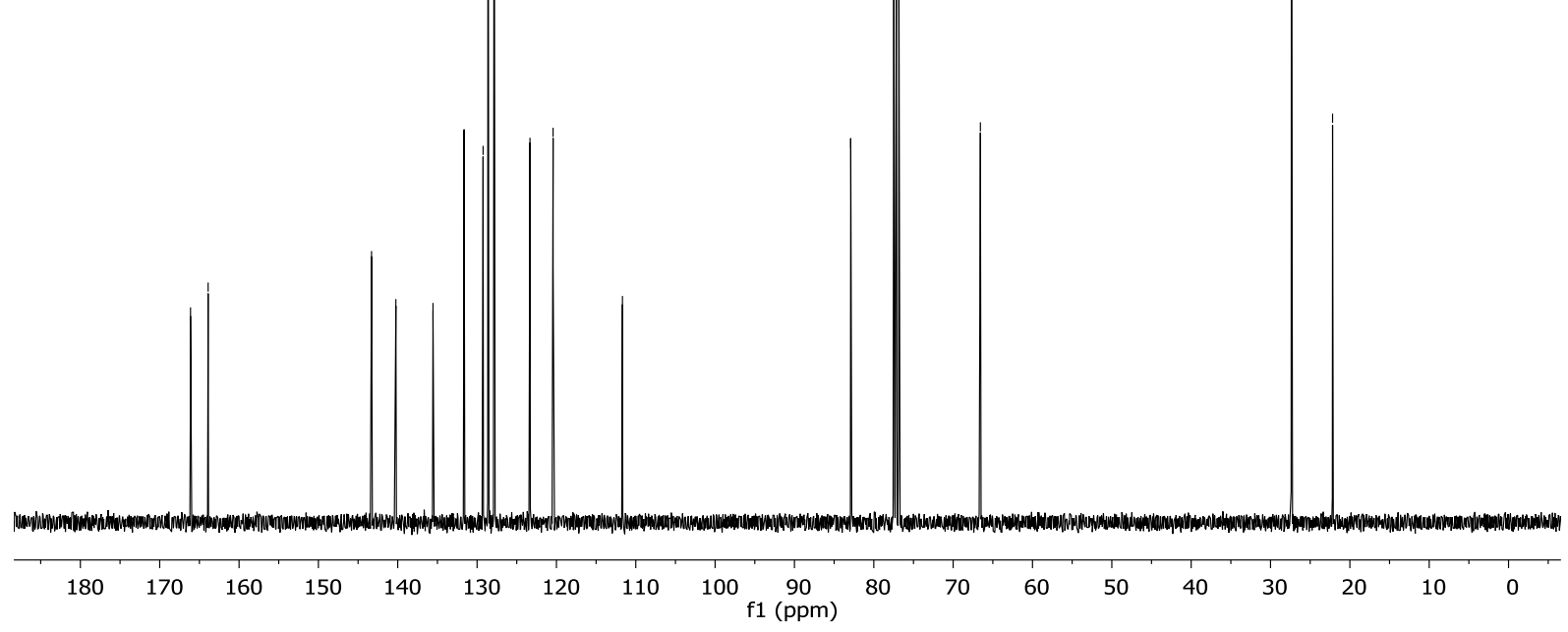




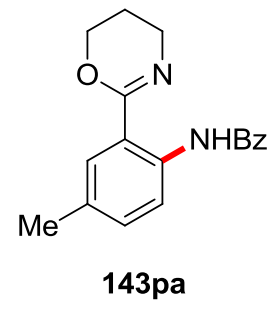

$\left(\mathrm{CDCl}_{3}, 300 \mathrm{MHz}\right)$
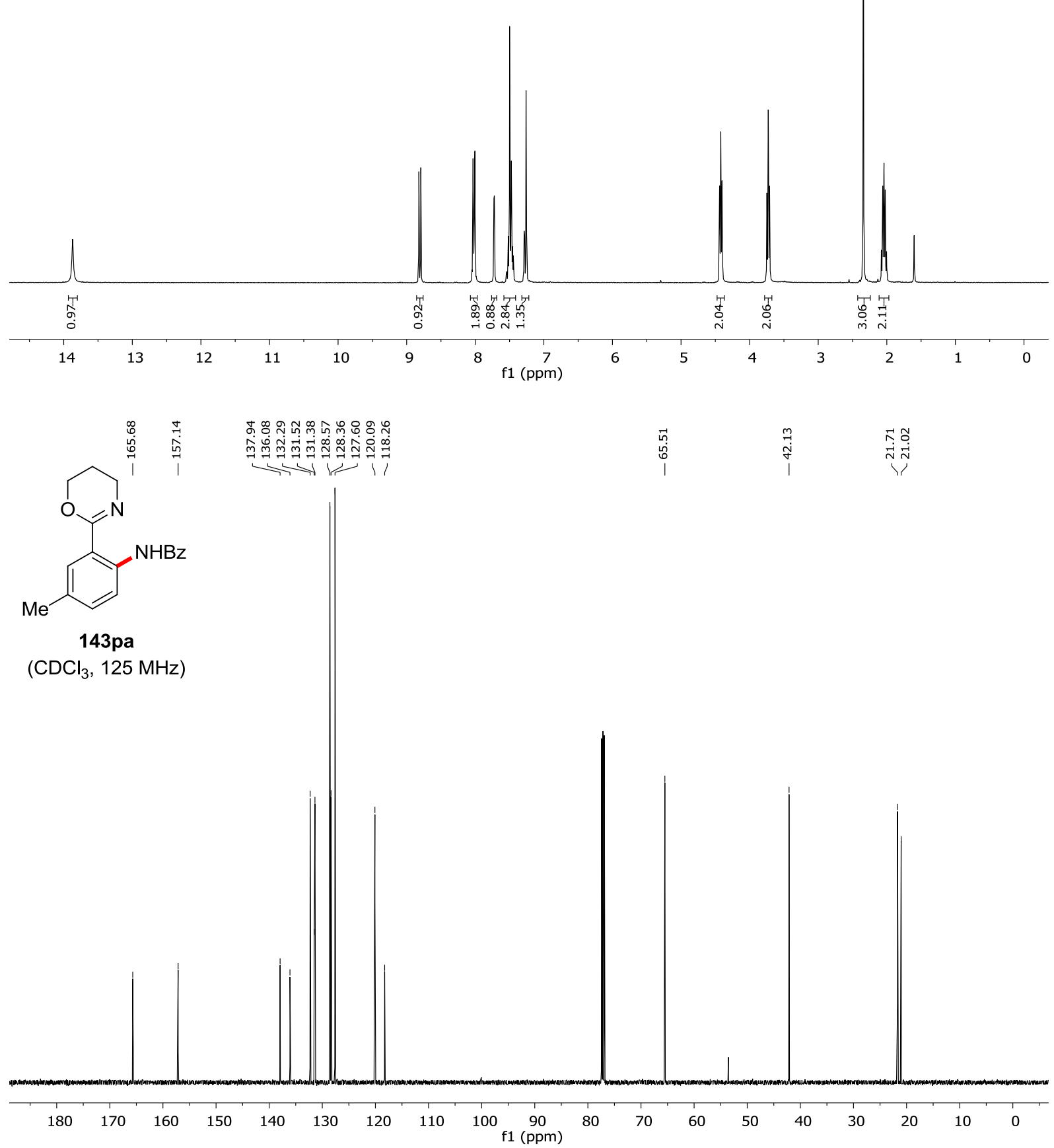


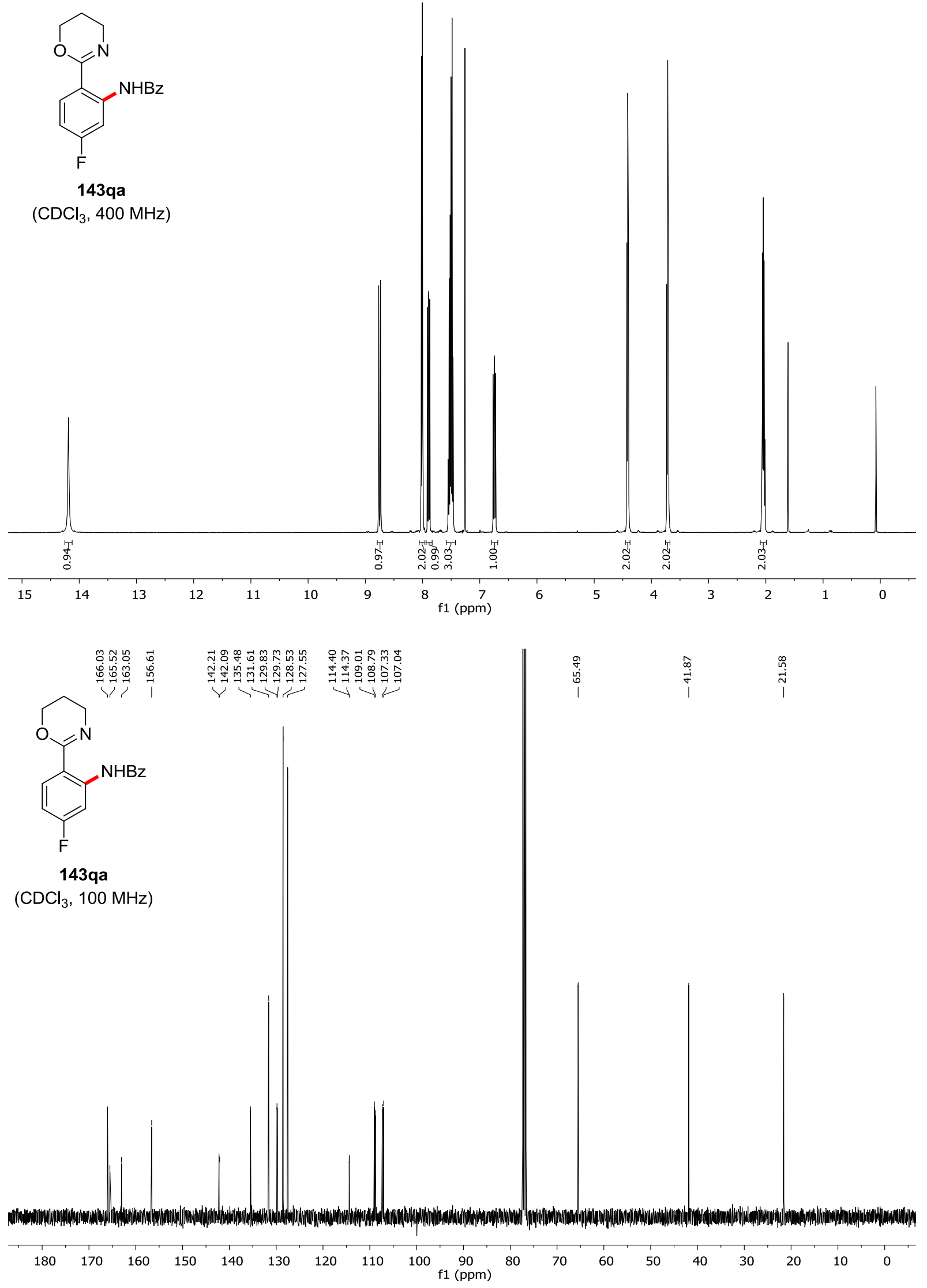




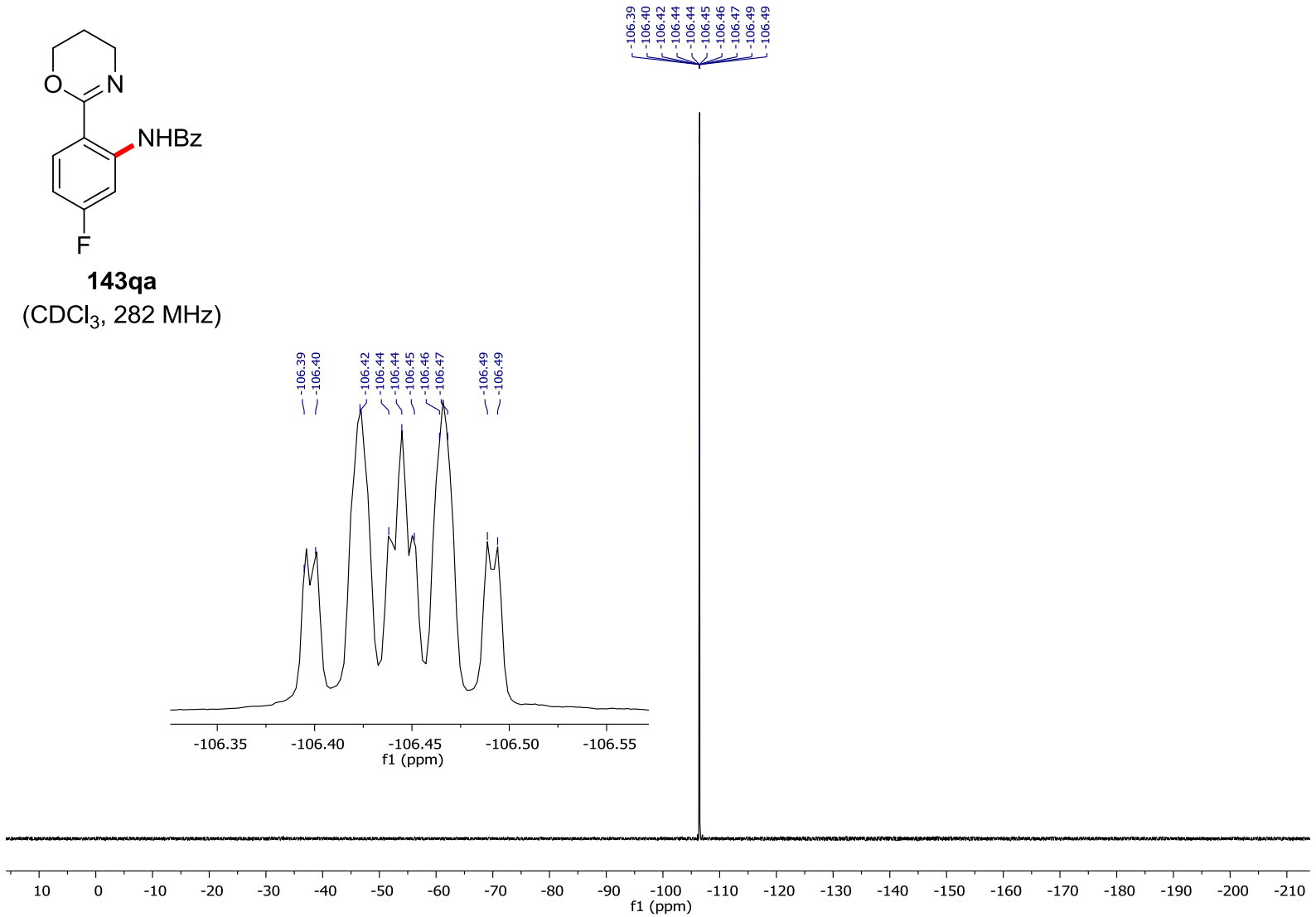



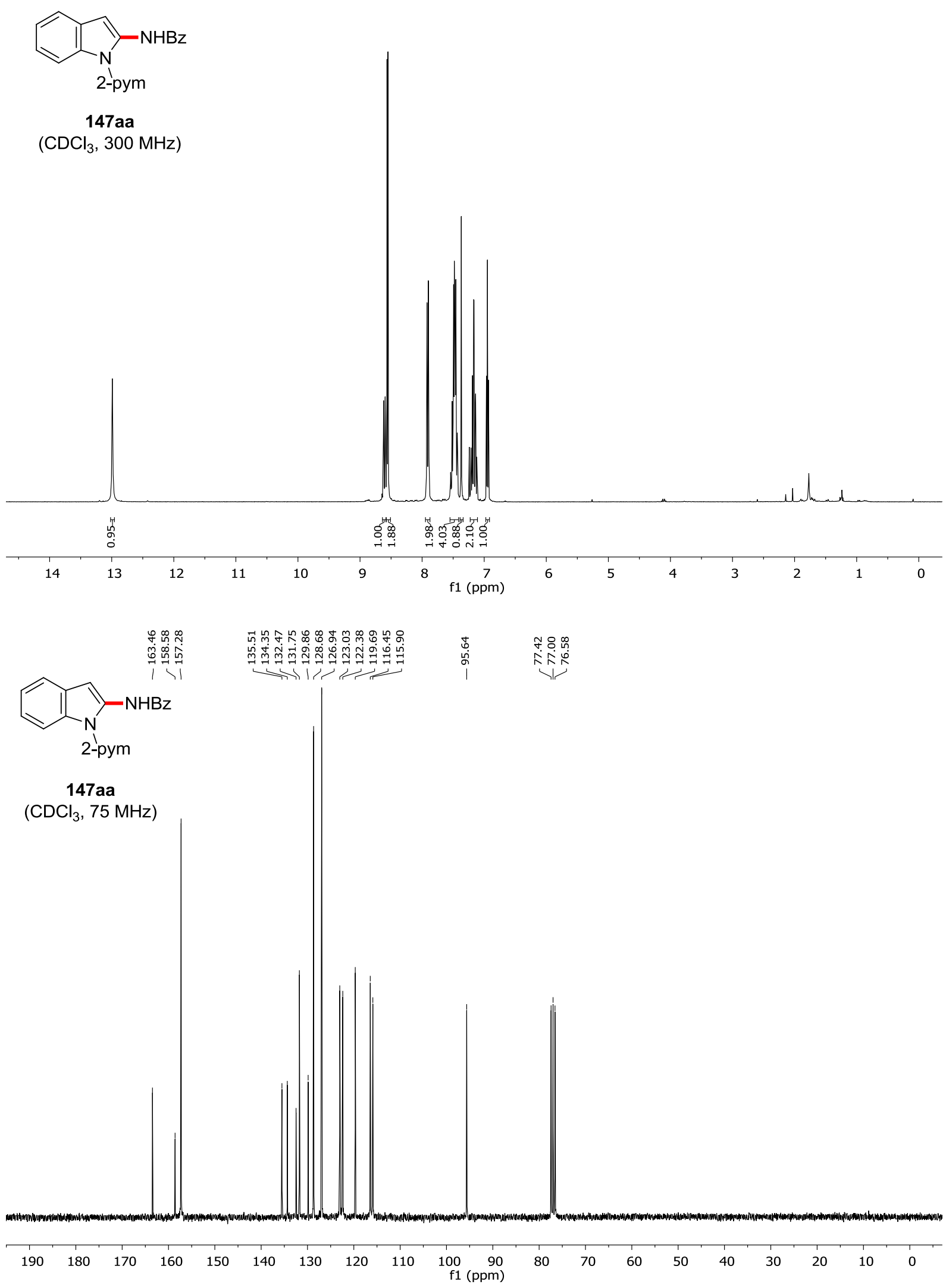

353 


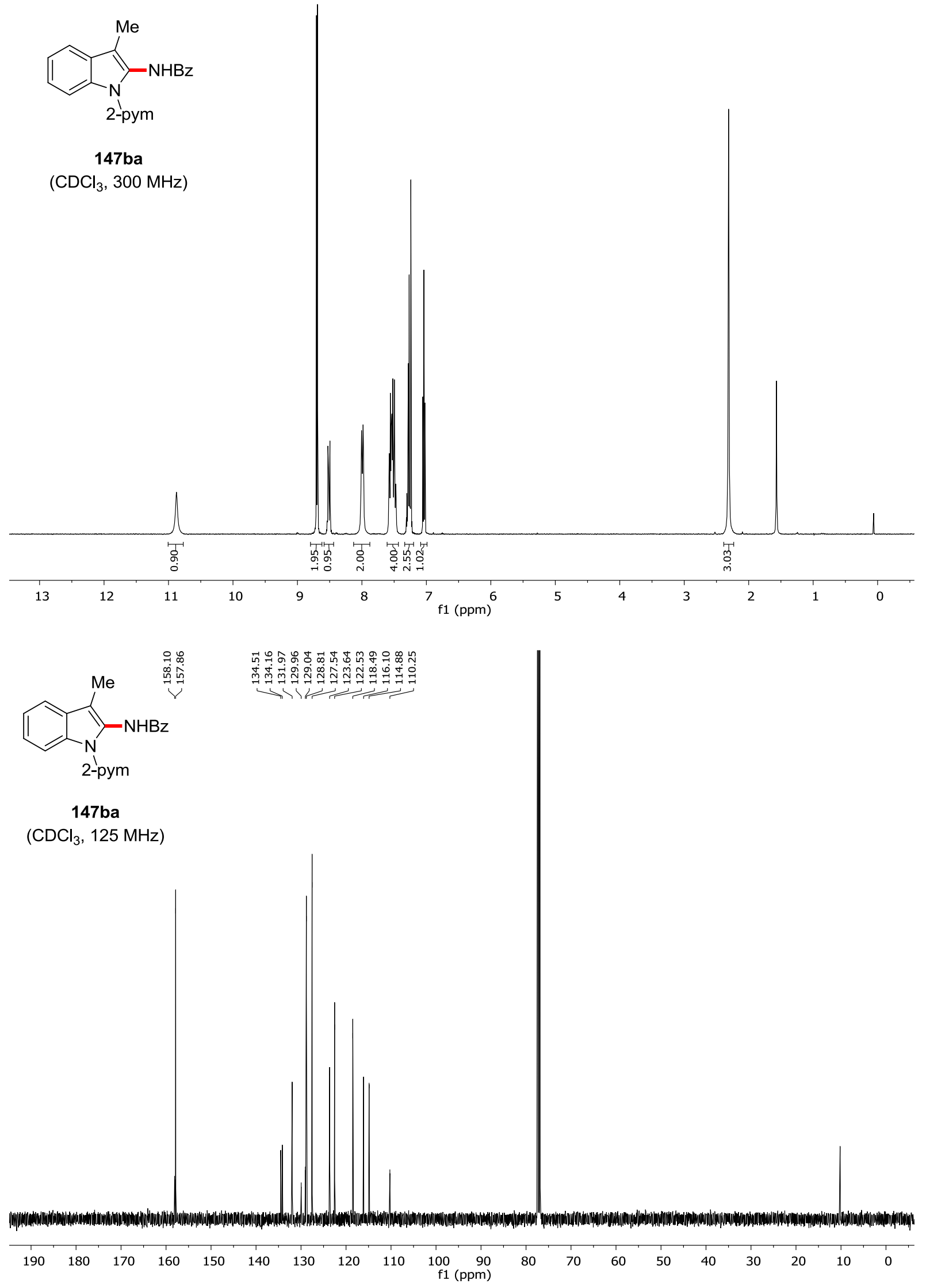



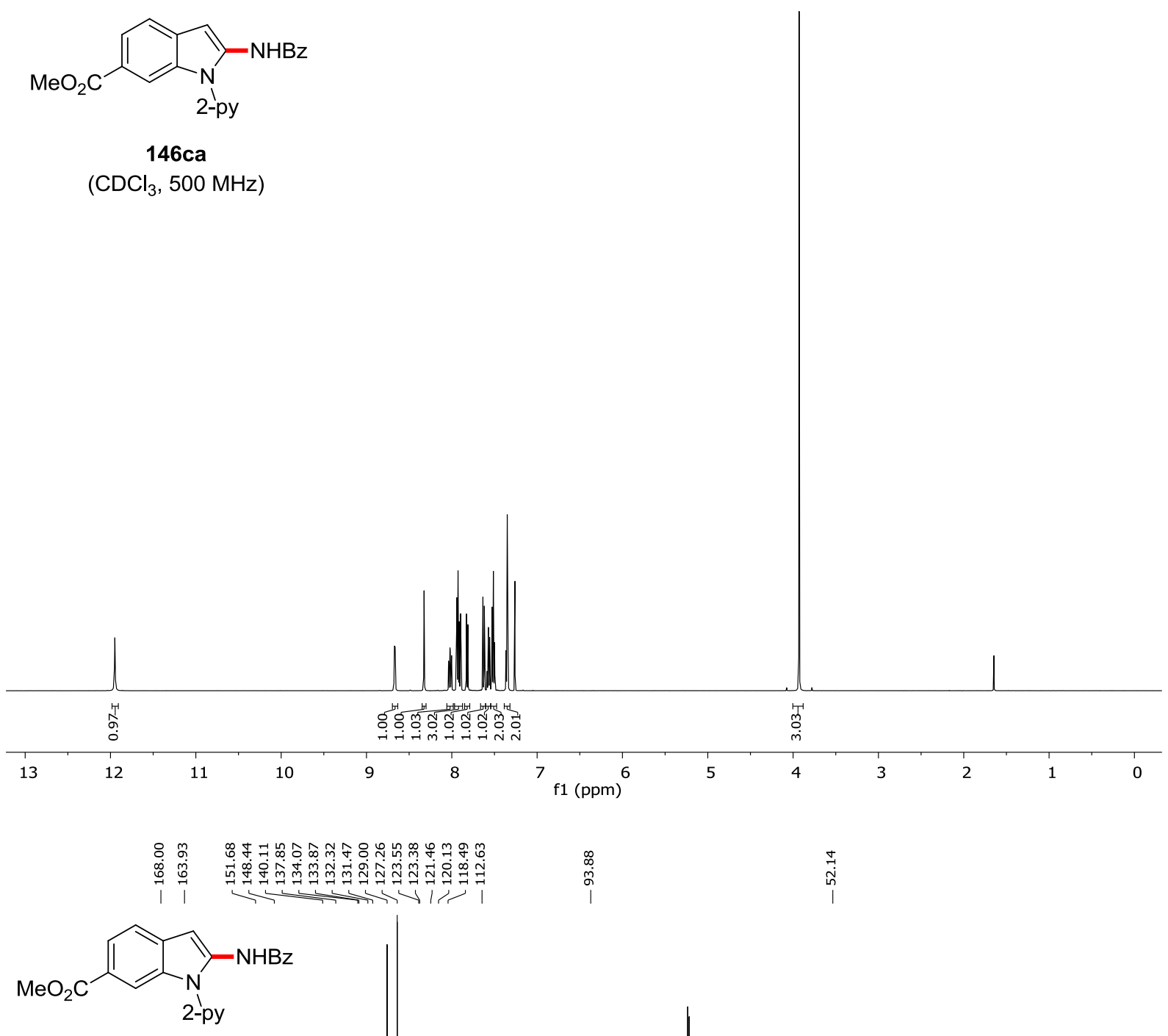

146ca

$\left(\mathrm{CDCl}_{3}, 125 \mathrm{MHz}\right)$

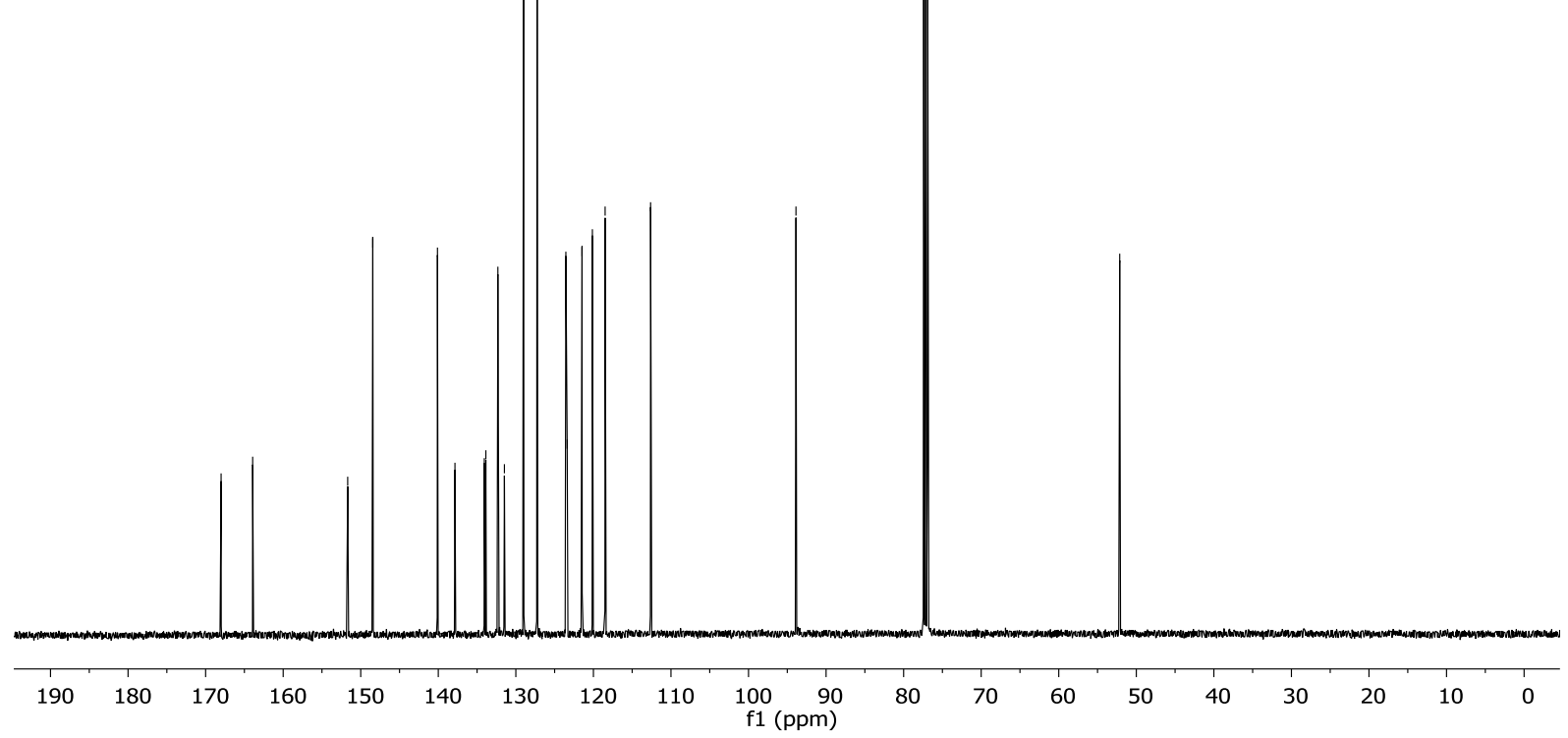




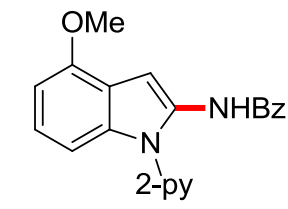

146da

$\left(\mathrm{CDCl}_{3}, 500 \mathrm{MHz}\right)$
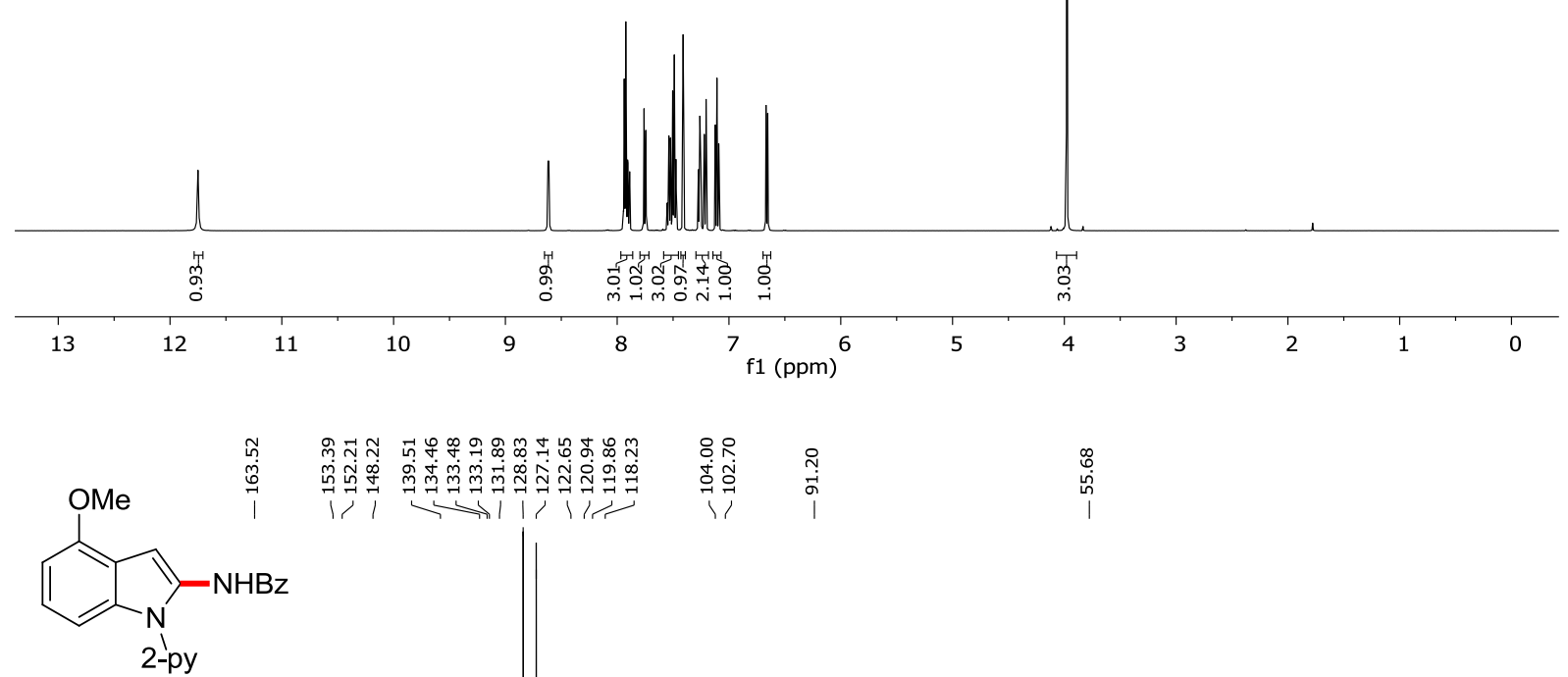

146da

$\left(\mathrm{CDCl}_{3}, 125 \mathrm{MHz}\right)$

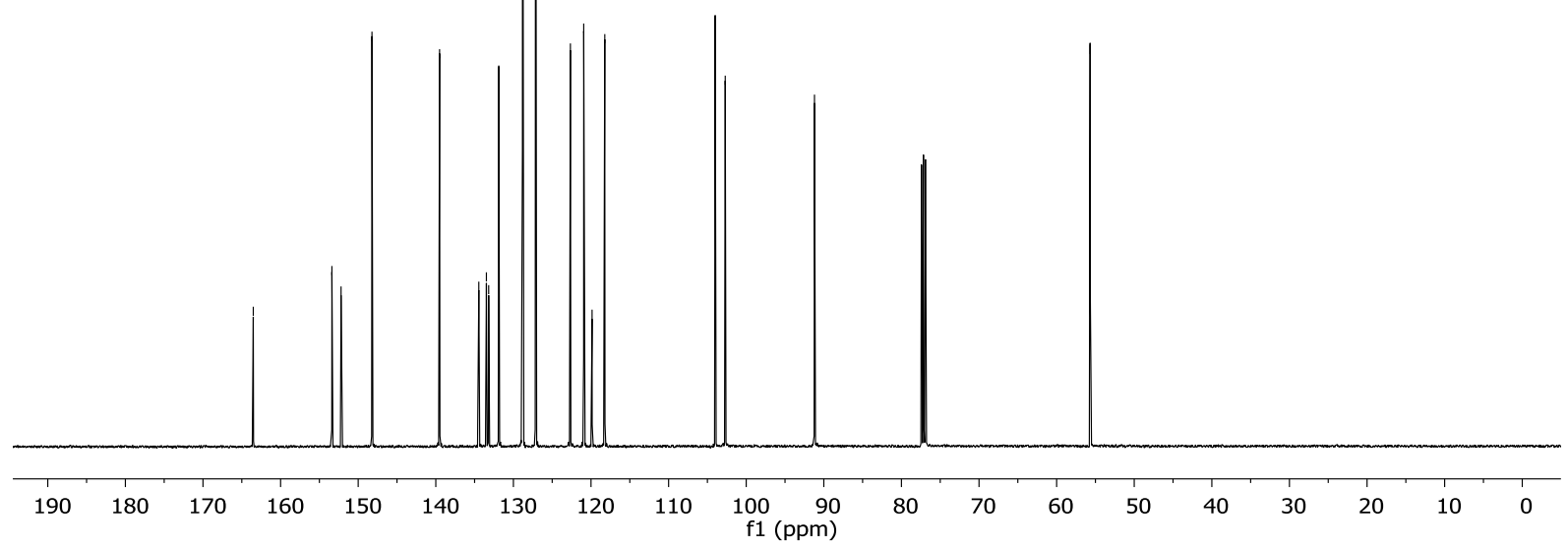



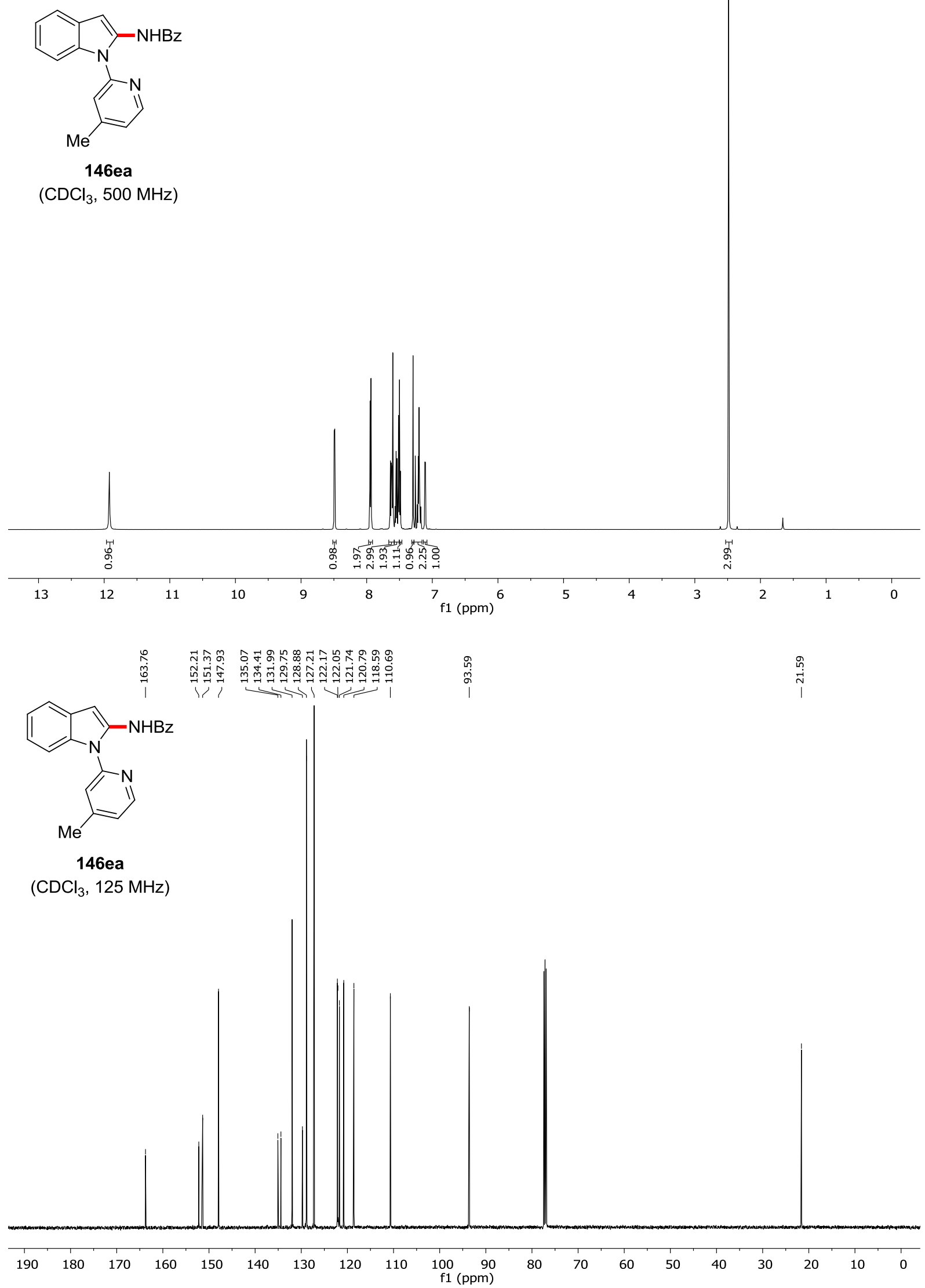


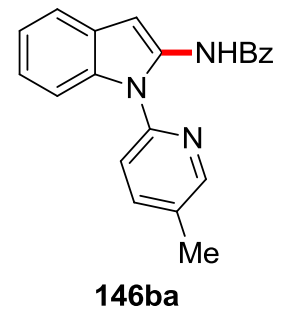

$\left(\mathrm{CDCl}_{3}, 300 \mathrm{MHz}\right)$
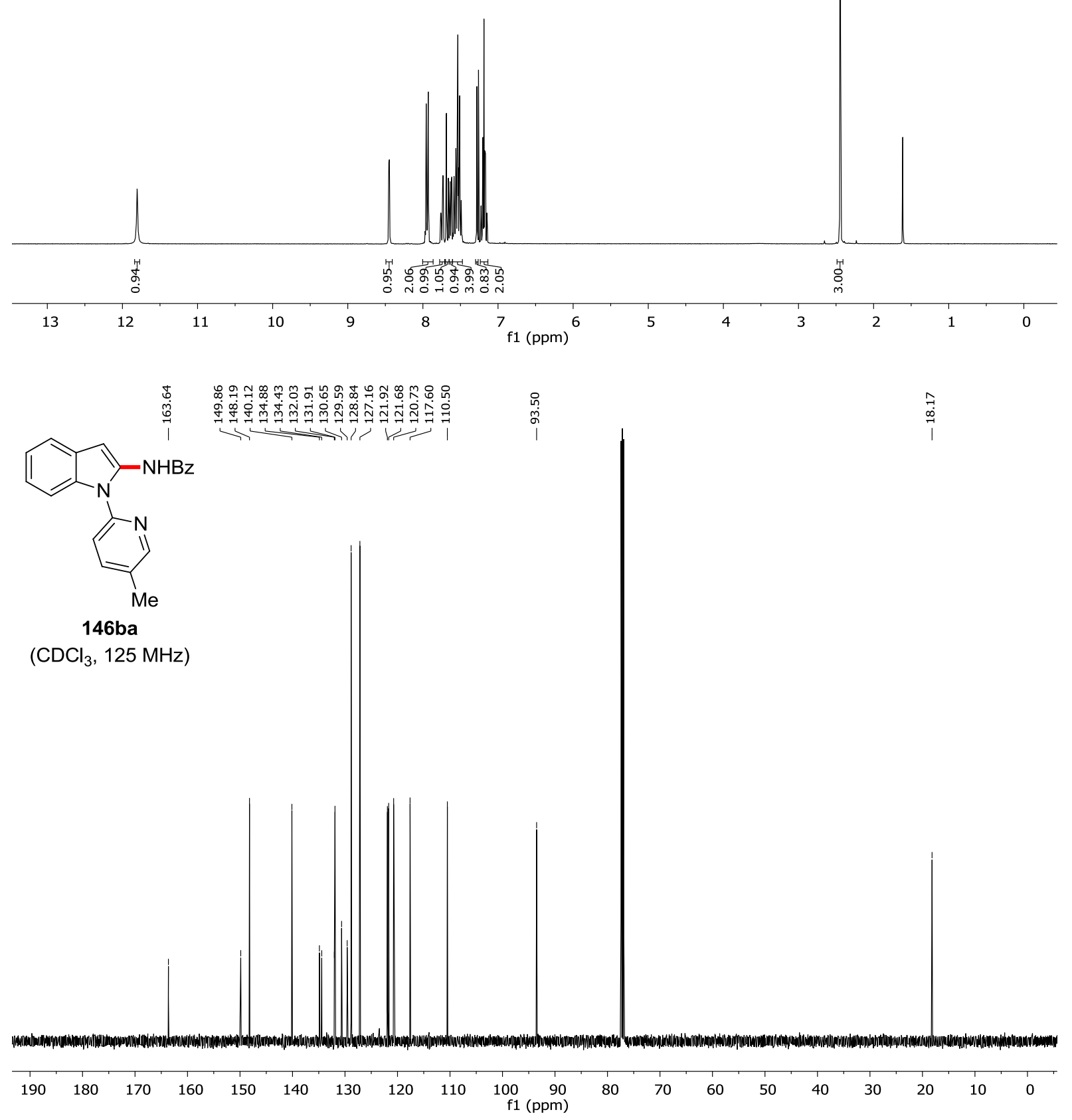

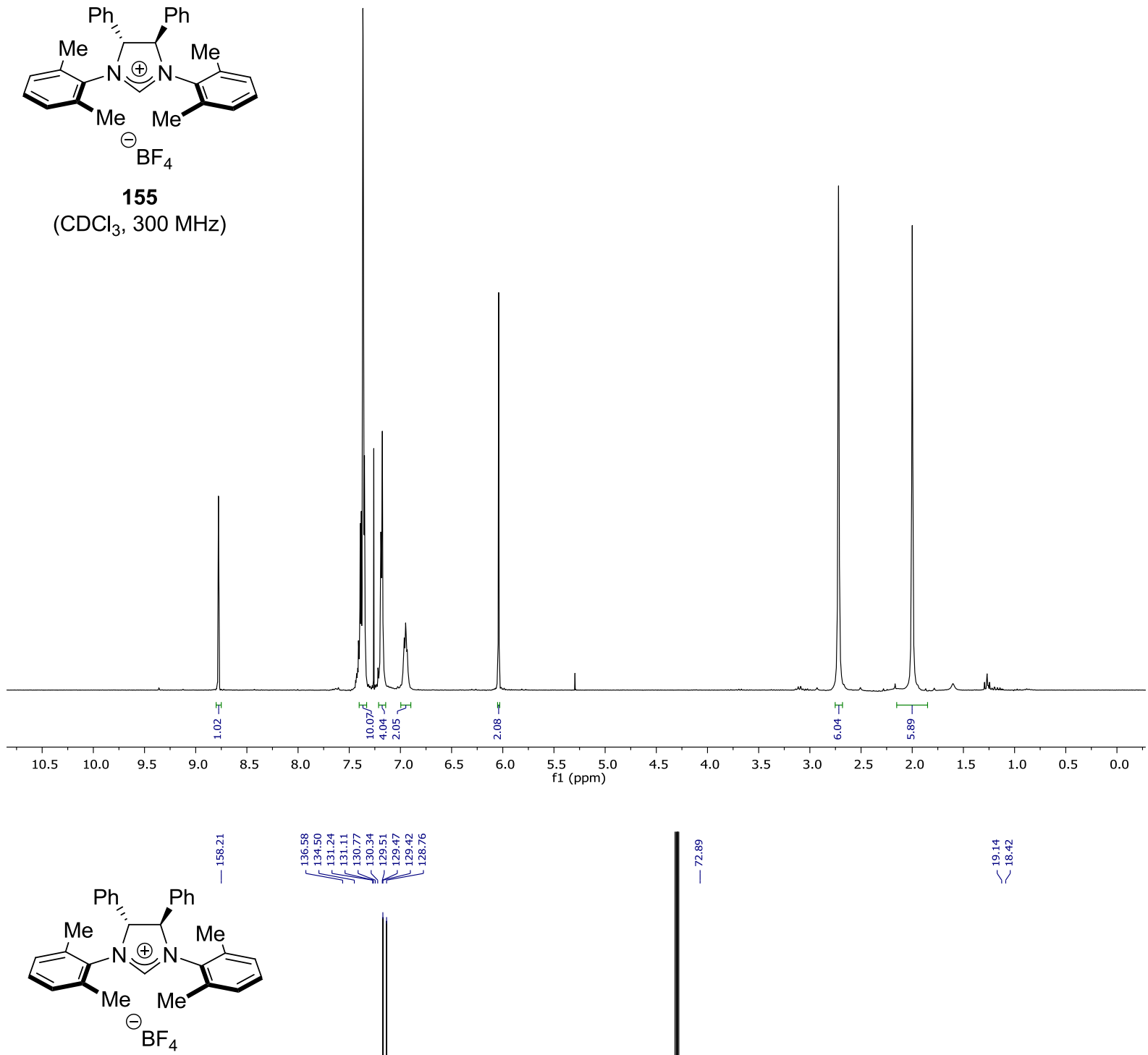

155

$\left(\mathrm{CDCl}_{3}, 125 \mathrm{MHz}\right)$ 


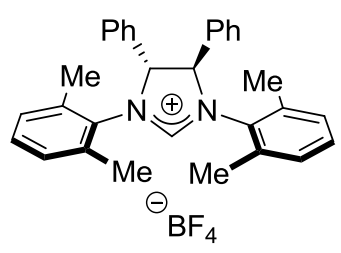

155

$\left(\mathrm{CDCl}_{3}, 282 \mathrm{MHz}\right)$

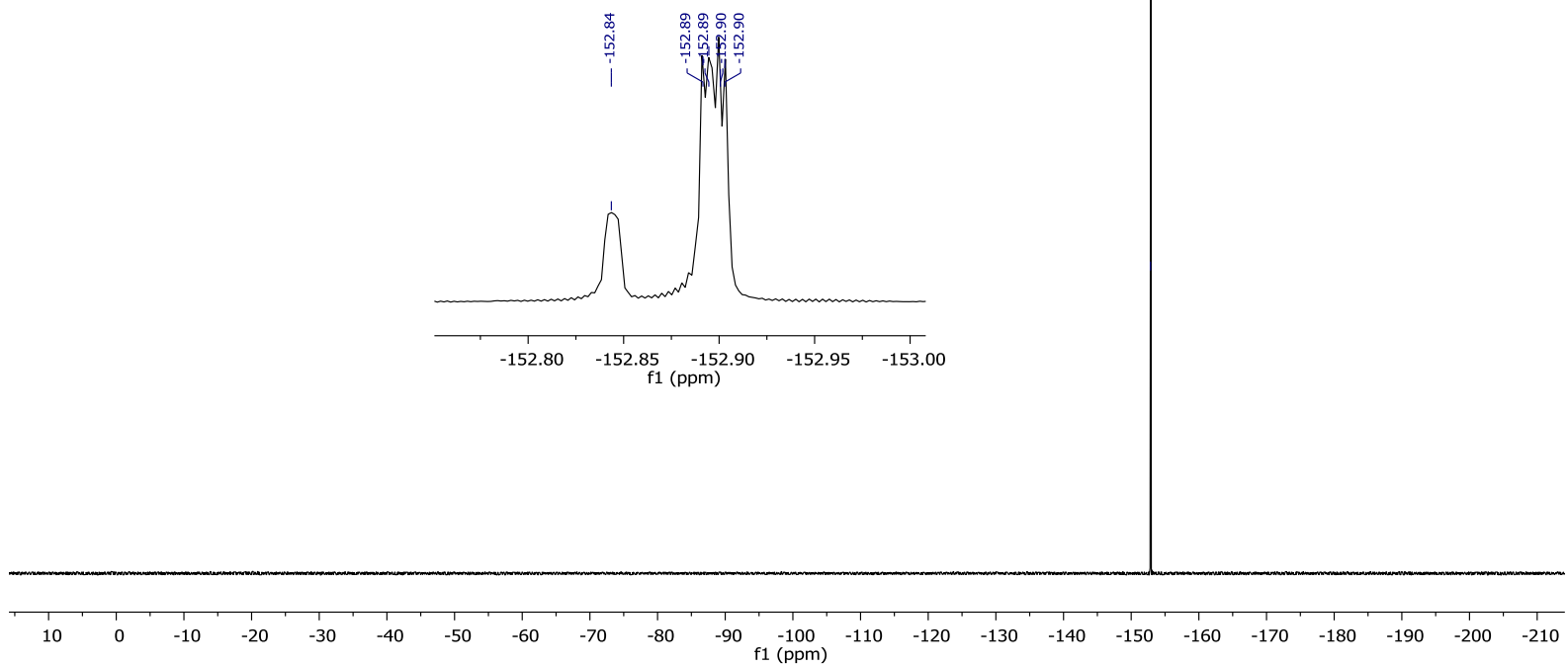




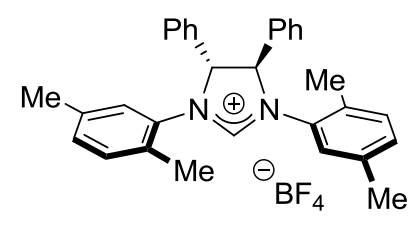

176

$\left(\mathrm{CDCl}_{3}, 400 \mathrm{MHz}\right)$
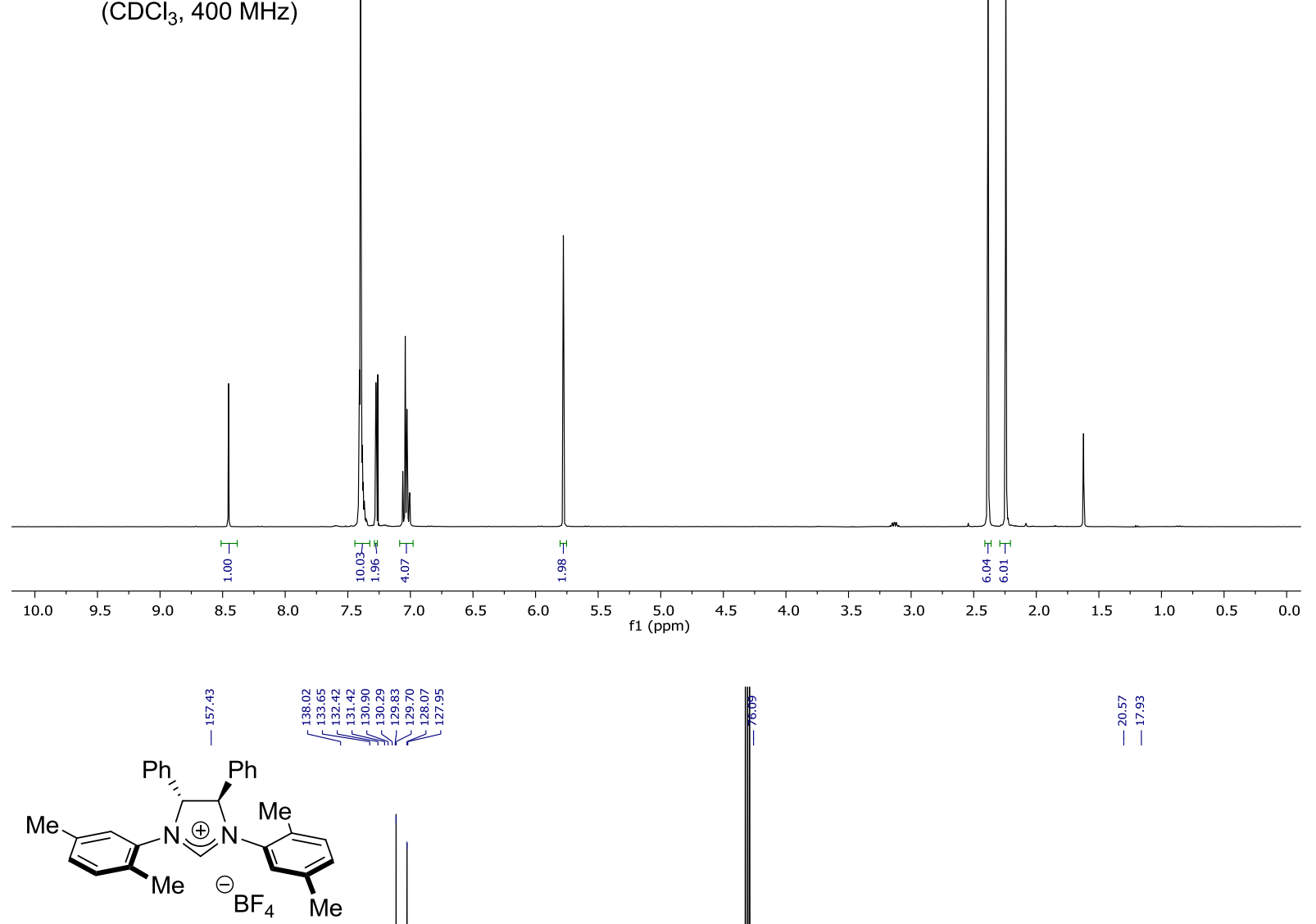

176

$\left(\mathrm{CDCl}_{3}, 100 \mathrm{MHz}\right)$

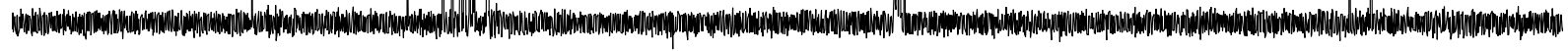

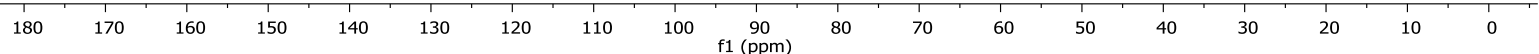




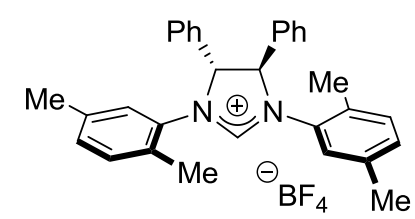

176

$\left(\mathrm{CDCl}_{3}, 282 \mathrm{MHz}\right)$

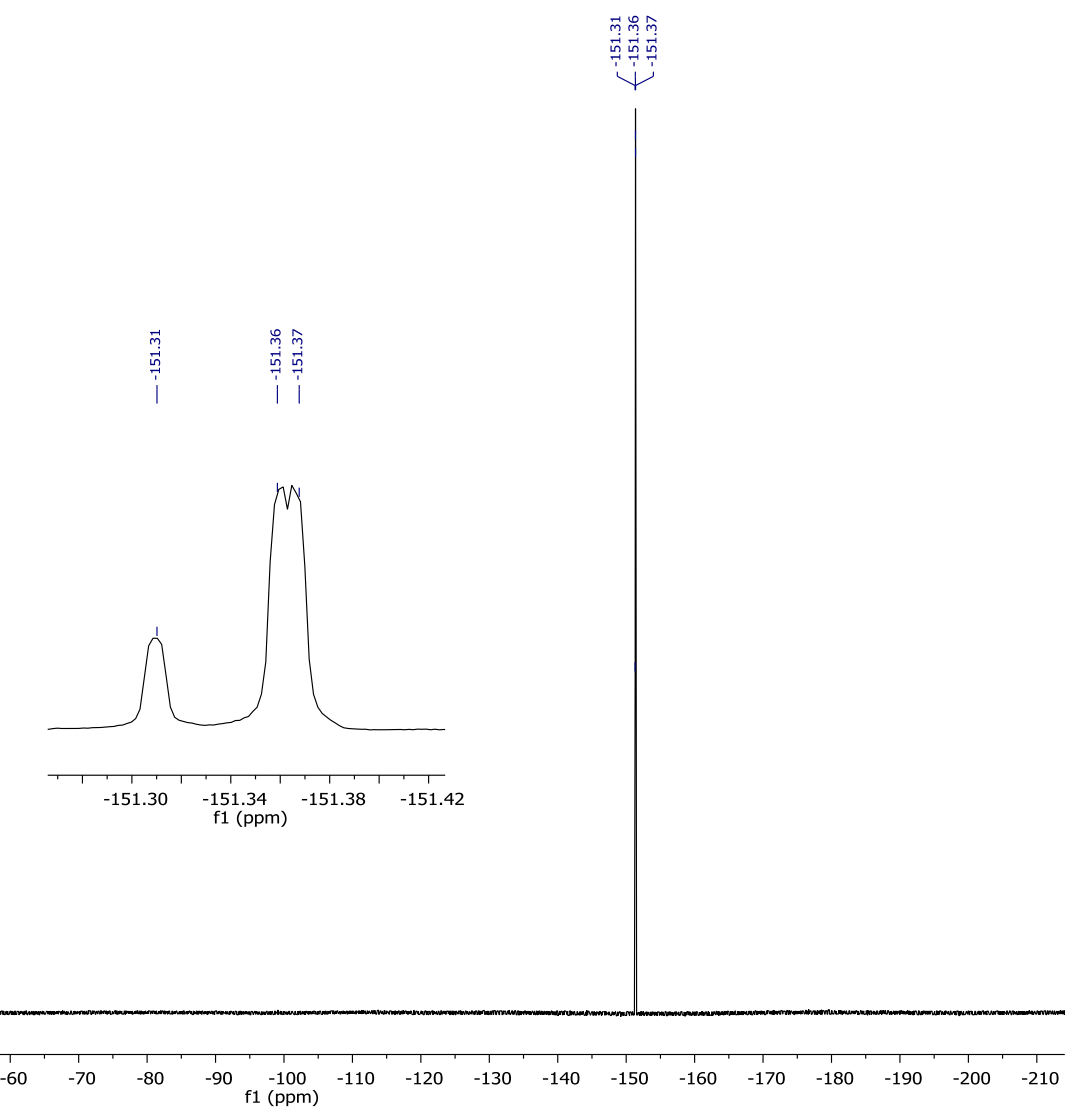




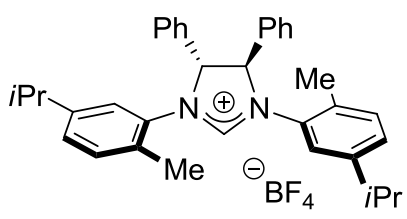

177

$\left(\mathrm{CDCl}_{3}, 500 \mathrm{MHz}\right)$
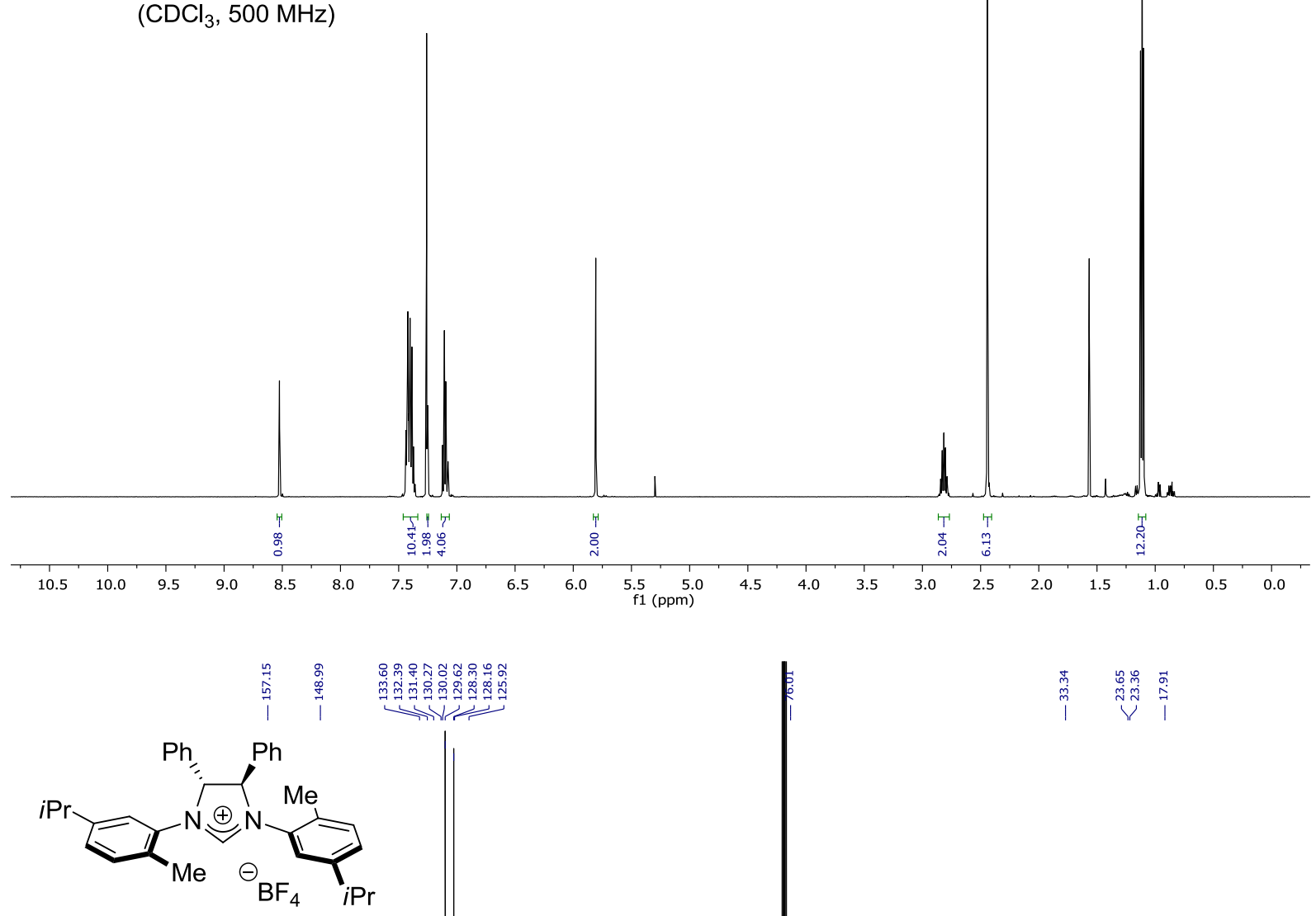

177

$\left(\mathrm{CDCl}_{3}, 125 \mathrm{MHz}\right)$

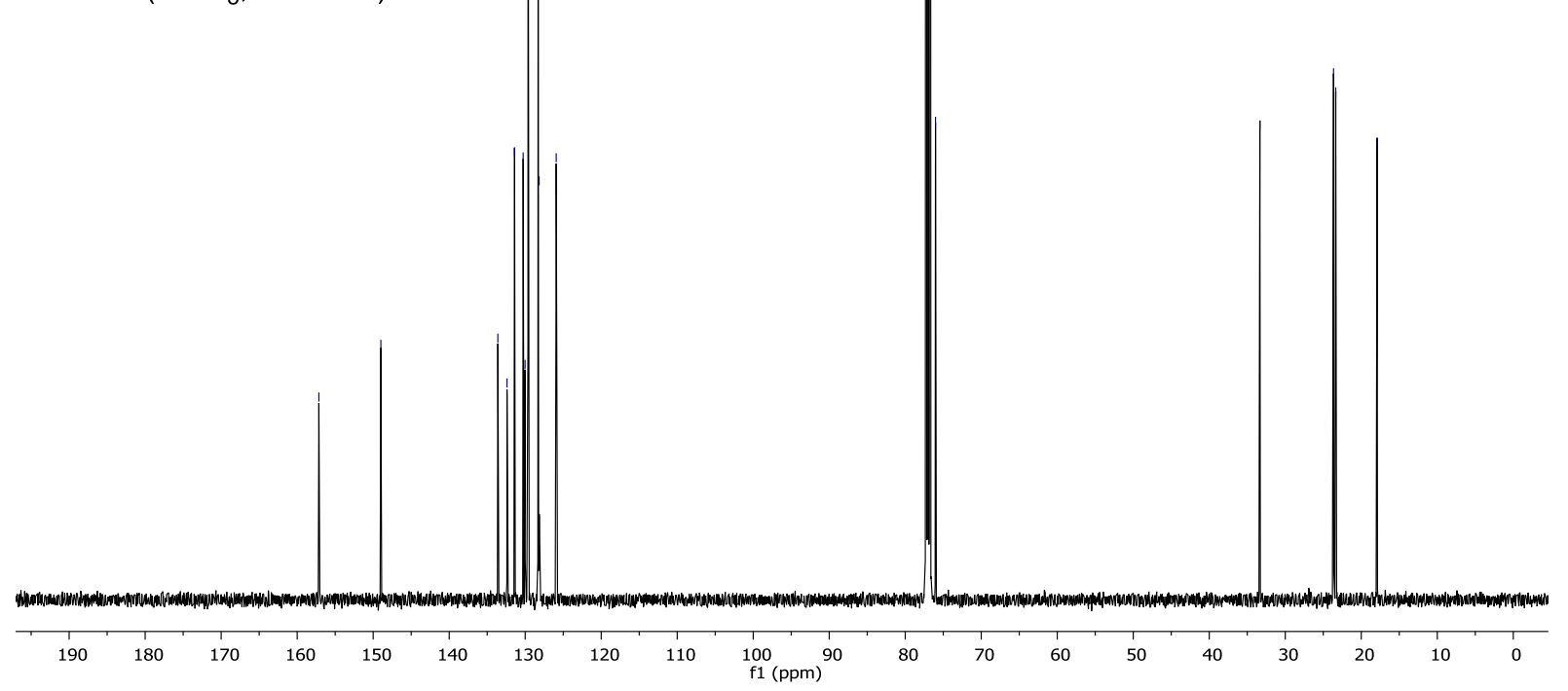



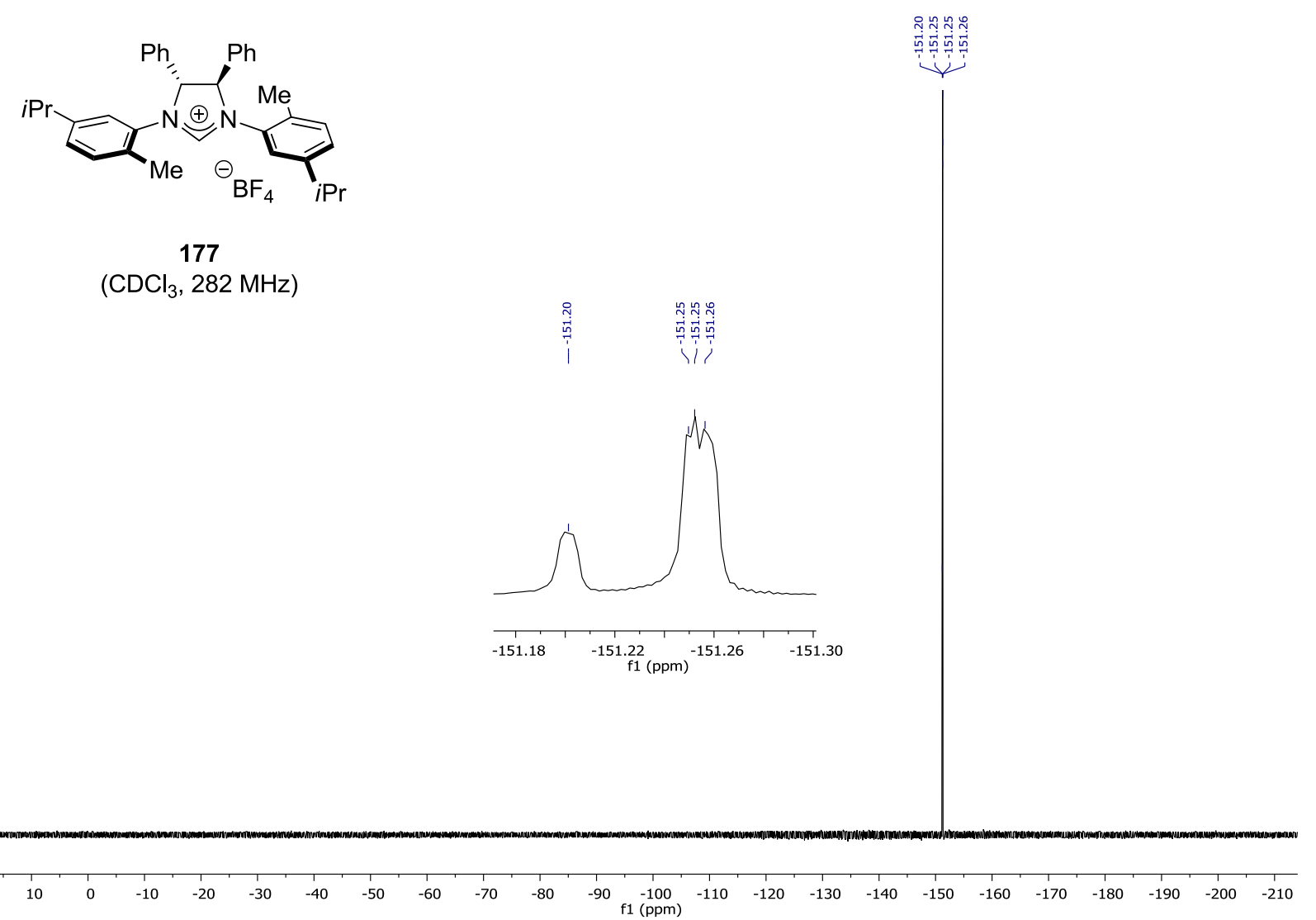

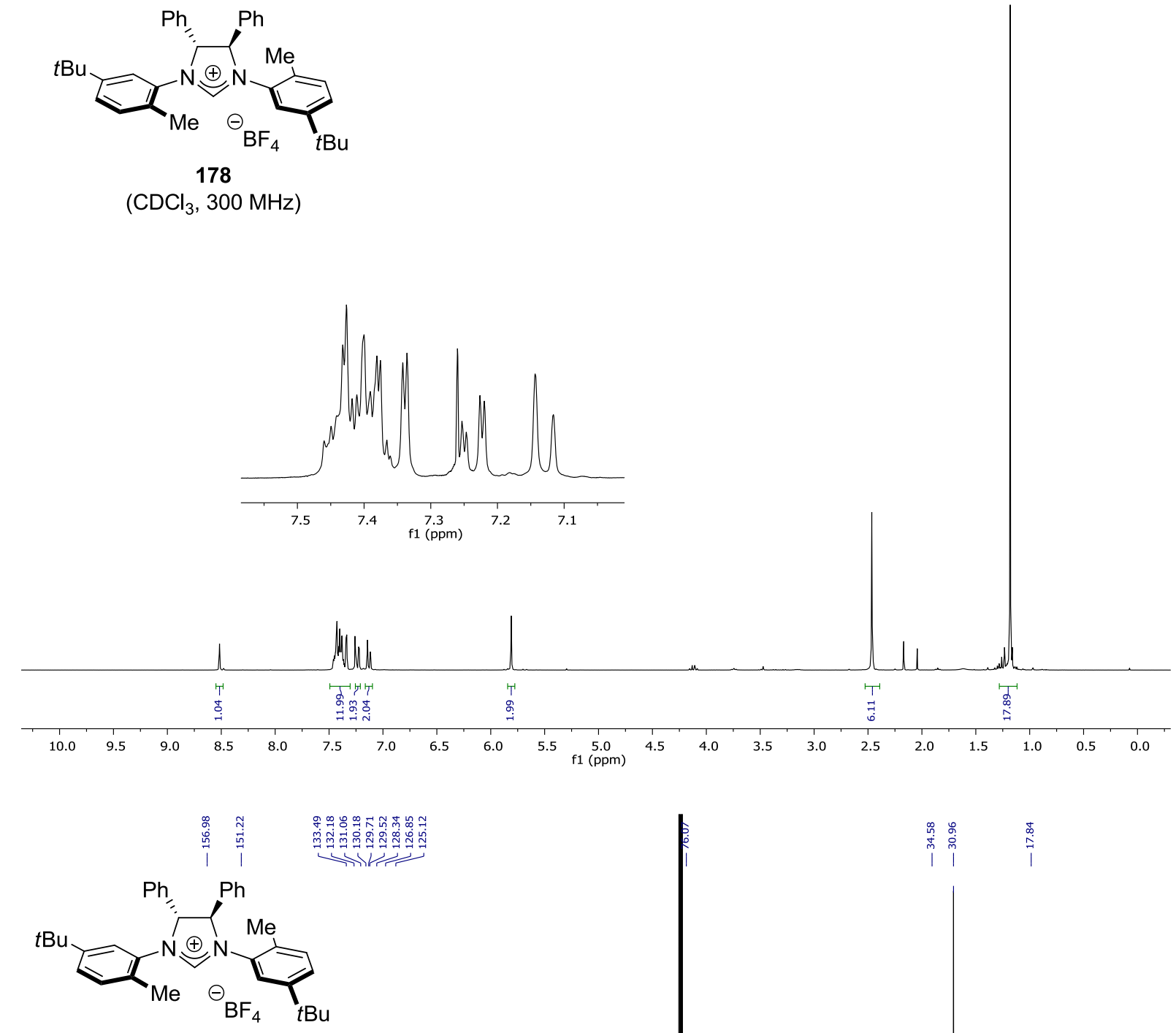

178

$\left(\mathrm{CDCl}_{3}, 125 \mathrm{MHz}\right)$ 


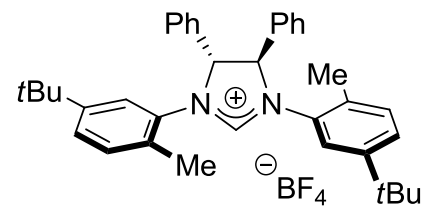

178

$\left(\mathrm{CDCl}_{3}, 282 \mathrm{MHz}\right)$

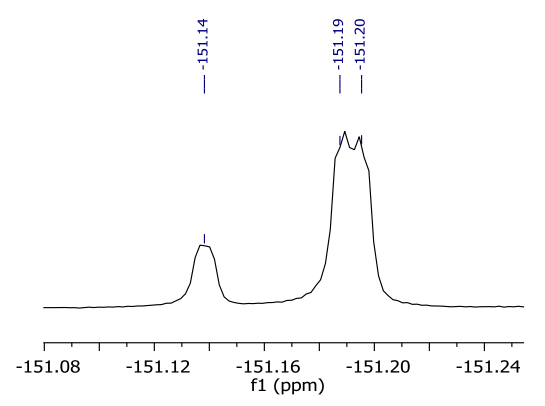




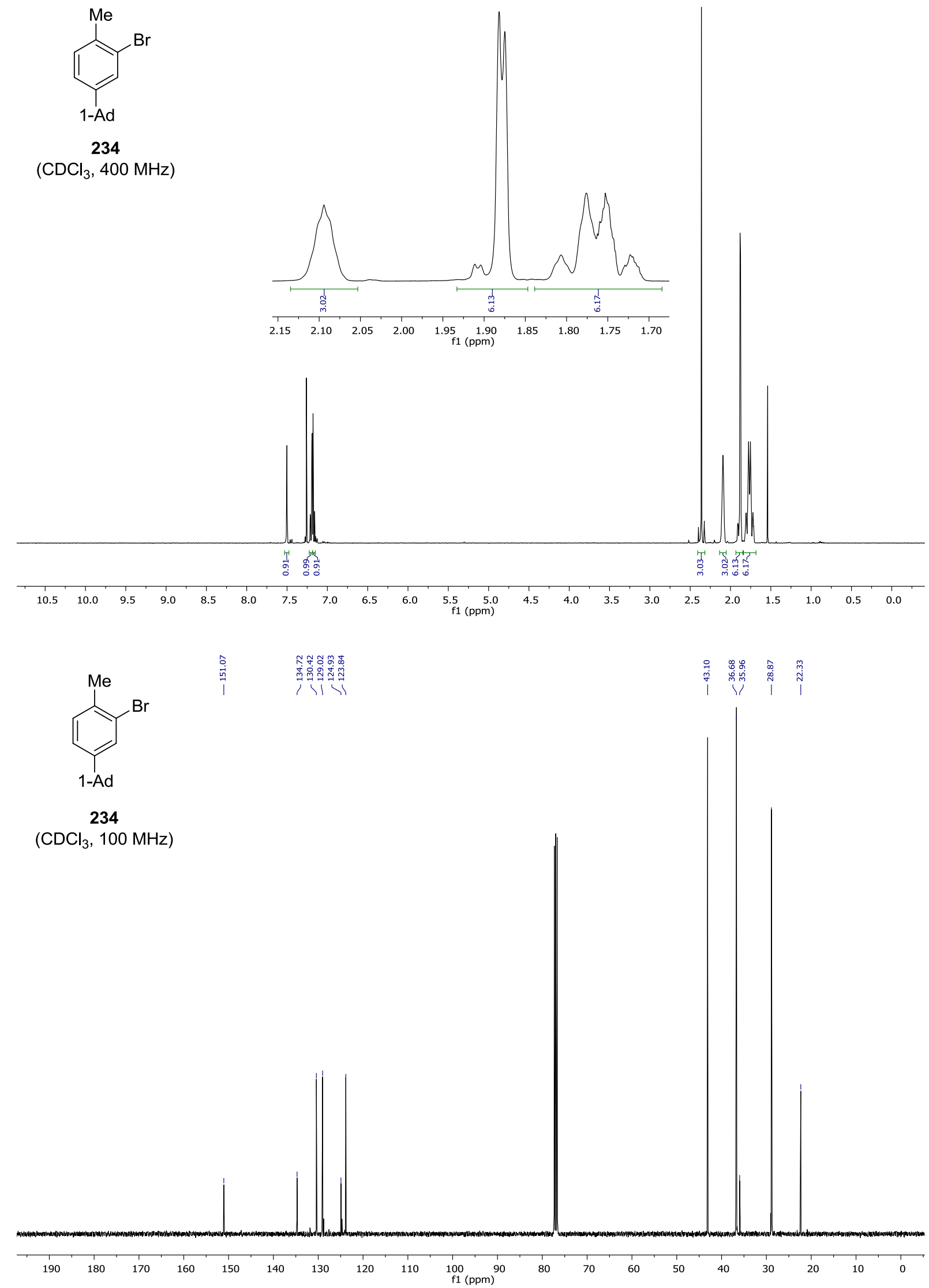



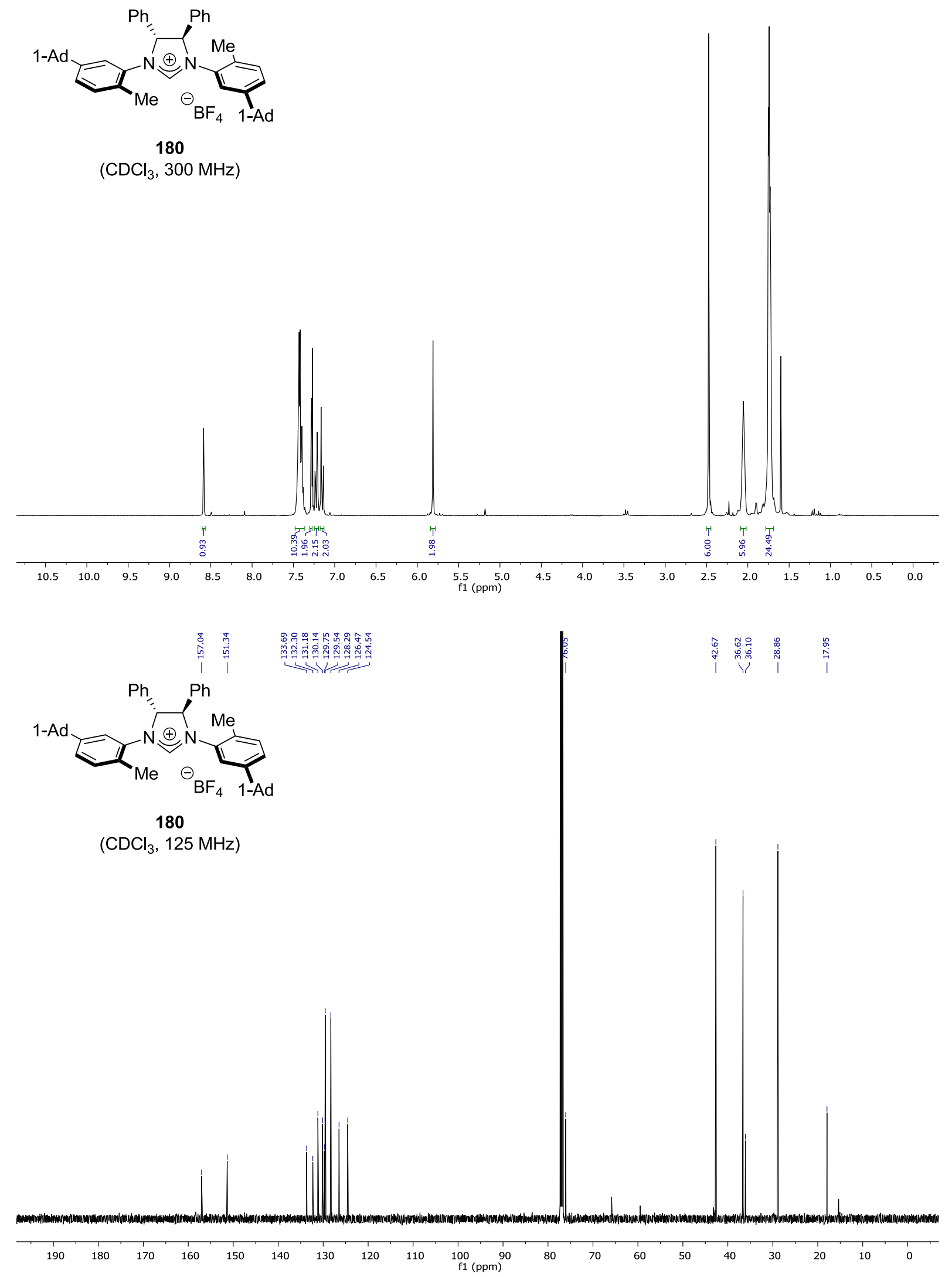

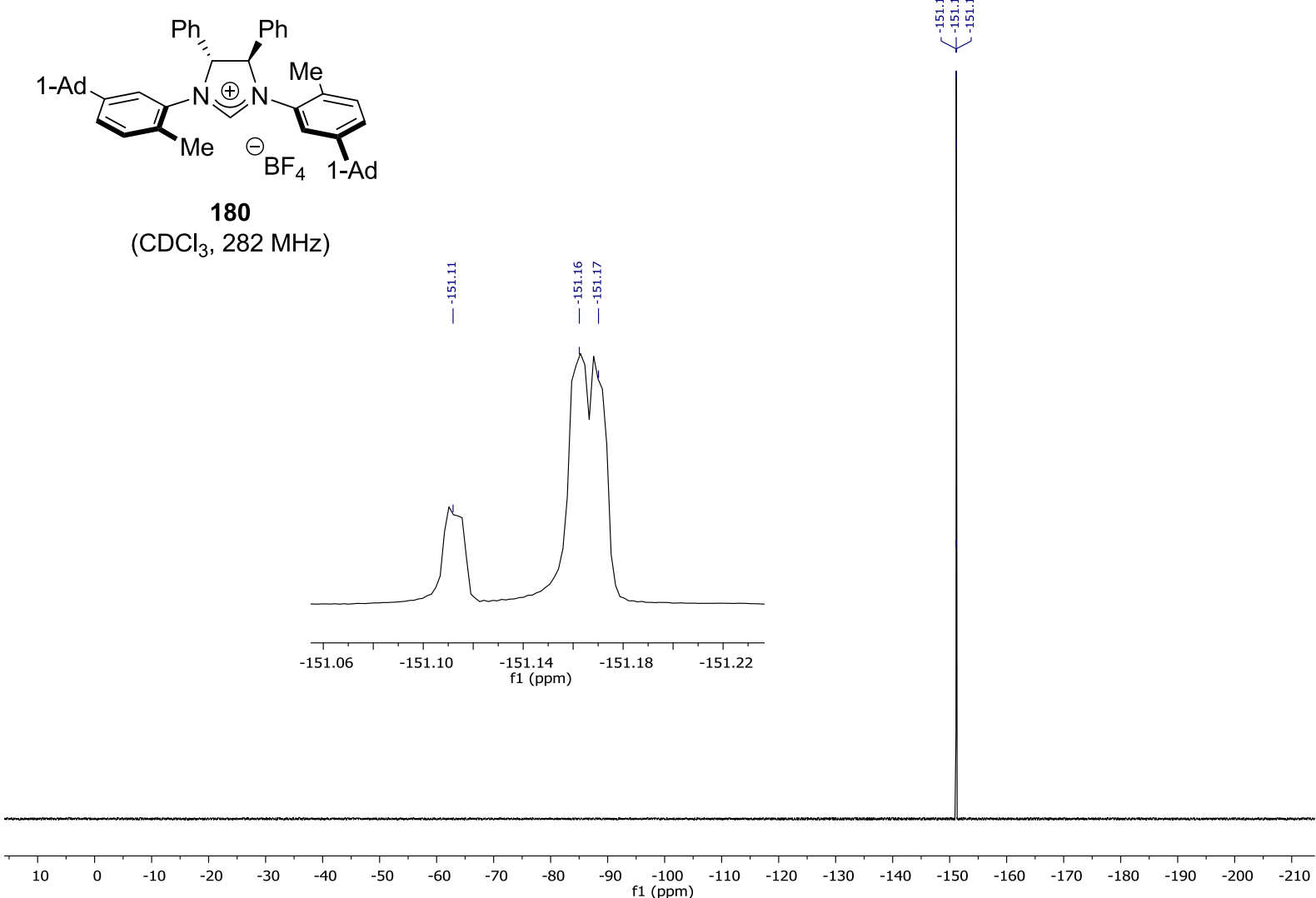


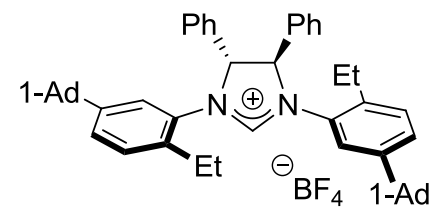

181

$\left(\mathrm{CDCl}_{3}, 500 \mathrm{MHz}\right)$
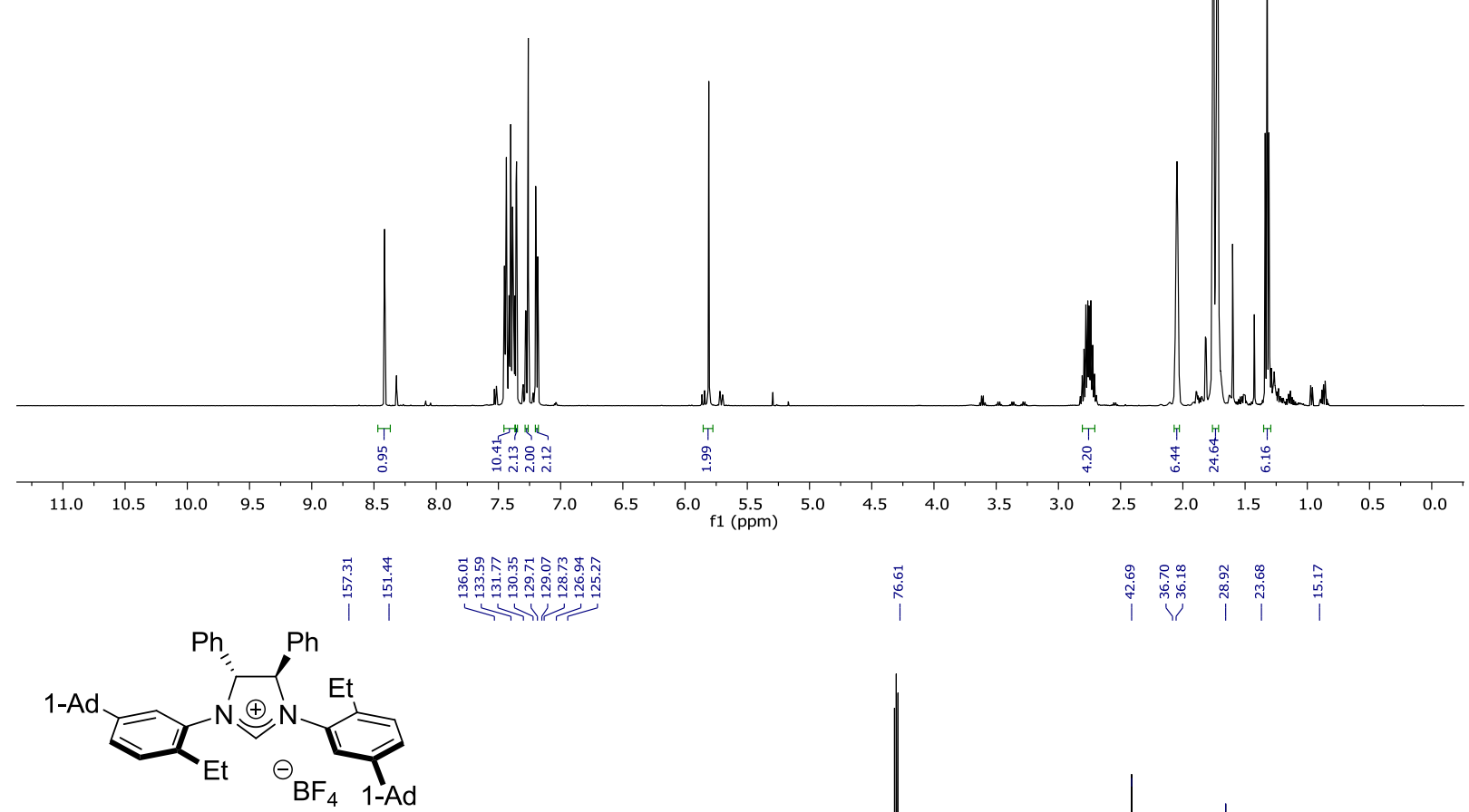

181

$\left(\mathrm{CDCl}_{3}, 125 \mathrm{MHz}\right)$

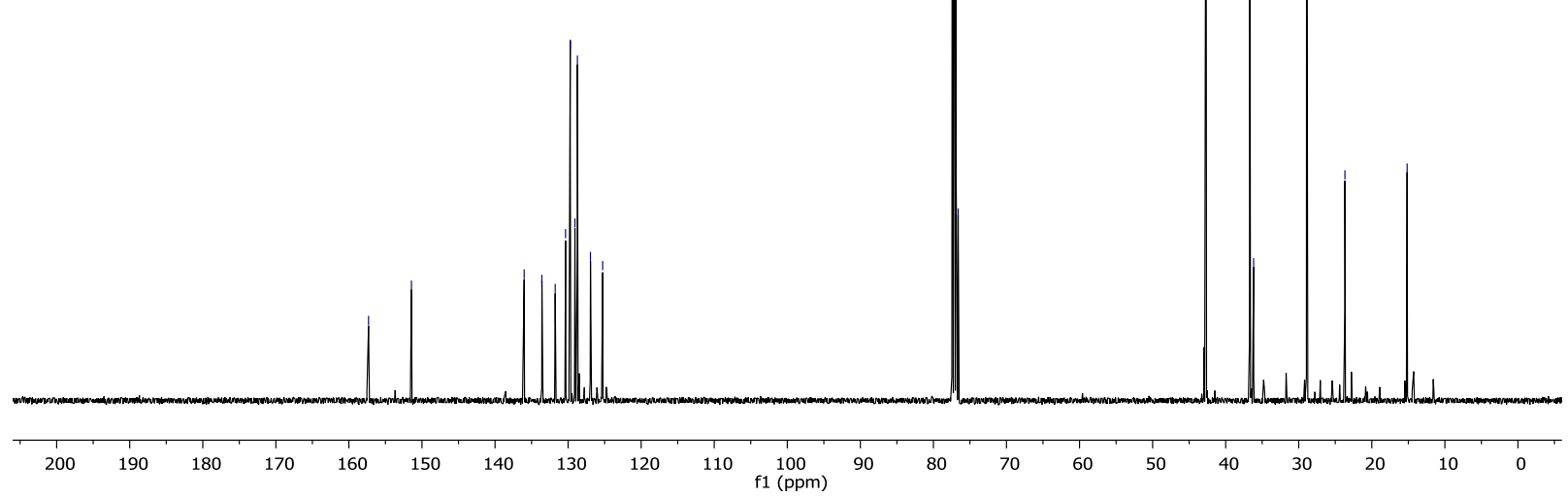




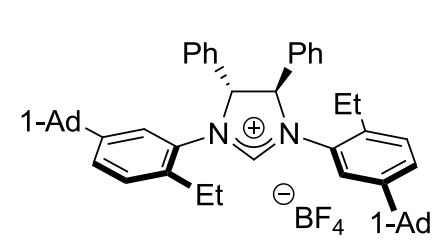

181

$\left(\mathrm{CDCl}_{3}, 471 \mathrm{MHz}\right)$

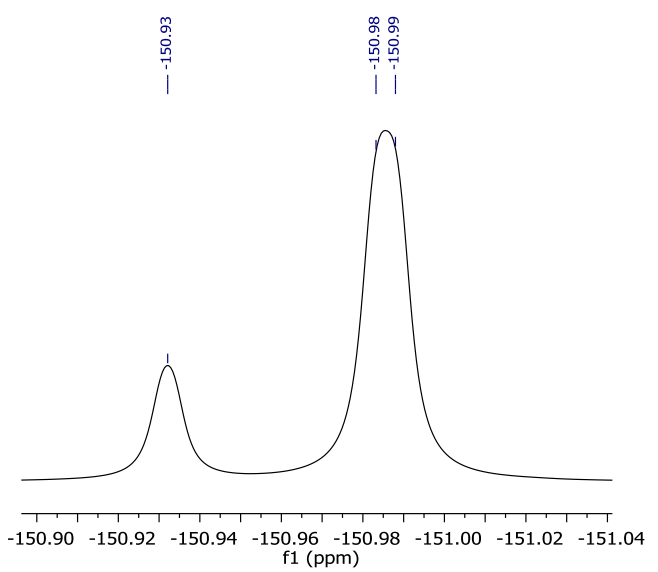

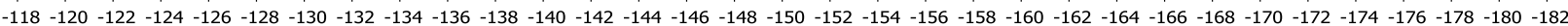
$\mathrm{f} 1(\mathrm{ppm})$ 


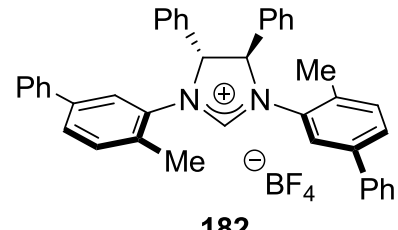

$\left(\mathrm{CDCl}_{3}, 300 \mathrm{MHz}\right)$
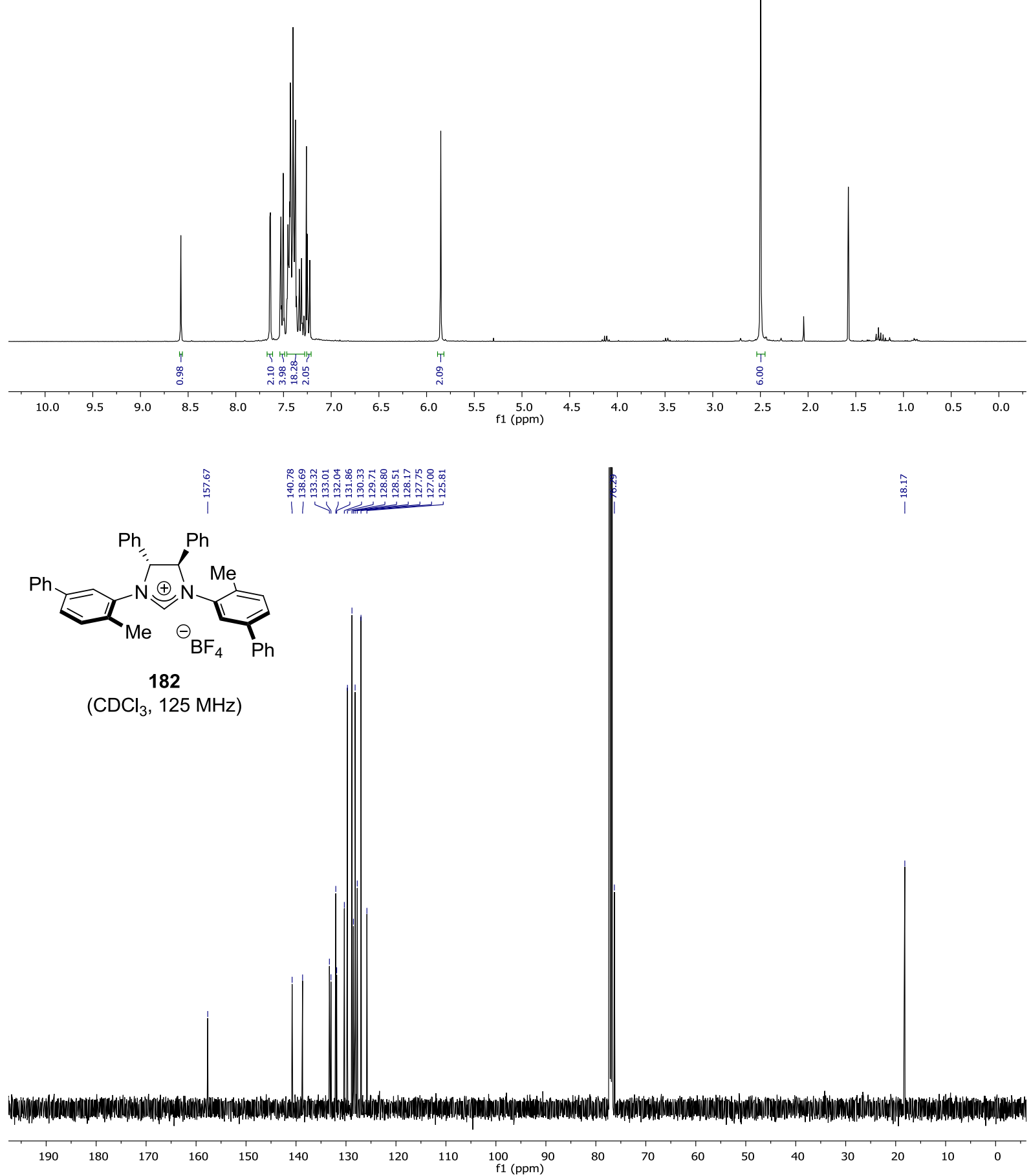


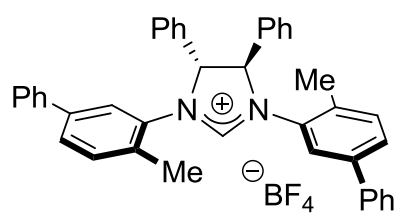

182

$\left(\mathrm{CDCl}_{3}, 282 \mathrm{MHz}\right)$

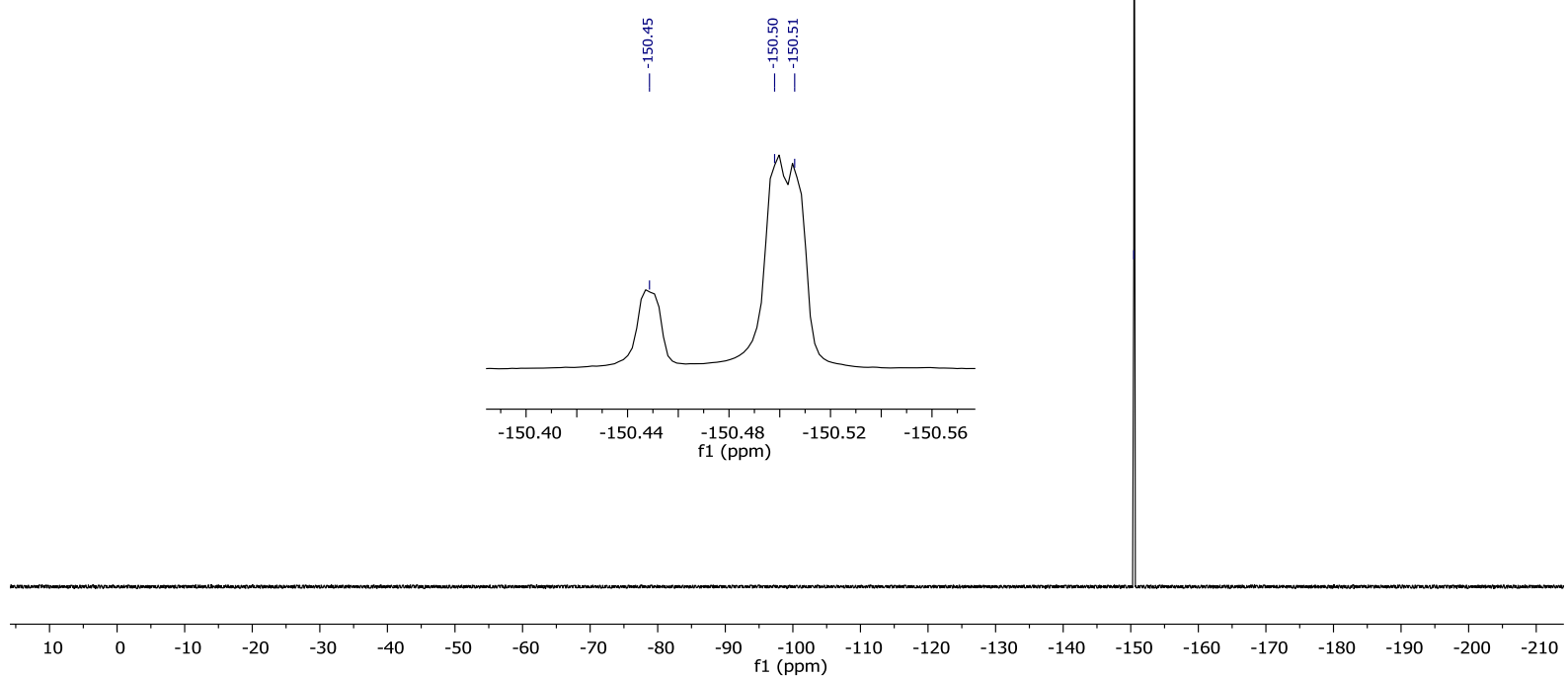




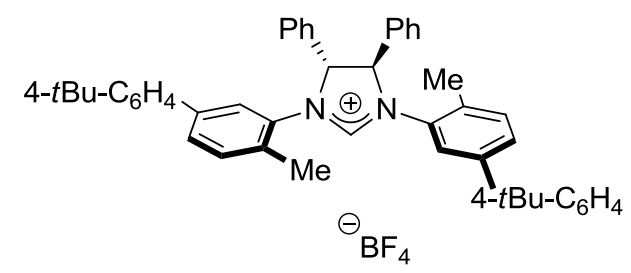

183

$\left(\mathrm{CDCl}_{3}, 400 \mathrm{MHz}\right)$
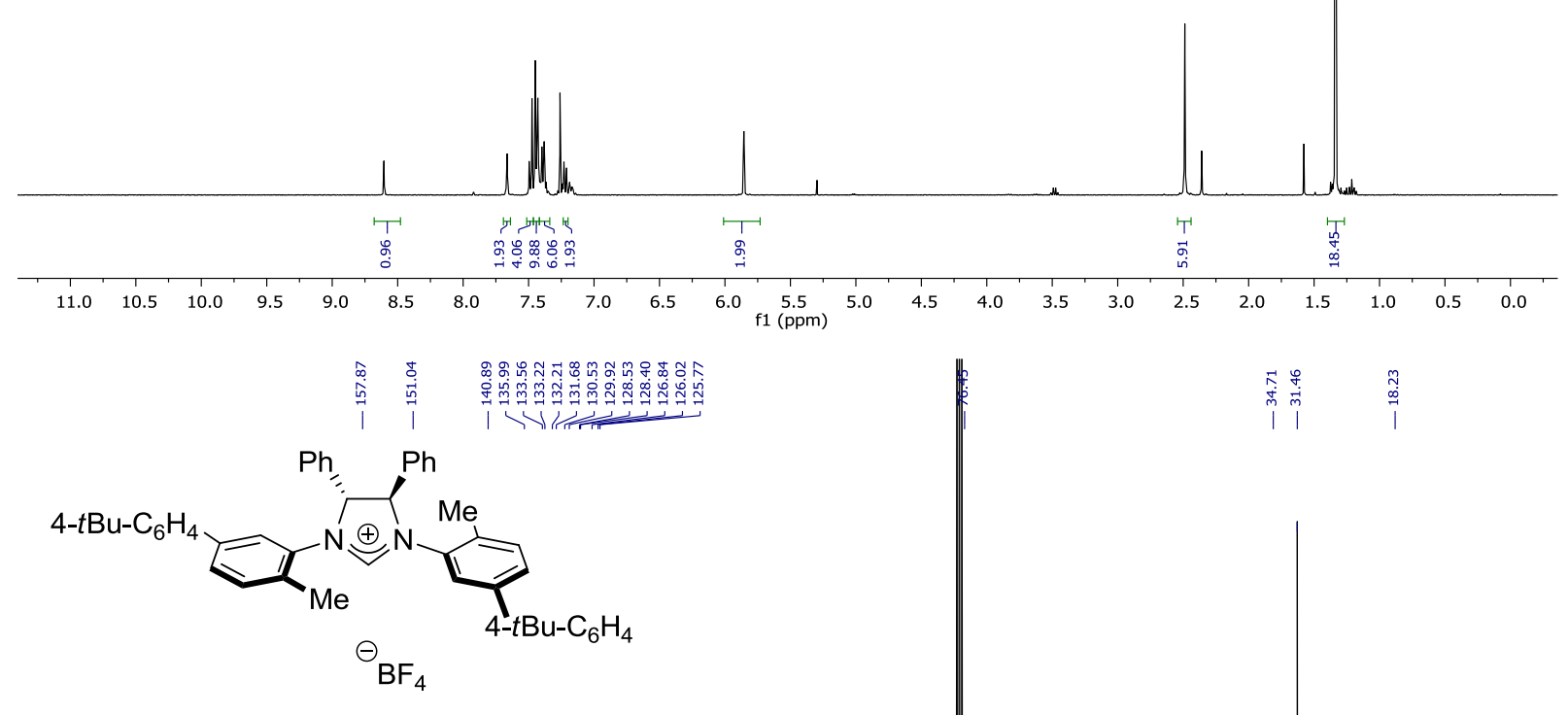

183

$\left(\mathrm{CDCl}_{3}, 100 \mathrm{MHz}\right)$

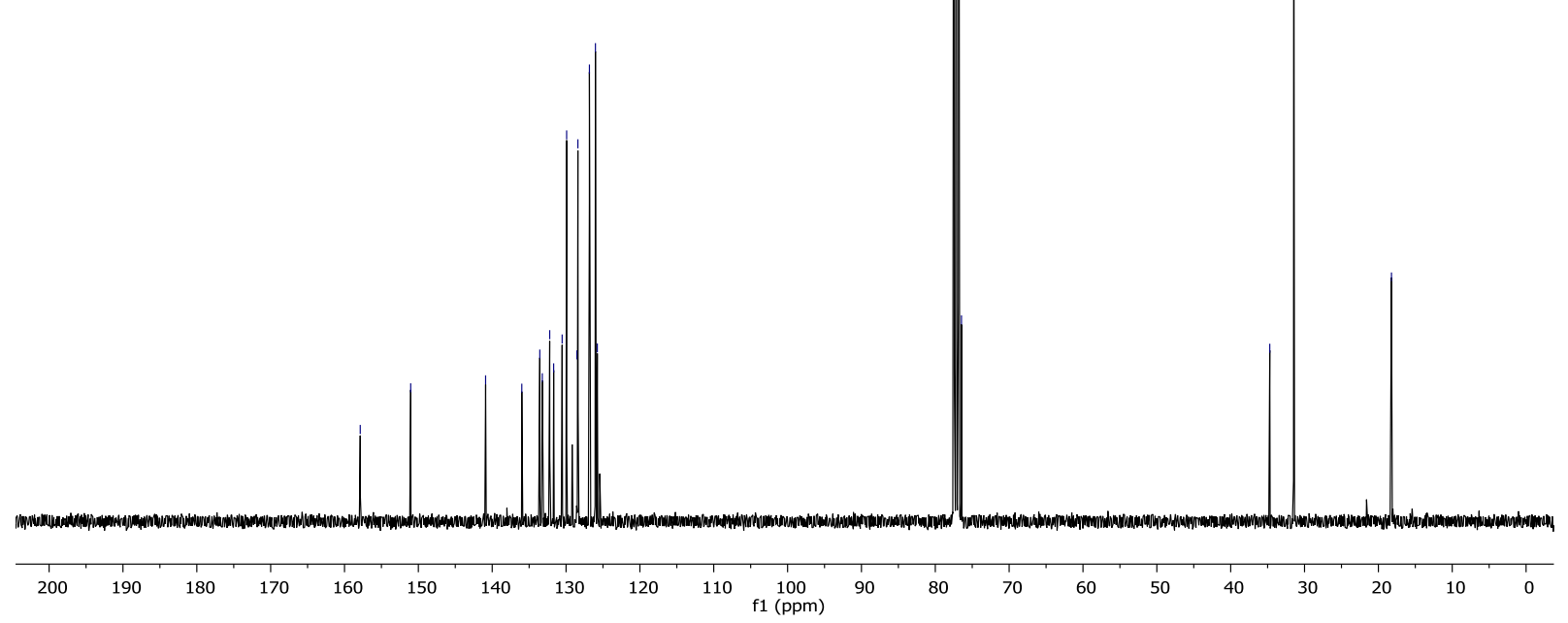




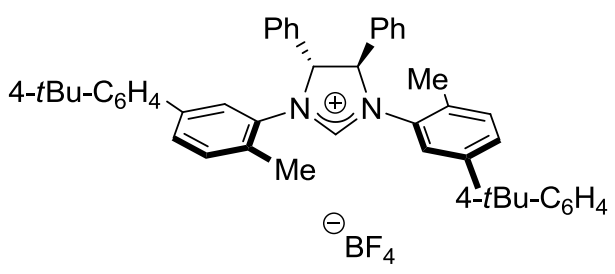

183

$\left(\mathrm{CDCl}_{3}, 373 \mathrm{MHz}\right)$

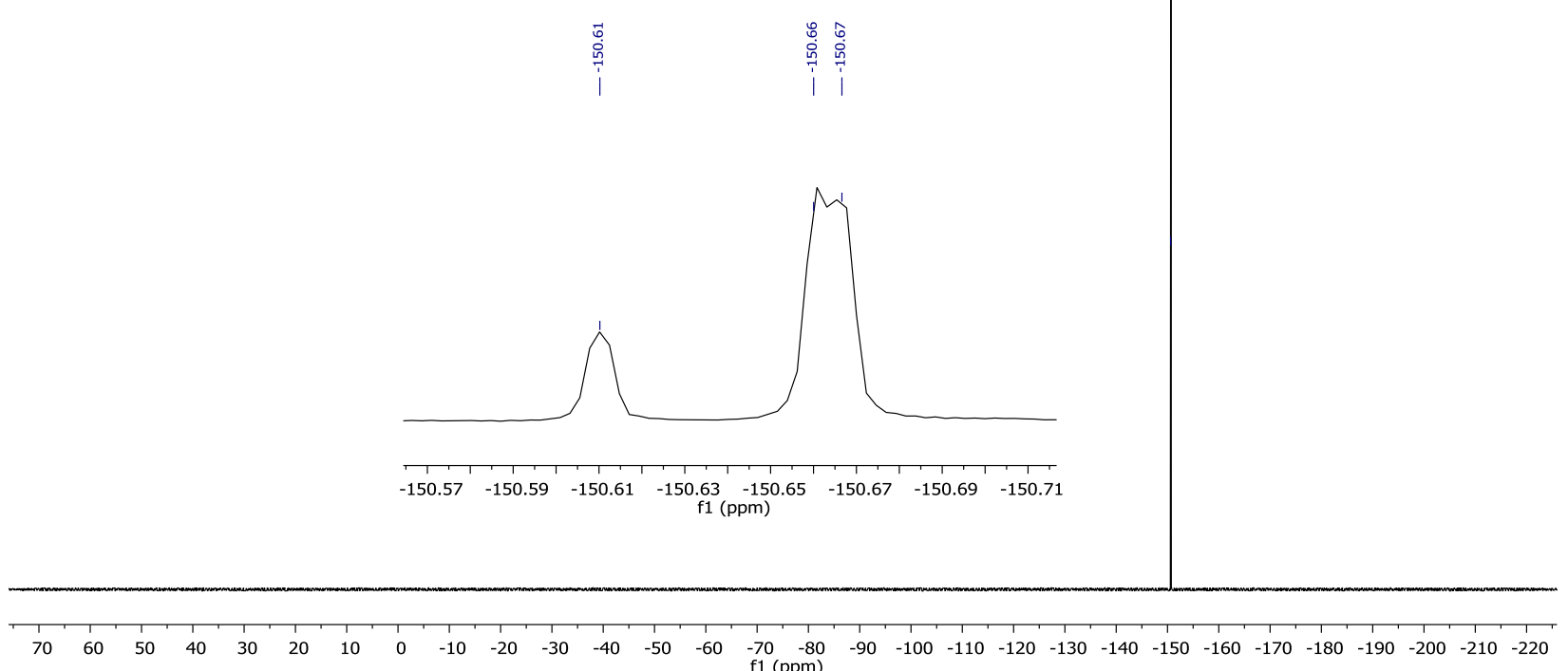




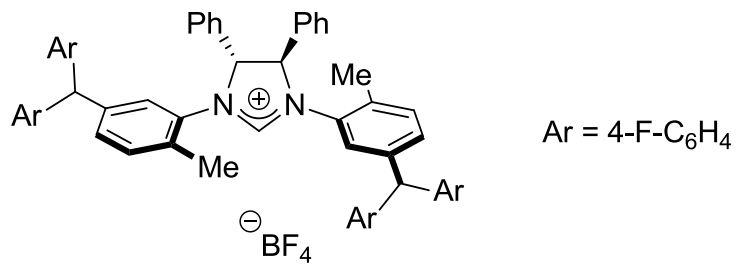

184

$\left(\mathrm{CDCl}_{3}, 500 \mathrm{MHz}\right)$
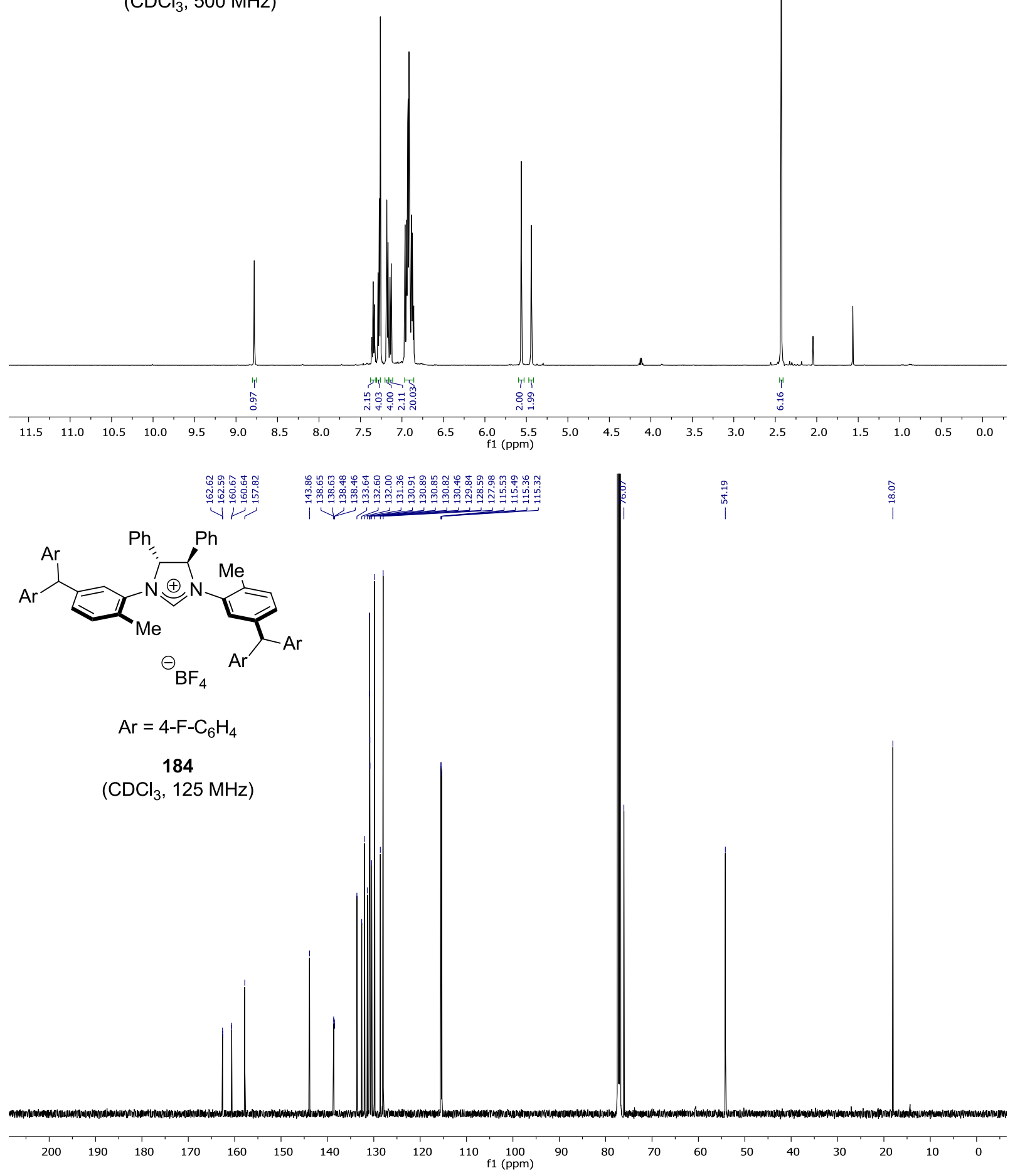


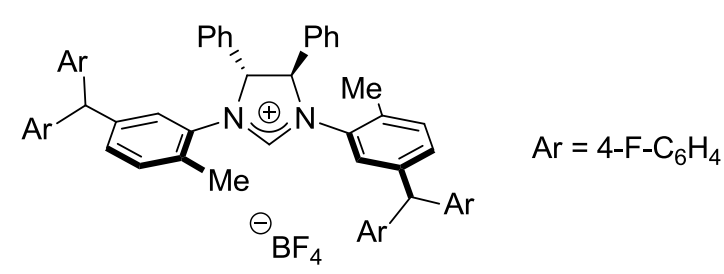

184

$\left(\mathrm{CDCl}_{3}, 282 \mathrm{MHz}\right)$

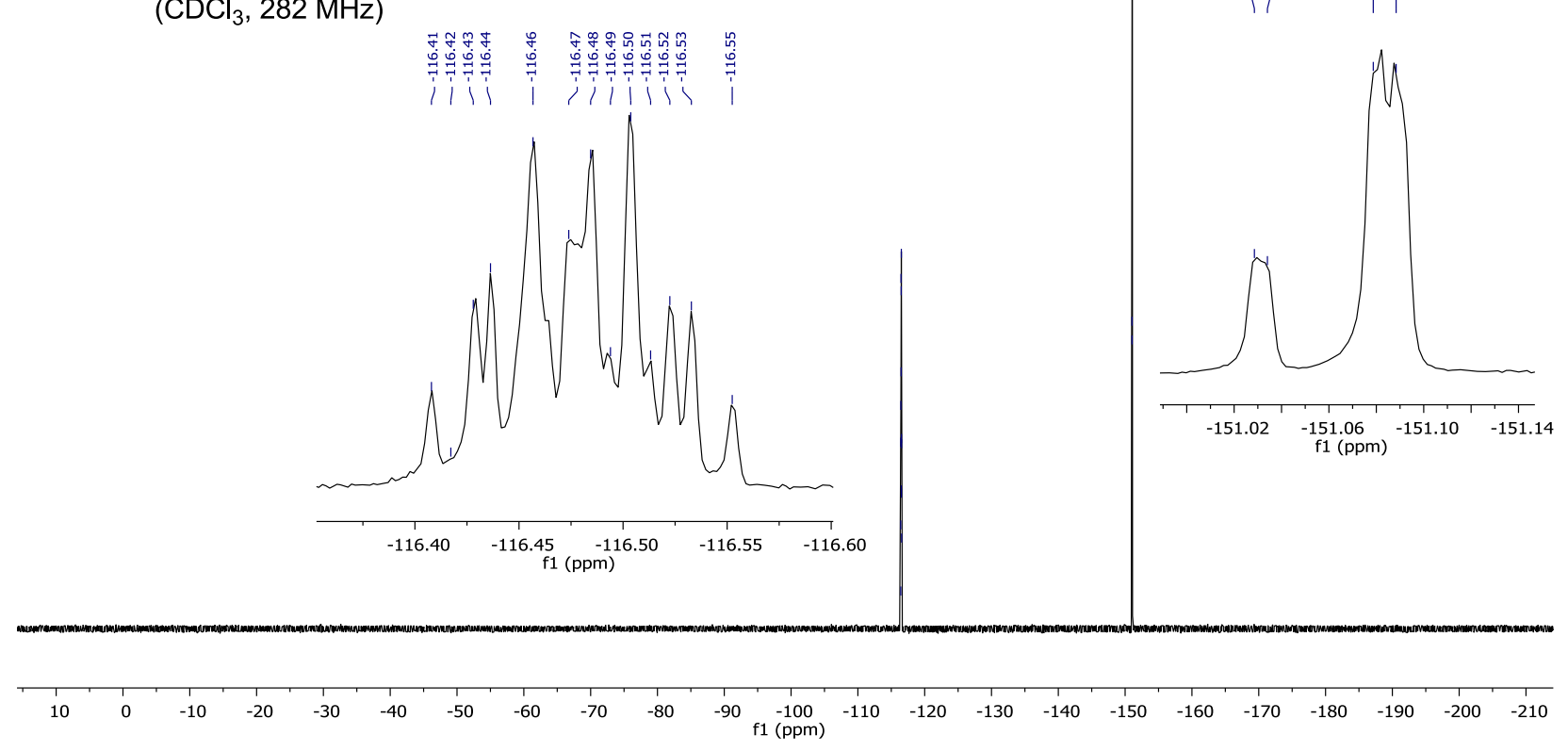




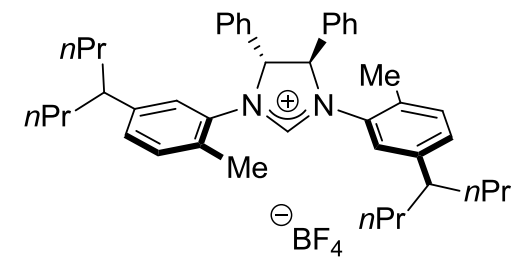

185

$\left(\mathrm{CDCl}_{3}, 500 \mathrm{MHz}\right)$
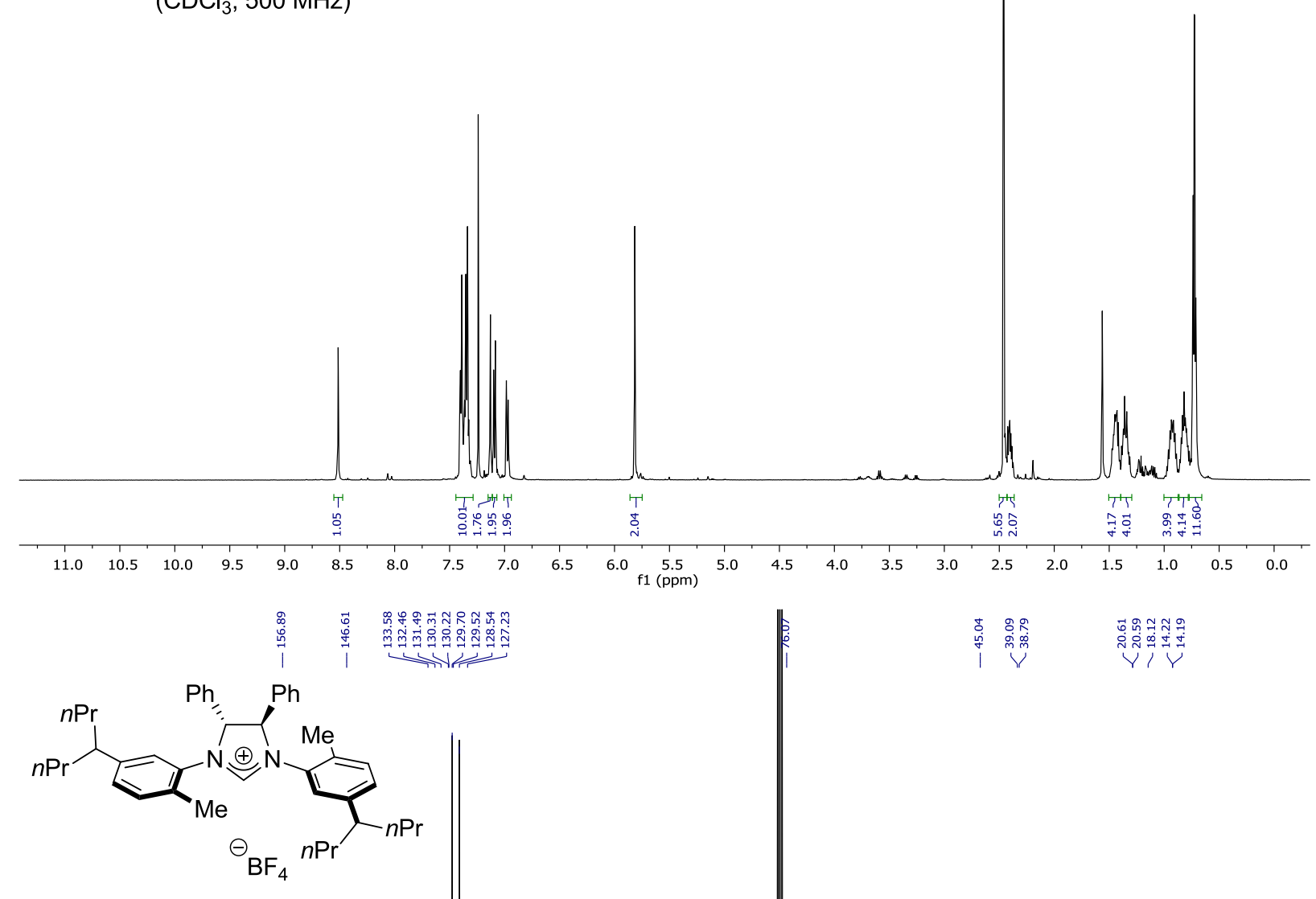

185

$\left(\mathrm{CDCl}_{3}, 100 \mathrm{MHz}\right)$

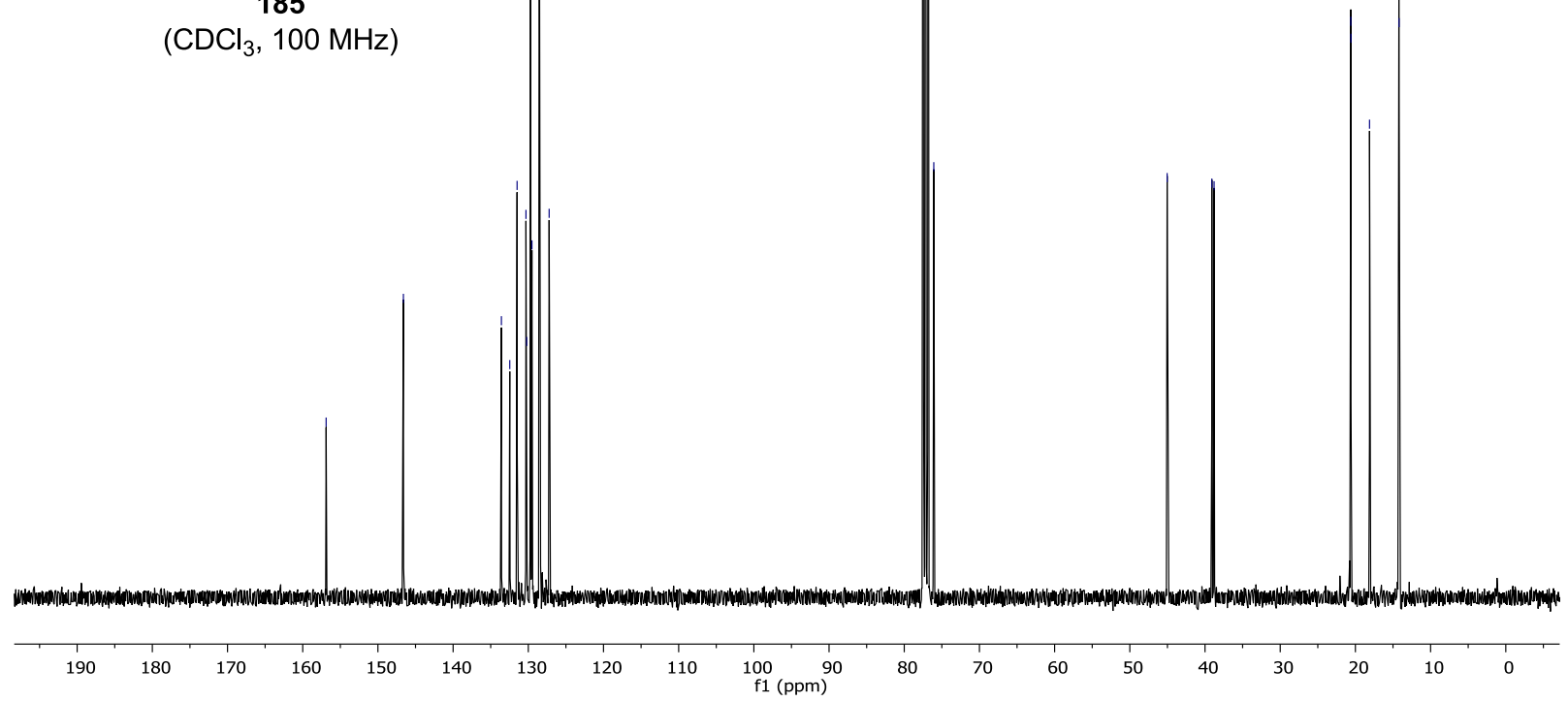




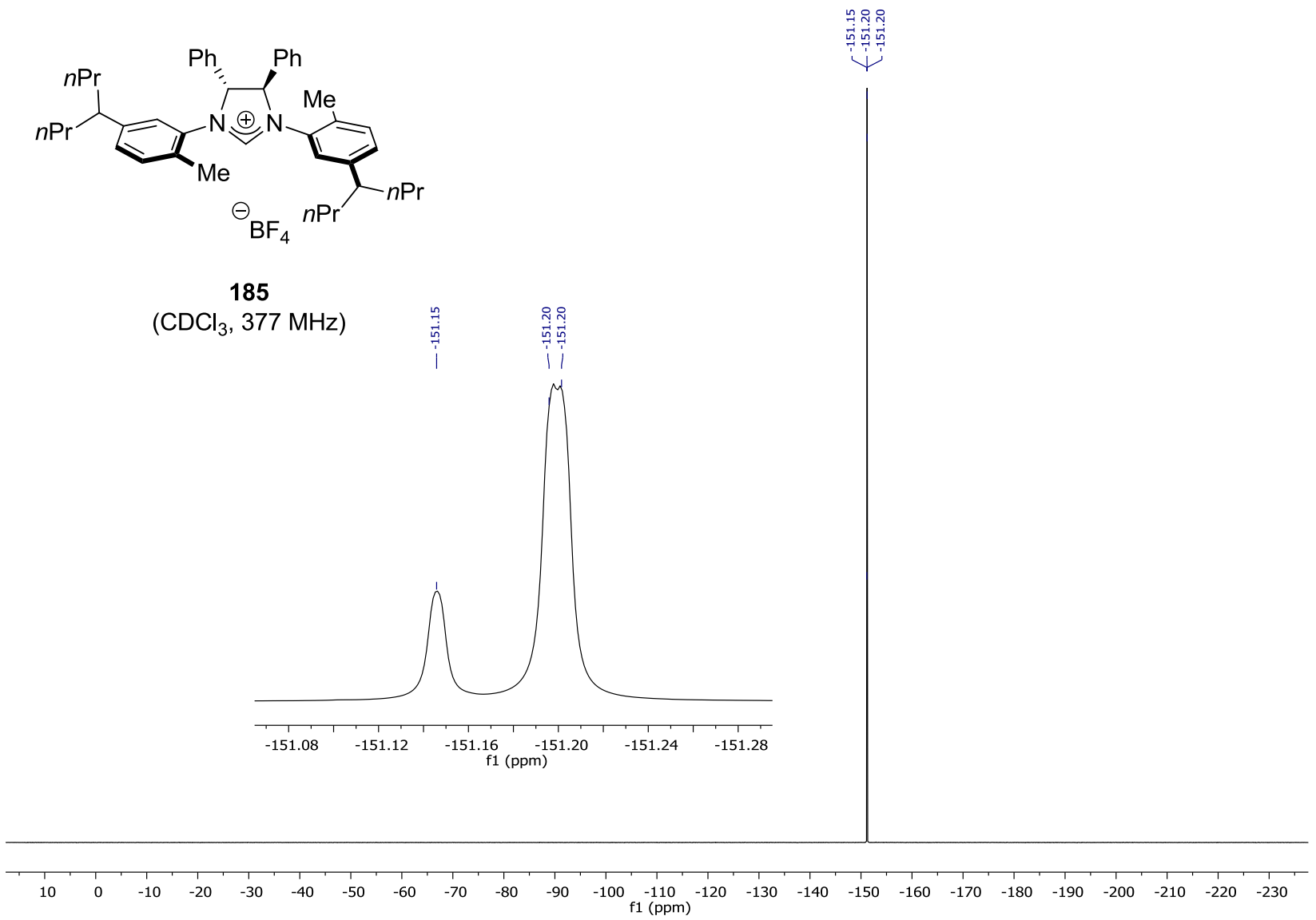




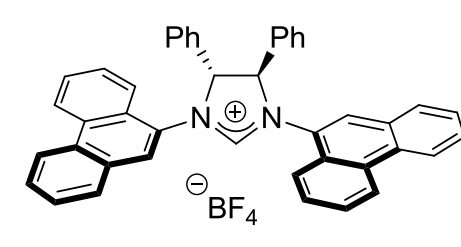

186

$\left(\mathrm{CDCl}_{3}, 300 \mathrm{MHz}\right)$
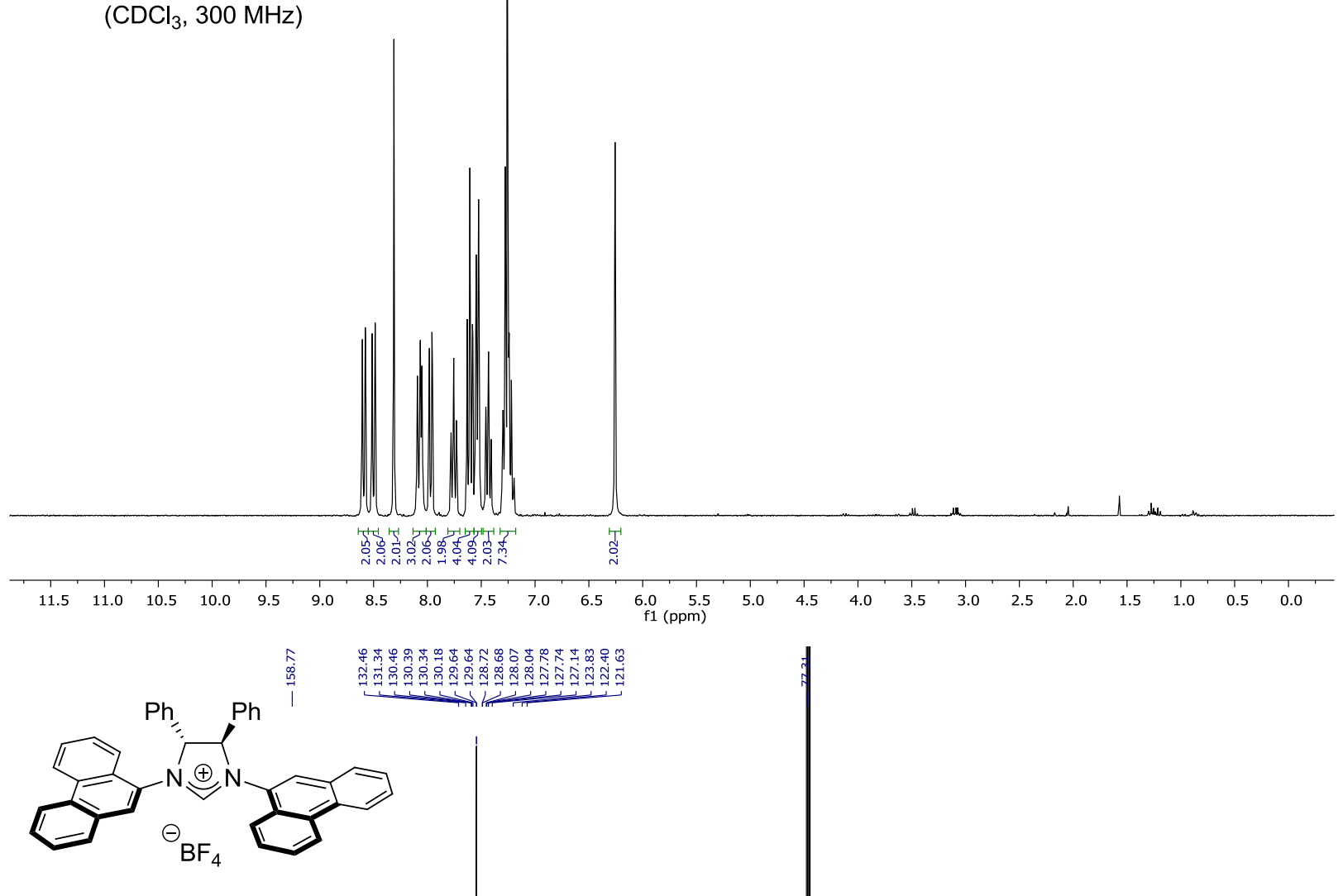

186

$\left(\mathrm{CDCl}_{3}, 125 \mathrm{MHz}\right)$

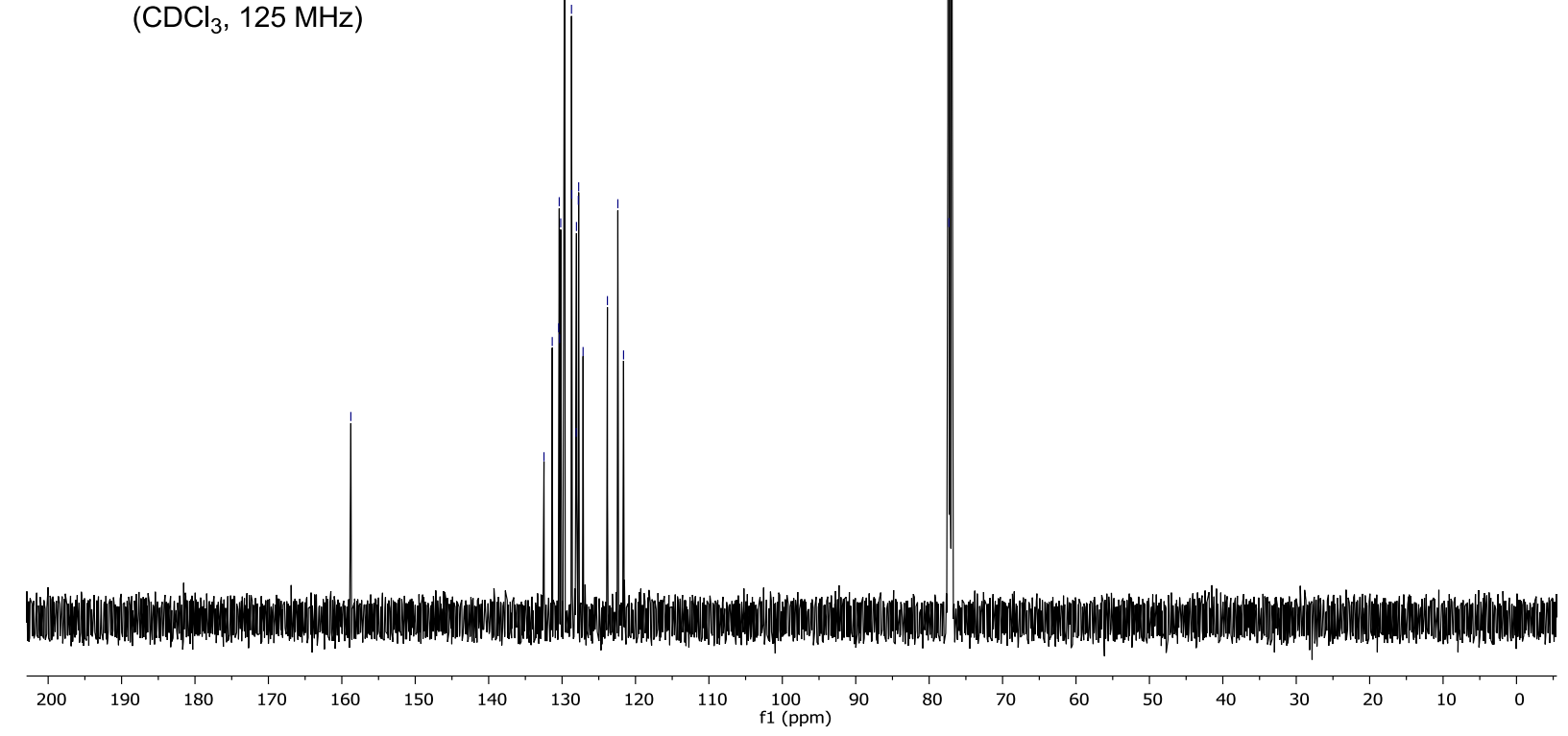




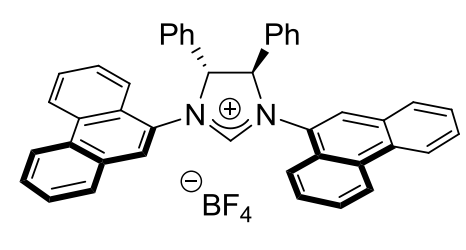

186

$\left(\mathrm{CDCl}_{3}, 282 \mathrm{MHz}\right)$

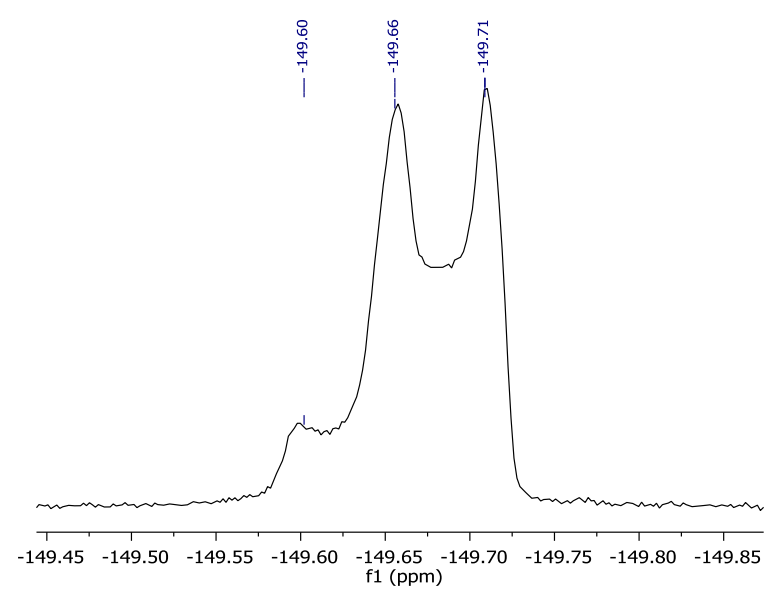

M.1. 


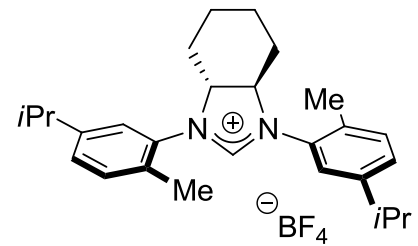

187

$\left(\mathrm{CDCl}_{3}, 500 \mathrm{MHz}\right)$
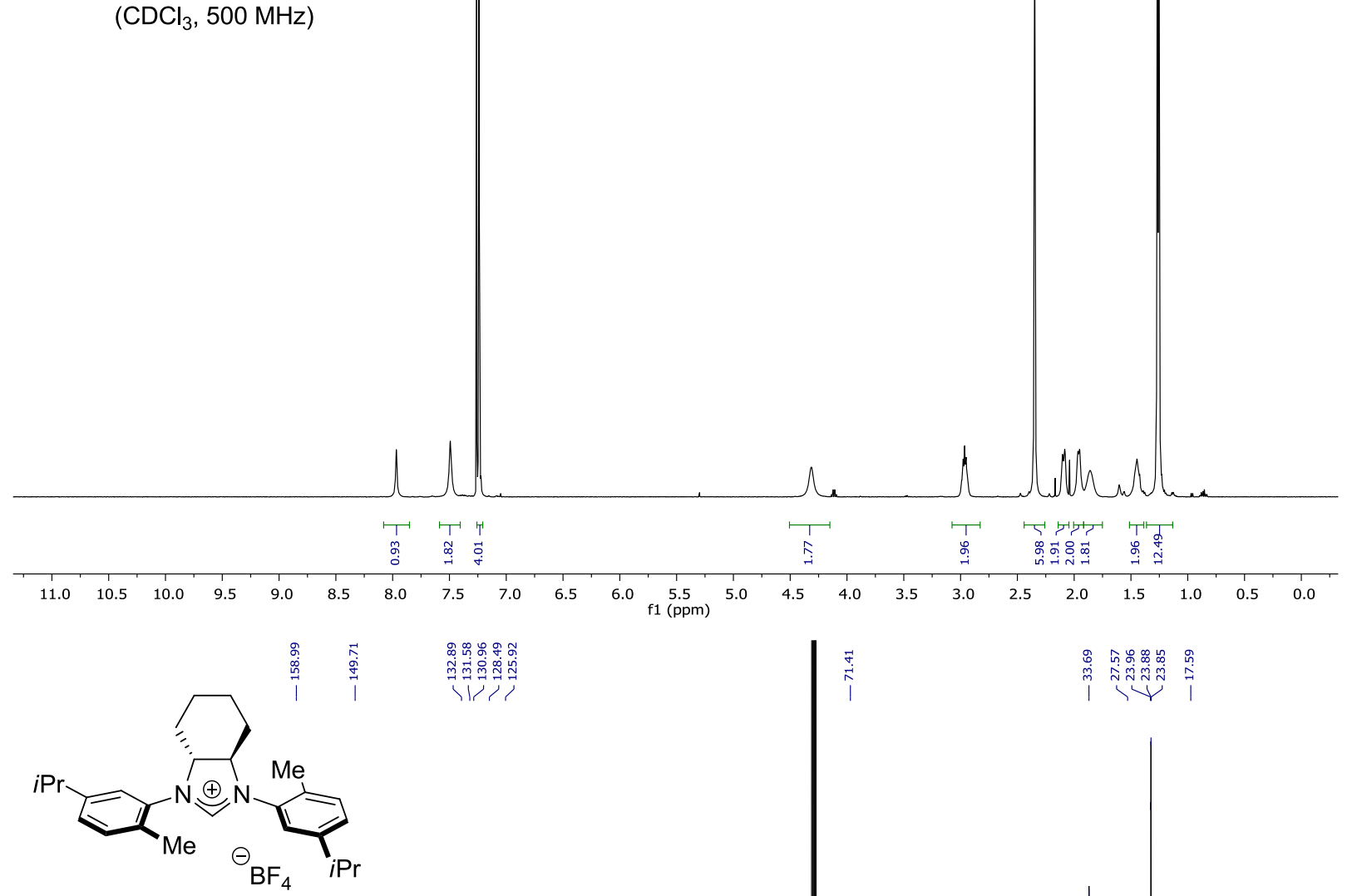

187

$\left(\mathrm{CDCl}_{3}, 125 \mathrm{MHz}\right)$

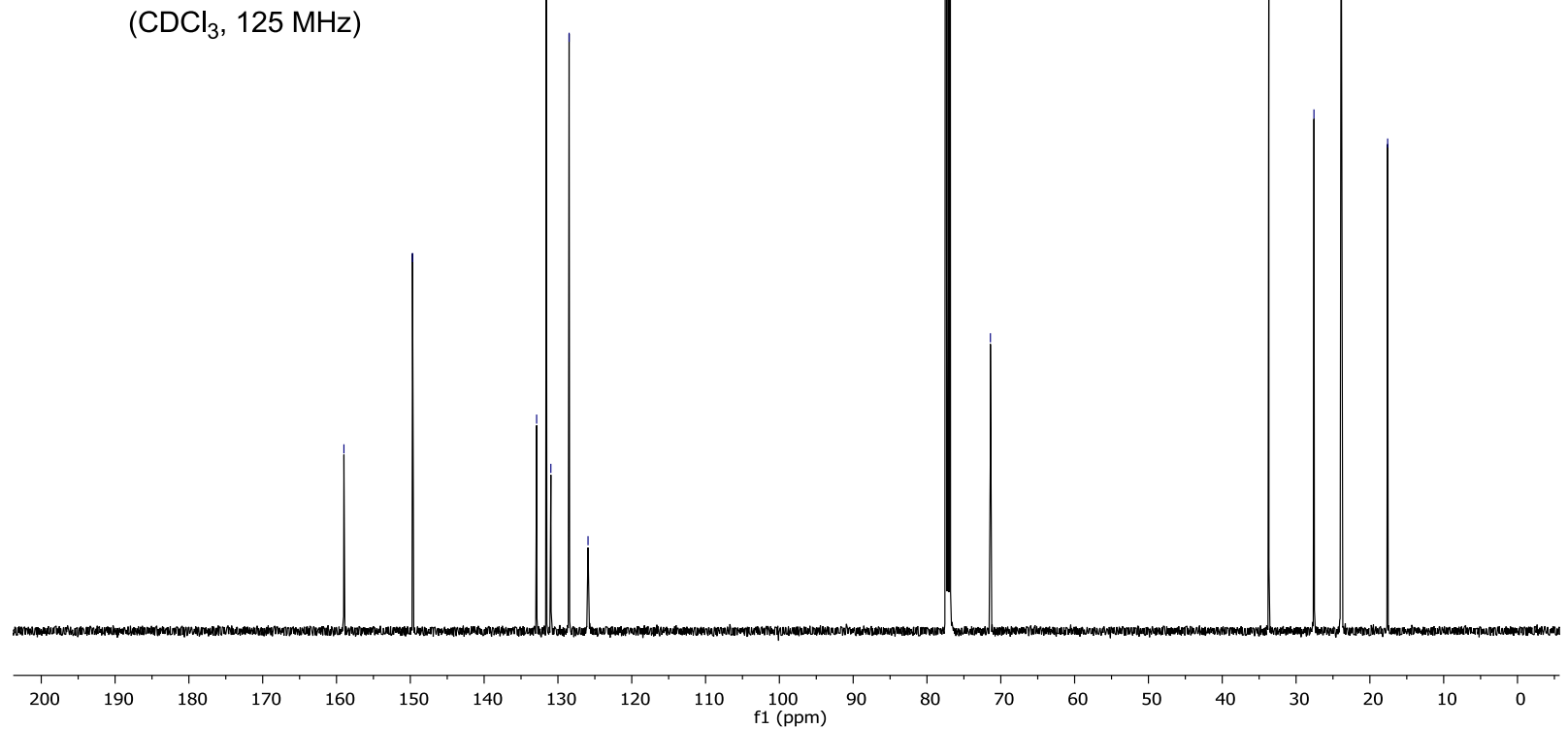




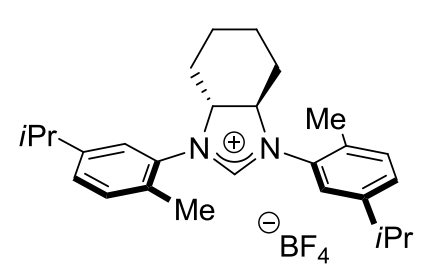

187

$\left(\mathrm{CDCl}_{3}, 471 \mathrm{MHz}\right)$

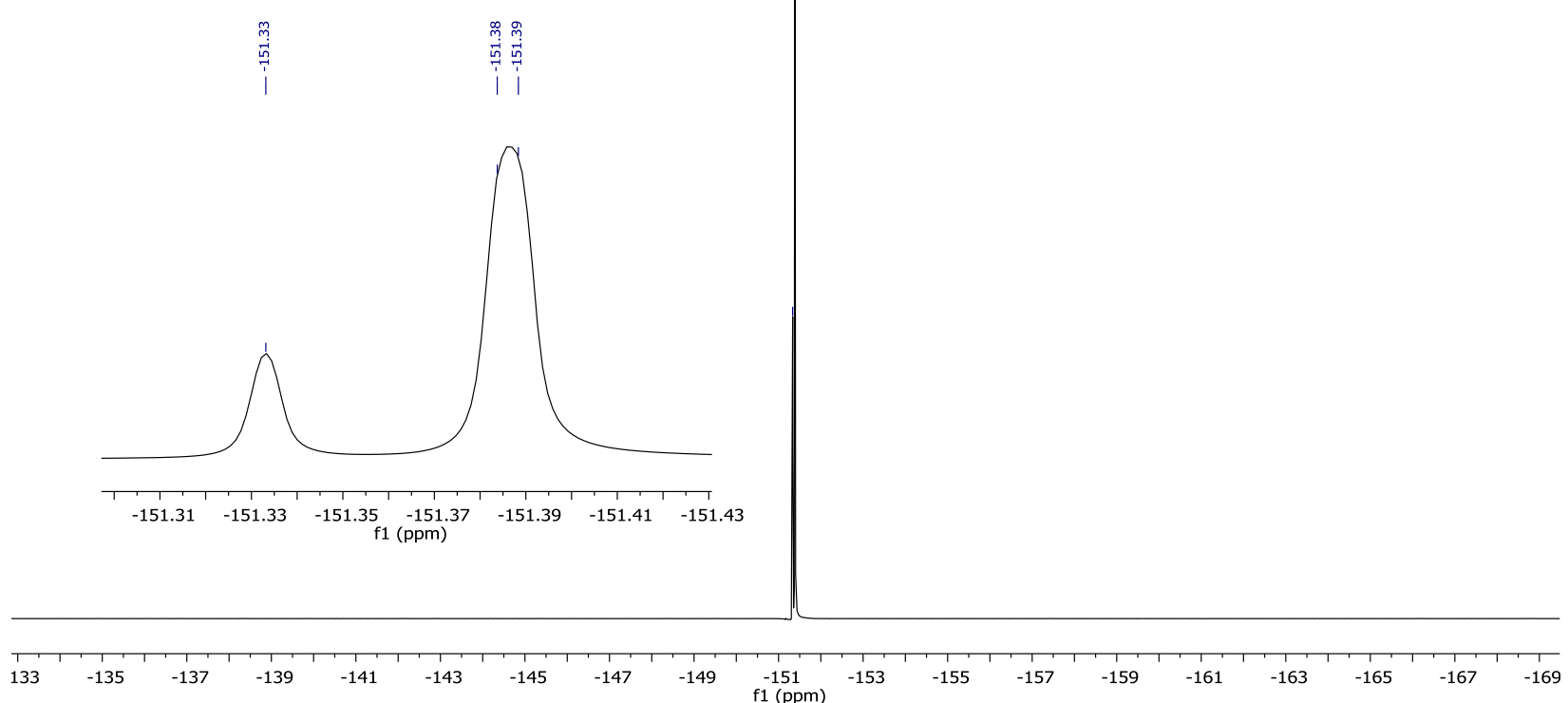



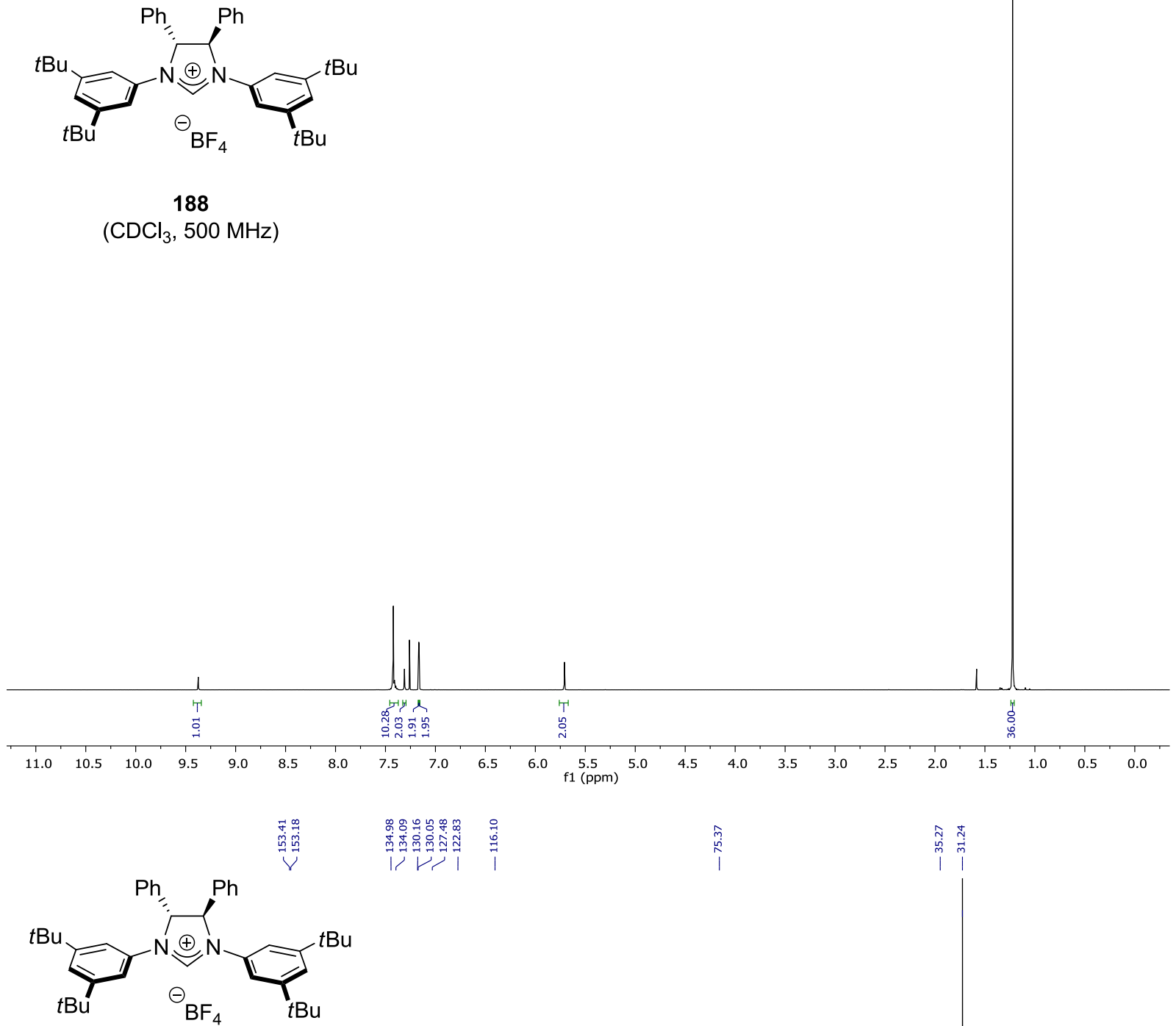

188

$\left(\mathrm{CDCl}_{3}, 125 \mathrm{MHz}\right)$

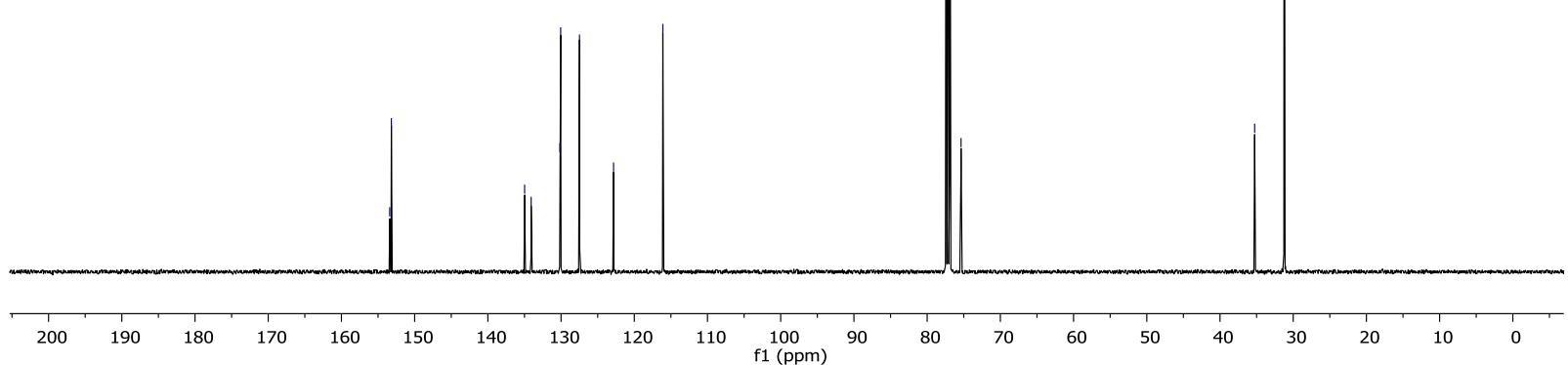




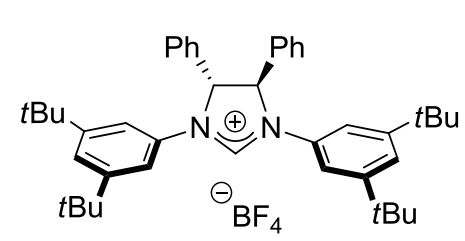

188

$\left(\mathrm{CDCl}_{3}, 471 \mathrm{MHz}\right)$

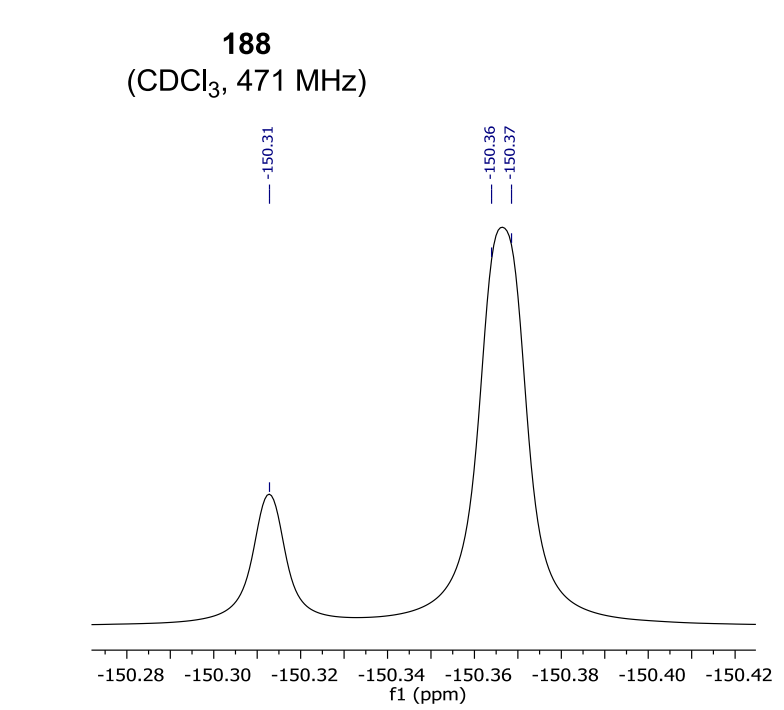

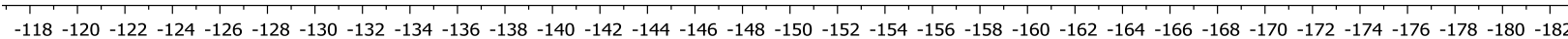
f1 (ppm) 


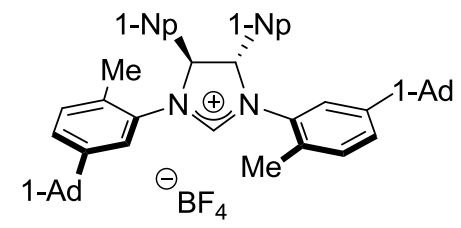

189

$\left(\mathrm{CDCl}_{3}, 600 \mathrm{MHz}\right)$
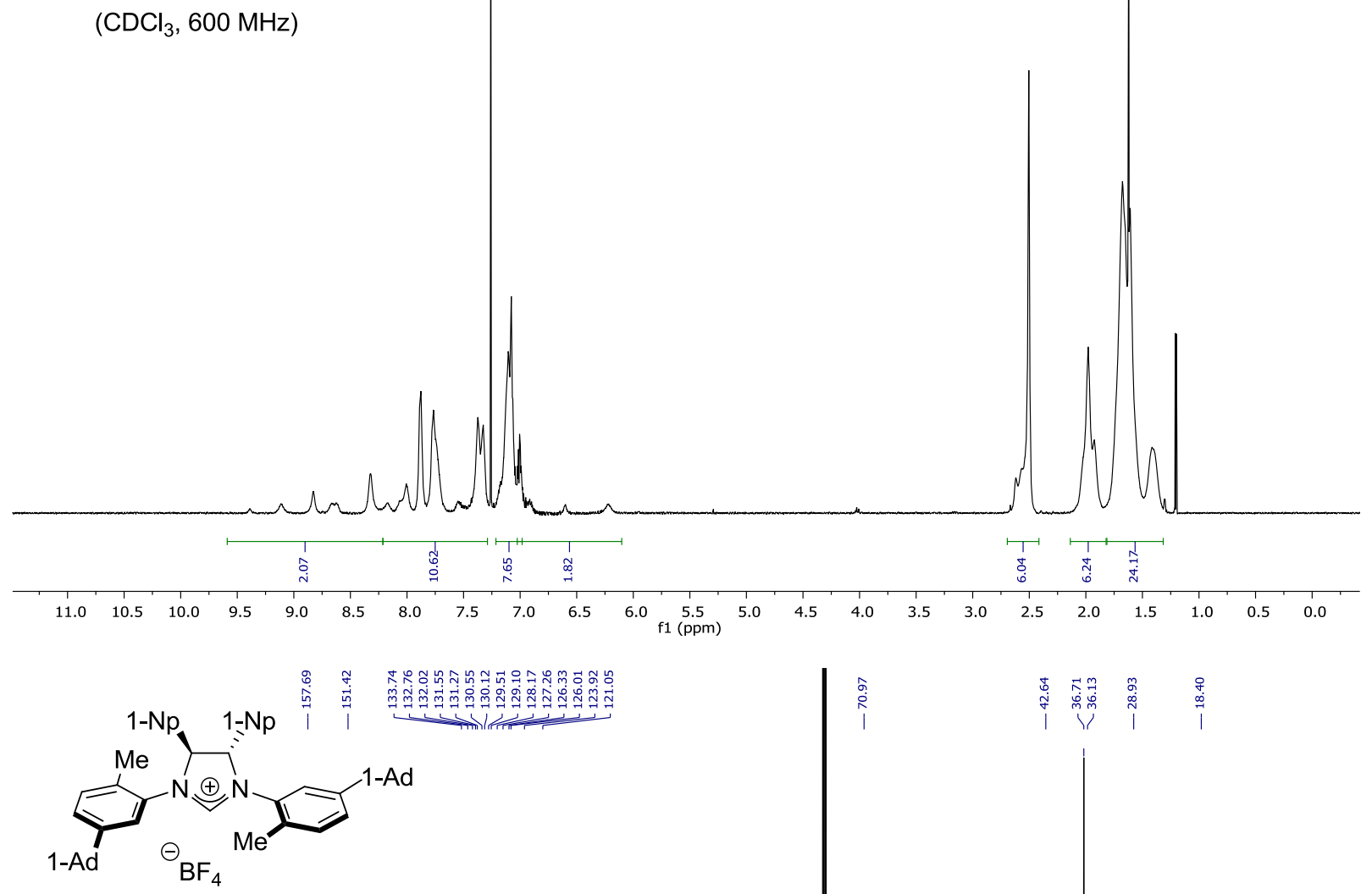

189

$\left(\mathrm{CDCl}_{3}, 125 \mathrm{MHz}\right)$

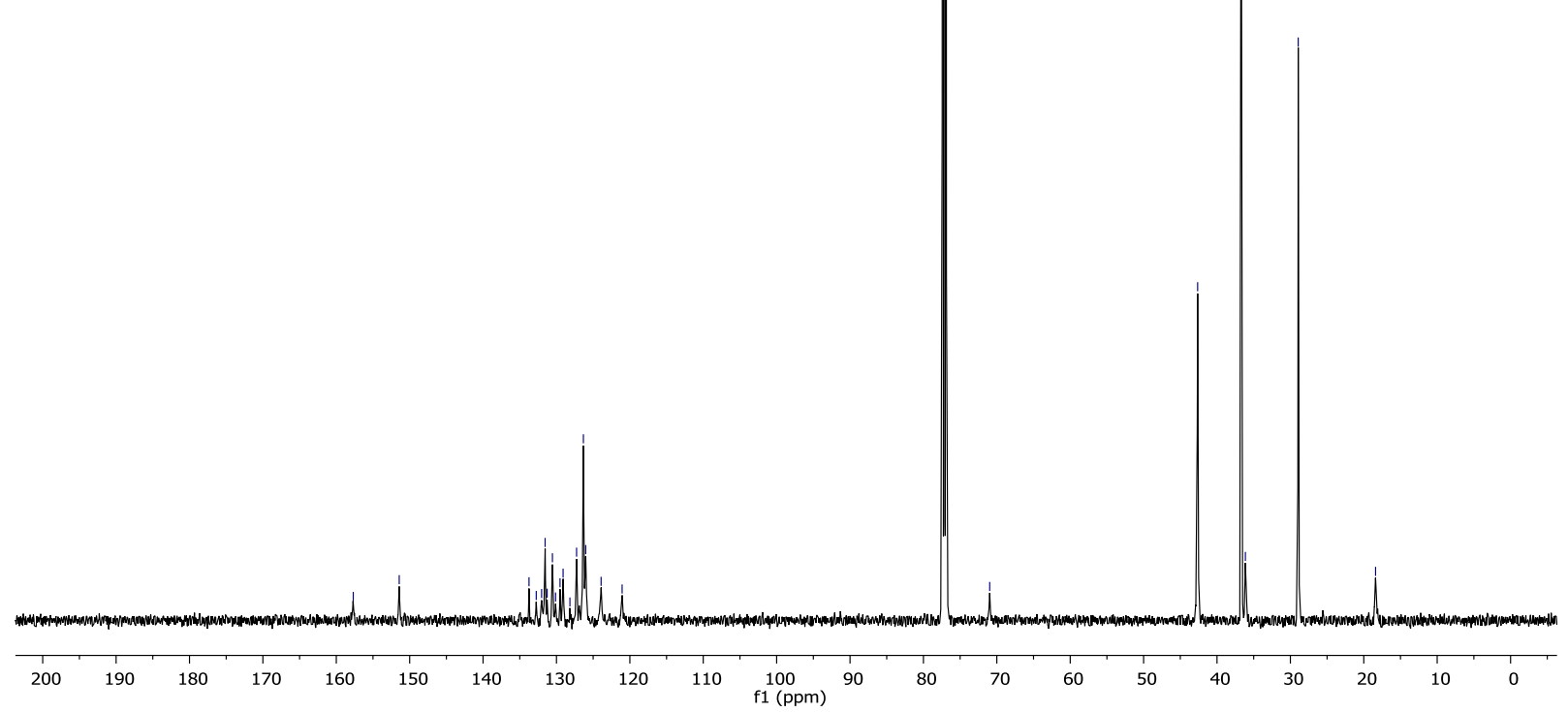




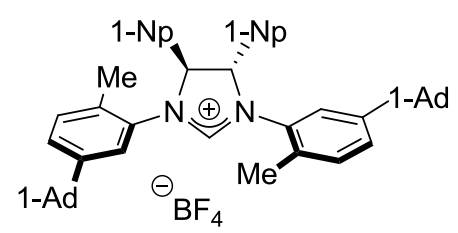

189

$\left(\mathrm{CDCl}_{3}, 376 \mathrm{MHz}\right)$
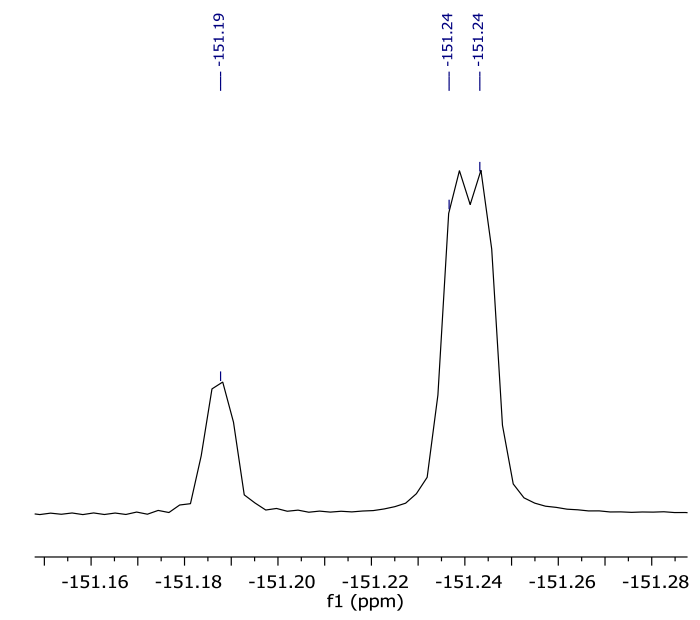

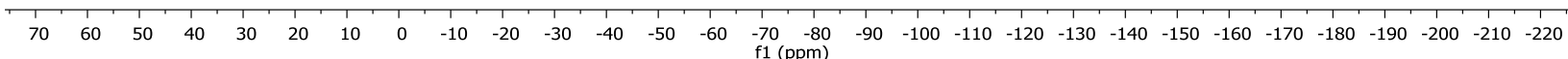




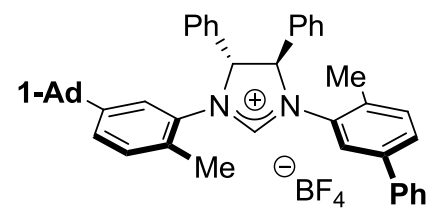

190

$\left(\mathrm{CDCl}_{3}, 400 \mathrm{MHz}\right)$
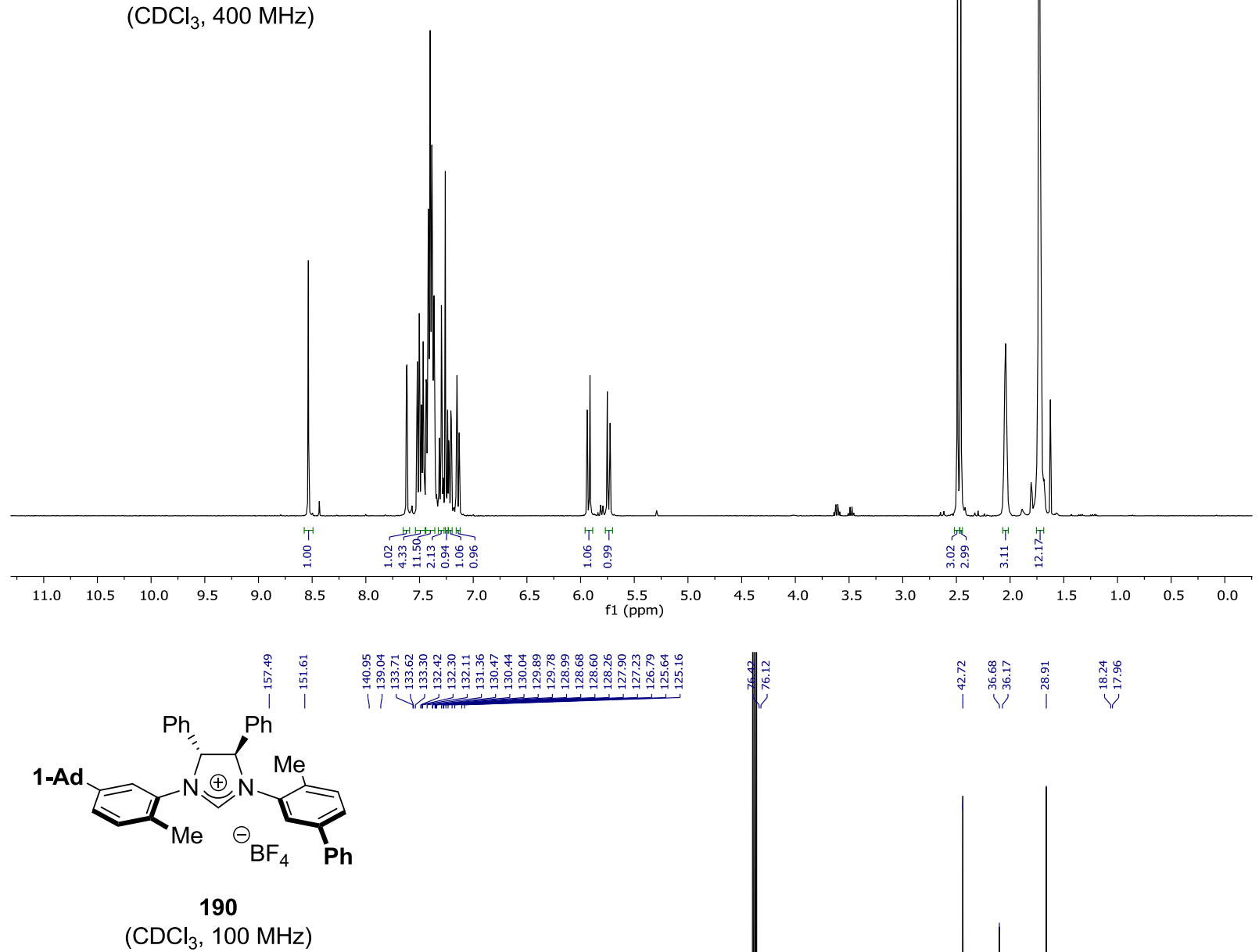

V

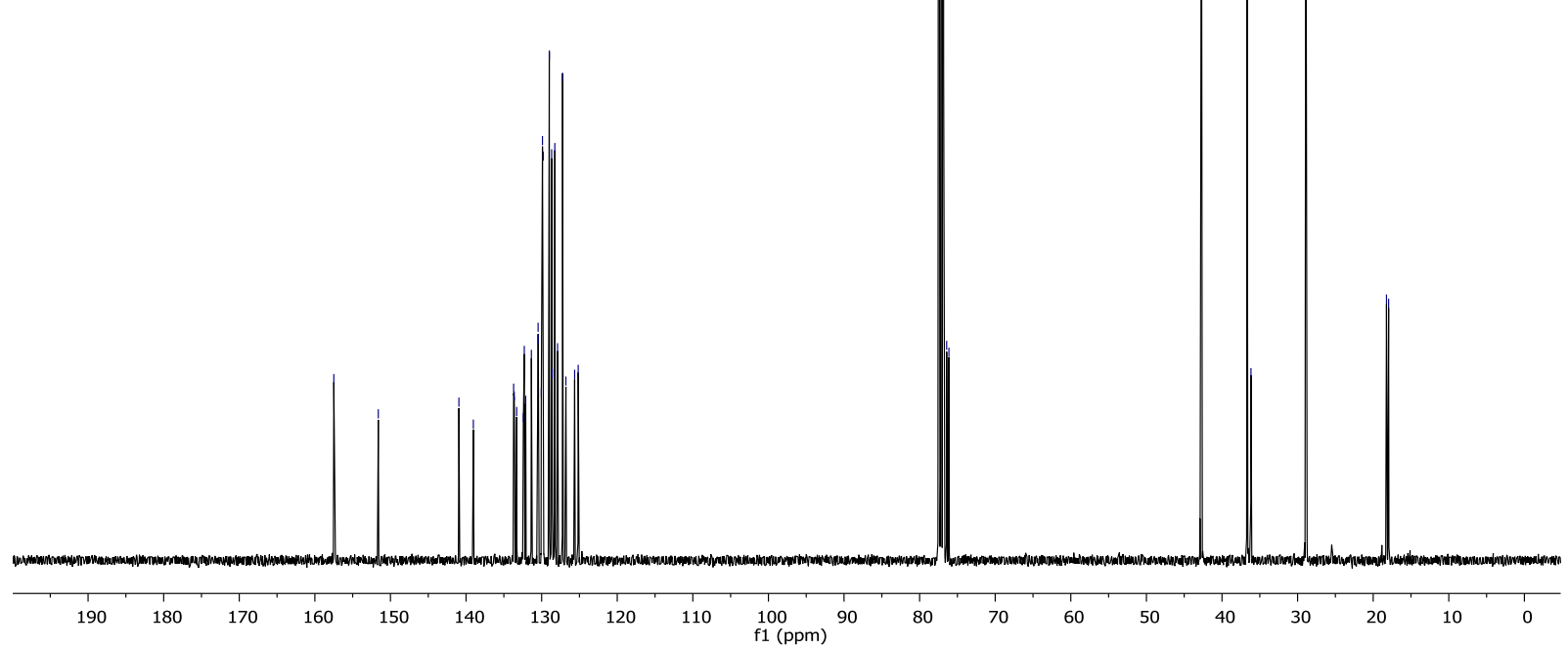




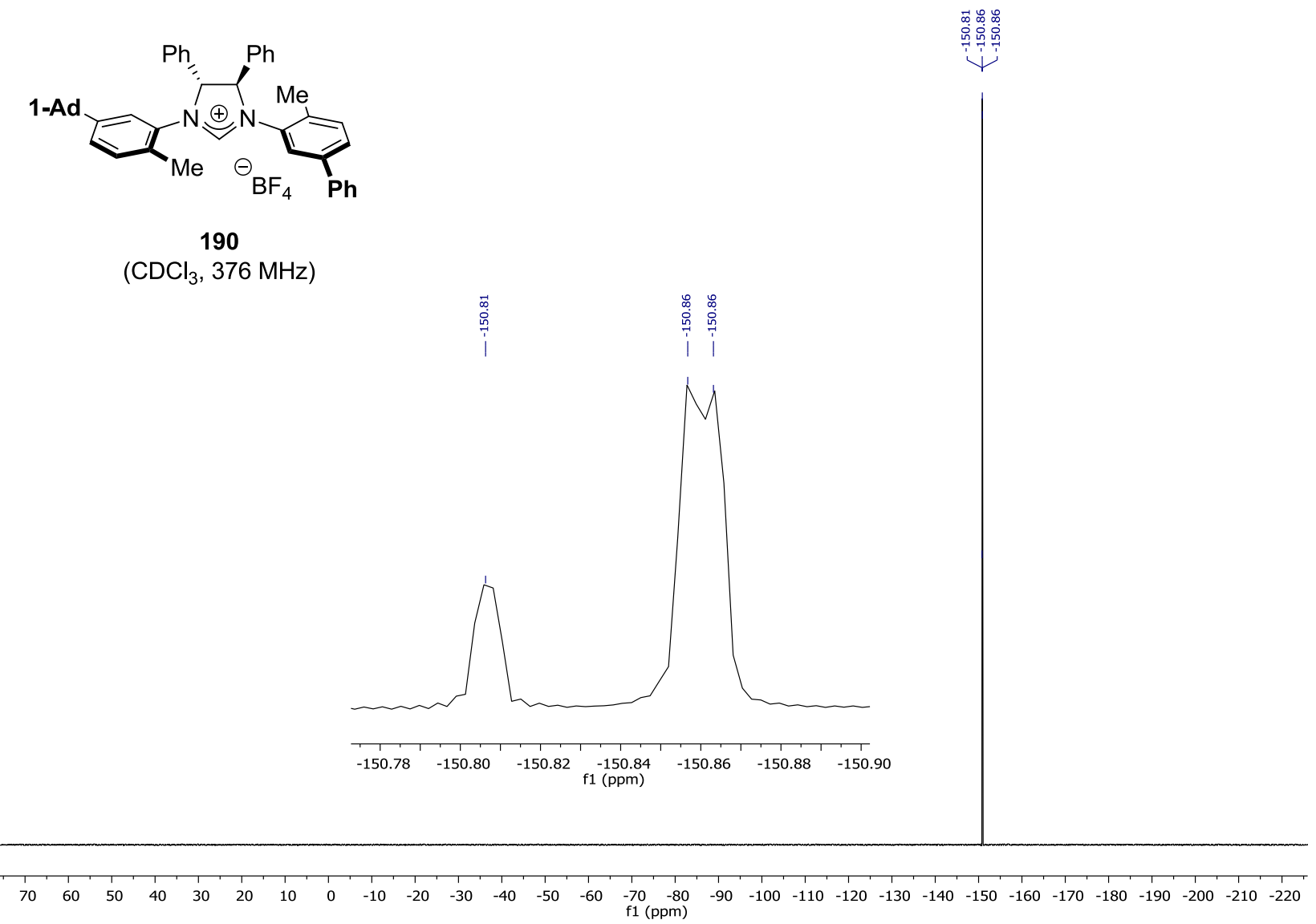




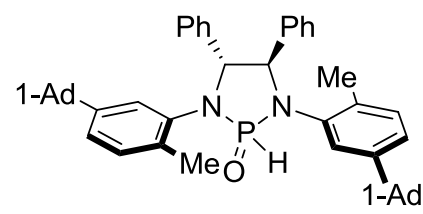

213

$\left(\mathrm{CDCl}_{3}, 300 \mathrm{MHz}\right)$
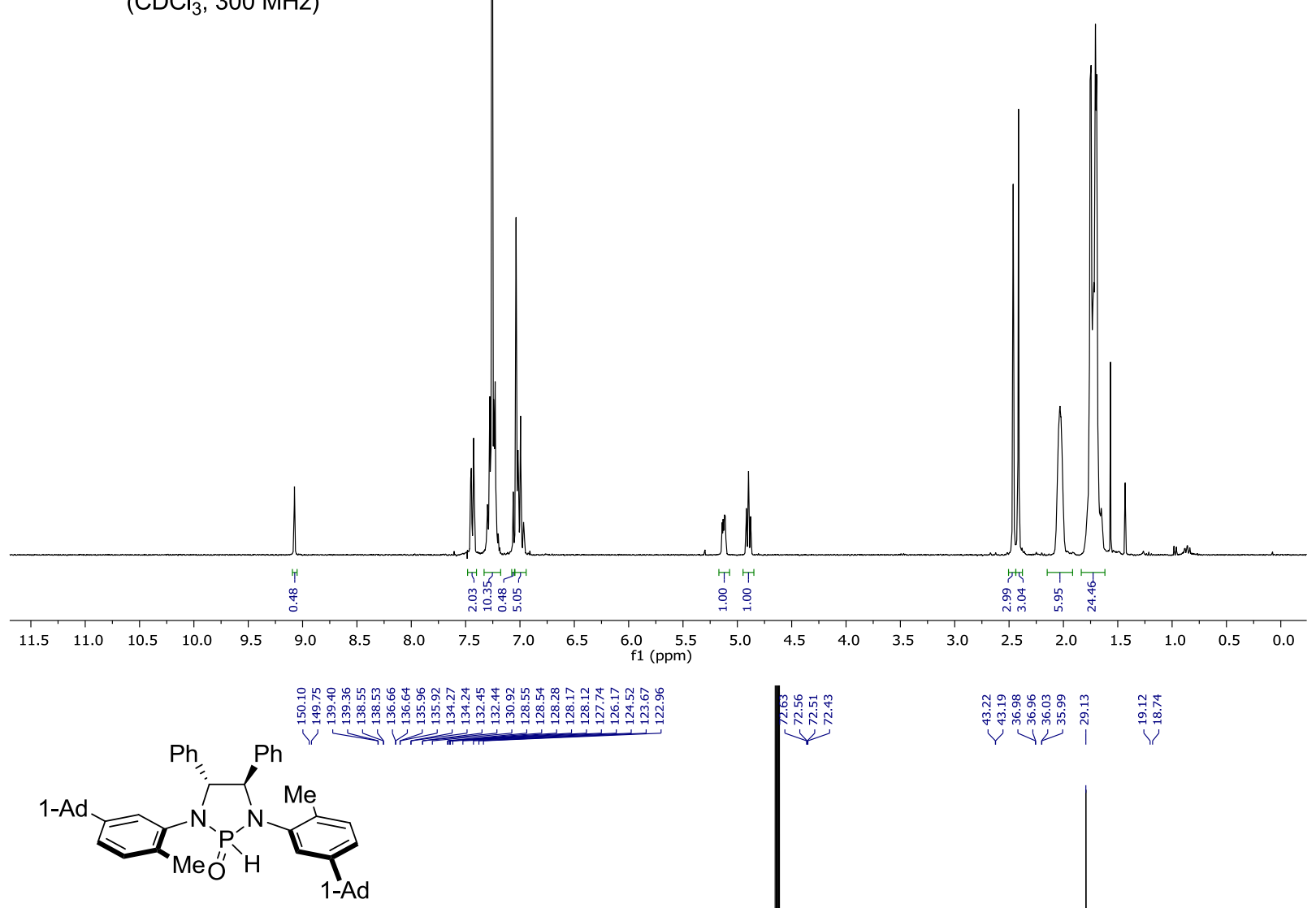

213

$\left(\mathrm{CDCl}_{3}, 125 \mathrm{MHz}\right)$

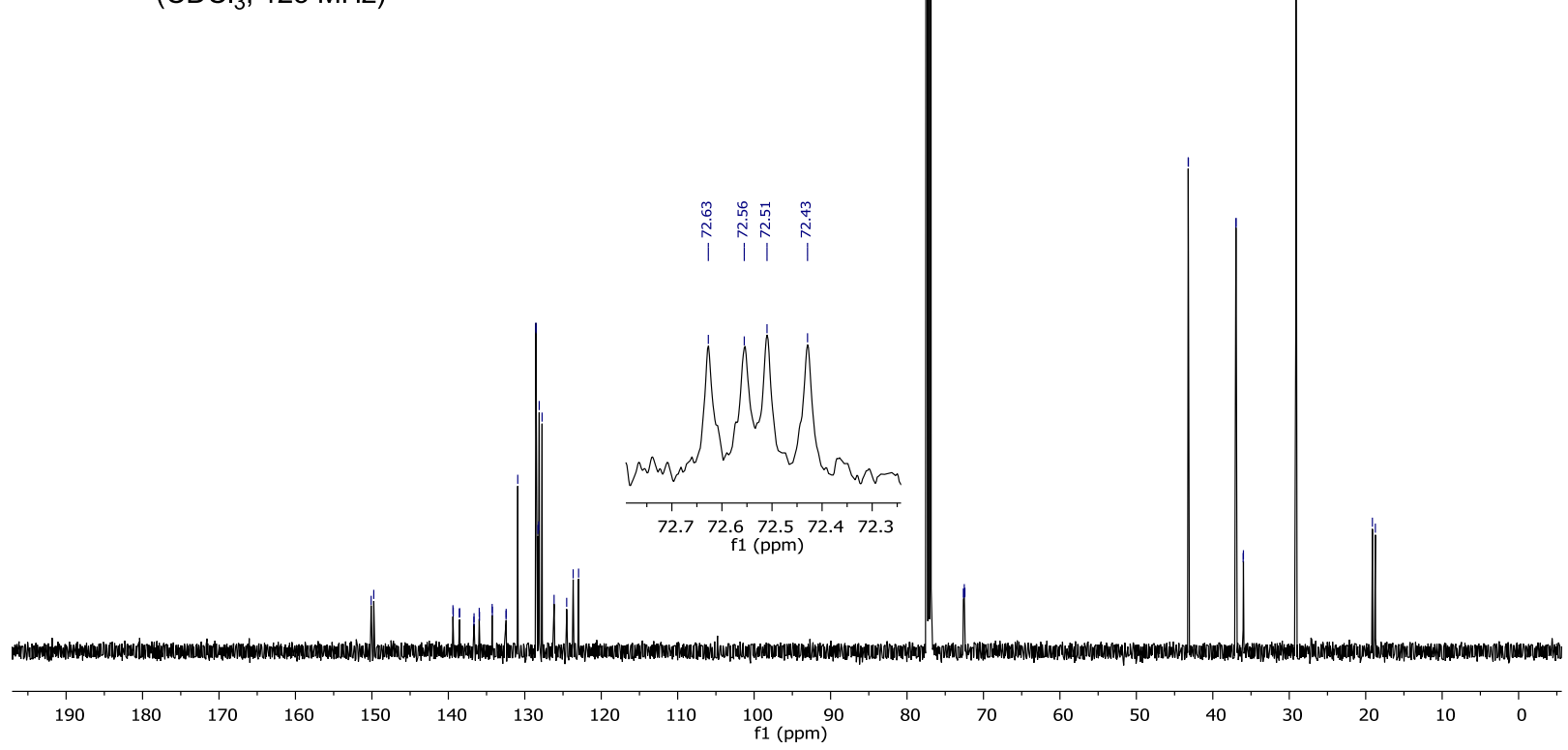




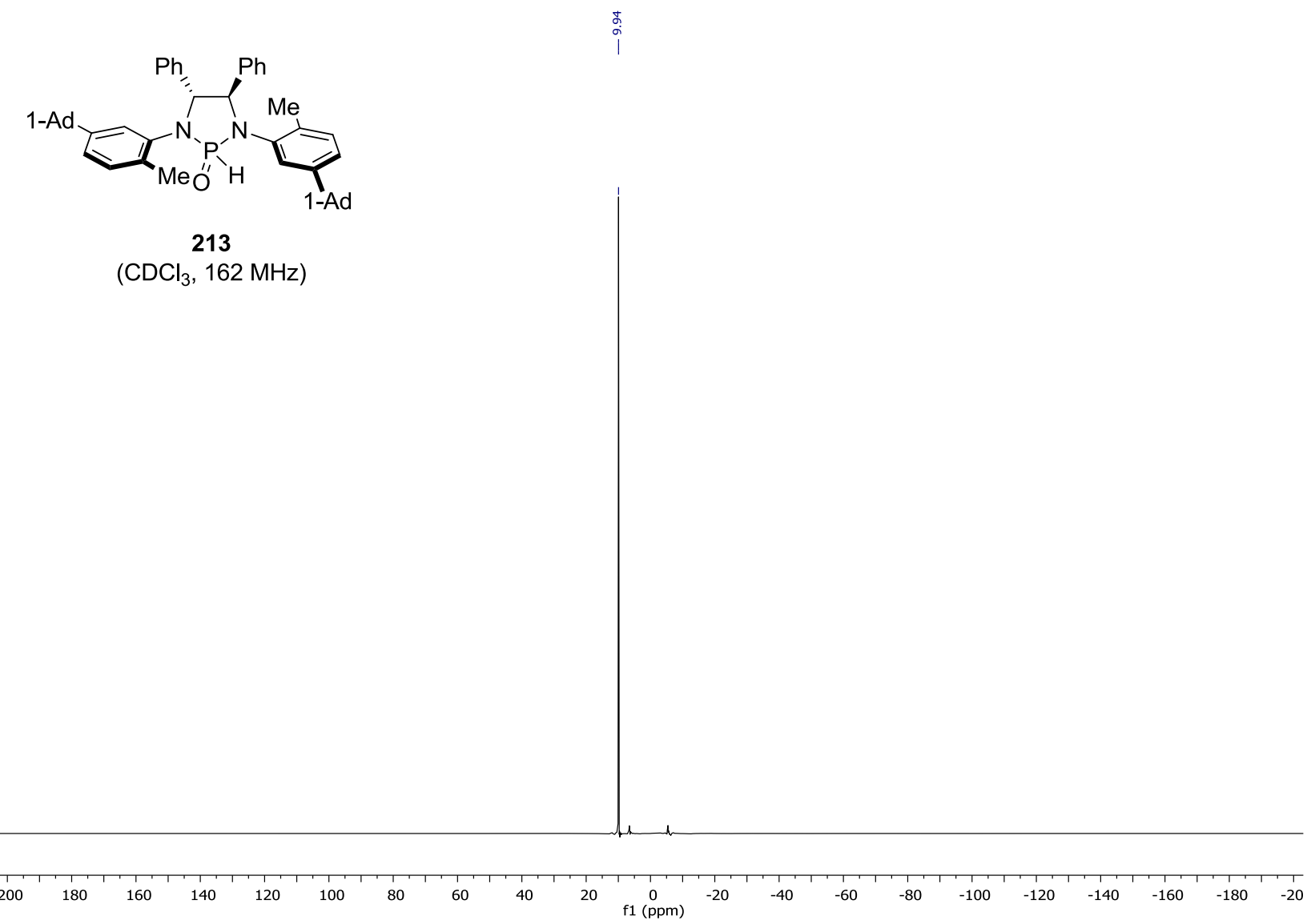




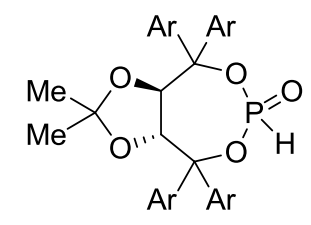

218

$\left(\mathrm{CDCl}_{3}, 400 \mathrm{MHz}\right)$
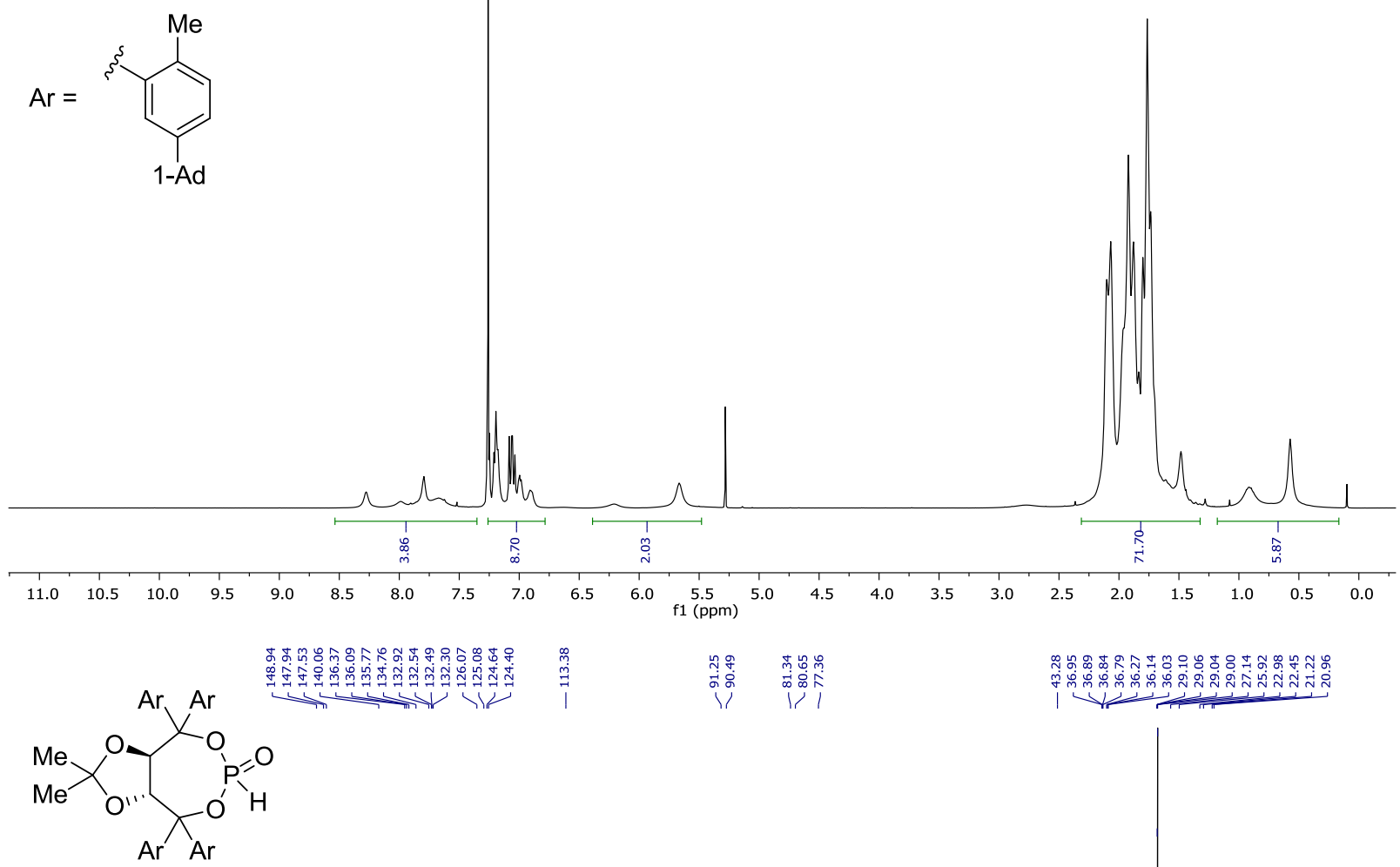

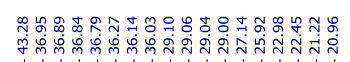

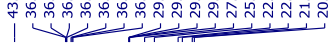

218

$\left(\mathrm{CDCl}_{3}, 100 \mathrm{MHz}\right)$<smiles>Cc1ccc([Al])cc1C=[Te]</smiles>

1




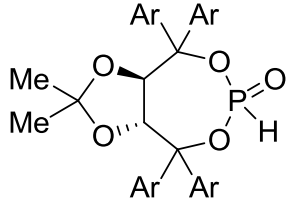

218

$\left(\mathrm{CDCl}_{3}, 162 \mathrm{MHz}\right)$

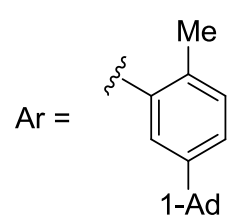

$1-A d$ 


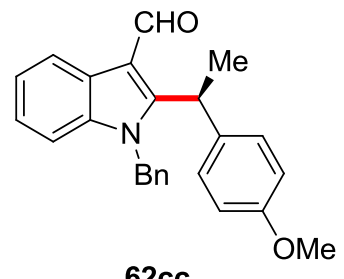

$\left(\mathrm{CDCl}_{3}, 400 \mathrm{MHz}\right)$
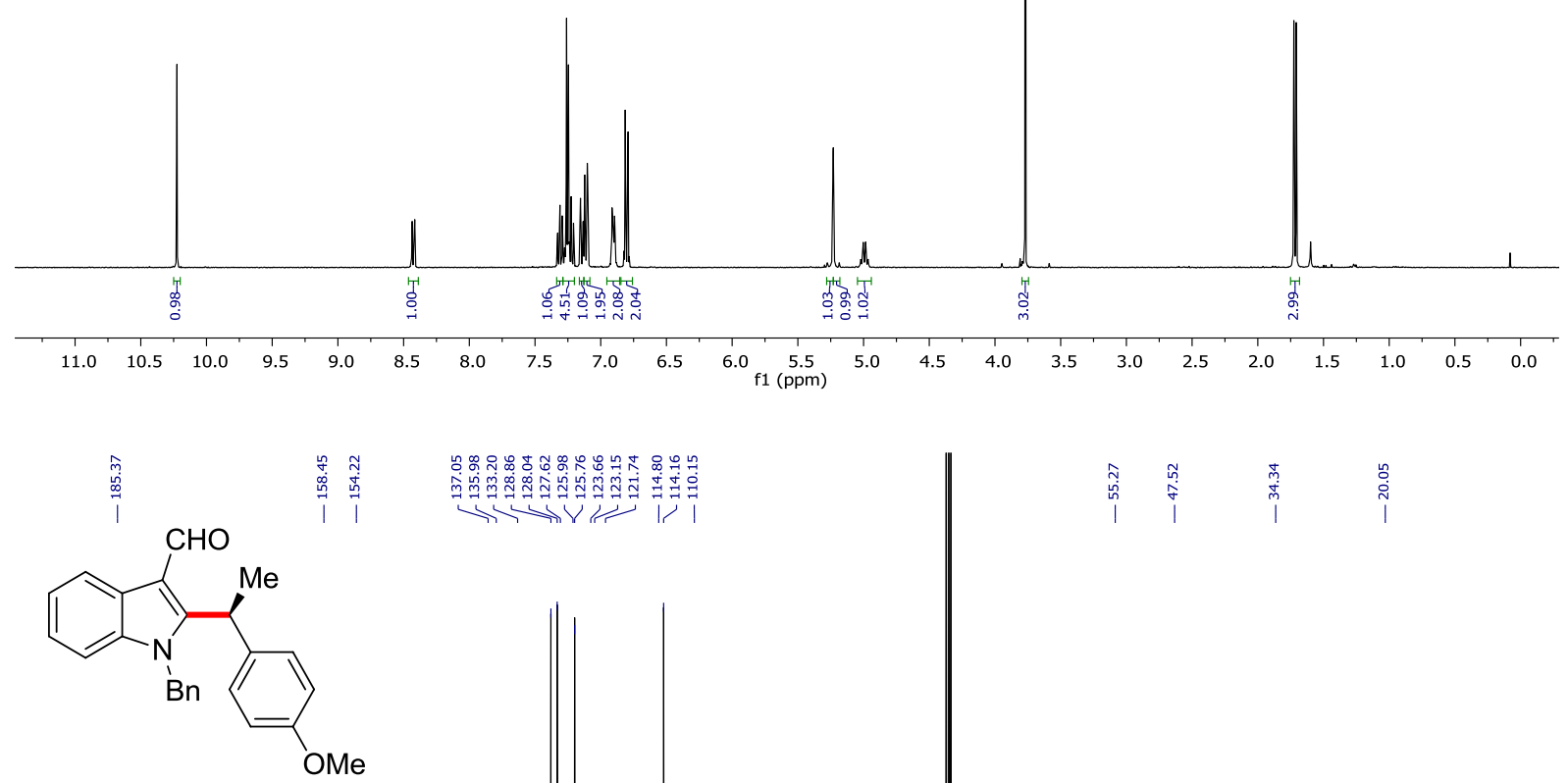

62cc

$\left(\mathrm{CDCl}_{3}, 100 \mathrm{MHz}\right)$

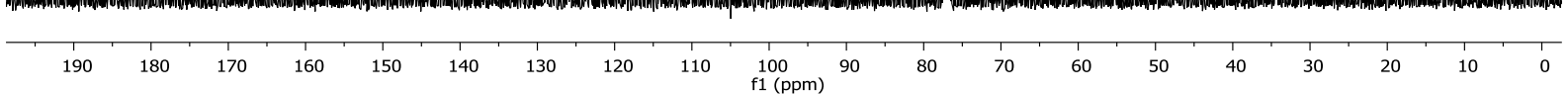




\section{Chiral HPLC of 62cc:}
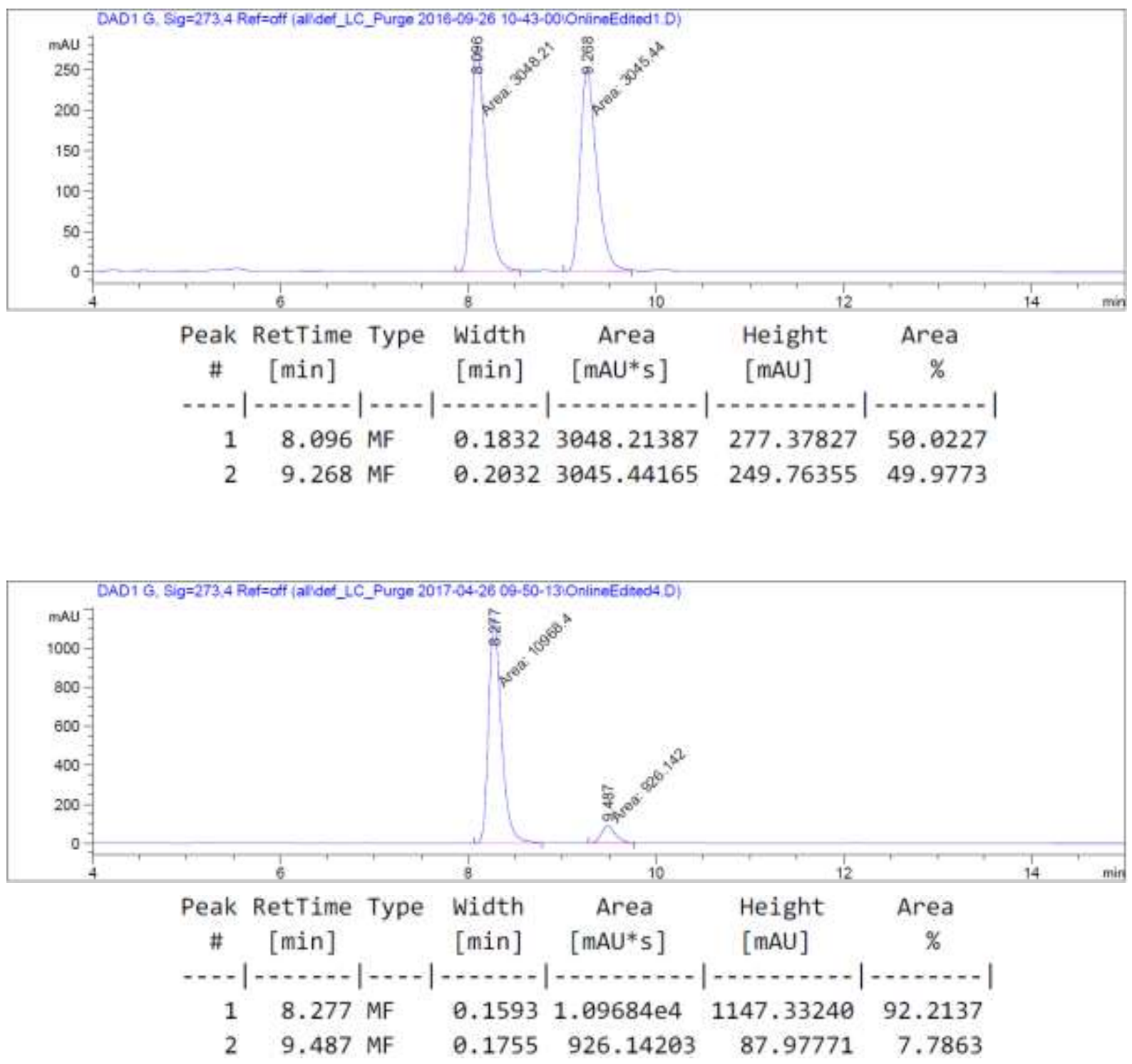


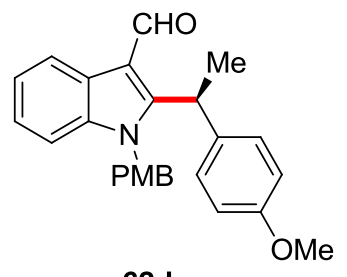

$\left(\mathrm{CDCl}_{3}, 500 \mathrm{MHz}\right)$
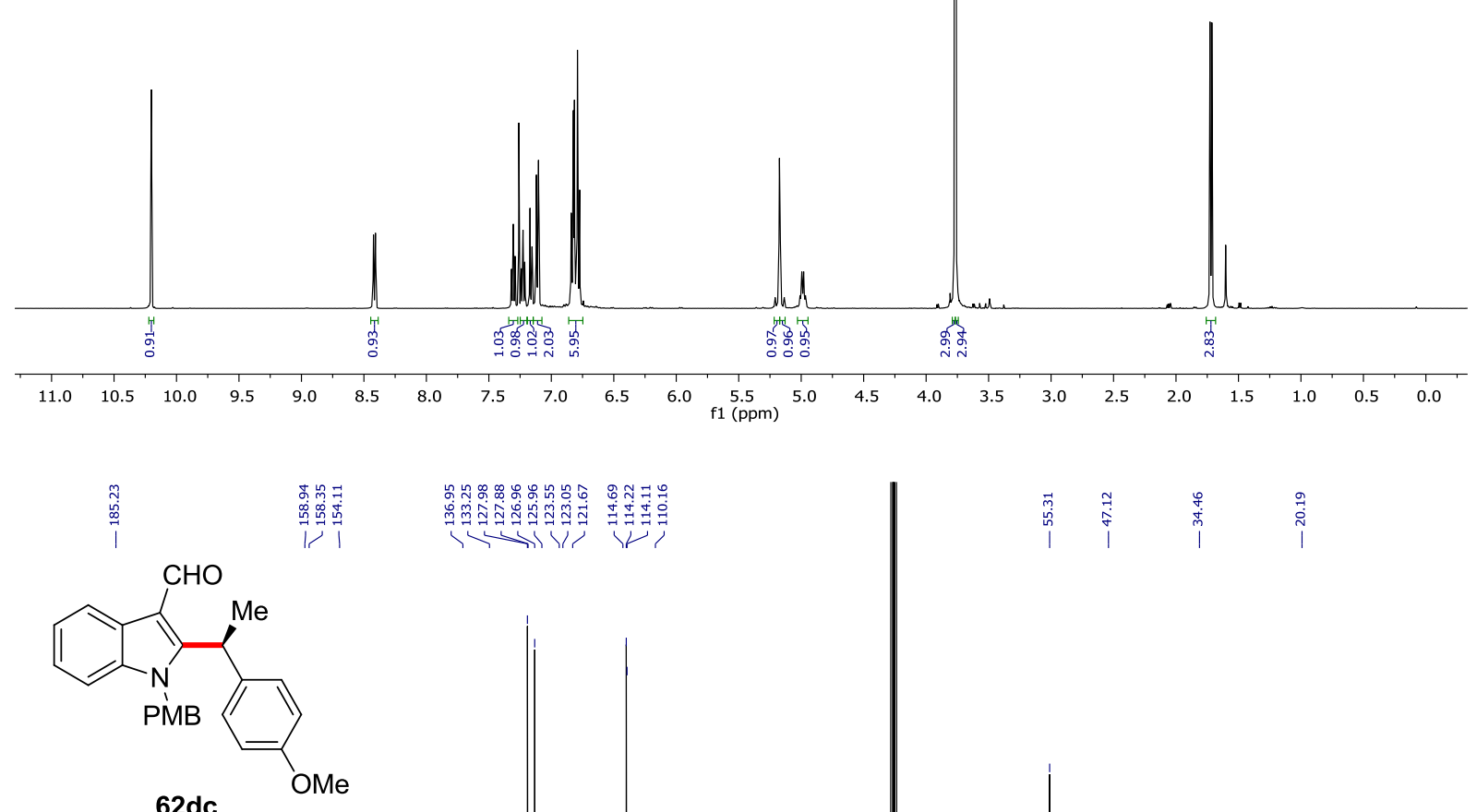

62dc

$\left(\mathrm{CDCl}_{3}, 125 \mathrm{MHz}\right)$

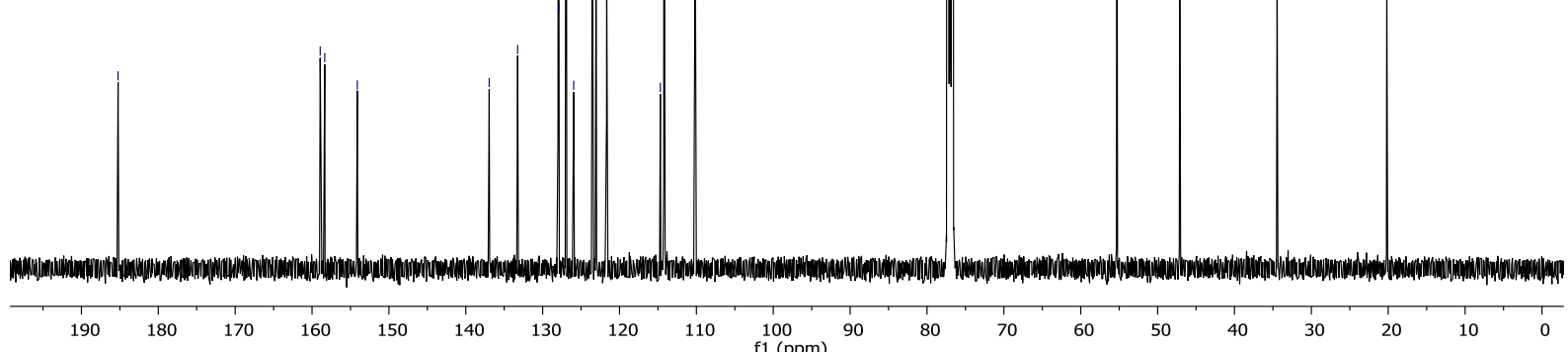

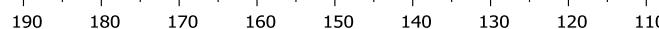

$\begin{array}{rll}100 & 90 \quad 80\end{array}$

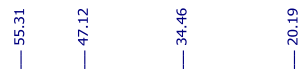




\section{Chiral HPLC of $\mathbf{6 2 d c}$ :}
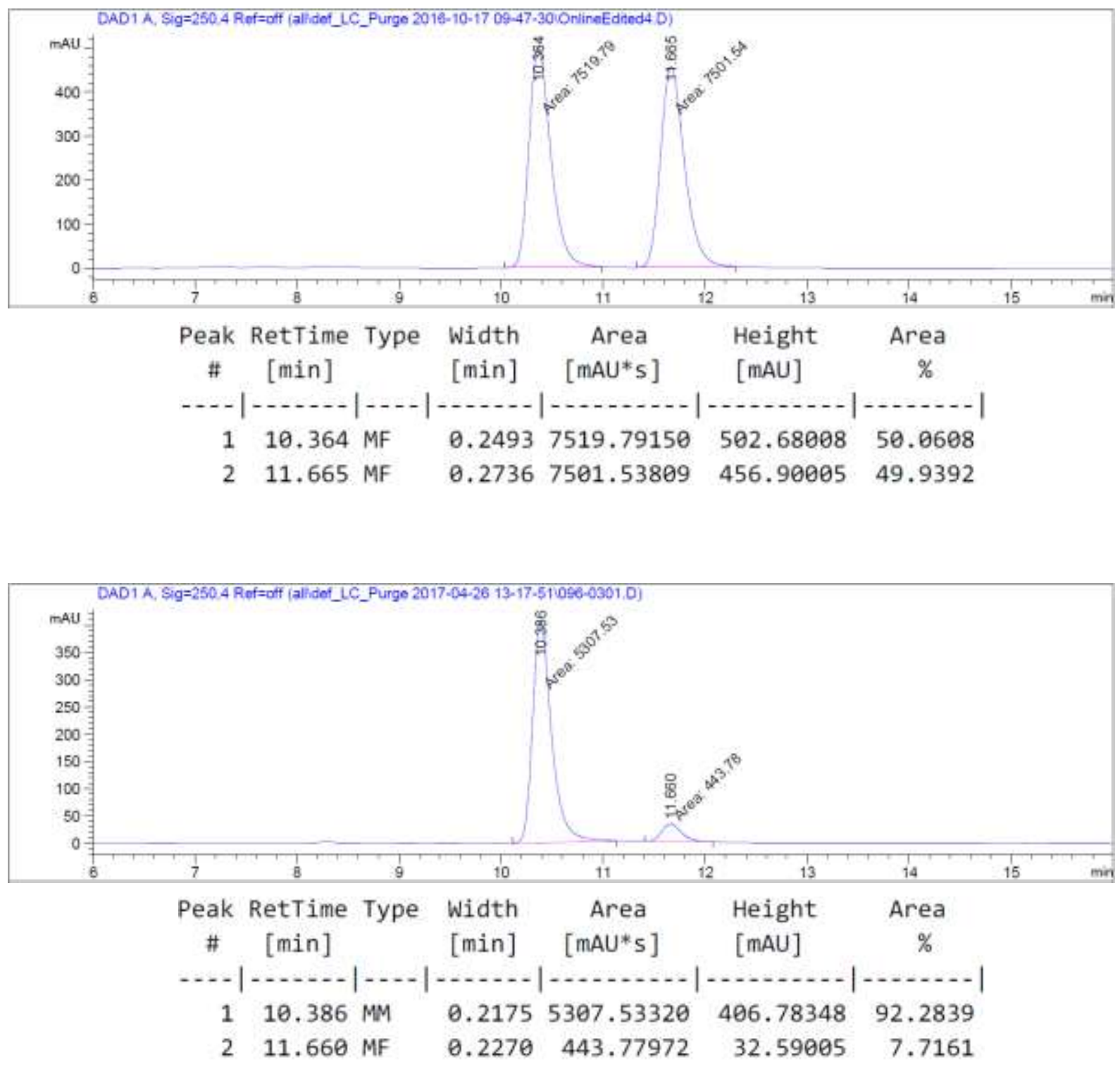

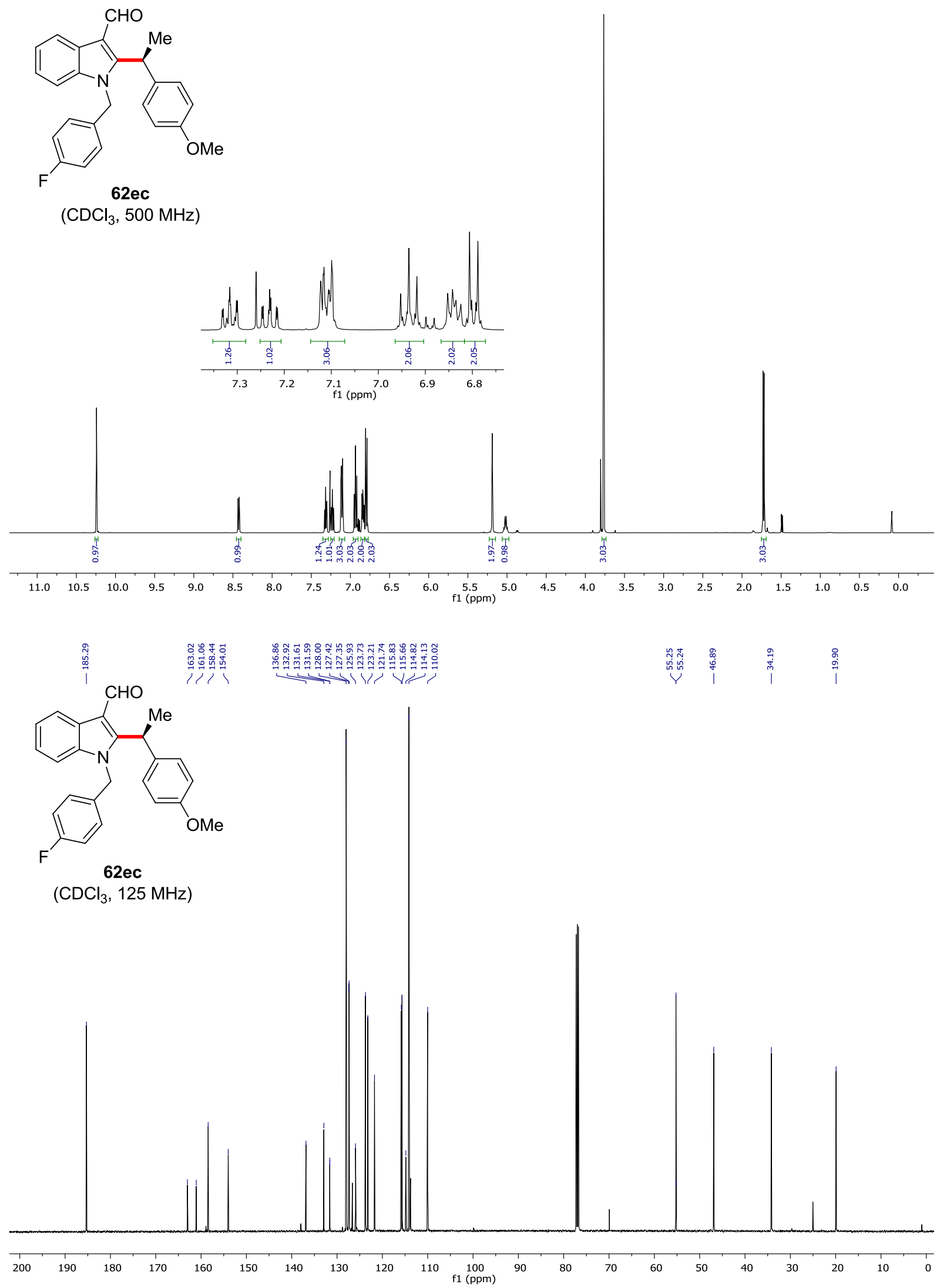


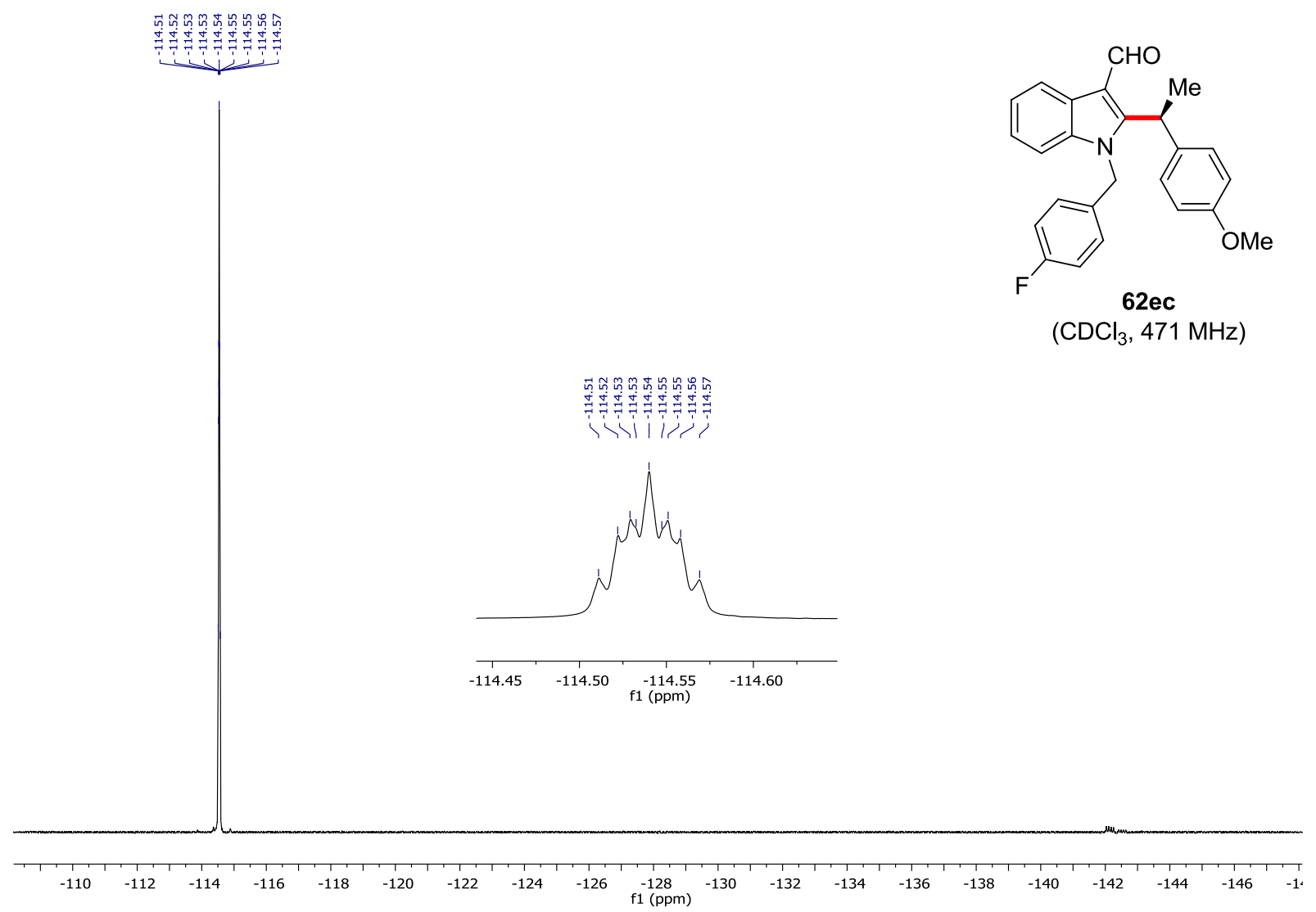




\section{Chiral HPLC of 62ec:}
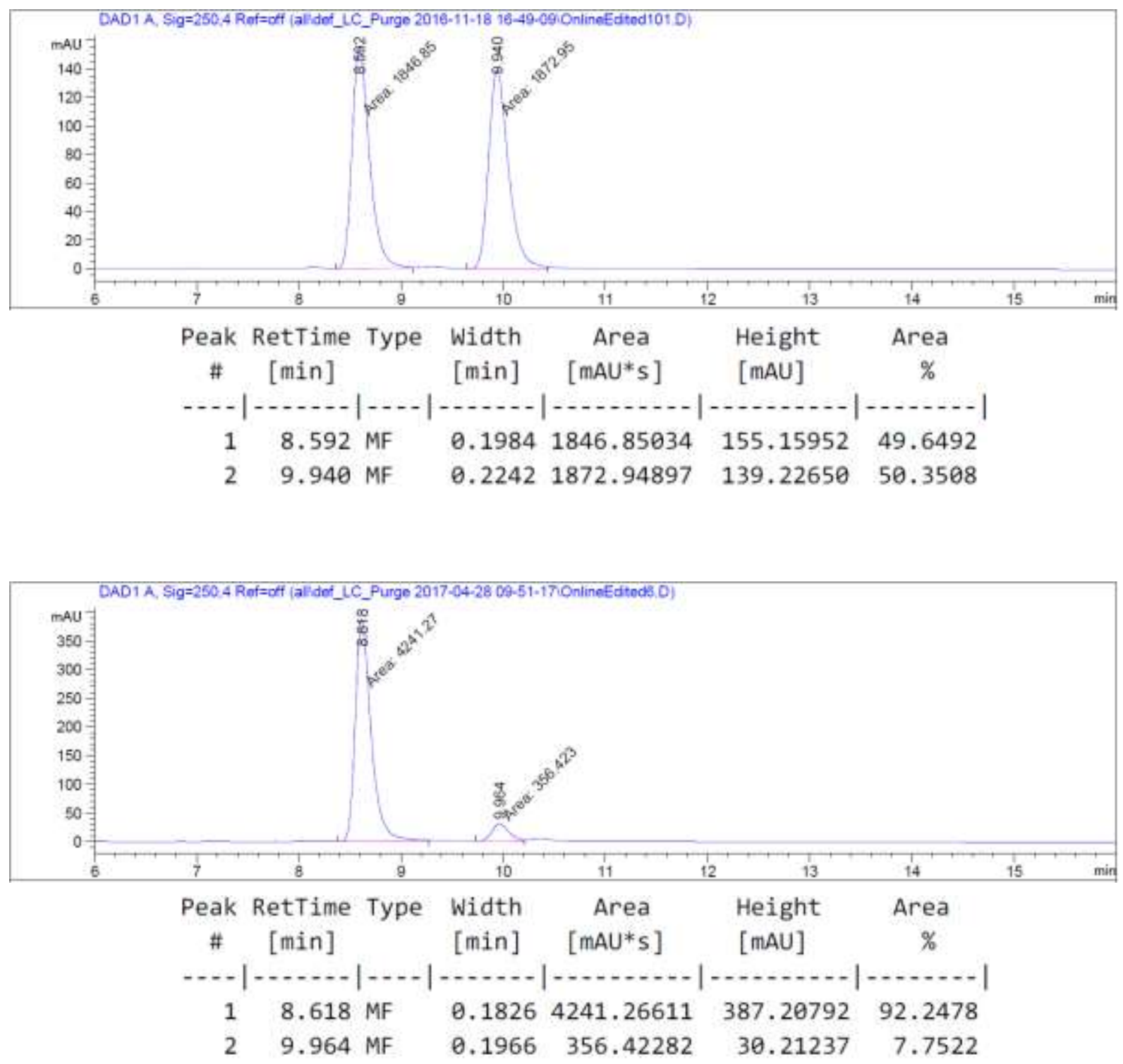


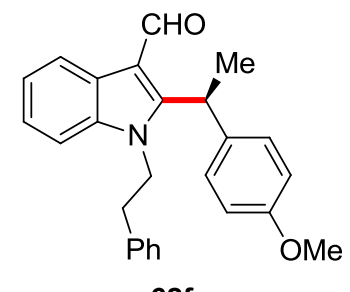

$\left(\mathrm{CDCl}_{3}, 300 \mathrm{MHz}\right)$
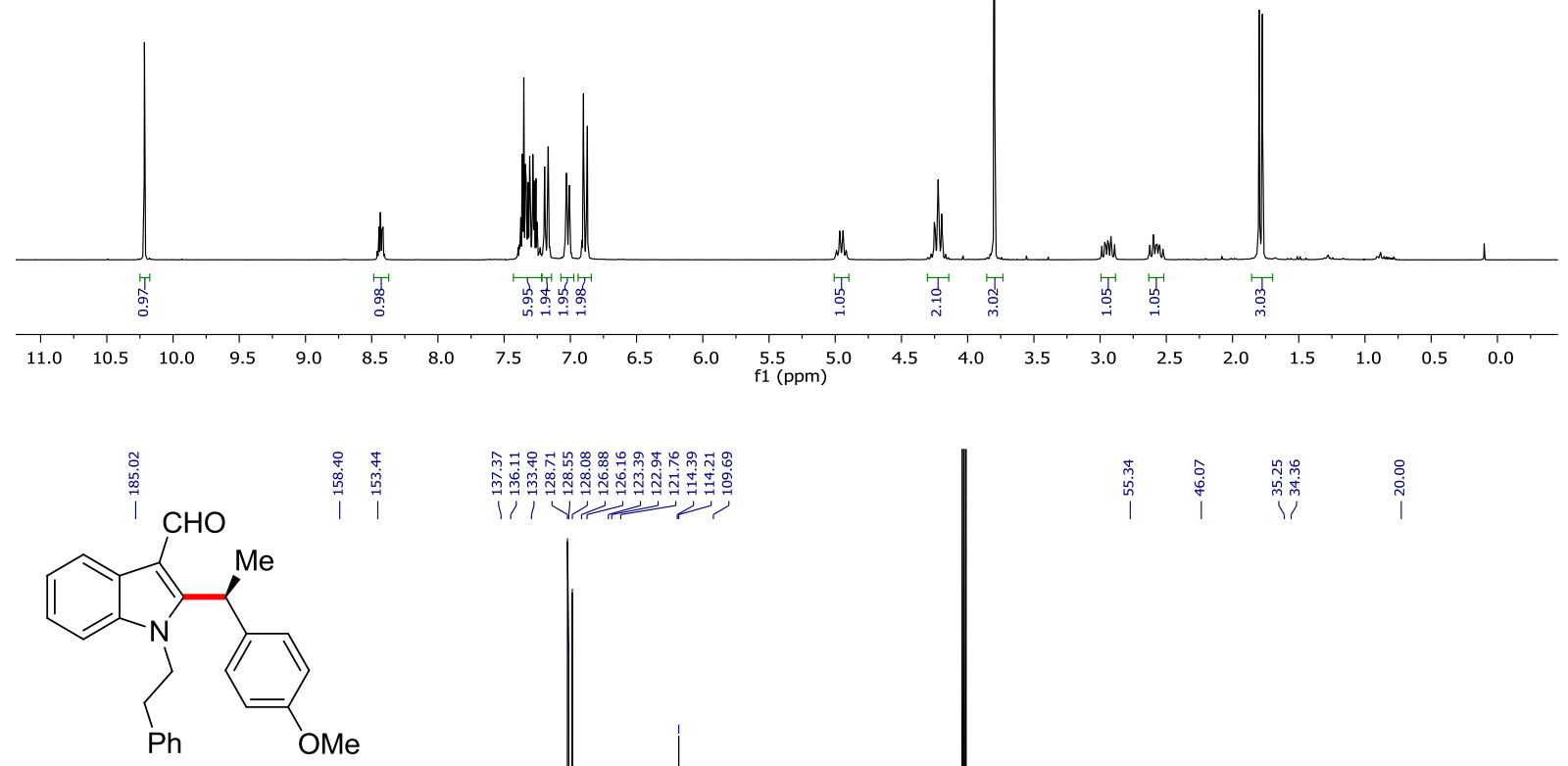

$62 \mathrm{fc}$

$\left(\mathrm{CDCl}_{3}, 125 \mathrm{MHz}\right)$

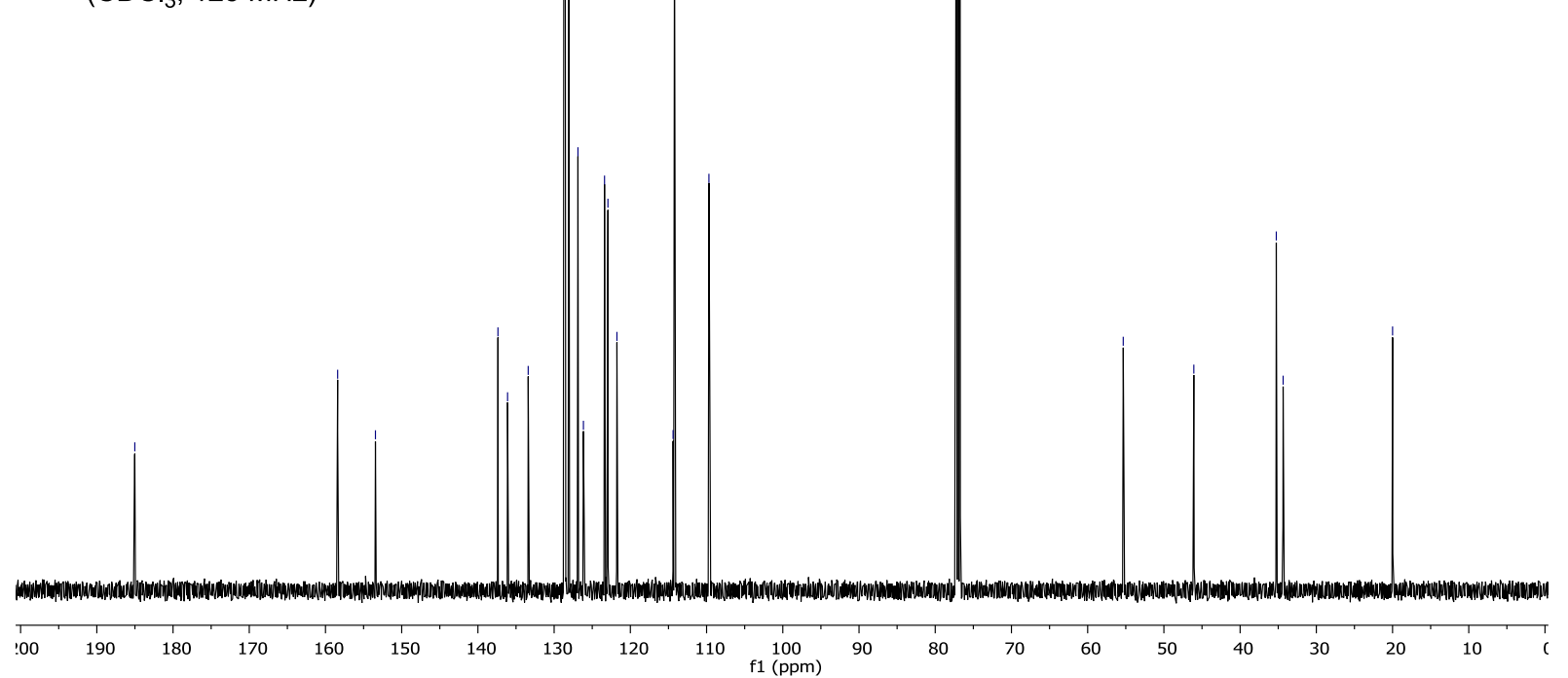




\section{Chiral HPLC of $\mathbf{6 2 f c :}$}
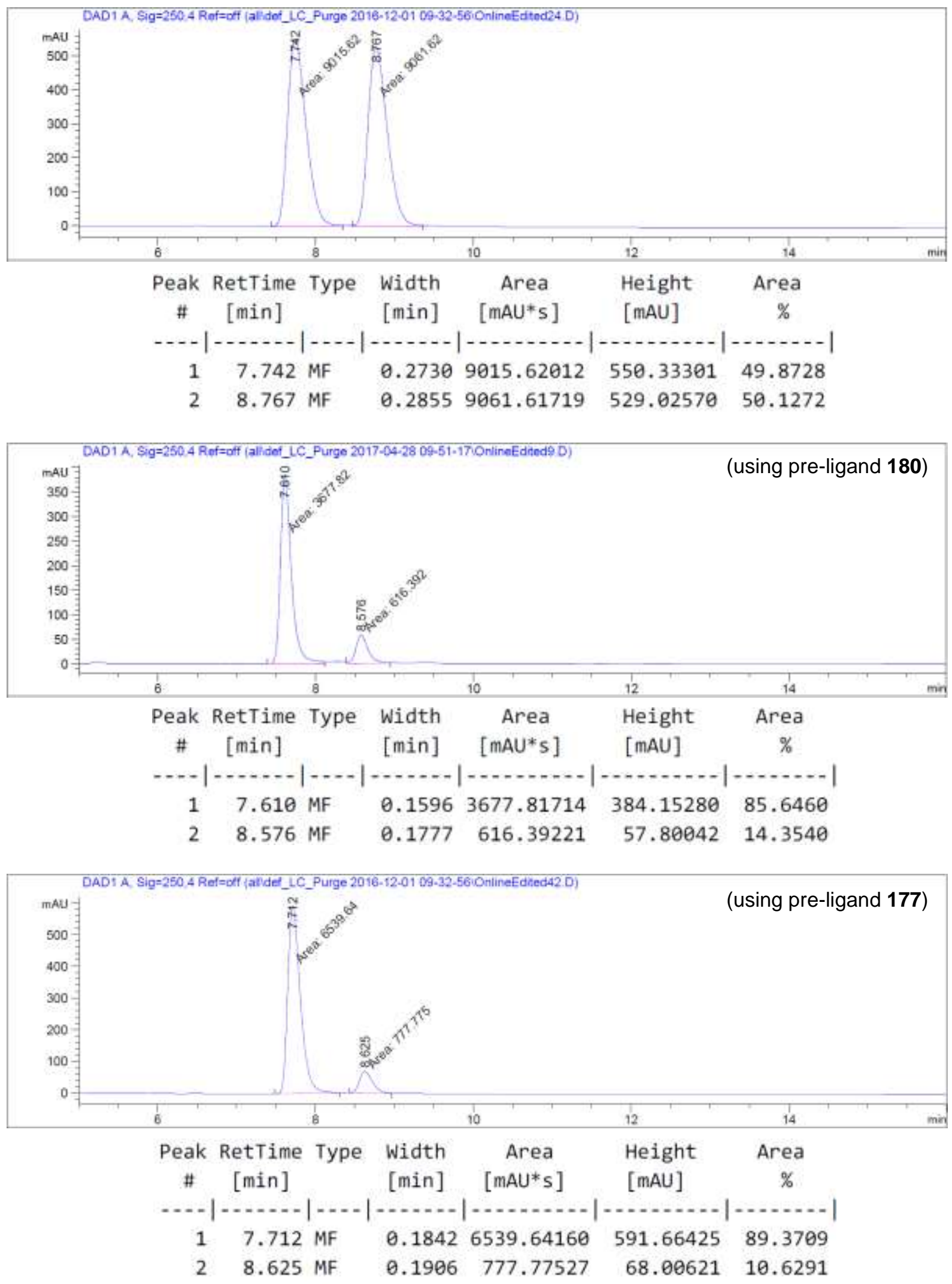


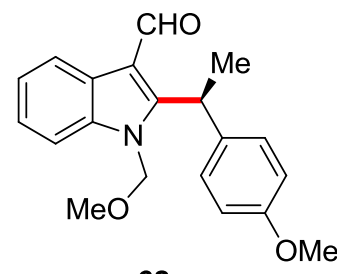

$62 \mathrm{gc}$

$\left(\mathrm{CDCl}_{3}, 500 \mathrm{MHz}\right)$
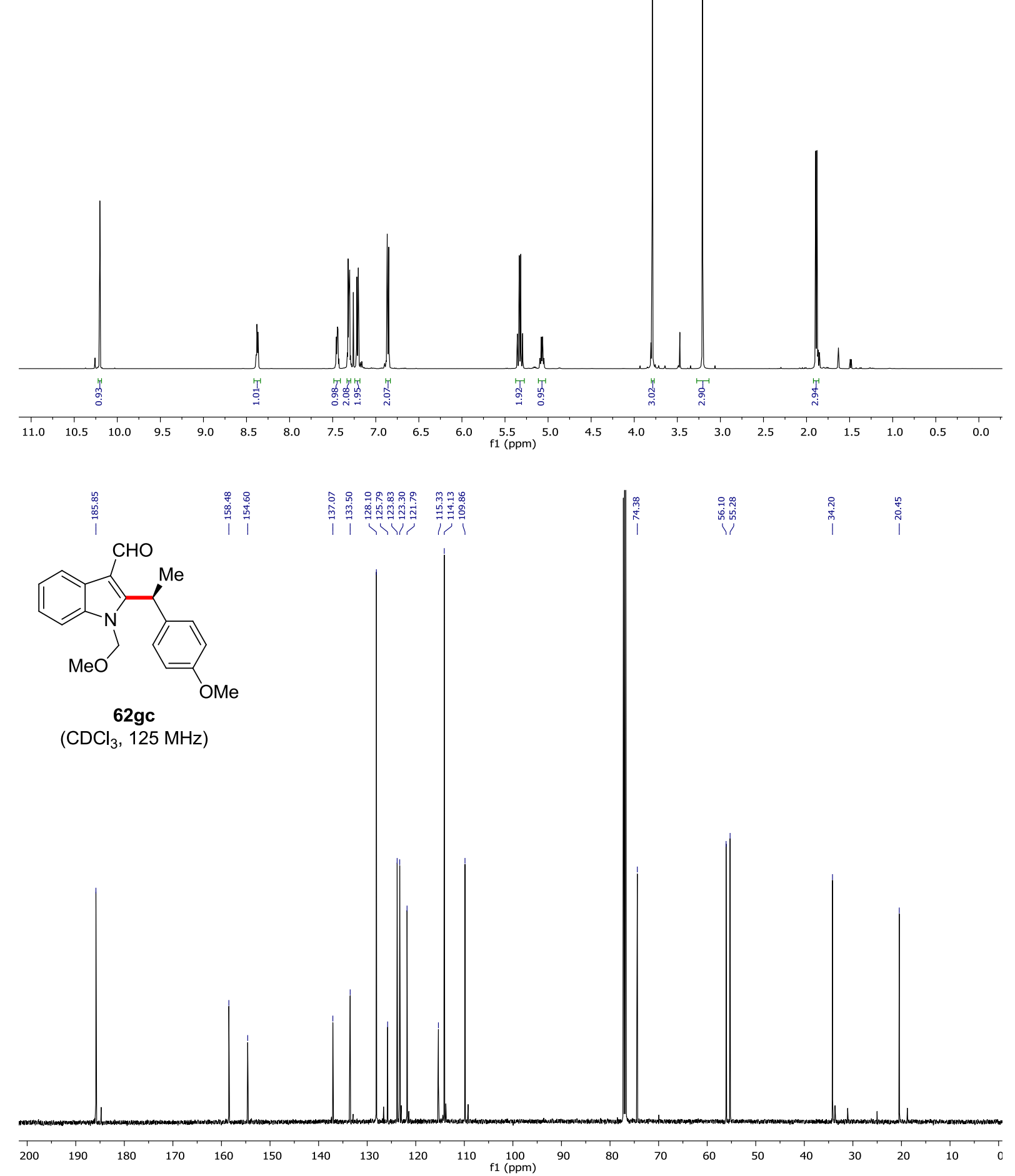


\section{Chiral HPLC of 62gc:}
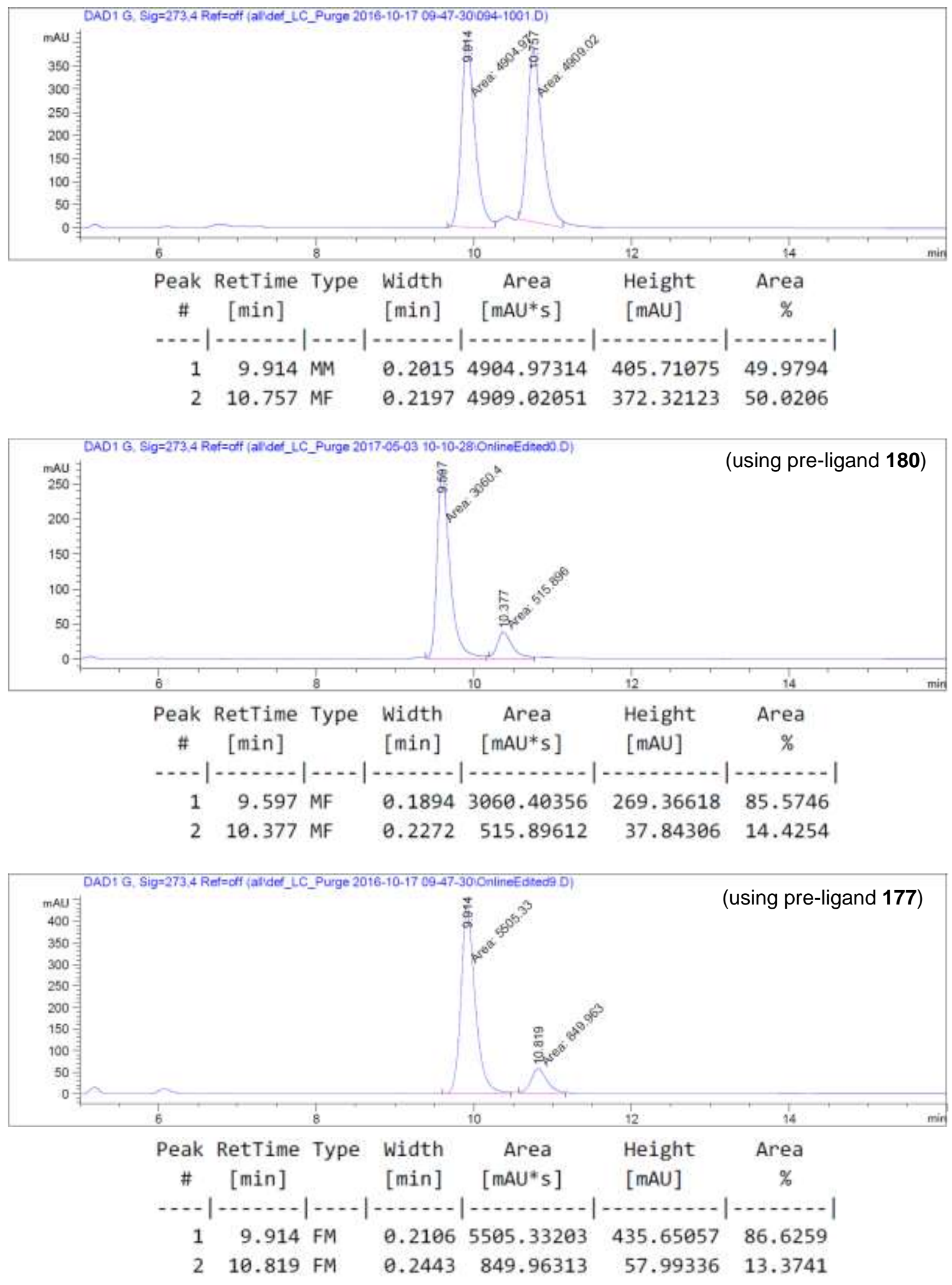


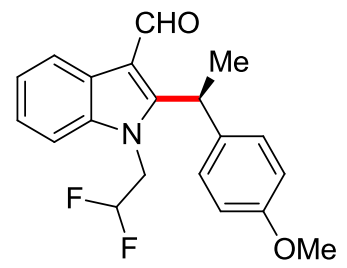

62hc

$\left(\mathrm{CDCl}_{3}, 500 \mathrm{MHz}\right)$
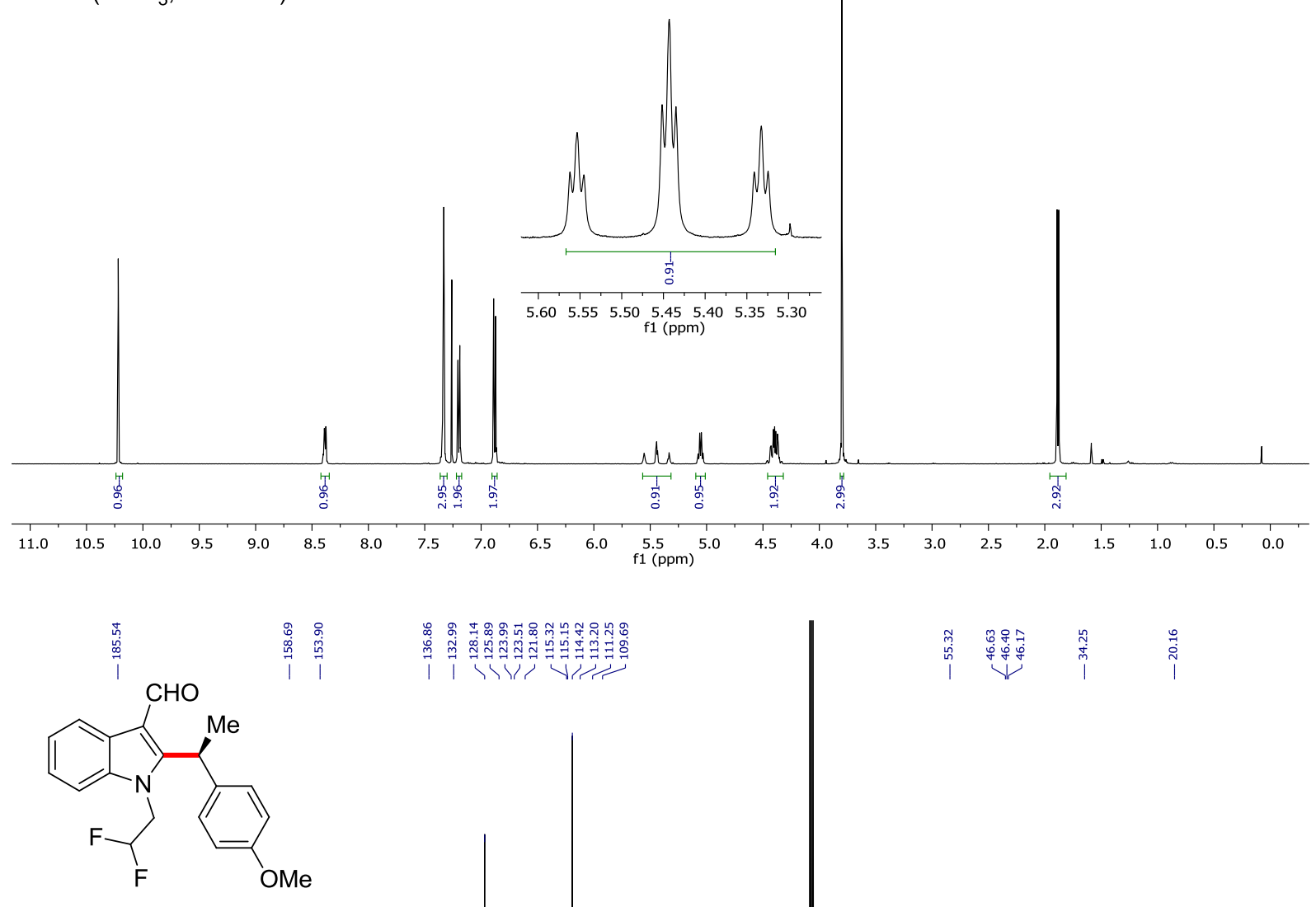

62hc

$\left(\mathrm{CDCl}_{3}, 125 \mathrm{MHz}\right)$

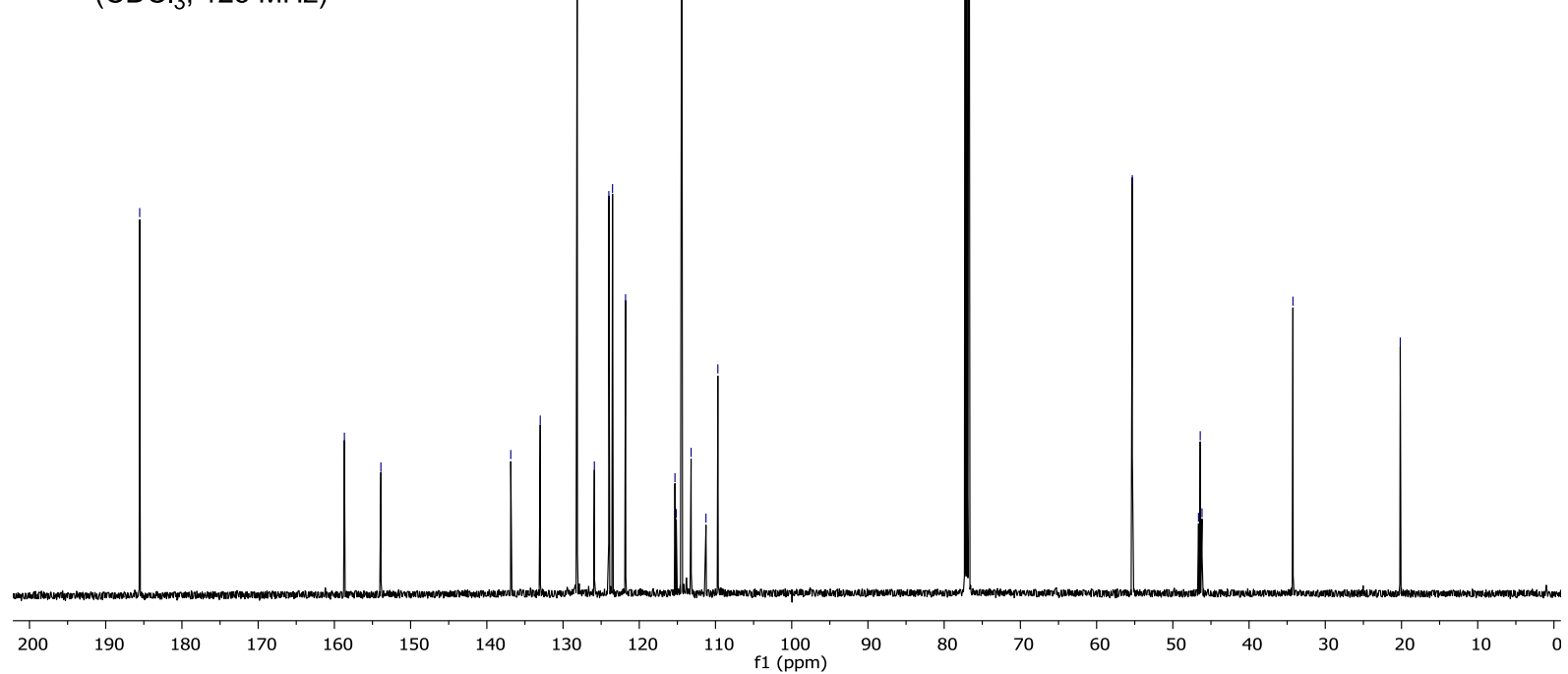




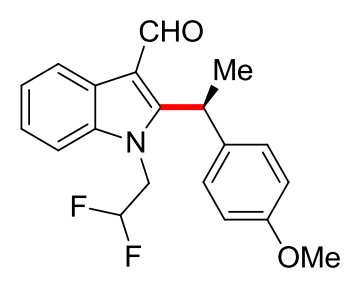

$62 \mathrm{hc}$

$\left(\mathrm{CDCl}_{3}, 471 \mathrm{MHz}\right)$

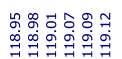

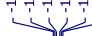

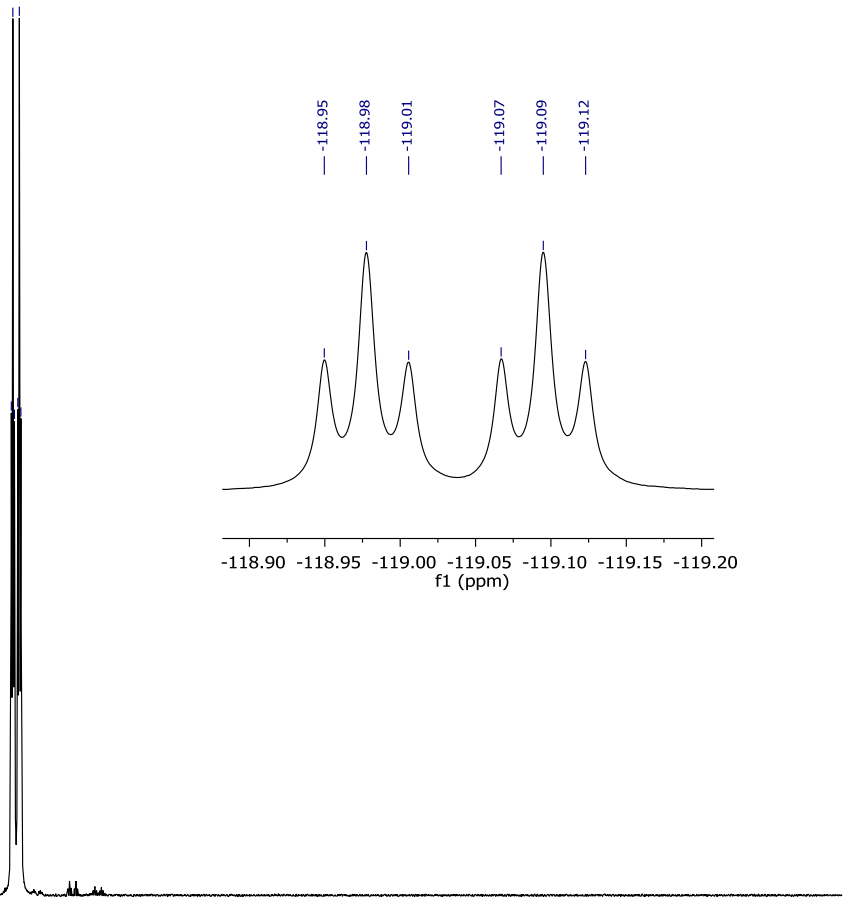

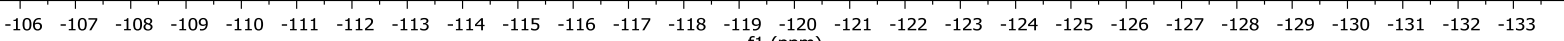




\section{Chiral HPLC of $62 \mathrm{hc}$ :}
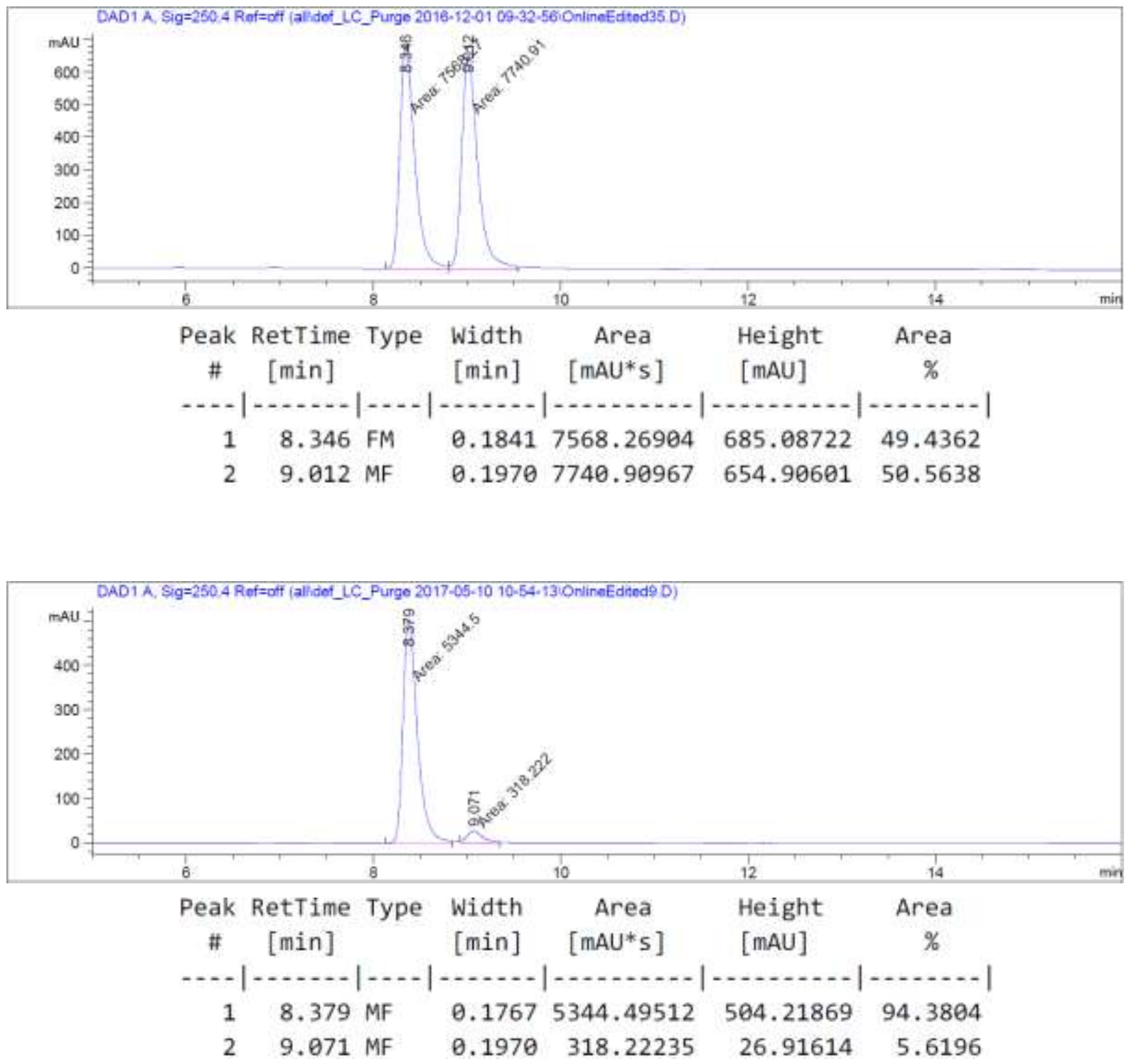


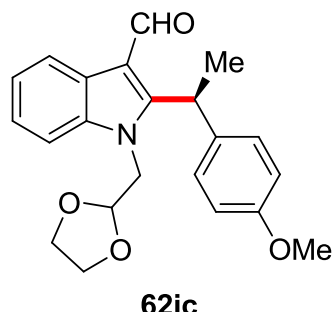

$\left(\mathrm{CDCl}_{3}, 300 \mathrm{MHz}\right)$
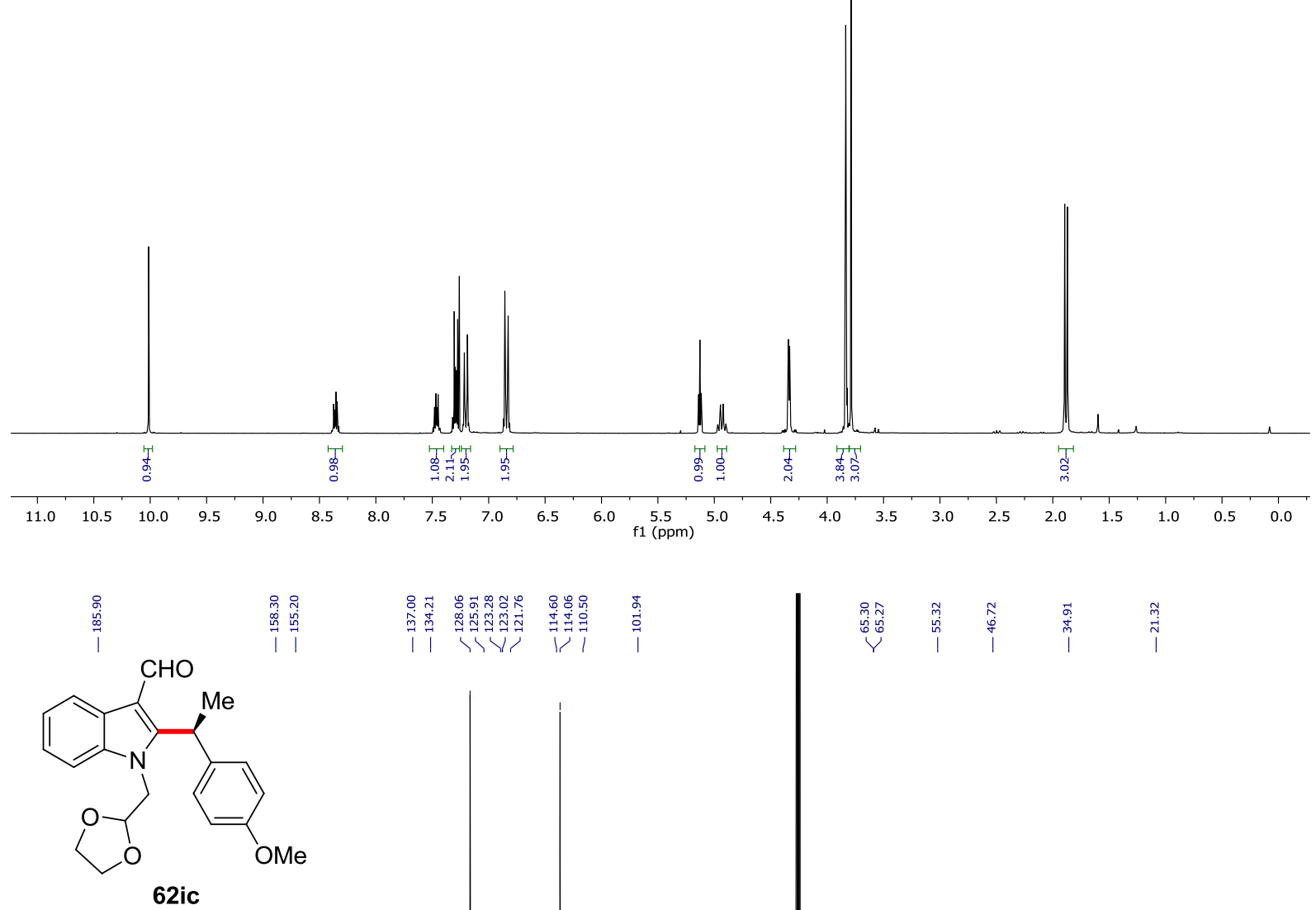

$\left(\mathrm{CDCl}_{3}, 125 \mathrm{MHz}\right)$

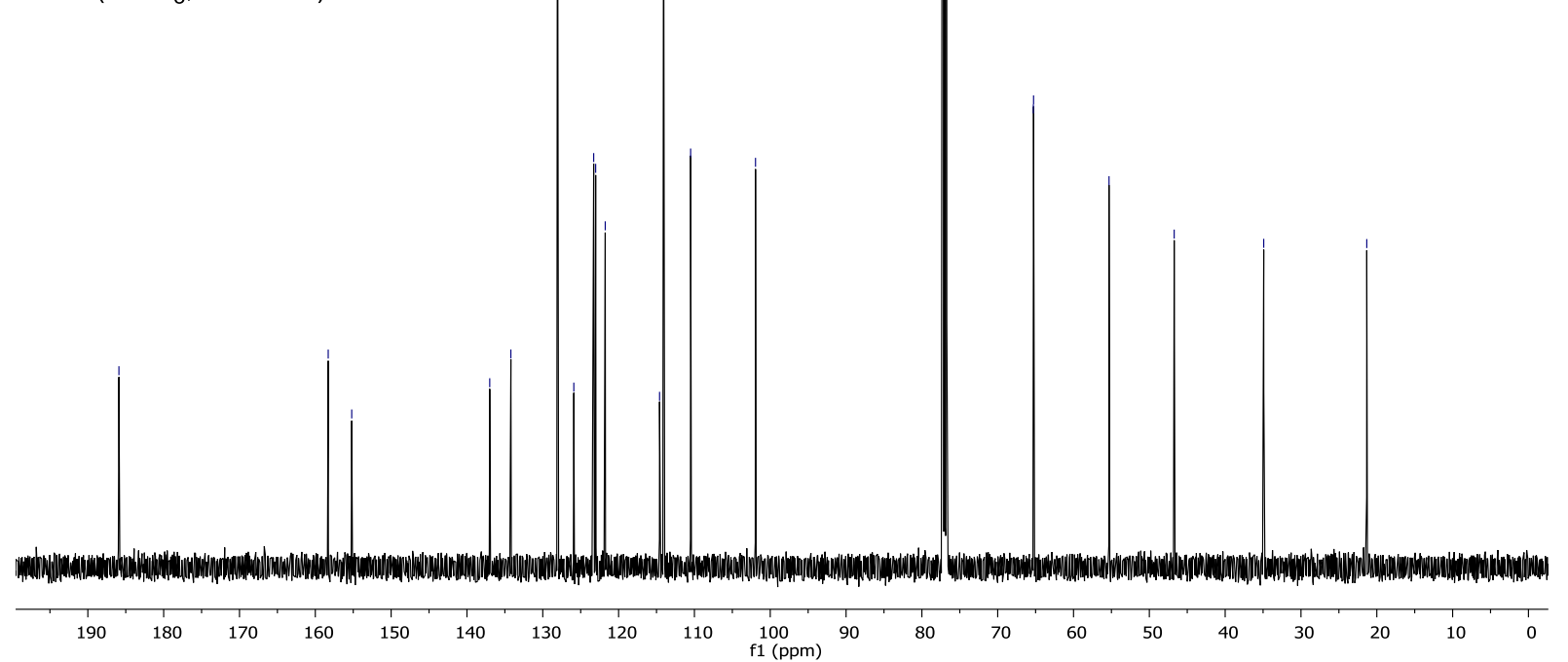




\section{Chiral HPLC of 62ic:}
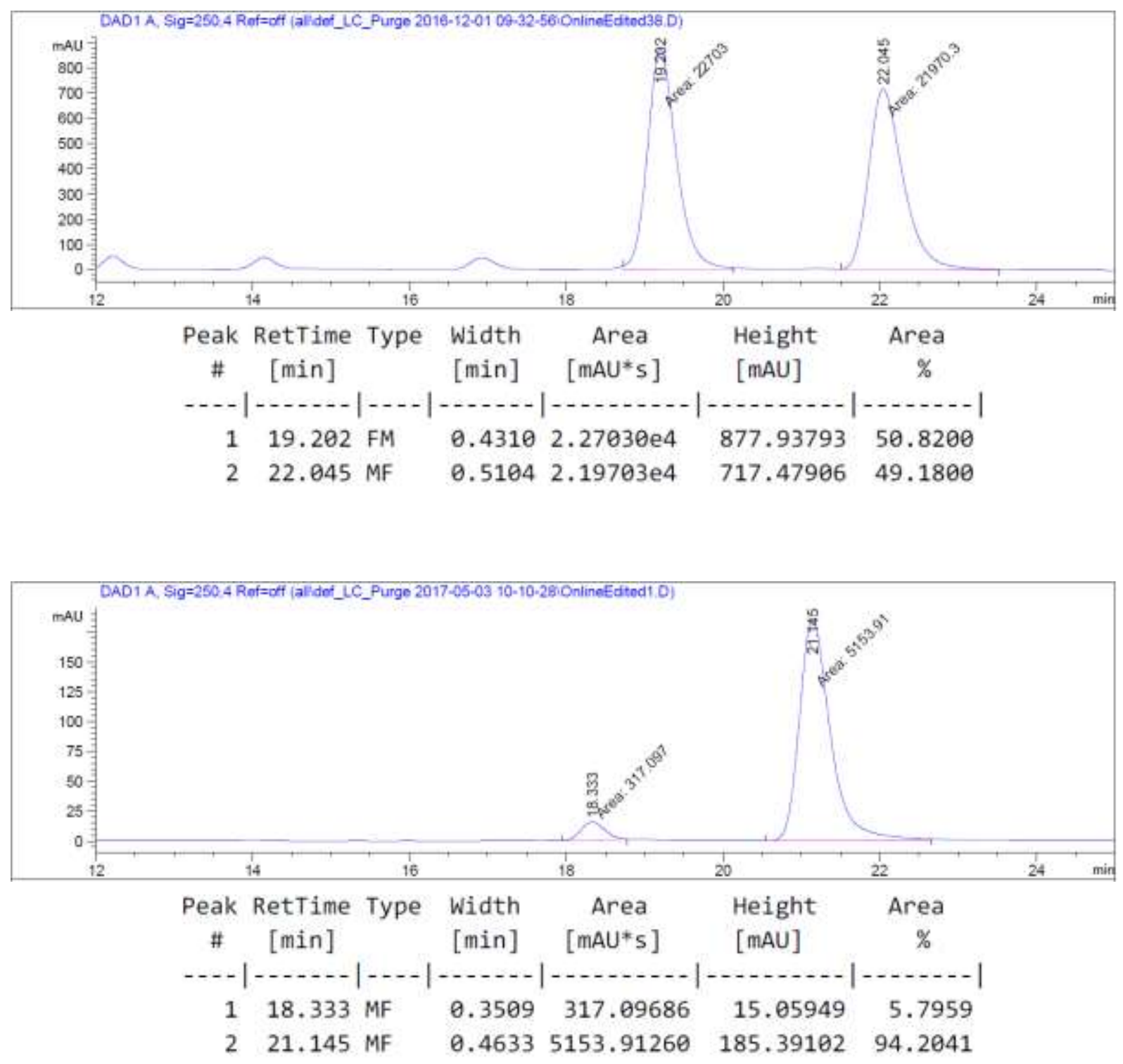

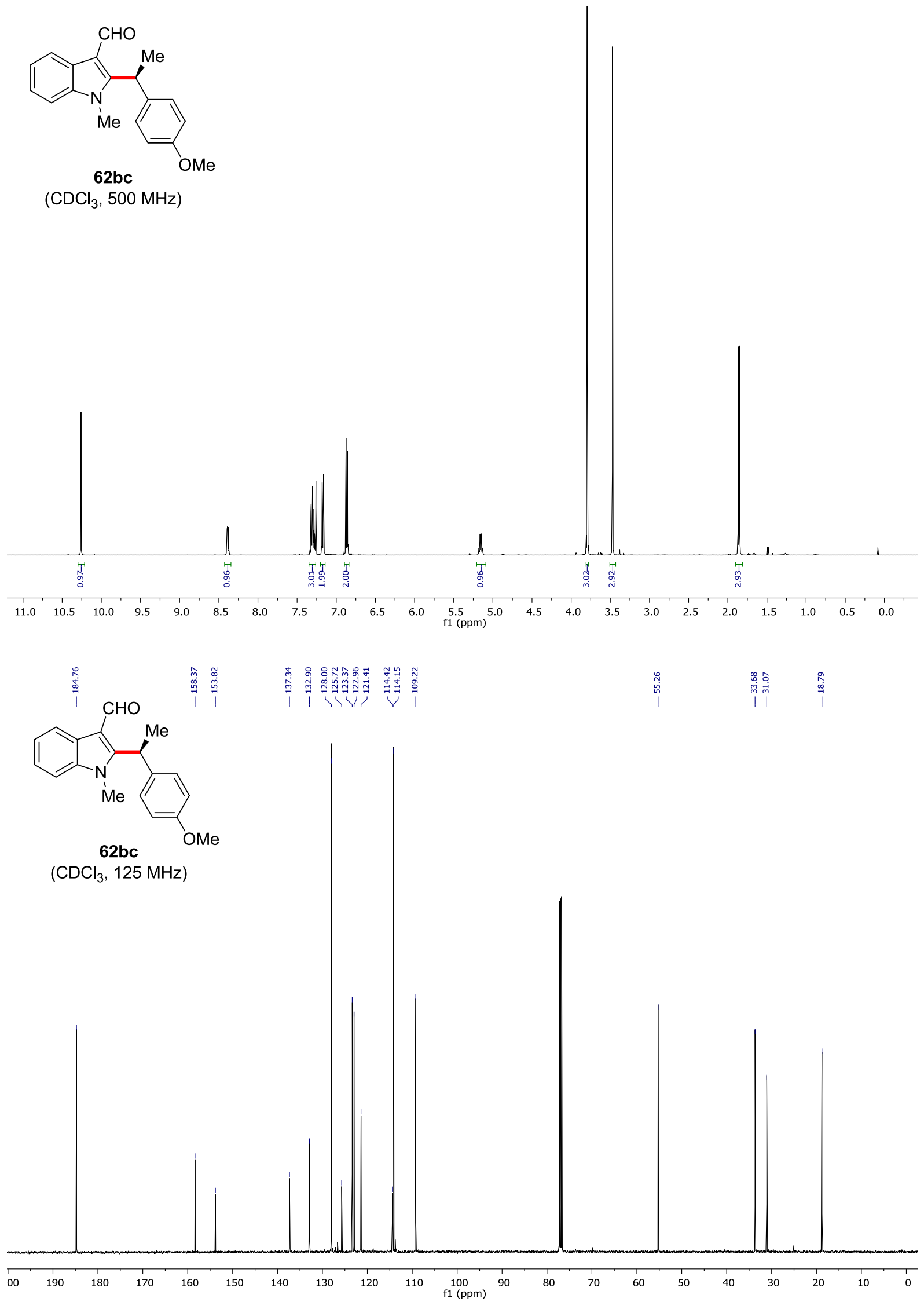


\section{Chiral HPLC of $\mathbf{6 2 b c}$}
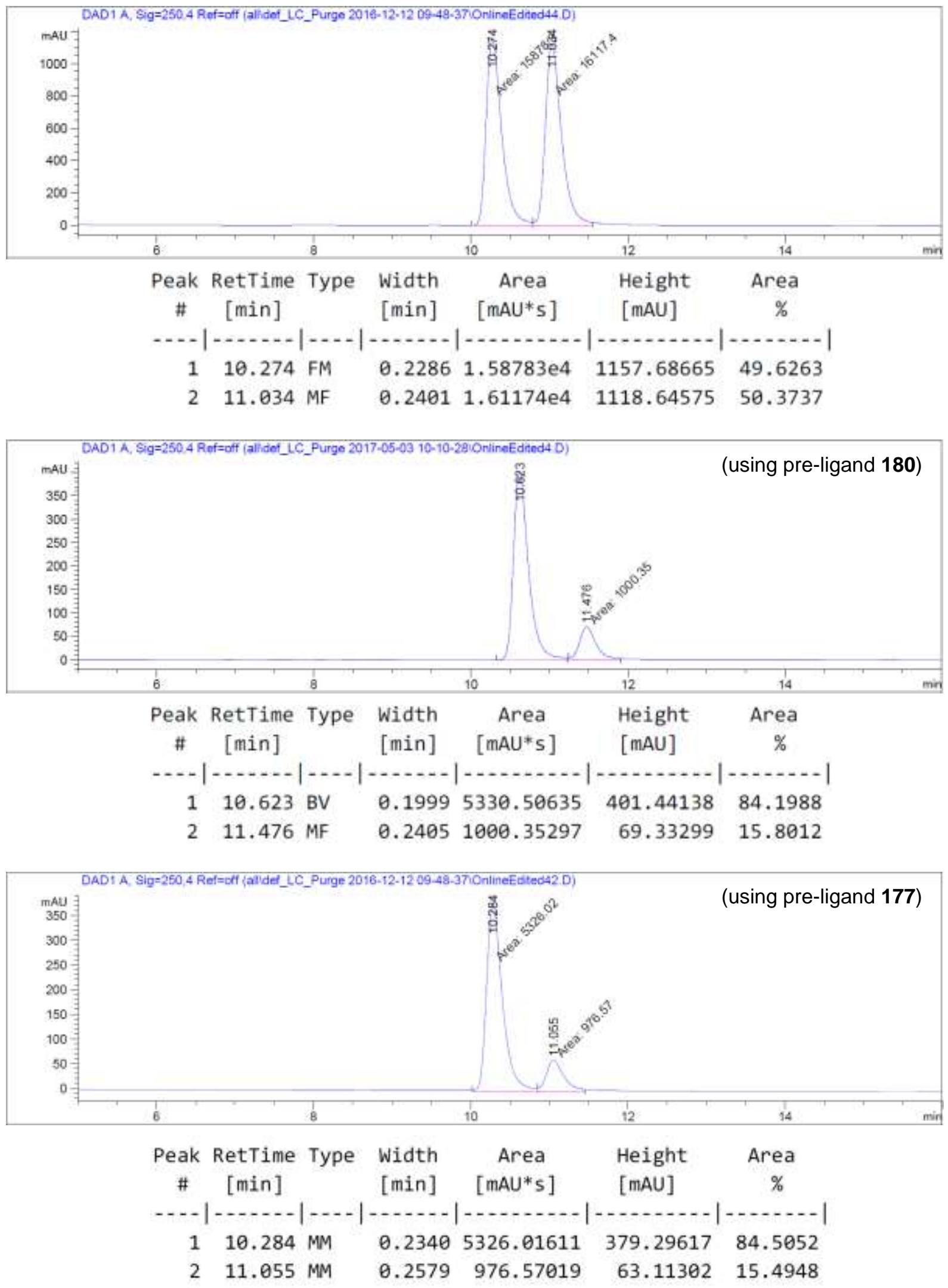

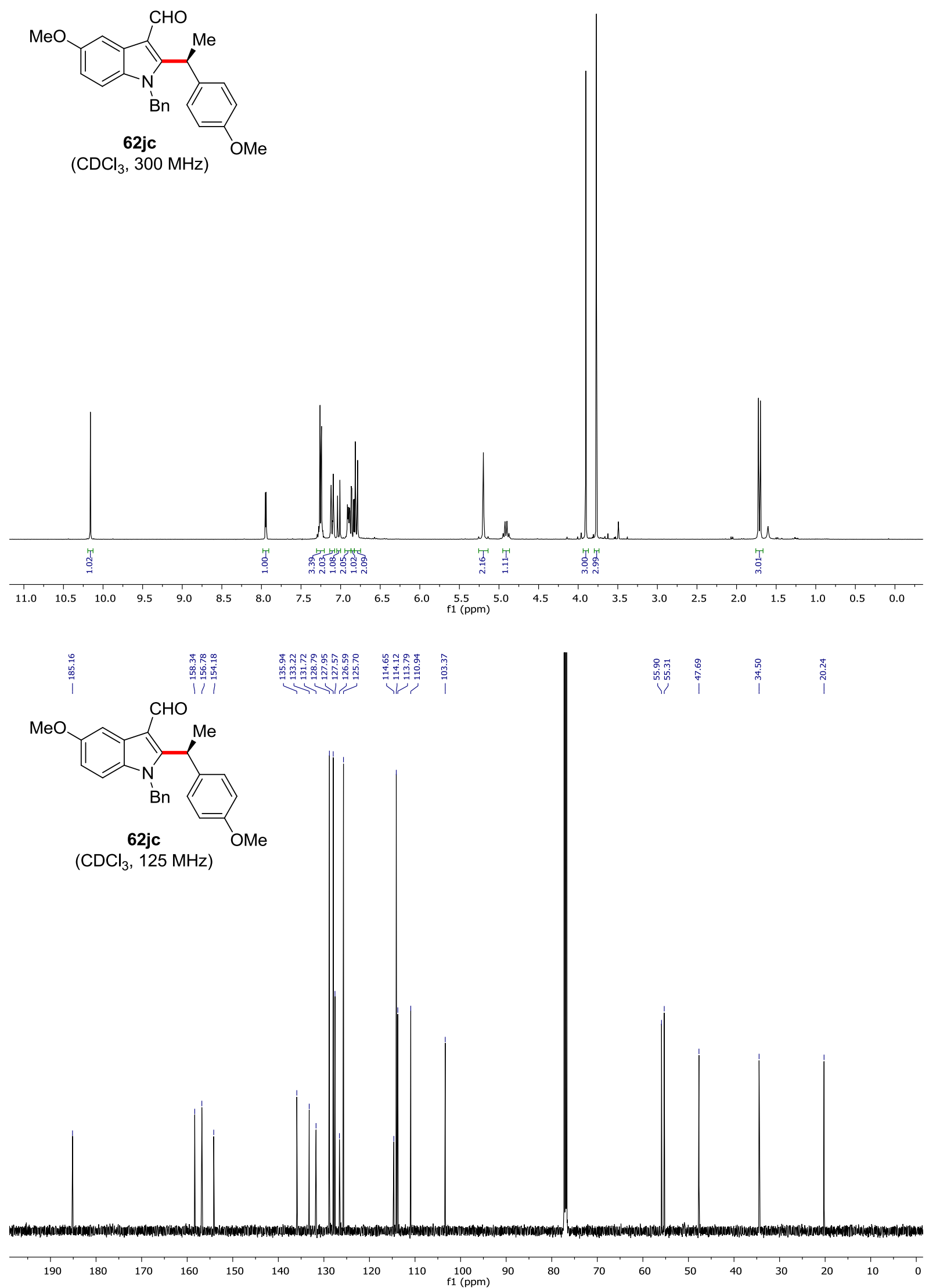


\section{Chiral HPLC of 62jc:}
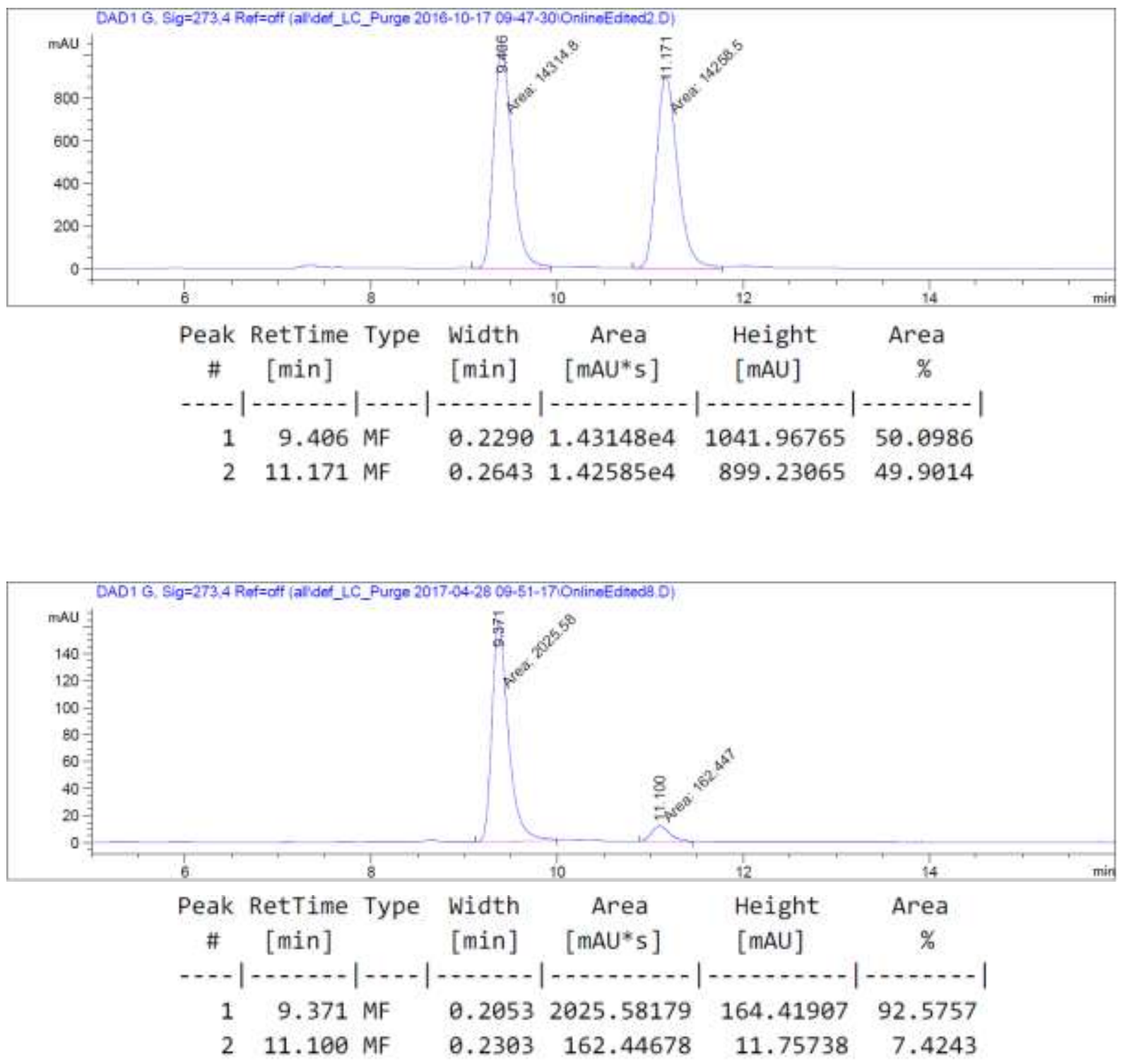

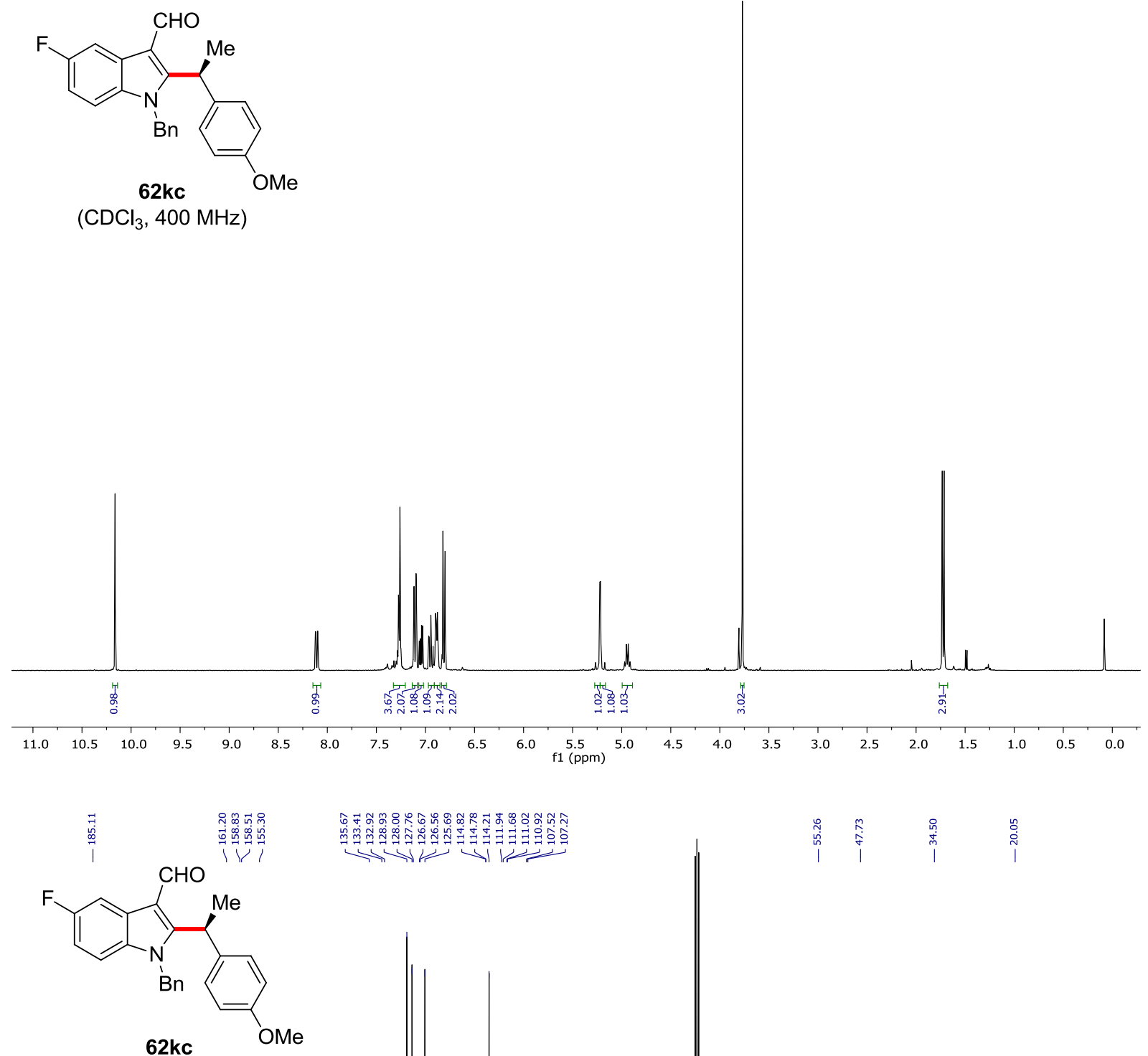

$\left(\mathrm{CDCl}_{3}, 100 \mathrm{MHz}\right)$

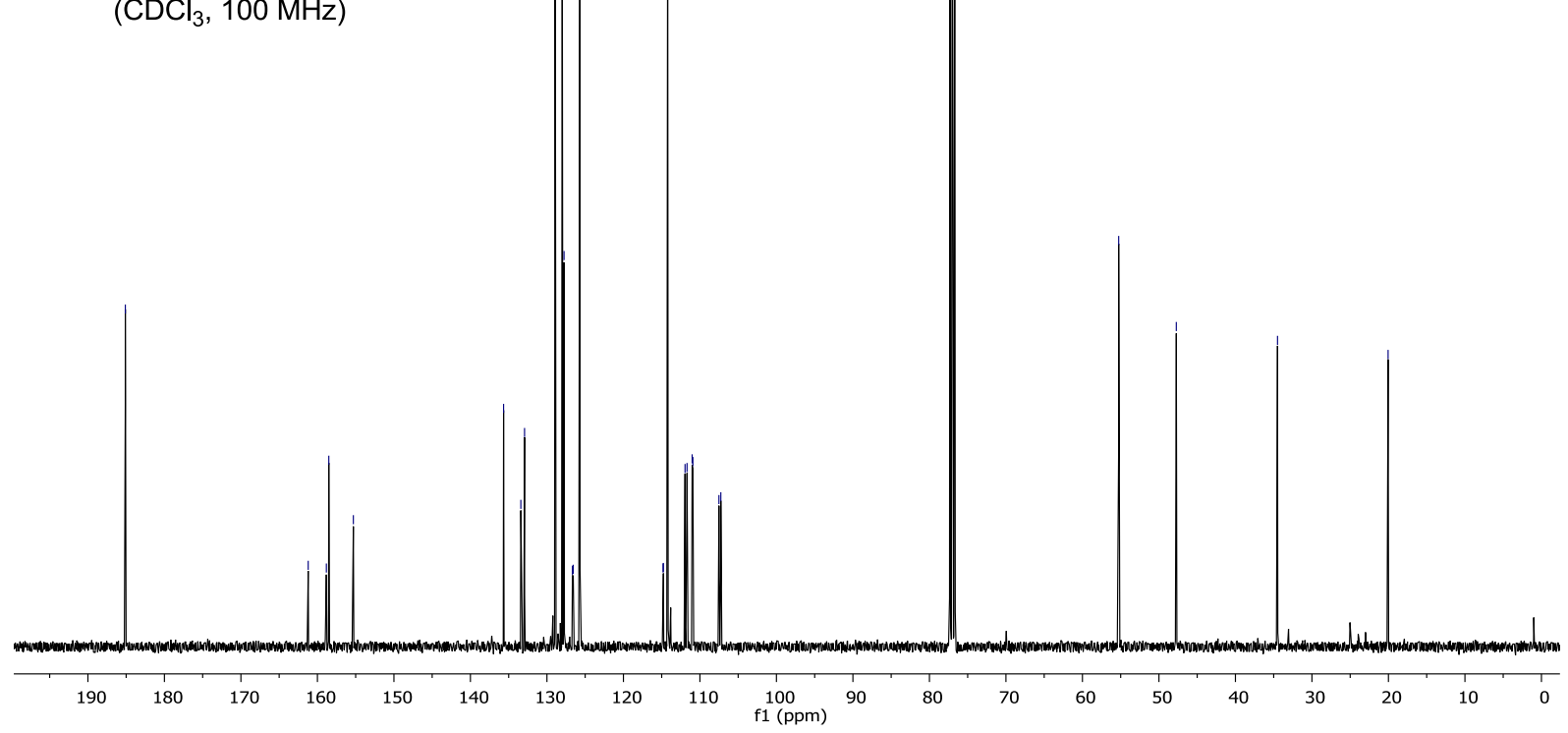



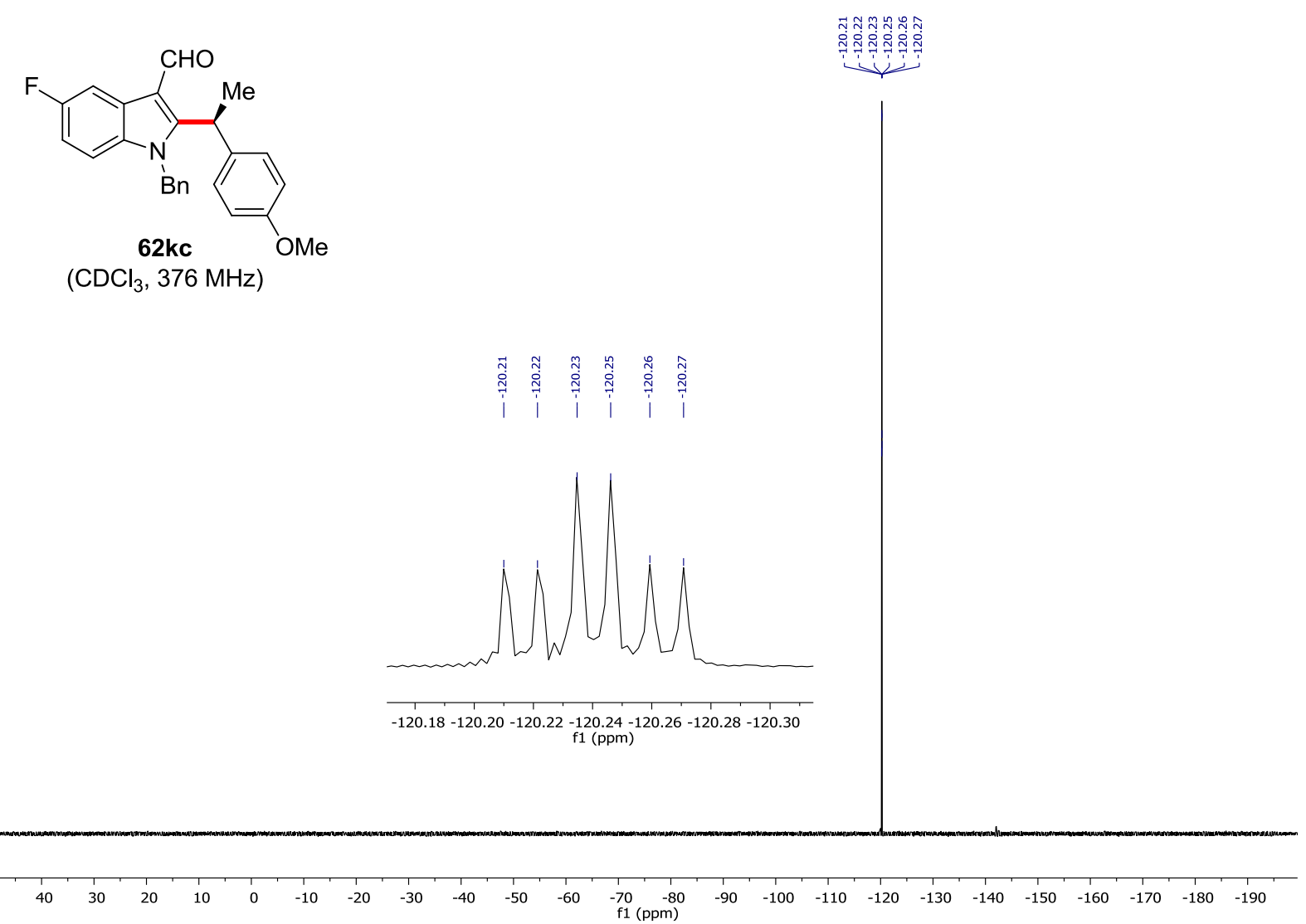


\section{Chiral HPLC of $\mathbf{6 2 k c}$ :}
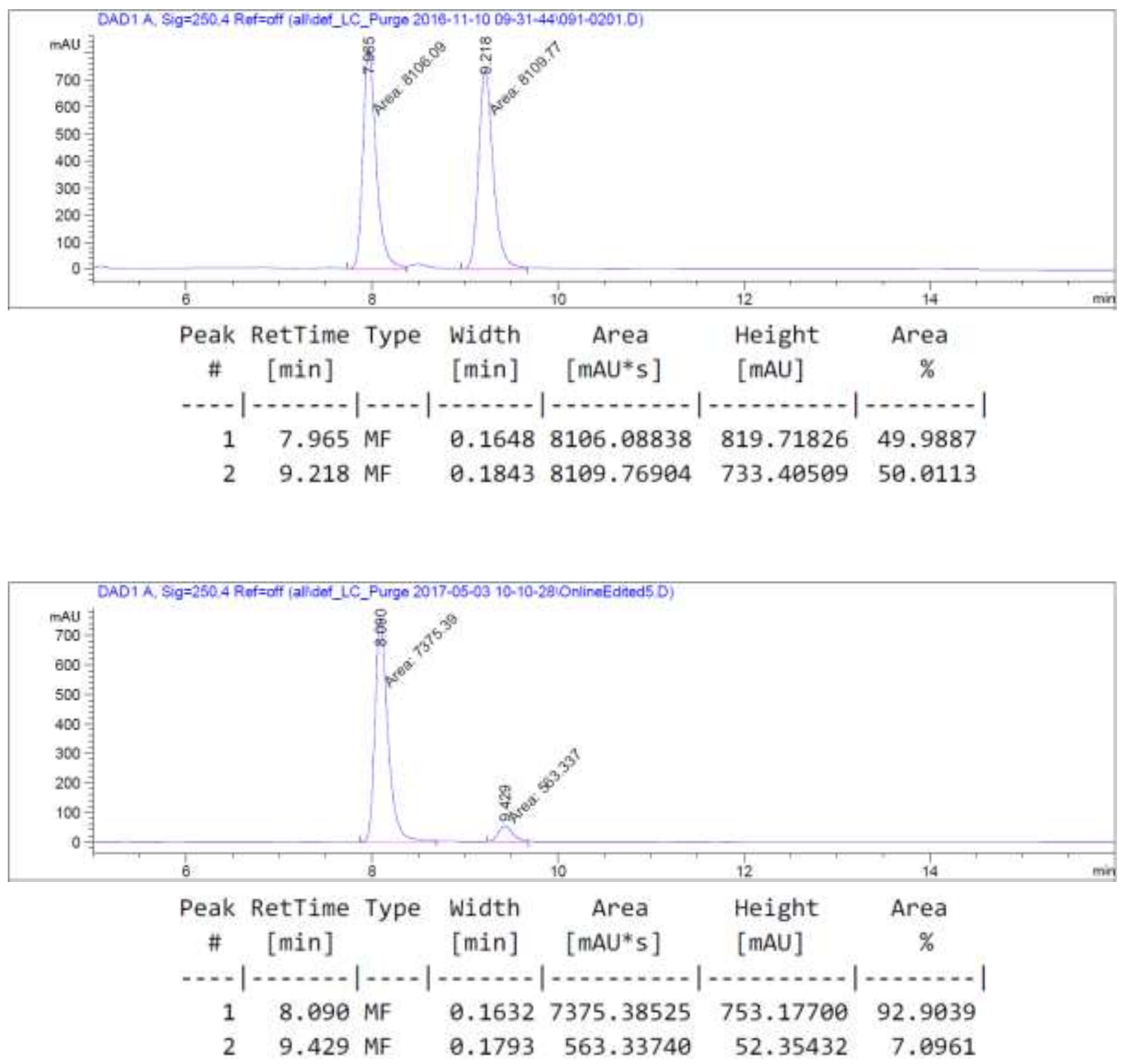


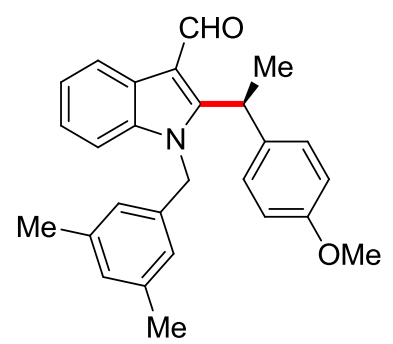

62Ic

$\left(\mathrm{CDCl}_{3}, 300 \mathrm{MHz}\right)$
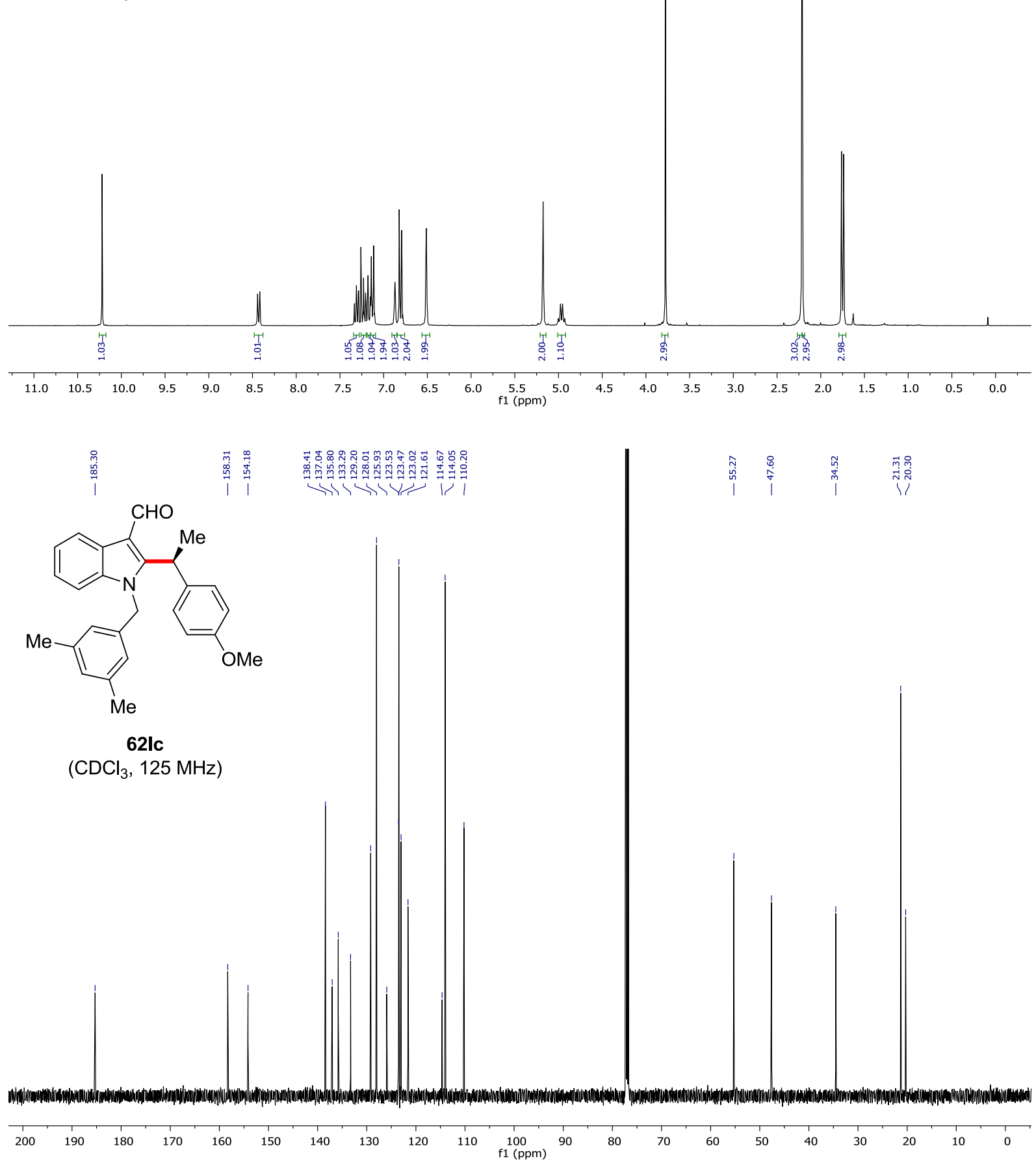


\section{Chiral HPLC of 62lc:}
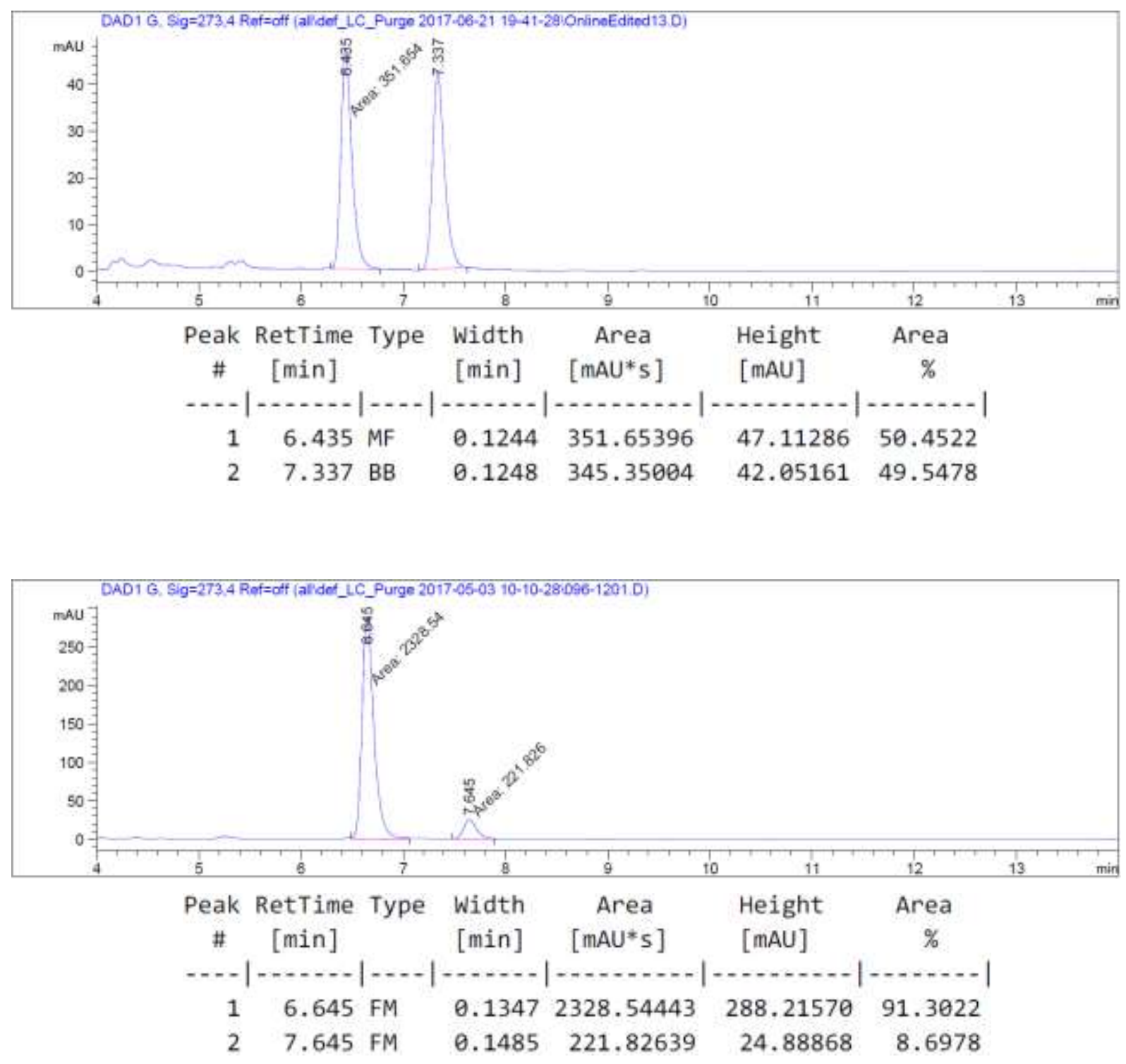


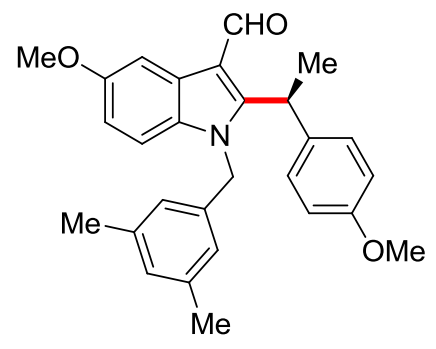

$62 \mathrm{mc}$

$\left(\mathrm{CDCl}_{3}, 300 \mathrm{MHz}\right)$
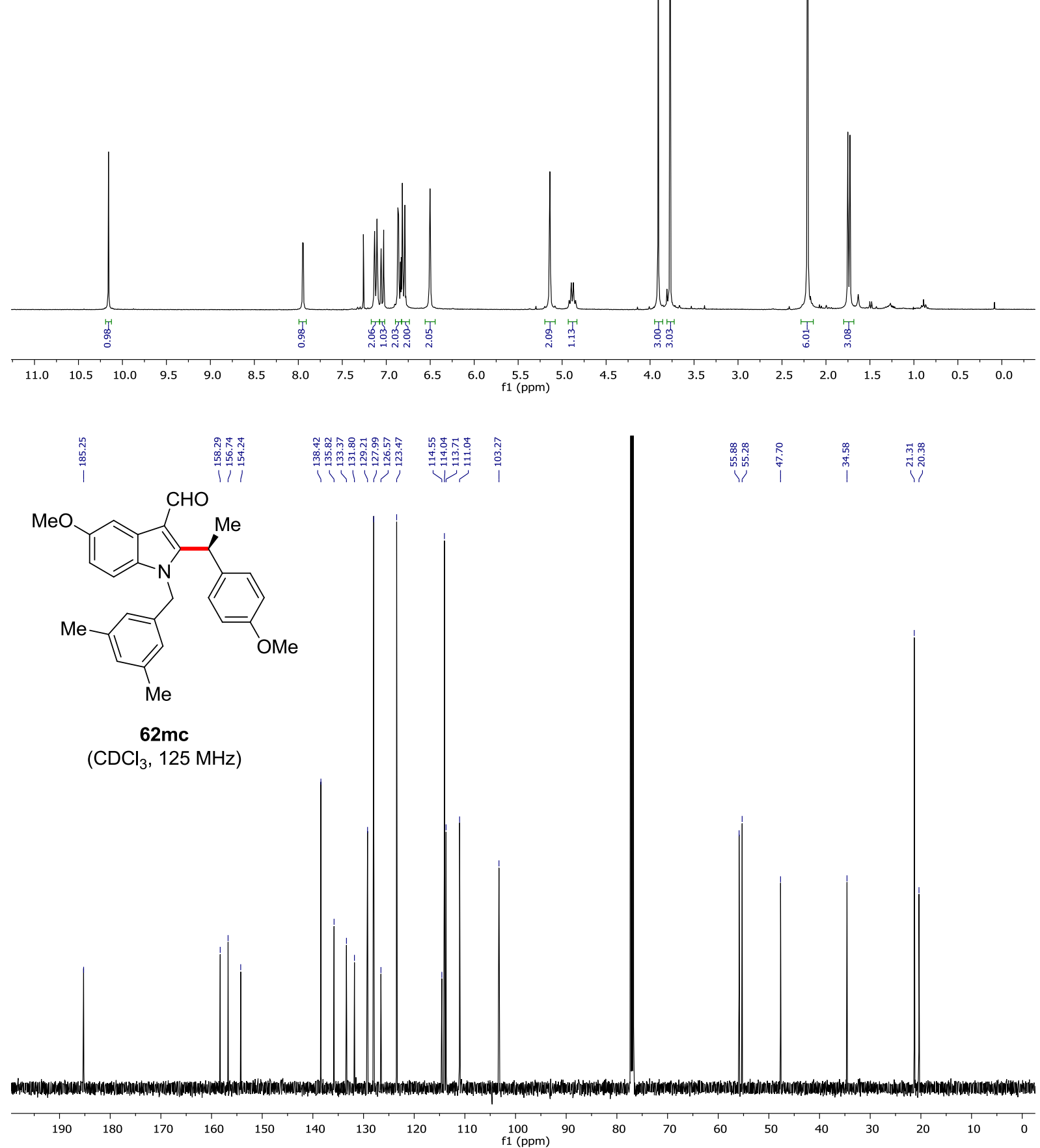


\section{Chiral HPLC of $62 \mathrm{mc}$ :}
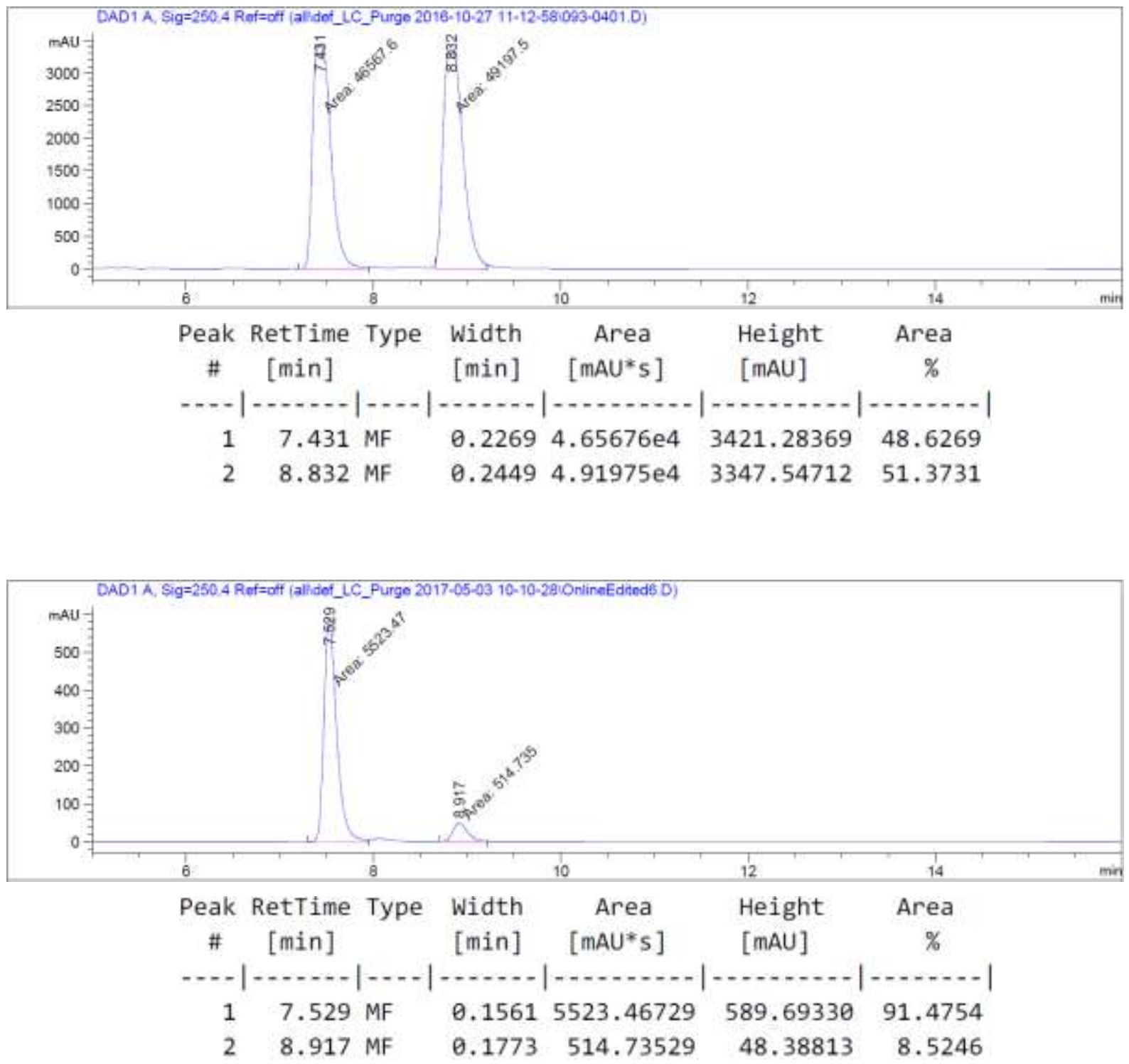


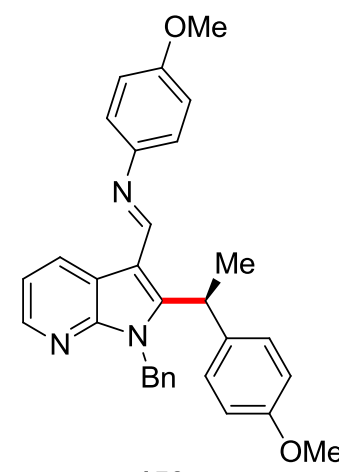

$152 \mathrm{nc}$

$\left(\mathrm{CDCl}_{3}, 600 \mathrm{MHz}\right)$
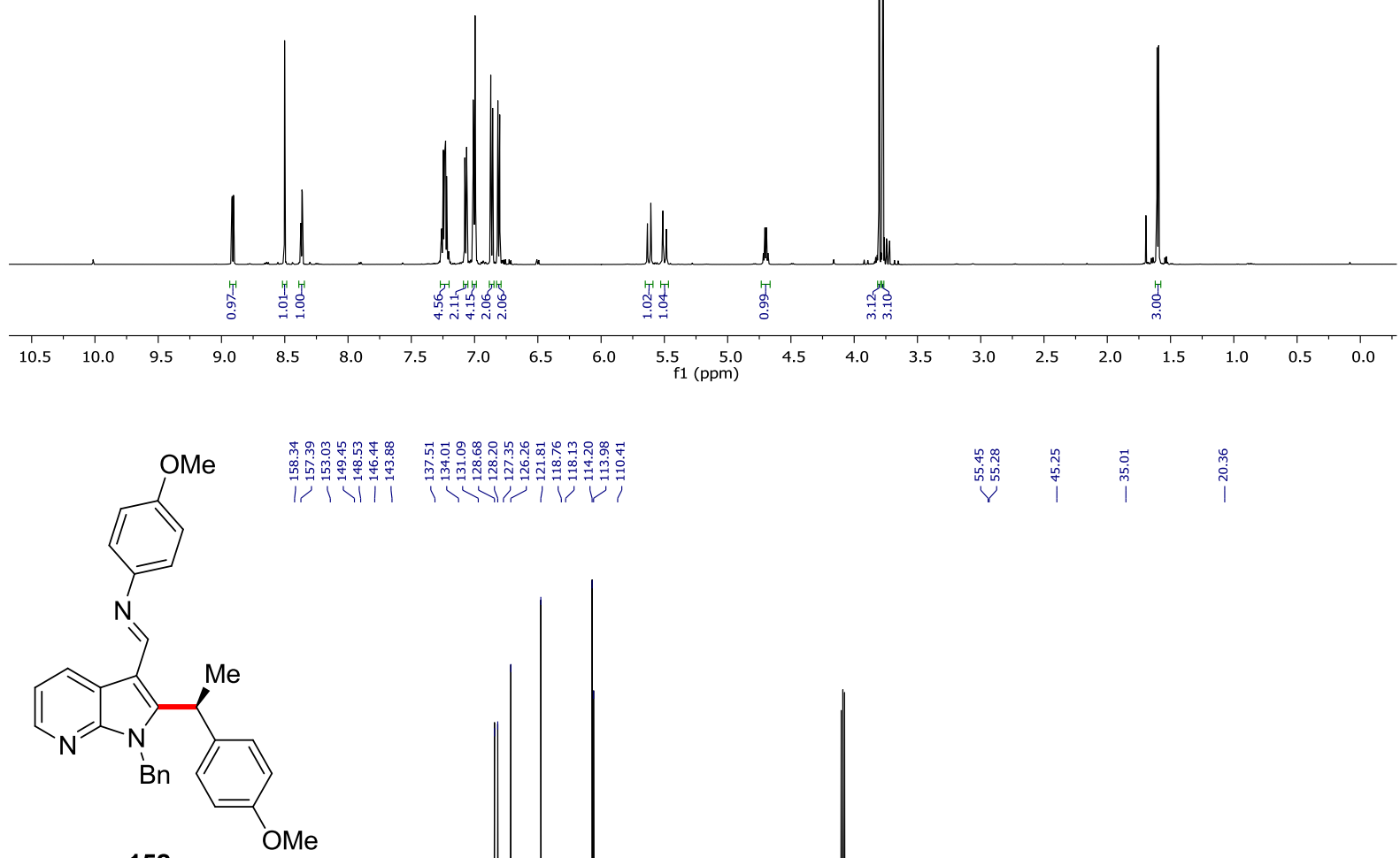

$\left(\mathrm{CDCl}_{3}, 125 \mathrm{MHz}\right)$

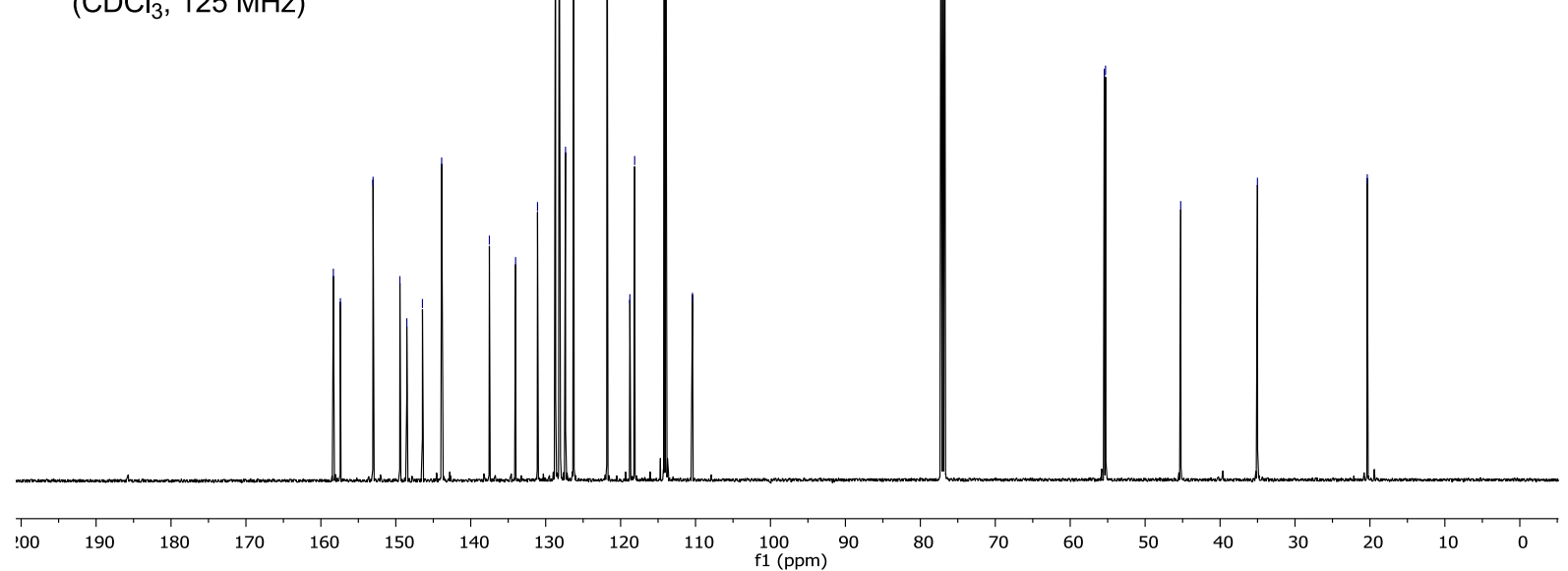




\section{Chiral HPLC of 152nc:}
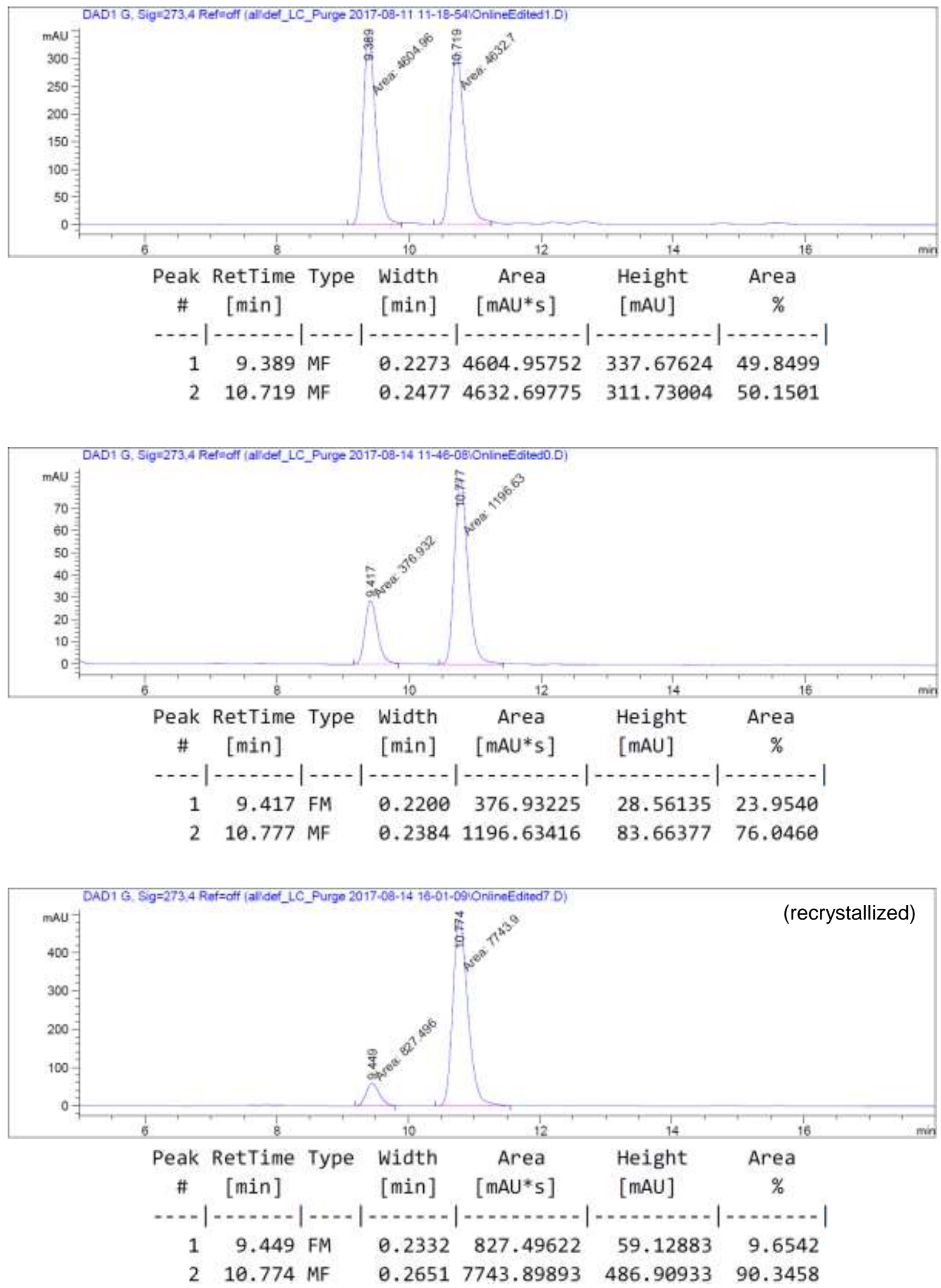

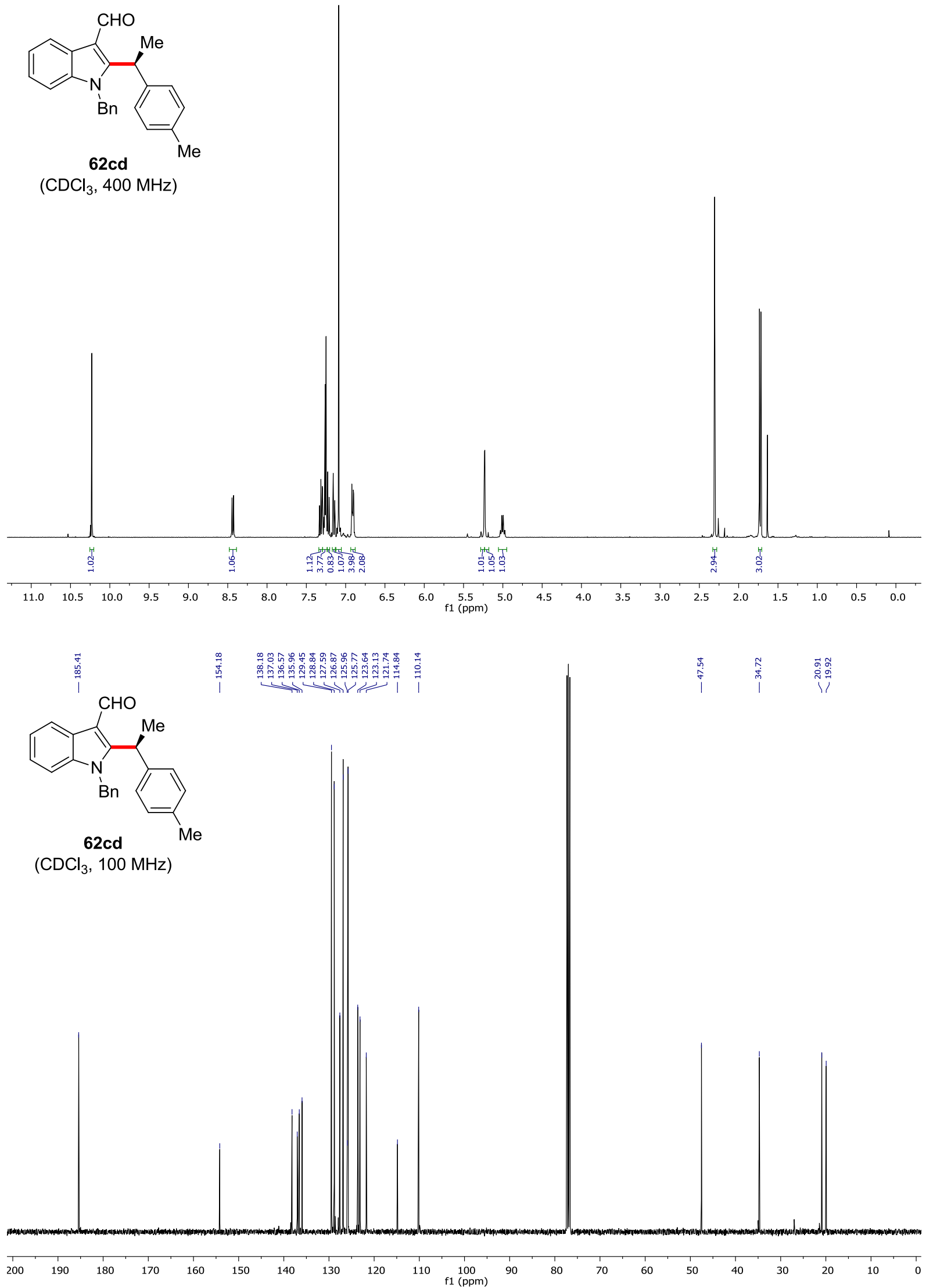


\section{Chiral HPLC of $62 \mathrm{~cd}$ :}
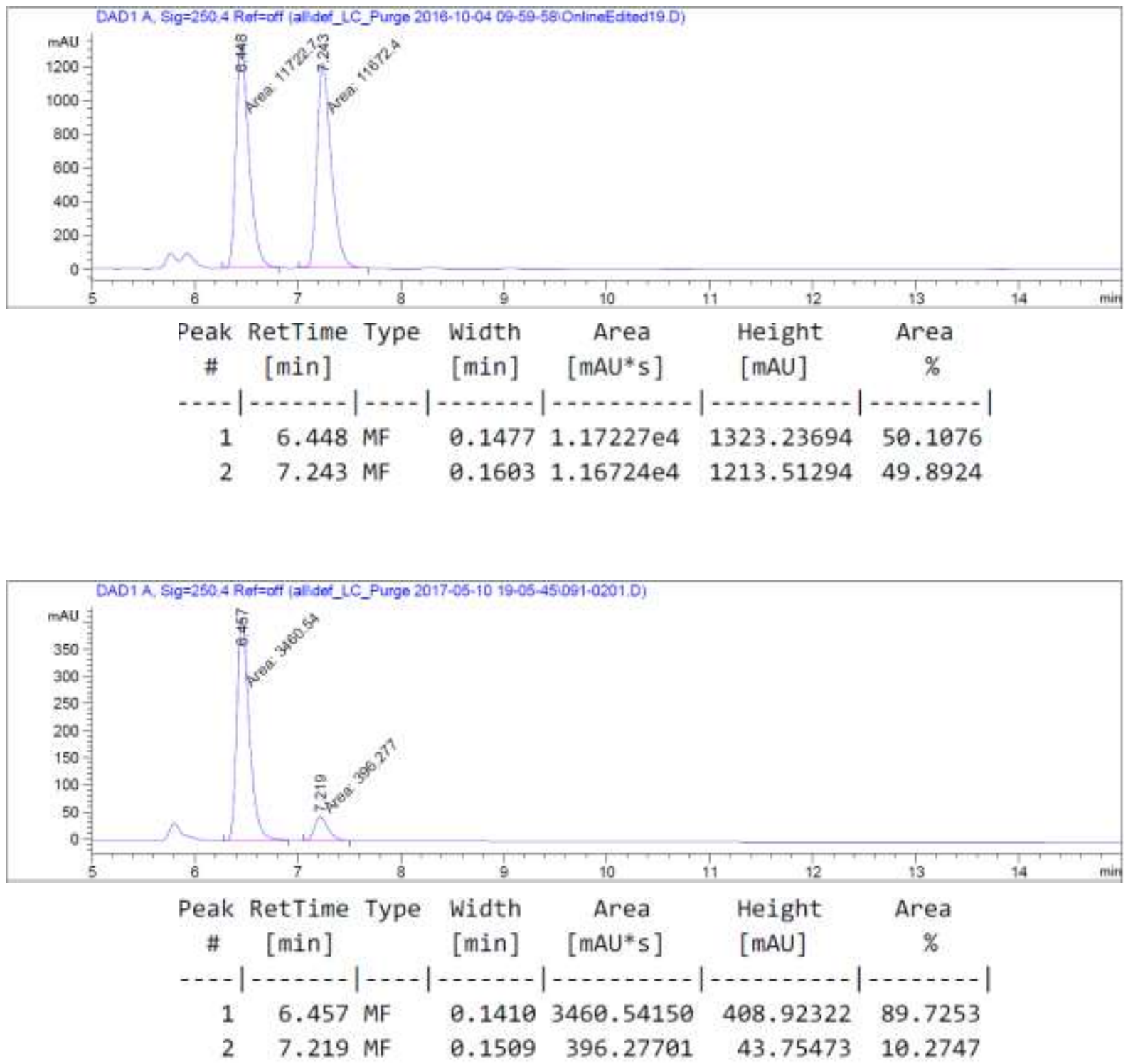


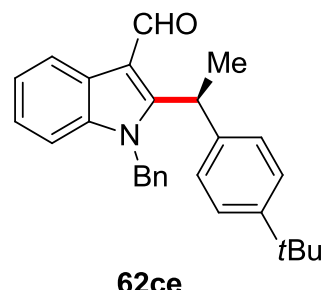

$\left(\mathrm{CDCl}_{3}, 300 \mathrm{MHz}\right)$
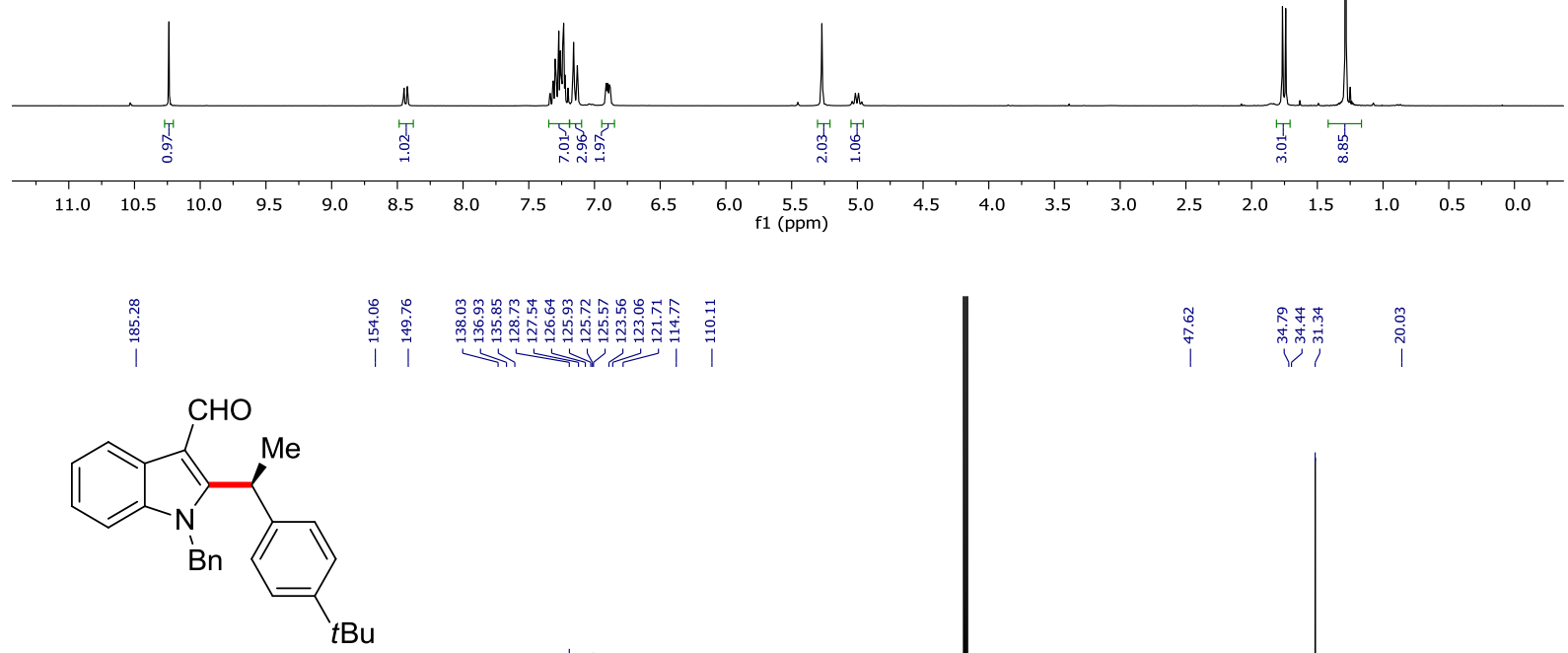

62ce

$\left(\mathrm{CDCl}_{3}, 125 \mathrm{MHz}\right)$

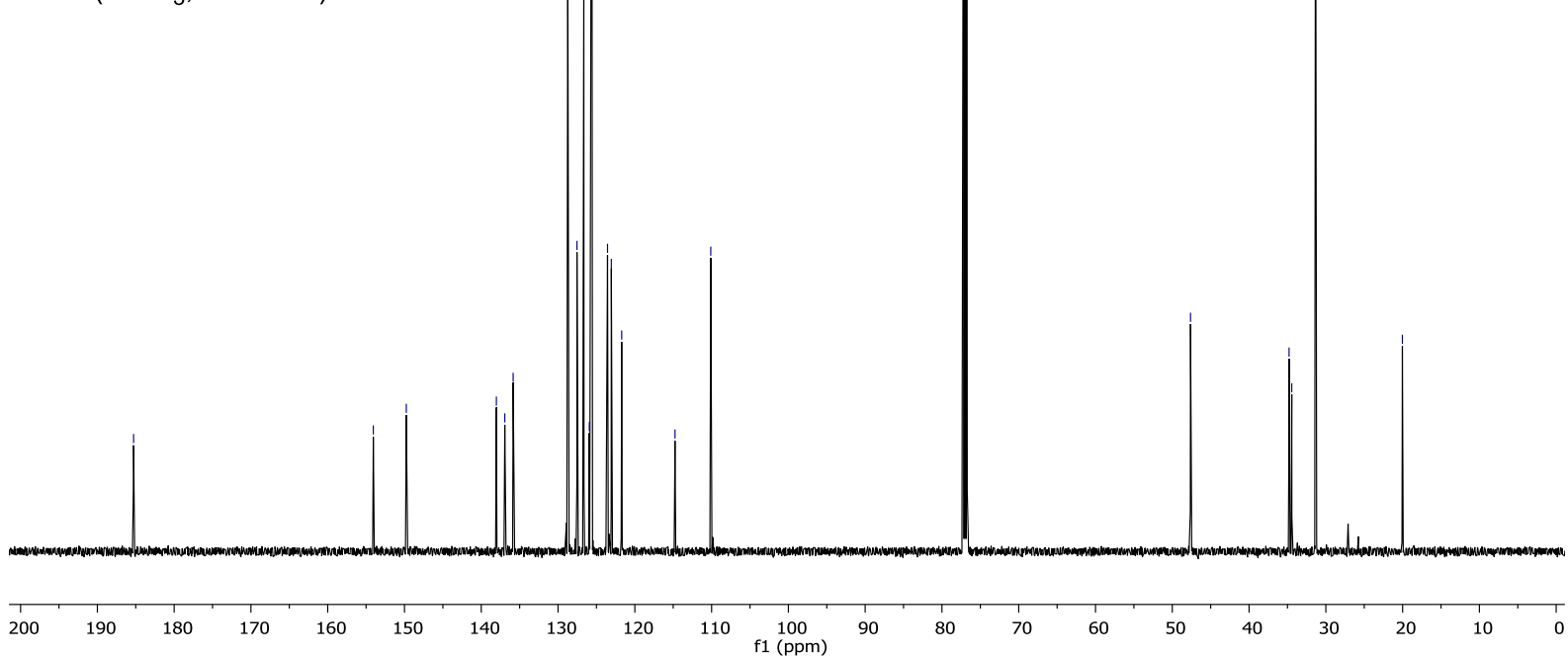




\section{Chiral HPLC of 62ce:}
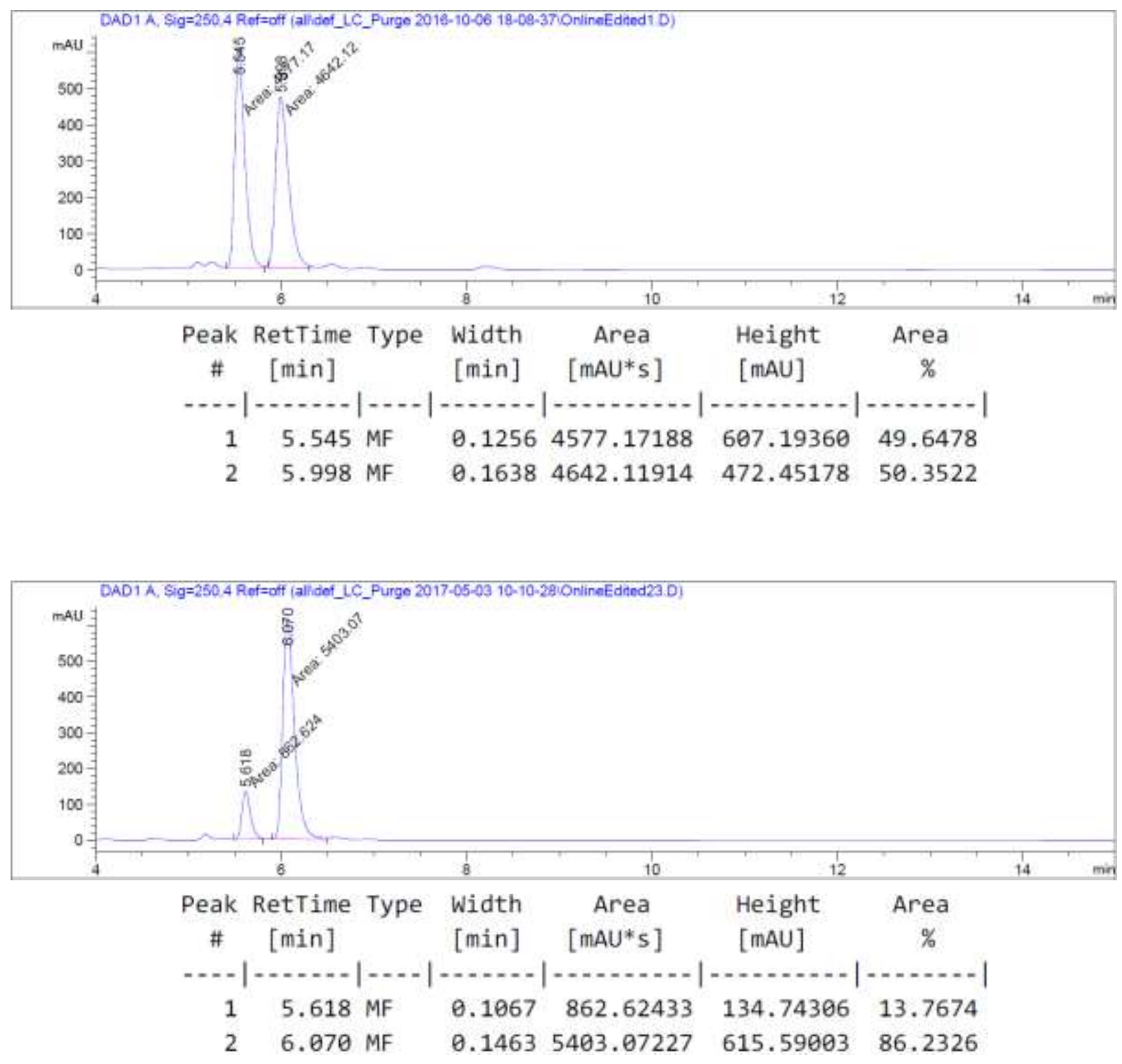


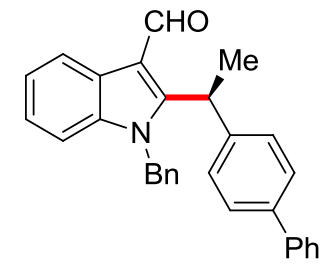

62cf

$\left(\mathrm{CDCl}_{3}, 300 \mathrm{MHz}\right)$
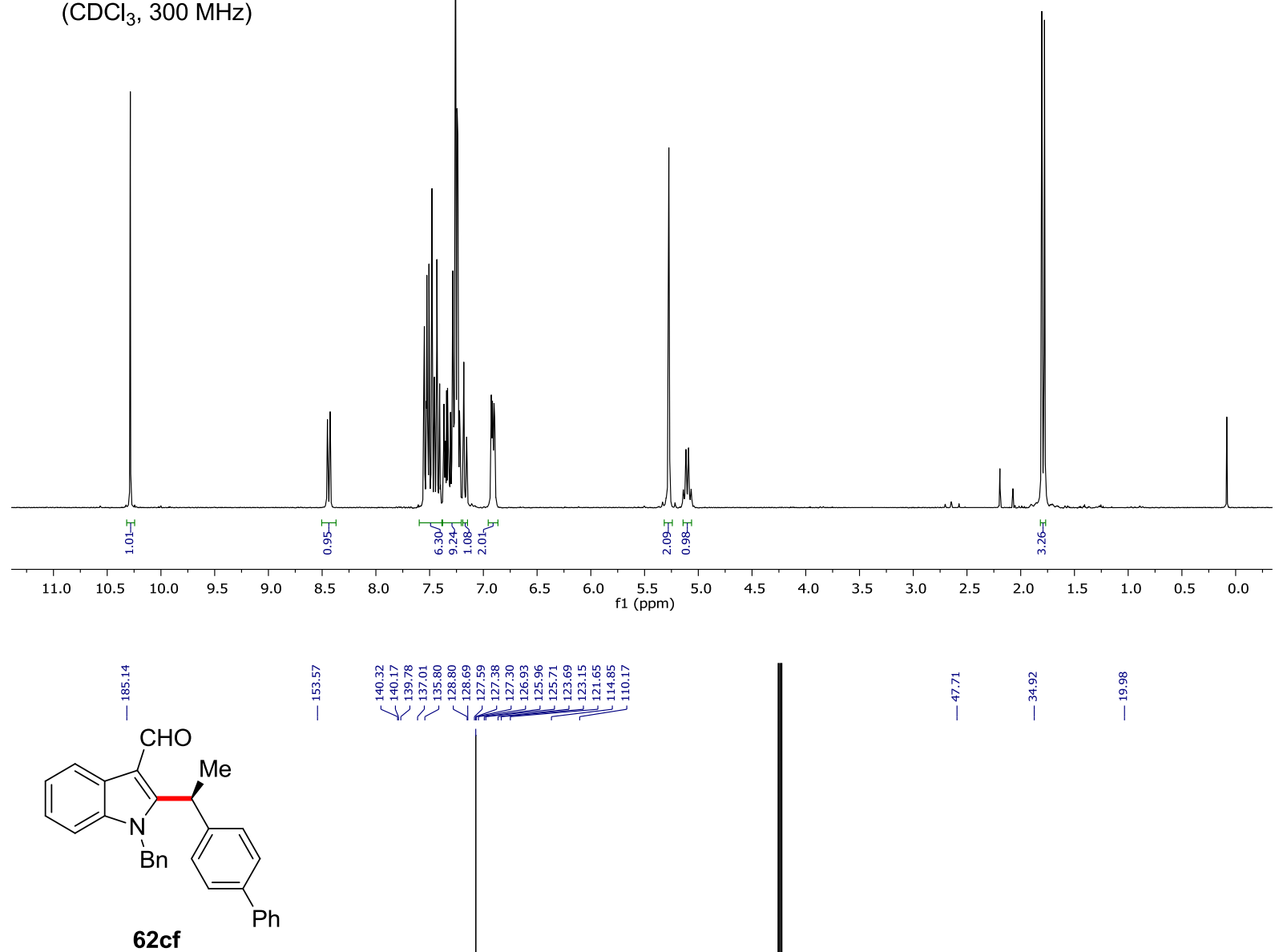

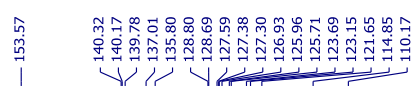

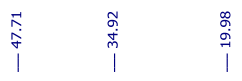

$\left(\mathrm{CDCl}_{3}, 125 \mathrm{MHz}\right)$

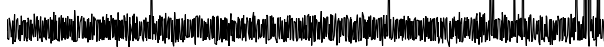

$\begin{array}{llllllllllllllllllll}200 & 190 & 180 & 170 & 160 & 150 & 140 & 130 & 120 & 110 & 100 & 90 & 80 & 70 & 60 & 50 & 40 & 30 & 20 & 10\end{array}$ 


\section{Chiral HPLC of 62cf:}
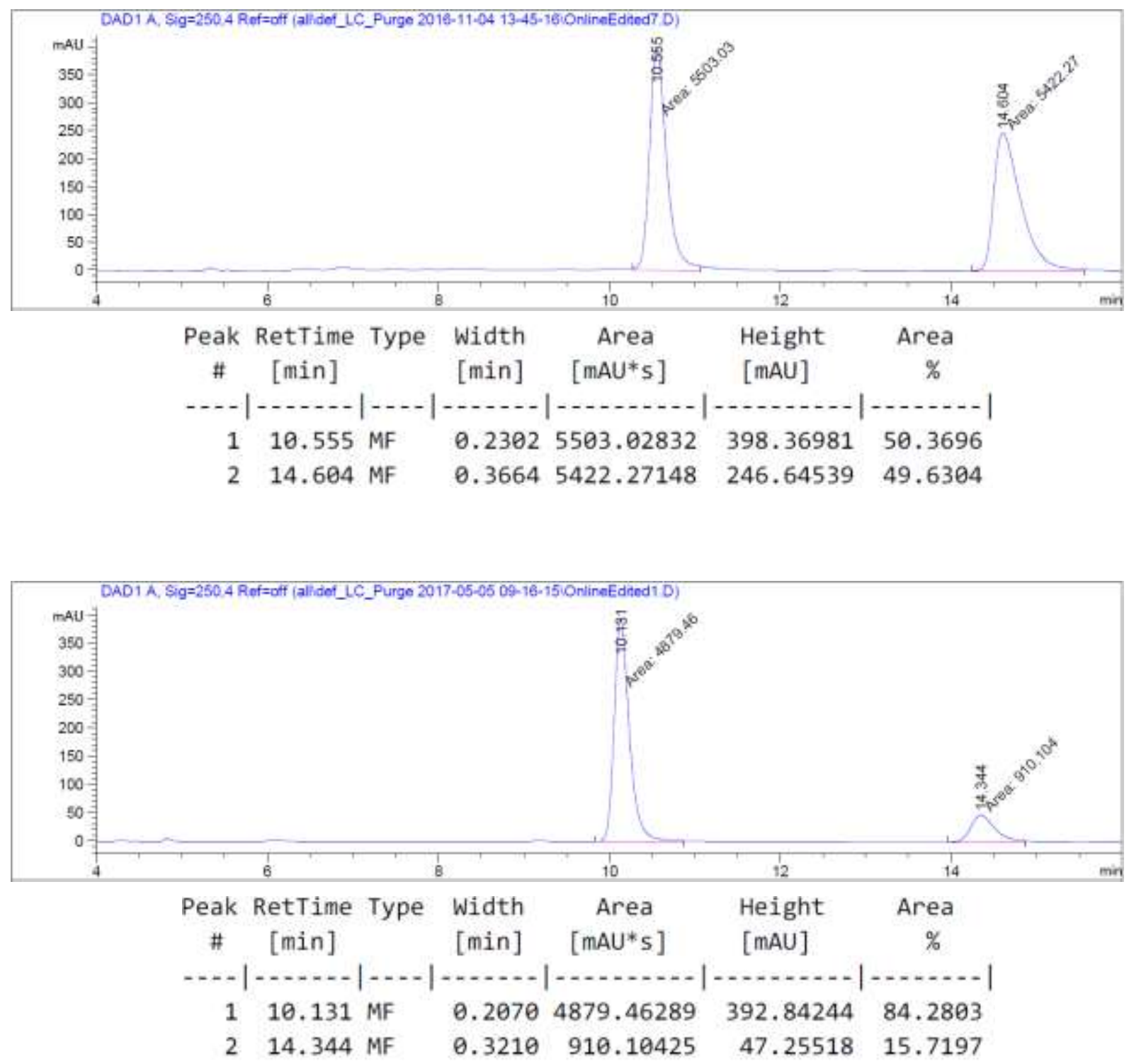

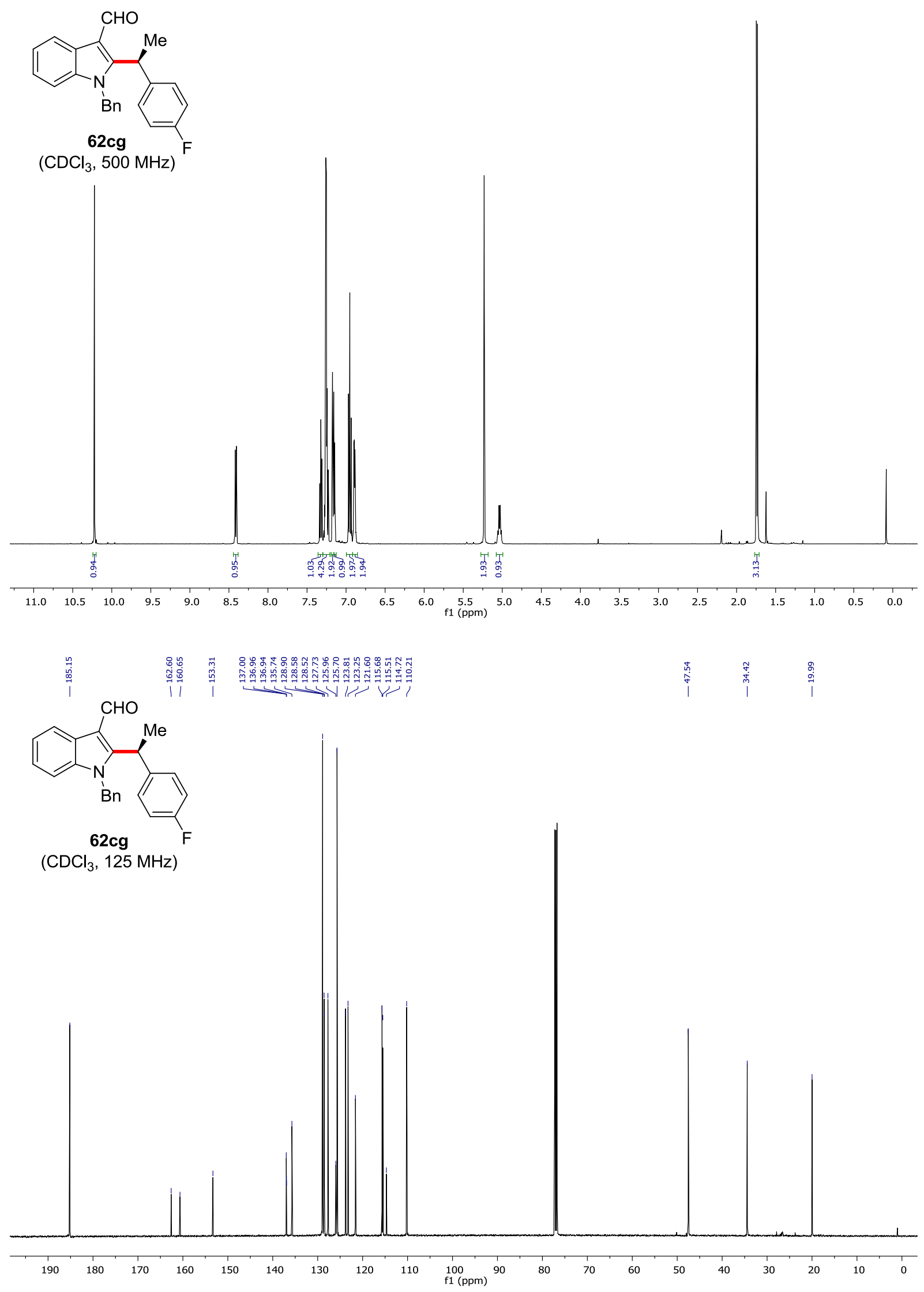


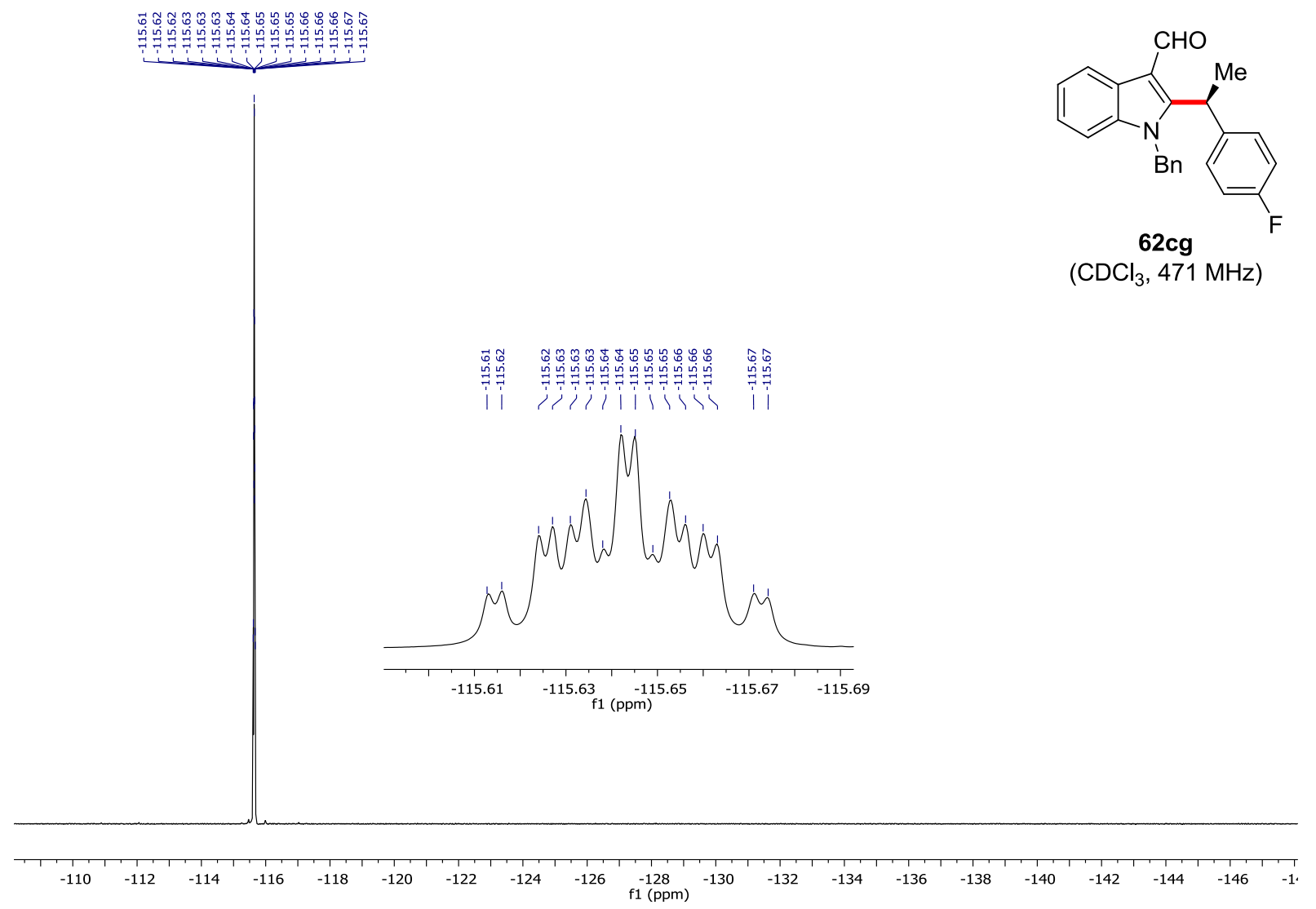




\section{Chiral HPLC of $62 \mathrm{cg}$ :}
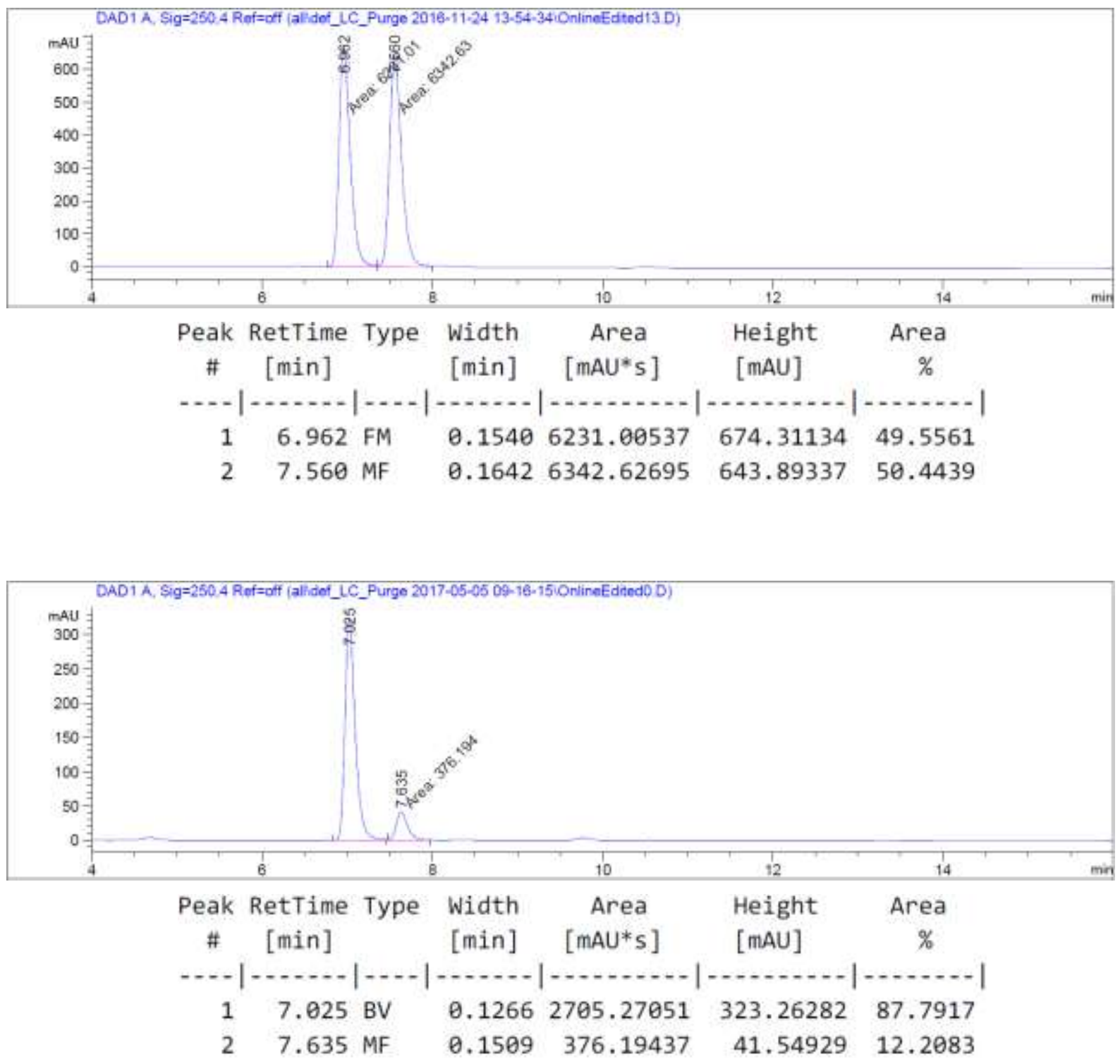


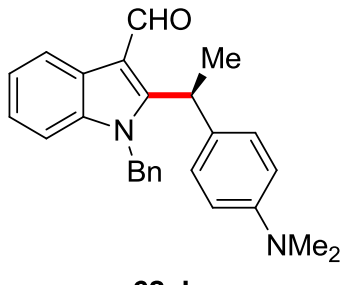

$\left(\mathrm{CDCl}_{3}, 300 \mathrm{MHz}\right)$
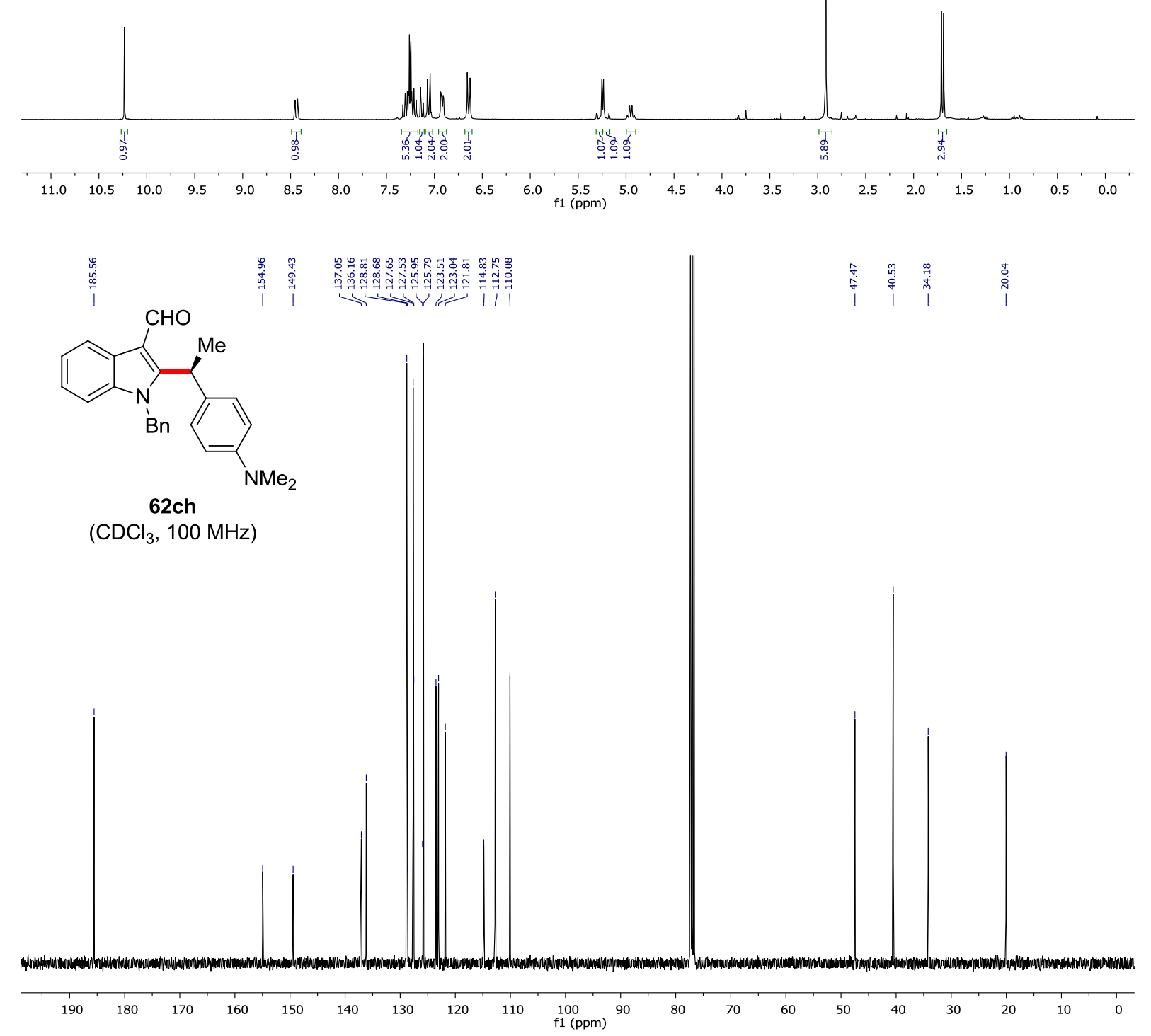


\section{Chiral HPLC of $62 \mathrm{ch}$ :}
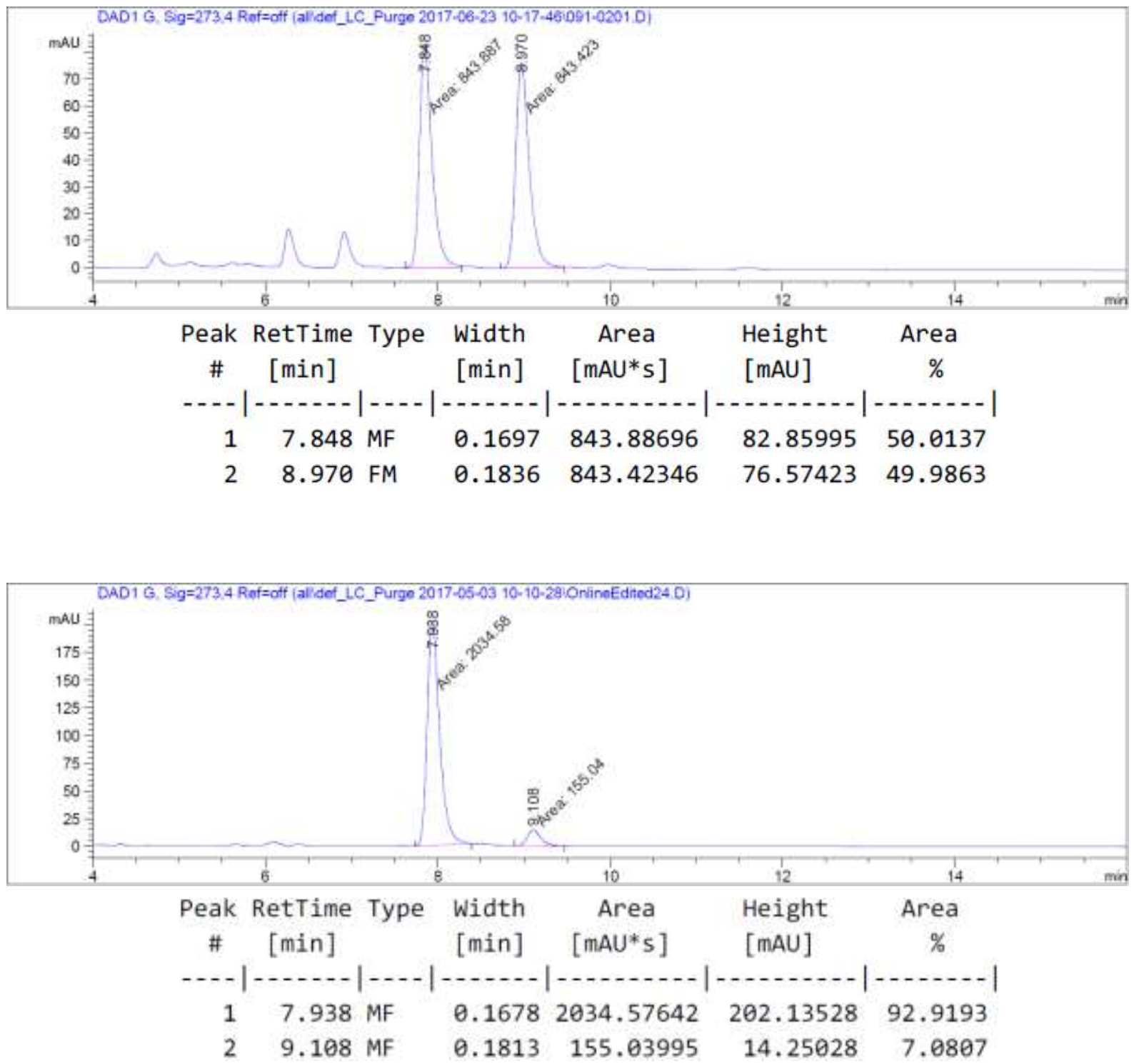

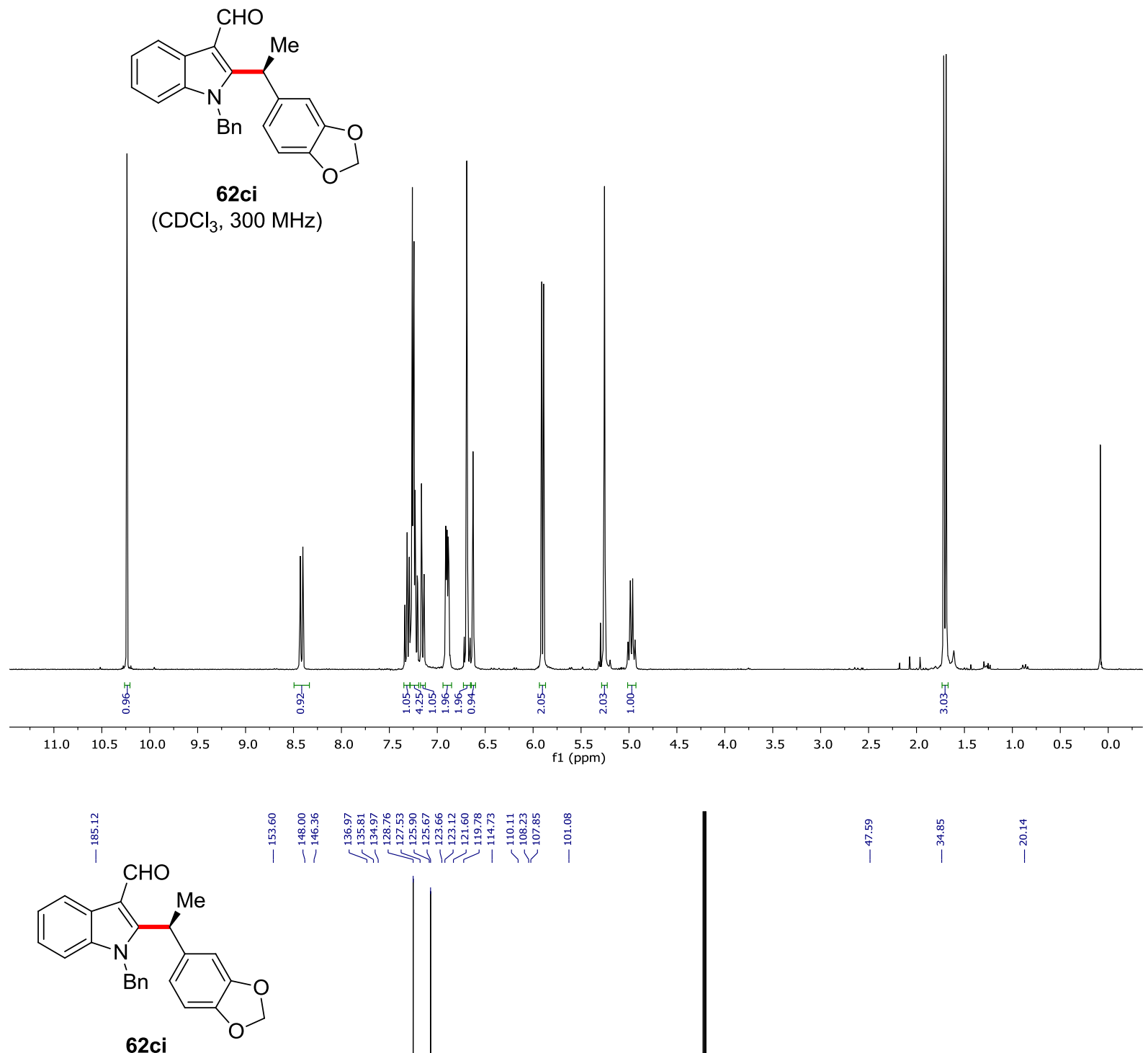

$\left(\mathrm{CDCl}_{3}, 125 \mathrm{MHz}\right)$

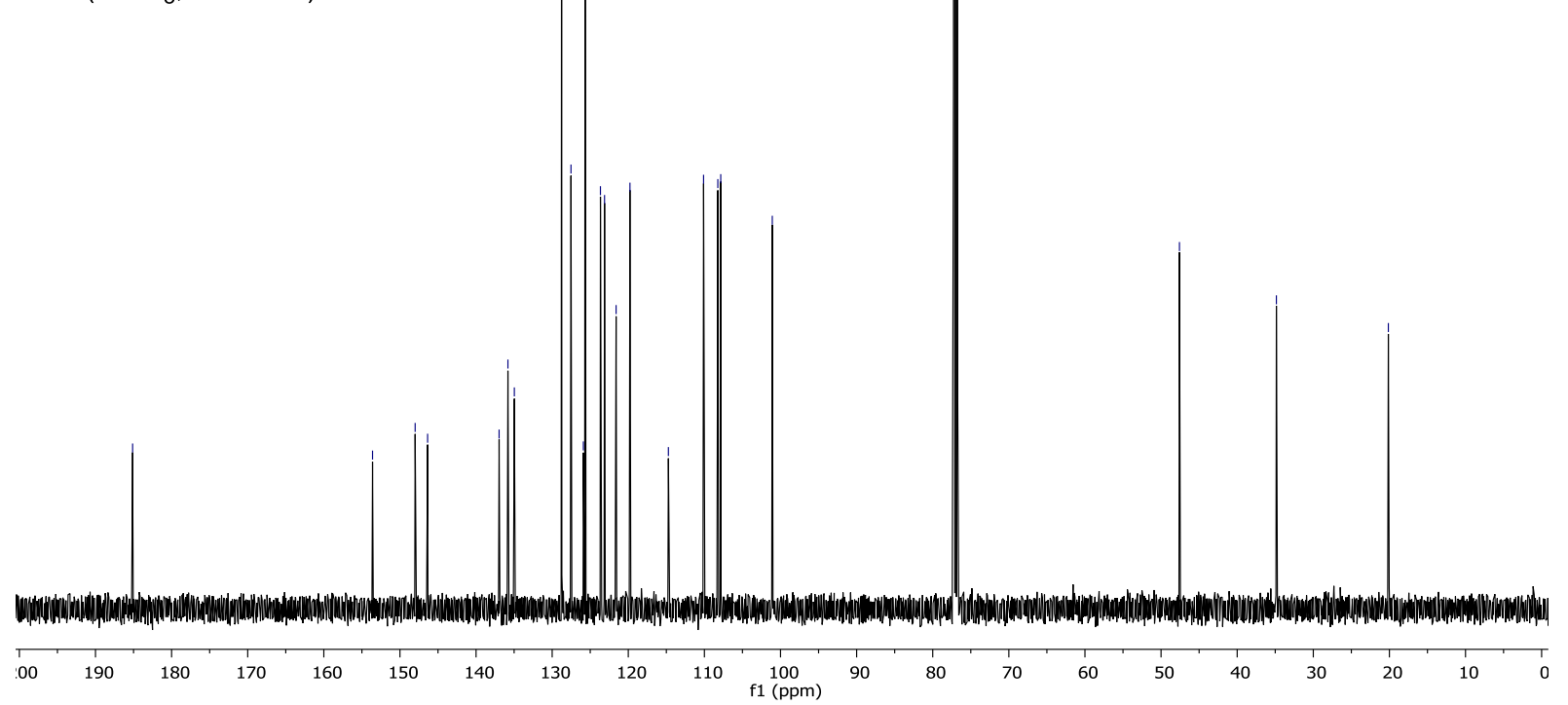




\section{Chiral HPLC of 62ci:}
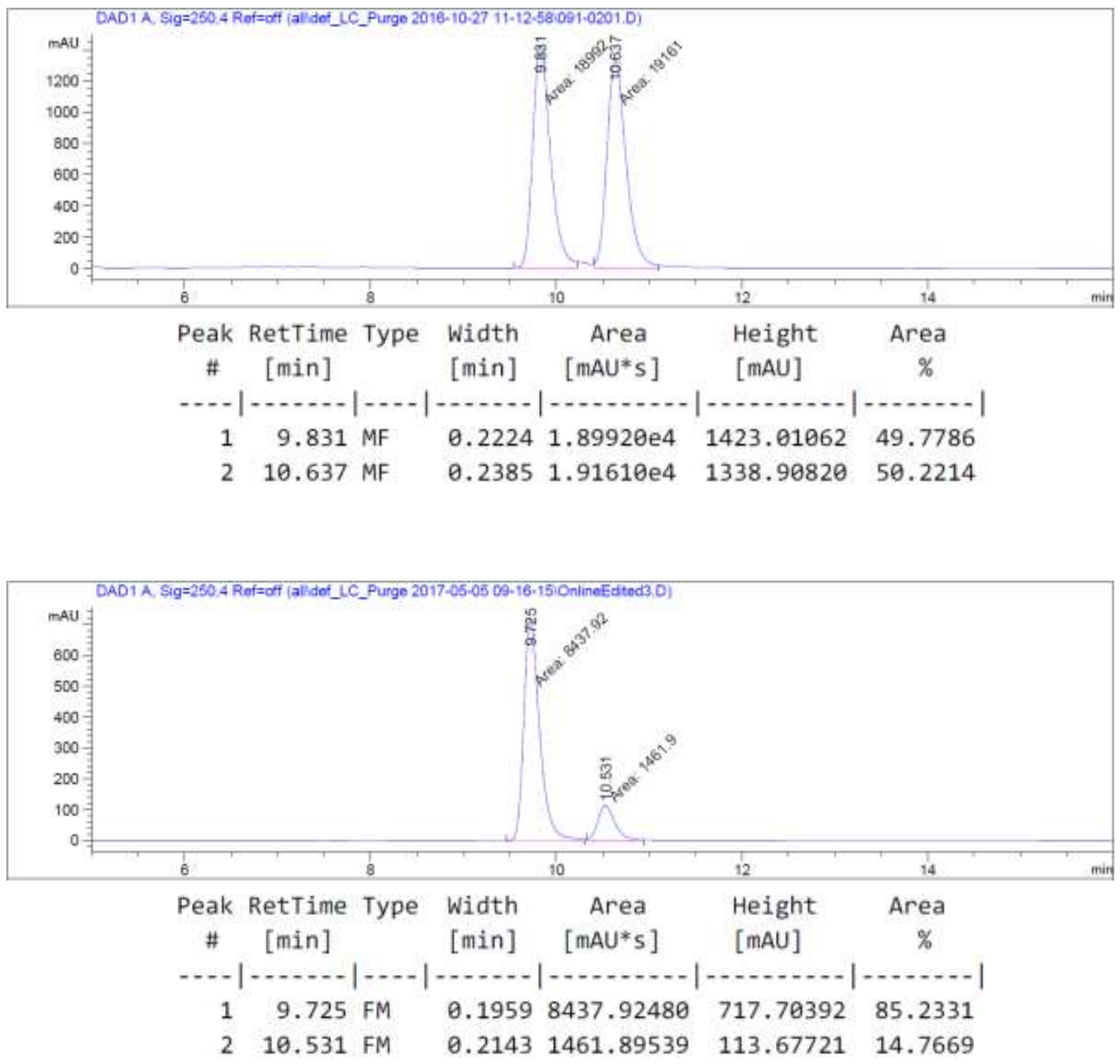


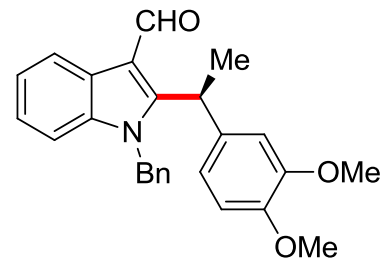

62cj

$\left(\mathrm{CDCl}_{3}, 300 \mathrm{MHz}\right)$
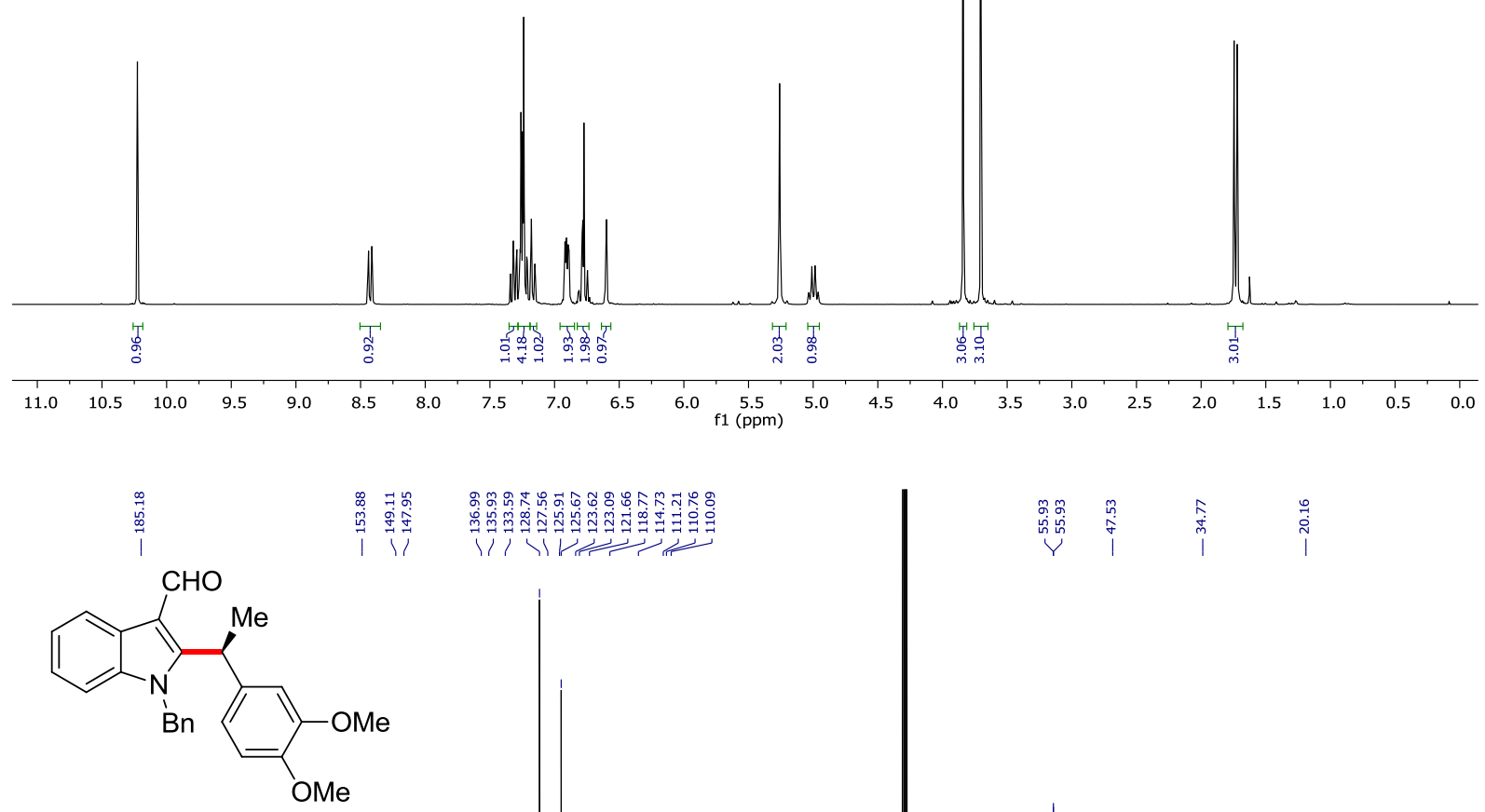

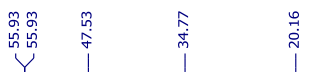

62cj

$\left(\mathrm{CDCl}_{3}, 125 \mathrm{MHz}\right)$

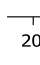

200

$\begin{array}{llllll}190 & 180 & 170 & 160 & 150 & 140\end{array}$

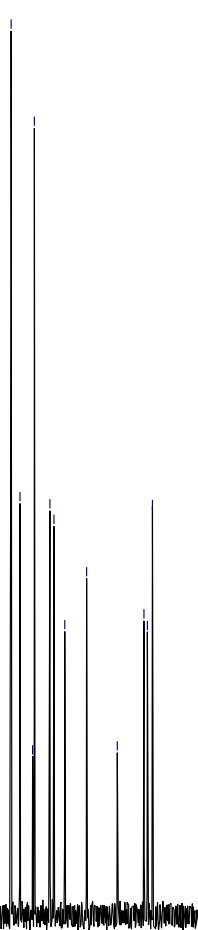

|||||||||||| 


\section{Chiral HPLC of 62cj:}
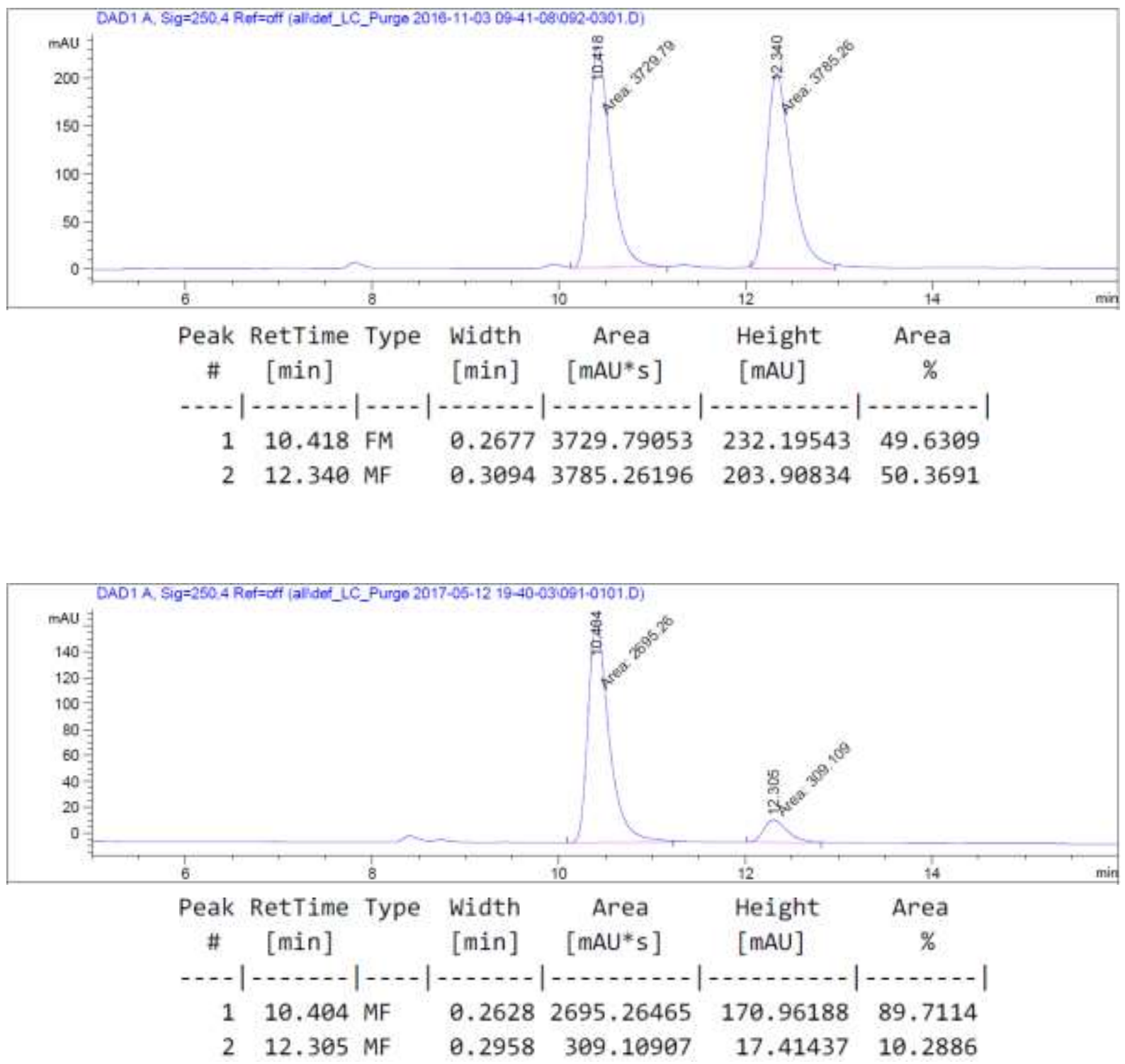


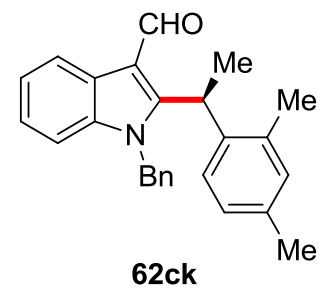

$\left(\mathrm{CDCl}_{3}, 500 \mathrm{MHz}\right)$
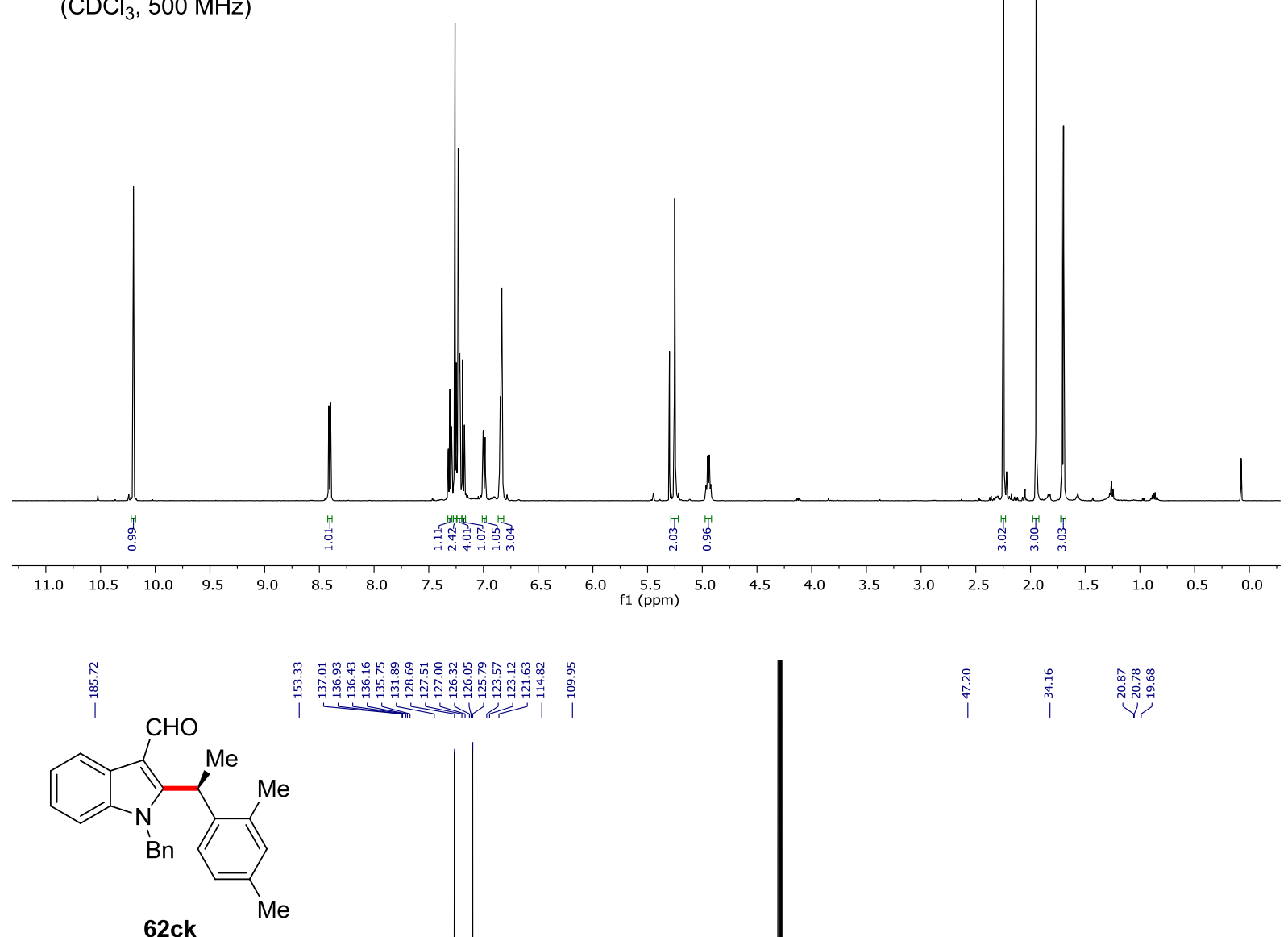

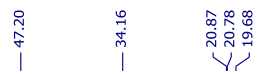

$\left(\mathrm{CDCl}_{3}, 125 \mathrm{MHz}\right)$

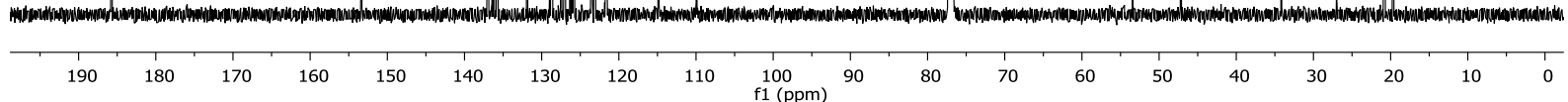




\section{Chiral HPLC of 62ck:}
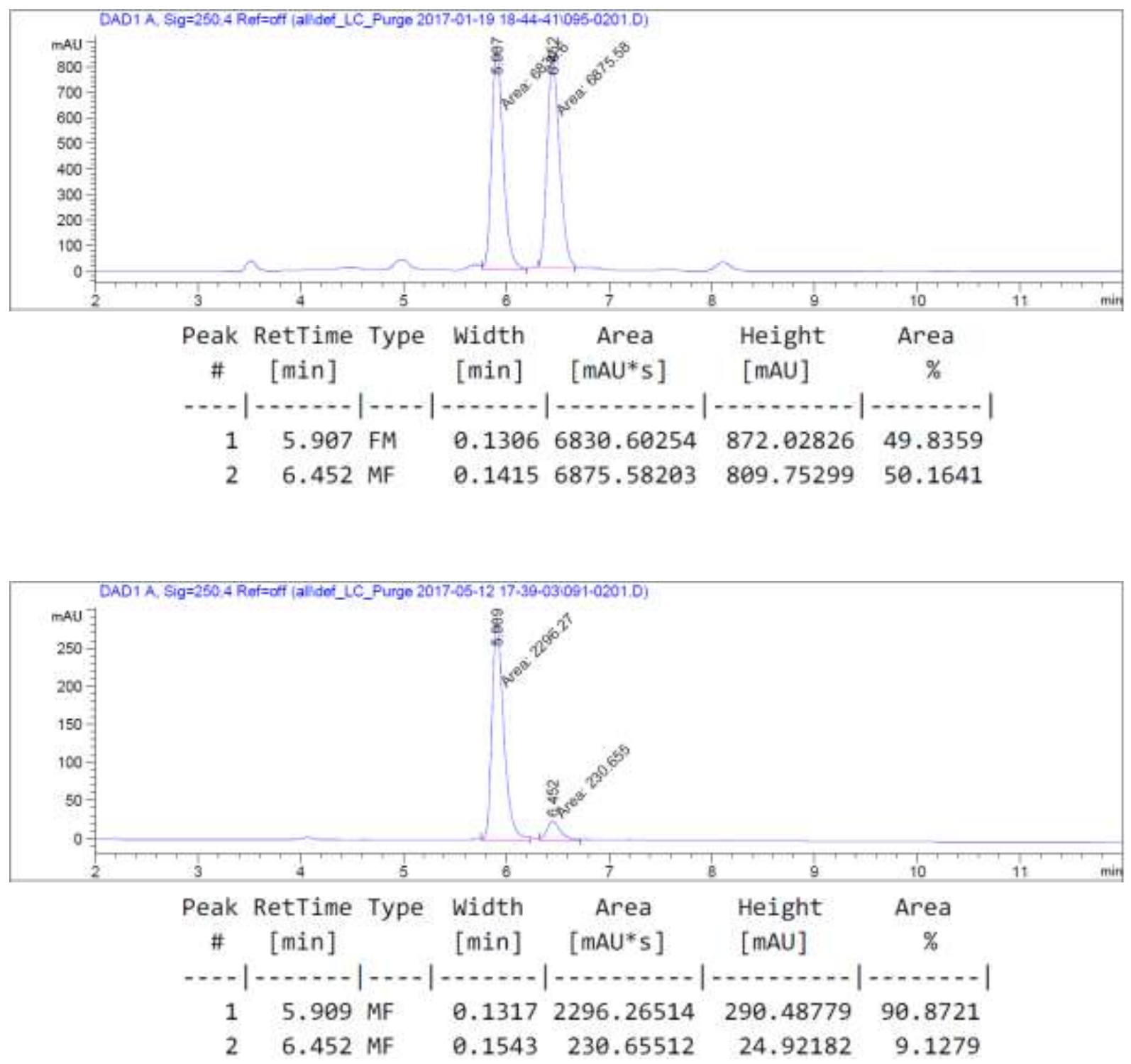


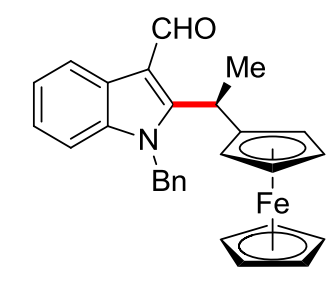

192ca

$\left(\mathrm{CDCl}_{3}, 300 \mathrm{MHz}\right)$
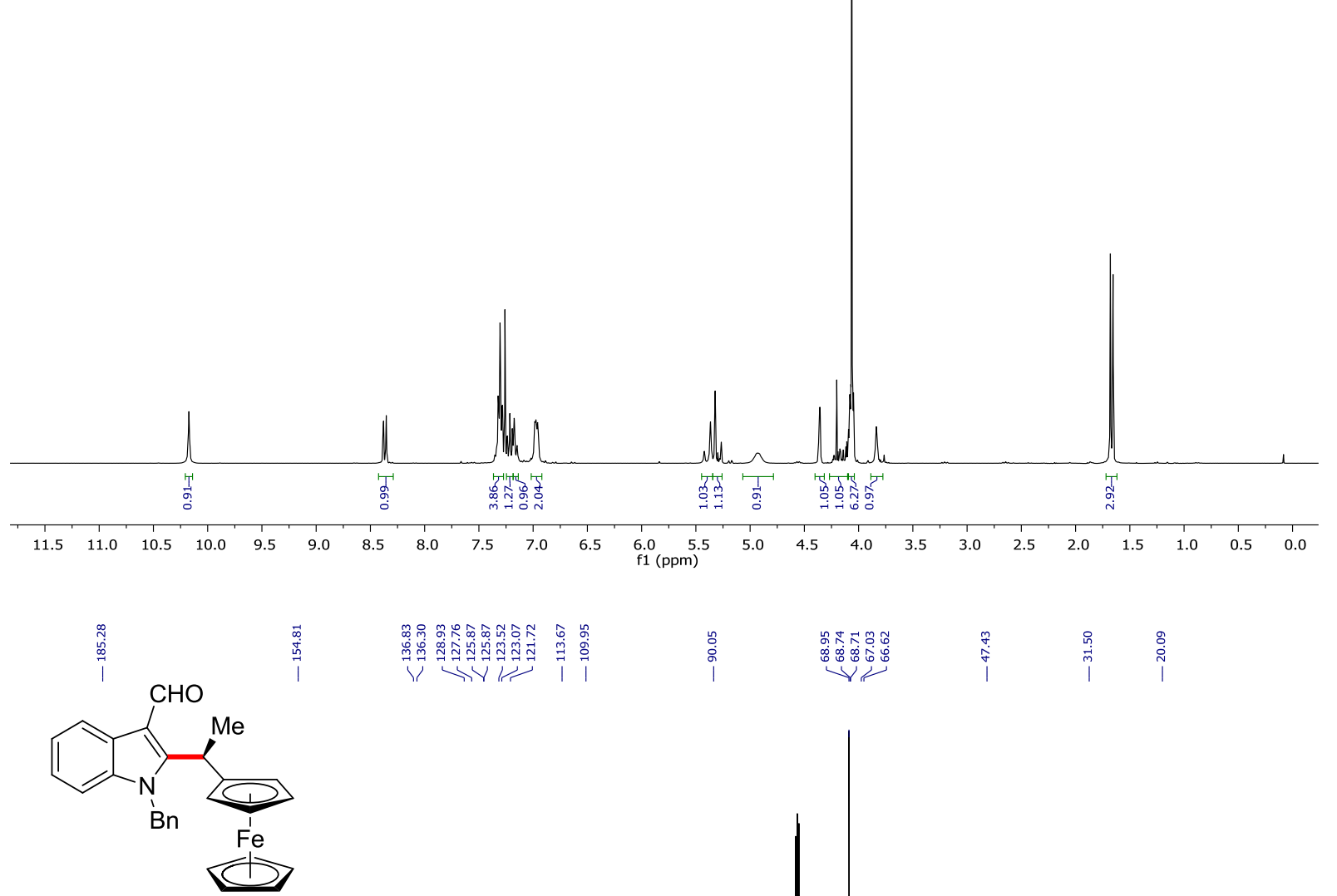

192ca

$\left(\mathrm{CDCl}_{3}, 125 \mathrm{MHz}\right)$

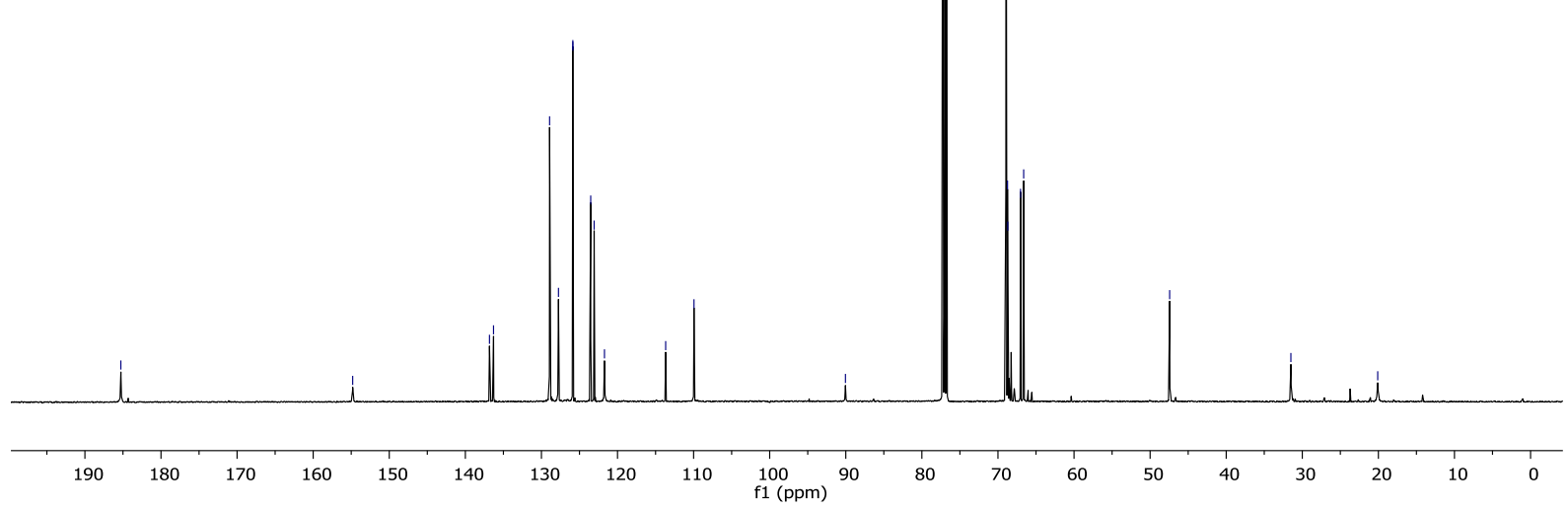




\section{Chiral HPLC of 192ca:}
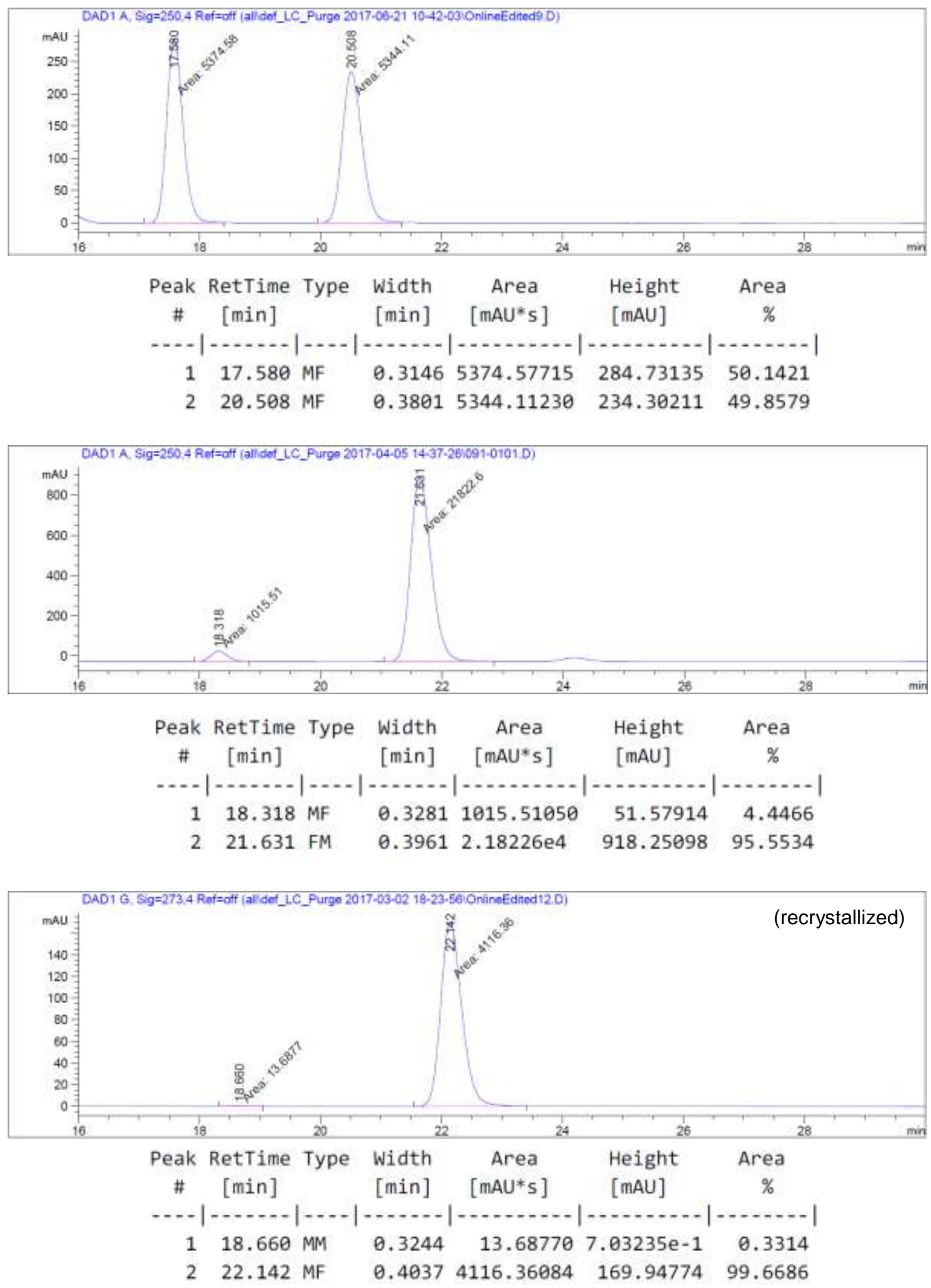

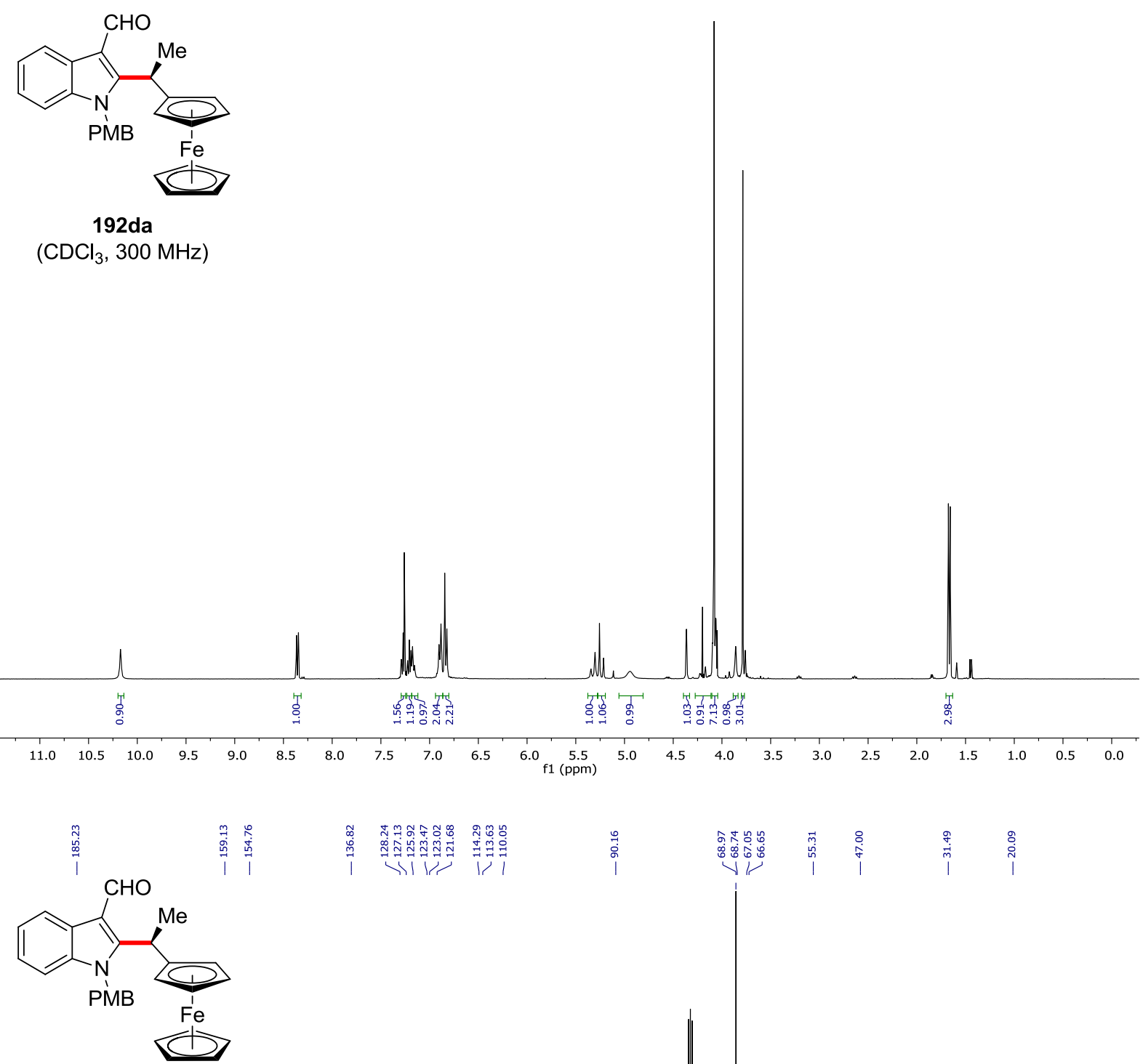

$\left(\mathrm{CDCl}_{3}, 100 \mathrm{MHz}\right)$

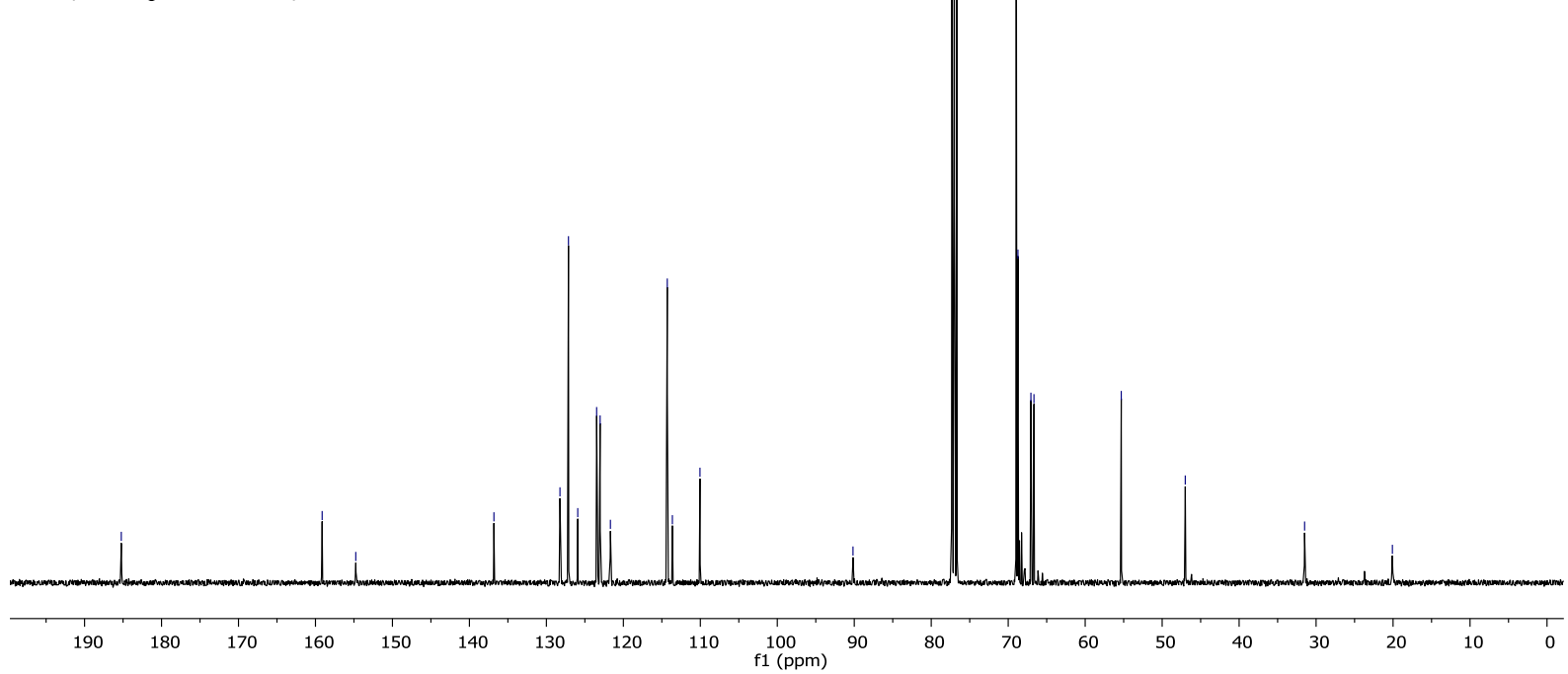




\section{Chiral HPLC of 192da:}
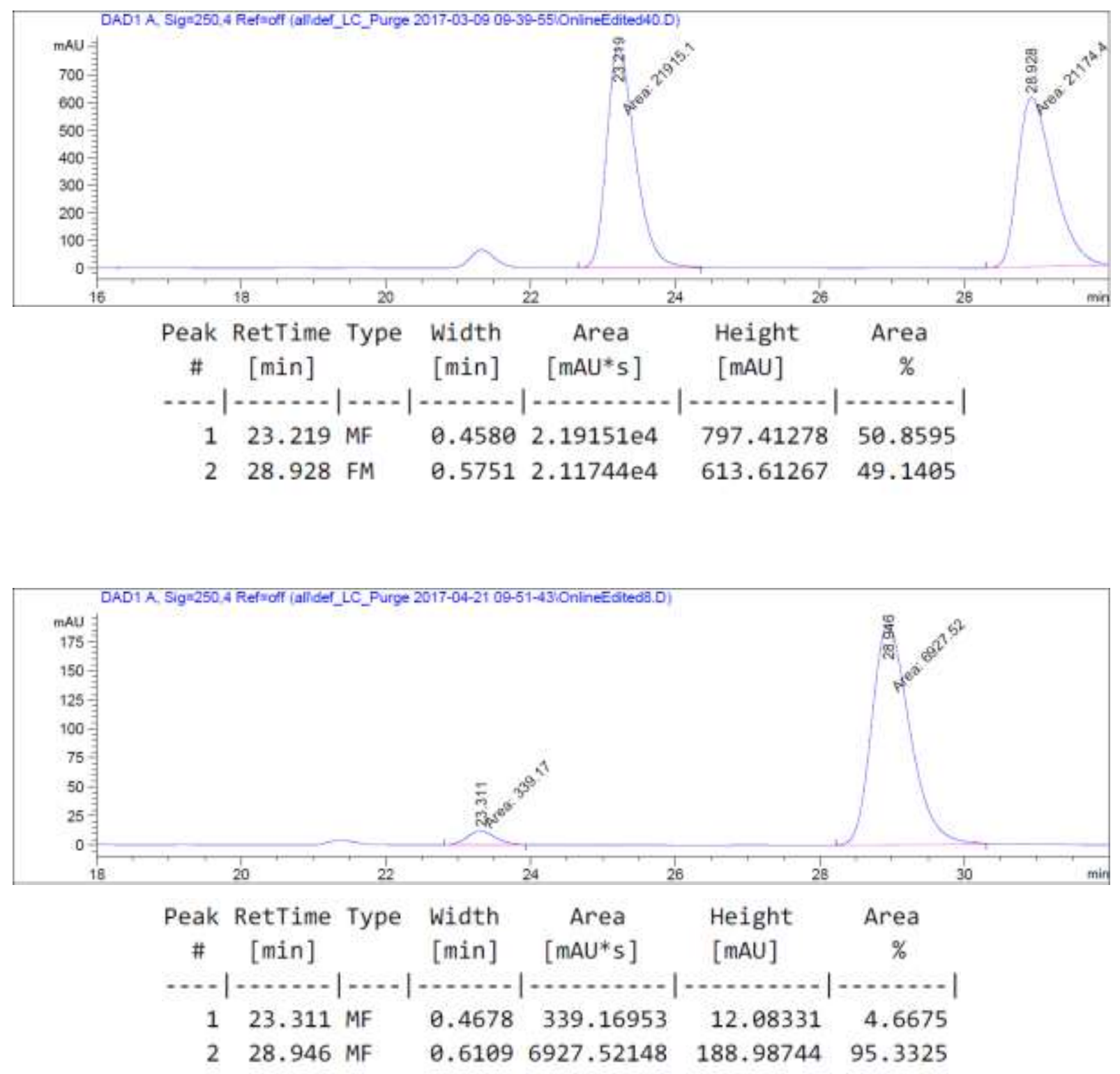

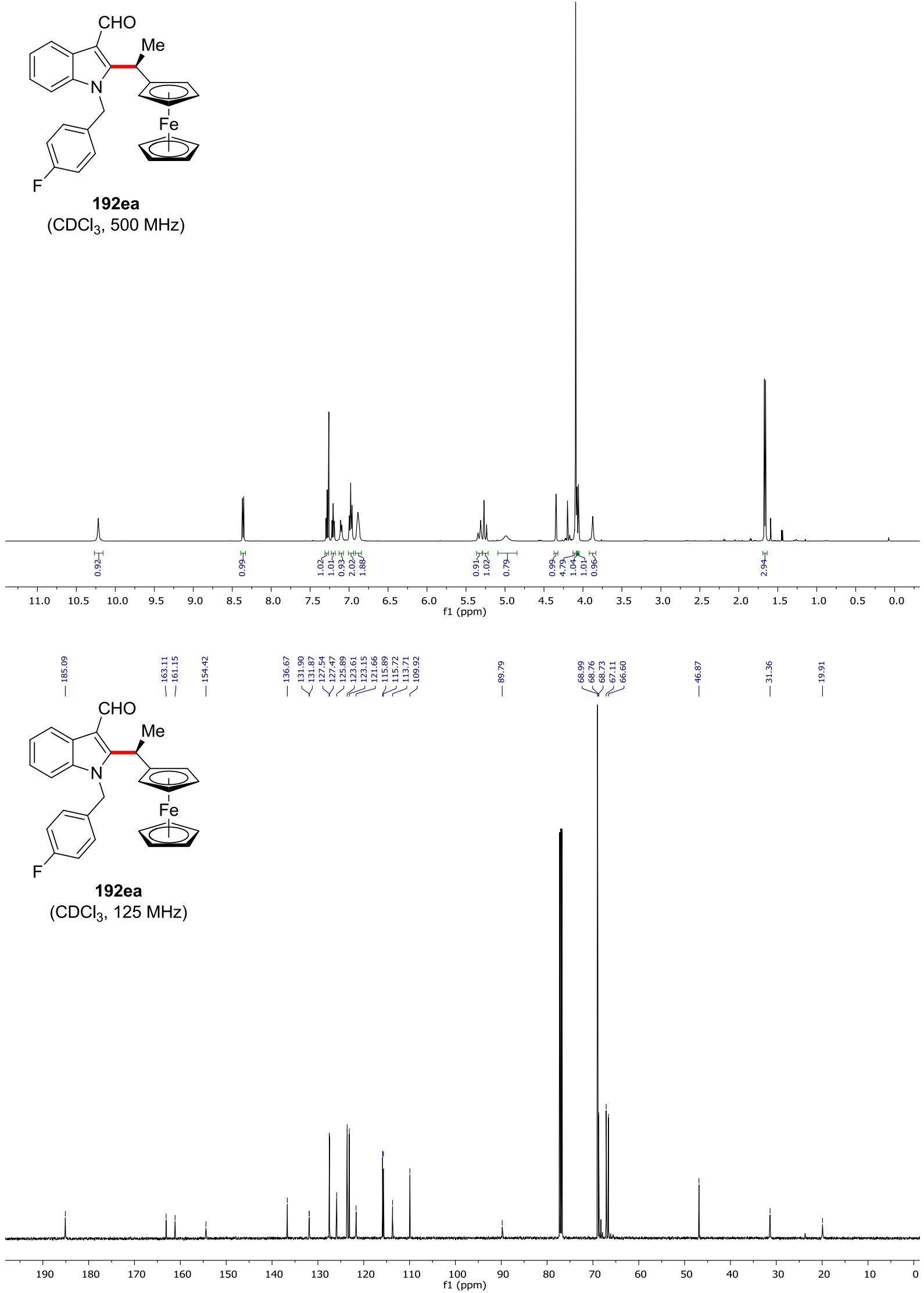


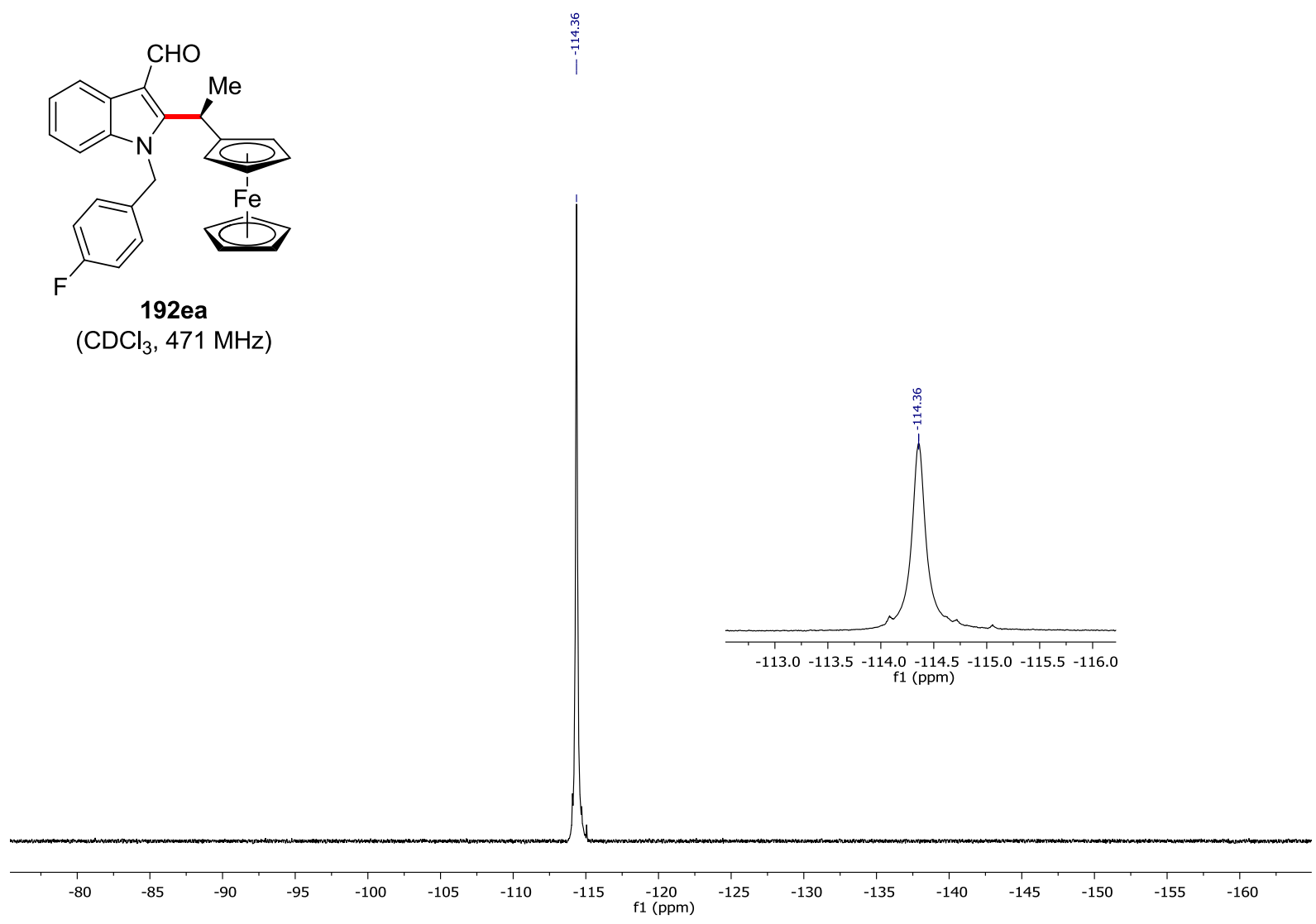




\section{Chiral HPLC of 192ea:}
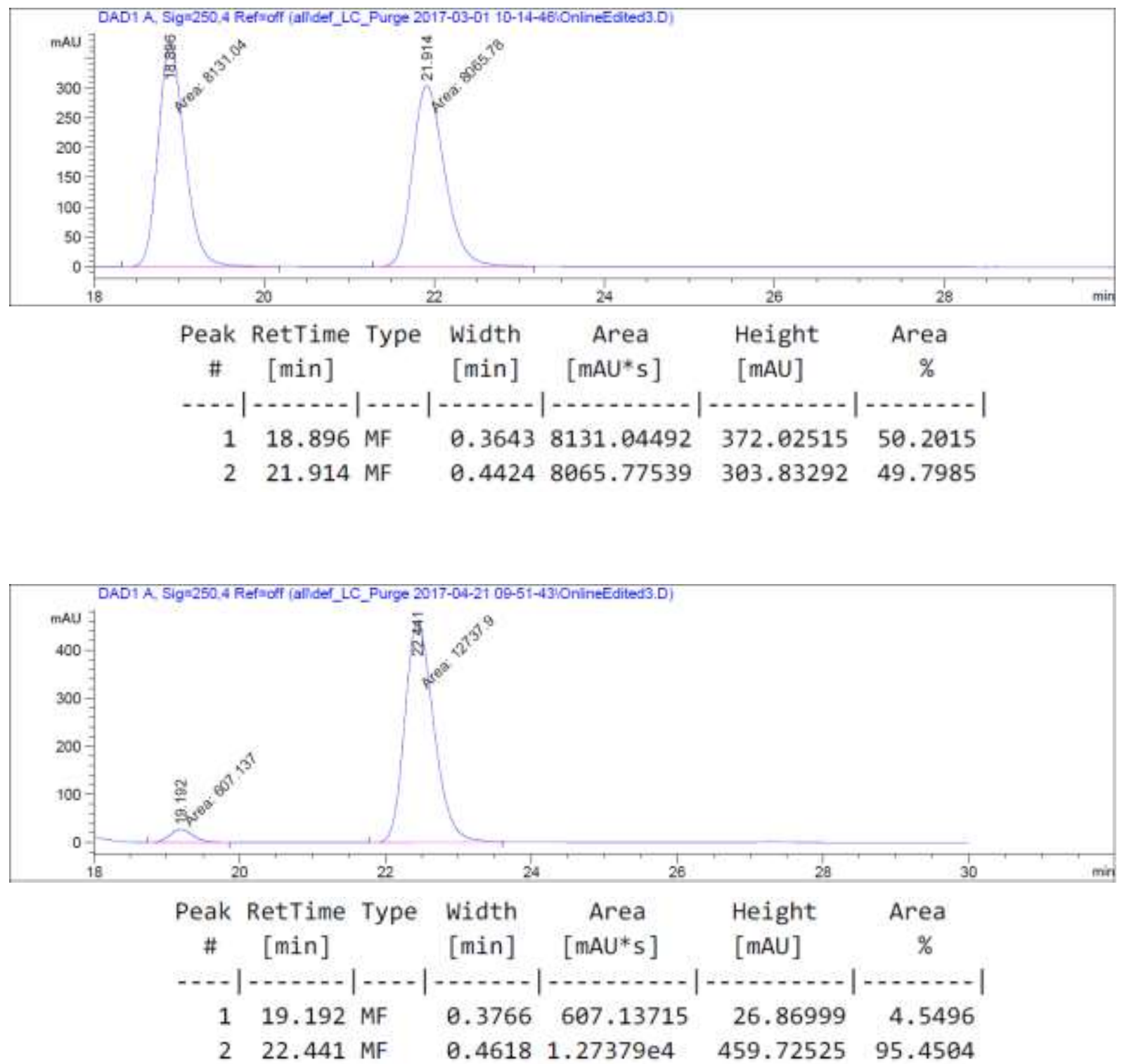


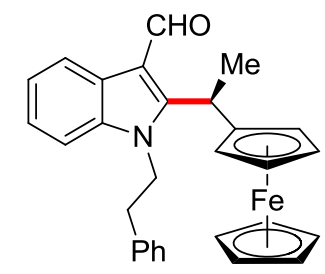

$192 \mathrm{fa}$

$\left(\mathrm{CDCl}_{3}, 300 \mathrm{MHz}\right)$
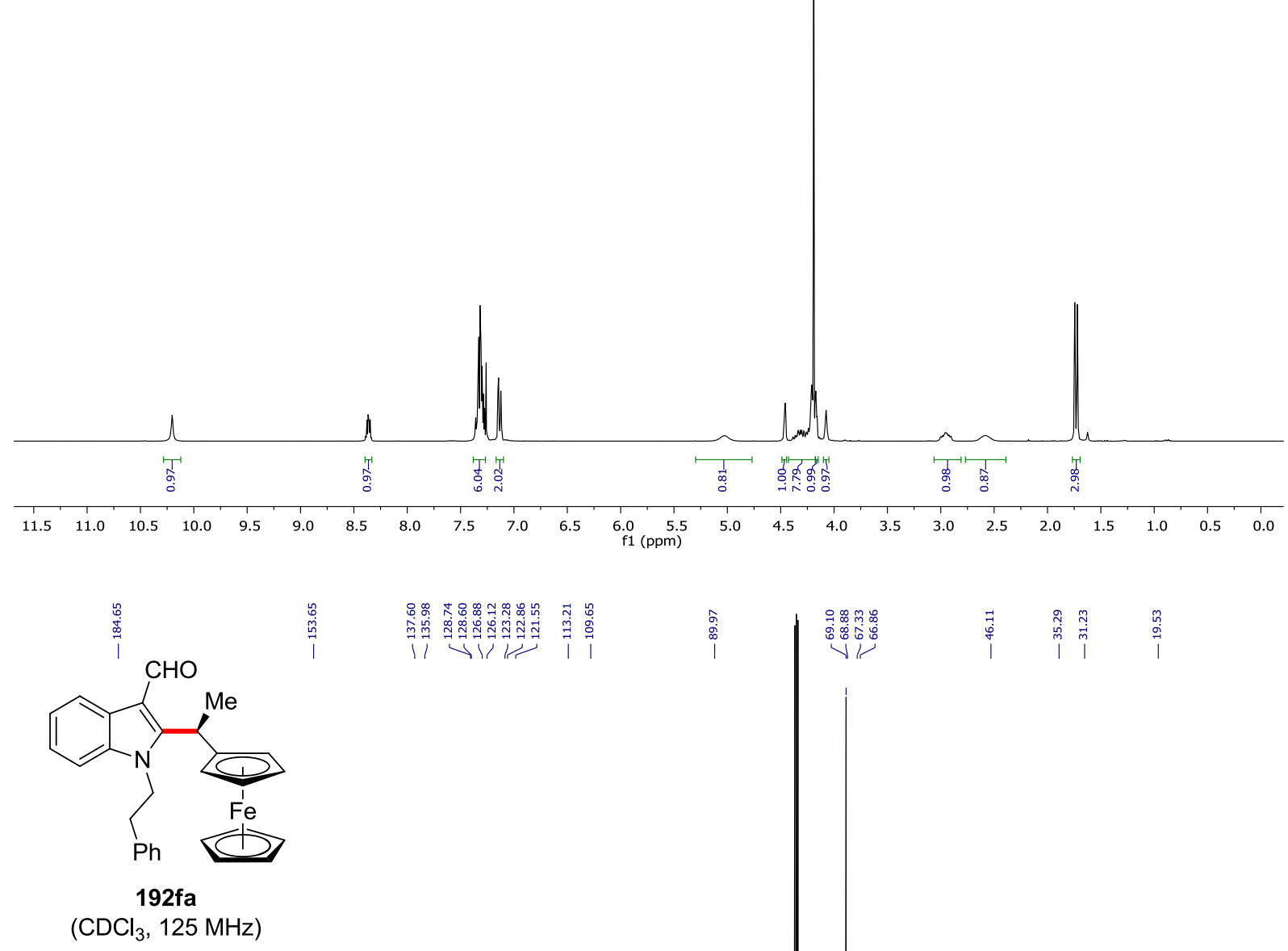

i

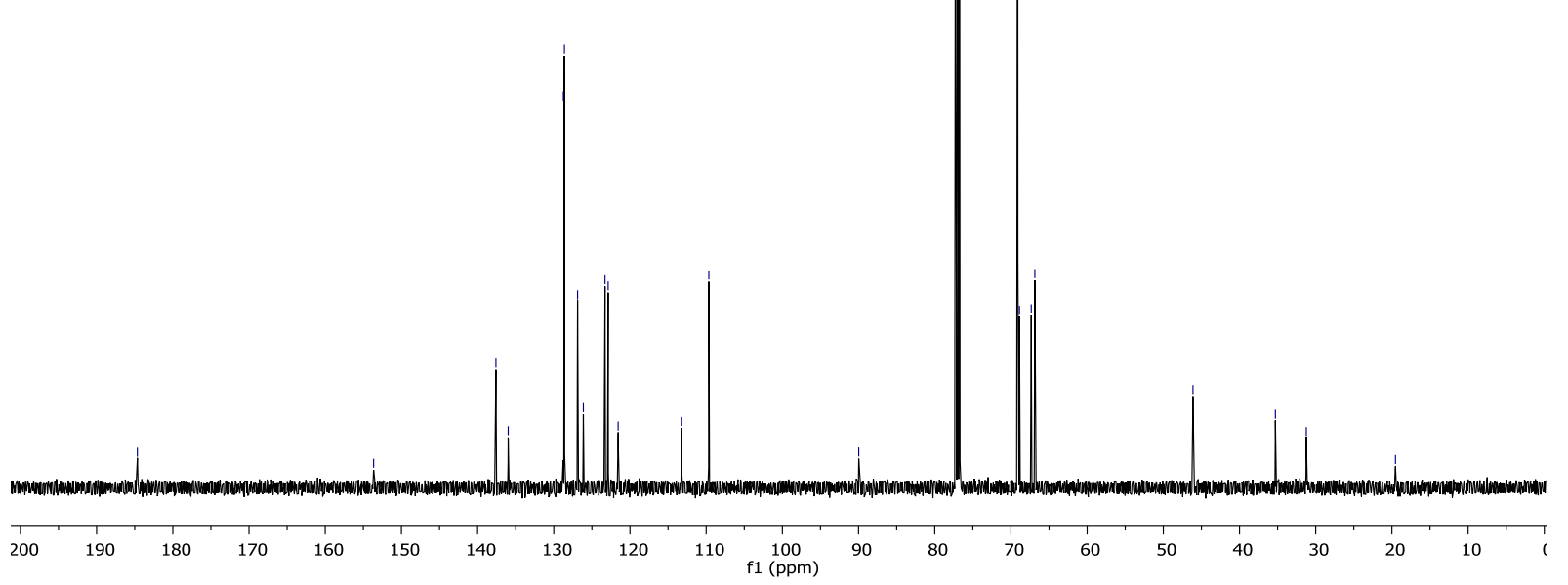




\section{Chiral HPLC of 192fa:}
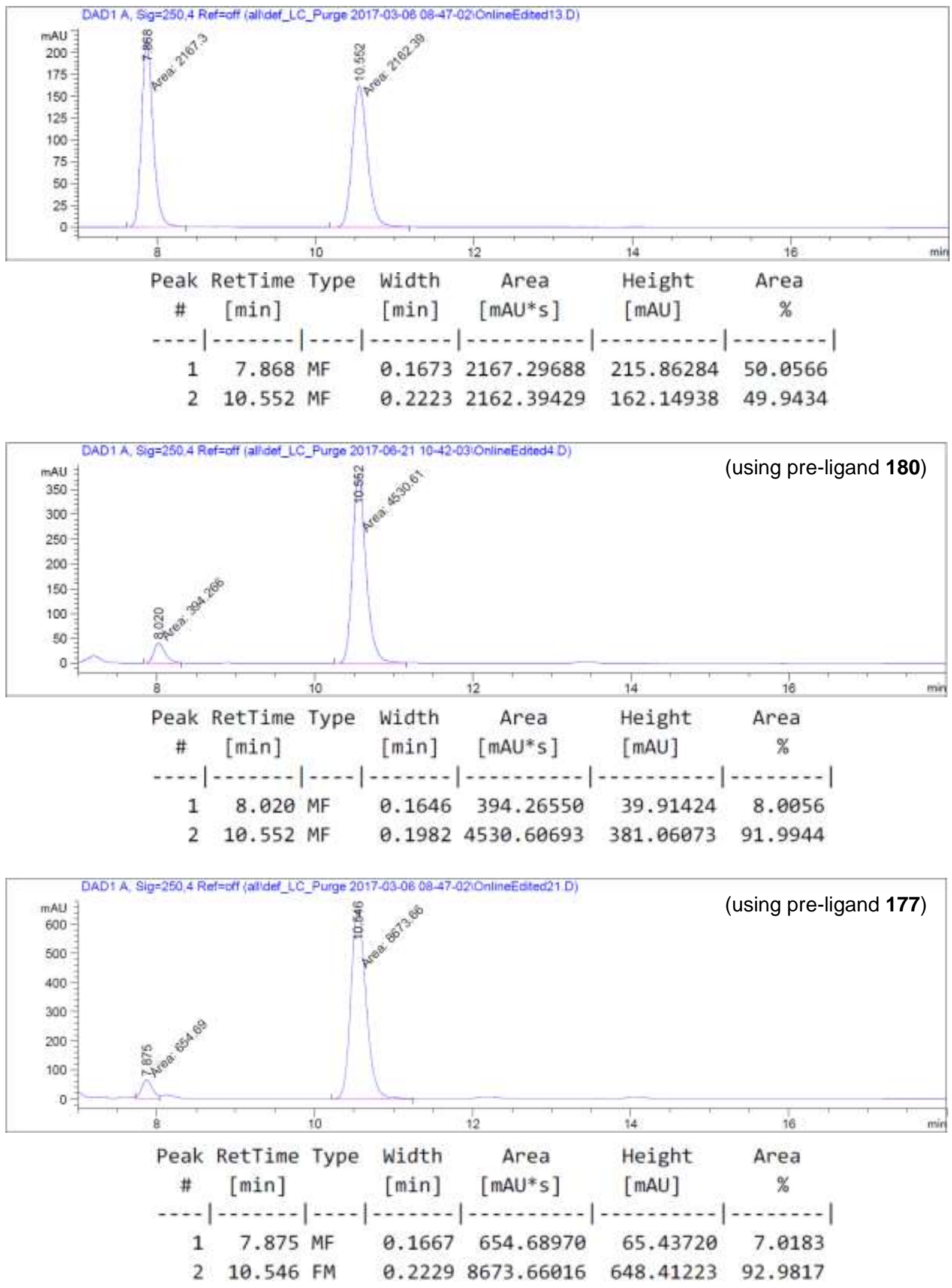

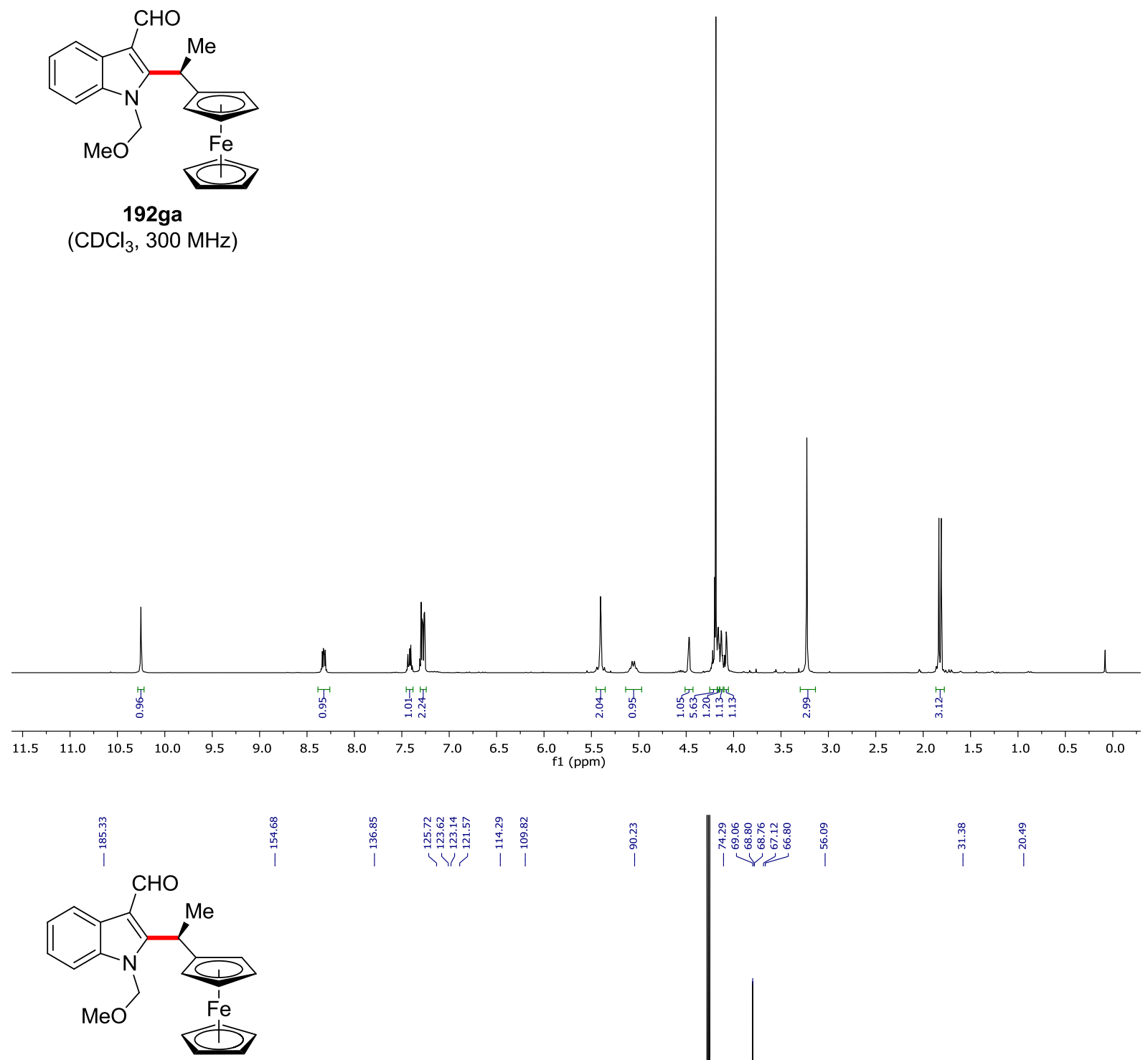

192ga

$\left(\mathrm{CDCl}_{3}, 125 \mathrm{MHz}\right)$

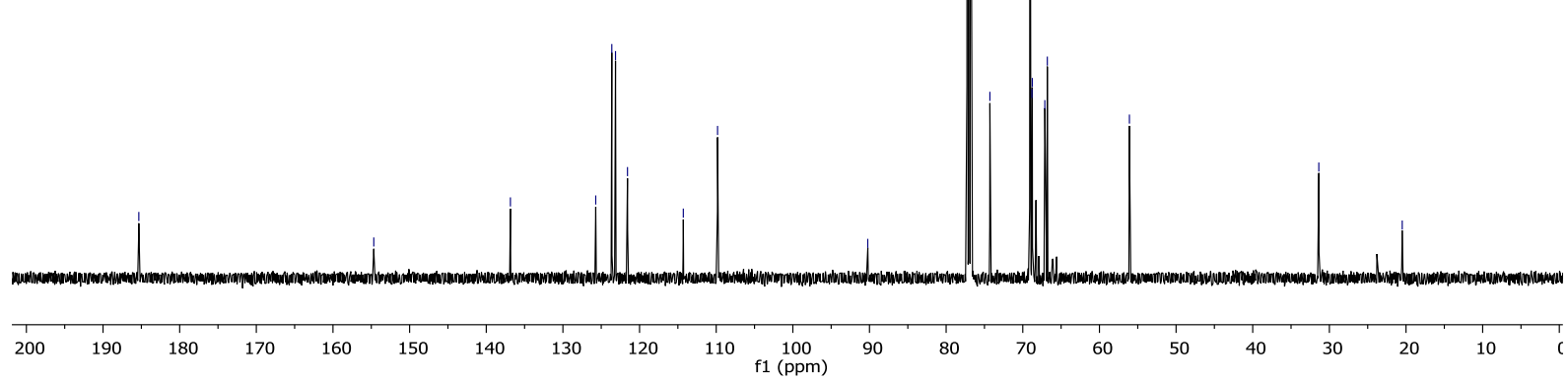




\section{Chiral HPLC of 192ga:}
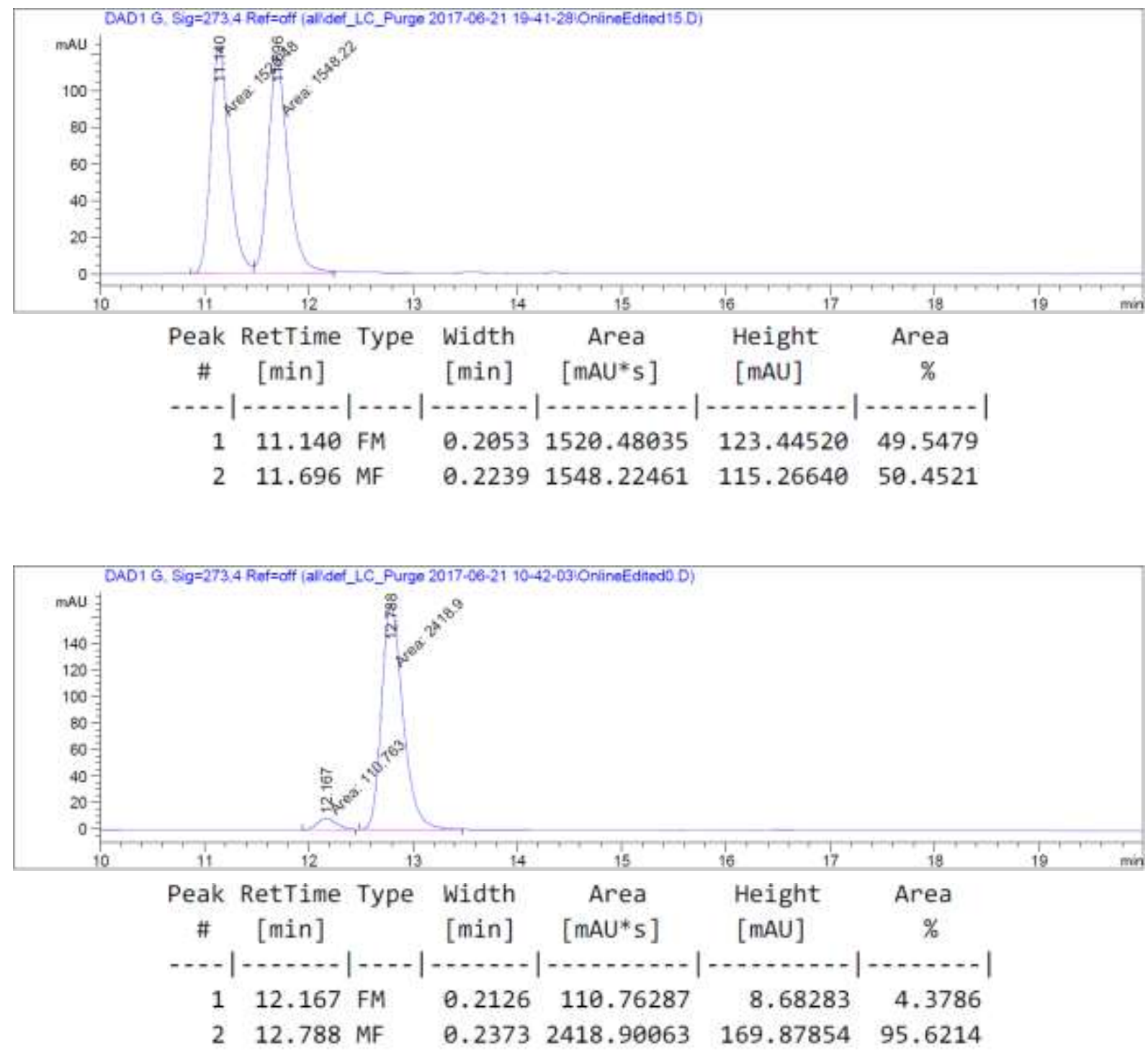


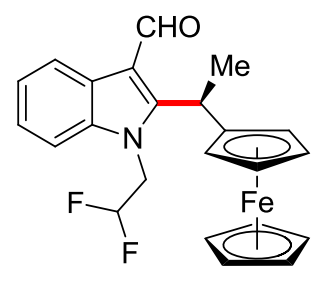

192ha

$\left(\mathrm{CDCl}_{3}, 500 \mathrm{MHz}\right)$
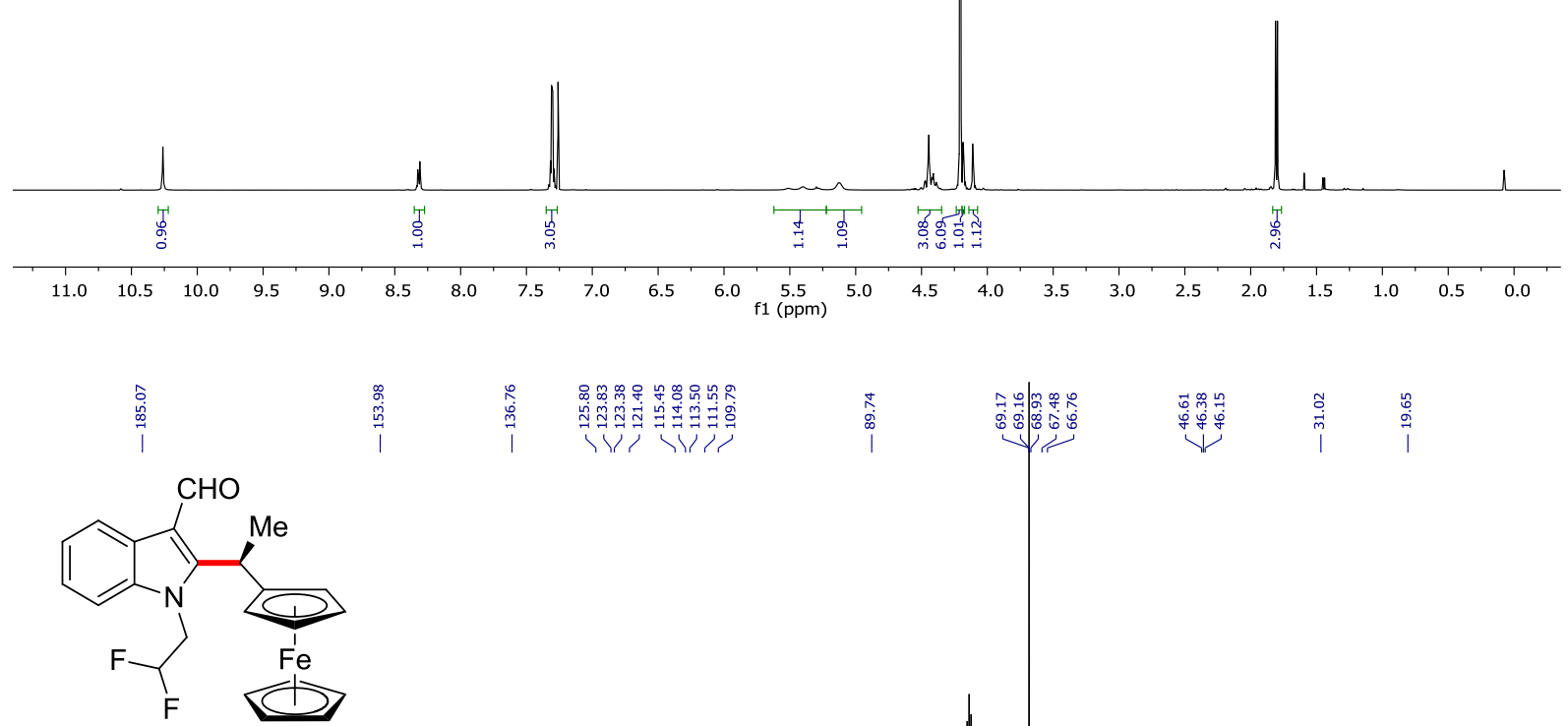

192ha

$\left(\mathrm{CDCl}_{3}, 125 \mathrm{MHz}\right)$ 

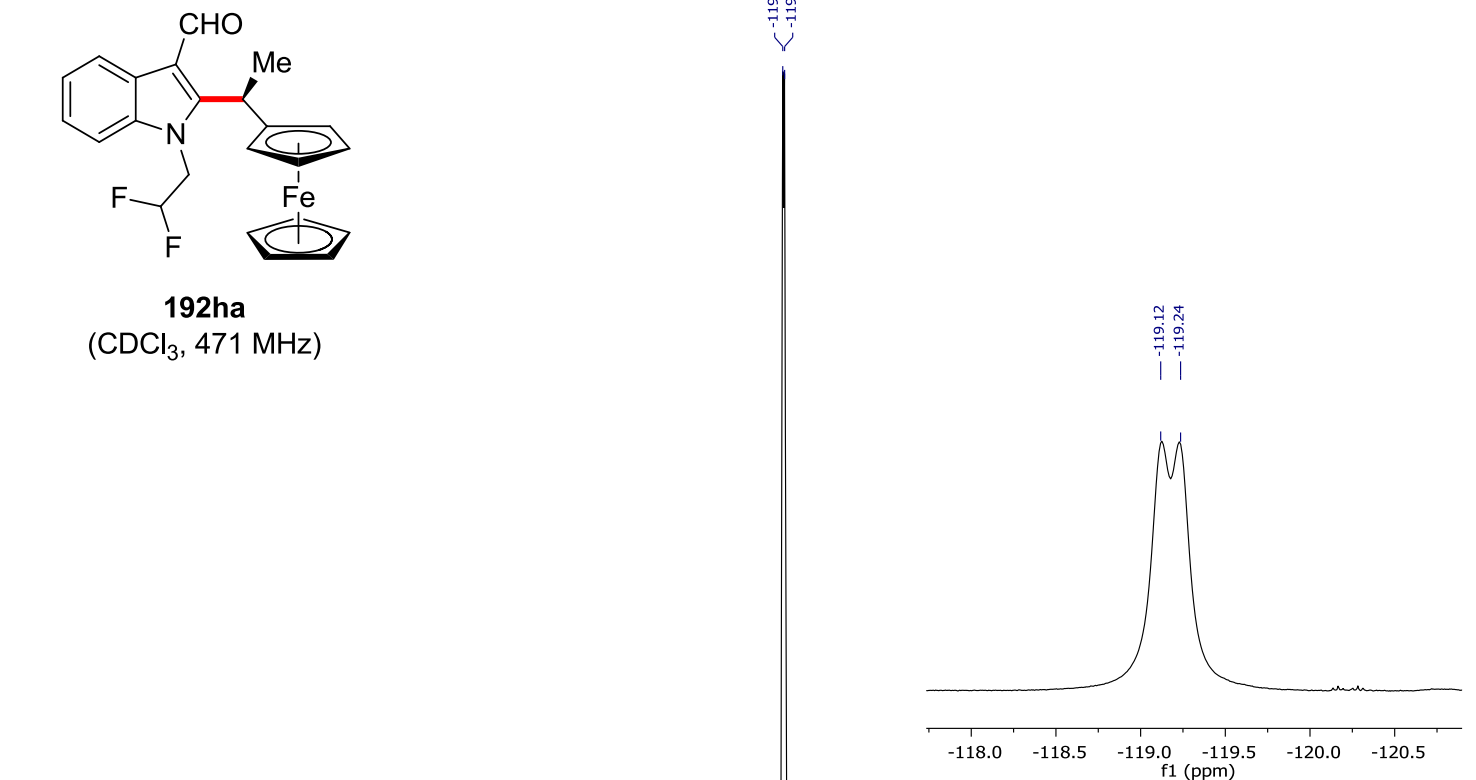

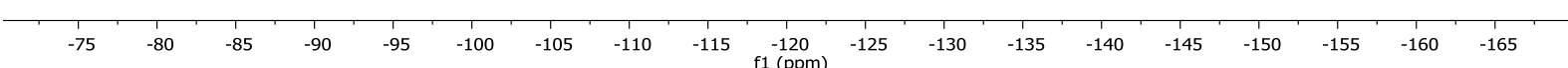




\section{Chiral HPLC of 192ha:}
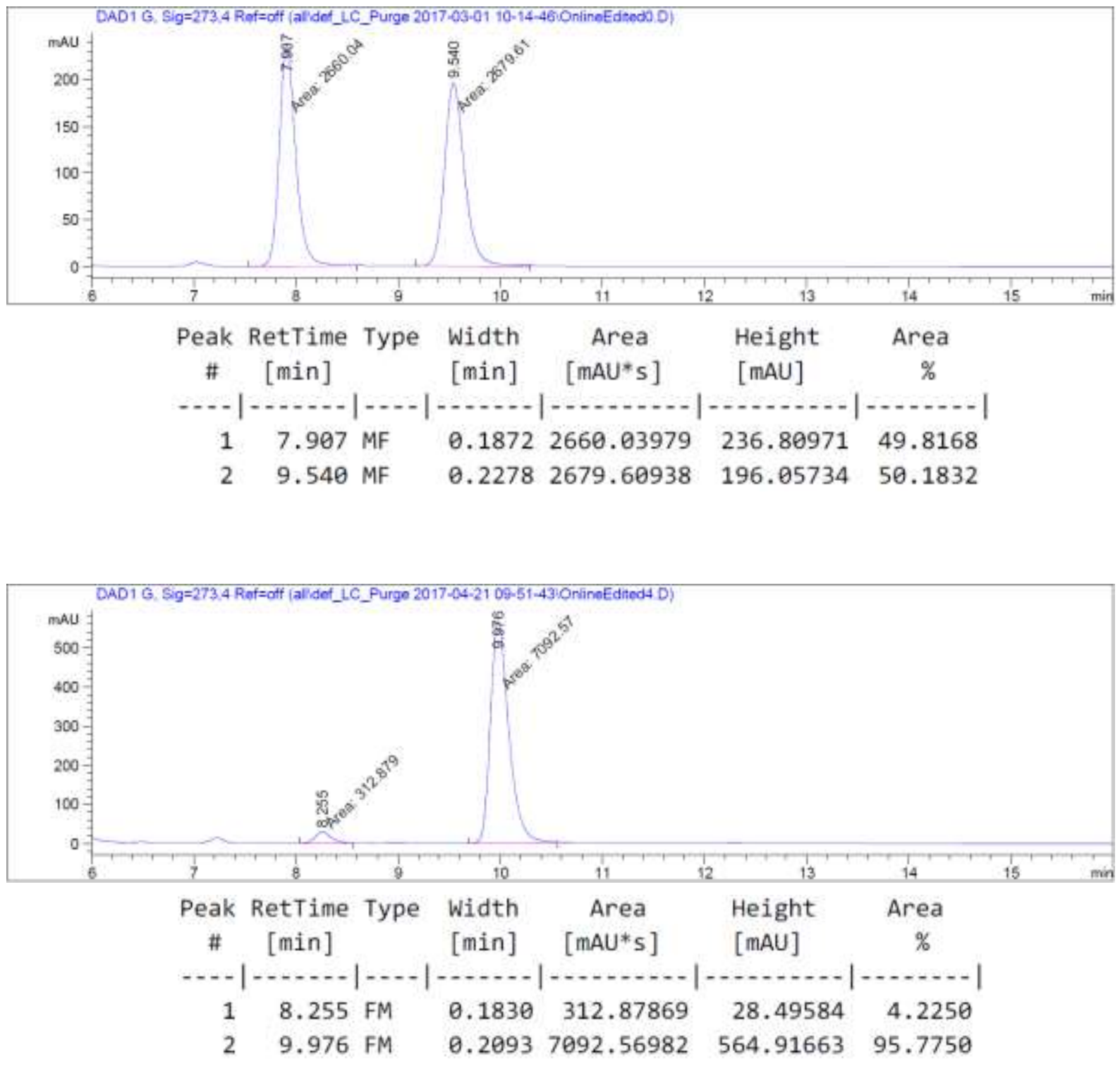

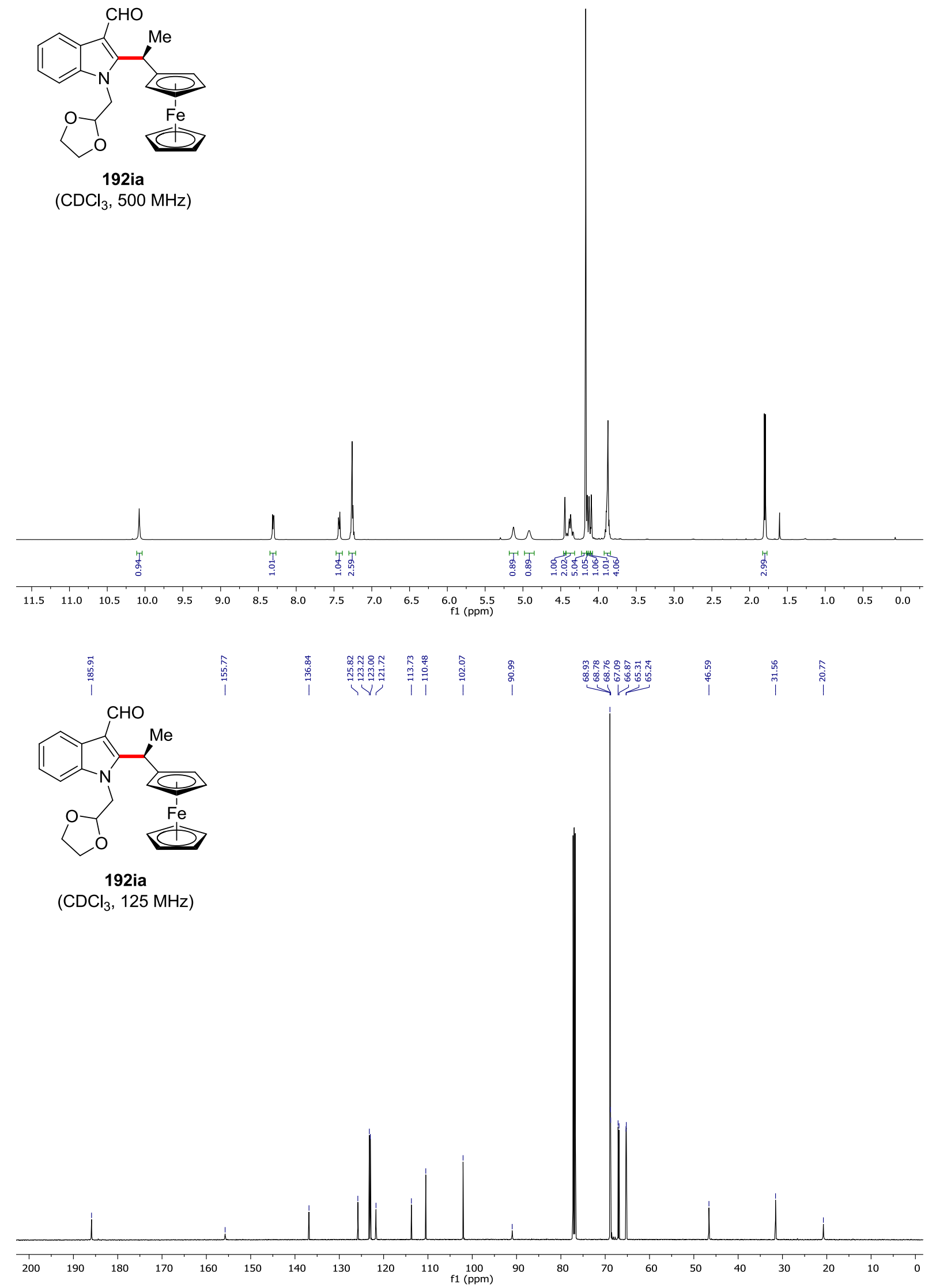


\section{Chiral HPLC of 192ia:}
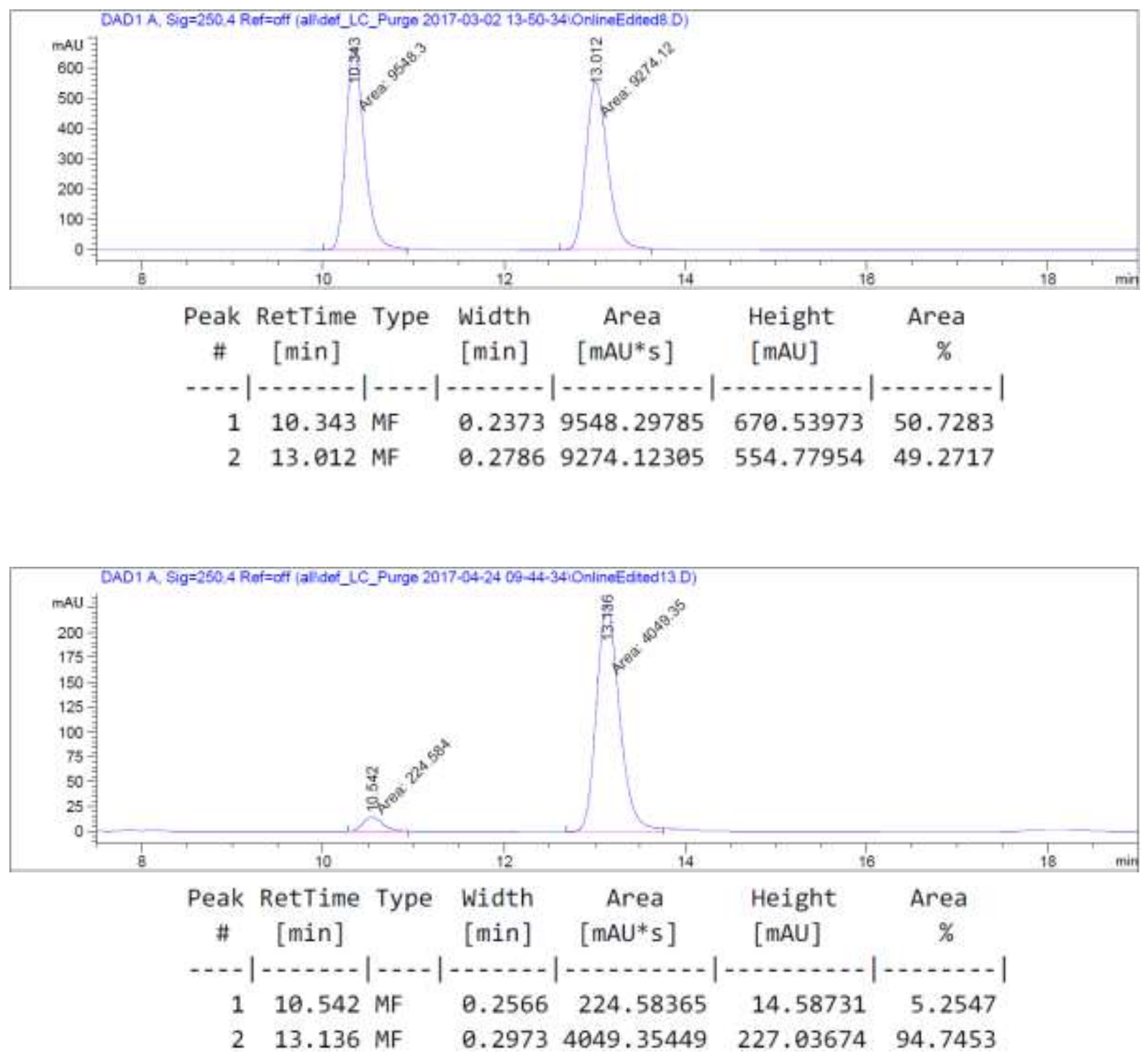


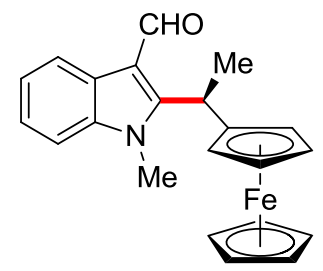

192ba

$\left(\mathrm{CDCl}_{3}, 300 \mathrm{MHz}\right)$
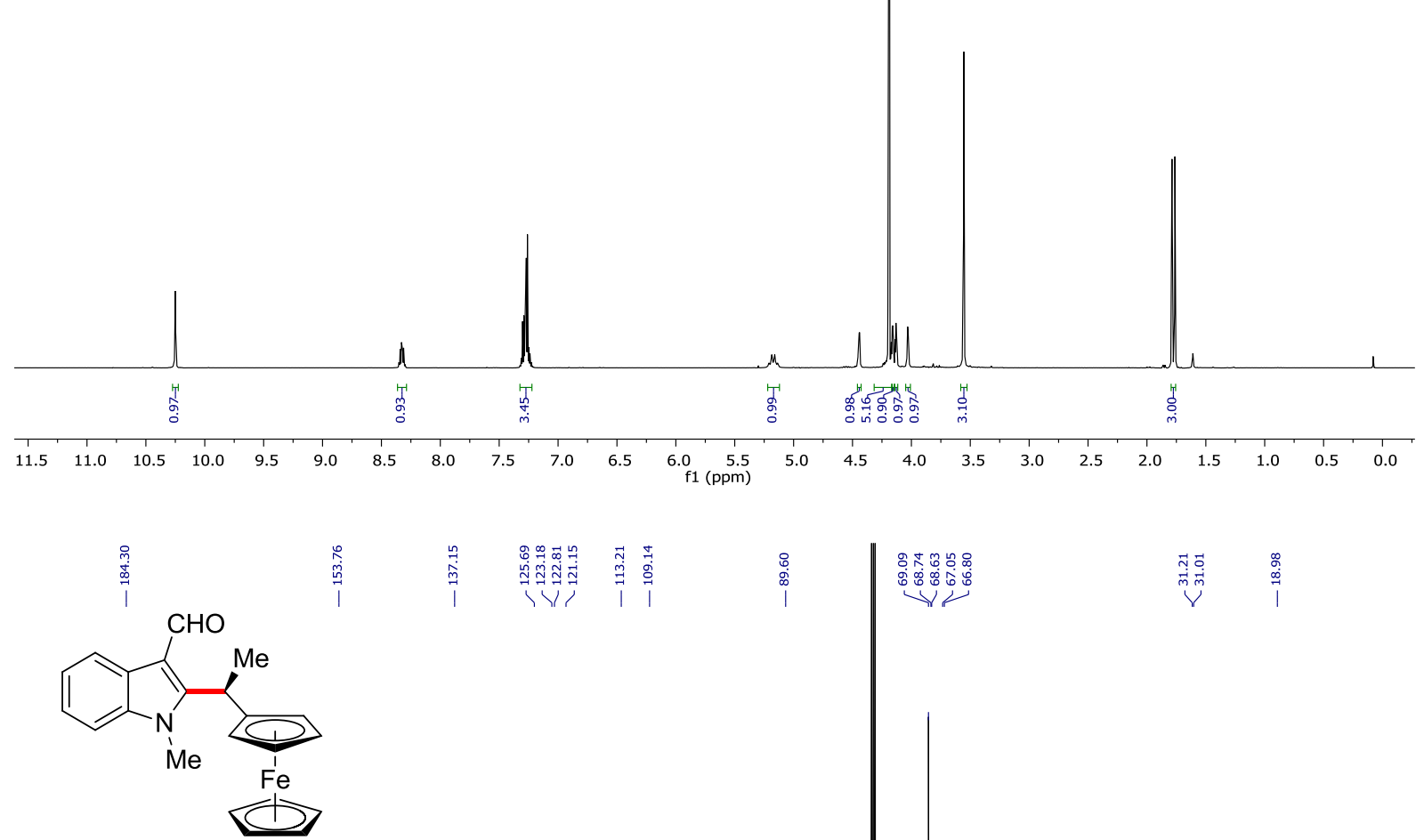

192ba

$\left(\mathrm{CDCl}_{3}, 125 \mathrm{MHz}\right)$

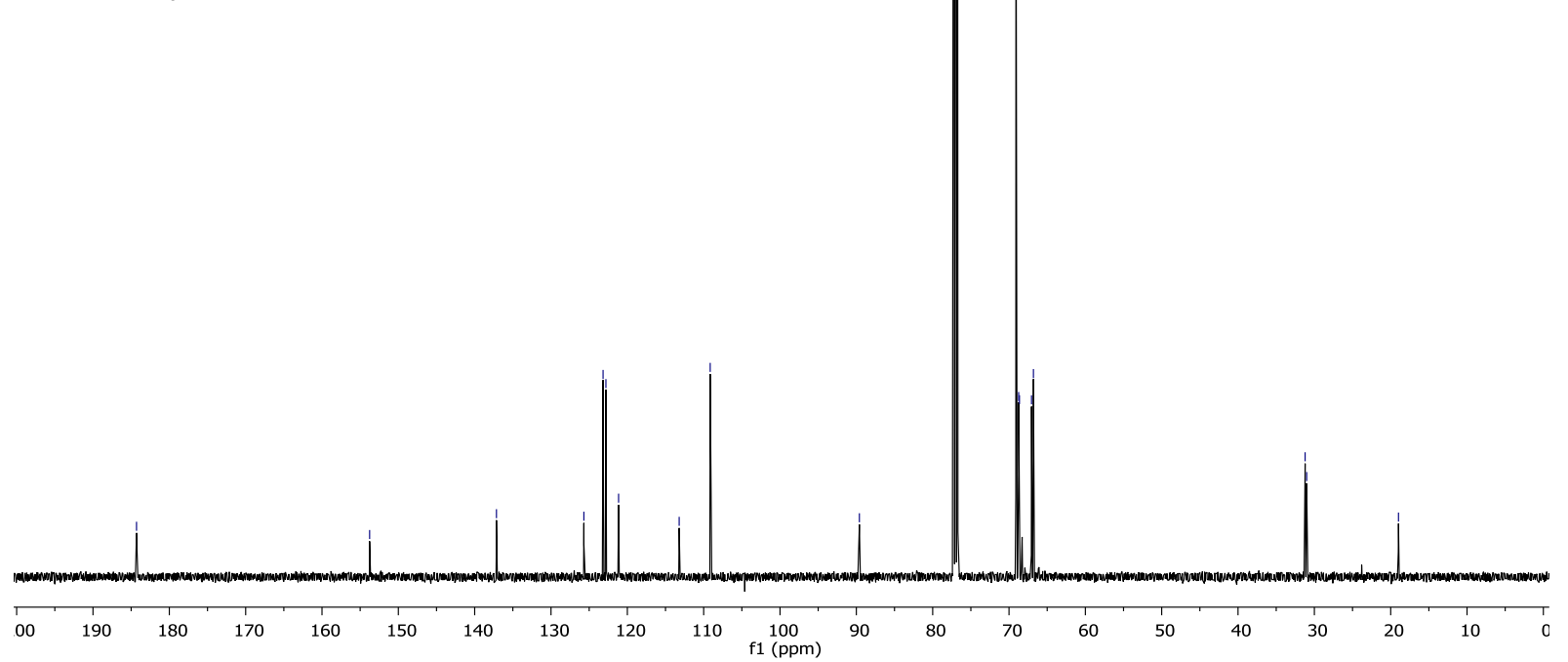




\section{Chiral HPLC of 192ba:}
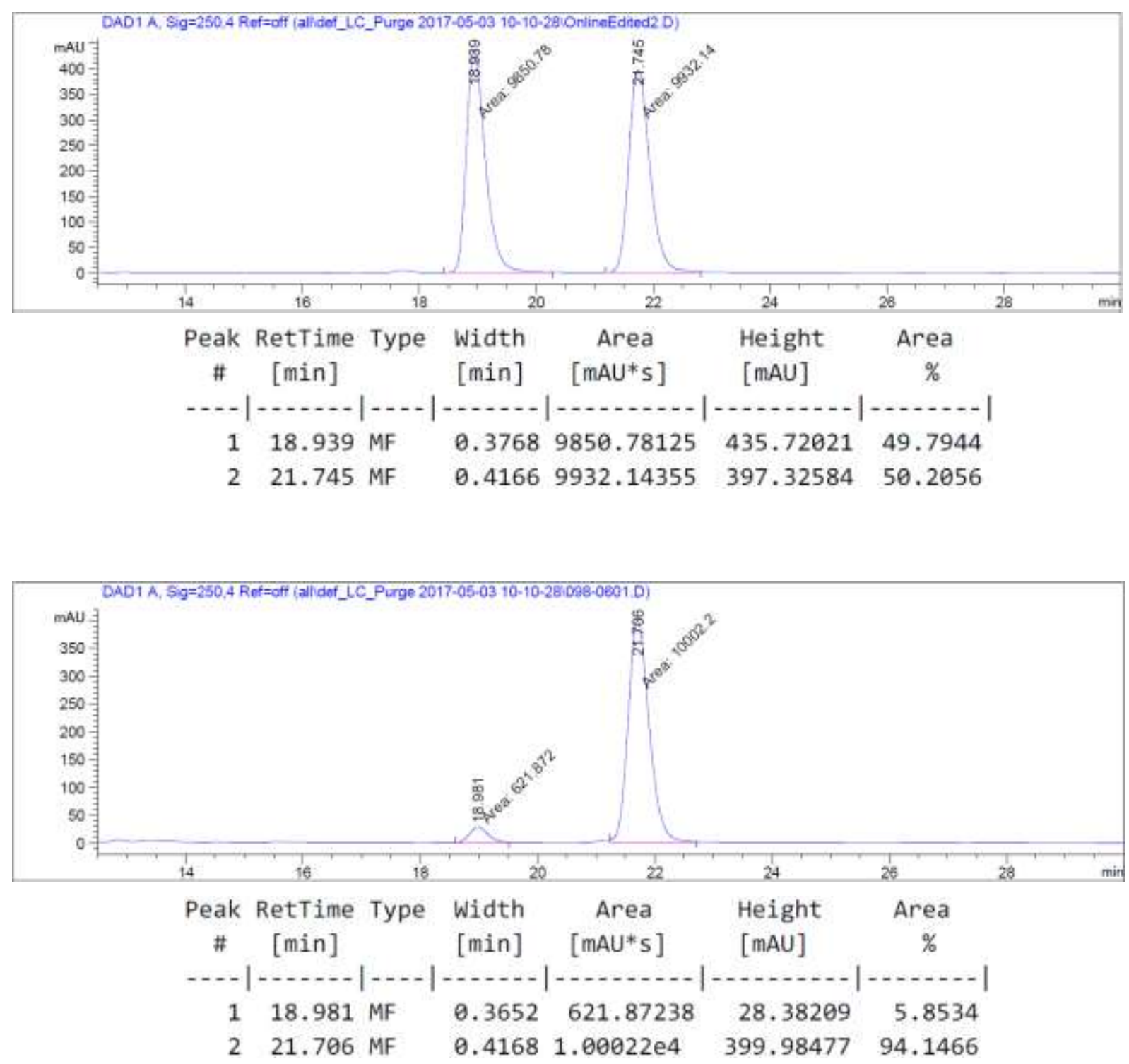

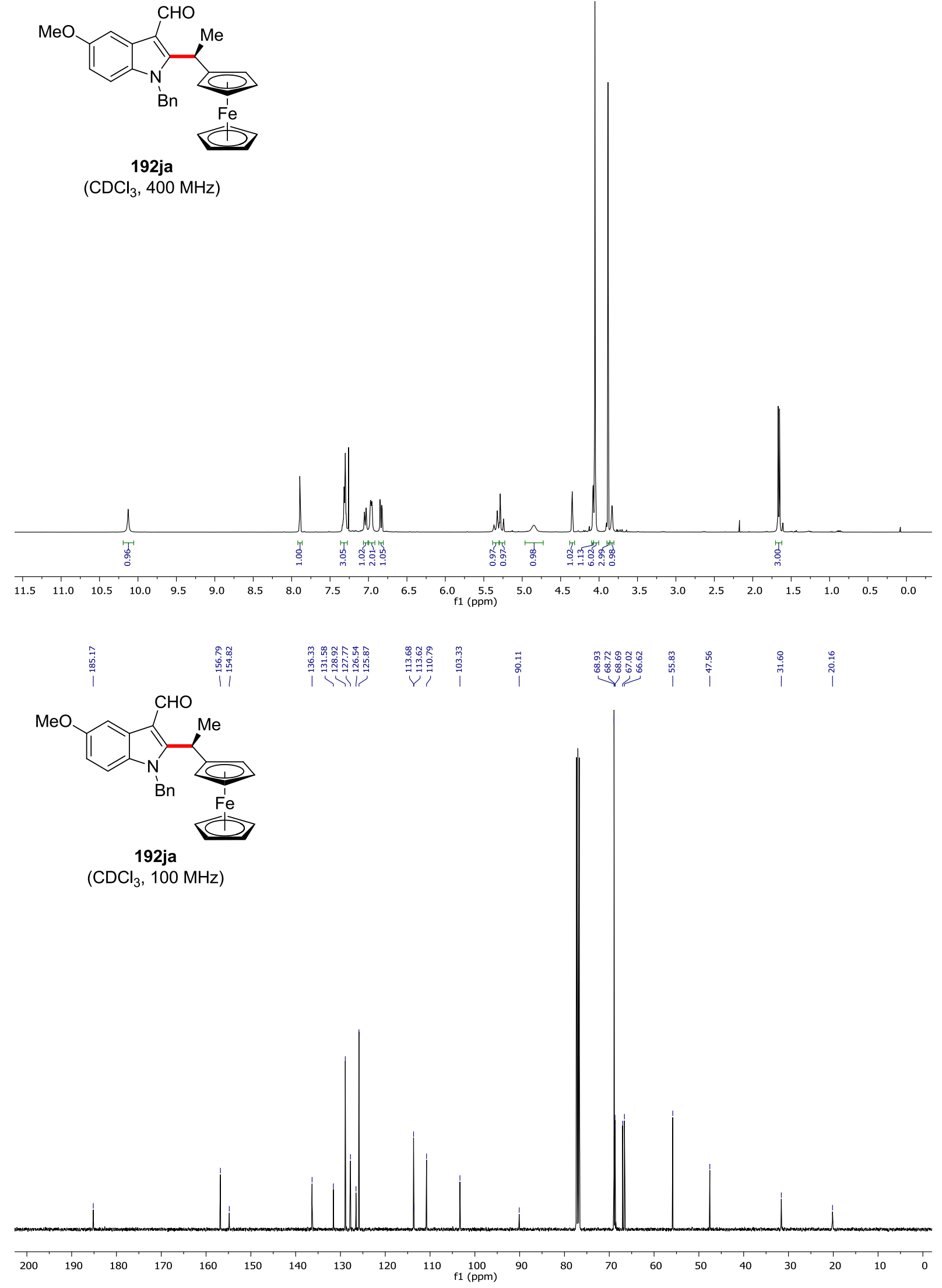

458 


\section{Chiral HPLC of 192ja:}
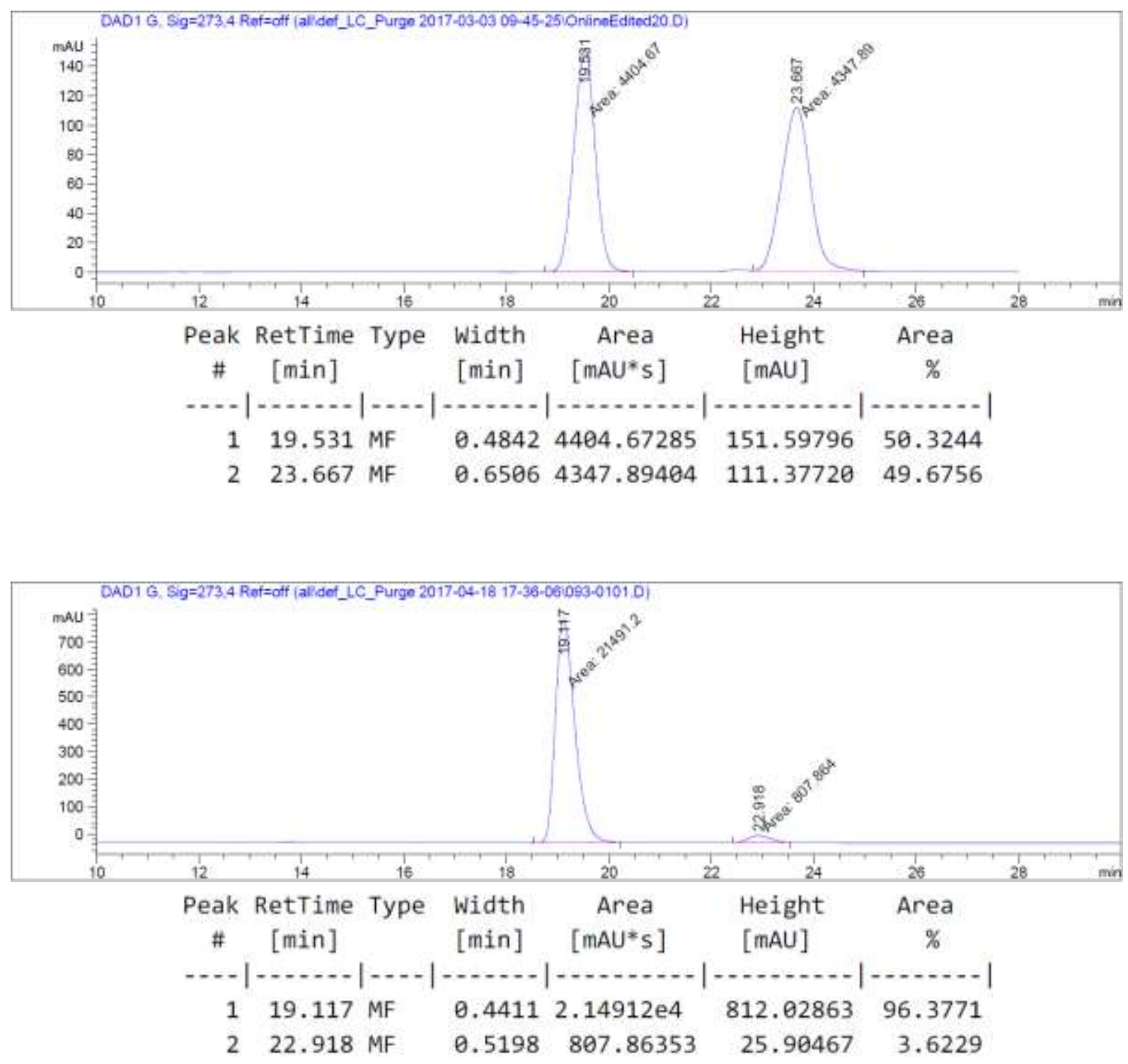

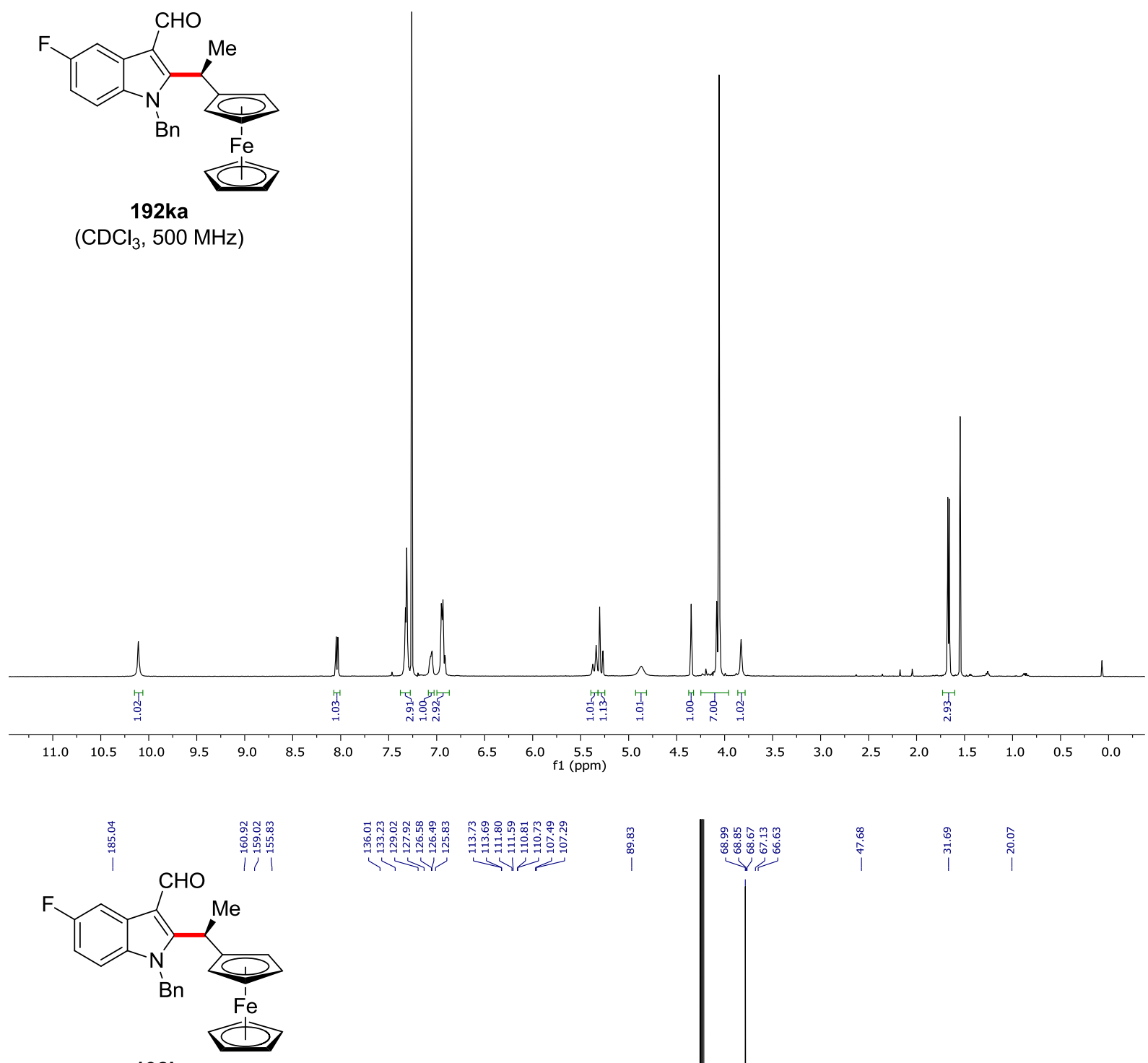

192ka

$\left(\mathrm{CDCl}_{3}, 125 \mathrm{MHz}\right)$

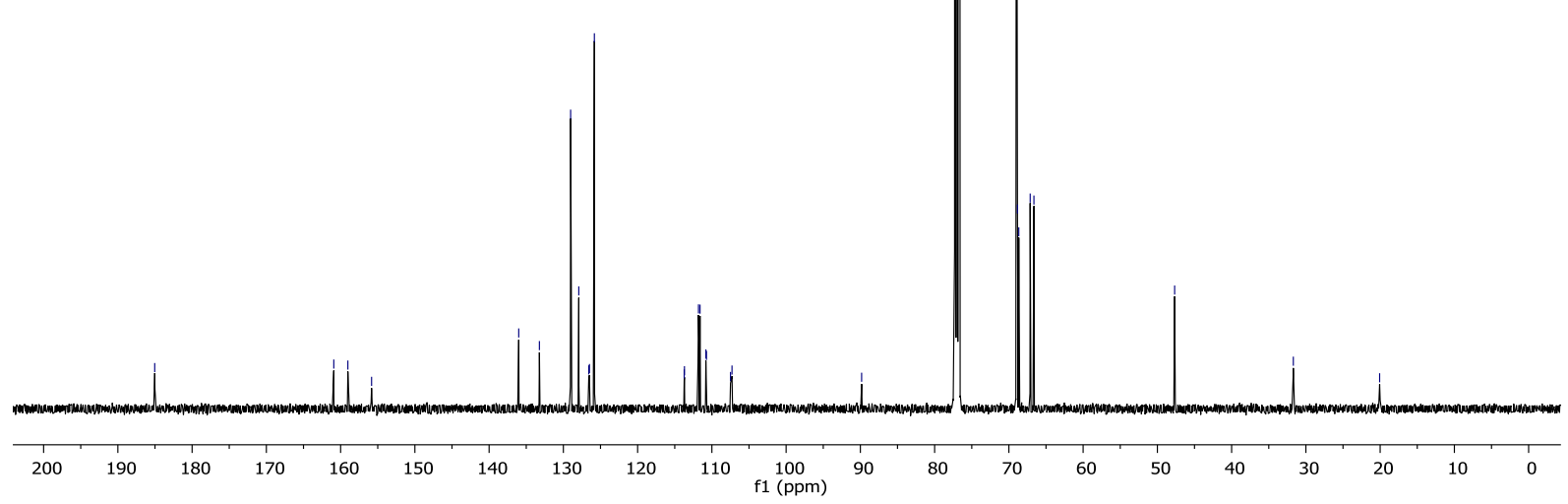




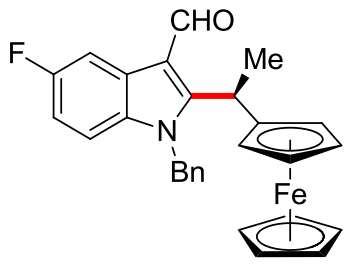

192ka

$\left(\mathrm{CDCl}_{3}, 471 \mathrm{MHz}\right)$

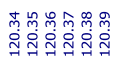

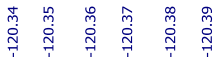

i i i i

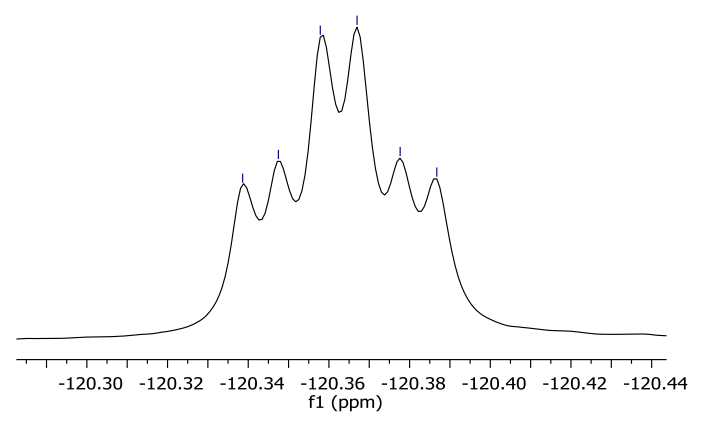

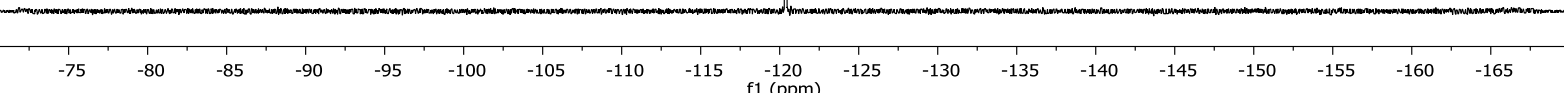




\section{Chiral HPLC of 192ka:}
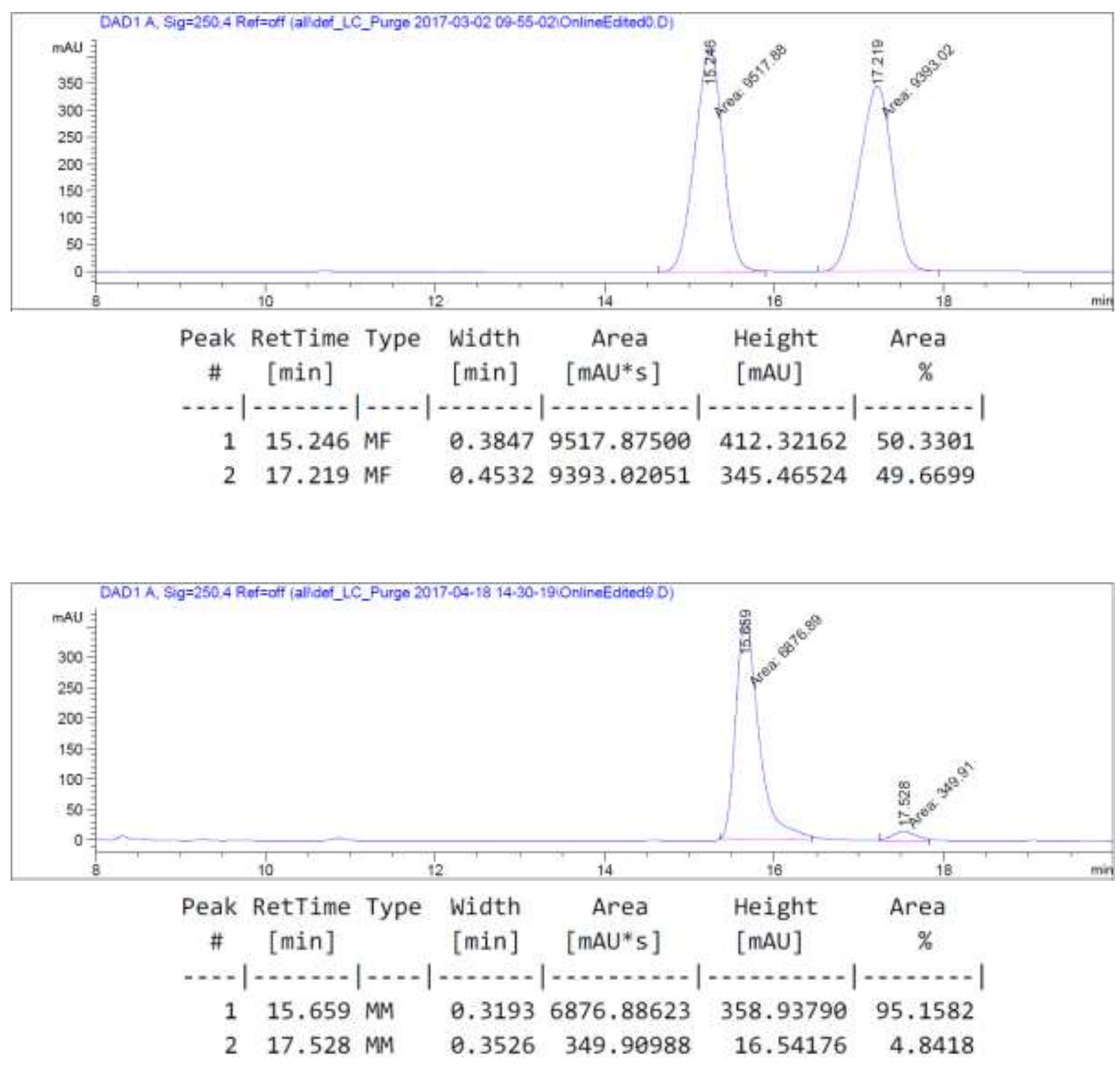

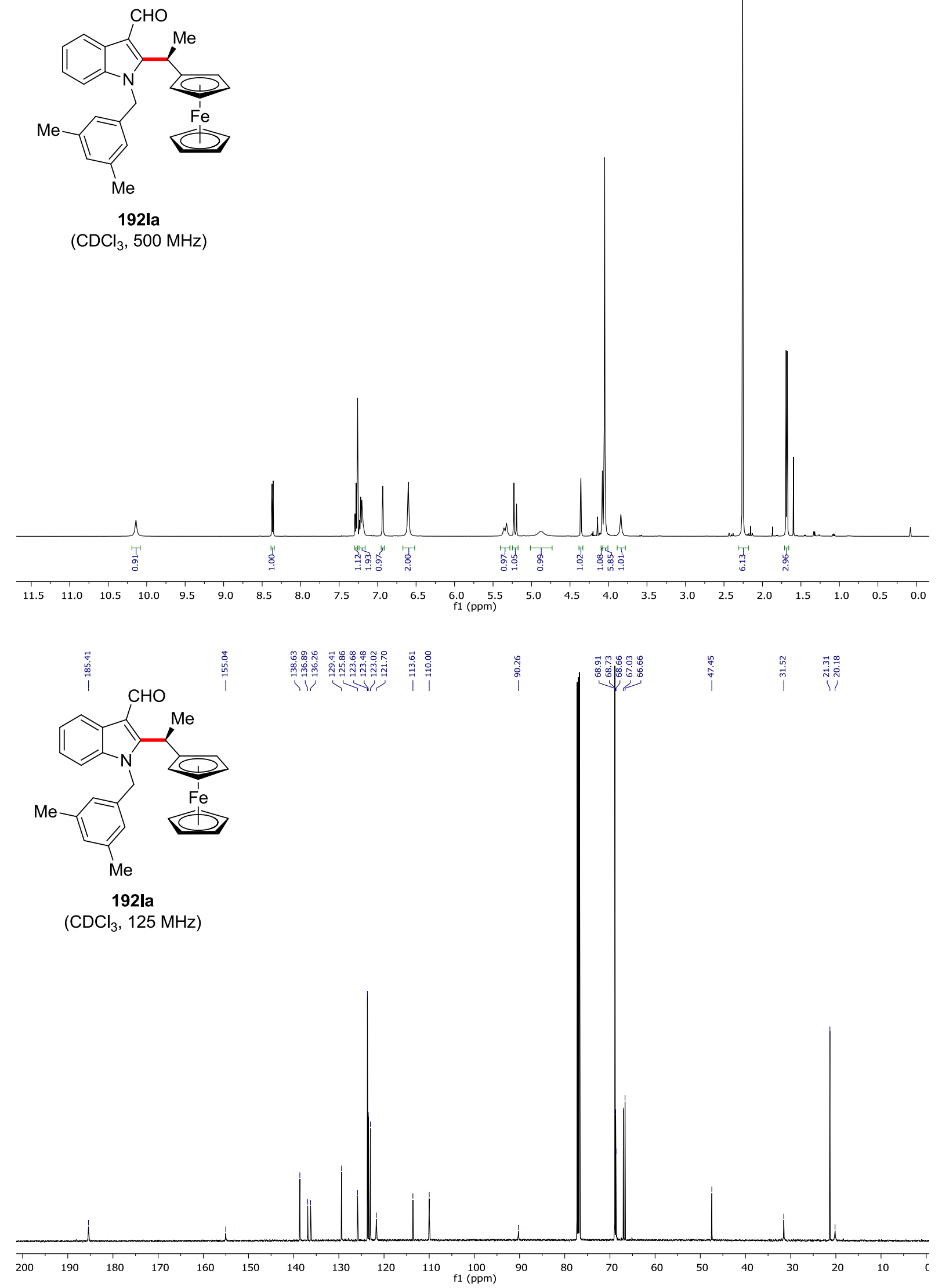


\section{Chiral HPLC of 192la:}
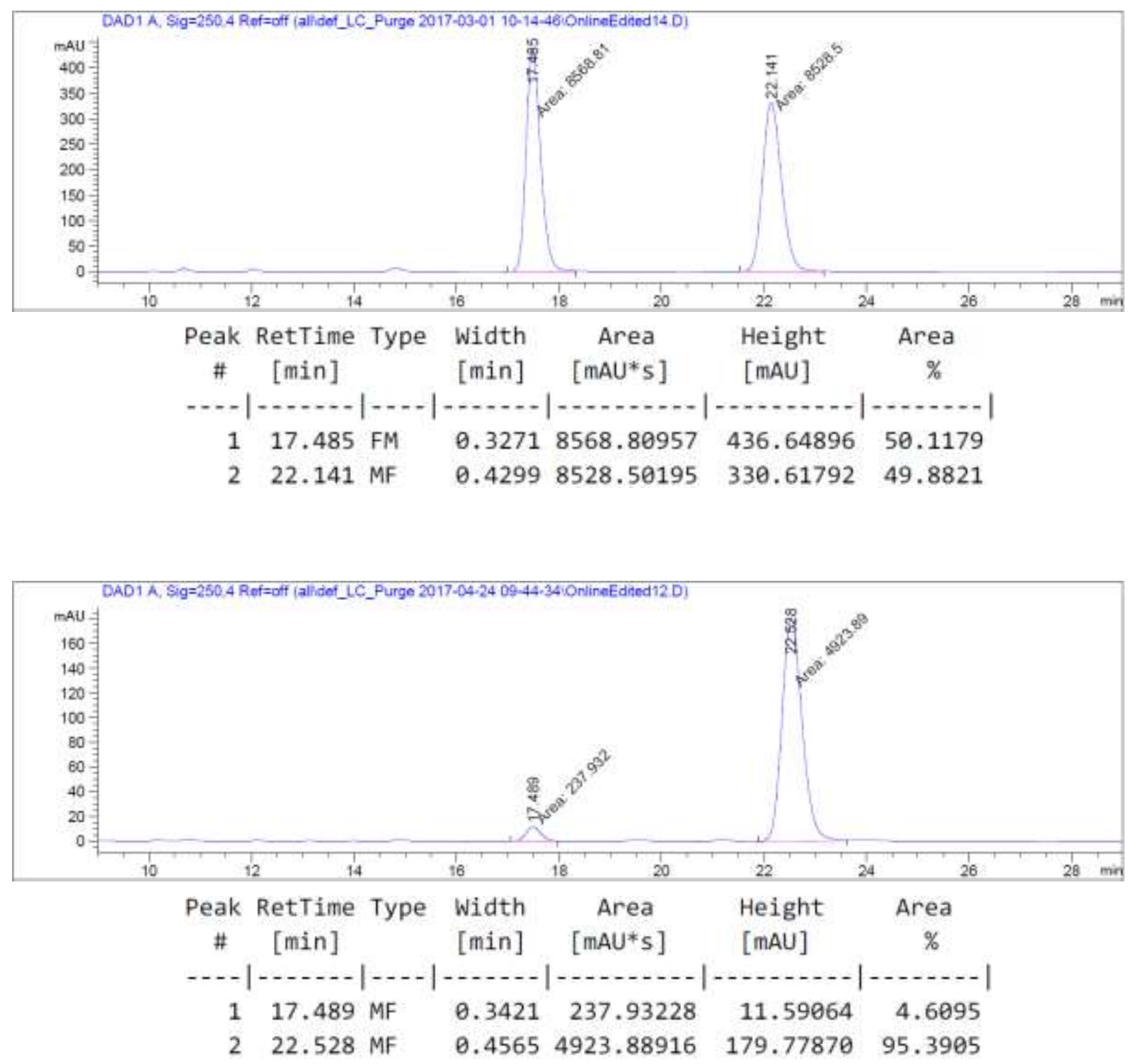

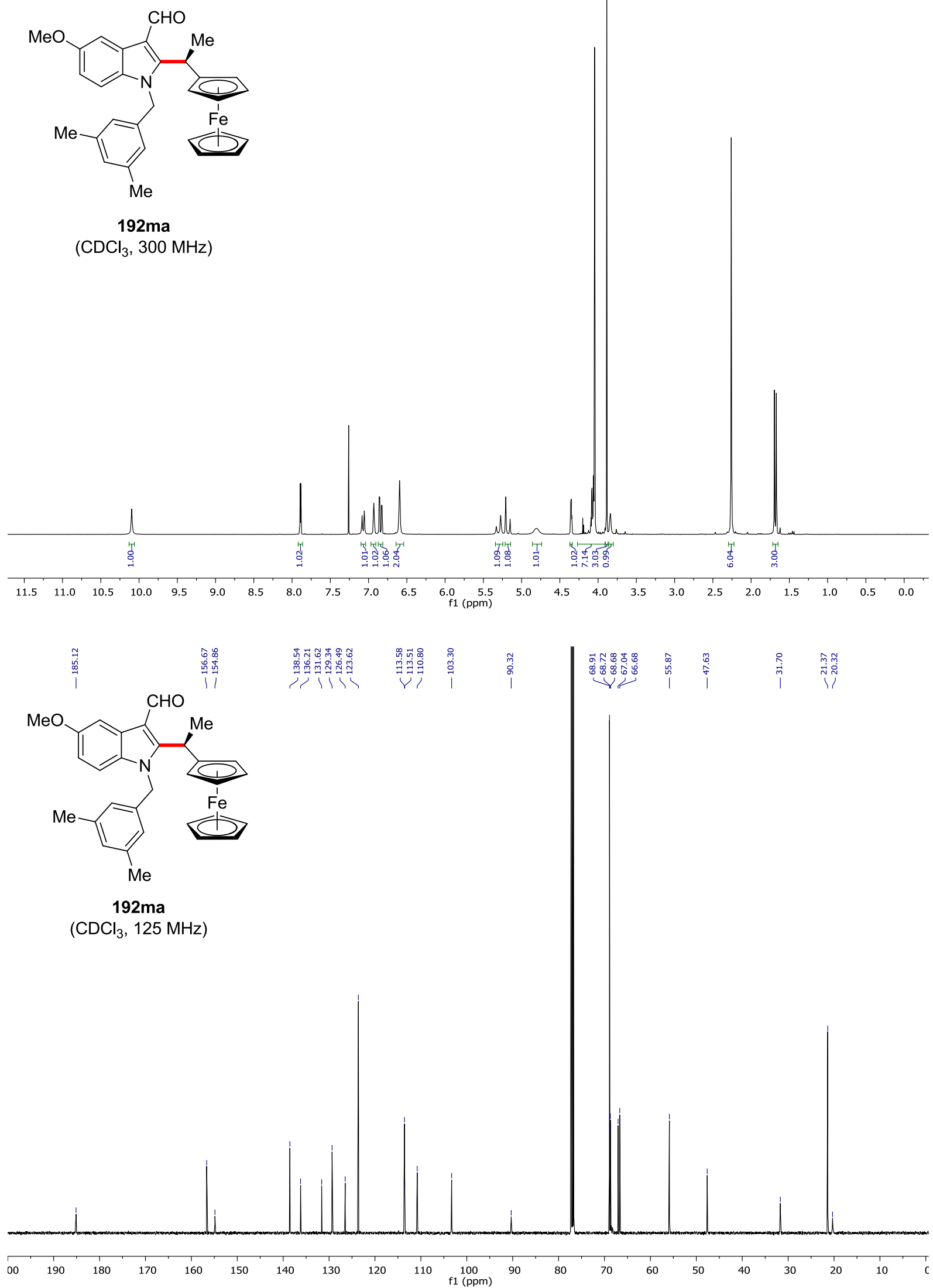


\section{Chiral HPLC of 192ma:}
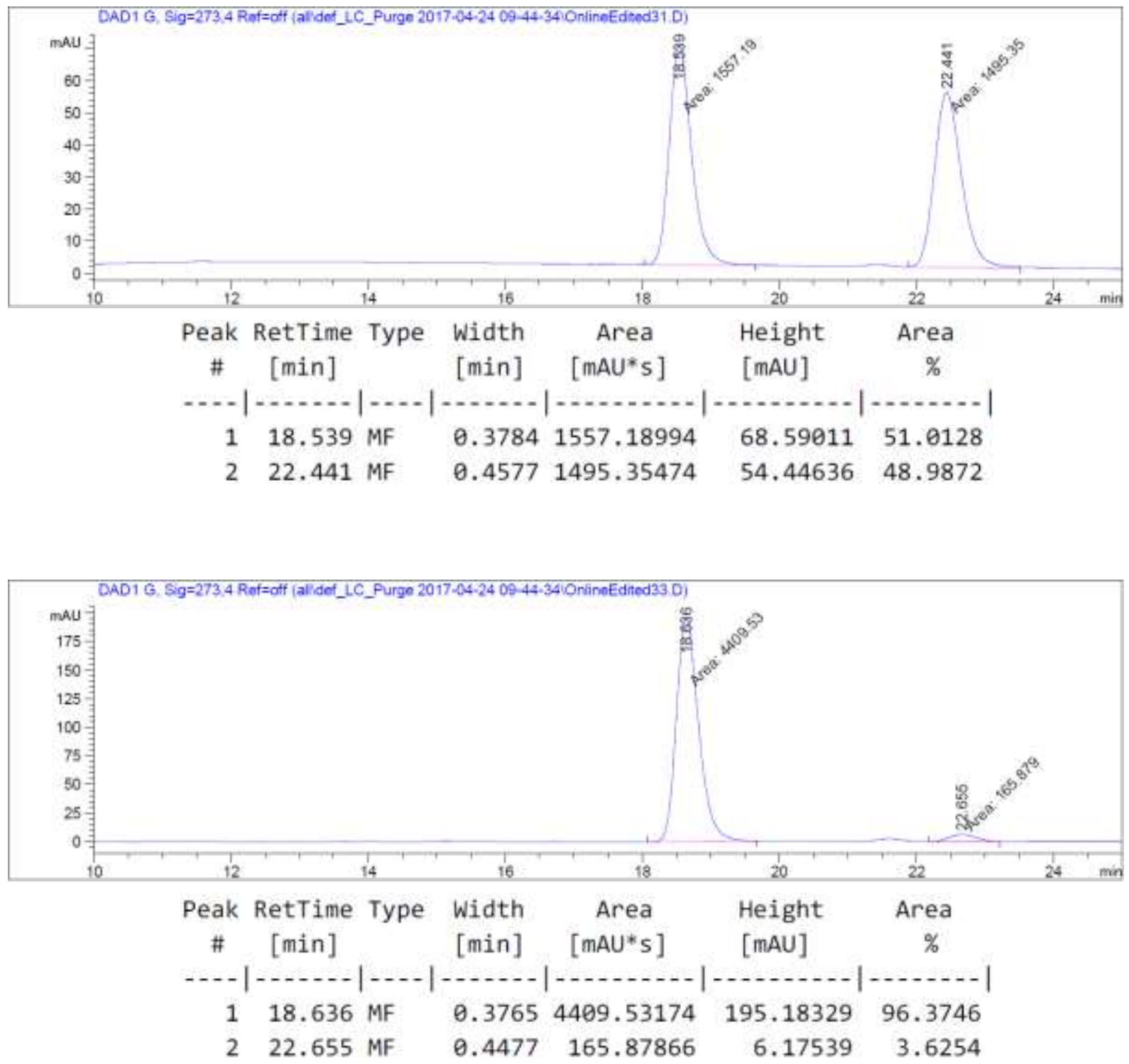

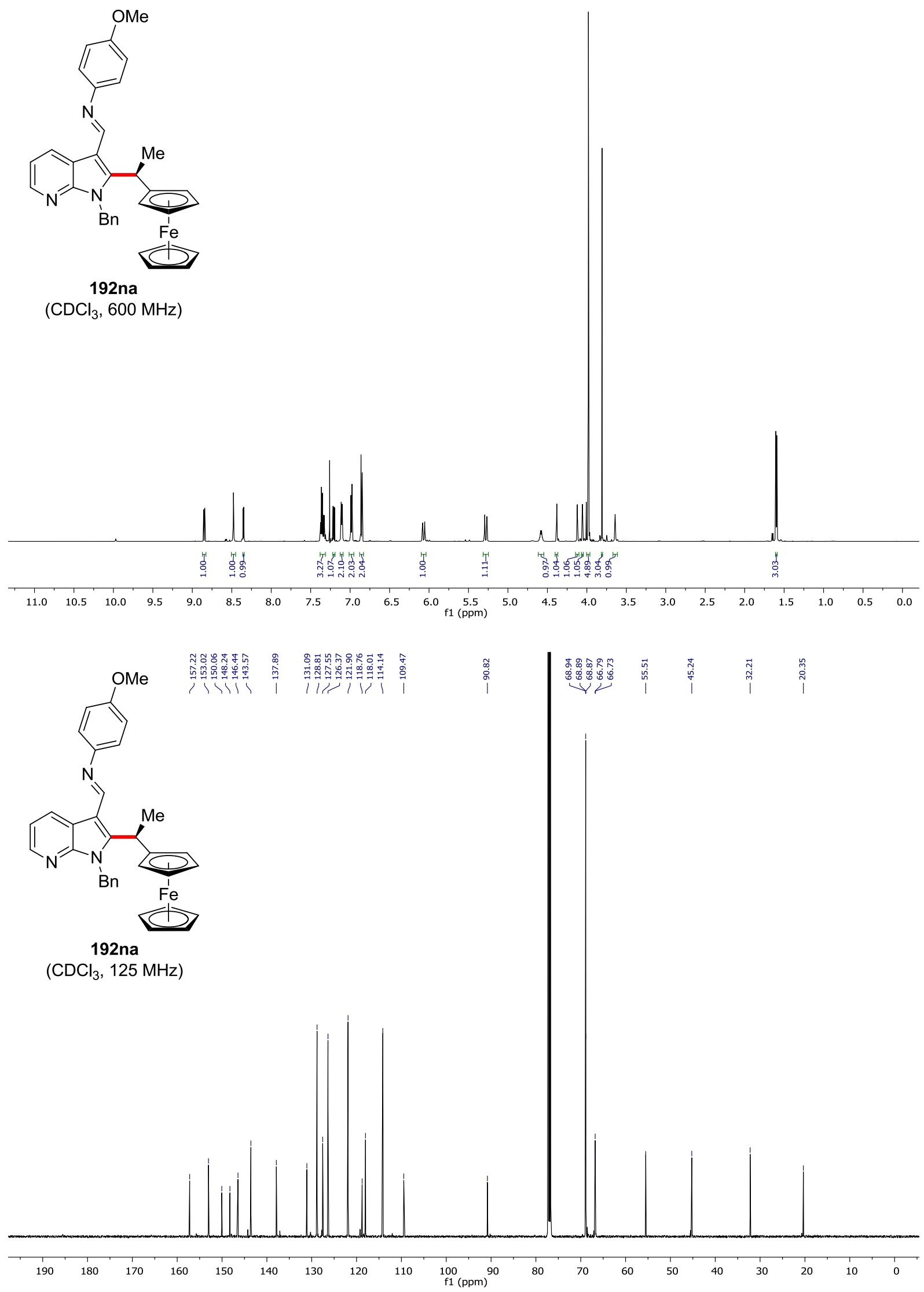


\section{Chiral HPLC of 192na:}
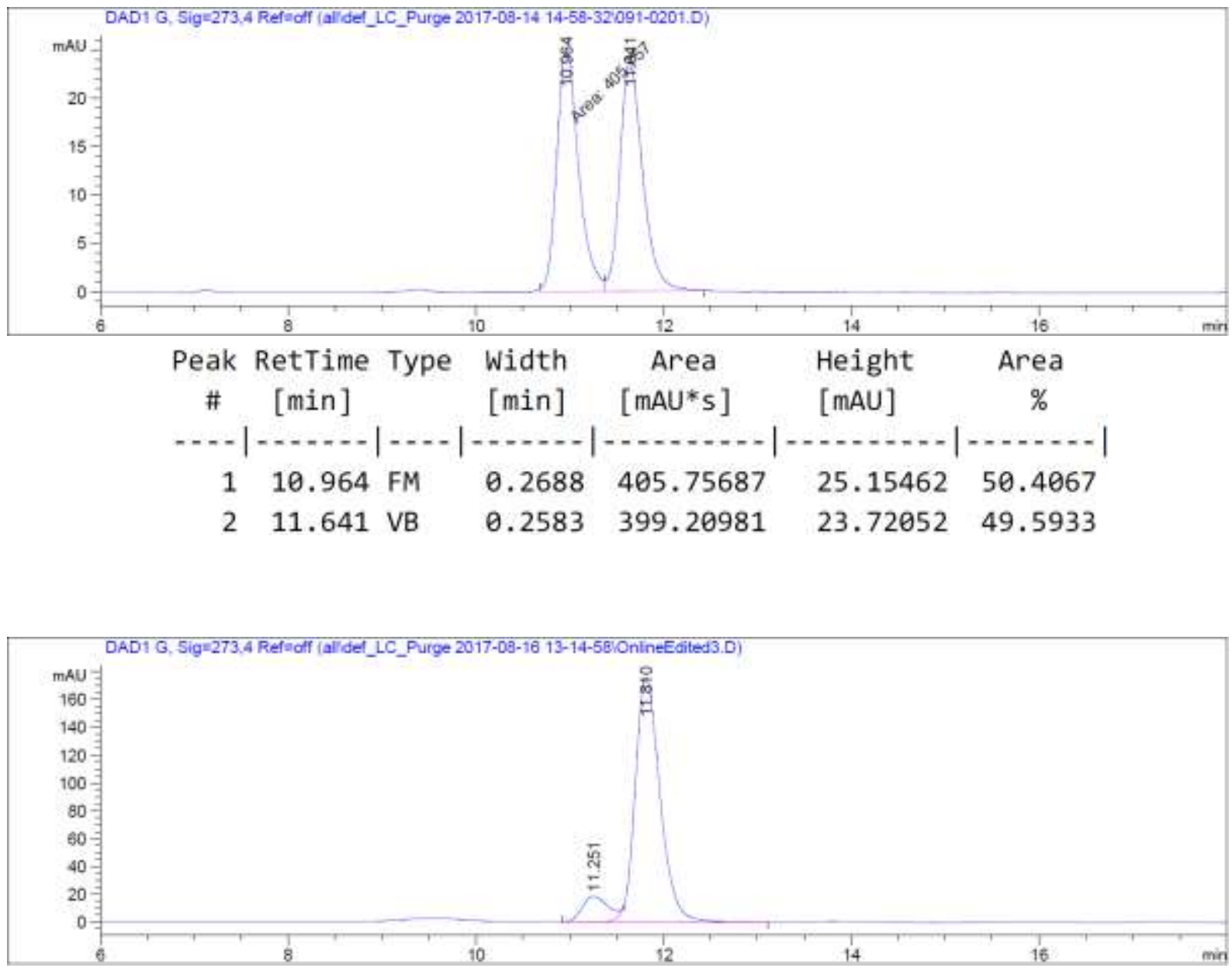

Peak RetTime Type Width Area Height Area

\# $[\mathrm{min}] \quad[\mathrm{min}] \quad\left[\mathrm{mAU}^{*} \mathrm{~s}\right] \quad[\mathrm{mAU}] \quad \%$

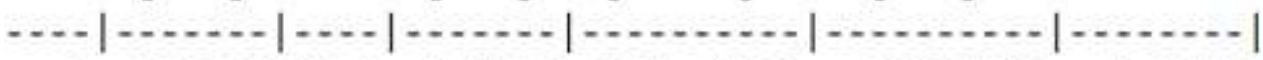

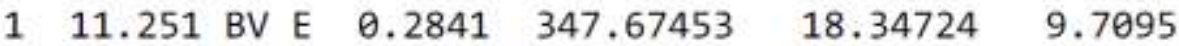

$\begin{array}{llllllll}2 & 11.810 & \text { VB R } & 0.2841 & 3233.10596 & 175.34357 & 90.2905\end{array}$ 

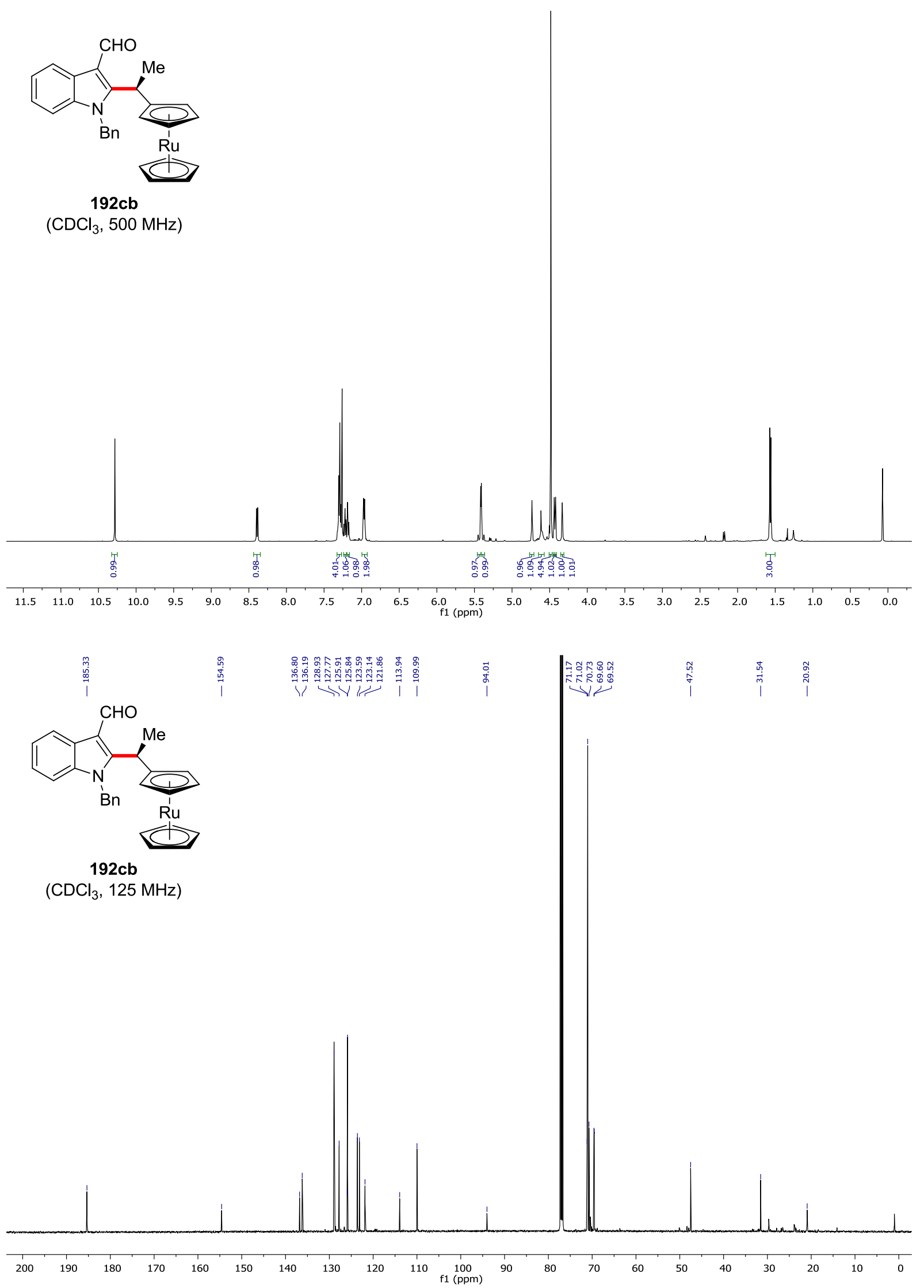


\section{Chiral HPLC of $192 \mathrm{cb}$ :}
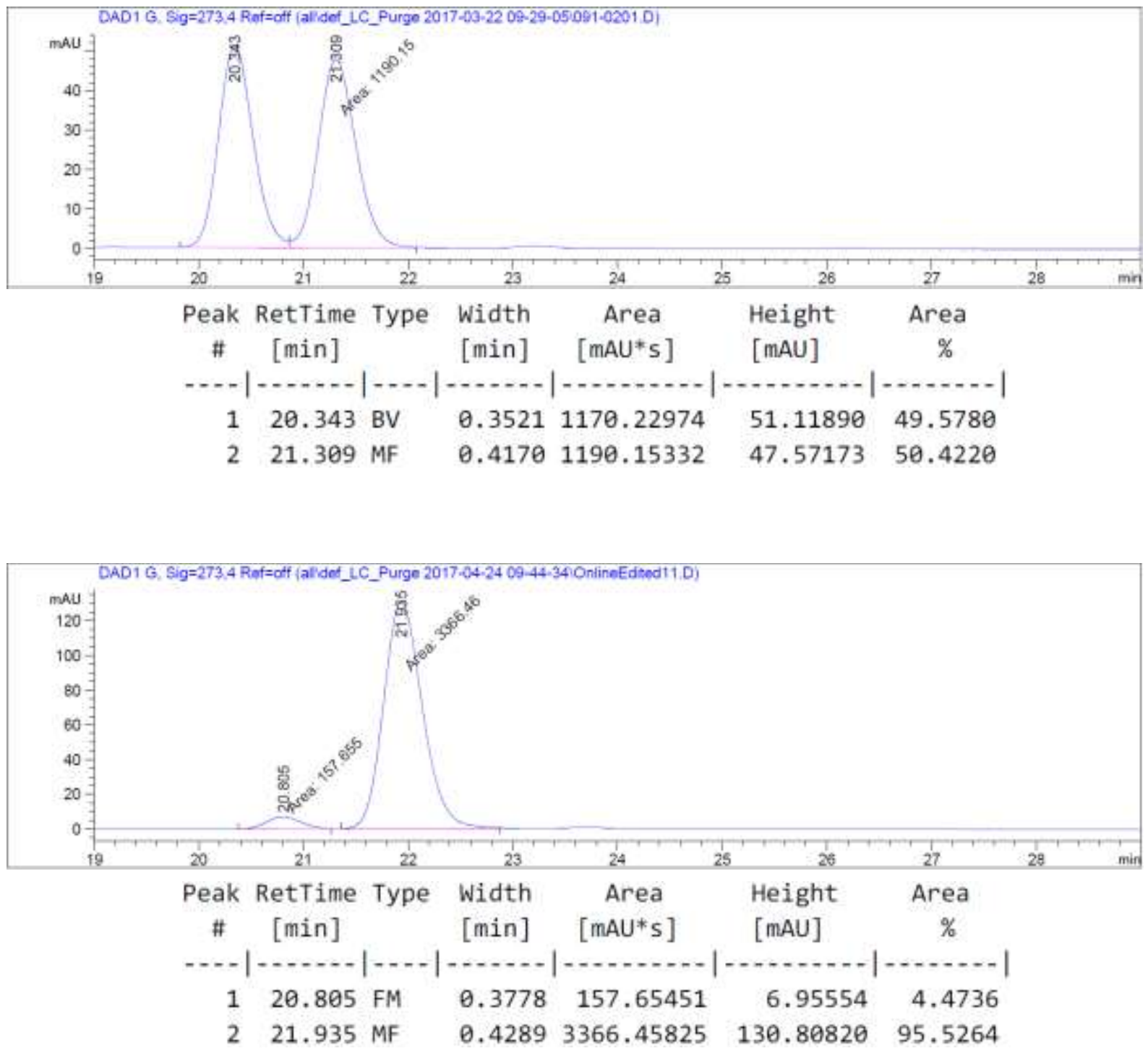


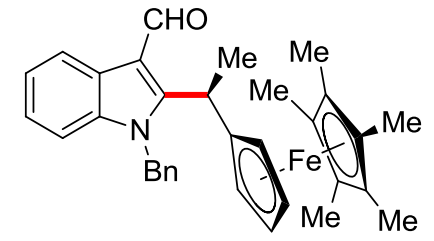

192cc

$\left(\mathrm{CDCl}_{3}, 500 \mathrm{MHz}\right)$
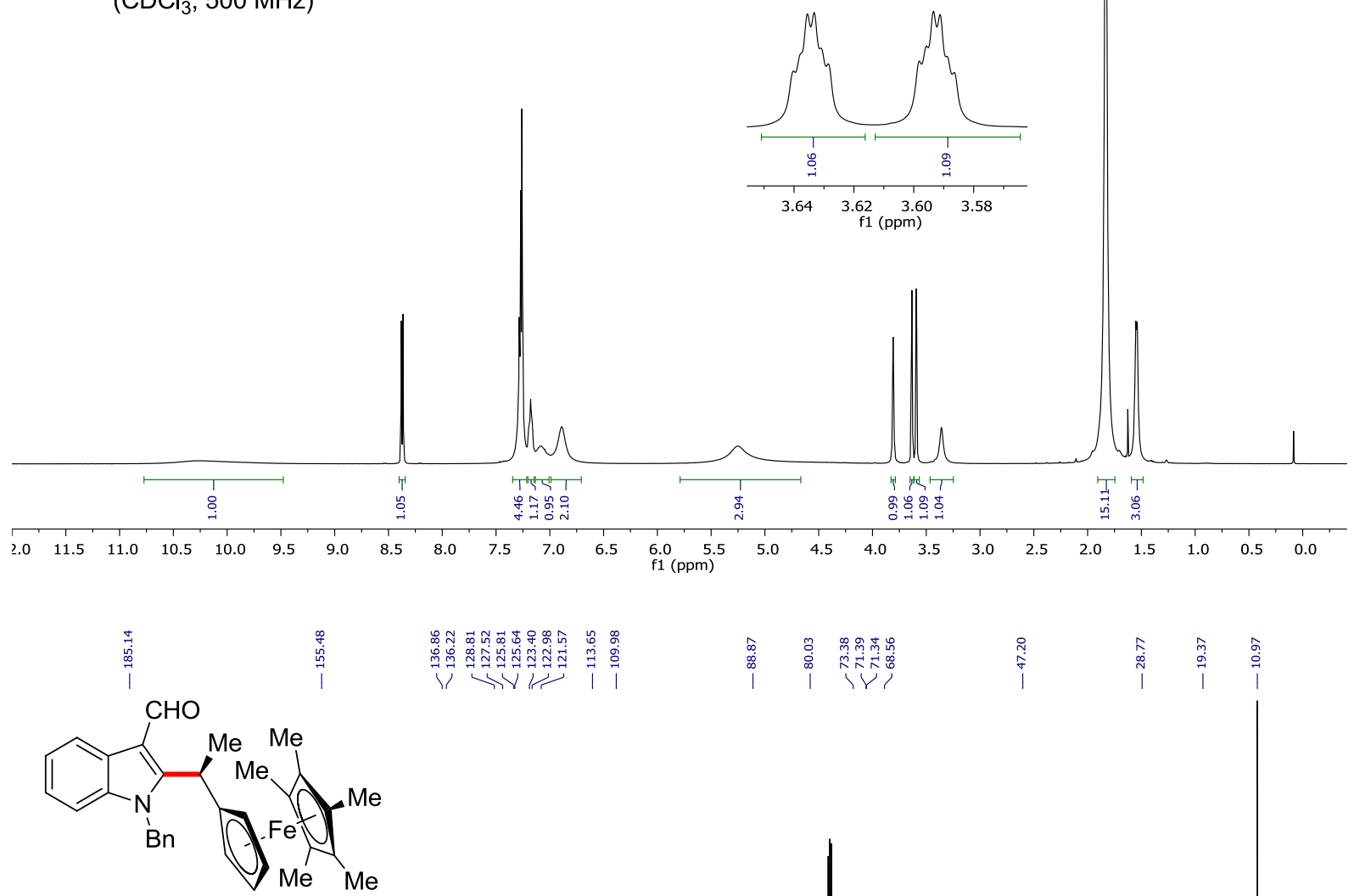

192cc

$\left(\mathrm{CDCl}_{3}, 125 \mathrm{MHz}\right)$

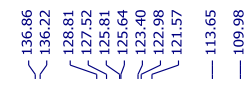

|

$\stackrel{\substack{\mathfrak{5} \\ \text { I }}}{i}$

$\begin{array}{ccc}\hat{\infty} & \hat{m} & \hat{a} \\ \stackrel{0}{0} & \hat{\jmath} & 1\end{array}$

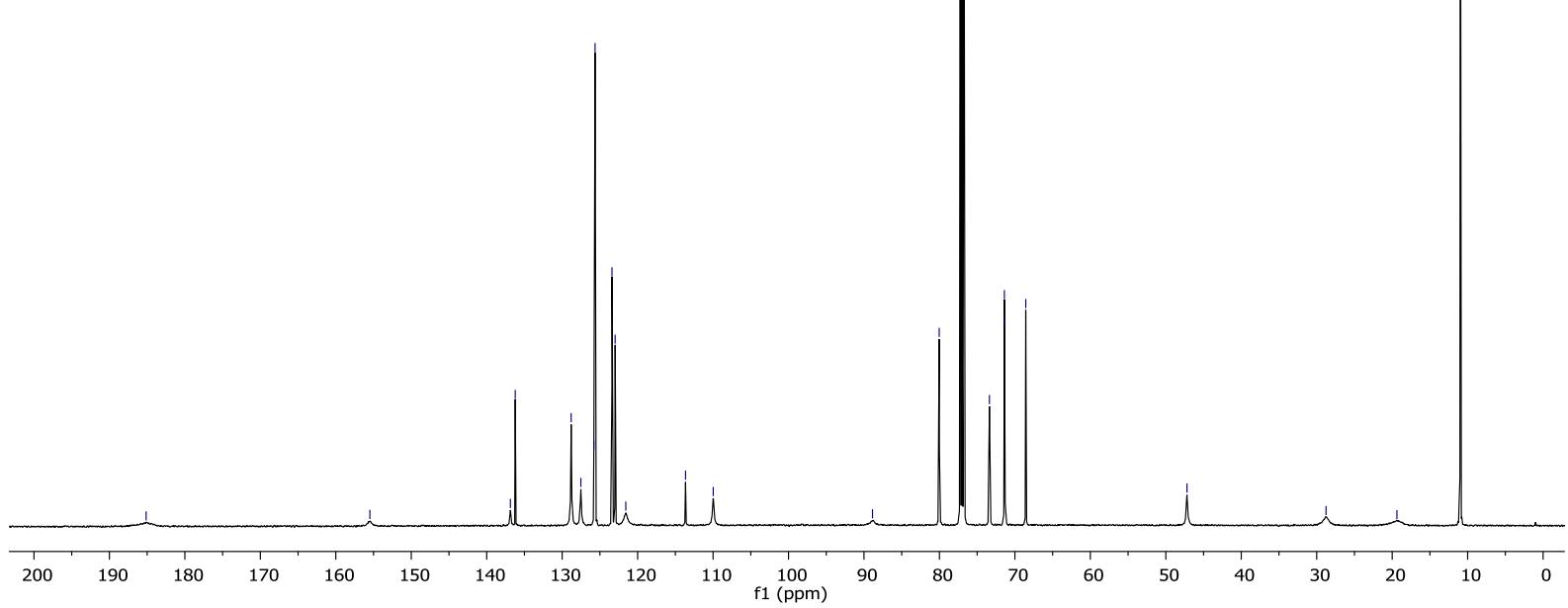




\section{Chiral HPLC of 192cc:}
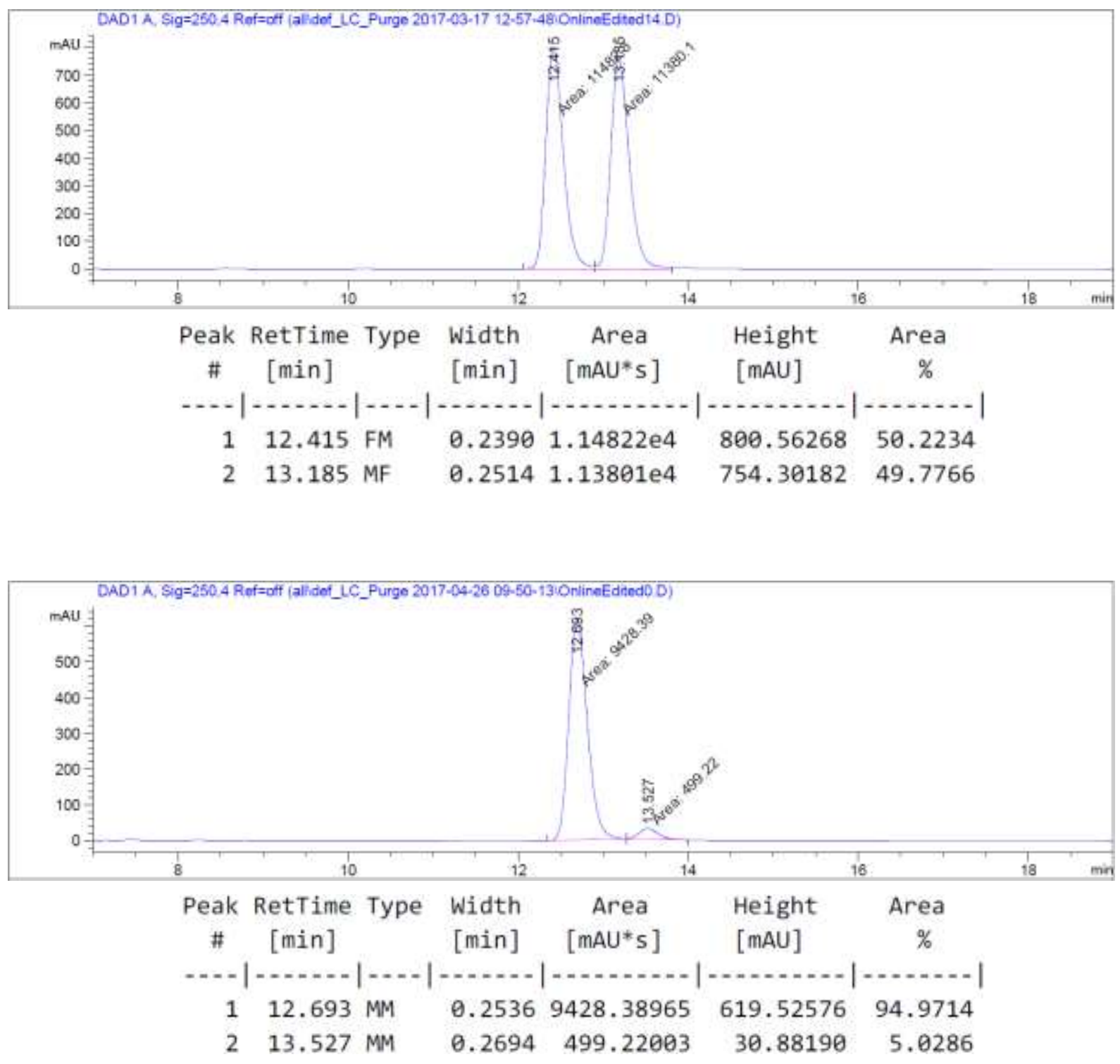


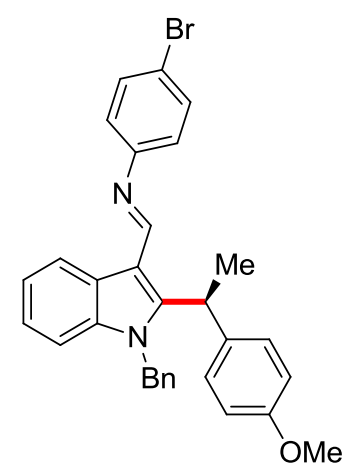

197

$\left(\mathrm{CDCl}_{3}, 300 \mathrm{MHz}\right)$
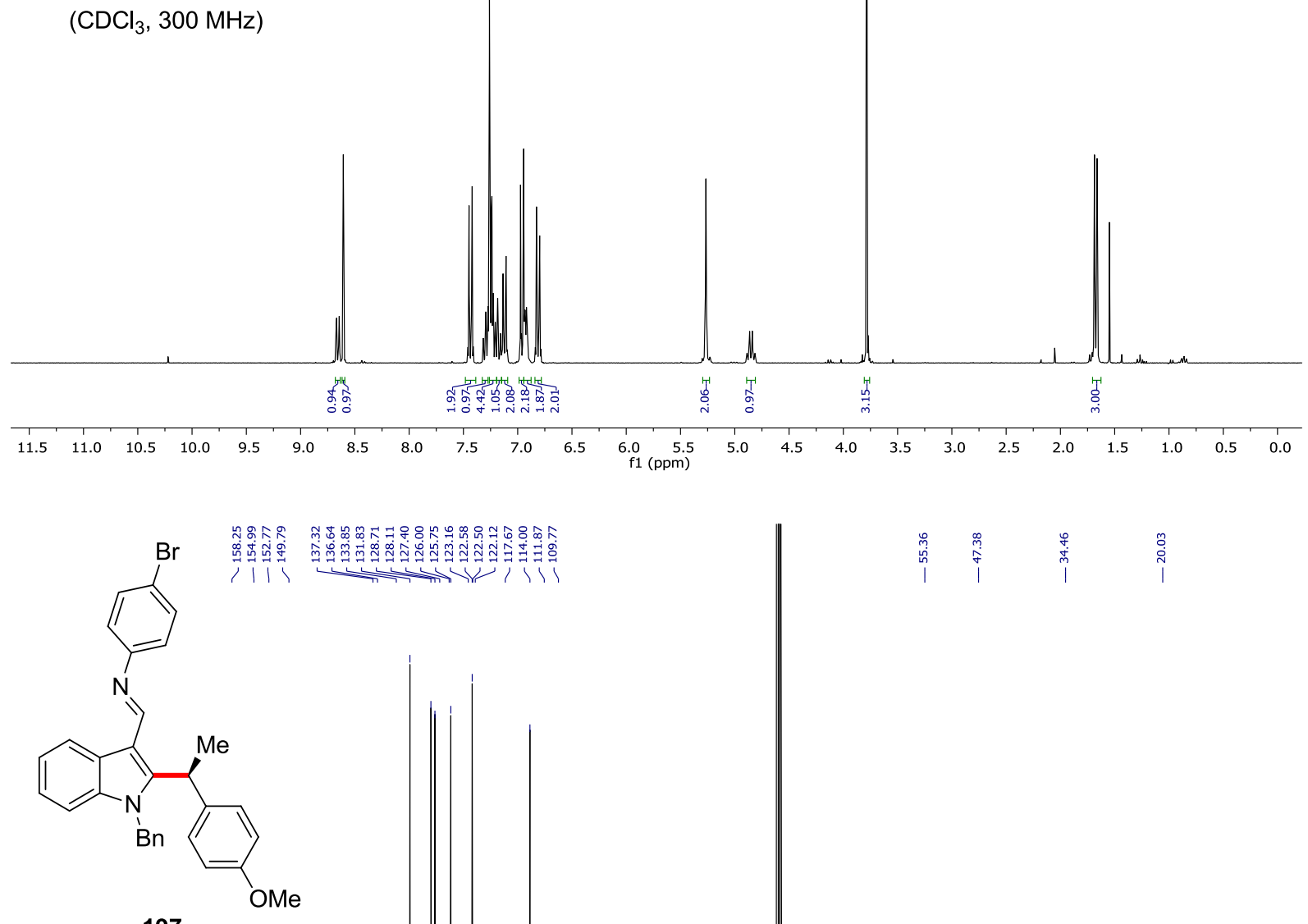

197

$\left(\mathrm{CDCl}_{3}, 125 \mathrm{MHz}\right)$

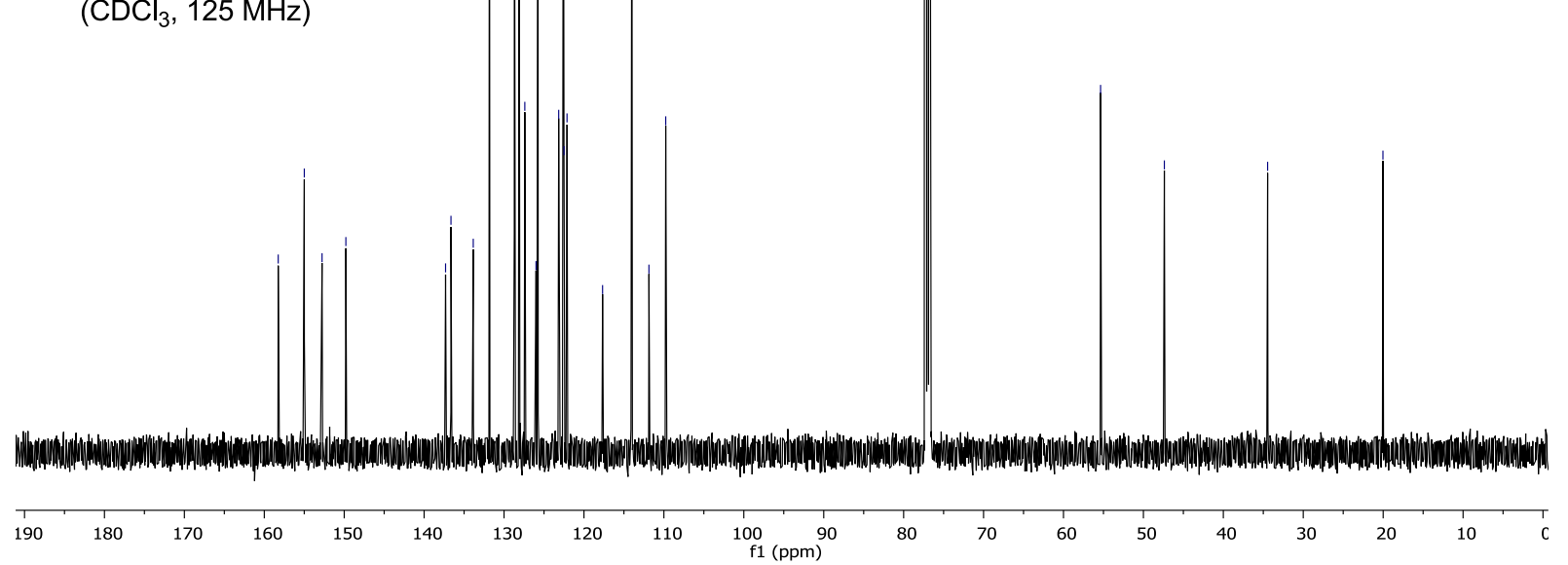




\section{Chiral HPLC of 197:}
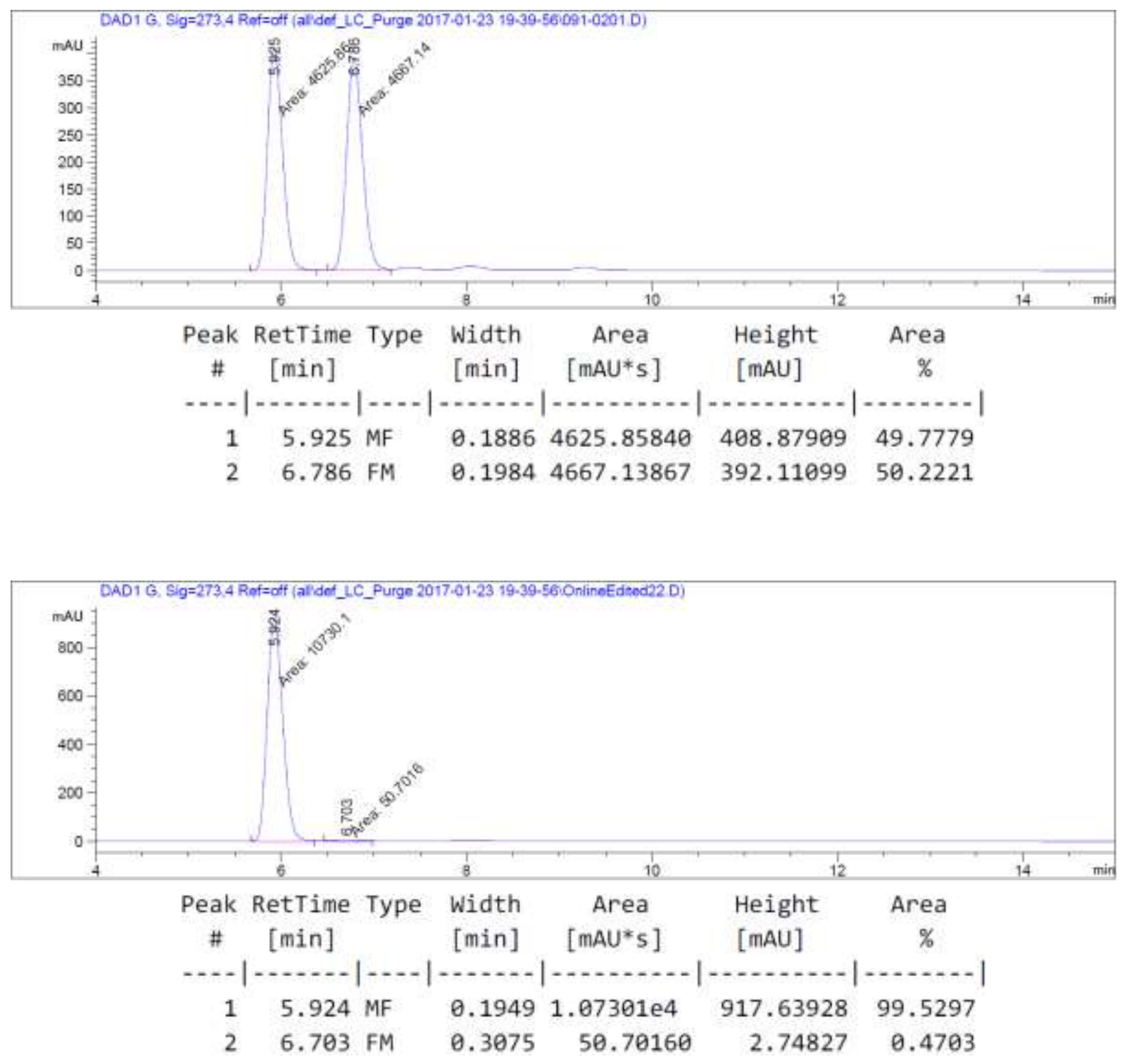


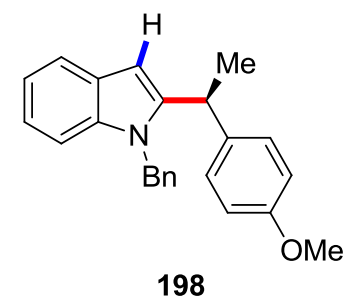

$\left(\mathrm{CDCl}_{3}, 500 \mathrm{MHz}\right)$
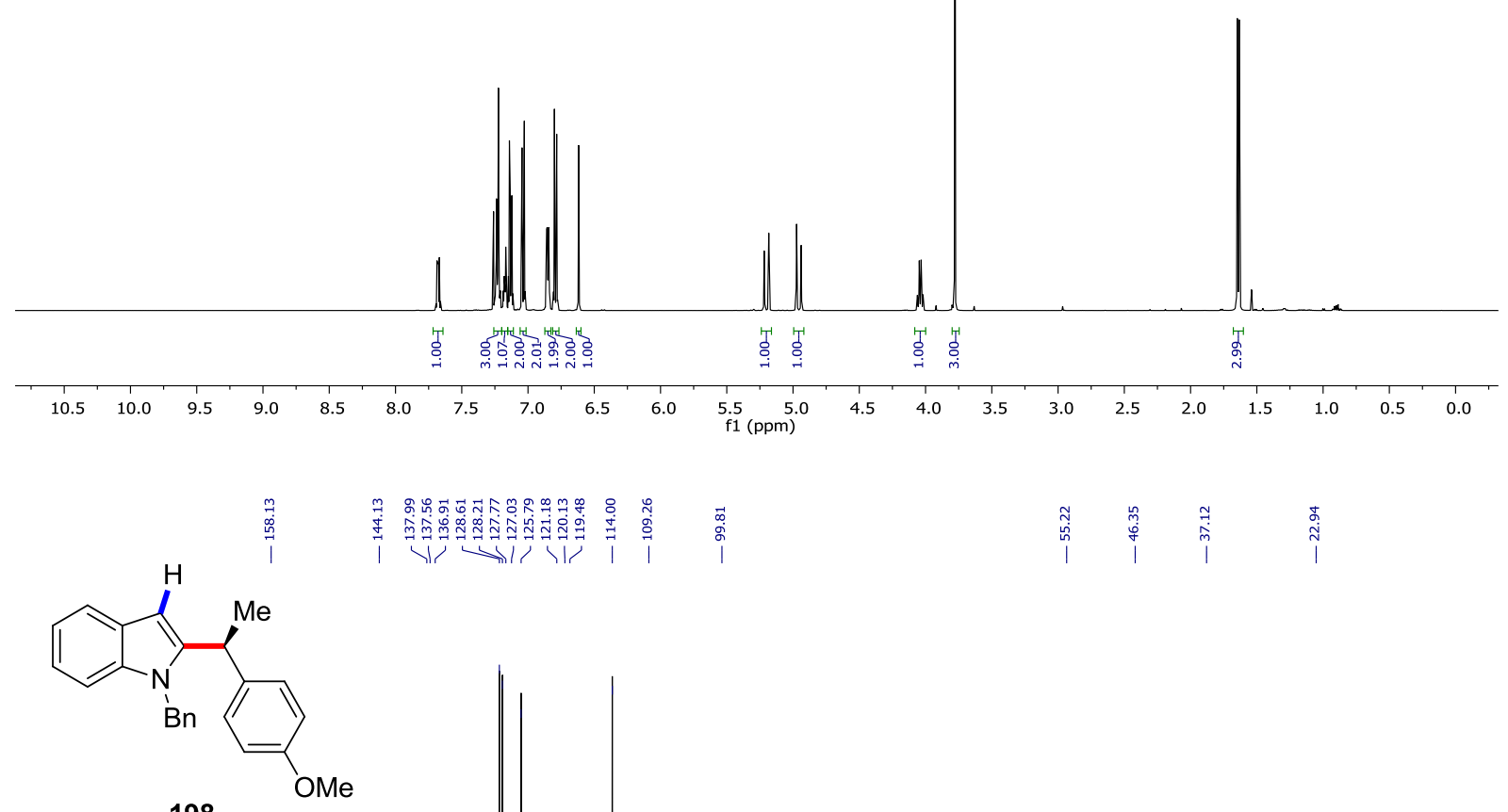

$\left(\mathrm{CDCl}_{3}, 125 \mathrm{MHz}\right)$

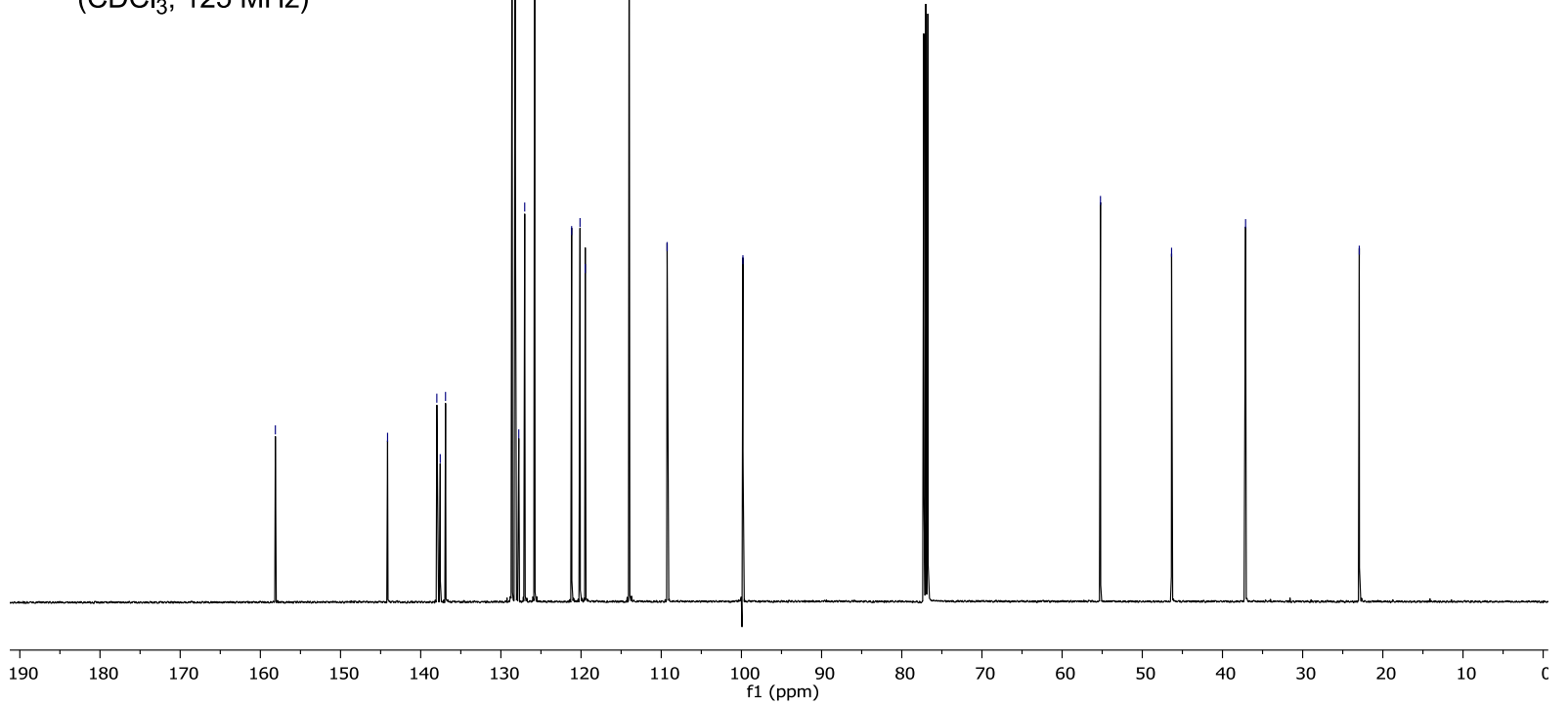




\section{Chiral HPLC of 198:}
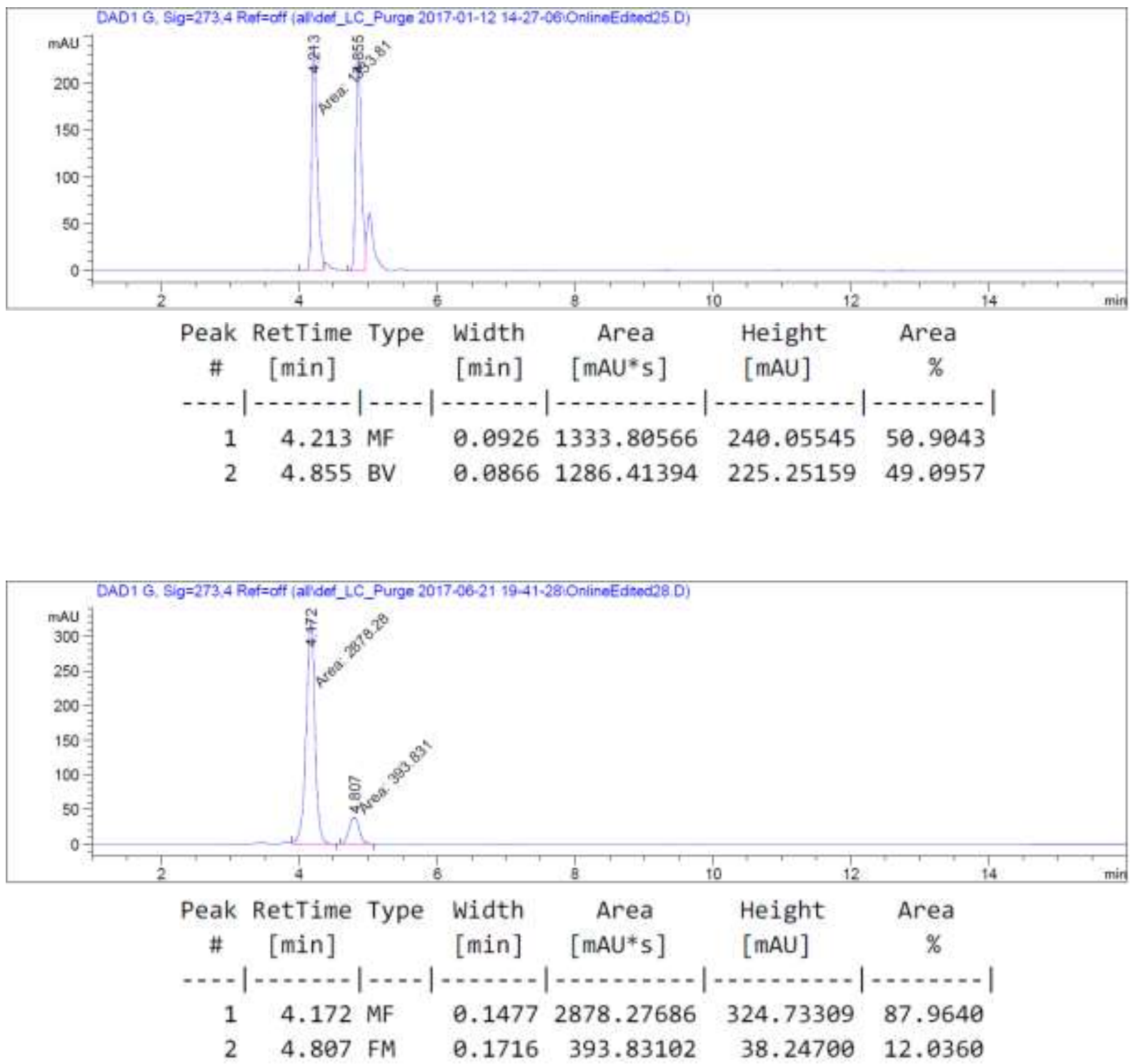


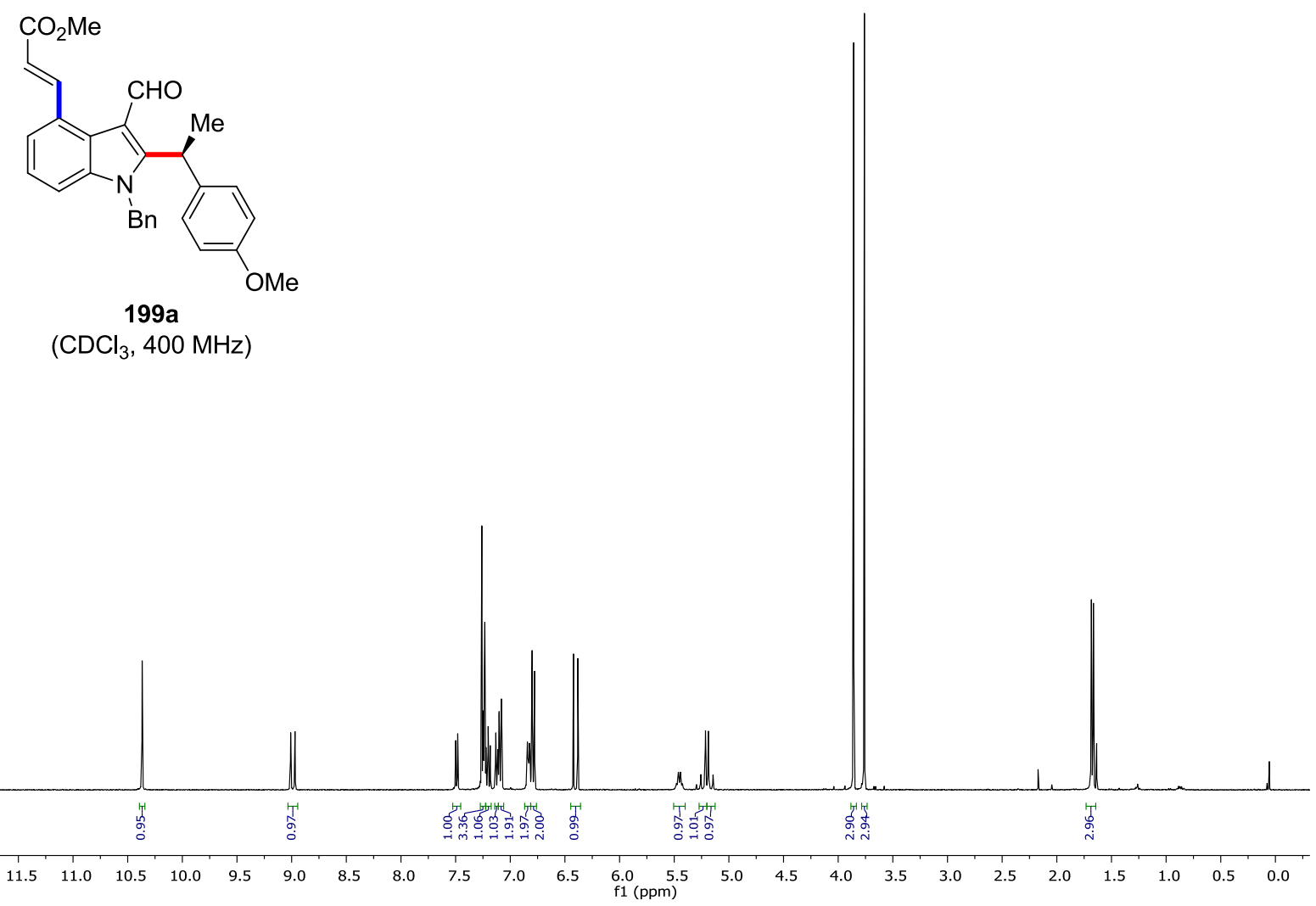

焉

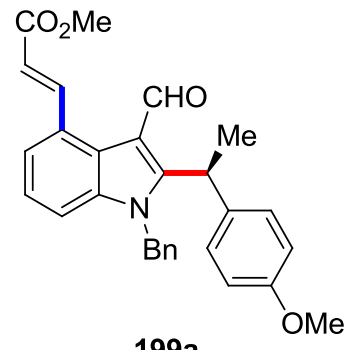

$199 a$

$\left(\mathrm{CDCl}_{3}, 100 \mathrm{MHz}\right)$

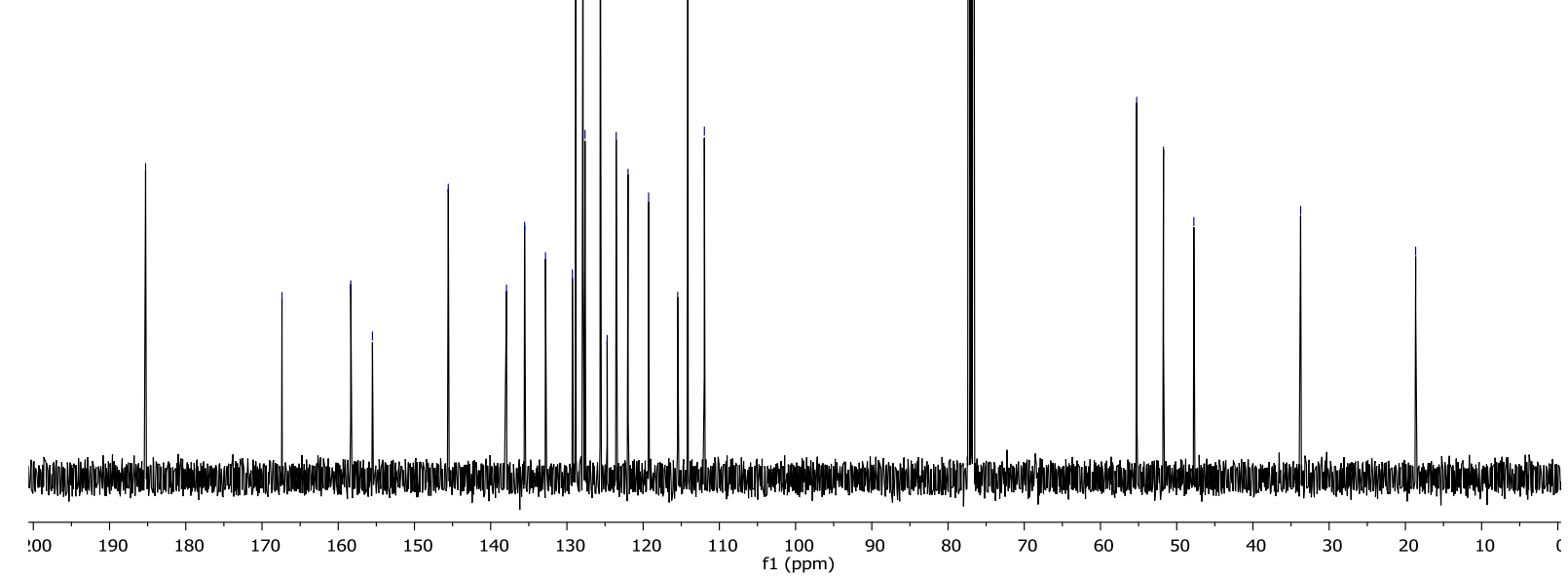




\section{Chiral HPLC of 199a:}
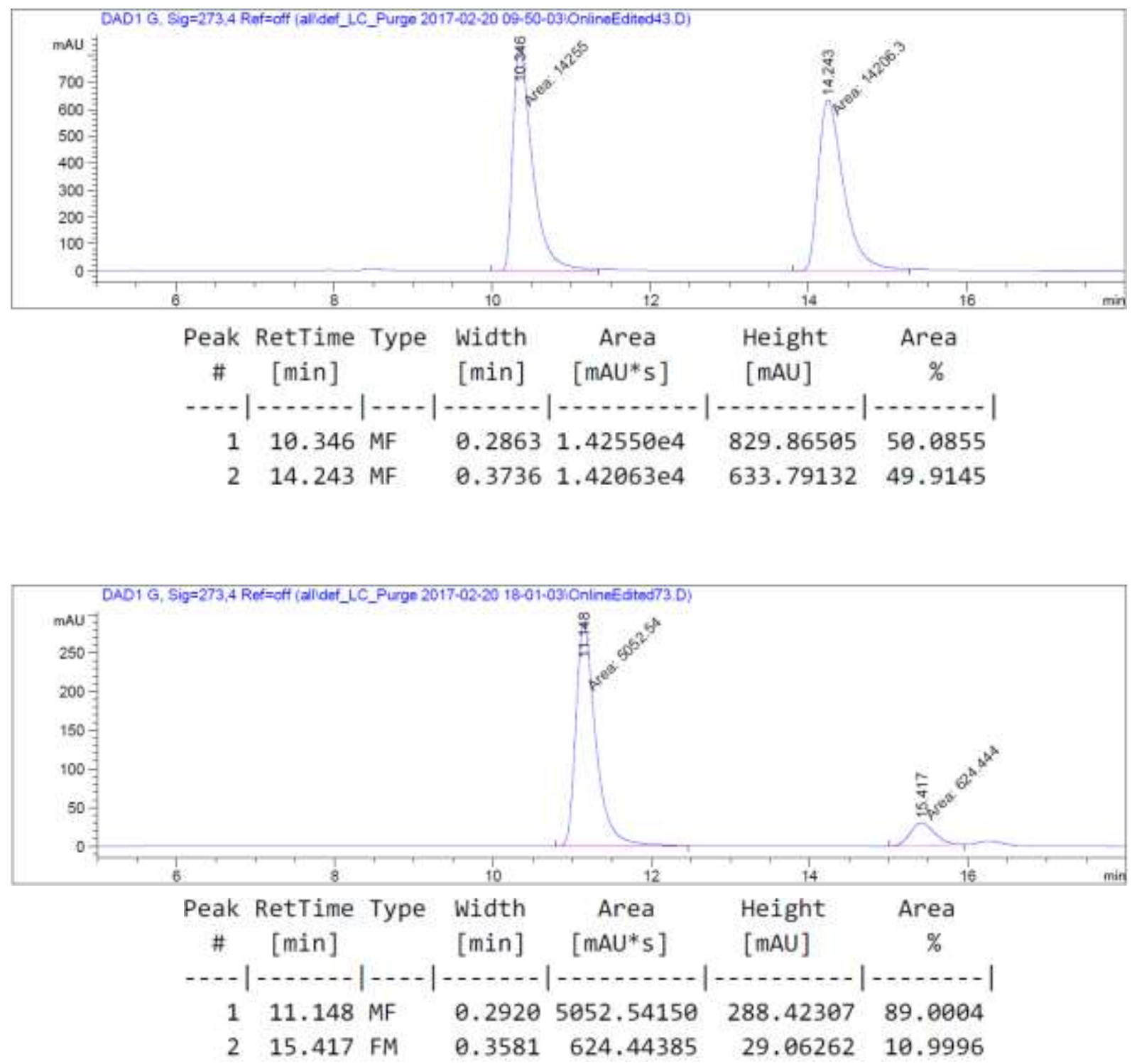


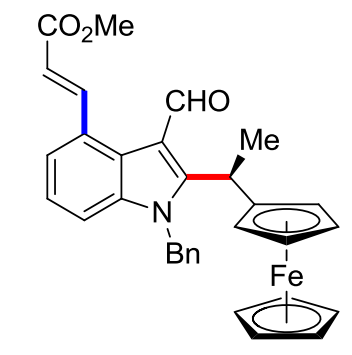

199b

$\left(\mathrm{CDCl}_{3}, 300 \mathrm{MHz}\right)$
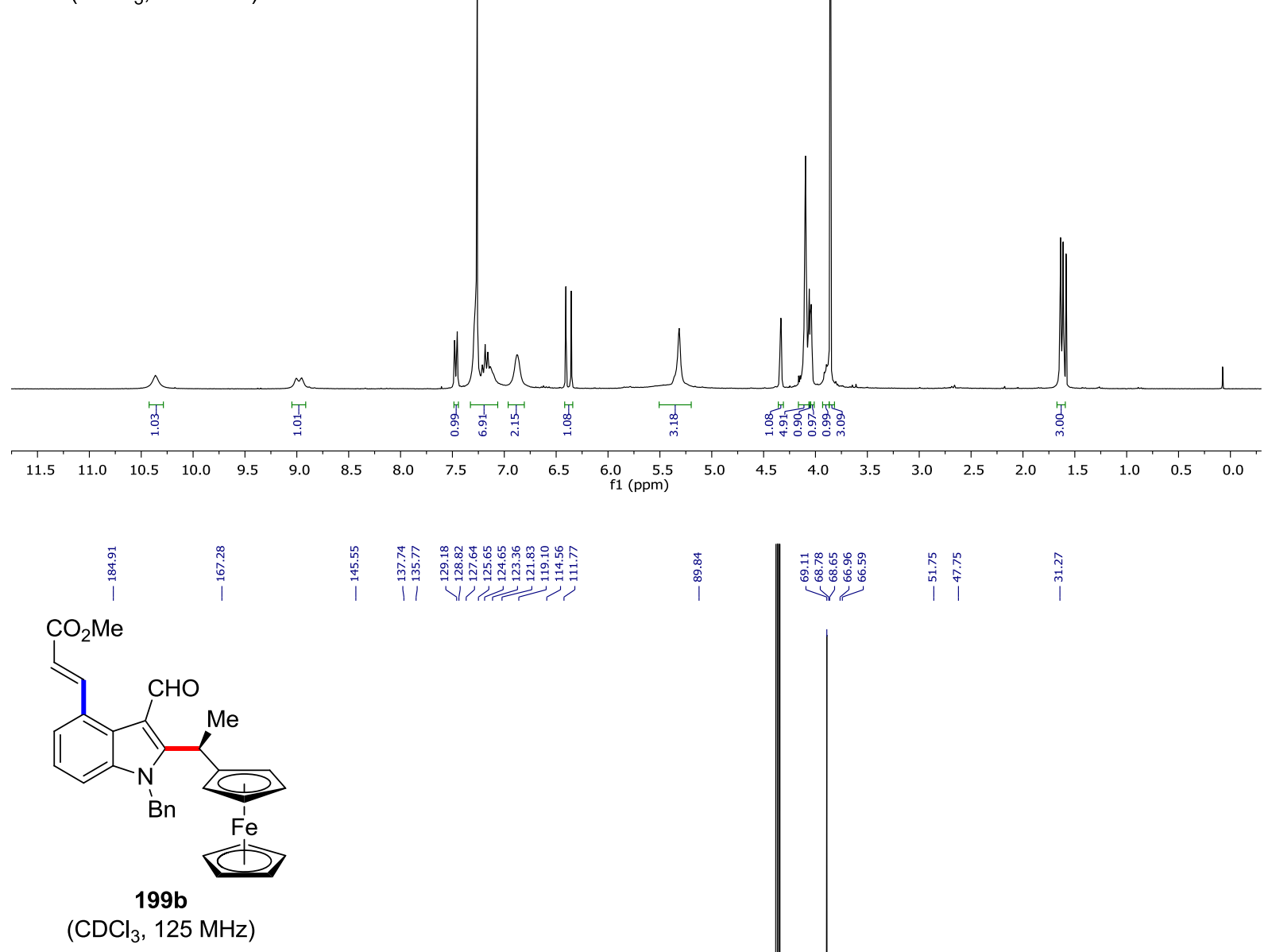

$\left(\mathrm{CDCl}_{3}, 125 \mathrm{MHz}\right)$

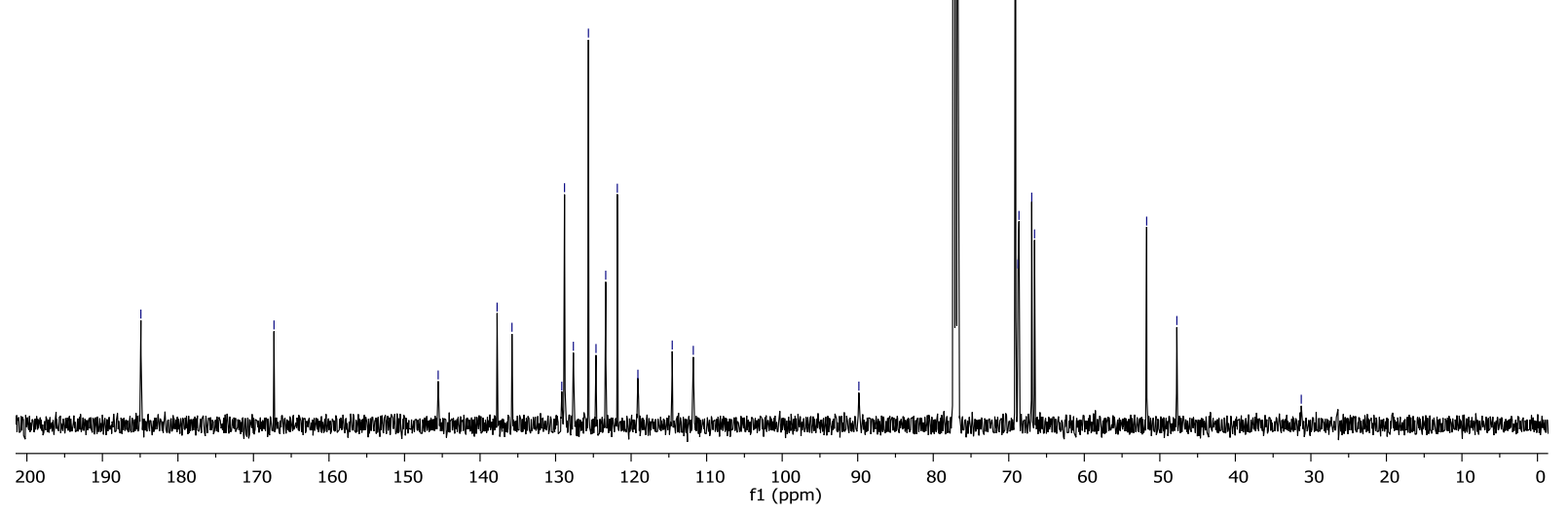




\section{Chiral HPLC of 199b:}
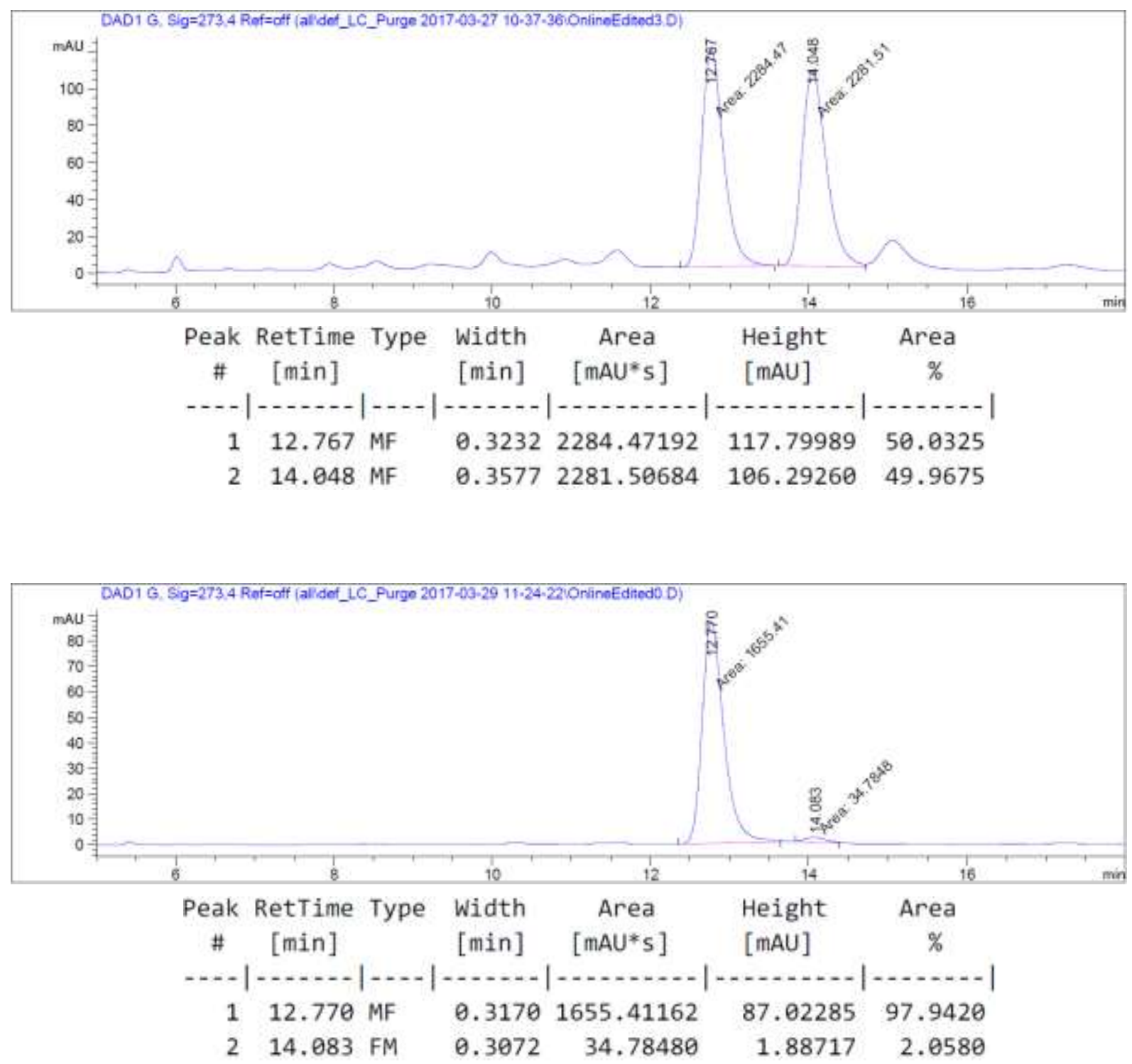


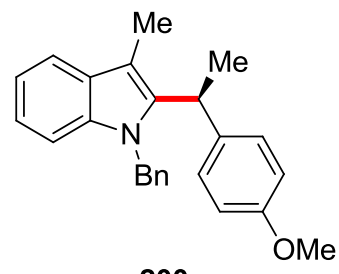

200

$\left(\mathrm{CDCl}_{3}, 300 \mathrm{MHz}\right)$
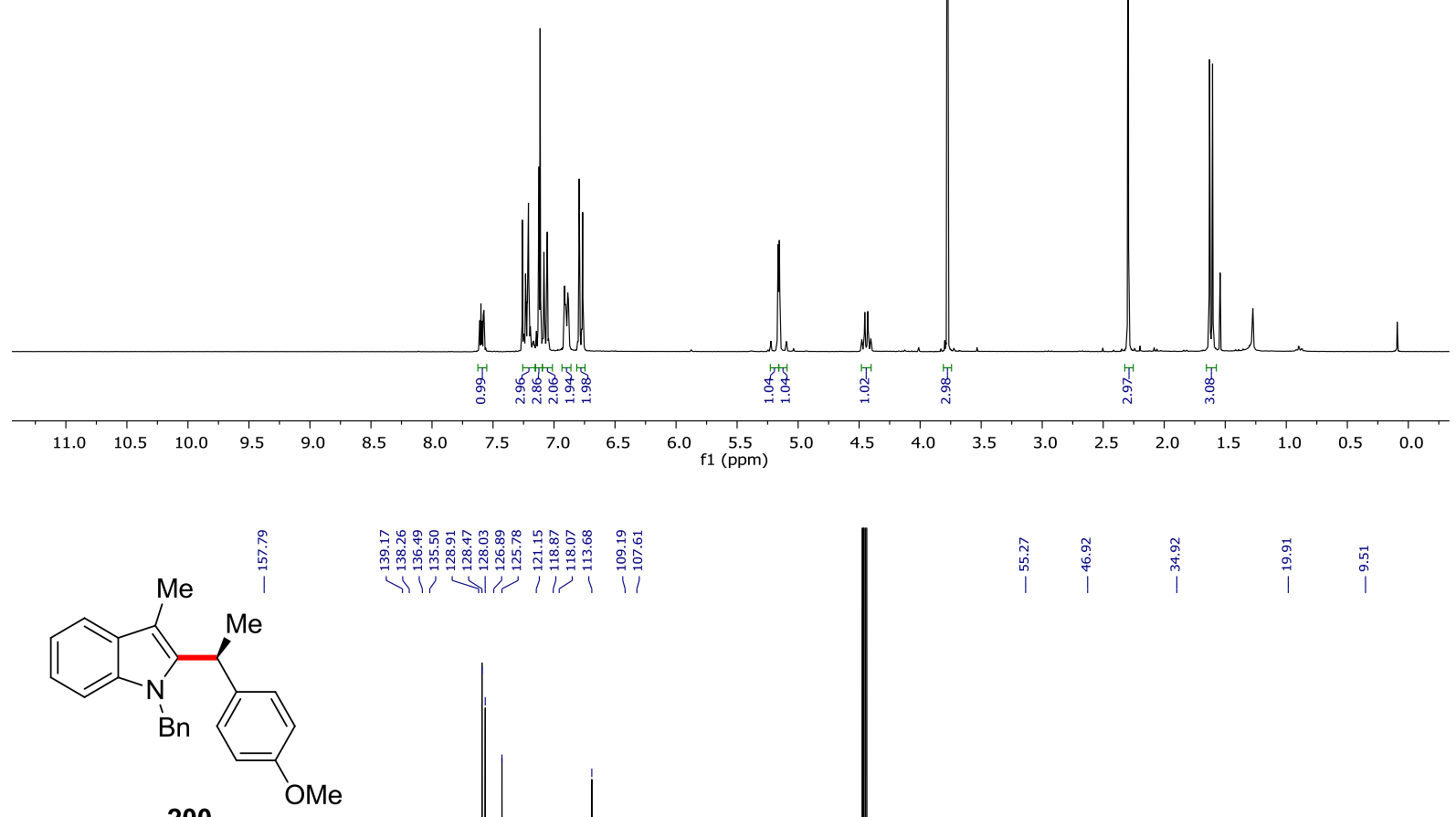

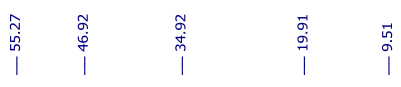

$\left(\mathrm{CDCl}_{3}, 125 \mathrm{MHz}\right)$

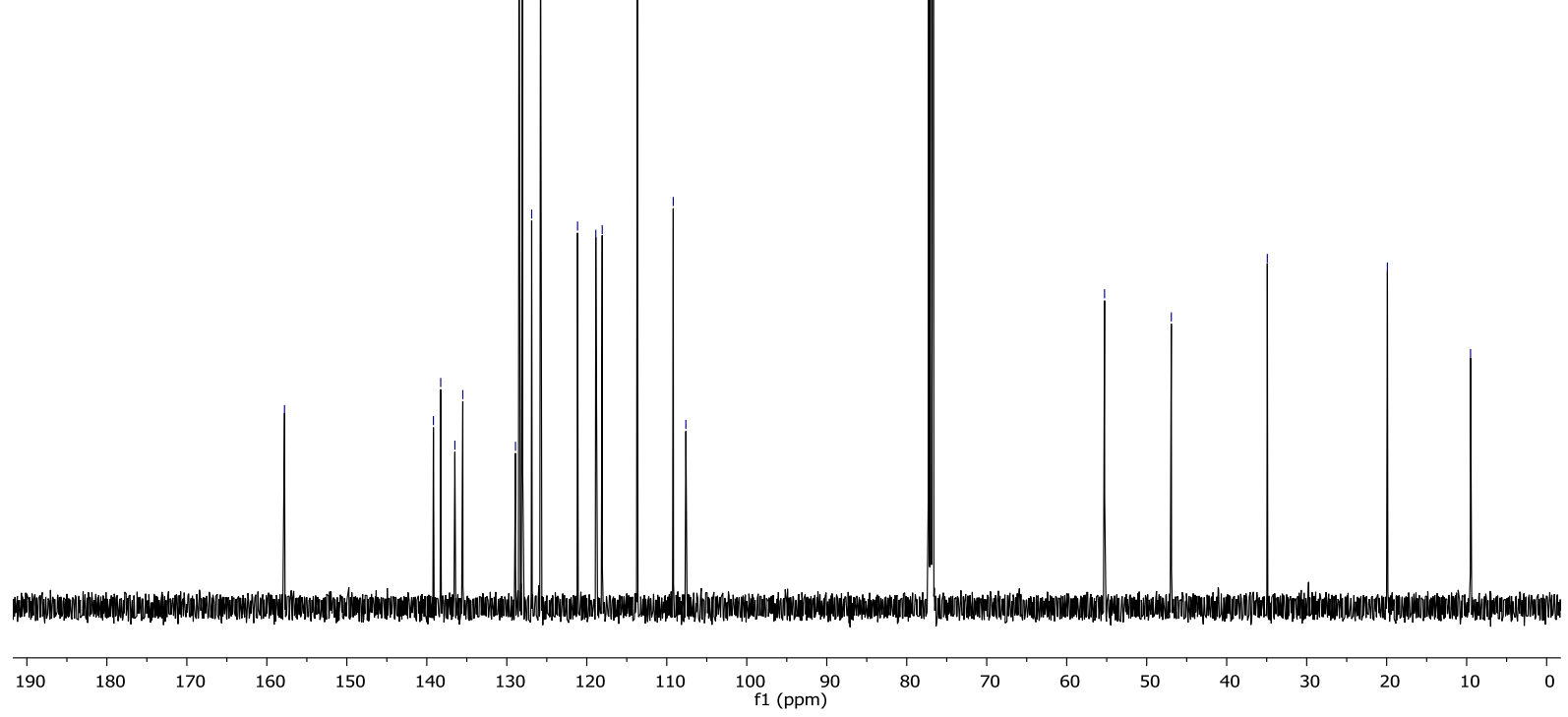




\section{Chiral HPLC of 200:}
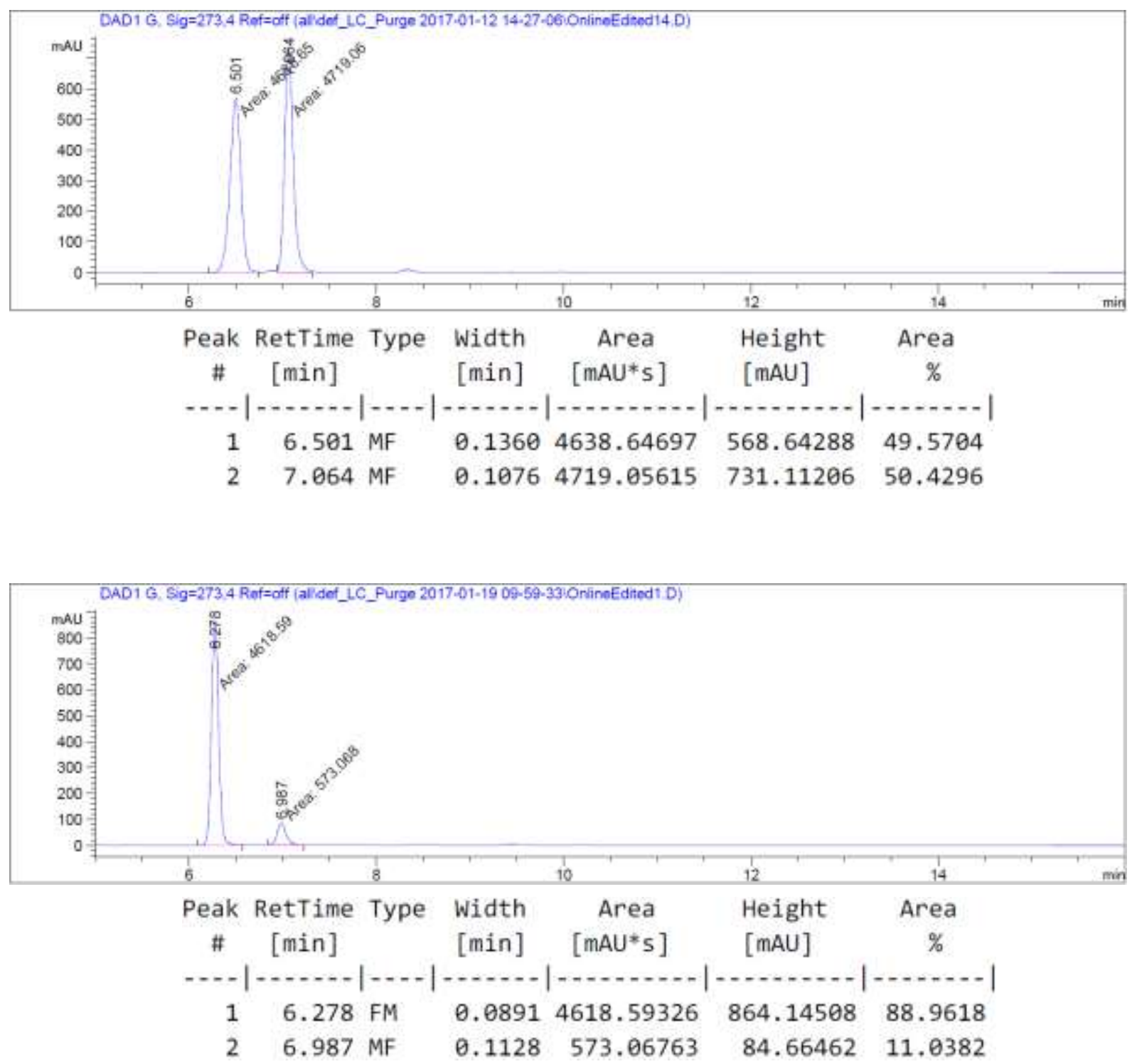


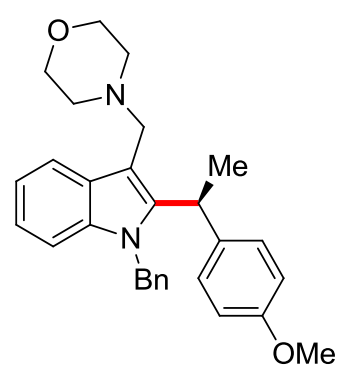

201

$\left(\mathrm{CDCl}_{3}, 300 \mathrm{MHz}\right)$
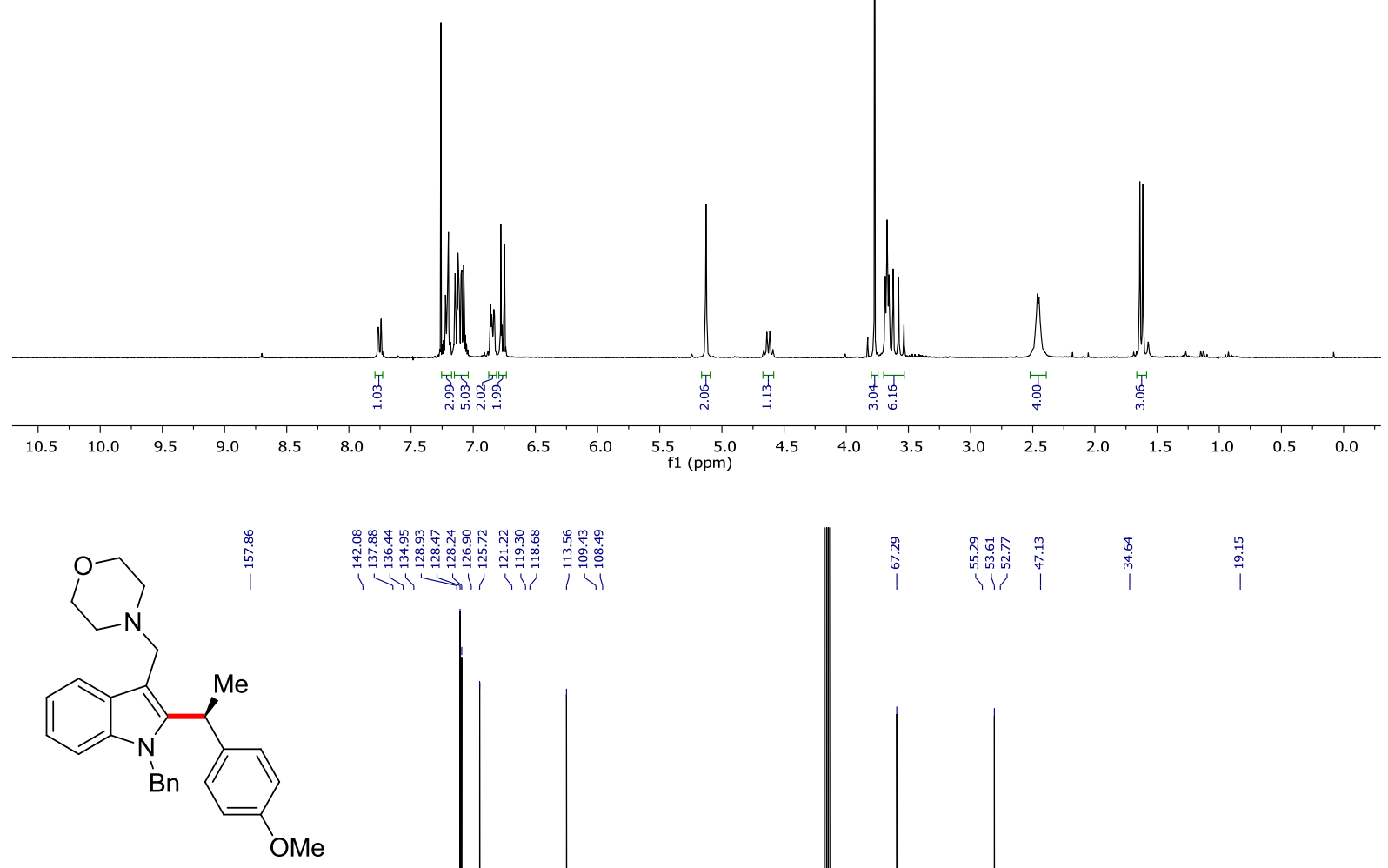

201

$\left(\mathrm{CDCl}_{3}, 125 \mathrm{MHz}\right)$

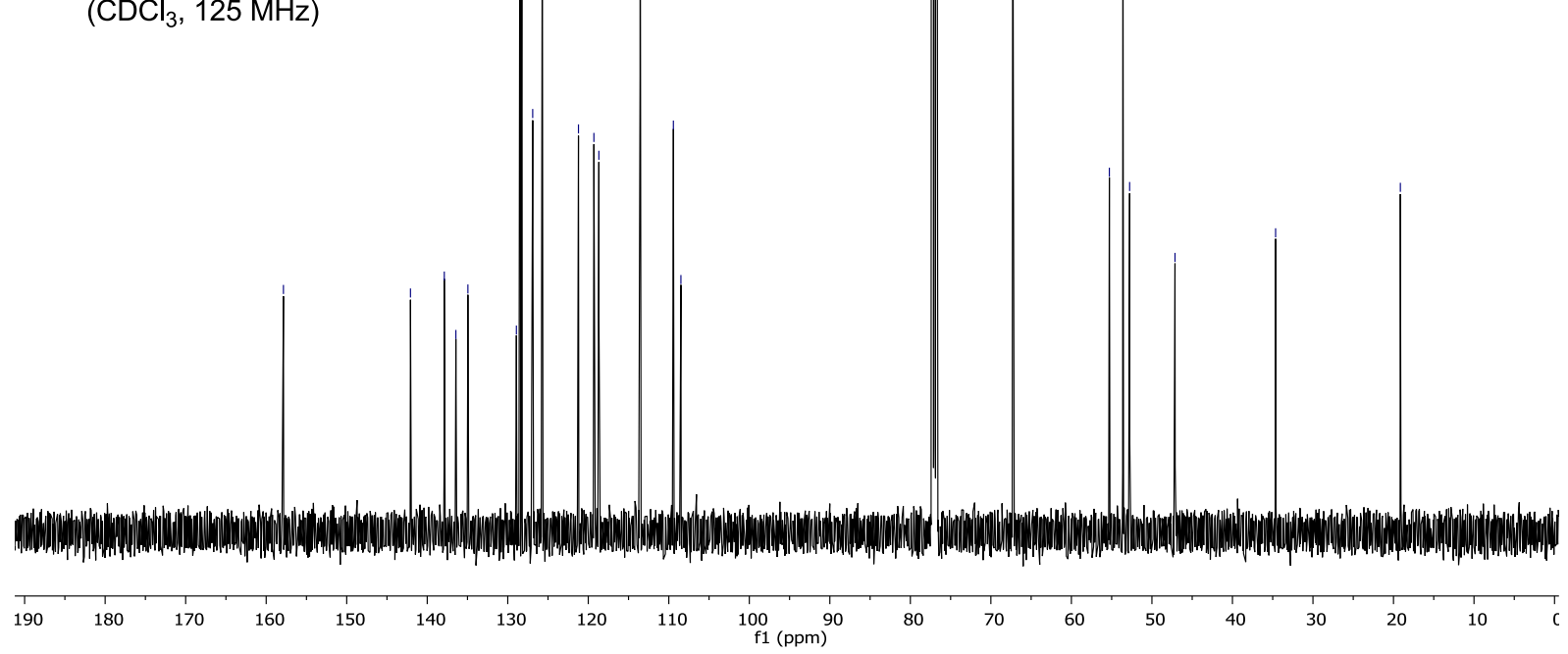




\section{Chiral HPLC of 201:}
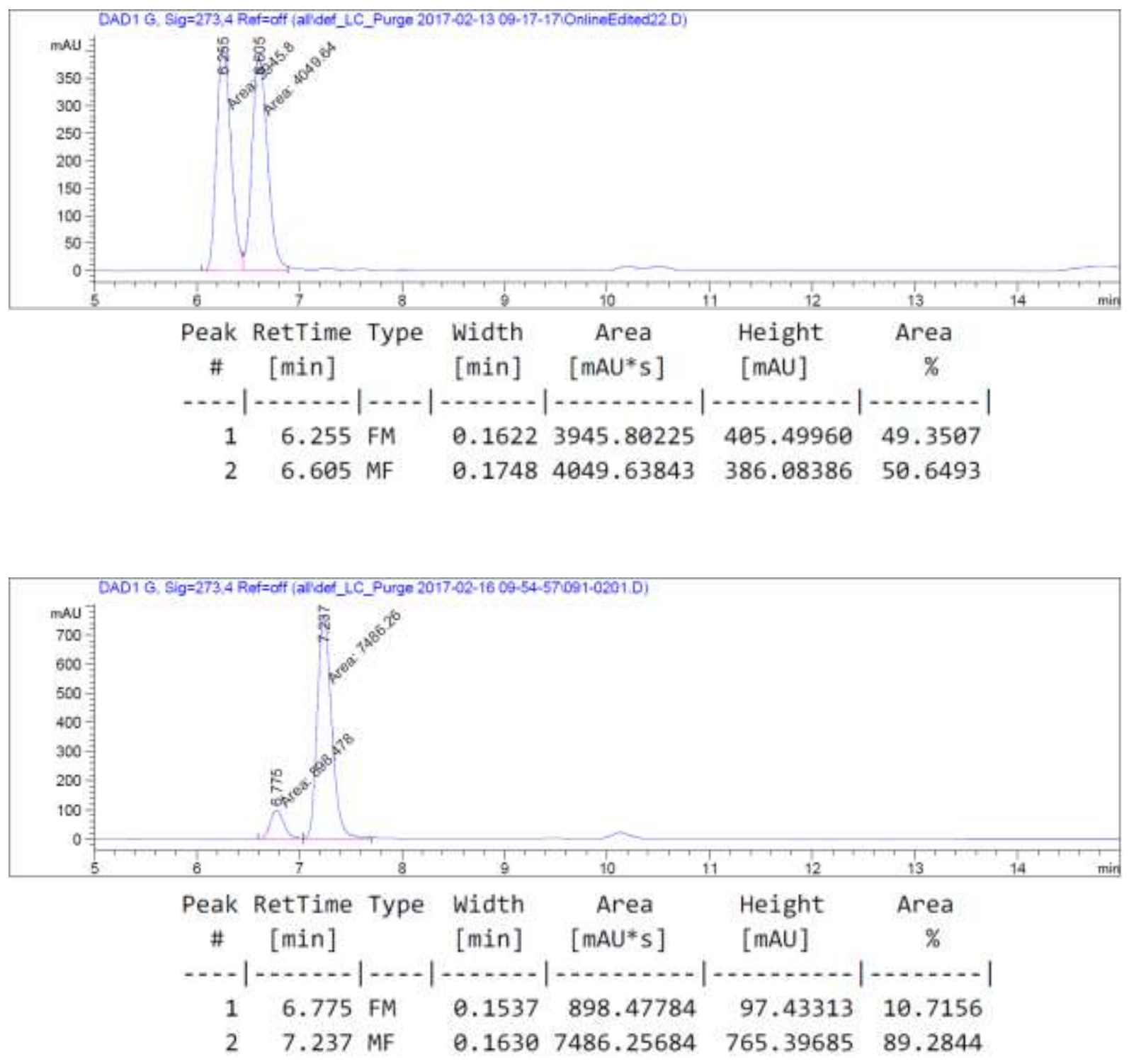

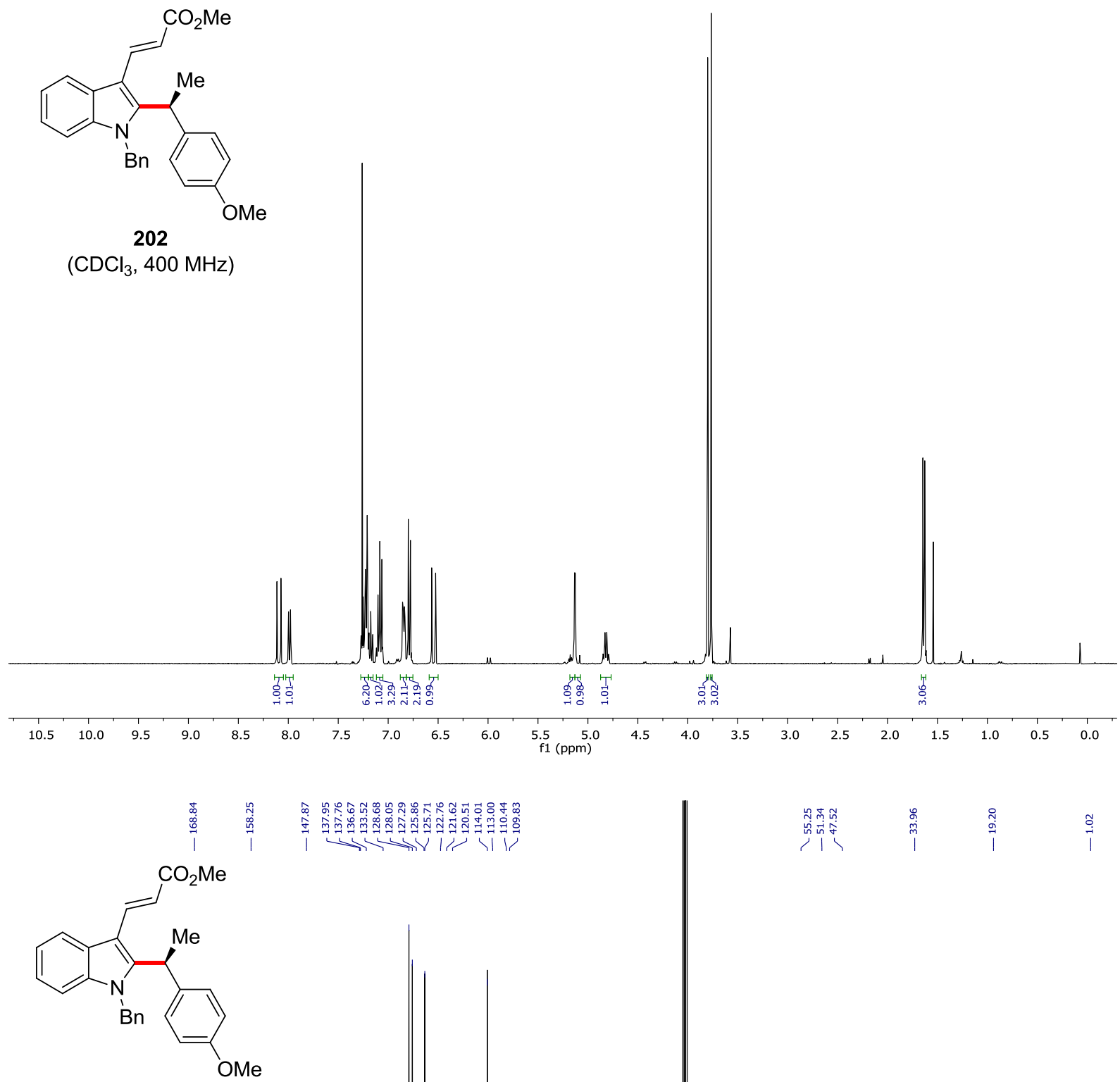

202

$\left(\mathrm{CDCl}_{3}, 100 \mathrm{MHz}\right)$

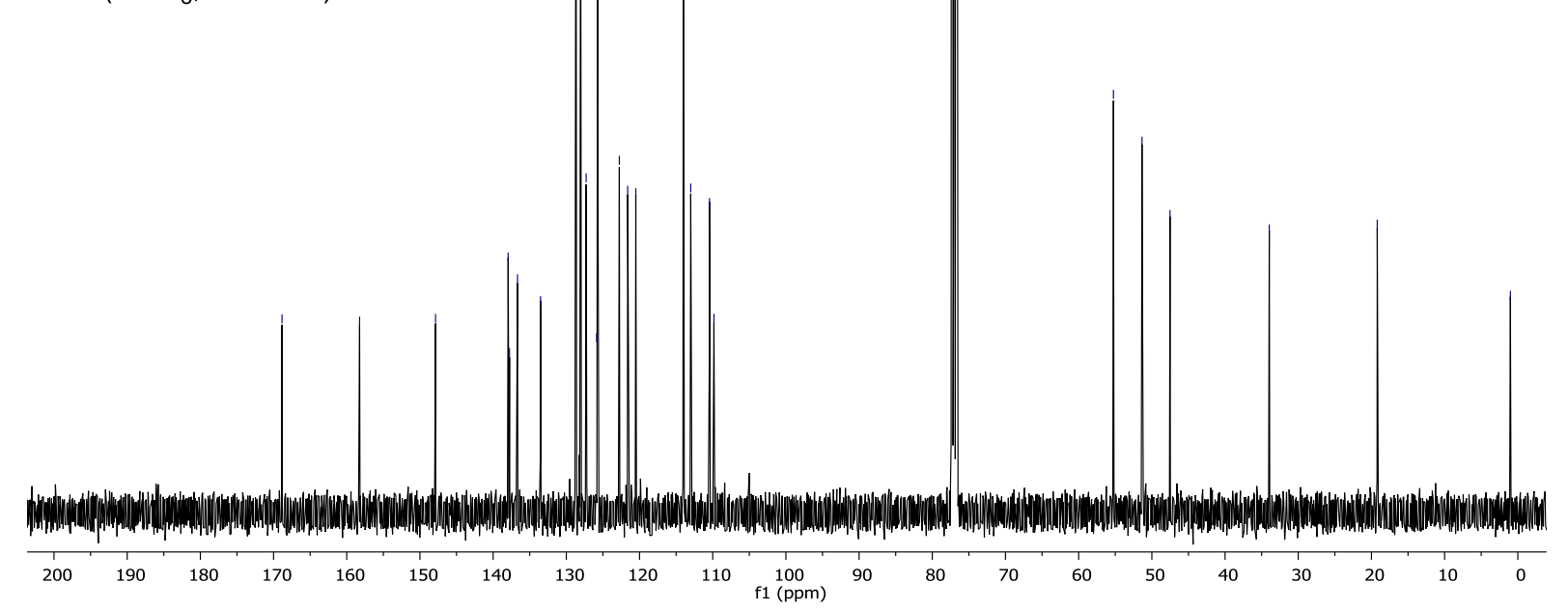




\section{Chiral HPLC of 202:}
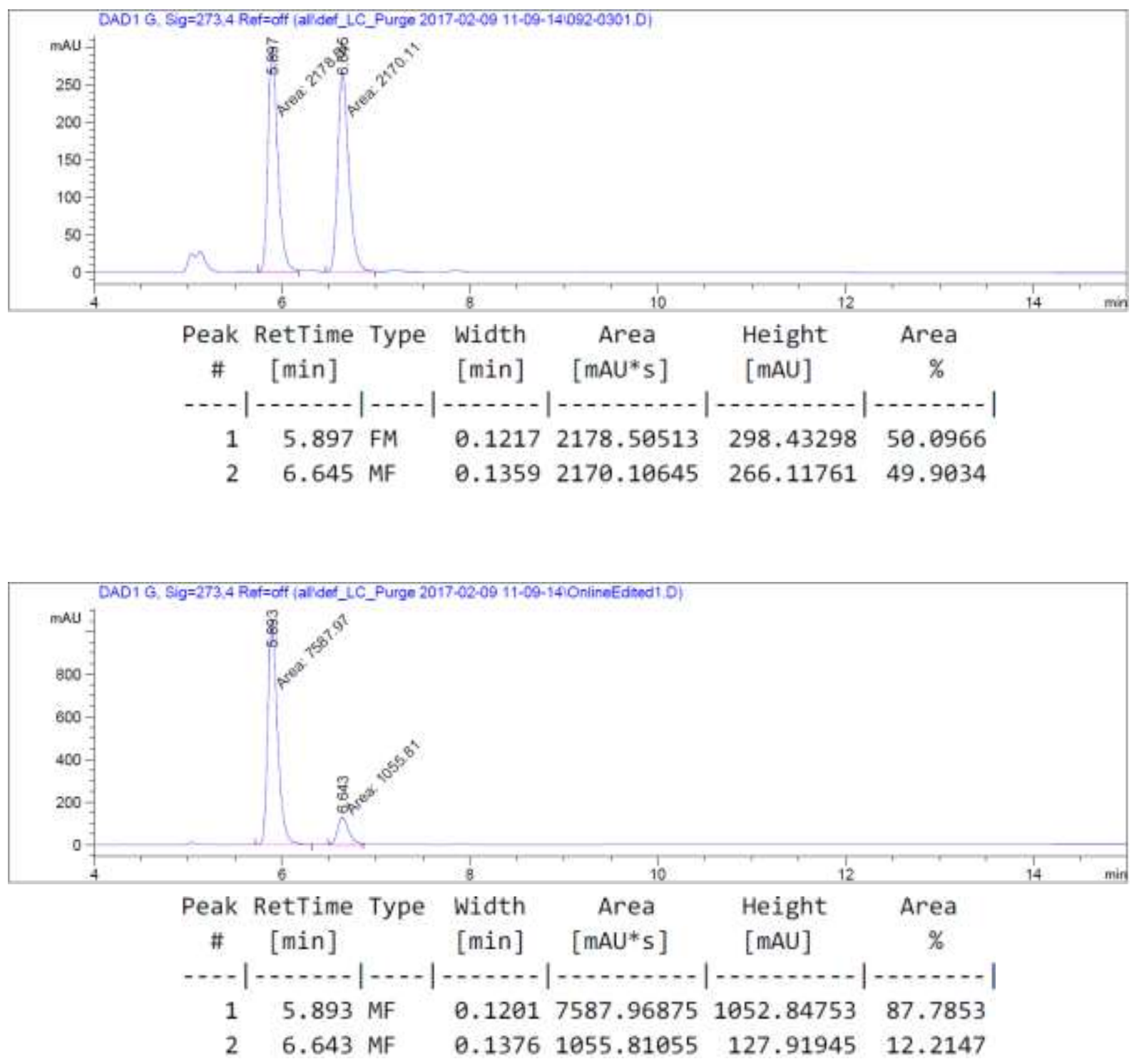


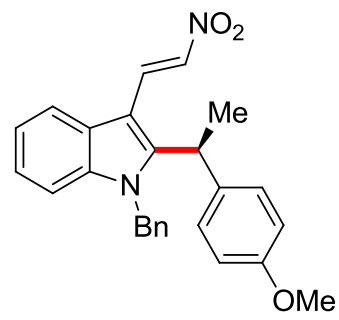

237

$\left(\mathrm{CDCl}_{3}, 400 \mathrm{MHz}\right)$
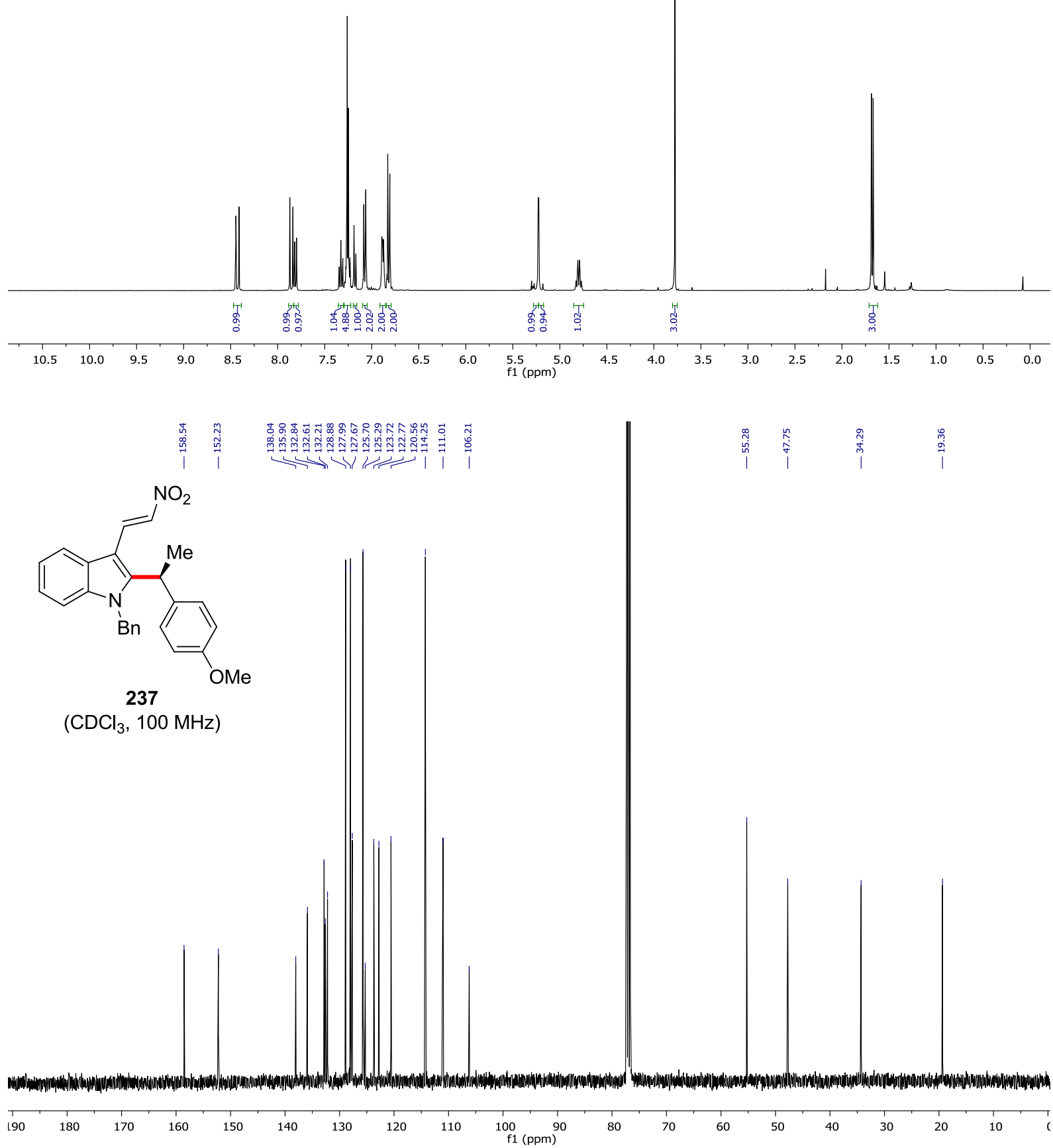


\section{Chiral HPLC of 237:}
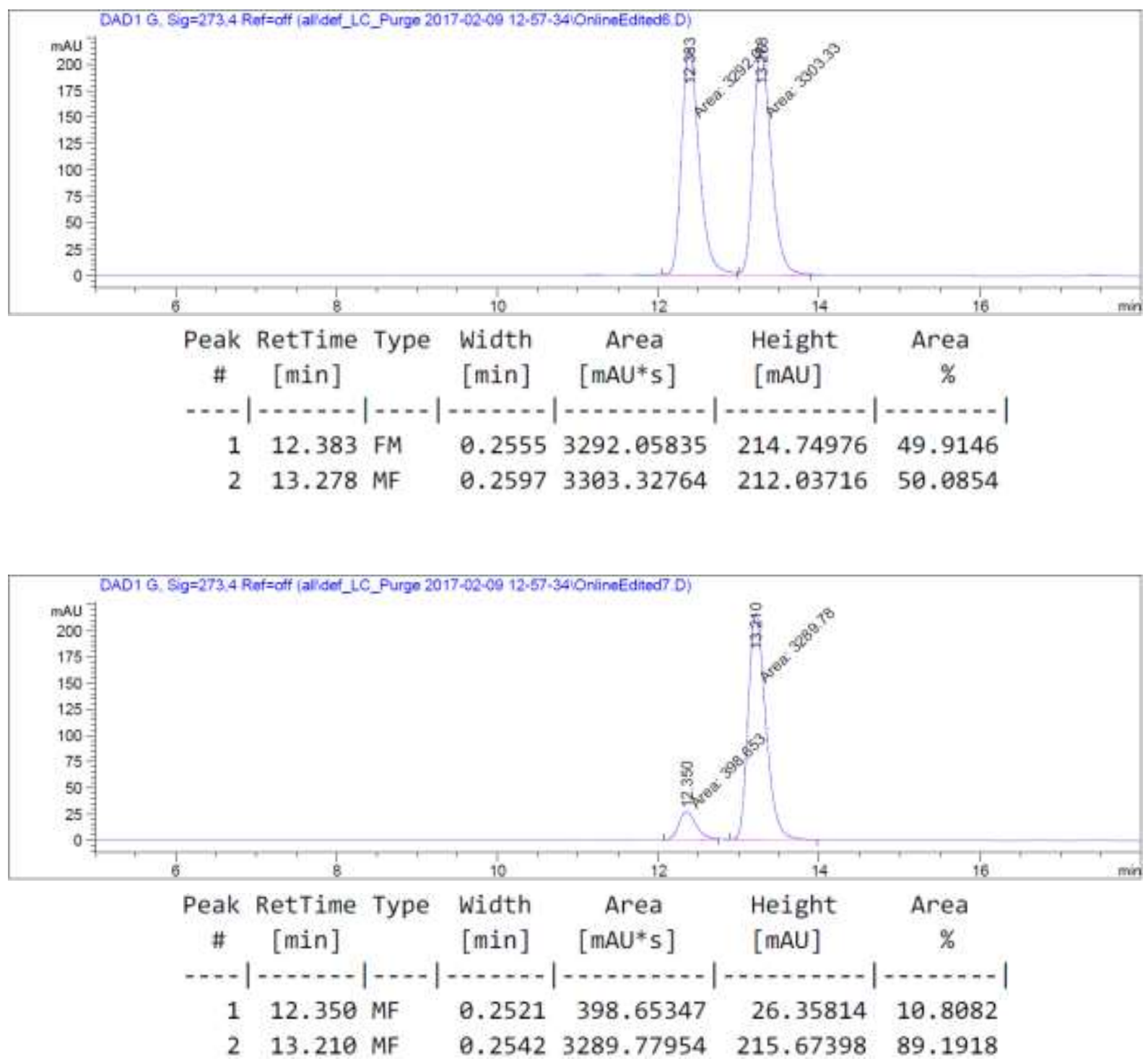


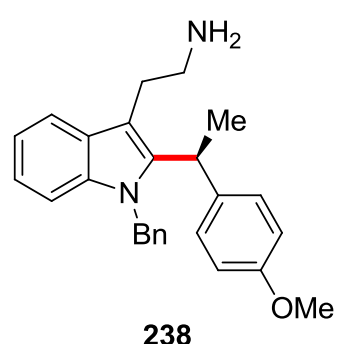

$\left(\mathrm{CDCl}_{3}, 400 \mathrm{MHz}\right)$
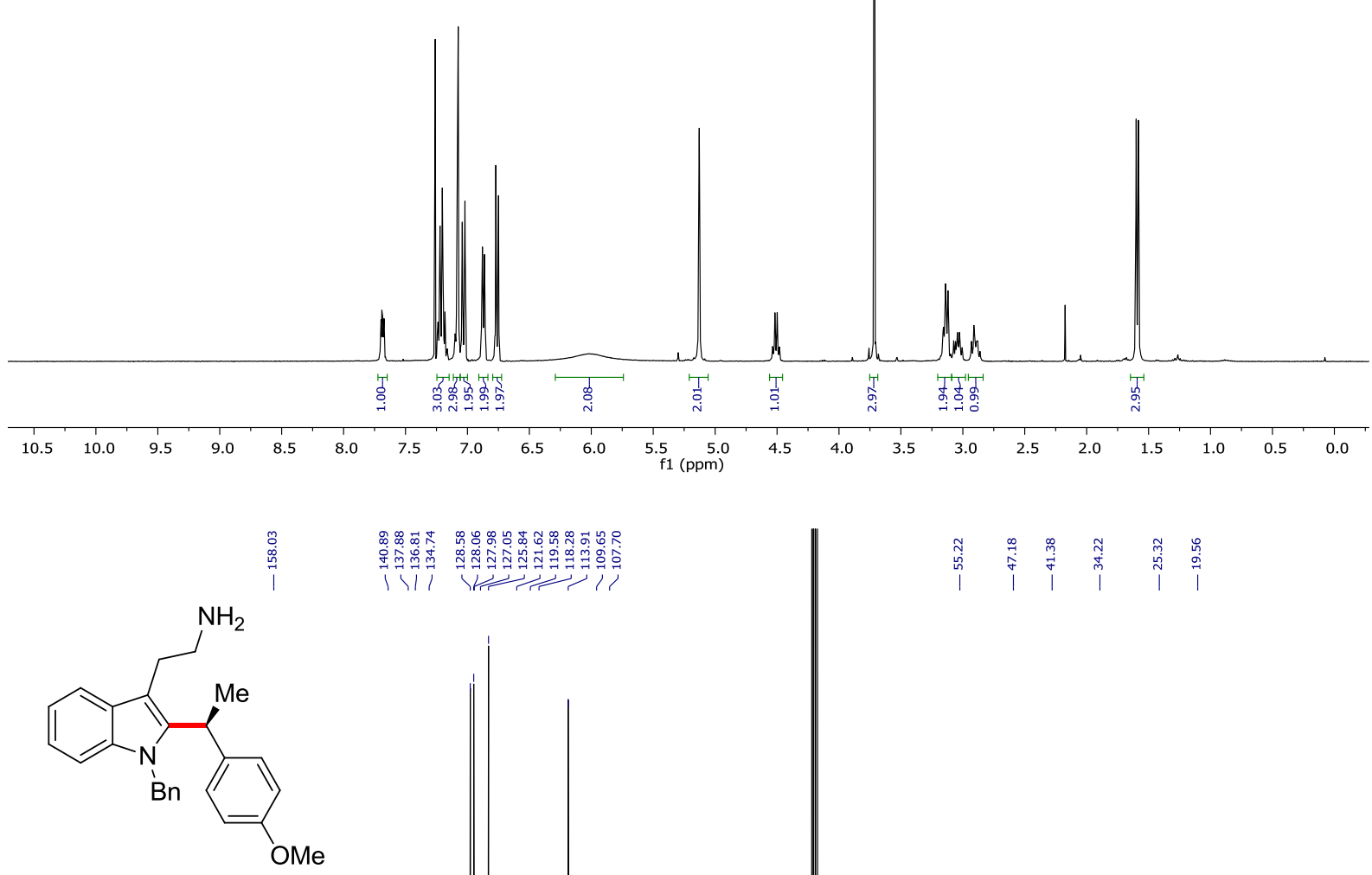

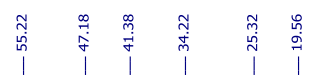

238

$\left(\mathrm{CDCl}_{3}, 100 \mathrm{MHz}\right)$

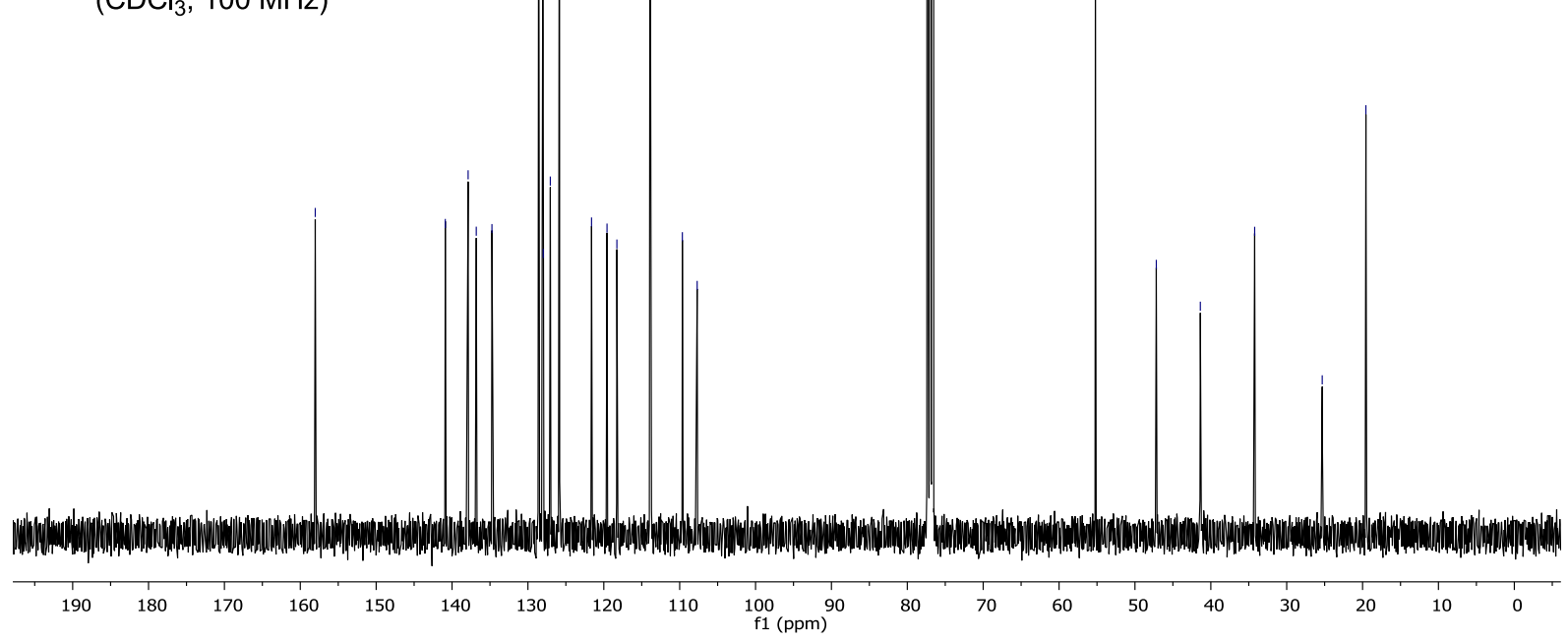




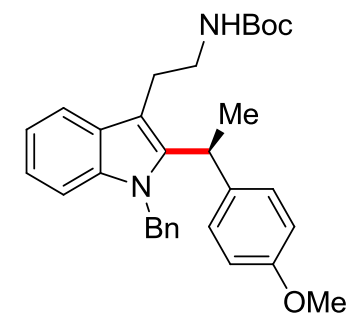

203

$\left(\mathrm{CDCl}_{3}, 500 \mathrm{MHz}\right)$
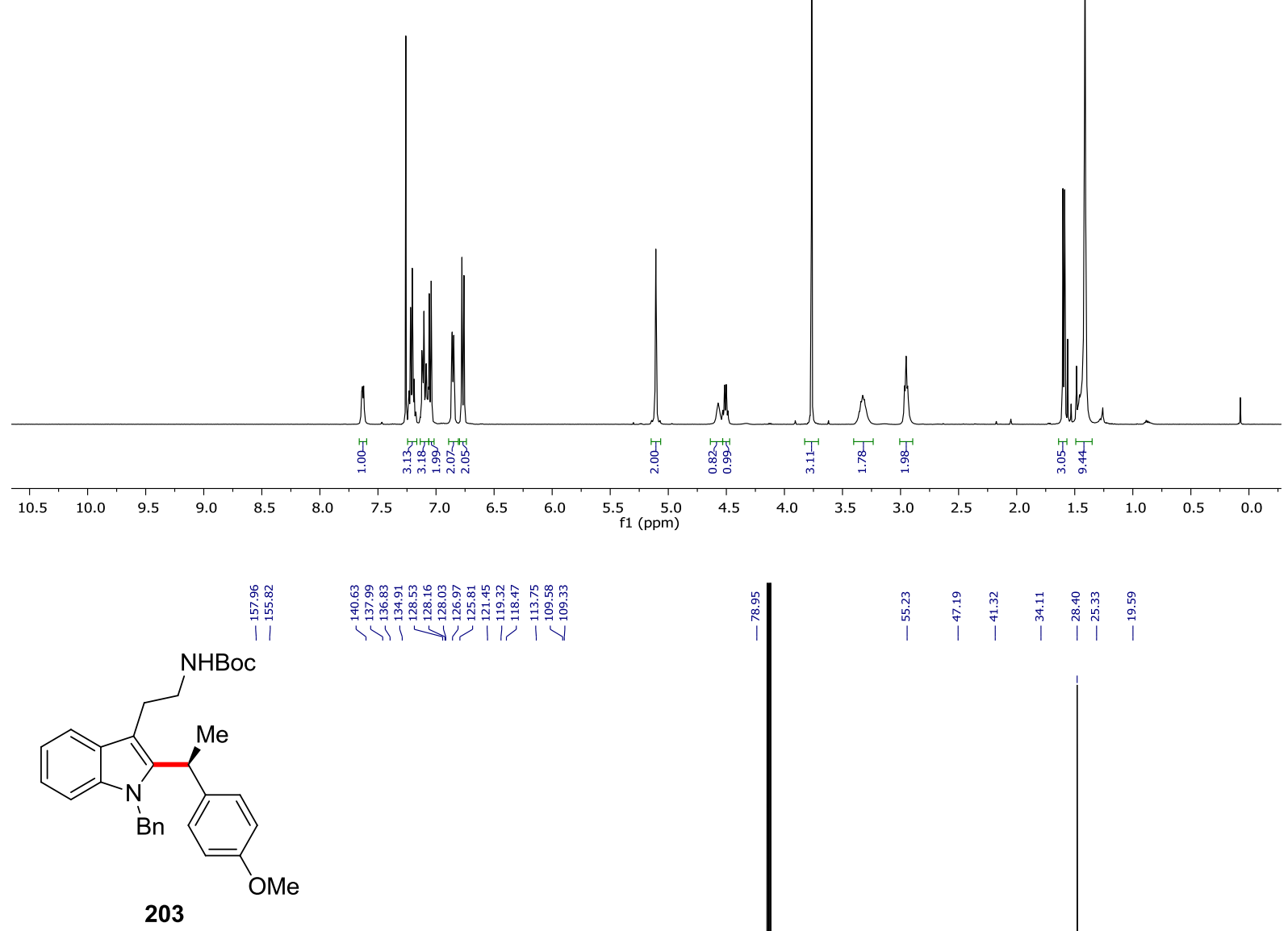

$\left(\mathrm{CDCl}_{3}, 125 \mathrm{MHz}\right)$

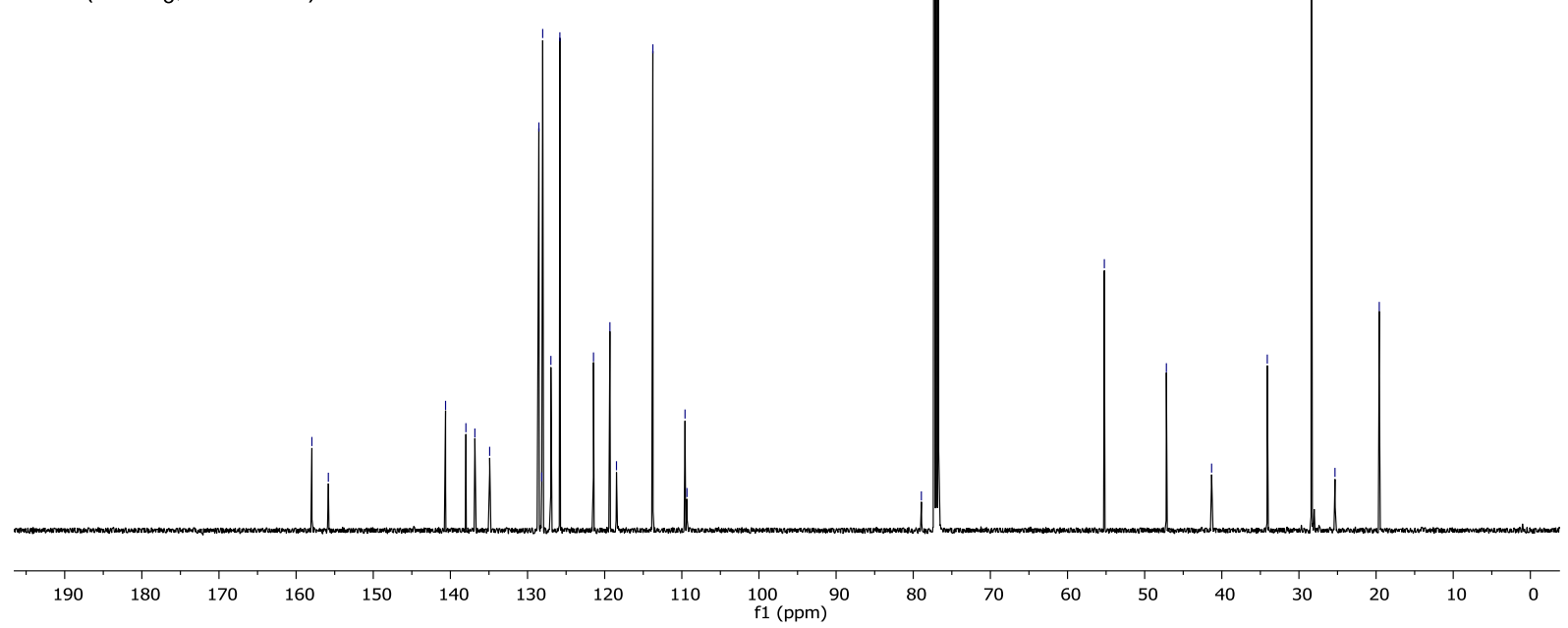




\section{Chiral HPLC of 203:}
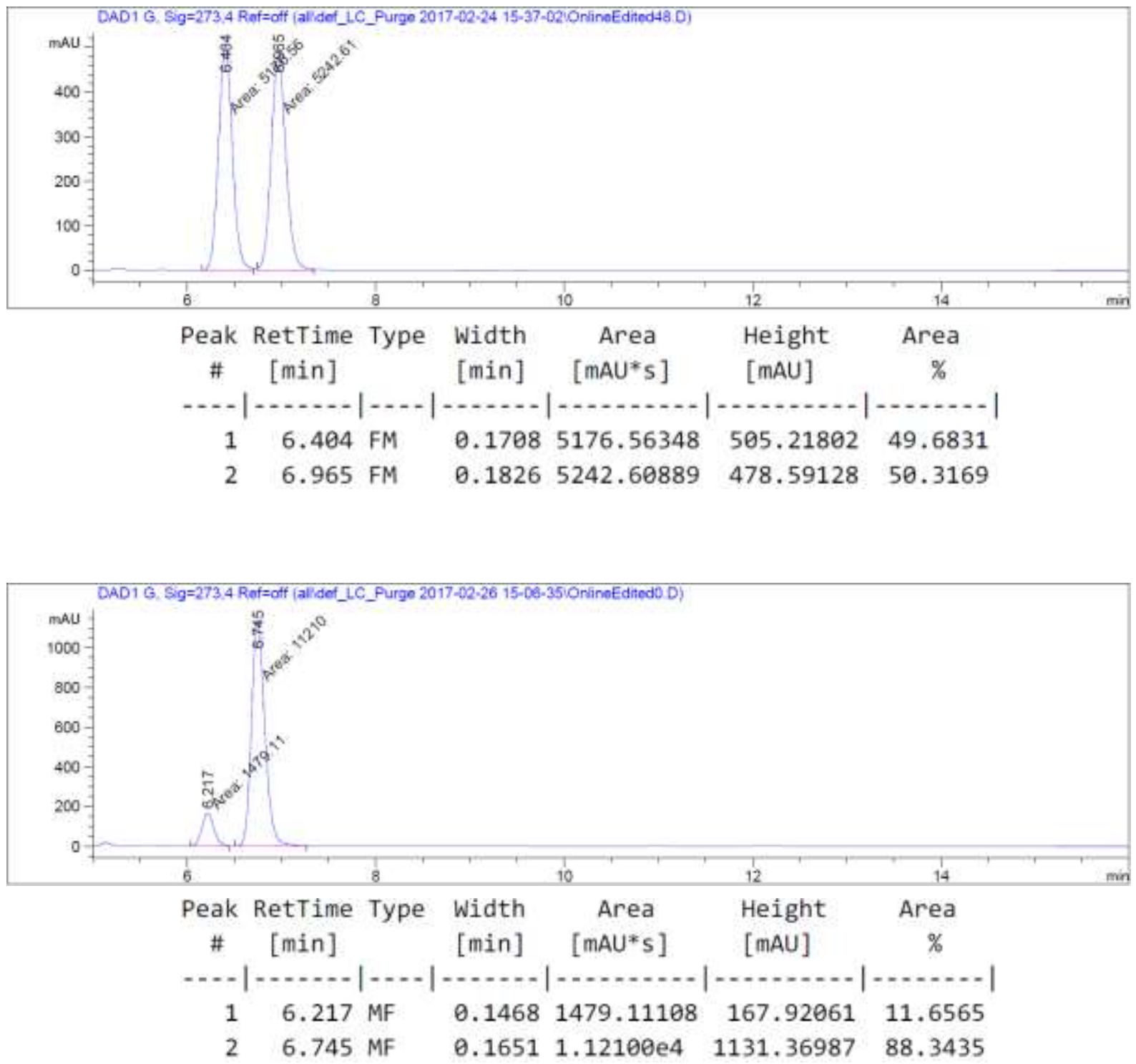


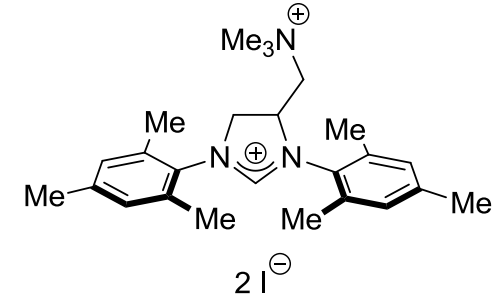

207

(DMSO-d, $300 \mathrm{MHz}$ )

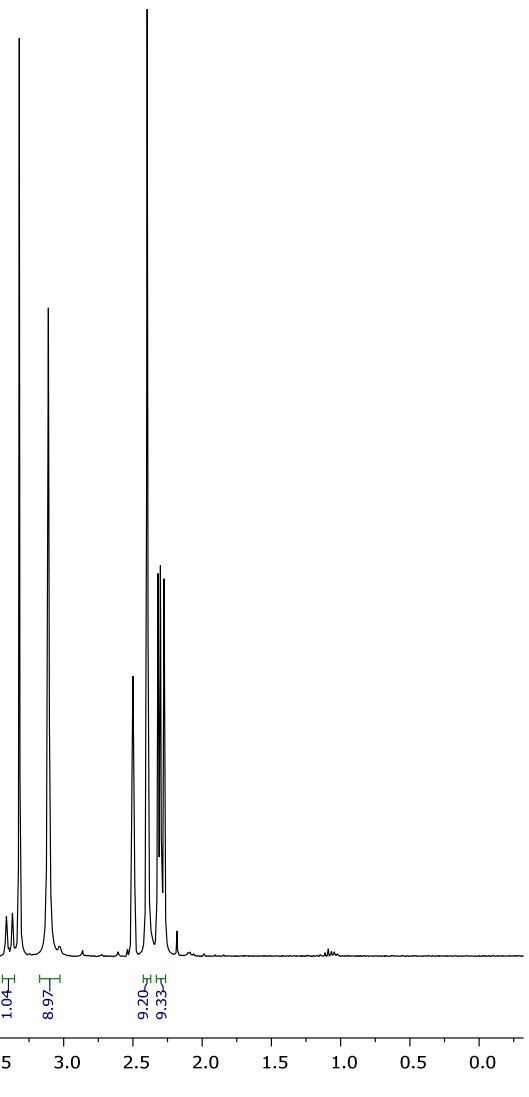

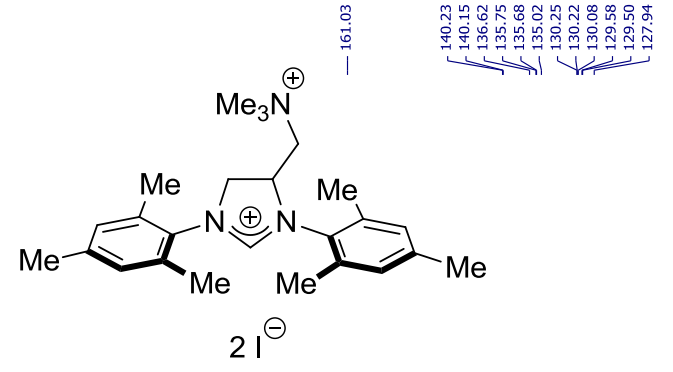

207

(DMSO-d $6,125 \mathrm{MHz}$ )

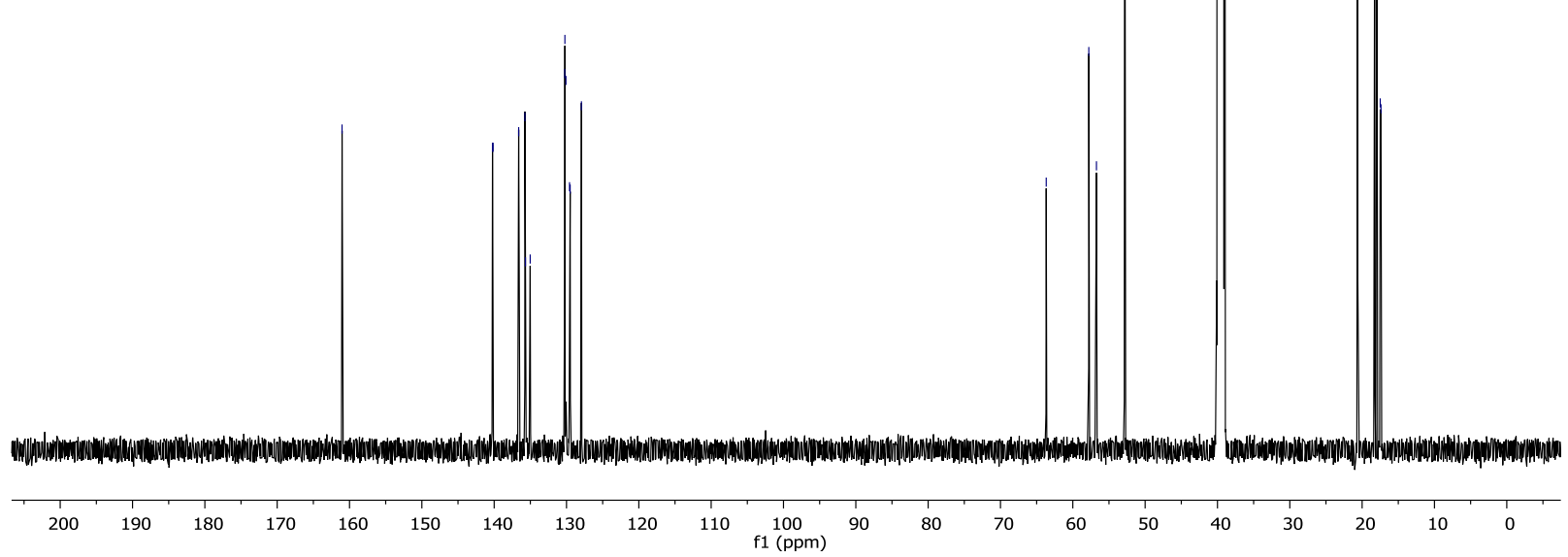




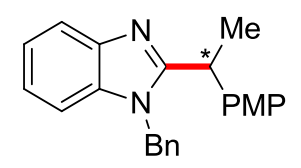

$106 \mathrm{bc}$

$\left(\mathrm{CDCl}_{3}, 400 \mathrm{MHz}\right)$
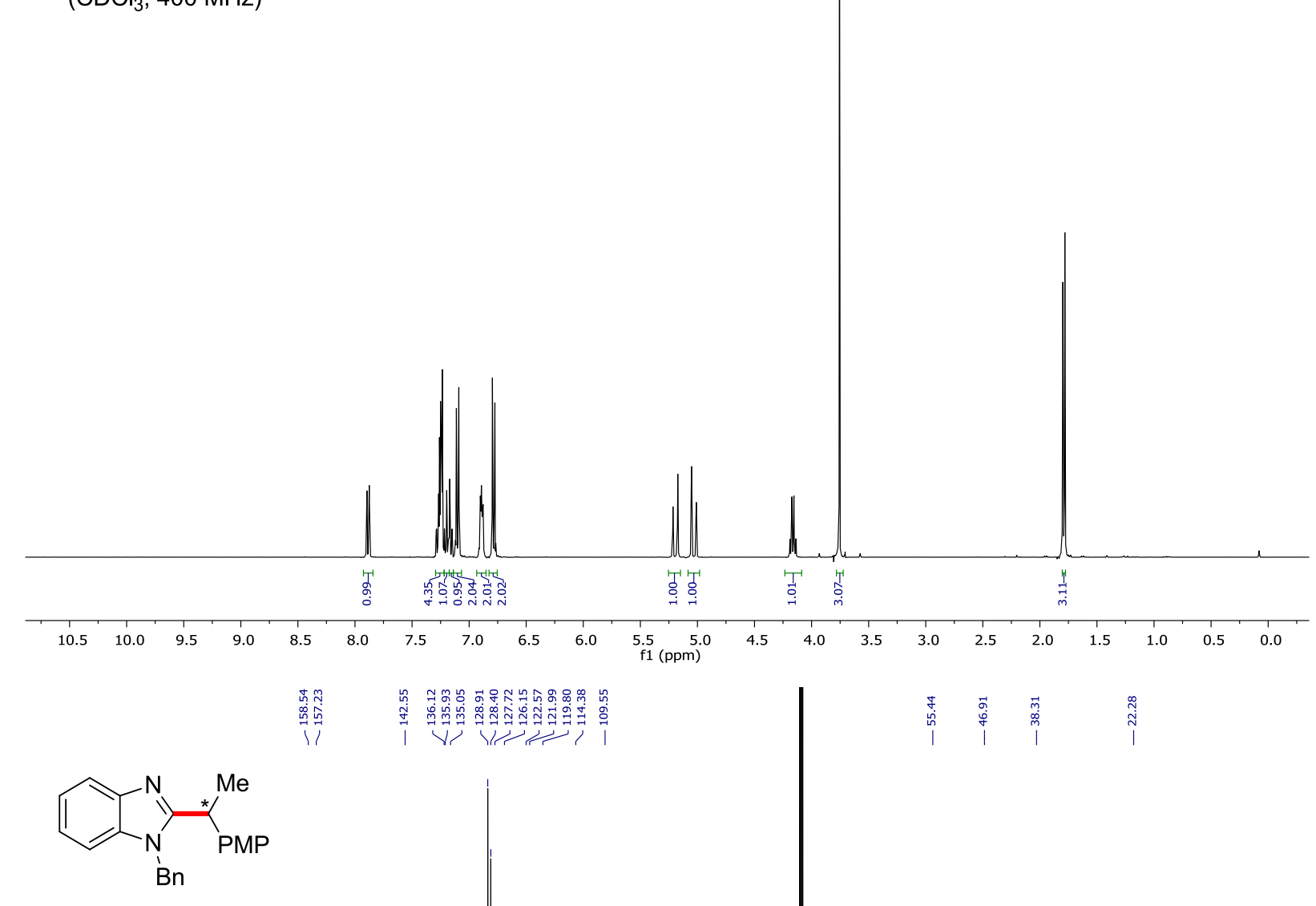

$106 \mathrm{bc}$

$\left(\mathrm{CDCl}_{3}, 125 \mathrm{MHz}\right)$

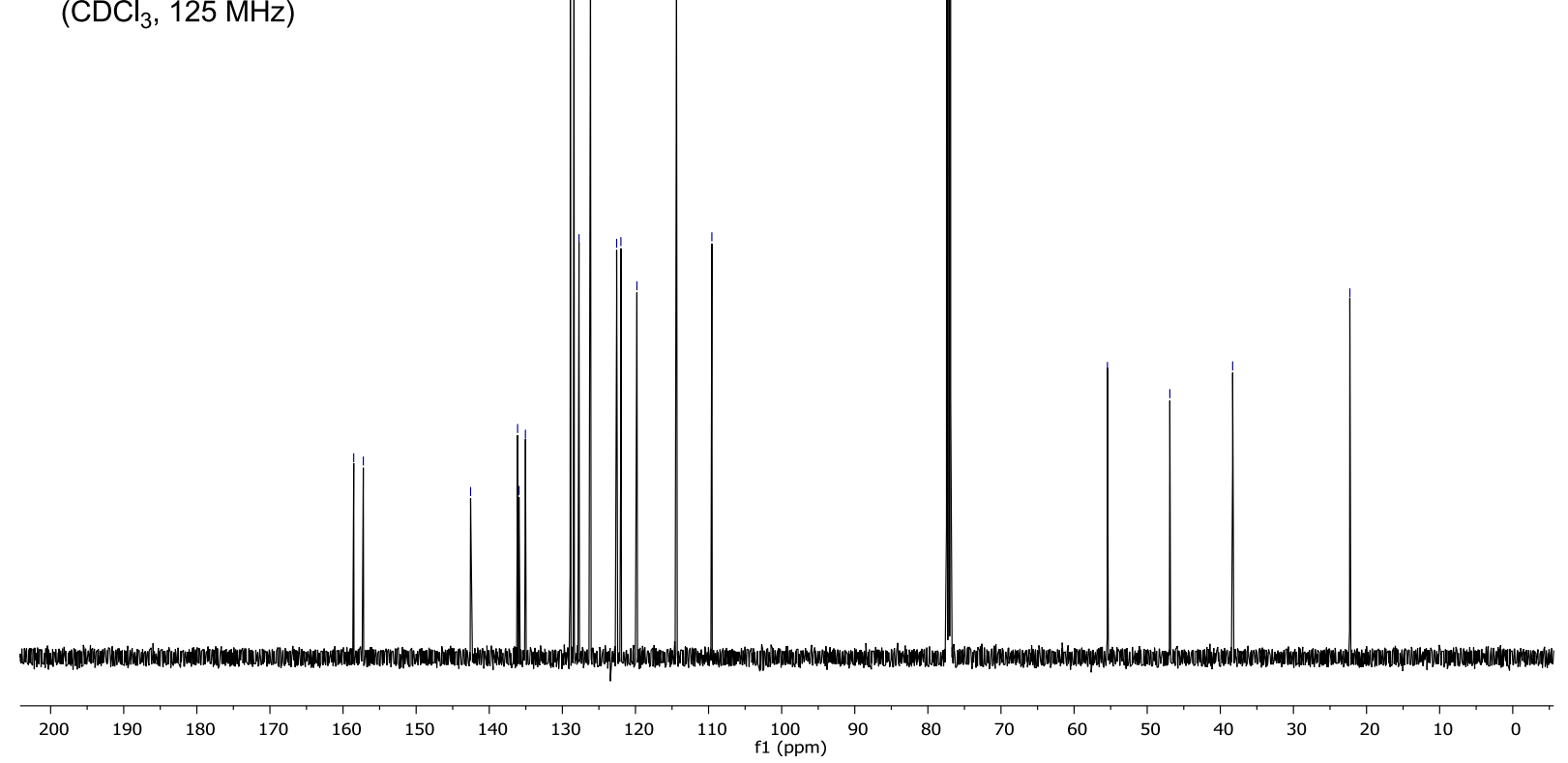




\section{Chiral HPLC of 106bc:}
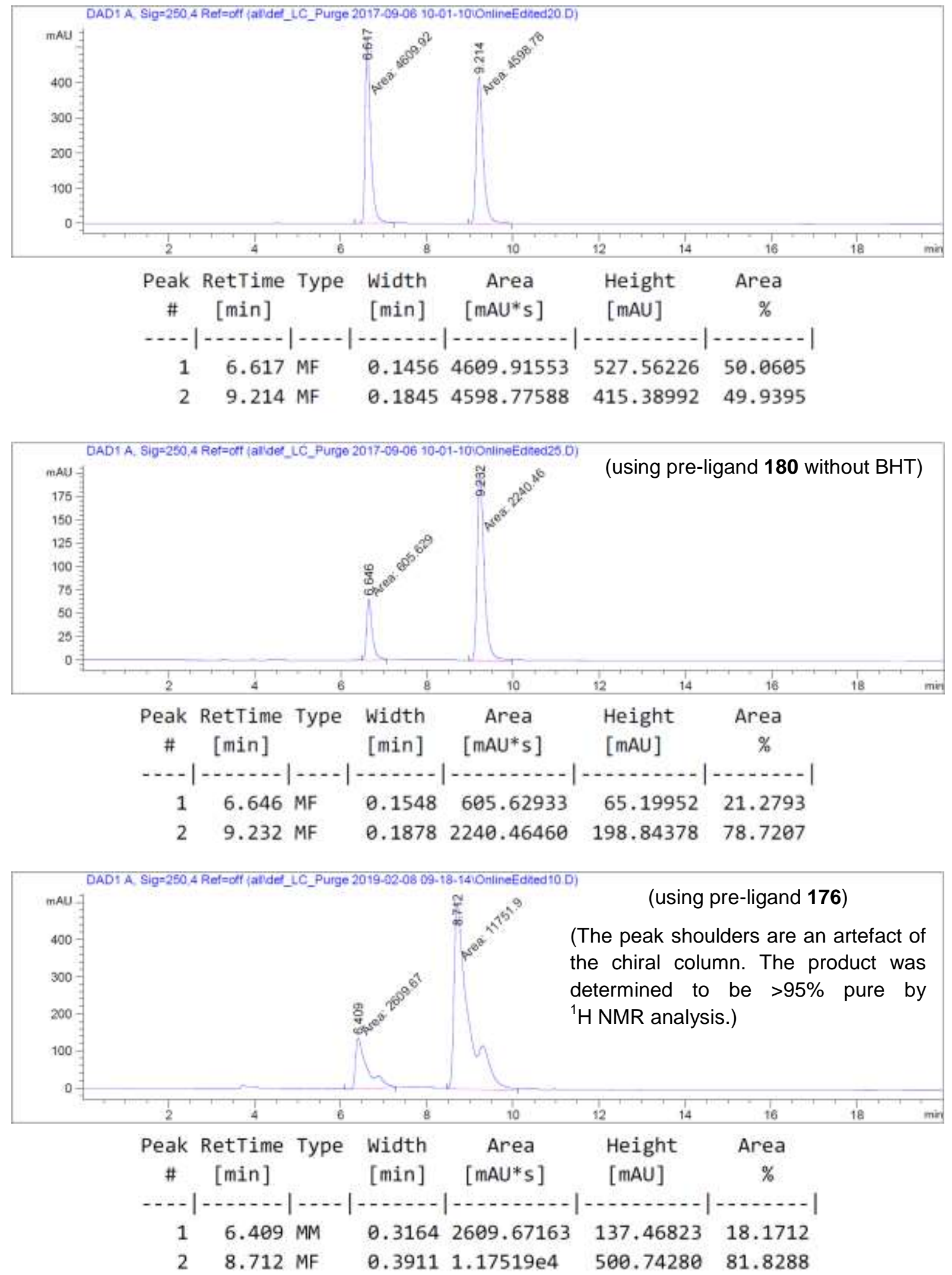


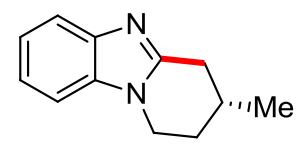

$145 a$

$\left(\mathrm{CDCl}_{3}, 300 \mathrm{MHz}\right)$
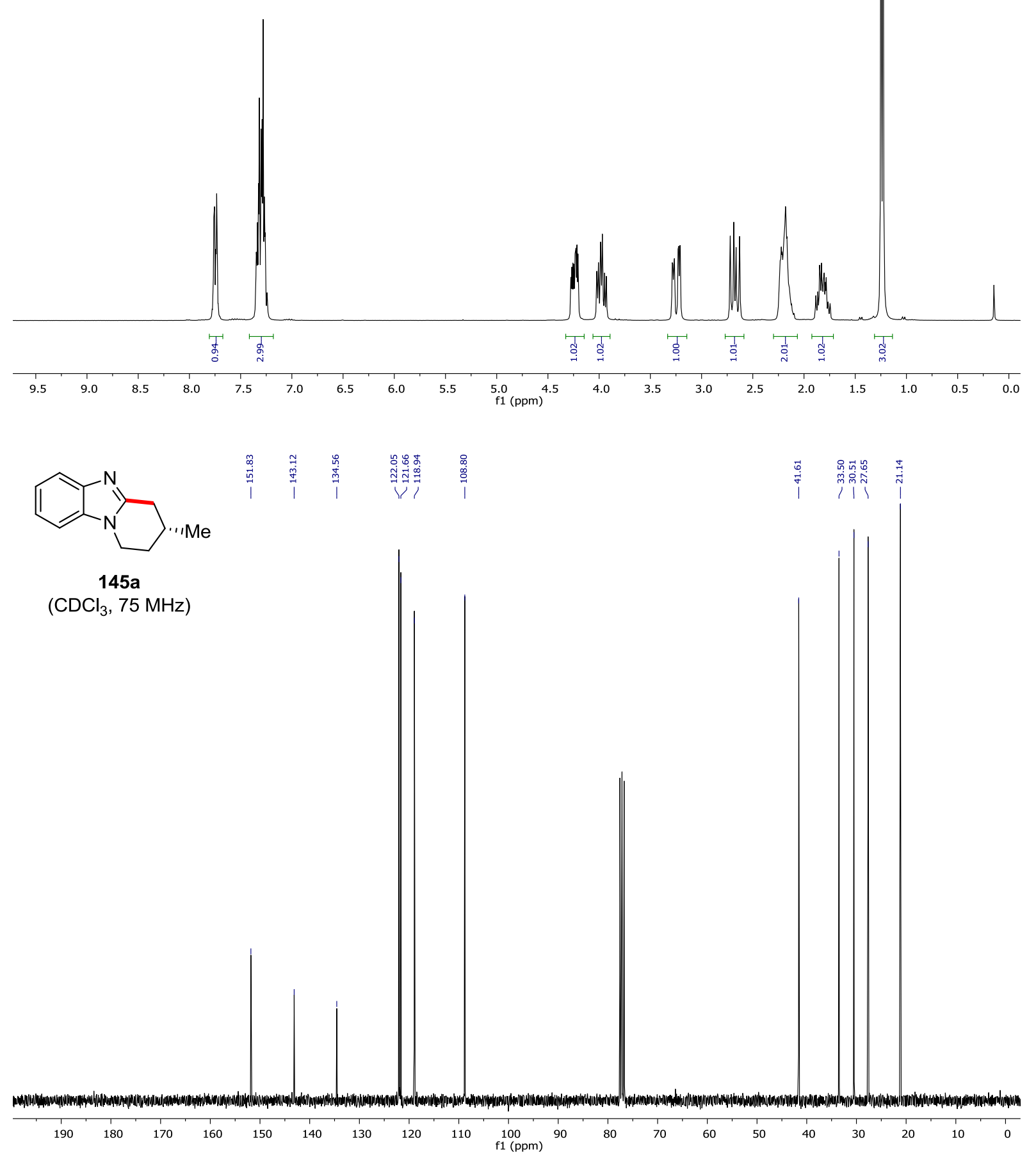


\section{Chiral HPLC of 145a:}
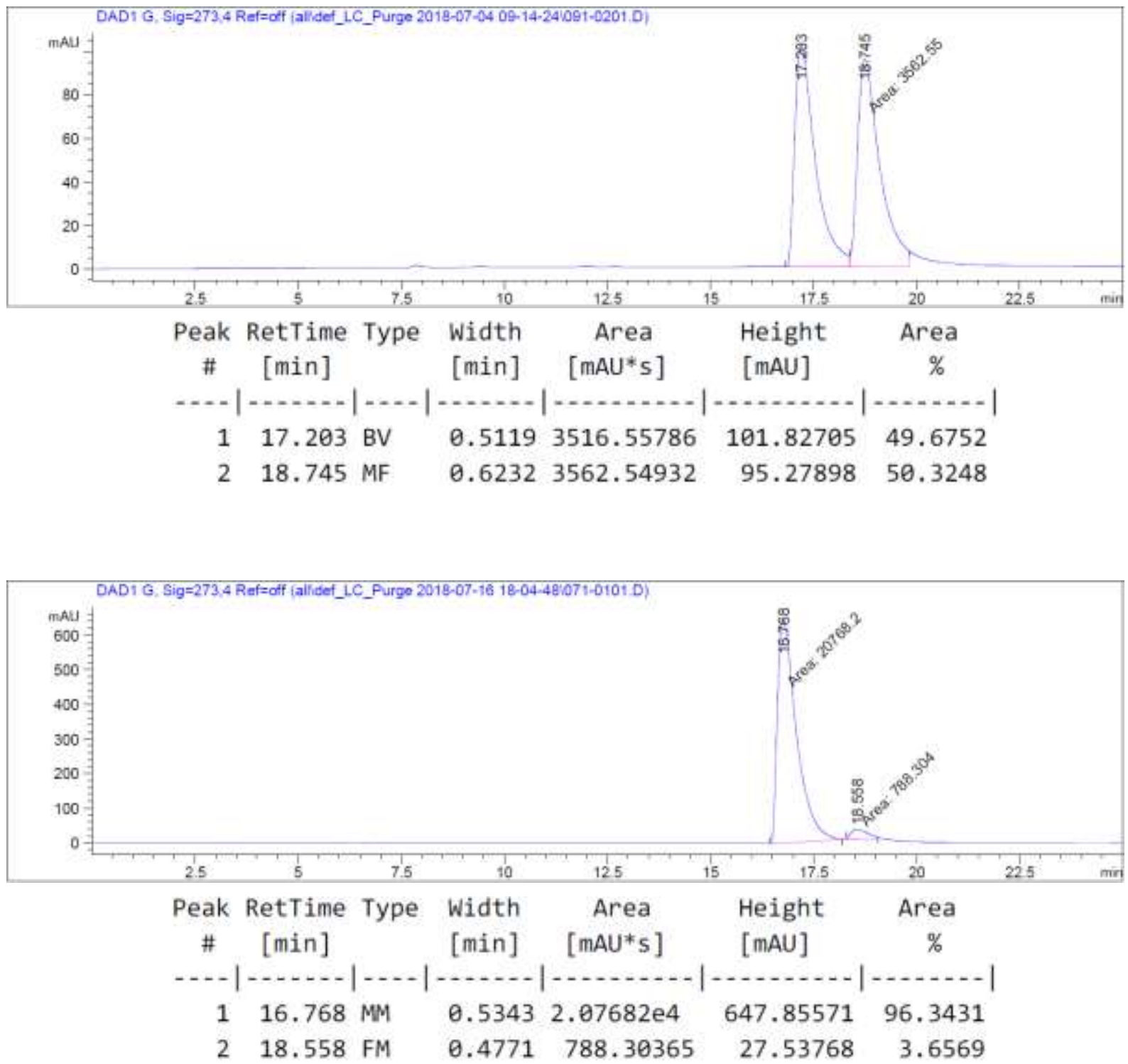

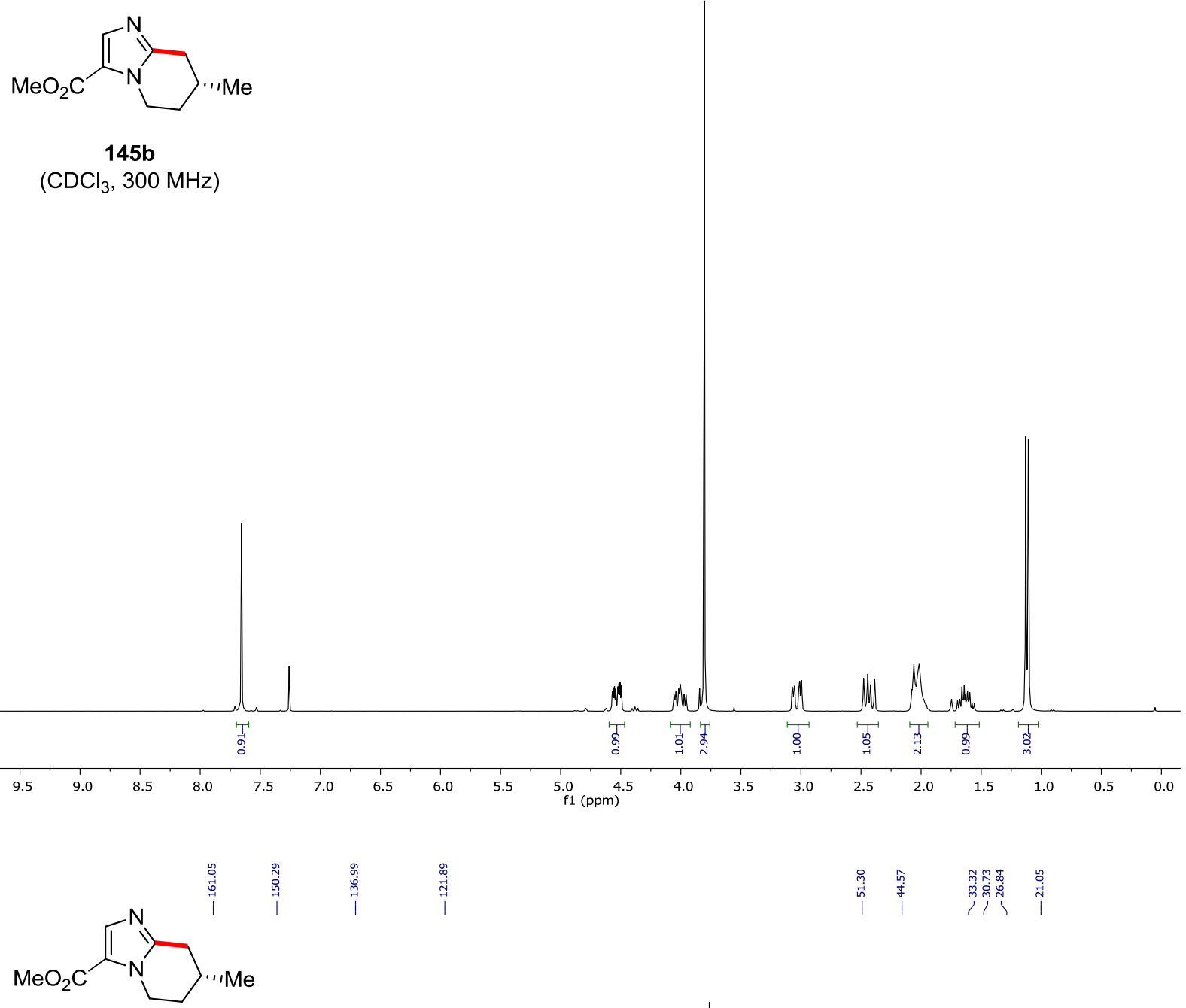

145b

$\left(\mathrm{CDCl}_{3}, 75 \mathrm{MHz}\right)$

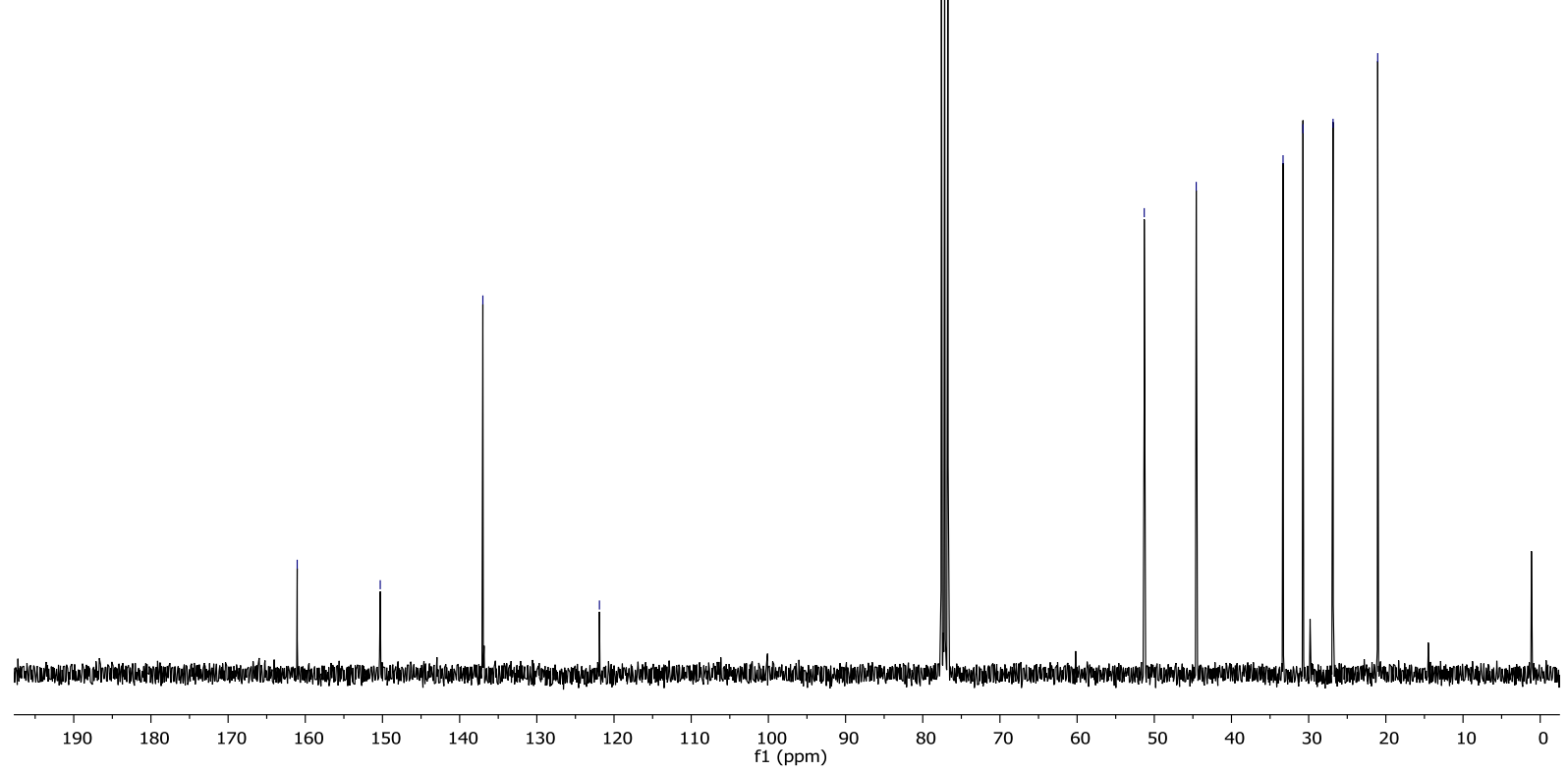




\section{Chiral HPLC of 145b:}
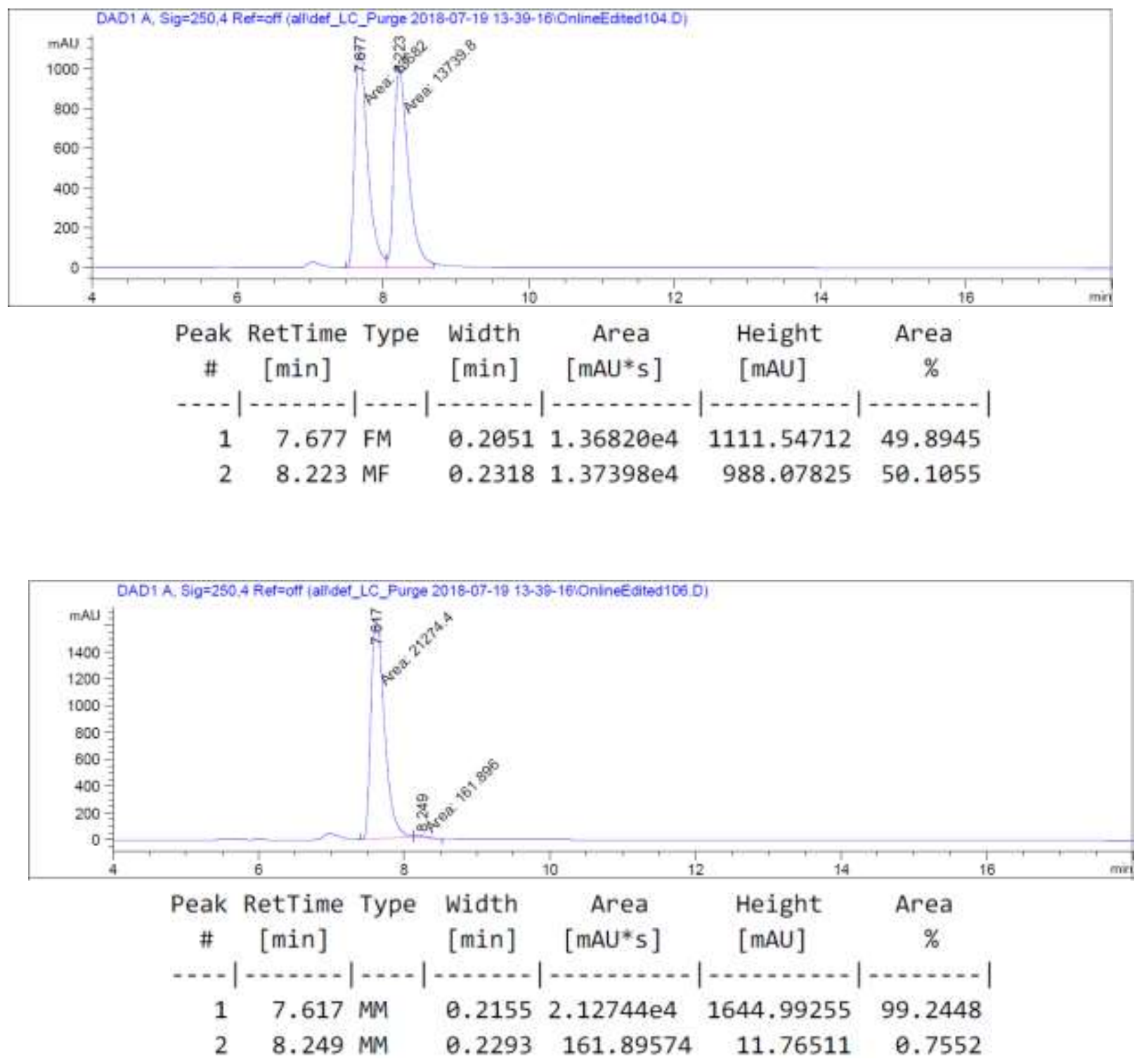


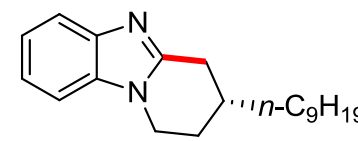

$145 \mathrm{c}$

$\left(\mathrm{CDCl}_{3}, 300 \mathrm{MHz}\right)$
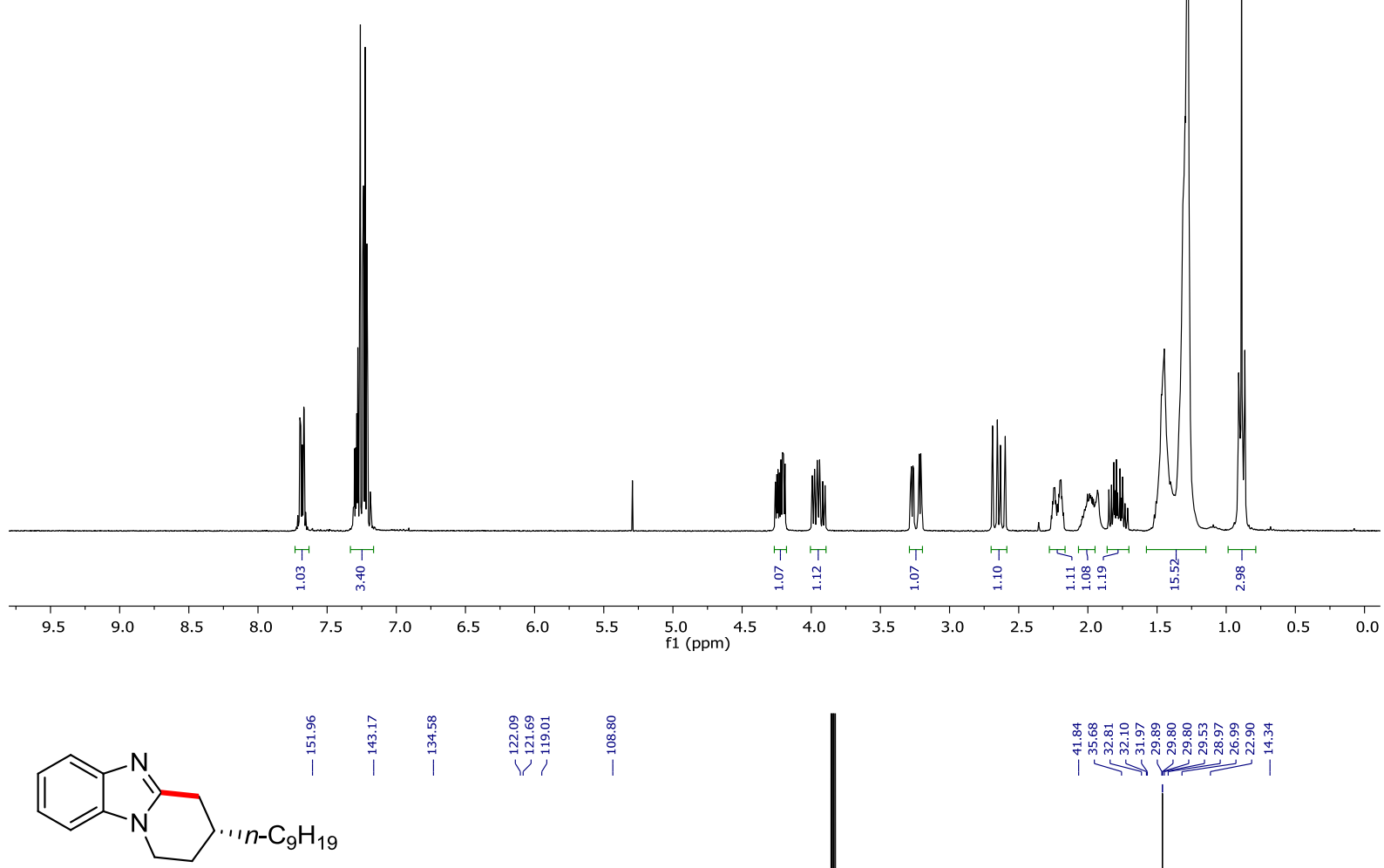

145c

$\left(\mathrm{CDCl}_{3}, 125 \mathrm{MHz}\right)$ 


\section{Chiral HPLC of 145c:}
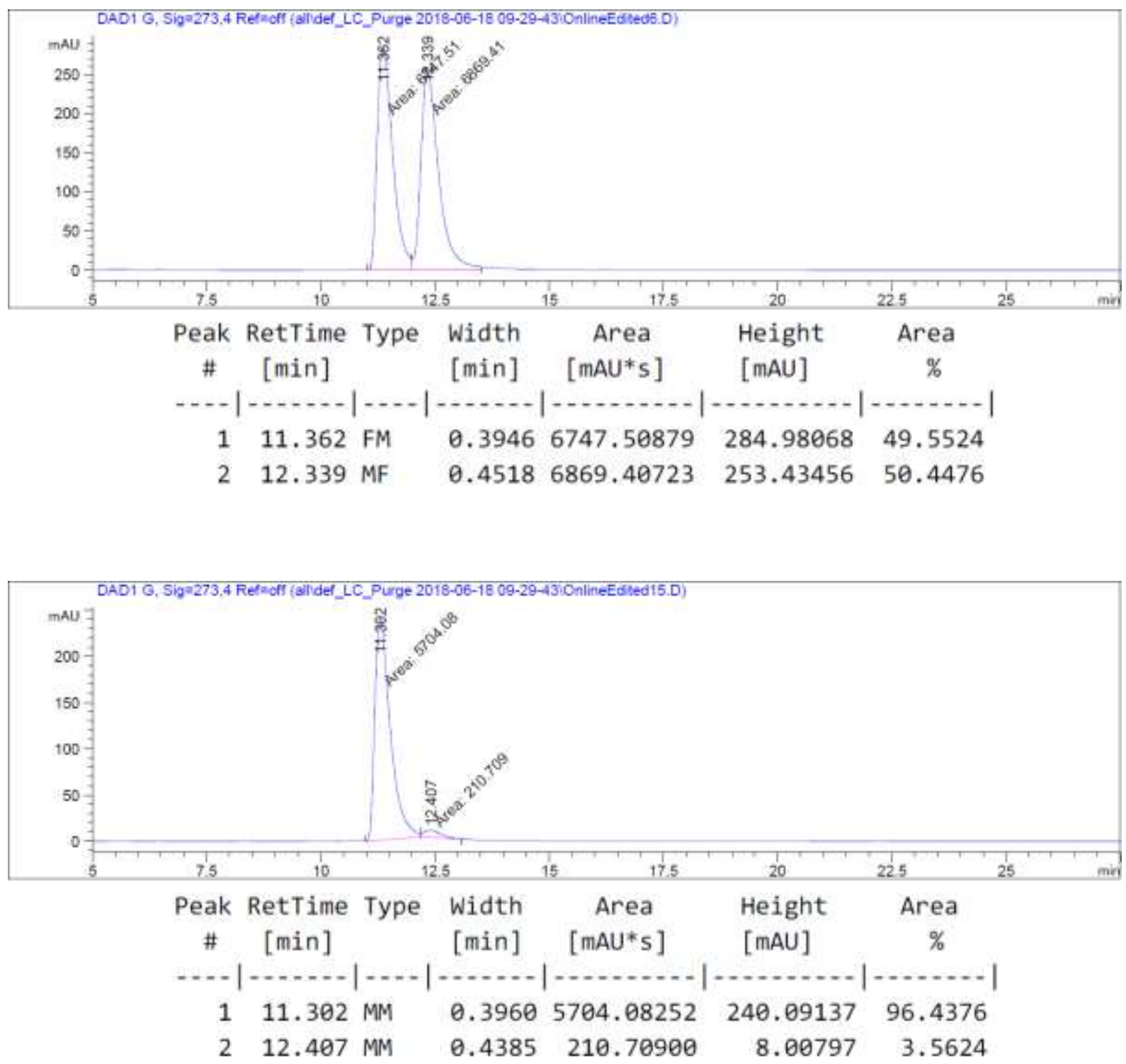

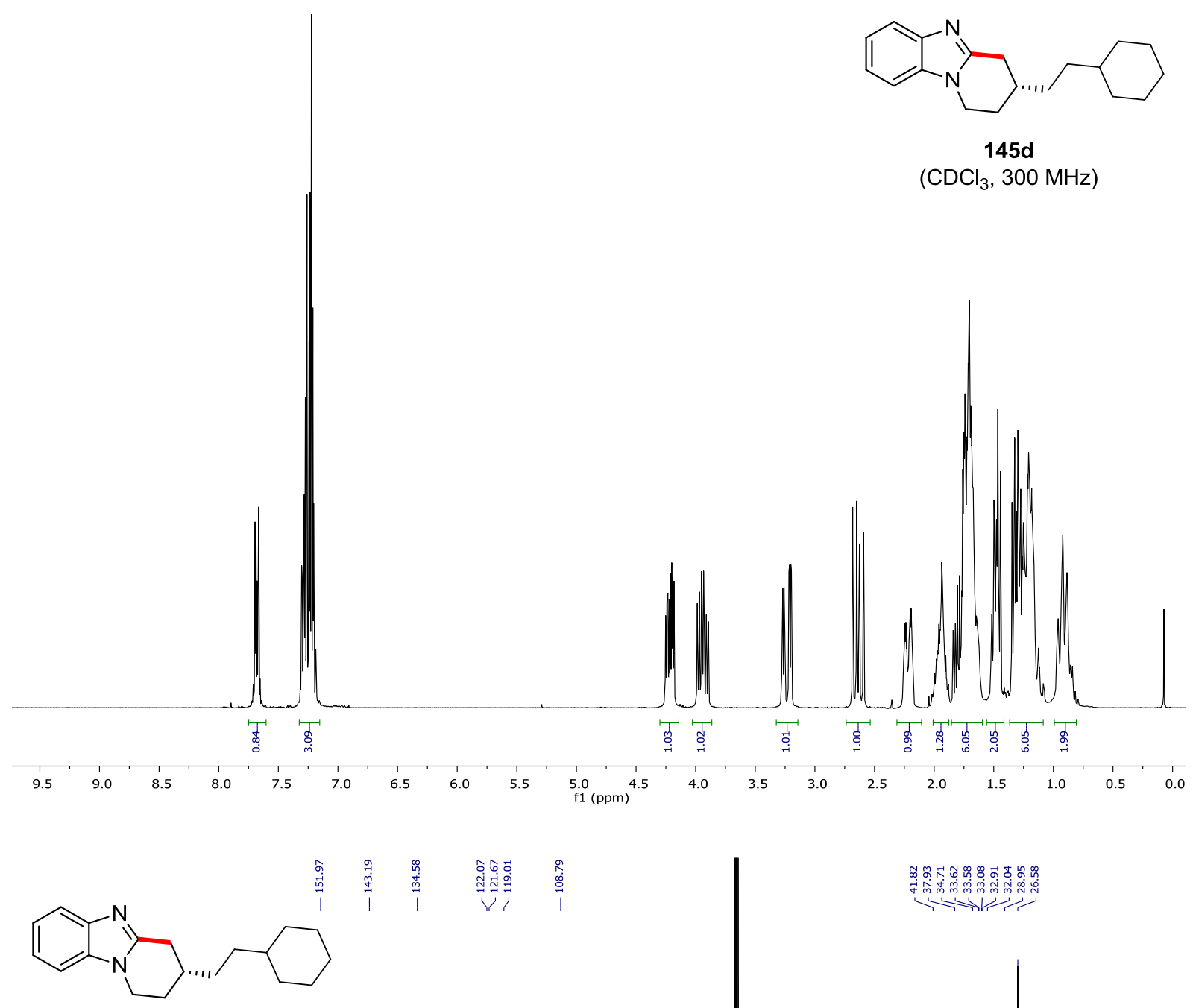

145d

$\left(\mathrm{CDCl}_{3}, 125 \mathrm{MHz}\right)$

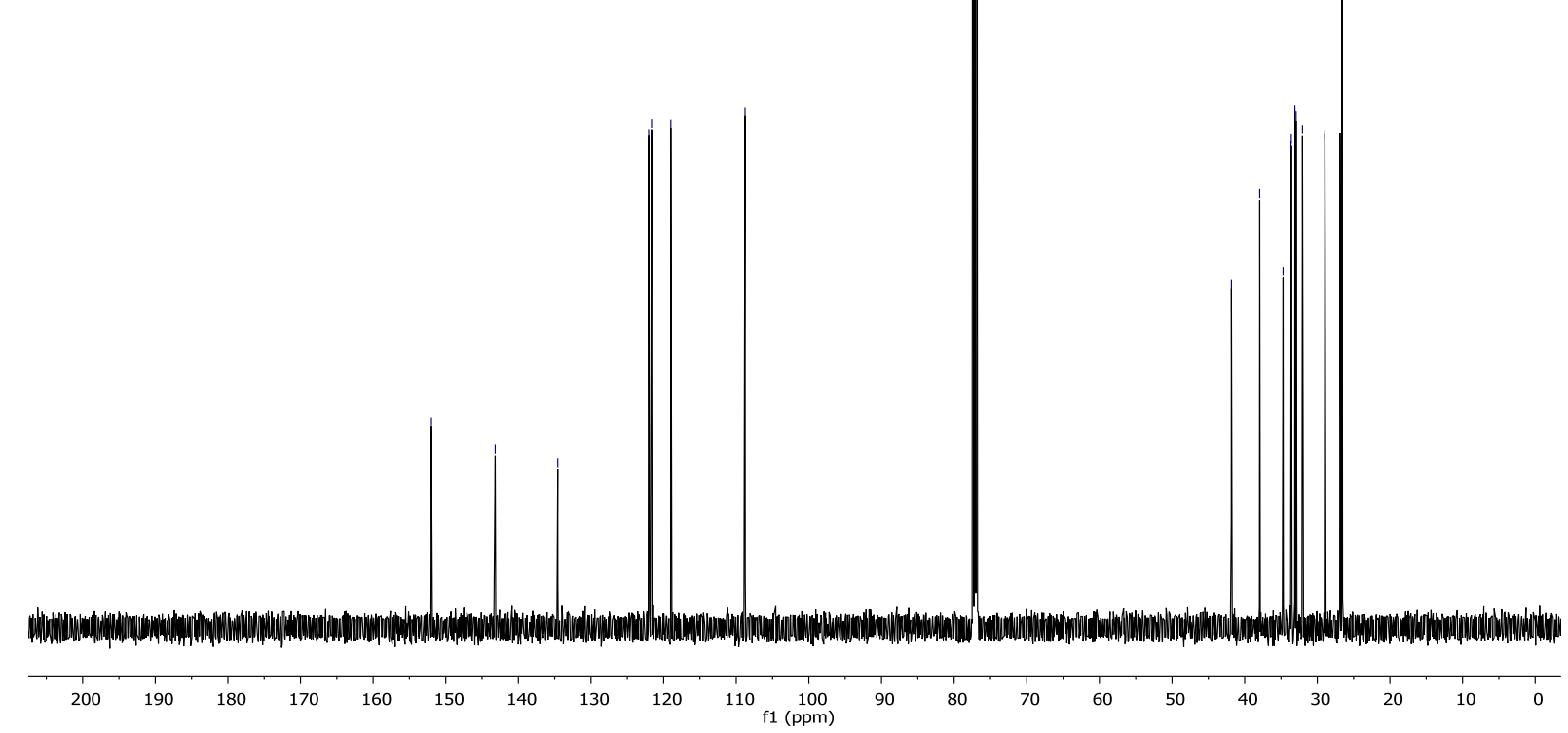




\section{Chiral HPLC of $\mathbf{1 4 5 d}$ :}
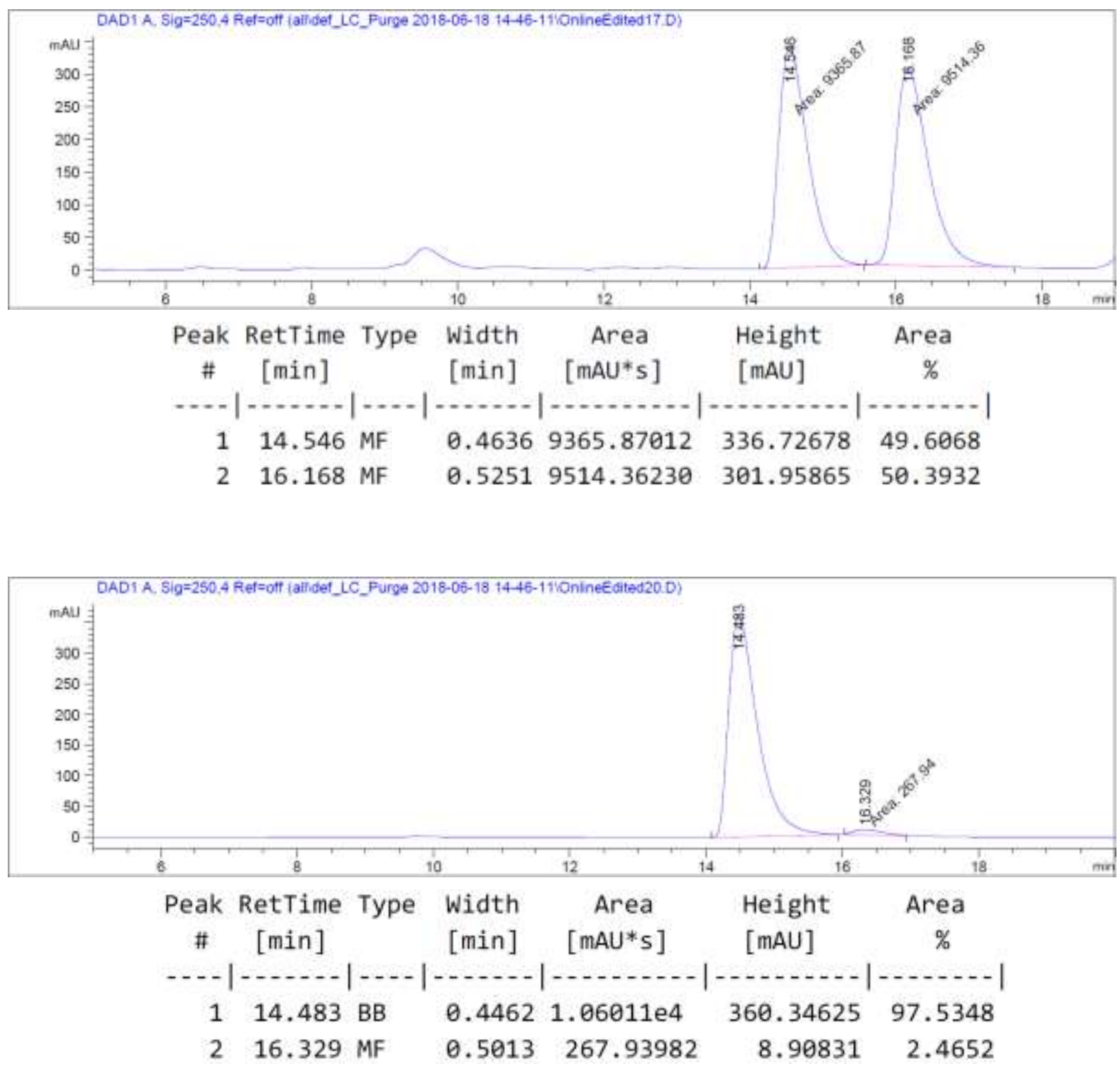

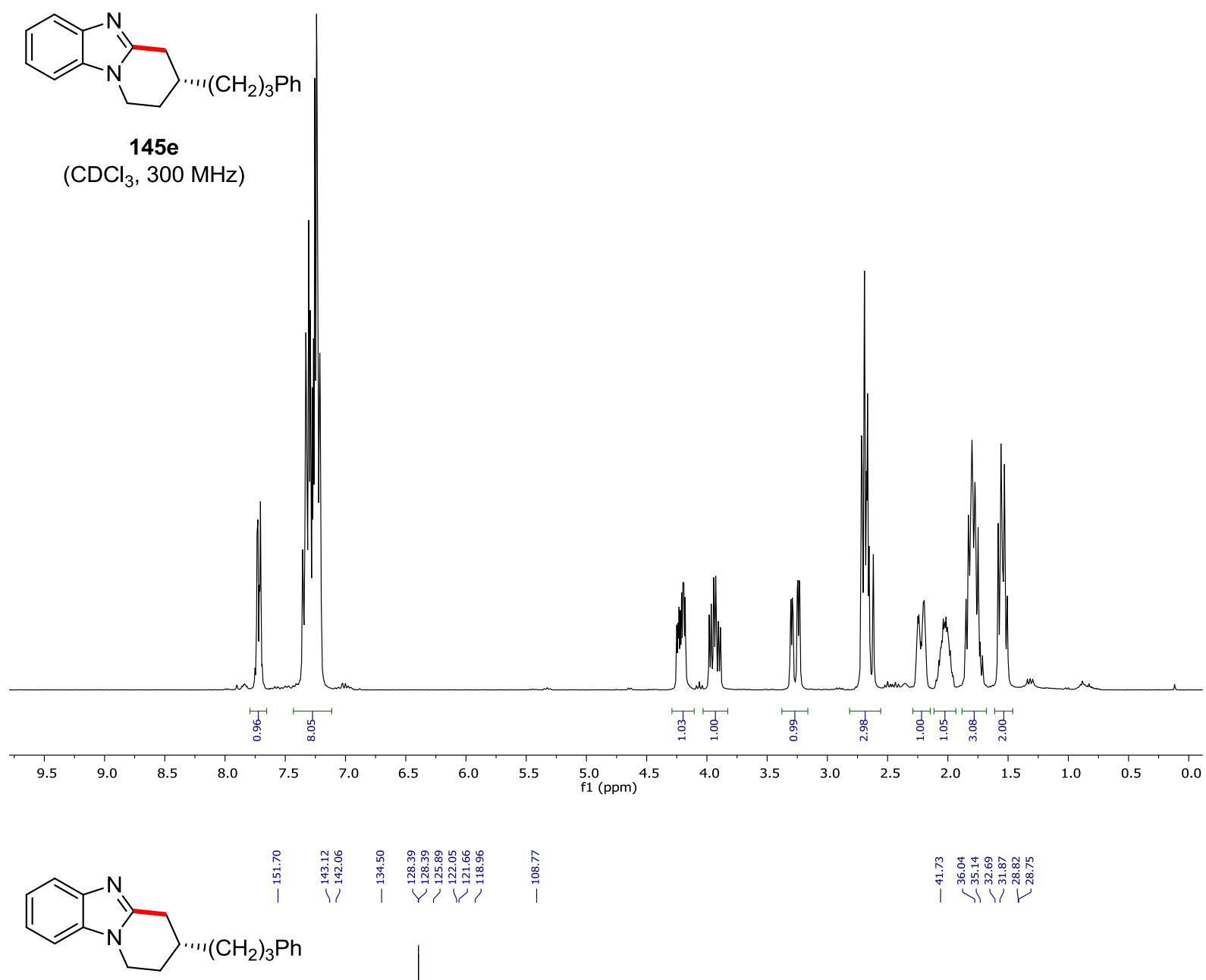

$\left(\mathrm{CDCl}_{3}, 125 \mathrm{MHz}\right)$

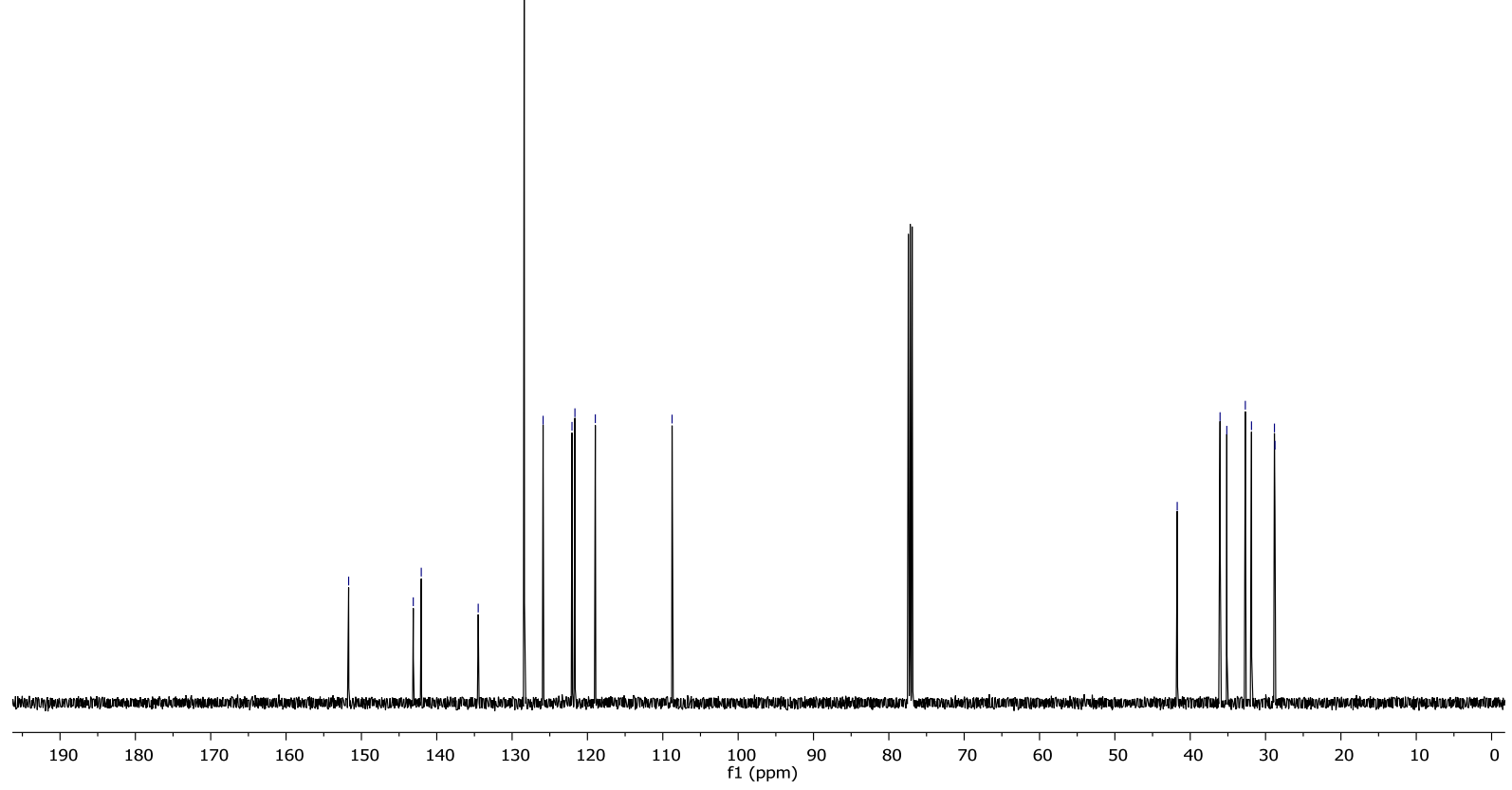




\section{Chiral HPLC of 145e:}
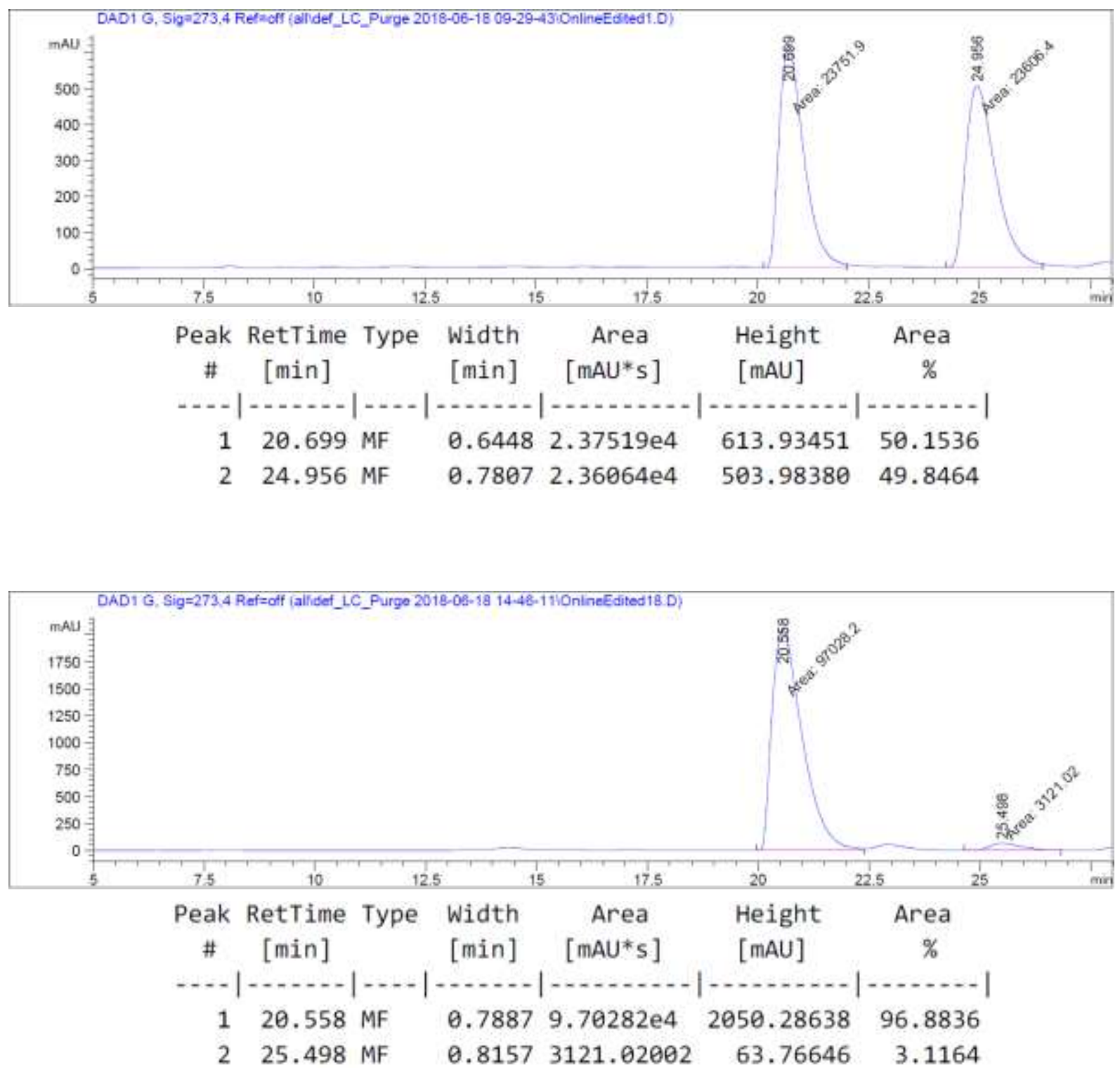

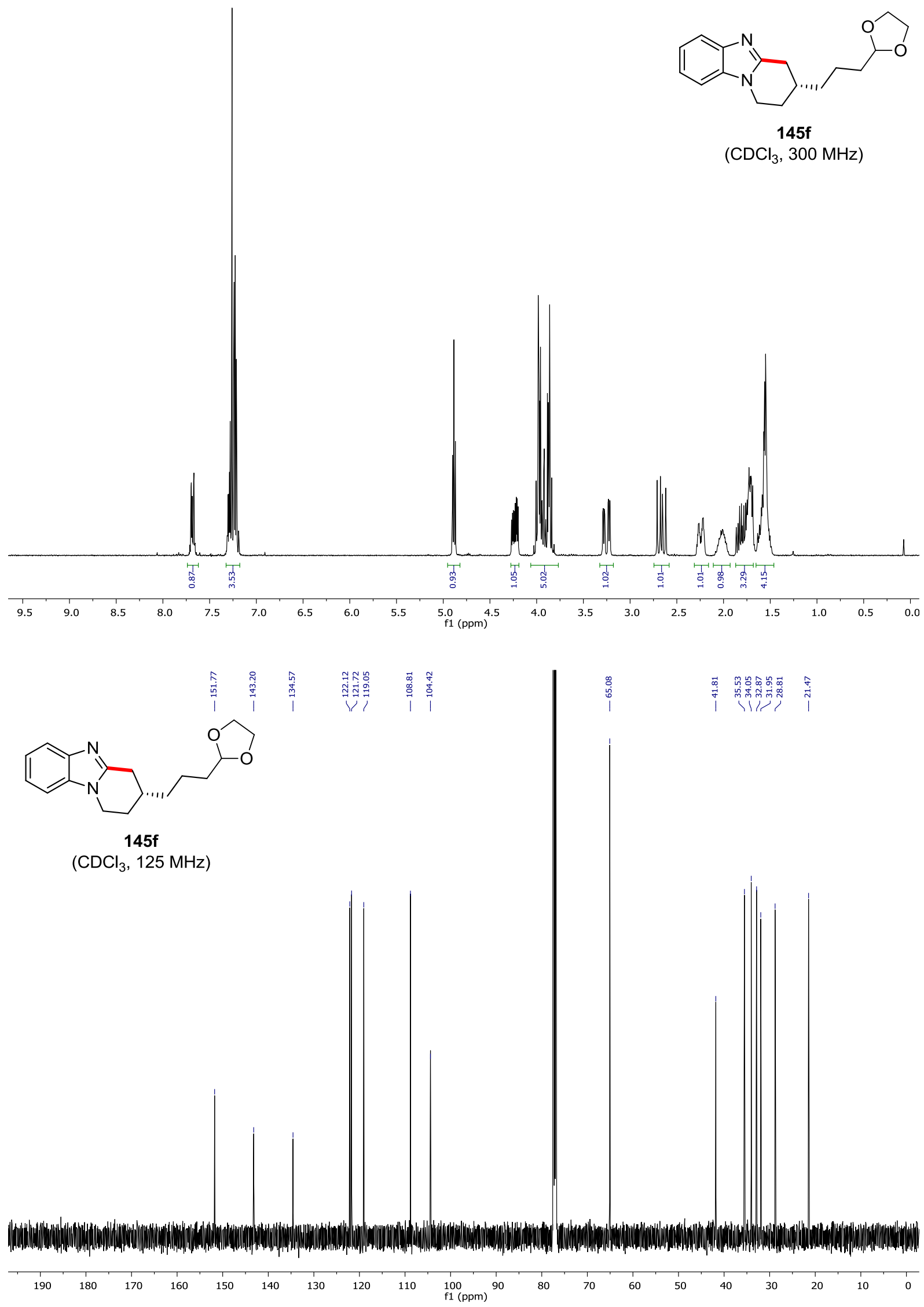


\section{Chiral HPLC of $\mathbf{1 4 5 f :}$}
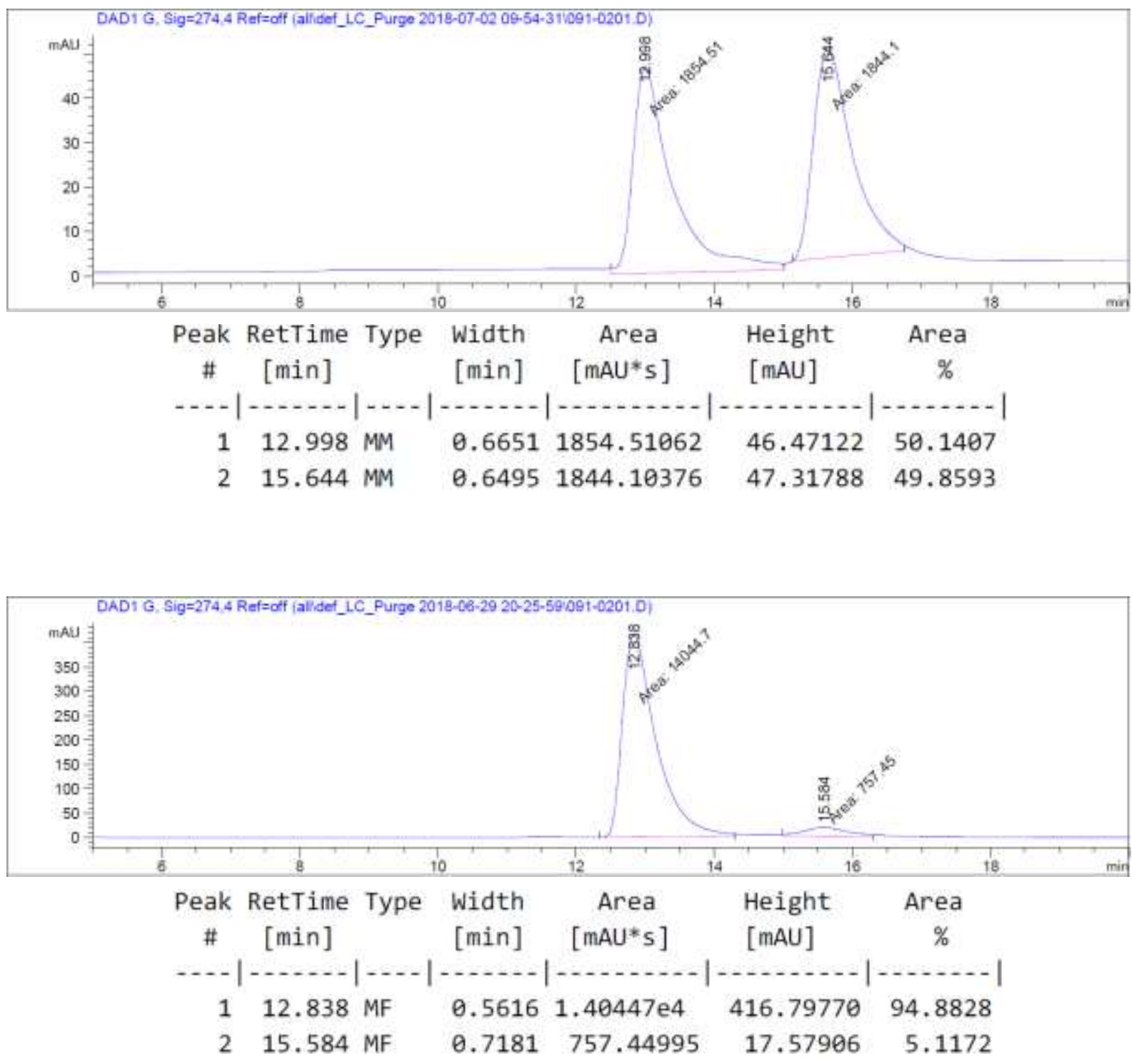

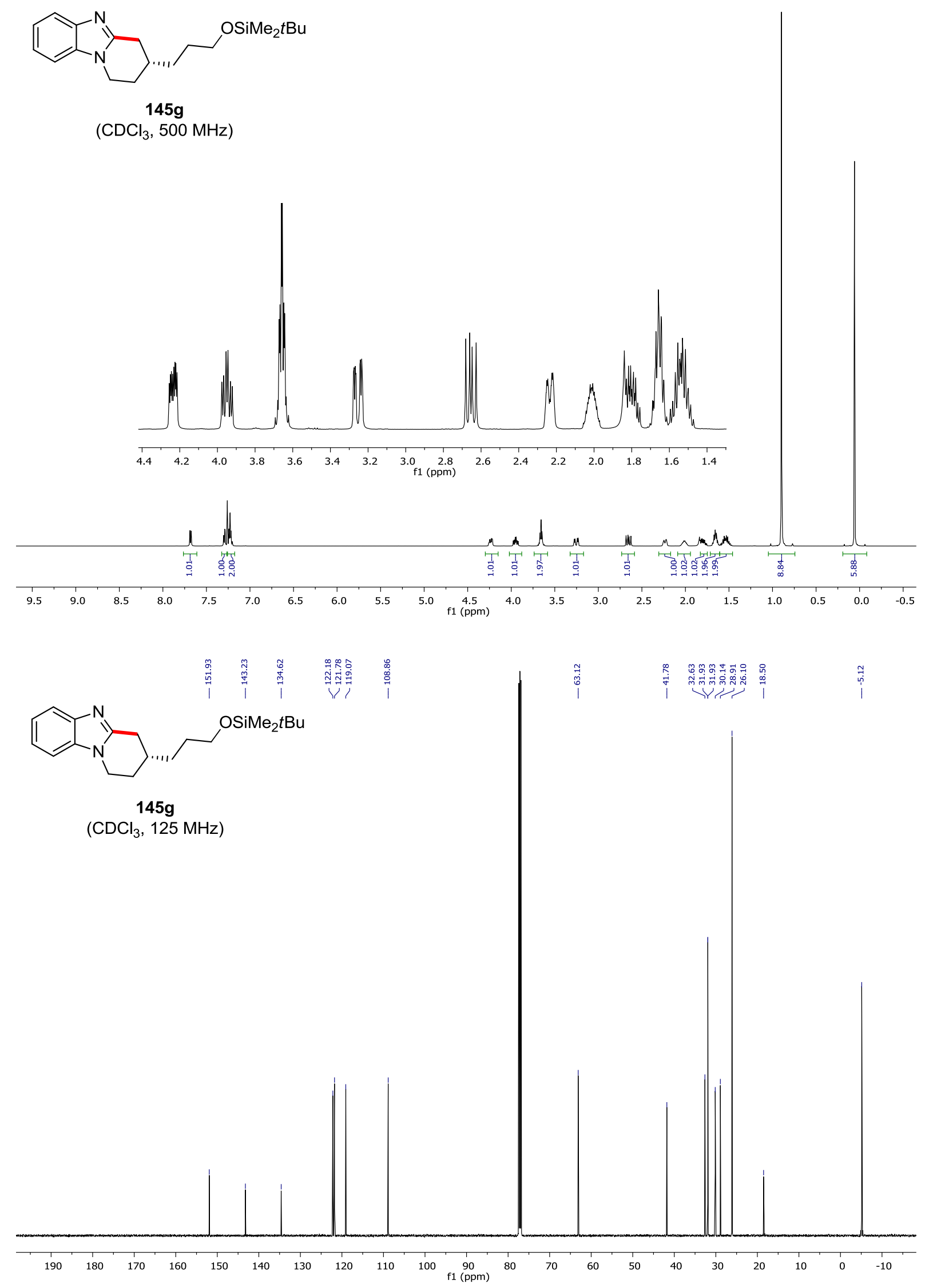


\section{Chiral HPLC of $\mathbf{1 4 5 g}$ :}
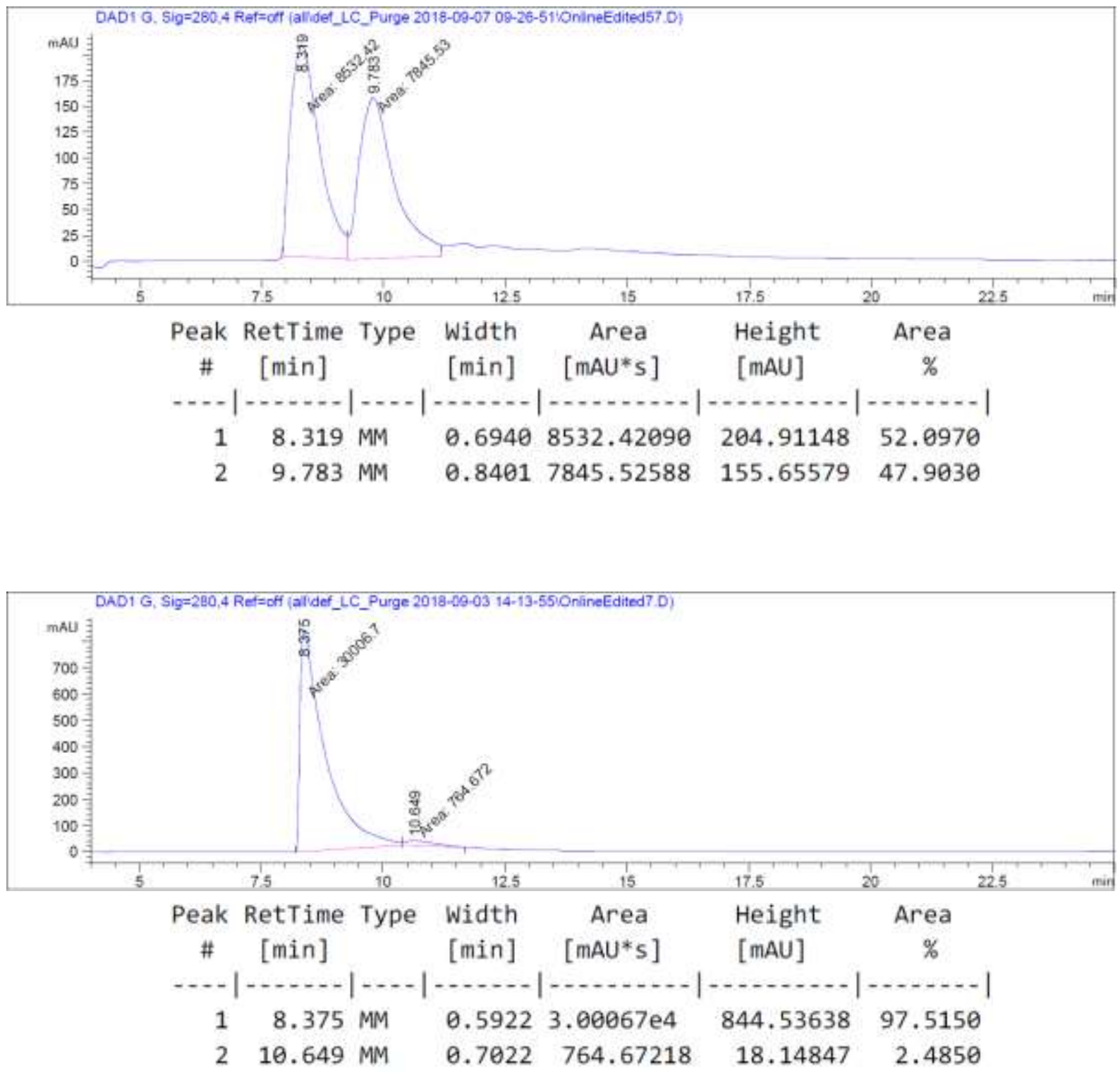

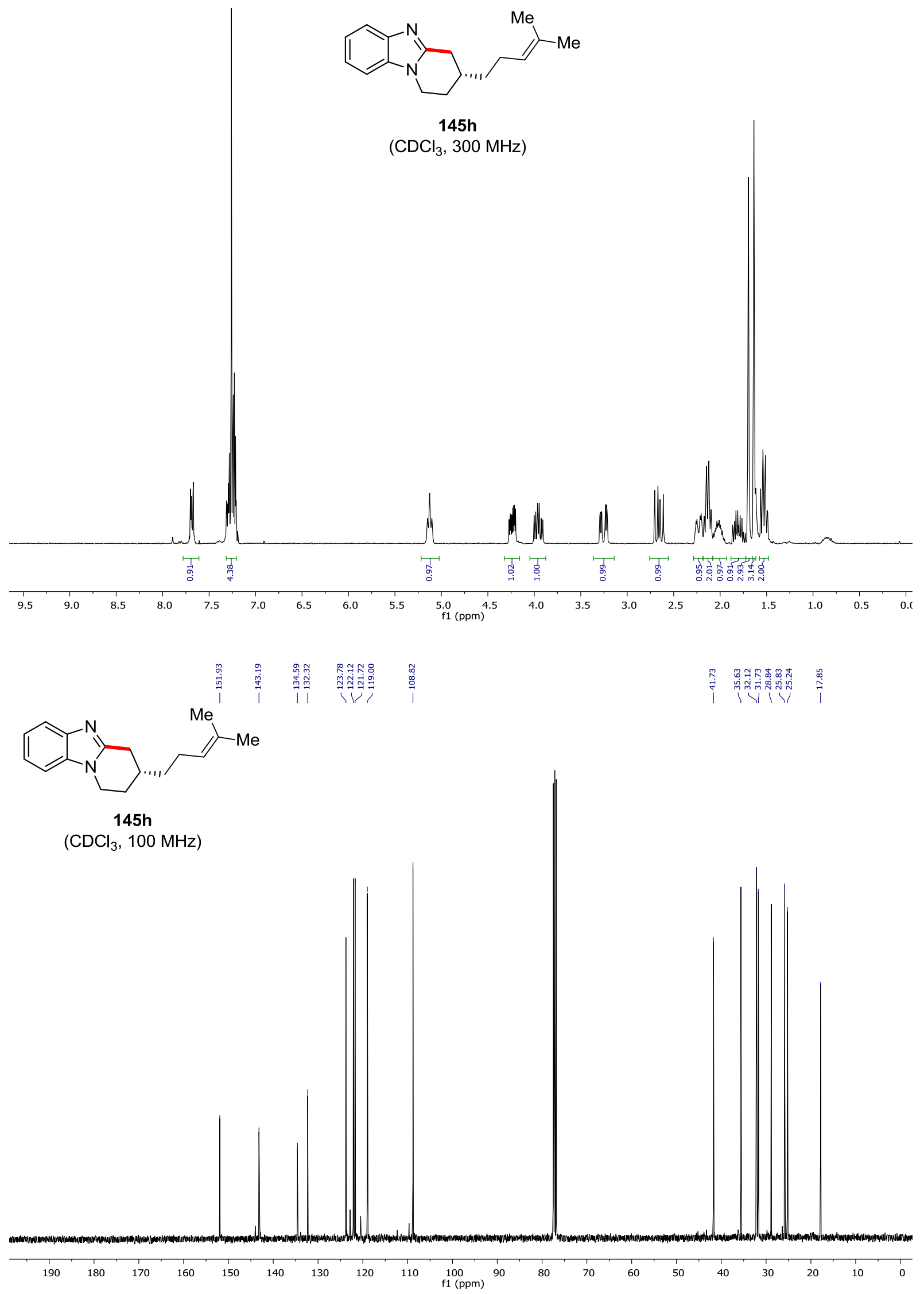


\section{Chiral HPLC of 145h:}
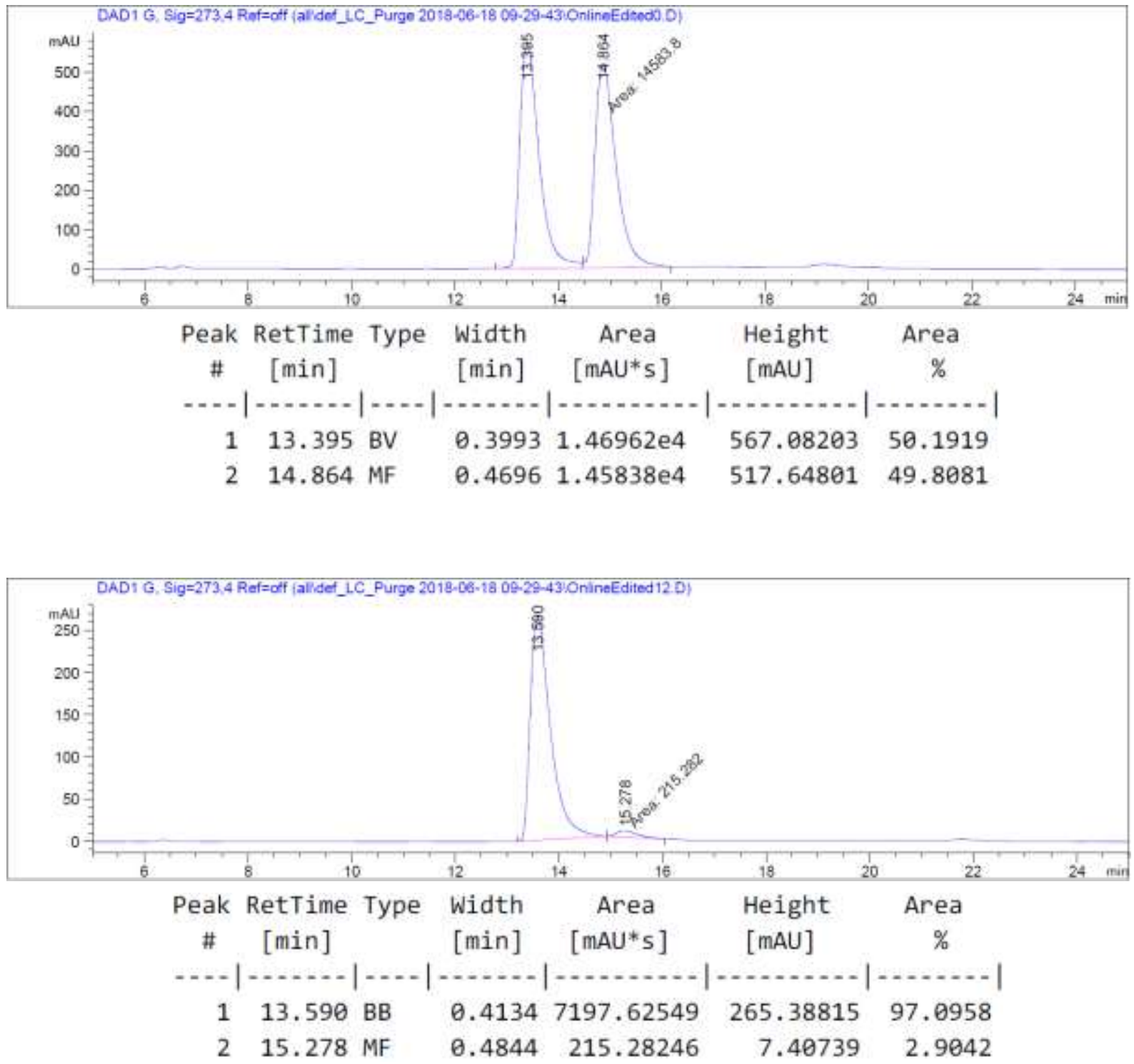

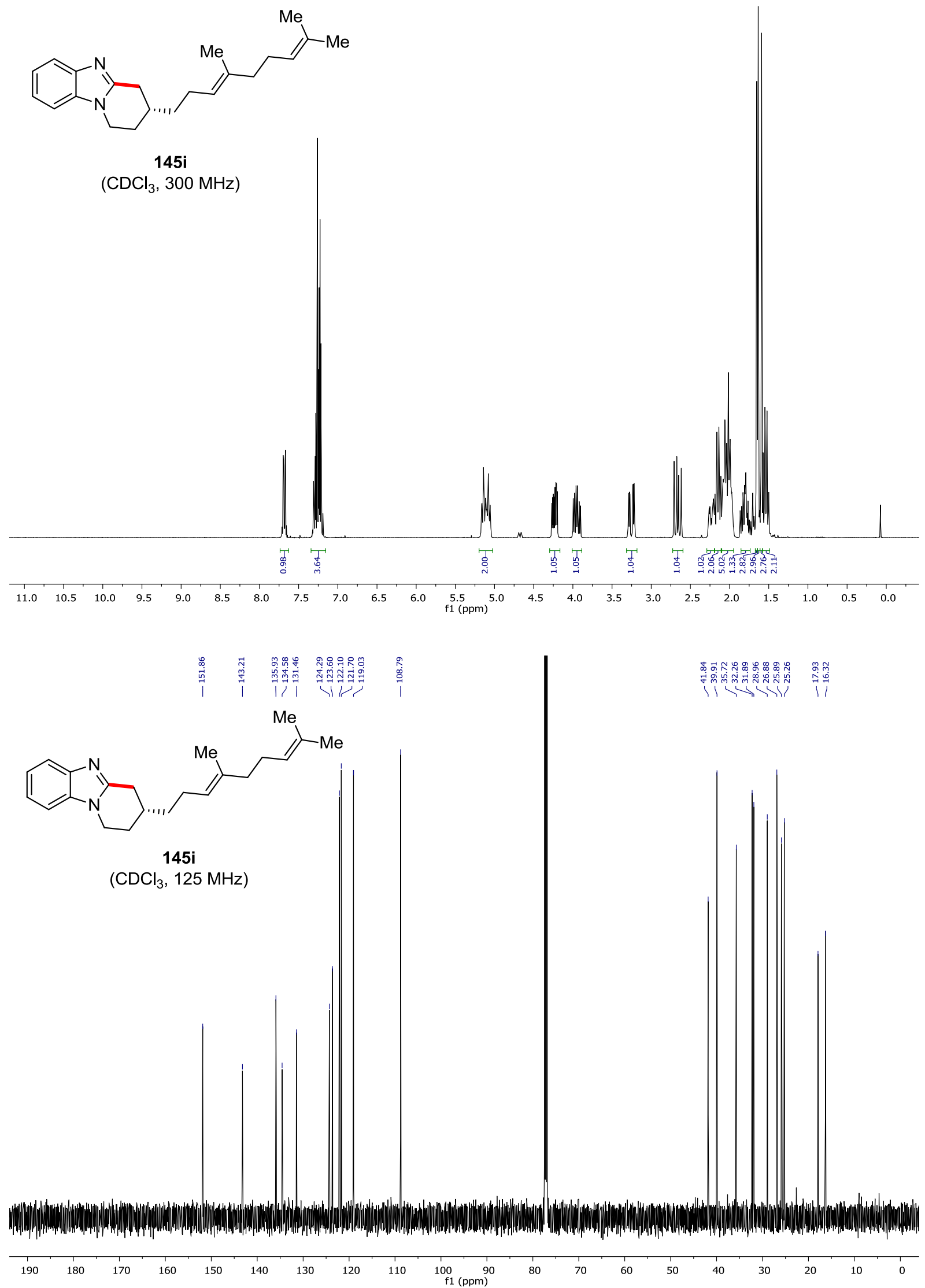


\section{Chiral HPLC of 145i:}
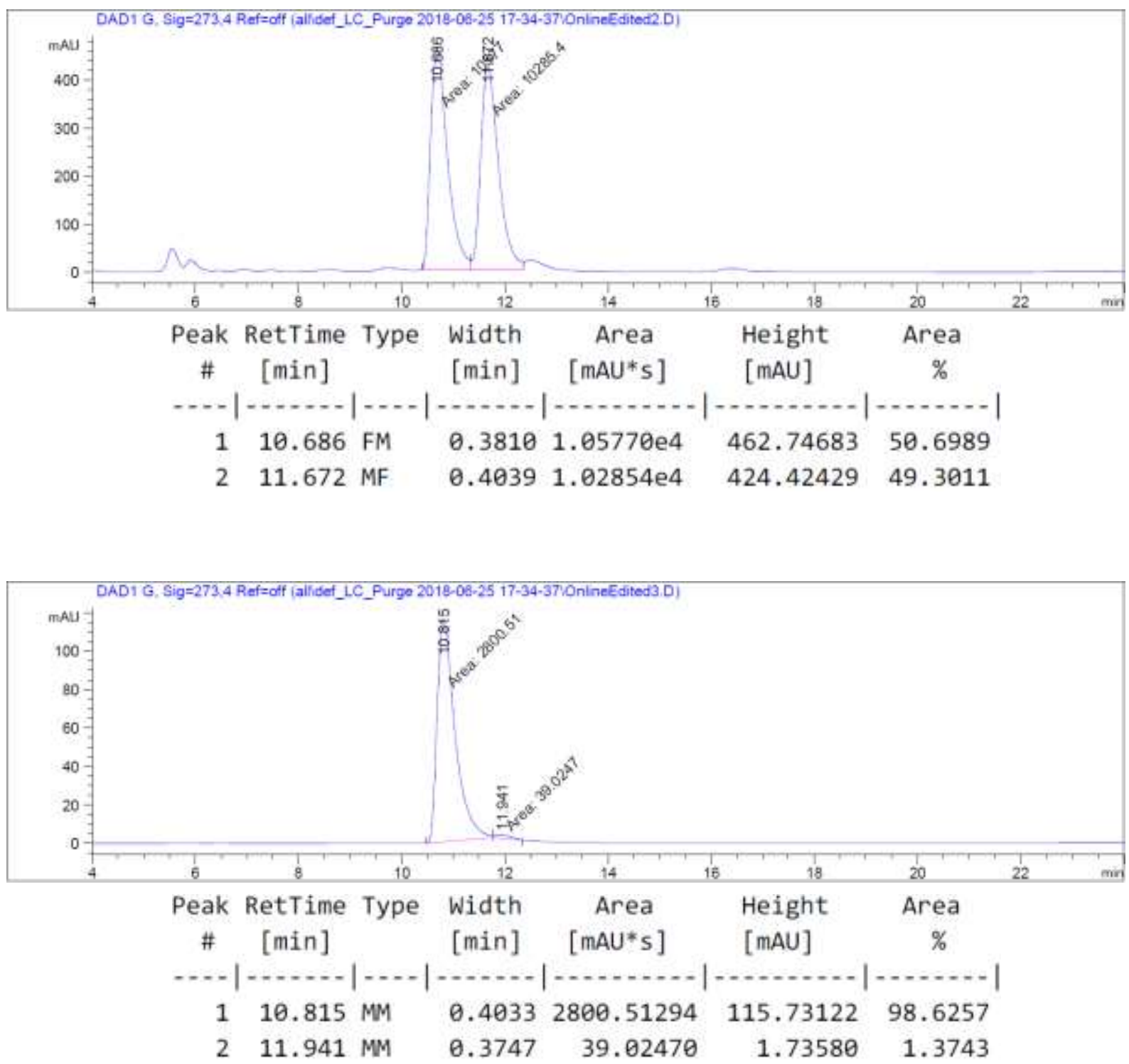

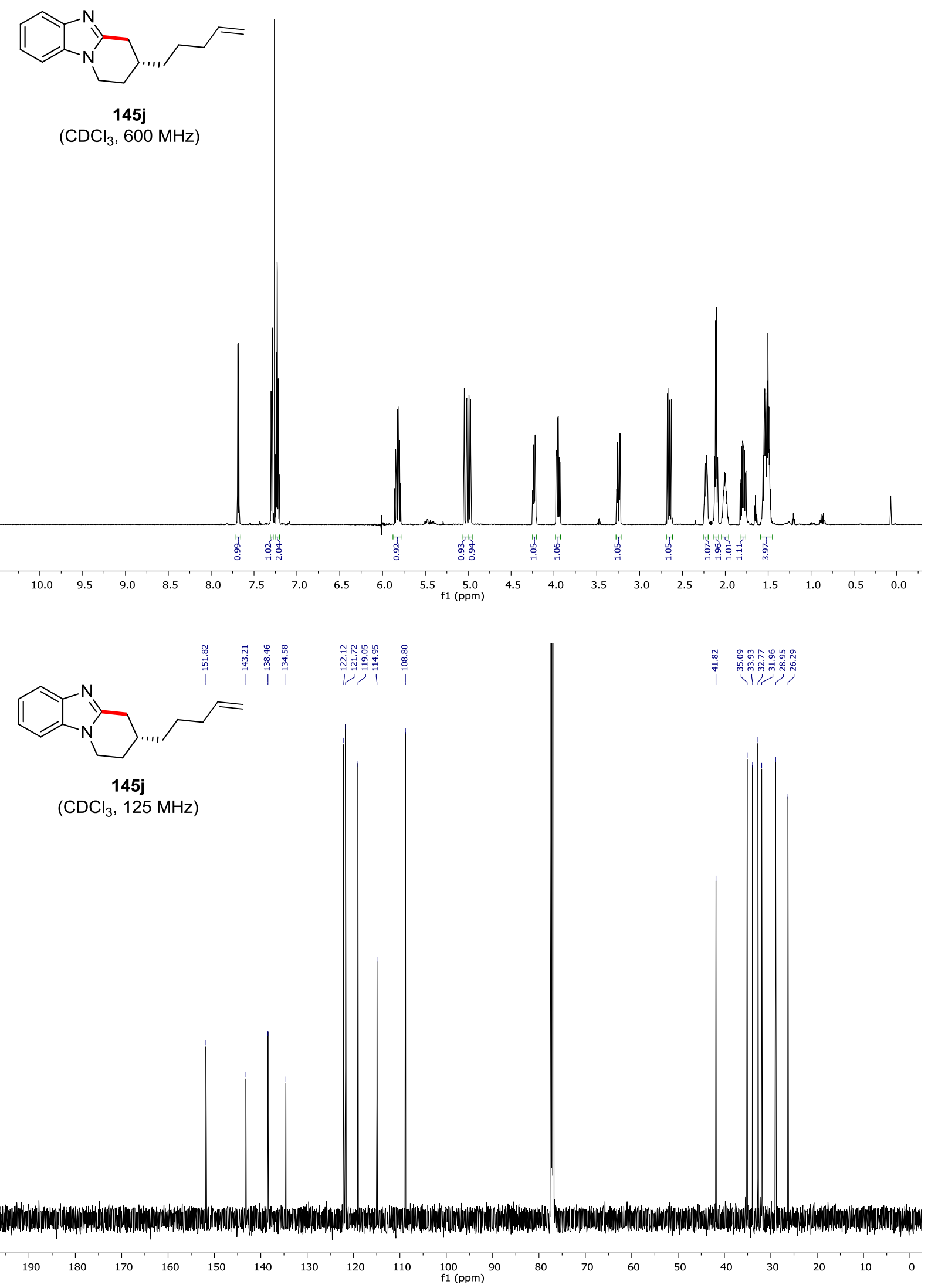


\section{Chiral HPLC of 145j:}
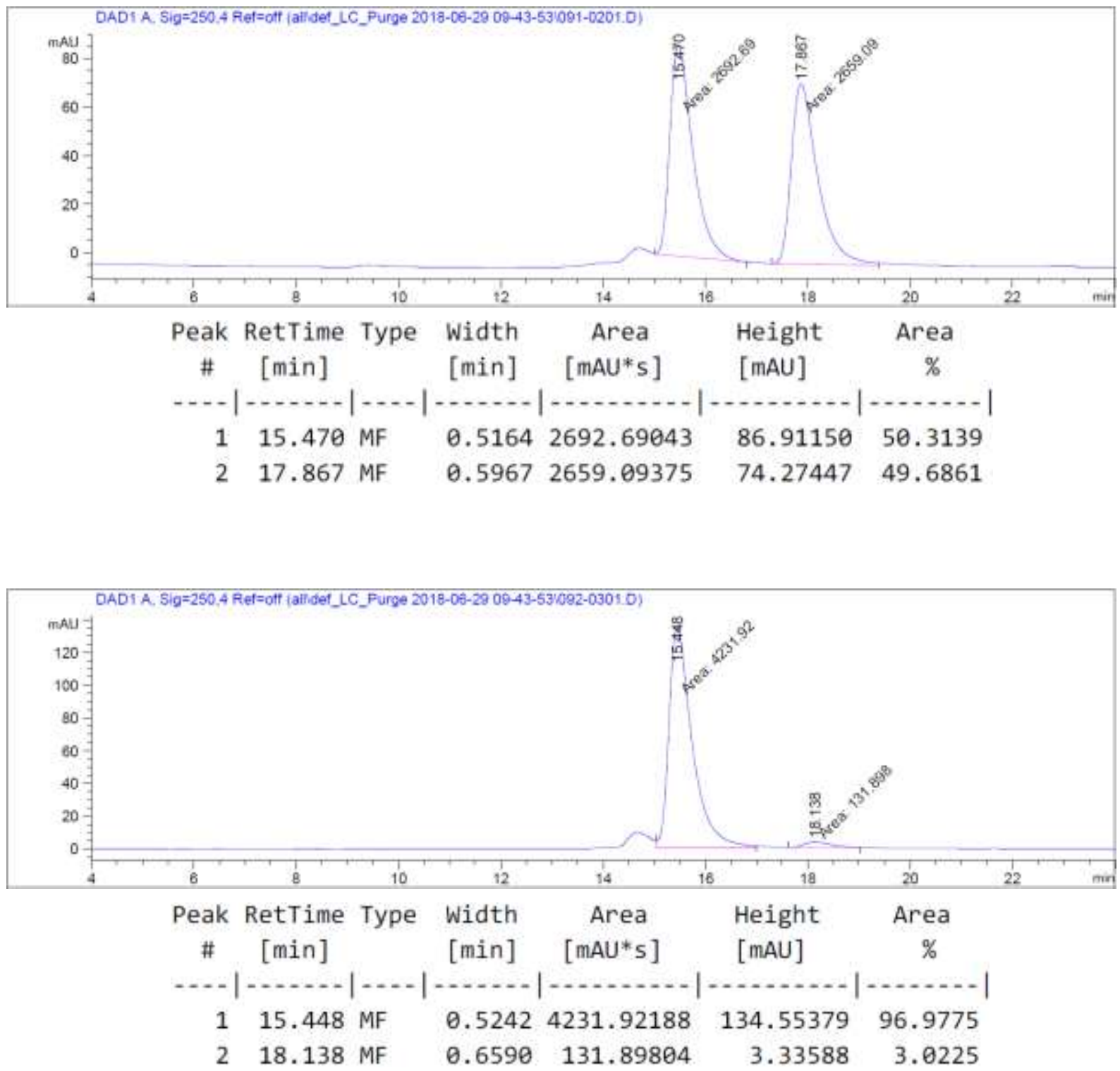


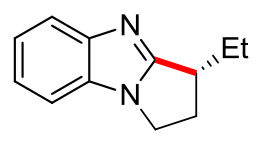

$145 q$

$\left(\mathrm{CDCl}_{3}, 300 \mathrm{MHz}\right)$
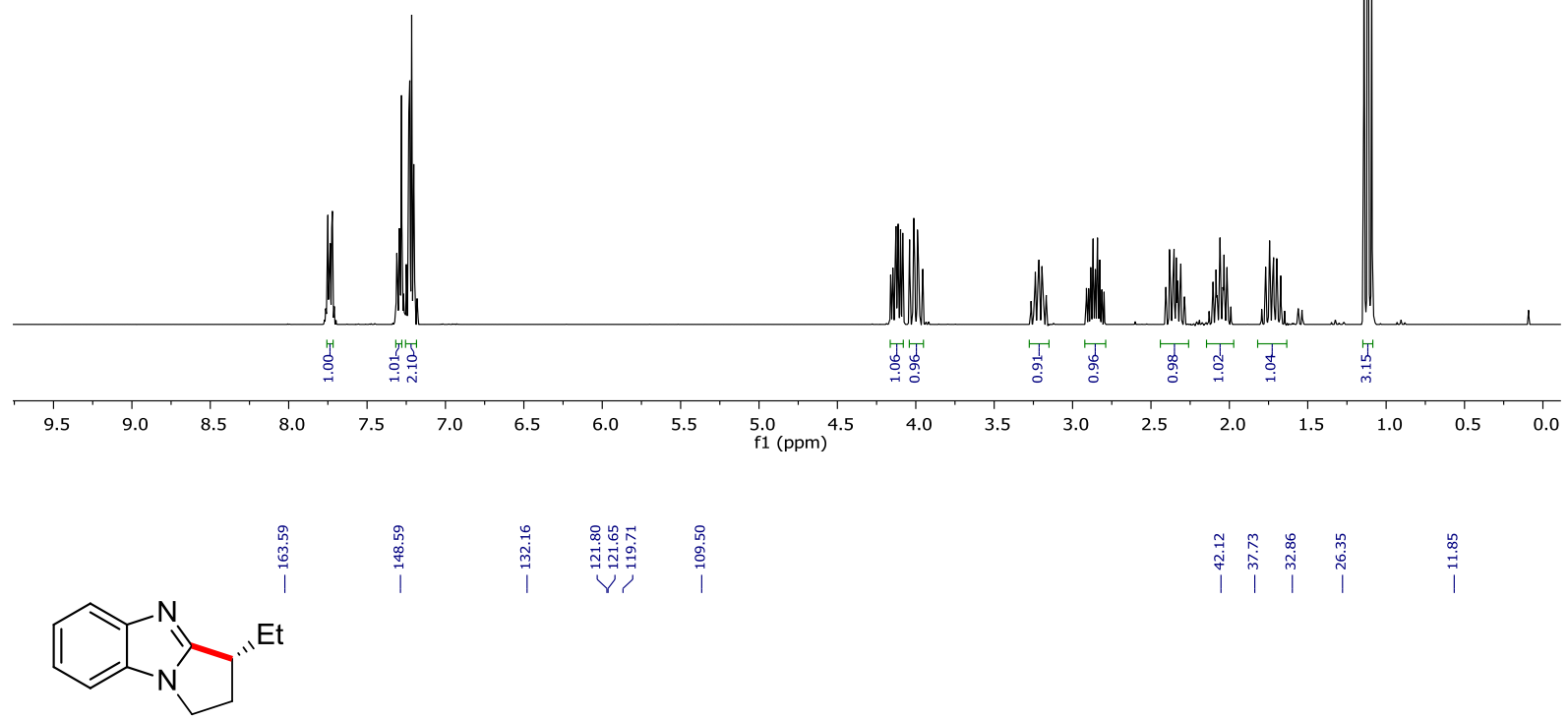

$145 q$

$\left(\mathrm{CDCl}_{3}, 125 \mathrm{MHz}\right)$

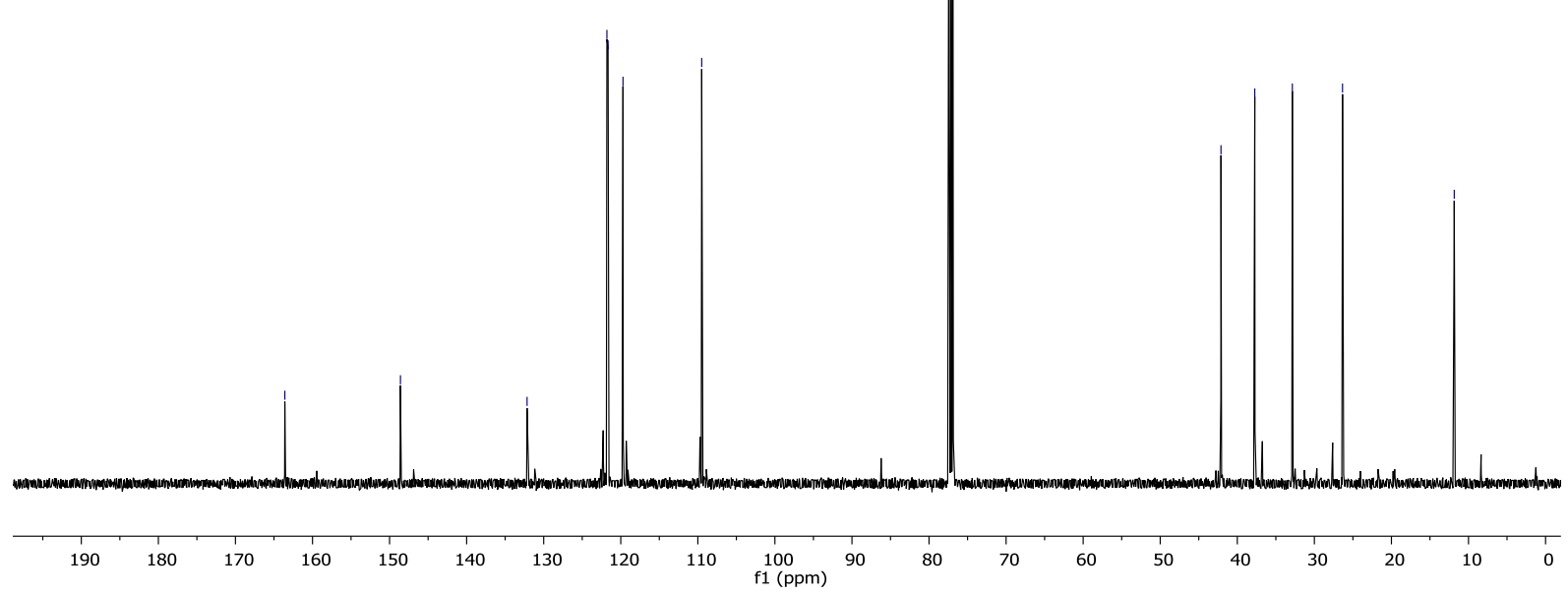




\section{Chiral HPLC of 145q:}
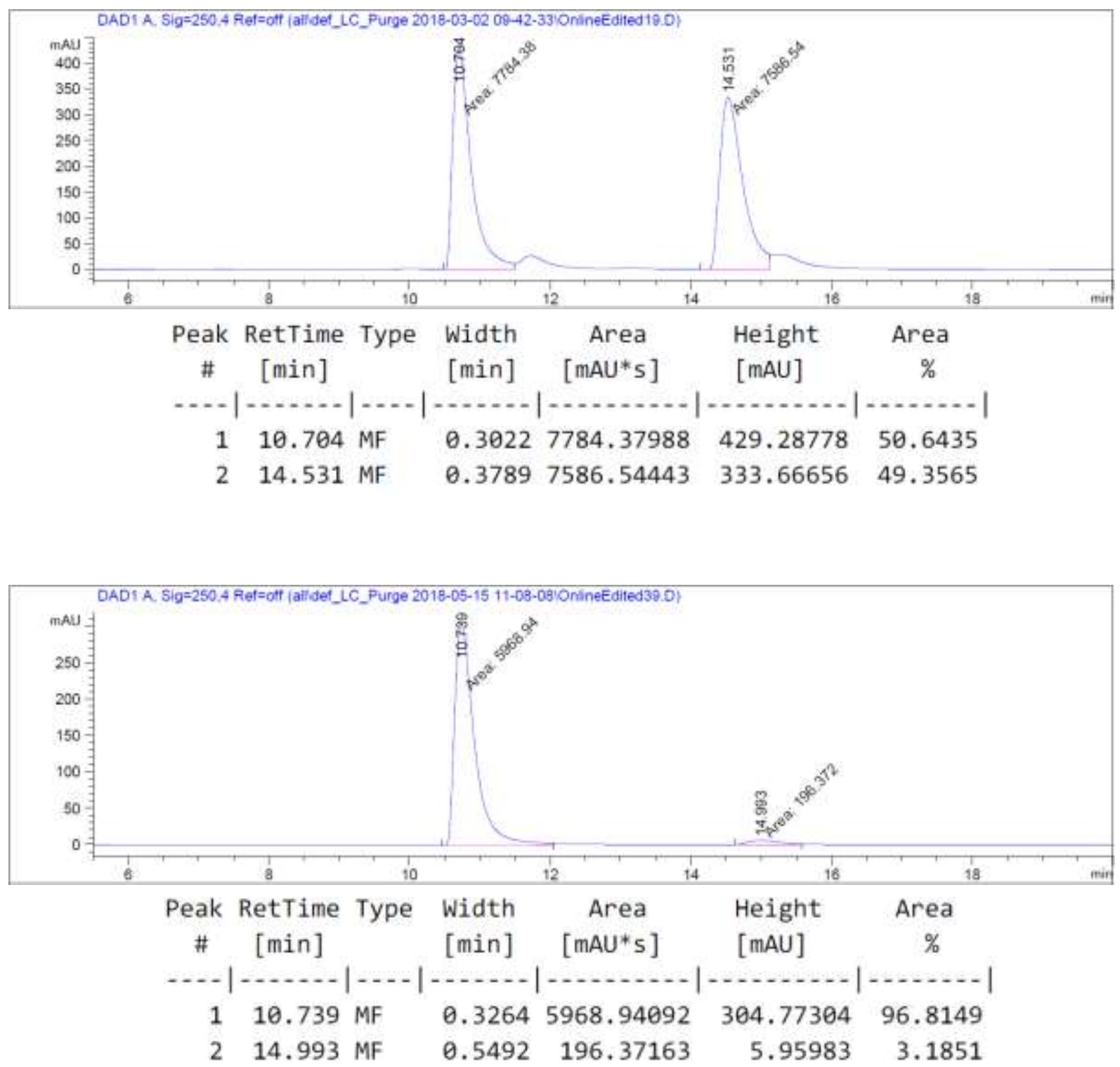


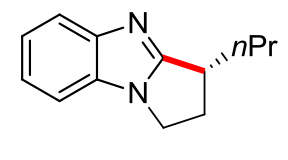

$145 r$

$\left(\mathrm{CDCl}_{3}, 300 \mathrm{MHz}\right)$
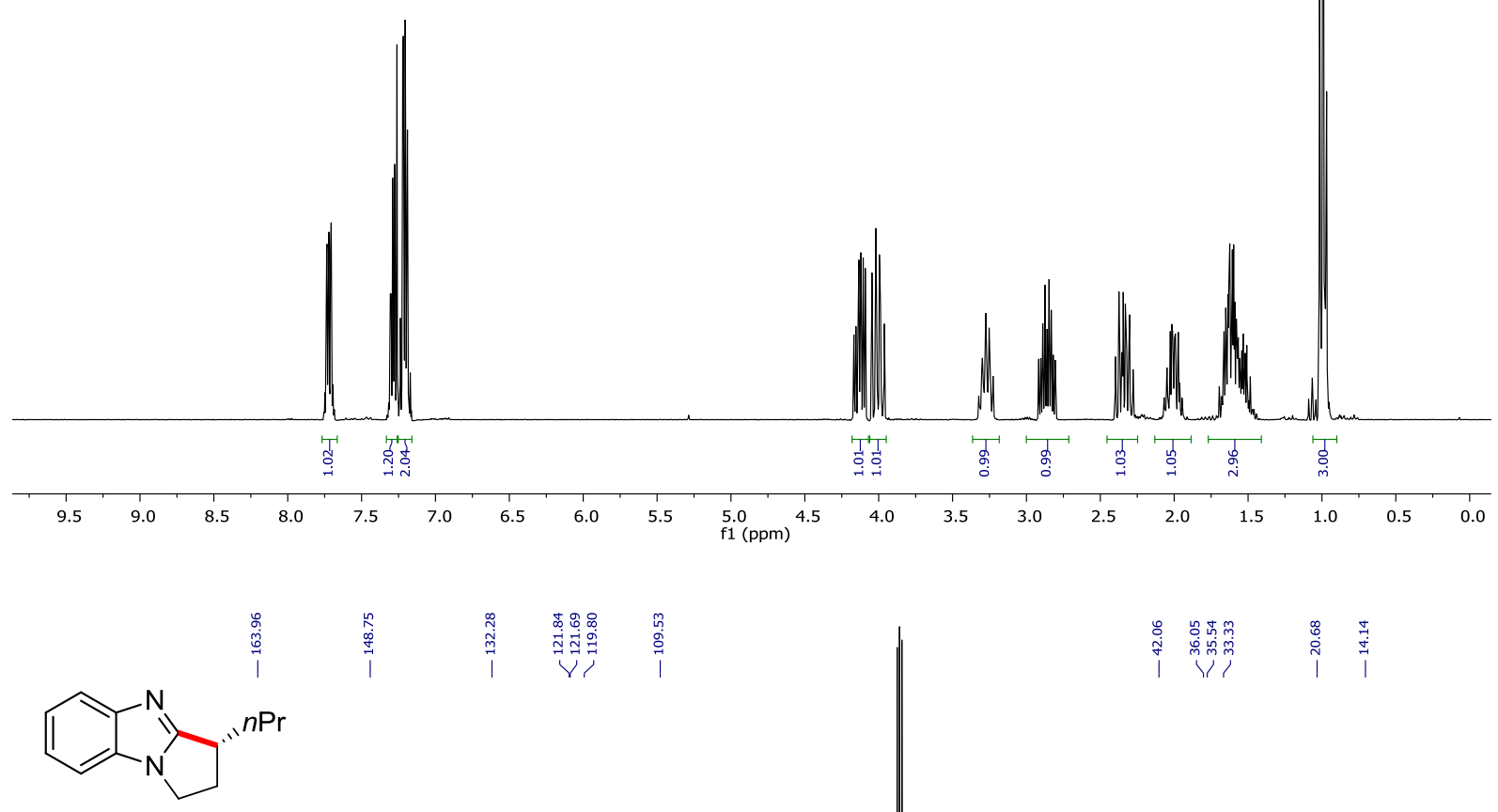

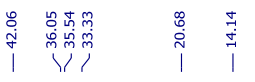
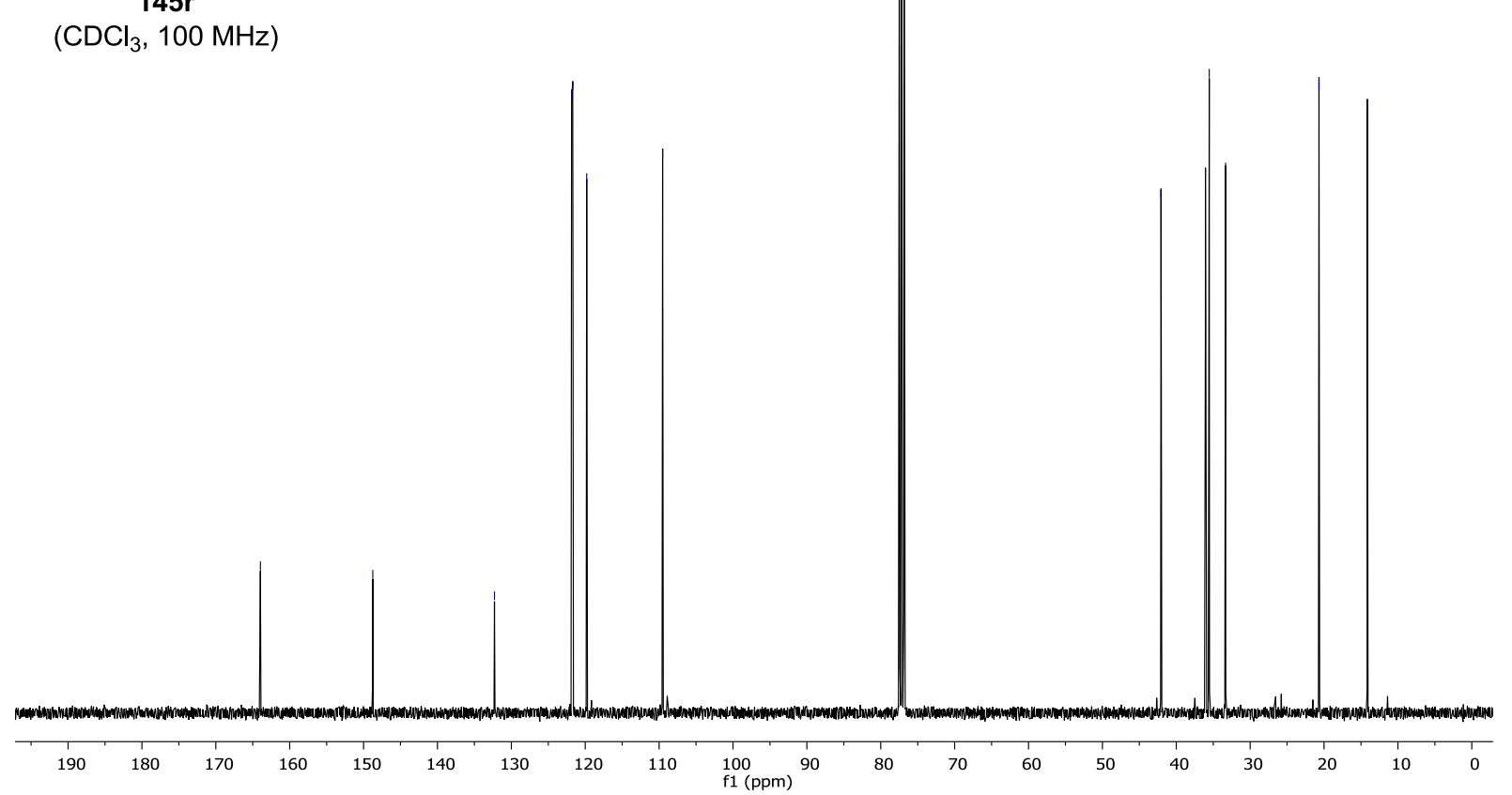


\section{Chiral HPLC of $145 r$ :}
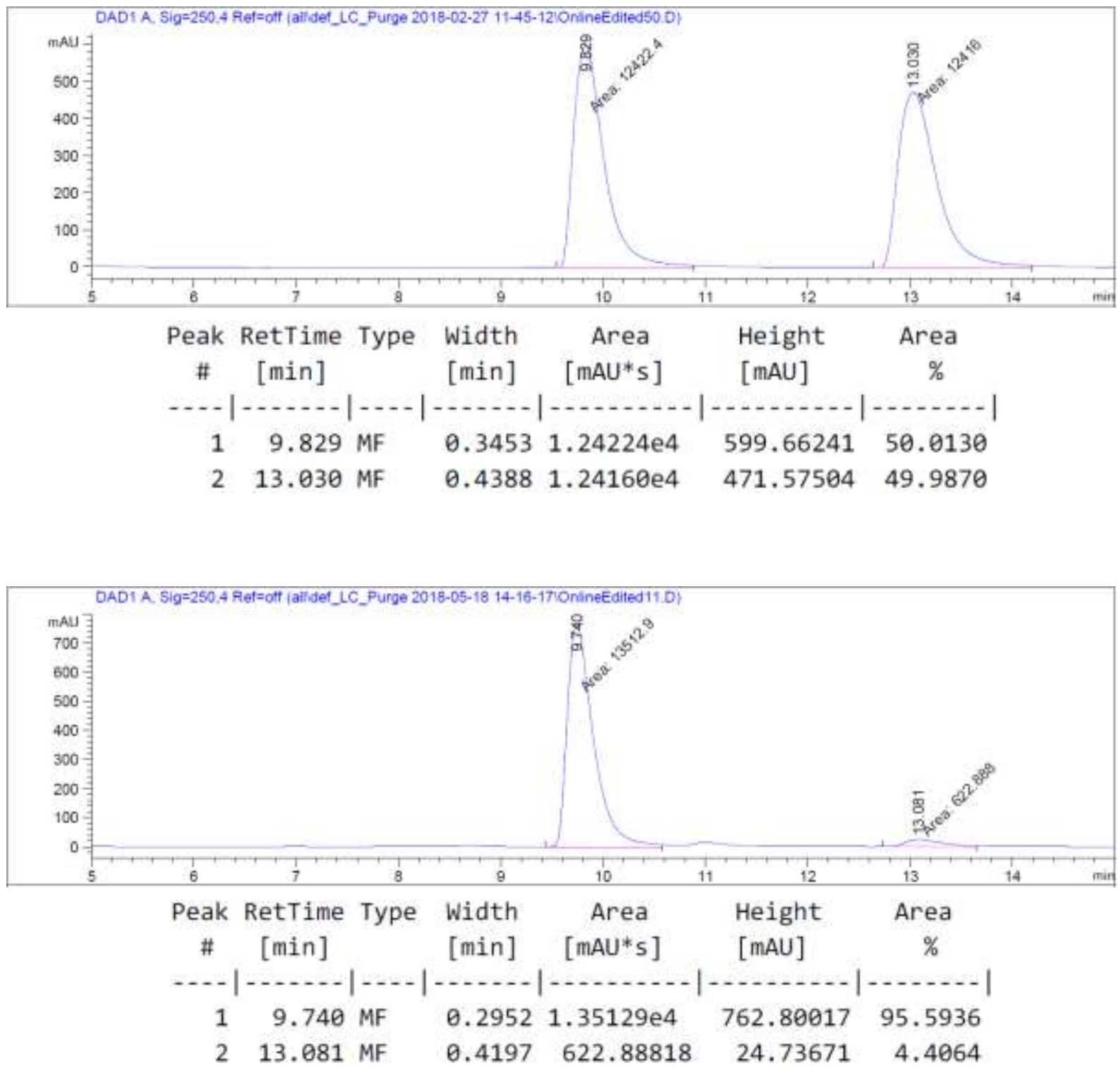

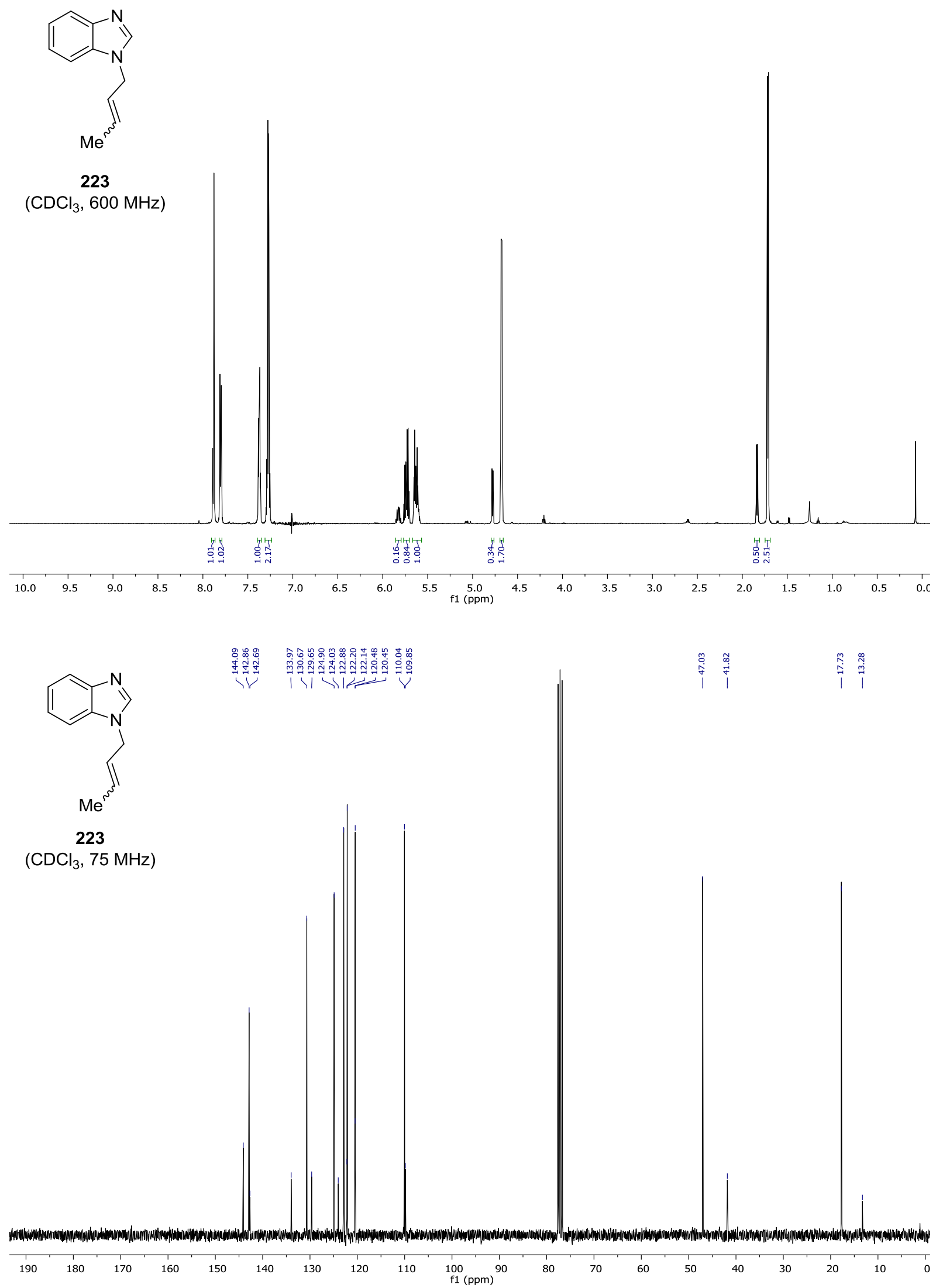


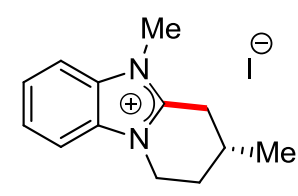

$$
\begin{gathered}
230 \\
\left(\mathrm{CDCl}_{3}, 300 \mathrm{MHz}\right)
\end{gathered}
$$
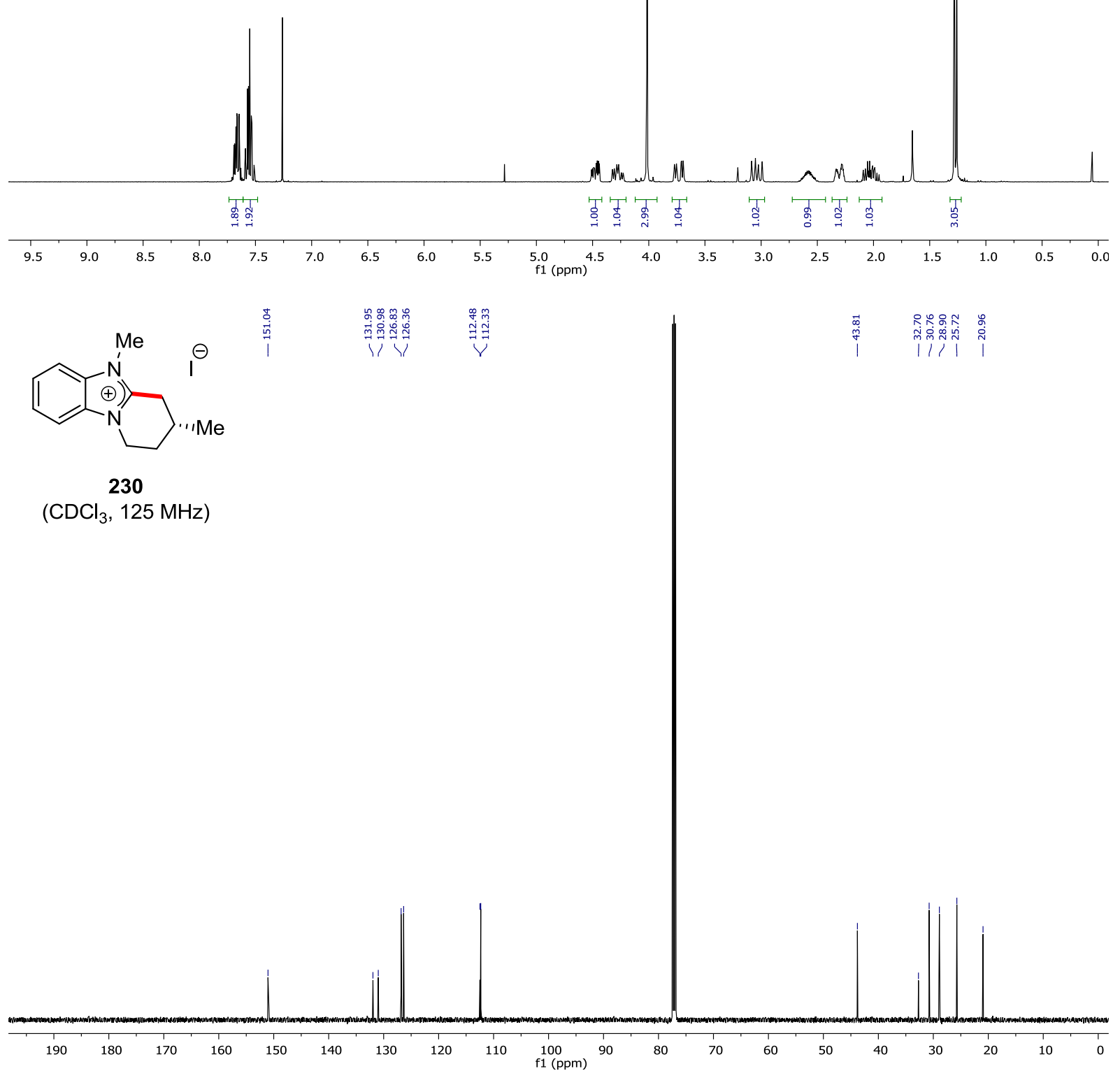

520 


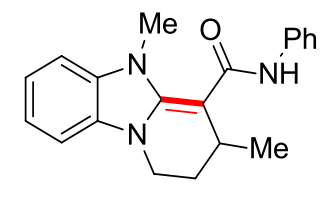

231

$\left(\mathrm{C}_{6} \mathrm{D}_{6}, 300 \mathrm{MHz}\right)$
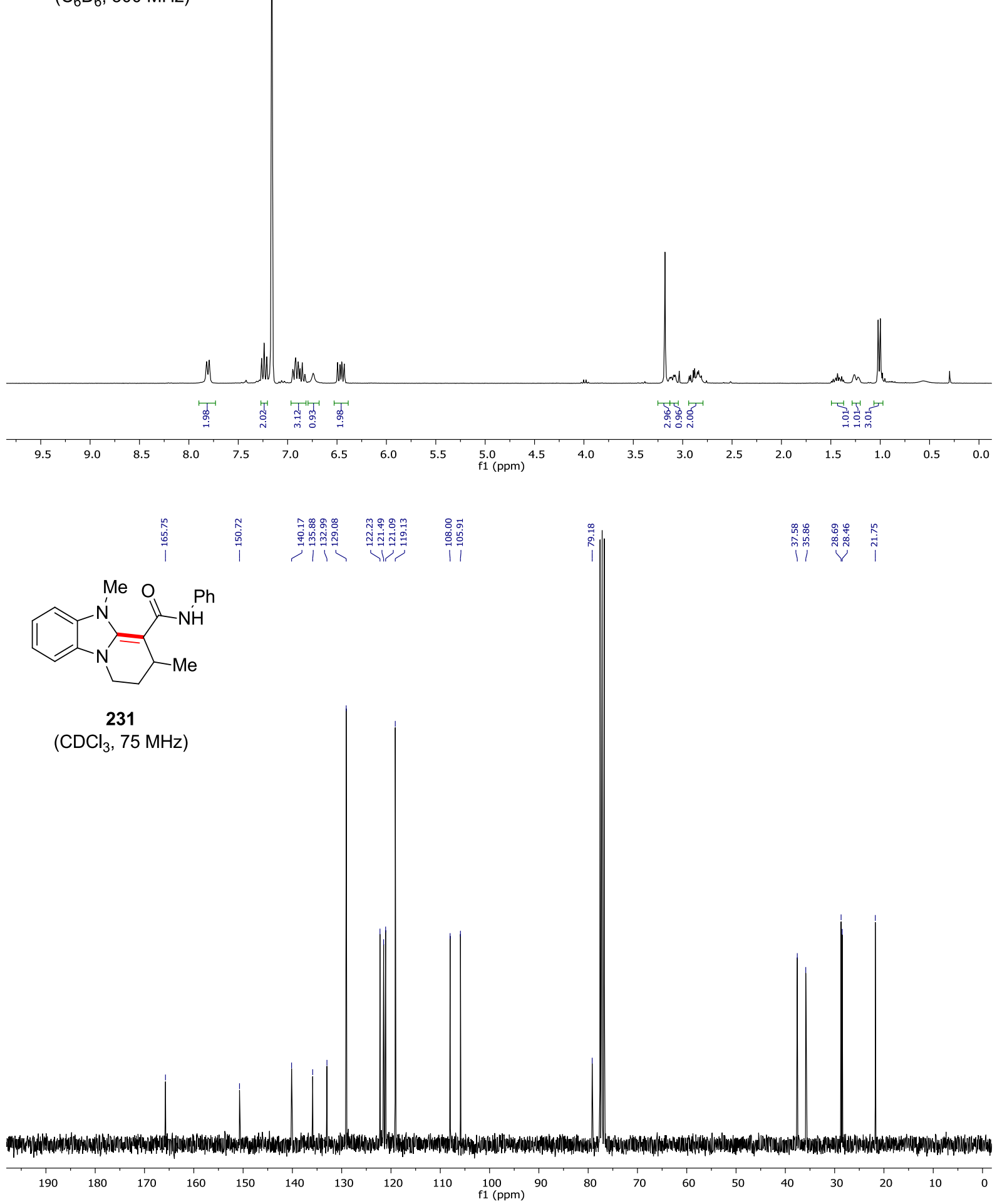


\section{Chiral HPLC of 231:}
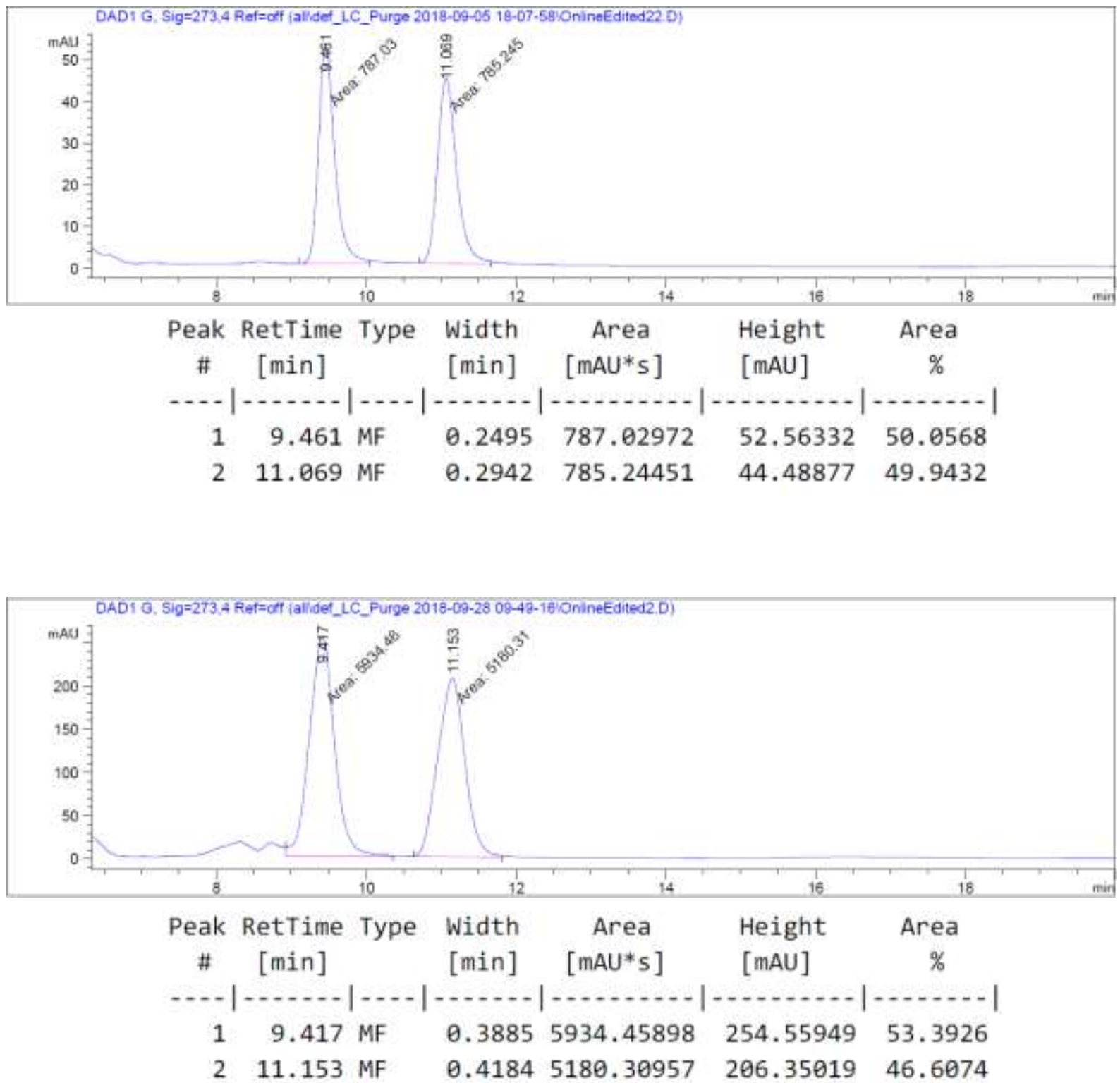


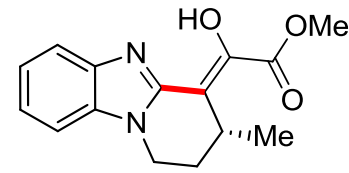

232

$\left(\mathrm{CDCl}_{3}, 500 \mathrm{MHz}\right)$
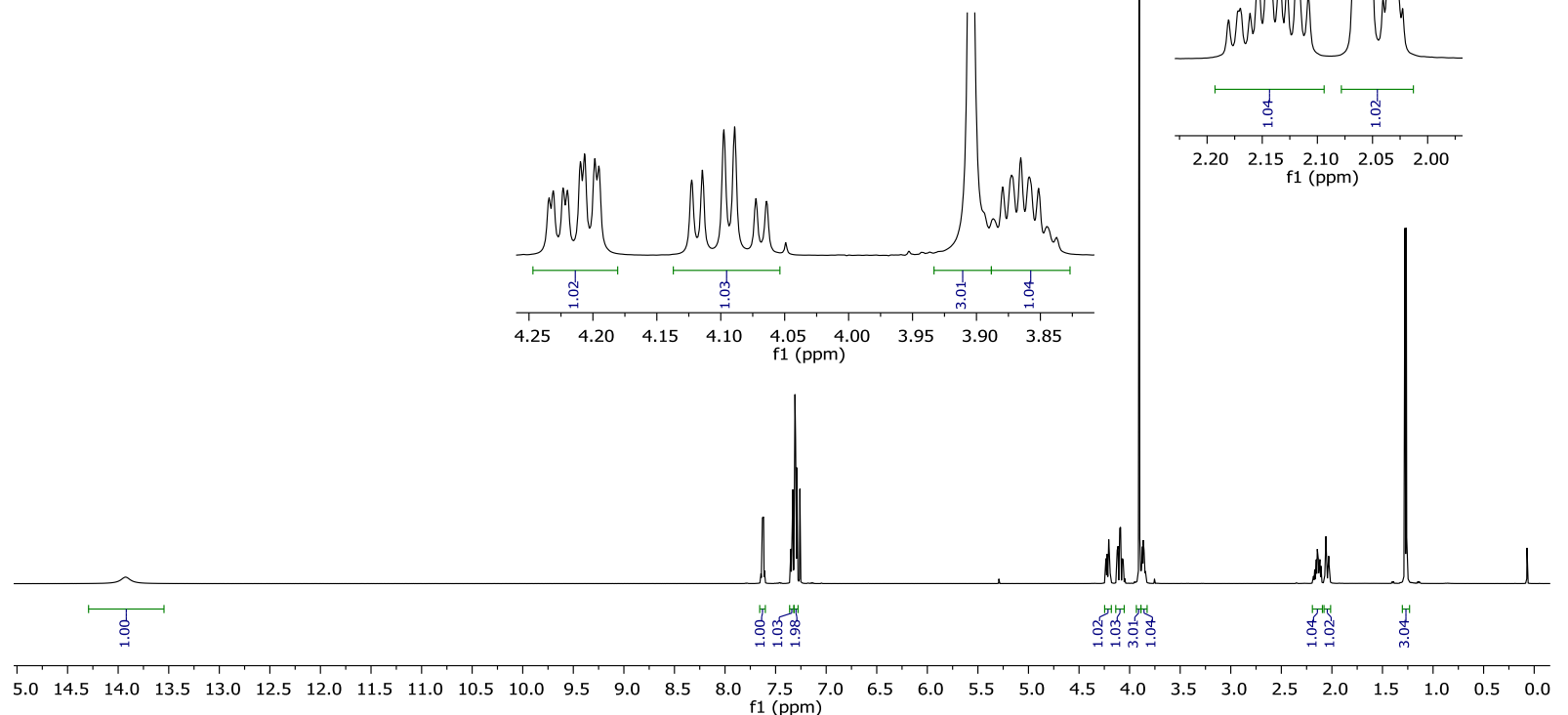

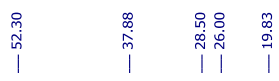

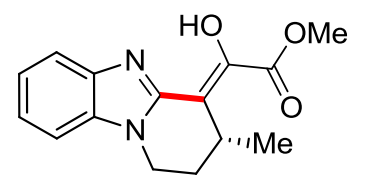

232

$\left(\mathrm{CDCl}_{3}, 125 \mathrm{MHz}\right)$
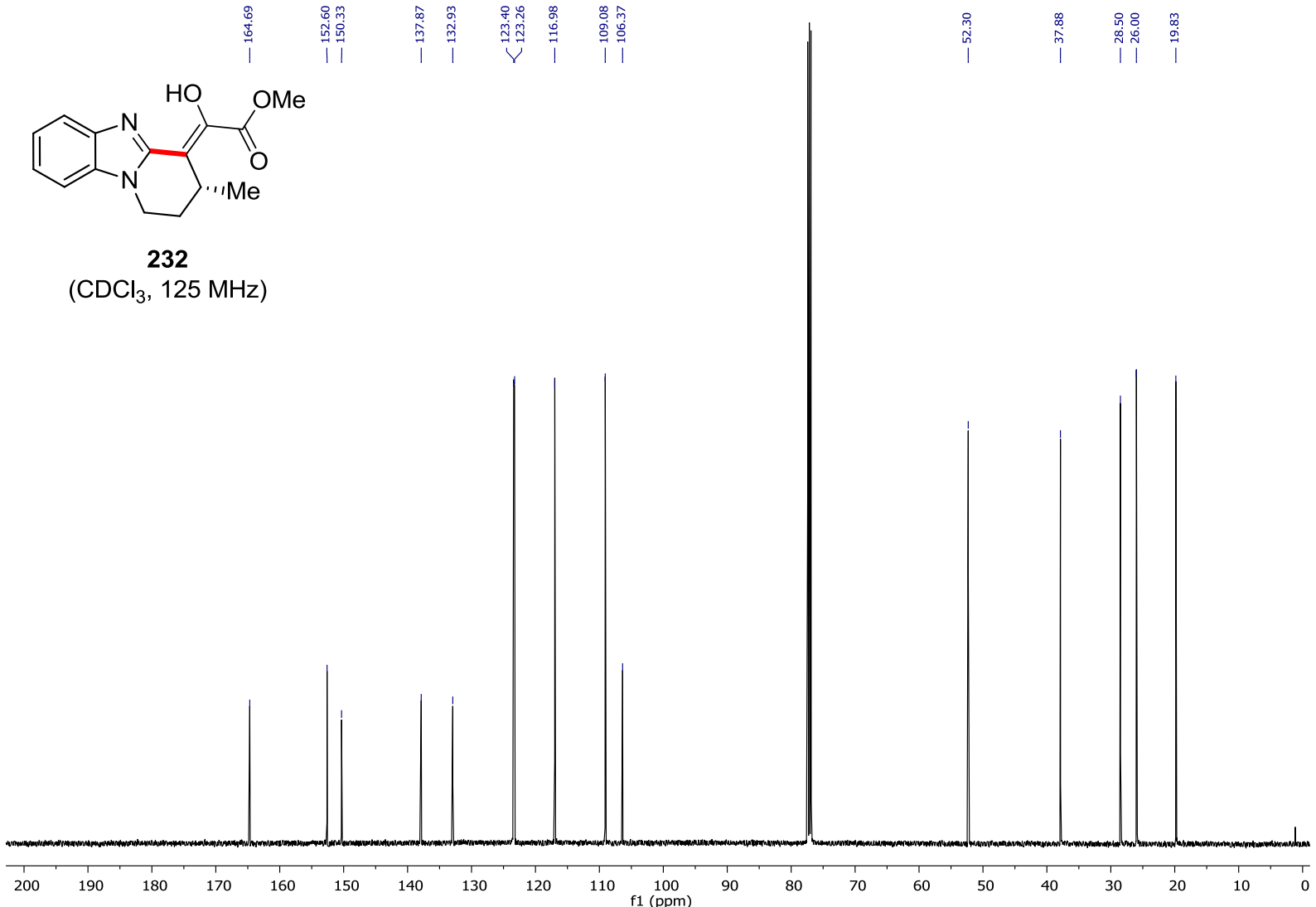


\section{Chiral HPLC of 232:}
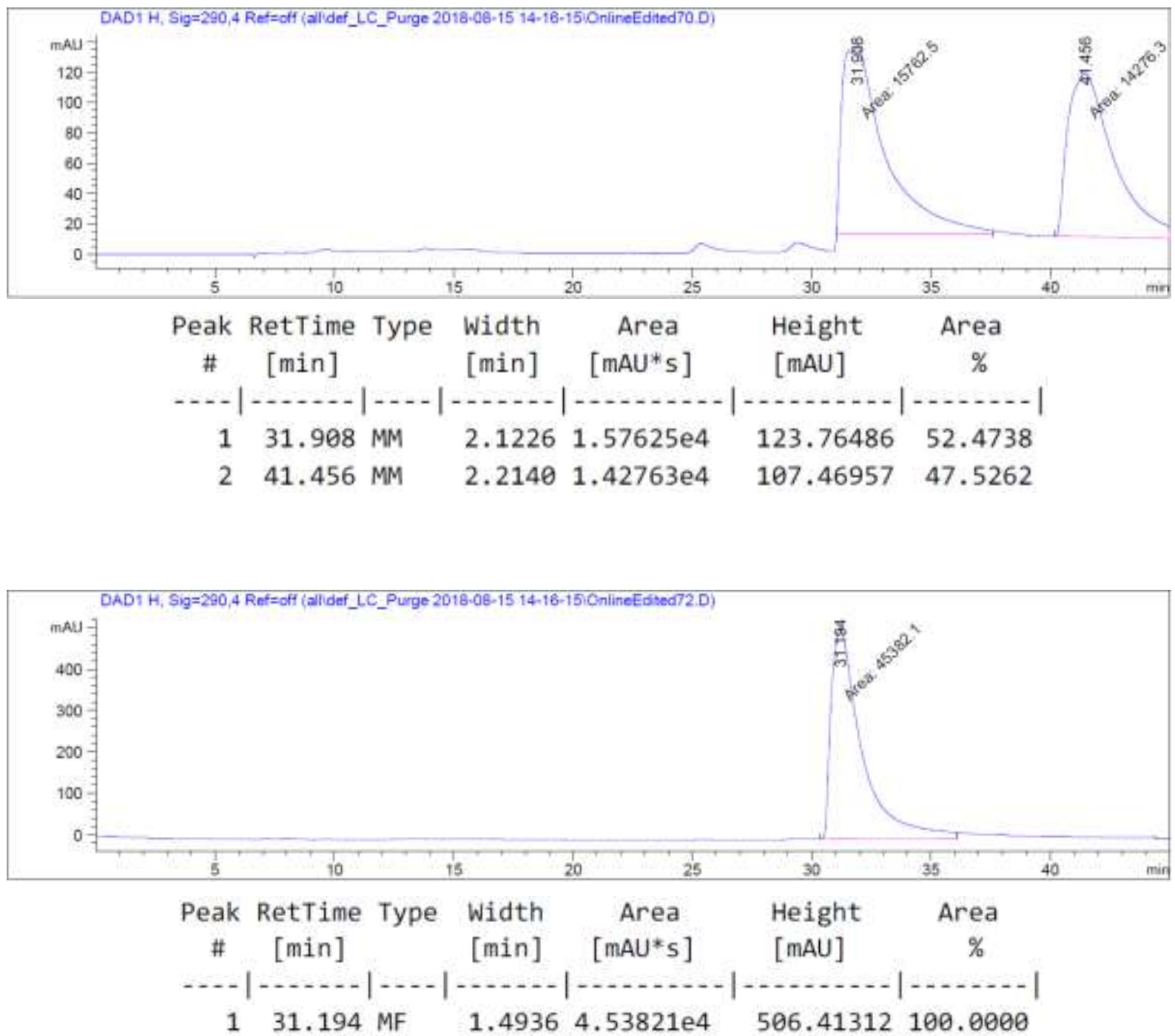